Supplement to the Thesaurus Syriacus of R. Payne Smith

Compiled by

Jesse Payne Margoliouth 



\section{Series Foreword}

This series provides reference works in Syriac studies from original books digitized at the ICOR library of The Catholic University of America under the supervision of Monica Blanchard, ICOR's librarian. The project was carried out by Beth Mardutho: The Syriac Institute and Brigham Young University. About 675 books were digitized, most of which will appear in this series.

Our aim is to present the volumes as they have been digitized, preserving images of the covers, front matter, and back matter (if any). Marks by patrons, which may shed some light on the history of the library and its users, have been retained. In some cases, even inserts have been digitized and appear here in the location where they were found.

The books digitized by Brigham Young University are in color, even when the original text is not. These have been produced here in grayscale for economic reasons. The grayscale images retain original colors in the form of gray shades. The books digitized by Beth Mardutho and black on white.

We are grateful to the head librarian at CUA, Adele R. Chwalek, who was kind enough to permit this project. "We are custodians, not owners of this collection," she generously said at a small gathering that celebrated the completion of the project. We are also grateful to Sidney Griffith who supported the project. 



$$
\begin{array}{r}
275.85 \\
M \quad 329
\end{array}
$$

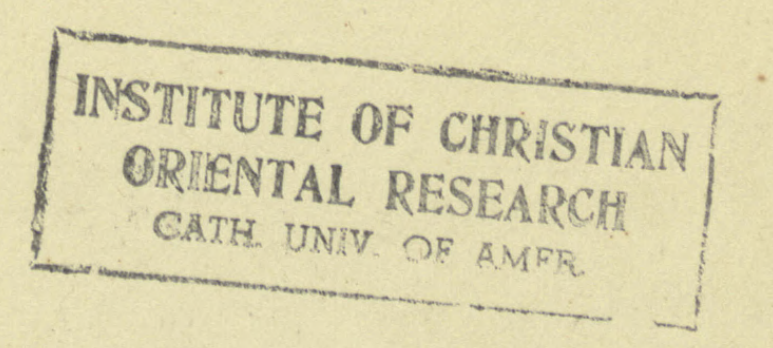

WOF' HYNRRNAT, D. N., Chalhotic Unizersity of 4 merion, DI I I N I T O N D. C. 


\title{
15720
}

\author{
OXFORD UNIVERSITY PRESS \\ London Edinburgh Glasgow Copenhagen \\ New York Toronto Melbourne Capetown \\ Bombay Calcutta Madras Shanghai \\ HUMPHREY MILFORD \\ Publisher to the University
}




\section{SUPPLEMENT TO THE THESAURUS SYRIACUS \\ OF R. PAYNE SMITH, S.T.P. \\ Formerly Oriental Sub-Librarian of the Bodleian Library, then Regius Professor of Divinity later Dean of Canterbury

\author{
COLLECTED AND ARRANGED BY \\ HIS DAUGHTER \\ J. P. MARGOLIOUTH
}

INSTITUTE OF CHRISTIAN
ORIENTAL RESEARGH
CATH. UNIV. OF AMFE.

O X F O R D

AT THE CLARENDON PRESS

1927 

sires sarel retareflanas :

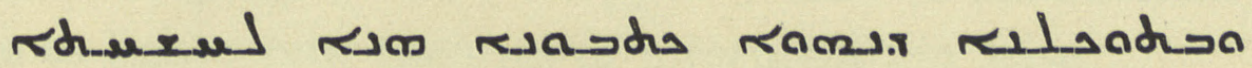

$\therefore$ Resian Rirl Rrä̈a. 



\section{P R E F A C E}

WEITHER a thesaurus nor its supplement can be final, even when of a dead language; MSS re-appear; new or insufficiently treated words come to light; new theories of interpretation arise.

Except in a few cases I have omitted (a) notes on the native Lexica, as students now have M. Rubens Duval's edition of Bar Bahlul with his full and scholarly notes; (b) dialects, such as Palestinian Syriac and Modern Syriac, on which full information is now accessible.

The following book is compiled (a) from collections, left by my father for this purpose, of words from books published too late for use in the earlier parts of his work ; $(b)$ from corrections and lexicographical theories in magazines, such as the Journal Asiatique and the Zeitschrift der Deutschen Morgenländischen Gesellschaft ; $(c)$ from glossaries and separate words noted by many editors of Syriac MSS, e. g. Dr. Wright's glossary to Kalilah-w-Dimnah ; Dr. G. Hoffmann's invaluable geographical study on Acts of the Martyrs; the notes of M. Chabot in his Recueil de Synodes Nestoriens; the full and valuable footnotes to Sir E. A. Wallis Budge's Historia Monastica ; $(d)$ from notes kindly given to me by various scholars, such as those of Bishop Maclean on liturgical and ritual matters : and from references supplied by Messrs. E. W. Brooks, F. Nau, R. Gottheil, and A. Mingana. Of especial value are the references from M. Rubens Duval's copy of the Thesaurus, acquired after his lamented death by M. l'Abbé Hyvernat, who most kindly sent this copy for my use before adding it to his library. (e) Naturally I have excerpted various books, aided by my husband, who has been my constant referee throughout. Sir E. A. W. Budge allowed me the use of his fine MS., the Butyrum Sapientiae of Bar Hebraeus, still unpublished, and of his Book of Medicine before publication.

My thanks are due to the Delegates of the Clarendon Press for allowing the publication of this supplemental volume. Warm thanks 
are also due to the past and present readers and compositors of the Press for their great interest, skill, and attention, especially to $\mathrm{Mr}$. Ulric Gantillon for many a scholarly suggestion. Dr. A. Mingana has had the kindness to read proofs and make invaluable corrections and suggestions.

My father's constant desire, in which I associate myself, is that the present wider study of the Syriac language may result in increased attention to versions of Holy Scripture in this language, especially to Mrs. Lewis's valuable discovery, in the Convent Library of Mount Sinai, of a more ancient version of the Gospels than the Peshito, itself existing in older MSS than any Greek text.

JESSIE PAYNE MARGOLIOUTH.

OxFord, June, 1927. 


\section{ABBREVIATIONS OF BOOKS QUOTED}

Act. Apos. Apoc. Apocryphal Acts of the Apostles, ed. W. Wright. London, 1871.

Ahikar

The Story of Ahikar from the Syriac, Arabic, Armenian, Ethiopic, Greek, and Slavonic versions, ed. F. C. Conybeare, J. Rendel Harris, and Agnes Smith Lewis. London, 1898.

Ahrens \& Krüger Die sogenannte Kirchengeschichte des Zacharias Rhetor. K. Ahrens, G. Krüger. Leipzig, 1899.

AKGW.

Abhandlungen der Königlichen Gesellschaft der Wissenschaften. Göttingen.

Al'ykham

Al'ykham seu Linguae et Artis Metricae Syrorum Institutiones. P. Gabriel Cardahi. Rome, 1880.

Alexis.

La Légende Syriaque de St. Alexis, ed. A. Amiaud. Paris, 1889.

Alex.-Lied.

Das Syrische Alexanderlied, ed. C. Hunnius. ZDMG. Ix. 169.

A.M.B.

Acta Martyrum et Sanctorum, ed. P. Bedjan. i-vii. Paris, 1890-7.

Amél. or Amélineau La Géographie de l'Égypte à l'époque Copte. E. Amélineau. Paris, 1893.

An. Syr.

Anecd. Syr.

Analecta Syriaca, ed. P. de Lagarde. Leipzig, 1858.

Ant. Patr.

Anecdota Syriaca, ed. J. P. N. Land. Leyden, 1862-75.

Aphr.

Eine Syrische Liste Antiochenischer Patriarchen. Bruno Meissner, WZKM. viii. 404, 1894.

The Homilies of Aphraates, ed. W. Wright, London, 1869. Syriac paging refers to this edition; Arabic numerals to D. I. Parisot's edition, Patr. Syr. i. 1894.

Apis

Apollinarist.

The Book of the Bee, ed. E. A. Wallis Budge. Oxford, 1886.

Apollinaristische Schriften, ed. J. Flemming \& H. Liebmann. Berlin, 1904.

Arist. Cat.

Arist. Apol. Ar.PflnN.

Ar.FischN.

Azazail

The Categories of Aristotle. Brit. Mus. Add. 14658.

The Apology of Aristides, ed. J. Rendel Harris. Cambridge, 1891.

Aramäische Pflanzennamen von Imm. Löw. Leipzig, 1881.

Aramäische Fischnamen. Von I. Löw in Nöld. F. S.

BA.

Histoire de S. Azazail. Paris, 1902. Bibl. de l'École des Hautes Etudes, fasc. 141.

BA.

Bar Ali, quoted Thes. Syr. passim. See Lexx. Cod. Goth. id. from a MS. at Gotha, ed. G. Hoffmann. Kiel, 1874.

Babai

Babai Senior, Archimandrita. $\quad$ C. S. Xt. Or. Série ii, tt. $61,62$.

Bahira

A Christian Bahira Legend. R. Gottheil, ZA. xiii.

Badger, Nestorians The Nestorians and their Rituals, by Rev. G. P. Badger. London, 1852.

Bar Ce.

Moyses Bar Kepha. C. S. Xt. Or. Série ii, tt. 77-82.

Bar Choni or Khoni Theodore b. Choni. See Cosmog. Manichéenne; also quoted in Pognon's Hippoc. q.v.

Barhad.

Mar Barḥadbšabba d'Arbaya, Évêque de Ḥalwan. Cause de la Fondation des Écoles, ed. Mar Addai Scher, Patr. Or. t. iv. fasc. 4. 
Bar Penk.

Bar Sal. in Lit.

B. or Bar Sud.

Batt. Const.

BB.

$\mathrm{BH}$.

BH. Col.

BH. Chron.

BH. Chron. Eccl.

BH. Eth.

BH. in -

BH. Gram.

BH. Nom.

BH. de Pl.

BH. de Sap.

BH. Stories

B. Nin.

Book of Shem

Brev. Ant. Syr.
BH. Carm.

\section{ABBREVIATIONS OF BOOKS QUOTED}

Bar Penkayé : Sources Syriaques, Vol. i, ed. A. Mingana. Mosul, 1906. Dionysius Bar Salibi. Expositio Liturgiae, C. S. Xt. Or. Sér. ii, t. 93. Stephen Bar Sudaili, the Syrian Mystic, ed. A. L. Frothingham. Leyden, 1886.

Omelia di Giacomo di Sarug sul Battesimo di Constantino Imperatore, ed. A. Frothingham, R.A.L. celxxix. Rome, 1882.

Bar-Bahlul: see Lexx. DBB. id., ed. Rubens Duval. Paris, 1888-1901.

Gregorius Bar-Hebraeus.

Carmina ed. Scebabi. Rome, 1877.

Liber Columbae, ed. G. Cardahi. Rome, 1898.

Chronicon Syriacum, ed. P. Bedjan. Paris, 1890.

Chronicon Ecclesiasticum, ed. J. B. Abbeloos \& T. J. Lamy. Louvain, 1872.

Ethicon seu Moralia, ed. P. Bedjan. Paris, 1898.

Des Gregorius Abulfarag Anmerkungen zu den Salomonischen Schriften, A. Rahlfs, Leipzig, 1887; Scholia in Ruth \& Apoc. Danielis, ed. A. Heppner, 1888; in Epistulas Paulinas, Maximilianus Loehr, 1889; in Leviticum, G. Kerber, 1895.

Euvres Grammaticales d'Abou'l Faradj dit Bar Hebreus, ed. l'Abbé Martin. Paris, 1872.

Nomocanon, ed. P. Bedjan. Paris, 1898.

A List of Plants and their Properties. From the Menarat Kudhse of Bar Ebhraya, ed. R. J. H. Gottheil.

Carmen de Divina Sapientia. Rome, 1880.

The Laughable Stories collected by Bar-Hebraeus, ed. E. A. Wallis Budge. Luzac's Semitic Text and Translation Series. Vol. i.

Rogation of the Ninevites. Press of the Archibishop's Assyrian Mission. Urmi, 1896.

Some Early Judaeo-Christian Documents in the John Rylands Library. A. Mingana. Bulletin of the above Library, Vol. iv. 1917. Breviarium juxta Ritum Ecclesiae Antiochenae Syrorum tt. i.-iii. Mosul, 1886.

Brev. Chald. Breviarium Chaldaicum, ed. P. Bedjan i-iii. Paris, 1888.

Brev. Chald., Maclean A MS. copy of the same, references supplied by Bp. Maclean.

Brief Xti Der vom Himmel gefallene Brief Christi. Max Bittner. Vienna, 1906.

Brit. Mus. Or. British Museum Oriental MSS. No. 1593 is a MS. of Chimie, q. v.

Brook's Chron. A Syriac Chronicle of the year 846, ed. E. W. Brooks, ZDMG. li.

Brook's Chron. Can. The Chronological Canon of James of Edessa, ZDMG. liii.

But. Sap.

BH. Butyrum Sapientiae: MS. belonging to Sir E. A. Wallis Budge containing Economica, Ethicon, Isagoge, Logica, Nat. Hist., Peri Hermenias, Philosophia, Politica, Theologia.

Byz. Zt.

Byzantiner Zeitschrift, ed. Karl Krumbacher, Leipzig.

Can. J. Tell.

Canones Johannis bar Cursus, Tellae Mauzalat Episcopus, ed. C. Kubercyk. Leyden, 1901.

Card. Thes. i. q. Poet. Syr. q. v.

Catholicos W. H. Browne. S.P.C.K. 1892. 
Cat. Cod. Syr. Catalogue of Syriac MSS. in the Bodleian Library. R. Payne Smith, Oxford, 1865 .

Caus. Caus.

Das Buch von der Erkenntniss der Wahrheit oder der Ursache aller Ursachen. C. Kayser. Leipzig, 1889.

C.B.M.

Catalogue of Syriac MSS. in the British Museum. W. Wright. London, 1870.

Charms

Chast.

i. q. Protection, q. v. Also Congr. Or. xi, sect. 4. Paris, 1898.

Le Livre de la Chasteté par Jésusdenah Évêque de Baçrah, ed. J.-B. Chabot. Rome, 1896.

Chimie

Chron Min.

L'Alchimie Syriaque, t. ii, La Chimie au Moyen Âge par M. Bertholet, avec la collaboration de M. Rubens Duval. Paris, 1893.

Cong. Or.

Chronica Minora, ed. Guidi, Brooks, Chabot. C. S. Xt. Or. Série iii, t. 4.

C. S. Xt. Or. Corpus Scriptorum Christianorum Orientalium. Paris.

Cosmog. Manichéenne La Cosmogonie Manichéenne d'apres Théodore bar Khoni, ed. F. Cumont. Brussels, 1908.

Coupes

Inscriptions Mandaïtes des Coupes de Khouabir. H. Pognon. Paris, 1898.

Cyrillona

D BB.

Die Gedichte des Cyrillona, ed. J. Bickell, ZDMG. xxvii.

De Astrolabe

De Goeje B.

Dion.

Dion. Ined.

Diosc.

See BB.

Severus Sebokht de Astrolabe, ed. F. Nau. Journ. As. 1903.

Feestbundel aan Prof. M. J. de Goeje. Leyden, 1891.

Chronique Syriaque de Denys de Tell-Mahré, ed. J.-B. Chabot. Paris, 1895.

Diosc. ed. Nau

Dionysii Inedita. F. Nau in R.O.C. ii (1897).

Pedanii Dioscoridis De Materia Medica. Leipzig, 1829.

Histoire de Dioscore, Patriarche d'Alexandrie, ed. F. Nau, Journ. As. 1903.

Doc. Mon.

Documenta ad Origines Monophysitarum illustrandas, ed. J.-B. Chabot. C. S. Xt. Or. Sér. ii, t. 37.

Dozy

Duval, Gr.

Supplément aux Dictionnaires Arabes. M. Dozy. Leyden, 1881.

Ebed J. Card.

El. Nis.

El. Nis. Chron.

Traité de Grammaire Syriaque par Rubens Duval. Paris, 1881.

Paradisus Eden, ed. P. J. Cardahi. Beyrout, 1889. Cf. Par. Eden.

Eliae Nisibeni interpres in Lag. Praet. Miss. q. v. Göttingen, 1879.

El. Nis. Gram.

El. Tirhan

Eliae Metropolitae Nisibeni Opus Chronologicum, ed. E. W. Brooks, C. S. Xt. Or. Sér. iii, t. ii. Paris, 1810.

A treatise on Syriac Grammar by Mar Elia of Soba, ed. R. Gottheil. Berlin, 1887.

Syrische Grammatik des Mar Elias von Tirhan, ed. Fr. Baethgen. Leipzig, 1880.

Ephr. ed. Bick.

Ephr. ed. Lamy

Carmina Nisibena, ed. G. Bickell. Leipzig, 1886.

Ephr. Jos.

Ephr. Ref.

Sti Ephraemi Syri Hymni et Sermones, ed. Th. J. Lamy. Mechlin, 1882-1902.

Histoire Complète de Joseph, par St. Ephrem. Poème. Paris, 1891.

S. Ephraim's Prose Refutations of Mani, Marcion and Bardaisan, ed. C. W. Mitchell. London, 1912. 
xii

Epiph.

Eranšahr

Eus. ed. Maclean

Fils Jon.

Flor. Vogüé

Fremd.W.

Galen

G. Busâmé

Gaza or Gezza

Geop.

Georg. Ar.

Gest. Alex.

G.G.A.

Greg. Carm.

\section{ABBREVIATIONS OF BOOKS QUOTED}

Epiphanius de Mensuris et Ponderibus, ed. Lagarde. Göttingen, 1880.

Eranšahr, nach der Geographie des Pseudo-Moses Xorenaci, Marquart, AKGW. 1901.

The Ecclesiastical History of Eusebius in Syriac, ed. W. Wright \& N. Maclean. Cambridge, 1898.

Les Fils de Jonadab fils de Rechab \& les Isles Fortunées Jac. Edes., ed. F. Nau. Paris, 1889.

Florilegium, dedié a M. le Marquis de Vogüé. Paris, 1909.

DieAramäischen Fremdwörter im Arabischen von Siegmund Fraenkel. Leyden, 1886.

Proben der Syrischen Uebersetzung von Galenus' Schrift über die einfachen Heilmittel. A. Merx, ZDMG. xxxix.

Ganath Busâmé = the Garden of Delights. A Commentary on Holy Scripture. Brit. Mus. MS.

E.-Syr. Service Book of variable anthems and hymns.

Geoponicon in sermonem Syriacum versorum quae supersunt, ed. P. de Lagarde. Leipzig, 1860.

Die Astronomischen Briefe Georgs des Araberbischofs, ed. V. Ryssel. ZA. viii. 1893.

The History of Alexander the Great, being the Syriac version of Pseudo-Callisthenes, ed. E. A. Wallis Budge. Cambridge, 1889.

Göttinger Gelehrte Anzeigen.

Sti. Gregorii Theologi Liber Carminum, ed. P. J. Bollig. Pars II, ed. H. Gismondi. Beyrout, 1895.

Greg. of Cyprus. Gregory of Cyprus, Rylands Library MS. 42.

H. and Brooks or Hamilton and Brooks. See Zach. Rhetor.

Hebr. or Hebraica Hebraica, a Quarterly Journal of Hebrew Study. Chicago.

Hex.

Versio Hexaplaris.

Hippoc.

Une Version Syriaque des Aphorismes d'Hippocrates, ed. H. Pognon. Leipzig, 1903.

Hist. B.V.M. The History of the Blessed Virgin Mary and the History of the Likeness of Christ, ed. E. A. Wallis Budge. Luzac's Semitic Text and Translation Series. Vol. iv.

Hist. Édesse Histoire d'Édesse jusqu'à la Première Croisade. Rubens Duval. Paris, 1897.

Hist. Mon. The Book of Governors; the Historia Monastica of Thomas, Bishop of Marga, ed. E. A. Wallis Budge. London, 1893.

Hist. Rabban Hormizd The Histories of Rabban Hormizd, the Persian, and Rabban Bar 'Idta, ed. E. A. Wallis Budge. Luzac's Semitic Text and Translation Series. Vols. ix, $x$, xi.

Hormizd

The Life of Rabban Hormizd. A metrical discourse, by Wahle surnamed Sergius of Adhorbaijan, ed. E. A. Wallis Budge. Berlin, 1894.

Hultsch See Metrologie.

Ined. Syr.

Inedita Syriaca, ed. E. Sachau. Vienna, 1870.

Inscript. Sém.

Inscriptions Sémitiques de la Syrie, de la Mésopotamie et de la Région de Mossoul. Pognon. Paris, 1907. 
Is. Ant.

S. Isaaci Antiocheni opera omnia, ed. G. Bickell. Giessen, 1873.

Is. Nin. B.

Mar Isaacus Ninivita de Perfectione Religiosa, ed. P. Bedjan. Leipzig, 1909.

Is. Nin. Ch. or Chabot. De Isaaci Ninivitae Vita, Scriptis et Doctrina: accedunt tres sermones, ed. J.-B. Chabot. Louvain, 1892.

Ishodad

The Commentaries of Ishodad of Merv, ed. Margaret Dunlop Gibson. Horae Semiticae X. Cambridge, 1913.

Išoyahb Išo yahb III Patriarchae Liber Epistularum, ed. Rubens Duval. C. S. Xt. Or. Sér. ii, t. 64 . Paris, 1905.

Ishoyahb Lett. The Book of Consolations or the Pastoral Epistles of Mar Isho-Yahb of Kuphlana in Adiabene, ed. Philip Scott-Moncrieff. Luzac's Semitic Text and Translation Series.

JAOS. Journal of the American Oriental Society.

J. As. or Journ. As. Journal Asiatique.

Jab. or Jabal.

Vies de Mar Jab-Alaha, de trois autres Patriarches et de quelques laïques Nestoriens, ed. P. Bedjan. Leipzig, 1893.

Histoire de Mar Jab-Alaha et de Raban Sauma, ed. P. Bedjan. Paris, 1888. The refs. are to the 2nd edition.

Jac. Edes. in Arist. Jacobus Edessenus in Cat. Aristotelis, ed. Schüler.

- Can.

- Hex.

Die Canones Jacobs von Edessa, ed. C. Kayser. Leipzig, 1886.

Études sur l'Hexaméron de Jacques d’Édesse. A. Hjelt. Helsingfors 1802.

- Resol.

Dissertatio de Syrorum Fide et Disciplina in Re Eucharistica: Joannis Telensis Resolutiones Canonicae Syriacae. T. J. Lamy. Louvain, 1909.

Jac. Sar. Hom. Homiliae Selectae Mar Jacobi Sarugensis, ed. P. Bedjan. Paris, 1905-8.

Jesus-Sabran Histoire de Jésus-Sabran par Jésus-Yahb d'Adiabene, ed. J.-B. Chabot : Archives des Missions Scientifiques vii.

Jo. Tell.

Jos. ed. Engel

Jos. Narses

Het Leven van Johannes van Tella, ed. Kleyn. Leiden, 1882.

Jos. ed. Weinberg

Die Geschichte Josephs, ed. Meier Engel. Berlin, 1895.

Homiliae Mar-Narsetis in Joseph, ed. P. Bedjan. Leipzig, 1901.

Josephus VI.

Die Geschichte Josefs angeblich verfasst von Basilius dem Grossen aus Cäsaria. Magnus Weinberg. Halle, 1893.

Jo. Eph.

Das sechste Buch des Bellum Judaicum, ed. H. Kottek. Berlin, 1886.

Third Part of the Ecclesiastical History of John of Ephesus, ed. W. Cureton, Oxford, 1853.

Jos. Styl.

The Chronicle of Joshua the Stylite, ed. W. Wright. Cambridge, 1882.

J.R.A.S.

Journal of the Royal Asiatic Society.

Jul. Hoff.

Julianos der Abtrünnige : Syrische Erzählungen, ed. J. G. E. Hoffmann. Leiden, 1880.

K-w-Dim. or Kal-w-Dim. The Book of Kalilah \& Dimnah, ed. W. Wright. Oxford, 1884.

- - ed. Bick. Kalilag und Dimnag. G. Bickell. Leipzig, 1876.

Lag. Agathangelos. A.K.G.W., Vol. 35: Arm. Stud. Armenische Studien, id. Vol. xxii. 
xiv

Lag. G. A.

-- Praet. Miss.

- Symm.

L.E.S.

Lexx.

\section{ABBREVIATIONS OF BOOKS QUOTED}

Gesammelte Abhandlungen von Paul de Lagarde. Leipzig, 1886.

Praetermissorum e recognitione ejusdem. Göttingen, 1879.

Symmicta ejusdem. Göttingen, 1877.

Lives of the Eastern Saints, ed. E. W. Brooks, Patr. Or. t. xvii. fasc. 1 , t. xviii. fasc. 5 , t. xix. fasc. 2 .

The Syro-Arabic Lexica of Jesus Bar Ali, Jesus Bar Bahlul and another, known as Hunt. clxx.

Lidzb. or id. Handb. Handbuch der Nordsemitischen Epigraphik von Mark Lidzbarski. Weimar, 1898.

Loof's Nest. Nestoriana, die Fragmente des Nestorius, ed. Fr. Loofs. Halle, 1905.

Maclean

The Right Rev. A. J. Maclean, Bishop of Moray, Ross, and Caithness. Head of the Archbishop's Mission to Assyrian Christians, 1886-91.

- Dict.

A Dictionary of the Dialects of Vernacular Syriac. A. J. Maclean. Oxford, 1901.

Mand. Gram. Mandäische Grammatik, Th. Nöldeke. Halle, 1875.

Mar Aba II. La Lettre du Catholicos Mar Aba II aux membres de l'école patriarchale de Seleucie. Congr. Or. xi. Paris, 1898.

Mar Bassus La Légende de Mar Bassus, martyr Persan, ed. J.-B. Chabot. Louvain, 1893.

Mar Benj.

Mar Kardagh

La Vie de Mar Benjamin. V. Scheil, Z.A. xii.

Acta Mar Kardaghi, ed. J. B. Abbeloos. Brussels, 1890. Die Geschichte Mar Abhdišo und seines Jüngers Mar Qardagh, ed. A. Feige. Kiel, 1890. Quotations are from Feige's edition unless otherwise stated.

Mar. Marc.

Marquart

Mart. Luc.

Maus.

Med.

Merx. Gram.

La Mort de Mar Marcos, ed. V. Scheil, ZA. xii.

See Eranšahr.

Martyrium Lucae, ed. F. Nau, R.O.C. iii.

Biblia Sacra Vers. Pschitta. Dominican Mission. Mausil, 1887.

The Book of Medicines, ed. E. A. Wallis Budge. Oxford, 1913.

Metrologie

Historia Artis Grammaticae apud Syros. Adalbert Merx. Leipzig, 1889.

Griechische und Römische Metrologie, von Fr. Hultsch. Berlin, 1882.

Mich. Syr.

Chronique de Michel le Syrien, Patriarche Jacobite d'Antioche 1166-99, ed. J.-B. Chabot. Paris, 1900.

Mon. Syr.

M. or Mt. Singar

Monumenta Syriaca ex Romanis Codicibus collecta a P. Zingerle. Innsbruck, 1869.

Monte Singar: Storia de un popolo ignoto (Yasidiani). S. Giamil. Rome, 1900.

M. Z.

Nars. ed. Ming.

Msiha-Zkha. Sources Syriaques, Vol. i, ed. A. Mingana. Mosul, 1908.

Narses W.

Natur

Nest. Chrest.

Nest. Hérac.

N. Hist.

Narsai Doctoris Syri Homiliae et Carmina, ed. A. Mingana. Mosul, 1905.

Syrische Wechsellieder von Narses, ed. Fr. Feldmann. Leipzig, 1896. Das Buch der Naturgegenstände, ed. K. Ahrens. Kiel, 1892.

Syriac Grammar with Chrestomathy. Dr. E. Nestle. Berlin, 1889.

Nestorius: Le Livre d'Héraclide, ed. P. Bedjan. Leipzig, 1910.

Natural History: part of But. Sap. 
Nöld. F. S.

- Syr. Gram.

Odes Sol.

Op. Nest.

Or. Xt.

Ost. Syr. Bisch.

Pallad.

P.A.O.S.

Par. Eden.

Par. Pat.

Patr. Or.

- iii. 1

- - iv. 4

- v. 5

Pers. Mart.

Pesh., Psch,

Pet. Ib.

Phet.

Philox.

Philox. de Trin.

Physiol.

Pléroph.

Poet. Syr.

Probus

Prognostics

Protection

QdhamW.

R.A.L.

R.A.S.

Reg. Mon.

R. O.C.
Orientalische Studien Theodor Nöldeke gewidmet. Gieszen, 1906.

Kurzgefasste Syrische Grammatik. Th. Nöldeke. Leipzig, 1880.

The Odes and Psalms of Solomon, by J. Rendel Harris. Cambridge, 1911.

Opuscula Nestoriana, ed. G. Hoffmann. Kiel, 1880.

Oriens Christianus für die Kunde des Christlichen Orients. Rome, Propaganda, 1902.

Ostsyrische Bischöfe und Bischofsitze im v., vi., und viiten Jahrhunderte. Ig. Guidi, ZDMG. xliii.

The Book of Paradise : being Histories and Sayings of Monks of the Egyptian Desert, ed. E. A. W. Budge. London 1904.

Proceedings of the American Oriental Society.

Ebed-Jesu Sobensis Carmina Selecta ex libro Paradisus Eden, ed. H. Gismondi. Beyrout, 1888.

Liber qui inscribitur Paradisus Patrum, ed. O. F. Tullberg. Upsala, 1851.

Patrologia Orientalis, ed. R. Graffin \& F. Nau. Paris.

Histoires d'A houdemmeh et de Marouta suivies du Traité sur l'Homme, ed. F. Nau.

See Barhad.

Légendes Syriaques, ed. F. Nau, 1910.

Auszüge aus Syrischen Akten Persischer Märtyrer von G. Hoffmann. Leipzig, 1880.

Peshito or Pschitta Version of the O.T.

Petrus der Iberer: Syrische Übersetzung einer um das Jahr 500 verfassten Griechischen Biographie, ed. R. Raabe. Leipzig, 1895.

Historia Sti. Mar Pethion, ed. J. Corluy. Brussels, 1886.

The Discourses of Philoxenus, Bishop of Mabbogh, A.D. 485-519, ed. E. A. Wallis Budge. London, 1894.

Philoxenus de Trinitate et Incarnatione, ed. A. Vaschalde, C. S. Xt. Or. Série ii, t. 27.

Physiologus seu Historia Animalium xxxii. O. G. Tychsen. Rostock, 1795.

Les Plérophories de Jean, Évêque de Maiouma, ed. F. Nau, Patr. Or., t. vii. fase. 1. M. Nau's notes are in R. O.C. iii.

Liber Thesauri de Arte Poetica Syrorum. D. G. Cardahi. Rome, 1880.

Le Traité du Philosophe Syrien Probus sur les Premiers Analytiques d'Aristote par A. van Hoonacker. Journ. As. 1900.

The Prognostics of Daniel. Brit. Mus. Or. 2084.

The Book of Protection : being a Collection of Charms, by Hermann Gollancz. London, 1912.

The Book of Qdhamu Wathar=Before and After, i. e. Daily Offices of the East-Syrian Church. At the Press of the Archbishop's Mission. Urmi, 1892.

Reale Accademia dei Lincei. Rome.

The Royal Asiatic Society, Journal of. London.

Regulae Monasticae Saec. vi, ed. J.-B. Chabot. Rome 1898. R.A.L.

Revue de l'Orient Chrétien. Paris. 
xvi

Rylands MS.

Sassanidi

Schatzh.

Schulthess Probe

S.C.L.

S. Dan.

Sette Dorm.

Sev. Ant. Hom.

Sev. Ant. Hymns

- - Vit.

Sev. Lett.
S. George
S. Maris
S. Pelag.
Sindban

Stat. Schol. Nis.

Stud. Sin.

Stud, Syr.

Syn. II Eph.

Syn. Or.

\section{ABBREVIATIONS OF BOOKS QUOTED}

MSS. in the John Rylands Library, Manchester.

Un nuovo testo siriaco sulla Storia degli ultimi Sassanidi, ed. Ig. Guidi. Congr. Or. viii.; also Chronica Minora, C. S. Xt. Or. Série iii, t. 2.

Die Schatzhöhle, ed. Carl Bezold. Leipzig, 1883.

Probe einer Syrischen Version der Vita Sti. Antonii von Fr. Schul hess. Leipzig, 1894.

Collection of Letters of Severus of Antioch, ed. E. W. Brooks, Patr. Or. xiv.

History of Mar Daniel the Healer, Brit. Mus. Or. 4404.

I Sette Dormienti di Efeso. Ig. Guidi, R.A.L. cclxxxii. 1895.

Les Homiliae Cathédrales de Sévère d'Antioche trad. Syr. inédite de Jacques d'Edesse, ed. R. Duval, Patr. Or. t. iv. fasc. 1, t. viii. fasc. 2.

The Hymns of Severus and others in the Syriac version of Paul of Edessa as revised by James of Edessa, ed. E. W. Brooks, Patr. Or. t. vi. fasc. 1 , t. vii. fasc. 5 .

Vie de Sévère, Patriarche d'Antioche 512-519, par Zacharie le Scholastique, ed. M.-A. Kugener, Patr. Or. t. ii. fasc. 1, et par Jean, Supérieur du Monastère de Beith-Aphtonia, ib. t. iii. fasc. 3.

The Sixth Book of the Select Letters of Severus Patriarch of Antioch, ed. E. W. Brooks. Text and Translation Society. London, 1902.

Brit. Mus. Or. 4404.

Acta S. Maris, ed. J.-B. Abbeloos. Brussels, 1885.

Acta S. Pelagiae, ed. J. Gildemeister. Bonn, 1879.

Sindban oder die Sieben Weisen Meister. Fr. Baethgen. Leipzig, 1879.

Statuta Scholae Nisibenae in Giornale della Società Asiatica Italiana, Vol. iv. (1890).

Studia Sinaitica, ed. Agnes Smith Lewis and Margaret Dunlop Gibson. Cambridge University Press, 1894-1916.

Studia Syriaca seu Collectio Documentorum hactenus Ineditorum ex Codicibus Syriacis. Ignatius Ephr. II. Rahmani. Mt. Lebanon, 1904.

Secunda Synodus Ephesia. S. G. F. Perry. Oxford, 1875.

Synodicon Orientale ou Recueil de Synodes Nestoriens. J.-B. Chabot. Paris, 1902.

Syr. Rom. Rechtsb. Syrisch-Römisches Rechtsbuch, von K. G. Bruns und Ed. Sachau. Leipzig, 1880.

Tabari

Geschichte der Perser und Araber zur Zeit der Sasaniden aus der Arabischen Chronik des Tabari. Th. Nöldeke. Leyden, 1879.

Takhsa

The Liturgy of the Holy Apostles Adai and Mari with two additional Liturgies and the Order of Baptism. Urmi at the Press of the Archbishop of Canterbury's Mission, 1890.

Talm.

Tiekkaf The Talmud.

Test. Dñi.

Das Gedicht Tekkaf l'Arestotalis, ed. S. Samuel. Halle, 1893.

Testamentum Domini Nostri Jesu Christi, ed. Ignatius Ephraem Rahmani Patriarcha Antiochenus Syrorum. Metz, 1899. 
Test. Ephr.

Theod. Mops.

- - in Jo.

Tim. I.

T. L.Z.

Tristram

Vit. Mon.

Warda

WZKM.

Yezidis

ZA.

Zach. Rhetor.

Le Testament de Saint Ephrem. Rubens Duval. Journ. As. 1901.

Theodori Mopsuesteni Fragmenta Syriaca, ed. E. Sachau. Leipzig, 1869.

Commentarius Theodori Mopsuesteni in Evangelium D. Joannis, ed. J.-B. Chabot. Paris, 1897.

Zwei Synoden des Katholikos Timotheos I. Prof. Oscar Braun in Or. Xt. ii.

Theologische Literaturzeitung.

The Natural History of the Bible. H. B. Tristram. S.P.C.K. London 1868.

Vitae Virorum apud Monophysitas Celeberrimorum, ed. E. W. Brooks. C. S. Xt. Or. Sér. iii, t. 25. Leipzig, 1907.

Hymns of George Warda, MS. belonging to Mr. Athelstan Riley. Id. ed. Aladar Deutsch.

Wiener Zeitschrift fur die Kunde des Morgenlandes.

Notice sur les Yézidis, ed. J.-B. Chabot. Journ. As. 1898.

Zeitschrift für Assyriologie und Verwandte Gebiete. Leipzig.

The Syriac Chronicle known as that of Zacharias of Mitylene, translated by E. J. Hamilton and E. W. Brooks. London, 1899.

Zach. or Zach. Rhet. Brooks Historia Ecclesiastica Zachariae Rhetori vulgo adscripta,

Zach. Bull. Il Testo Siriaco della Descrizione di Roma nella Storia attribuita ed. E. W. Brooks. C. S. Xt. Or. Série iii, tt. 5, 6. a Zaccaria Retore. Bulletino della Commissione Archaeologica di Roma, fasc. iv. (1884). Roma, 1885.

ZDMG.

Zeitschrift der Deutschen Morgenländischen Gesellschaft. Leipzig. 


\section{ABBREVIATIONS}

\begin{tabular}{|c|c|c|c|}
\hline $\begin{array}{l}\text { obrev. } \\
\text { cs., absol. } \\
\text { tj. } \\
\text { av. } \\
\text { o, a f. } \\
\text { chem. } \\
\text { tep. } \\
\text { ph. } \\
\text { r., Arab. } \\
\text { trol. } \\
\text { tron. } \\
\text { ssyr. } \\
\text { o., bpric. } \\
\text { m. gen. } \\
\text { hald. } \\
\text { em. }\end{array}$ & $\begin{array}{l}\text { conjugation. } \\
\text { ology. } \\
\text { onomy. } \\
\text { an. } \\
\text { bishopric. } \\
\text { nender. } \\
\text { compare. } \\
\text { ic. } \\
\text { mistry. } \\
\text {, usually referring } \\
\text { hes. Syr. } \\
\text { ively, collective } \\
\text { h. } \\
\text { und. } \\
\text { position. } \\
\text { ation. } \\
\text { ct state. } \\
\text { cted form. } \\
\text { inative. } \\
\text { d, derivative, deriva- } \\
\text { ative. } \\
\text { astical use. } \\
\text { ally. } \\
\text { tic state. } \\
\text { h'al conjugation. }\end{array}$ & $\begin{array}{l}\text { Gr. } \\
\text { gram. } \\
\text { Heb. } \\
\text { Hex. } \\
\text { ib. } \\
\text { imp. } \\
\text { impers. } \\
\text { inf. } \\
\text { in loc. } \\
\text { interj. } \\
\text { intrans. } \\
\text { i. q. } \\
\text { irreg. } \\
\text { Jac. } \\
\text { l., ll. } \\
\text { Lat. } \\
\text { lit. } \\
\text { Lit., liturg. } \\
\text { logic. } \\
\text { Mand. } \\
\text { m., masc. } \\
\text { med. } \\
\text { metaph. } \\
\text { n. } \\
\text { N. Heb. } \\
\text { N. Syr. } \\
\text { Nasor. } \\
\text { Nest. } \\
\text { opp. } \\
\text { Pa. } \\
\text { par. } \\
\text { pt., part. } \\
\text { pass. }\end{array}$ & $\begin{array}{l}\text { thpe el conjugation. } \\
\text { taph'al conjugation. } \\
\text { llowing. } \\
\text { minine gender. } \\
\text { ture tense. } \\
\text { nder. } \\
\text { geometry. } \\
\text { reek. } \\
\text { ammar. } \\
\text { ebrew. } \\
\text { the Hexaplar version of } \\
\text { the O.T. } \\
\text { idem. } \\
\text { perative. } \\
\text { personal. } \\
\text { finitive. } \\
\text { the passage. } \\
\text { terjection. } \\
\text { transitive. } \\
\text { em quod. } \\
\text { regular. } \\
\text { cobite or West-Syrian. } \\
\text { ee, lines. } \\
\text { tin. } \\
\text { erally. } \\
\text { urgically. } \\
\text { logic. } \\
\text { andaean. } \\
\text { asculine. } \\
\text { the middle, in medicine. } \\
\text { etaphorically. } \\
\text { te. } \\
\text { o-Hebrew. } \\
\text { odern Syriac. } \\
\text { soraean. } \\
\text { rticiple. } \\
\text { ssive. } \\
\text { posite, opposed to. } \\
\text { ragraph. } \\
\text { ran. }\end{array}$ \\
\hline
\end{tabular}




\section{ABBREVIATIONS}

Pe.

pen.

perh.

Pers.

pers.

pl.

pr.n.

prep.

pres.

pret.

prob.

pron.

ref., refs.

refl.

Rit.

sect.
Péal conjugation. penultimate.

perhaps.

Persian.

person.

plural.

proper name.

preposition.

present.

preterite.

probably.

pronoun.

reference, proof passage.

reflexive.

Ritual, usually of the

East-Syrians, some-

times Jacobite.

section. ser.

Shaph.

sing.

st.

suff.

Syr.

subst.

Suppl.

T. or $t$.

Thes. Syr.

trad.

trans.

Turk.

ult.

var.

VHh.

W.-Syr. series.

Shaphel conjugation. in the singular number. state.

suffix.

Syriac.

substantive.

in this Supplement.

Tomus.

Thesaurus Syriacus.

traduction.

translation.

Turkish.

last line.

variant reading.

Harklensian version of the N.T.

West-Syrian. 



\section{S U P P LE M E N'T T O THESAURUS SYRIACUS}

$\therefore N \therefore$

\section{Remarrs}

10:01) "Oarıs, the Oasis of Egypt, BHChr.

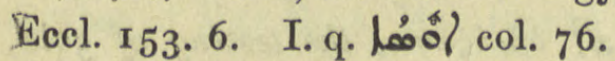

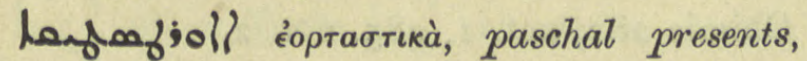

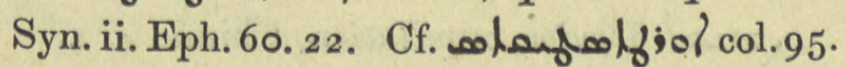
कastil Aetius, an archdeacon of Constantinople, BHChr. Eccl. I 77. I.

Ja入l ảnón, aloes, Chimie 5. 14. Cf. J)?, coll?, and ja>l.

;] $)^{\circ}$ col. 3. Metaph. an air, appearance, إحمبا

oolojl? Aerius, bishop of Zephyrion in Cilicia, Nöld. F. S. i. 470.55 .

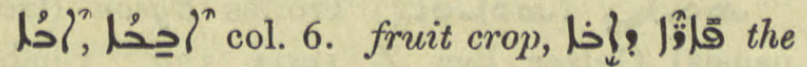
fruits of the season, ripe fruit, Pallad. 619. 4 .

إحصى Benus, an Egyptian hermit, C.B.M. (Gr.) $650 a$, he is called $\leftrightarrow$ A Abban, Pallad. $37 \times \mathrm{f}$.

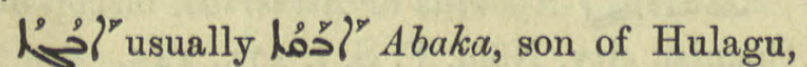
the Mogul Khan, Jab. 25.2 and note, 94. pen.

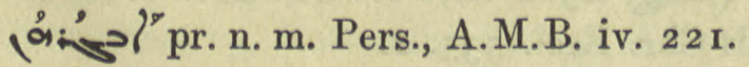

"ج اجب حه : col. 8. Addite adamavit," Abbeloos, BH Chr. Eccl. 585. 6.

jow 286. 17 .

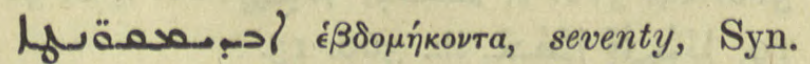

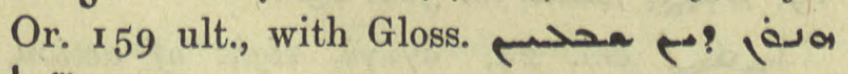
. مذتما.

;a)l Abaršahr, the capital of Khorasan, Pers. Mart. 292 ; G. Busâmé 6o. 5 af.

a) col. ro. Add: : or pelican, Med. 59 I. 8.

|ื่ 2719

\section{mias}

トُa?! a bar of goid, Kal-w-Dim. ed. Bick. 37. 22.

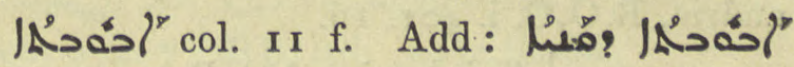
a hollow reed, Med. 103. 16 ; blow powdered drugs through a tube, ib. 162.23. The fore-arm a a

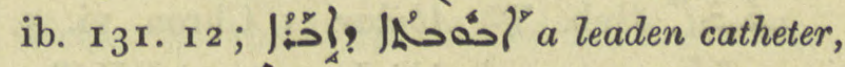
Anecd. Syr. ii. 223. 22, 26, ZA. xi. 212.

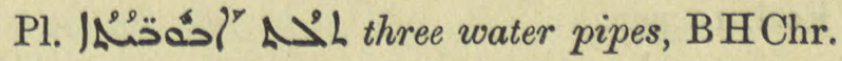
Eccl. 839. 30. .

นُนُธ่อ์จ tubular, hollow, cuttle-fish have |

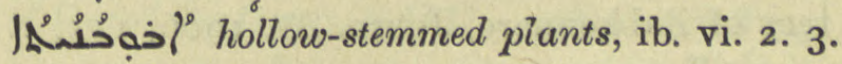

ian an alembic. Nawaw $\rightarrow 0$ ? asalo asoofo Brit. Mus. Or. 1593. $35 r$, this is a manuscript of La Chimie au Moyen Age.

, : xii. Syr. $\mathrm{r}, 9 \mathrm{r}$.

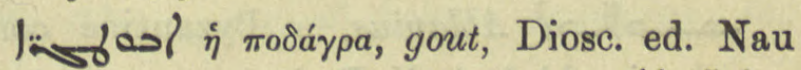
94. 9, 299. not. 2. Usually written la

ne⿻l pr. n. m. Abui, Mich. Syr. ii. fasc. ii. 3 5 . b. 4 af. $=$ transl. $255 . a 16$ note.

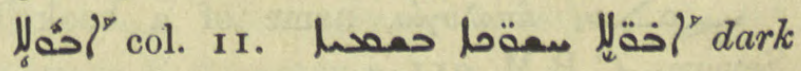
columns in heaven, N. Hist. v. 4. 3 ; an en-

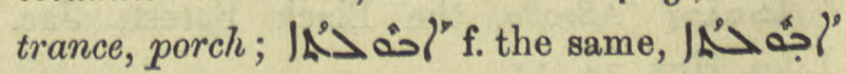

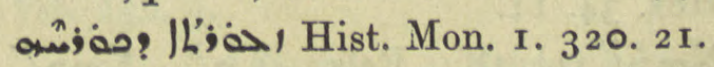

;م्ح| 'A north of Khorasan on the border of Russia, Syn. Or. ro9. 6, Pers. Mart. 29 I, f., ZDMG. xliii. 403.5 .

glos pr. n. m. Pers. A.M.B. iv. 239. 


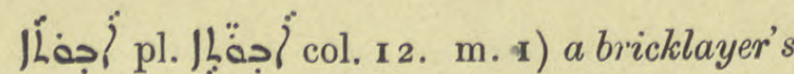

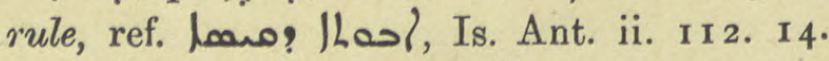

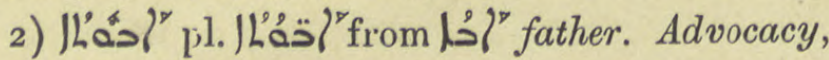
pleading or defence in a court of law, with

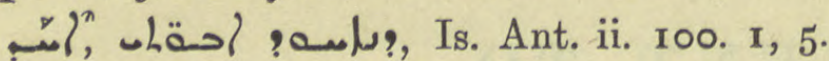
Lat. patrocinium.

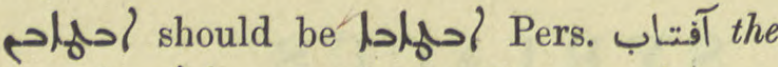
sun, Anecd. Syr. iv. 9. 2.

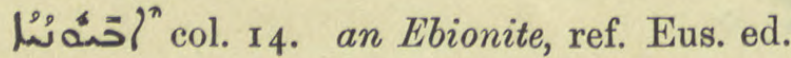

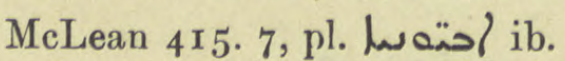

إحلا Abila, a bishopric in Syria, De Goeje B. 64 . II.

إحده مـ Abilius, third bishop of Alexandria, R.O.C. i. 401 . I6.

مهenal Epiphanius, Ant. Patr. 295. 3 af. pard Abikam, the name which Ahikar took in Egypt, Ahikar s. 8, I I, . I, 6, \&c.

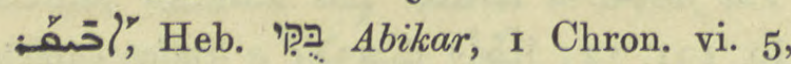
i.q. حقّ verse 51, Bukki, Lxx. Boкki, BHChr. Eccl. ro pen.

أحشh or o a village and monastery, C.B. M. $706 a$, Doc. Mon. I62. 10.

a ( Mar Abkoš, a monk in the monastery of Rabkennare, Journ. As. 1906, I I I. I.

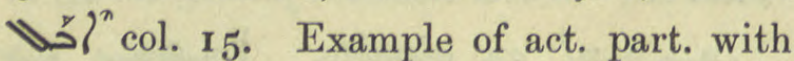

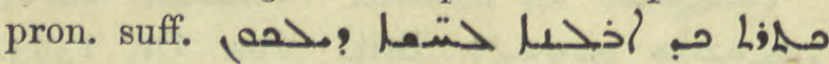
I remained lamenting your sufferings, Isoyabh, ed. Duval, 91. I5.

$\mathrm{lol} \mathrm{m}$. the pole of a bier or litter, Hist. B.V.M. I13. 5, 129.16 bis.

أحلاحس Ablavius, a Byzantine commander-in-chief, A. M. B. iv. 294, حخحمص father of Olympias, Pallad. 202. 5. I. q. أlol. 16 .

una la a Severus, C.B.M. 91 ז. a.

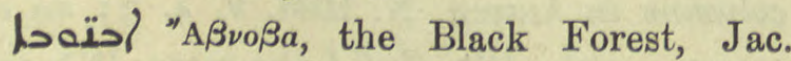
Edes. Hex. 34. Ir.

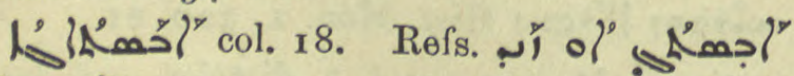
$12 n=0]^{\prime}$ the Avesta or Zend-Avesta, A.M.B. ii. $576.12,579.19,589.15$, Phet. 26.7.

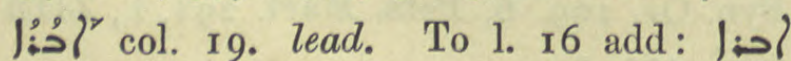

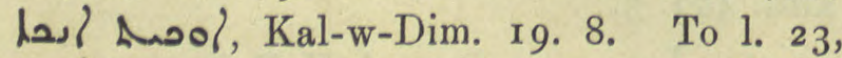

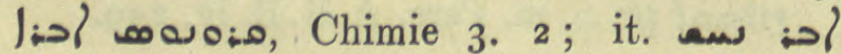

" molybdochalque, plombcuivre," ib. 4. I; .

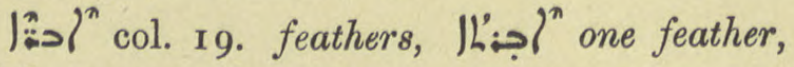
Med. 59 1. I 7 .

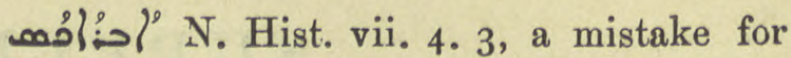
a a a Fisch N. 4 I.

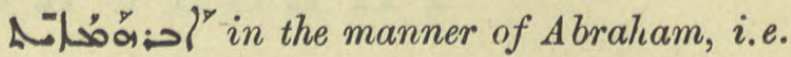
hospitably, Pet. Ib. 46.14.

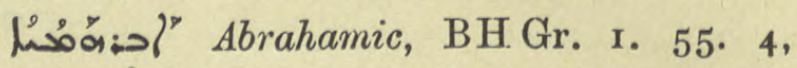

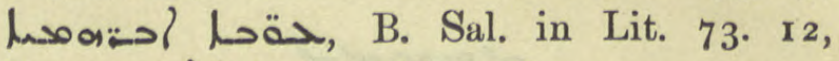

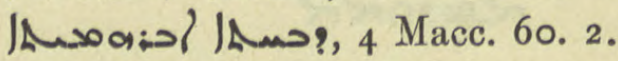

|l pr. n. Pers. Jab. 232. 10.

m Chimie 282, not. I, 285 . not. 2 .

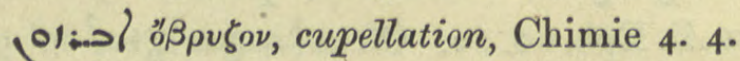

H:Dl Abarne, hot springs in N. Meso-

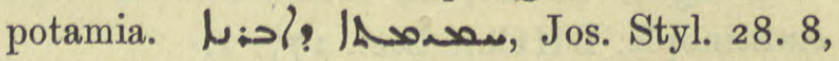
Anecd. Syr. ii. 2 ro. 7 , Jac. Edes. Chron. Can. 295, Dion. Ined. 463. I.

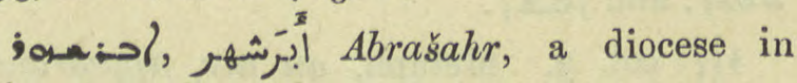
Khorasan, Syn. Or. 34. 2 I, 37. 3, 43. 19, ZDMG. xliii. 396. 13, 398. 1 7, 4 or. 6.

أخ: a drug, Med. 601. 4.

oak (colbo-子) Troilos Egeon (Geonensis) Gelzer Byz. Z. 1903, 127. Chabot

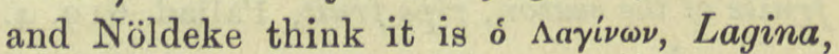
a bishopric suffragan to Perga, ib. and Nöld. F. S. i. 472,83 .

sa) ? Agarius or Aetherius, bishop of a Spanish city, Nöld. F. S. i. 475 · 145.

la ${ }_{\mathrm{S}}$ Aghaban, a town in Greater Armenia, BH Chr. Eccl. 535. I.

of $\rightarrow \longrightarrow$ Gagas, a river of Lycia, Chimie 9. 2 .

L'ب col. 23. ferula asafoetida, Med.

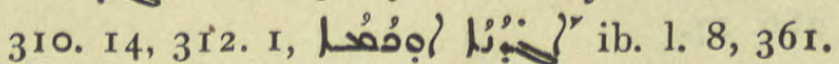

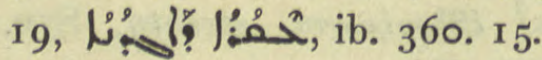

loa col. 24, à $\gamma \omega \gamma \dot{\eta}$, right of action, add refs. Syr. Rom. Rechtsb. 29. 9, I5, pl. $\infty_{\text {a }}$, ib. 18. 3 .

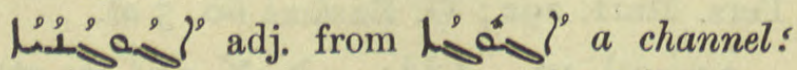

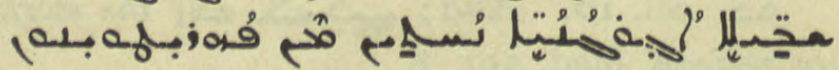
passages of the nature of ducts descend from the peritoneum, N. Hist. vii. I. 3 infra. 


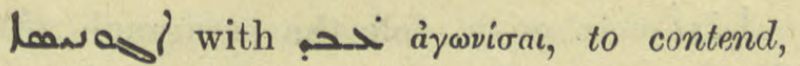
agonize, A.M.B. v. 359. paen.

هـ

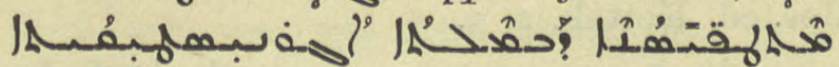

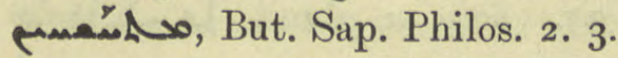

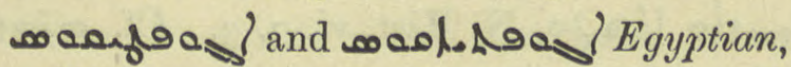
Jac. Edes. Hex. I I. I I.

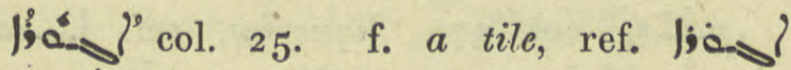

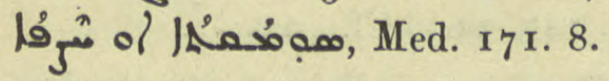

j;a m. probably an infirmary, hospital, Jos. Styl. $3^{8 .} 3$, I 5 .

a aulona.y pr. n. f. Pers. Agusqanuš, mother of Mar Saba, Mar Bassus xii. 5 Syr.

L Charms 83. 6.

nong the Egean Sea, Sev. Ant. Vit. 324. 1 .

an ${ }^{\circ} \rho \circ o v$, wool, and? épiov, a woollen rug, A. M. B. iv. $3^{1} 3$ ult., Chimie 305 , cf. anga ib. 274. For change of $r$ to a guttural see Hoffm. ZA. xi. 237, xvii. 88.

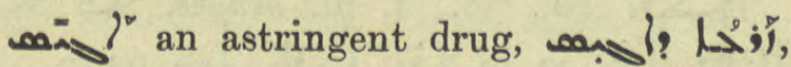

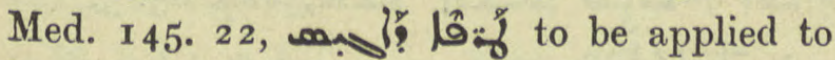
loose gums, ib. $175 \cdot 22$.

Hengl Agapetus, Pope, BHChr. Eccl. 207. 8, incompletely written lang ib. 1. 2I, 209. I, I 3 .

L.: ? descendants of Japheth, Chron. Min. 355. 10.

esfegla $?$ Aglaophôn, a work on the Resurrection by Methodius Bishop of Lycia, C.B. M. 917 a.

(a) >ل col. 27. agallochum, ref. Med.

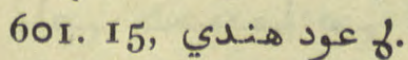

Aglaïdas, name of a lecturer, A.M.B. iii. $3^{2} 5$.

manasoly for manand col. 27. an eclipse of the sun, Anecd. Syr. iii. 225.1 .

$7^{n}$ name of a monastery in the diocese of Mardin, B.O. ii. 228. 6 af.

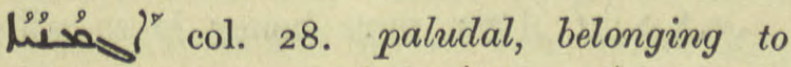

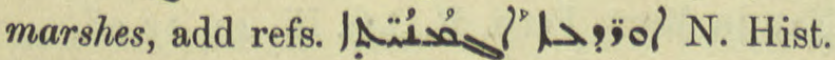
vii. 4, 5, 7; Lexx. under محّم col. I539.
L and turn inwards, Med. 94. 8.

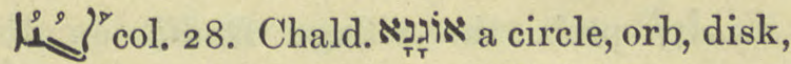
Buxtorf., a bowl, Cosmog. Manichéenne 3I, note 2.

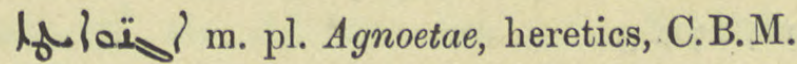
$798 a, 935 b$.

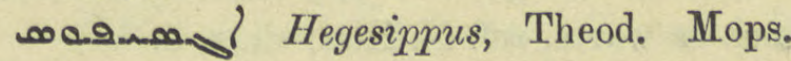
10. 3 .

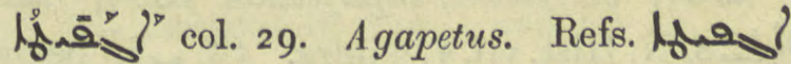
Pope of Rome, Sev. Ant. Vit. 287 ter., Ha , ib. 28I. 3, empa ?, Anecd. Syr. ii. I7. 5. ZDMG. xliii. 395. 2. See frang, Suppl. supra.

Aa Ar. Agrimonia eupatorium, Med. 602. 7 ; BB. sub ja;aflsoa.

$10\}$ : 2 Agraca, a city whence arsenic was procured, Chimie $3 \circ 3$. ult.

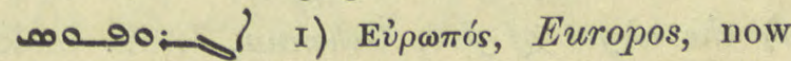
Girbas, on the West side of the Euphrates, opp. Kennešrin, C.B.M. 970 b, a bpric. under Antioch, Sev. Ant. Vit. 3 19. 10, 325. 9, Or. Xt. ii. 2 70. 282 note 5 , but no90jol ib. 275 , Jos. Styl. 84. 9. Cf. Pers. Mart. I6r sqq. and Thes. Syr. of Herod the Great, R.O.C. i. 398.8,

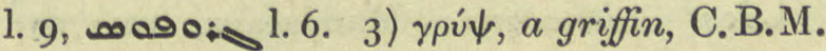
I $192 b$.

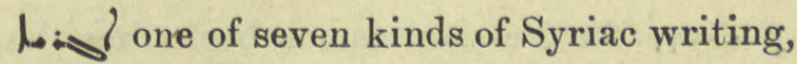
El. Nis. 96. 75 .

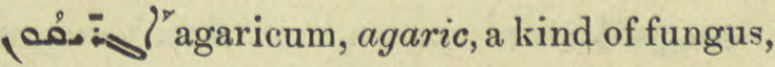
Med. 49. 2, I4, 50 ult. 5I. Iо, 19, I36. 2, 299. 2I, اغـاريـقــون boletus igniarius, ib. 601 . II.

مA an E.-Syr. bishopric, ZDMG. xliii. 395. 8. I.q. e. Syn. Or. 36. I6. jog A Agathodorus, bishop of Isun? De Goeje B. 65 antep.

lawohy pr. n. f. Agathonice, Sev. Ant. Vit. 258.3 .

- $\rightarrow$ A Akhtamar, an island in Lake Van, BHChr. Eccl. 535. I.

J?\} pr. n. m. Ada? Jab. 314. 6, T. L. Z. 1894. 2. 43 .

- $\nmid ? !$ o 'A $\delta a ́ \delta \omega \nu, ~ A d a d a$ in Pisidia, now Kara Bavlo, Nöld. F. S. i. 473. 108. 
๓ol?? pr. n. m. Euodius, Ant. Patr. 297. 9 af.

- 4 ? prob. the village Dadih on the northern slope of Jebel Riha, ZSVG. 1922. 172. C.B.M. 706 b, 707 b; Doc. Mon. 164 . I2, 17 1. 3 .

- oljáị̂l col. 34. ảóvives, glands, excrescences, such as warts, ref. N. Hist. vii. r. 6.

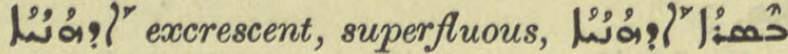

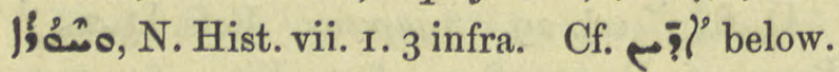

o!? Ado, founder of the sect of lïo? Adonaeans or ii. $154 \cdot 3$.

L'ớ? col. 34. I) an oven, add refs.

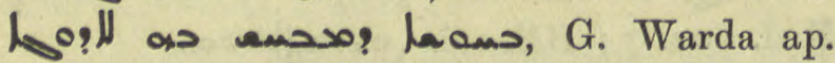

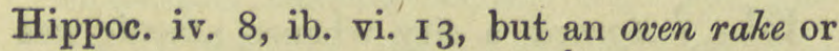

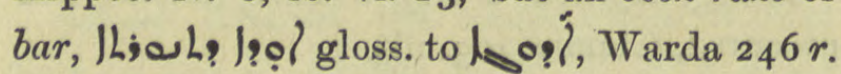
2) the abyss of fire, Gehenna, Lexx. under

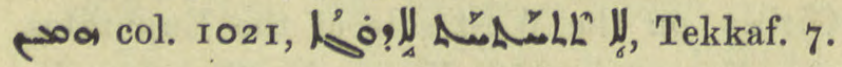

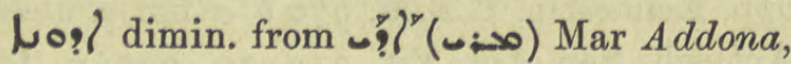
Metropolitan of Elam, Chast. 59, the monastery named after him was in Qardu, ib. $5^{8}$ ult.

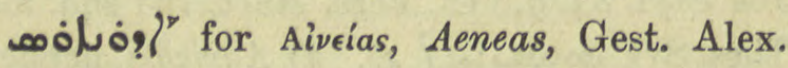
3०. 2.

Lio?? Adonaeans = Mandaeans, from og? founder of the sect, Coupes ii. I54. I8.

-jo! for Persian names beginning thus,

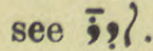

?- 10ibio? pr. n. m. Aderbaruzgard, Mar Bassus xii. 5 Syr.

9áljọ? Adorzahad, governor of Adiabene under Ardašir, M. Z. 3 I . 74.

o.f;o!l adoratores, worshippers, i.e. the Magi, G. Busâmé 64 b. 4 .

a:த้;ọ?l' Adorpareh, governor of Adiabene, Jab. 228.3 af., M.Z. 56. 20.

ló:@้;ộl’ Adarparwa, Mart., A.M.B. ii. 5, iv. $17 \mathrm{I}$.

:- :Beit Arabaye, A.M.B. iv. 224.

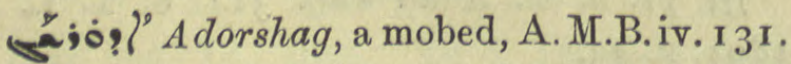

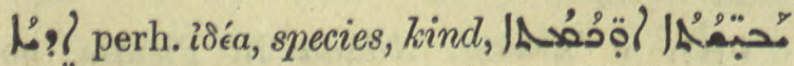
L.??:, Med. 587.8.
? cols. $3^{6-7}$, dele the paragraph after the first line. I. q. Gr. ä $\sigma \tau v$, the suburbs opp. أمخدا Jos. ed. Kottek. 2. 5 .

ب? col. 37. ảo ץ. Thes. and Suppl. supra.

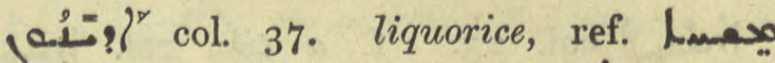

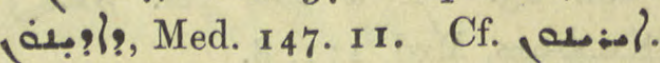

| Med. I70. 9 .

حـ Plane-tree spring, a village near Maaltha, Nars. ed. Ming. Pref. $4 \mathrm{I}$, note.

$ل_{9} ?^{n}$ col. 37. lepidium latifolium, pepperwort, refs. Med. 137. 5, 1о, 141. $23,583.18$, .

مه 61. $4,62.1$.

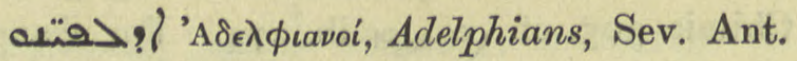
Vit. 106. r3 $_{3}$, id. Lett. 6r. 9 .

أ?

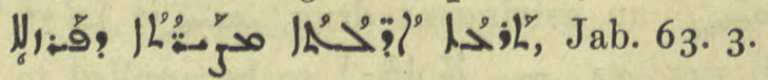

A_to? in the manner of Adam i. e.

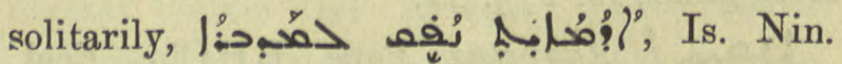
B. 266 ult.

nas? A damas, The Light, i.e. the heavenly man, a Manichaean equivalent of primal man, Cosmog. Manichéenne 22. note 3, 23. note 2, jas sox? one of the five sons of the Living Spirit, Coupes ii. 128. 4, 130. 7 .

IAmono? (ملامل) of adamant, BH. ap. J. As. $1898,77.20$.

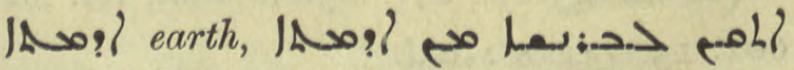

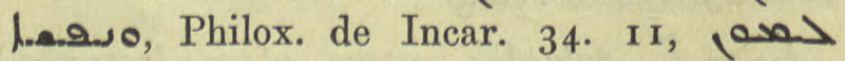

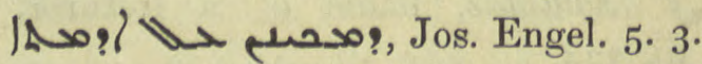

 major, the plaintain, Hunt. clxx under

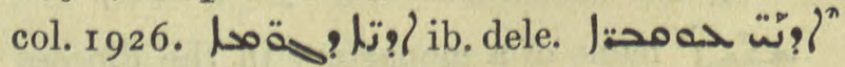
myosotis, ref. Med. 53. 12, 55. 24.

(0. o. ? Attic, pure honey is so-called, Nau in Mart. Petri, R.O.C. iii. 49. 12 af.

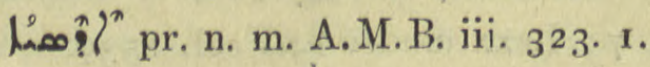




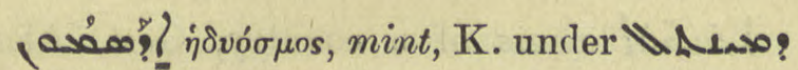
col. 9 I 8.

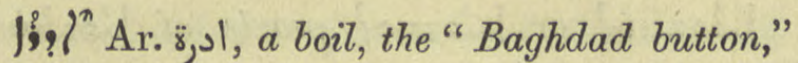
Med. 557. 19, $5^{8} 3$ four times, $5^{8} 5.3,4$, Charms 3r. 20, 22, 32. r, 82 med.

;? and preceding and following names are compounded, is the Pehlewi jٓ ذ̄ ātūr fire.

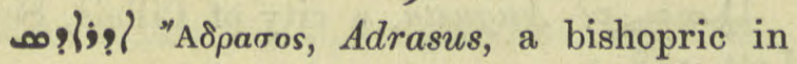
Arabia, Nöld. F. S. i. ${ }_{469}$. 37. Adra, Mich. Syr. I transl. 3 I 4.

iv. I 7 I, Pers. Mart. 36 ff. 283,289 .

هi!? Idribt, name of a fortress, Anecd. Syr. iii. 256.25 .

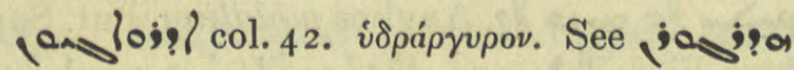
col. 977 and ojoyoj909 Suppl.

Leog? corr. Leogll col. Iro, Osroene, Nöld. F. S. i. 470.

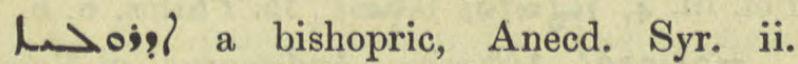
37 I. I 2.

Lojgl col. $5^{2}$ end of par. Pers. مدرو an inner apartment, Act. Apost. Apoc. مخد. 2, Sassanidi 63. 20.

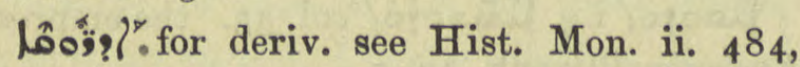
note 4. Fire altars of the Magi, ib. i. 262, Mar Kardagh ed. Abbeloos 62, ed. Feige 52,57 .

:

सs:i? one of the great fosses which fortify the city Shuster is thus named after isa? who dug it, Sassanidi $3^{2}$.

L.j?? Adraye, a village of Beth Garmai, Chast. 22. 5.

olig?, na;!l the Adriatic Sea. Col. 42, I I af. dele the words et Ponto to usurpatur, and col. 43. 5 the words Pontem includere. See Nau, Journ. As. 1896, 3i fo f. Add: BH. applies the name Adriatic to that part of the Mediterranean which is opposite to Tanis in Egypt, Chr. Eccl. 359. 19.

abi? Pers. of اذه Adarmeh, a bishopric between Nisibis and Mosul, Pers. Mart. 203, note 1607 , Hist. Mon. i. 37 pen., Chast. 8 ult., х 2. 4, asojo?l, Syn. Or. 6r9. 5 .
Woigl n. pr.f. Adramanag, foundress of a monastery in Hirta, Chast. 70. 4 .

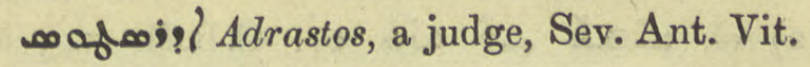
36. pen.

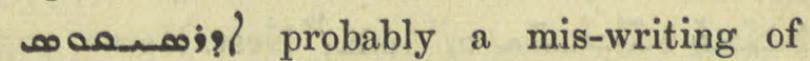
صomigl the Adriatic sea, q.v., Schatzh. I26. 11 .

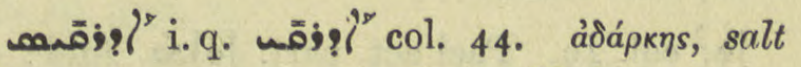
efflorescence on marsh plants, Med. I 45. 21 , 147. 10, nasi? ib. 149. 4 and col. 44 .

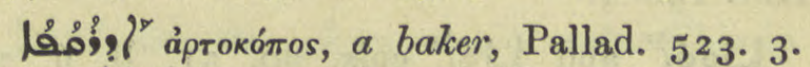

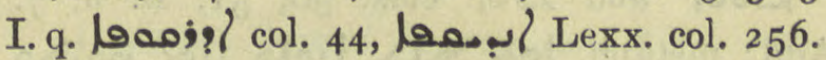

ơ a pestle. So l. 23 should be $\sim \hat{\hat{q}}\}^{\prime}$ q.v. col. 4 Or. Dele from "Etiam" to "percussit" 11. 23-26.

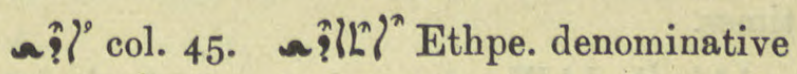
from la?l, to take specific shape, be formed, añ pt. 5 .

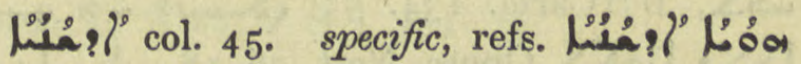
the origin of a species, But. Sap. N. Hist. iii. ii. sect. 3 ,

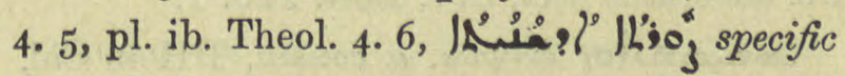
form, id. N. Hist. iii. ii. sect. I, IL'an

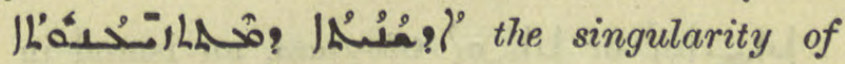
movement pertaining to that species, ib. iii. 5 .

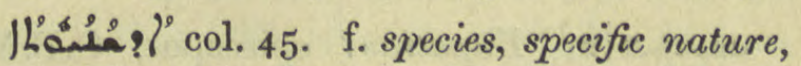

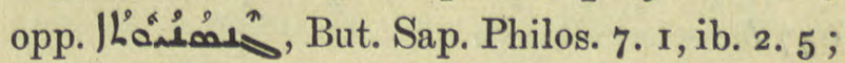

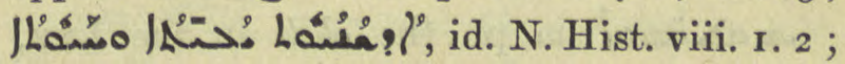

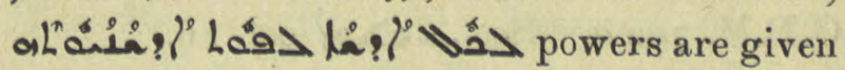
to each species according to its special nature, ib. iii. ii. sect. 4 infra, ib. Isag. ii. 2.

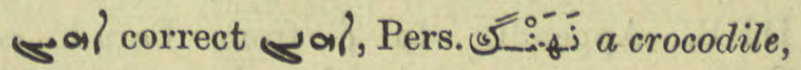
Kal-w-Dim. ed. Bick. 26. 15, 28.3 .

is $>$ al title of a high Persian official. Cf. rabbinic אמרכל with $ל$ for ר, Syn: Or. 77. 14 and note.

مa Pers. آهلك lime, quick-lime, BB. under 10:0.

فjal probably ابو هارون a name for the nightingale, Natur 33. 7 ; cf. Qazwînî i. 406.

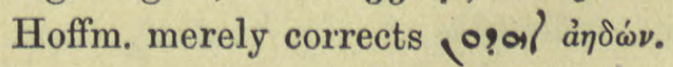

counoial pr.n.m. Pers. A. M. B. ii. 579. I 7 . 
의 col. 47,f. Add : I) §, Omega, Galen. ap. ZDMG. xxxix. 248, 289, Pallad. 495. 3, 4 . 2) $\epsilon \hat{\text {, }}$, 3) For emphasis, indeed, entirely,  j'ai admis tout uniment (le nom) de Mère de Dieu, Nau transl.

Jol col. 49. Pael act. part. Jổ. Add : to

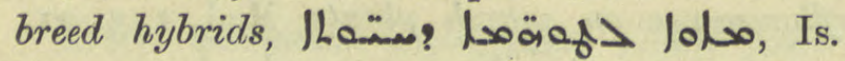
Ant. 198. 4.

Lioo: Add: metaph. Joa la lols lool pando oomac1n he was on intimate terms with them, Jab.233. I5. A compartment or chamber of a glass furnace, Chimie roo. 22, ro r four times.

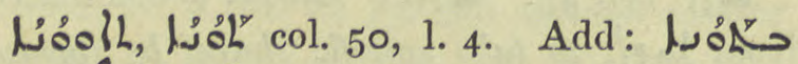
ه. spite in its inner heart, Natur 18. 12, Iól

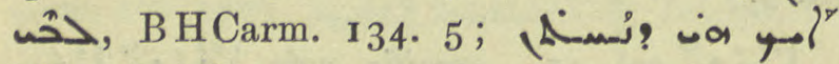

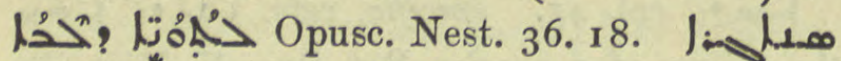
Wool? advocate of the treasure-chambers, i.e. advocatus fisci, Sev. Lett. 220 . I.

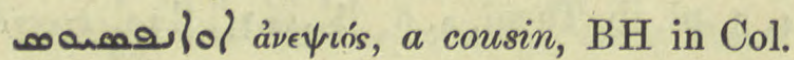
iv. ro: see s s.engr? below.

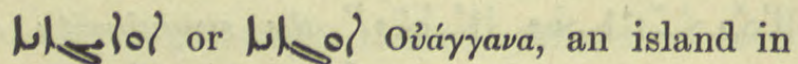
the Red Sea, Jac. Edes. Hex. 20. 6.

andolol Eupatius? episcopus, C.B.M. $647 b$.

no>asol m. pl. the obeli in the Lxx, C.B.M. $79^{2} a$, See na col. 16.

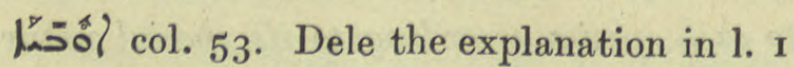
of par. ' Bia, $0 /$ violence, or woe is me, DBB. Appendix to $?$ from Parad. ed. Tullberg 28, note I3; lخol, Pallad. 159. 2, 3 ; Hist. Mon. ii. $35.5,6$; A.M. B. vii. 86 ,

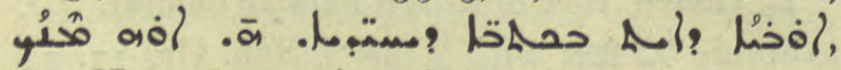
Op. Nest. 6. 15, Anecd. Syr. ii. I35. 14.

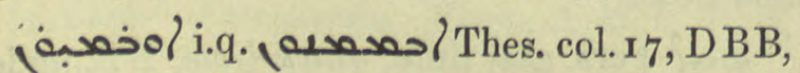
col. $\mathrm{I} 7$, a wood so hard that it will not burn and insects do not attack it, Gest. Alex. 2 I 9. 17.

↔ol Bishop of Hamadan, ZDMG. xliii. $403 \cdot 9$.

en/onsol Copt. пбогc, O Lord, Pléroph. 104. I, R.O.C. iii. ${ }_{3}^{6} 3.19$ in loc.

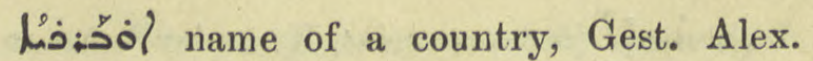
I80. 14 .

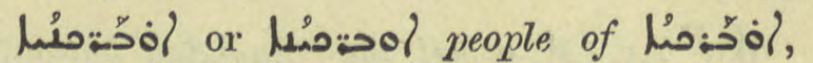
Gest. Alex. 18r. I.

l’ốl col. 53. rhus coriaria, sumach, ref.

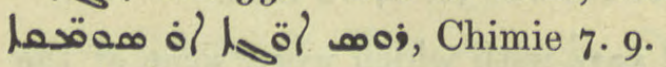

ofeloy of Augusta, the province wherein are Mabug and Samosata, Nöld. F. S. i. 470.

Hea ol Augusta, a city of Cilicia, Sev. Lett. I $28 . \mathrm{r}$.

coflocy of col. 54.2$)$ 2) the month of August, ref. ZA. viii. 7. $2 \mathrm{I}$.

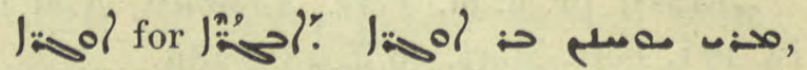
Protection 54. 3. See col. 7. 1 I af.

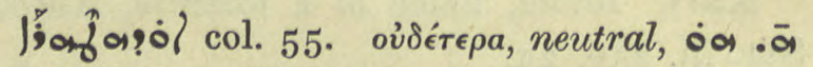

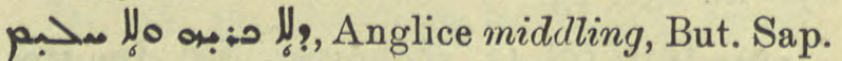

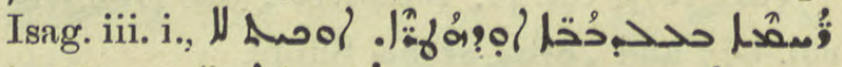

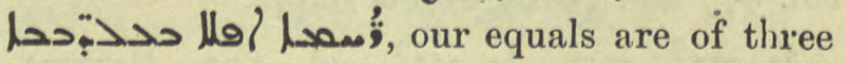
kinds, friends, enemies, and the indifferent, ib.

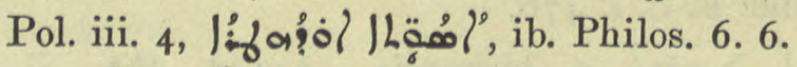

colvogol Aủdvvaios, Audynaeus, a Macedonian month, Sev. Ant. Hymns 255 tit.

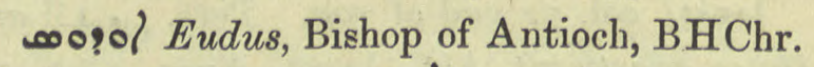

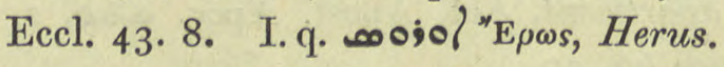

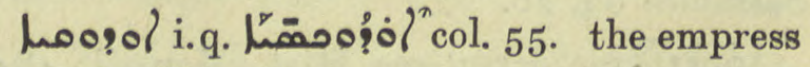
Eudoxia, Pléroph. 23. 5 .

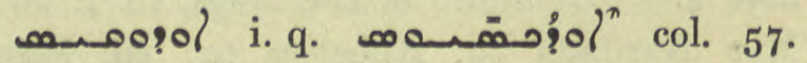
Eudoxius, Nöld. F. S. i. 474. ${ }^{1} 3^{2}$.

no.pol Euodius, Bishop of Antioch, BHChr. Eccl. 31. 18, ल.9lo?, ib. 39. 23 . na?aol, R.O.C. i. 399. 12.

j;̣ol ṽ $\delta a$, hydra, water serpent, BB. under (0)000i?a.

nojanj\};̣ol col. 57. quicksilver, ref.

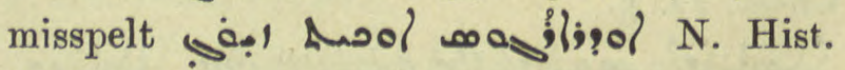
iii. ii. sect. 2 .

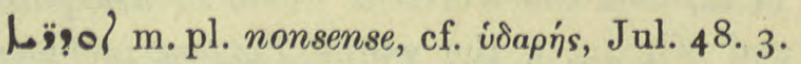

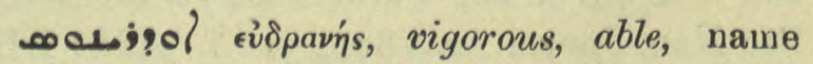

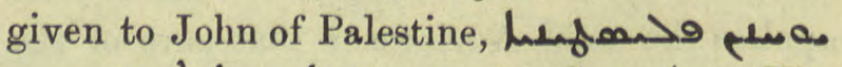

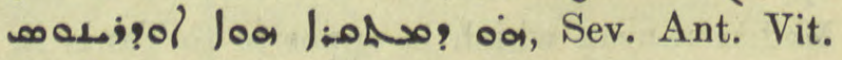
63 ult.

nof Uch, a village where Mar Bishu was buried, A.M. B. iii. 620 .

nofo? the North-West wind, Med. 533. I. it must be a corruption of some Greek word. 


\section{orelretafor}

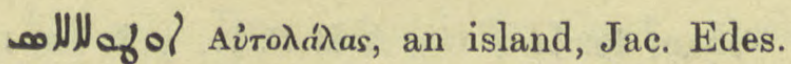
Hex. 19. 12.

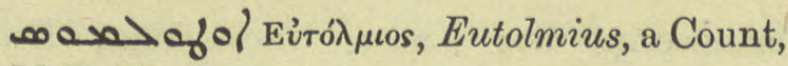
A.M.B. iii. 327 .

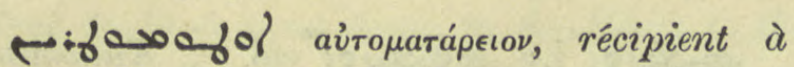
tirage spontané, Chimie 240 , note 4, ए:- مofol, ib. 26 I, note I, I7. I 4 .

लóf̣l a bird which mimics man, odjol

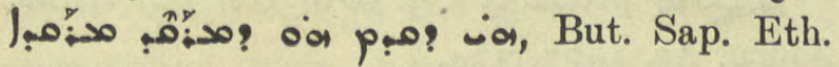
3. I, N. Hist. vii. 4. 2. Perhaps àzós, the horned owl.

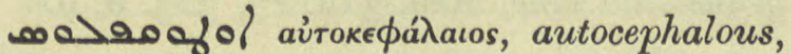
gloss. مصبص: Syn. Or. 182. 27 ; लaحarafol, ib. 148. 2.

lefol what? "flowers of the red vine,"

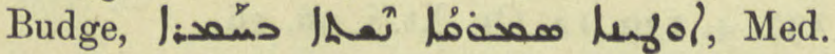
569. 10.

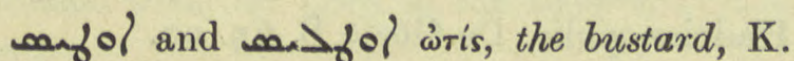
under مُحمُ col. 1544.

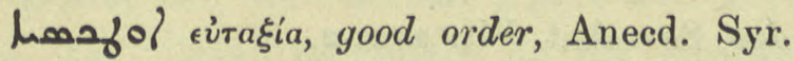
iii. II8. 22.

लáf-jol Otreius, bishop of Melitene, Nöld. F. S. i. 47 1. $6_{5}$, of Arabissus, ib. 66.

| A.M.B. ii.576.n.2. Usually written l ina? q.v. col. 18 and above.

Hool col. 63. Euchaita. Add: a third city of this name is in Egypt, Stud. Sin. ix. IOI. I3.

lyـتol m. pl. Eủxîtal, heretics called Adelphians, C.B.M. 926 b, 956 b.

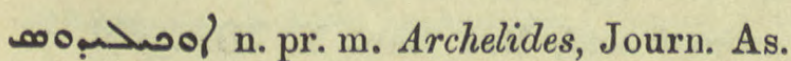
I907. 418.

as/onol Auxenon, a presbyter of Apamea, Nöld. F. S. i. 473. I 17.

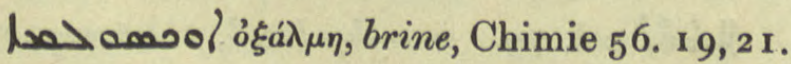
namsol var. nanosol, the river Oxus, Jac. Edes. Hex. 13. 13, 26 , 10.

nowool col. 64. Dele the sentence in 1. 3 of par. « $\xi$ ios, worthy, B H Chr. Eccl. 799 . 24. $\S$ ii. 553 .

heovol ò $\xi \hat{\epsilon} a$, sharp accent, Epiph. De Mensuris 7. 6.
ץ.

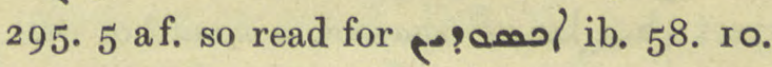

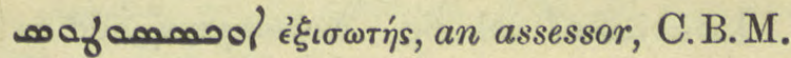
$7 \mathrm{I} 8 b$.

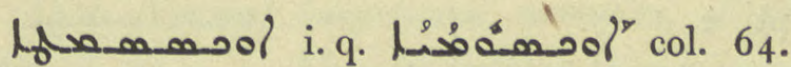
inhabitants of Axoum in Ethiopia, Aneed. Syr. iii. 330. 2.

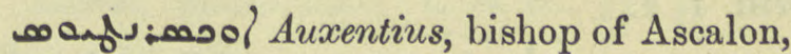
Nöld. F. S. i. 468 . 18.

l: 00 col. 64. $\ddot{\chi} \propto a$, ochre, refs. Chimie 2. II , 44. 24 , 人: 0 ol ib. 279 , note I.

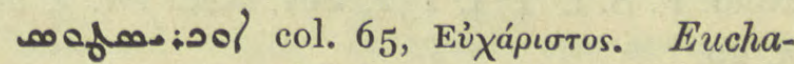
ristius, pr. n. m. C.B.M. 1070 b.

م 6. 4 .

l

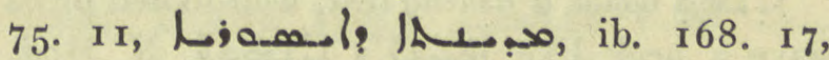

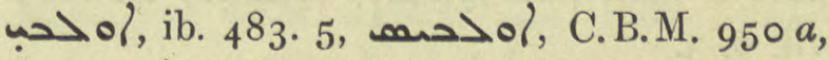
مس 20 / Nöld. F. S. i. 47 I. 77.

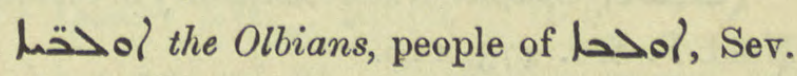
Lett. 20.8.

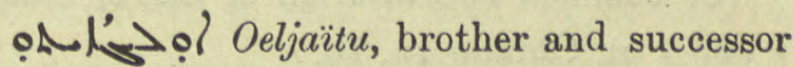
of Kazan, Jab. 147. med.

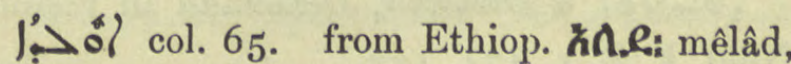
to gather together, collect. I) a heap of chaff, Lexx. 2) a heap of corn, store, Is. Ant. ii.

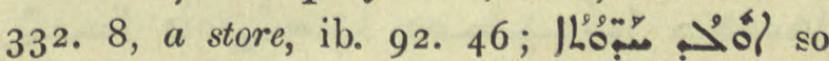
correct $\boldsymbol{L}^{n} \mathbf{l}^{\prime}$, winnowed wheat stored in pits, Hist. Mon. i. $2_{5}{ }_{5}$ infra; Sassanidi $63 \cdot 3$ o.

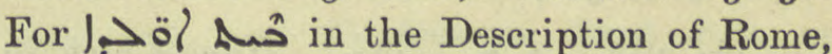

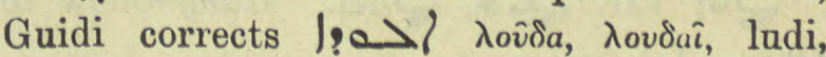
games, Zach. Rhet. Bull. Arch. Romae xii. 222. Cf.

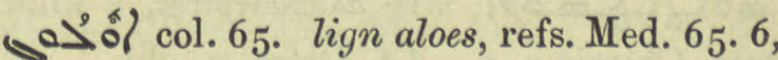
601. 20, مصe, B.V.M. I42. 8, I 43.4 .

nof col. 67. the river Karun, refs. Sassanidi 33. I I, Coupes ii. I54. 7 .

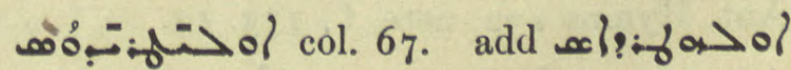
a girl flute player, Tim. 1, ed. Braun 2,

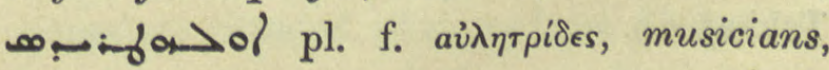
Hippoc. xxi. ult. and 4 a f., مبr مa> o?, ib. xxii. $1,2, f$. note 6 . 
a. frool BH. ap. Hebraica iii. 25 I.

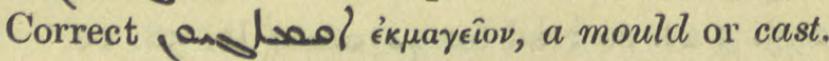

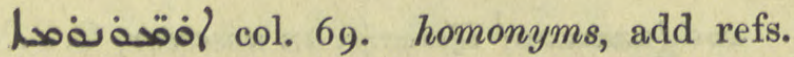
Jac. Edes. in Arist. 21. 2, But. Sap. i. pt. 2 .

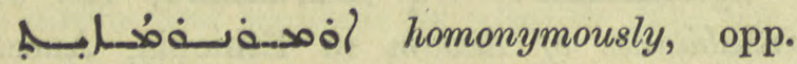

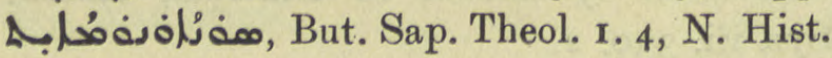
viii. $1,2$.

m-mosol Ammonius, philosopher of Alexandria, Sev. Ant. Vit. I6. 8, 22, I 3.

noluesool ó Ev̉paveias, Eumenia in Phrygia, Nöld. F. S. i. 474. 132, Sev. Ant. Vit. 3. 26, 5. 20.

no:- ool Homer, Coupes iii. I 10.

↔ $\infty$ of Aymerus, a Frank, BHChr. Eccl. 597. 23 .

مُن ơ ó ócú, a fishing line, web-footed birds

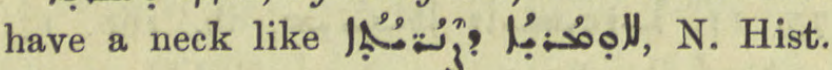
vii. 6.3 .

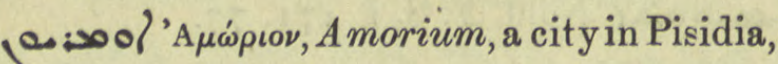
Nöld. F. S. i. 473 . 16.

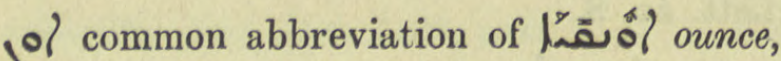
? है 4 oz. Chimie 12. 19.

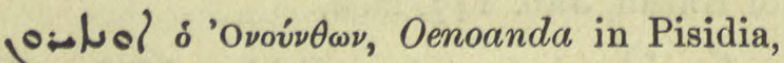
Nöld. F. S. 1. 474. 123.

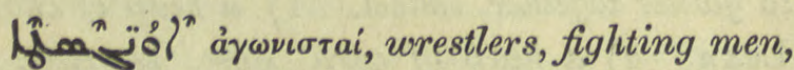

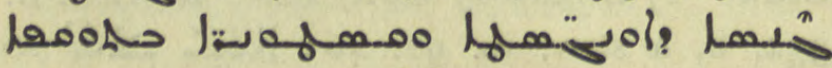
, But. Sap. Pol. I. 3 .

एकं var. स्a, woa. Hang-Chow in China, Jab. 29. I.

nowa.sol col. 72. 1. Add: nona. of

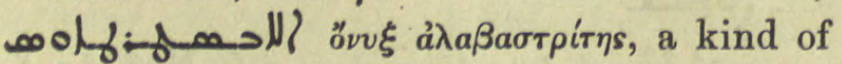
talc, Chimie 9. 6. Cf. Diosc. v. clii.

lonsovol ä $\gamma X^{\circ v \sigma a, ~ a n c h u s a, ~ a l k a n e t, ~ m a l l o w, ~}$ Chimie 2. 9.

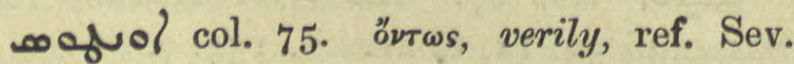
Ant. Hymns 1 32, note, f., I73. 12.

acrivol var. jaoffol the Henoticon? Tekkaf. 79 .

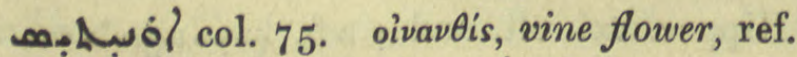
Med. 212. 14, 18. Cf. Lefol above.

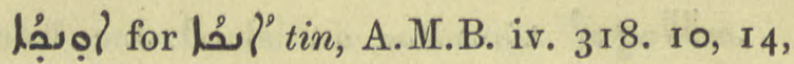
but $\mid\}^{y}$ ll. 8, 12, I5, 319. 1, 4. One manuscript has fnoo? throughout, Bedj. in loc.

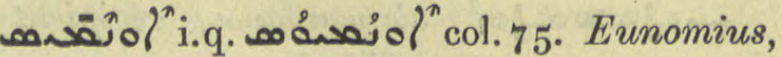
heretic, Theod. Mops. סص 5 5ars. ed. Ming. 360 , note 4 .

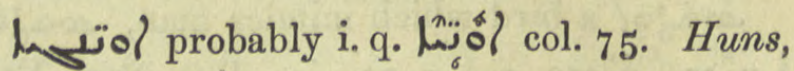
Journ. As. $1907,334$.

مـن oivá $\nu \theta \eta$, wine made from the flowers of the wild vine, Med. 322. I3, 323. 6, 9.

loof col. 76. 2) the Oasis of Egypt, add refs. Anecd. Syr. iii. 100. 2, I I 9. 18, leolo?

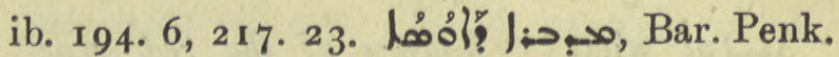
137. 4, 139. 12, Or. Xt. i. 96 . 16.

Lusool pr.n.m. Eusebianus? bishop of Hadrianopolis, De Goeje B. 65.20.

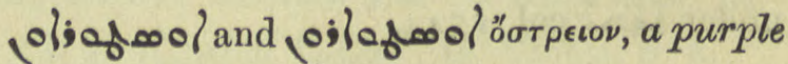
dye, Sev. Ant. Vit. 2 I 2. 2.

s anaffool pr.n.m. a priest commemorated on June 23 , Or. Xt. i. $3^{1} 5$ med.

entsool Eustathius, bishop of Metropolis,

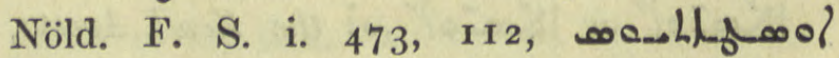
Eustathius of Brusa, ib. $475,139$.

صaـool Hosius, bishop of Cordova, Sev. Lett. 322 . 1 .

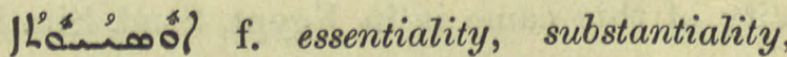

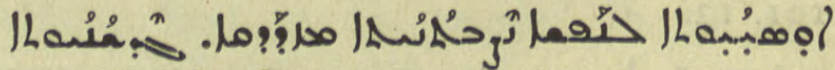
| to the vegetable soul, fortuitousness to the animal soul, But. Sap. N. Hist. viii. I. 4 ;

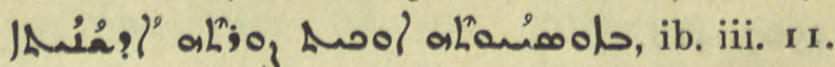

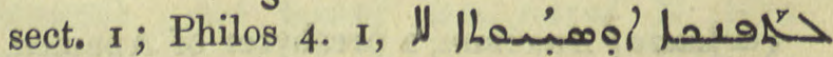

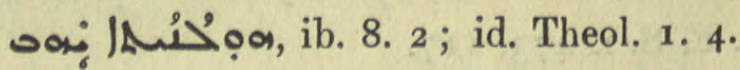

لlمól col. 79. marshy land, a swamp, ref. Hist. Mon. i. 229 ult. 23 o. 3 .

nolutool Eusanius, bishop of Eucarpia, Nöld. F. S. i. 474 . I30.

eneool Ausonius, bishop of Sebennitis, Syn. ii. Eph. I 2. 22.

Lulonool wecanria, Greek form of luad Hosanna, C.B.M. $543 b$ bis. 


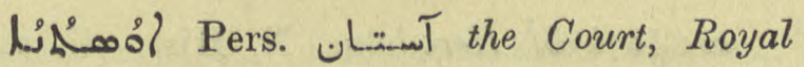

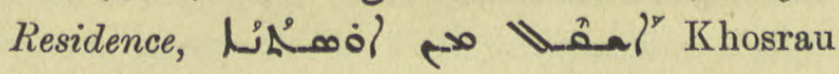
Nushirwan left the Court, Jab. 227. I3; كَn the Shah-in-Shah went down to the Court to the Cities, i.e. to Seleucia-Ctesiphon, ib. 266 . I3. Aool? IAs Maison de la Dynastie, Chabot, but see 1 (م) 0?. A place in Armenia N. of Arzanene, Syn. Or. 34. 9, 38. 3, ro9. I 5 , also called oli\}? A Aoo?, ib. 43. I7, ZDMG. xliii. 396. Iо. See Marquart I69.

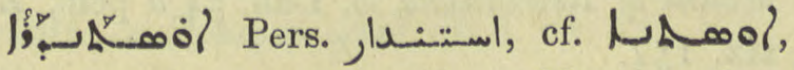
Governor of a province, Pers. Mart. n. 832, Tabari 448, Syn. Or. 77. 14.

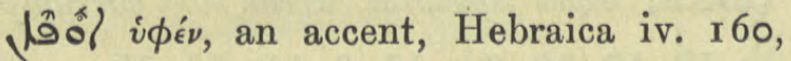
Syr. 2 .

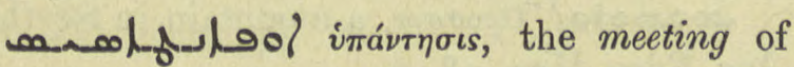
Simeon with our Lord in the temple, C.B.M. $273 a$.

イ 54. 14: الغُدَين al-Fudain on the Khabur, Nöld. note to transl. 46. Or rò 'Anáóvas of Procopius, perh. Tell Âbâd in Tur Abdin, Hoffm. ib.

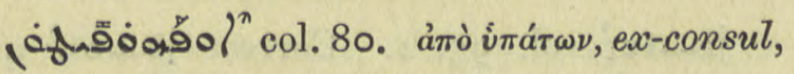
ref. Pallad. 192. 20.

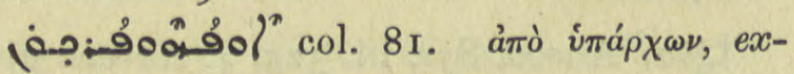
prefect, ref. Pallad. 202. 6.

uา Hebraica iv. 168 , Syr. 2.

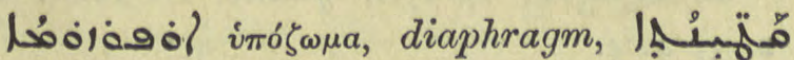
. 3 .

م mark -, Epiph. 7. 9 .

ex.m.ja.sol m. pl. aphorisms, Hippoc. 2, 3 tit.

100\}, Heb. זָ, Uphaz, Hex. Jer. x. 9 ; Psch. ڤึo์ Ophir.

Sysol pr.n.m. Optatus, A.M.B. iii. $3^{2} 5$.

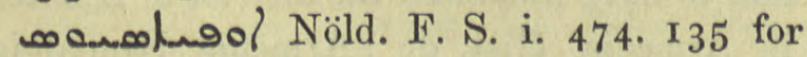
م

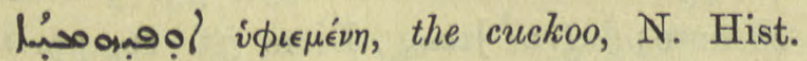
vii. 3.2.

(فं9o) col. 83. opium, ref. Med. 60r. ro. Usually

2716
| which are barren, N. Hist. vii. 5. 4 .

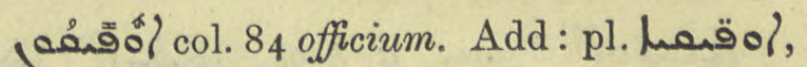
Syn. ii. Eph. 32. 22.

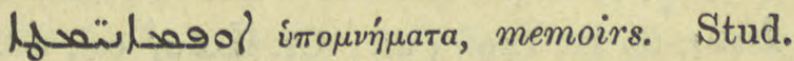
Syn. ii. 15. I, 3. Usually written fos q.v. col. 997 .

hesol place-name, C.B.M. 7 1 $b$, Doc. Mon. 2 1 8. 29 .

lę̄ol ỏnós, juice, Chimie 37 . I.

f:و ố col. 86. amphora, a wine jar. Add : a liquid measure equal to Heb. Nebel, see $\| \hat{\jmath} \mid \hat{n}$ col. $225^{8}$. It equals $I_{50}$ sextarii or pints according to Cyprian measures, 22 according to the Ascalonian, 18 with the Azotians, and 14 with the inhabitants of Gaza, J:- 0 ? oa إ

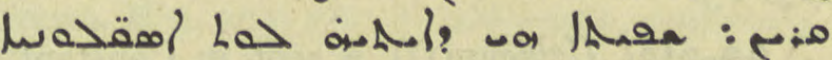
La)

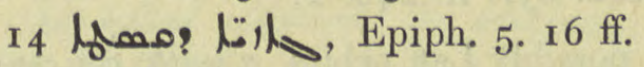

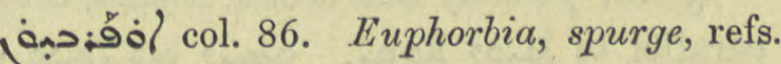
N. Hist. iii. ii. sect. 2, Med. 49. 8, 601. 16 ;

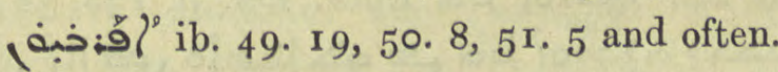

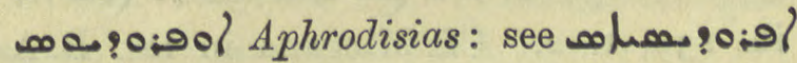
below.

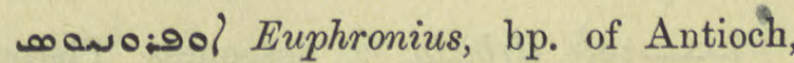
BН Chr. Eccl. 81. I 2 ; Ant. Patr. 298. 40. 26 ; Syn. ii. Eph. 14. 1.

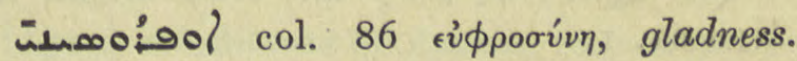
Add: pr. n. f. Euphrosyne, Stud. Sin. ix. 6r ff.

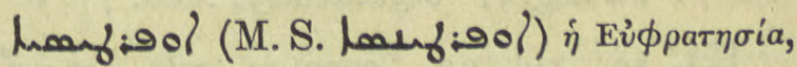
Euphratesia, i.e. the province Commagene with the Cyrrhestica, Sev. Ant. Vit. 270. 9.

م Sev. Ant. Vit. 104. 9, 106. I0; abbrev. mo:00/ C.B.M. 1049 b.

nouscool pr.n.m. Oecumenius, Count, Sev. Lett. 1 2. 7, 92.4 .

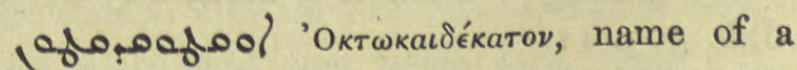
monastery situated 18 miles from Alexandria, Sev. Ant. Vit. 24. I, 89. $\mathrm{I}_{3}, \mathrm{Nau}$ in loc. R. O.C. v. 83 . 
مُمْفِ N. Hist. v. 5. 2.

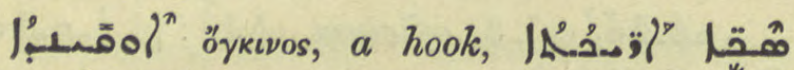

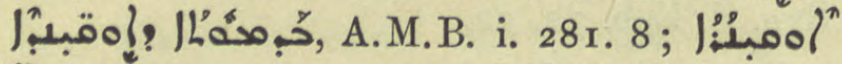

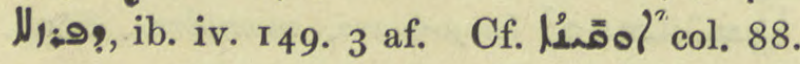

IRمُ الوقاية, a veil of silk and gold tissue, Dozy, Suppl.; Praet Miss. 20. 33; a veil of net, col. 2502 under مِحُم.

L 1 :0ol Eucarpia in Phrygia, Nöld. F. S.

i. 474.130.

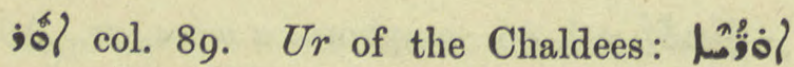
the people of Ur, Abraham is called läjo Warda $103 r$.

jol ' $\Omega$ pos, Horus, son of Isis, Arist. Apol. m. 12.

منص Urbicius, a minister of the Emperor Anastasius, Jos. Styl. 78. 19; Syn. ii. Eph. 25. 13.

aمajol Urbicum (?), a spot in Constantinople, Anecd. Syr. ii. 246. 2.

As;ol possibly Lat. orbita, a path, course; parallel to مبر مهلم loa Al foiol ha Ephr. Ref. ii. I38. 38 ;

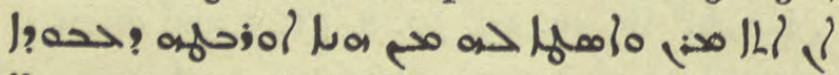
ib. r39. 1, 2. Cf. Mingana in loc. R.A.S. Oct. 1922.

husiol Urbicia, a deaconess, Pléroph. 94. 12.

joingol ápyúpıov, an obol, Diosc. ed. Nau 8r. r.

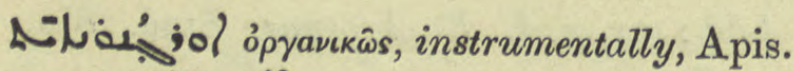

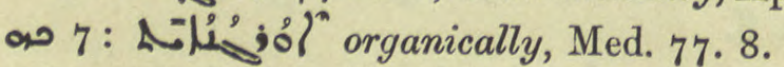

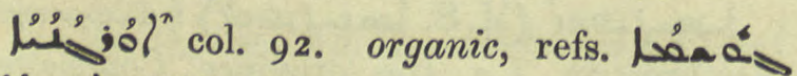

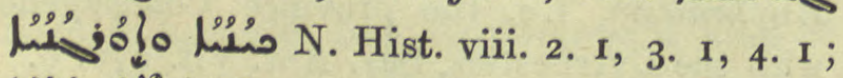

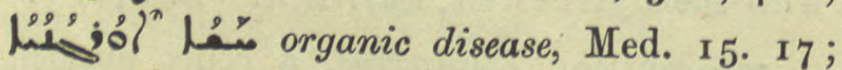

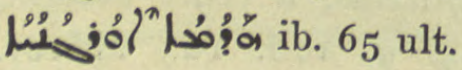

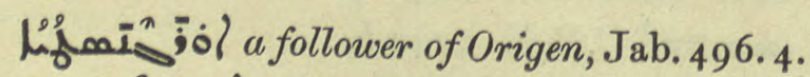
See

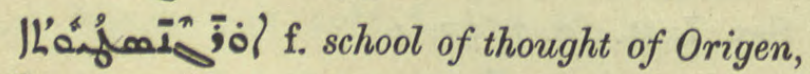
Jab. 503. II.

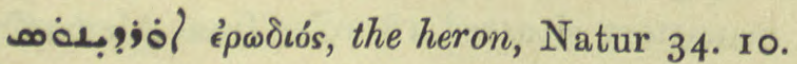
This passage is quoted in BB. col. 92 .

s.o이 pr.n.m. M. Z. 2 10. 19. ๓áḍojol Herodotus, N. Hist. vii. 2. I infr.

yojol a mountain in Beit Garmai, Chast. 5. $14,8.13$.

(ojol Uranius, bishop of Himerion, Syn. ii. Eph. I r. I3.

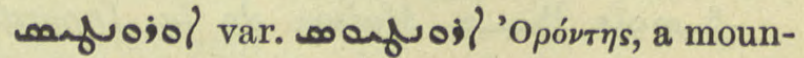
tain in Media, Jac. Edes. Hex. 37. 10.

๓ojol' col. 94. ö pos, a mountain, ref. God called Moses to the Mount of Revelations, S. Dan. 54 a pen; ZA. xix. 154 .

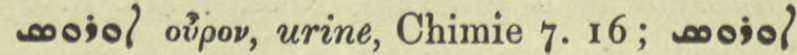

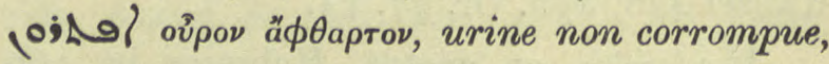
ib. 16. 10, 20, 24, 17. 2, I 5, 55. 21.

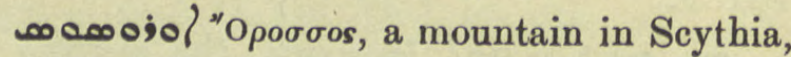
Jac. Edes. Hex. 38. 6.

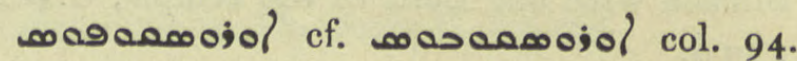
horoscope, Georg. Ar. 25. 16, 19.

masojol col. 94. Europus on the Euphrates. See nosoin?

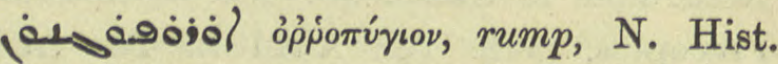

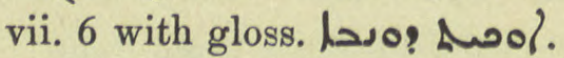

IAnjol col. 95. I. Correct IAwo; q: v. sub /معis.

H;ol Anecd. Syr. iii. 325. 4, Ahrens gives

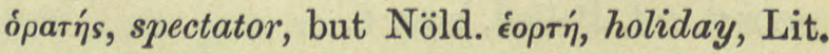
Zentralblatt. 1899, p. $13^{6} 4$.

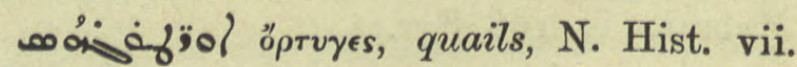
2. $3,4.4$.

ufjol aoprí, the aorta, N. Hist. vii. 1. 3,

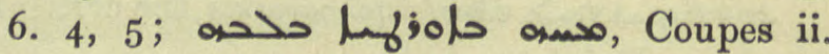
130. 9 .

1ำํㅇำ col. 95. Correct Urartians; the form Urtu was used for Urartu, P. Jensen ZA. vi. 66 note. This people is supposed to have been a remnant of Aramean autochthones, and to have inhabited the district of Anzitene in Armenia, Hist. de l'Arménie, Sandalgian i. 249. Add refs. Jos. Styl. 33. 9 transl. 28 note; lyjol /

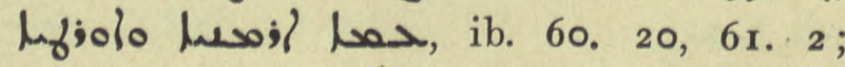

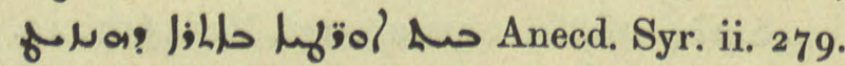

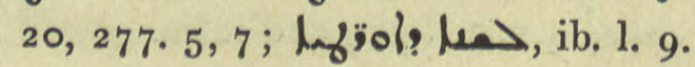




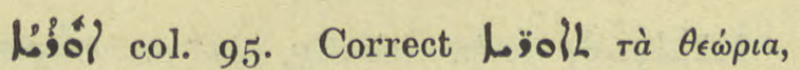
shows, races, Zach. Bull. Archaelog. Roma xii. 222.

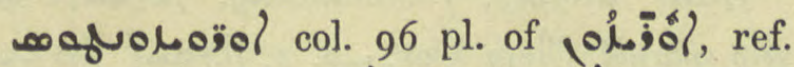
De Astrolabe 84 ; nöanduriol N. Hist. ii. iii. sect. 4 .

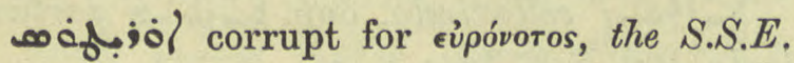
wind, Med. 533. 11 .

y'0l Heb. ㄱํ, 'O Uruk, now Warka, W. of Larsa, Syn. Or. 246. $7,32,247$. 19 traditionally i. q. jó?;

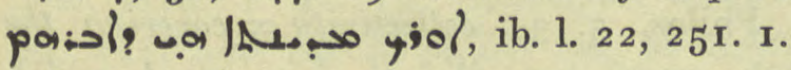

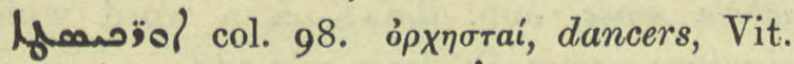
Mon. 89. II ; noflewiol C.B.M. $335 a$; miswritten mamoiol ib. $342 a$.

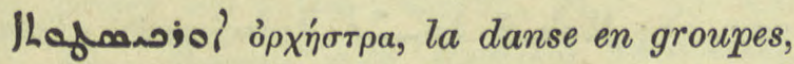
Sev. Ant. Hom. 54. 12.

pjol col. 98. Oü probably Rum Kala, Or. Xt. ii. 270; Sev. Ant. Vit. $325 \cdot 7$.

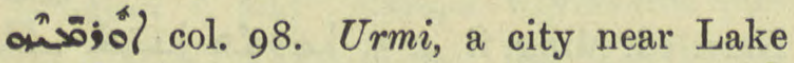
Urmi in Azerbaijan, now written wộl, Pers. أُرْمى Ar. Ar. Arld. N. Syr. Gram. xxii. note, Jab. 43. 12.

lusojol place-name, C.B.M. $705 a$, Doc. Mon. I57. 2 I.

jol perhaps abbrev. of oủántos, Uranius, bp. of Himerion, Doc. Mon. 253.7 .

Hoiol col. 98. river Orontes in Syria, refs. Stud. Sin. 96. 21 ; Dion. Ined. 46r. 6, 477. 16 ; enfigol, Jac. Edes. Hex, 25 . I.

مafojol name of one of the soldiers set to guard Our Lord's sepulchre, Journ. As. 1906, 283.6 .

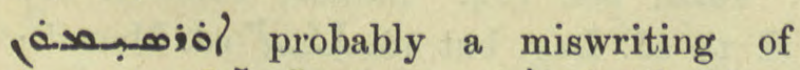

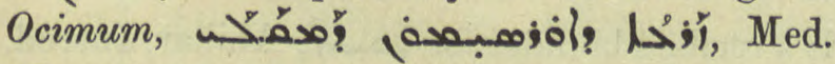
I82. 18 .

no9jol col. 99. Orpheus, add: noldiol, C. B.M. 427 a, صهి;ol Coupes iii. 10.

niol col. 99. Europe, Jac. Edes. Hex. 26. 9 ; u90jo?, ib. 3 1. 13 bis, $34.7,{ }_{3}$.

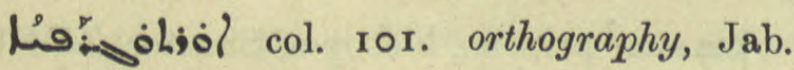
I. 22 note.

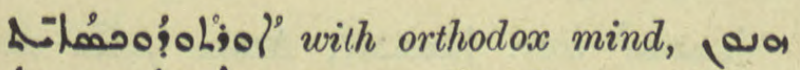

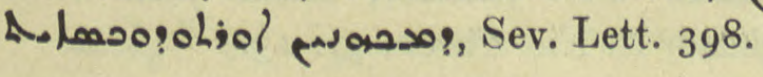

jikol or JilLoj? i 'E $\rho v \theta \rho a$, the Erythrean Sea, Jac. Edes. Hex. 14. 8.

jojlíol åpoov, a limb, N. Hist. vii. 3. ז.

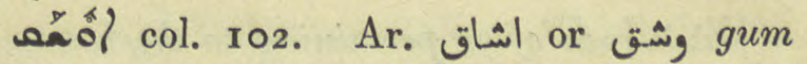
ammoniac, assafoetida, refs. Med. 56. 9, 9r bis, 587. 13, 602. 2.

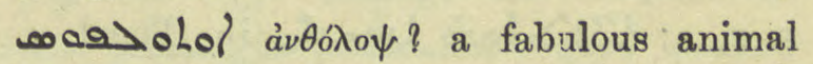
with sharp horns, J: مس م. Anecd. Syr. iv. 48. I I f.

nosolol pr. n. m. Autunus, BHChr. Eccl. 347. 7 .

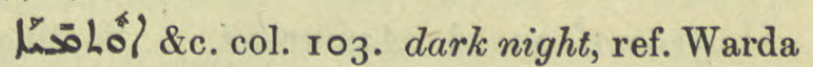
$246 r$, gloss. معeم

Lijulol" col. 103. l. 6 of par. his own power or authority, hishols dلا (oa) ? Syr. Rom. Rechtsb. 9.

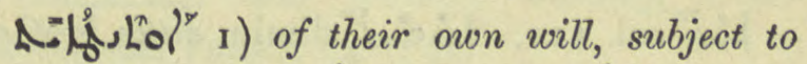

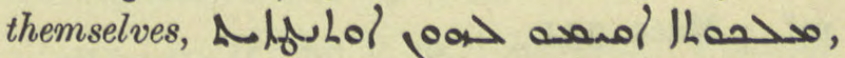

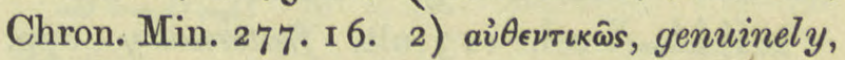
colophon, ap. R.O.C. 1912, 88, Syr. I.

lofulol ai $\theta \epsilon \nu \tau i k \dot{\eta}$, the original, loyulol 1 Lind? C. B.M. $7^{2} 5 a$, Syn. ii. Eph. 78.4 .

(a)

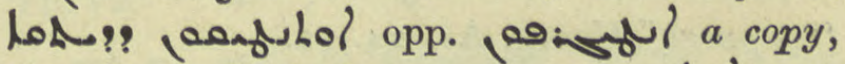
Syr. Rom. Rechtsb. 23 ; مـ nod l it is authentic, Syn. ii. Eph. 79. 2, 26 ; Nöld. F. S. i. 476 . 16.

|د:sll col. 103 ult. Dele and run on

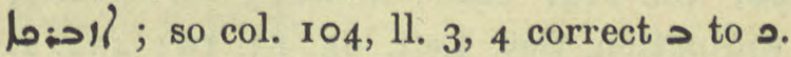

. H.joll inhabitants of Azotus, Epiph. 5. 19. a $>$ a) 901 barbarous or corrupt words in A.M.B. iii. 593. 4.

1! Izad, name of God, بـ!? la- l,

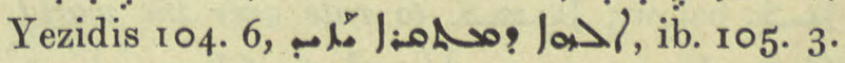
Y./l" a Persian town, A. M. B. iv. 217 , C.B.M. I181 $a, y$, ib. $1182 b$.

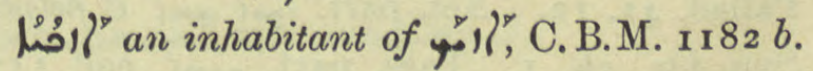

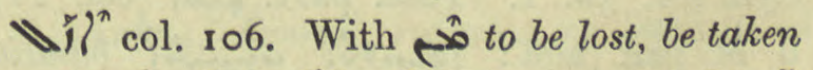

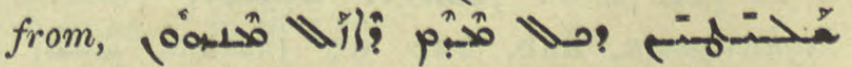
טo' thing which had been taken from them, Jab.

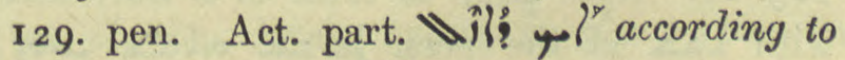
the current price, Dion. I83. I 2, I84. II. Pass. part. fem. hinم Isis goes 
to bathe, Isis wants a bath, said in derision when idols were brought out, Sev. Ant. Vit. 35. 6; Nau in loc. R.O.C. iv. 549 n. 3 .

年 of or pertaining to the heavenly bodies, But. Sap. N. Hist. Bk. ii. v. sect. 2 ; pl. ib.

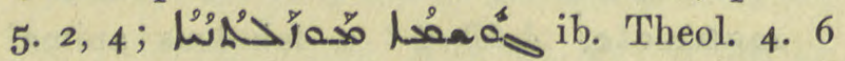
tit., pl. ib. 5. 3;

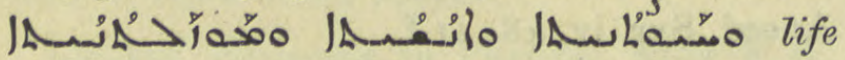
vegetative, animal, human, and celestial, $\mathrm{N}$.

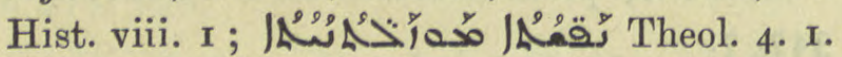

hyosoll m.pl. Azmurtaei, a Scythian warrior race, Ephr. ed. Lamy iii. 195.

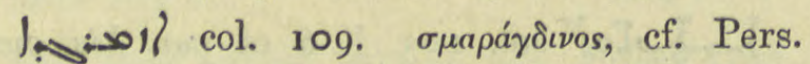
زمـردين, an emerald, Anecd. Syr. iii. 336. 3 ; Lexx. under مهند col. I 584 .

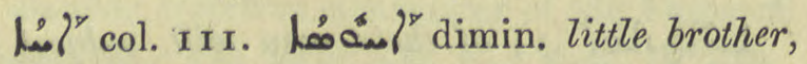
ref. 4 Macc. $35 \mathrm{I}$.

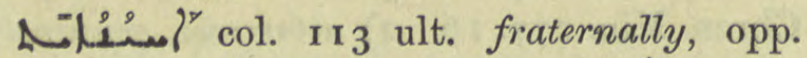

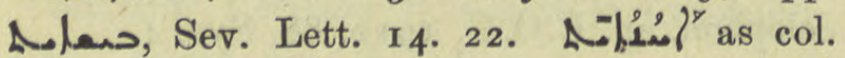
I14. I, Anecd. Syr. ii. 258. 2, A/uta? ib. 278.1 .

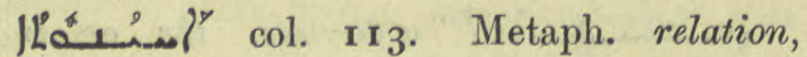
"rapport," Georg. Ar. 24. I 2.

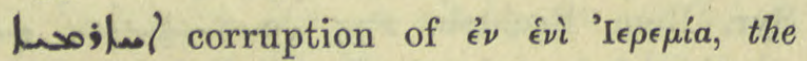
Book of the Prophet Jeremiah, Eus. ed. McLean 416. II, ZA. xii. I25.

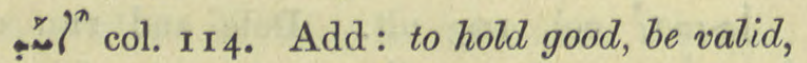
of ordination, Sev. Lett. $65.13,16 ; 45^{2} .2$. With "حمب to be incumbent upon, Loo alosaf Wh, Ephr. Ref. i. 35. 9, 41. With

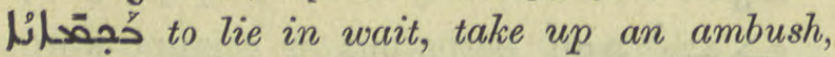
Natur 14. 2; رُ to regain self-control,

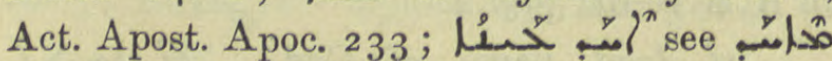

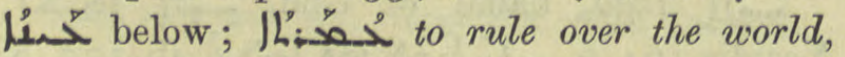
Gest. Alex. 34. 2 ; خـمخ

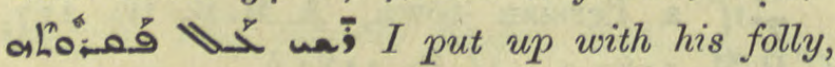

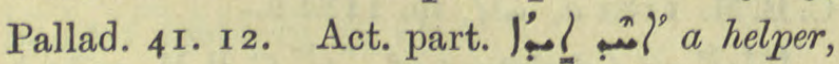

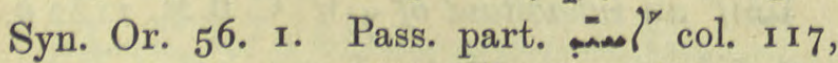

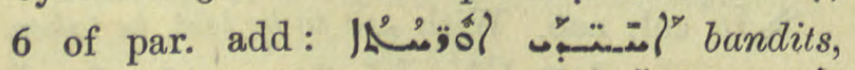

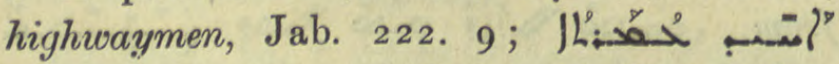

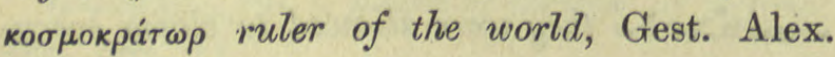
225. Ir. Aph. nool" to order of a workman with $L a\rangle$ of the pers. Hoin ala $\rightarrow$ al a customer ordered Joseph the carpenter to make a couch, Apoc. N. T. I5. 7.

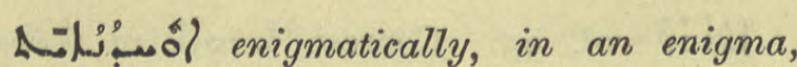
Syn. Or. 186. 14.

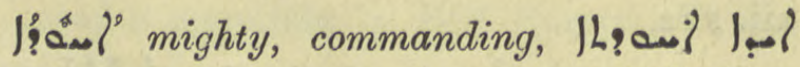

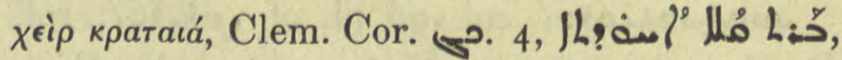
But. Sap. Periherm I. 5.

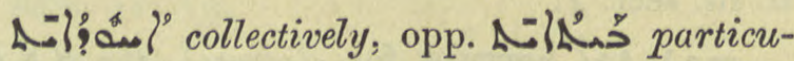
larly, But. Sap. Isag. r. 3 .

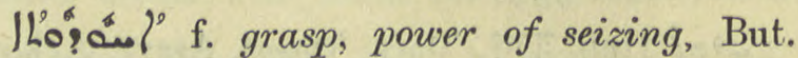
Sap. N. Hist. vii. 6. 6 ; closing, restraining of the womb, ib. 5.2 ; setting a limit, limiting, ib. Philos. 5. 3 ; collectivity $=$ covering the

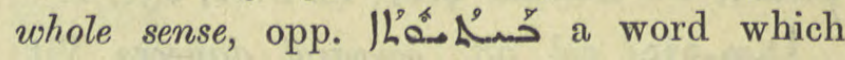
signifies a part, ib. Isag. I. 3 ter.

$1 L^{\circ} 0^{\circ}$ 3) self-restraint, abstinence, Pallad.

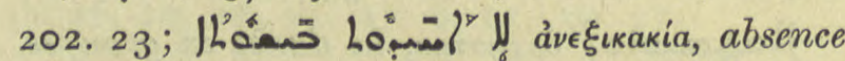
of resentment, forbearance, Sev. Ant. Hymns $5^{8}$ note $b$.

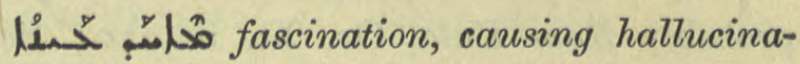

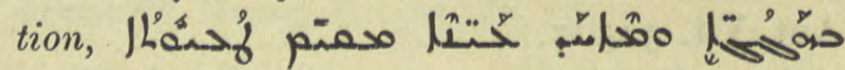

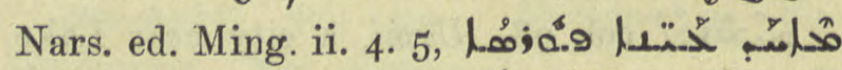

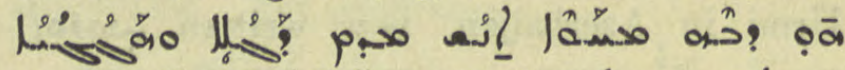

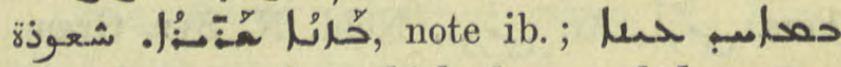
"مد- sorceries which they work by means

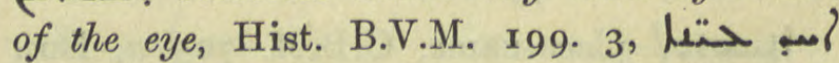
od? लans, ib. 204. 7.

/معם interj. Oh, if the reading be correct,

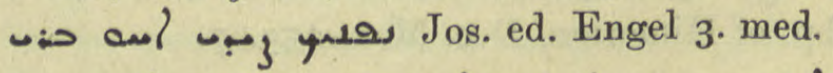

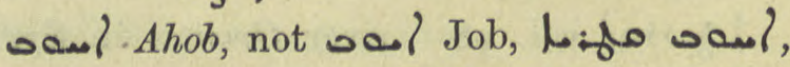
B.O. iii. i. note 4 ; see Chabot on this name, Journ. As. 1906, 273 sq. DBB. Proem xix.

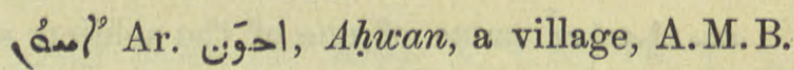
ii. 5.9

;onat col. 124. Ahikar, Tobit i. 22, f.,

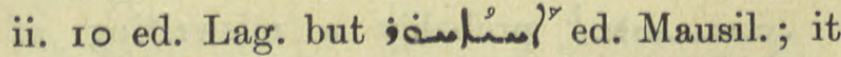
is a scribe's blunder for معمب?, Ahikar, Introd. xxix. See Meissner, ZDMG. xl. I9r, f.

אחי אחר the other brother = pr.n. Ahikar; cf. אחירם Num. xxvi. 38; Pers.

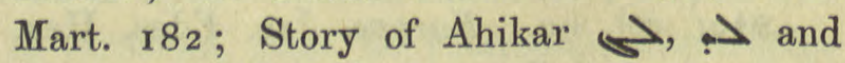
passim; Ebed J. Card. 100. I r.

\مane? pr.n.m., bishop of Shuster, ZDMG. xliii. 406.9 .

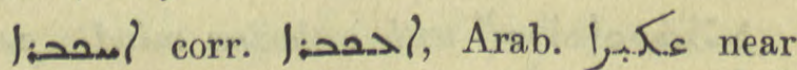
Baghdad, El Nis. Chron. 71. 13. 


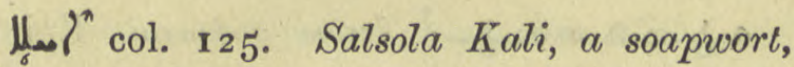
add refs. Med. 560. 6, مع a stem of

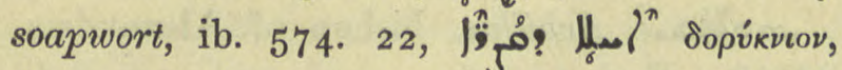
Saponaria, soapwort, fuller's herb, ib. 63. I 8, 22, 17 I. 7 .

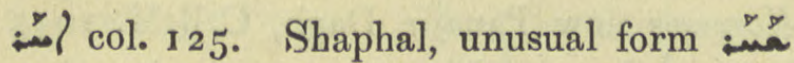
to leave, wa Jhoghl o:ma they leftabandoned-the country round Mosul on account of famine, Dion. I76. $21,177.5$.

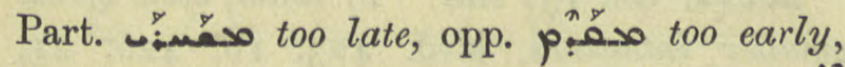

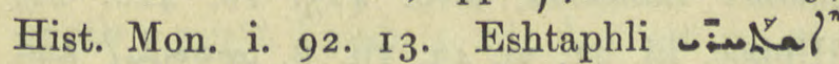
col. I 28, add ref. to be left out, be lacking,

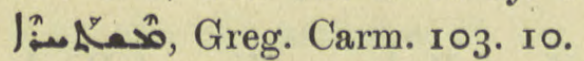

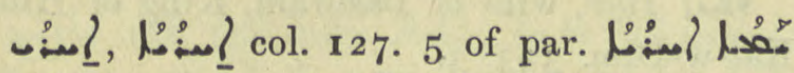
the farther sea = the Caspian, M.Z. 33. 43,

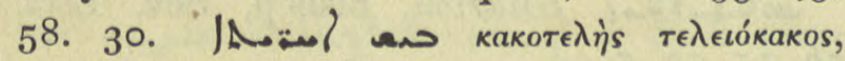
utterly bad, Pelagia 7. $3^{2}$. Rit. the second

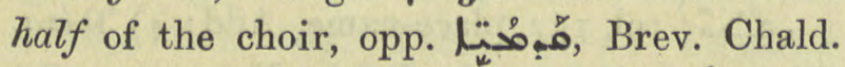
i. 26 pluries; Qdham W. 7, ff. Cf. مبُ

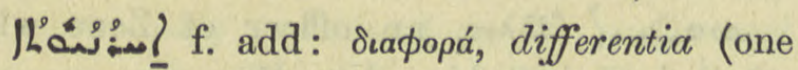
of the subjects of philosophy), But. Sap. Philos.

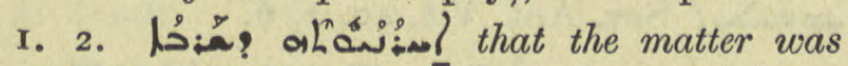
otherwise, Is. Ant. B. 85. I 2.

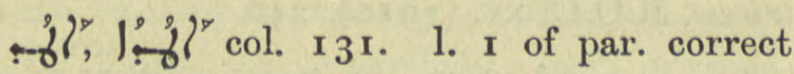

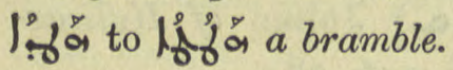

ofl a part of Arabia, Doc. Mon. 2 I3. 25 , C.B.M. $709 b$.

us a-fl a red dye, Chimie xlvii. i. q. u० q. v.

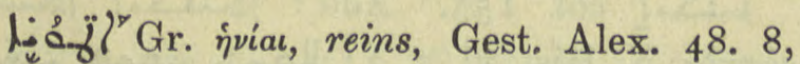
I 35,4 , ZA. xxii. 205 .

oofl Atys, lover of Rhea, Arist. Apol. \&, $7,9$.

ufl Ati, a village in Adiabene, Chast. ro ult., 16. . 8 .

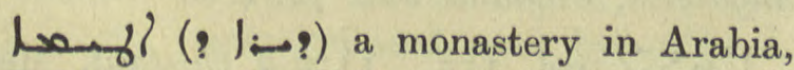
C.B. M. 7 I $1 b$, Doc. Mon. 218.25 .

coofl Atticus, a philosopher, ВН. ap. Hebraica iii. 25 I. I I.

hayl col. 132. Attic, Tim. i. ed. Braun

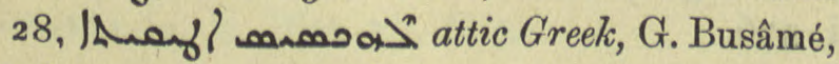
5 b. 4 af.

a fly col. 132. astron. the zone of the heavens, the heavenly sphere, lyo o أم D, N. Hist. ii. 3, sect. I, I I a

(an) Italicum, Italian, Chimie 10. 5. Usually for. See col. 146 .

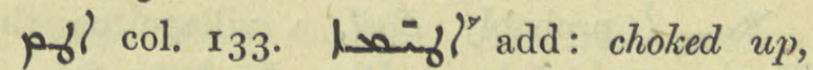

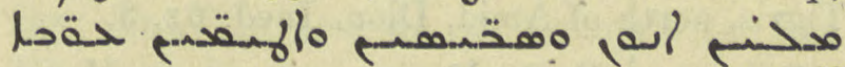

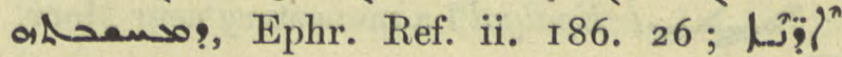

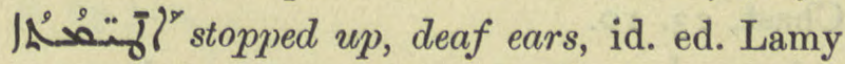

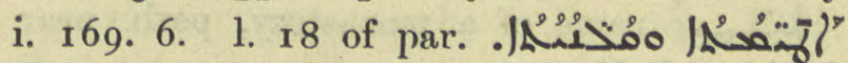

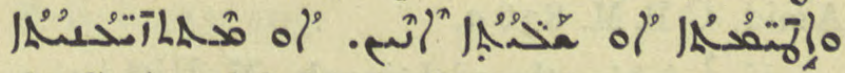
the Syriac alphabet is divided into consonants and vonvels and the consonants are either quiescent or vocalized, But. Sap. Philos. 6. 3 .

J'ásorifl add metaph. solidity, trustworthiness, Pallad. 94. 3 af.

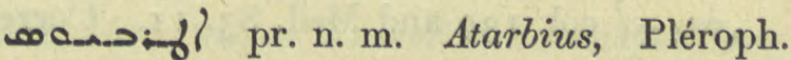
22. 9 .

150:fl\%. col. 134. lemon or orange, add

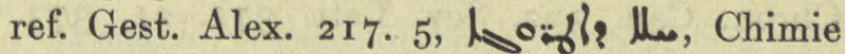

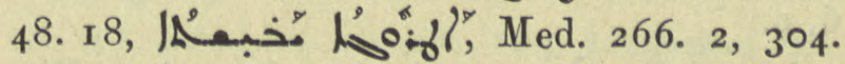
II, I 2 .

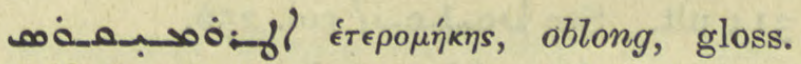

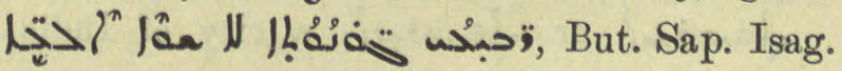
2. 4 .

boفij.fl col. I34. heteronyms, ref. But. Sap. ii. I, pt. 2.

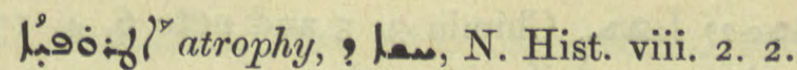
sas. fl Trajan, Emperor, Stud. Sin. ix. 93. 5, 94. 4 af.; ; : 8 ib. $1.7,94$ ter. and col. I 5 I 9 .

of interj. woe, Men an ol, Dion. I5 7 . 7 .

eafimole? the river Jaxartes, Jac. Edes.

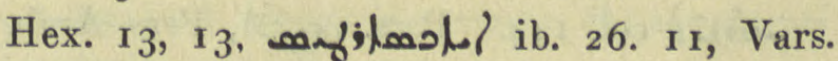

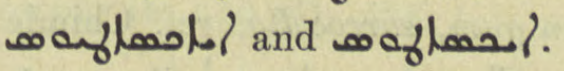

uسمام inakoai, refrains or burdens of hymns, C.S.B. 273 .

امح:|مام/مس Ibrasakis, transliteration of ABPA $\Xi A \Sigma$. The numerical value of the Syriac as of the Greek letters is 365 . Basilides called this name the name of God, Coupes ii. I07, tir 5 . 


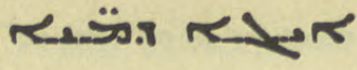

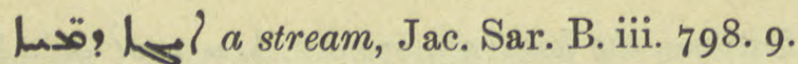
For Assyr. deriv. see Hoffm. ZA. xi. 24 I.

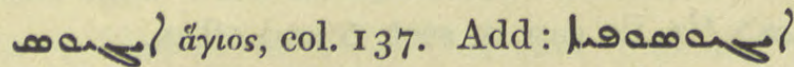
áyia roфia, Hagia Sophia, name of the great church at Edessa, BH Chr. Eccl. 65. 2.

$\checkmark$ l probably Égil, a village on the Tigris, north of Amid, Dion. Ined. 6r. 8.

I Egalgal? a monastery near Merv, Chast. 23. 10.

إ name of a monastery, perh. near Amid, C.B.M. $836 a$.

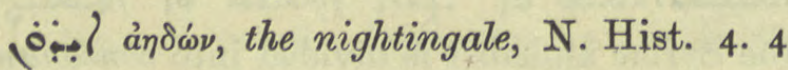
infra.

of C.B.M. $94 \mathrm{I} b$.

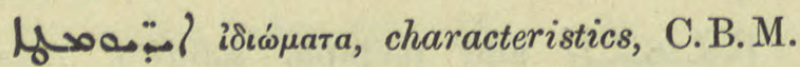
94 I $b$.

أبب بـف col. I39 and Med. 83. I1. Correct

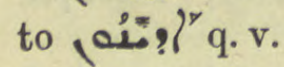

أمبمعام col. I39. an edict, refs. Jac. Edes. Chron. Can. 302, Sev. Lett. 372. $21,373$. 6,9 .

|

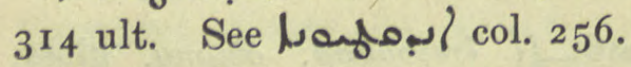

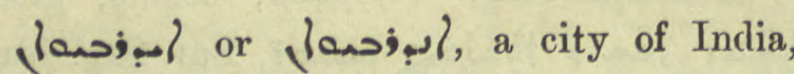
Kal-w-Dim. ed. Bickell r r6. 9.

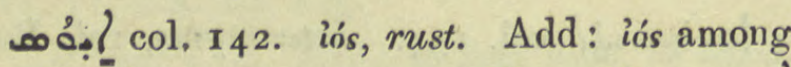
the Greeks means any metallic oxide, $\infty$ o. ?

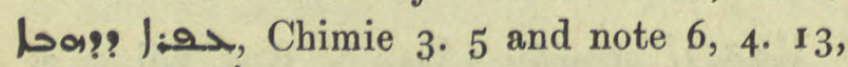
written 0 il, ib. 277 . not. I.

ตอัด! Joses, Mat. xxvii. 56 VHh. in marg. acos.

๓ofooe? Justus, Acts i. 24, xviii. 7 VHh.

a?k? a wheaten loaf, BA. under loos, col. $\mathrm{r} 693$.

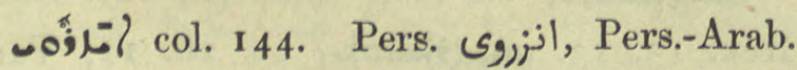
.ع. Persian gum, sarcocolla, ref. Chimie 7. 10 as gloss to 1000 : Oftener Lo; ح.v q. in Suppl.

Hon. 1 m. pl. Lat. intima, intimata? Tekkaf. I I5. Samuel translates Forschungen, investigations. But prob. /s

l $>$ l Attala, a bishopric in Pamphylia, Pléroph. $137.8,138.4$.

\section{dasors}

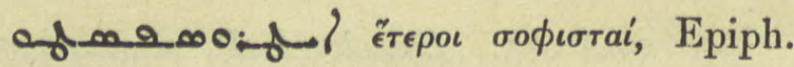
I8. 8 .

\& Achillas, bishop of Alexandria, BHChr. Eccl. 67. I6.

?محلص name of the mountain of Ephesus, Koressos now Panajir Dagh, C.B.M. 1045 , ib. 1048 b.

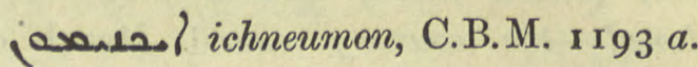

م smaller intestines, Med. 423. 16, 426. 21 , Hippoc. iii. 2 I, vi. 43 , vii. 8 , noalll and cola>.l, ib.

jلll Ilar, wife of Bashram, King of Hind, Kal-w-Dim. 333. 3 .

mas;lll Hilarion, bishop, Sev. Lett. 76. $15,77.5$.

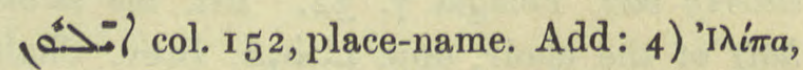
Ilipla, inland from the Baltic, Natur 45. 18.

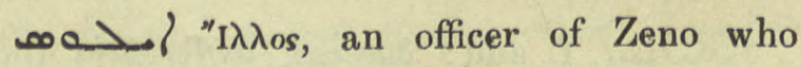
rebelled against him, Vit. Mon. I0. I3, I5.

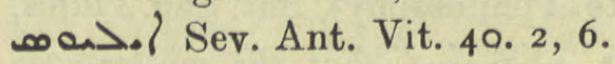

مa:- Lat. illustris, title, illustrious, R.O.C. xv. (1910), 240.

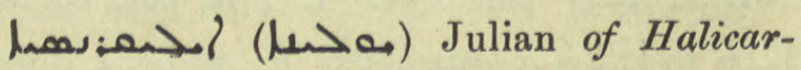
nassus, Anecd. Syr. ii. 246. 28.

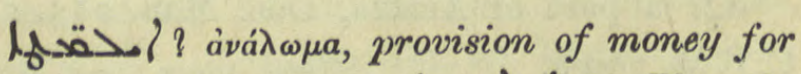
a journey, with gloss. إof, Pléroph. 48, ult. Syr. See loa

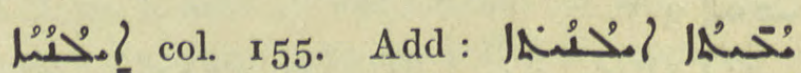

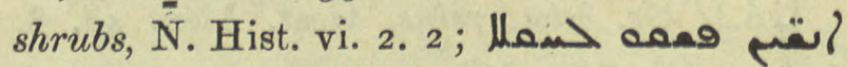
الخدشA| ofome explain the field (Gen. xxi. 33) as a plantation, others as a cornfield, Anecd. Syr. iii. 298. 4.

| Angleterre, England, Jab. 72. 4, 6.

l:"? col. I55. I) =a chestrut, Med.

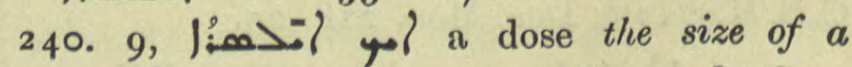
chestnut, ib. 197. I, 2 I, 3 18 ult. and often. 2) Corylus avellana, the hazel nut, filbert, Ar. Pfln N. No. 23 , Jac. Edes. ap. Nöld. F. S. i. 576 .

Lox, Imouth, name of a book on chemistry, by Zosimus, Chimie 214 , n. I. 


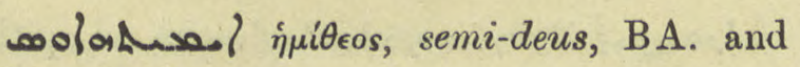
BB. under Johsoa.

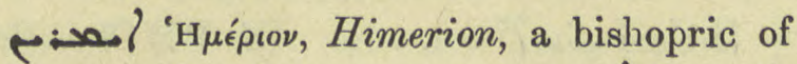
Osrhoëne, Doc. Mon. 253. 9, بـ/, Syn. ii. Eph. 11. I3.

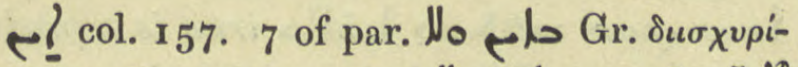

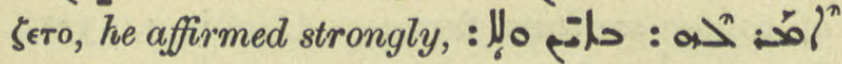
A.M.B. v. 80.7 ; ib. iv. 32 1. $16,612.6$.

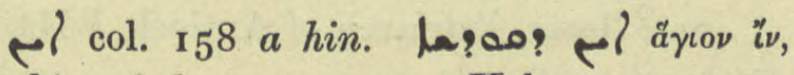
the hin of the sanctuary, a Hebrew measure, equals 9 pints, Epiph. 5. 20; lo; م od the greater hin is just double, i.e. 18 pints or a quarter of a metretes, ib.

(2) \{\}$^{n}$ col. $5^{8}$ infr. Add: to be influenced,

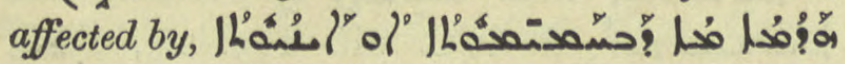

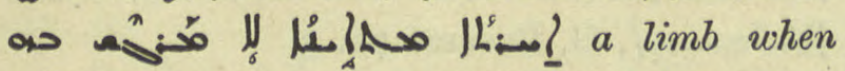
affected by heat or some other cause loses

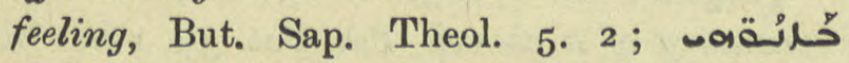

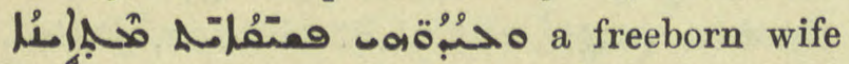
is more easily influenced in her ways and manners, ib. Econ. ii. I ; N. Hist. viii. 3. 2. infr.

مُ $\gamma^{\prime \prime}$ col. I 58 ult. relating to quality, opp.

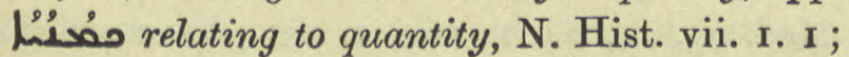

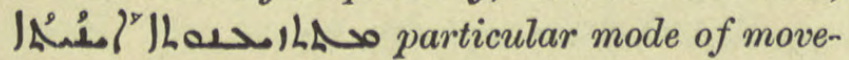
ment, ib. i. 1. 3 .

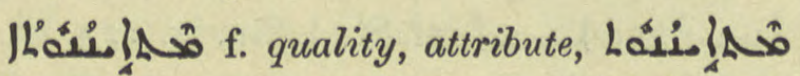
;)

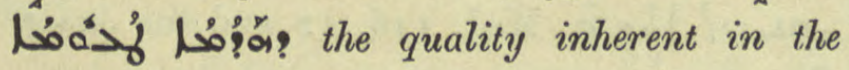
organ of taste, ib. Theol. $5 \cdot 3$.

أئين بد. an official, a torturer, Jab. 242 , pen. gloss. :

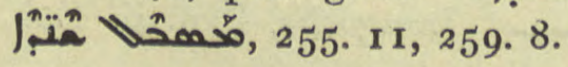

sox.1, pr.n.m. Indimus, bishop of Irenopolis, Syn. ii. Eph. 10. 25 .

sole? col. I59 is, ives, fibres, the four

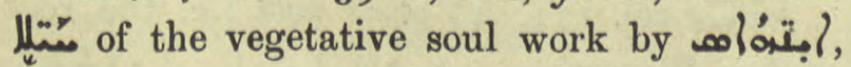
But. Sap. N. Hist. vii. I. 7, viii. 2. 2, Eth. I. 3 ;

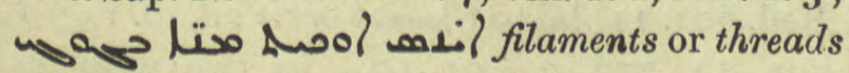
of a spider's web, De Astrolabe 74.

خَمسن: إبـة? بمه

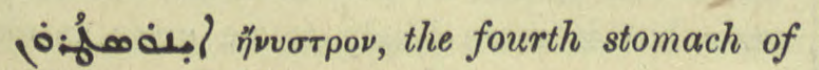
ruminants, N. Hist. vii. 6. 5. nous? Inzus, bishop of Corna in Lycaonia, Nöld. F. S. i. 472.96.

إ i. e. procession. C. B. M. $335 a$, miswritten lam>al, ib. $342 a$.

నْ col.16r. a copy. A list of signatures, Sev. Ant. Vit. 320. 8. A bishopric in Asia Minor, perh. a mistake for Isaura, De Goeje B. 65 pen.

مزasaura, a city of Lycaonia, Nöld. F. S. i. 473 . 103.

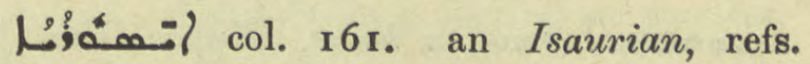
Pléroph. 47. 10, Sev. Ant. Vit. 28. 3 r.

Jisofeal a stylite, pillar saint, C. B. M. $707 b$ bis, Doc. Mon. 172. 3, 4. Usually written l: of ol $^{\text {q.v. }}$

l. books, Job, Psalms, Proverbs, Koheleth, Song of Songs, Epiph. II. 20, I L ib. 12. 4. Cf. eمendeo col. 2599.

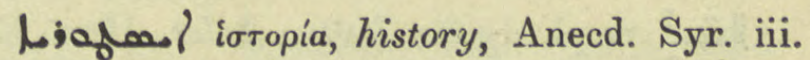
r6. 4 ; Liofol ib. I8. Io; Lident ib. II9. I.

صofifon? oirrpos, the gadfly, N. Hist. vii. 2. 2 .

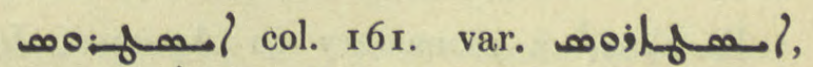

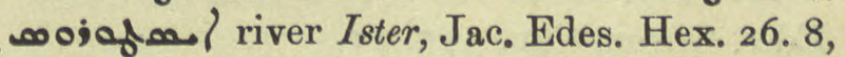
34. 10.

hiasenen? col. 162. equinoctial, ref. C. B. M. $\mathrm{I}_{44}$, De Astrolabe 8I.

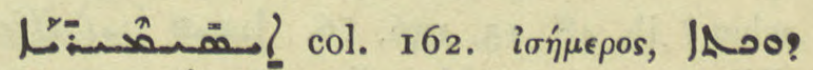
sic J:varem ?? the equator, De Astrolabe 268. 6.

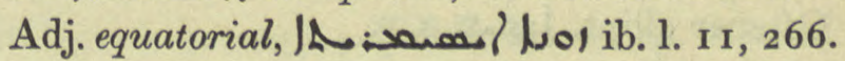

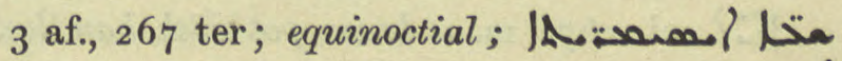
ib. $257.6,261$. 12, 262. 6.

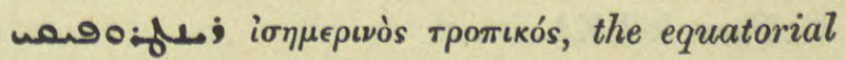
tropic, ib. 270 ult., 27 I. I.

|مسeم col. 162. Isis, Egyptian goddess,

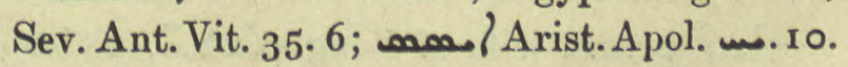

إمقهار m. pl. Spaniards, T? |jLl, Jac. Edes. Hex. I r. 3 .

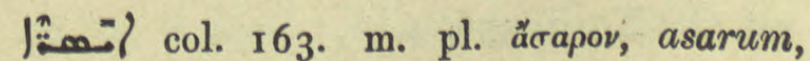
a kind of aristolochia or birthwort, Med. $\mathbf{1}_{5} \mathrm{I}$. I2, 17 I. I I, 238. 5 and often. 
4. DA. name of a monastery, C. B.M. $706 a, 707$ b, Doc. Mon. 163. 29, I 7 1. 25.

i for lin greedy, Aneed. Syr. iii. 243. 24 ; احس ib. 244. 26.

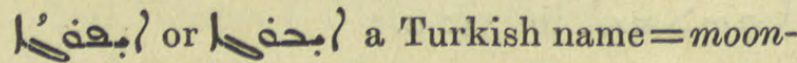
worshipper, Jab. I5. 5 .

م vii. $5 \cdot 3$.

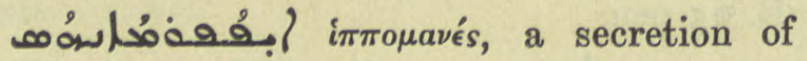
mares and sows, sought for enchantments, N. Hist. vii. $3 \cdot 3$.

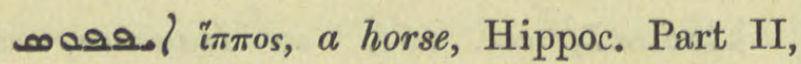
viii. n. 2 ; Warda $245 r$.

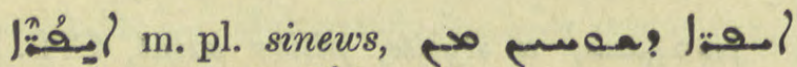

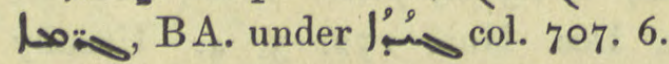

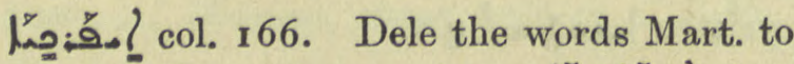
the end. The reference is to Apost. xxiii. $34 \mathrm{VHh}$.

mo: $65^{8} a$ ult.

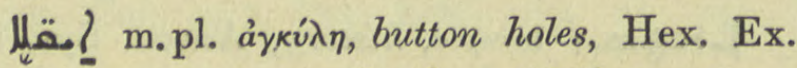

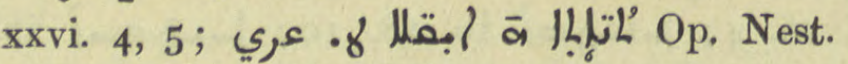
in loc. 137. 8. Cf. للمع col. 360 and infra.

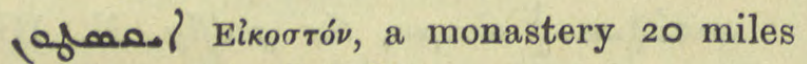
from Alexandria, Pet. Ib. 64. 19.

ANo:م:? a monastery in Apamea, Jac. Edes. Chron. Can. 575. II.

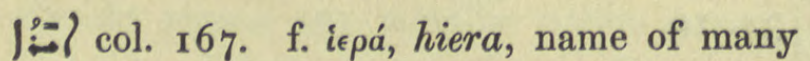
medicines or recipes, of Galen, Med. 49. II, 21 ; L what? ib. 48. 15, 136. 16. إب:ا وبم: Hiera Pikra, a stomachic, ib. 45. 16, 137. 13. Oftener ellipt. Jen q.v.

f:l col. 167. m. a brass pot with handles. Samaritan איר, var. of Heb. סיר Ex. xvi. 3. S. Fraenkel ZA. ix. I.

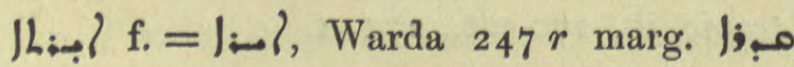

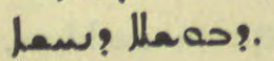

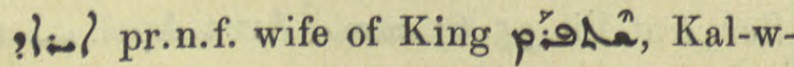
Dim. ed. Bick. 95. 20.

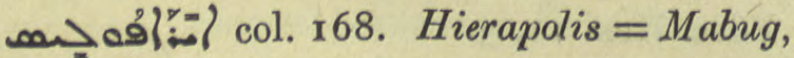
ref. Or. Xt. ii. 356.4 .

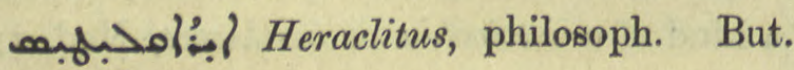
Sap. Philos 2. 3 .

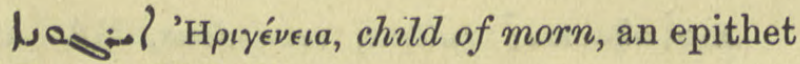
of Eos, A.M.B. iii. 2 279. 16.

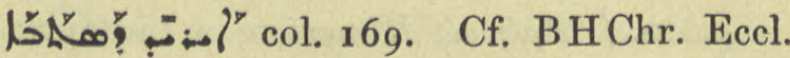
ii. ro9. 9 and n., Pers. Mart. 108 n. 97 I.

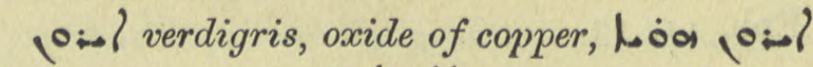
ofrun is a product of copper smelting furnaces, Chimie 4. 13/14. I.q. ب. إم and.

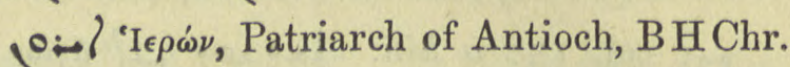
Eccl, 4I. I4.

ل/ع gloss hus or a a a ib. 58. 20.

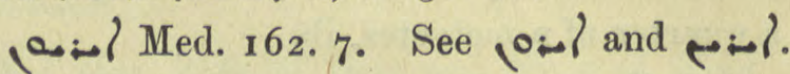

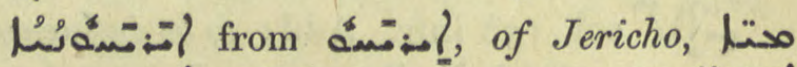

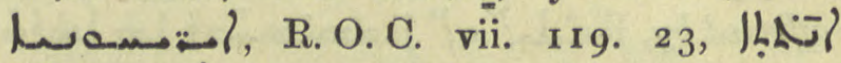

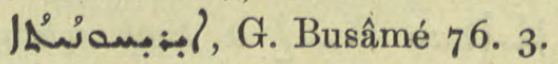

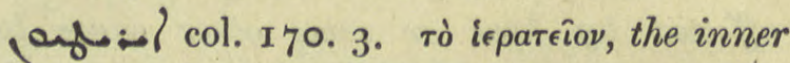
sanctuary of a church, Par. Pat. 39. I but in the text \{af; = A.M.B. vii. I02, Pallad. I 7 I ult., 569.7 .

أم- col. 170. i.q. _o:.l. rust, verdigris, زنجار Chimie I29, note 2, mediaeval alchemists call verdigris Jarim. Used in medicine, Med. 63. $21,64.1,169.1,13,560.24,601$. 19, J.A.O.S. xx. 196. 13,14 .

(col. I70. 2) Irene, virgin and martyr,

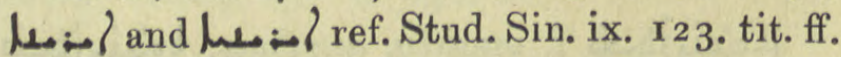
(a._-i ipıvov, lily oil, so correct for

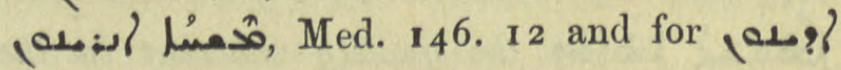
ib. 147 . 11 .

إبتبك. pr.n.m. a Mongol Emir, Jab. 149.

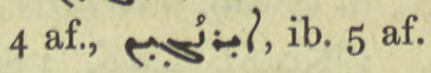

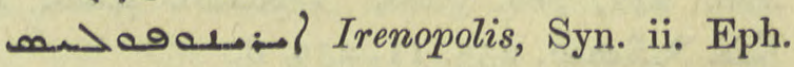
II. 16 .

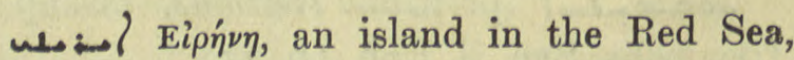
Jac. Edes. Hex. 20. 7 .

(-) ( col. I71. I) orris root i.e. the root

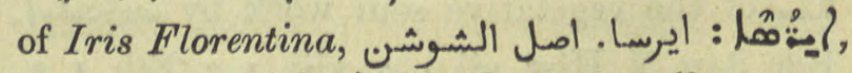

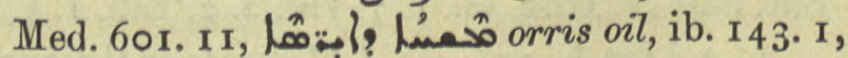
251. $13,25^{2} .3,401$. 10. In two passages, Med. 161. 7 and 182. 4, translated by Budge, "irises" and "husked barley" i.e. fío î?", the meaning may be the flour of darnel or tares but is more likely to be orris as elsewhere. 
(و) iepákıo, Hieracium, hawkweed, Med. 412. 20; (هلم) ib. 414. I3, 434. 2.

إمعel Aisouma, a hill near Amid., Dion.

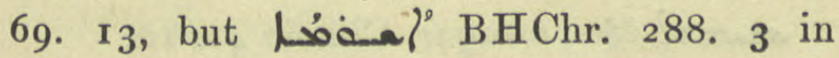
col. 406 .

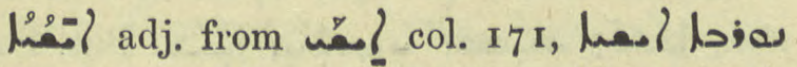
a shoot out of the stock of Jesse (Jes. xi. I), Warda I $v$.

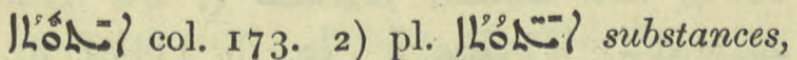

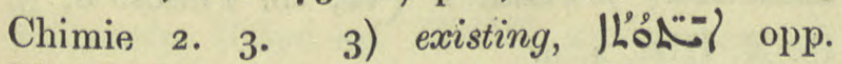

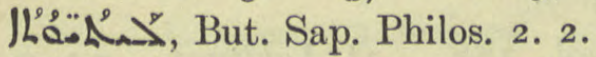

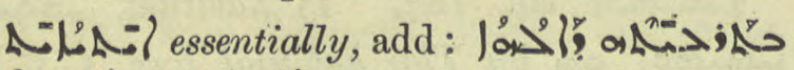
م央 essentially in the mind of God, Caus. Caus. 197. 10.

uola.? the island Ithaca, Jac. Edes. Hex. I2. 8 .

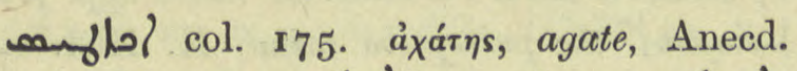

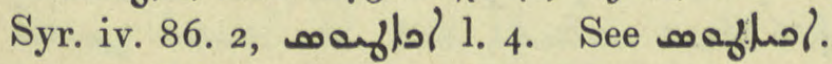

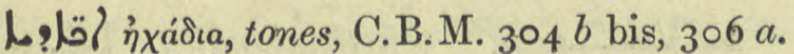

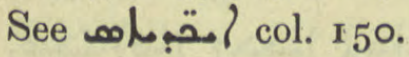

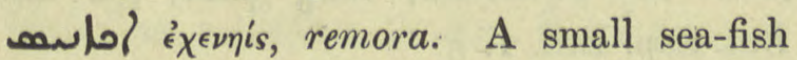
credited with the power of delaying ships, Anecd. Syr. iv. 97. 26, صanlo\}, ib. 98. 2,

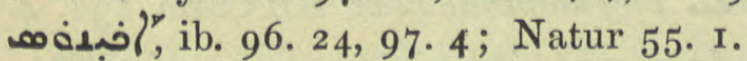

ใิ?

|ُ Pallad. I 12.4.

مشّا لl Warda $247 r$. See

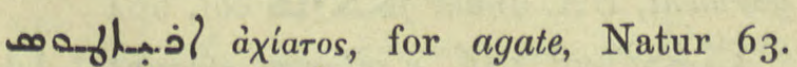

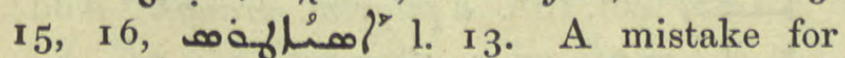
m. less absurd passage of Physiologus, Anecd. Syr. iv. 86.

w) Akhi, son of Ḩamselim, King of Persia and Elam, Ahikar, Introd. xxxiv, 49. I3.

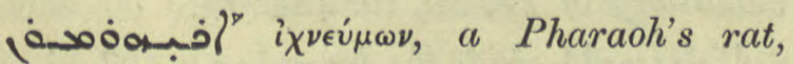
N. Hist. vii. 4.3 , j- ف-

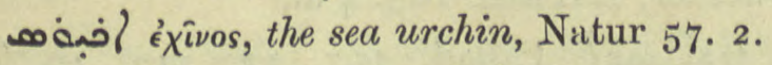

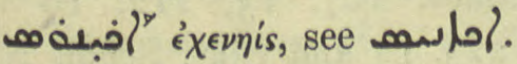

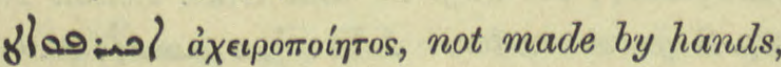
a picture of Our Lord was so-called, Anecd. Syr. iii. 324 . 16 .

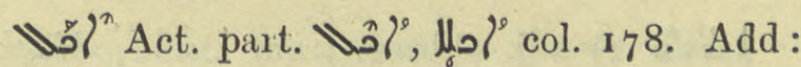

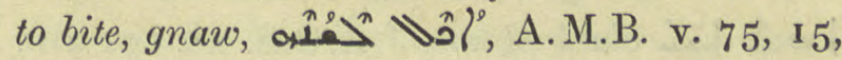

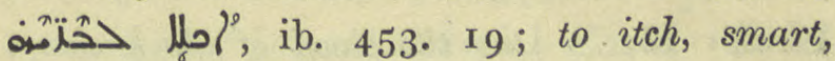
أl", Kal-w-Dim. ed. Bick. 5. 9; Ar. Pfln N. 9, ZDMG. xxxi. 538. 2 r, مَّم

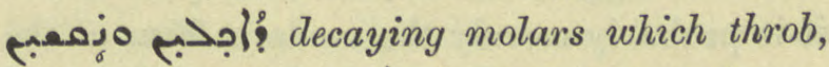

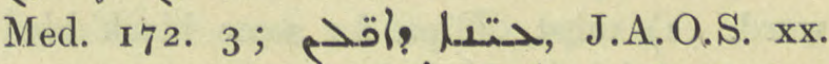

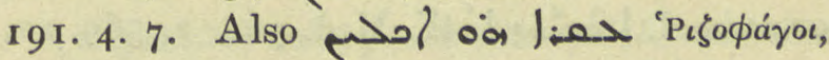
a people of Ethiopia, Anecd. Syr. iii. 330. 2. Pass. part. add : a angl his head was eaten away with cancer, Pallad. I5r. 6. Aphel

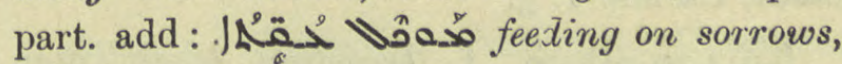
Jab. 195. 3 af.

$\mu_{0} ?^{\prime}$ col. 180. I) not rust but a gnawing worm or moth, Journ. As. 1894,568 ; لl ahoula p: Jac. Sar. ap. ZDMG. xxxi. 383. 4 .

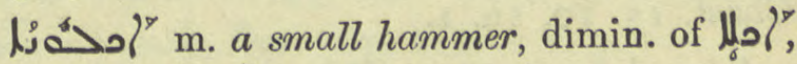

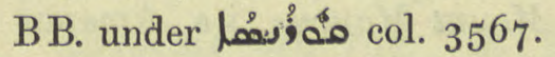

|' 200. $6,201.5$.

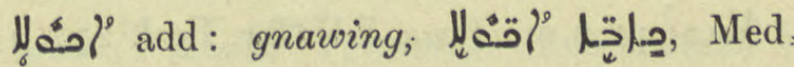
$2 \mathrm{I} .16$.

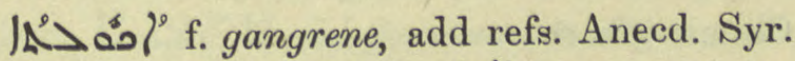

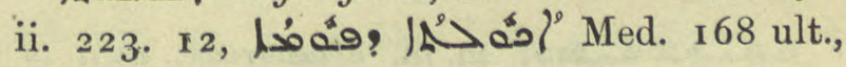
I 69 ter, 170 ter, I 7 I quater, 562.20.

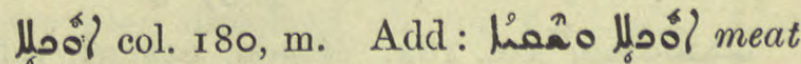
and drink, $\mathrm{BH}$. in Prov. viii. $2 ; \mathrm{ZA}$. viii. 6. $18,3^{6 .} 16$.

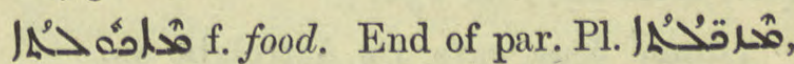
Duval Gr. $\$ 246 b$, I Tim. iv. 3 , Jul. I ro bis, مقدي ib. 1. 5.

مخاقليا

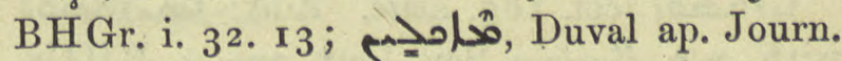
As. 1894,568 .

مسملا m. food, fodder, 193. II.

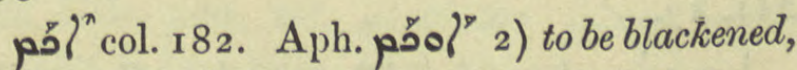

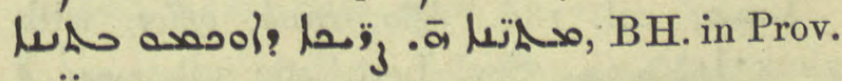
xxii. 29.

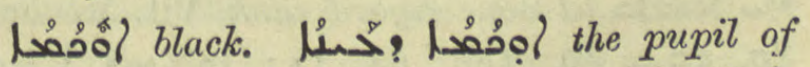

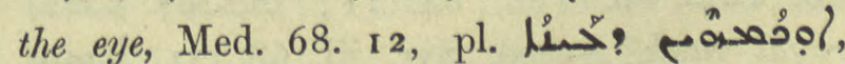
ib. 79. 9. | |

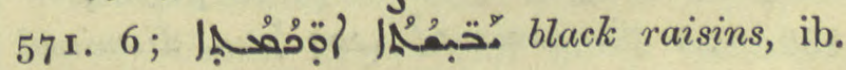
573. 13 . 
1.

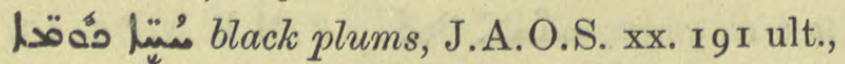

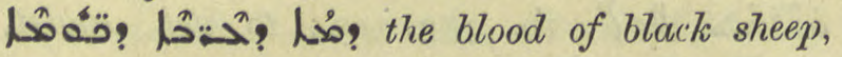

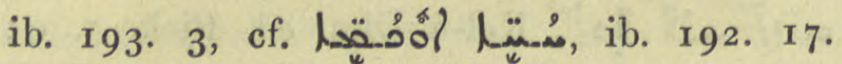
Gottheil suggests |xóơól in both places, but it is probably merely the modern form of the word. 2) subst. ellipt. for some black bird

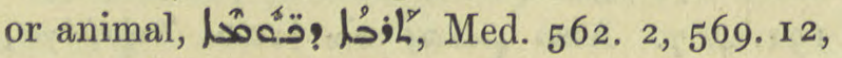

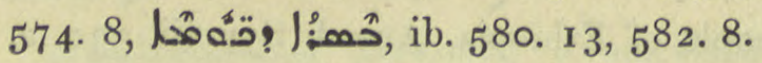

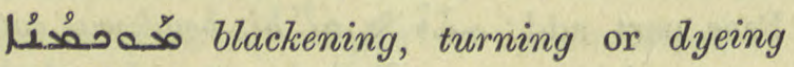
black, Chimie I. 5 .

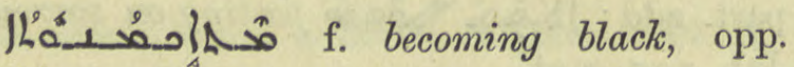

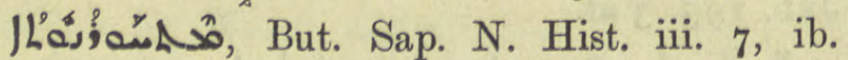
Isag. ii. 9 .

山ผํْơ pr.n.m. Uchama, col. I83. Add: Journ. As. 1907, I63. 5, A.M.B. iii. 473 , Chast. I5. 10, 19. I.

bovol jof Mount Maurus, i.e. Amanus N. of Antioch, Dion. I3. 5 .

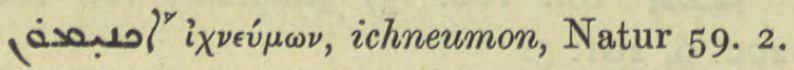
leol pr.n.m. Axus, BH Chr. Eccl. 277 . I5.

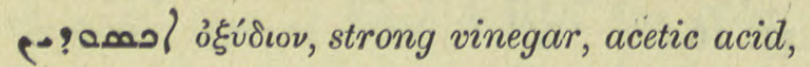
Chimie 58. 10.

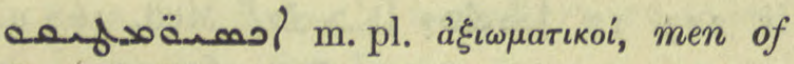
high rank, Syn. ii. Eph. 8. 19; 37. 3.

u> Chimie 16. 21, Med. 39. 20, 45. I3,

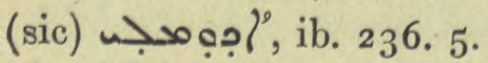

|

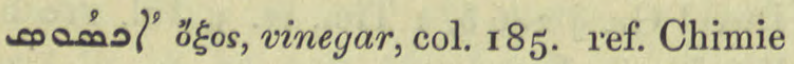
7. 14.

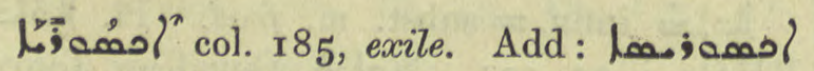

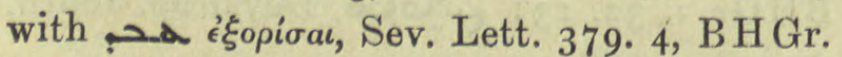
i. $47.2 \mathrm{I}$.

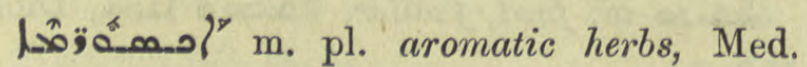
211.13 .

he..? col. 186. àkia, rank, office, with gloss /fلs ;

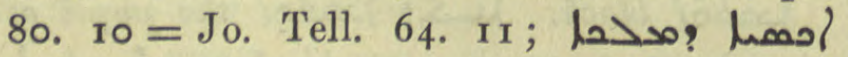
royal rank, Anecd. Syr. iii. 68. 2 I, h hol lands\}? the office of the imperial prefect, ib. 259. 25 ; pl. pe dismissal from office, ib. 3 I 5 . 6 .

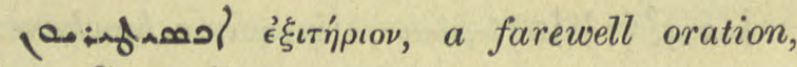
Greg. Carm. ii. 27 . 14.

coserenp? Aximus (Maximus?) emp. Stud. Sin. ix. ror.

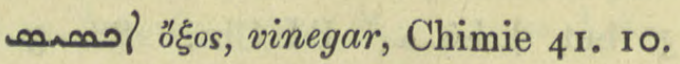

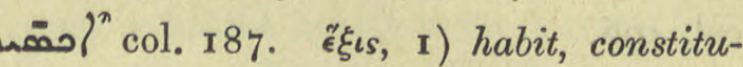

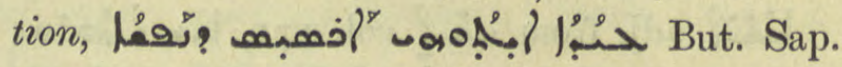
Eth. r. 7 ; opp. om II. 4 , va a . ib. N. Hist. 4. 9.

حسه دy hemal oa by Thy habitual love, C.B.M.

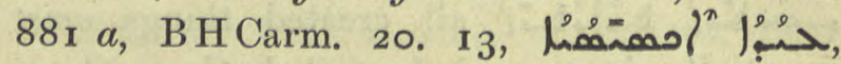

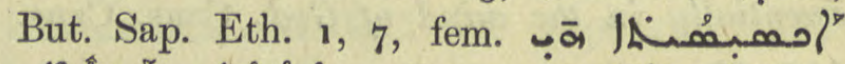

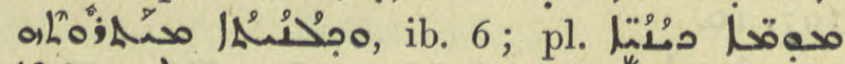

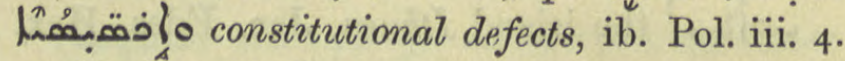

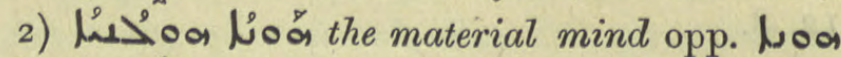

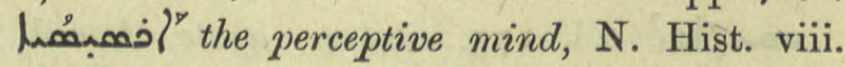
4. 6 bis.

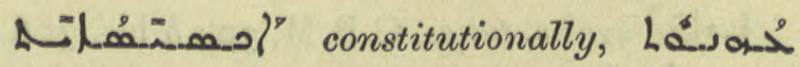

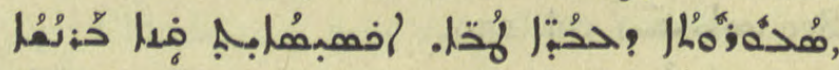
But. Sap. Eth. I. 5 .

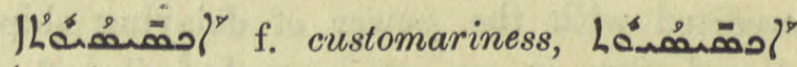

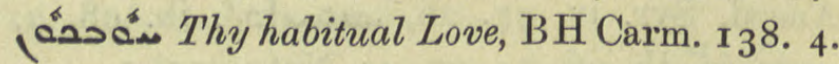

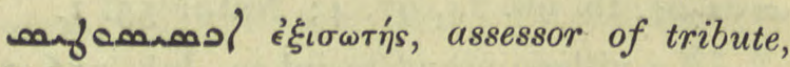
C.B.M. 424 b.

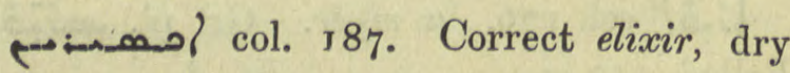
perfume.

r.o.t an old ragged and patched garment, B A. under IA ?: 5 col, 604.

hinol" col. I88. a pilgrim, stranger. Add :

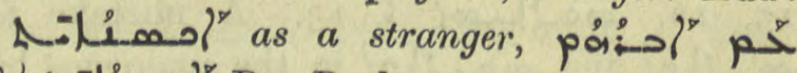

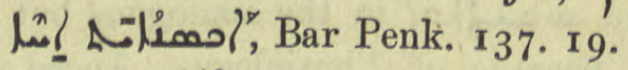

(1)

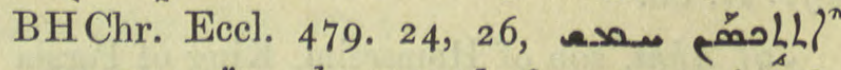
منتم sayed five years in the monastery of Peskin, ib. 485.13.

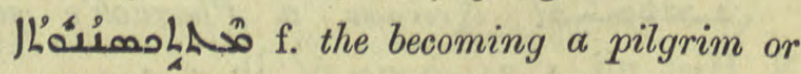
traveller, BHEth. 105. 4 af.

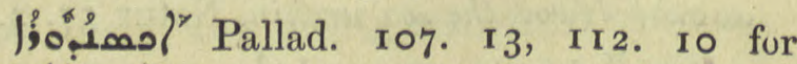
|

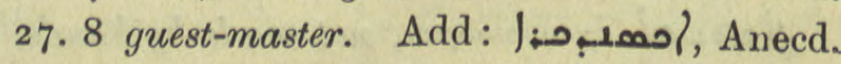
Syr. iii. 2 16. 2, Zach. tr. H. and Brooks 167 ,

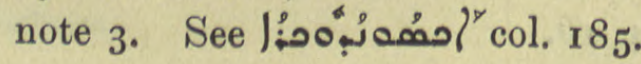




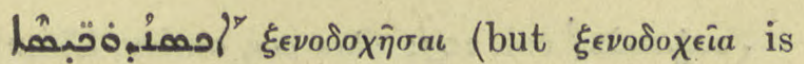
meant), hospitals or guest-houses, Pallad. 200. I I.

wedton? Xanthippe, wife of Albinus, R.O.C. iii. 4 I. 6.

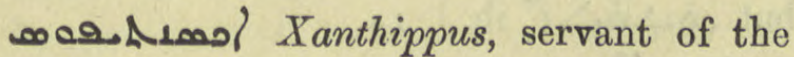
Apostle Thomas, Act. Apost. Apoc. a خ;. 23.

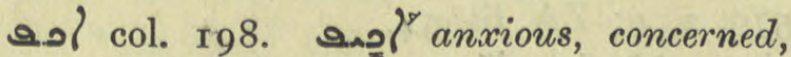

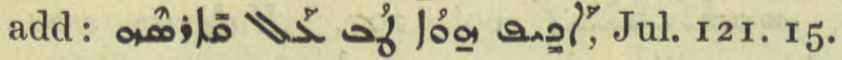

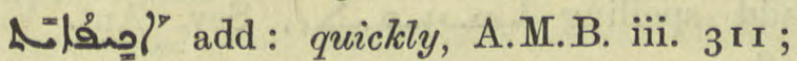
diligently, Galen ap. ZDMG. xxxix. 244. I8,

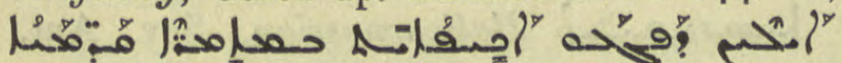
those who have diligently studied the earlier treatises, ib. 283. 4. Urgently, Sev. Ant. Vit. 285.8 .

|L'áa_ol" f. add: anxiety, doubt, Lóa لٕ

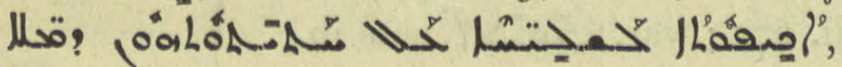
Theod. in John i. 36 .

|فْ-ố m. a pack-saddle, DBB. 6r. II,

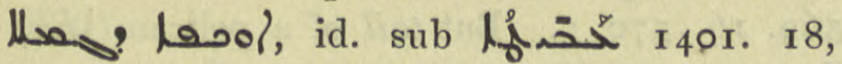

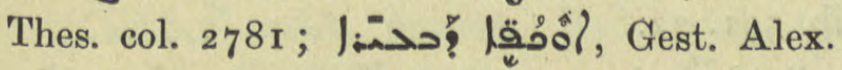
23 I. 6.

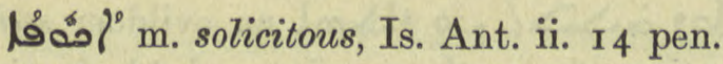

|이" need, add: : Loo lool wo what need was there? Ephr. ed. Lamy iii. 483

|جُج col. r89. pack-saddle, ref. G. Busâmé, ${ }_{4}$ b. 2.

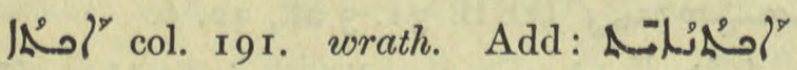
angrily, Sev. Lett. 453. 1.

اختيار الدين name of an Emir, BH Chr. 38 r. 7 , 457. I.

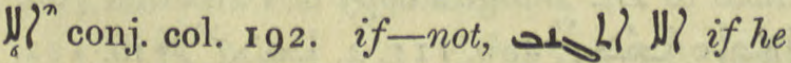
was not deceived, Ephr. Ref. ii. 87. 4. Cf. Matt. xi. 6 Curet. us Mash V/ woosof.

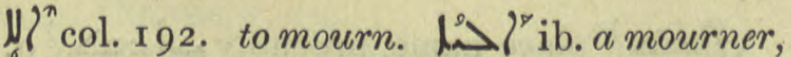

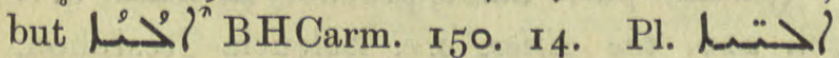
Charms 8I. II.

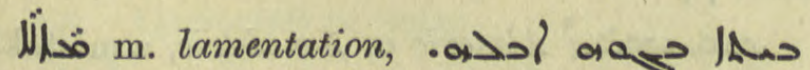
a 7 pen.

الإل and Red Sea, Jac. Edes. Hex. 20. 8.

olyll i.q. لi ji col. 194 and Jab. 9r, Ala Dagh $=$ high mountain, near Tabriz, note ib. and 97 . moirsasalr

الحصنمثم Ar. Ar. 508 Bahrein, El Nis Chron. I73. 2.

/ خذاda he-goat, Chimie 24 I n. I ; Warda

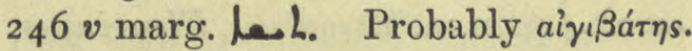

) >حسL Albina, daughter-in-law of S. Melania the Great, Pallad. 201. 2, mis-written |usal\}, ib. 196. 7 ; Pet. Ib. 27.19.

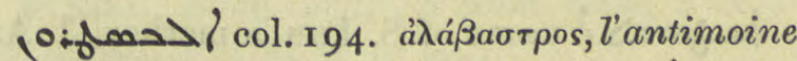

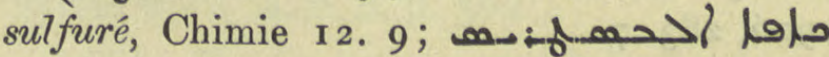
alabaster, ib. 4I. 8 ; cf. 72 n. I ; Act. Apost. Apoc. مدص. 12.

o $\rightarrow$ ? Helladius, bishop of Caesarea, Mich. Syr. i. Fasc. ii. 160 c. 2 .

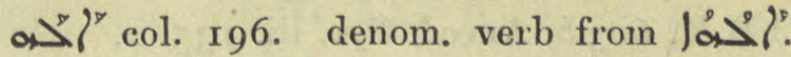

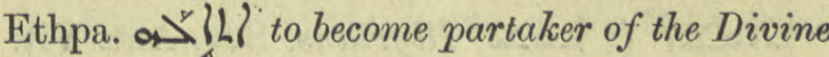
nature. Add: Greg. Carm. ii. 49. 20. Part. pl. f. تُقْهِ

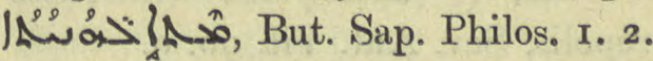

Lwoal col. 197. m. dimin. a godlet, godling, Jac. Sar. Hom. iii. 797. 4, Mar Bassus 7. 82 ; Hormizd. 29 I 4.

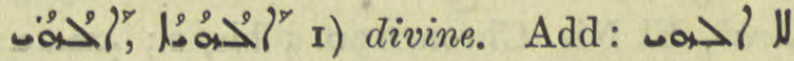
godless, Jul. 62. 25. 2) for Gr. $\theta$ eiov, sulphur;

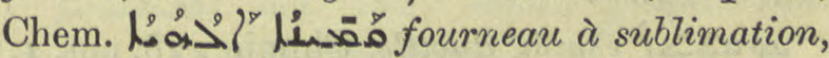

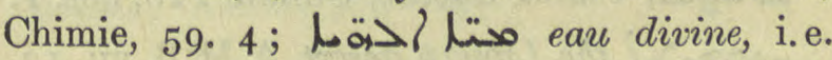
white of egg wherein sal ammoniac and alum have been dissolved, ib. 39.5 ; متر la , مقةمعا, ib. 42. I4; prepared with sulphur, ib. $59 \cdot 2$.

Ihema $\rightarrow$ l divinity $=$ Holiness, title of veneration addressed to a bishop, $\mathrm{BHChr}$. Eccl. 16r. 2 I.

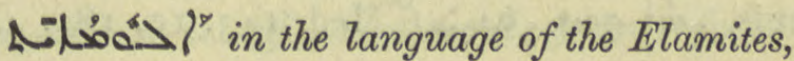
C.B.M. 901 a.

lsa>l Olba in Isauria, De Goeje B. 65. I9;

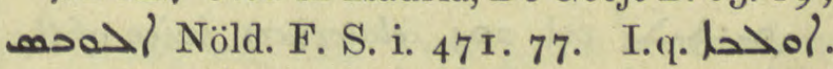

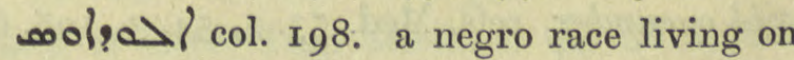
the Nile, add. ref. riohso! أ), Jac. Edes. Hex. 32. 10.

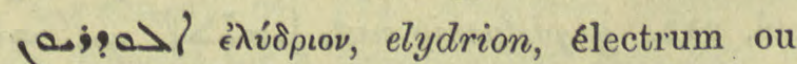
chélidoine, Chimie 3.9 and note ${ }_{3} 3$, explained as "soufre marin" from BB. under (a);a) col. $20 \mathrm{r}$; ib. II. 2 I, I7. 16, 18. 8.

مo: col, 200 : pr.n.m. Olympiodorus, Warda $247 r$ with gloss. IAsooso, R.O.C. $x v .23 \circ \mathrm{f}$. 


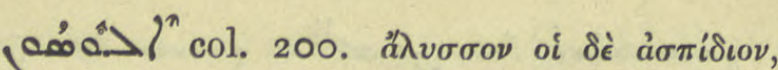
Diosc. i. 444, useful to heal those bitten by a mad dog, Chimie 5. 20.

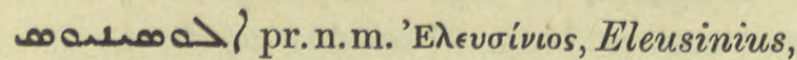
Sev. Lett. 93. 4.

م i. 47 I. $5^{8}$.

lefloal name of S. Paul's mother, J. As. 1906, 283.7 .

مaسمal pr.n.m. Aqyllos, Nöld. F. S.

i. 475.146 .

$\infty 0 ; 0)$ lailiovpos, the Weasel, nickname of Timothy the Great, Bishop of Alexandria, C.B.M. $603 b$, Anecd. Syr. iii. $135 \cdot 2$.

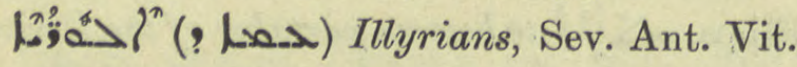
I5. 9, 57. 12.

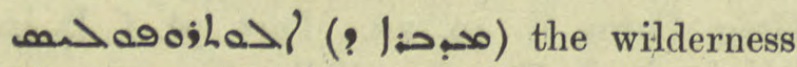
of Eleutheropolis, Sev. Ant. Vit. 96. 9.

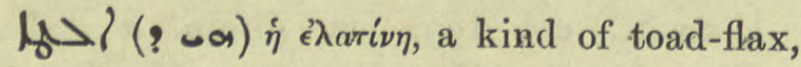
Diosc. iv. 40, Galen ap. ZDMG. xxxix. 240.

الحيل Arab. "إلطائى Al-Täi, the Tayaya, Arab, El Nis. Chron. I90. I bis, 2 ; مa مع: - 193.7.

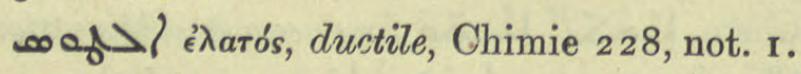
w >alibyan desert, A.M.B. vii. I 2.

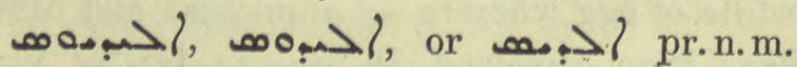

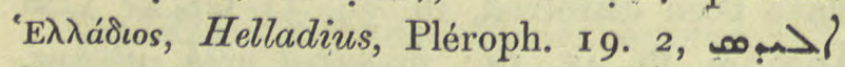

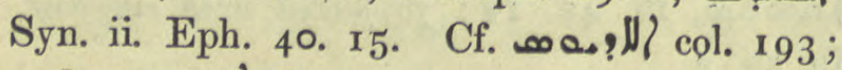
and

I" so corr. misprint, col. 205. I.

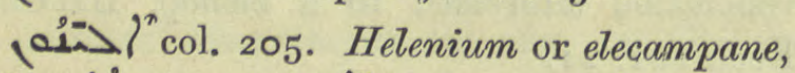
refs.

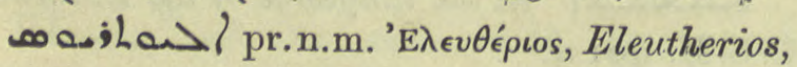
Sev. Lett. $72.17 \mathrm{f}$.

(a) wild cucumber, refs. Med. 53. 5, 54. 3, 198. 6, 379. 5 .

I"

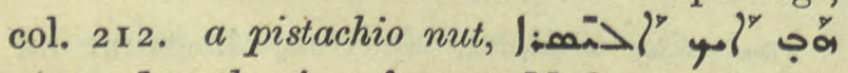
give a dose the size of a nut, Med. $3 \circ 5 \cdot 5,3 \circ 8$. 18, 3 10. 16.

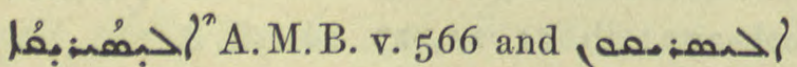

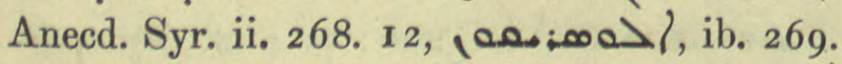

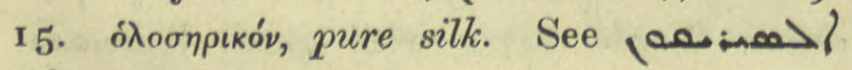
col. 2 I I.

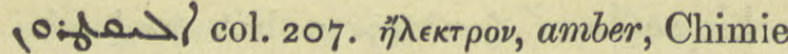
2. 4, 3. 4, 9 pen. An alloy of gold and silver, ib. 236 , note 4 , recipes for compounding electrum, ib. 260-266. Usually printed

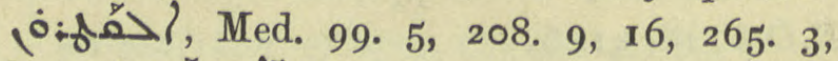

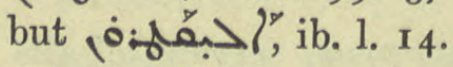

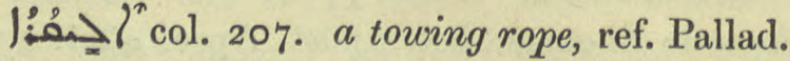
579. 2, 4 ; ZA. iii. 54 .

م

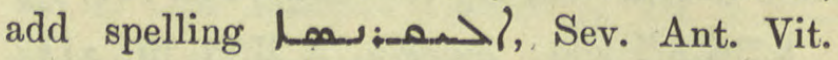
284 . 4 .

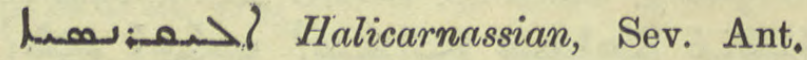
Vit. 275.10.

L 1 in Alerianus prob. for Valerianus, Bishop of Neo-Caesarea, De Goeje B. 65. I 8.

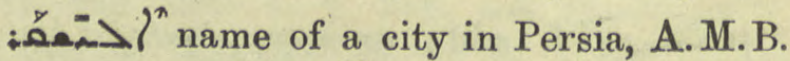
ii. 671 ; var.

| add: 569. 16, 570. 5. But tail of a pelican (مُمْم) ib. 591.22.

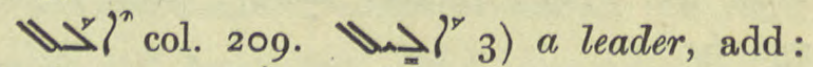
|lous evildoer seeks protection from those who uphold justice, Philox. I30. I; you have made your city

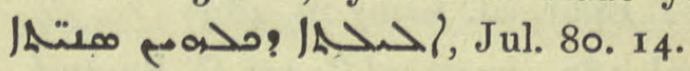

حا col. 2 ro. ä $\lambda \lambda \eta$, another; see under

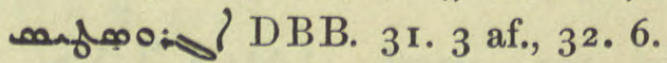

زٔمَّم :

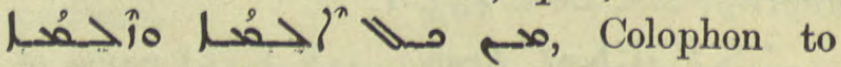
G. Busâmé, ditto to Hist. B. V.M. I $5^{2}$ ult., f., ditto to Dr. Budge's copy of Palladius 768. 2.

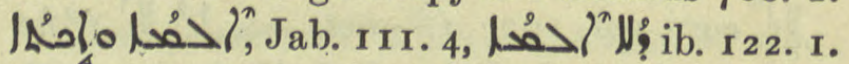
L. 7 of par. insane or furious lust, BH. Eth. 247.

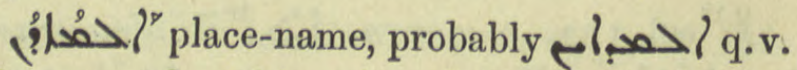
col. 2 10. the cities, i. e. Seleucia-Ctesiphon, Jab. 74 .

/ Almeric or Amalaric, a mistake for Theodoric the Goth, Anecd. Syr. iii. 287. 14 .

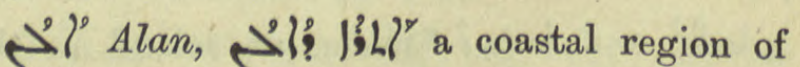
the Caspian Sea, near the Volga, BHChr.

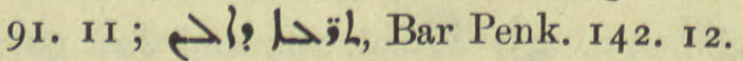

Aiح'? in the tongue of the Alani, C.B.M. $901 a$. 
إحب Alinda, a bishopric in Caria or Asia, De Goeje B. 65 . I 5 .

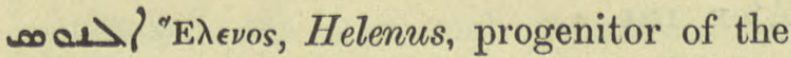
Greeks, Arist. Apol. $\checkmark$ I I.

a) ä̃as, salt, sal ammoniac, Chimie 4. $6, \mathrm{I} 5$.

مै

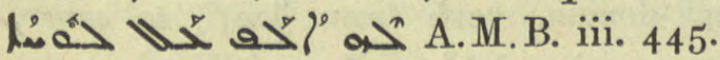

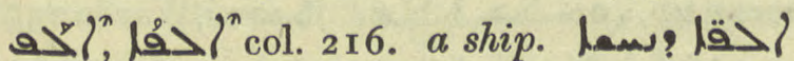
brazen vessels, this occurs in a list of utensils, C.B.M. 49 I $a$.

ןalyony, Med. 264. I, probably a confusion of $0 ; 09>$ l and l:

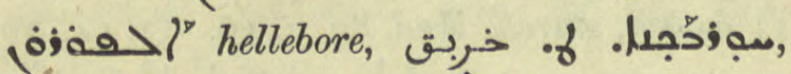
Me2̂. 601. 17. See 10:9>l col. 2 I 7 .

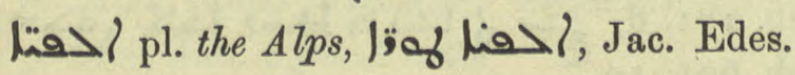
Hex. 34. 9 .

مa name of an Arabian abbat, C.B.M. $714 a$.

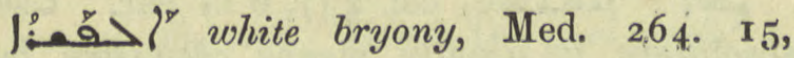
هـزار جشان , ib. 6or. 18.

$1 L^{\circ} 0_{5}^{\prime}>l^{\prime}$ col. 2 19. f. disgust, disgustixgness,

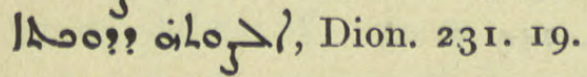

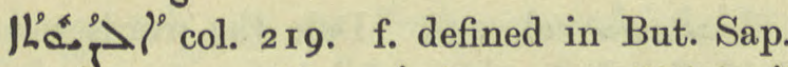

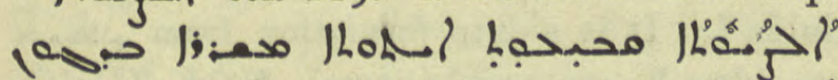

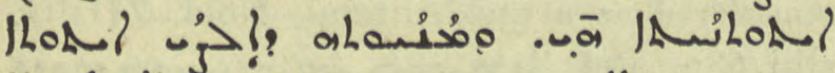

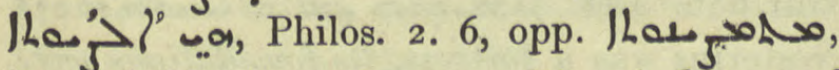
ib. and Theol. 4. 6; compelling influence of stars, George Ar. 24. 9, 29. I, 12.

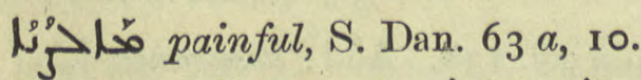

wolما i. q. 220.

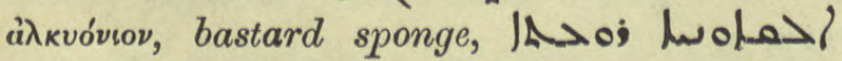
les?, Chimie 7. 14.

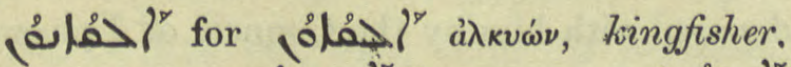

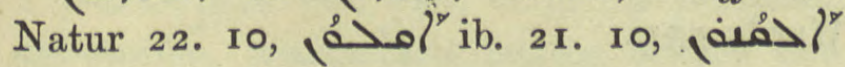

1. 16. Corrected in nates.

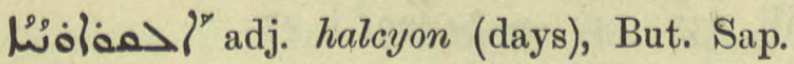
N. Hist. vii. 3. 1 .

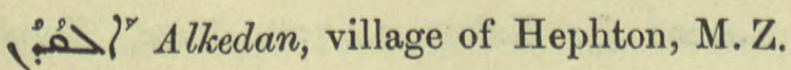
207. 16.

إم 14. 10. (0)0000入) dross of silver, ib. II. 5 .
Heم_ Alcmena, mother of Hercules, Arist. Apol. مص I7 7 .

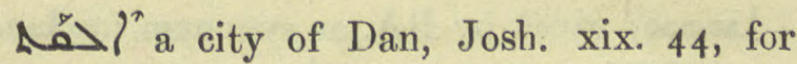

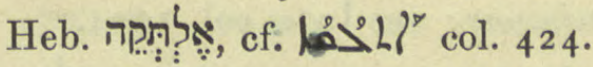

l. $>$ pr.n.f. Eleria, daughter of Zeno, Journ. As. $1907,402$.

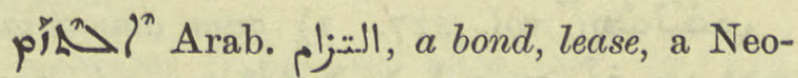
Syriac word, A.M.B. ii. $5^{69}$.

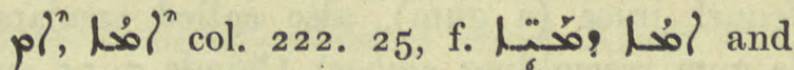

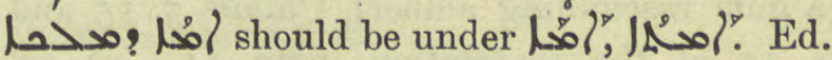

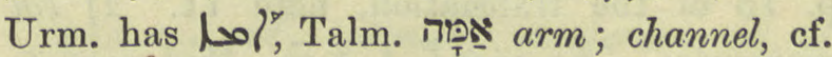
Angl. an arm of the river, R. D. Journ. As.

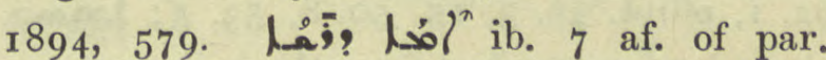
Arabism the crown of the head, Med. 58. 8, Dion. 2 I 6. 8, Anecd. Syr. ii. I56 5 .

| / Y / Yamnia, a bishopric in Palestine, Mich. Syr. $5^{8} 8$ c pen.

(a)bol Mount Amanus, Jac. Edes. Hex. II. $6,37 \cdot 3$.

م.

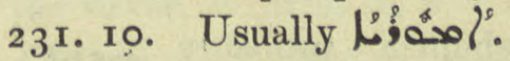

Lanol col. 224. ambo, pulpit, add: (مد), Q.B.M. $705 b 2$.

| 31. I I, I 2, مصمعهم), ib. 26. I 5, 28 antep., 29. I3.

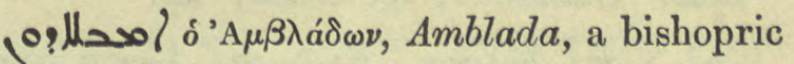
in Lycaonia, Nöld. F. S. i. 473. 104.

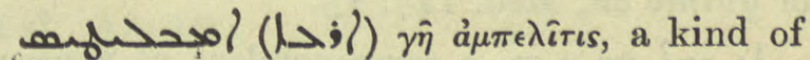
earth smeared on vines to kill insects, JAsojos à saffron coloured, yellow, Chimie 8. 2.

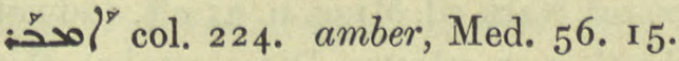

إمص: col. 22 24. Pers. heap of chaff, Lag. Mitth. i. 44 infra,

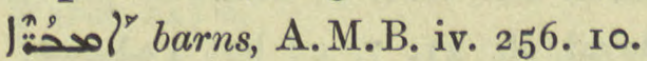

| of a draught for a consumptive, Med. 220. 9, 226. 9 .

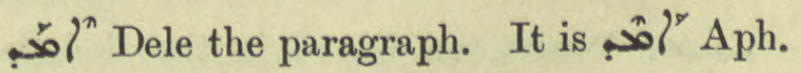
of 0 q.v. col. 2010.

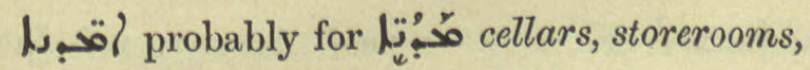
Ephr. ed. Lamy ii. 805. 7 . 
Masol Amul, a bishopric in Tabaristan, N. of Demavend, Syn. Or. rog. 24 ; Marquart ${ }_{3} 6$.

loos given by BA. as synonym for lola a small fish, minnow; see lola col. 1001.

was (? : : :) a monastery in Arabia, C.B.M. 7 I 1 b.

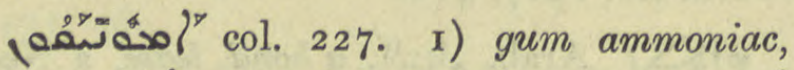

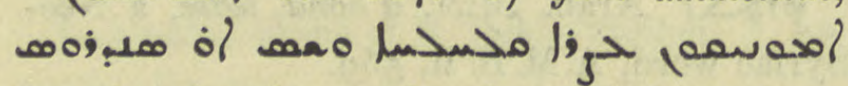
fennel juice (a gum), also called sandarac (a gum resembling amber), Chimie 5. I $_{5}$ and p. Io of the translation, note II. 2) $\varepsilon a l$

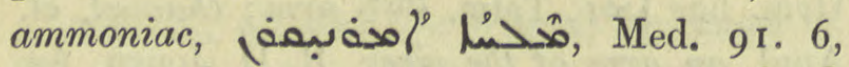
92. I, ellipt. 49.3, I $5,50.8,53.5$;

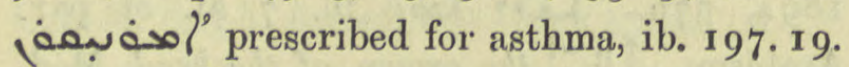

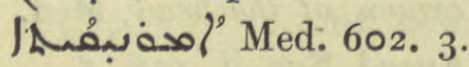

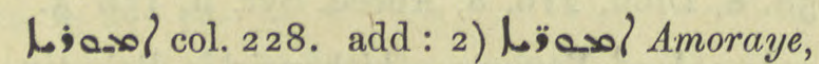
a people coupled with Persians, Gest. Alex. 167, 176. Perh. Himyarites. Other sug-

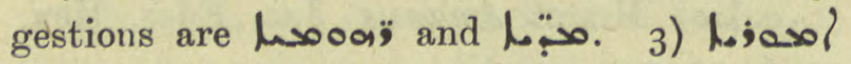
Amorion in Asia, De Goeje B. 65. 20. I.q. (ס्) col. 228.

(مa) Himerion, De Goeje B. 65. 5 .

a.jol lapis aimaritns, bloodstone,

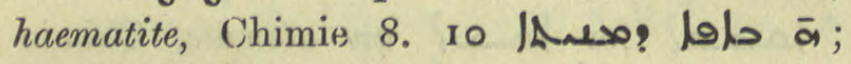

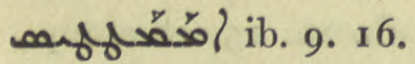

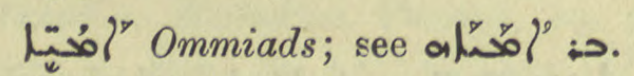

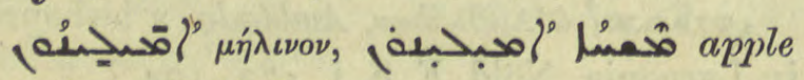
or quince oil, Med. 147.15.

I of pr.n.m. a correspondent of Severus of Antioch, C.B.M. 95 I $a$.

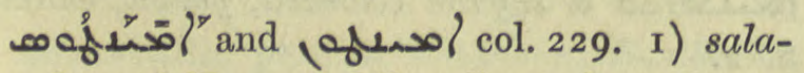
mander, ref. Nars. ed. Ming. ii. 35 ult. and note. 2) amianthus or asbestos, Diosc. v. clv; Hist. Mon.i. 273 ; ofuso/ olo Chimie 9. 7, 18, an inferior kind of talc, ib. 1. 9; chaux de pierre

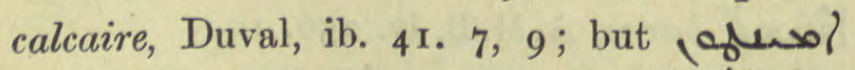

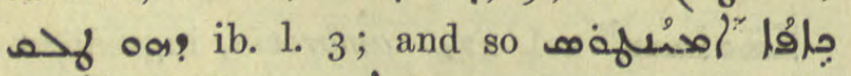

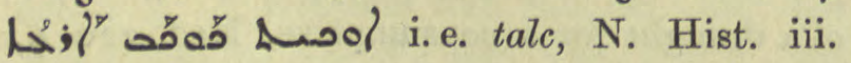
cap. $\mathrm{I}$, sect. 2 .

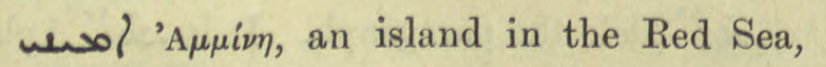
Jac. Edes. Hex. 20. 7 .

101 col. 230. a pool, swamp; ref. Jac. Edes. Hex. 23. I 5 .
Rُ. sol" col. 230. a spade, shovel, S. Dan.

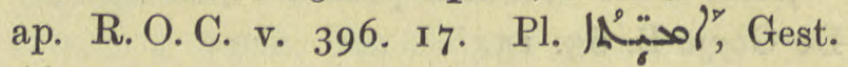
Alex. ror. 4.

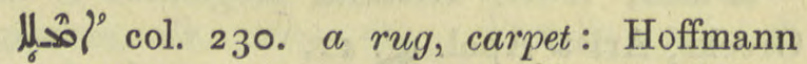
thinks this word and $\mid$

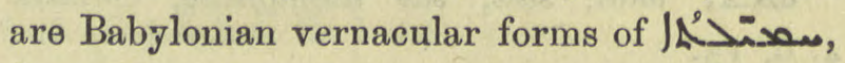
Pers. Mart. n. 206.

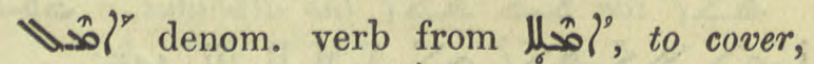

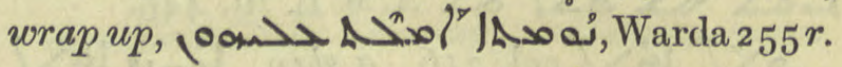

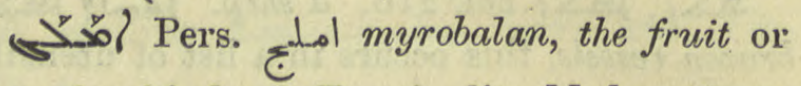
nut of $a$ kind of Terminalia, Med. ${ }_{3} 8.7$, 239. $13,393.7$.

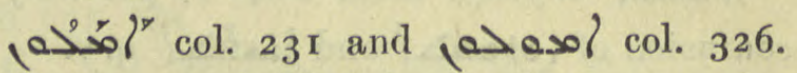

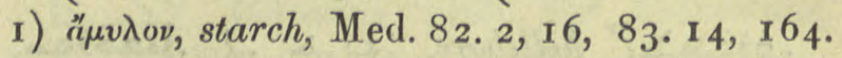

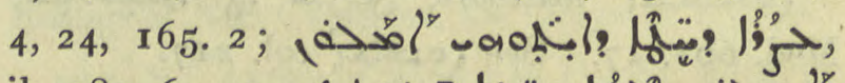
ib. 181. 6, ib. 601 . 19.

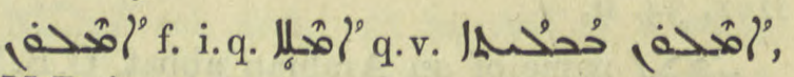
A.M.B. iv. 239 . I.

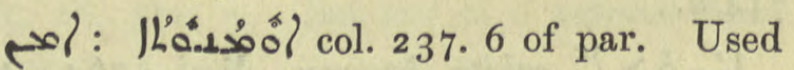
absolutely it usually. means chemistry but sometimes astrology, Spic. 9. I.

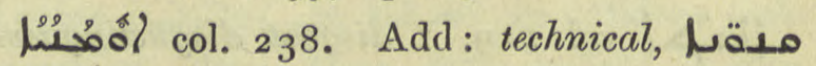
hisool, Galen. 244. 7.

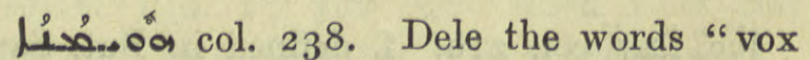
dubia". It is a late formation from "ấ considered as a quadriliteral, Nöld. ZDMG.

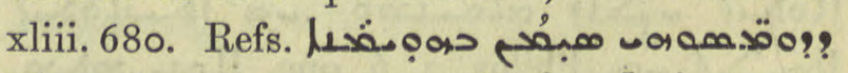
Nestorius was a fortress, its foundations were laid on faith, Warda $5^{2} r$, Parad. ed. Card. where the word is wrongly vocalized lišo.

لل

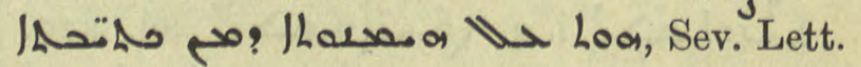
I19. 9 .

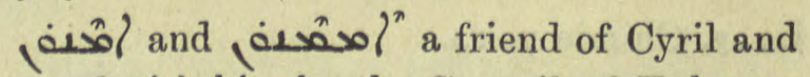
deposed with him by the Council of Ephesus, Warda $55 r$.

enfer. pr. n.m. Amantius, Anecd. Syr.

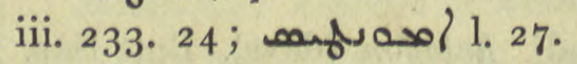

|

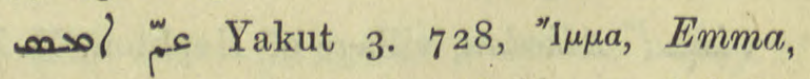
a town between Antioch and Aleppo, C.B.M. 708. 2, Doc. Mon. I 72. 10, Sachau ZA. xii. 48.

Hä inhabitants of Amasia, see col. 240. I, Sev. Lett. 247 . 16. 


\section{extag}

anfogsol ä $\mu \pi \omega \tau \iota s$, ebb of the sea, Bar Ce.

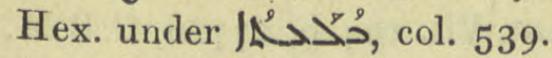

Asóasol' col. 240 and so correct for ja I 4 n. I. I) a skylight, window light or pane, |

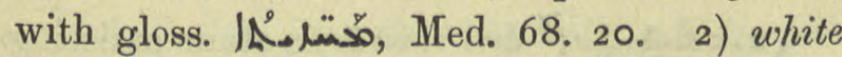
lead or gypsum, Chimie 136. 1; 49. 5, 12,

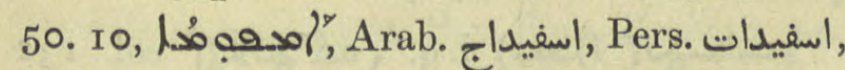
Med. 7 16. 4. 3) a clarionet, Mt. Singar 54. 5, 55. 4 .

hasol col. 24I. Guidi corrects nymphea, fountains, Bull. Arch. Romae xii. 222.

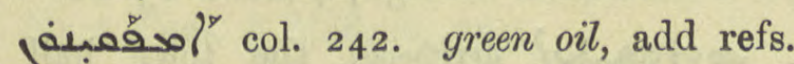
Med. 60. 8, 213. 9 .

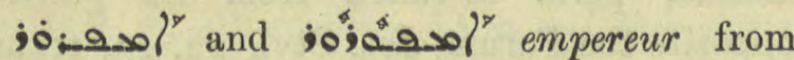
imperator, Jab. 63 and note.

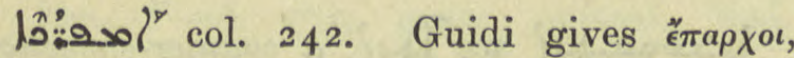
vico-magistri, city guards, Bull. Arch. Romae xii. 223. I and note. Ref. Sassanidi 63. 40.

أمع: col. 243. Delete the paragraph.

- $\infty$ col. 243 :

'"asol' col. 245.8 of par. the officiating priest, Qdham W. I74. 9.

J

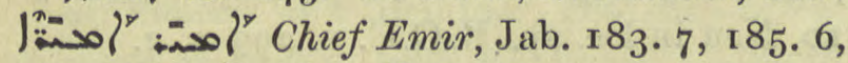

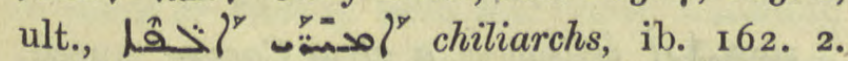

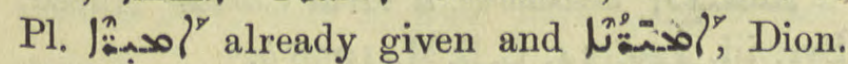
I25. $20,128.15,234.8$ bis.

|Lo'o:-isol" f. emir-ship, El. Nis. Chron. I35. $24,137.7,1_{42}$. II.

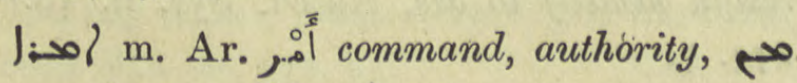

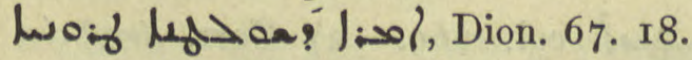

r. Syn. ii. Eph. II. I3. See r-wa.?.

ILُם affirmation, /her: 0 la o Lasen of the Son

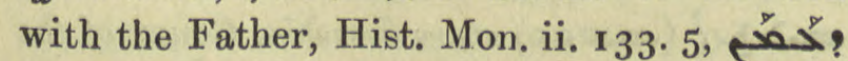

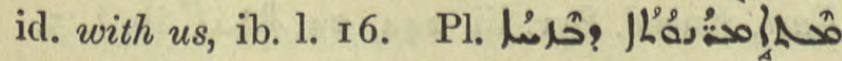
ridiculous discourses, Or. Xt. i. 82, 4.

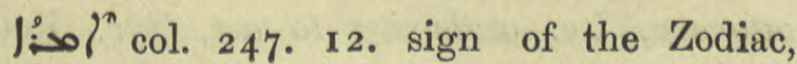
Aries, used as a name for Marcasite, Chimie 6. $\mathrm{I}_{4}$.

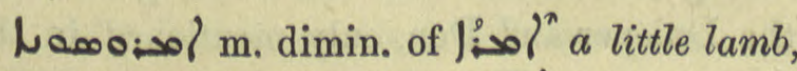

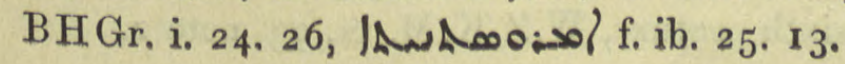

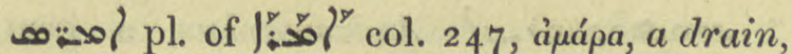

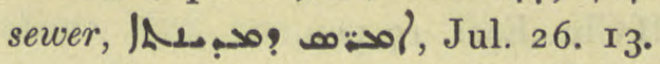

ool.s; $\rightarrow$ l i.q. 470. 45 .

L: 10 / Lat. amaracus, marjoram, but in the Gk. karia, Act. Apost. Apoc. \$1. I 2. Cf. (20)

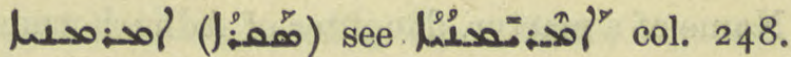

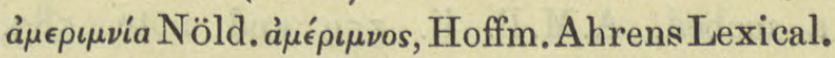
an open cheque, unlimited credit, Anecd. Syr. iii. 2 I 4. 19, 2 I 5. 22.

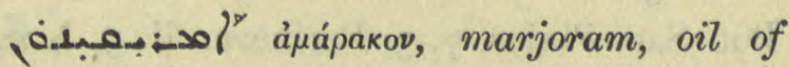

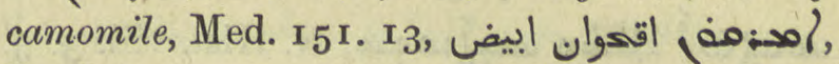
ib. 60I. I 2.

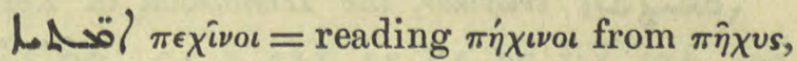
people a cubit high, pygmies, Anecd. Syr. iii. 330. 3 from Ptolem. Geog.

| marized, BHGr. i. 47. 25 .

(2)

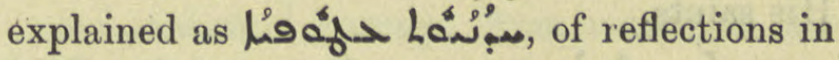
the water, N. Hist. v. 2, I ter.

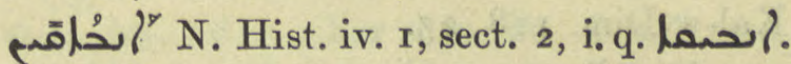

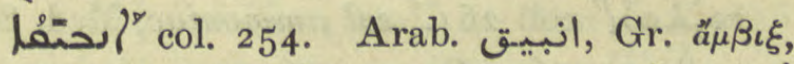

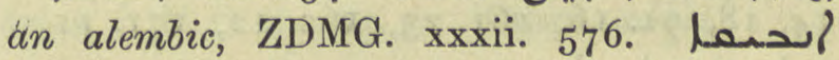
I $\mathrm{K}$ a blind alembic, i.e. without a head, Chimie 33. 20 and often. Also written

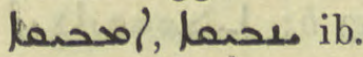

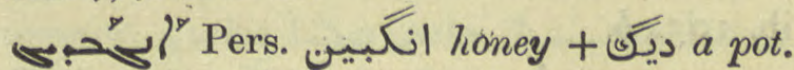
Name of a medicine compounded with honey, Med. 3 I 2. 10.

col. 2276 and Suppl. infra. Pers. نار مـشاك Dozy says it is a small pomegranate of Khorasan; others an Indian fruit with fragrant flowers; Med.393.9. Lي

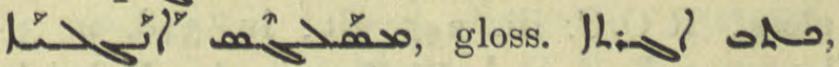
Warda $247 v$.

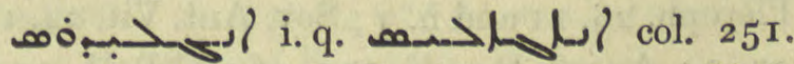

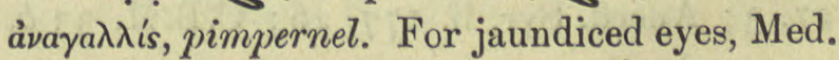
392. II ; Chimie 50. 5 ; إكبـ ib. 227 , n. 2.

u Astrolabe 270 pen.

م Chimie 10. 21 ; مـ 


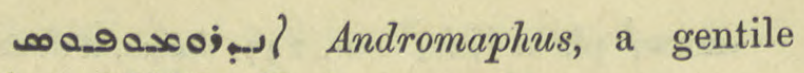
priest, B H Chr. Eccl. I 7 pen.

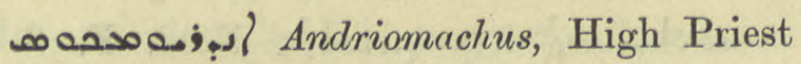
of the Jews, BHChr. Eccl. 19. 6.

مoul col. 258: Zend. Anâhita = undefiled, a personification of unsullied waters, hence goddess of waters. Later the planet Venus. Name of a martyr, daughter of Adhurhormizd, a mobed, A.M. B. ii. 559 and note, $5^{8} 3$, Tabari 4 .

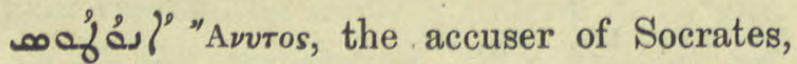
But. Sap. Philos. 7. 3, Periherm. ii. 5, ممحّح

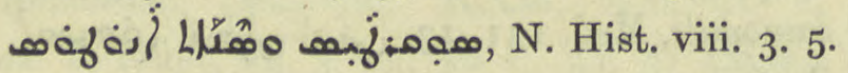

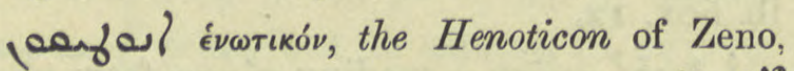

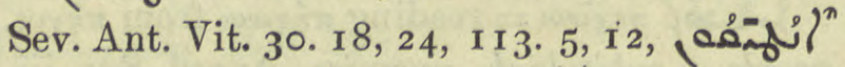
ib. I13. 2. Usually fasa or సّ̆a.

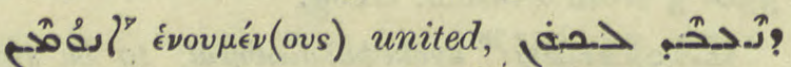

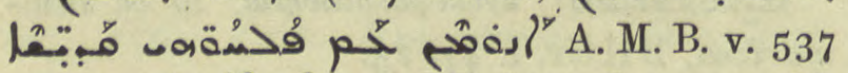
note His saints.

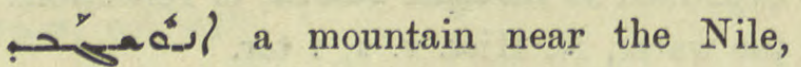
Kal-w-Dim. I 8 8. 25.

jơُaُaُ? col. 26r. sal ammoniac, Med. 89. 14, 18, 91. 16, 161. 23, 162. 12, 171. I1.

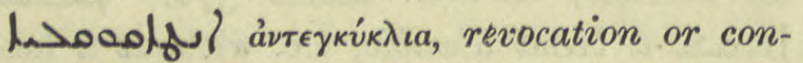
tradiction of an encyclical letter, Anecd. Syr.

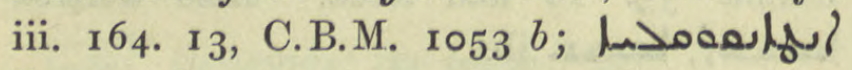
ib. $1054 b$.

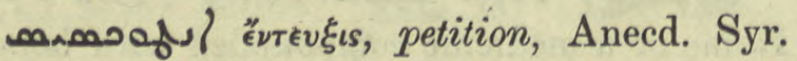
iii. I76. 7, Zach. tr. H. and Brooks I 6 note.

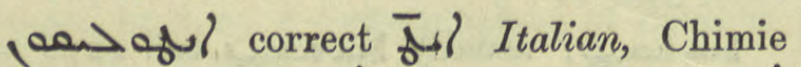

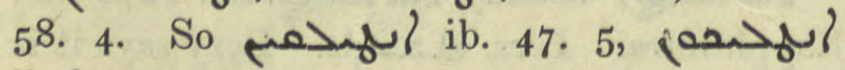
ib. 1. II and Io. I4.

(क) "Evarov, name of a monastery nine miles from Alexandria, Pet. Ib, 64. I8 and gloss., R.O.C. iii. 245 ult.; iv. 348 , n. 3 ,

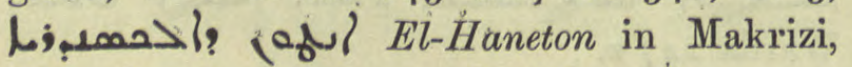
Pléroph. 28. 1 I and n. 3 ; Sev. Ant. Vit. 24 . 1 I, 27. 3 ; Anecd. Syr. ii. I 77. 26 ; C.B. II. $33 b$, $34 a$ and note.

a soul fortress of Antigun, perh. Antigous in Pisidia, see Ramsay Hist. Geog. of Asia Minor 14I. Jac. Edes. Chron. Can. 575. 4, 583 . note 5 .

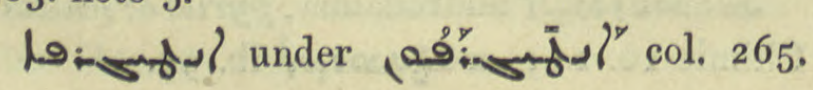

11. 2 and 3 of par. dele "minus recte" and add refs. Sev. Ant. Vit. 194. 9, 277. 6, C.B.M. ro59 $b$.

حخحس ? مLa with gloss. ص versaires de Marie, Coupes ii. I39.

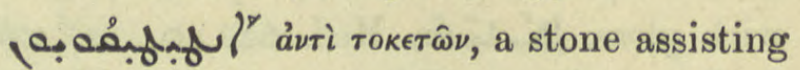
parturition, Natur 24. 4.

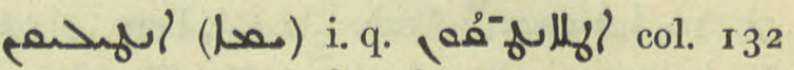
the Atlantic, Anecd. Syr. iv. 97. 24.

conful prob. for mand Antony,

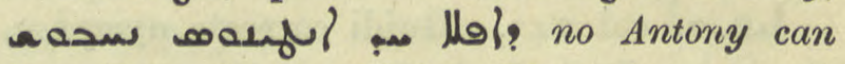
force Jerusalem on high, 4 Macc. 63. 20.

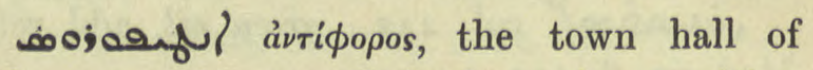
Edessa, perh. so-called from its position opposite the Forum, Jos. Styl. 22. 15, transl. I 8 and note.

W letter, Anecd. Syr. iir. I 74. 24.

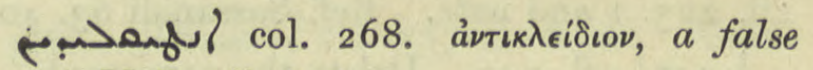
key, A. M. B. vii. 808.

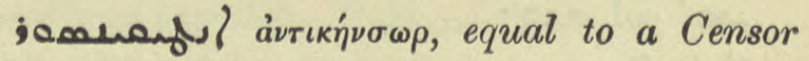

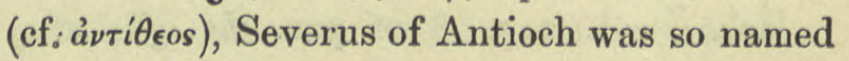
by his fellow students on account of his proficiency in Civil Law, Sev. Ant. Vit. 2 I 5. ro, Nau in loc. ap. R. O.C. v. 293 n. 2.

: Syr. iii. 229. 24 , C.B.M. $105^{8} b$.

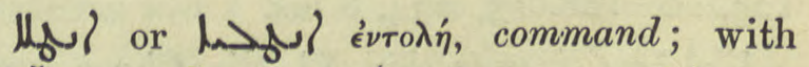
Vمع or to receive the command i.e. to resign himself to die, Anecd. Syr. ii. 40. 7, 274. 7.

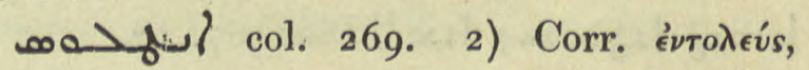

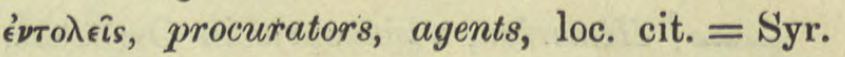
Rom. Rechtsb. 7: 16.

. ref. not 10. 22 but 192. 12. Add: 2)

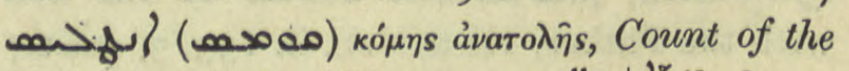
East, Anecd. Syr, iii. 244: 24, lلئ't ib. 1. 5.

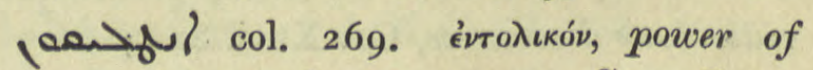
attorney, i.e. authority to act, Syr. Rom. Rechtsb. 18. 7, 8.

ल the Dnieper and Don, only mentioned in the sixth century, W.Z.K.M. ix. 93, note 2. 


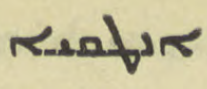

hoful col. 269. Dele medicamentum, the word means Attic, one attic mina, Duval ap. Journ. As. 1 894, 570.

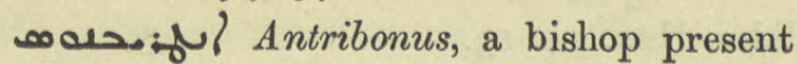
at the Council of Constantinople, A.D. 382 , Nöld. F. S. i. $475,146$.

lo of col. 270 , correct: with àvarpo$\pi \hat{\eta} \sigma a t$, to bring a suit to recover money given to a physician or advocate, Syr. Rom. Rechtsb. 34 . colfoilfu? 'Avтарктко́s, the Antarctic zone, De Astrolabe 84, male saمfo:fl ib. 82.

") col. 270. 7. Anais, Anaitis, a goddess, i. q. بal supra and wanaia, col. 2387. N. Mart. Pers. A.M.B. iv. 184 .

ifsoul a city near Kars, Bedj. suggests the Jab. 25 ult. reading of ow they came to Ani, see col. 270.

|col. 271. anise, aniseed, Med. 40. I6, 99. 4, 144. 18, I61. $6,542.4$, 554. I.

ب abs. form of أحُّ col. 272. tin, Chimie 97. 3. Usual name with alchemists, Zeus, ib. 3. 4, col. I IO4 under $\infty 01$ and col. 5 I 8 under Vh, also Hermes, col. 1053 under emasor, Chimie 2. I2, and Mars, ib. I26. I I ; col. $3^{81}$ under مصف?

崩 denom. verb from |al, Peal conj. to tin,

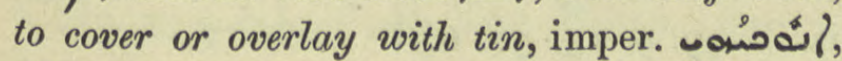

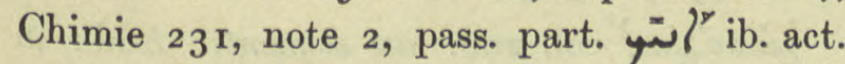

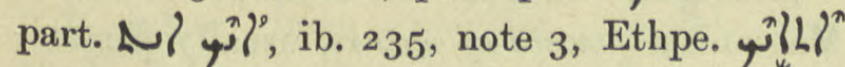

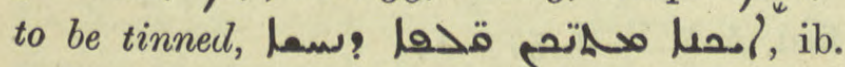
236 , note $I$.

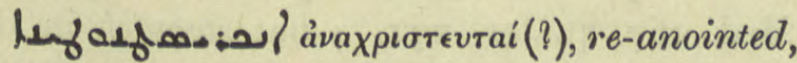
Anecd. Syr. iii. 172. I 5 .

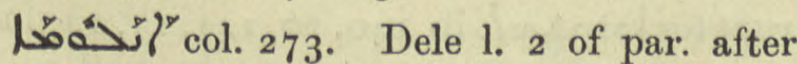
ref. to BH. 78. 7. Dele ll. 4, 5, 6 after the ref. to Jo. Eph. I 24. 18. Run on 1. 6 at It. to the end. Add: pl. الدحة مار victuals, provisions, Anecd. Syr. ii. 297. 6, iii. 129. 6 ; |مهار Ii. II8.

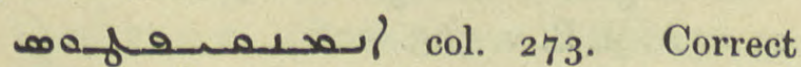
oflawas emancipati, sons freed from the father's authority, Syr. مسترן, Bruns and Sachau, Syr. Rom. Rechtsb. 4.

| and add refs. Jul. 234. 24, B HCarm. 105. $1_{3}$, I26. 5 , I I.

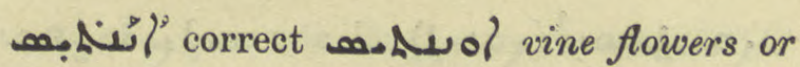
shoots, Med. 292. 3.

" in $^{n}$ col. 275 ult. Dele the last line from "Male". Ethpe. add refs. Pallad. 62 pen., Jul. 47. 23 .

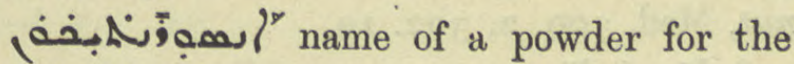
mouth, Med. I70. I 2.

yodos? a country, Doc. Mon. 163. 29, I7 I. 25 .

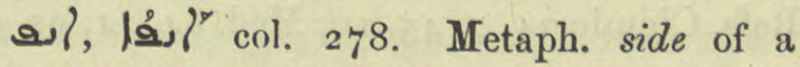
حسم:| محتمبم حم:ل

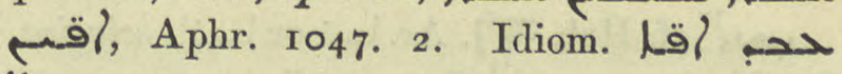
ILo: Jul. 53. 20.

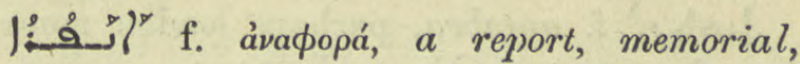
A.M. B. iii. $34^{2}$, pl. ${ }_{5}$, C. B. M. $1028 a$.

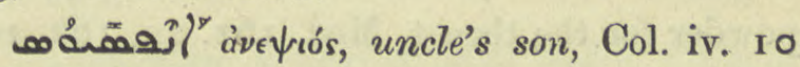
VHh., J!? is à BH. in loc., Sev. Lett. 3०5. $\mathrm{I}$.

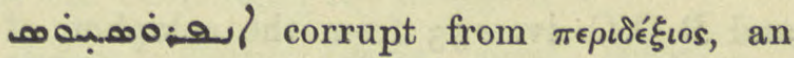
Indian fruit-tree, Natur 62. Io.

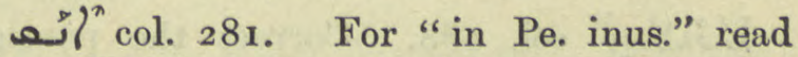
fut.

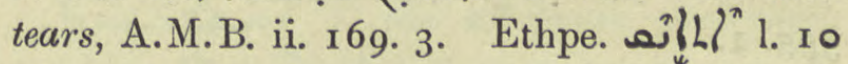
of par. dele the words "mensuram" and "aliquantisper".

" procession, a figure of Our Lord should be carried in procession through the cities, Anecd. Syr. iii. 324 . $23,3^{2} 5 \cdot 4$.

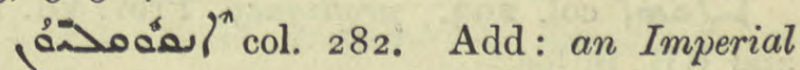
rescript, Pet. Ib. 79. 3, C.B.M. $936 b$.

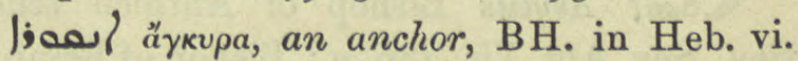

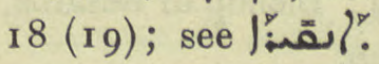

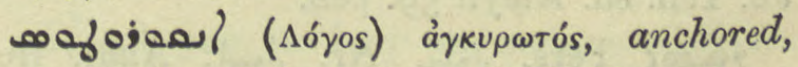
a tract by Epiphanius, C. B. M. $960 a$,

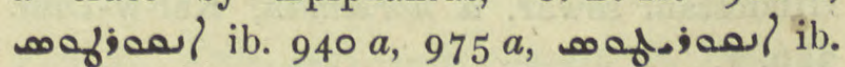

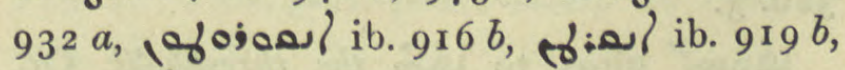
(of in ib. $960 a$.

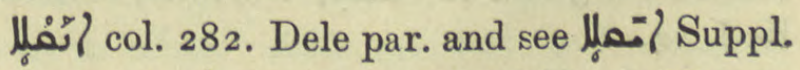
of R. O.C. i. $40 \mathrm{r}$. I3, I7.

صandentis Innocentivis, Pope, Alexis 20. 9 . 


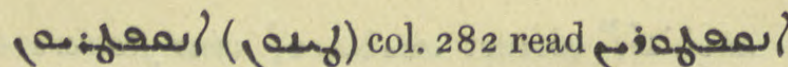

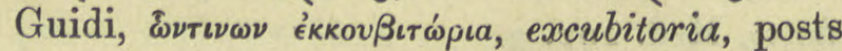
where guards were stationed, Zach. tr. H. and Brooks 318, note 14 .

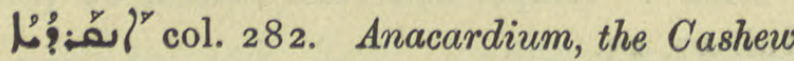
nut, Med. 309. 2, 392. 19.

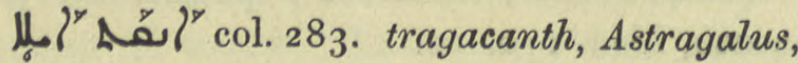

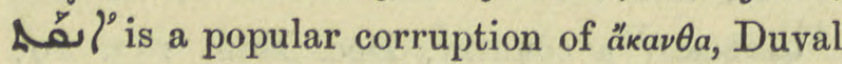
ap. Journ. As. 1894, 570; Ar. Pfln. 49. Refs. Chimie 34. 7, 45. 19, Med. 84. 22, 92. 6, 353. 22.

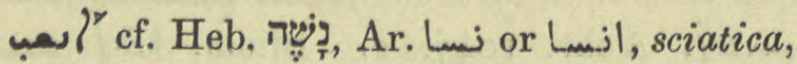
Op. Nest. 130. 7 in Gen. xxxii. 32.

inta monastery, C.B.M. 706 b.

l: $\sim$ ? f. antabra, perhaps leaden money,

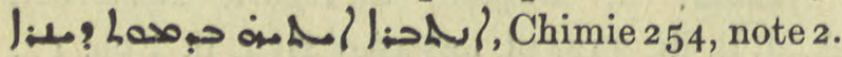

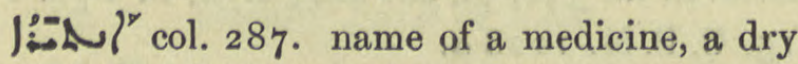
powder for the throat, Med. 161. 22, 162. Io.

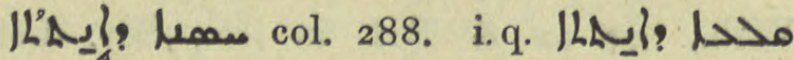
the Castle of Atta near Mardin, B.O. ii. 462 and R.O.C. iv. $I_{53}$ from the colophon of a MS. of B. H.

L'RLئly col. 288. Correct the passage

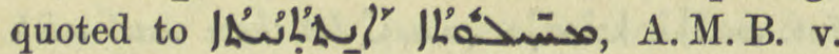
355. $\mathrm{x}$.

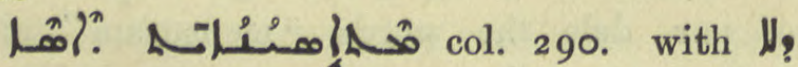
incorrigible.

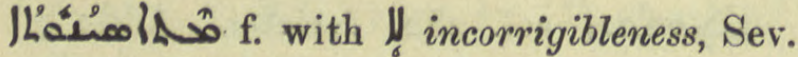
Lett. 28.3 af.

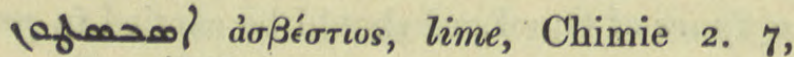
(a)fach), ib. 4. 1 .

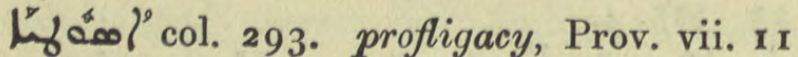
and $\mathrm{BH}$. in loc.

Maol Asylus Bishop of Antioch temp. Sev., Sev. Ant. Vit. 286. 9 ; Bishop of Resaina, Jo. Tell. ed. Kleyn 59. pen.

اسوار, Ar. Angl. from Hindostani sowar, a horseman, Kal-w-Dim. 326. 3 .

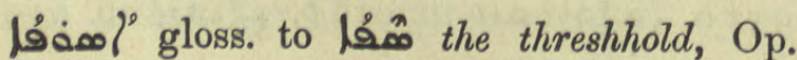
Nest. I 16. 3 and to "Magl" entrance, vestibule, ib. 1. 14 .

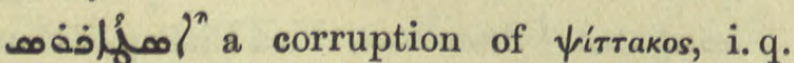
a (حد) a parrot, N. Hist. vii. 4. 2. I. q. col. 299 . c-sms.r castiafor

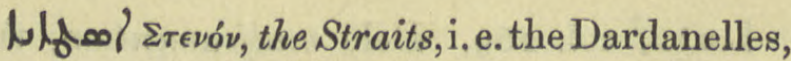
Pet. Ib. 23.15 ; لiffor ib. 1.7.

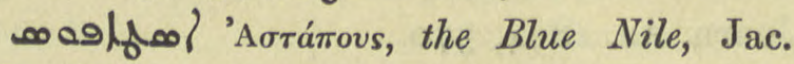
Edes. Hex. 24. 13.

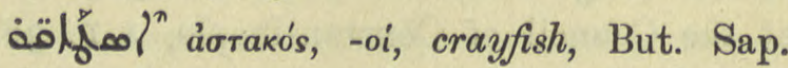
N. Hist. vii. 3. I, 6. 6.

صojlfifol oyster, Natur 64. $1_{3}, 1_{4}, 65.15$. See $\infty$ olifit?

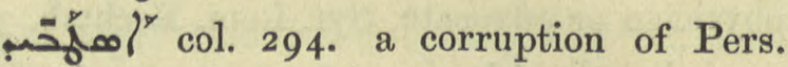
spahbedh or spahpat, a General, Tabari 96, I 44, \&c. Jos. Styl. 58. 16, 75. I 1, 22, 76. 5, 8. إستبرك و gold brocade, Gest. Alex. 200. 9.

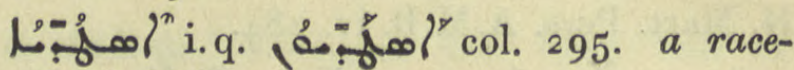
course, A.M.B. v. 2.

5aflol col. 295. Astahr or Istahr, a city in Fars, near Persepolis, Chabot, Syn. Or. 37. 4, 43. 25, 53. 8, 1 2, ZDMG. xliii. 396. 16. A province and diocese of Persia, Chast. 5. 4 . Written 20رo/ Sassanidi 26 ; cp. Tabari 397 ; ;ofjl, Mon. Syr. ii. 67. 26.

ofeol "A $\sigma \tau v$, a village in Egypt, Sev. Ant. Vit. 18. 9.

صọفة nightshade, Ar. عنب التعلب, Med. 601. 13, إم حتى à myrtle berries, ib. prob. wrong; it. ib. 49. 9, 15,5 o. 6.

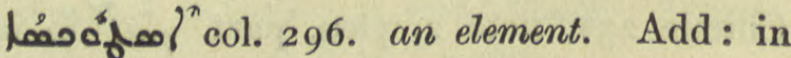
Magianism powers of nature, usually personified, A.M.B. ii. 577 . 17.

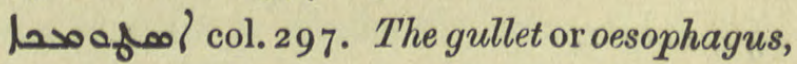
Med. 267. quater, 27x. 4 but stomach in the following pp. Mentioned as a drug, prob. corrupt for lo; ofol, ib. 140.20, 14 I. 17, 145. 16. |

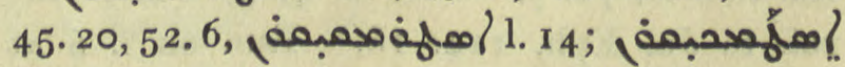
ib. $308,10,322,6$.

infol see iafol.

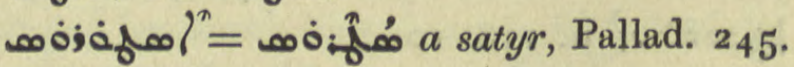
$2 \mathrm{I}=$ A. M. B. v. 565 .

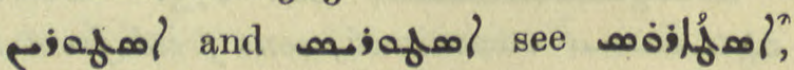
Anecd. Syr. iv. 86. I I.

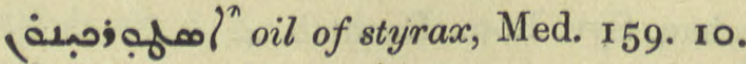

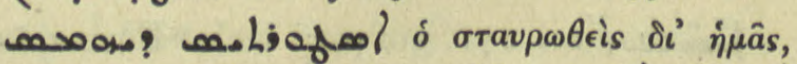
Anecd. Syr. iii. 219. 20. Cf. $2 \gamma_{3}$ l? larl مخهم ib. 2 I7. ult. 


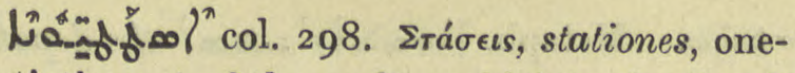
sixtieth part of the psalter which in the Greek

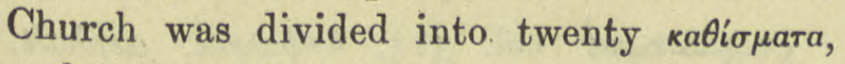

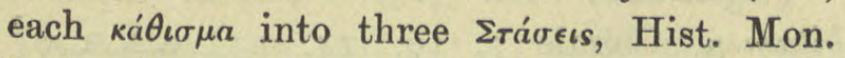
i. 142 , ii. 292 note, Is. Ant. ii. 54. 7 af.

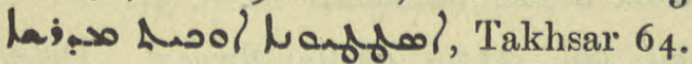

ल homilies were addressed by St. Chrysostom, C.B.M. $478 b$, $\infty$ a. - 50 ib.

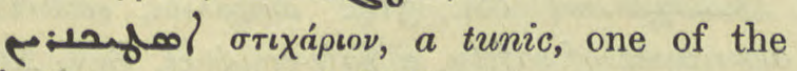
priestly vestments, A.M.B. v. 557. 17.

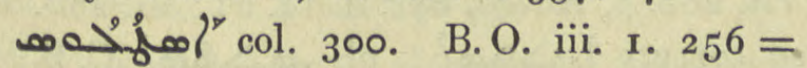
Card. Thes. 45.6 is $\sigma \tau \hat{\eta} \lambda a u$, pillars, but why

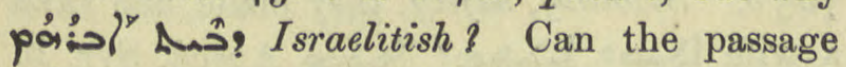
refer to the carvings of Jewish trophies on the Arch of Titus?

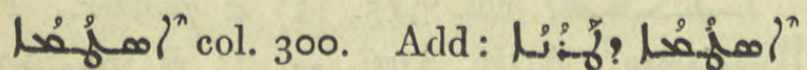
steely flint, Jac. Sar. Hom. ii. 65 , Hist. Mon. i. 329. 2 .

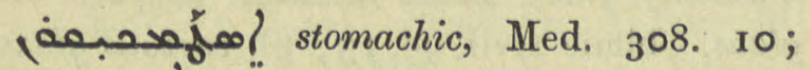

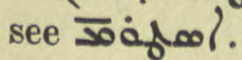

moyeol Ostanes, a Median philosopher, Sev. Ant. Vit. 62. 4.

linfol col. 299. ostiarius, doorkeeper, Anecd. Syr. ii. 236.9 bis.

مenfol col. 300. Refs. Ephr. ed. Lamy i. 469.3 ; حتس / Nest. Hérac. 269. 19 , 397. 4 .

|:

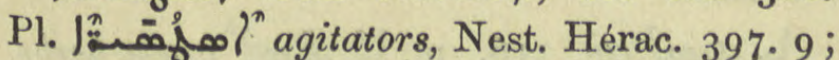
roughs, blackguards, B H Chr. Eccl. $725 \cdot 3$.

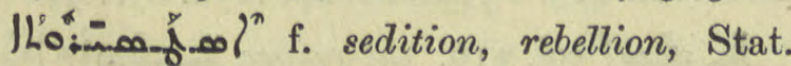
Schol. Nis. 2 r. 5 , forbidden by Canon iii. ib. 183. 2,3 .

.

|fî̉. first line of par. and run on l. ro at " non nisi in pl." add refs. Schatzh. 30.9 and passim, Med. 148. 18, 602. 9.

- 10 l pr.n.m. Asterius, Syn. ii. Eph. 32. 3 ; A. M.B. iii. $33^{6}$.

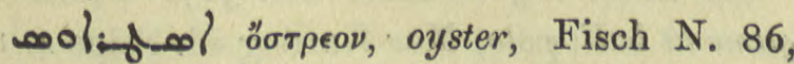
Anecd. Syr, iv. 91. 4.

إ. See h>0.jol col. 302 and Med. 181. 2 I, 23I. 2 I.

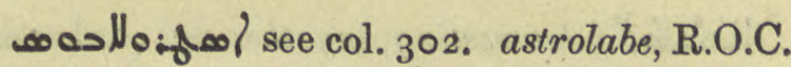

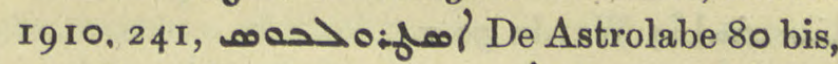
usually written jaص o. ff, 86 bis. Miswritten (a) "of ib. 80. 4 .

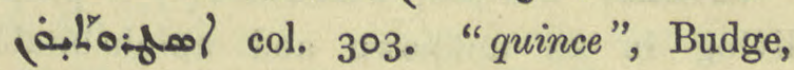
Med. 197. I 6.

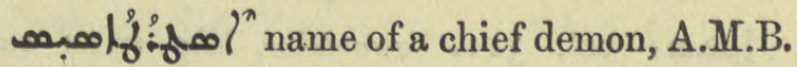
v. $445.4,8,447$. I I, I 5 .

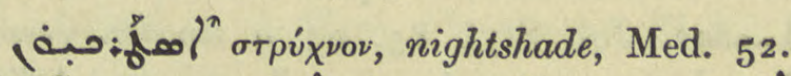

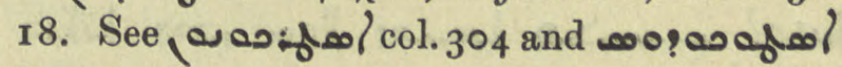
Suppl.

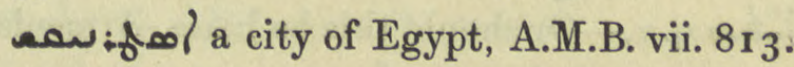
/ 'A Aravoi, A siatics, Syn. Or. 5, 168.6= 427 note 5 .

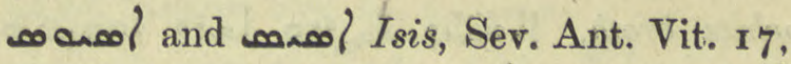

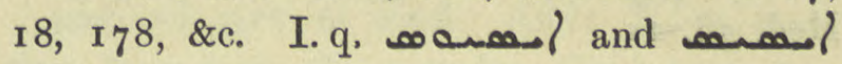
col. 162 .

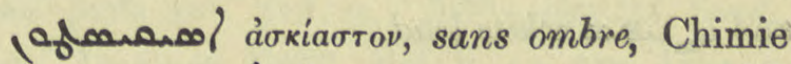
10. 7 , סممسחم:, ib. II. Io, ib.

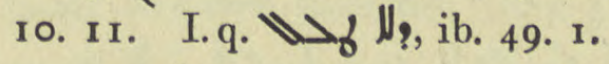

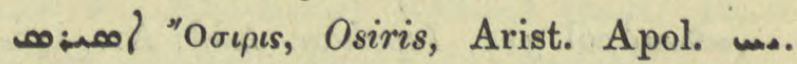
I1, 22.

Vanol To words derived from $\sigma_{\chi}{ }^{\circ} \lambda \dot{\eta}$ add:

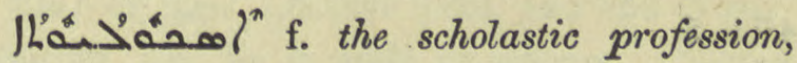
teaching, Stat. Schol. Nis. 30. 7, 31. 19, 192.7. 193. ult., Ihan خaمal? I: $\rightarrow$ ? Isoyabh, ii. ed. Duval 32. 20

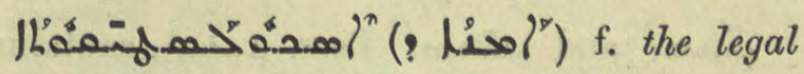
profession, Sev. Ant. Vit. $21.24,92$. I.

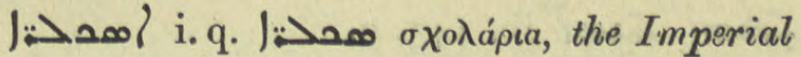
Guards, Aneed. Syr. iii. 233. 20.

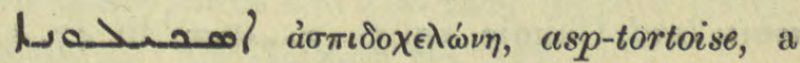
fabulous beast, Anecd. Syr. iv. 90. 4, Fisch N. $7 \mathrm{I}$.

مه

ली col..309. to heap up, metaph. C.B.M. $45^{2}$ b. 19. Act. part. m. pl.

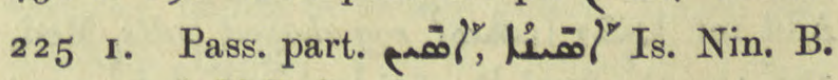
293. 18, A.M.B. i.

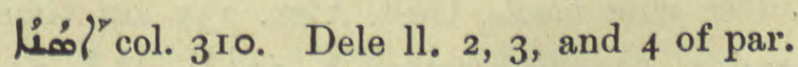
as far as cibus. Add refs. BH. in Prov, xxxi. 1 1, Gest. Alex. 75. 6. A reservoir of rain,

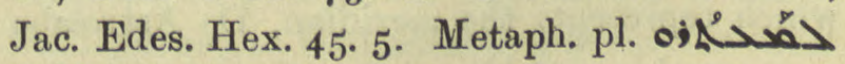

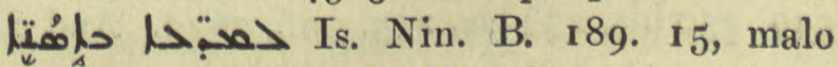
sensu of pride, \&c., Is. Nin. Ch. 74 n, 
रमa10\%

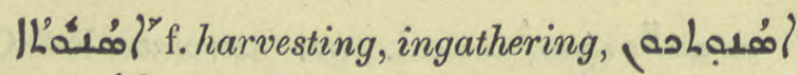

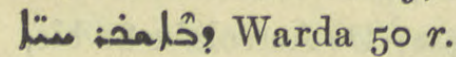

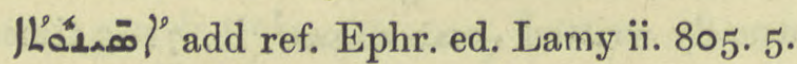

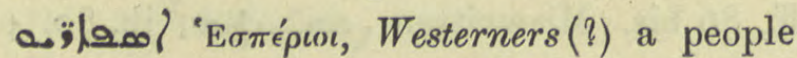
living in an unknown and uninhabitable country, Jac. Edes. Hex. 32. I I.

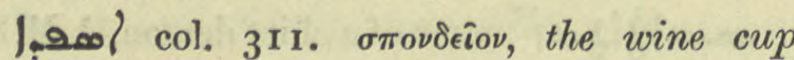
used for libations; a liquid measure; إمues. Jos. Styl. 85. 9; a wooden vessel, هـاتلا ?

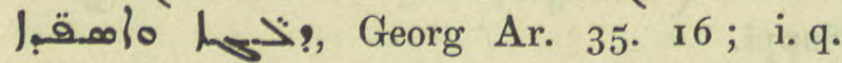
مهفْ: armenian unu'/nm?

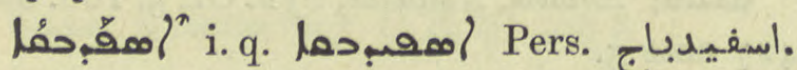

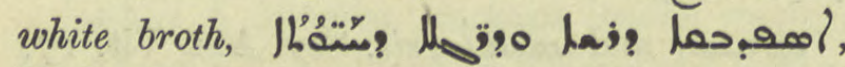
Med, 58. $2 \mathrm{I}$.

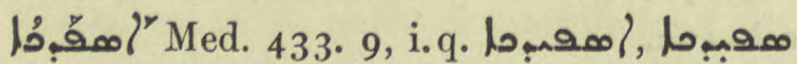
and owite lead.

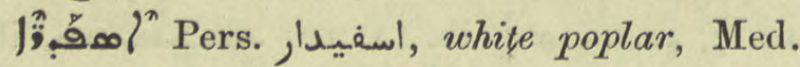
566 .

(اo Ptol. 'A $\pi \pi a \delta a ́ v a$, Syn. Or. 43 antep., 62 antep., 67. Ix, \&c., ZDMG. xliii. 396. I5, 403. 2, 404. 5 .

warol Spanish, Chimie 53. 18.

nollareol a bishopric in Arabia, Neapolis? Nöld. F. S. i. 470.39 .

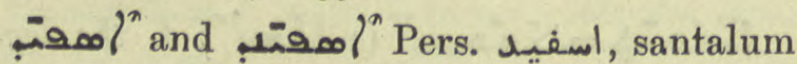
album, sandal wood, Med. 88. 4, 602. 4 ; أمثتب "أمفتب: ib. 145.9.

اسفيداج cors, ceruse,

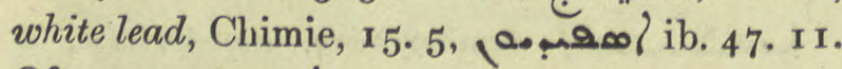
Oftener written مصبمرا.

|مقاق . Theoph. 1. 8.

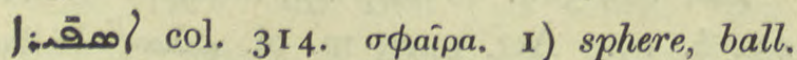

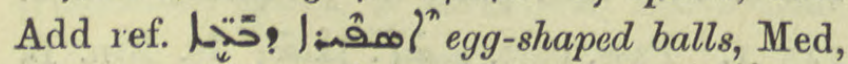

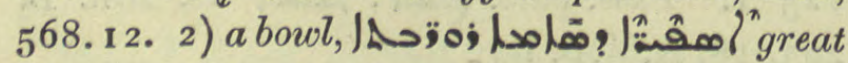
silver wine bowls, Gest. Alex. 237 six times.

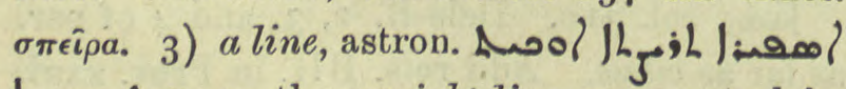

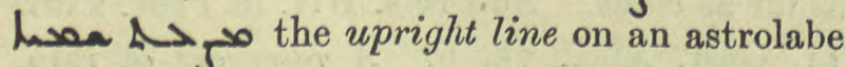
which represents the meridian, De Astrolabe 249,250 bis, 253 bis, 255.5 af., $262 \mathrm{f}$. And $\beta$

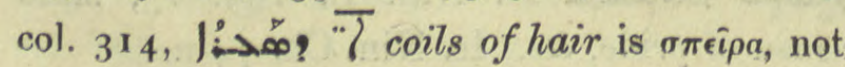

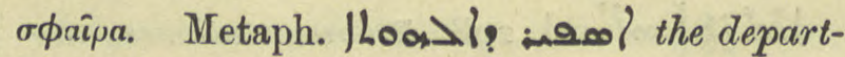
ment of Divine worship, Jul. 65. 26.

|s bitumen, Chimie 7. 19, 14. I3, ه-1.

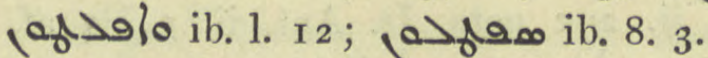

| the article and substitute $\psi$ a Fremdw. II5.

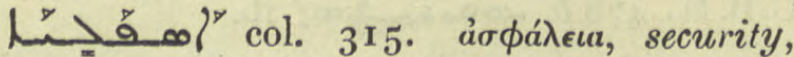
assurance of safety, a safe-conduct, Sev. Ant. Vit. 282. 5 , Anecd. Syr. ii. 14. 2 I ; iii. 280.24

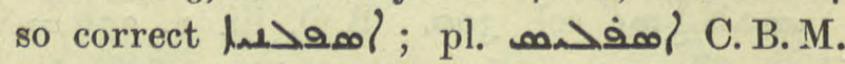
705 b. Stability, safety, they made him take

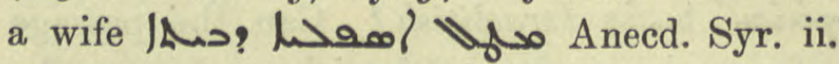
189. 19.

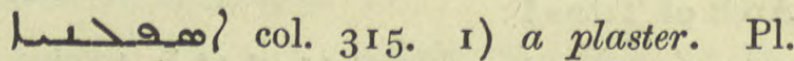

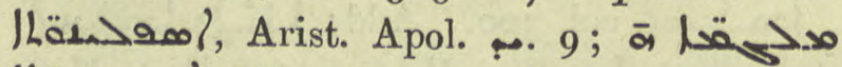

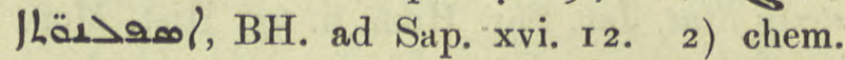
a coating, glaze, , ofapphire blue glaze, Chimie xlvii, 99.15 ; pl. various glazes, гог. 6. 3) spleenwort, Med. r०3.

heoel Aspona, a bishopric in Galatia, BH Chr. Eccl. ${ }_{57} 7.7$.

|م | Medicago sativa, lucerne, Med. 392. 19. See Tabari $244 \mathrm{n}$.

. I4. 2, 33. I5. Also written هوملاملا, مومحس, and 1 log.

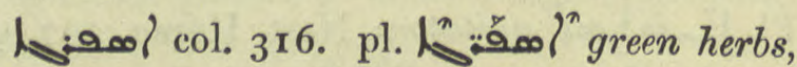
B HNom. 2 I 5 . 15 ; Galen ap. Ined. Syr. 94. 13.

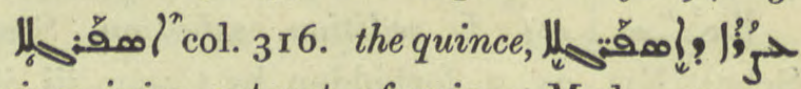
quince juice, extract of quince, Med, 207.12 ,

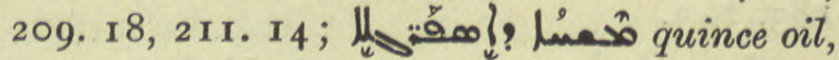
ib. 212.16.

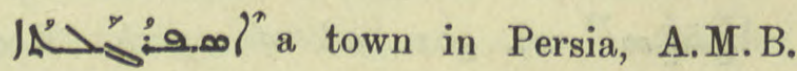
iv. 137 .

لlof: 00 i col. 317. Lat. sportula, a little basket in which largesse was distributed, $h$. a gift, Syr. Rom. Rechtsb. 32 .

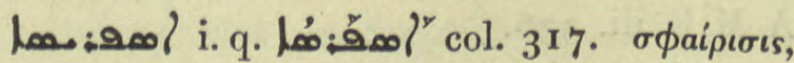
game at ball, polo, Mar Kardag ed. Feige 4. I 3 and note by Hoffmann. Or should Arm. wuryun'5q hippodrome be compared? Cf. ZDMG.xliv. 532. Dele "Aspharsa urbs" and the two sentences beginning "suspicatur." 
إمa:q. q. pol. 27 ro.

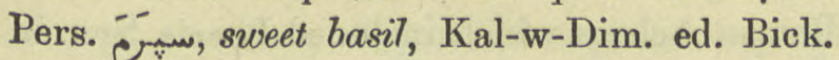

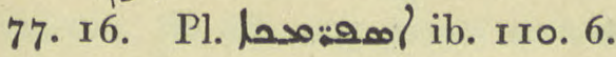

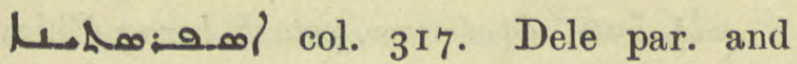
substitute: with حصب $\sigma \phi a \imath \omega \theta \hat{\eta} \nu a$ from $\sigma \phi a i p a$ a ball or button at the end of a weapon, thongs or gauntlets loaded with metal; to beat with armed gauntlets, Jo. Eph. I5. 1 2, I 5 .

|Aaol col. 317. $\sigma \pi a ́ \theta \eta, a$ spoon, spatula, Chimie 51. 5, 54. 7. The rule or pointer of an astrolabe, De Astrolabe 75 .

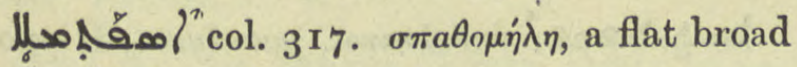
probe, Med. 214 . 19.

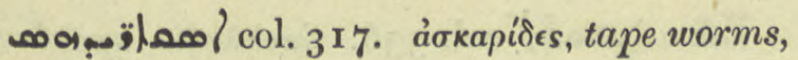
Hippoc. iii. 25 .

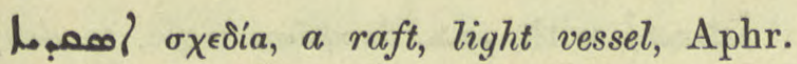

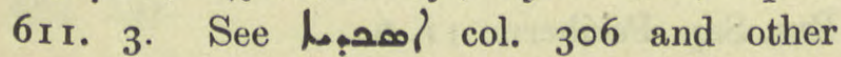
spellings.

(م) Skodarin, a lake in Roman territory, ZDMG. xxix. 92, Chron. Min. 73.6 .

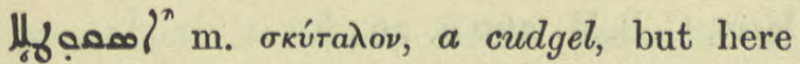
$a$ wedge of stone, David chose five smooth

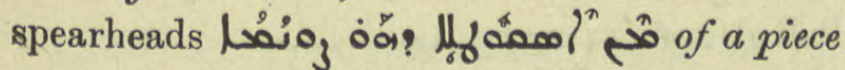
of that hard stone, Jac. Sar. Hom. ii. 65 .

Jasoofly.pl. possibly secundicerius, the second offieer; servants of the Governor, A.M.B. v.' 53 I. 3 .

h

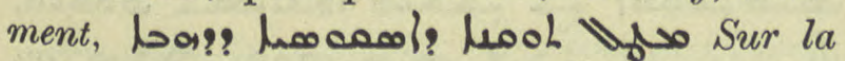
préparation de l'or, Duval says it is a variant, Chimie Io. $4=19.4$ and note $\mathrm{I}$.

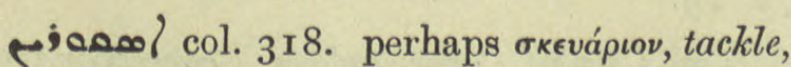
ropes (supplied by basket-makers), Anecd. Syr. ii. 106. 9. Cf. Jimal DBB. 24I. 5 and note 2 .

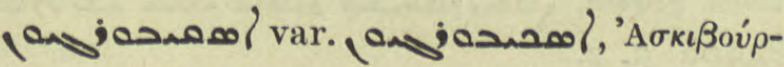
rov, not a mountain as Jac. Edes. supposed but a city, Asciburgium on the left bank of the Rhine, Jac. Edes. Hex. 34. I 2.

M.م-ol à $\sigma \kappa \eta \tau \dot{s}$, an apprentice, learner,

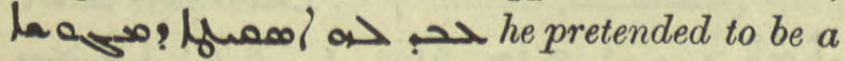
student of Magianism, Kal-w-Dim.ed.Bick.8.9.

(a.entanool col. 318. Dele par. and substitute, a book on the Ascetic Life, by

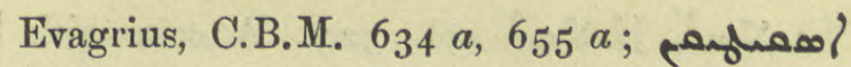
the Asceticon of Pachomius, ib. $727 b$, I $100 a$,

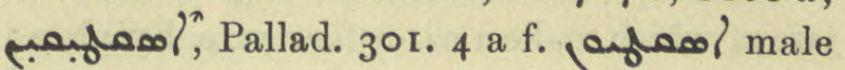
ib. 343 . 10.

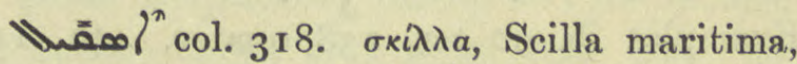

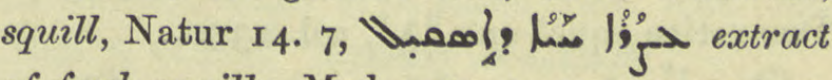
of fresh squills, Med. 197. 4, 199. 7, 299. 2, 4, I7, 601. I 2 .

/ مقدحص m. pl. Slavs, Slavonians, El Nis. Chron. 140. 24.

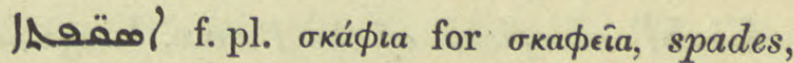
Act. Apost. Apoc. 10. 2.

|

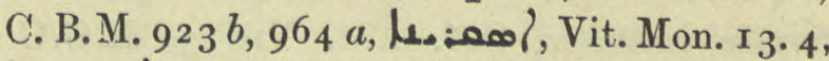
أمم:م: Anecd. Syr. iii. 354 2.

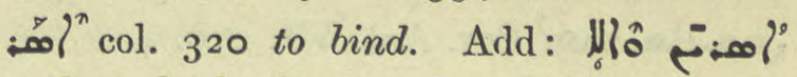
they loop back the curtains, Takhsa 7, I3. Pass. part. col. 322. 9. Add: limited, local, معod إمسن localized substance, ib. i. 5 I. 47 ; fixed,

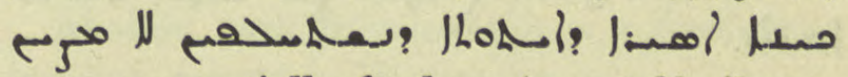
natures essentially fixed are immutable, ib. 77 . I5, 99. I I ; "bound in essence," ib. ii. 2 I 7. 33 .

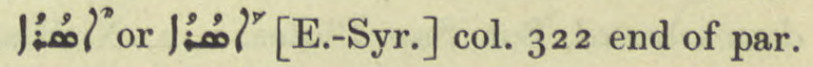
Binding i.e. by a spell or charm, Charms passim, Protection 7 and passim. A tendon, ligament, Med. 109 ter, 380 ult.

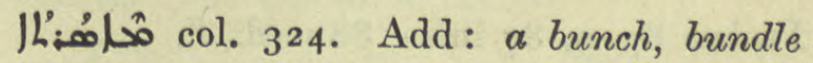

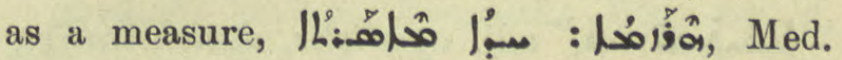
299. 10.

: roo fla $\lambda \epsilon \pi \tau a ́$ and roo /

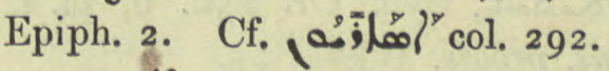

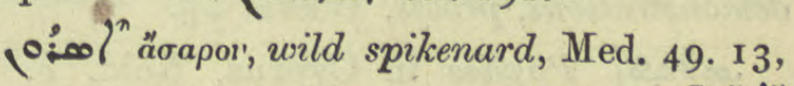

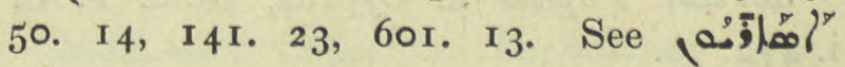
col. 292 .

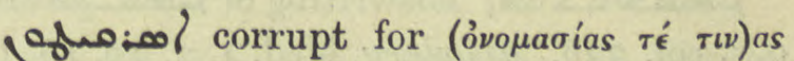
ả $\chi \chi^{\prime} \nu \tau \omega v$, names of certain rulers, Theod. Bar Konî ap. Hippoc. v. I ff., Coupes ii. II 9 in Epiph. Anacephalaeosis.

IA 8 " pr.n.f. Astha or Esther, mother of Heli son of Matthat and of Jacob son of Matthan, so Joseph was physical son to Jacob but legal son to Heli according to the Levitical law, $\rightarrow$ ol $\overline{5}$ IACl, BH. in Luc. iii. 25 ; Hist. B. V.M. 5. 2. 


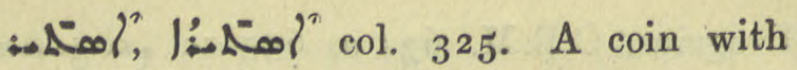
head of Ishtar, Jensen ZA. xiv. I 83. A measure two of which make I oz. or four zuze, Epiph. 2 ;

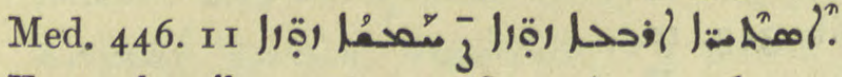
Examples ib. 90 ter, I $3^{8}$ quater, 446. II. Two estire equal one لـ

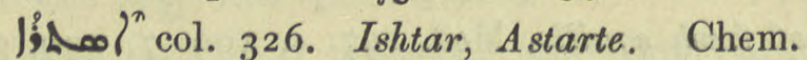
copper, Chimie 2 I I note $\mathrm{I}$.

lol an Arabian monastery, C.B.M. $71_{3} b$, Doc. Mon. 223. 19.

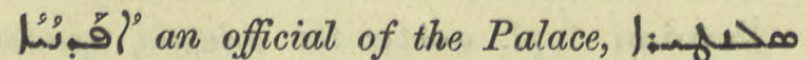
إר Anecd. Syr. iii. 256. 17.

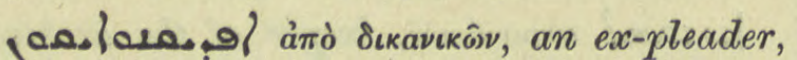
former barrister, Sev. Ant. Vit. 3, R.O.C. 98 , n. 2, Anecd. Syr. iii. 226. 3, 245. 6 ;

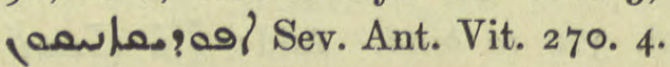

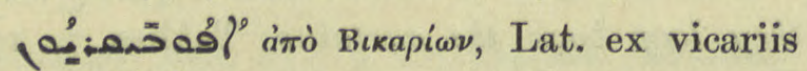
fecit, Pallad. 20r. r 7 .

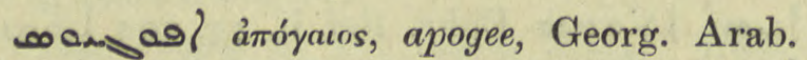

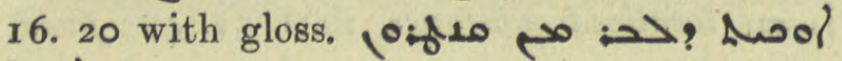
1;?.

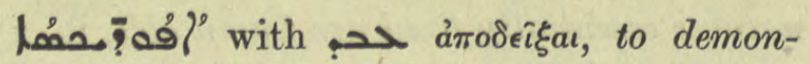
strate, Nöld. F. S. i. 48r. 2 ; with loa to be demonstrated, But. Sap. Philos. I. 2, N. Hist. ii. I, sect. I, v. 2. I infra.

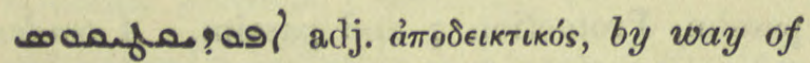
demonstration, one of three kinds of syllogism, Probus 83. 9, 87 infra, 88 med., ff.

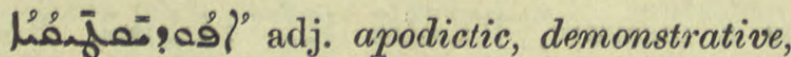

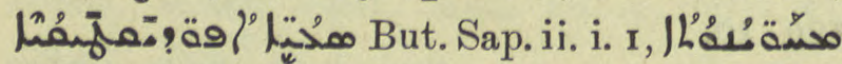

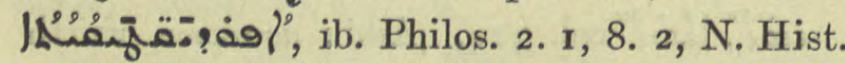

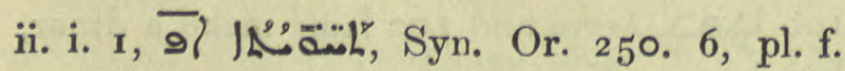
demonstrations, proofs, Tekkaf 25 .

مoا Paphos in Cyprus, Nöld. F. S. i. 472.78 .

أlima q.v. col. 336. apoptexy, Med. 4. 2, 10. 16, 17.

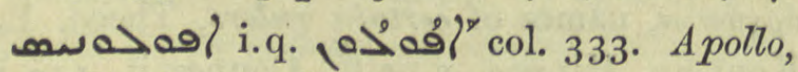
Stud. Sin. ix. 97 ult.

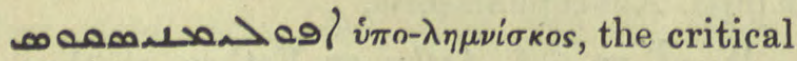
mark —, C.B.M. $792 a$.

. Rom. Rechtsb. 55 .

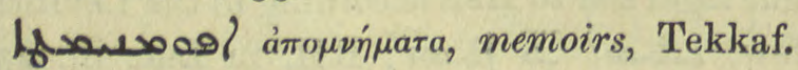
114.

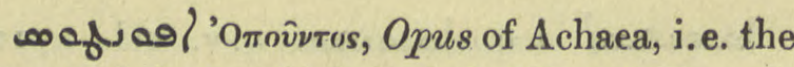
Roman Achaea which included Locris, Syn. ii. Ephr. II. 14.

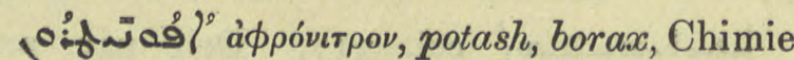
4. 12, Med. 147. 10, 18, 149. 2, 160. 1.

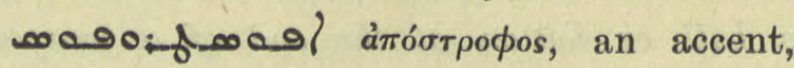
Hebraica iv. 168 Syr. 2; Epiph. 7. 6.

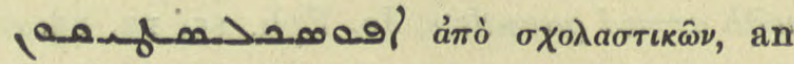
ex-barrister, Pet. Ib. 81. 20.

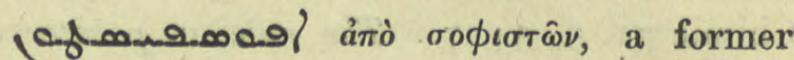
sophist, Pléroph. 1 13. 5, R.O.C. v. 475 in loc.

l. vii. 37 .

| Hippoc. ii. 42 , iii. $15,19,22,30$, vi. 54 .

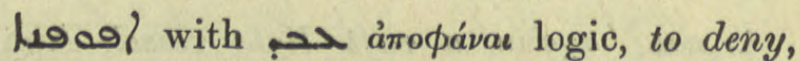
But. Sap. Periherm, i r, 6.

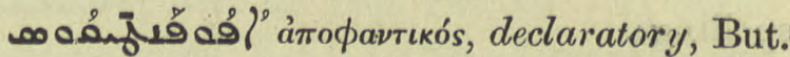
Sap. Periherm. 2. I.

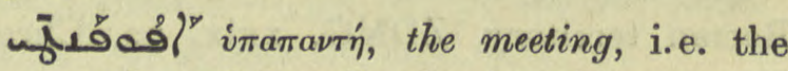
presentation of Our Lord, when Simeon met Him, C.B. M. $260 a, 264 b$.

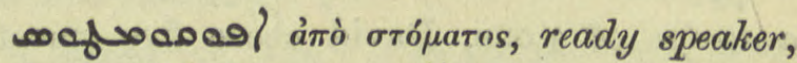
Pallad 206.' I I marg. حاضر للمجي.

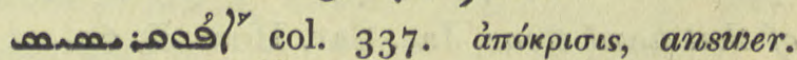

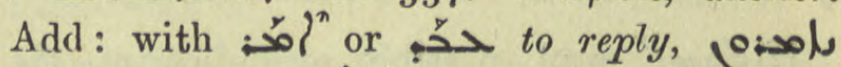
דيl

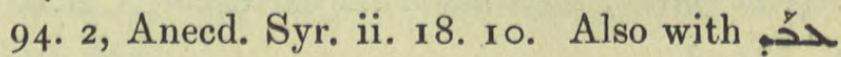
to act as agent, transact business, Ma>L onlo,

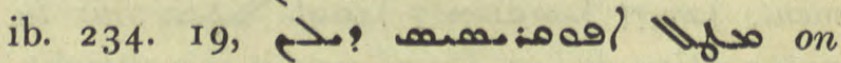
account of our affairs, ib. 1. 26; Sev. Lett. 72. 19 .

| agent, C. B.M. 977 b, Doc. Mon. 169. 25 .

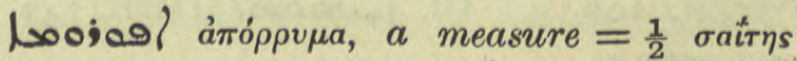

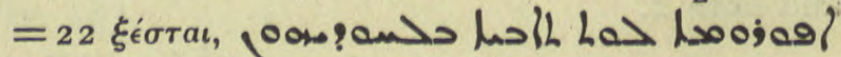
Epiph 5. 2.

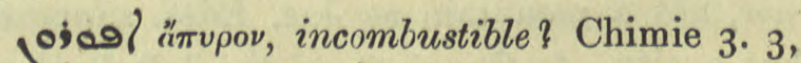
10:0) ib. ult., 0;00\}, 10. 19.

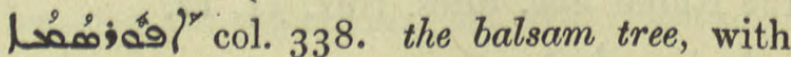

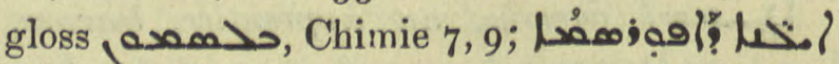

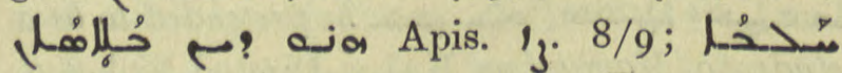

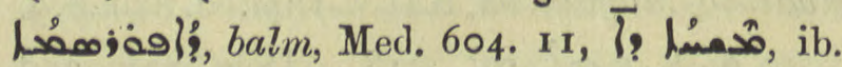

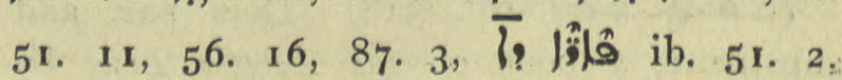




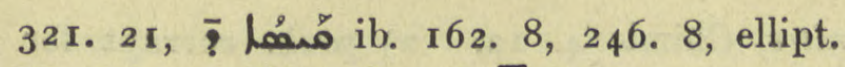
ib. 87. 16, liọ! â- $\widehat{T}$ T half an oz. of balsam, ib. 88. 20 ; given as a rendering of A Aa ans' ib. 609 ult. Trs. hither BH. de Pl. f. 6 col. 3074 ult.

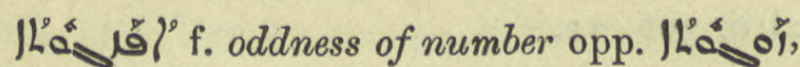
But. Sap. Isag. ii. 4, iii. I infra, Philos. 6. 7,8 .

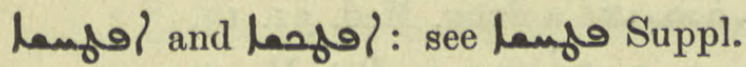

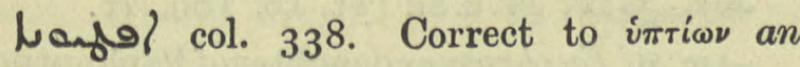
officer, Krauss, L. W.

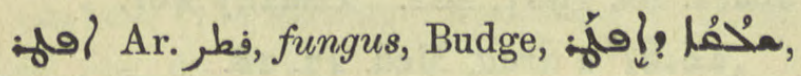
Med. 51. I6.

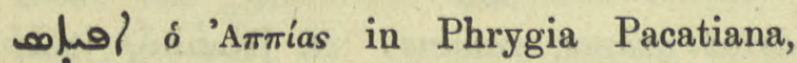
Apia or Appia, now Avia, Nöld. F. S. i. 474. I3 1 .

L.j/ Apiaria, ancient name of Macedonia, El Nis Chron. 34. 8, 10.

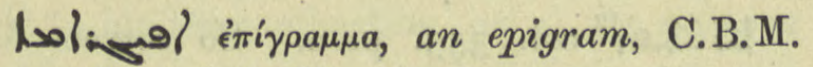
545 a, Sev. Ant. Vit. 33 r. 6.

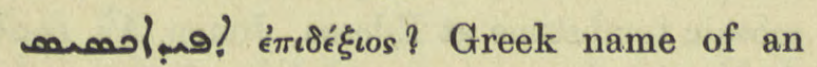
Indian tree, C. B. M. I 193 a.

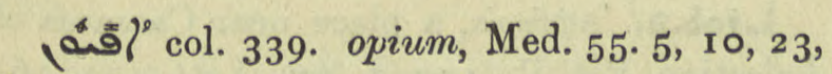
60. 9 \&c.

of 1 ? Apiton, one of many names of a female demon, Charms 84 .

cof C.B.M. ror $3^{b}$.

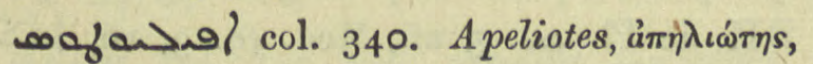
the East wind. Cf. Löw, ZDMG. xxxvii. 472 . Add ref.

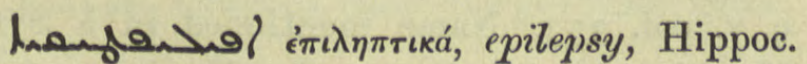
v. 6 .

no:-on-ol Apsimar, usurper of the Byzantine throne, A.D. 698 ; Jac. Edes. Chron. Can. 572. $\mathrm{x}$.

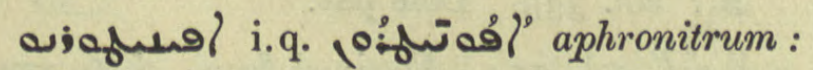
see above.

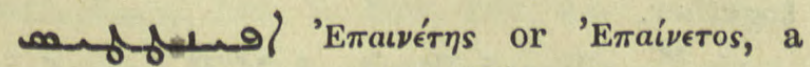
scholasticus of Alexandria, Pléroph. 28. 12.

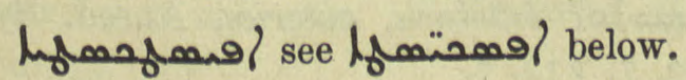

I I I a Pisidian, Sev. Ant. WZKM. ix, 203 .

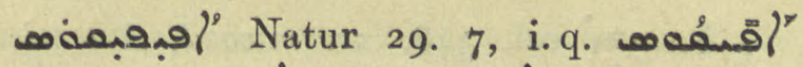

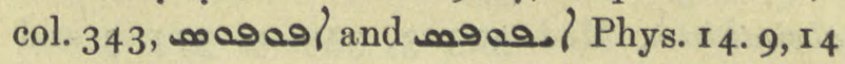
є $\pi \circ \psi$ hoopoe.

مa Epiphanius, Bishop of Tyre, brother of Flavian, Patr. of Antioch, Sev. Ant. Vit. 100. $7,114.6$. Usually

Learl col. 343. i.q. Jants, ref. Ephr. Ref. ii. 3r. 4 O.

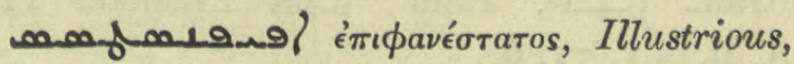
Anecd. Syr. iii. 56. 16, 163. 18, 167. 13; Kugener in loc. R.O.C. v. 463 med., C.B.M. $1053 a$.

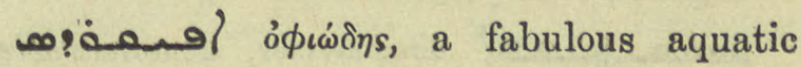
creature, A.M.B. iii. 276. го. Perh. same as the following word.

أومفة a fabulous sea creature but it has the effect of a basilisk, Natur 57. 16.

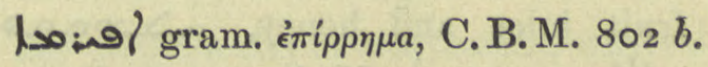

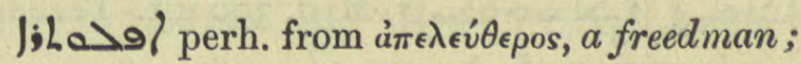
inscript. quoted in Hippoc. xx. n. $\mathbf{r}$.

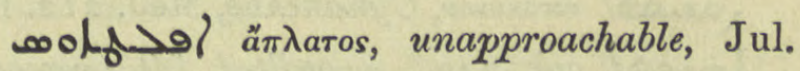
33. 19.

L. $\infty$ ol Apamria, Monoph. monastery where Achudemmeh baptized the son of Chosroes, Patr. Or. iii. i. 34. I I.

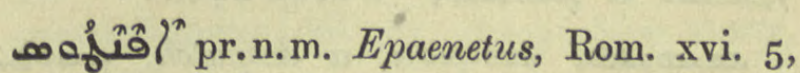
BH. in loc., Jul. 56. 16.

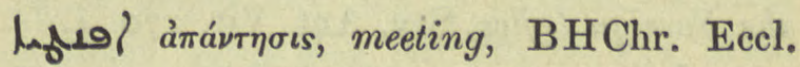
207. 11 .

of 177. 10.

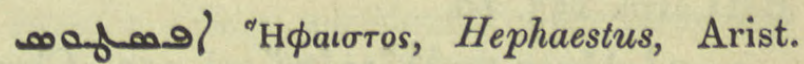
Apol. ب 2 I.

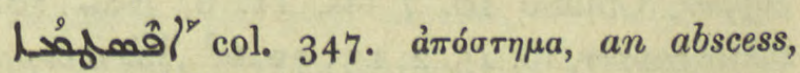
pl. |حear, ib. 100. 20 , on the lungs, ib. 194. 19, 195. 8 , in the belly, 282.16 .

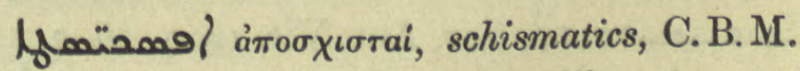
1053 $a$, I055 $b$, Anecd. Syr. iii. 165. 15, 188. 16, 189. 14, \&c., hfonfoul ib. 182. 20, C.B.M. $1055 a$, Hمصond Anecd. Syr. iii. 194. 10, Af 
بول? col. 348. wormwood, Artemisia absinthium, Med. 3०3.3, 359. 9, 377. 3 and often; BH. de Pl. $f$ ro.

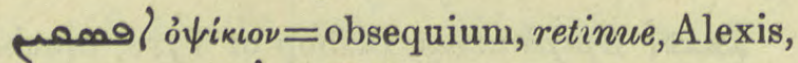
18. 2 ; همبمىi, B HChr. I 20. 3.

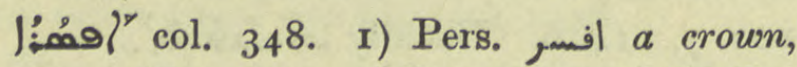
headgear like a crown of Syr. priests, F. Müller WZKM. viii. $364 . \quad 2)$ Pers. سِ halter, Aram. אפסטר Fraenkel II5, A. M.B. v. 7 I. 6.

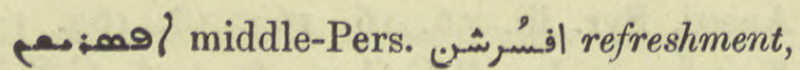
cooling, Lag. Symm. 9I ; a powder to strengthen the eyes, Med. 92. 44.

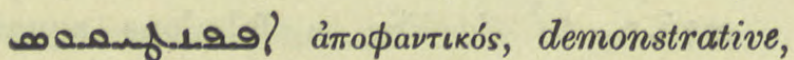
with $1: 0$ a syllogism, Syn. Or. 250. I.

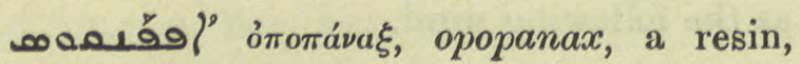
Med. 49. 18, 50. 9, 54. 6.

" with fruit juice and honey, ب-

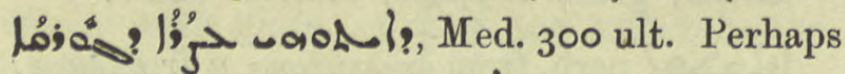
a miswriting of wamol.

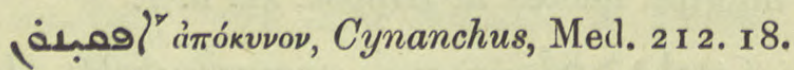

إم col. 349. a quilted felt overcoat,

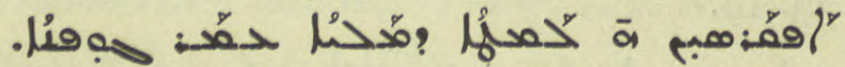

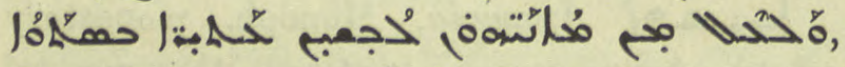
Op. Nest. 96. 1 2 f. in Jud. xiv. 1 2, 13 ; إهم้: مبر aَ" Warda $246 r$.

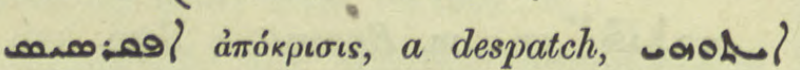
ILan oa he was in the Foreign Office, Sev. Ant. Vit. 270.4 .

J:Q? a place in Arabia, C.B.M. 7 I4 $a$, Doc. Mon. 224. 10.

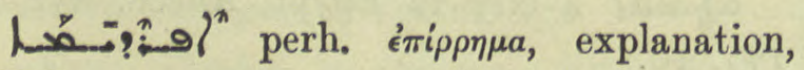
BH Carm. 1 55. 10.

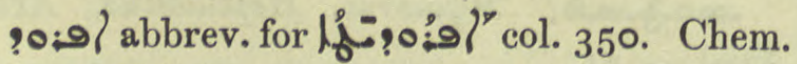
copper, Chimie 10. 7 bis, I1. 8,

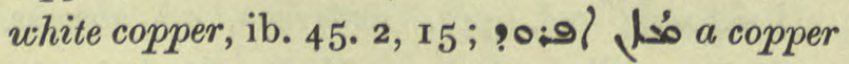
vessel, ib 48.3 .

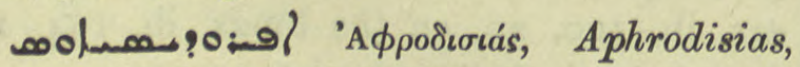
capital of Caria, Nöld. F. S. i. 474. I33;

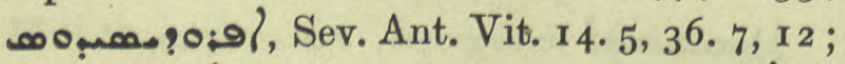

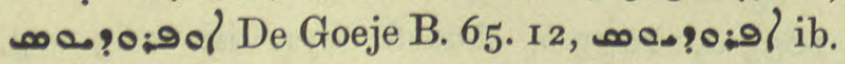

, Euphronius, Bishop of Antioch, Or. Xt. i. 86. I 7 .

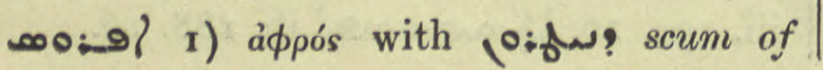

nitre, Chimie 4. I2. 2) prob. corrupt for Ev̉pos the South East wind, Med. 533. 3.

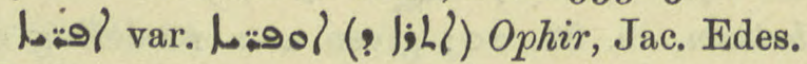

Hex. 2 r. 5 .

: xliii. 396. 12.

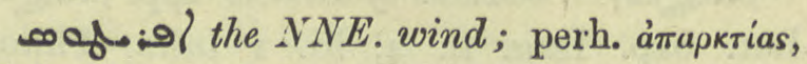
Med. 533. 6.

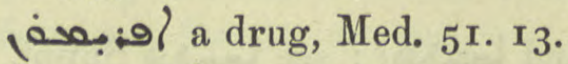

مه

ulo: $\{$ Euphrosyne daughter of Paphnutius, Journ. As. 1907, 402 . Usually $\overline{9} 0\}$.

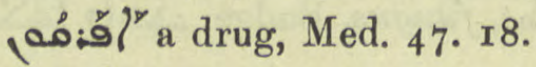

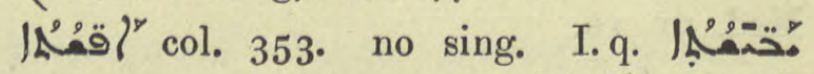

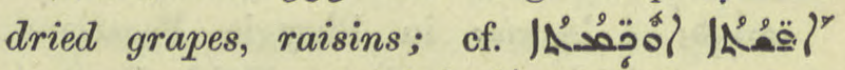

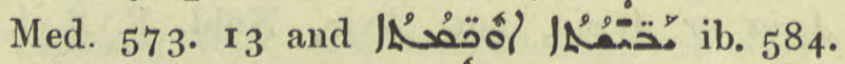

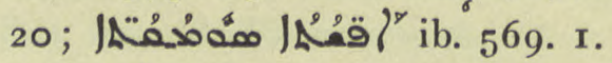

Nol Afta, a village of a Saltou, Pléroph. เоo. $8=$ R.O.C. iii. $3^{61}$ infra.

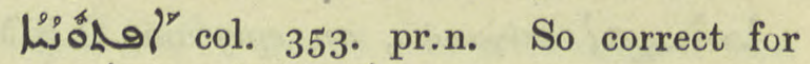
10010/ and loods?, pr.n.m. Aftōna, on a mosaic tombstone at Edessa, Journ. As. 1906, 283 .

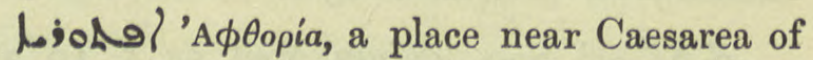
Falestine, Pet. Ib. 1 20. 7, Sev. Lett. 132. 16;

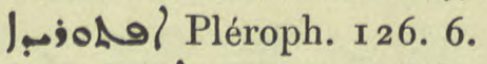

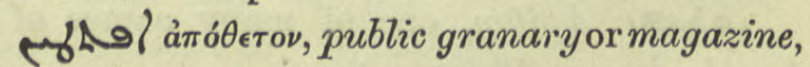
Jos. Styl. 35 ult., 64. 19, 77. I3.

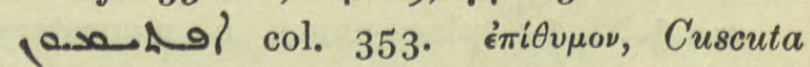
epithymis, a parasitic plant growing on thyme, BH. de Pl. محْمُ 5 af., Med. 49. 3, 16,

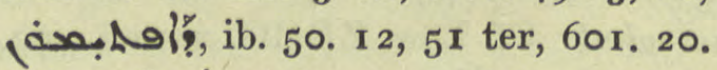

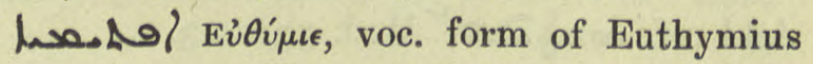
q. v. col. 353. Name mentioned in a Syr.Melchite All SS. litany, Or. Xt. iv. I 16. 7, I18. 10, R.O.C. iv. 156.

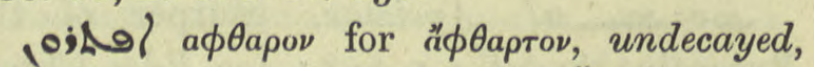

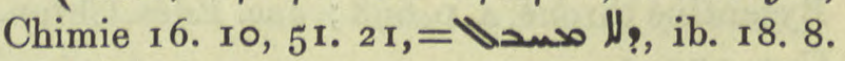
๑) col. 355. Dele par.

$1 \mathrm{Lo}_{3}$ ?' col. 354. Derived from 5 to press together.

;. of a village in Persia, A. M. B. iv. 2 I 6. 16.

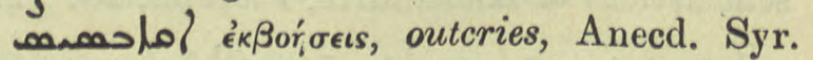
iii. $203.7,272.7$.

uق like a nettle, N. Hist. vii. 2. 2. 
ma N. Hist. vii. 4. 4.

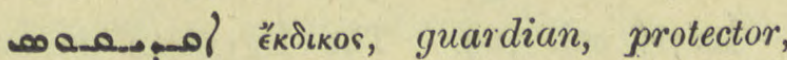

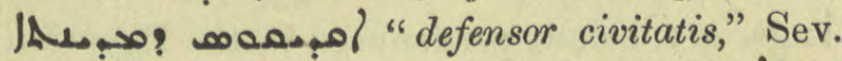

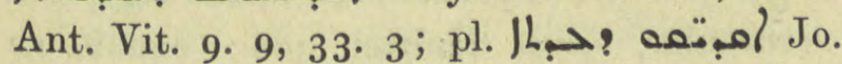
Tell. ed. Kleyn 73. I I, 74. 5, 82. 5, Vit. Mon. 87. 23, 94. 17. Cf. Cos col. 23.

إمب. place-name, A.M.B. I. 4 I 5 .

|

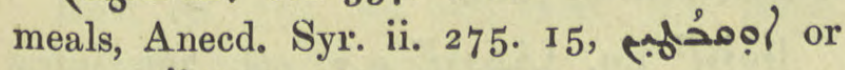
/' Gest. Alex. 51. 6, I 2.

(a. ? 9 (?) a surname, Accudaeus, BH Chr. Eccl. 175.6.

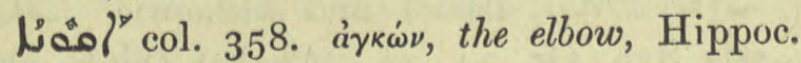
vi. 22, Med. I31. I 2. A cubit, Jac. Edes. ed.

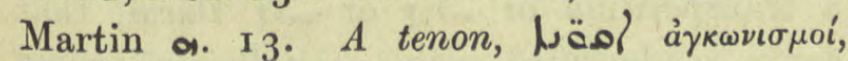
Hex. Ex. xxvi. I 7 .

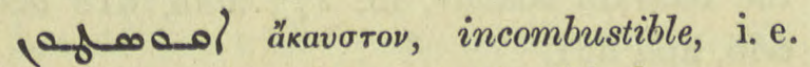
I) sulphur or pyrites mixed with honey or salt to hinder combustion, or 2) the unburnt residuum, afood as Chimie 16. I2, I 7. 2, \&c.

toal for مó אókкos, a berry, Med. 600. 5 .

(2) बfo. of

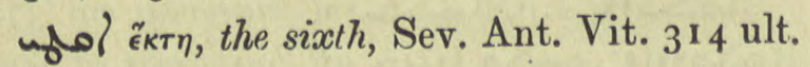

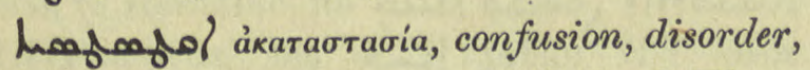
Vit. Monoph. 75. 7 , 80. 5 ; Jo. Tell. ed. Kleyn 58. $8,64.6$.

endel col. 359 and send the ocean, C.B.M. $427 a$.

م

Sin. ix. ror. 9 .

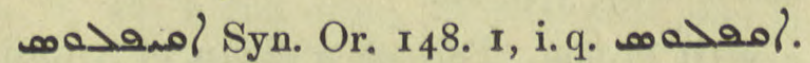

$\left.\prod_{0}\right)^{n}$ col. 360 . the fastening of a curtain,

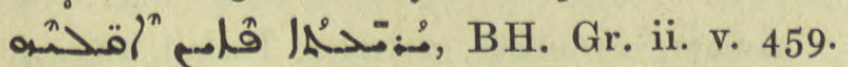
Cf. lla.p.

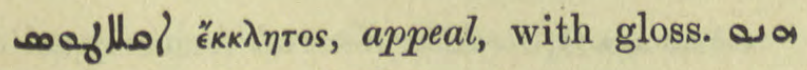
Ihaidisas, Syn. ii. Eph. 28 r. 24.

lol col. 360 Jo. Eph. I59. I3 corr. أl q.v. col. 363 . S. Fraenkel ap. ZA. xvii. 85 .

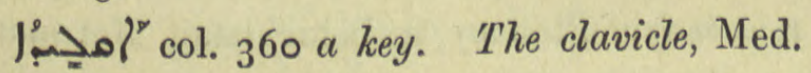
223. 3 .

271

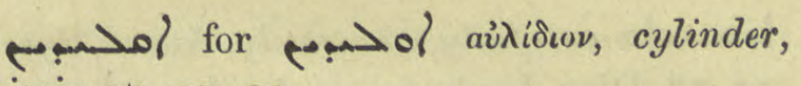
Chimie I7. $21,22$.

مشخ: cellaria, Fraenk. ap. ZA. xvii. 86.

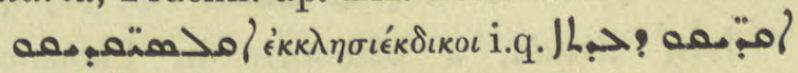
q.v. supra, churchwardens, Sev. Lett. I34. II.

?

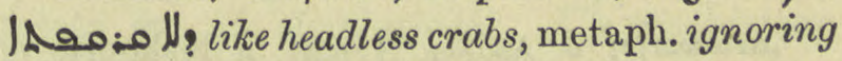
authority and procedure, Syn. Or. 182. 26, مه مصحه|?

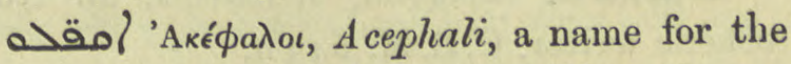
Monophysite party, Sev. Ant. Vit. 28. 34, I07. II.

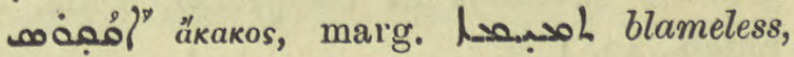
Warda $207 r$.

إقاقيـا Ákakia, acacia, Mimosa nilotica, Med. 83. 7, 170. 18, 22, I71. 2, 601. 4 ; misprinted ib. 64. 10, 65. 2, 169. I5; Ar.Pfln N. 197, Galen. I3v. Cf. /ملم/ col. $35^{6}$.

'مُج 'Akar, a city of Marmorica, where James

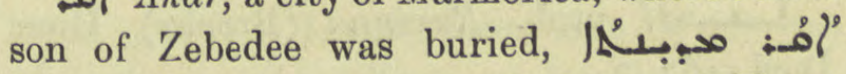

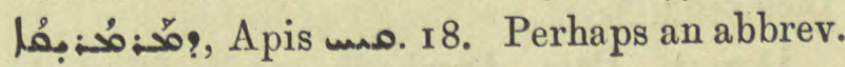
of the following.

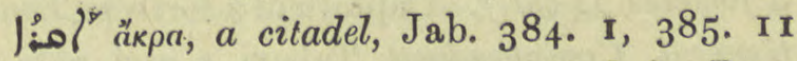
text and the same passage quoted in Pers.

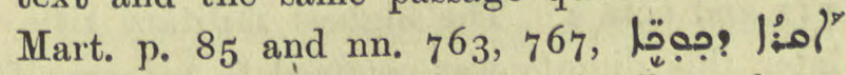
the fort of Cuche, i.e. of Seleucia, ib. 536. 10. Nabataean اقر, Comm. on Labid I 12.

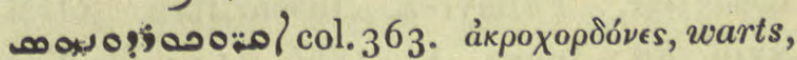
Hippoc. iii. 25.

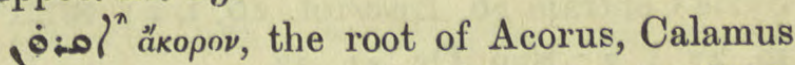
aromaticus, sweet flag, Ar. Pfln N. I3 I, Med. 51. 2, 10, 246. 9, 399. 9 .

ANoid a fortress on the Tigris, Patr. Or. iii. i. $3^{2}$. II, $48.6 \mathrm{ff}$.

;0:م a village mentioned in the colophons to Medicine and Hitist. B.V.M.

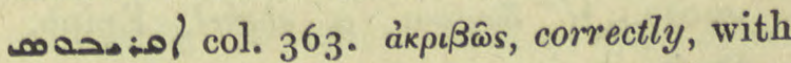
$N$ it is not the right thing, not according to etiquette, Vit. Mon. 9r. 2, Jo. Tell. ed. Kleyn 77. 18.

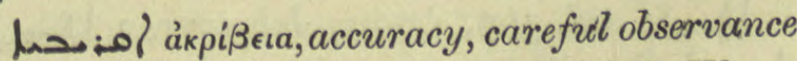
פمته Vit. Mon 75. 7, Jo. Tell. ed. Kleyn

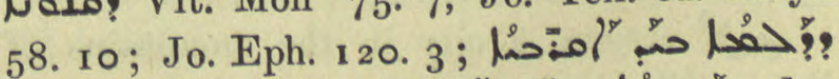

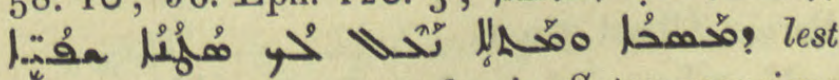
overclose attention to trade give Satan occasions of hindrance against you, Greg. Cyprus $9 a$; 
- محام carefully, accurately, Anecd. Syr.iii. I1. 1 8, Dion. Ined. 479.4. I.q. h إم: col. $3^{6} 3$.

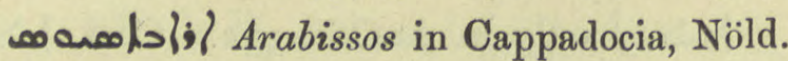
F. S. i. 47 I. 66. I.q. I?\}; Arada, a bishopric in Phoenicia, Nöld. F. S. i. 469. 19. I l: M.Z. 206. 2.

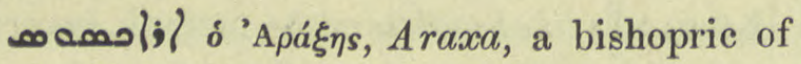
Lycia, Nöld. F. S. i. 474. I 28.

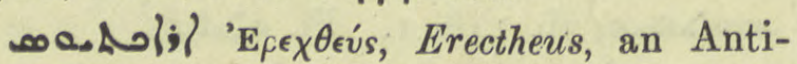
ochene writer, C.B.M. $643 a$.

|Rُ groins; swellings in the groins, Dion. 42. antep., 43. 1,3 .

|'خه place-name, A. M.B. i. 4 I 7 .

IA under col. 2968, Fisch N. 2.

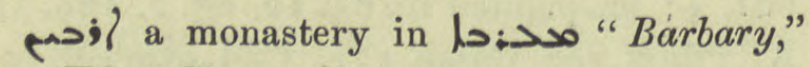
Jac. Edes. Chron. Can. 576. Io.

henosil prob. Ravenna (Brooks), Dion. Ined. 483.3 .

ار إ) a fortress بـ chief. Captain of a fortress, hence General, Commandant, Tabari 5 and note 3, I I I and note 2. The highest military title, later this dignity was confined to princes of the royal family; Palmyrene ארגבטא, Gr.

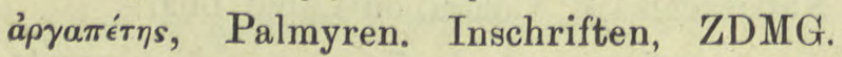
xviii. 89; Journ. As. 1866, 114 ; De Vogüú Syrie Centrale 26, Inscript. 26, 1.2 ; ib. $27,1.2$; Syn. Or. 2 1. 22.

Mas il a village in Qardu, Chast. 67. 3, A.M.B. iv. 163 .

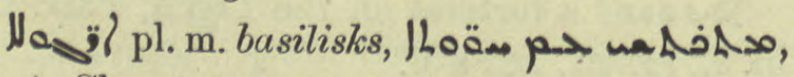
Nest. Chrest. fo. 3 .

a ? Aragon in Spain, Jab. 49 pen.

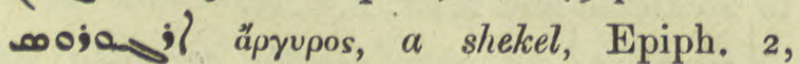

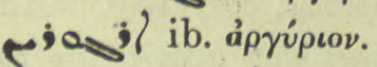

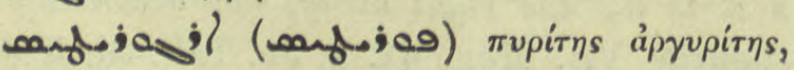
argentiferous pyrites, Chimie I0. 12.

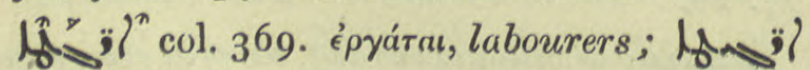
Anecd. Syr, ii. 319, 20.

(s) Argan, a city in the mountains of Persia, Chast. 6o. 6.

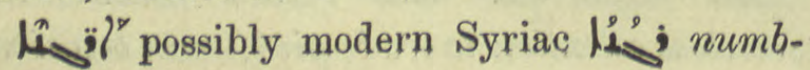
ness, Med. 600, 15.

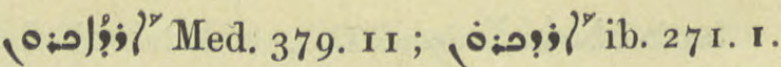
Prob. for joja j;!ol quicksilver, calomel.

L.sij col. 369. The Egyptian measure A $\mathbf{j}$; q.v. below, pronounced in the Egyptian vernacular, é $\rho т о \beta$, Hultsch 366 ; equals 12

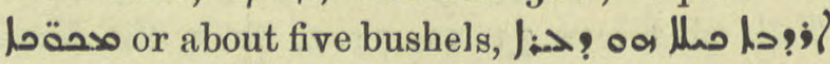

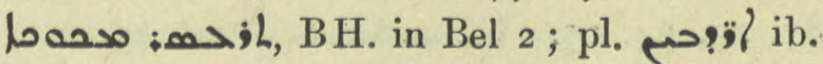

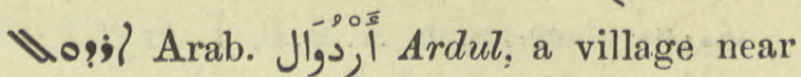
Wasit, C.B.M. 788 b.

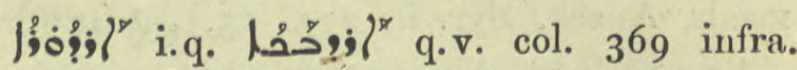
a tambourine, ljogjl" nad l?

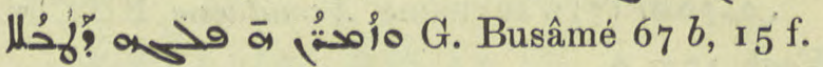

;) Ardai, island and bishopric, IL̈30 -9;: Syn. Or. 34. 23. Chabot thinks this a transposition of $\mathbf{j}$ ? ? or $\mathbf{m}$ ! Darai, that again is identified with م- Deirin, one of the Bahrain islands, ib. 273 note, 618 note, 666 , and $67 \mathrm{i}$.

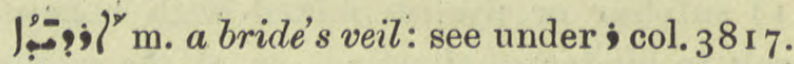

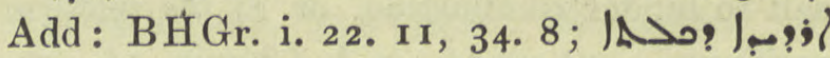
حط ' Anecd. Syr. iii. 34. 23, Alexis I3.

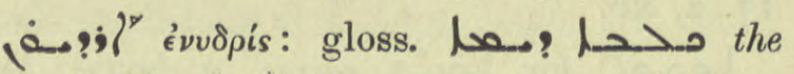
sea-otter, Natur 58.8.

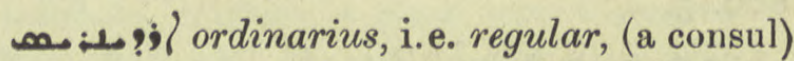
elected in the usual manner, Syn. ii. Eph. 29.26.

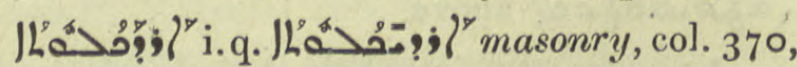
|l all buildings of hewn stone BB. under los col. $207 \mathrm{I}=\mathrm{DBB}$. 1057 .

5 i? what? to be boiled in sheep's oil for a hard cough with blood and phlegm, Med. 248. 23.

magredient of a plaster against chills, Med. 378. го.

\$) j pr.n.m. Perhaps a dimin. of Ardašir. SeeNöld.Pers.Studien 31 ; ZDMG.x́liii.396.15.

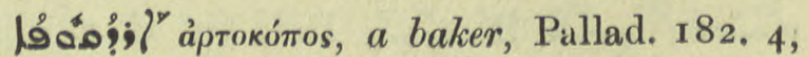
A. M. B. vii. I 16.

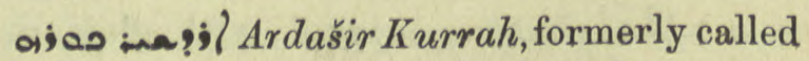
Gôr, then Perozabad, now Fer ozabad, 63 miles s. of Shiraz, Tabari I I, note 3 , Marquart $3 \mathbf{I}$. Syn. Or. 44. 25,7 1 , 27, 79. 7.

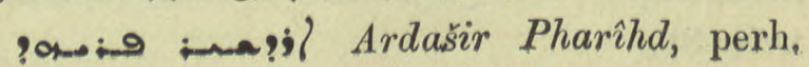

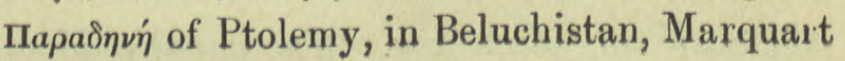
3i, Syn. Or. 37. 7 . 
909] col. 37 I. 3 of par. Orosius: see under sa.l col. I40.

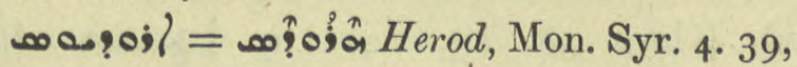
Patr. Or. iii. i. 47.6.

110;? col. $37 \mathrm{I}$ ult. is the BabylonianHebrew form of lio's rice, q. v. col. $3^{84}$. lio'ól is transcribed from the Greek opo and ${ }^{\prime} \rho v \zeta a$ is borrowed from Dravidic arisi, Gustav Oppert, Kohut, Studies 398.

'oól' col. 372. an ark, chest. I.q. ligó? a mummy case, col. 935.

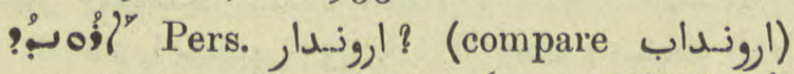

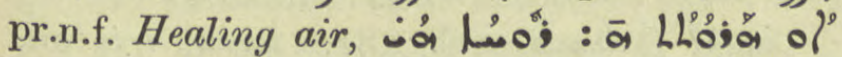

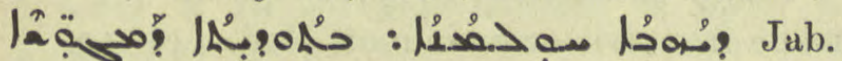
246. I I Bedjan's note.

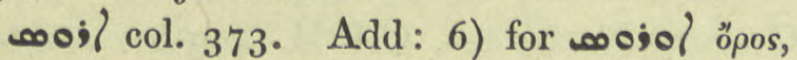

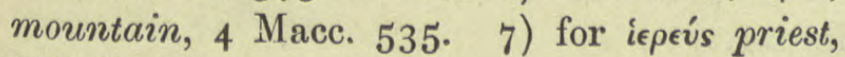
C.B. M. $5^{87} a$

hoooj\} $\ddot{\epsilon} \rho \omega$ with Syriac termination, erotic,

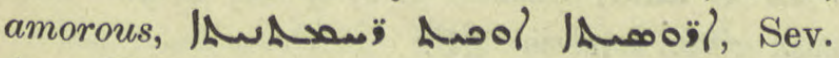
Ant. Hom. 54. I3.

LLoi\} 'E $\rho v \theta i a$, an island off the African coast, Jac. Edes. Hex. I9. I I.

بأl col. 373. Pers. Add: Tabari 45I, note 3 , Jab. 2 Io. 5 af. with note, the official who presents petitions; A.M.B. ii. I3 I. I4.

i.? name of a hero, i.e. Arjuna, Kal-wDim. ed. Bickell 76. 25, Benfey Introd. lxxiv.

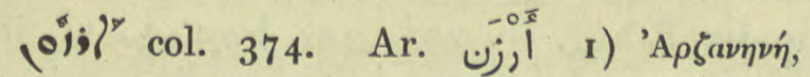
Arzanene, a province of Armenia, N. of Mesopotamia, and the town Arzun on the م: a tributary of the Tigris: see Marquart ${ }^{2} 5,306$, Syn. Or. under Anol and 391, note 3 , Pers. Mart. I 3 o, Nöld. on this and the following, ZA. xiv. I69. Refs. Syn. Or. 34. 8, 36. 2 and often, ZDMG. xliii. 394 f., Chast. 13. 20, A.M.B. iii. 513. 2) oftener (j). and po; (ji) on the river Araxes,

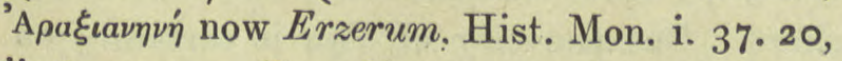
ii. 67 , note 3 , Pers. Mart. I 359 , ZA. as above. wil for month, Jos. Styl. 3r. 7 and

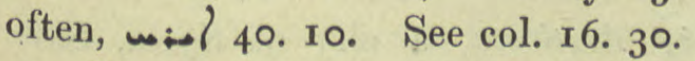

w. lم́:'s to journey, travel, depart. 2) metaph.

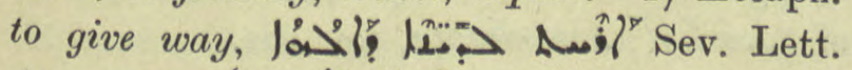
I02. 5 ; ó, ib. 294. II. 3) denom. from lí; a traveller, to come as a

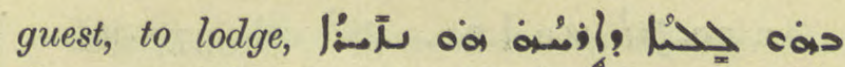
the very night when that Nazirite lodged at her house, Kal-w-Dim. 31. 9.

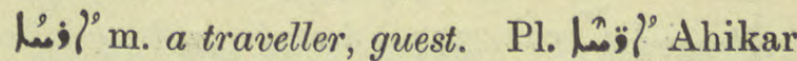
w, $I_{4}$ is wrongly translated "tiles"; the words two children were taught to cry out

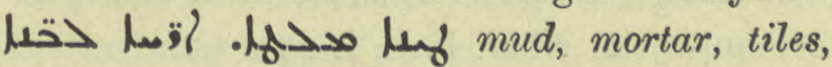
bricks are an abridgement. Cf. the history of Ahikar MS. Sachau (Berlin) 336. 4 I $v$.

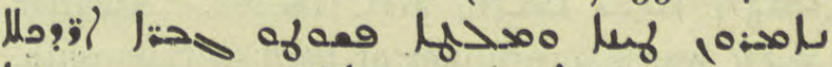
施 they will say: give out mud and mortar, the masons, guests of

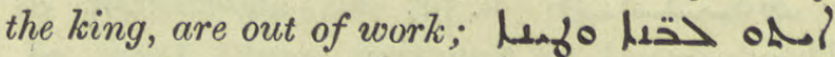
o ib. 42 r. F. Nau ap. Journ. As. 1907 , Jan.-Feb. ${ }_{5}$ \%.

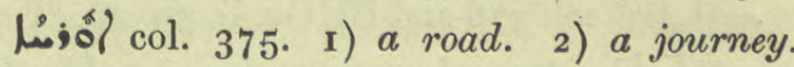
3) a custom, habit. Add: 4) with oas to

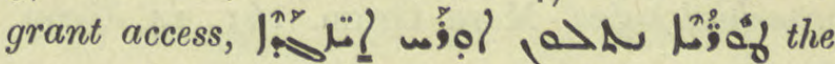
mountaineers (holding a fortress) shall grant access to ambassadors, Jab. 195. 4. 5) Heb.

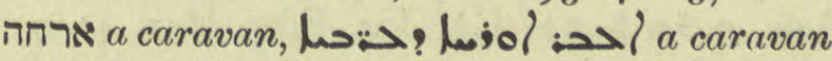
of Arabs passed by, Ephr. ed. Overbeck 285.4 $=$ id. Joseph. 26. 4 .

f place-name, Doc. Mon. I 72.4 C.B.M. $707 b$.

$\mathcal{H}_{\mathbf{j}} \mathbf{l}=\mathbf{b}$, , a tree growing in sandy deserts, willow-like flowers, fruit like a jujube ; from its root-bark ink is made, Freytag, Lex. Arab., a willow. C.B.M. $5^{80} b$, Is. Nin. ed. Chabot 29.

Mj\} col. 376. à $\rho \epsilon \tau \dot{\eta}$, virtue, ref. Anecd. Syr. iii. $17 \cdot 7$.

manoly; Artemius, Bishop of Titiopolis, Nöld. F. S. i. 47 I. 74.

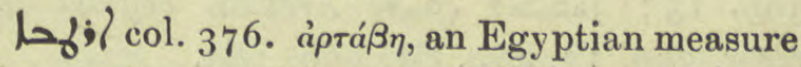
of capacity equalling four 1500 bushels or seventy-two /o مants, Epiph. 3 and 4 مar equal one-tenth of an laj; ib. Pl. Pُ

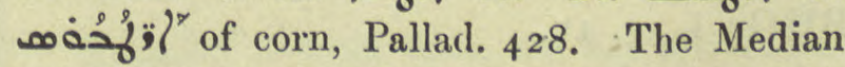
artaba, usually called Persian, is one and a half times larger than the Egyptian

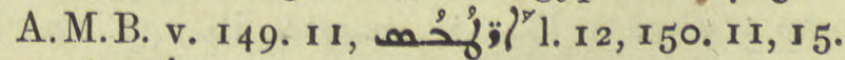
Cf. la?;?

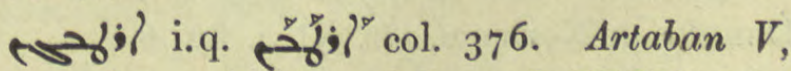
maternal grandfather of Sapor I, ZDMG. lvii. 563. 2. 


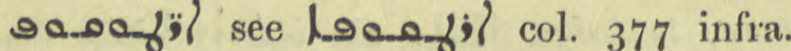
m.pl. а̊ртоко́по, bakers, Sev. Ant. Vit. 243. 7 .

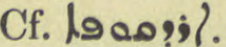

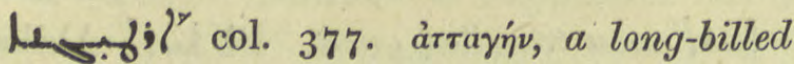
wader, Natur 27. 9.

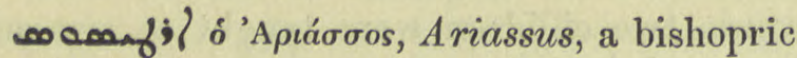
in Pamphylia, Nöld. F. S. i. 472. 91.

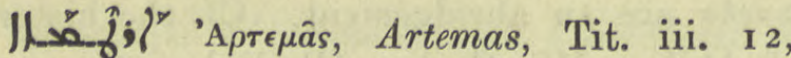
Lof;? Wo. hof;? BH. in loc. Another lof; was a forerunner of Paul of Samosata, Doc. Mon. 3०. 16, 1 12. 28, 333. 5 .

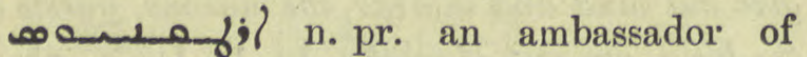
Theodosius II to Egyptian monks, Diosc. ed. Nau 22. 14.

क

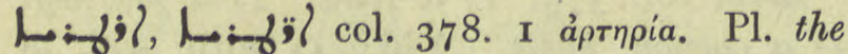
bronchial tubes, Merx Gram. 161. 14, Geop. 5I. 18.

L;? Aria or Herat, a province of Central Asia, a ja Anool A;\} Jac. Edes. Hex. 4I. 9;

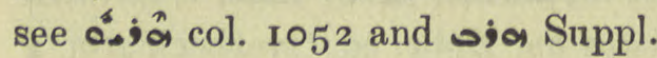

L:; col. 378, a lion. A sign of the Zodiac, 1. I 8 of par. correct for maligranati granum, milii granum Jeمo?! Jh:9. Ref. Med. 488. I, 490. 2, Chimie 6. I3. To R';" the disease

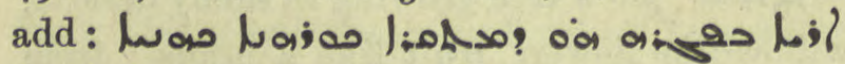

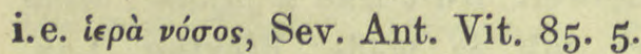

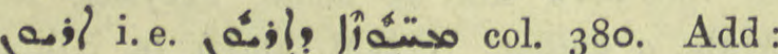
situated in Beth Garmai on the Lesser Zab, it was a Suffragan See to the Metropolitan See of Karka d' Beit Selok, Syn. Or. 33, 34 and often, Pers. Mart. notes 443, 2 1 80. Hist. Mon. i. 69. 1 2, ZDMG. xliii. 394. 9, 399. 15 ; 1 רد: Arewan beyond the river, 396. 13, Syn. Or. 666, 285 .

صasi? Arius, col. $3^{80 .}$

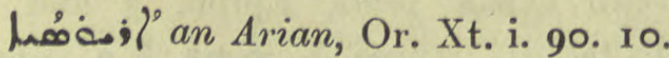

| BHGr. i. 47 . 17 .

M. Manichee, C.B.M. 9I 7 bis, pl., Sev. Lett. 6

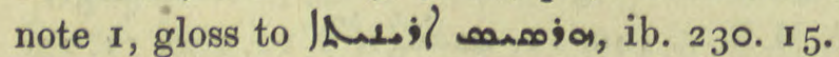

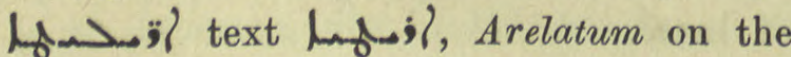
Danube in Noricum, Or. Xt. i. 82. 6.

Lwo; Ariminum, now Rimini, Anecd. Syr. iii. 175.19.
(Aalso;i) I) an Ariminite, adherent of the Council of Rimini, Syn. Or. I32. 30 ; Jo. Tell. ed. Kleyn 58. 13. 2) i. q. /fw1so. il:

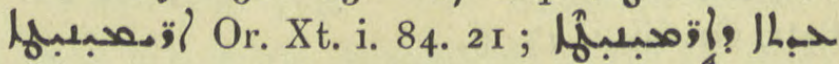
Warda 54 r; Anecd. Syr. iii. 287. 3 ; مَبر Ebed J. 200, 201 ap. col. 392. Dele the rest of the article.

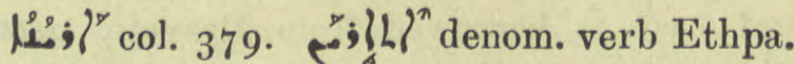
to become leprous, $\mathrm{cf}$. R. Duval on the authority of Imm. Löw. No ref. given.

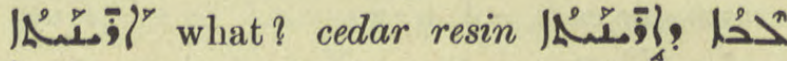

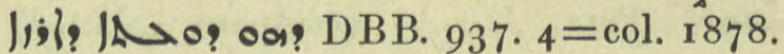

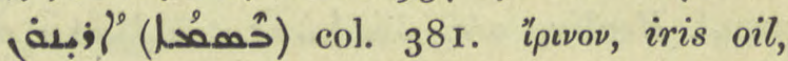
made from iris or orris root, Med. I48. I3.

ols;) for Nazianzus, the birthplace of Gregory Nazianzen, C.B.M. $423 b$; ols.ul ib. ter.

c. il col. 381. "A $ل_{1: 0}$ a sign given here is $\tau$.

h.m.;? from the preceding word. ferru-

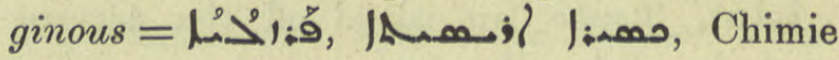
53. $18,54.2$.

ool-on_of Aristeas, quoted for the statement that the LXX were chosen from the ten tribes, seven from each, Epiph. 17. 3 .

10a ofom; aristolochia, Chimie 6.2,

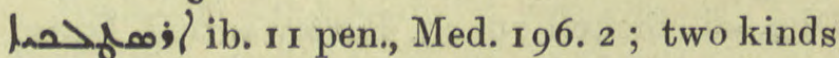

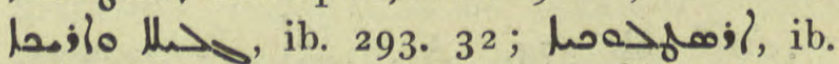
144. 2 I, miswritten 20.

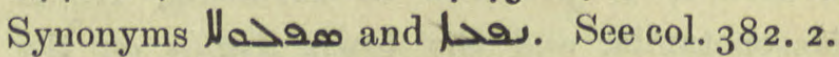

I. Aretissa, Pliny, Jac. Edes. Hex. 25. 3 and trans. 7 I note, lafong; and and Syr, Arm. and Arab., BHChr. Eccl. 535. I a note,

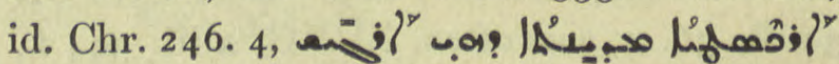

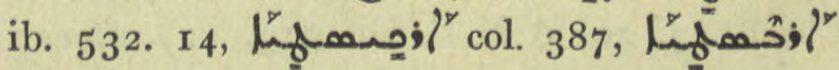
col. $3^{67}$; Lake Van and a city on its Southern shore. But the city of Van is certainly on the Eastern shore and Arjesh is marked on the Northern shore.

Nem; Arethusa, a city of Syria, Sev. Lett. 8I. I6.

Hase:; BHChr. Eccl. 437.2, miswriting

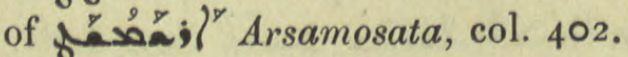

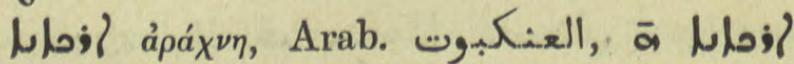
un Gall. araignée, the upper table of an 
astrolabe held together with fine network so as to show the lower table, De Astrolabe 74, 85,248 ter.

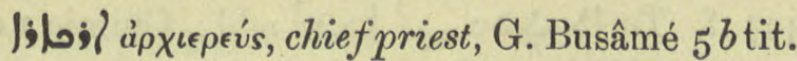
, the troop of Archons born of the Evil Principle according to Manichaean doctrine, Coupes ii. 130. 3 af.

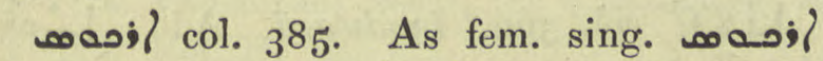
IAwo:f = the devil, Chald. Brev., Maclean.

L

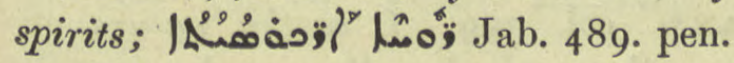

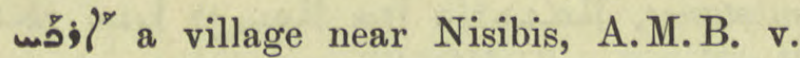
443. I 2.

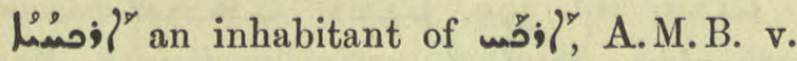
449. 10, 458. 14.

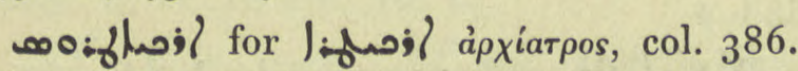
chief physician, Sev. Lett. 92. I I, Probus 76.

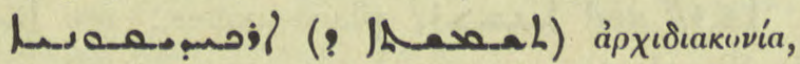
archidiaconal functions, Syn. Or. I 53. 2 I.

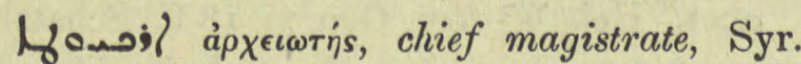
Rom. Rechtsb. 23. pen., 24. 3 .

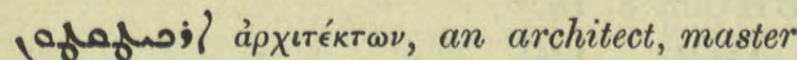
builder, Anecd. Syr. iii. 289. I9 = Sev. Ant. Vit. 287. 3; pl. plest. Alex. 74. 4 .

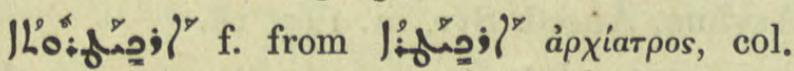
386. the art of a physician, Jab. 208 ult.

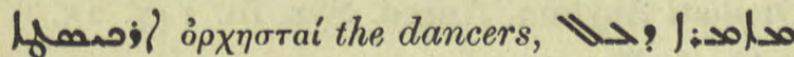
Mowo;?, C.B.M. 682 a. Monoj? a dancer, Jos. Styl. 22. ro, pl. As A. 186. 5 where Thes. has Homoi?, col. 400 infra and under lemsens col. 2093.

L. arcuballista, cf. Old French arbaleste and

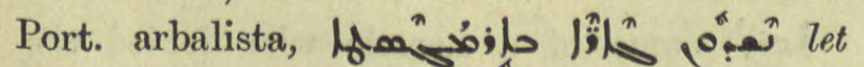
them discharge arrows from an arbalest, Gest. Alex. 268. I 4 .

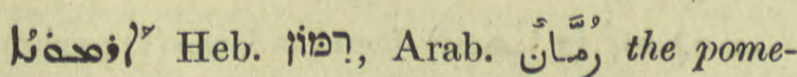

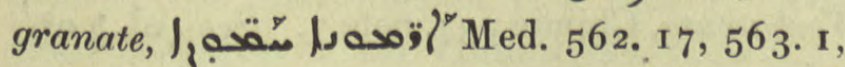

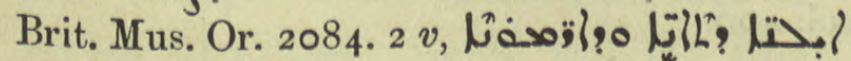
ib. $5 v, \mathrm{I} 2 r$.

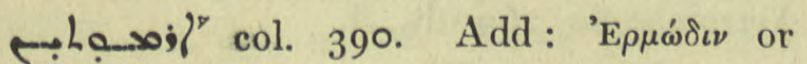
'E $\rho \mu \omega \nu \theta i s$, Arab. ارموتيم Armutin, a town in the Thebaid, A.M.B. v. I 48 . $4=$ Pallad. 322. I. Cf. Amélineau 60.

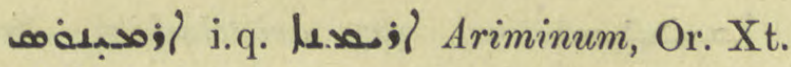
i. 90.8 .

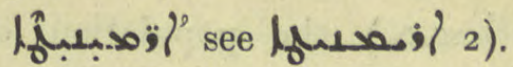

(1) 39 1. Hermes. Chem. mercury

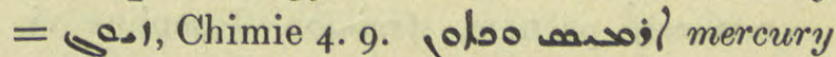
and lead but translated molybdo-chalque, i.e. a compound of lead and copper, ib. I I. 9.

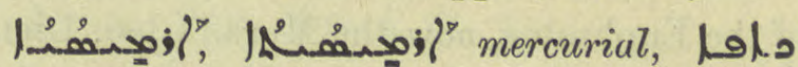

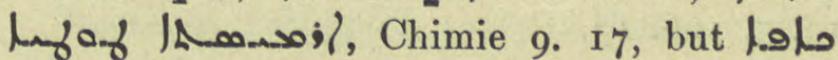
|A.sid, BH. ap. Journ. As. I898, 77. 20.

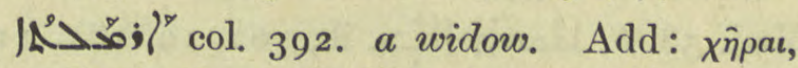
an order in the early Church, see I Tim. v. 9. Jac. Edes. Chron. Can. 266, G. Fraenk. in loc. ZDMG. li. 534, BH. Nom. $97.8 \mathrm{ff}$.

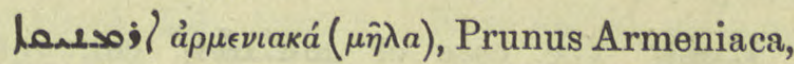
the apricot, Geop. I3, 23, Ar. Pfln N. I5o.

if col. 393. Add: Ar. أراّ Albania, the country north of the rivers Araxes and Cyrnus, now the Kour, west of the Caspian, 'A $\lambda$ ßavoi of the Greeks, Marquart I I 6, Syn. Or. 276. 619 .

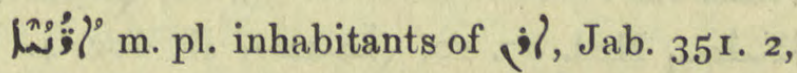
359. 12 .

'jil" now wil Arna, Arni, a bishopric in حم:J Jab. 40 note 5 ; Pers. Mart. 204.

|خُ

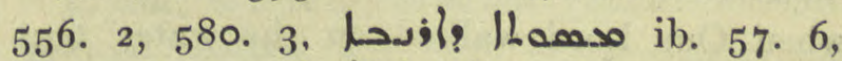

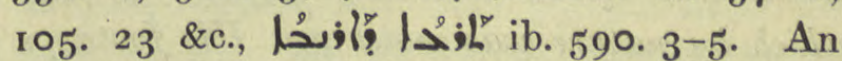
adipose tumour, BB. under /flsooflatfo

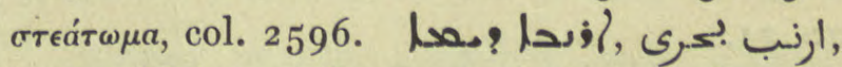

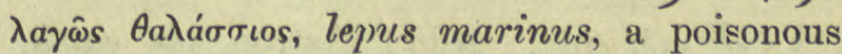
sea fish colour of a hare, Fisch N. $8_{4}$, DBB. 93. $\mathrm{I}$.

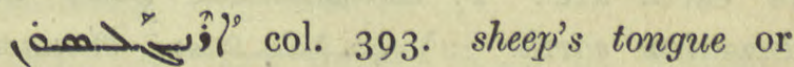
plantain, Med. 65.5 , I59. 9, I 7 I. 3 \&c.

ص

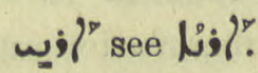

LLo:birds, Schatzh. 24. 2, J Lofosj?, id. ib. 1. 15 ; G. Fraenkel in loc. ZDMG. lvi. roo.

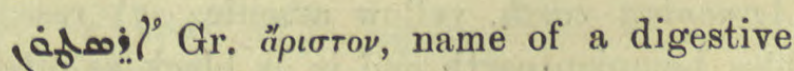
prescription, Med. $35^{8 .} 5^{\text {. }}$

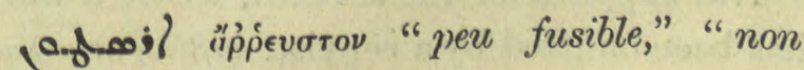
fluide," Chimie I r. 19. 
|? loc ofen; above.

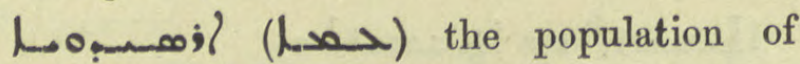
Arsidonia (?), Dion. 108. 19.

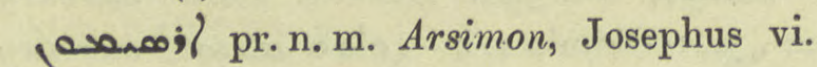
16 ult.

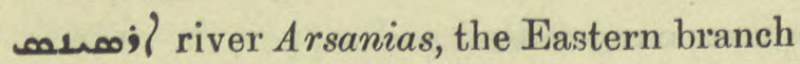
of the Euphrates, now the Murad Chai, Dion. 13. 5 , 106. 13.

นُمُ0;" col. 395 infra. barley water, refs.

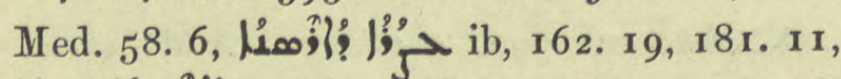

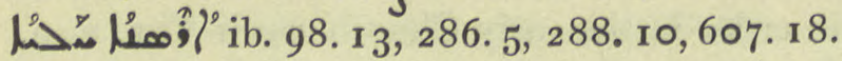

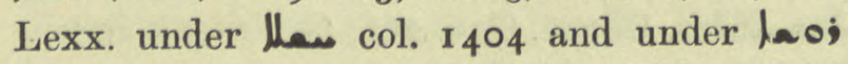
col. $3^{87}$.

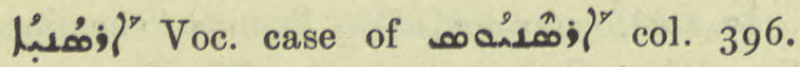
Arsenius the hermit, Pallad. 446. 20, 469. 3, M. Z. I73. 14 .

L A A rsinoe, De Goeje B. 65. pen.

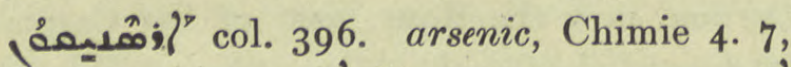

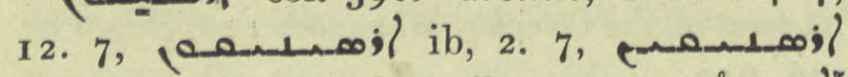

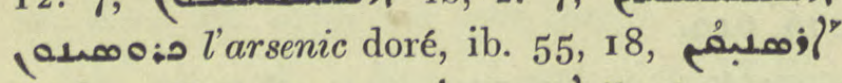

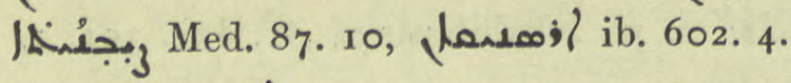

๓ De Astrolabe 254 ult.

uil col. 396. Add: Ethpe. WilLi) to meet,

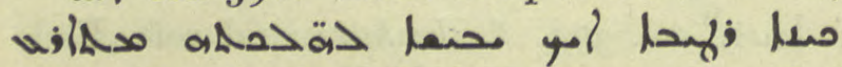
when Our Lord walked on the water that which by nature was moist met His footsteps $a s$ if it were a dry substance, Doc. Mon. 20. 21 .

L'il col. 397. Add: Astron. the earth, Chimie 6. I5, 7. 4; Chem. the seven kinds

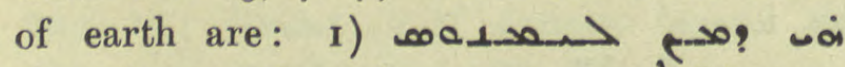
Lemnian earth. 2) (a) (-) bitumen.

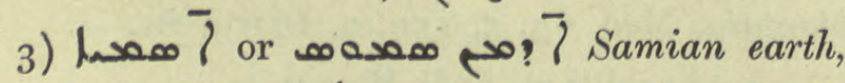
perh. talc. 4) مئ Cimolian earth, a soapy silicate; it inhibits inflammation and

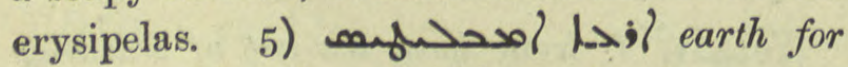
vines, a bituminous schist rubbed on vine stocks to keep off insects. 6) (میم ? Armenian earth, yellow arsenic. 7) resembles Lemnian earth and is as black as pitch. Duval supplies for the seventh, where a word has been omitted by a copyist, $\dot{a} \sigma \beta \hat{\delta} \boldsymbol{\lambda} \eta \eta$ or $\mu \dot{\epsilon} \lambda a \nu$, soot from Diosc. v. clxxxi. Chimie 7. $16 \mathrm{ff}$. infra roloir

and 8 supra, see pp. I4-I 8 of transl. and notes. Nos. $3,4,5$, and 6 with glosses from the Lexx. are already given under $1>$; coll.

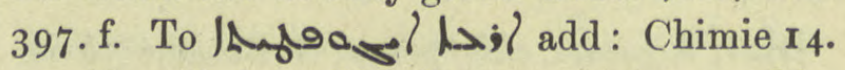
I3, identified as Cimolian earth, i.e. marble,

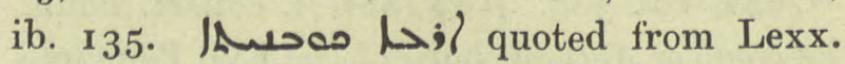
col. 398 , should rather be “terre étoilée," ib. I35. f.

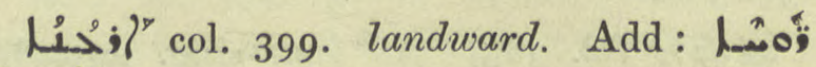

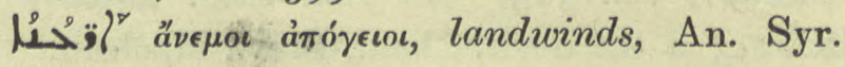
142. 22.

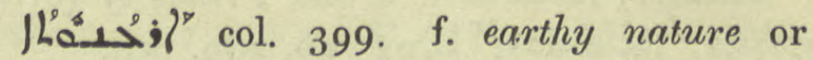

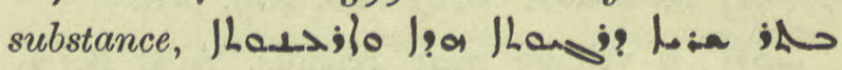
Bar Koni ap. ZDMG. liii. 504.

| кatà tónous local or native medicines, Med. 553. 4 .

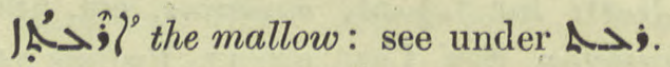

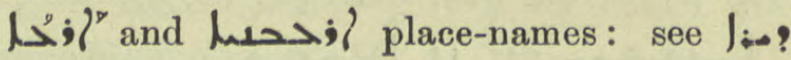
in Suppl.

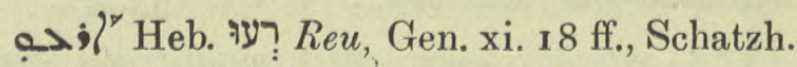
I 20. 18, 122. 3 f. \&c., 'Payav̂ Luke iii. 35, BHChr. 8. 16.

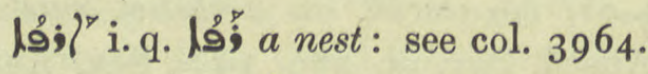

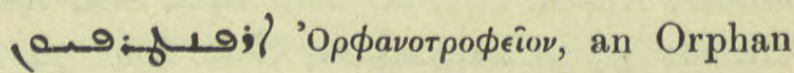
Asylum, Anecd. Syr. iii. I47. 18.

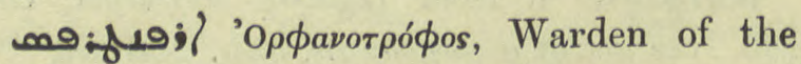
Orphan Asylum, Anecd. Syr. iii. 147. 25.

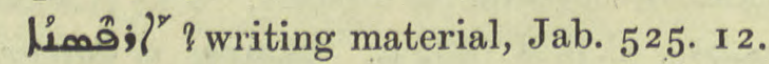

| А or Caesarea Libani. A town at the foot of Mount Lebanon 12 miles from the sea, Pet. Ib. 104. 22 and note, Pléroph. 85. II and note.

Lo; name of a heretic who denied the Third Person of the Holy Trinity, ZA. xv. 106, 9, 13 .

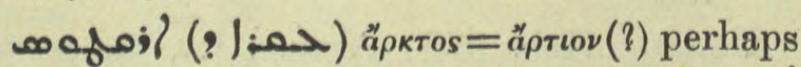
Verbascum, Chimie 237, note 7. See olufoi? col. 400 .

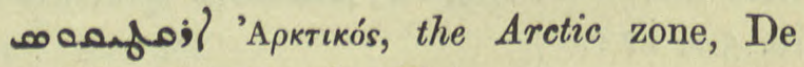
Astrolabe 82, 269 ult., ff.

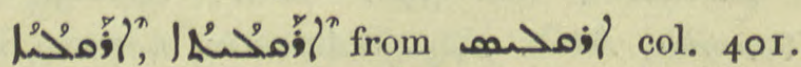

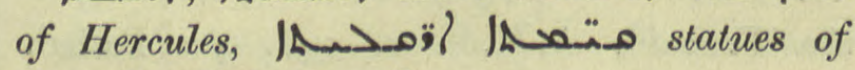
Hercules, Hebraica vii. 47. pen. 


\section{ontaloir}

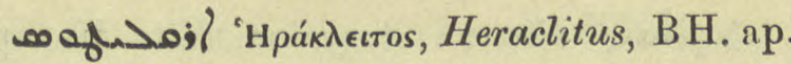
Hebr. iii. 45 o. 7 .

pail name of a village, Hormizd $155^{\circ}$. Cf. קמק The

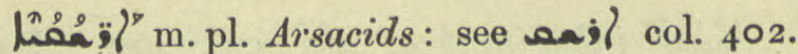
M.Z. 5. 28, 30. 6r. .

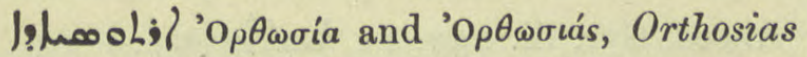
on the Phoenician coast, 12 miles north of Tripoli, Pet. Ib. I05 ult.

1. أl col. 403. Add: المغرفة a ladle,

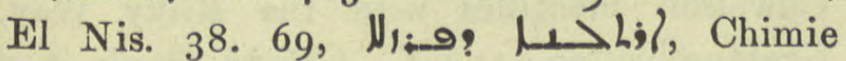
95. 19.

1-l Ashga, a village in the district of Susa, Syn. Or. $55^{\text {r. }}$.

l"Ashgar, a village of Adiabene, Chast. 8. I I, IO ult., I6. I 4 .

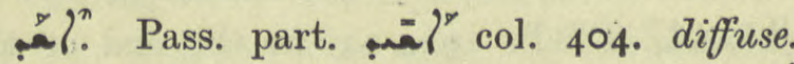
Add: scattering, dissipating energy,

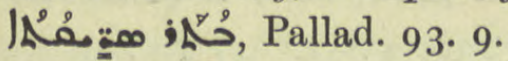

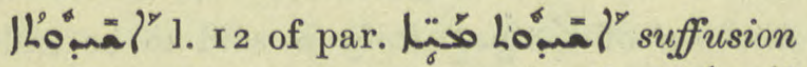
of the eye, Med. 56. 4, 75. 9, | the same, ib. 556. 19. Metaph. wandering thoughts, distraction, Pallad. 340. 4, LLo"nal"

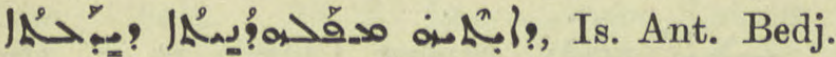

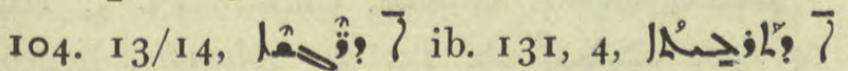

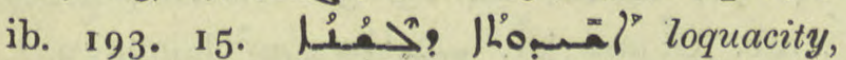
garrulousness, Isoyabh ed. Duval I42. 23.

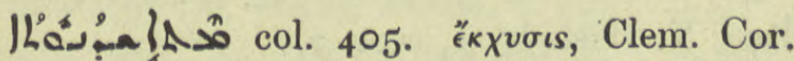
\$. 24. L L of desire, Sev. Ant. Hymns 177.

soal Zend arshokara maker of virility, epithet of Verethraghna, the Genius of Victory, Bahram Yast. xiv. 28, see Sacred Books of the East, xxiii. p. 238 . In Manichaeanism the Supreme Being is conceived as a tetrad. Mani is thought to have adopted this from the Mazdeism current in Mesopotamia, F. Cumont, Cosmogonie Manichéenne, i. 8, note 2. The four principles of the Zoroastrian faith are: i:

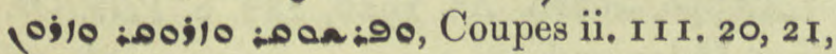
WZKM. xii. 354 in loc. Later these were considered by Christians to be four gods, A. M, B, ii, 577. 6 f., Parhad, 366. 3 ;. . مئمة;

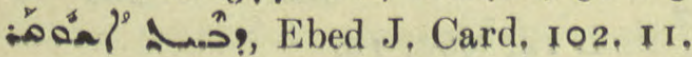

hy 0 fal col. 406. Fraenkel says this word should be of Pers. $\div$ (صواحان) ص and Arab. should place the $t$ sound before the sibilant. It is the game of Polo. Fremd W. 29I.

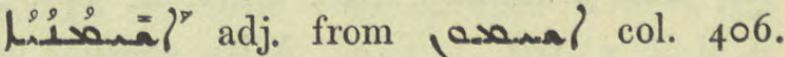
مrob. a, desert like that of Jeshimon, Hist. Mon. i. 77. 2.

ไharal A shkaphil, a city in Beit Aramâyé, Chast. 48. 2, 7 .

Anosal Shamunith, mother of the seven Maccabees, Or. Xt. iv. Ir8. Ir. Usually written uمَّa q.v. col. 4213 .

م ? $^{n}$ a Mongol town seven farsaqs south of Urmi, Jab. I50. I. Now called مeạ? by East Syrians and اوشني لجمَ by Persians, note ib., Ušnu (Ušnukh), Pers. Mart. 23r, 24 I \&c.

uب اشفعنى اشعفنى or Ashfegani wife of Ahikar, Ahikar Introd. xxxiv. 52. I I, 18.

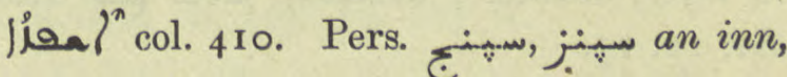
the $n$ is kept in Mandaic שפינז, Nöld. Mand. Gram. 5I, abode, dwelling, alaal? معب90

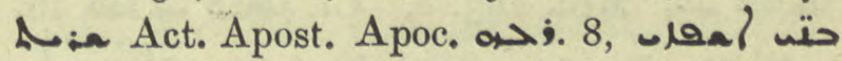

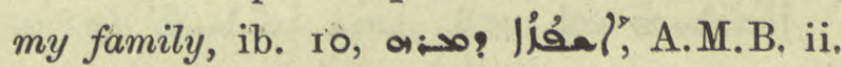
230. 4 and 6 af.

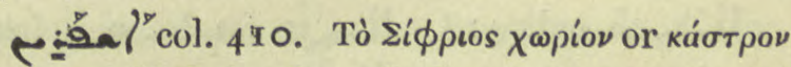
"I $\sigma \phi p \iota s$, Siphrîs, Jos. Styl. 55. $3=$ trans. 46 note, 75. 14. See Procopius de Bello Persico I. 8 .

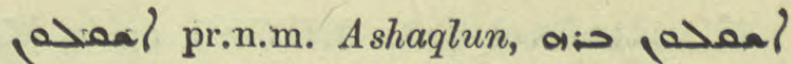
bow, Coupes ii. I30. 16. Perhaps derived from Mon to sustain, uphold, Cosmog, Manichéenne 75 , note 6 .

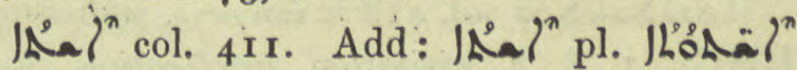

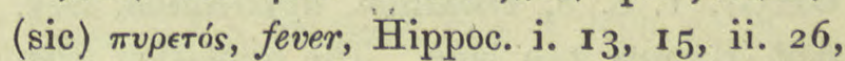

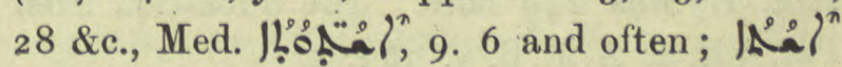

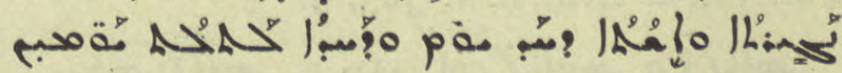
chronic, diurnal, and tertian fevers, $4^{8}$ ult. f. I, |นُمْ

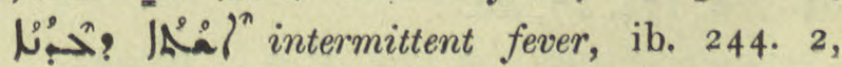
297. 12 ; $\mathrm{BH}$. de Pl. $\longleftarrow 84$.

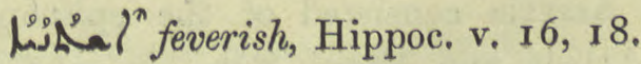

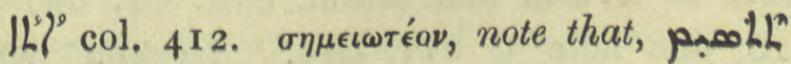

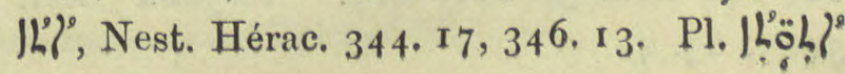


Rit. an alphabetical psalm, esp. a portion of Ps. cxix said at ferial evening service, Qdham W. $46.16,53.12$.

$\left.1 h^{\prime}\right\}^{n}$ act. part. $\left.\left.\mid L^{n}\right\}^{\prime}, L^{\prime} h\right\}^{\prime}$ col. 4I5. Add: ILإِ صمُ gradually, Act. Mart. ii. 347. 23, A.M.B. v. IoI. II, I2. With another verb expresses continuity of action, Sho they continue to preach, Ephr. Ref. ii. 209. 40. Cf. similar use of W, col, ro6 infra. لح:h

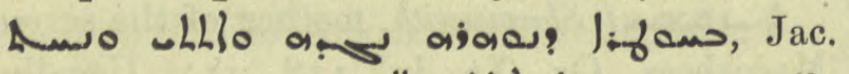

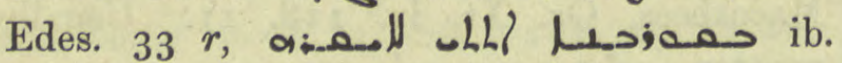
Aph. col. 415. Add : to hold in

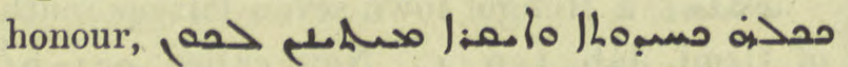
we hold you in all veneration and honour, Sev. Lett. I

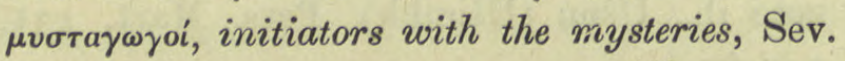

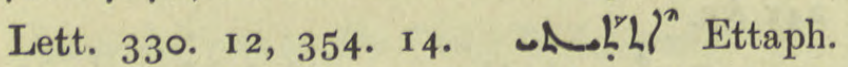
col. 4r6. Add: to be brought to the point, to infer, deduce, , asilhl loja to Georg. Ar. 12.13.

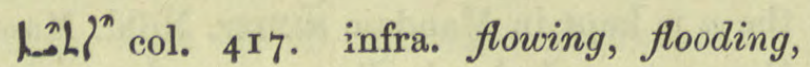

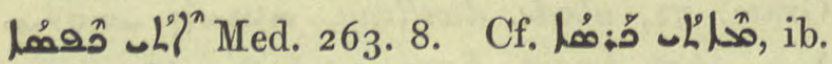

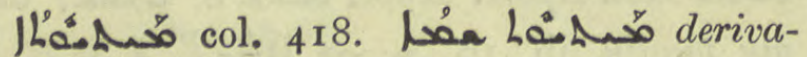
tion, etymology, B. Sal. in Lit. 25 pen.

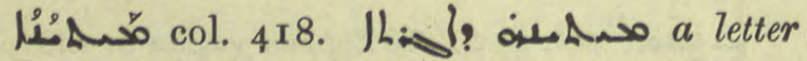
carrier, postman, A.M. B. v. 3. 2, 4. 19. 1 Aphr. $3^{8} 5 \cdot 25$.

مِّتب

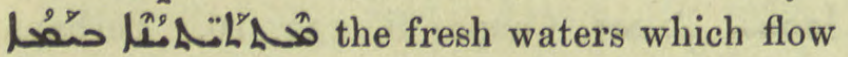
into the sea, But. Sap. N. Hist. iv. 5, sect. 2.

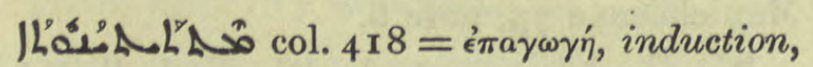
BHGr. 1. 8. 8, visitation, application, Sev. Ant. Hymns 249 note. The quotation from Pat. Vit. = A.M.B. v. 363.5 .

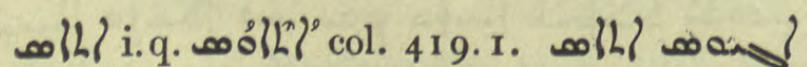

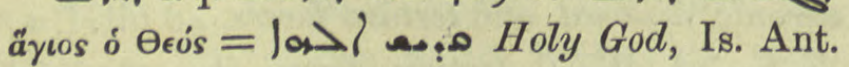
84. $7 / 8,174.17$, \&c.

jôl'? Assyria, col. 420 . Eccles. The province of Assyria consisted of the episcopal sees of Arbela and Mosul, Syn. Or. $667 a$ : 10, 608 note $3,611,619$. Isoyabh Lett. $54 \cdot 7,63 \cdot 4$.
A'Gốl\}' like an Assyrian, Vit. Monoph. 67. 17 .

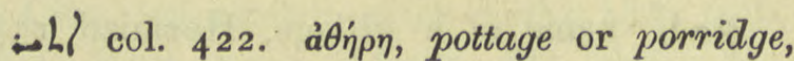
Kal-w-Dim. 2 I0. 2 I.

حسم

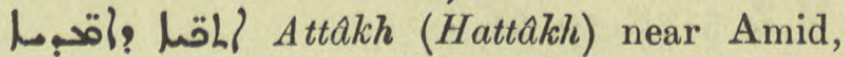
Anecd. Syr. iii. 259. I I, hol\}? |jLl, I. I3.

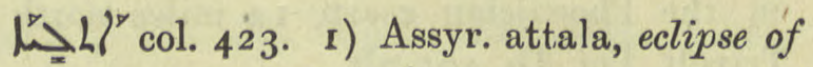
the sun. 2) New-Heb. תל the Dragon, which Chwolson identifies with the Milky Way, Grabinschriften, 122 f. Others think it a constellation and BHCarm. $3^{8}$. I I , quoted

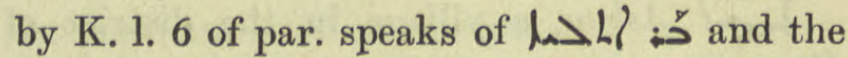
مضُ as, different. 3) R. Duval thinks $\Lambda>L C$ is derived from Vlith a Greek termination, cf. food from eos and compares ILanall DBB. 192. 24, at the end of the

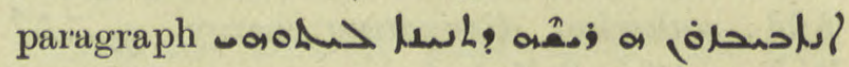
h $ح$ L R.O.C. xv. ${ }^{23}$ O. 2 Syr. 4) In Jab. 53

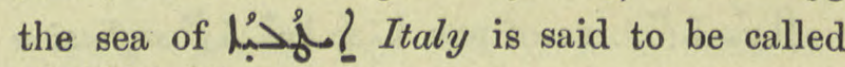

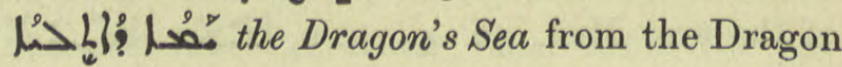
which was supposed to produce the flames of Etna.

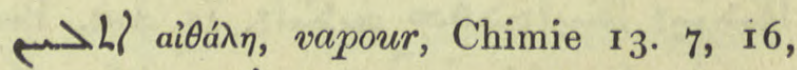
14. 18, 4 ใ ib. 15. 3. Alembic, hence Arab. أثال/Chimie I 9. 2,

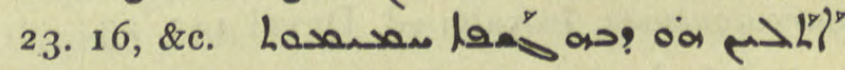
Ja, N. Hist. iv. I, sect. 2.

lan $>$ (; jof) Pallad. 630. 7 probably the same as the following name.

"A a city in Lower Egypt, A.M. B. vii. I09.

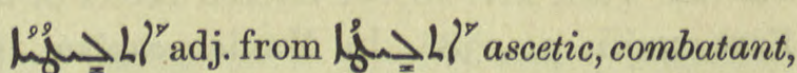

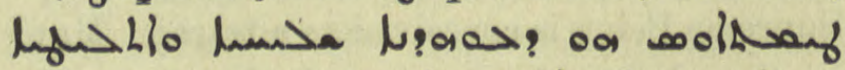
Sev. Lett. 362. 16 ; lof id. Hymns 177 .

مa saly perh. the country of the harh: see col. 4472 ; nomads in the neighbourhood of Aleppo, Journ. As. 1900, 287. 6, Patr. Or. iii. I Index and 28. I I.

Limes $\{L\}^{n}$ äavaria, immortality. Name of a liver medicine, Med. 356. 18, 357. 5,

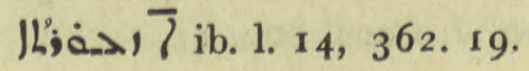


L'Fhl" col. 426 infra. Add : rustic, hemand LiLho, El. Tirhan 3. 6.

no\{il\} for uo\}il Thrace, Jac. Edes. Hex.

II. 15 .

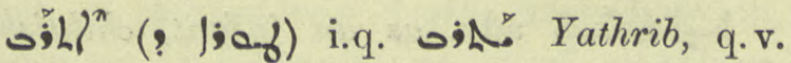
Bahira 206. 4 af.

hojhl a native of Yathrib, Pet. Ib. I42. 5 .

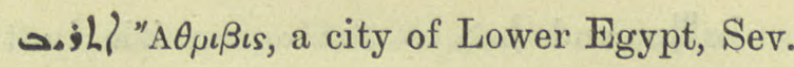
Lett. $384 \cdot 3$.

L $; \mathcal{L}$. name of a monastery in Armenia, Anecd. Syr. ii. 57. 7,

m. $L L^{n}$ ' col. 428. 'Artis, Attic, a kind of

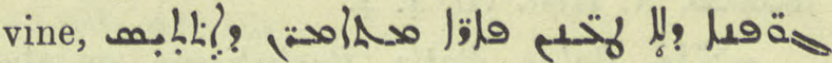
N. Hist. vii. 3 . I.
$\Xi$

o prep. col. 430. Add: $\sigma u ́ v$, Loof's Nest.

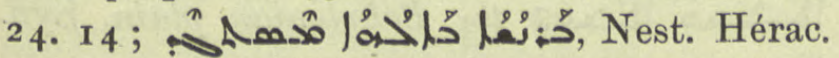
3I4. I3, 3І 5. 2. Col. 432. Rit. ellipt. for حمُ to the tune, Qdham W. 30. I, 33. 7; o ox they repeat the doxology to the

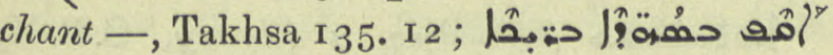
proceed to the chant The Blessed Martyrs,

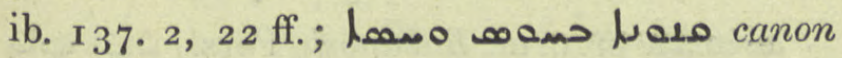
to the chant Pity and Pardon, ib. 62. 4, I 43. I, Brev. Chald. i. 88. 8 ; معاحا. حمف سم Motwa to the chant, Clap your hands, ib. iii. เо3. 18.

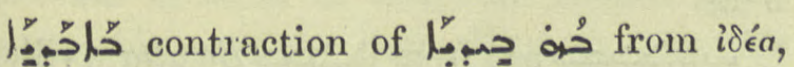

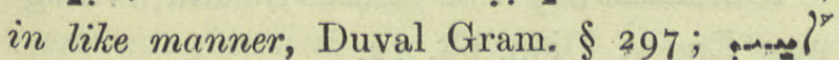

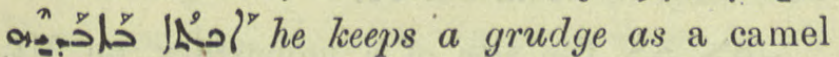
does, Anton. Rhet. ap. BH. Gr. i. I 75 . II,

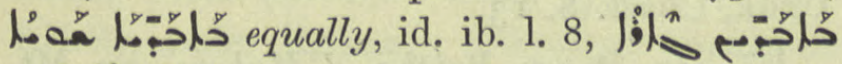

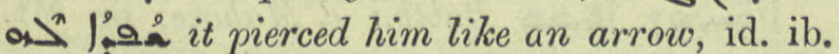

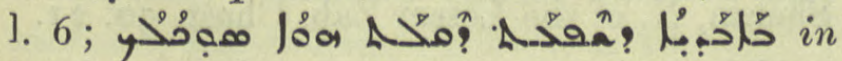
like manner as the Tigris is copious so is thy understanding, Hormizd 2210 , ib. 57, 1983 , 2945. Without ceasing, continuously, yiv? حادب tourne sans cesse, Chimie I6. I4.

Vash $>$ col. 432. Bishop of Antioch, B H Chr. Eccles. 53. 6 ; حلحمالمام Aut. Patr. 298, 13. I.q. Na col. 443 .

a Babylonia, a province of Asia, Jac. Edes. Hex. 4I. 5 ; hulas ILoghl, ib. 15.2.

Lol-1.s Bagavana, a mountain in the eastern part of Armenia, Chimie $3 \circ 3$ pen. 2716

\section{casensts=}

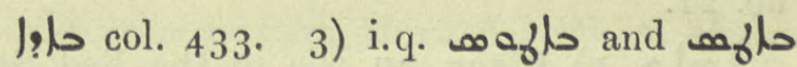
the measure bath, it contains 50 /f and equals a j;ad, ;0xos a small omer, Epiph. 4.

usols the Egyptian month Paophi, Mart. Luc. R.O.C. iii. $165.12,167.6$.

10ool z river Bausa in the neighbourhood of Mardin, C.B.M. 275 a.

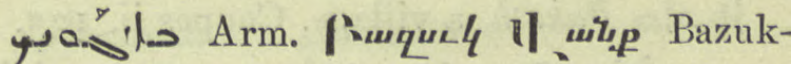
Vankh. Bazgaunoch, an Armenian monastery in the district of Guba, BH Chr. Eccl. 433. 4.

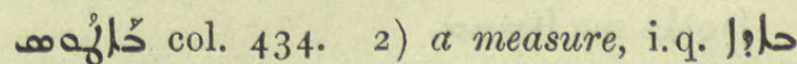
a bath q.v. 4) Báros, Baris, a kind of ray, probably Raja rhinobatos, found in the Arabian Gulf, N. Hist. vii. $3 \mathrm{I}$; Ar. Fisch N. 47.

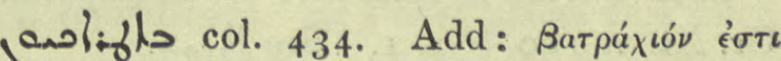

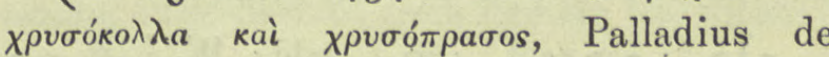
Febribus, ed. Bernard, Leyden, I 745 . So Duval, Chimie I 3, n. 5 to (a.0:pate d'arsenic.

مارهم Bias, violence, Syn. ii. Eph. 2 I. 3.

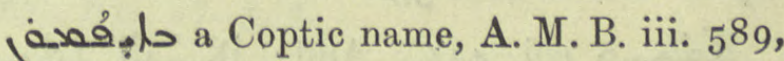
perh.

a violent fit of coughing, Dion, ed. Tullberg محهa 6 .

حمد C.B.M. 274 b. See under col. 487 .

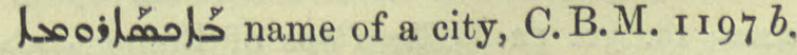

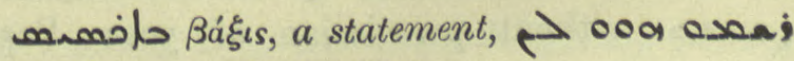

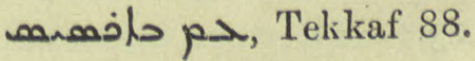


ماحما вала́ка, an isle in the Red Sea, Jac. Edes. Hex. 20. 8.

m river Halys, now the Kizil Irmak, A.M.B. iii. 274 and note.

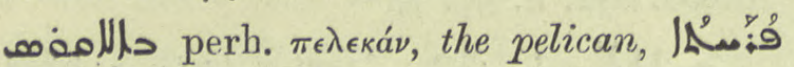

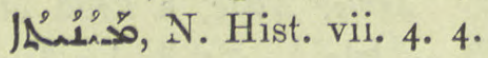

מُاخحم Balas, Balis, Barbalissus, on the right bank of the Euphrates, BHChr. Eccl. 249. 2 I n. I. q. حذa; see below.

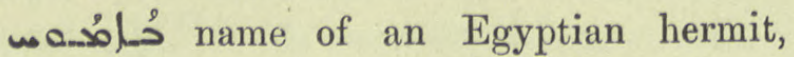

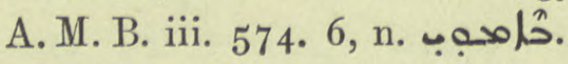

Lُ Moringa nux behen, the behen nut, Med. 40. 18.

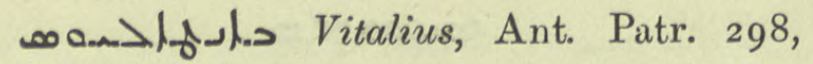
no. $2 \mathrm{I}$.

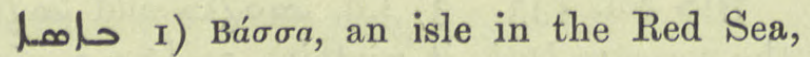
Jac. Edes. Hex. 20, 8. 2) name of a presbyter of Edessa, Syn. ii. Eph. 43. 27. 3) f. a martyr

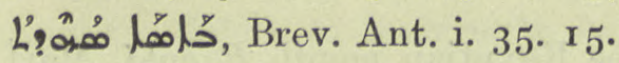

basalt, Chimie 245, n. 4.

s/osilides, bishop of Byblus, Mich. Syr. ${ }_{59} a$.

دمامي Bakath, a village, Coupes ii. 125.

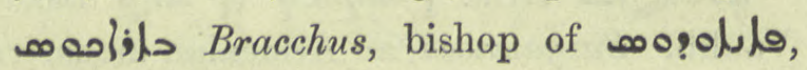
Nöld. F. S. i. 468. 16.

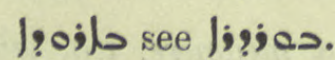

ملزم Pers. I 7, 19.

صاف Birta, a bishopric in Mesopotamia, Or. Xt. v. I00, Syr. I. See JL.

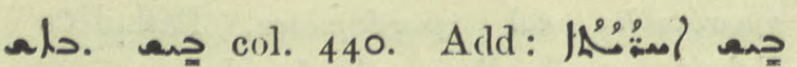

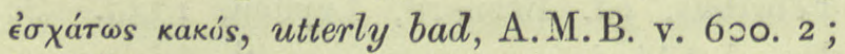

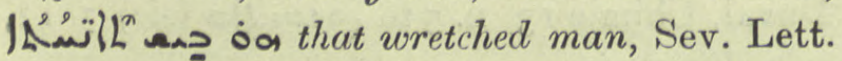

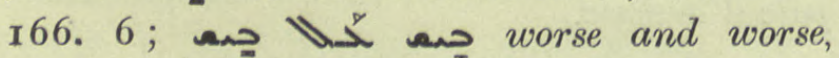
Ephr. Ref. ii. 200. 35 .

|Las wretchedness, misery, Pet. Ib. I 16.5 .

L 1 Battis, bishop of Tella, Nöld.F. S. 470.

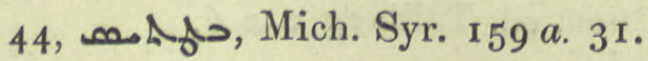

nolla see nolles.

مد้ papa, Kal-w-Dim. ed. Bick. 29. 30;

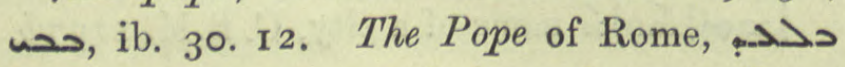
sحصa without permission of your Pope, BHChr. Eccl. 739. 13 . a for ase a buffalo, Chimie 49. 6.

ححمِ to be eaten with honey after food, Med. 542. 3. Perh. Pers. بب. mimosa.

1 حُحَّ pr. n. m. Babusa, B.O. iii. i. 142.

حُحكَمْ adv. as an infant, childishty, Syn. Or. I 76. $24,183 \cdot$ I 5, 184. 5 .

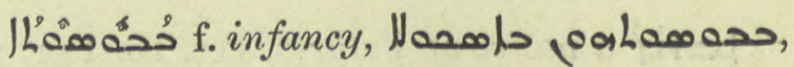
Georg. Arab. ro. 8, I 2.8.

ไมُ

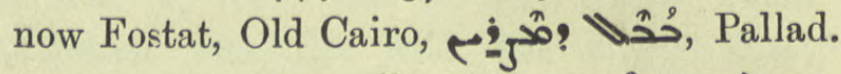

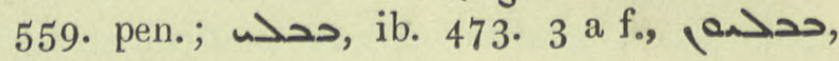
ib. 428 ult.

Jathe hill of Babylon i. e. Mt. Mokattam, Pallad. 237. 6 a f.

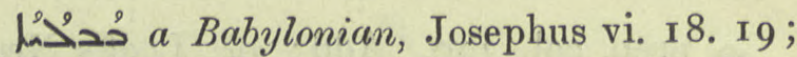

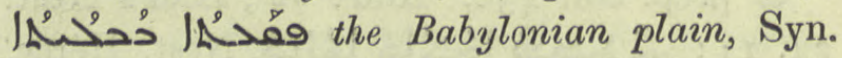
Or. $131,24$.

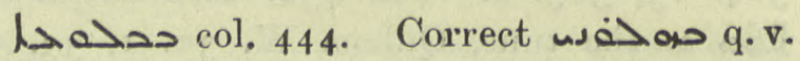
infra.

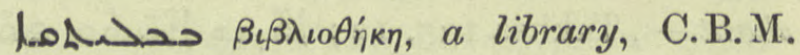

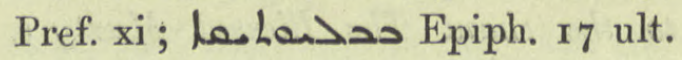

باب انوش pr. pr. Babanosh, Pers. whose father is blesssed, Hist. Mon. i. 227 , ii. $429 \mathrm{n}$.

حح山 a village of Khuzistan, Syn. Or. 323 .

محخد Baicalta, a village near Emesa, Dion. 68. 12.

כב $\Delta_{\mathrm{J}}$ the site of a monastery, on Mt. Uruk, Chast. 8. I3.

בحA Babtha d'Mahoze, on Mt. Sheran, N. of the Lesser Zab, Chast. 50. 2 I.

lُ Sassanidi 23. 16.

|ُู rumbling sound in the lung, Med. 217.13 ; 

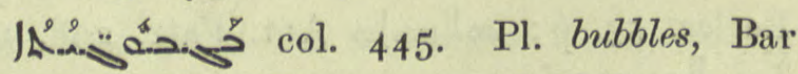
Choni ZDMG. liii. 505 on Hesiod's account

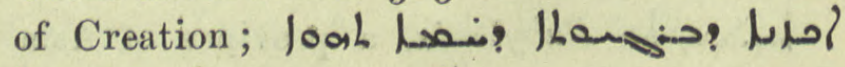

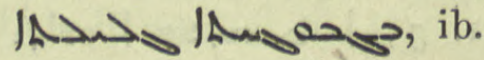

$\|_{\infty}>$ col. 446 . Add: Ethpe. $\|_{3}^{n}>l^{n}$ to

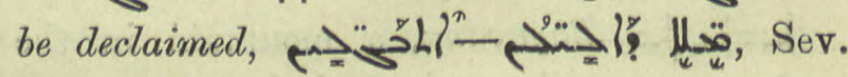
Lett. 248.6 . 


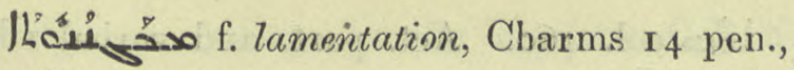
9I ult.

باذنقيا حسمبر or perh. Be Niqya near Hira. See Yakut. The place of Alexander's death, near Babylon, according to Sassanidi 30.3 af. ap. Cong. Or. viii.

s col. 448. 2) to be emaciated. Act. part. نُ opp. 3 ;

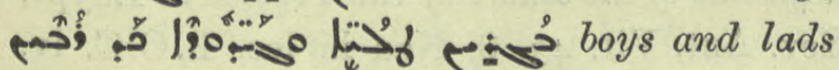
grew thin as they were growing up, ib. To fall ill, Anecd. Syr. ii. 50, 22, Dion, 220. 4 ; with $\leftrightarrow$, a

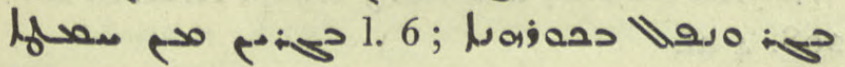
ib. 218. 22. Pass. part. a $a$. (כ) some were attacked over five times by this illness, ib. 2 16. 20.

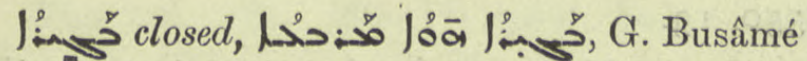
7. 2 af.

D: I $\rightarrow$ or(?) hail like pebbles, Dion. 194. 19.

نُ xliii, 397. 8. 2) $=\leadsto \leftrightarrow$ ( $\leadsto$, Chast. I0. 12 .

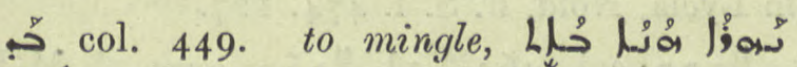

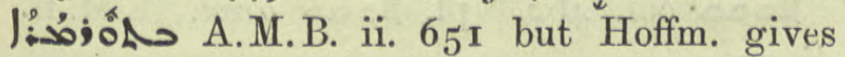
, 13 in this passage, Pers. Mart. n. 627 this river mingles with the Tormara.

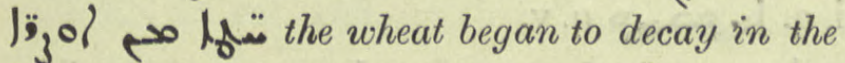
barns, Dion. 60. 17. Read (..)? ? but cf.

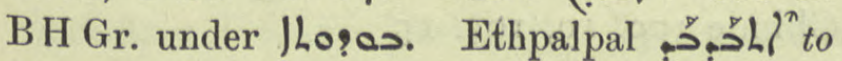

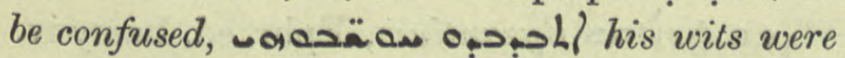
confused, Kal-w-Dim. ed. Bick. 2. I8.

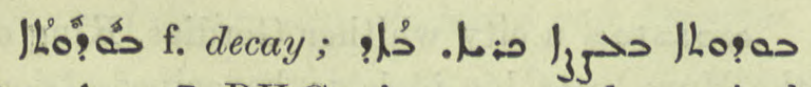

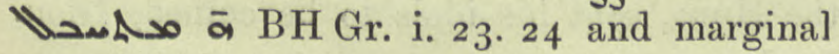
note.

J. Ethp. जo falsely or foolishly composed,

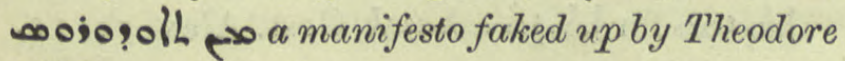
of Mopsuestia, Sev. Lett. 338. r.

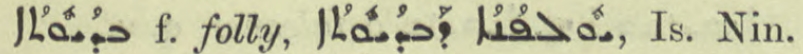
B. 6 I. 2 ; BA. under مُ مُ201. 2245.

 $\sigma \kappa \epsilon \lambda \iota \sigma \mu o ́ s$ a $\sigma \kappa \epsilon \lambda i \zeta \omega$, varico, supplanto. An Fallacia? Field's note.
A.

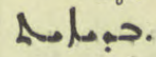

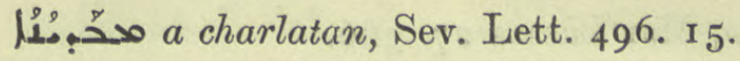

حب perhaps البون Al Baradān, Badarun

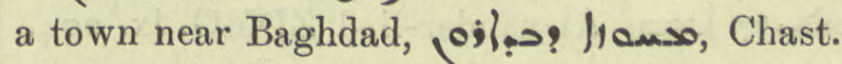
46 ult., 47. 13 .

دers. Pبحدباد a sail h. a flup or lappet of a garment, Arab. البنة gore or front of a shirt, El. Nis. 36. 24 , Fremdw. 54 note.

Mo $ح$ col. 450 infra. Case letters: see El. Nis. Gram.

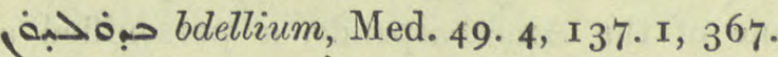
I0, I7, \&c.; مبه حبح Chimie 7. 12. Syr. مأم

حُ حُبّ pr.n.m. Pers. Mart. A.M.B. iv. I63.

Bdigar, a fortress in Adiabene, M.Z. 54. I68, A.M.B. iv. 128.

مب a city in Egypt, Badyssus? A.M.B. iii. $23 \mathrm{I}$ and note.

neaps a corruption of Bâdhgês to the north of Herat, capital of the Hephthalite Kings, Marquart 77, Syn. Or. $4^{2} 3$.

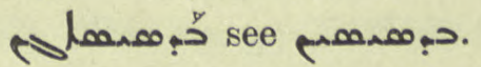

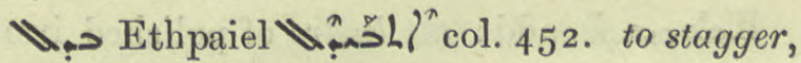
reel, Ephr. Ref. i. I 76. 34 .

لِ بيذار بَنِر

col. 452. and مبهمبمب Pers. prob. convulsions. R. Duval places this word among those derived from Persian but without the Persian equivalent. Ref. Med. 56. 22 .

حُبْنمْ col. 454. probably a professor of philosophy, Barhad 398 f. and note, Stat. Schol. Nis. 27. 3, 5 .

ox col. 455ou who

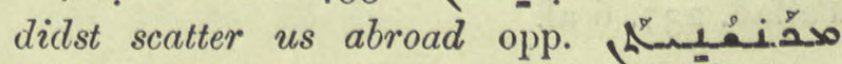
BH Carm. 79, II.

as a softened form of a in names of places.

: Seleucia, rebuilt by Ardašir I. Tabari 16 nn. $I$ and 4 , Syn. Or. 667 ; ib. $37.25,76.29$, Sassanidi 17.7 , Jab. 232.9 , 4IO. I 3 . 


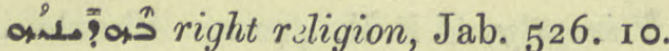

Mâs col. 456. Ethpe. Mâshl" add: to be calm, Mar Bassus 27. 366 .

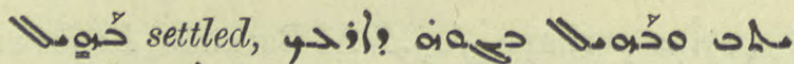
"204. 3I 4 .

لِ incessantly, Aphr. \$هL ult.

بـ vii. $3 \cdot 3$.

(⿻ars Pers. I) Bahman i.q. Vohuman, one of the Ameshaspends, a personified attribute of Ahura Mazda, A.M. B. ii. 577. 18. 2) name of the middle month of winter. 3) name of a plant which flowers in

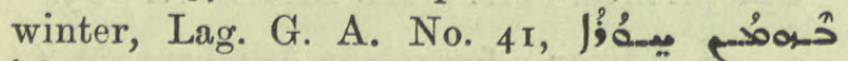
|

pluas pr.n.m. Ant. Patr. 305 no. $13^{8}$.

lمْ

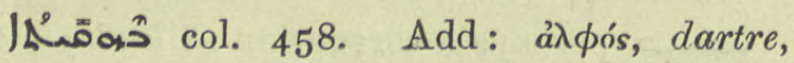

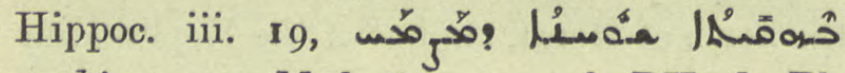
a shiny sore, Med. 602. I4, pl. BH. de Pl. no. II 7 .

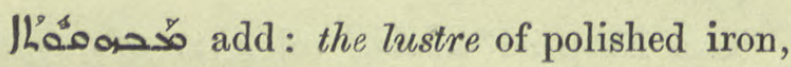
Is. Nin. B. I90. I I.

, Behqawad, a district of Beit Aramaye; Kufa and Hira belong to it, Chast. 26. II ; Sassanidi 29. 6.

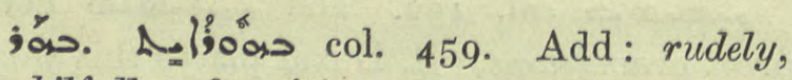
unskilfully of writing or preaching, Anecd. Syr. ii. 2. 13, Theod. Mops. 57. 6.

plias col. 459. Late Persian and Arabic form of jajo q.v. Bahram, son of Sapor I, BHChr. Eccl. ii. 45 ; pjas Mon. Syr. ii. 67.8.

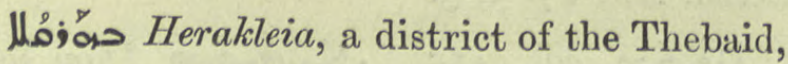
Pallad. 237. 6 af.

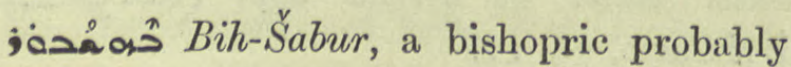
in Fars, Syn. Or. 670: ib. 7 r. 27, f.; ZDMG. xliii. 402 ; A.M.B. ii. 559, tit., 565.9 .

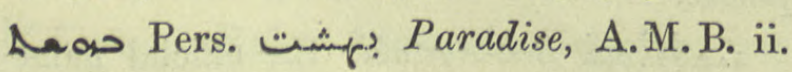
576. 16,578 . I1.

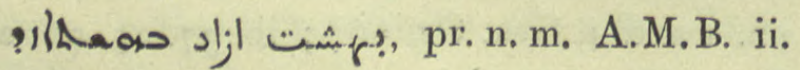
27. 12.
La col. 460. Act. part. and verbal adj.

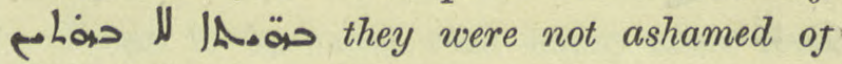
shameful actions, Jul. 43.

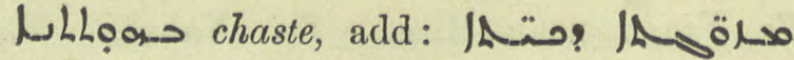
(sic) IA ILoas, Theod. Mops. in Jo. xxi. I 7.

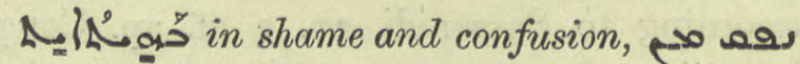

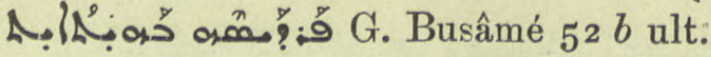

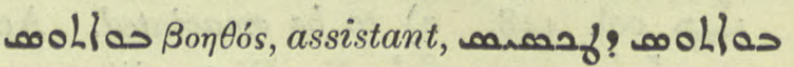
adjutant of the cohort of the Prefect of Egypt, Sev. Ant. Vit. 43. 8, Nau in loc. R.O.C. iv.

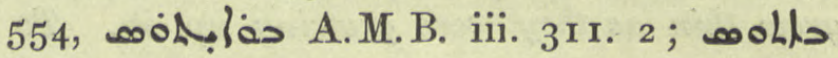
ib. $312 \mathrm{n}$.

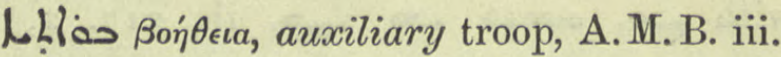
298. 4 af.

bes I) col. 462 . I. a tunic لإ bes lsana $\rightarrow$, G. Warda ap. Hippoc. ii. p. vi. I5. 2) حبوجا a kind of comet, Med. 550. 13.

a abbrev. of ll oxen, Chimie 48. 16, כحه ib. 49. 6.

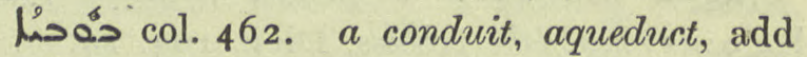
ref. الحال Jich. Syr. 258. 7 af.,

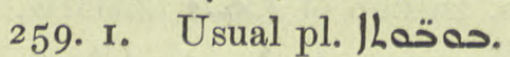

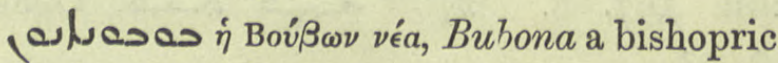
in Lycia, Nöld. F. S. i. 474. I 27.

لمه saxen, A.M. B. vii. $7 \mathrm{I}$.

1ُ حُ col. 462 and Hippoc. iv. 73, v. 60 i. $q$. lores q.v.

بو a a crucible, Chimie 100. 19, 102. 15.

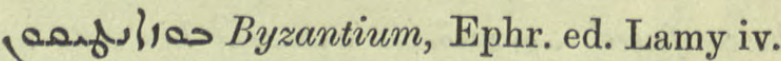
36r. n. 1. I 2.

م a city whither Gordias, King of the Huns, sent his idols to be coined, Dion. Ined. $475 \cdot 7$.

حف من name of the daughter of Herodias, Apis 0. 9.

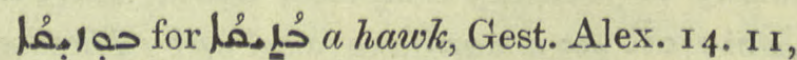
I 5. 7 ; BH. Stories 73.3 af., 74. 9.

إم a a falconer, q. v.

fo Pers. a crucible, Chimie 21. I4, 24. 20.

oja-fas cf. col. 464. butter, Chimie I6. 17. 


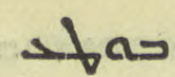

Ufos a village in the Hauran, C.B.M. 7 II $a$, Doc. Mon. 2 I 7.12.

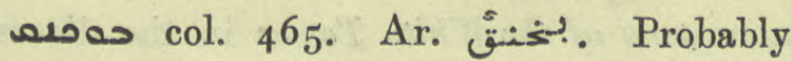
from Coptic. A kerchief worn by young girls, tied under the chin, to keep their oiled and perfumed hair from dust and to save their Khimar or head veil from contact, Dozy, Vêtements Arabes.

l col. 488 and The ref. is to Philox. ed. Budge ro. I9,

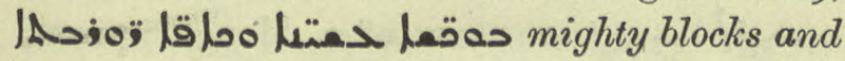
great stones.

Woa Arab. بـ a Bactrian camel, Kal-w-Dim. 335. 5, 339. I 2 ,

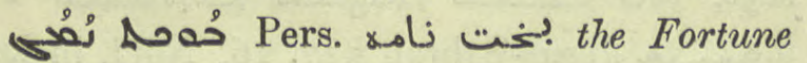
Book, Jab. 234. 2.

مa col. 466. Boג

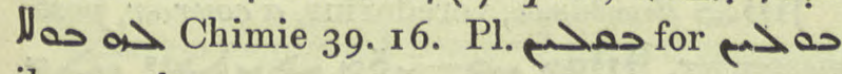
ib. $5^{2}$ ult.

ل్

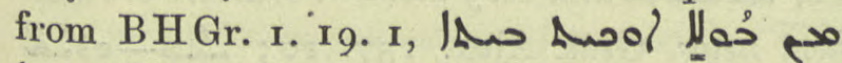

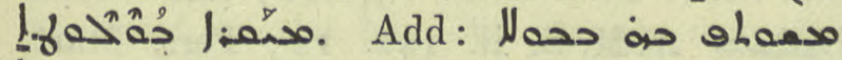
IAL. family, Sev. Ant. Vit. I r. 6.

| Med. 314. II, 602. I5.

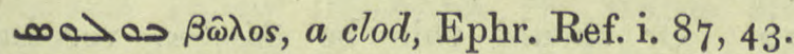

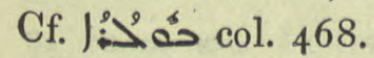

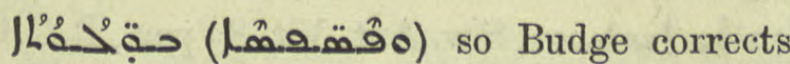
ل a game. Fraenkel thinks it must come from

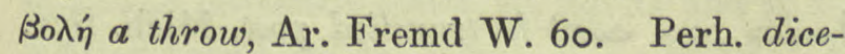
box and dice or draught-board and draughts.

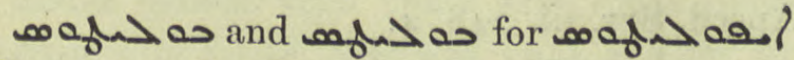
Hippolytus, Or. Xt. ii. 193.

maxn>col. 468 . ravenous hunger, Med. 5. 4, 280 . II.

(ander see porforanate, Med. 6r. I6.

إ 240 n. 3. BB. under pasoga col. I746.

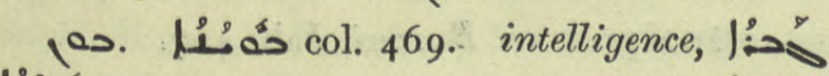
هد: atsa sa ib. 61, 4. For

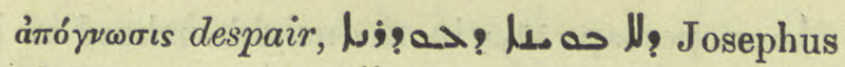
vi. 2. 2 ; probably Jمه

它 (exhortation to read a canonical letter), Isoyabh ed. Duval 22 I, I3.

| obtuseness, add: But. Sap. Eth. iv. 7 ; a disease of the soul, ib. 3 .

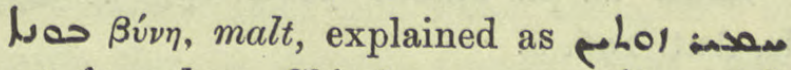

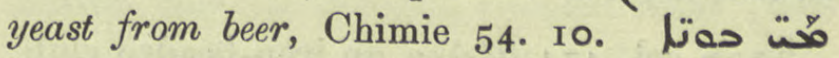
Ar. مرى Med. 606. 5 .

م حفóvaros, wild ox, N. Hist. vii. 4. $6,6.5$.

رeمes dim. of lopes q.v. col. 47 I. little balls or pellets, Chimie 30.4 .

ל Bardekdar, an Egyptian lord, BHChr. Eccl. 77 I. 14.

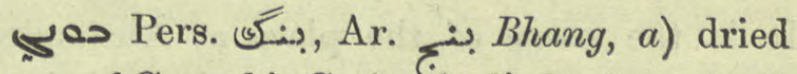
leaves of Cannabis Sativa. b) henbane, Med.

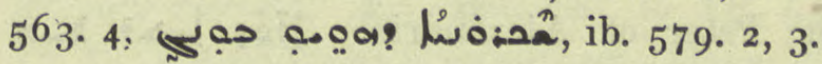

حمe pr.n.m. A.M.B. iii. 472.

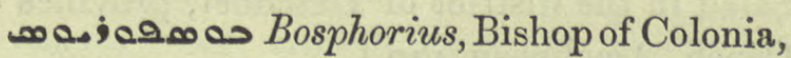
Vit. Mon. 13. 3; Nöld. F. S. i. 47 1. 62.

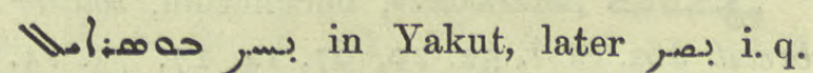
Buṣr el-harîrî, Nöld. ZDMG. xxix. 435, a village under the rule of Damascus, C.B.M. 7 I $a$, Doc. Mon. 2 I 7 35.

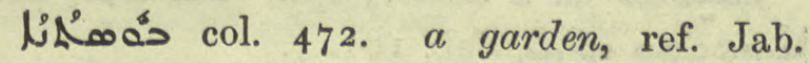
I 39 pen.

I

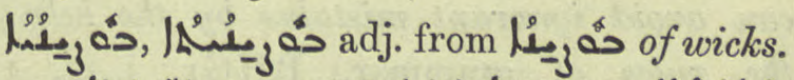

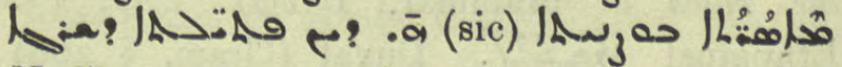
M. Singar 3 I. 5/4 af.

$4, \infty$ a village and monastery in the Hauran, C.B.M. $71_{2} b$ bis, $71_{3} a$, Doc. Mon. 22 I. $18,21,23,29$.

|ुु

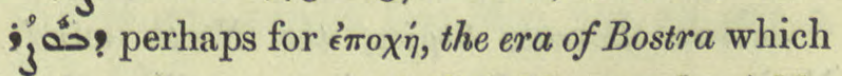
reckons from A. D. 106, C.B.M. $1072 b$. Add : 5) $\mathrm{j}$;L ; 3 as Pa-Asan, a temple of Osiris in the Delta, Pallad. 57, 3 af., but Bedjan has

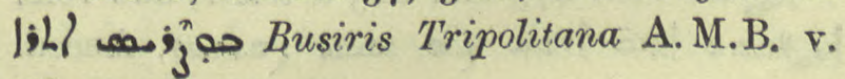
76. 5 . 
بوقة in the neighbourhood of Antioch C.B.M. 818 a. See Sachau Hist. Geogr. Syriens $\mathbf{5} 5$.

حفهُْ correct حفقيُنسم, Gest. Alex. 134. I6r. I3.

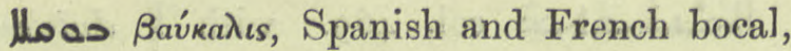
a wide-mouthed bottle, Chimie 26r. note 2 .

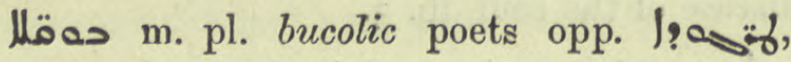
Is. Ant. 296. 6.

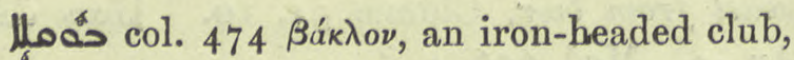
Pers. دبّوس add: El. Nis. 4I. 27.

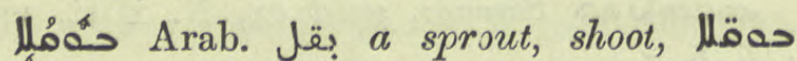

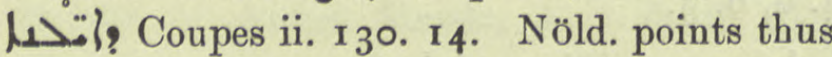
after B.A. Cod. Goth 3475 ; see WZKM. xii. 357 .

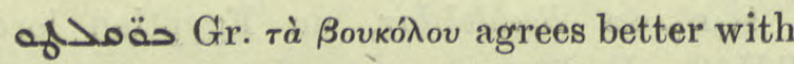
the variant 0 o s s.M.B. v. 552. 14. It is the name of the place at Alexandria where St. Mark was martyred, and later St. Peter of Alexandria. Jió? حمد

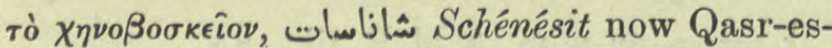
Saiâd in the district of Deschnet, province of Qéneh, Amélineau $430 \mathrm{f}$.

ली bread, Jos. Sty]. 52. 20, 67. I4, I7, 73. I4.

إlon baculus, a rod, Nest. Chrest. 96. 209.

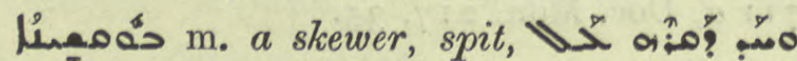

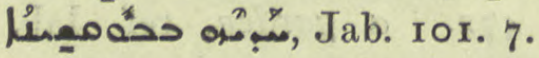

;os col. 474. Add: to be uncultivated in mind, the reader can avoid ignorant mistakes by the help of the rules of grammar, BHGr. i. 2. I3.

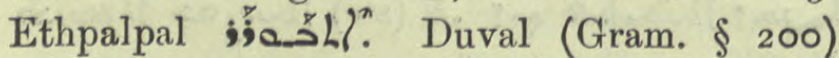
considers this verb a denominative from J

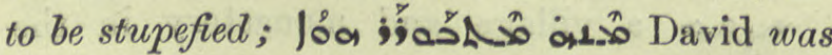
amazed by the water fetched for him from the well at Bethlehem, 4 Macc. Io. I I, ملحمز; 1- besotted, Jos. Styl. 87. 7.

مa Boreas, Bopéas, the North wind, Med. 533. 4. See various spellings on col. 475 .

so cols. 475 and $6 \circ 3$ a tower, add refs. "עarg, Aneed.

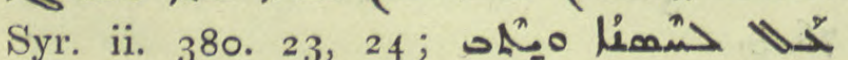

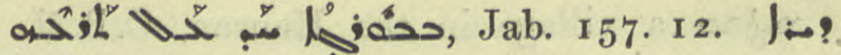

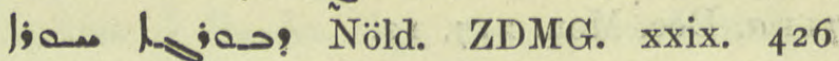
monastery of the White Tower in the diocese of Arabia, C.B.M. 7 10 $a$, Doc. Mon. 2 14. 26 ;

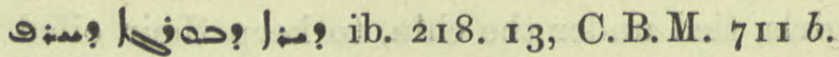
Astron. a sign of the Zodiac, add refs. Med. 465 ult., $483.22 \mathrm{f}$.

hy galgaria, Brook's Chron. 575. x.

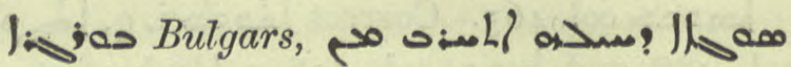
lis jor, Brook's Chron. 575. I.

بो wife of Ulus Ogotai, Jab. I 20 , 1 .

| rags, Joa oa JLam a Jesus-Sabran 527. 16, Nöld. in loc. WZKM. xi. 189.

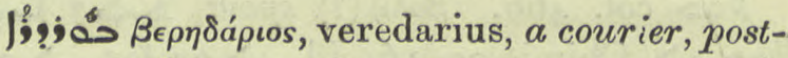

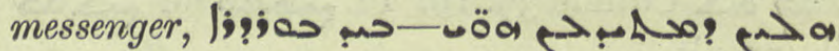

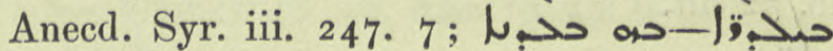
oll ale $>$ at that very moment couriers brought

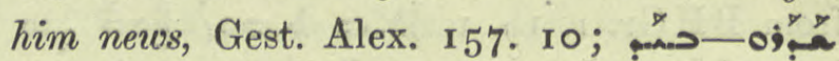
ح.د. A.M. B. ii. 142. 12 ; Pers. Mart.

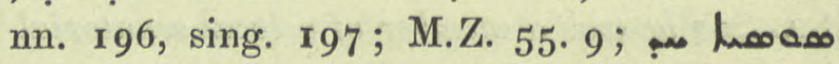

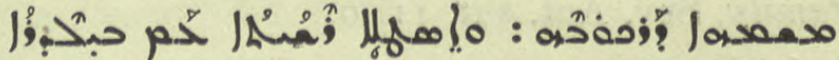
the royal gifts were a high-bred saddle horse (and a choice robe) with his groom, Jab. I45. 12. Or should this be $\beta$ '́p $\eta \delta o s$, veredus, a post-horse as the following? wa מט פסגم: Sev. Ant. Vit. 83 pen., R.O.C. v. 80. 3 and n. $\mathbf{I}$ in loc. Cf. a similar passage in Pléroph. I 26. 4 where hood is used. (مصد he travelled post, Mich. Syr. ${ }_{25}$ I $b, \mathbf{1}, 2 ;$ oll l;90; they travelled post, Anecd. Syr. iii. 248. 4 .

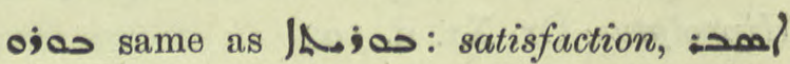

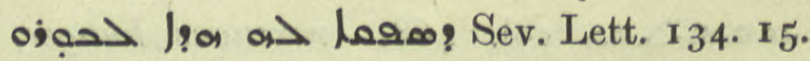

๓ofojos Beyrout, Sev. Ant. Vit. 54. 8; oofo: ح, ib. 47. 2 ; مof०ص, ib. 89. 5 q.v. col. 606 .

?\$: pr. n. m. Burzad, a man of Be Nuhadra, M.Z. ז99. 2 I.

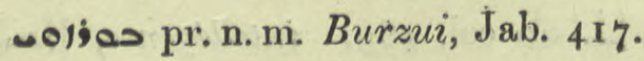

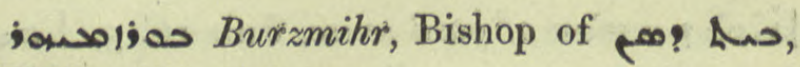
ZDMG. xliii. 406. 10. 


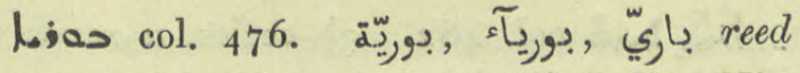
matting, Op. Nest. 89. 2 ; البارية El. Nis. 37. $5 \mathrm{r}$.

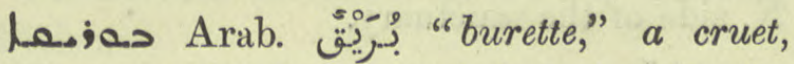
flagon, esp. the flagou used for wine for the

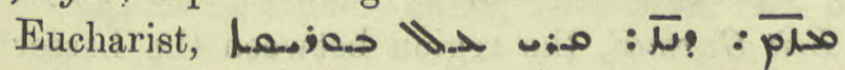
معصس أ PN. Ivii, read it over the flagon and you will derive bodily and spiritual help, ZDMG. xlii. 458. 16.

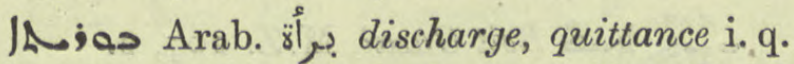
محل A 1 Joong he accepted the office of tax collector, Mich. Syr. 49 I. Ir. af., 5I6. 4 .

bio see borax.

1 ب. a large cooking pot hollowed out of stone, Chimie 4I. 20 but / פarthen pot, ib. 33. 19.

בajerenice? name of the woman with the issue of blood, Journ. As. 1906, 283. 4.

| Zend baresma, a date spathe, a branch of pomegranate or of tamarisk. This was held in the hand in Magian worship; Mar Kardag F. 66. I, 68. 8, 71. 2, 78. 4, Iutrod. i 1 n. I6; Pers. Mart. nn. 838, roo4. Cf. Ephemeris ii. 222 , Lag. G. Abh. I59, Arm. Stud., Spiegel Eran. Alterthümer iii. 57. I. G. Warda ap. Hippoc. ii. p. iv. 5 af., Jab. $43^{6}$.

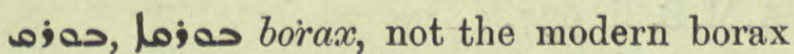
but a mediaeval flux, Chimie passim : حموم h. مته goldsmith's fux, ib. 54. 2 I and n. 2 ; written ib. 37. 4 \&c.

حهزم

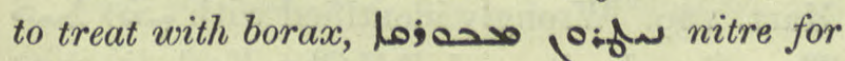
soldering, il. 26 antep.

pasa (asprint for paras prob. Cochin, R. O.C. 1912. 76. 4, 9 bis, and

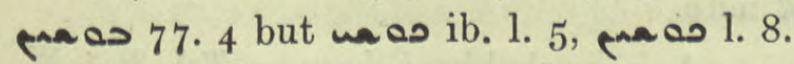

JLٌ حُ col. 478. Add: pl. حقام so correct

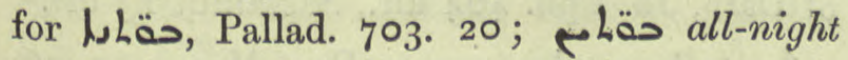
vigils, Anecd. Syr. ii. 47. I4. I) passing the

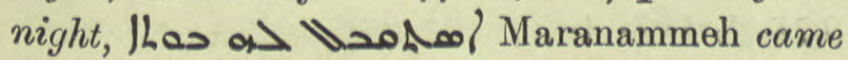
to a place where he could pass the night, Sassanidi 28. I1; a night's stay i. e. one stage

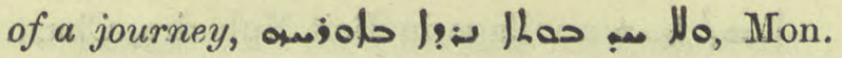
Syr. i. 2 2. $3^{2}=$ Jac. Sar. Hom. iii. I 93.16.

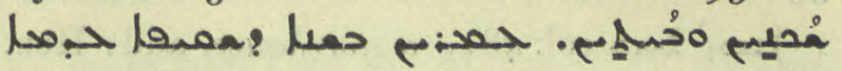
إمئم they settle and remain, they dwell on a rocky peak until they die, this is called keeping vigil, Poem on mountain ascetics, Ephr. ed. Ov. I 20 . I $3 \mathrm{f}$.

12น́s col. 479. 4) astron. a House, one of the twelve sections into which the sphere of heaven was, in thought, divided IÄ! (⿻上丨

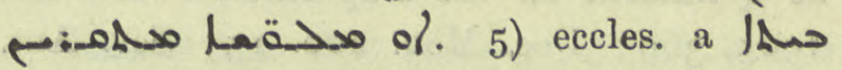
seems to be as much as was repeated at one time whether long, a strophe, or short, a versicle. Lamy says a حمA is a strophe, not a verse, that with Ephrem a مu may contain as many as fourteen verses though sometimes only $t w o$, Ephr. ed. Lamy iii. p. ix and note. A versicle,

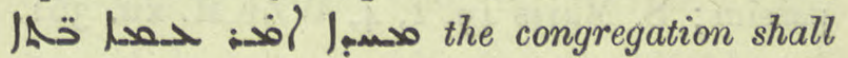
thereupon say the versicles viz. at the conclusion of the Litany they repeat "Thy body which we have received," \&c., Bar. Sal. in Lit, 94. 13, 14; a subdivision of an anthem, Qdham W. I74. $10 ; 257.2$; ر مد a canon, Brev. Ant. i. 54 a I 3 ; 发次 12ำ after each verse, ib. 59 a II, I 2;

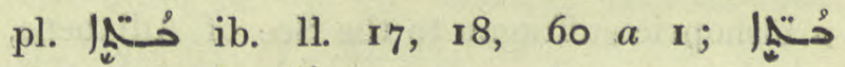

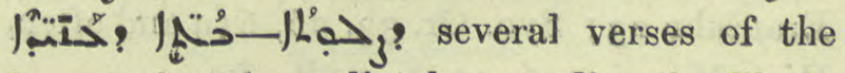
long anthem immediately preceding the Gloria verse, are so called, Maclean. 6) a case,

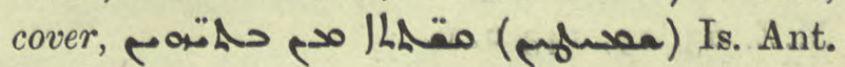
ii. $27^{8}$ pen.

Compounds with add :-

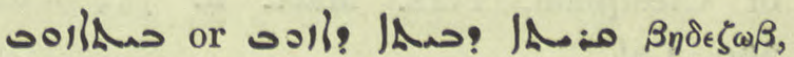
House of hyssop, lool? IA Josephus ap. Hebraica iii. I 45 .

ح ح the Common Room of a monastery, Hist. Mon. i. lxv. I,

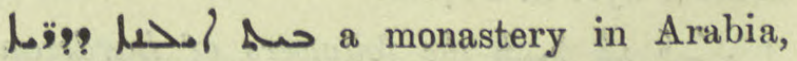
C.B. M. 7 I $2 a$ and $b$, Doc. Mon. 2 20. I 8, 22 I. 5, 222, I 2 .

حص / name of a monastery, C.B.M. $706 b$.

| ed. Guidi 222 ult., 233 n. 
حم /مصر a village in Beit Nuhadra, Chast. 53. 18.

/ Ex. xvi. 23.

حق 22.

10 ) neighbourhood of Henaita and Ma'altha, Chast. 28. 8/9.

Losol Beit Aphthonia, a monastery at Qennešrin on the Eupbrates, Journ. As. 1905, 369; Doc. Mon. 200. 15; Sev. Ant. Vit. $207 \cdot 5$.

objl حu col. 48r. archives, Ephr. Ref.

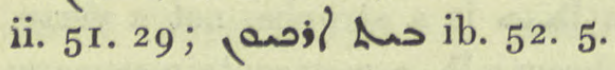

حم col. 481. the central part of Mesopotamia, Suristan, Syn. Or. 667 and passim; S. Maris 49. I, 4, WZKM. xii. $3^{6 r}$.

Jiß حس Beit Arnê, Chast. 70. 8.

village and monastery, Doc. Mon. 146. 8, I52. 21 , حصA حصe?, ib. 162. $16,163.20$ and often.

بادغيش col. 48r. Add: Ar. حس ح and باغاش Beit Bagaš, a district on the banks of the Great Zab, Pers. Mart. 227.f.; a bishopric suffragan to the See of Adiabene, Eranšahr 23; Syn. Or. 33. 26, 619 and often, Hist. Mon. i. 106. 12, 145 ult., \&c.

 M.Z. $63.19,143$ n. 6.

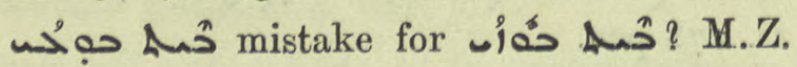
207. 6.

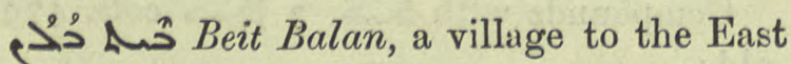
of Ctesiphon, Pers. Mart. n. $744=$ Jab. 369. 3 af.

حِي Nuhadra, Patr. Or. iii. i. 66. 7 .

: פמ 2 name of a family of Amid, Anecd. Syr. ii. 287. 8.

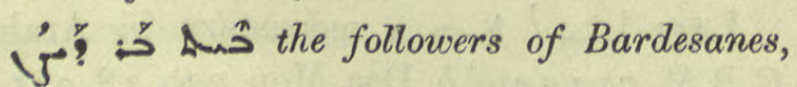
Georg. Ar. 19. 21.

م: Sas col. 482 . i.q. Eleutheropotis, in Palestine, Mich. Syr. Tom. i. Fasc. ii. Transl. 248, No. 33 .

ly a name of a village? Doc. Mon. I 7 I. 24 .

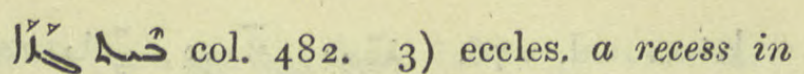
the North wall of the sanctuary, for the sacred vessels, Takhsa 5. 13, 145. 22. Hence the $\mathrm{N}$. side of the sanctuary.

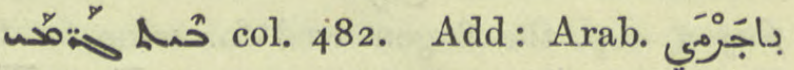
Beit Garmai, the region between the Lesser Zab and the Diala, East of the Tigris, Syn. Or. 668; ib. I9. $1 \mathrm{I}$ and often; Pers. Mart. 253 \&c.

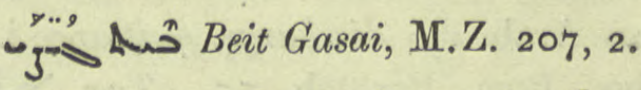

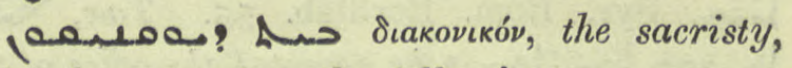
Pléroph. r 2. 9. i.q. the following.

فم حص the serving-place, the vestry to the S. of the Sanctuary, Takhsa rog. 20; Hist. B.V.M. I79. 8.

IA Pet. Ib. 102. 4 .

מاسس Ar. Beit Dasen, a village in Hakkiari, West of the Great Zab and South of Amadia, Pers. Mart. 202 ff. a bishopric suffragan to Adiabene, Eranšahr 23 f., Syn. Or. $668 ; 33.26$, I 10. I7 \&c., M.Z. 63. I 8.

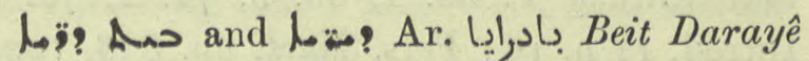
now Bedreh or Bedrai, 15 o kilometers East of Baghdad, Syn. Or. 668; ib. 37.6, 43. 21 and

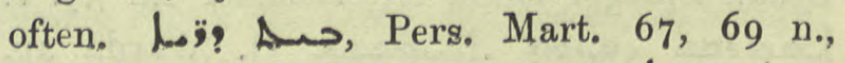

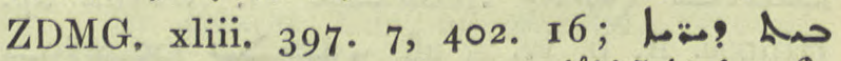

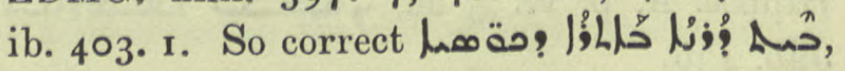
A.M.B. ii. 637 .

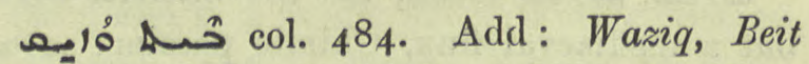
Waziq, or Bawaziq is situated on the West side of the Lesser Zab, a bowshot above its confluence with the Tigris, Yakut and Pers. Mart. 189. Wrongly identified with Thes. Syr. 1. cit. and BHChr, Eccl. ii. 123 ; the latter place is four parasangs to the South, Pers. Mart. 190, S. Maris $3^{\mathrm{I}}$ Syr. ult. and note.

nac va probably Bebasa =Tel Beš W. of Dara, Jo. Eph. 404 ult., Sassanidi ${ }_{3} 3$ ult.

Las place-name, Doc. Mon. I63. 26, I 7 I. I, I82, 4 .

حمA Beit Zaite in the district of Birta in Marga, Chast. 10. I4. 


\section{R-Jid}

لإ. i. $44^{2}$.

حمب مo the South side of the sanctuary, Maclean.

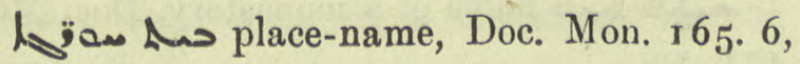
I7 7 . 22.

حt col. 485 . so correct for

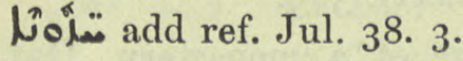

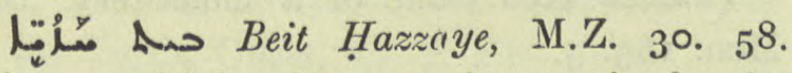
Mingana thinks this may be a mistake for Läa حصA.

Beit Hêātēe, a monastery near Mosul, Hist. Mon. ii. 102 n. 7, but see Pers. Mart. 7I. Chast. 43. 5, 6r. I6, Doc. Mon. 214.

حמל ملمa a village in Adiabene, M. Z. I 79 ult.

Löof An Turkestan, Or. Xt. i. 308 pen. (letter of Tim. i.).

lefy an prob. surname, of the family of Tatian; cf. lend below, Pléroph. 18. 7.

lagf th a village five miles North of Jerusalem, Pet. Ib. 98. 3 .

מع earlier form of the

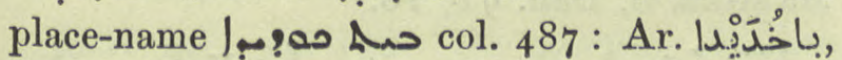
four days journey SE. of Mosul, Pers. Mart. 178; Jesus-Sabran 578. 21 , Nöld. in loc. WZKM. xi. 188.

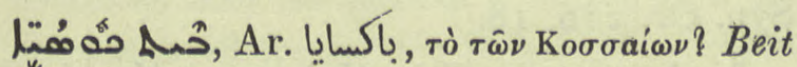
Kussaye, below Baghdad, Pers. Mart. n. 824,

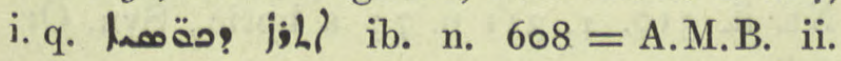
637. I2; Nöld. ZDMG. xxviii. Iог; Jab. 4I4. I.

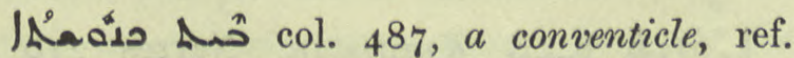
Jul. 5. 18.

la i. 94.40 .

Yakut. Beit Laphat, capital of Khuzistan and Metropolitan See. Later called Gunde Šapur; now the ruins of Šlahabad between Susa and Suster, Tabari 4I. note; Syn. Or. 668; 33 bis, 34. 3 ; 6I6-618 and passim.

- مدA name of a village, Chast. 22. 8. 2716

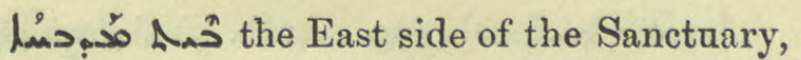
Maclean.

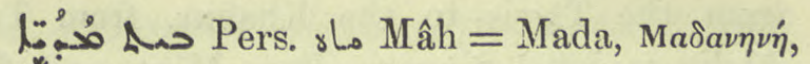

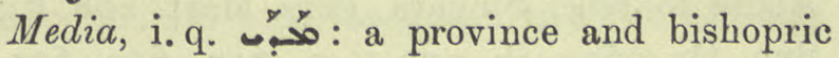
stretching from the pass of Holwan to the neighbourhood of Hamadan, Eranšahr I8, Pers. Mart. 107; Syn. Or. 669. Ib. 34. 20, 37. 3, 53. I I, 2 I 4.9 and often. ZDMG. xliii. 397. I I ff., 401. 3, 403. I, 406. I 5, M.Z. 206. I8; Jab. $517.9,14$.

Lioa Da Bat Mahqurt, a bishopric in the province of Adiabene. Its extent answers to the territory of the Kurdish tribe الماجردان in Azerbaijan, Eranšahr 23, f., Syn. Or. 669; ib. 33. 27 ; ZDMG. xliii. 394 n. 5 .

jas the the House of Mitran, one of seven noble Persian families, of Arsacid blood, Tabari I 39 n. 3, 439 and n., lsoa of م jas o. Jab. 350. 10; cf. $3^{67}$ pen. and see names in joux, Suppl.

مu Pers. Mihra-Kān-Katak = House of the Mihrs, Eranšahr 20; oxwa ib. a bishopric united with that of Ispahan, ib. 30, ZDMG. xliii. 399. 2 ; Syn.

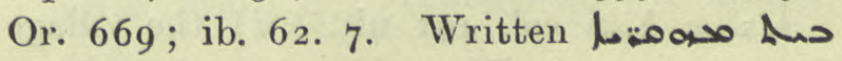
ib. 1. 26, 67. I I ; ZDMG. xliii. 399. n., 400. I,

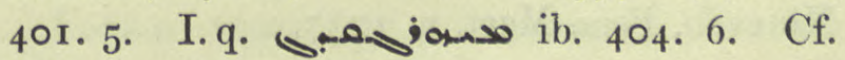
below.

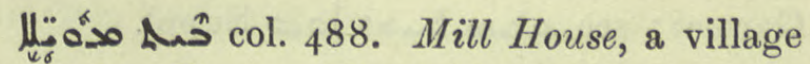
near the Tigris, Hist. Mon. i. 238 . II ; A.M.B. 1. 455 .

مد مas Moxoene, now the district of Moks, South of Lake Van, a bishopric Suffragan to Nisibis, Syn. Or. 669 ; ib. 32.22 \&c.; ZDMG. xliii. 394. 2/3, 396. 14.

. Beit-Mihr-Bozed, prob. in Khuzistan, Syn. Or. 669; 79. I6 and so

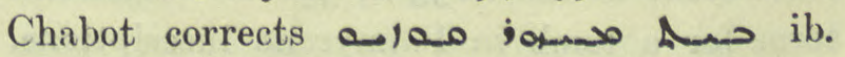
77. 11 .

ILen on col. 489. the Imperial Court, Sev. Ant. Vit. 106. 9 ; Pléroph. 1 1. 12.

90ja wis of name of a village, Doc. Mon. I63. 28.

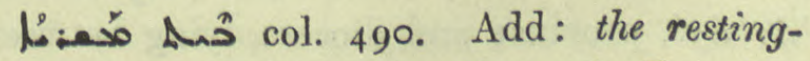
place = grave, Nöld. in Pognon's Inscript. Sém. No. $4^{8}$. ZA. xxi. $5_{5}^{6}$.

H 
Rirmas dus

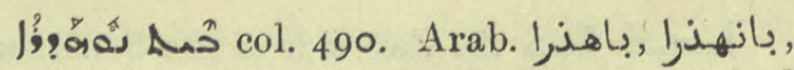
1. Beit Nuhadra. This diocese reached from the Tigris to the Khabur, from Tur Abdin to Jebel Singara, Pers. Mart. 208, ff.; Syn. Or. 669 ; ib. 300 n. 2, 617, 619, 621. Arbela is the See of the Metropolitan of Beit

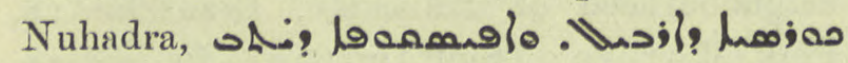

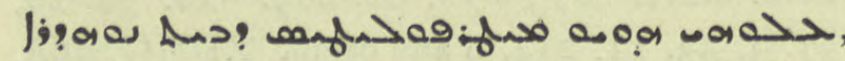
ib. 33. 25 ; 62. 9, \&c.; BHChr. Eccl. ii. 69. I, MZ. 143 n. 3 ; jigas or. Xt. i. 3 I0. 5 .

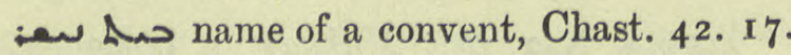
J:9N ح Beit Nethperê, a village in Adiabene, Chast. 26 pen., 27 ter.

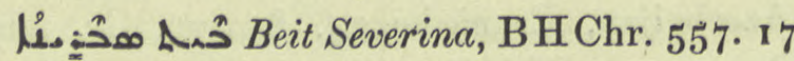

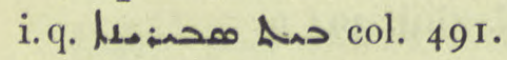

محسى Doc. Mon. 215.13 .

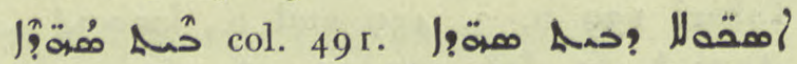
" 5 " prob. schools dedicated to the memory of holy martyrs, Chast. 26. I, 6.

Ihanos and and androom,

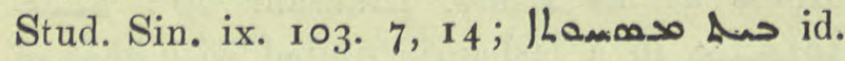
BH Cand. col. 2596 .

حس col. 49r ult. 2) the village Ba Sahra on the West side of the hill above Nineveh, Pers. Mart. n. 1737 .

y. o. Dus Beit Slōk, capital of Beit

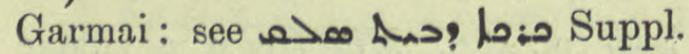

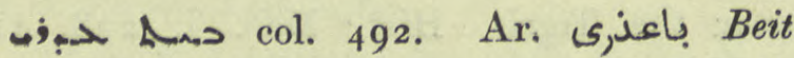
'Edrai, East of Alqosh, Syn. Or. 668, transl. 300 n. 2, 53I n. 3 \&c., ib. 61. 3, 63. 21 ;

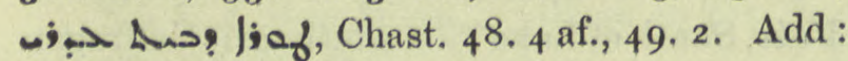
Pers. Mart. 2 I, 208, 2 I 2.

בית על Koh. xii. 5 ; Tg. Jon, to Is, xiv. 18 ; Levy NHW.; Lidzbarski Handb. 235 f., 342 a. Technical term for a tomb in Palmyrene inscriptions, Chabot ZDMG. 1x. 283. Adopted by Greek Christians as oikos aívitos, C.I.A. iii. $35 \circ 9$, f. (4th-5th cent.) ZA. xix, 133 n. 2, Flor. Vogüé 233.

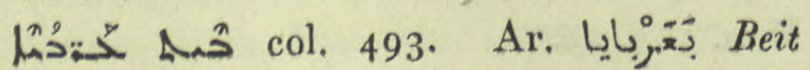
'Arbayê, province and diocese, lying between Mosul, the Tigris, and the Khabur, Syn. Or. 667 ; 66. 2 ; Nöld. ZA. xxi. 153 ; Pers. Mart.
23,131 ; Hist. Mon. i. $63.21=$ ii. 115 n. 4. It included Tur Abdin, ib. and Mar Bassus

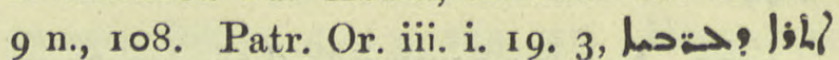
ib. 20.8 ; A. M. B. iii. 45 o.

and name of a monastery, Doc. Mon. I71. 19 .

مas the household of the Patriarch, ZDMG. xxxvi. 349.

مas name of a monastery, Doc. Mon. 165.5 .

J 2 place-name, Doc. Mon. 172. 12.

1 Doc. Mon. $219.20,29$.

$\operatorname{lag}_{3}^{n} \hat{\Omega}^{n}$ a building near the Palace, prob. the Mews, Jab. 255.4 af.

مُ مَّ of the Sanctuary, Maclean.

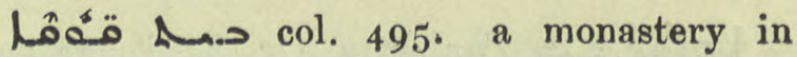
Adiabene, seven hours West of Arbela, on the Great Zab, Hist. Mon. i. 23. 6, 89. I5, 90, 6, $3^{81}$ r. ${ }_{5}$, Chast. $3^{6 .} 2$ r, M.Z. I 7 I. 2.

una مa Beit Quši, a village near Hira, Sassanidi 9. 16.

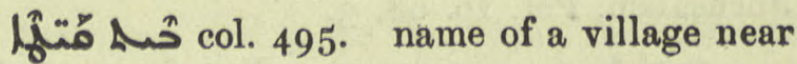
Ma'altha, S. Dan, $9 b, \mathbf{l} 8$.

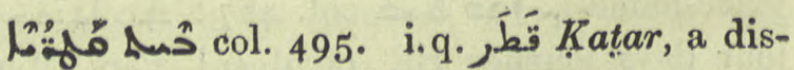
trict on the Arabian coast of the Persian Gulf, Hist. Mon. i. 86. 7, 95. 13, Chast. 64. IO, Syn. Or. 2 I $6,18$.

مل perh. Beit Hale near Mosul, Or. Xt. ii. 3 ro, 4,3 х $~ n .7$; a bpric., Syn. Or. 608. 13.

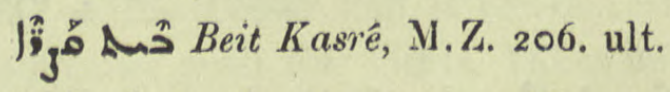

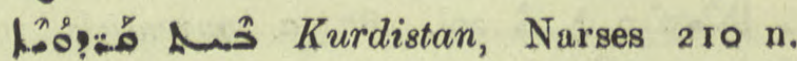
Cf. 0 col. 373 I.

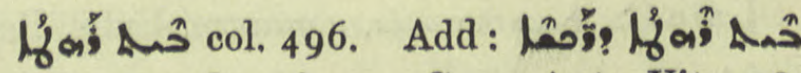

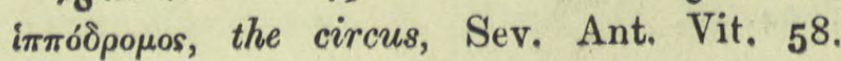
$7,13$.

Lonog (? |jhi) near the (? |jhi) Kashkar, Chast, 62. 2, I1.

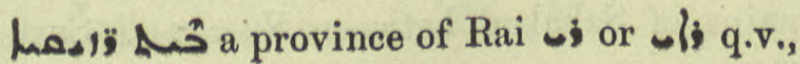
in Upper Media, nearly corresponding with the ancient satrapy 'Payıav', Syn. Or, 669; ib. 34 . 


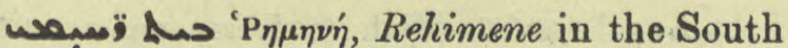
part of Armenia. As a bpric. it was suffragan to Nisibis, Syn. Or. 669: ib. 33 \&c.; ZDMG. xliii. $394 \cdot 3$.

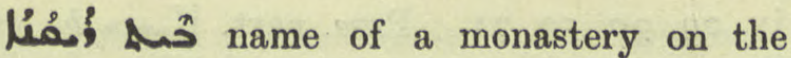
Tigris, M.Z. 209. 17, 22, 213. 20. Cf. ح. koj near Haditha, Chast. 62. $21,63.2$.

م col. 496. Beit Ramman i.q. Pers. مـ Q Dardilabad which is Sena on the Tigris, Chast. 29. 7 , Hist. Mon. i. 79. 23, 292. 3 ; Pers. Mart, 189 f., 253, 257.

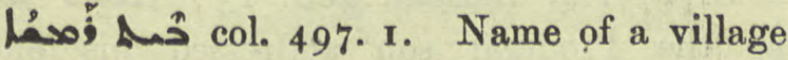
in the diocese of Aleppo, Sev. Lett. 69. 5 .

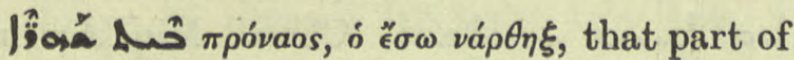
the church where nocturns were sung, Hist. Mon. ii. $431 \mathrm{n}$.

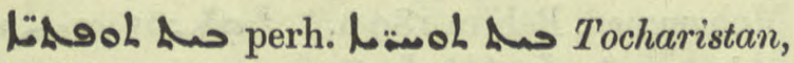
Or. Xt. i. 308 ult. (letter of Tim. i.) and note p. 3 II

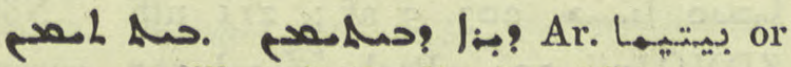
a village in the district of Damascus, ZDMG. xxix. 428 ; C.B.M. 7 I 2 a, Doc. Mon. 220. 7,9 .

حit Beit T'arle, a village in Beit Nuhadra, Patr. Or. iii. i. 66. 6.

Lib th col. 498. the doorway, Takhsa 145. 19, 20; the West side of the sanctuary, Maclean.

Aaval su the part of a church in front of the sasere service is held, Gr. $\sigma \omega \lambda \lambda^{\prime} a$ Du Cange, Hist. Mon. i. 3 I2. 8 and ii. 55 I n. 2 .

כ̌ denom. verb. col. 499. 4) to admit.

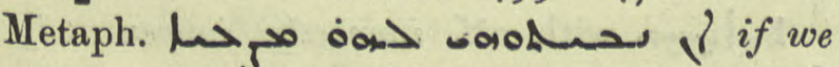
admit a principle intermediary between the Good and the Evil, Manicheisme 95. I.

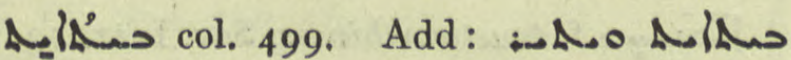

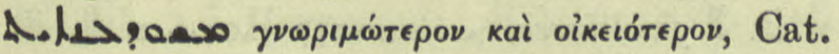
Ar. Jac. Edes. 26. 4 and 6 af.

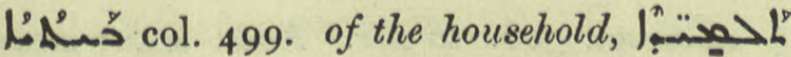

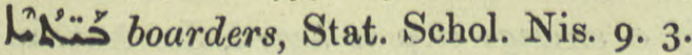

notes Boethius, BH. ap. Hebraica iii. 25 I. 4 af.

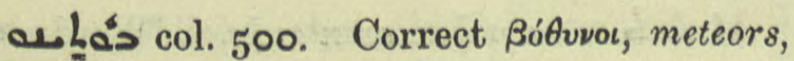
Lag. Symm. i. 93, G. Abh. 65. 28. tح́ col. 50r. Pass. part. حِ N. B. construction; | حــ churches and monasteries have been plundered by him,

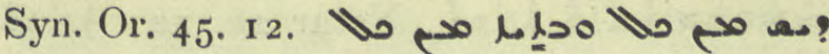
trodden under foot and despoiled by all,

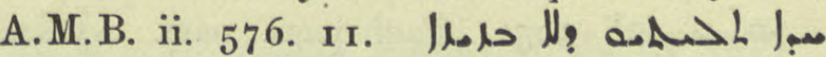
one inviolate Trinity, S. Dan. 5 I a 9. Pael

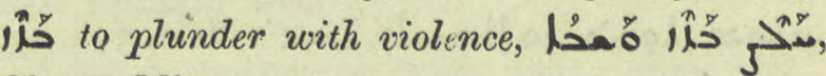
Chron. Min. 334. I.

เร้ $B a z$, a district in Tiari, A.M.B. i. 4 I 7.

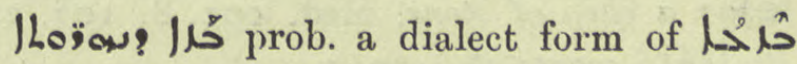
a cleft; Talm. ביוץ a parting of the waters; name of a monastery in Kashkar, Sassanidi I1. I 4, Nöld. in loc. Cf. Is. xviii. 2.

| word ; cracks round the eyes, Med. 92. 2.

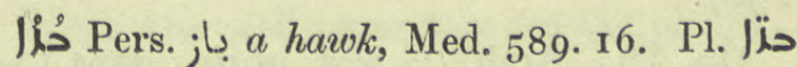
Kal-w-Dim. ed. Bick. 6. I6; ul, ib. 31. 9 pen., 32. 1, 2 .

(a b Bazgun, a country adjoining the land of the Huns and the Gates of the Caspian, Anecd. Syr. iii. 337. 7 .

Q S. Daybreak, pr.n.f. A.M.B. ii. $5^{88 .} 9$.

Loh name of a monastery, Brook's Chron. 578. 3 af. Cf. hsolas col. $5 \mathrm{I}_{7}$ and BHChr. Eccl. 329 pen.

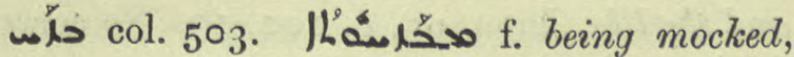
set at naught, مصحl, Alexis 67. I, 5 .

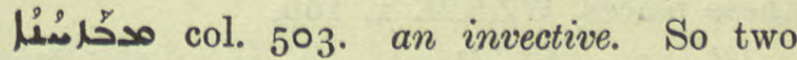
orations of Greg. Nazianzene on Julian are called, C.B.M. $429 a$;

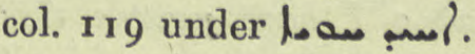

ـ حِمِ col. 503 infra. a hawk, falcon.

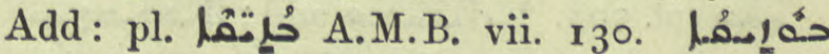
Gest. Alex. I 4. I I, I5. 7. The Lexx. have both forms under If col. I 449. Dele the last line of par.; Lagarde said later that his Sanskrit was wrong, G.A. 2 I. 45 .

حِ col. 504. Pa. wh้ add: to bore,

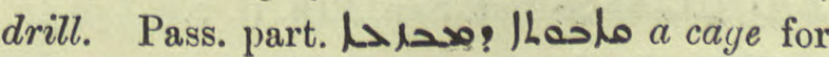
lions with many air-holes, BA. under $\mid L^{2}$ col. 2386 . 
L

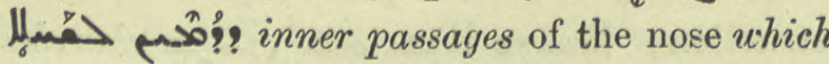
act as strainers, Med. 62. I I; a siren has

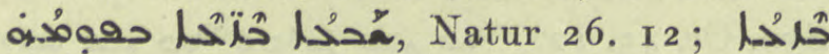
أُ an ant-hole, N. Hist. vii. 2. 3.

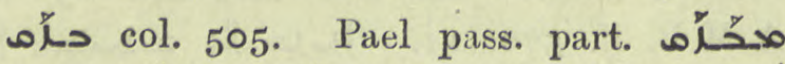

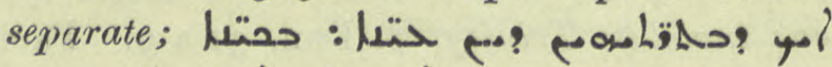

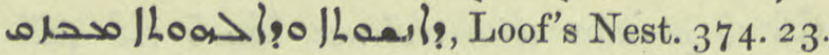

grón a pebble. Add: gloss to //3rit, gravel, |مْ? a chip of stone, Med. ror. 8, 107. 23.

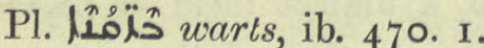

دمرَّم Bezqin, a Jacobite monastery, Pers. Mart. 21, 182; Hist. Rabban Hormizd 54.

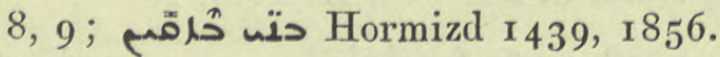

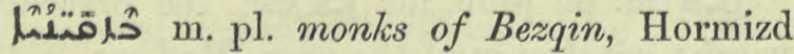
1507 .

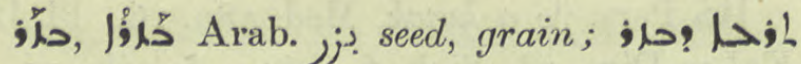
Verses on grain, Protection 90. I7; l9ق

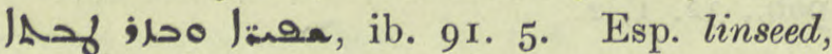

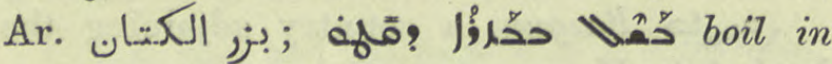
linseed oil, Med. 577. I ז, ellipt. ib. 586. 14 ;

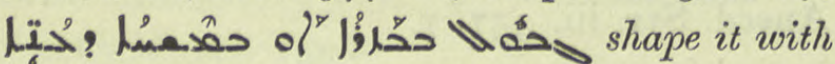
linseed or sheep's oil, ib. 601.7/8. الدهن المشثر, El. Nis. 76. 89.

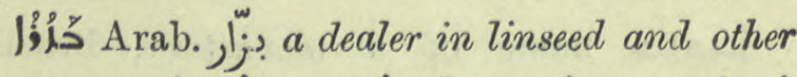

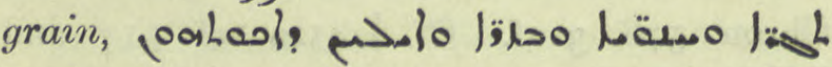
traders and innkeepers and corn-chandlers and the like, Dion. 95. 6, roo. 13. So correct for li:so مته م. ib. 234. I8. Nöld. suggests

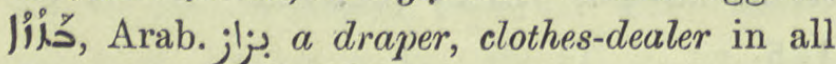
these places, WZKM. x. I 66.

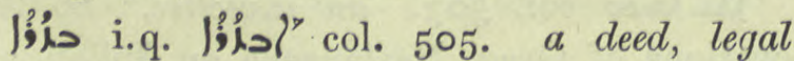

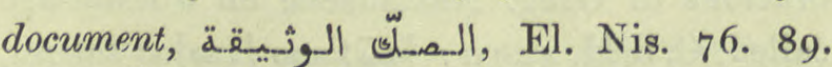

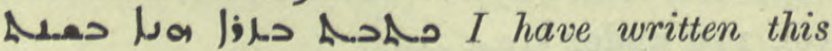
receipt in the year ..., C.B.M. $33^{8} a$.

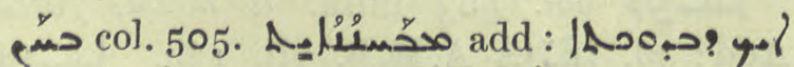

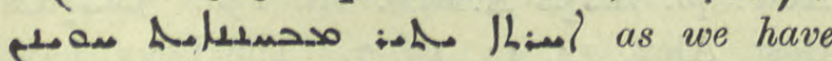
elsewhere more exactly discussed, An. Syr.

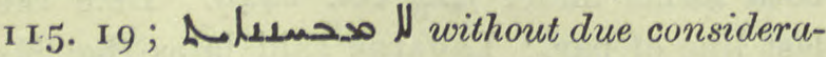
tion, Manichéisme, I 20. I.

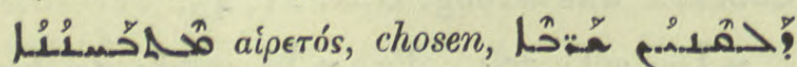

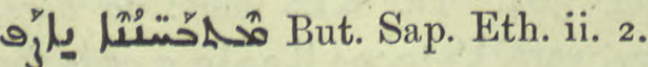

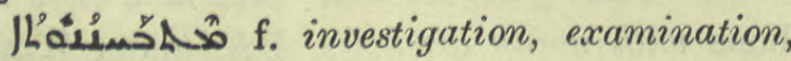
Nest. Hérac. 182. I $7 ;=$ = $1.20 ; 235.16$. sur col. 506. Med. to bring on a crisis, induce the turning-point of a disease, Hippoc. iv. 36. Ethpe. an illness, Hippoc. i. 1 I. 19, ii. 23 , iii. 27 , iv. 29, 30, 59, 7r. Pass. part. حسئن who has passed the crisis of a disease, Hippoc. i. I9,

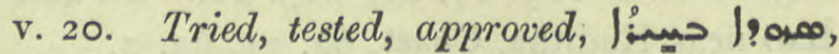
Sev. Ant. Hymns r 91, Narses ed. Ming. ii.

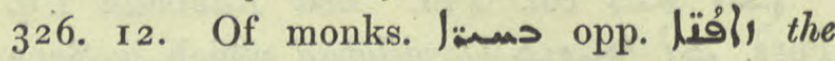
tested and the lapsed, R.O.C. vii. I 7, pen. and

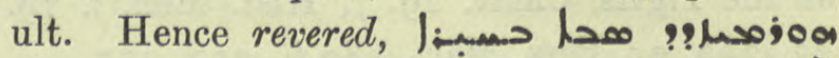

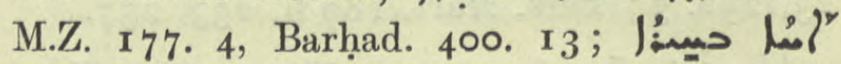
so the Prodigal son addressed his elder brother, Ebed J. Card. 46. 6 ; ممشع/ Ja

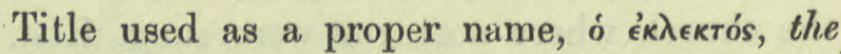
Elect, cognomen of Sergius, the teacher of

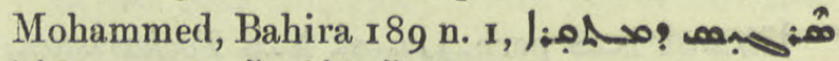

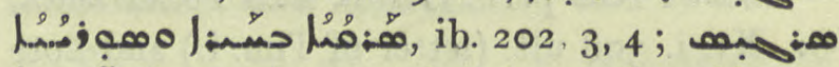

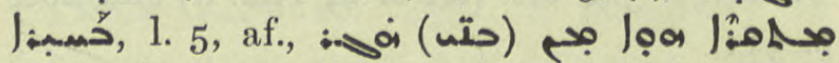
مبنم: 203. 3 af.; 2 II ult. Nöld.

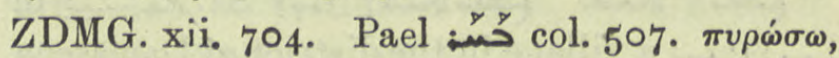

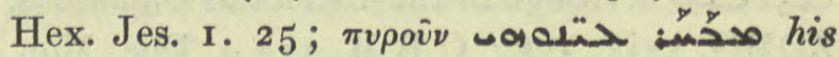
eyes sparkled, Jo. Tell. ed. Kleyn 65. 9.

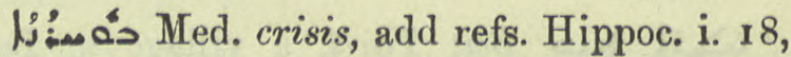
ii. $\mathbf{1}_{2}, \mathbf{1}_{3}$, iii. 8 , iv. $5 \mathrm{r}$ and p. 7, n. 3 . Chem. test h. the melting pot, ארم in the crucible, Chimie 98 ult., 99 ter.

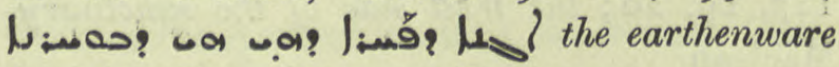
bowl i.e. the melting-pot, ib. ror. I 2.

حُنَّة Med. bringing on a crisis, Hippoc. v. 20. Astron. pl. heliacal rising of Sirius by which other dates are defined, Georg. Arab. 26. 8.

هيمن with wexactly, inaccurately, BH. in Koh. I. I ; with ل! improperly, Sev. Lett. 427 . 1 .

IL'o 0 f. trustworthiness, Sev. Lett. I3.5; with لl faultiness, wao:so?! Jlow ll, ib. 262. 9 .

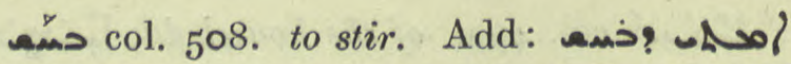
L:Dos if a strange step is heard, Is. Ant. ii. 280. I. Aph. I52. 2 , 2 IO. $\mathrm{I}$.

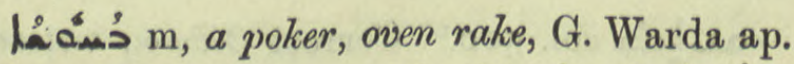
Hippoc. Pt. ii, vi. I 2 with gloss j;ash? l?:0?. 


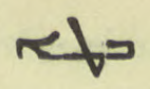

53

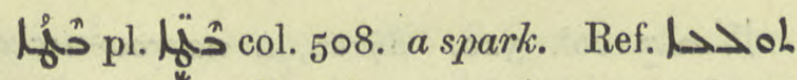

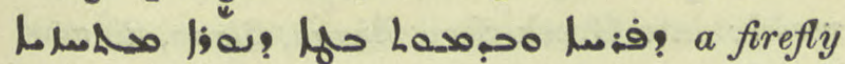
which looks like a fiery spark, Kal-w-Dim. ed. Bick. 28. I3.

Is perh. i.q. Lل Lattis, bp. of pac, ZDMG. xliii. 394. 9, /ه, ib. 395. 7 .

A s col. 509. eighth par. Delete: it is IR.

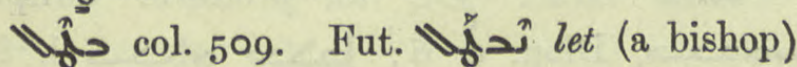
be deposed, lit. let him desist, Conc. Sel. 33. 5. Past. part. ح 1 I have leisure $=I$ am attending to, Reckendorf, ZDMG. xlii. 400.

 from heaps of corpses brought the army to a standstill, Josephus, vi. I. 6 .

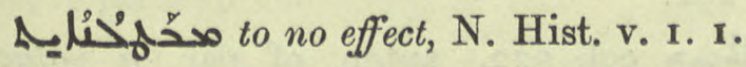

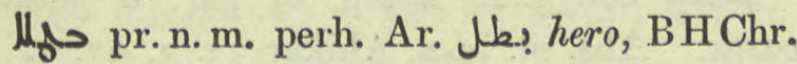
Eccl. 84I. I.

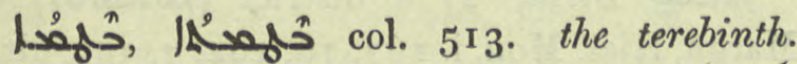

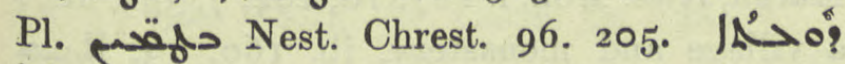
IRำ

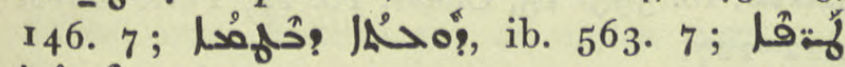

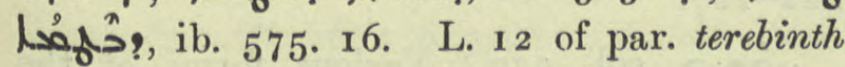
nut or berry, حسّة

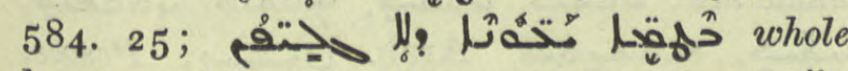
berries which have begun to germinate, ib. 586. 5 .

حمatmin, a village, Pet. Ib. 96. I 7 . Raabe identifies it with Bothnin in the territory of Gad, trans. 92, n. 4 .

ते col. 514. Add to Aph. Âे to breed, ظُمعُما stallions, Op. Nest. I1 4.4.

lifs m. the embryo, foetus up to two months of gestation,

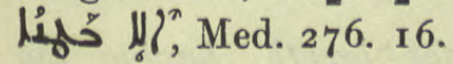

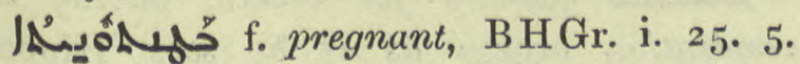

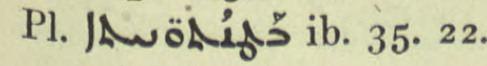

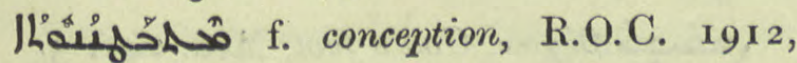
86. 6.

حُ 46. 7 .

ho Bia, violence, Syn. ii. Eph. 23. 2 I, 28. I 4, 16 i. q. Jiofo ib. 29.4.

\section{afule}

ho pr. n.m. Syn. ii. Eph. 56. 12.

حمارم Bayazid, name of an Emir, BHChr. Eccl. ii. 523 .

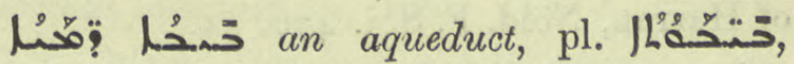
Anecd. Syr. iii. 207 bis, 209. I.

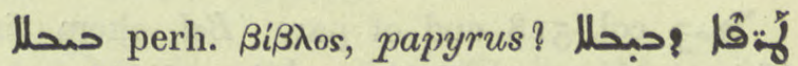
مed. 577 . 1 .

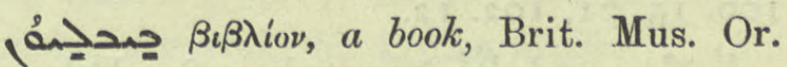
$3327.35 r$.

דمحتم Pers. m. pl. panthers, Dion. I94. 14.

حس Vigilius pope of Rome 537-555, Anecd. Syr. ii. $3^{8} 5 \cdot$ 16, $\rightarrow$ ib. 1. 8.

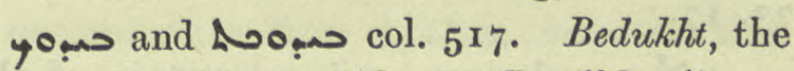
Assyrian goddess of love. Possibly Noo? the Virgin Belthi. Both names wDo? and are used separately for the planet Venus, Pers. Mart. 128 ff. So Noo $\mathrm{B} \hat{\eta} \lambda \theta i s i \dot{\eta} \kappa i ́ p \eta$, Cumont, Textes et Monuments, 134, Lag. G. A. 16; Symm. 1. 94 f. حَنبه" among names of Greek deities, A.M.B. ii. 656. 5. BB. says this is the Persian name, ح the Chaldaean, and Jisel the Aramaean, col. 542. 6. Cf. Chimie 123.

حب a grandson of Hulagu who reigned for a few months, Jab. 94 pen. כ is the name of a Mongol Emir.

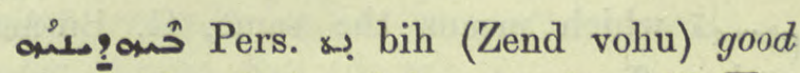
+ حaith, religion, Jab. 524 ult. Cf.

; see jabove.

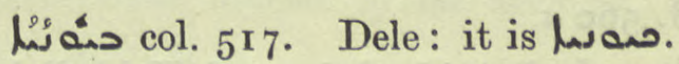

جִ Med. 237. 7 .

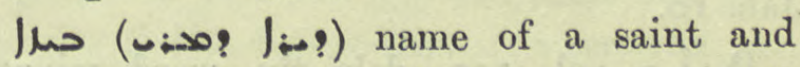
monastery, C.B.M. 708 b, Doc. Mon. I 25. 8, I $27.27,128.12,173.1$.

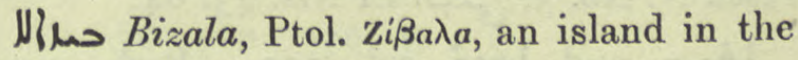
Red Sea, Jac. Edes. Hex. xx. 9.

colv Bí̧os, Bizus, bp. of Seleucia in Coele-Syria, Nöld. F. S. I. $469 \cdot{ }^{2} 5$.

Iho for IA a phial, Chimie 26. 4,

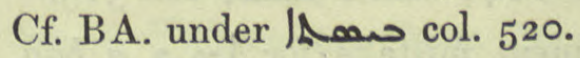

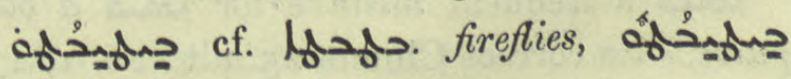

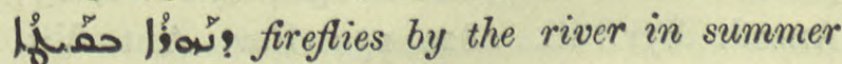
(Mingana), Med. 598. 6. 


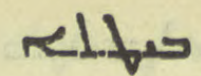

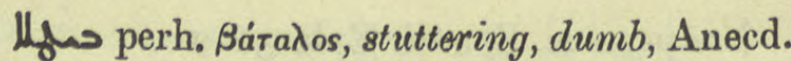
Syr. iii. 139. 14.

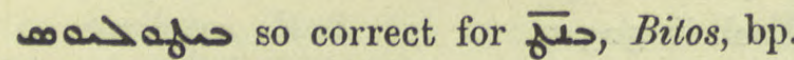
of Harran, Nöld. F.S. i. $470.4 \mathrm{r}$.

(?: Doc. Mon. 214.23.

D. col. 518 end of par. Bel, chem. tin, Chimie xv. I25; tha of ib. 99. I5, 100. I5, 21, 102. I 2.

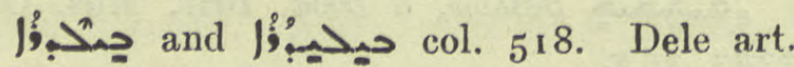
and see fi!jo a courier.

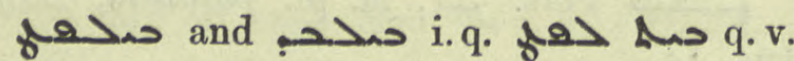
S. Maris 83 and note; Sassanidi 9. 6.

Ind a native of Beit Laphat, Syn. Or. 73. 17.

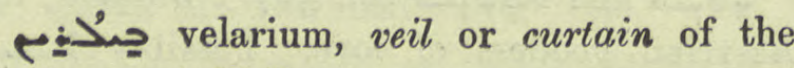
sanctuary, A.M.B. v. 577 pen.

حمصُ col. 519. 4. Rit. add: E-Syr. I) usually the raised space between the sanctuary doors and the dwarf wall in the nave parallel to them ; pä? (rubric) prayer or praise said from this raised platform, Brev. Chald. i. 74. 4 af., 325. ult. 2) rarely the sanctuary. Maclean.

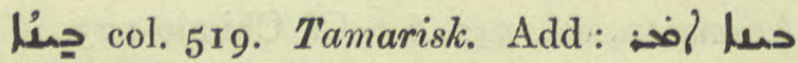

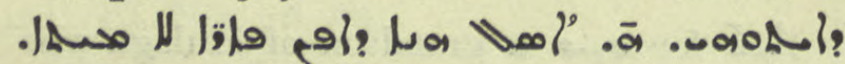
i.e. Artemisia; wormwood or southernwood and some say it is قيـ. which means the same, G. Busâmé $57 b$ × $3 \mathrm{ff}$.

Bivôckes, vindices, solicitors, C. B. M. $560 a$.

" כسبرا'Bainiel, father of Yahballaha, Jab. 10.

مLin col. 520, dele the word minuti. A kind of carp, Streck, ZDMG. 1xi. 635 f., Fisch N. No. 3, البُّنّي El Nis. 43. 84.

حيسمُ and a board, plank, i. e. the boards of a cage for wild beasts, into which the bars are fastened, $=$ =

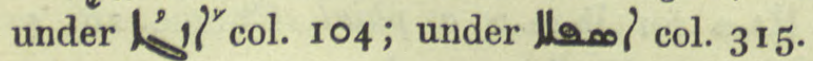

حمل a frequent mistake for lo a a bag, purse. So correct Chimie 25. ult., $26.1,13$; Anecd. Syr, iii. 282. 15; Sev, Ant. Vit. 283.8 .

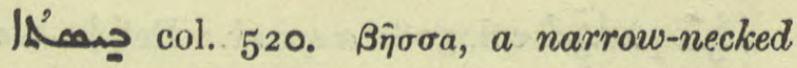
vessel; late Greek $\beta \hat{\eta} \sigma a, \beta \hat{\eta} \sigma \sigma a, \beta i \sigma \sigma a, \beta i \sigma \sigma \iota \nu$, Du Cange r96. f.; Fraenk. ap. ZA. ix. 3 ; Can. Jac. Edes. I 17.

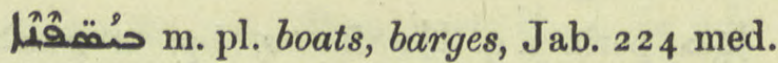

| Jab. 270 ult. and n., 272 . $x$.

Sarab. بَّ to to proclaim Caliph. 1. 8; .

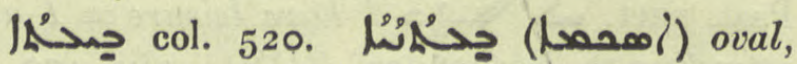

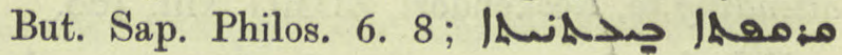
the arched crown of the head, Caus. Caus. 30. 20.

ح name of a place in Lycia, A.M.B. iv. 292.

ILin col. 522. antep. of par. a palace, castle. Abs. pl. (صن, Ahikar 33. 3, 37. 9.

ILirta, 2) a district (10100ز) of Marga, Hist. Mon. i. 167. 10, 194. 7, 359. ult. ; a village and monastery, ib. I60. I3, Ihin /م: ib. 395. I5, Chast. Io. 12, Pers. Mart. 222-227.

جِ col. 522. Various forms of the

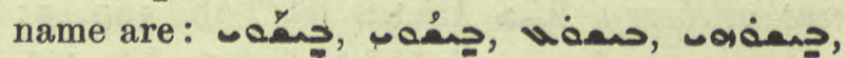

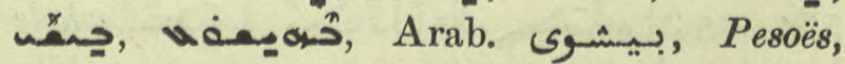
Pesaeus ; Bhishu, A.M.B. iii. 572. ff., ZA. xv. 104. 4, 16. The village named after him, Mar Bhishu, is two days journey W. of Urmi and one day from Gawar, Pers. Mart. 229.

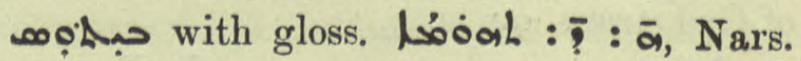
ed. Ming. 360 n. 2 , one of the three (Gnostic)

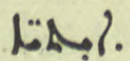

حمل حملحم see under.

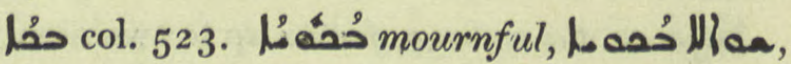
BHGr. 464.2 a f.

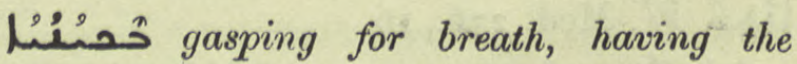
respiration broken as if by sobs, Hippoc. vi. $5^{2}$.

|

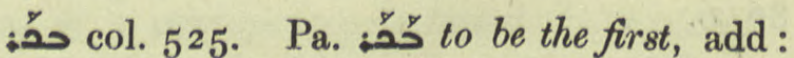

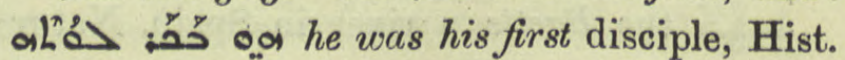
Mon. i. 24. I4; Qdham W. 238. I1. Ethpa.

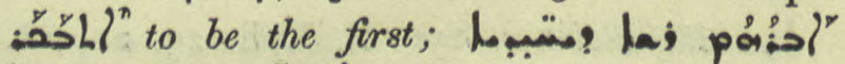

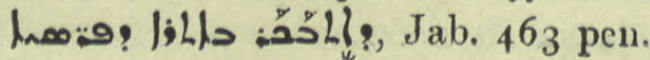


risas

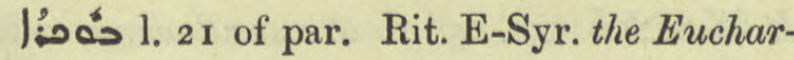
istic bread or wafer, half an inch thick and two inches in diameter; round, leavened cakes

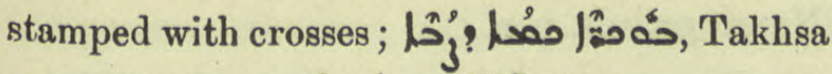

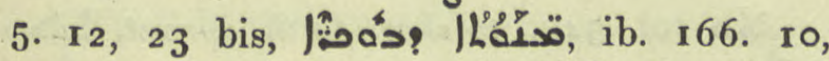

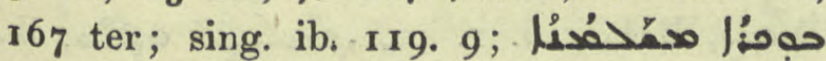
an entire loaf, ib. 168. I 2.

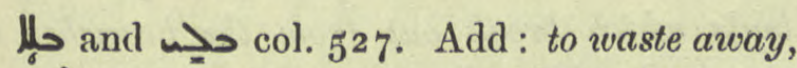

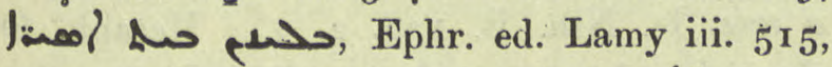

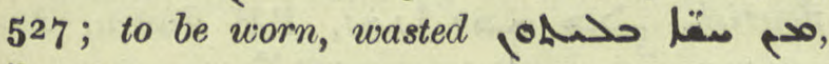
ib. 383 .

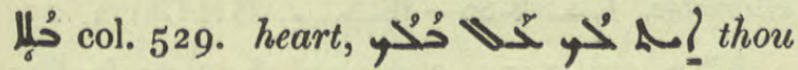
art concerned about, A.M.B. v. 111 . I var.

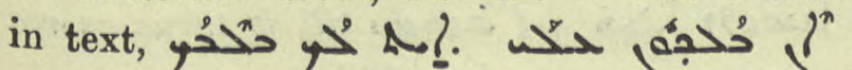
if you are well-inclined to me, ib. 117,7 ;

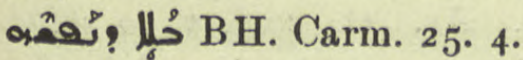

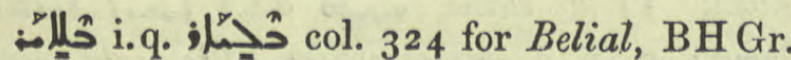
i. 25.16 ; id. in 2 Cor. vi. 15 ; سجة

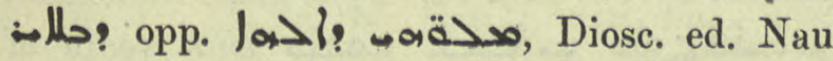
52. 9 ; حلار Georg. Ar. Carm. 30. 738.

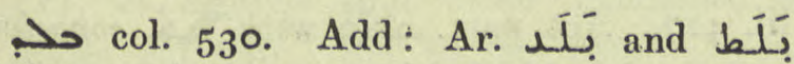
Balad, now Eskî-Mosul 40 miles north of Mosul, Syn. Or. 667; 619, 621 ; 62 bis, Pers. Mart. 97. 217 ; Patr. Or. iii. i. 19. 4 ; Chast. 26. 4 ; Dion. 146.17 .

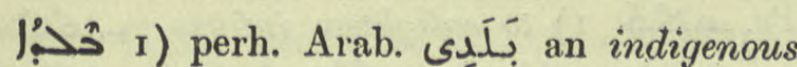
plant; see Dozy 109 and Ar. Pfln N. 28 r, n.; Med. 468. 3. 2) prob. corrupt. Brooks, قحب| أزما long planks (?) to make an engine of war, Chron. Min. 73. 28.

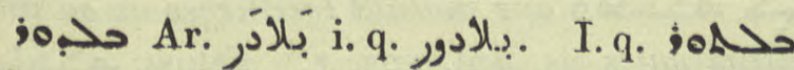
col. 54I and Ar. Pfln N. 72. Semecarpus anacordium, Med, 239. 12, 306. 12, 19,

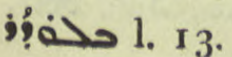

خ scol. 53 I. to astound, terrify. With acc. with terrifying news, Jul. 13. 9. To hasten,

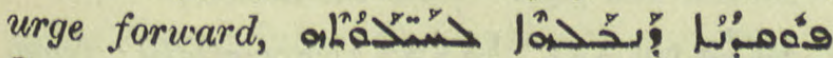
Jovinian was commanded to bring up his forces speedily, Jul. 162. 3, I66. 26.

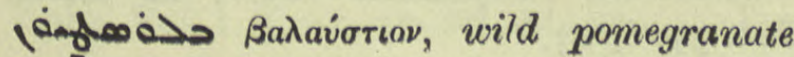
blossom, Med. 159. 9, I62. I, 2 I 1. I, 4, 212 ter; مله Eloyptian pomegranate, Chimie 7. 9.
55

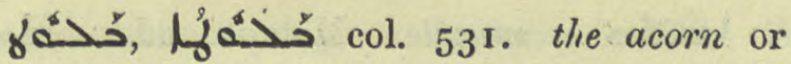
chestnut? N. Hist. vii. 4. 4, passage quoted below under مدرُهُ teucrium chamaedrys, Med. 372. I, 602. 9.

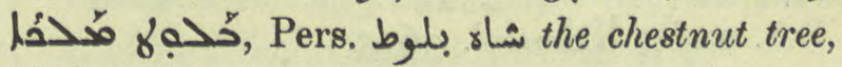
Med. 578. 17, 602. 10.

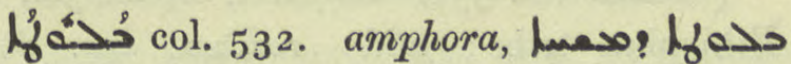
Hist. Mon. i. 353. 17, an acorn-shaped vessel

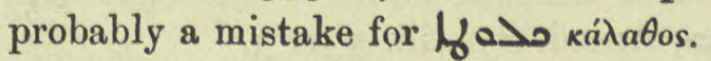

حكل col. 533. Add: 3) to spring up,

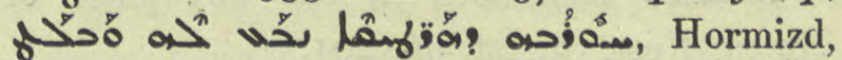
1922 ; ef. $9>$. Ethpe.

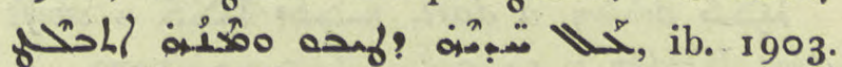
Pa. כ Hoffm. suggests that this may be

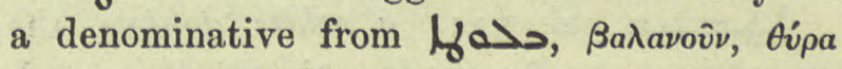
$\beta a \lambda a v \omega r o ́ s$, vereicheln, to sport your oak, tr. to bolt, ZA. ii. 57 ; لحخد id oo? they forced the bolt home, Vit. Monoph.

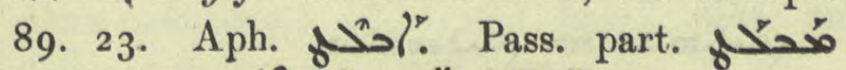

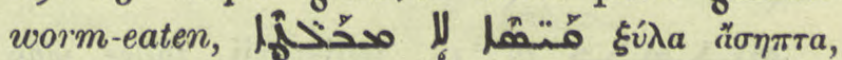
Hex. Ex. xxv. 5. Iо; A.M.B. v. 605. I6. To spring up, give rise to, of Hormizd, 189o. L. 6 of par. to have protrud-

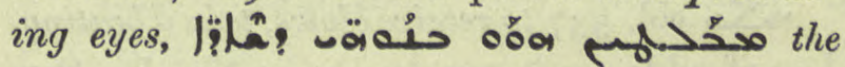
little devils' eyes started out of their heads, S. Dan. 61 $a$. 18.

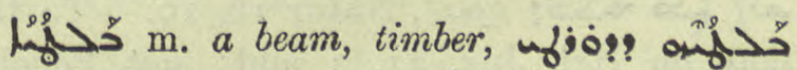
the wood of my spear, Gest. Alex. 42. 5 ;

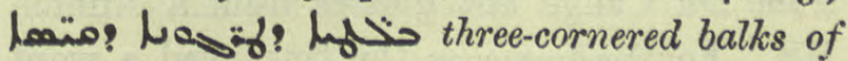
timber, Anecd. Syr. iii. 257.4.

o correct I chartularius, Keeper of the archives, Anecd. Syr. iii. 233. 5 .

حُخس col. 535 and 532. the city Balkh, Kal-w-Dim. I61 ter, 162. 9.

. Mr. 602. 12, but perh. .بليدلق Terminalia bellerica, ib. 138, 6, зо6. i I ult., $3^{61}$ pen., 376.5 .

ححسגis name of a village, C.B.M. 22 I $a$. حُ r) a man of Balkh, Kal-w-Dim. 162. 2. 2) Pl. 1 كَخ Scythians and Serbs, Wellhausen suggests Ba入 Хoi, Wallachians, Caus. Caus. 96. 17.

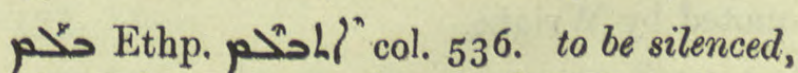

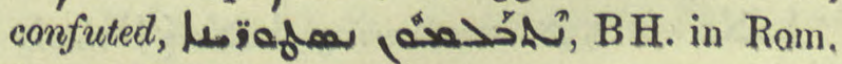
ix. 5 ; Georg. Ar. 3 I. 1 I. 


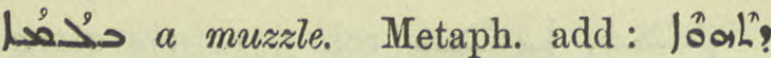

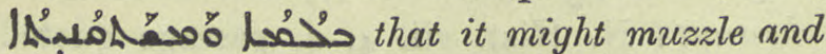
silence opponents, Hist. Mon. i. 56. I5; Jab.

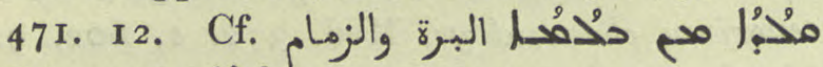
BA. under مُحْج? col. $3^{625}$.

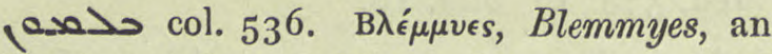
Ethiopian tribe; add: هحقهם, Jos. Styl. 17. 5 and n. to transl., WZKM. ix. 97; ح>l, Anecd. Syr. iii. 330. 3.

م mentioned as the birthplace of Hosea, Nest. Chrest. 96. 20 r.

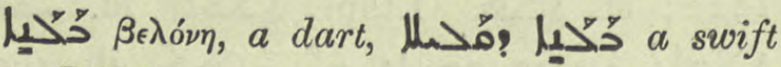
dart, BHGr. i. I4. 22. Cf. Mas above.

M. s. col. 537. a white donkey, the angel was seen by the حُجْهِ (N. B. points), not by Balaam, G. Busâmé 14. I2; 'L خص

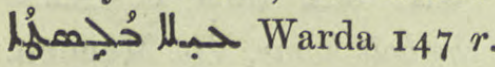

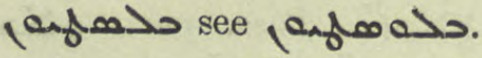

م $\mathrm{m}$. pl. balistarii, those who discharge the ballista, Chron. Min. 72. 27 ; ZDMG. xxix: 92. 13, 97. n.

حخح 537 to receive blows, take a beating.

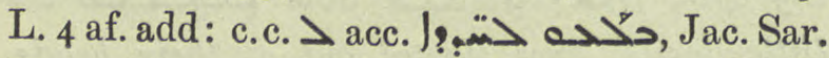

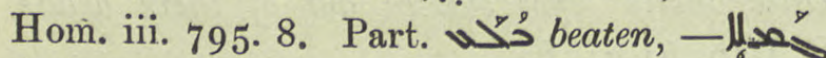

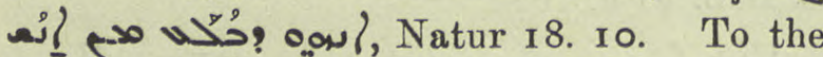
note on Rel. Jur. 3I. 2 add: not Scholia from $\mathrm{Du}$ Cange but glosses from a Munich

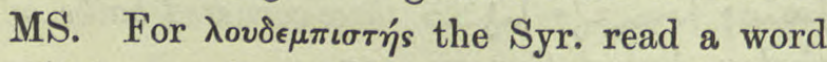

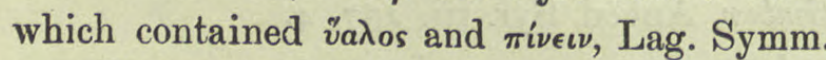
95. Part. Hex. Ex. v. 16. Metaph. absorbed in, entirely devoted to, Jhopas on on jong, Warda

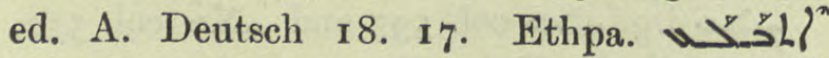
astron. to be obscured, occulted, U W ।

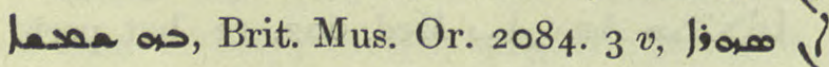

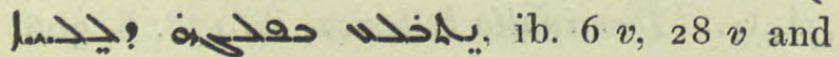
often.

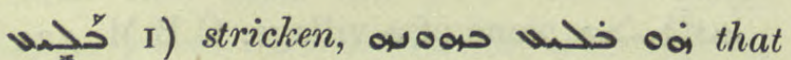
lunatic, Sev. Lett. 240. I6. 2) swallowed up,

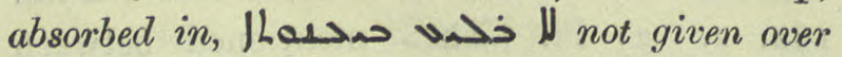
to gluttony, Kal-w-Dim. 47. 4, خلبدمبه, ib. 306. 9 ; جلحبا, Bar Ali ed. Hoffm. 2464 quoted by Wright.

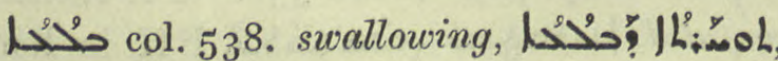
Med. 270.8.

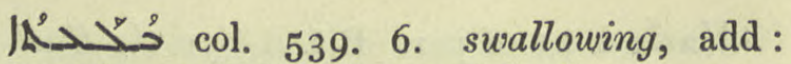

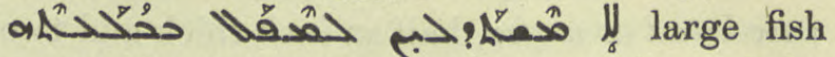
cannot be enticed into the gorge of a whale, Natur 55. ult. pen.

حل 5 col. 540. to shoot forth, sprout, ת

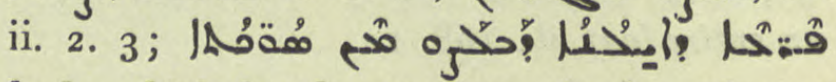
buds which break out from the branches of a tree, Is. Nin. B. I 32. 2. 3. Metaph. to

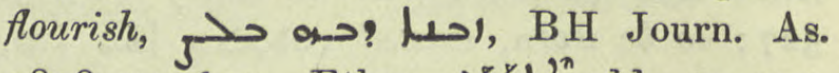

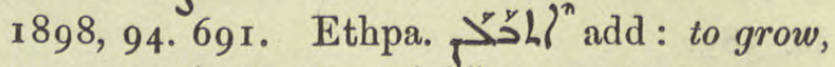

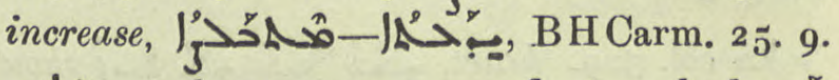
حـ

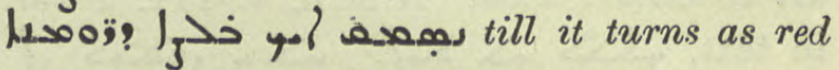
as a pomegranate shoot, Chimie roo. 4.

بلق conj. v. to shine, flash. 1) to shine, u jaor a corner where little light came, Anecd. Syr. ii. I 19. 18. 2) to come unawares, with years of famine great damage befel, Ephr. ed. Lamy

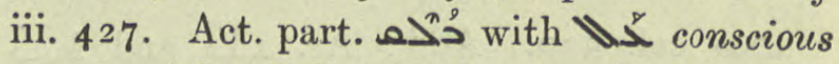

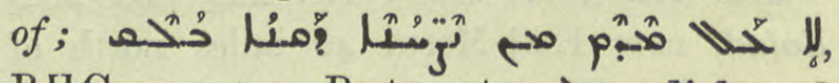
B HCarm. 25. 5. Past. part. حلم enlightened, . 108. 4 ; with th conscious of, ib. 92. 2.

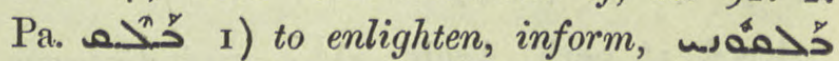

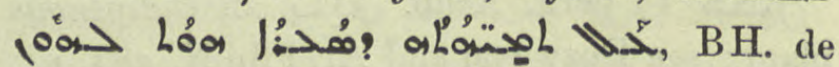

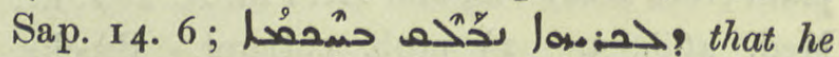
may instruct the sick with wisdom, Warda

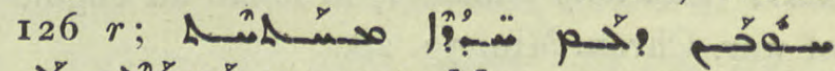
خ illuminates us, Jul. 105. 5. Ethpa. محَّhin to desire, be eager for, Ephr. Ref. ii. 57. 25 ;

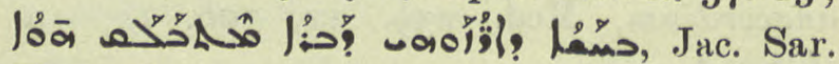
Hom. iii. $212.4,225.7$.

مَٔ col. 54 I. dele occursus, Shining,

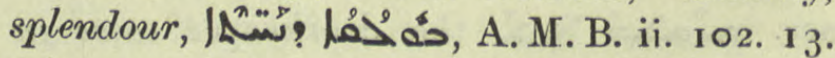

col. 54 I. Dele the article.

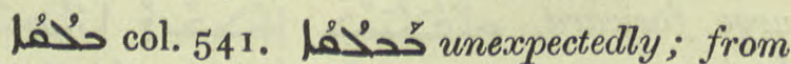
an accident, $\mathrm{BHChr}$. 18 . 19.

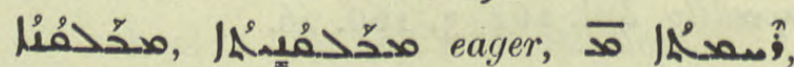
Jul. $77 \cdot 6,27$.

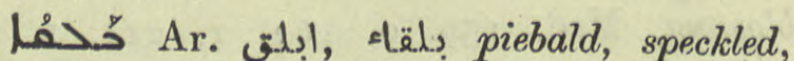

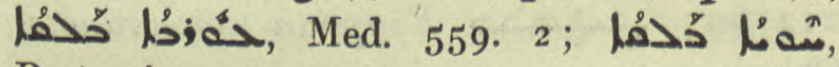
Protection 23. I 2, 88. I I. 
anals

כै pr.n.f. Balkis, Queen of Sheba, $\mathrm{BH}$. in Luc. xi. $3 \mathrm{I}$.

حل col. 54 I. Dele absorptus est. To try, experience, endure. sense to be smitten, afflicted.

حض col. 54 I. I) Balash, brother of Peroz and uncle to Kawad, Jos. Styl. I 5. 14, 16. 3 , Tabari I33, f., Pers. Mart. 46, \&c. 2) Balis, a fortress on the Euphrates in the province of Aleppo, add: Dion. 26. 19, 22, Jo. Tell. 48. 3.

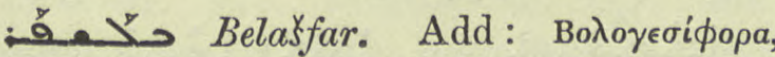
a province and Arsacid city near Holwan, Pers. Mart. n. 592, Tabari ז 34 , Phet. 42. 2, A. M.B. ii. $559.3,565.9$, Syn. Or. 37,44 \&c., ZDMG. xliii. 396 . $\mathrm{r}$ ff, and often.

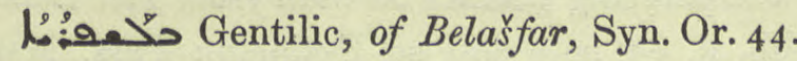
24 , ZDMG. xliii. 396 ult.

col. 54r. Pointed

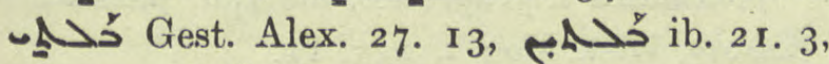
26. 7 ; حخدي Is. Ant. i. 2 I0. 99. Bîn $\lambda \theta i s$, consort of Baal, title of Astarte, ZDMG. xxxi. 727, of Astarte and other goddesses see jas col. 326. Ref. to this title on monuments, Euting, loc. cit. Other refs., Pers. Mart. 128 ff., A.M. B. vi. 38 pen.; قمًّ ה BH. in 2 Cor. xii. Chem. copper. Bilati (Vénus) cuivre, Chimie Introd. xv.,

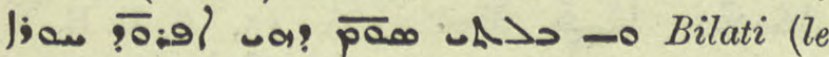
cuivre rouge) c'est-à-dire Vénus, le cuivre blanc, ib. 6. 7, 0:0:0 take Cyprian

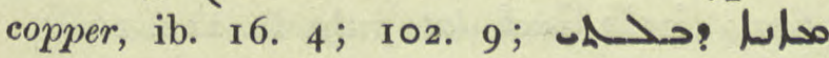
a copper vessel, ib. 5 I. 4 ; ب. بـ a flask of ointment, Hist. B.V.M. 35. 8.

מحم . For letter $n$ suffixed to proper names see ZA. xi. 17 on Hoffmann's Aramäische Inschriften.

$\leftrightarrow B a n$, one of the second Triad of Manichaean teaching, محد سمت: من: مبر the Friend of Light created Great Ban and Great Ban created the Living Spirit, Coupes ii. I 28. I, 2, I 29. I 8 , Cosmog. Manichéenne 20 n. 4; خ Ban, architect and builder of a grave for darkness, Ephr. Ref. i. 3. 32, 39. 20, 94. $4^{2}$.

حُ col. 542. Pa. حُ

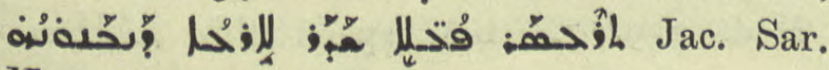
Hom. iii. 8 ro. 13.

\section{rdialeasdrs}

Liُn sin col. 543. a building. Add: the

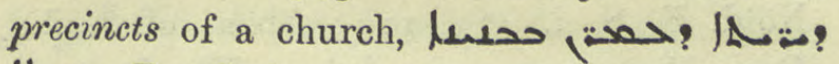
Ih: Bar Sud. 44. I3.

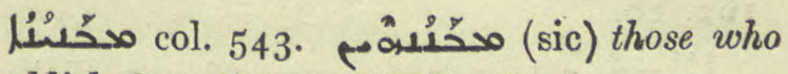
established traditions, $\mathrm{BHGr}$. ii. 2. 4 .

ح حدب0 pr.n.m. Bindoë, Sassanidi 8. I7.

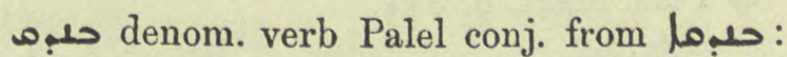

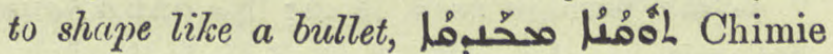
30. 3 .

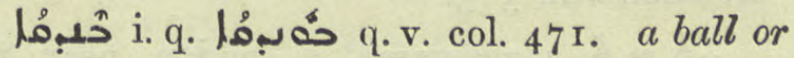

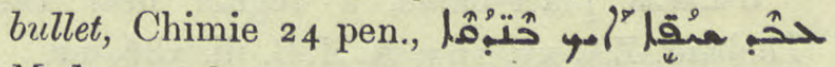
Med. 59. 18.

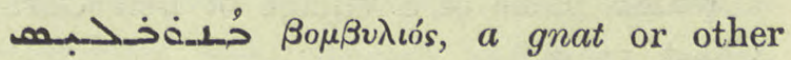
humming insect, Natur 42. ult.

خَّ i.q.

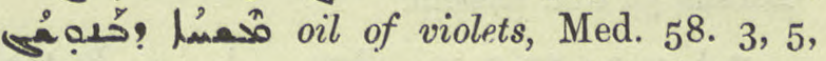
67.4 and often. Two kinds of this ointment

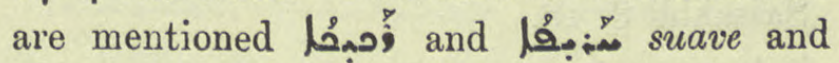

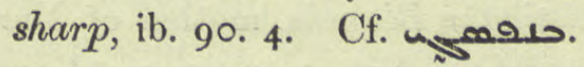

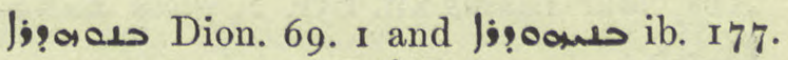

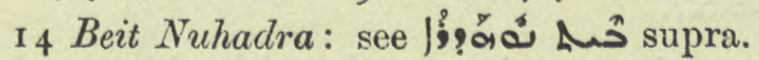

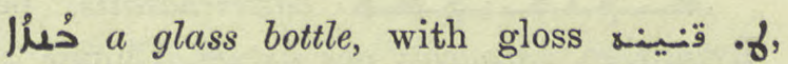
N. Hist. iii. 9.

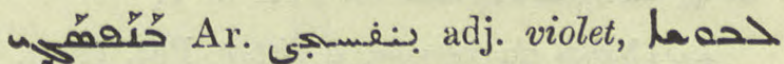
waglo כh Hist. B.V.M. I 5 o. 4 af.

ح col. 545. the keeper of a bath, add: BHGram. i. 18. 24 . Ref. A.M.B. vi. 91. I3.

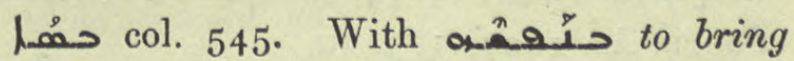
himself into contempt, act despicably, Pallad.

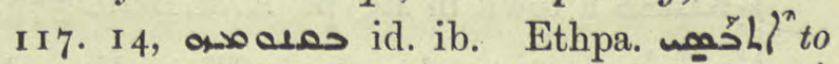
be despised, add: : $100 ; \infty$ ! the commands of your lord have been treated with contempt, Jul. 19. I7.

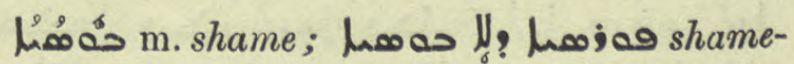
less exposure, Warda, ed. Deutsch. 9. I 7.

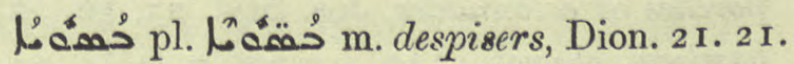
A حم col. 546. without due reverence; carelessly, negligently, add: Jul. $3^{6}$. 12, Pléroph. 35. 8.

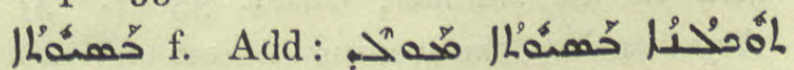
familiarity breeds contempt, Is. Nin. Bedj. 193. 14.

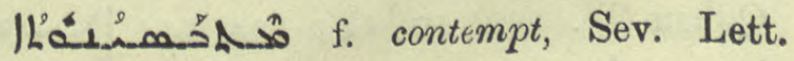
I 54.20. 
l⿳⺈ مُ pr.n.m. col. 546. Add: a presbyter, Nöld. F. S. i. 473. I19; Abbat of 1 Doc. Mon. 223. I3, صفئl ib. I62. 10.

حمبُْ

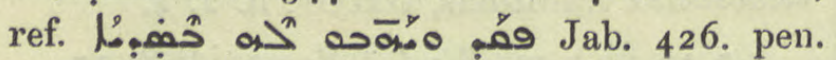
$=$ Pers. Mart. n. $84 \mathrm{I}$.

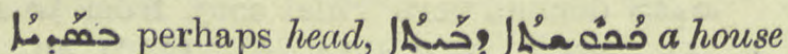

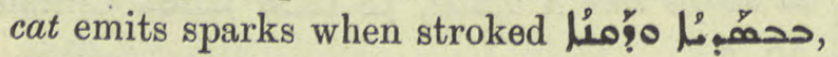
N. Hist. viii. $3 \cdot 3$.

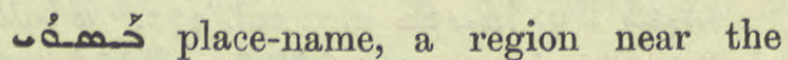
Thebaid, ZA. xv. 105, A.M.B. iii. 586. I I.

حame of a village of Beit Garmai, Chast. 53. 2.

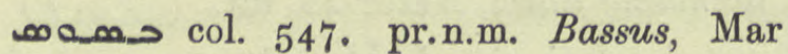
Bassus 59 and passim.

$\stackrel{\rightarrow}{\rightarrow}$ pr.n.m. Pers. Bistam, Sassanidi 8. 18.

دمastina = Blanche, mother of Mar Aba, Mar Bassus xii. Syr. 2, gloss A

م Bastrichanitis, perh. Byzantine, rasouchti aes ustum, Chimie $2 \mathrm{I}$ I.

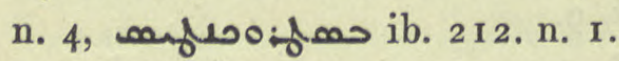

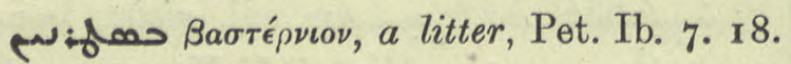

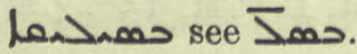

حمسمدا Bassima, founder of a monastery in Qardu, Chast. IO. I 4, גarem, Journ. As. 1907, I63. 10.

حمبعحبُ

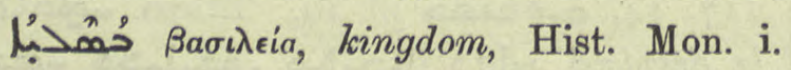
139. 16, ii. 287 n., Lexx. col. 547 ult.

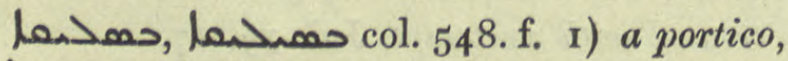

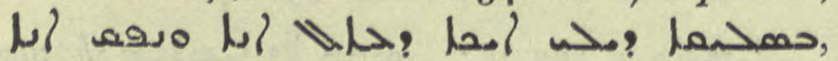

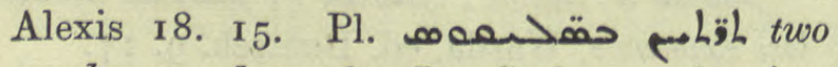
porches or colonnades, Jos. Styl. 25. 16. 2) a basilica, church, :

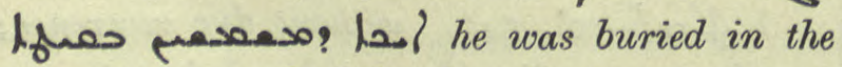
inner courtyard of the church where service is held in the summer, Chast. 35. 7. 3) the Royal anthem, the evening anthem on Sundays, Festivals, and Memorials is so-named, Maclean, Daily Offices 78. note; Takhsa 78. 14, 21, Qdham W. 123. 10, 14; 124. 14;

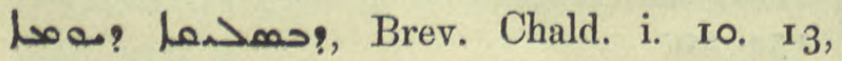

96. $8,3^{2} 3$ ult., 54. I, 65.5 af., 323. 5 af.;

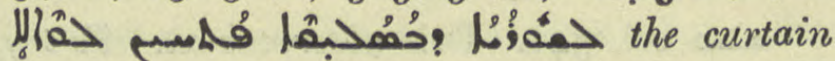
is drawn back when they arrive at the preface to the Royal anthem, ib. 325 ; Or. Xt. ii. 2 I 5.

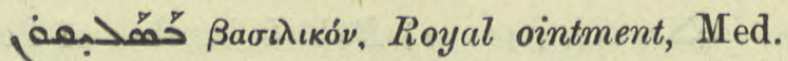
89. 10, $16 ; 25^{2} .7$. The hazel nut, ib. 103.3.

حصم col. 549. 3) to burn incense, cense,

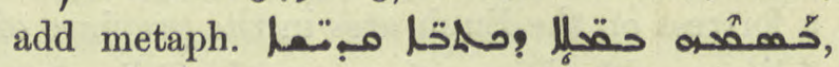
Patr. Or. iii. i. 34. 10.

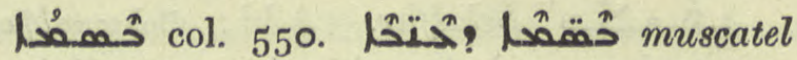
grapes, Med. 58. 8.

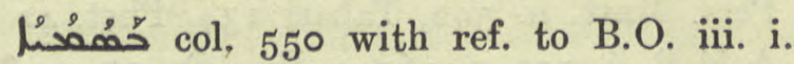
$472=$ Hist. Mon. i. 49. I2. Budge suggests

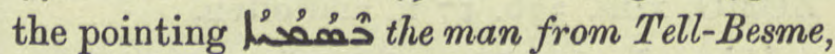
ib. ii. 85 note and so Hoffmann Pers. Mart. II 5 .

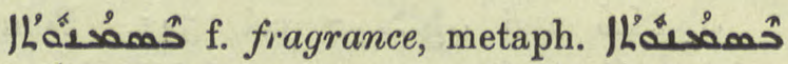
I:200\%?, Hist. Mon. i. 235. Ir.

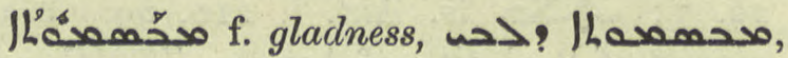
Kal-w-Dim. 336. 10.

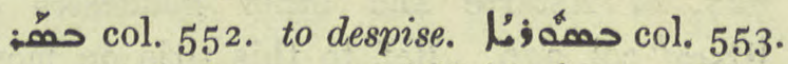

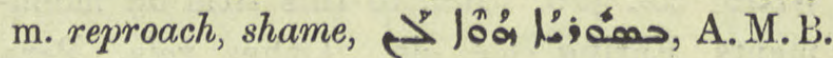
v. 434.13 .

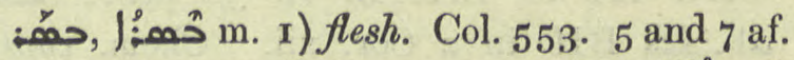
Pl. muscles or fleshy parts,

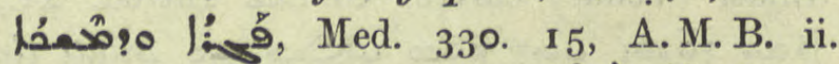
595. 9. 2) pulp, pith add: $\int_{\tilde{J}}^{n}$ قै? galls, Med. 6r. 16 ; حُمْئ asparagus, ib.

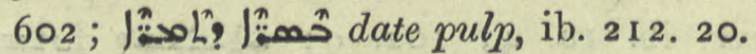

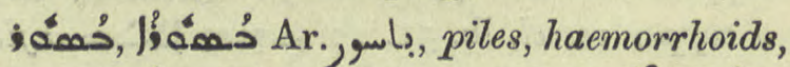

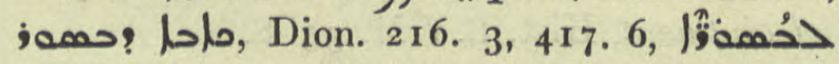

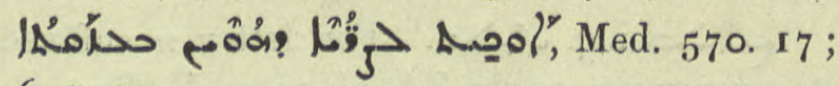
600. 23 .

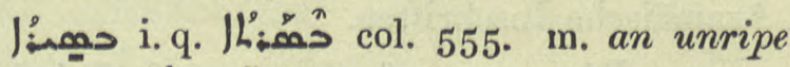
grape, pl. حَّ Med. 558. 2, 566.8.

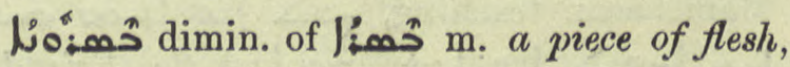
C. B.M. 593 a, Kal-w-Dim. 308.5 .

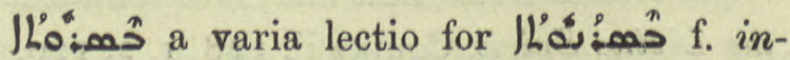

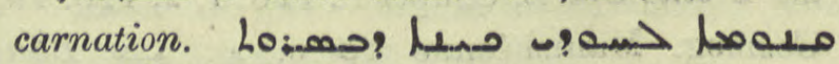
Lloa >l, Loof's Nest. 369. 7.

Lُ dropsy affecting the whole body is so called, Med. 339. 7. BA. spells this 1' same col. 


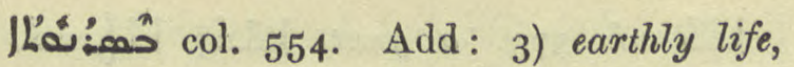

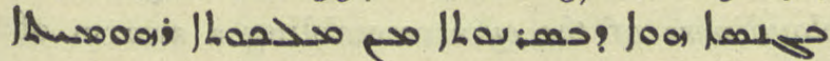
as for earthly descent, he was of Roman Imperial race, ZDMG. xlvi. 757. I9.

10ْمَ col. 555. an earthenware or glass vessel, Chimie 4ז. 4.

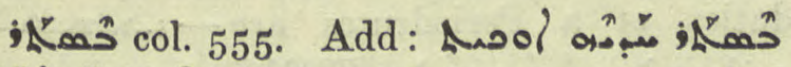
هُ blade, But. Sap. Econ. iii. 3 infra.

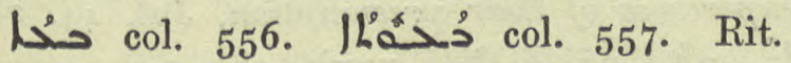
Rogations are of three kinds i.e. in three metres, that of Ephrem, of James of Sarug, and pentesyllabic, Brev. Ant. i. $57 a$ tit. and ter. twenty days before the Great Fast, B. Nin. I tit., 3 tit., 5 tit.; Brev. Ant. i. $3^{8 .}$ I, 5 ; Qdham W. 181 tit.; ellipt. ib. 182.6; Takhsa ${ }_{5} 6$ infra, $1_{57}$. 6. East Syrians also have

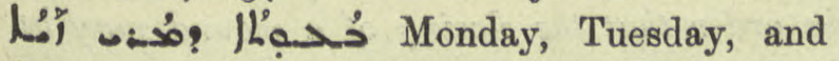
Wednesday of the week before Christmas week-this fast is almost obsolete - and the

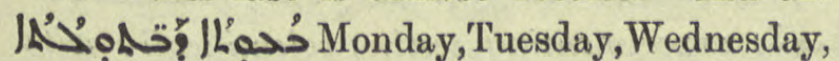
of the second week after Epiphany (observed locally by girls), Maclean.

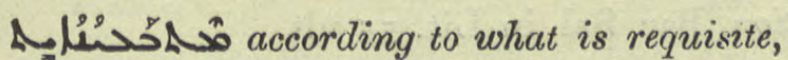
Syn. Or. 149. 20; G. Busâmé 42. I5; with a But. Sap. Periherm. i. 2.

مُحْ from Ar. col. 878 . r, 5 .

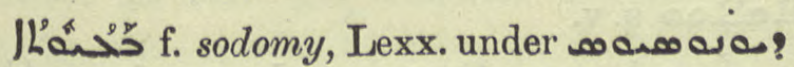
col. 878 .

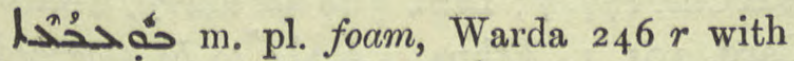
gloss

حُحتفهـُ f. pl. col. 559. bubbles as on boiling broth, Med. I3. I4, I6, Brit. Mus. Or. I593. II $v$.

مخحخبُأمي . col. 559 afar, remotely, Sev. Lett. I53. 6.

Laص (ه) col. 559. Add: Rabban Baith, founder of a monastery in Beit Nuhadra, Chast. 54. 4, I 7 ; Hist. Mon. i. 237 ult.

حخّن

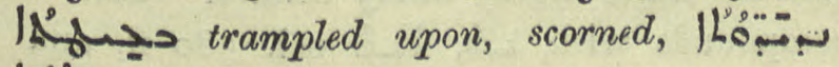

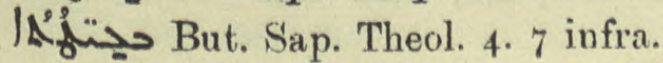

| blows with the fist, fisticuffs, Sev. Ant. Hom. I5. 8.

1มำ ح. a kick, Syr. Rom. Rechtsb. 60.

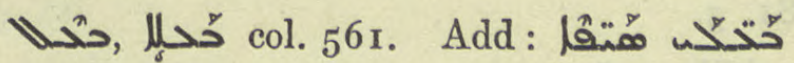
swordsmen, Protection 9r. I 2.

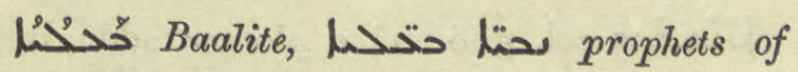
Baal, Ephr. Ref. ii. 55. 5.

סحדد name of a female demon, Charms 83,8 af., JAOS. xv. 289 . I 2.

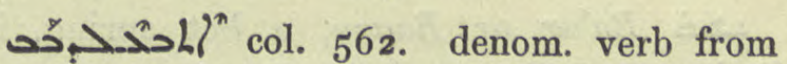
حارli to be an enemy, to oppose,

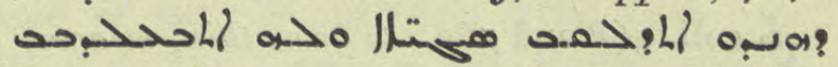
John withstood many in India and they became his enemies, Išodnah, ZDMG. xlvi. 758. 12.

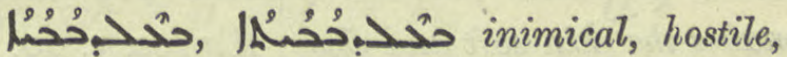

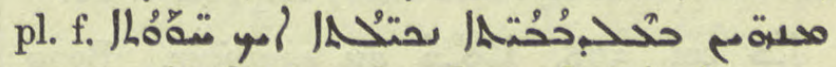
some animals are hostile and injurious as snakes, opp. مسَححُتحُما friendly, N. Hist. vii. I. $\mathrm{I}$.

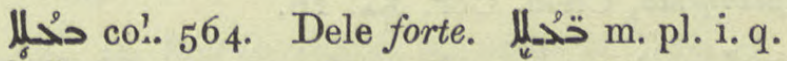
حَخَّه late fruits which do not fill out and ripen, Rylands 44 , fel. 4 a.

حُحهم col. 559 should be transferred

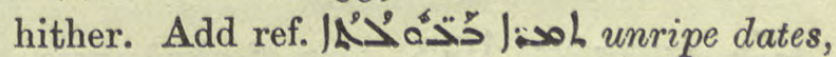
Anecd. Syr. iii. I 77 . 12.

col. 564. i.q. 536 and Suppl., Blemmyes, Pallad. 326. 12, f. 8.

col. 564. Baaltan, a village in the district of Emesa, BHChr. Eccl. 319. 23, Jac. Edes. Chron. Can. 577. 14.

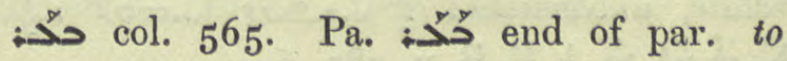
pillage, devastate with

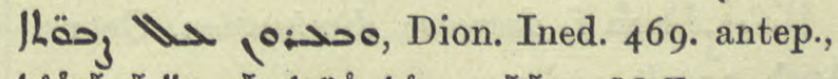

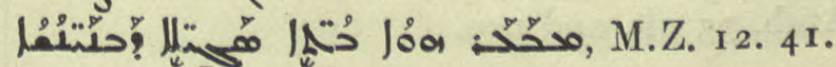
Ethpalal to a wild state as trees, Pléroph. 32 . I I.

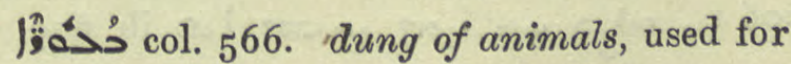

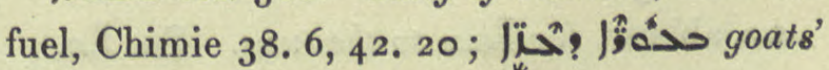
droppings, ib. 24.8 .

ححهة J

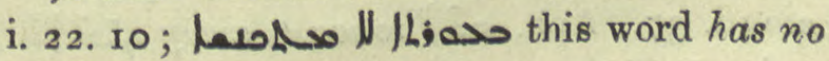
plural, ib. 34. 7 . 


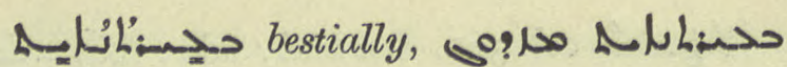
Sassanidi 12. 22.

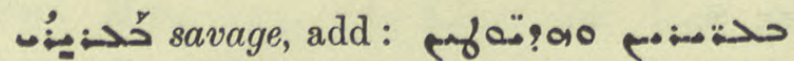
ignorant and like beasts of the field, S. Maris 8I. 5 .

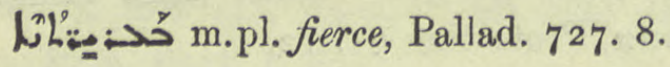

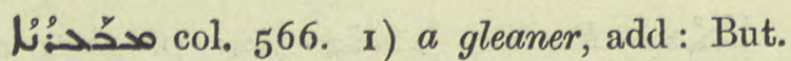
Sap. Eth. iv. 2 infr. 2) a despoiler,

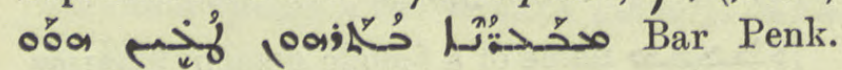
I6 I. 8 .

ذ ذ $^{\circ} a^{i} a r$ or Baaru, a hot spring, in Arabia, P. E. F. ${ }_{905}, 224$, Pet. Ib. 85.2.

$5_{5}^{5}$ coll. 568 and 569 . to grow thin, meagre,

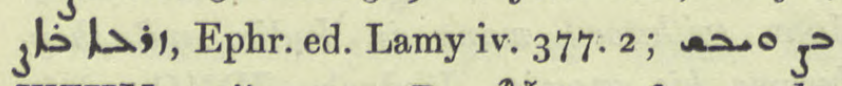
WZKM. xvii. 199. Pa. $25^{n y}$ to refine, render clear, r35 makes the skin soft and clear, Hippoc. v. 20.

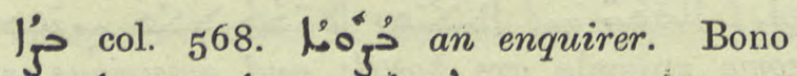

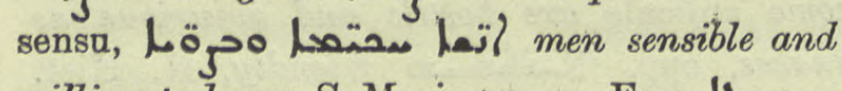
willing to learn, S. Maris 79. 4. Fem. I $\mathrm{Ag}_{\mathrm{g}}$

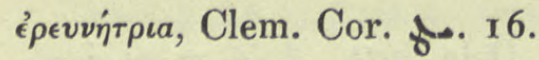

is col. 569 .

ILiog 5 f. coverlet, rug, BA. under lenas, BB. under IA Ran as col. 693.

$\sim \widetilde{J}$ imperfect, lad Hex. Ex. iv. Io.

Aي 2 col. 572. less opp. I Georg. Arab. 16. 6 ; in some measure, more or less, Josephus vi. 8. I3.

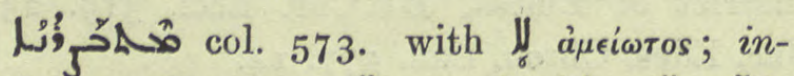

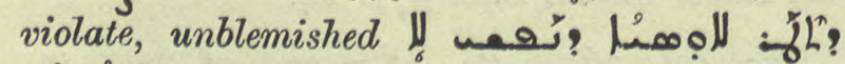

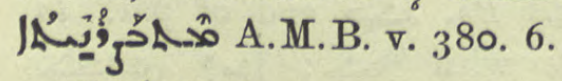

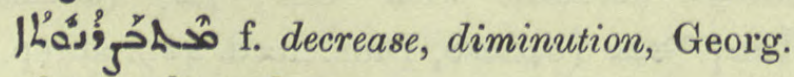
Arab. 35 ult. and pen.

a's i.q. J's col. 573. Basra, modern name of Prat' d' Maišan, Syn. Or. 601. 9,

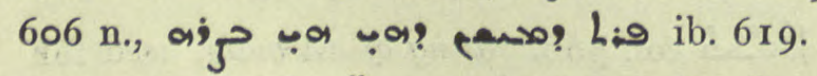

مص abbrev. for

خ̌ col. 573. to be gnawed, worm-eaten. Ref. Lamy i. 37. Aph. Ẩ to waste away

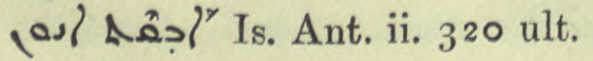

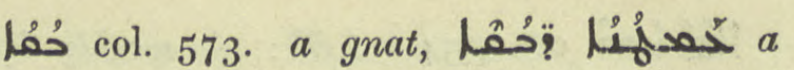
cloudiness of the eyes resembling a swarm of gnats, Med. 75. 15, 78. 22.

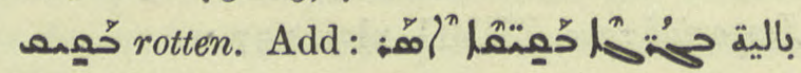
BH. de Sap. 37.

مُ col. 573. to examine into. Add: the daties of a Periodeutes are - $>$ > ZDMG. xxxvi. 349. Past. part. oahesi- or we have full experience of their presumption, Jul. 44. 28.

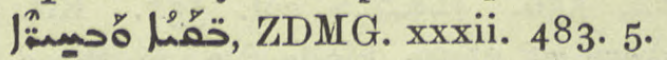

مُ

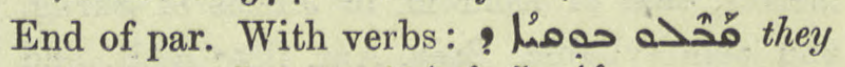

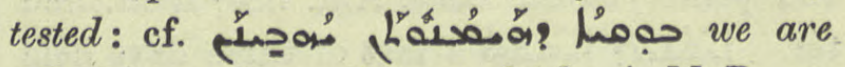
offering a test of our faith, A. M. B. v. 102. 10.

مصمعه Ined. Syr. 92. 2 I correct

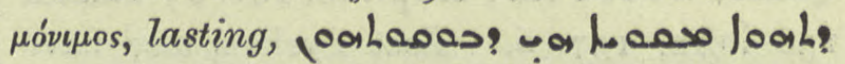
in order that the closing of a wound may be lasting ....

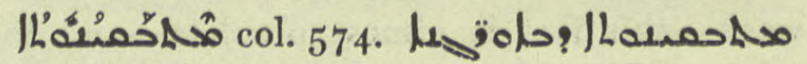
شexperimenting, testing scientifically with brass instruments, Georg. Arab. 18. 19; ILA الا ميلحمس want of exact examination, Bar. Sal. in Lit. 55 ult.

ן pectorale, a pectoral, Anecd. Syr. ii. $268 . \mathrm{I} 3$.

حمخيم Chimie 52. 22. Corr. No q.v. Jast. Alex. 89. 10 and n. Corr. Jheخ q.v.

حمّ col. 575. Pa. to search, examine, add: BHChr. 177. 7 ; 㪿 do not worry, Ebed J. Card. ror. I. Ethpa. :حُhiْ to be searched, BHChr. I77. 5 .

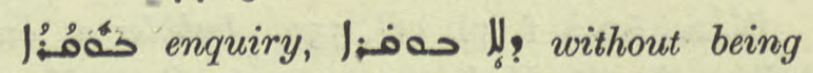
called in question, Jac. Edes. ZDMG. xxxii. 482. 12.

دهتم in shoals

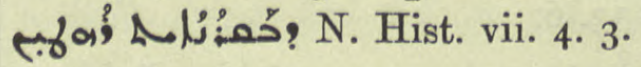

-

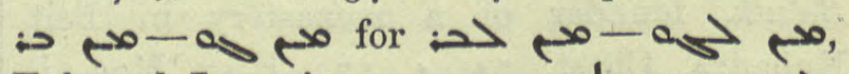

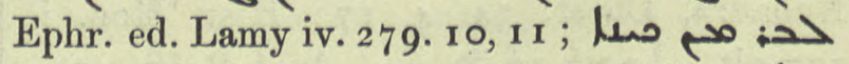
extraordinary, Natur 7. 4. L. 9 af. his N $>$ L

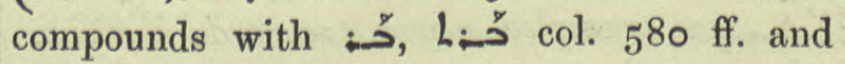
Suppl. 
Pael. خُ denom. col. 576. infr. to be apart,

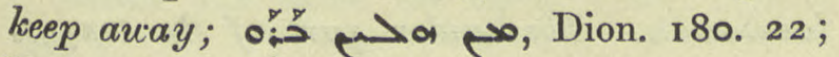
مמمع out, absent, away

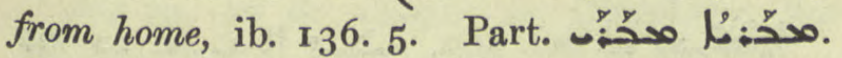

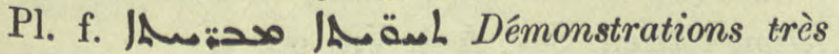
claires, Ant. Tagrit. ap. Nöld. F. S. i. 483 . Ethpa.

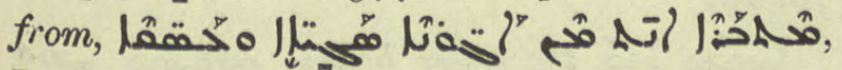
Is. Nin. B. 80. 2 .

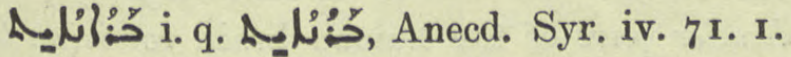
A col. 578. med. Dele "malim semper

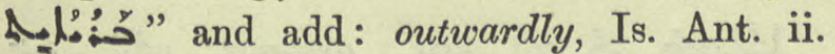
I6. I $_{3}$ Anecd. Syr. ii. 29. 9, N. Hist. iv. iii.;

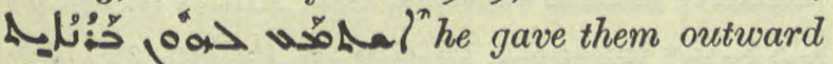
obedience, Jab. 6, 14.

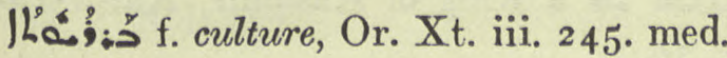

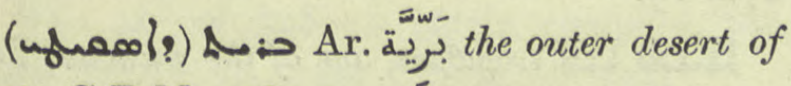
Scete, C.B.M. ro80 a.

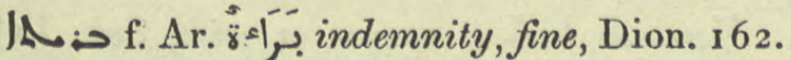

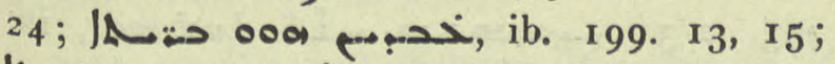

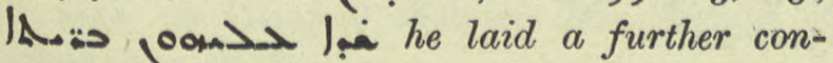
tribution upon them, ib. 232. 9, 2 I, 233. 23 .

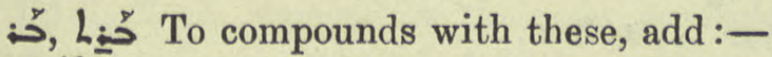

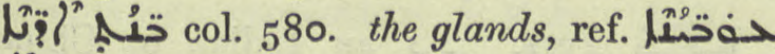

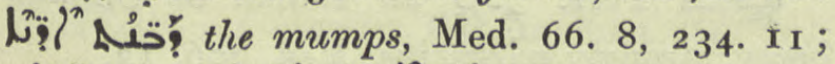

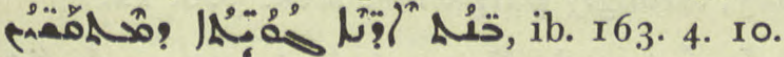

| corr. Ib. 25.2 .

9lojol Pers. Pـ a weasel, Kal-w-Dim. 259. I7, 262. 2, 264. 8 ; ! !bol ص ib. 313. 8.

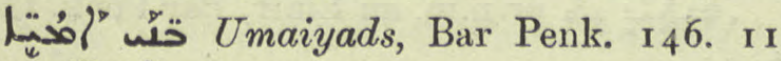

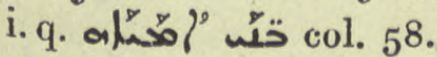

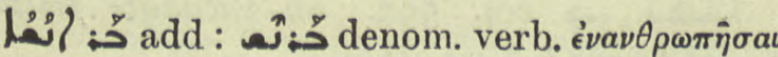

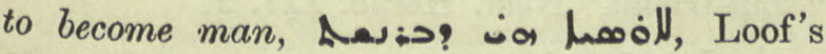

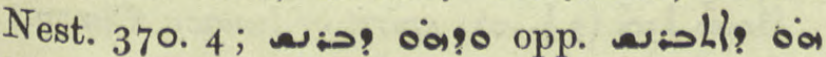
ll. 5 and 6 ; 59. 317 .

هةمُمَما

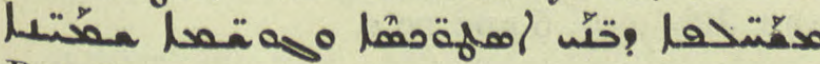
But. Sap. Theol. 4. 8.

معزمحسر earthworms, Lexx. under خُّ col. 3568 .

L $>4$ s perh. the constellation of the Dragon, B H Carm. 38. 11 ; Nöld. in Chwolson, ZDMG. xliv. 526 med.

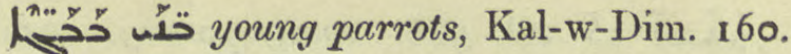
21,16 I. 19, I63. 3 .

每

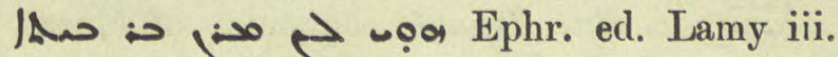
2I3. II.

col. $5^{8} 3$ infr. ref. a censer, Sev.

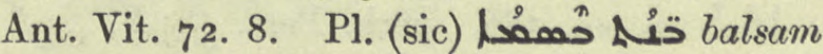
berries, Med. 50. 14, 5 I. I 2, 52. Io.

مَّْ col. 584. a fellow-creature, A.M.B.

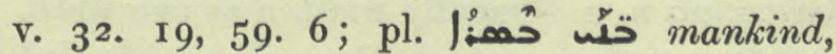
Philox. 9.

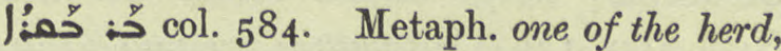
used of heretics, pl. Jab. 5 18. I4 $4=$ Pers. Mart. n. 965 ow on offm. says he found a $\triangle$ faintly written above the 0 .

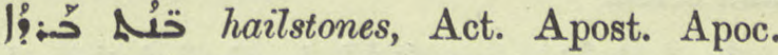
مصن. Io; Med. 220. 2 I, 24.

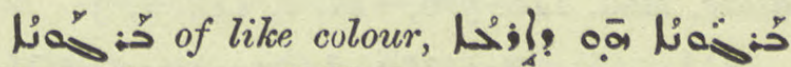
it is earth-coloured, Natur 32. I.

ILR $\rightarrow$ col. $5^{84}$. Dele par. It is Ihl or Lhays: see col. $3^{808}$.

น Bar Ganawai, surname of John of Tagrit, Bar Sal. in Lit. 3 I. note.

1ُ2 حُ col. 585. mustard, Med. 602. 14. | A.M.B. v. 136. 9. 1. phoeniceum, sap of the scarlet horned poppy,

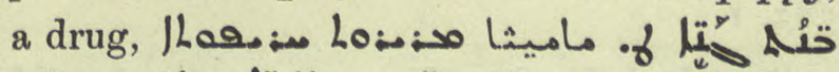
م. N. Hist. iii. ii. 2 .

those of his house, his fellows, Aphr. 692. 24 .

فo: col. $5^{8} 5$. pr.n.m. Iso. iii. ed. Duval 22. 13 .

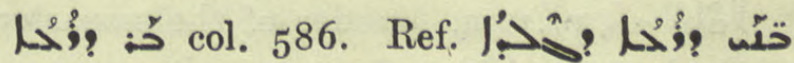
leathern arm-pieces, Gest. Alex. I 72. 5 .

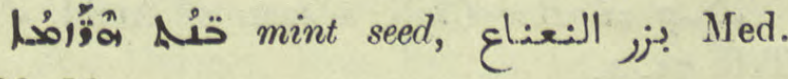
602. 13 .

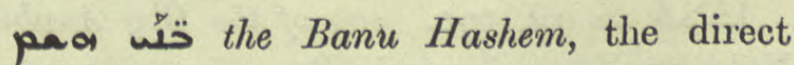
descendants of Mohammed, so named from

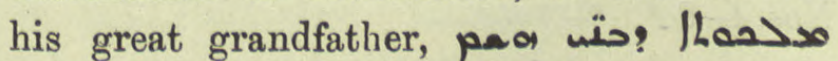
prob. the Abbaside Caliphate, Bahira 205. 13 but see trans. and note; Chast. 66. 6 . 
أحـ col. 586. contemporary. Add: temporal, pertaining to time, opp. eternal, of the B.V.M. Mar Bassus 33 .

مَّ Bar Zinayé, cognomen of Maran Ammeh, M.Z. 209. 10, 2 10. 7, 8; of Ishuyabh ib. 2 10. 20.

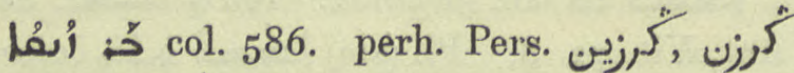
crown of the ancient Persian kings i. e. fillet, diadem h. bandage, wrappings, Nöld. Mand. Gram. 20 n. 2. Usually written as one word, BHGr. I. 23. ro, pl. ib. 34. 24, حَ: מa:" strips wound round the arms, Gest. Alex. I 29. I but puttees, leggings, greaves,

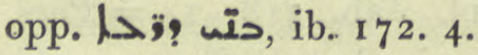

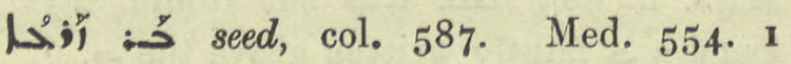
and often.

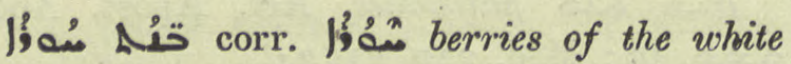
alder, Med. 140. 5/6, I4I. 2, 143. I, 3 .

arlan $\rightarrow$ Bar Hotiph, cognomen of Mar 'Abda the younger, Chast. 44. ro.

م م Bar Haziz, Chast. 48. 3.

مُش col. $5^{8} 7$. pr.n.m. of a bishop, ZDMG. xliii. 395. 4 .

مُحْ col. 587. fennel, Med. 554. 2, 555. 8, 575. 15, JAOS. xx. I88, WZKM. xii. 85 .

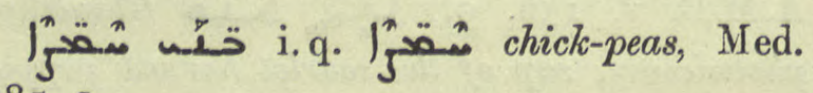
$5^{8} 5.1$.

1A. of $^{6}$ Lin col. 588. a sore on the choroid tunicle of the eye, Med. 77. 19; 12.7of : لl

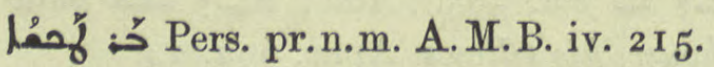

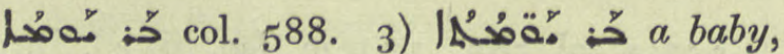
A.M.B. v. $625 \cdot 3$.

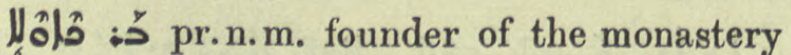
of Caphartuta, Chast. 8. 2 I, 20 ult.

حَ: Bar Chili, a man of Amid, Jo. Tell. 7 I. I.

مُح col. 590. son of the Dog Anubis, Nöld. ap. ZA. xxi. 157 in Pogn. Inscript. Sém. Nos. 6-8.

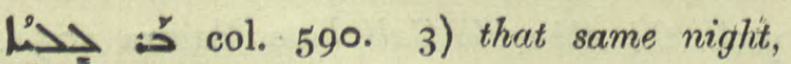
Kal-w-Dim. 137. 10.

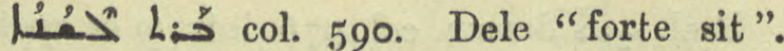

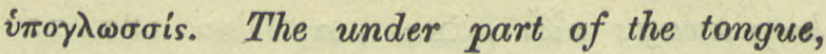
Med. 203. 17.

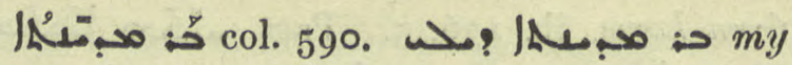
fellow-citizen, Sev. Ant. Vit. 90. 3 .

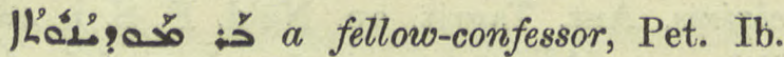
70. 14 .

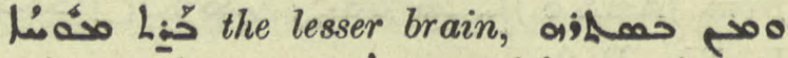

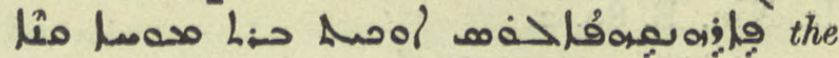
hinder part of the human brain contains the парєүкєфа入 is or cerebellum, N. Hist. vii. I. 3.

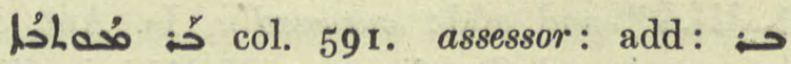

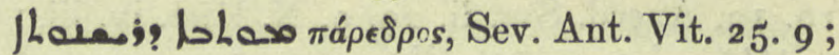
, Pallad. 201. 18.

م a King of Kashmir, Kal-w-Dim. 272. 19.

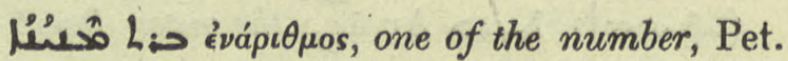
Ib. II 5.20.

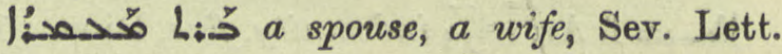

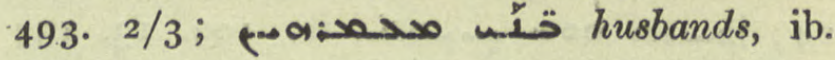
510. 22.

col. 59r. colocynth seeds, Med. 54. II, 198. 2.

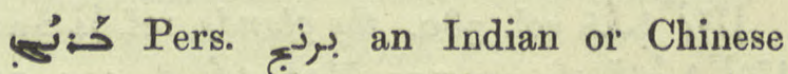
berry, strongly purgative, Med. 306. 12.

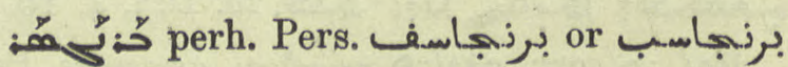
Artemisia, mugwort, Med. 31 I. 17.

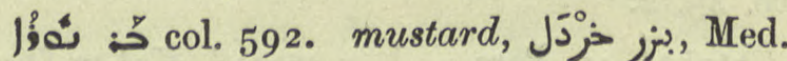
602. 12.

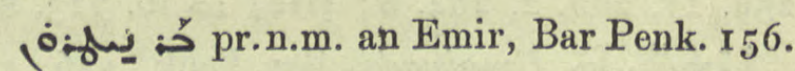
6 , II.

col. 592. an intimate friend, "soul-friend," Nest. Hérac. 44 I. 4.

|' spirit of lunacy, Charms 9. I5, 90. 6 af., 9I. I; JAOS xv. $29^{2}$ pen.

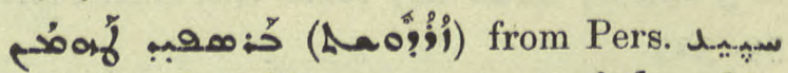
Zaradusht, the white man $=$ son of the white race, Jab. 240. 6 and note.

حمد my compatriot, Sev. Ant. Vit. 88. 3 .

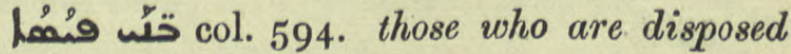
to believe, Aphr. 1045. 4.

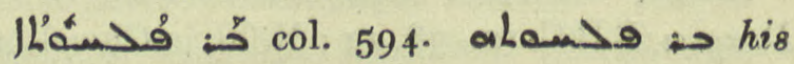
fellow-minister, Pet. Ib. 33. 20. 
ت1

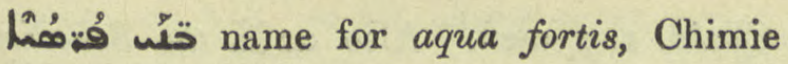
35. 3 .

ات (Stephen) Bar Sudaili the Syrian mystic, Bar Sud. I0. I and $\mathrm{n}$.

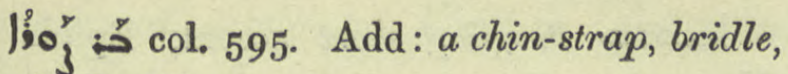
الزيق El. Nis. m. $3^{6.24}$.

surname of Mar Elisha who succeeded Narses as Head of the School of Nisibis, Barḥad 387. 4, Narses ed. Ming. 35. 18.

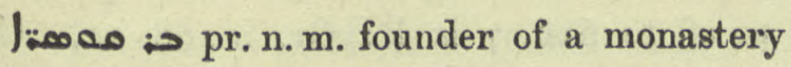
in Mosul, Chast. $3^{2 \cdot} 3$.

Aar Sabta, a follower of Apollinaris, Nöld. F. S. i. 477.

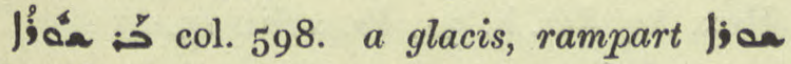
IN Jian iso, Nest. Chrest. 92. 133.

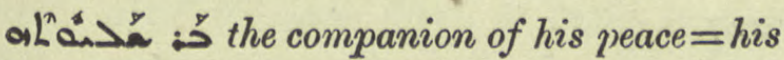
fellow monk, Pet. Ib. 45. 7 .

Habiba Bar Shenaye of Bethkoka, M.Z. 2 I 5. 3.

$1 L^{\prime} a^{*}\left\langle L^{n}: \nu^{2}\right.$ engaged in the same profitable undertaking $=$ martyrdom, A.M.B. v. 349 .

ILóL'ó: f. sonship, BH. in Rom. viii. 19.

S col. 600. to create.

Á  69. 7 .

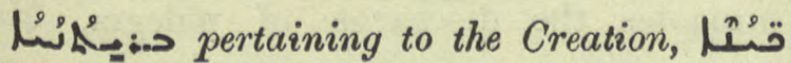
حتئش created things, Op. Nest. I 23 ult. in Gen. I. 2.

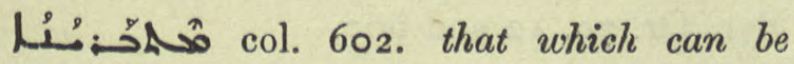

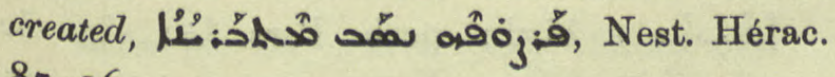
87. 16.

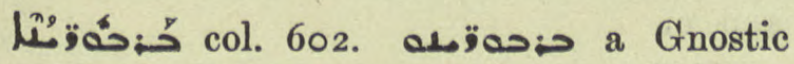
sect, Coupes ii. 115 ; the followers of Simon Magus, Theod. Mops. in Jo. 327 ult.

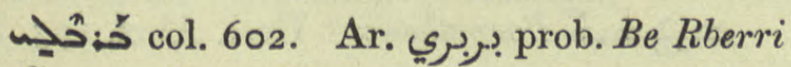
in Gawar, Pers. Mart. 222 n., Badger Nest. i. 397 .

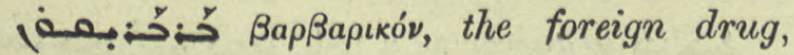
a purgative, Med. $3^{6} 5 .{ }_{3}$.

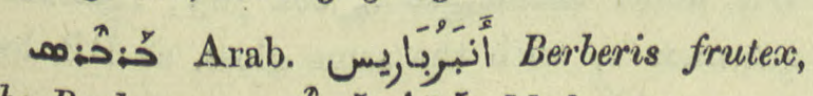

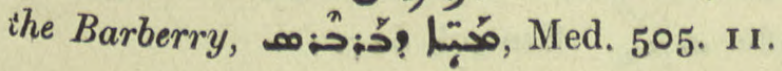

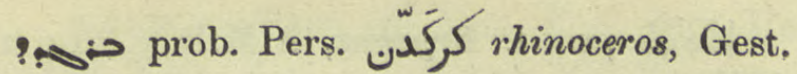
Alex. 2 II. I5.

Lay col. 603. a currycomb.

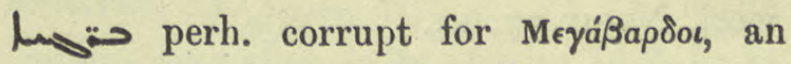
Ethiopian tribe, Anecd. Syr. iii. 330. 3.

حُ a drug, Med. 468. 2.

Dop: a monk of Mt. Izla, Iso. iii. ed. Duval 22. I3.

(c)?: the plank or wheel of a threshing instrument, lisis ? Nest. I I 2. I5 on Amos ii. I3.

لِّم: حَ a medicinal plant, Med. 571. 7.

هi col. 604. Ar. Armenian Partav, Barda'ah North of the Araxes, Syn. Or. 620 and note.

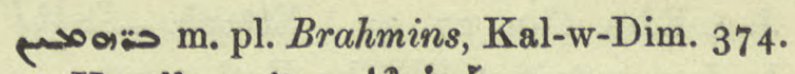

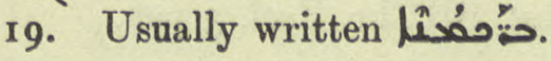

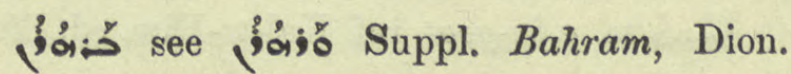
Ined. $65 \cdot 7$, Warda $166 v$.

ل or sister of Seroi, son of Chosrau Parvez, Sassanidi and Nöld. trans. $3^{2} \mathrm{nn} .4$ and 5 .

i\}o: $\rightarrow$ Berwar, a district of Kurdistan. Title of the Hymns of Sabrishu, J. As. 1907, $3^{89}$.

M 0 o: a village South of the Great Zab, S. Maris 3 r. 8.

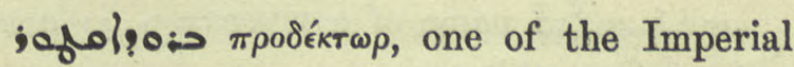
Life Guards, Mart. Luc. ed. Nau 162. $2=$ R. O.C. iii. 162. 2.

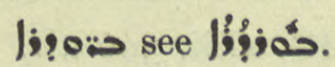

ano: $\rightarrow$ the Bruchium, a quarter of ancient Alexandria, Epiph. I8. I.

po: $\rightarrow$ Barom, a village in Beit Garmai, i.q. حـA A. M. B. ii. 679. $5=$ = Pers. Mart. 78 n. 7 Io.

0:000 000:Barōm Ḥusrawan, an estate in Beit Garmai, Mon. Syr. ii. 67. 5= Pers. Mart. 49 and $n$.

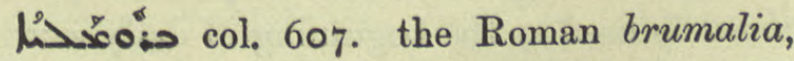
h. any feast day. ص: Sev. Ant. Hymns 35. 3, pl. 34 I $a, 34^{2} b$. 


\section{مesaiv}

64

Hơ

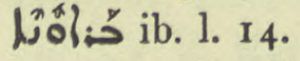

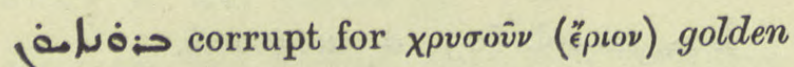
wool, Natur 57. 12.

100: Baruqa, a village and monastery, Chast. 45. I, II, 6I. 18.

hoo:s place-name, Doc. Mon. 2 I6. J 2.

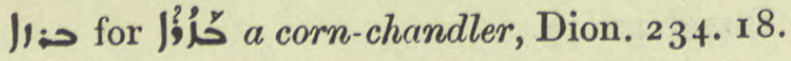

$N^{\prime}:$ or $ح$ name of a city, Kal-w-Dim. I48. 8 .

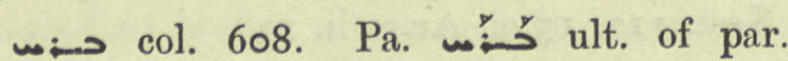

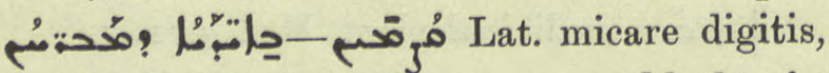
to hold some of the fingers up suddenly for another to guess how many; this was a game and also a way of settling doubtful matters, Jac. Edes. Can. 27. 20. Ettaph. w'shlil to be clear, manifest, BHChr. Eccl. 3 pen.

مُ 69. 7 .

perh. misspelt. a bishopric of the province of Adiabene, probably identical with

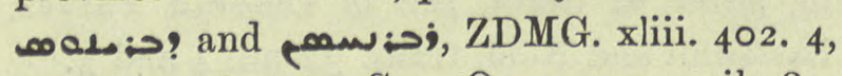
404. 5,406 . 13 $=$ Syn. Or. $33^{2}$ n. 3 , ib. 89 . 24, I IO. 21,214 I3.

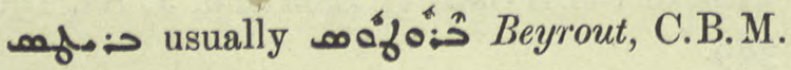
$725 a$.



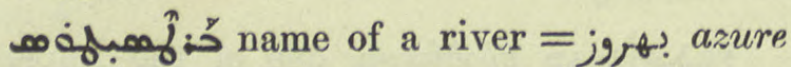
crystal, Gest. Alex. 204. 14.

حم: pr.n.f. Veronica, Stud. Syr. ii. 9. 2. ح pr.n.m. ZDMG. xliii. 396 ult.

م صم pr.n.m. A bishop present at the Council of Isaac, 4 ro. Syn. Or. 34. 14, 36. 10, ZDMG. xliii. 395. 6.

ح- col. 609. fasting. See under ف)

col. 610, 8 of par. correct حمُّهُ for for ح.a. Pael y

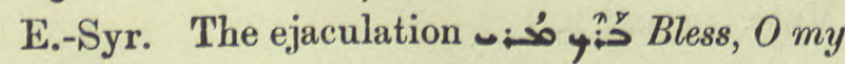
Lord is used at the beginning of daily service and in the Liturgy at the beginning of the Eucharist proper, Takhsa I I.4. Often written as one word,

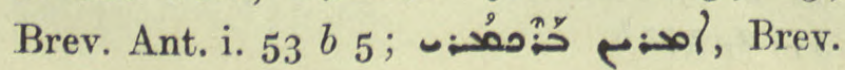

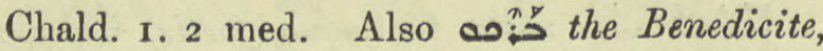
$O$ all ye works of the Lord, bless ye the Lord, Brev. Chald. i. 36. twelve times ff.,

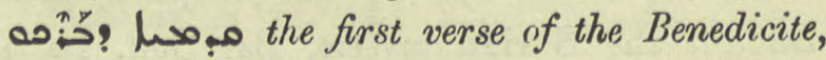
ib. 9 af.; 37.15 .

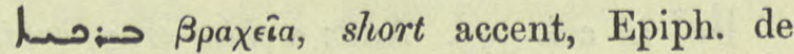
Mensuris 7.6 .

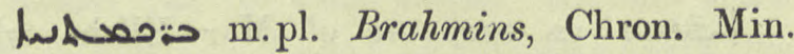
356. 22, Usually spelt Lxas: col. 6r5.

$p_{i}^{\prime} \rightarrow$ particle, col. 6r5. The quotation ro af. to 6r6. 7 is BHGr. i. 168. 2 r. Col. 616. 6 correct 100.

המם Hex. Aq. Jer. li. 34, Ps. xviii. 15.

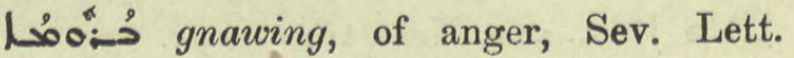
227.17.

bojos col. 6r7. Dele par. It is given under ā Thes. and Suppl.

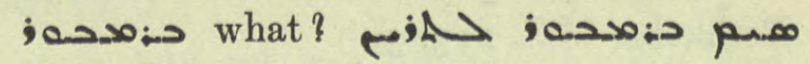
?حمةدو Charms 23. I7.

م: m. pl. Brahmins, Kal-w-Dim. ed. Bick. 99. 9, I 4, 100. 4 \&c. See Sم: above.

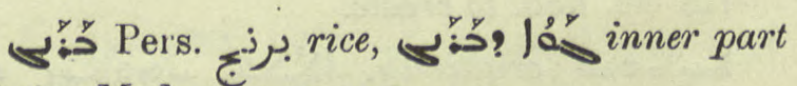
of rice, Med. $21_{5}$. 5, 430. 4, 9.

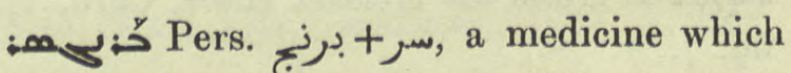
promotes the discharge of phlegm, Med. 3II. 17.

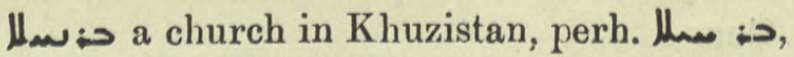
Syn. Or. 79. 12 and 667 .

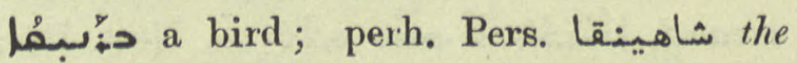
royal white falcon, Natur 25.2 , trans. 50 and $\mathrm{n}$.

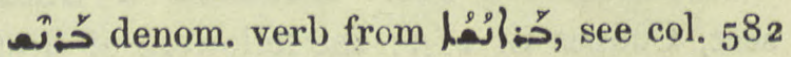
and supra.

. خِ col. 6r 7. See Ar. Pfln N. 279.

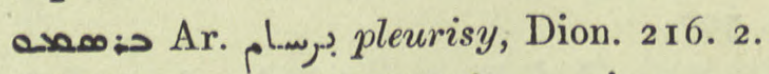

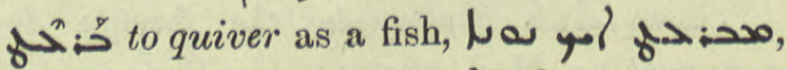

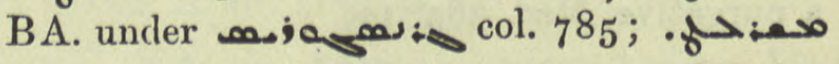
مص:خد 3 with the same gloss. as BB. has under als. See col. 6r7. 
$3^{\circ}$ : col. $6 \mathrm{r} 8$. to hit, pierce as an arrow or spearhead, add: M.Z. 6. 4. Metaph. $\int_{6}$

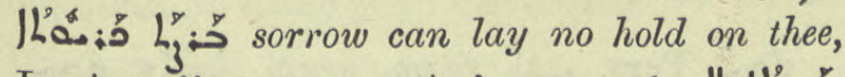

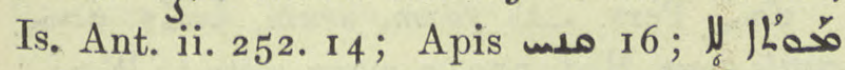

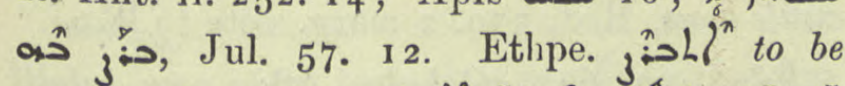
penetrated, perforated, $\}_{3}^{\prime}$ Lóa Med. I56. I 5 .

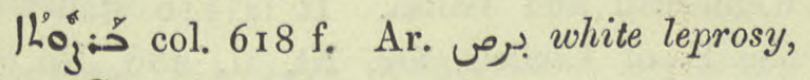
Caus. Caus. 42 . 16.

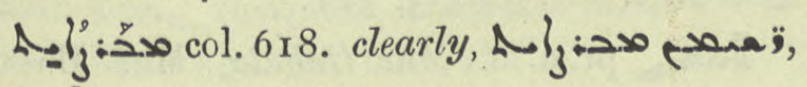
Syn. Or. I 49. 22.

حُنْف م col. 618. to shine, flash. Add

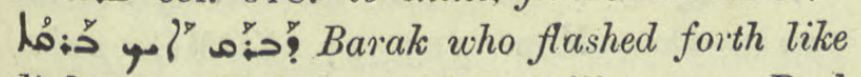
lightning, Ephr. ed. Lamy iii. 233. Pael

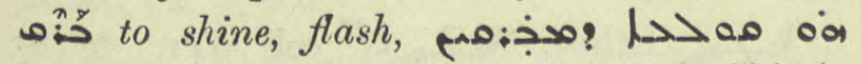
"minerai d'or des polisseurs," Duval, Chimie 4. 4 and trad. 8 or minerals which shine. Aph. مiّ to make flash, to make to shine,

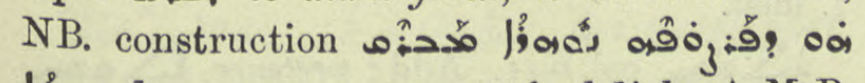
Jóg whose countenance sent forth light, A. M. B. v. 546. Ettaph. $5:\left.0 L L\right|^{n}$ to shine: to be

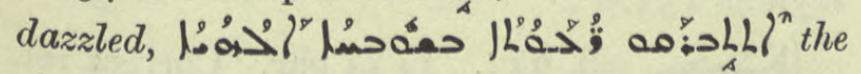
shepherds (at Bethlehem) were dazzled by the divine glory, Sev. Ant. Hymns 6.

100: a sudden pain, 'م5 إ lumbago, agma a sudden sharp pain took him in the back, Charms 8. 9, I 4, Protection 84. I.

مخة ال لـ Ref. i. I 20. 46.2$)$ electric, 2) electric ray, torpedo, Lexx. under لمـ col. 2470.

人ُ دُ pl, m. gravel, grit, Chimie 37. I 2.

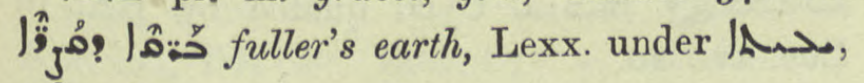
col. I6 I I.

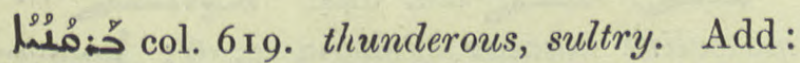

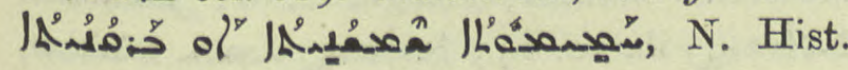
v. 4. I.

Leais pr.n.m. an E. Syr. bishop present at the Council of Jaballaha A. D. 420, ZDMG. xliii. 395 . infra. a a a village, col.622. Refs. Pers. Mait. 224 ; Hist. Mon. i. I36. 12 ; Mar Bassus 125.

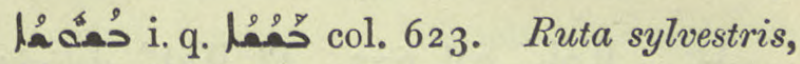
wild rue, Ar. حمل, Med. 54. I 2, 55. 20, 56.8;

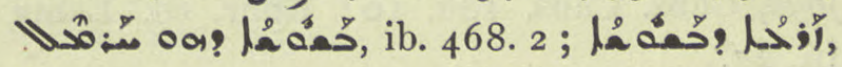
ib. 559. 4, 570. 23; JAOS. xv. 194. I4; WZKM. xiii. 10.

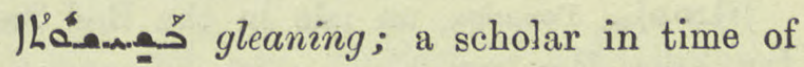
harvest must not neglect his work and go out to glean for greediness, yogow Stat. Schol. Nis. 29. 9, I 9 I. I9 =Giorn. Soc. Asiat. Ital. iv. I 9 r.

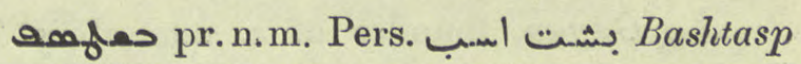
i. q. Vishtasp, Barhad. $3^{6} 5$. ult.

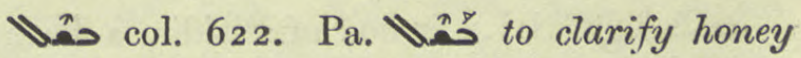

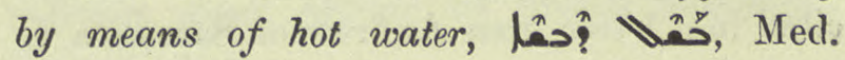

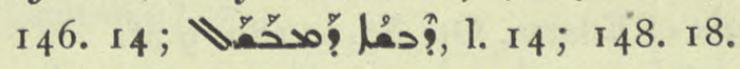

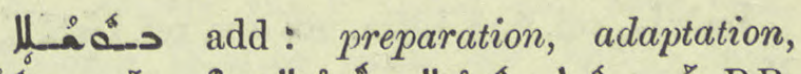

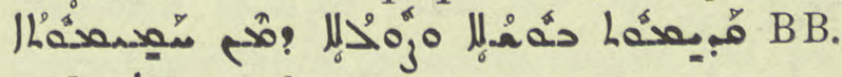

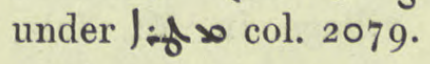

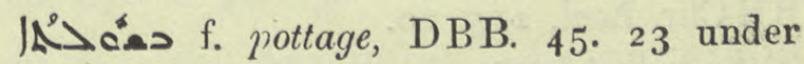

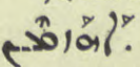

A. virgin-born, Philox. ed. Guidi 478 a $2 \mathbf{r}$.

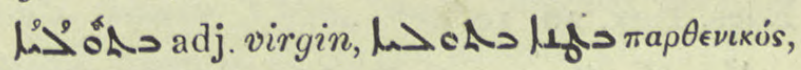
AKGW. 1904, 25. 25 .

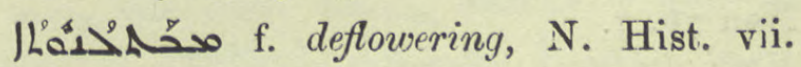
I. 3 infra.

iيُ col. 625. Rit. the second half of a choir, with us the Cantoris, Qdham W. passim.: see is.so م- Suppl., Brev. Chald. i. tit.,

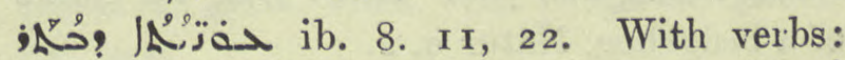
col. 626 4⿳亠口冋l" to turn back, defeat, |a>?

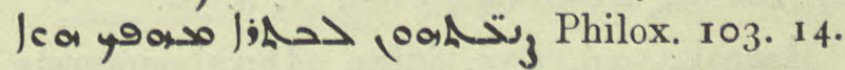

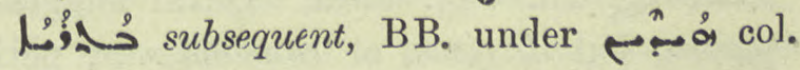
1002 .

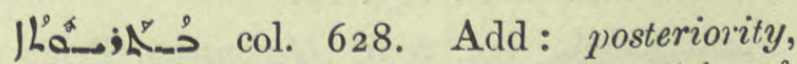

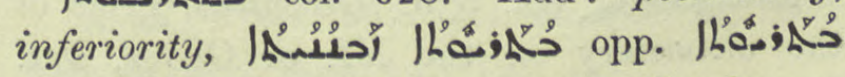
IAُAْ Bü. Sap. Theol. I. 5, N. Hist. iv. I.

amois Barpáxıo, copper treated urith sulphur, "malachite dorée ou chrysocolle," Chimie r6. 9. See حاره: Suppl. above. 


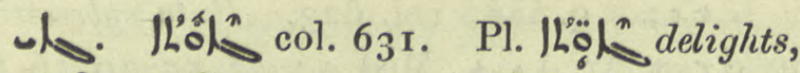
joys, Caus. Caus. 186. 10; Ephr. ed. Lamy iii. 27.

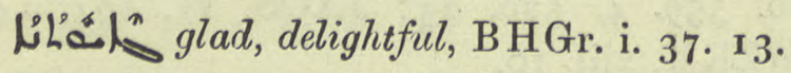
j) Jac. Edes. Hex. 20. 8.

|. حخر, to geometrize, BHGr. i. 47. 18.

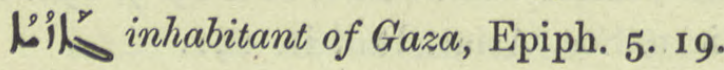

Hayan, founder of a monastery in Kashkar, Chast. 69. 2 I.

لخ G.1.i.s. who? of Pisidia, a Father cited in a letter from Andrew of Samosata to Rabbula of Edessa, Or. Xt. i. r8o. 9 af.

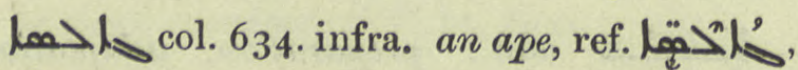
Med. 335.8.

lasoly Gamua, a village in Mt. Izla, B H Chr. Eccl. 2 I 5.20.

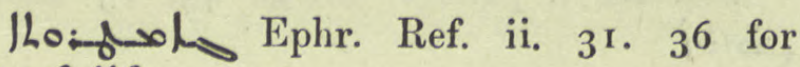

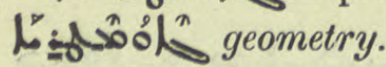

angls var. consts, the Ganges, Jac. Edes. Hex. 26. 10.

Gangra in Paphlagonia, Diose. ed. Nau 76.7 .

llow mis-print for lenful $\gamma \in v \tau \imath a \nu \eta \dot{\eta}$, gentian, Chimie T. 12.

$J_{3}^{\prime} \sec J_{j}^{20}$.

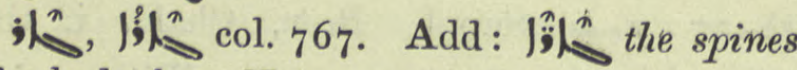
of a hedgehog, Natur 9. 2.

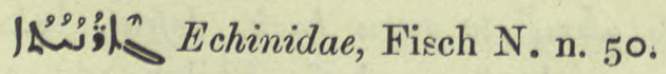

1 soojls col. 635. infra. Duval suggests $\pi \lambda \dot{\eta} \rho \omega \mu$, but detritus, or conglomerate, perh.

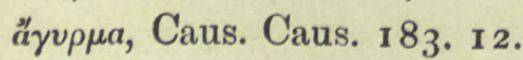

sandoils col. 636. Gerontius, Prior of the monastery on the Mt. of Olives, Pet. Ib. 3i. 2 .

Lesils Germania, Germany, Jac. Edes. Hex. 32. ult., 34. 7 .

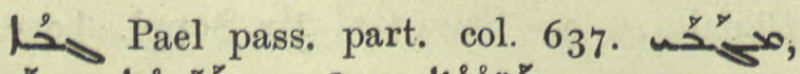
مa select. ? from synods, Journ. As. 1906, 57 pen.

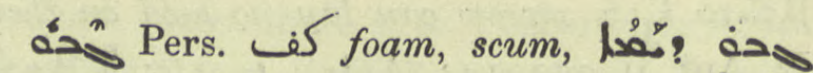
cuttle-bone, Med. 559. 2 marg. note to |حُم

لِ on the Euphrates, a village halfway between Kennešrin and Balisa. It is r 10 stades to the E. of Chalcis, Or. Xt. ii. 280 , No. 3 ; Sev. Ant. Vit. 320. 3, $3^{21}$. 7 .

لva pr.n.m. Mar Gabula, Mar Bassus xii. Syr. 5 .

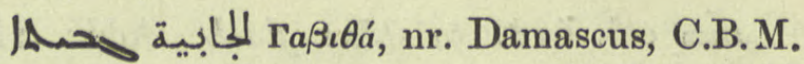
7 10 $a$, Doc. Mon. 215. 17, ZDMG. xxix. 78. f., $43^{\circ}$.

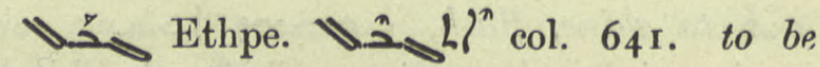

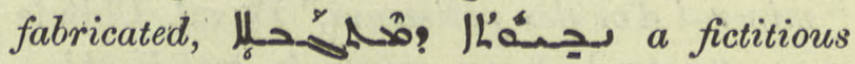
prophecy, Nest. Hérac. 374, quoted Pléroph. 7. med.

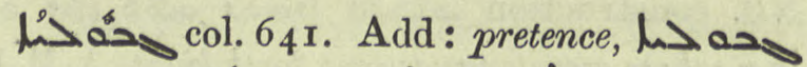
Ilexaman looj:0 La>nalo, Sev. Ant. Hymns I 53 .

1Rำ to Rom. ix. 20.

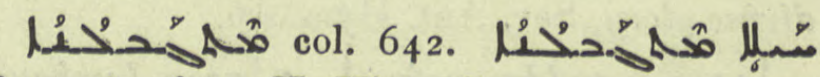
formative force, N. Hist. vii. 5. I.

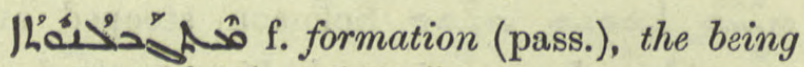
formed, |R่ใช of drops from moisture in the air, N. Hist. v. 1.3 .

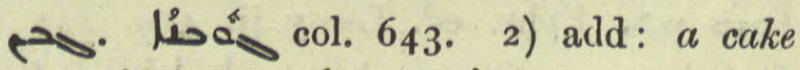
of figs, Op. Nest. 97. 7 in $\mathbf{I}$ Sam. xxv. 18.

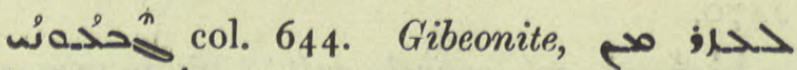
م: هAl Cyril quoted by BA. in Luc. xvi. 20.

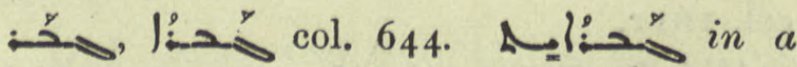
masculine manner, Pelagia 2. 6 ; محص

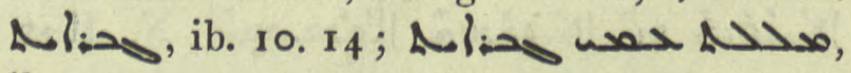
ib. II. I3:

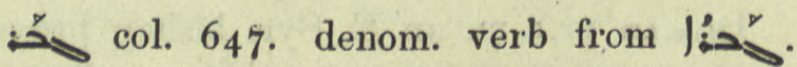
Pael conj. $\stackrel{\text { }}{\longrightarrow}$ to strengthen, invigorate, קatr. Or. iii. i. 107. 6, 114. 4. Ethpa. :ُy li $^{n}$

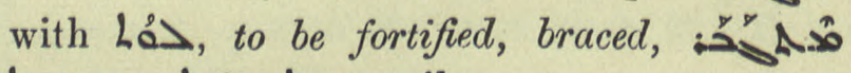
I 


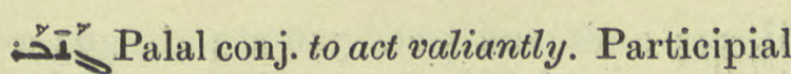

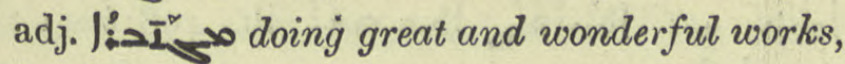

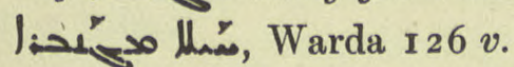

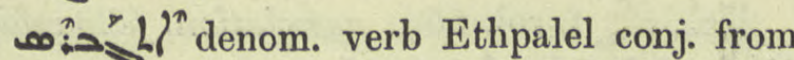

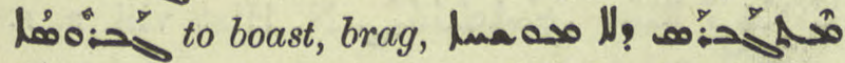
he bragged immoderately, BHGr. i. 48. 19; Tekkaf 36. 187 .

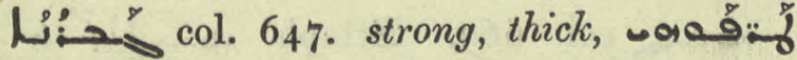
1ำ leaves, Med. 599. 20, 600. I8.

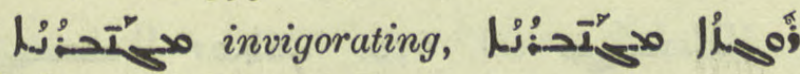

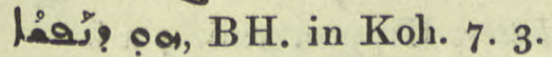

İy Chast. 62. 3, 13 .

Ho: $=-y^{\prime}$ Gabrona, founder of the monastery of Shmona, Chast. 15. 3, 32. 8 ; A.M.B. iii. 473. 1 .

خِخ: Pers.

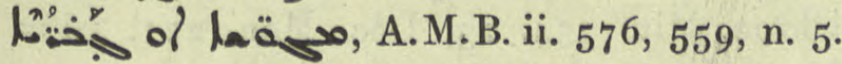

$\because$ col. 650. Act. part. \& $\stackrel{2}{\circ}$ erect, lofty, Ephr. ii. 457 A.

$\because l_{>}^{\circ}$ col. 649. fortune. L. ro of par.

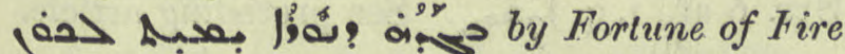
I have sworn to you, Nars. ed. Ming. ii. 402. 18. A statue of Fortune, M

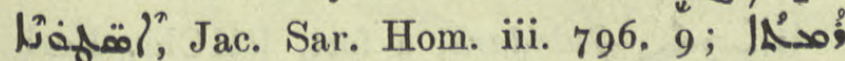

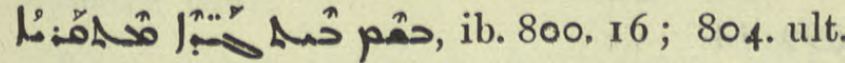

I?: col. 652. wormwood. Metaph. bitter-

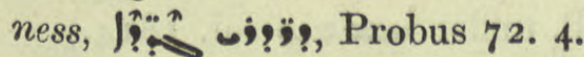

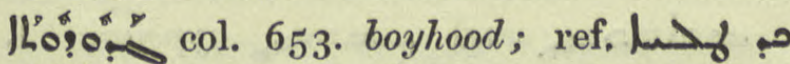

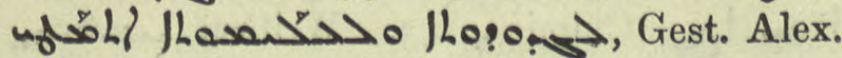
24. 2 .

$\because$ name of a river on the borders of Andrion, BHChr. Eccl. 671. 2. Andrion is not identified. Variants was and a.so?.

Dans from $7 \lambda$ a Syrian god. Pr.n.m. of a fourth century martyr, ZDMG. xlii. 474, B.O. i. I $2 b$, Wright's Martyrol. 10.

I col. 653. Add: a strait, lim

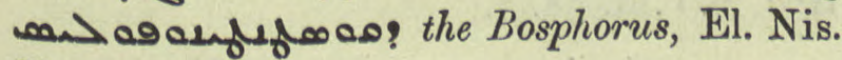
Chron. 184. 26.

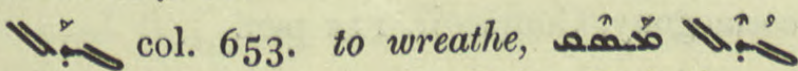
smoke ascends in wreaths, Ephr. Ref. ii. 35. 24 but ef. l's. Pass. part. $\|_{6: \infty}$ over-full,

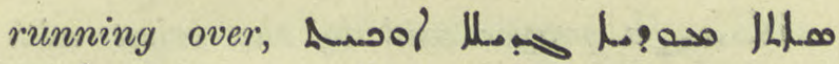

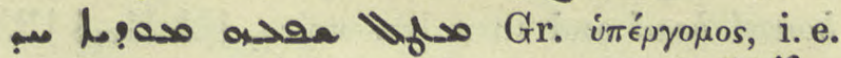

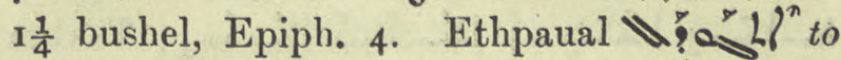
be over-full, stuffed, M!a hlo w $>$ ohl, Anecd. Syr. ii. 209.8 .

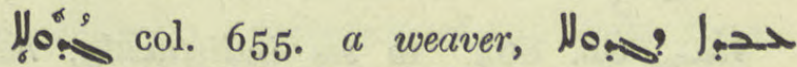

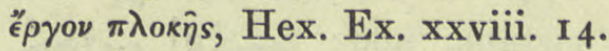

مل: : Is. Ant. i. 2 I 4.4 ; Wellhausen, Skizzen iii. 28.

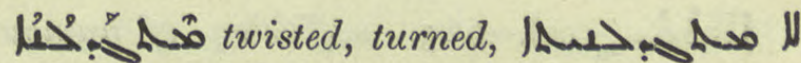
>م a golden chain not twisted of many strands, ${ }^{\mathrm{BH}} \mathrm{Gr}$. i. I4. 19.

Poc col. 657. Act. part. pọ? mélange pour obtenir un bronze tranchant, Chimie 97. 2.

; correct ill ?

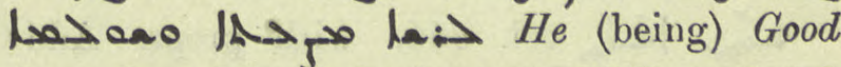
decreed good for the beginning, middle, and end, Mon. Syr. ii. $3^{6 .} \mathbf{3}$.

象象 659 infra. Löw corrects to

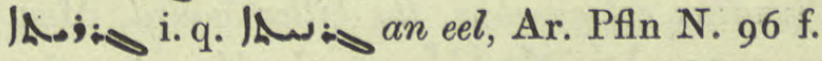

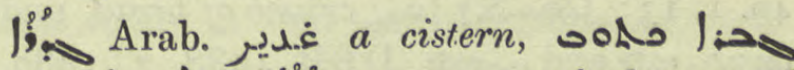

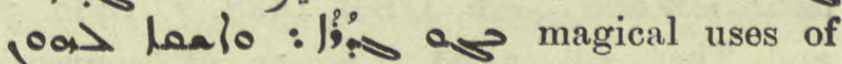
Psalms, Kays. in Ps. xxxiv, ZDMG. xlii. $45^{8 .} 4$.

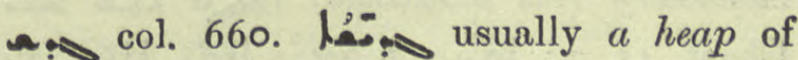

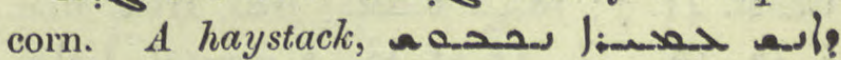
la.s, Anecd. Syr. ii. 319. I9.

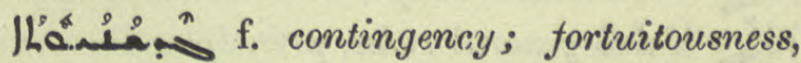
Is. Ant. B. 175.3 af., But. Sap. Isag. i. 5, Theol. 2. 3, N. Hist. viii. I. 4: see under L'ánio ơ?.

lom Aph. won 2) to be quit of, to get rid of, with $e^{\infty}$ of the pers. ow asay

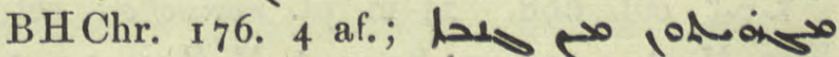
Ephr. ed. Lamy iii. 545.

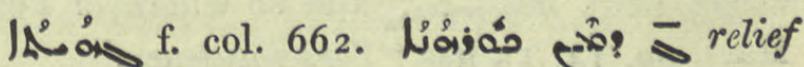
from sickness, Med. 147. 14.

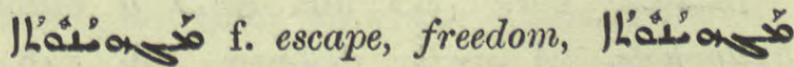
fำ

ilay Pers. زج a carrot, Med. 266. 7, I I.

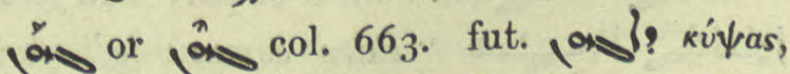
lit. that $I$ should stoop, Jacobite reading Mark 1. 7, Studia Biblica, Oxon, iii, 68. 


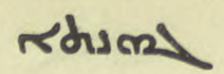

68

IR่ós a prayer said by the priest with

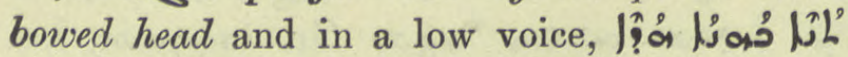

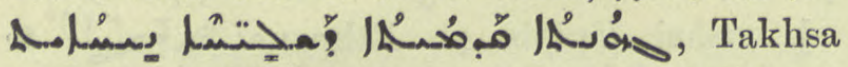

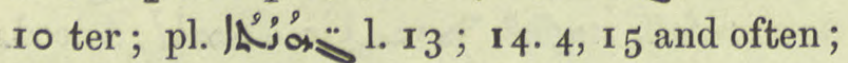

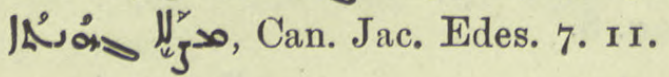

;â-s col. 664. to see indistinctly, have

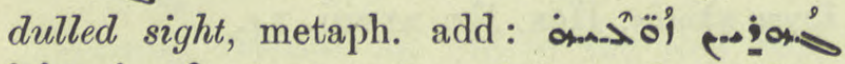

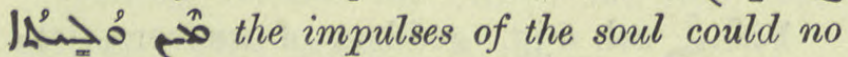
longer perceive what is right, Jac. Sar. Hom. iii. 8 I 7 . 19.

JLo'ó col. 665. suffusion of humours over the eye, dim sight, mistiness, Med, 14. 20,

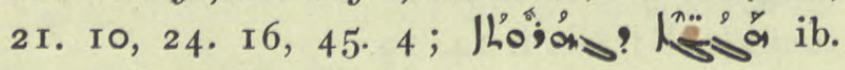
72. $17,20,73.16,74.9$.

Jar Jebel Gara or Ghara, five hour's SE. of Amadia, Badger, Nestorians I. 254; A.M.B. i. 4 I 4 .

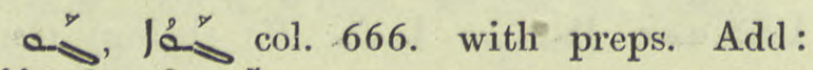

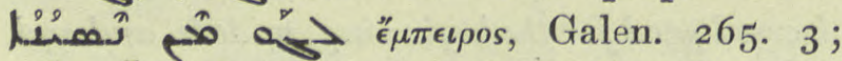
مै

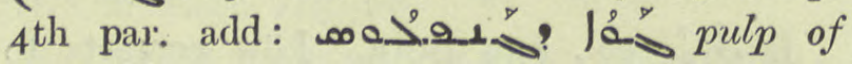
Citrullus colocynthus, the bitter gourd, Med.

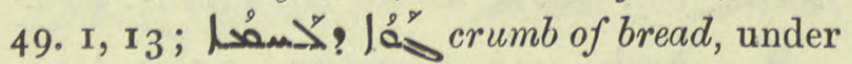

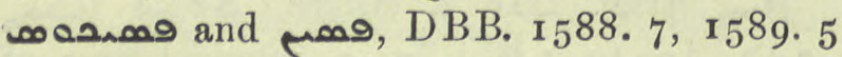
and Thes. col. 3 I88. L. 19 af. convent, monastery (the buildings) loso l. Chast. 4I. 7; Ja้ i. 47. I6. L. I5 af. lå general, lỉilás lay general advantage, ZDMG. xxiv. 268. 2 ; | 244. 17 .

$\because$ ố

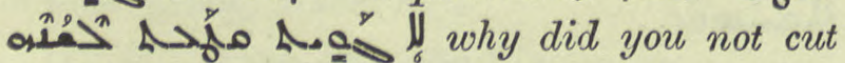
out his tongue by the roots, A.M.B. iii. $27 \mathrm{I}$ ult.

L’ós col. 668. 2) m. pl. eunuchs; ref. Pers. Mart. p. I 3 , n. $89=$ A. M. B. ii. I 9. I where Bedj. has Mart. pp. 14, I5 has "Verschnittener."

مers. buttonhole. A girdle, drawers, Jab. $546=$ Pers. Mart. Ior I.

นُนُ

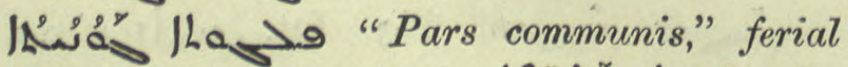

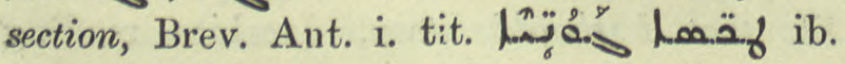
Index; the Commune Sanctorum, ib. 164 , ii. $343 a$ ult. and 5 af., $346 a$ af., $362 a_{5}$;

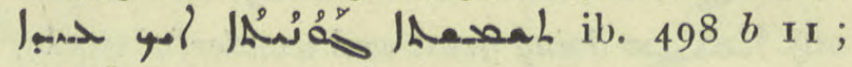
5 I 7 b 8.

LLْنُaُy f. col. 669. association, fellowship, .

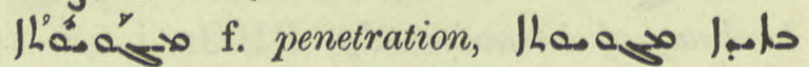
ILof 0 AKGW. vii. (1904) 4, 54. 2.

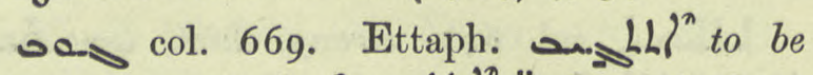
answered, p:s on answer, Or. Xt. i. 308. I3.

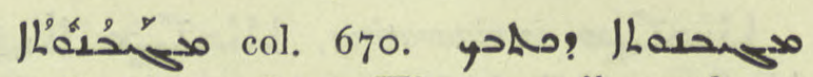
a reply to your letter, Hippoc. xxii. 4 a f.

1’ْ a pit, cistern. col. 670.26 of par. dele "vas, hydria."

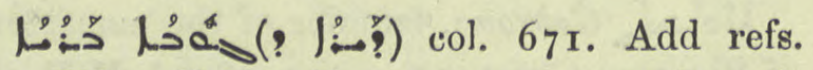
Jac. Edes. Chron. Can. 575, antep., 578.4 af., Ant. Patr. 3 ог. 5 af., BHChr. Eccl. 337.

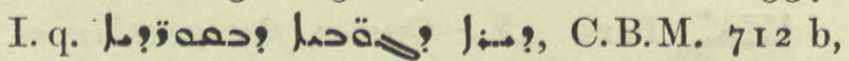
Chron. Min. 254. 23, Doc. Mon. 221 . $16=$ ? 1.22.

liٌُ col. 671. a Gubbite, inhabitant of 今ُ Syr. 6 af., i. q. Sحُتِ

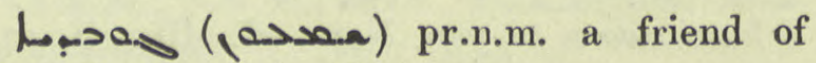
Marutha, Patr. Or. iii. i. 7I. 5 .

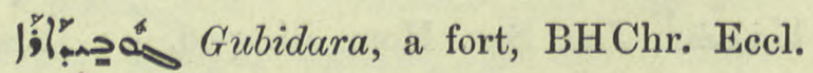
599. 14.

J Sorbus domestica, the Service-tree, BB. under م: م: col. I8I4.

nolessas and pensos gypsum. I. q.

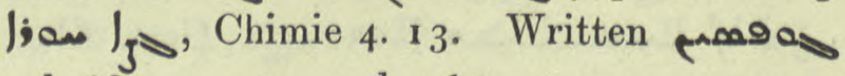
col. 687 ,

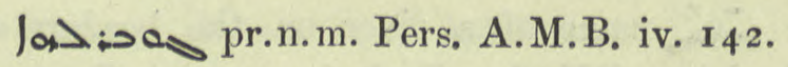

Lُ col. 672. perhaps moaning, ailsos?

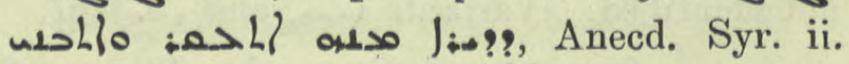
284. 15 .

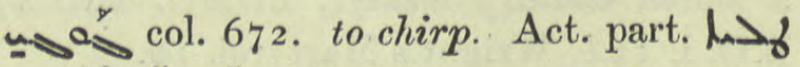

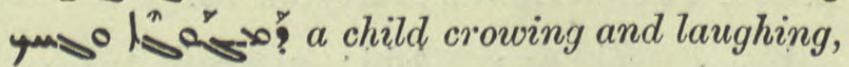
Hist. B.V.M. 58. 7 .

unas Gugi, a personage of Manichaean cosmogony, Coupes ii. I I 2 pen.

u’ýs col. 672 infra. f. a spider's web,

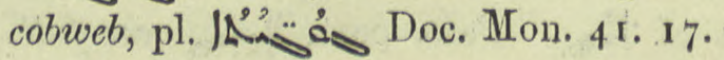




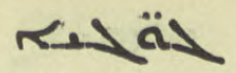

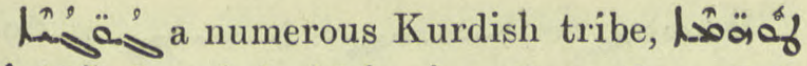

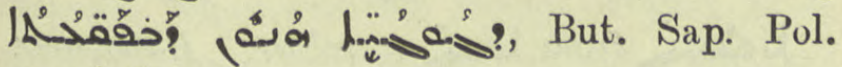
I. 3 infra.

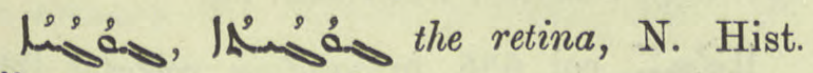
vii. I. 2.

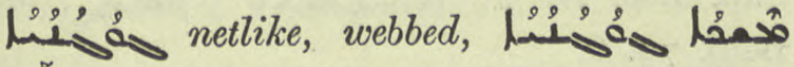
?. the nautilus has a thin web between its feet.

"रू Goghmal, an estate in the valley of Marga, Hist. Mon. r. I64. I.q. M⿻ as q.v.

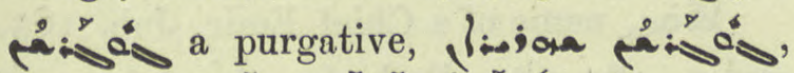

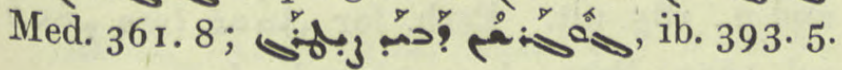

9 Arab. swift, name of a horse, Kal-w-Dim. 335. 2, 339. 4 .

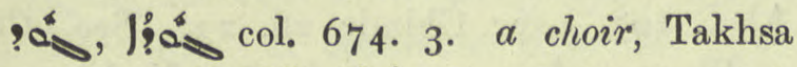
$52,147.5$; 1 , which begins = our Decani, opp. أه the following or latter choir = Cantoris, Brev. Chald. i. 25. 7, 26. quater; Qdham.W. 7 ter, 8. I, 9. I ; lệs by choirs, l. ult., ro. 6 .

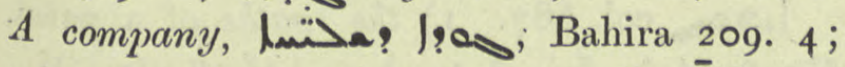

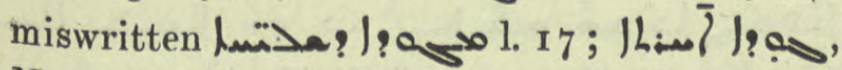
Nest. Hérac. 35. I.

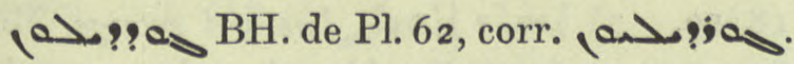

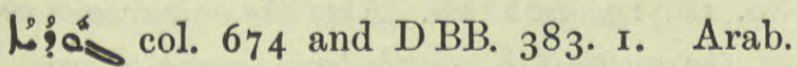
جوذّ prob. NS. l!? as a tunic. Cf. the following word.

IRْ? B.V.M. $57 \cdot 3$.

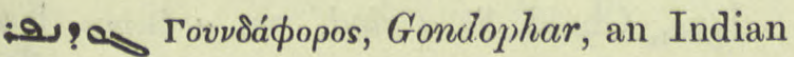
King, Act. Apost. Apoc. 4 , 4 , 4 ,

las. liang failing, running dry, opp.

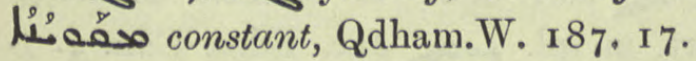

L'ơ์ col. 675. f. deficiency, Lớ Jhasy; lack of moisture, N. Hist. viii. 2. 2.

$1 a^{y}$, liay col. 675 . a nut, walnut, $1 \mathrm{~A}^{\mathrm{y}}$

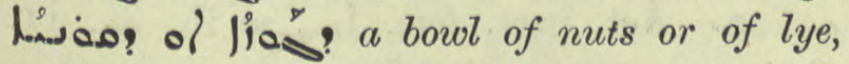
perhaps pounded walnuts or walnut oil, Med. 55. 15 .

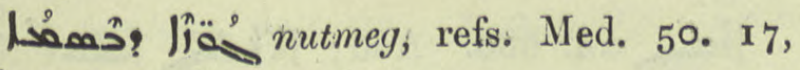
56. I 5, I74. II, 307. 4 .

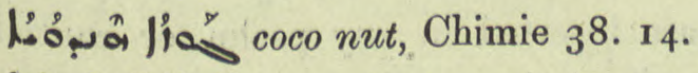

| I4I. $23 / 24,3 \mathrm{I} 3.19$.

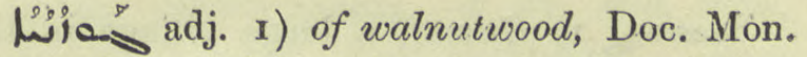
I7. I, hulas lida l. 4 in Isaiah's vision: cf. Pesh. 2) nutshaped, Hex. Ex. xxxvii. I 7 ed. Lag.

jos col. 676. Gozan I) a country. 2) the river Hulwan a tributary of the Diala, Pers. Mart. n. 544, Phet. 20. 8=A. M.B. ii. 6r3. I3.

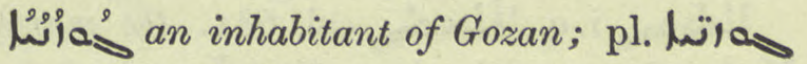
Coupes ii. $158.8, \mathrm{f}$.

was Aphel part. me col. 677. 8 of

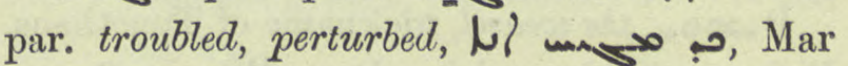
Aba ii. I I 5.

L Journ. As. 1895,185 f.

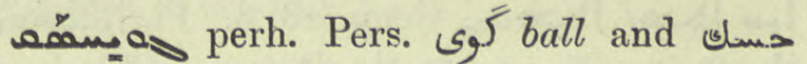
Eryngium, the round ball of the Eryngium, a medicine for diarrhoea and heartburn, Med. 294. 9 .

between Emesa and Damascus, C.B.M. 6r 3. $a$.

uِg جوخى Guki, a bpric. mentioned with Ctesiphon, so it was in BeitAramaye, not in Beit Garmai, Abbeloos, Act. Maris 67 and n., Pers. Mart. 259, 277 ; Coupes ii, 152. med. Or. Xt. ii. 3 I0. 2.

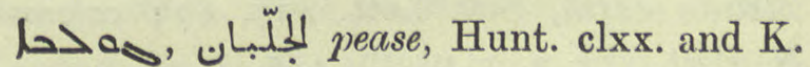
under lao; col. 387 I.

$\infty a>a s$ i.q. $\infty a>d s, l a\rangle$ \&c. an ape, Sindb. I7. 19.

جولنار , col. 680. pomegranate blossom, Chimie 7. 9.

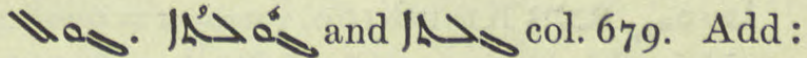

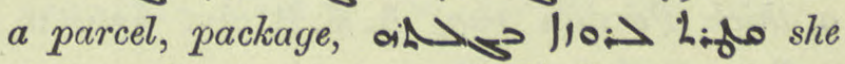
tied up the rice in a parcel, Sindban Io. 8, I I,

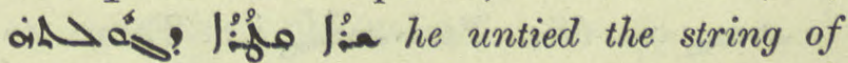
her parcel, 1. I 2. Rit. an altar-cloth, व I46. 18 .

koó col. 680. 9. of par. a bean used as a measure, مَ administer a diaught the size of a bean, Med. 99. 6 .

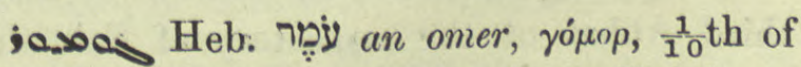
an ephah, Hex. Ex. г6. 16, 18, 36, Epiph. 4; ; jaxas ib. 
$\checkmark s 0$, I) Gumal, a large village in the Marga valley, BHChr. Eccl. ii. I2 I. I5. 2) Gomel river, Pers. Mart. 194-1 98.

ر. to the south of Lake Moeris, Pallad. 120. 3 . Budge gives Geomastike and says Bedjan reads

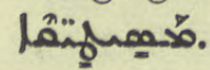

1. Med. 589. 5 with gloss. ;ف of par.

Ji: $\infty \mathrm{os}_{\mathrm{S}}$ the weasel, nickname of Timotheus, Bishop of Alexandria, Doc. Mon. 228. 13, 23r. 22. Cf. Nöldeke's note ZDMG, xxxv. 235 .

مدف:م

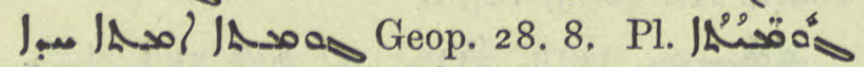

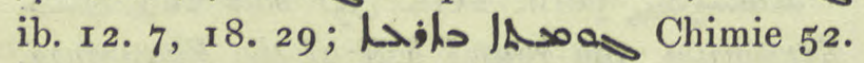
12; a hole or hollow in a mixture, as abe IA 0 as ib. 25.15 .

Noos name of a town near Mt. Izla, Anecd. Syr. ii. 372. 2. Cf. JAsoas? IA م: a village in the marsh, ib. 211.3 .

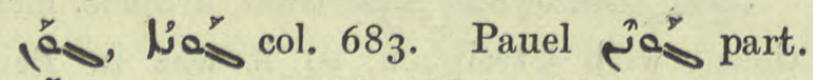

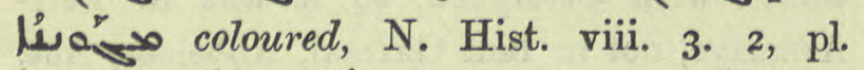

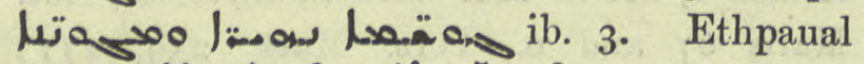

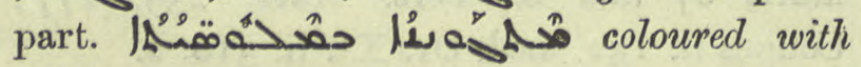
Melian earth, Sev. Lett. 427. I7; coloured, N. Hist. viii. 3. 2, pl. ib. 4. 5.

way some kind of garment perh. embroidered in colour, pl, 1 Anecd. Syr. ii. 268. I3, Jhäas ib. I7.

مơ⿱

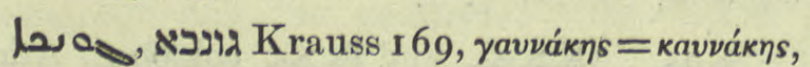
a thick Persian rug, A.M.B. iv. 336. n., 338. n.

eos col. 684. Pael $\infty a_{y}^{n y}$ i.q. Ethpa. to take refuge, have recourse to, eấ ahad Mar. Benj. 91. I 2 unless this should

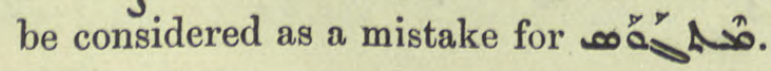

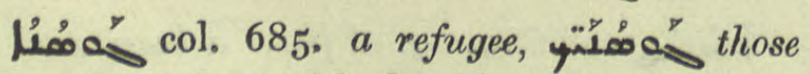
who take refuge with thee, Act. Apost. Apoc. مصد 18 ; Anecd. Syr. iii, 247. 25.

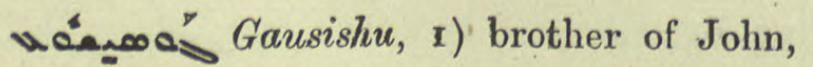
third Abbat of the monastery of Sabrishu, M.Z. 198. 3. 2) Bishop of Beit Nuhadra, ZDMG. xliii. $404,18$.

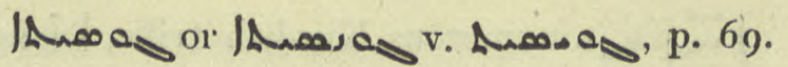

lióng from lơ⿱ ravening, BH Chr. Eccl. 80 I. 18.

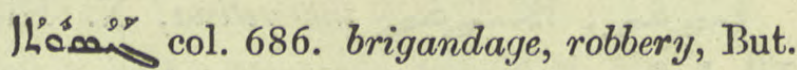
Sap. Eth. iv. 7; Loufon o lhomy, Mar. Benj. 79, 6.

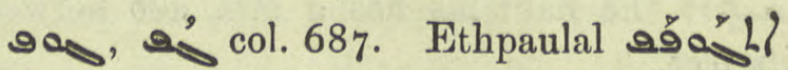

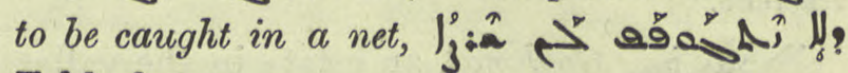
Tekkaf $\mathbf{1}_{4}{ }^{2}$.

لهُ

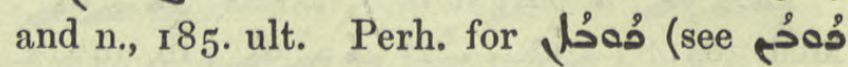
col. r690) Pers. حوبان a shepherd.

ヘำ ró rútos, gypsum, lime, plaster, Gest. Alex. 193. 3, 7; Chimie 28. 23 . See other spellings under ${ }^{2}$ col: 765 ., Med. 303. 6 .

Aه A A village and convent in the district of Damascus, C.B.M. $709 b_{25}$, Nöld in loc, ZDMG. xxix. 434.

J: 9 a col. 687 . I) the spathe of a palm,

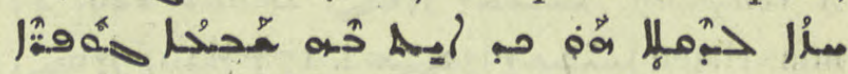

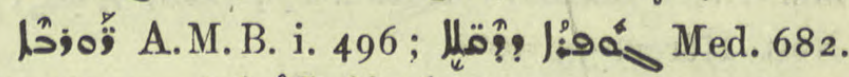

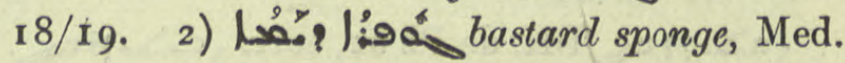
89. 12, 19, 602. 18. Also the catcareous shell of the cuttle-fish, cuttle-bone, sepia staire, ib. 92. 10.

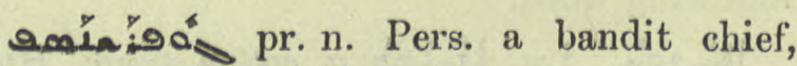
temp. Bahram III. M.Z. $3^{6 .}$ I 3 .

I as Chald. is a clod. m. a lump, ljas

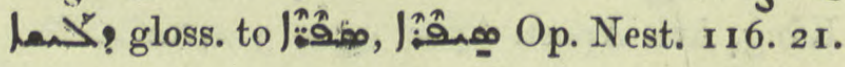

;os, col. 688. to commit adultery. Act. part. m. pl. ↔ Jac. Edes. Can. 4. 7 . Aph. I) to deflower, ravish, of Greek

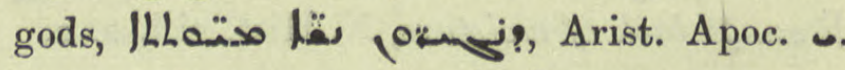

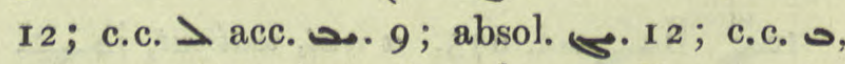
ค. 20. Act. part. fem. J $\mathrm{S}^{-\infty}$ ib. ๙. $1 \mathrm{I}$. 2) to cause to commit adultery, $-\mathrm{n}$ - a.oa

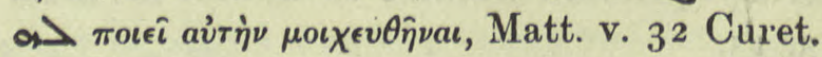

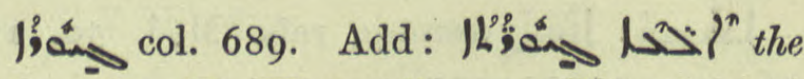
false ribs, N. Hist. vii. 13. Rit farcing, a clause inserted in any psalm or canticle,

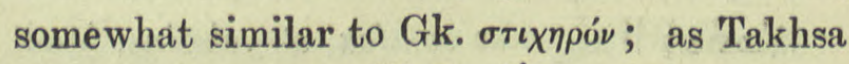
21. 10; ib. 55. 8; Ihes, lián Ps. cxxii. I 
rias

farced, when said in Church opp. a different response when evening service is said in a private house, Qdham W. 28 ult., |jạA $\rightarrow$ so: one to four psalms, farced, ib. I35. I2.

jag Ar.

†ón perh. = lim col. 7 ro. quicklime,

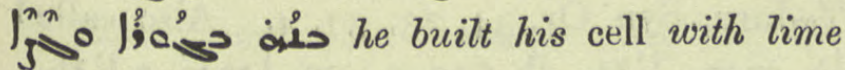
and fine plaster, Jab. I 50. 9.

läas col. 69o. shoes. Add ref. Diosc. ed. Nau 94. 7, 1 .

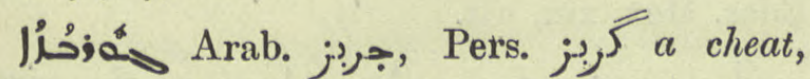
sharper, Kal-w-Dim. 30 bis, 34. 8.

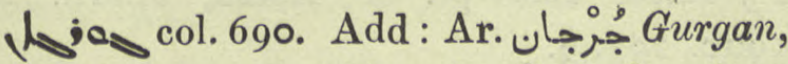
i. q. Hyrcania. A bishopric, Syn. Or. 672 ; 43, 62 bis, 67 , 110; Or. Xt.i. 3 10. 3 , ZDMG. xliii. 399. 2, 400. I; Nias Kal-w-Dim. 259. 20.

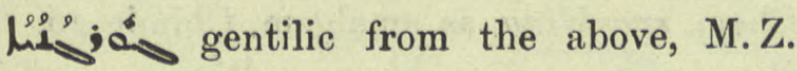
33,42 .

fُ of Peter of Azerbaijan, A

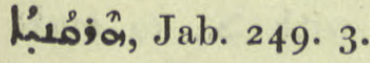

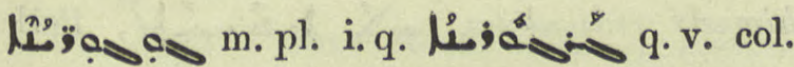
774. Melilotus, a trefoil, Med. 372. 6, 397. 7 .

(6) so correct

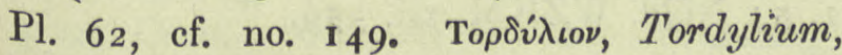
Diosc. i. 56, ZDMG. xliii. I25, Ar. Pfln N. 226.

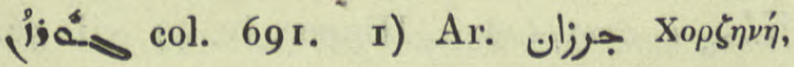
Georgia or Iberia, Eranšahr I 16, Syn. Or. 672,37 ; ل Jijas va! ג, Chron. Min. 28r. 6. 2) Arzanene, Anecd. Syr. iii. 337. 2 ; S. Dan. $10 b_{4}$ af. 3) i. q. Jebel Ghara, S. of Tiari and Amadia, jos as liaf jiob, A.M.B. i. 4I4.

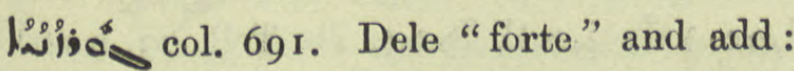
Pl. Georgians, Jab. 351. 2, 359. 12. Prob. men of Arzanene, ib. 227. 3 a f. The language of Gurzan, Coupes ii. III.

hias prob. a miswriting of bogay or boor : :
1

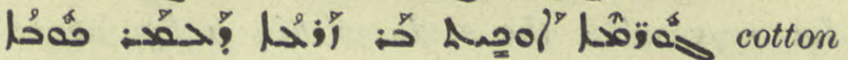
seed, Jab. I96. 4 but hoo: ed. i. I77. 8,

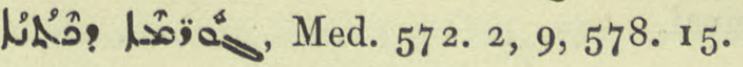

lwig dialect of Tagrit, a plane, BB. under زما:

fagos a village in Cilicia, $\mathrm{BH}$ Chr. Eccl. 783.18 .

| some cooling draught: the recipe contains grapes, cardamoms and crocus, Med. 300. 23.

anjos pr.n.m. Pers. A.M.B. iii. 5I 5 .

i|jonias pr.n.m. Pers. the father of Mar Benjamin, Mar Benj. 69 n. I.

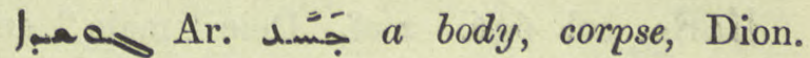
193. $21,215.7$.

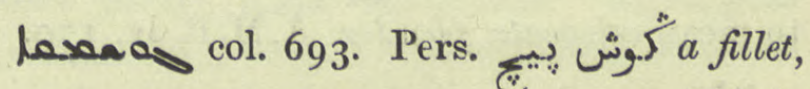

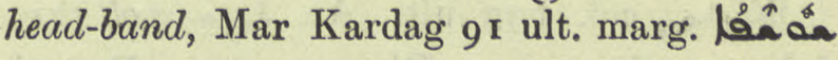
and Abbeloos gives this word in the text of his edition, ror. I. See DBB. Appendix 2 I 7 under lanas.

vacos pr.n.m. Pers. A. M. B. ii. 648 .

? Gusnyazdā dh, Nöld. ZDMG. xliv. 534 in Mar Kardag. 65.1 , 66. 5, 77. 8, A.M.B. ii. 636 ult. $=$ Pers. Mart. 68.

w 0 unas. pr.n.f. Pers. A.M.B. ii. $637=$ Pers. Mart. 69, nn.

كشمب استب a stallion. pr.n.m. Gushnasp, A.M.B. iv. 2 29, Pers. Mart. n. 606, ZDMG. xliv. 673 .

inames pr.n.m. Pers. A.M.B. ii. 642 $=$ Pers. Mart. 69, $\mathrm{nn}$.

aclowas pr.n.f. Pers. A.M.B. iv. 229 $=$ Pers. Mart. ${ }^{2} 5$, n. I9I.

ในใน Pers. Mart. 15, n. $100=$ A. M. B. ii. 26 , ? Ia OS Protection 52. 5 .

a.olnay pr.n.m. Pers. Gushtasp, the protector of Zoroaster, Coupes ii. I I I.

hoy pr.n.m. Gyphthaios, Josephus vi. 25.20 . 
HLa sol. 694. I. Geop. 64. 4

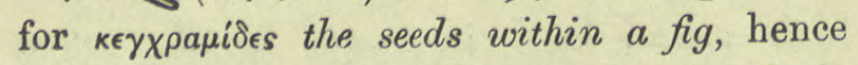
Whä must be pl. of llas pulp of a fruit, R. Duval.

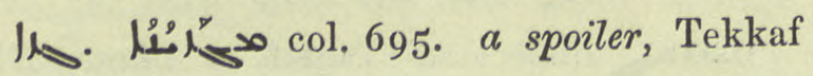
I 2.

1 ${ }^{y}$ col. 696 . m. treasury. Eccles. the Geza or Gaza, a large service book containing hymns and anthems proper to festivals, /

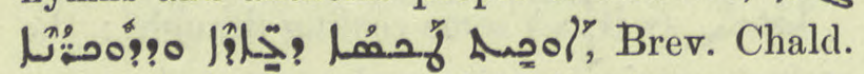
i. 286 tit., ib. 53 tit., la امو QdhamW. 132. I 2. L. ro of par. the holy elements, add:

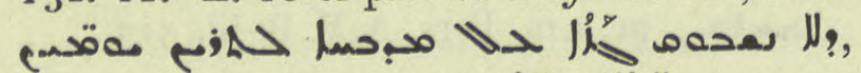

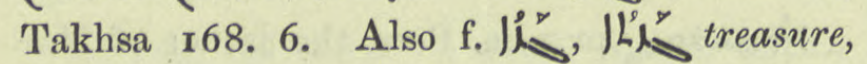

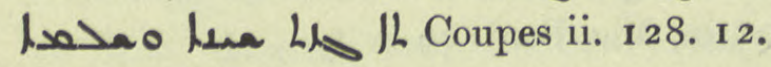

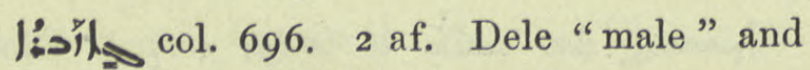

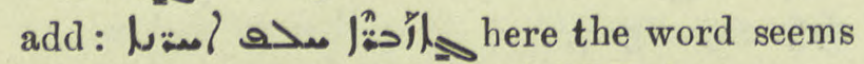
to mean advocate, pleader, A.M.B. iv. 170.

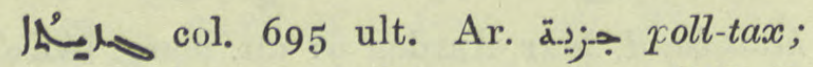
Jab. I I 1 ult. and n., Dion. ro ult. = B, O. ii. ro4 quoted in Thes. See transl. ro. note for history of this tax; ib. 122. 4 ; A الs tax gatherers, ib. I24. $21,128$.

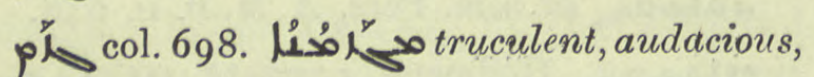
Dion. 136.12.

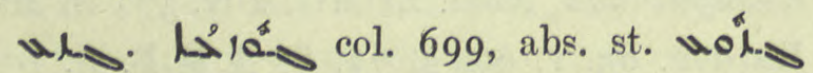
a tree-stump, hewn-down tree, nols awa

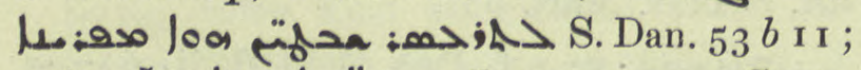

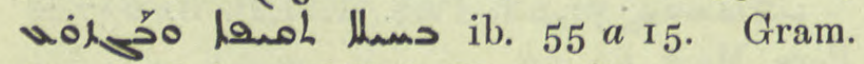
a radical form, B H. in Matt. vii. $2 \mathrm{I}$.

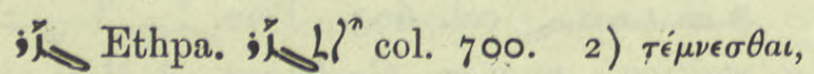
"recevoir une incision", Hippoc. vi. 26. Aph.

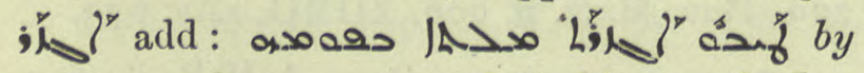
grace words were granted to his mouth, Jos.

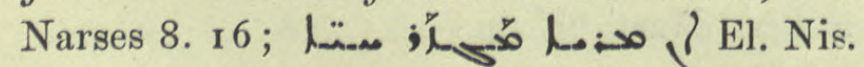
Chron. 70. 7. To cut, cut short, act. part.

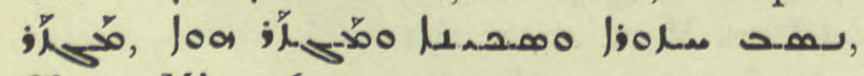
Chron. Min. 56. I r.

- $-\infty$, I) decreed, add. ref. oa is حasephus vi. 9. II. An executioner, Charms 79. I7, but 1

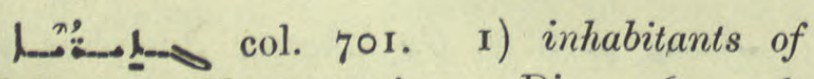
Al-Gezira, Mesopotamians, Dion. 69: I6,

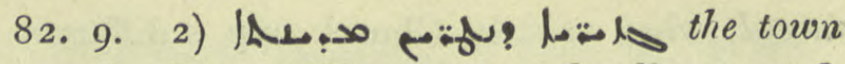
guard, Chron. Min. 2. 21 ; beadles, guard, Jab. 242 pen.; ZDMG. xxxv. 233.

L'숭 col. 702. an island. Place-names: I'Fُ absol. the isles of the Persian Gulf, Ardai, Darai (these two names are probably variants), Deirin, Marmahig, Ruha, Talwan, Toduru, Syn. Or. 34. 23, 37. 4. Also called | isles of the Indian Ocean, l. 12.

A Anogh f. pl. var. Aasoligly islets, Jac. Edes. Hex. xix. ult.

fuy or pay Ar. Arsu the river Pyramus, Inscript. of 'Enesh, Journ. As. 1900. $287 \cdot 3$.

1.

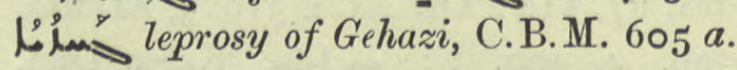

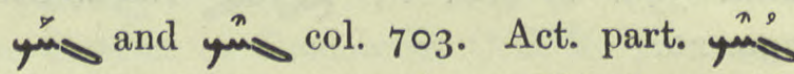
Chem. sparkling, as amalgam, Chimie 26. 18.

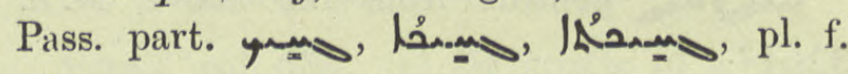

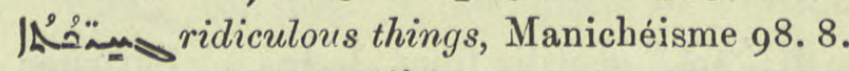

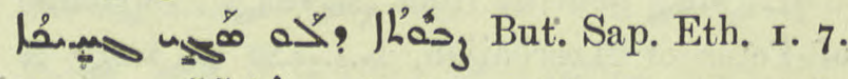
Ethpa. yyㅐ reflexive with $>$, to make himself ridiculous, I 65.2.

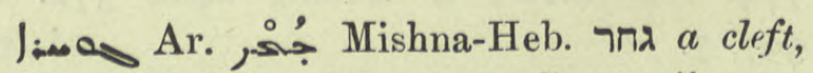
cavern, Theod. bar Khoni ap. Coupes ii. 145. I, 5 ; Nöld. WZKM. xii. 359.

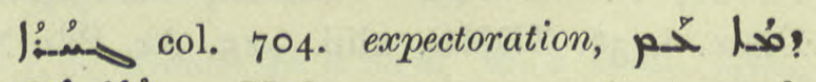

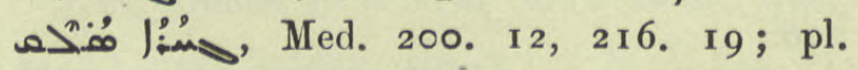

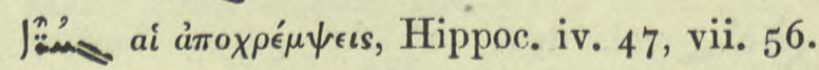

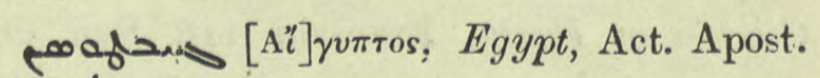
Apoc. lo. 6.

the Emir Jajak, son-in-law of Hulagu, Jab. 186. 3 and 3 af.

In perh. a suburb of Antioch or a neighbouring village,

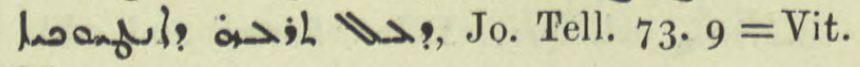
Mon. 87. 20.

罗 Add: to hew down, Is. Ant. ii. 270. II. Ethpa. on ib. 266 antep. 


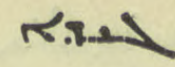

73

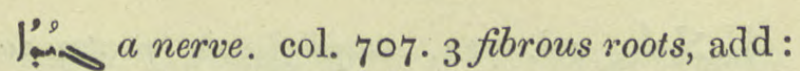

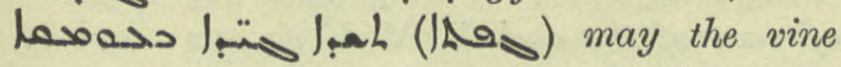
send rootlets downwards, Act. Apost. Apoc.

I 14.

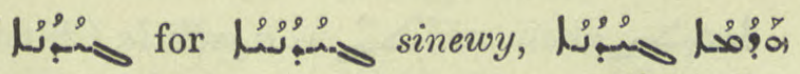
a sinew, Med. 26. 6; pl. 98. 2 ; $1 L^{\circ}$ م

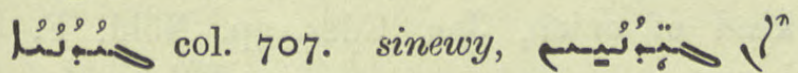
(น⿳⺈⿴囗十) , But. Sap. Econ. iii. 3 ; nervous, of the nature of a nerve, .

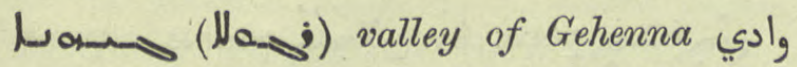
ج of Mt. Elpeph, now Jebel Maklub, Mar Bassus I 95.

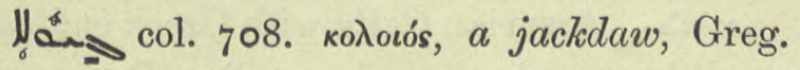
Carm. ii. 23 . 15 .

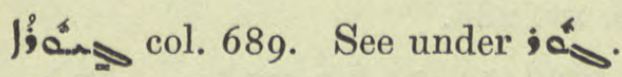

coung col. 708. end of par. Metaph. a Gihon = full stream, ILera> oo?, Is. Nin. B. $4^{6}$. I.

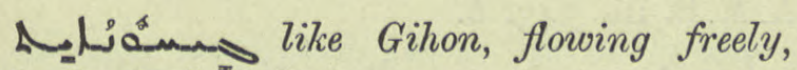

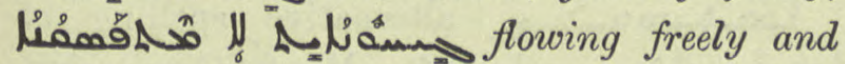
uninterruptedly, Chald. Brev. 424.

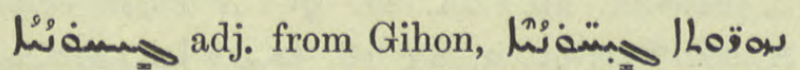
Lj, 50 Gihonic and Nilonic rivers, Babai 3 a 2 .

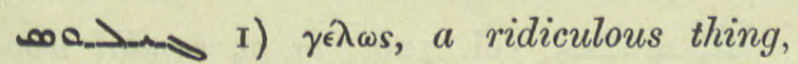
Greg. Carm. 100. 6. 2) col. 709. i.q. 1) and ${ }_{\text {S }}$ a monkey, Kal-w-Dim. 243. 9.

a the first part of this name must be a mistake, the context and note show that os. Jilu is meant, A.M.B. i. 4I7. Written a. $\mathrm{C}$ ib. pen.

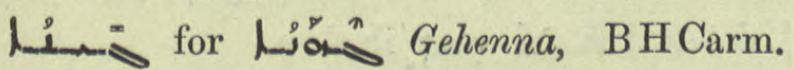
Proem. 3. 2.

إl Pers. soul, life. pr.n.m. Gina, Doc. Mon. I7 1. 6; 267. 4, 311. 2.

جمَهُ or jânavâspâr, soul-devoted or self-sacrificing, name of a picked corps of cavalry, Jesus-sabran 535 . I I, Nöld. in loc. WZKM. xi. I87.

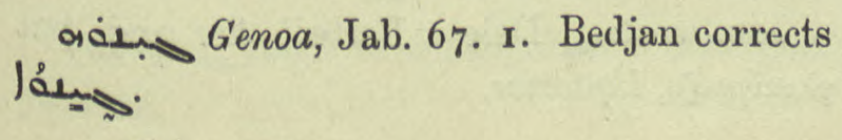

rals

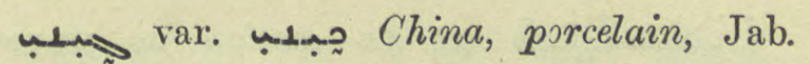
I 45 ult. and note.

en. $\rightarrow$ pr.n.m. Gaianos or Caianus, a Phantasiast, Sev. Ant. Vit. 286. I.

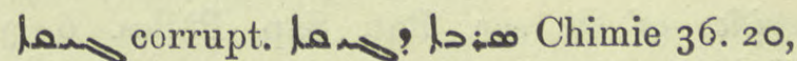
langib. I5. 6 where the Cambridge MS. has /AوI pitch; 289. II af.

lim col. 710 med. Add: ef. Ar. في R. Duval. Correct ref. BH. in Jes. xliv. I3. Jim for ib an arrow, Josephus vi. 5. 2.

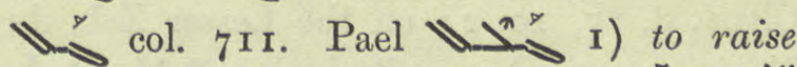
waves, make vibrations, hoزم ححبمى violent rains from the frigid zone agitate the air and cause wind, N. Hist. v. 3. I. 2) perh. denom. from $\prod_{6}>$ to dig a hollow for rain water, $\kappa \dot{v} \kappa \lambda \omega \phi$ 29. Ethpalpal

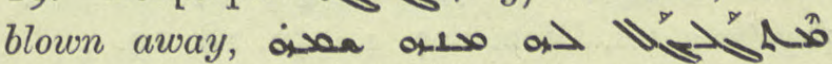
|lon if a king disregard justice the name of kingship will slide off him and leave that of tyrant, Jul. 34. 27.

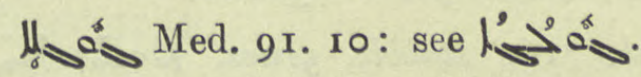

$\mu_{-\rightarrow}$ col. 7 I3. 4. I) the wheel, instrument of torture, A.M.B. v. 534. Add: 8) a stone rolling in a groove, to close a tomb, 9م , Act. Apost. Apoc. s. I3, Geiger in loc. ZDMG. xxvi. 800 .

هo the turning sphere of the heavens, $\mathrm{B} \mathrm{H}$ Carm. 36. I 2.

ر Chimie 50.20. Correct as MS. B. little rolls, twists or pellets.

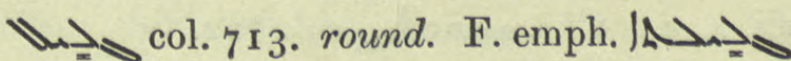
prob a round hut, דد דa Pallad.

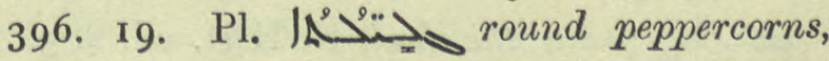

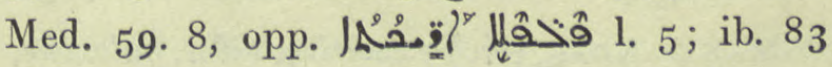
ult., 89. 18, 90. 2, 138. 7 .

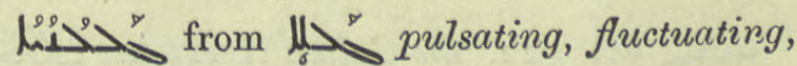
Med. 4I. I5.

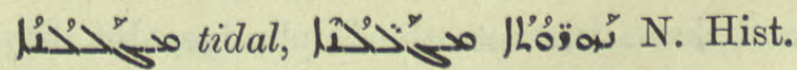
iv. $5 \cdot 3$.

1

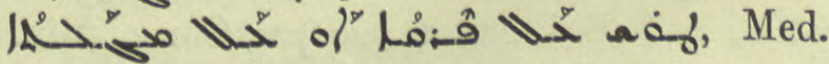
6r. 18. 


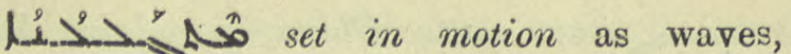

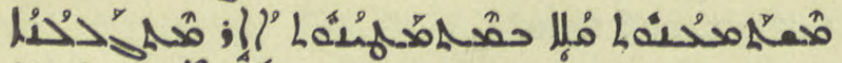

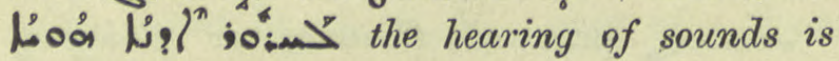
caused by the impact of air-waves against the orifice of the ear, But. Sap. Philos. 6. 3; N. Hist. viii. $3 \cdot 3$ bis.

رLُ N. Hist. iv. I. 2 ; of air, ib. viii. 3. I ; Philos. 6. 3 .

$\|_{\sigma} \rightarrow$ f. Gellala, a monastery in Qardu, Chast. 8. 17, ro. 2 ; on the Lesser Zab, Act.

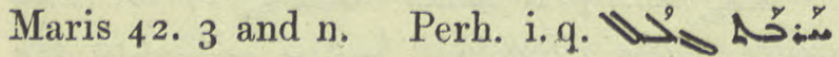
q. v. Pers. Mart. 26r.f.

$\prod_{0}$ Pass. part. col, 717, 1, 5 of par. a Bar Sud. 94.

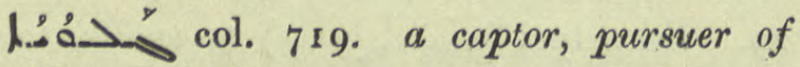
fugitives, ref. Dion. 127. 4, 152. 18, 19.

1ُ col. 720.6 of par. Ref.

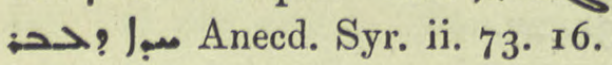

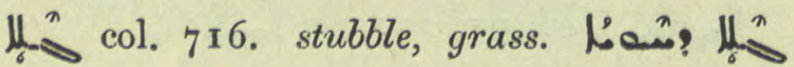
snake-grass, Med. 575. 7, $5^{8} 5 \cdot 13$.

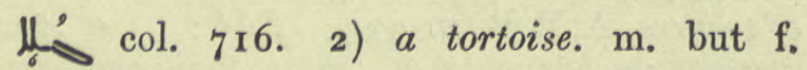
Kal-w-Dim. 75. 14. m. 1. 18; f. 76 several

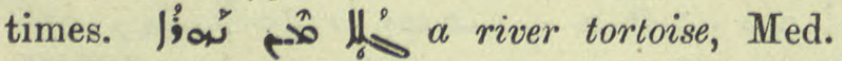
198. 15 ; a turtle, Chimie 6.17 ; حُص Natur 6o. 7, / Med. 86 pen., 87.1 ; 148, 6, 584. 13. A battle formation, testudo, -

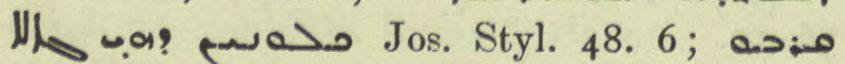
مa

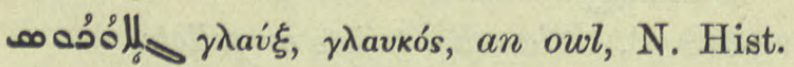
vii. 4. 3. Cf. 0000 ll col. 722.

ص .

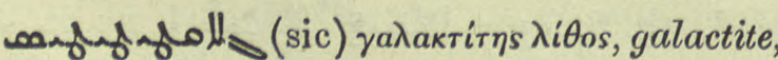
Chimie 8, 11 .

نُ col. 722. the Gallab river, Ar. to the East of Edessa, it runs into the Balikh and that again into the Euphrates, Jos. Styl. 57 antep., transl. $49 \mathrm{n}$.

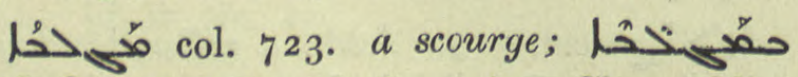
نح 247. I; BHChr. Eccl. 325. 3. cosioals

Lُ of part of the eyeball, Med. 83. 12; and so

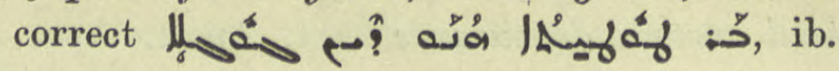
91. 10.

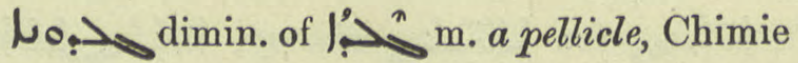
34. 9 .

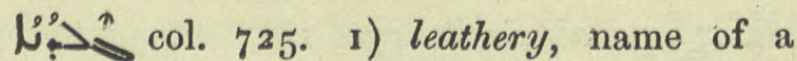
kind of onion, Jac. Edes, ap. Nöld. F. S. i. 575 .

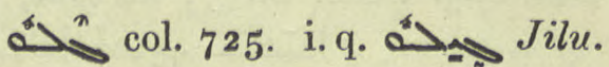

só a man of Jilu, Charms 31. 2.

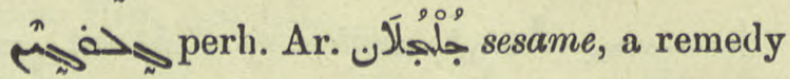
for scorpion sting, Med. 578. ×3. See Dozy, Suppl. 205.

م > Assyrian Gallu with Greek ending; a well-known demon, Charms 84 ; Protection 64, JAOS. xv. 288.

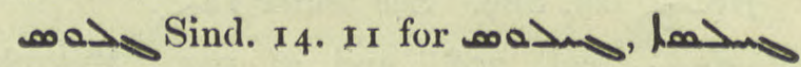
etc. an ape.

م. name of a river near Damascus, A.MI.B. iii, 343. 6.

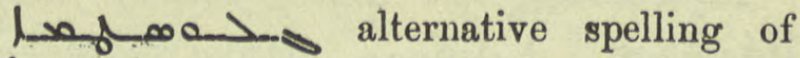

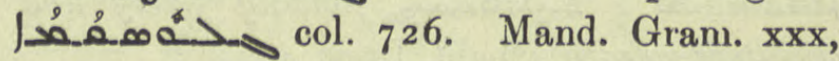
S. Fraenk. ap. ZA. ix. 9. A chest, coffin,

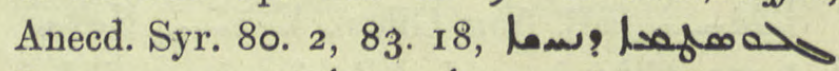

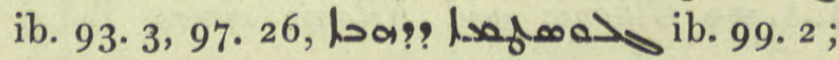
C.B.M. 491 a 23 .

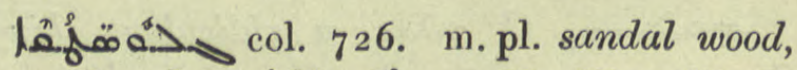
marg. "U. sect. 2.

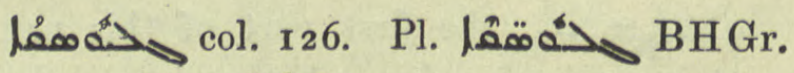

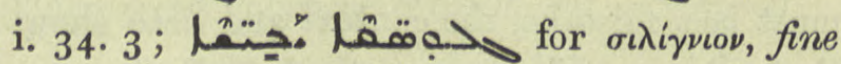
wheaten meal, Pallad. 131. 15, Med. 181. 6;

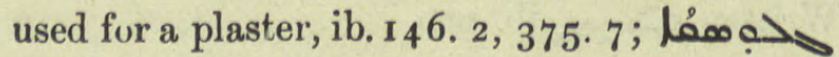
.

col. 727. glaucium, the juice of a plant, prob. the Horned Poppy, Glaucium corniculatum, Med. $8_{5}$ ult., I48. I4, I49. 6 ,

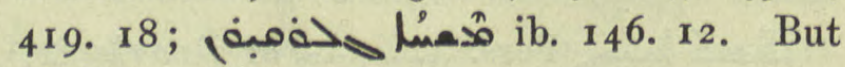
cf. next entry.

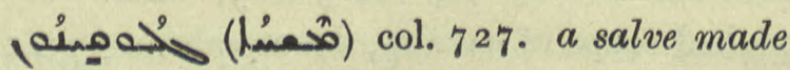
from must, add ref. Med. 53. 21.

D Da:Dele "Forte" \&c. and put

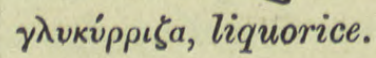


山

M. L Lơ one of Aristotle's three principles, BH. Hebraica iii. 25 I. I, Tim. i. ed. Braun 8;

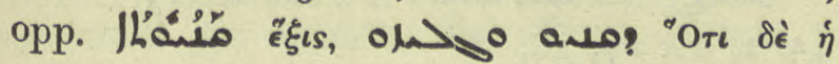

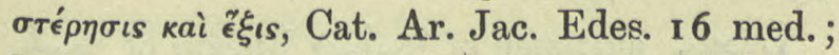

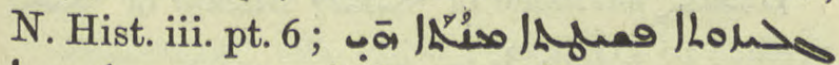

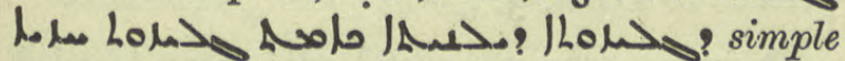
deprivation is a part of special deprivation such as the loss of sight, ib. Philos 2. 5 .

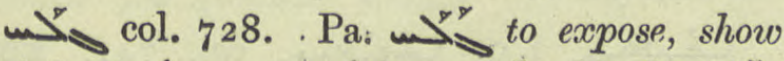

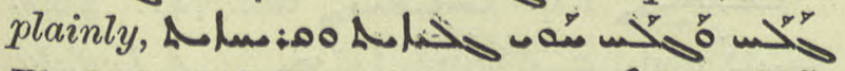

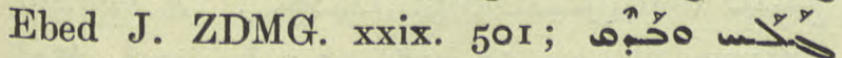

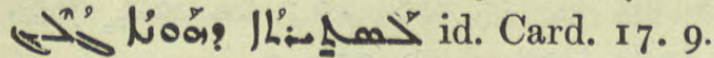

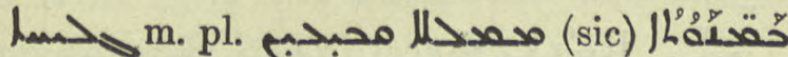
لهبma conjugations are set down and explained in the parts of speech, C. B. M. I $180 \mathrm{~b}$.

خ col. col. 729. Anecd. Syr. iii. i. 208. Io; Ahrens suggests ${ }_{\mathrm{A}} \mathrm{S}$ and compares

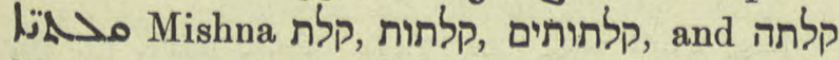
like кá $\lambda a \theta o s$, the panels of a vaulted roof, spaces between the ribs.

ํㅜㄴ mentioned in a list of heretics, A.M. B. ii. 150 .

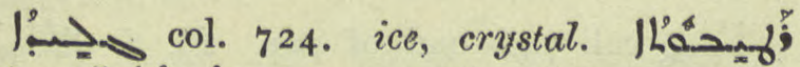
خ eye, Med. 68. I3, I5, 69. 16.

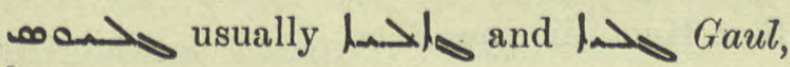
Jul. 7. 25 .

Darhaps Gallia, Gaul?

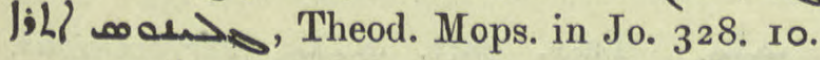

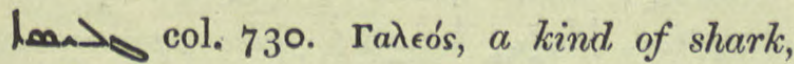
Ar. FischN. 74 .

in col. 730. antep. This quotation is from the Testament of S. Ephrem but Overbeck 1 43. 5 has $/$ jam, galearius. soldier's servant, camp-follower; ai- d-

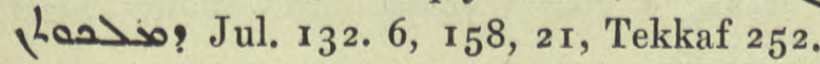

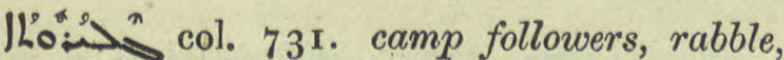
Is. Ant. i. 286.2.

lso> ool. 73x. One of the outlying parts of a monastery, perhaps a stone quarry,

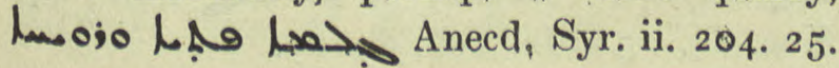
( $347.2 \mathrm{I}$

\section{rasad}

ال J $\infty \mathrm{L}^{\circ}$ ? Med. 580. I 2 .

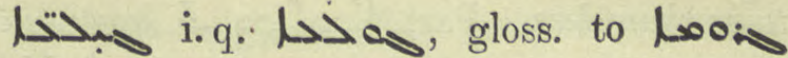
J:sol? BA. Cod. Goth. 2970.

a.> col. 732. I) Metaph. 900 strip off envy from your hearts, Nars. ed, Ming. ii. 397. $5 a$ af. Act.

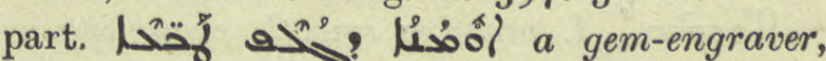
Pallad. 118. 6. 2) i.q. مخدص to shell, husk.

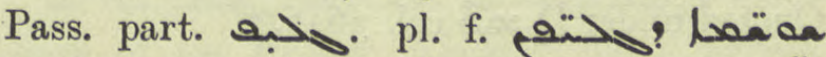

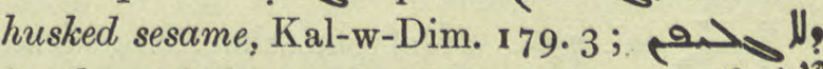
in the pod, 1. 4, 181. 6, 8. Ethpe. 20 4$\}^{n}$

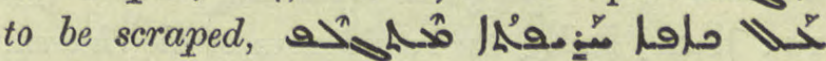
sandalwood scraped againșt a sharp stone Kal-w-Dim. 22. 19.

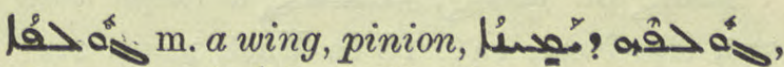

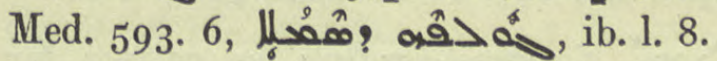

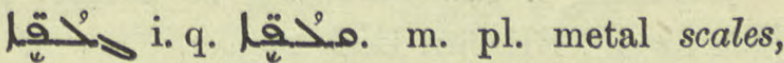
Chimie 257 n. 2,258 n. 2.

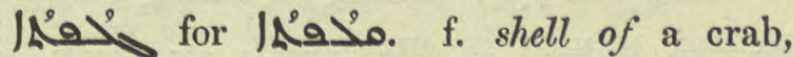
Natur 5 r. 4 .

Hiف교 col. 733. a feather, a wing; لُ Med. I63. Idiom or proverb دَّ

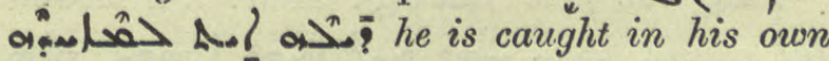
feathers, Sev, Lett. 14.

5 col. 733. Pa. 5. contract, wrinkle, Nöld. ap. GGA. 1882, p.

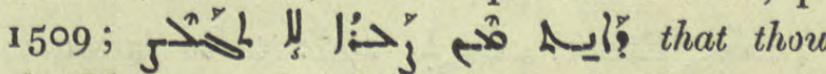
shouldest not shrink from insult, Ephr. ed. Lamy i. 28 I pen.

Ry col. 734. to lop off, cut down to the roots. Add refs. II J Jos.

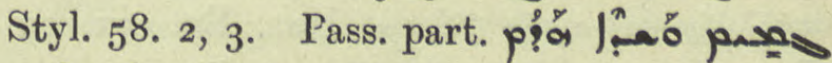

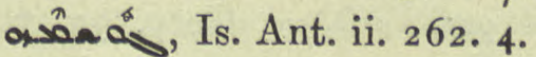

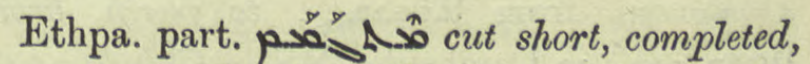

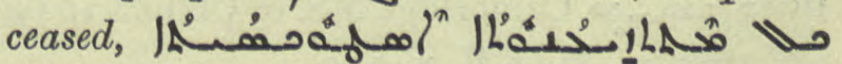

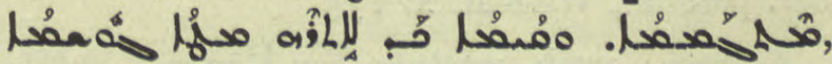
N. Hist. iv. 4 .

| lä? $\}^{\prime}$, N. Hist. v. 5. 2.

Los rá $\mu \mu$, letter $\gamma$, C.B.M. $594 a$.

جمهادى Gumada, an Arab month, | 
מم- col. 735. Add: to press smooth, |

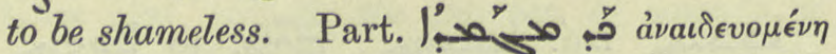

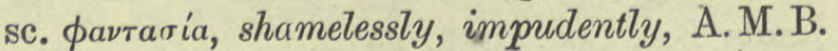
v. 158. 2. Aph. :impudently, Sev. Ant. Vit. I02. 8; Stat. Schol. Nis. 25 . 18.

L'ơ์ clothes, Op. Nest. I 59. 2 I in Eph. v. 27. Quoted under fُ مُ

llos col. $73^{6 .}$. II of par. the King-beam,

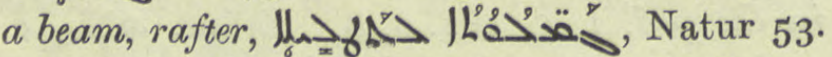
I2 ; /hب $\rightarrow$ ! ll Brief Xti 108. 18. 8, 22.

لl pl. ant, Op. Nest. 146. 18 in Matt. xxiii. 24.

$\mathrm{J}^{\prime a} \Delta \mathrm{a}^{\prime}$ f. camel-herding, so correct for IN⿴囗大), BH Chr. Eccl. 263.2.

W feeling, nausea in pregnancy, N. Hist. vii. 5. 2 bis.

امحُ f. a handful, cf. Ar. Chimie 99. 10.

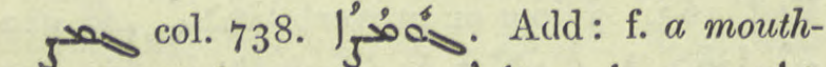

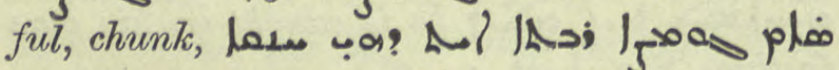
a) he put a large chunk in his mouth and perhaps it was that which choked him, Kal-wDim. ed. Bick. 85.7 .

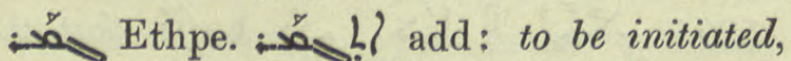

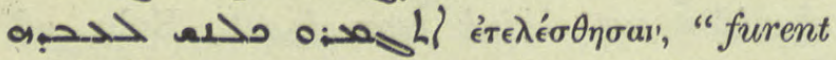
initiés," Coupes ii. 1 28. 23, Cosmog. Manichéenne 26 n. I. Pael מمّ col. 739. 3) add: c.c.

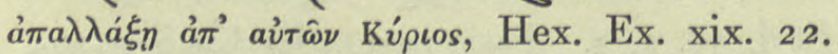

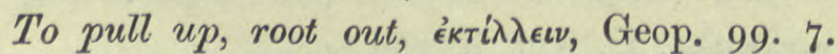
4) denom. from Jlisases. to parch, broil, م- Cambridge MS. of Chimie $104 r$ and passim., WZKM. x. 135 .

f: $\infty$ ay prob. mistake for l'ión fulfilment, opp. ILلl a figure of speech, h:sos of الم 1 o, Ephr. ed. Lamy i. 555. 20 " praeferenda est realitas figuris."

I'; inflamed pustules, on the eye, Med. 77. 21 ; 1.

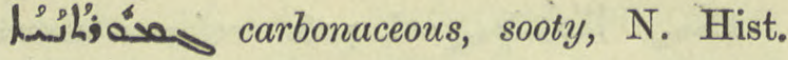
v. $4 \cdot 3$.

1"x ? 45. 2, 61. I7, 18 .

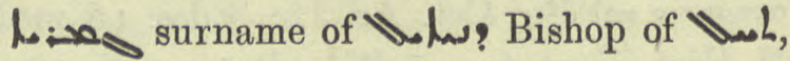
preface to DBB. 3 . 5 .

公 a mode of writing practised in that monastery, El. Nis. 96. 6.

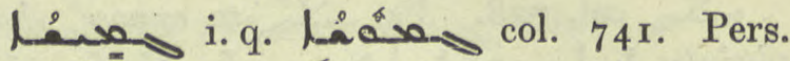

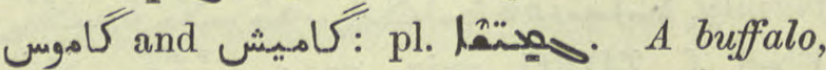
Charms 99. 9, I5, I8. 20 ; 1. buffalo bulls, Gest. Alex. 2 I 2.2.

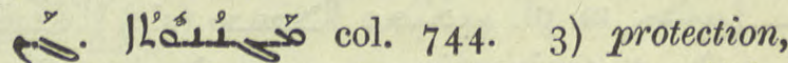
Anecd. Syr. ii. 245. II. 4) continuance fُóa

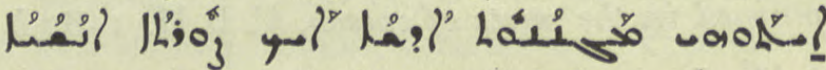
existence is the continuance of the species such as the human, N. Hist. iii. cap. I.

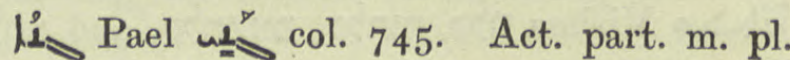
c. c. $>$ of the pers. bring blame and shame, Josephus vi. 7. Iо.

lُứ col. 746. 3. Add: a couch, bed, Hist. Mon. i. 226 ult.

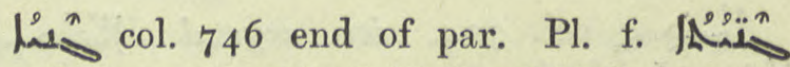

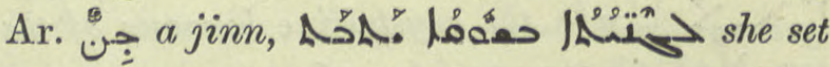
up images of jinns in the market-place, Jac. Sar. Hom. iii. 799. 8.

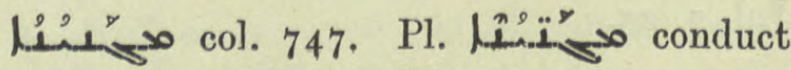
deserving blame, Kal-w-Dim, I 30.

P. Pass. part. deceptive, Pallad. 99. 3, 202. 19.

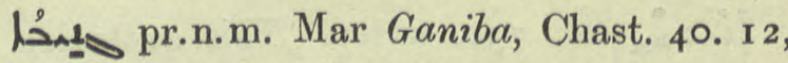
55. 14, I5, 56. 7. Protection 52. 8.

هي

مبْنُ a round vessel, Lexx. under col. 1680 .

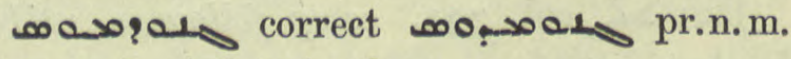
Ganymede, Arist. Apol. «. 3 .

lsoa.1 col. 750. Add: mark, character,

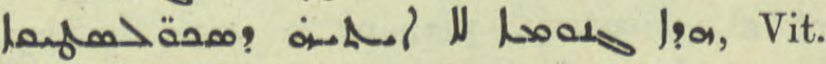
Mon. 84. $25=$ Jo. Tell. 69.19.

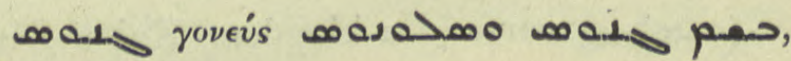
Charms 10. 19. 
canefeall

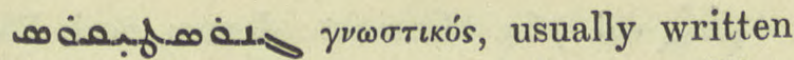
with $L$. Having the powers of a seer, Hist.

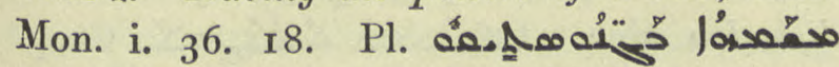
celebrated for wisdom, Bar Penk. i. note 3 =Journ. As. 1907, 169.

ח. An inventory, C.B.M. I. 23 b.

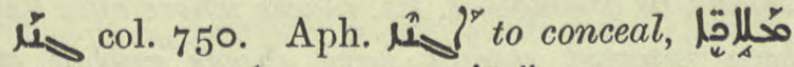

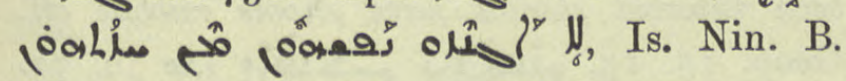
66. 5 .

Ju1 col. 751. a mysterious being, a spirit, lsos? the the spirit of the sea, Kal-w-Dim. ed. Bick. 25. 1, 18, 19, spirit of the air, 11. I7, I9; jof? / / s ib. 74. 21 .

y Maragha in Azerbaijan, Phet. 30. 4, Pers. Mart. 248 sqq., n. 564 , ZDMG. xliii. 398. I4. مُ Sapor I, M. Z. 34. 46 .

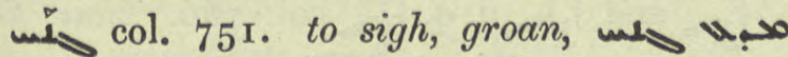
his mind was sorrowful, Cyrillona 566 antep.,

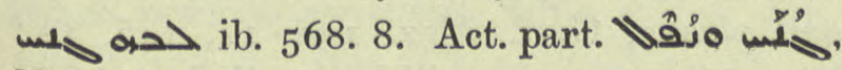
Natur I0. 8.

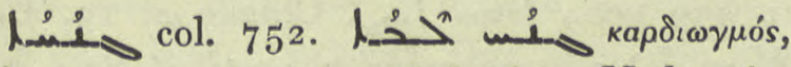
Hippoc. iv. 18.65 , palpitation, Med. $26_{2}$. I 2, 2 I, 263. 5 \&c., BH. de Pl. ro9. Metaph. terror, horror, Anecd. Syr. ii. I9. I4.

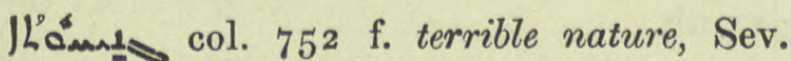
Lett. I 43. 12, Anecd. Syr. ii. 54. 12.

llofs and /

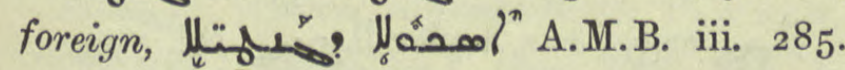

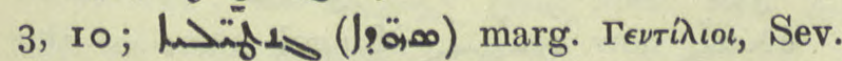
Ant. Hymns 153 tit., C.B.M. $33^{2} a$.

lifíg col. 753. Gentiana, gentian, refs.

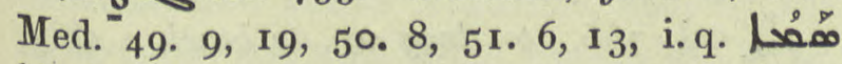

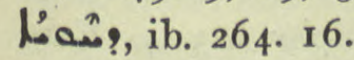

un pr.n.m. Gani, founder of a monastery in Beit Aramaye, Chast. 8. 14, 17. 20.

年,

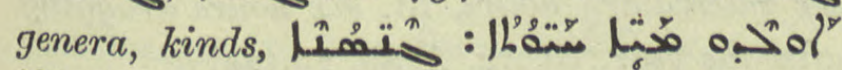

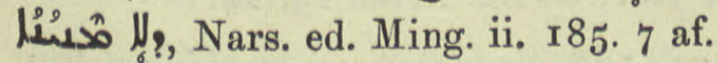

mi col. 754. denom. verb Pael conj.

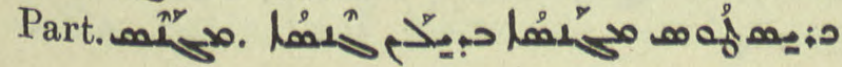

Christ our kinsman, of our race, Poet. Syr. 98. I5.

ILْفُ

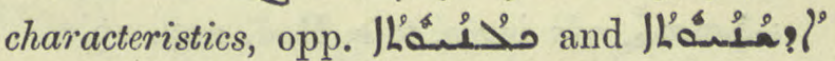
specific character and universality, But. Sap. Philos. 7. I.

Kล์ Sap. Philos. 2. 5 .

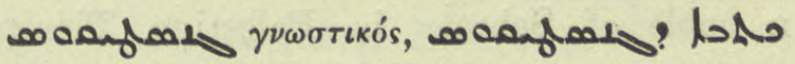
Book of the Higher Wisdom, by Evagrius, C. B. M. $445 b, 770 b$.

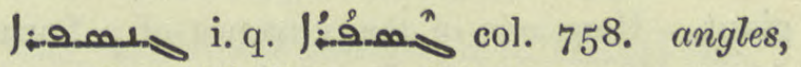
sharp edges of hailstones, Dion. I 94. 20.

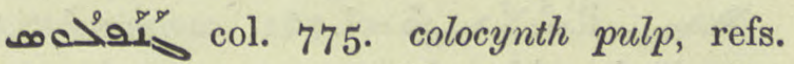
Med. 5 ०. 5,5 1. $23,5^{2}$ bis, $136.20,137$ bis. Cf. under låg above.

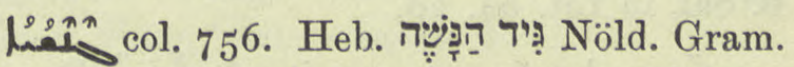
$\S \mathrm{I} 42$. The sciatic nerve; sciatica, Hippoc. iii. 2 I, vi. 56,57 , Med. 48 . 19,5 o. 5 .

Aـ Janiyeh, a village 15 miles NNW. of Jerusalem, Pléroph. 39. 8 and n., R.O.C. iii. $25^{2}$. I.q. LLA ib. 23. 10 and R.O.C. xiii. 186 in loc.

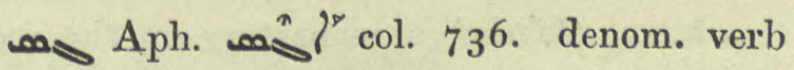
from $\lim _{n}^{n}$ the side, R. Duval. To lie down, recline, add: asam and he took his place on a couch for supper, Act. Apost. Apoc.

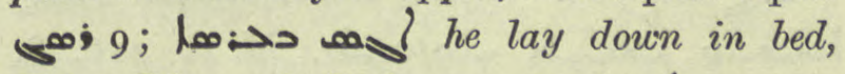
Kal-w-Dim. ed. Bick. 73. 8, An نـ 1.9 ; Anecd. Syr. ii.

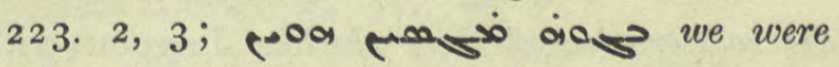
lying down indoors, Jos. Styl. 29. 10.

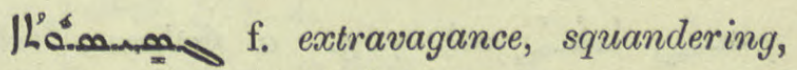
Syn. Or. 226. 5. Cf. لقر riches col. 757 2).

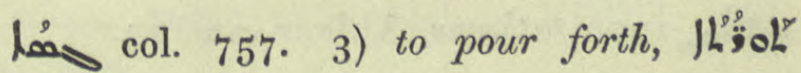

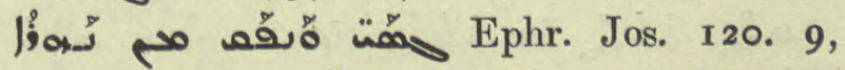
I25. 7. Pa. ".

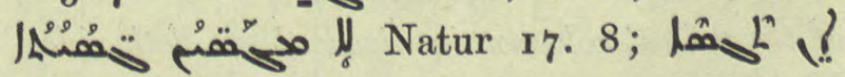

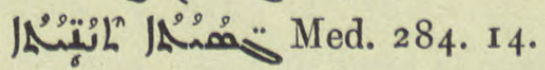

1ُ

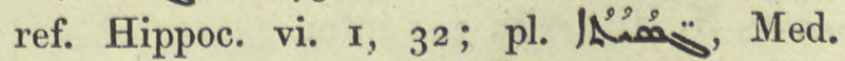
284 bis, mis-written /Aقמ 1. 8; Ephr. Ref. i. $28.9,15,24,47$. 


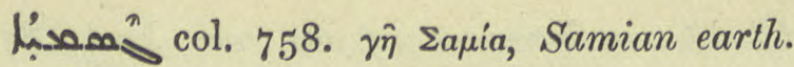
talc, Med. 207 ter, 208 bis, 209. 2, 4I3. 2,

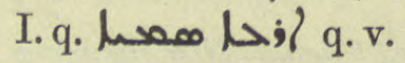

بd

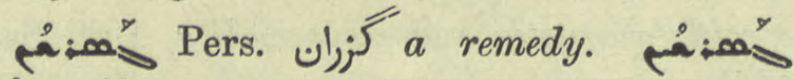

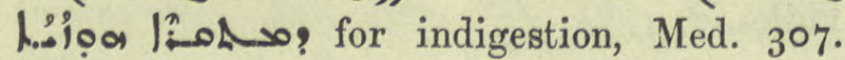
I $7 / 18$.

a $>$ Sáïos or Taüavós, Caius (Nau), a noble of Sebaste in Palestine, Pléroph. 127. 8.

حص probably Gyas, a hưhdred-handed giant. Here one of many names of a female demon, Charms $84=$ Protection 64 .

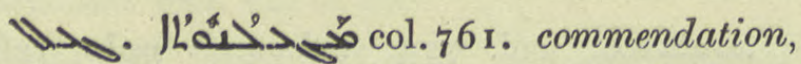
committal, add: Apis. مس. 12; the commendatory prayer at the end of the liturgy, B. Sal. in Lit. 94. 20.

po col. 761. to vomit;

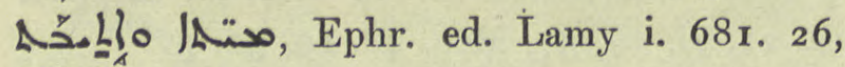
BHCarm. I35. 7 .

1.ำ col. 762. vomiting, retching. Pl.

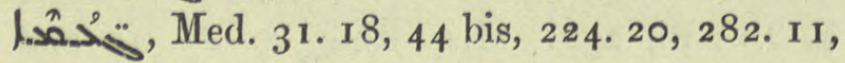
289. 8.

$\omega\lrcorner$ Gaitani, a monastery on the road to Tagrit, built to succour travellers, Patr. Or. iii. i. 32. Io, I r.

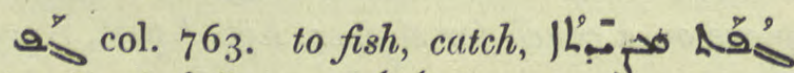

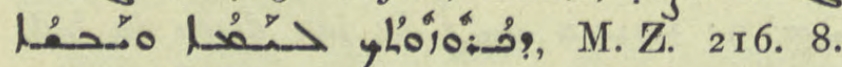
Aph. $\theta^{n}$ to set close together, cf. N. Heb.

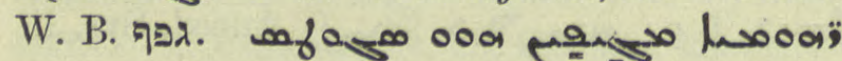
the soldiers locked their shields, Josephus vi. 24. II.

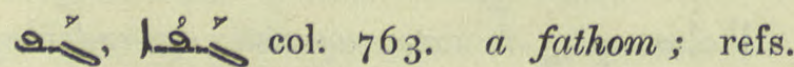

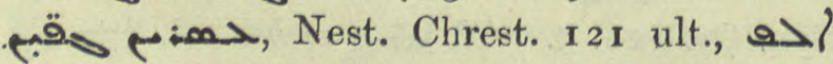

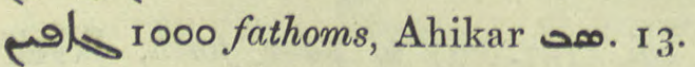

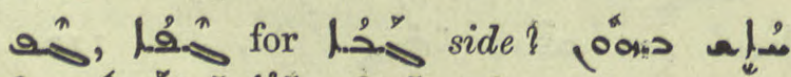

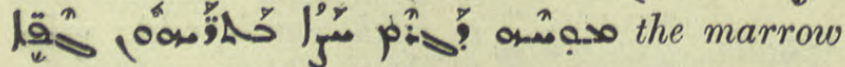
of the spinal column is diseased on both sides, Med. II3. II.

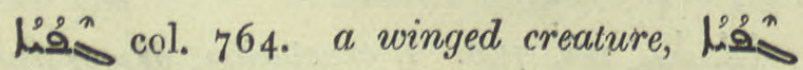
๘

1208 Gapitha, a village of Marga, M.Z. I 87.5 .

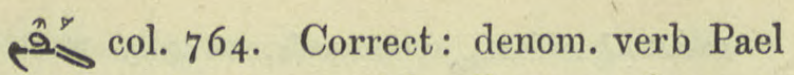

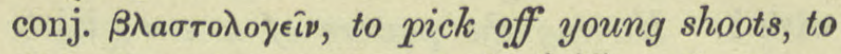

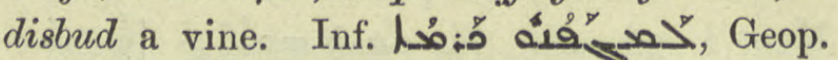
I4. I I ; to disbud an olive, 1. I8, 35. I 2 ;

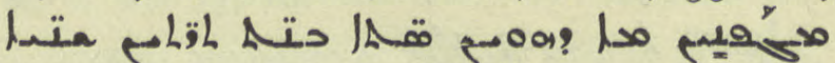

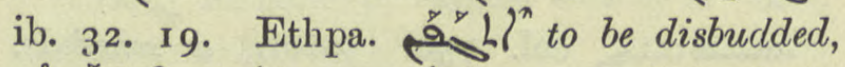

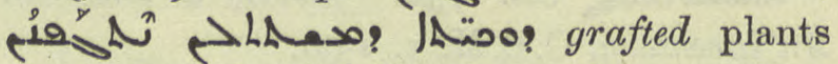
should be disbudded, lit. places where eyes have been inserted should have shoots rubbed off,

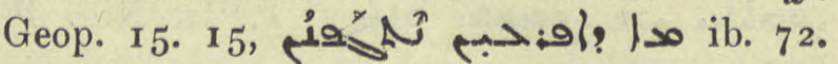
I6, 8 r. 28.

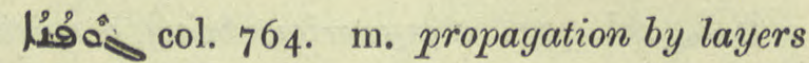

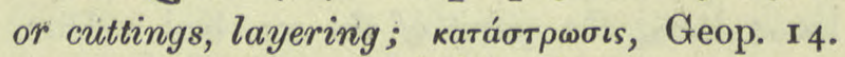

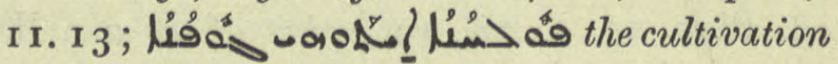
of vines is by means of layering, ib. 81, 27, 28 . But the word seems to mean disbudding,

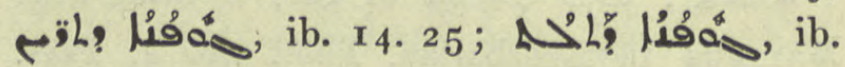
I5. I 2, 32. I 9 .

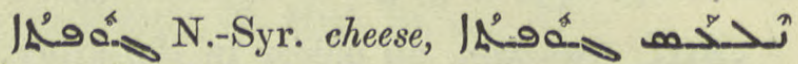
|حم

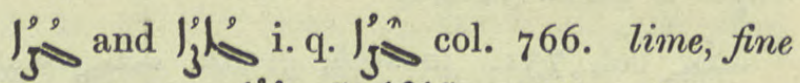

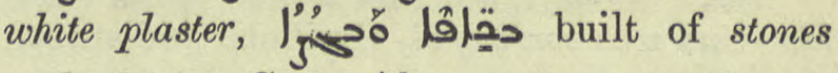
and plaster; Gest. Alex. 138. 14, 146. I2; Hist. Mon. i. I 24. 9 ; l'

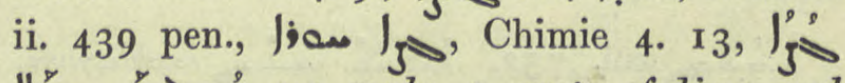

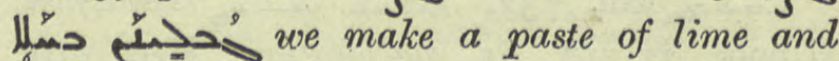
vinegar, Med. 65. 12, 602. 20.

1 IJ plastered? Name of a church, Jab. 2 I 5 ult.

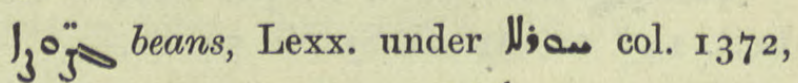
under e.

Ihys scab, Lexx. under ILjois, col. 779.

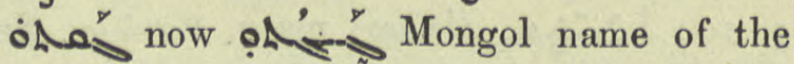
river Jagatai, the Persian name is 9o: 50 , Jab. I 5 2. 4 af.

$\therefore$ col. 766. to drag up, to beach a ship.

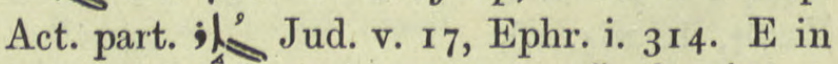
loc. To drag at, to tear, add: مै

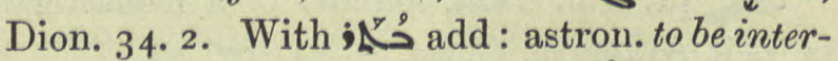
calated, inserted, مخل o six intercalary hours, lit. six hours dragging after the year, Georg. Arab. 25. 24.

's:- col. 768. dragging up, beaching a ship,

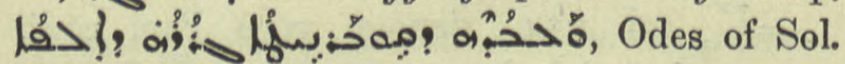
ii. 284 . 


$$
\text { Nis }
$$

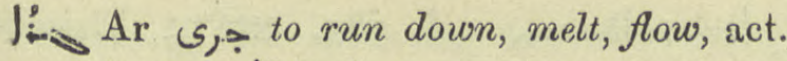

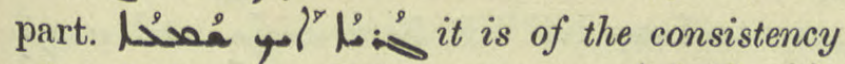

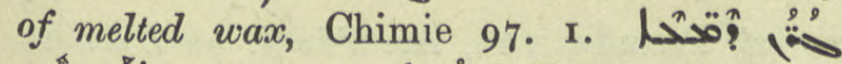

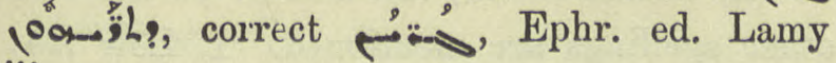
iii. 193 .

$\stackrel{f}{\circ}$ col. 769.5 of par. to urge, incite,

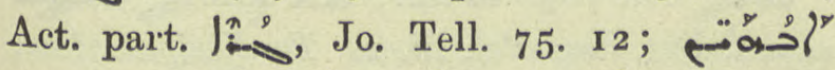
00ā 762. 15.

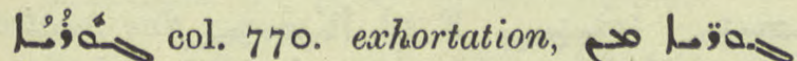
b/ م: "Paraeneses Mar Abae," Ephr. ed. Lamy iv. 88. 7 .

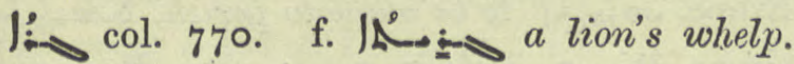
Pl. f. $/ L^{\prime} 8 \%$, Is. Ant. ii. 328.5 .

D Aph. leprous. Causal ast? the covetousness of Gehazi made him leprous, Ephr. ed. Lamy iv. 237. 4 af.

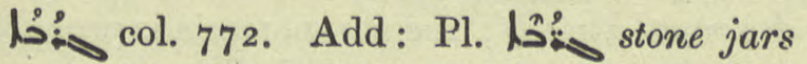
for oil, Jac. Edes. Hex. xliii. 5. Perh. Ar.

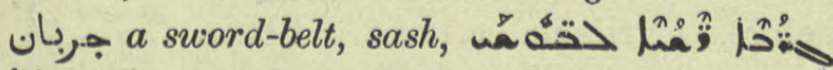
مُ I 43. 9, I52. 5. But cf. bias col. 690 .

(ד) $\rightarrow$ name of a village, mentioned soon after Beit Aphthonia, Doc. Mon. 200. I8.

ज. col. 773. Pali un to clash, knock, (000 birds clash their beaks, Caus. Caus. 255. 2.

Lias s col. 775. r. m. pl. Gregorians, i. q. Julianists, BHChr. Eccl. 299. I 8.

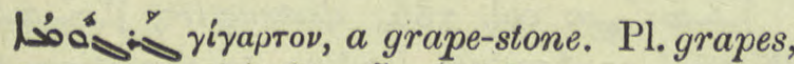

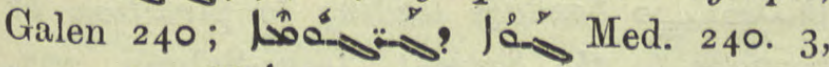
24I. I6. Cf. lsoo: $\rightarrow$.

hoas Arab. جَ the people of the Lebanon, now called Maronites, and, by the Byzantines, Mapoaîa, Nöld. ap. ZDMG. xxix. $8_{2}$ n., Sachau Hist. Geogr. Syriens ii.

J's col. 775. Eruca sativa, a colewort,

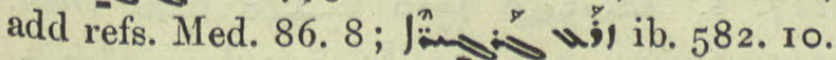
Ar. جرجي ib. 603. I.

nasay $\rightarrow$ pr.n.m. Pers. A.M.B. iv. I 45.

$\stackrel{\circ}{\circ}$ col. 776. Imper. 90-s scrape off, Chimie 38. 7. 51. I. 2) To dissolve, 2ح- ? ?

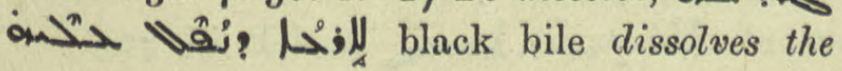

earth on which it falls, Med. 13. I3; J:م;

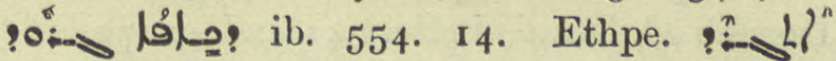
col. 777. I of par. Dele $\Delta$ the verb is $9 \rightarrow-3 L$.

(D)

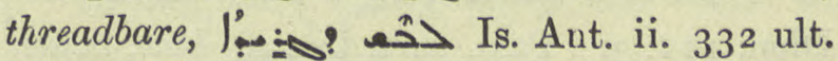
Articulate, distinct, clear, $\|^{n} \boldsymbol{L}^{n}$ he could not articulate distinctly and

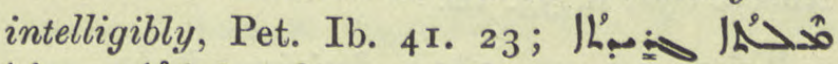

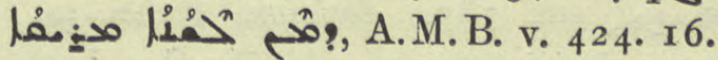

A. 42. 16 ; Anecd. Syr. ii. 27 O. I.

هُ Med. 406. ro; lå: ib. ter, sing. ib. bis, pl. 407 bis.

IL? col. 777. Add; 3) a scrap, scraping,

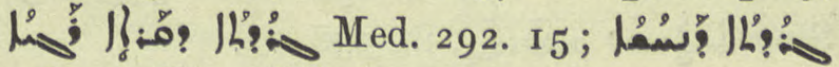
a brass filing, Chimie 240 n. 2.

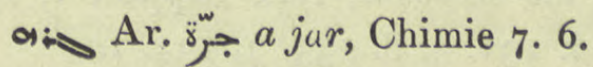

ک

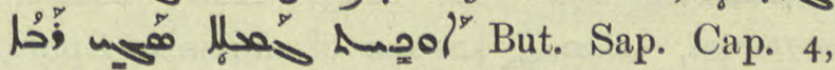
sect. 3 .

Le? name of a monastery near Antioch, Anecd. Syr. ii. 227.13.

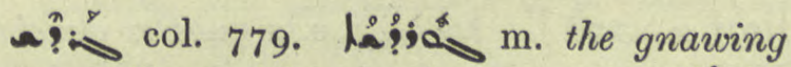
or crunching of bones, Lexx. under רא col. 2987 .

1L'án f. the gnawing or crunching of

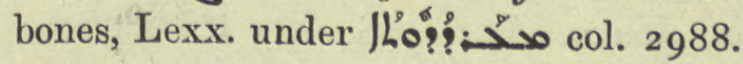

J!o: a rake or poker, مخه مه Kal-w-Dim. 276. 25.

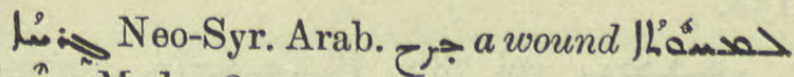

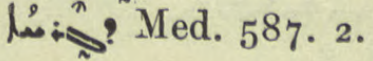

ofis pr.n.m. C.B.M. $906 b$.

$\leftrightarrow \rightarrow$ name of a bishop, ZDMG. xliii. 395 infra.

جريب from 780. Ar. Ar. Pers. اريو اريب El. Nis. 58. 46 ; Lag. Arm. Stud. $\S 536$, id. B.N. 45. a measure of capacity, then the piece of land sown with that measure, Tabari $242 \mathrm{n.2}$. It is equal to I $/ \mathrm{h}^{\prime} \mathrm{l}^{\circ}$,

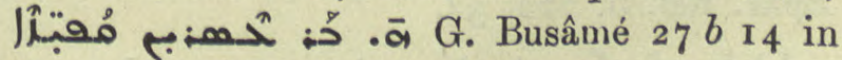
Gen. Cf. BH. in Hos. iii. 2, Thes. Syr. and Suppl. under ;o. 
lasidi.q. גריוא, גריבא Isoyabh ed. Duval, Letters $4 \mathrm{I}$ and 47, 70. 26, 92. 3, ed. Moncrieff 66.6 af., 87. 12.

p col. $78 \mathrm{r}$. to pay a غرامة fine, mulct,

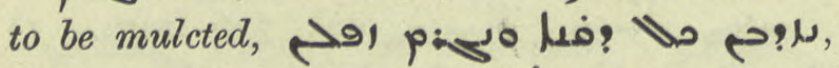
Dion. III. 3. Pass. part. Lمُ

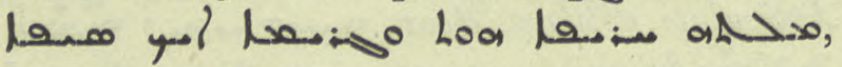
Anecd. Syr. ii. 53. 10.

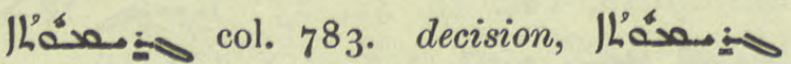
?חס 103. 14 .

pis, 100:

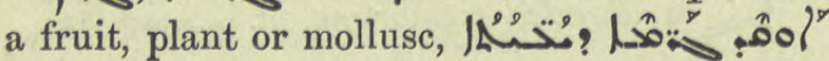

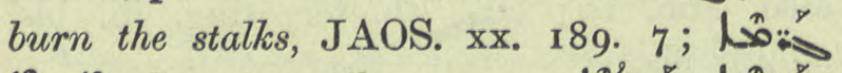

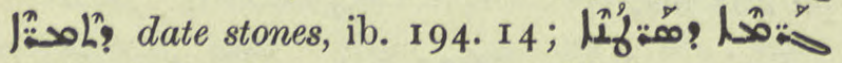
crab shells, ib. 193 antep., Med. 553. 13.

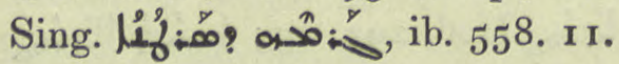

$f_{5}^{\prime y} p^{n} \rightarrow$ the backbone, refs. Hippoc. v. I5, vii. 33, Med. 132. 5, 8, 133. 7, 134. 10.

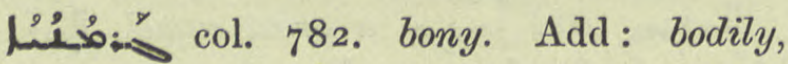

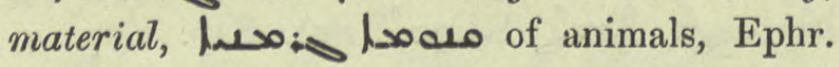
Ref. ii. 19. 22.

1. a scruple, equal to four carats, Chimie 69. I2, لأ i. e. a quarter drachm,

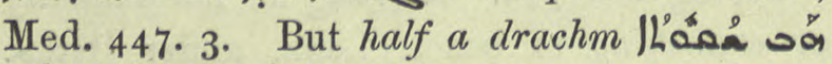

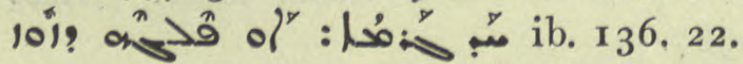

exis name on a mosaic tombstone at Edessa, Journ. As. 1906, 283 ff. Cf. Gr. rápuos and the latter half of the Edessene name pisa $\Sigma a \mu \psi(\gamma \in \dot{\rho} \rho a \mu o s$, ZDMG. xxxvi. 158.

andois col. 783 . Pl. andors writings of debts, Anecd. Syr. ii. 8. 8.

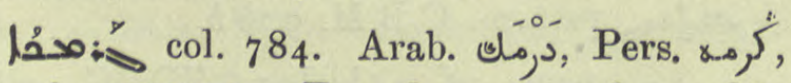
Mand. גראמכא, Fremdw. 33. fine white

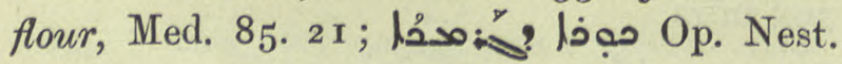
97. 12.

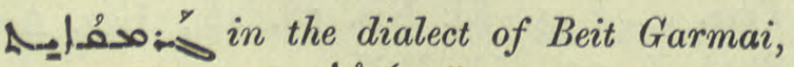

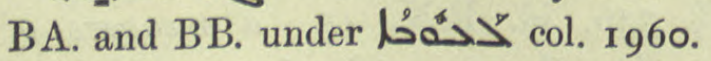

(حمل) $\rightarrow$ the dialect of Beit Garmai, Ephr. Ref. ii. 222.34 .

جرمقاني Arasshopper, Dozy, gutter-snipe, BHChr. Eccl. 337. 7 .

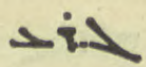

גוריתא . Fleischer note to Levy NHWB. Pers. قِريث an eel, Fremdw. I21, Ar. PfinN. 96 f. where other spellings are mentioned. I. Löw ZA. xxii. 86. Pers. مار ماهي Med. 603. I.

col. 785. to tear, ${ }^{2} \boldsymbol{A}^{n}$ 个:د', But. Sap. Pol. iii. 2. To dissipate, lose, معسل ?פח حه > Anecd. Syr. ii. 40. 3; Ephr. ed. Lamy ii. 465 . 3. Pass. part.

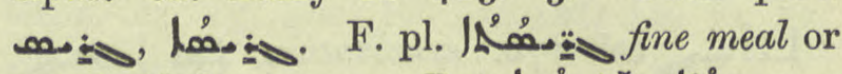

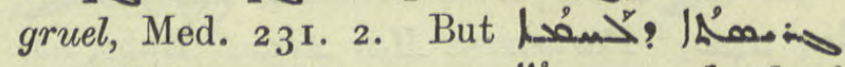
A.M.B. i. 402 . 10 correct $1 \mathrm{~L}_{\mathrm{S}} \mathrm{s}$ a flat loaf.

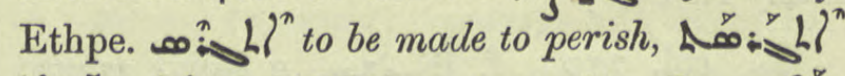

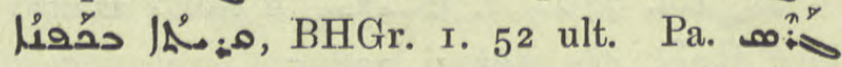
col. 786. 1. 2 of par. correct to part. fem. ט thou destroyest thy whelps, BH Carm. 102. 9. II. denom. from loois to become a snake, a destroyed himself; the paronomasia is probably intentional as the word ló: is used just before, Cyrillona v. 268. Ethpa. क्रु ? $^{n}$ denom. from ló: $\rightarrow$ to become a serpent, Tekkaf. 36. 187 .

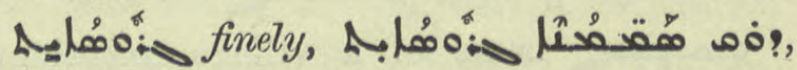
Med. 142 bis, $3 \circ 3$. 15 .

مقد

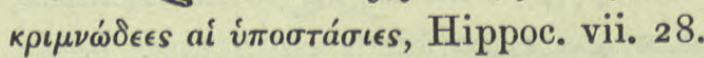

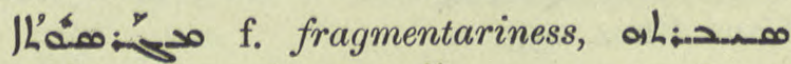

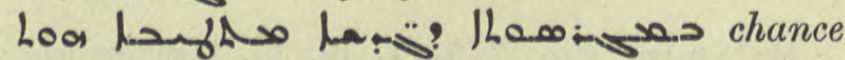
broken victuals, scraps, Jesus-Sabran, 522 . 14.

حَح | $>$ bondage leading to perdition or bondage to the Serpent, Jesus-Sabran, 507. I3 af.

Afoos prob. ä $\gamma \rho \omega \sigma \tau \iota s$, triticum repens, couch-grass, Jos. Styl. 4I. I3.

u. col. 787. Pass. part. 25 of par. a lad, servant, slave, Kal-w-Dim.

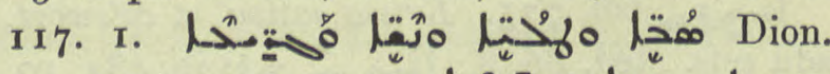

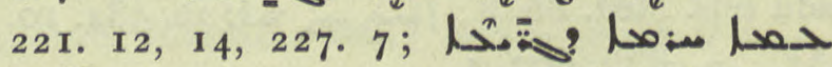
管? les miscréants Arabes, ref. to Jer. xxv. 23 , ib. III. 6. Shaven, tonsured; Jacobite monks, ib. 76. I, C. B. M. 945 b, Hormizd 2445,2463 and passim. Used of other monks A.M.B. iii. 404. 


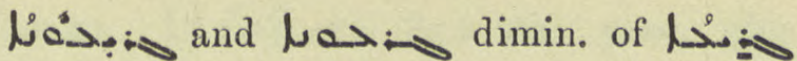
m. a lad, servant, Dion. 2 I3. 2, Lesi-

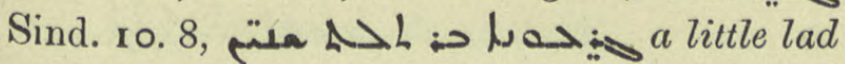
of three years, ib. 24. 3 .

ILo' $\rightarrow$ f. col. 787. collective noun, Jacobite monkhood, C. B. M. 945 b.

I'áas f. shaving, the barber's art, Kal-w-Dim. 33. 22.

1

๑

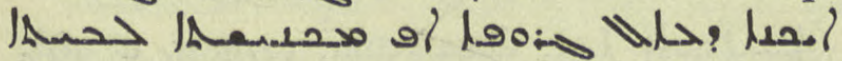

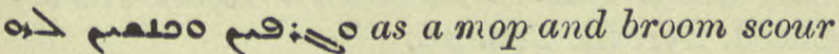
and sweep a house, Dion. I I 2 . 18.

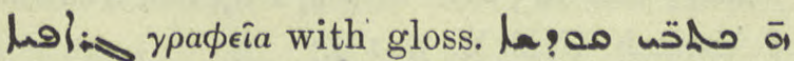
Hagiographa, Epiph. I I. 23.

L Journ. As. 1906, 280. 7 .

Li; of Gerar, Aim y yand, Ephr. ed. Lamy iii. 193.

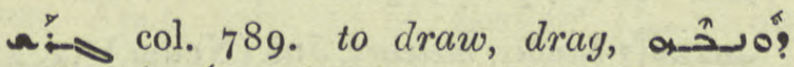
|

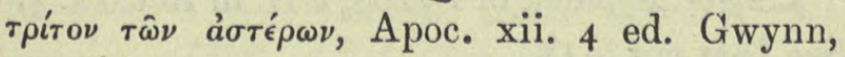

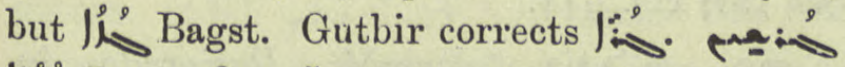

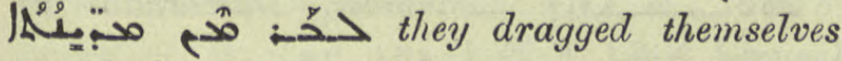
outside the plague-stricken towns, Dion. 42. 20. With lo متمْ to haul wood, ib. 87. r.

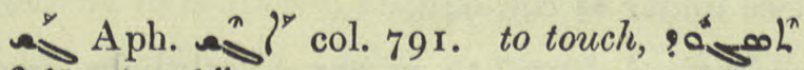

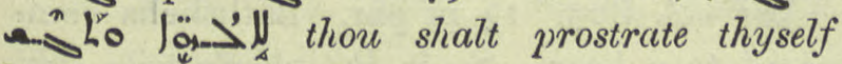
before the gods and touch the ground, A.M.B. ii. 58 r. 1 4. Palpel ${ }^{n}{ }^{n}{ }^{\prime}$ to grope,

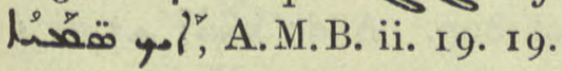

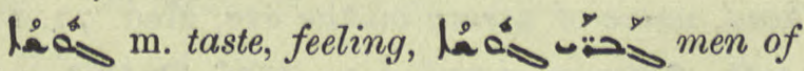
taste, the wise, BHCarm. 257. 5. Ar. ذوق.

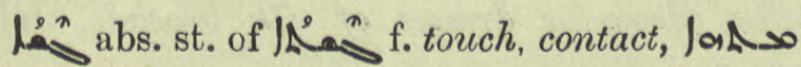
م001 Lestrains the evil in himself from action, Kal-w-Dim. 236. 2.

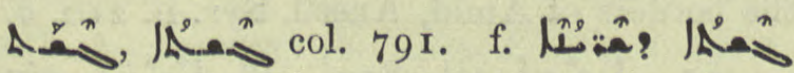
feeling the pulse, Med. 1 28. 16, 259 five times.

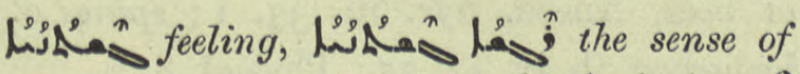

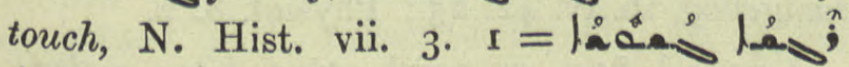
ib. ii. v. sect. 4 . rodds

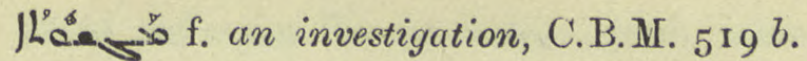

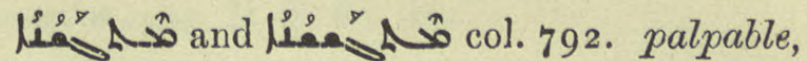

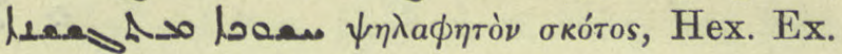

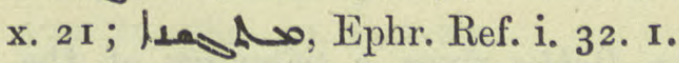

looa marg. $j$; arsenic, but Duval says "poix," Chimie $3^{6.22 .}$

wh col. 792. to skin, fluy. Add: Aم

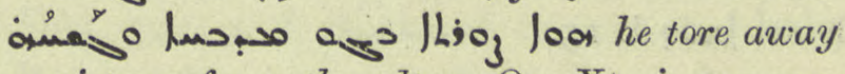
a picture from the altar, Or. Xt. i. 94. 10. Ethpa. wa 4$\}^{n}$ to be cut or torn away, as

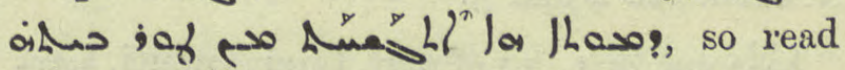
for An ${ }^{\prime} l^{n}$, Nest. Funeral Service, Hebraica, iv. 194.8 .

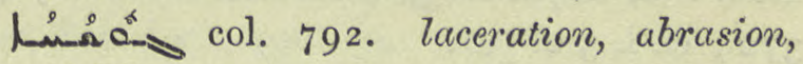
-

Na.o. Ar. Á honey-sweet; "homme mielleux," Martin Métrique 54.

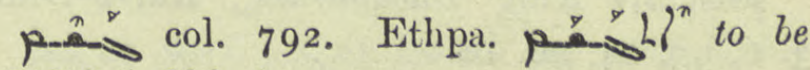
solidified, Chimie ro. ro.

A corporeally, materially, Is. Nin.

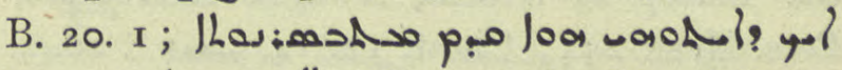

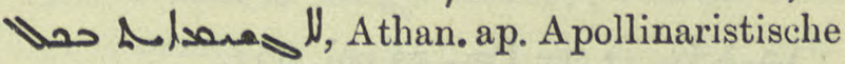
Schriften $45 \cdot 8$.

par. Ar. name of a village and monastery, Nöld. ZDMG. xxix. 429, C.B.M. $709 b, 710 b, 714 a$, Doc. Mon. 213. 22, 215. 1 $4,216.5,16,224.6$.

in col. 795 ult. Correct ref. 2 Sam. xix.

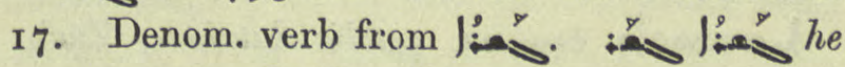
constructed a bridge, Gest. Alex. 206. 2, but

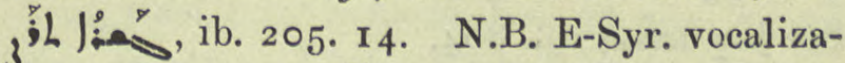
tion. Ethpe. : $12^{n}$ to be bridged over, fosoa هم: Ephr. Ref. i. 97. I 8.

lig or limeng rétvpa, Gisra, an estate two miles from Tripoli, Pet. Ib. 109. 23.

arts Zend gaetha. the world of living creatures, A.M.B. ii. 576.16.

wS col. 796. name of the LA: of Simon Magus, Coupes ii. I I 3 .

lhs i.q. IA, a village belonging to Eudoxia : see above. 
1: the Dabha river, a tributary of the

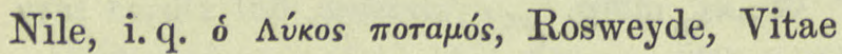
Patrum, 51. col. 2, Pallad. 60. I5, I 24 bis, A.M.B. vii. $4 \mathrm{I}$; Dion. Ined. 6r. 7 ; دا ib. 1. 10, مصل 1.15 .

bi? a kind of spider which does not spin, N. Hist. vii. 4 and 5 .

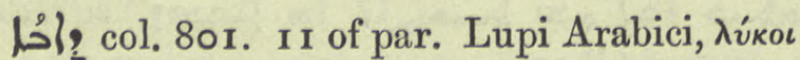
זฑิ 'Apaßias is a LXX mistake for זמבי ערב, Hab. i. 8, Soph. iii. 3, Nau Journ. As. 1905, 372 ; ef. 1.5 of par.

إُ דים

pjat-sl, King Dabdharam,, Kal-w-Dim. 3. $6,95.24,96$. 10.

joll: or ; the Bishop of Arbela, towards Azerbaijan, Pers. Mart. 244 ; Chast. I2. 14 , بح: 1. 20.

lonfoy ? see loy 0 ? col. $83^{2}$.

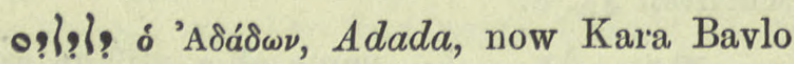
in Pisidia, Nöld. F. S. i. 473. 108.

r?: pr.n.m. a Governor of Azerbaijan, Jab. 239. 9.

ata 9ol? pr.n.m. Ant. Patr. 304. no. 122.

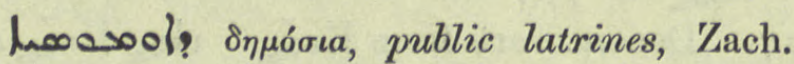
Rhet. 319 n. 6, Bull. Arch. Rom. 222 ult. See lọم col. 884 .

ojol, unknown place, "Doarenses," Wright, C.B. M. $429 b$.

Lol? for Loo? land of Dothan, Jos. ed. Engel 10. 12.

个? and Pers.-Ar. دين religion, Phet. 26. 7 ; Jlan a 5 ? Sassanidi 21 n. 5 ; A.M.B, ii. 576. 6.

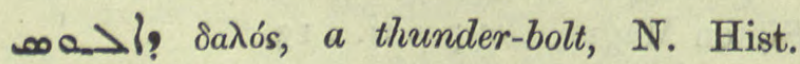
v. $4 \cdot 3$.

.

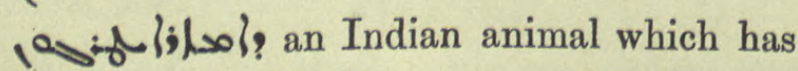
a human face with the paws and body of a lion, Brit. Mus. Cod. Add. xxv. $878 b$.
A. oم osil? publicly, Pléroph. 26. 3, R.O.C. iii. 244 infra.

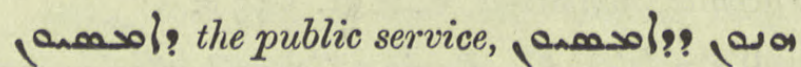
officials, Sev. Ant. Vit. 66. Io. See 10noses?, col. 884 .

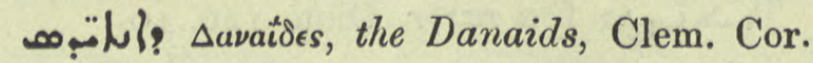
1. 2.

larfoul! Lat. donativum, largess, a gift, Anecd. Syr. iii. 21 9. 26.

Le/? col. 803 ult. $\Delta a ́ \phi \nu \eta, D a p h n e$, a suburb of Antioch, C. B.M. $54 \mathrm{I}$ a.

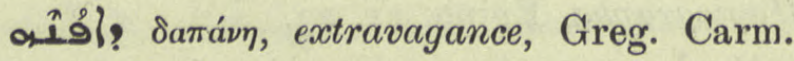
32. 12.

ma1ol? Daphnos, Bishop of Derbe in Lycaonia, Nöld. F. S. 473. ror.

إق? for pulse, QdhamW. r86. 20.

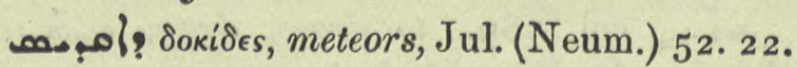
holi\}? $\Delta \epsilon \rho \beta$ aios, of Derbe, BH. in Act. xx. 4 . See loi? col. 944 .

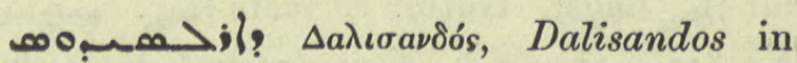
Isauria, Nöld. F. S. i. 47 r 72.

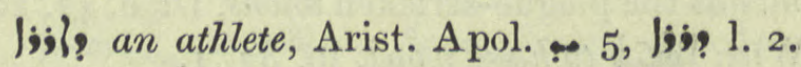
See under '? col. 942 .

1ُ col. 805. I4 of par. Bathsheba compared to a bear. So is Potiphar's wife, Jos. Ephr. 88. 4, 89. 2, 11 1. 6.

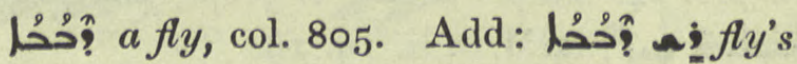
head, name of a sore on the eye, Med. 77. I7.

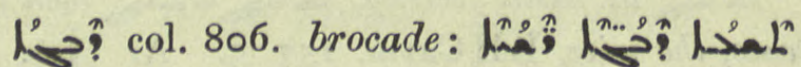
nine pieces or dresses of silk brocade, Jab. 9I. 5 .

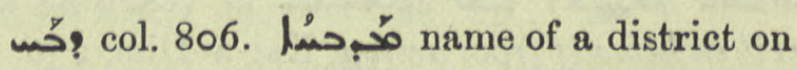
the borders of Amid, Anecd. Syr. ii. 2 10. 6.

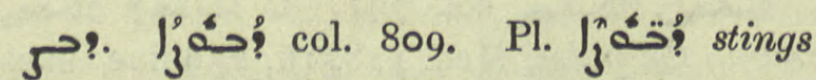
of bees, Anecd. Syr. iii. 33. I ; spines of a hedgehog, ib. iv. 42. 25 ; Physiol. 8. 2.

Jُ? col. 809. m. prick, sting, bite; a flea-

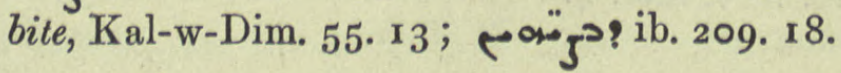
$1 L_{5}$ ?ُ0\% col. 809. f. sting of a scorpion, pl.

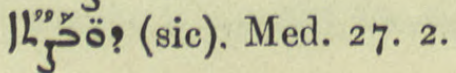




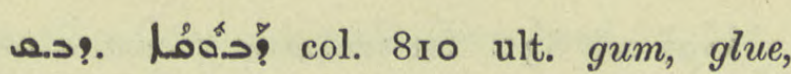
viscous suhstance, Med. 108. I, 358. 10, 401 .

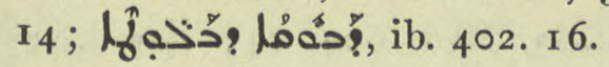

لحلام جِرّة Chimie 2. ro; 人ُa??

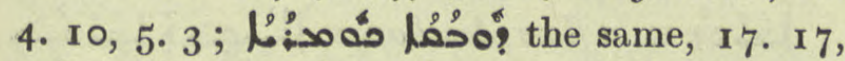
written /. 20 , ib. 18.8. 3) a volume,

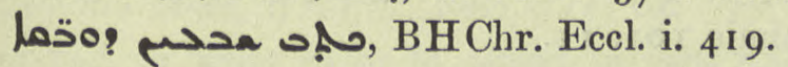

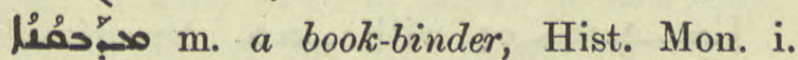
117 . 3.

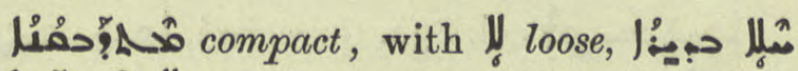

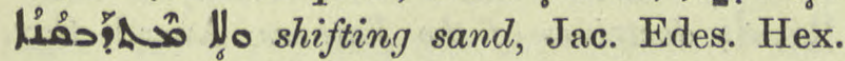
I7. 2.

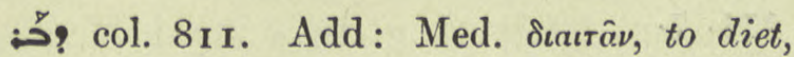
Hippoc. i. 7. 9. Chem. to treat, Chimie 10. 1 2, \&c. Ethpe. $\$$ šll / $^{n}$ Chem. to be treated, Chimie ro. 9 n. 4. Pael 1.20 of par. correct ref. Hex. Josh. v. I4,

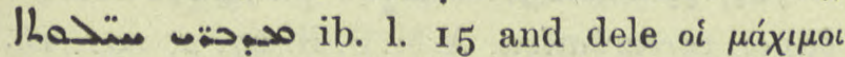

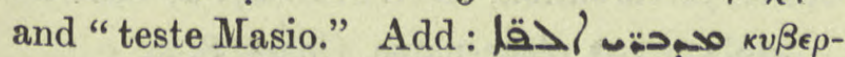

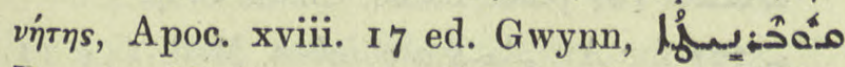
Bagst. Med. to prescribe a regimen, to diet,

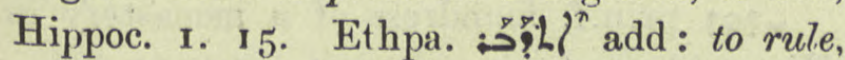
govern, govern your thoughts? Pallad. 532. 20. To be

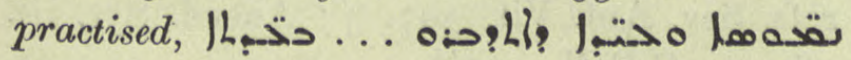
Sev. Lett. 179. I7. Med. to submit to a regimen, to be dieted, Hippoc. i. 15.

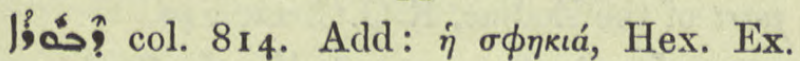
xxii. 28 Lag.

$\int_{\text {? }}^{\text {? }}$ (sic) but if it is Part. with act. sense it should be $\left.\right|^{n}$ ? Var. M. pl. lictors, officers, Apis 143.9.

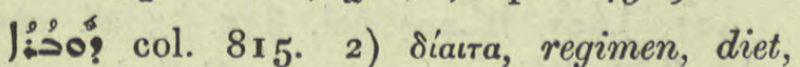
Hippoc. i. 4, 5, 7, 8, I5, ii. 45, Med. 29 I. 2 I. Chem. treatment, jow Chimie 20. 6 ; 90:0\} \$\$0?, ib. 25 . 17.

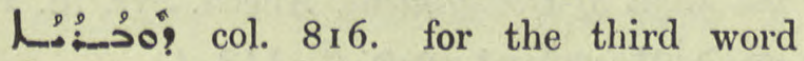

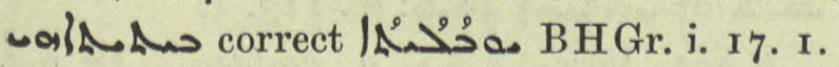

مُ of or living in the desert, Kal-wDim. 21 . 16.

Do:s? perh. synonym for thapsia or for Egyptian pomegranate, Chimie 50. 4, 252. I6.

? ח: معهم name of a bishopric in Adiabene, probably the same as صiم مدم Syn. Or. $670 b$ ult. ib. 33. 27 , Eranšahr 24.
Qُ suffragan See to Arbela, prob. same as م01.

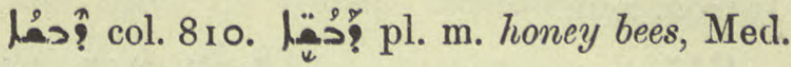
579. I1, 589. I9, 590. 24.

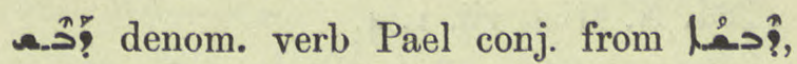
to become like honey, Chimie 100. 5 bis.

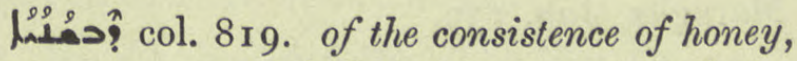

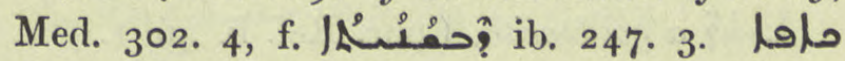
IALaney stone, mellite, Chimie 8. I 2.

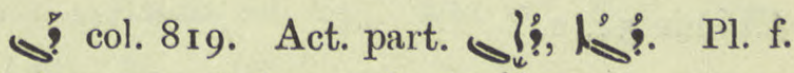

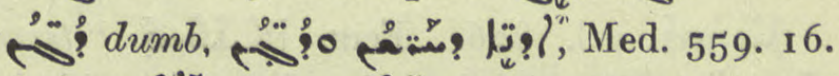

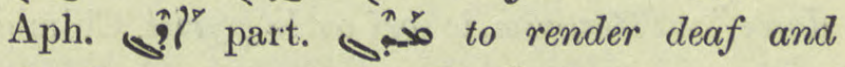
dumb, 0ah 0asas os they turn a deaf ear, Sev. Lett. 496. г3.

lío col. 820. dumb, epithet of lead, opp. tin which sounds. h. e. le plomb-cuivre, Chimie 254 n. I.

hyo? deaf, hayo? boolh lol deaf Father Thomas, Pléroph. 22. 10.

ํ? col. 823. to be blear-eyed, have dim

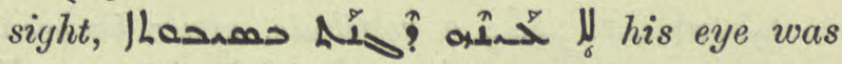
not dimmed by age, A.M.B. v. 1 $9.6=$

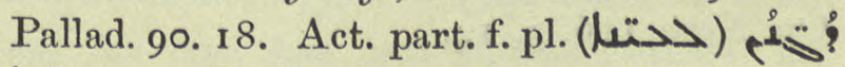

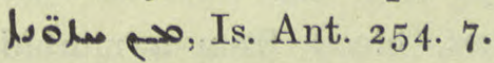

绝? BHGr.i.2 I.7.m. ỏ $\phi \theta a \lambda \mu i a$, ophthalmia

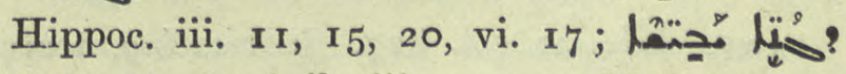

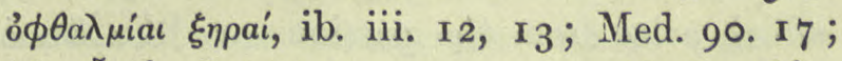

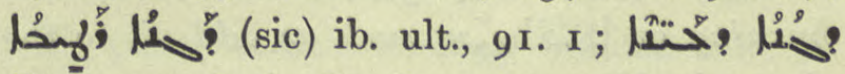
ib. $53.3,78.6 ; 82.5$.

? col.823. to touch, Say

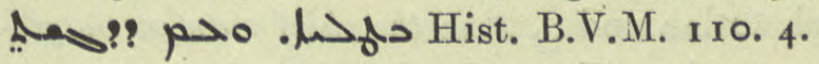

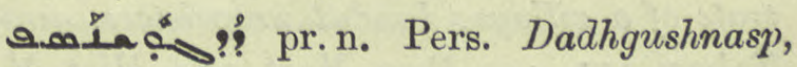
A.M. B. ii. 560 ult.

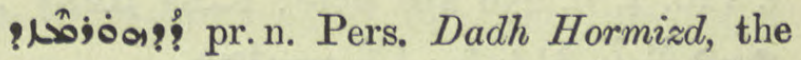
Chief Mage early in the reign of Khosroes Anushirwan, contemp. with the Catholicos Aba, Jab. 226. 12.

ơ?? pr.n. Mart. Pers. A.M.B. iv. ${ }^{4} 2$.

cas!? i. q. Zeus, Stud. Sin. ix. 97, 24.

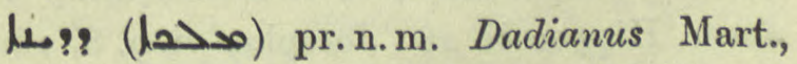
Warda 23,5 and often, S. Dan. $16 v$ bis.

9?: Dadar, a village in Qardu, Chast. 58 . 2o, 65.2 . 
J? ?ִ pr.n. of a king of Arzene, Kal-w-Dim. 348, Preface xxii, id. ed. Bick. 105. 5, Introd. xcii.

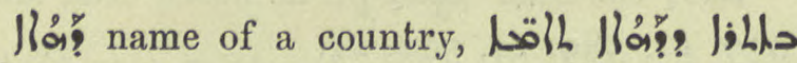
, مُخد, N. Hist. vii. I. 4.

uว้/á? or بخ่? a village to the E. of Maragha, Jab. I39.

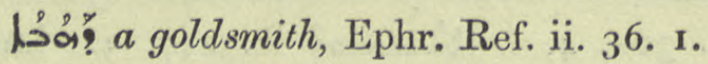

Liśa! col. 826. ellipt. for arsénique dorée, Chimie 54. 2.

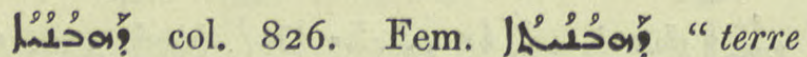
dorée" = l'électrum, le sori ou la pyrite, Chimie 3. $6,4.5,8.17$.

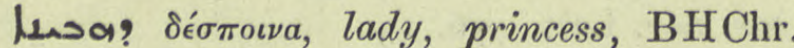

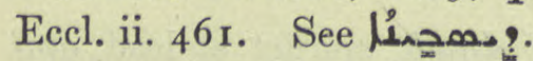

Lیa? col. 826. الذّهـبـانة Dahbana, a village to the S. of Edessa on the way to ar-Rakkah, Jos. Styl. 6 r. 8 transl. 53 note.

hoa? Kurd. Jas m. a drum, Yezidis 103. 4 .

خa" Ar. Ar a vestibule, space between

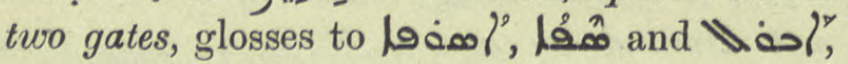
Op. Nest. I 16.3 , 14.

lis?a? slow, inert, blood from a morbid growth is of this character, Med. 204. I9.

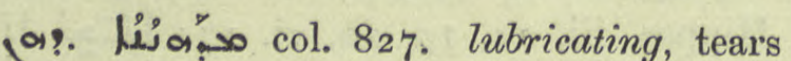

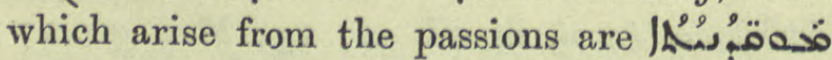
burning or Is'an such as add oil to the flame, Is. Nin. B. 245 . I7.

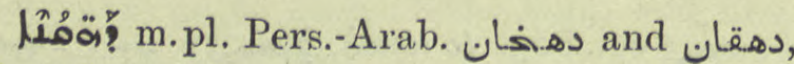
pl. دهاقين the chief man or magistrate of a village; landed proprietors, gentry, Tabari 35I, 440, Pers. Mart. 239, Hist. Mon.

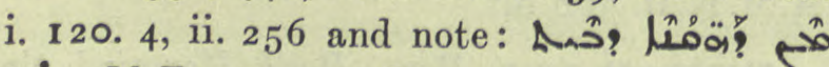

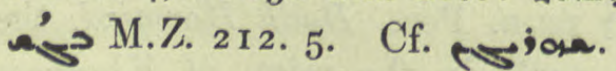

ఎ0?, ?: col. 830. Pael act. part.

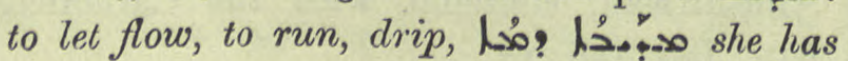
a flow of blood, Hippoc. v. 29. Pass. part. م:" drained of strength, weak, ib. ii. 35, v. 6o. Ethpa. fever, Hippoc. ii. 28 ; Josephus vi. 9. 8, 16. 9 ;

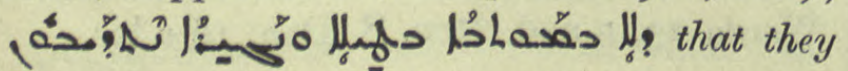
be not worn out by a long and useless session, Sev. Lett. 62, I 2.

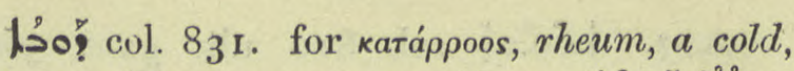
Hippoc. iii. 12,30 , v. 22 , vii. 35 ;

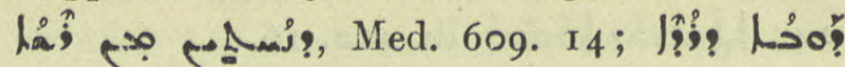
iL 0 dripping which runs down from a halfroasted liver, ib. 88 ult.

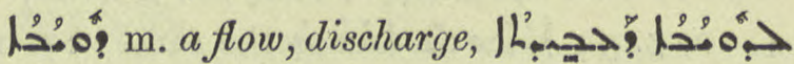
a spout for the discharge of wrought glass, Chimie ror. I I.

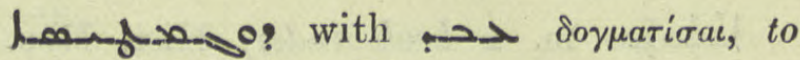
dogmatize, Sev. Ant. Vit. 25 2. 9.

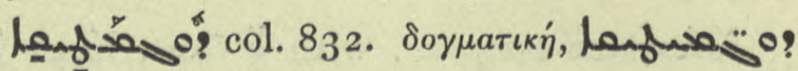
Jhäl: doctrines of the Fathers, Anecd. Syr. ii. 162. 26 ; lonfoy ? Ihis?, Sev. Ant. Vit. 271.7.

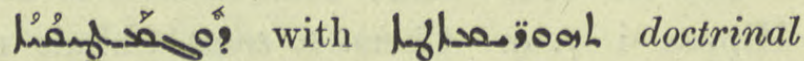
theories, Sev. Lett. 405 . I.

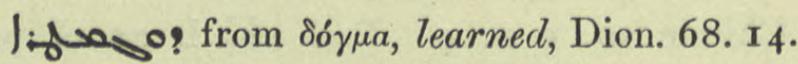

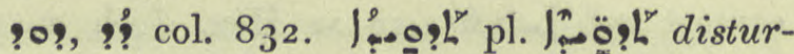
bance, perturbation, A.M.B. ii. 67.2 , го2. 16. م. place-name, Chast. 8. 7.

909 for Ar. دجاج a chicken, Med. I 44. I I. ч! ! pr.n.f. foundress of a monastery in Hirta, Chast. 69 pen.

foro! col. 834. the Twelfth Indict, add refs. Sev. Ant. Vit. 307. 3 ; Anecd. Syr. iii. 287.7 ; C.B.M. 423 b.

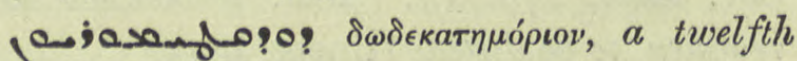
part of the Zodiac, R.O.C. xv. $24 \mathrm{I}$.

- -0 ? col. 834-5 and l? Psalter, Na l,o this Psalter, C. B. M. I33a;

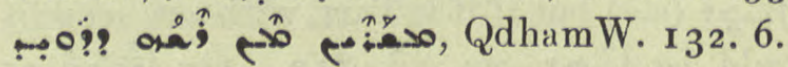

بం? a village in Dinahwar, A.M.B. ii. $5^{60}$.

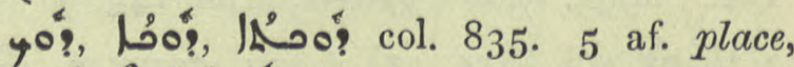

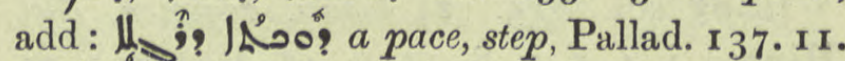

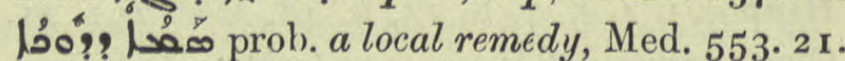

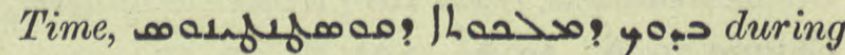
the reign of Constantine, Anecd. Syr. iii. 22 ;

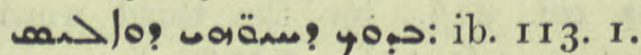

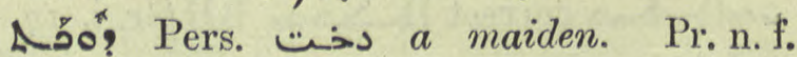
A.M.B. iii. $5^{1} 5$.

Мo!, \: to be moved. col.837. $34 \mathrm{ff}$. C.c. ๑,

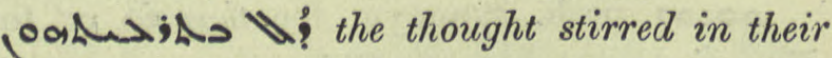
minds, Mar Bassus I5. I9I ; معل دAd loa M!? her feelings were deeply moved,

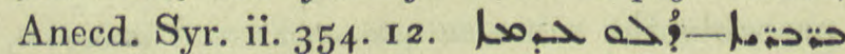

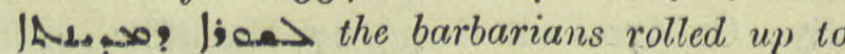




\section{Rl.}

the wall of Constantinople, Dion. Ined. 483 . I I.

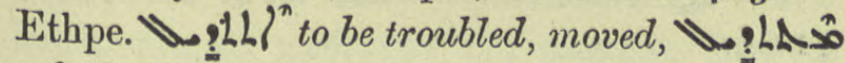
 "'? to serve. R. Duval derives this from

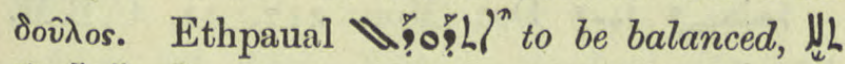

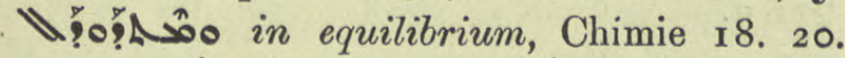

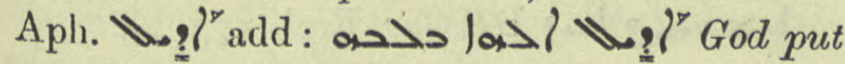
into his heart, BH Chr. Eccl. I4 I. 24 . L. 16 of par. to bring in or to attend to, wait upon travellers, Anecd. Syr. ii. I 39. I.

لب⿱ v. bis, vi. quater, vii. bis, Med. 65 ult., 209. 4 ,

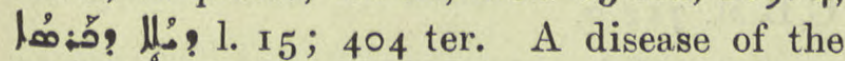

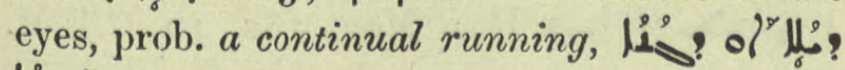

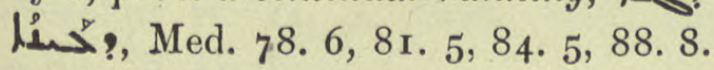

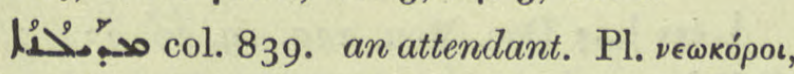
Pléroph. 1 2. 8 ; Aneed. Syr. ii. 2 1. I6; Dion. Ined. 67. 4. It. adj. a subsidiary and servile office, Sev. Lett. 3०3. 12.

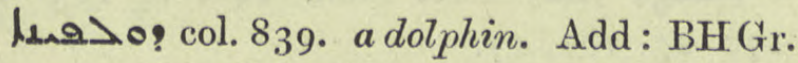
i. 24.5 ; Jac. Edes. Hex. $44 v$; Ar. Fisch N. 48. 13 .

لمُ0? perh. Pers. A measure of capacity, مَّمَ 4 measures of water, Med. 138. 8, 142. 2 , 2I 3.5 .

Loo! Ar. دُ, Dumma, a town near the mouth of the Diiala, A.M.B. ii. 668.

lsoo! for sonsoo! q.v. col. 840. 1500? foro legendary names of the two robbers crucified with Our Lord, Stud. Sin. ii. 9. I5 ;

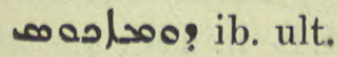

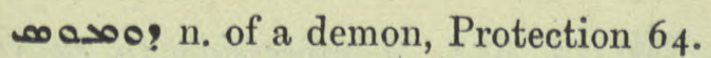

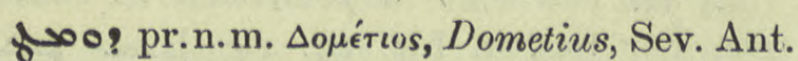
Vit. 207; Bishop of IA an ZDMG. xliii. 396. I 2 .

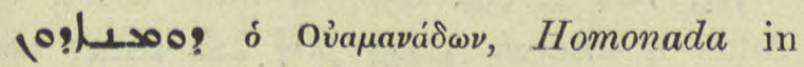
Lycaonia, Nöld. F. S. i. 472.93.

lansoo! col. 840. For évðó $\mu \eta \sigma t s$, Apoc. xxi. I 8 ed. Gwynn; Bagst. Lharmsoo?.

hemsoo? i.q. hooso! col. 917. publicly, homsoo? sot public opinion acquitted them, Pallad. 630. 17.

(فnosoog the Secretariat; public offices, Jab. $3^{6}$ and n. See panosas? col. 884 .

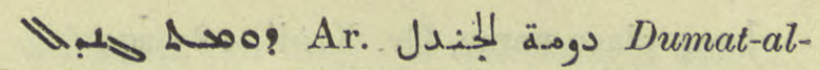
Jandal, a part of Arabia Petraea, thought to be the same as Duma, Sassanidi 36. $7=$ Chron. Min. 38. 23.

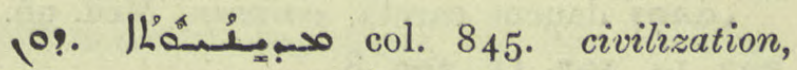

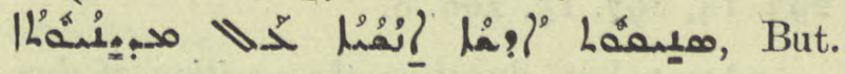
Sap. Pol. I. I, N. Hist. vii. I. I.

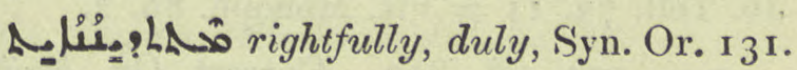
27 , R. O.C. iv. 257,5 af.

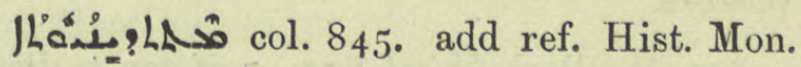
ii. I4I. I2.

10? or e०! Dun, a small town in the diocese of Dinahwar, Pers. Mart, 67 , Phet. 42. 9 .

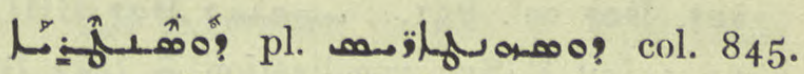
dysentery, Hippoc. iii. iv. v. vi. vii. often,

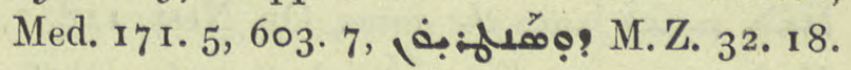

 quent name for Mandaeans, Coupes i. 6, I 2 n. I, ii. I54. 3, น100? 1. I7, Mich. Syr. ix. cap. 6 ; Nöld. ap. WZKM. xii. 357 . Cf. متبم ال?

J0\% ef. Pers. دوس or water in which red hot iron has been plunged, others say scoria, dross of iron, Chimie 296. 9.

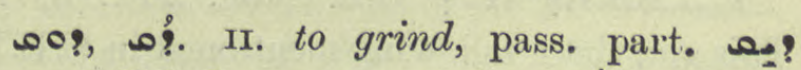
ground, Chimie 37. 3, 4. Aph. As? to rise up, ,

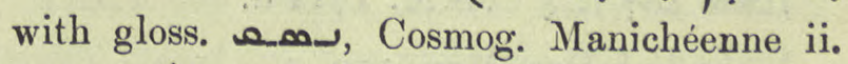

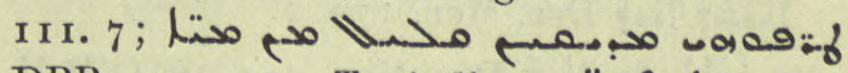

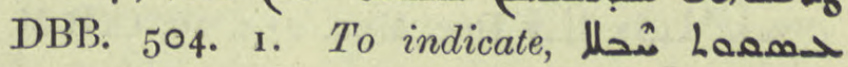
? Isoyabh iii. ed. Duval

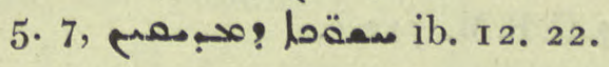

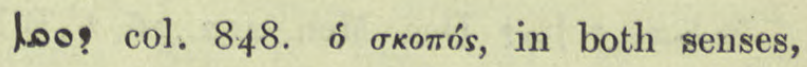
a) a watchman, sentinel, Sev. Ant. Hom. $25 \cdot 3$, 26 five times. b) a mark, target, 100? A.00?, ib. 25 . 10, 26. I. Astron. 100?

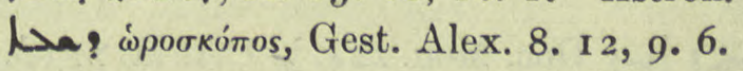

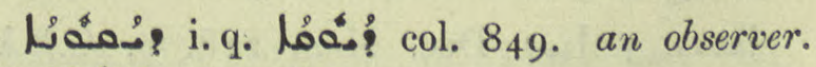

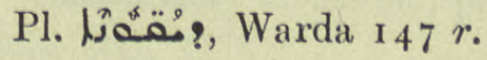

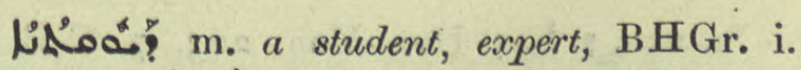

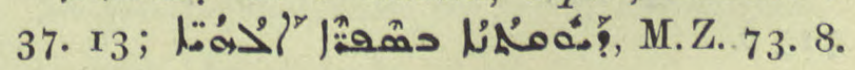

Loo! pr.n.m. Bishop of Susa, ZDMG. xliii. 395 . 
Loo? place-name, Or. Xt. ii. 183 sqq.

م o col. 849. a wallet, trs. to col. $20 \mathrm{r}_{4}$ i.e. place under $p$.

פم daucus carota, parsnip, Med. 99. 4, 293. $14,297.15,299.8$.

of 100 ! Lat. ducatus, the position of dux Jo. Tell. 72. $1 \mathrm{I}=$ Vit. Monoph. 89. 2 .

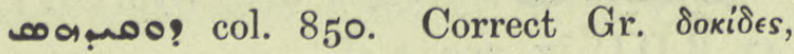
meteors.

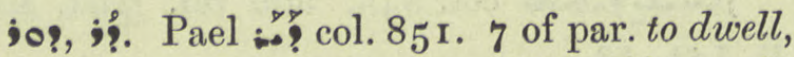

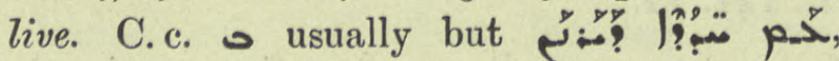

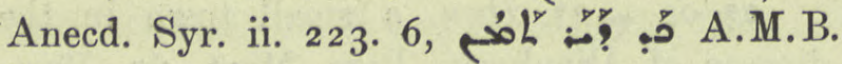
iii. 490 .

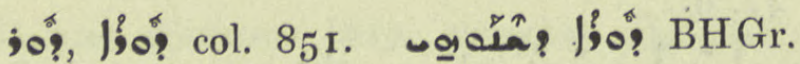
i. 44. 4, but E.-Syr. vocalization à liñ? j;o?

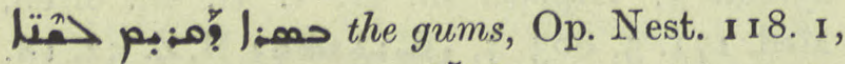
Med. 167. 9 marg., ผä̋̈०? 1. 12, 169. 3, 18; . بُ swollen gums, ib. I68. 8.

|

J" a monastery. Add to the list on col. 853 . -

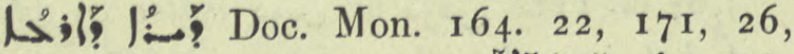

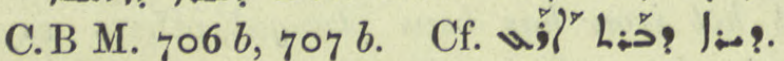

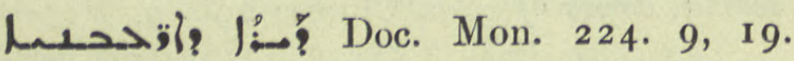
This and those following mentioned in C.B.M. are in Arabia.

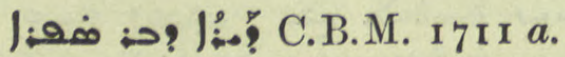

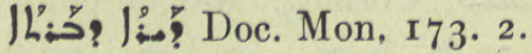

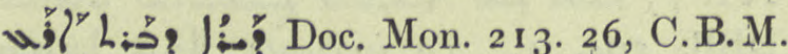
$707 b$.

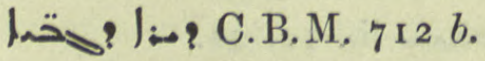

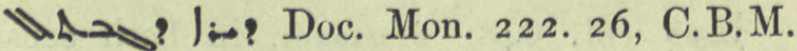
$713 a$.

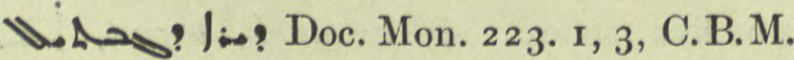
$7 \times 3 a$.

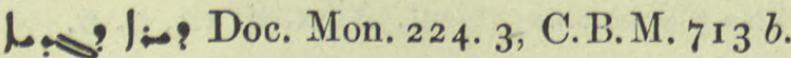
? Int Doc. Mon. $2 \times 8.9$, C.B.M. 7 II $a$.

Unoes I I 2 , Doc. Mon. 223. 7 , C.B.M. $713 b$.

A190, /6, Doc. Mon. 214 * I3, C.B.M. $709 b$. hon? I- Doc. Mon. 2 I9. 2, C.B.M. $7 \mathrm{II} b$.

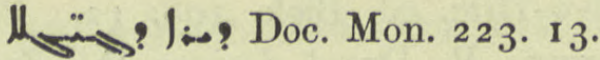

las ? In? Doc. Mon. 21 7. 22, C.B.M. 7 II $a$.

h 1000 ? I: 2 BHChr. Eccl. 273. 25.

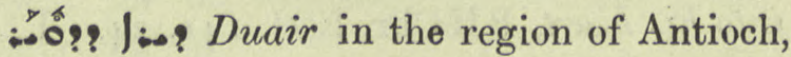
BHChr. Eccl. 473. 2, 48I. 2 r.

jo?? :

Whio?! Jis? Doc. Mon. 2 I9. 30, 220. 3, C.B. M. 7 I $2 a$.

en: Doc. Mon. I82. 10, C.B.M. $708 b$.

@:?!? Doc. Mon. 220. 19, 20, 232. 2, 3, C.B.M. 7 I $2 a$.

? ? In In Edessa, Hist. Édesse, Duval i 8 ; Patr. Or. v. 5. 7 ro. Ir.

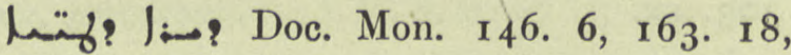
I70. $25,181.27$.

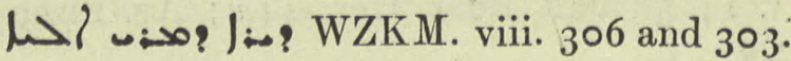

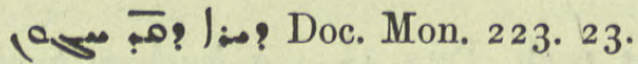

(م: Mar Audishu, near Amadia, now ديرى cf. Münster, minster, Kal-w-Dim. ed. Bick. I 26, Introd. xxviii.

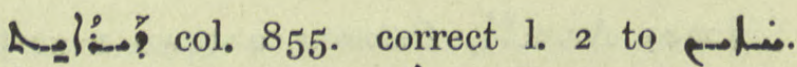

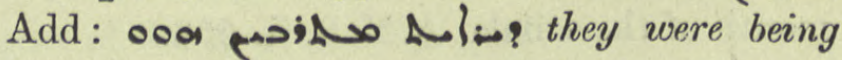
brought up in a monastic school, or to the monastic life, Sev. Lett. 248. 18.

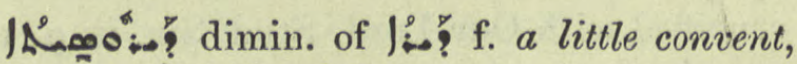
|

;\{, Jُ col. 855. a procession in church, refs. Bar Sal. in Lit. 19. 24, مبمهامي حبزן, ib. f., 25. 26.

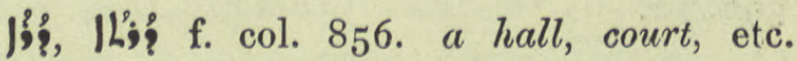

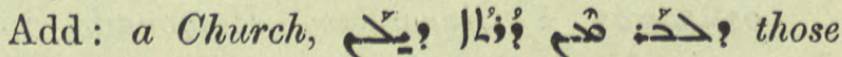
who do not belong to our Church, Georg. Arab. 34. I7. Astron. a house, mansion, 隹 Brit. Mus. Or. 208.4. 6 r.

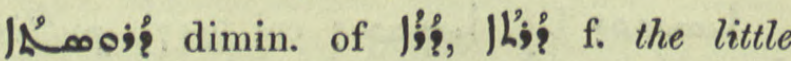
enclosure or yard of a monk's hut, Hist. Mon. i. 297. 2, Anecd. Syr. ii. 45. 26, lحih a Pl. A Ano:? Doc. Mon. 164. 2. 
jo? Dura on the Euphrates, add: Pers.

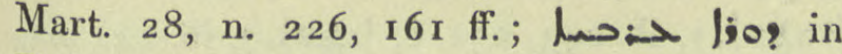
Tirhan, Chast. 54. 2 ; wa j;o? Chast. 48. 7 , 62. $\mathrm{I}$.

ß乡̧o? col. 857. Add: a deed of gift, Syr. R. Rechtsb. 18, 5 ff., /Lowso! Kio! free gift of ownership, ib. ll. 16, 20 .

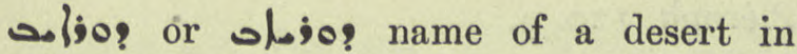
India, Kal-w-Dim. ed. Bick. I 16.8.

lojo! a village between Beit Garmai and Belesphar, Chast. 37. 4 af.

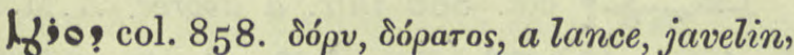
Josephus vi. 14. 4, Gest. Alex. 100. I4; ufjo? ib. 42.6.

צ'ơ a mountain near Jilu, A.M.B. i. 4 I 7.

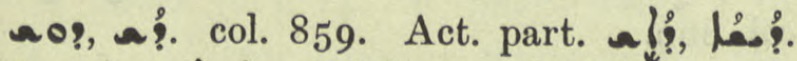

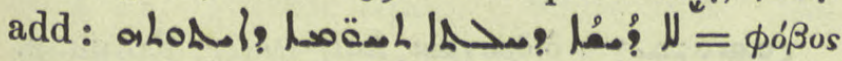

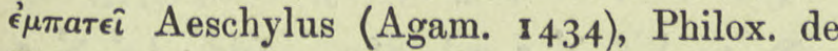
Trin. 18. 8.

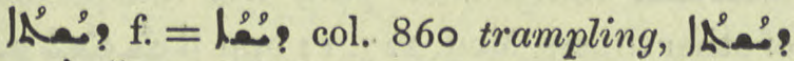
Kheas\}? IN. S. Dan. 9, $v$ 14.

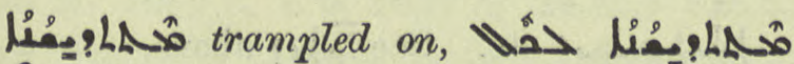
党 3 open to all comers, Isoyabh iii. ed. Duval 22 ult.

"ִ col. 86r. Pael part. worm pulverizes the skin, Greg. Carm. ii. 16. 18 .

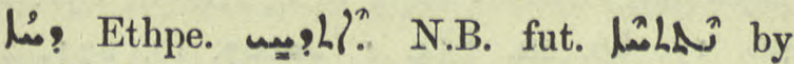
assimilation of ? to L, An. Syr. 142. I4,

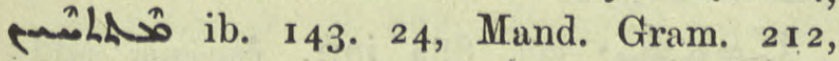
n. 3 .

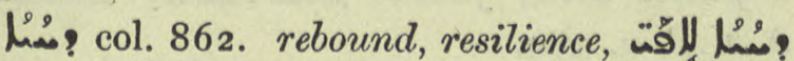

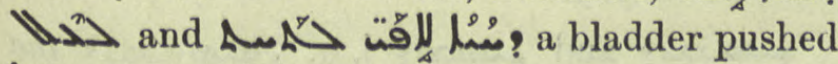
into the water rebounds upwards and a stone hurled into the air tends doumwards, N. Hist. v. I, Philos. 6. I.

"Nechakh, Emir of Gezirtha, Brook's Chron. 575. 13.

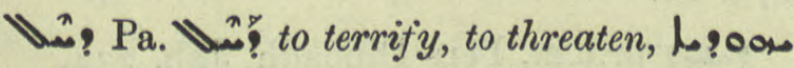

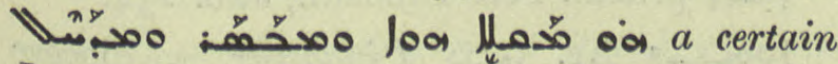
Jew used insulting and scornful and alarming language, Josephus vi. 29. 3 .

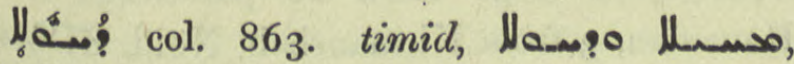
Manichèisme 107. 4 .
لإم_ col. 865. a scarecrow, horror;

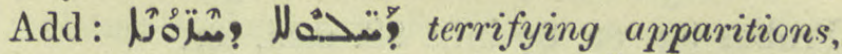

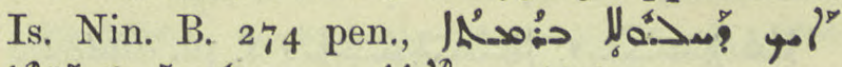

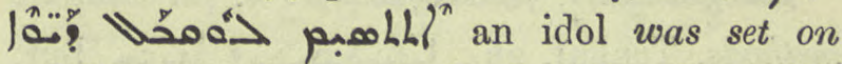
a hill to scare away devils, Jac. Sar. Hom. iii. 802. II.

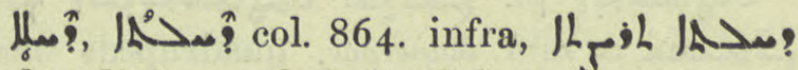
right religion, orthodoxy, Jul. 54. I3.

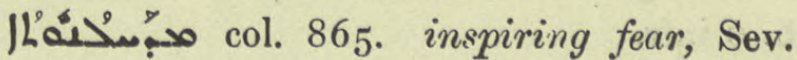
Ant. Hom. I6. II.

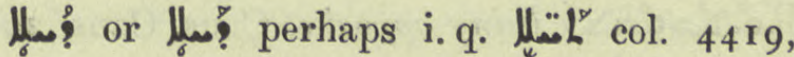
both are carminative. Pl. sativum, pepperwort, Med. 563. 3, 567. 12,

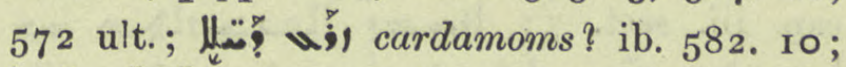

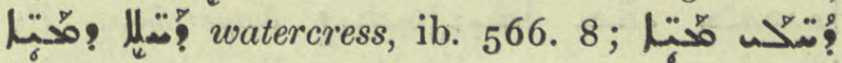
(sic) $569 . \mathrm{II}$.

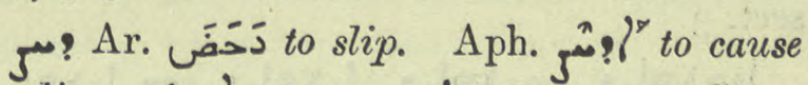
to slip, odu lo Greg. Carn. i. 24. I.

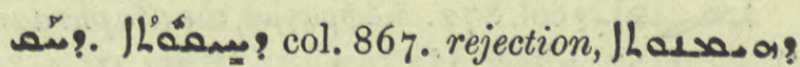
12 gh, Pléroph. 25 . 12.

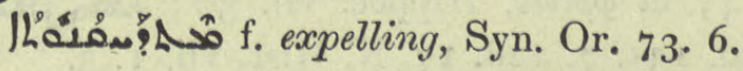

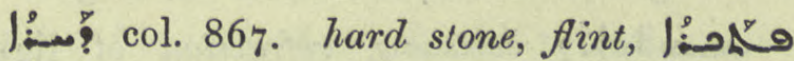
?ְְִ: Jac. Sar. Hom. iii. 797. 2.

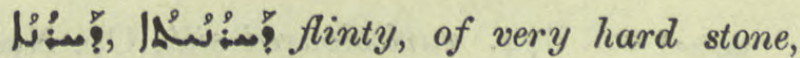
ماقا ?olack flints; basalt, Jac. Edes. Hex. xliii. 2.

A.j.? see Suppl. under A.? and Thes. under $\mathrm{N}_{\mathrm{O}}$ ? col. 892 and dele the article.

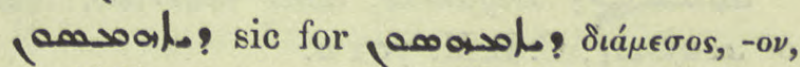
the middle plane of the zodiac, De Astrolabe 247 bis; amsole, ib. 84.

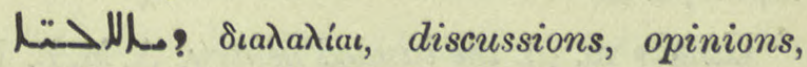
decisions, Syn. ii. Eph. 294. 24, f. 4;

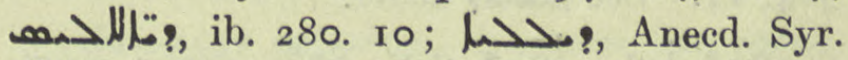

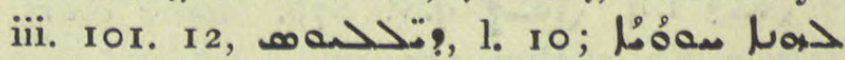
, But. Sap. on b, civ. p. 3 .

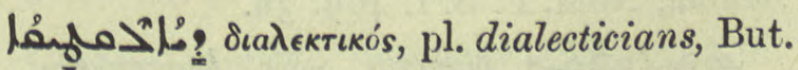
Sap. Eth. iv. 3 .

|? But. Sap. Philos. I. I.

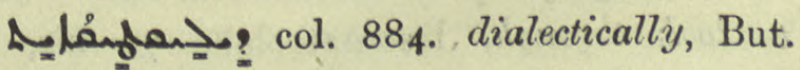
Sap. Periherm. I r. 6. 


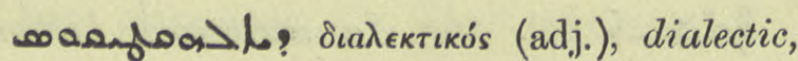
Probus 83. 10, 87 infr. 88. med. ter.

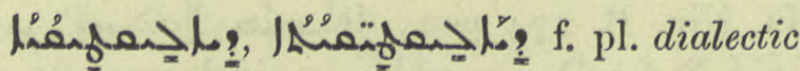

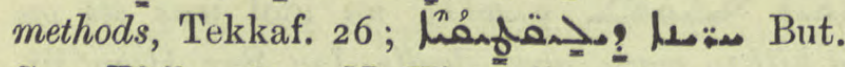
Sap. Philos. 8. I, N. Hist. vii. r. 6.

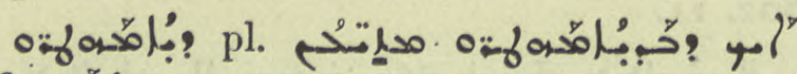

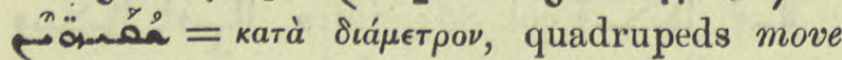
their legs cross-corner-wise, N. Hist. vii. 6. 6 infr.

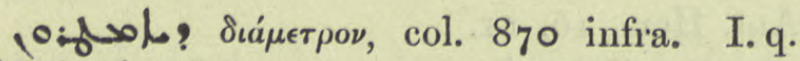

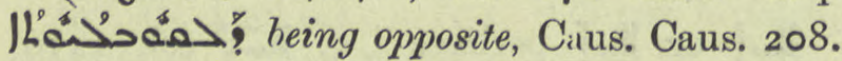
18: pl. Jifolol opposite positions, Georg. Arab. 26. 23; Oifaxe?, But. Sap. Bk. ii,

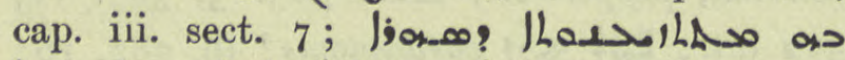

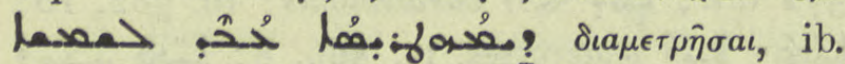
Theol. 2. 5 infr.; N. Hist. Bk. ii. cap. iv. sect. 3 .

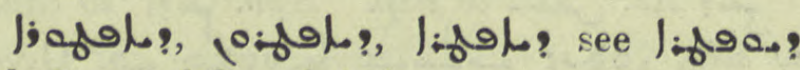
col. 879 and Suppl. infra.

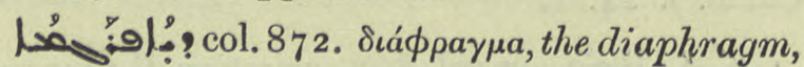

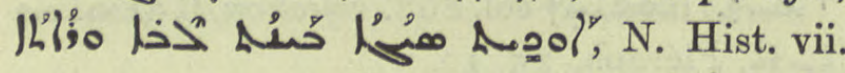

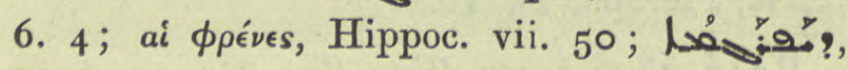
Med. 13 г. 7, 228. 17, N. Hist. vii. 1. 3.

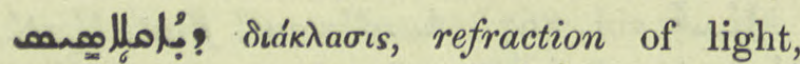

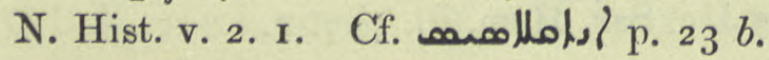

- col. 874. varicoloured silk, Op. Nest.

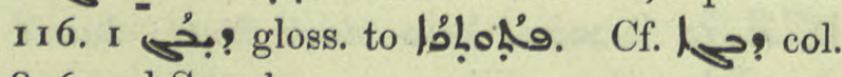
806 and Suppl.

? محن name of a region: see ;os\}?.

صaxs? Digamus, twice married, name of $\mathrm{sol}$ (م), C. B. M. 70r $a$.

@ Eph. 78. 8.

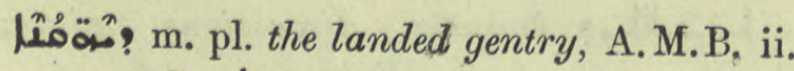
584. II. See lioa? above.

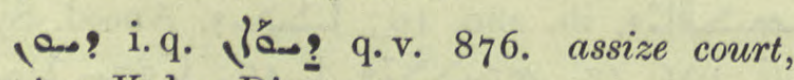
session, Kal-w-Dim. I 5 0. 10.

م Arabia, Nöld. F. S. i. $469 \cdot 36$.

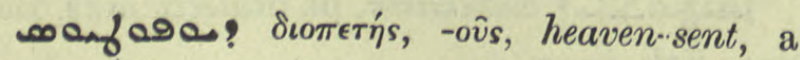
name of Venus, col. 542 under 2 s.

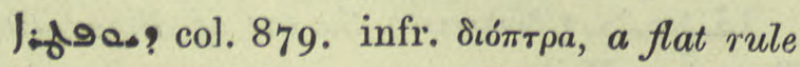
with an upright piece at each end with a hole to look through, De Astrolabe 76 bis, f.,

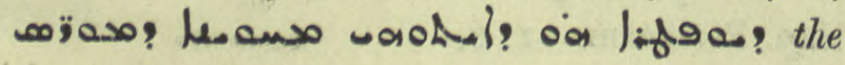

\section{inตls.}

diopter called the index, ib. 86, also written

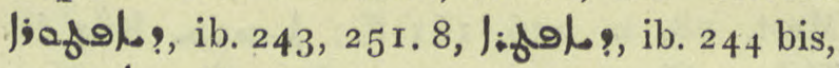
(0)-fol., ib. 239.

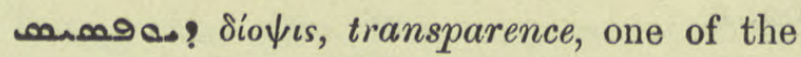
names for mercury, Chimie 46 . I I.

hwos? surname of John, an Egyptian bishop and archimandrite, perh. $(\tau \hat{\eta} \varsigma)$ Siakovias, Pléroph. 120. 9.

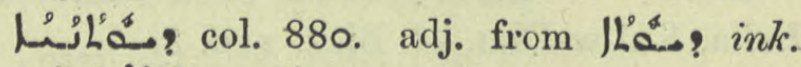
"us sat we may make known with ink instead of lips, Apis. 9. 6.

1.

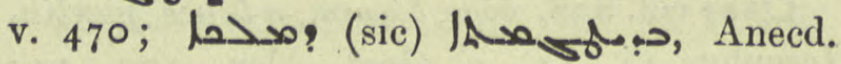
Syr. 1 29. 9 .

cosfe? name of a book: C.B.M. $3^{88} b$;

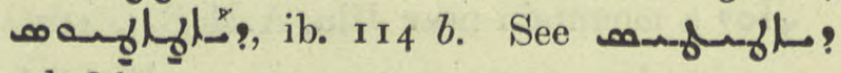
col. 869 .

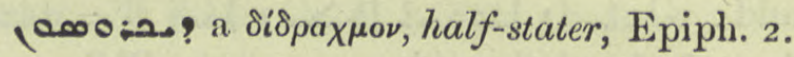

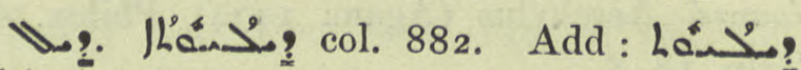
JLaم independent pain i. e. independent of other illness, Med. 227 . I opp. /Lمْ sympathetic pain, ib. l. Iо.

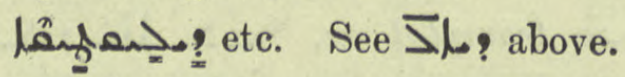

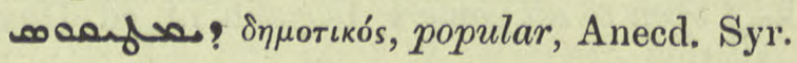
iii. 145.24 .

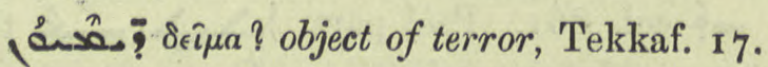

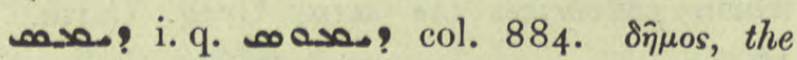
populace, C.B.M. 1051 $a, b$, Anecd. Syr. iii. 56. 17, 66. 26, 254. r, م-9, ib. 27 I. 23 , C.B.M. $1059 b$.

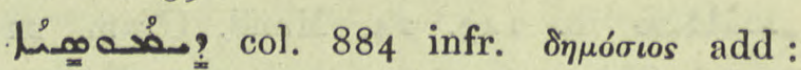

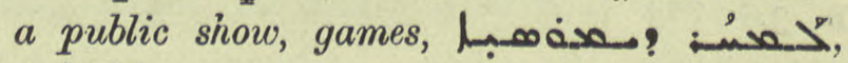
A.M.B. v. $5^{89}$.

poser? and i.q. poreseres the problic bath, Jos. Styl. 25. 16, 26. 2, 70. 20.

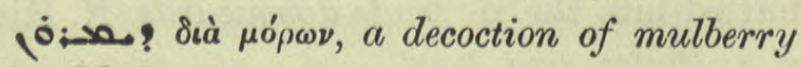
juice, Med. I60. I2, I6 I. I5.

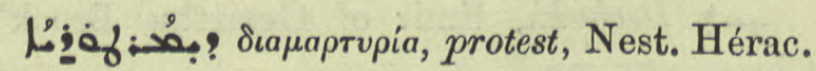
I 73 . 16.

?ִ pr.n.m. Pers Dindadh, Jab. 234.

joal. Ar. دينور Dinahwar, a diocese suffragan to Hulwan, Pers. Mart. 259; cf. 67 ; formerly a large town 20 parasangs from Hamadan, 3 from Kirmanshah, Nöld. ZDMG. xxviii. $102 ;$ A.M.B. ii. 560. 


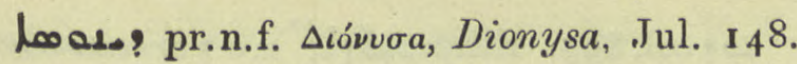
20, 149. 2 .

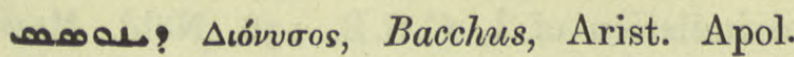
a.s. 3 .

Pعehlevi from seligion: a man of religion, a Brahman, Kal-w-Dim. ed. Bick. 69. 5, 74. 23, Introd. lxxiii.

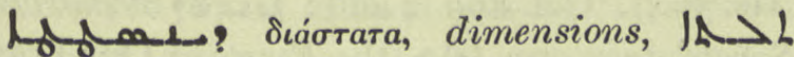

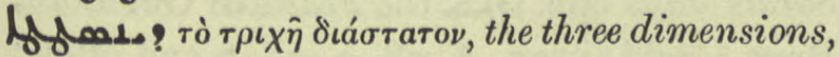
Ephr. Ref. ii. 9, 22, i. q. متمصم (N) I 4 bis, etc.

بـ Ar. pr.m. دينار an Arab general, Brook's Chron. 572 ult.

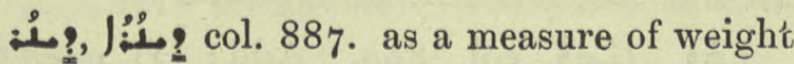
a dinar equals 6 grammes, Chimie 69. I2: or 24 carats, ib. l. 14. 6 dinars weigh I lb. ib. l. I 4. I dinar equals 6 white zuze. Jab. 38. $12 \mathrm{f}$.

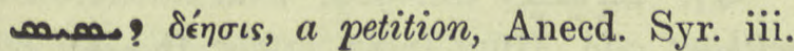
132. 2, i.q. /Lْة حسة 1. 9, I40. I. Ib. I34. 8, I39. 18, 19, טم:حم/ ?" מחسم, ib. 163. 24,

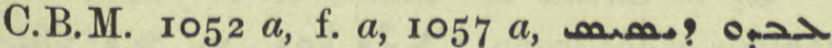
they framed a petition against heretics, Sev. Ant. Vit. 268 ult.

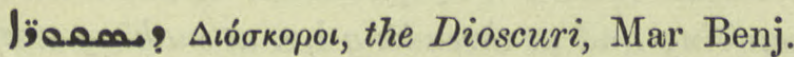
64 n. I.

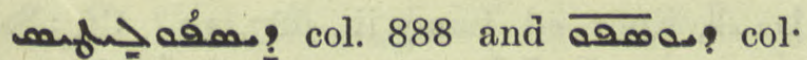

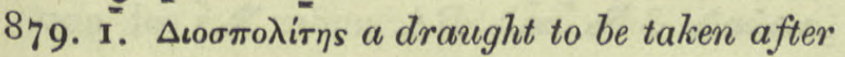
a purge, Med. 45. $21,290.8,304.22$.

اللُّد . Diospolis i.

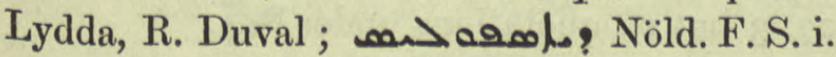
468. 4 .

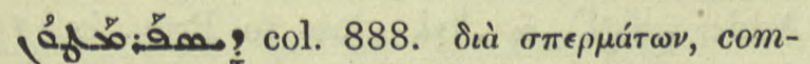
pounded of seeds, Med. 32 I. 5, I2, 408. 2 I.

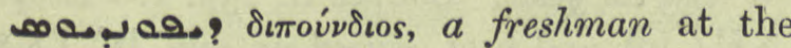
University of Beyrout, pl. aصa ?, Sev. Ant. Vit. 48. 2 ; marg. note to 47.4 .

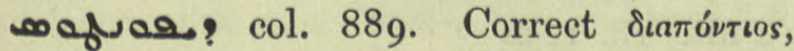
beyond the sea, Syr. Rom. Rechtsb. I9. 4 af.

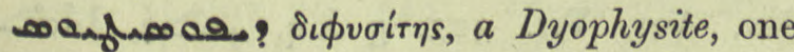
who declares two natures in Christ, Sev. Ant. Vit. 274. I, Anecd. Syr. iii. 230. 3; pl.

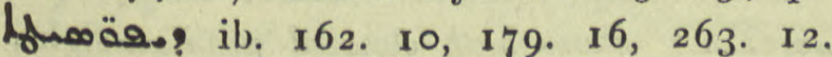
Cf. Ahemoas? col. 880 .

2716
ผ0829? col. 889. diptychum, a twofold writing tablet, Act. S. Pelag. 5. 3. Add :

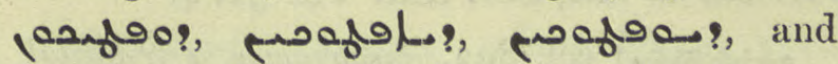
مرجم a diptych in the eccles. sense.

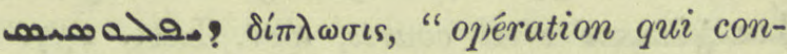
sistait à doubler le poids des métaux ou à changer les métaux en or ou argent," Chimie 27. 9, ib. 1. 2 I. Cf. ib. translation, $5 \mathrm{I}, \mathrm{f}$.

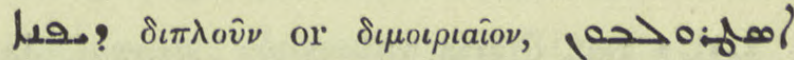
لـ !? a double astrolabe, De Astrolabe 74.

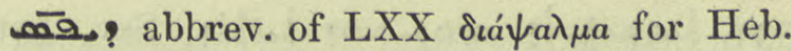

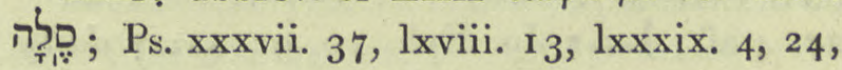
Lee.

coly우요 col. 889 infi: a mineral produced by art, sediment of molten copper, etc. Chimie 4. I3, 5. 16 . See ib. p. 128.

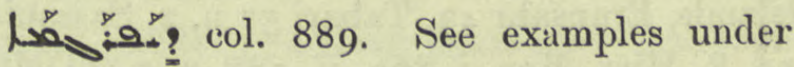
|say:ol. Suppl.

مerh. Ar. ذفرى the parts of the skull which stick out behind the ears, BHGr. i. 23. 16.

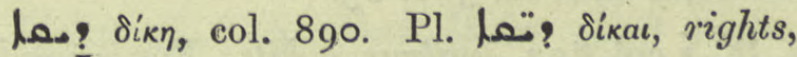
privileges, Aneed. Syr. iii. I64. 4 ; تمص

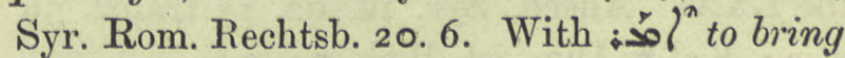
a lawsuit, plead, ib. 7. 16, I 7 .

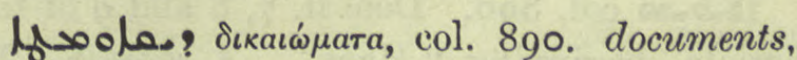

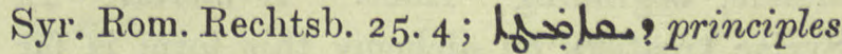
of right, maxims of law, ib. 39 tit.

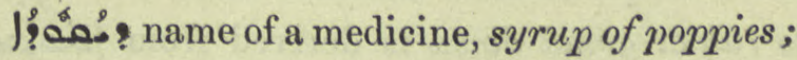
see under sمب.

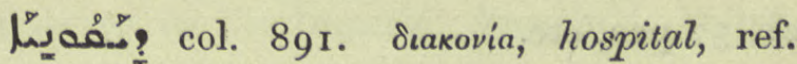
Anecd. Syr. ii. 237. 2 I ; pl. معقدم ?, ib. 24I. I2.

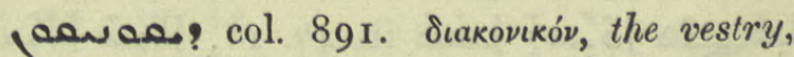
Pet. Ib. 109. I.

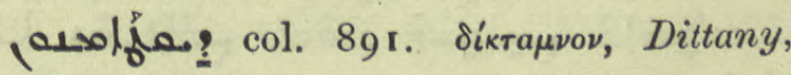
N. Hist. vii. 4. 4.

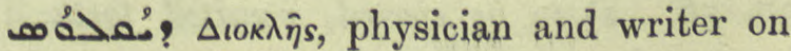
medicine, Med. 19. 2, 4 .

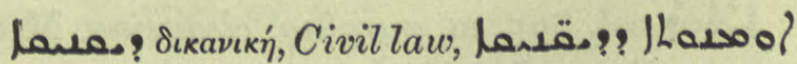
the law (profession of), the bar, Sev. Ant. Vit.

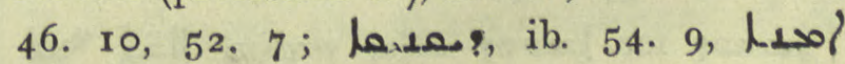
لمعـe.?, ib. 92. 7 . 


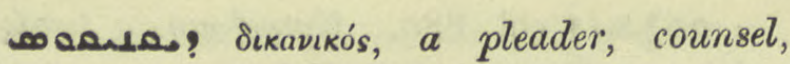
barrister-at-law, Sev. Ant. Vit. 52. 4 .

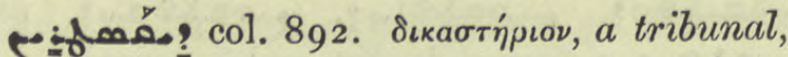

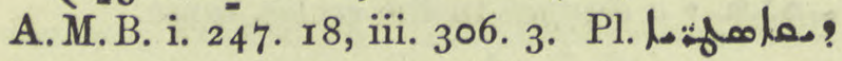
ا ib. 434. 10, 13.

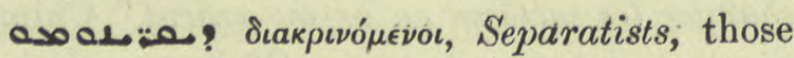
who did not acknowledge the Council of Chalcedon, B H Chr. Eccl. 227 . I3, n. 3.

!): Lat. diaria, allowance, stipend, Jac. Edes. Chron. Can. 266, Fraenk. in loc., ib.

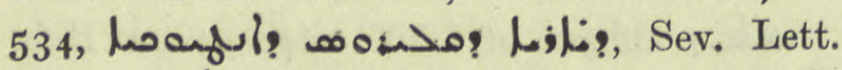

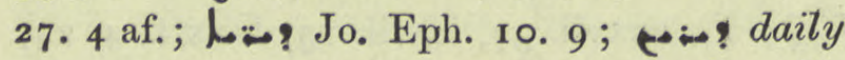
allowance made to monks, Anecd. Syr. iii. 219. 4, Chron. Min. 288، 2.

(م-2) I) see preceding word. 2) col. 892. دَرَّنْ Diren, one of the Bahrein group of islands, Eranšahr 43, Tabari 57 n. 2, Sassanidi 1 3. 8, Hist. Mon. i. 869 , ii. 188, n. 5, ZDMGG. xliii. 406 ult., 407.

A? pr.n.m. Dajath of Amid, Anecd. Syr.

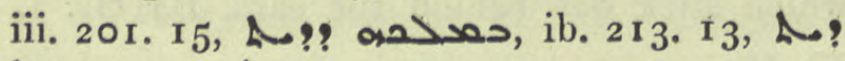
1201, ib. 326.13.

12. col. 892. milvus, a kite. For ikrîvos in the LXX. Anecd. Syr. iv. 62. I3 transl. 59 n. 2 ; Kal-w-Dim، ed. Bick. 74. 24.

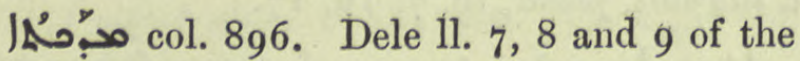
article, beginning "Haec." A mortar. In Syriac treatises on alchemy this form is far more frequent than مَّبْ

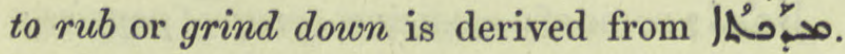
R. Duval. Refs. Chimie 22. 17, 19, 47. 19, 2I, $5^{2}$ pen, etc.

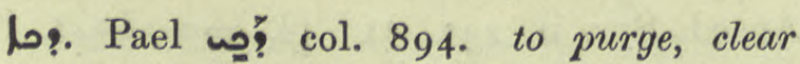
away, Hippoc. ii. 9, iv. 8, 1 2. Med. 45. 14, 19;

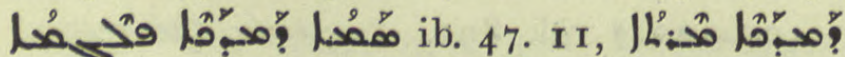

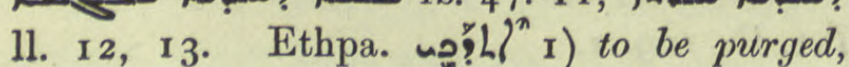
مُ1, Med. 45. 9, 10, 23, Hippoc. ii. 36, 37, vii. 38. 5 . 2) to be ejected, vomited forth, ib. i. 2.24 , iv. 3.1 , note 2 .

นُْْ col. 895. m. purging, cleansing, Med.

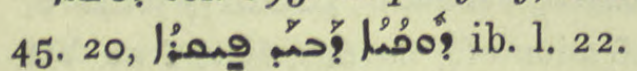

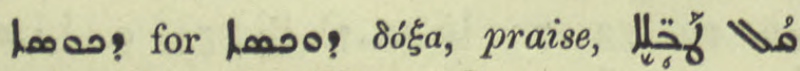
م ه
-

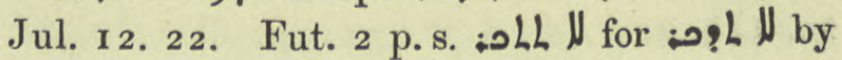
assimilation of L, C.S.B. 158 , Nöld. Mand. Gr. 2 I 2 n. 3.

H:مo' col. 898. 9 af. ff. the Eucharist celebrated in commemoration of a saint, Jac.

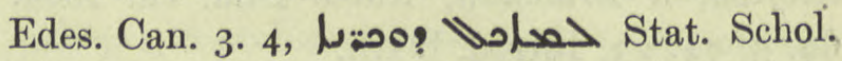
Nis. 30. 4. Col. 899. 2 add: L:صo! memorials, Saints' days opp. I حل حل the Feasts of Oúr Lord, Brev. Chald. i. 25. 6, 28. 9, 29. 7 and often;

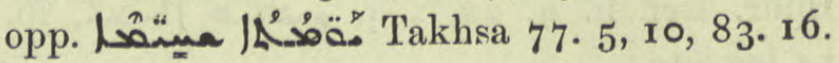

جذه memanda for oculists, a text book by حلى ح: حسم Ali Bar-Isa, BHChr. Eccl. § iii. 277.

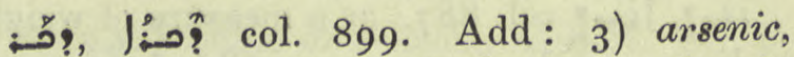

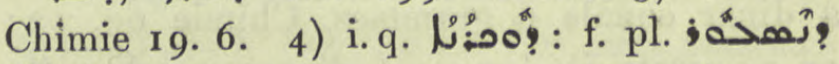

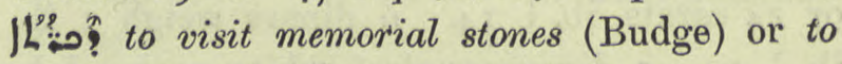
perform memorial services, Pallad. 86. I 7 .

\!. without ado, Hunt. clxx under fAnes col. $33^{1} 5$.

W: to thread the shuttle or to fasten the

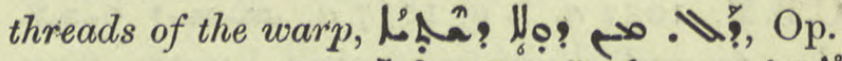

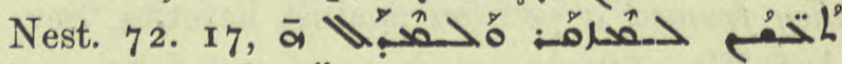

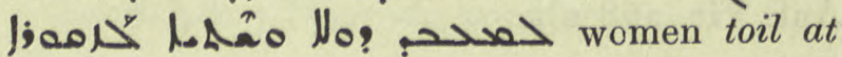
weaving and at threading i.e. at making warp امت م: لامتر, Ephr. ed. Lamy iii. 687. 17. Cf. : W?

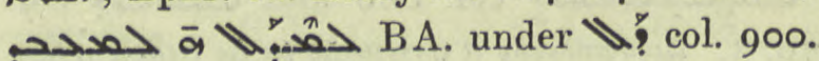

لل

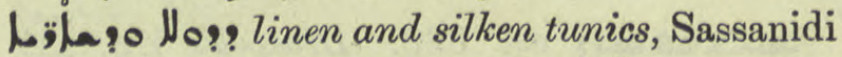
27. 3 but cf. quotations under W?.

لإ? col. 903. Pa. u’ to draw up, lift out.

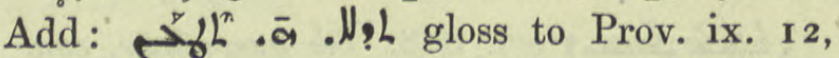
Op. Nest. 100. 16, N.B. רaran "il leva les yeux," Coupes ii. I55. I. Ethpa.

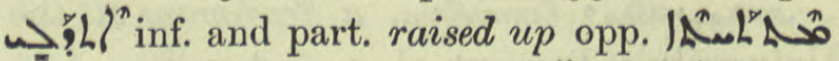

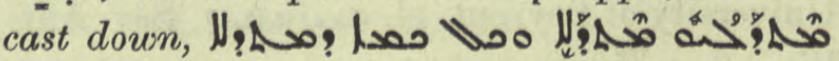

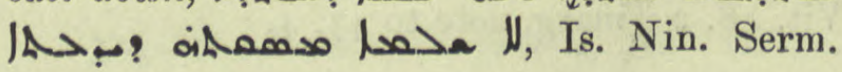
I6 ult., I8. r. 2 .

\ه: Dolichê, a bishopric in Euphratesia, Sev. Ant. Vit. 325. 5 i.q. 4ם col. 906 .

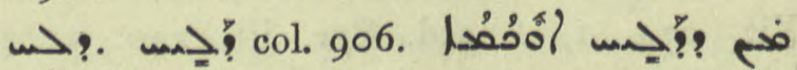

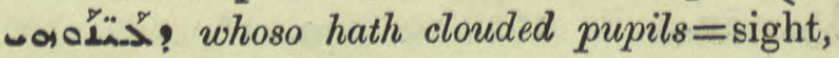
But. Sap. Econ. iii. I. 


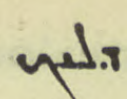

خص Dolichê: see Nod?. Nöld. F. S. i. 470 . 5o, Or. Xt. ii. 273,280 , Gelzer 44. 875 .

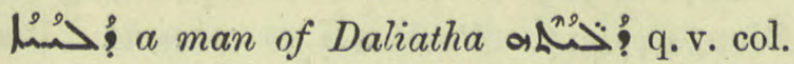
908. مش: Protection 54. 3.

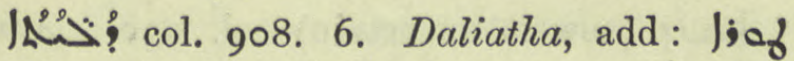

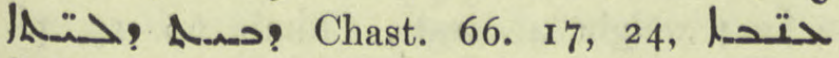

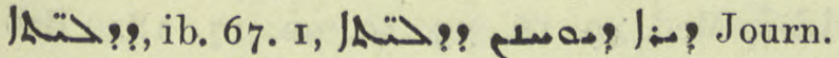
As. $1907,164.15$.

مه : Dalmatius, a Dalmatian, Doc. Mon. $269.8,28$.

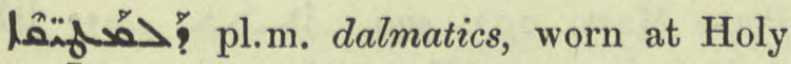
Communion, Anecd. Syr. iii. 49. 27.

?רחم) a village near Heliopolis, A.M.B. iii. 348 .

ใִ col. 908. According to Op. Nest. it

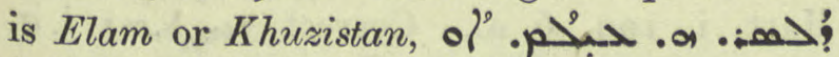
Liö A I28. I3; female idols were

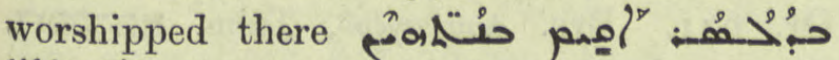

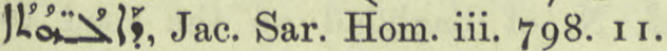

"خ col. 908. to drop, drip. Act. part. ref. 57. 17.

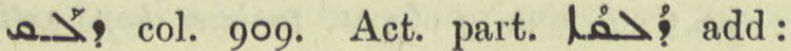

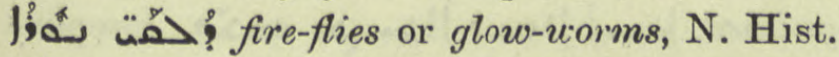
vii. 2. 2 .

Lـo

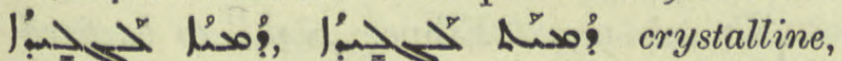

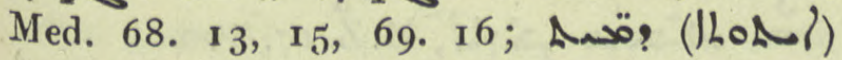
inal homogeneous, Ephr. Ref. i. 4. 36.

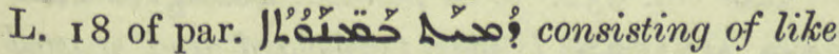

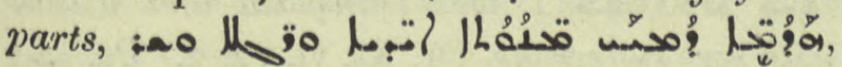
N. Hist. vii. 6. I, Hebraica iii. 250 . I3. Pa. uso? add: to see a likeness, to recognize,

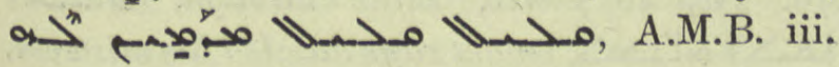
235.18.

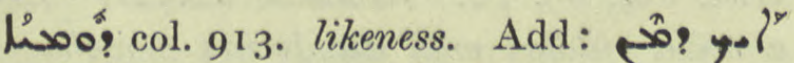
hisoo! by comparison, Pallad. 331. го.

ئ similarly, Hist. Mon. i. $3^{27}$. I7.

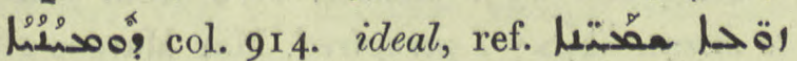
مut. Sap. Theol. 4. 3 .

áso!, /Lán' col. 914. Pl. of our Lord Lïr

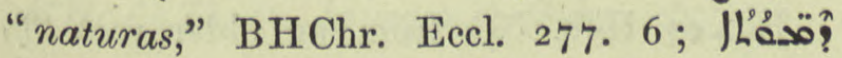

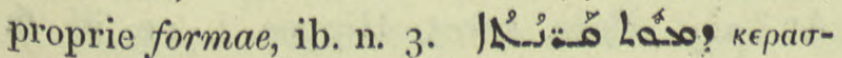
фópos, horned, Gest. Alex. 20. 8.

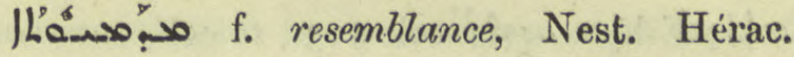
4 II. 3 .

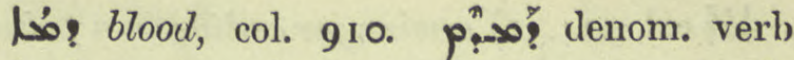

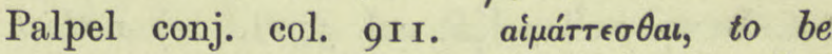
covered uith blood, to be bleeding, -90.?, his hands would be covered with blood, A.M.B. v. I74. 12 = Pallad. 34 I ult.

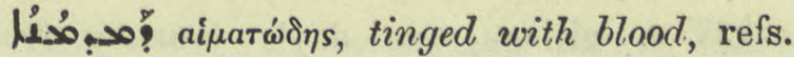
Hippoc. iv. 47 , vii. 56 , Med. $33^{8}$. 1 .

| vii. 56 .

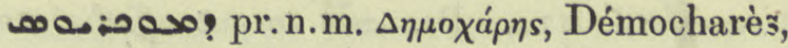
Sev. Ant. Vit. 39. 10.

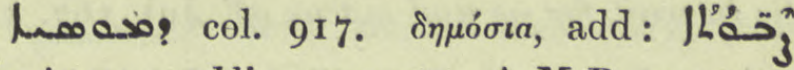

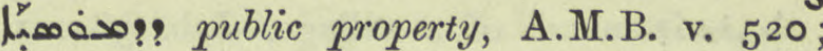

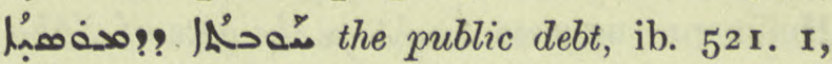
?: wheat belonging to the community, Pallad. 322 ter; ; publicly attested, Pléroph. 25. 3; 26. 6.

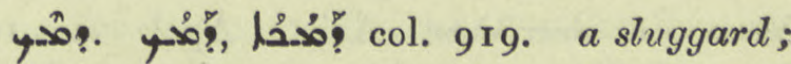

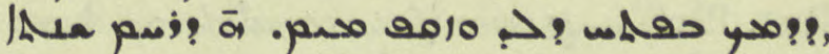
BH. on Prov. xxiii. 2 I.

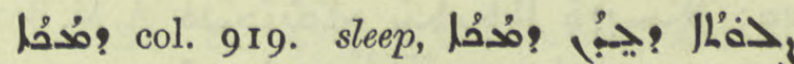
gloss. to لمحمِ prayers at bedtime, QdhamW. 242. 2.

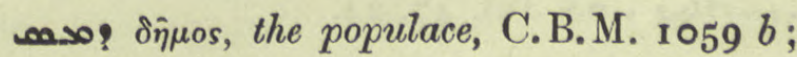
Anecd. Syr. iii. 271, 23. Usually written enses.

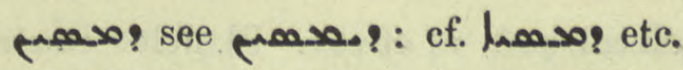

(م) Lat. domesticus, -um, belonging

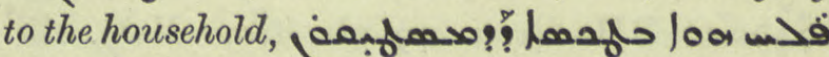
he served in the Imperial household, Pallad. 226. I3.

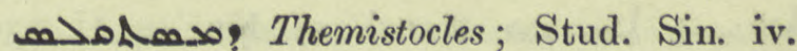
II, $12 ; 17.14$.

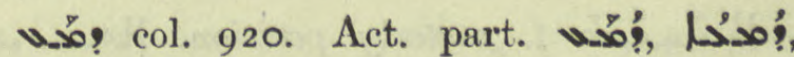
f. pl. whose eyes run, Med. 55. 19.

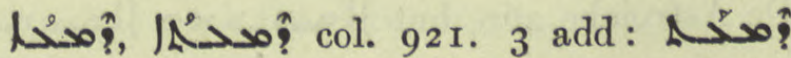

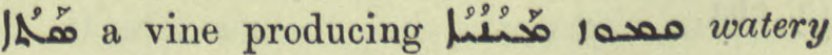
gum. N. Hist. vi. 3. 3 .

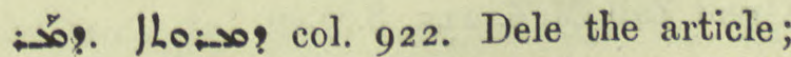
it is |ho:- o? the things = business of the Lord. 
L' Ethpe. w. li $^{n}$ col. 923. to call, summon, Aula Josephus vi. I2. I 4.

L’⿳⺈ col. 924. r) a wine-jar ending in a point; ref. Lexx. under $\int_{5}^{\prime}$, col. 3701. 2) a thick

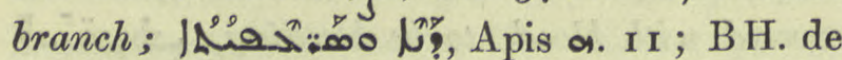

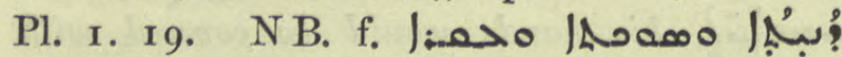
branch, stem, and root, N. Hist. vi. 2. I.

L'? a village one day's journey from Ctesiphon, Jab. 375 .

"ومادحم ? devil, A.M.B. v. 457. I5, 19.

פ col. 924. Pa. Pa" to cut off the rear,

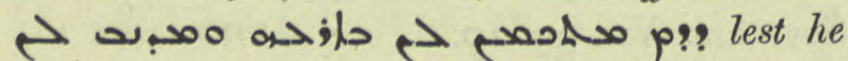
lie in uait for us and cut us off, Jul. I67. 4.

S:a' name of a village, Jab. 384 . I. Hoffmann suggests (N口) and adduces names of other gates named after goddesses, Pers. Mart. n. 764 .

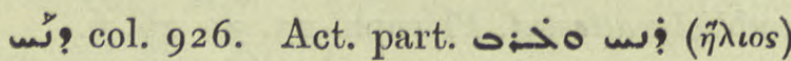

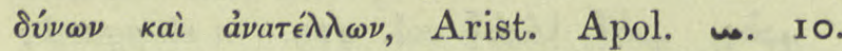
Pass. Part. פחמ

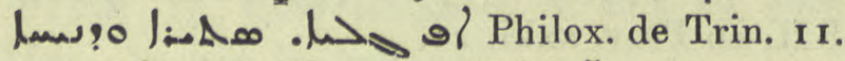

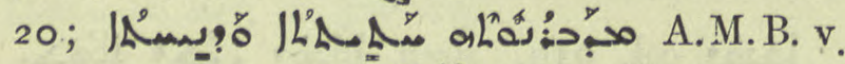

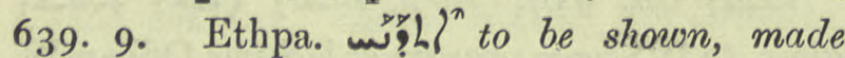

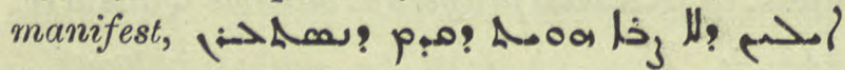
•

1L'as col. 928. the making clear of an affair, Kal-w-Dim. ro3. 6.

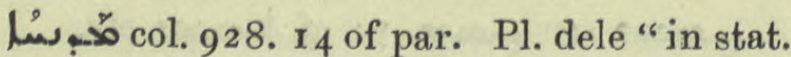

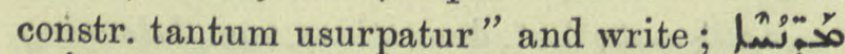

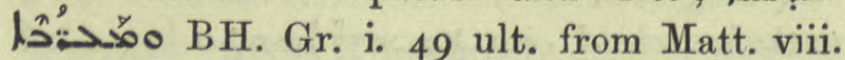
I $\mathrm{I}=$ Luc. xiii. $29 \mathrm{VHh}$. Add the direction

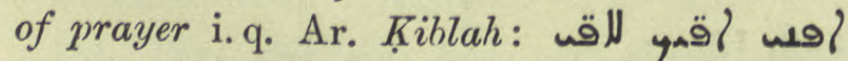
م- مب transl. of Kur'an II I39, Mingana 89 or 27 .

مبس

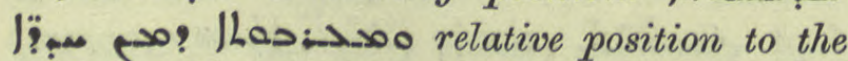
East and West of each other of two cities,

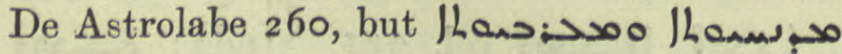
ib. $26 \mathrm{I}$.

งُ" a place on the left bank of the Great Zab, M.Z. 2I 5. 8. 9.

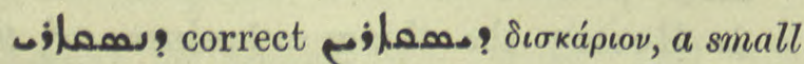
salver or paten, A. M. B. iv. 299. 5.

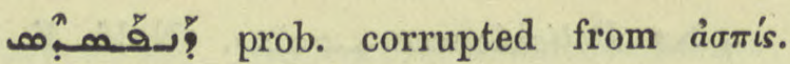
A kind of viper, Med. 279. 2 I.

'ُ col. 929. to torture. Act. part. lóg Dion. I47. I7. Pael to torment. Pass.

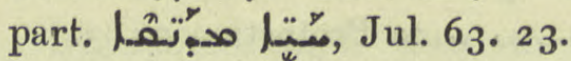

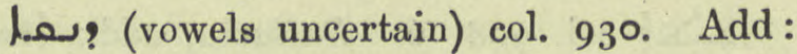
a 'roighs 4 carats, Chimie 69. I5, pl.

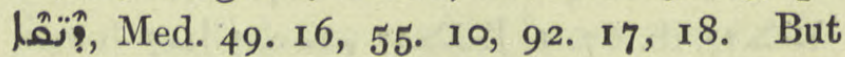

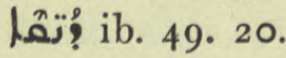

คُ extending from Daudiya to the Upper Zab,

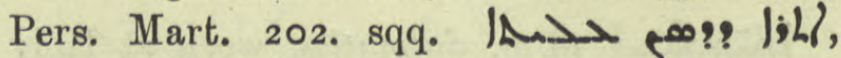

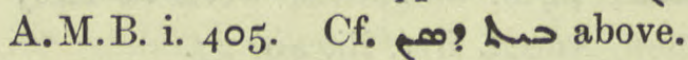

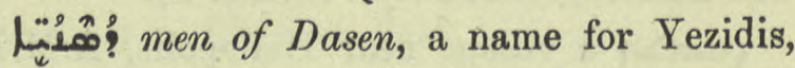
Monte Singar tit., 5 ult., 44 med.

; ' 9 col. 930. ro af. quoted from Act. Mart. i. 199. 6 af. Correct ? :

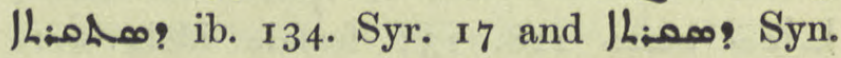

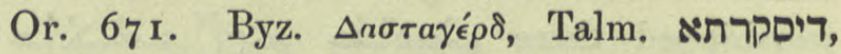
Dastagerd, N. of Baghdad on the road to Media. Now Eski-Baghdad, Tabari 295 n. I, Pers. Mart. I 20, n. 1075, a bishopric, Syn. Or. 37,44, ZDMG. xliii. $39^{6}$ pen.

पते? col. 93 I. 9 of par. to have hope extin-

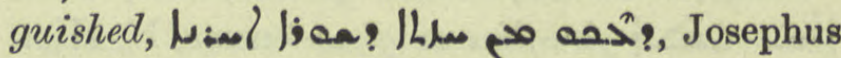

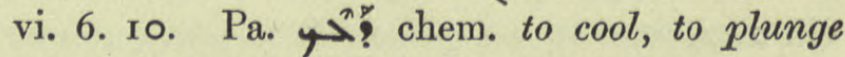
into cold water, Chimie 96. 9.

$5^{x^{2}}$ : col. 932. Ethpe. $5^{n}+h^{n}$ to be fixed,

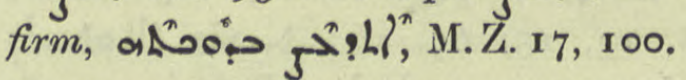

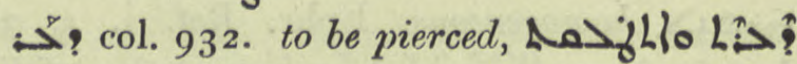
I was transfixed and wasted

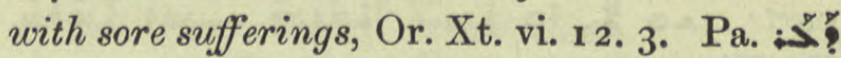

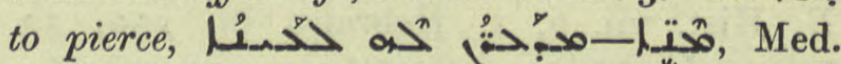
79. II ; to pierce, shine through, do: $\rightarrow$ ?

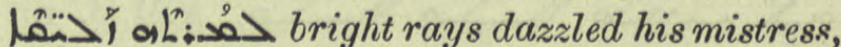

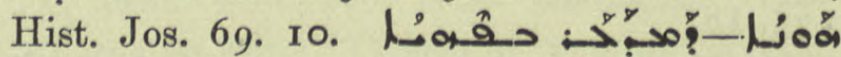
a mind riddled with wandering thoughts, Is. Nin. B. 284.8 .

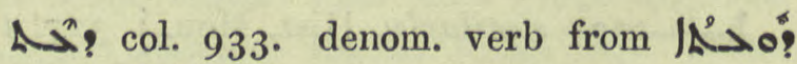
to sweat, $L_{A} \rightarrow$ ? I perspired, Mar Marcos I64. Pa. An? to perspire freely. Part.

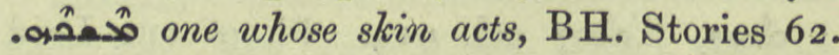
232.

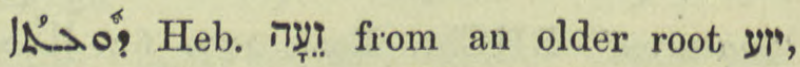

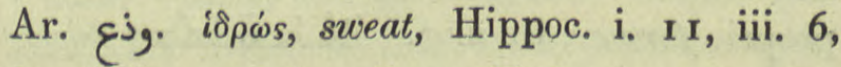
iv. 6 times, v. 67 , vii. 3 ; Med. 330. 16,360 . 
20, 538. 12, 13. Metaph.

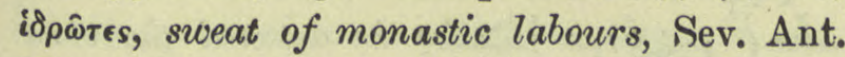
Vit, 227. I. Gum, resin, Med. $5^{8} 5$ ult.,

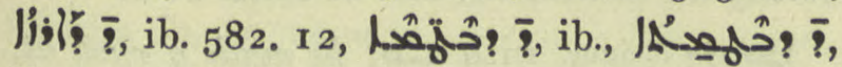

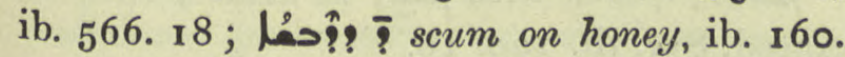

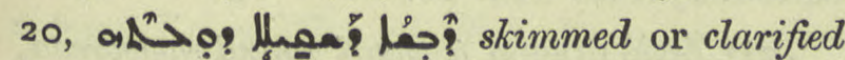
honey, ib. 3 оr. $7 / 8$; /R'? ? gum of the olive,

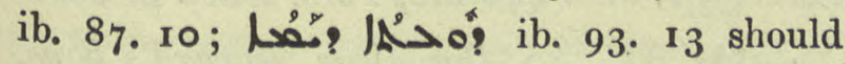
probably be كفئ

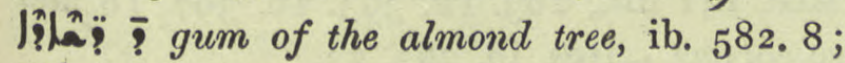
I's'ol"? ? what? ib. $56_{5}$. 17 .

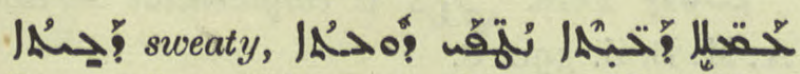
toilsome labour dripping with sweat, Hist. Mon. i. $343 \cdot 9$.

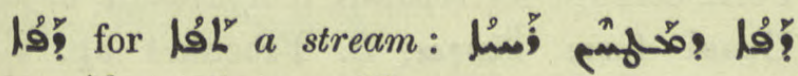
Gest. Alex. 261. 1, 268. I 7 .

16’ col. 934. I I of par. an altar slab, Takhsa 146. 4, 165. 25, 129. 19. Col. 935. 2 leaf, page, Brev. Ant. $3^{6}$ pen., ص on p. -; ib. $61 a_{13}, 14,16,191,196$.

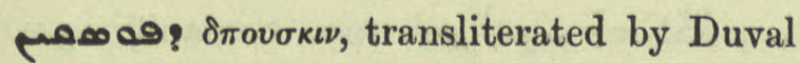
without explanation, Chimie I4. II.

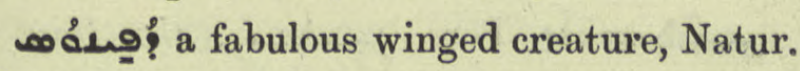
58. 14.

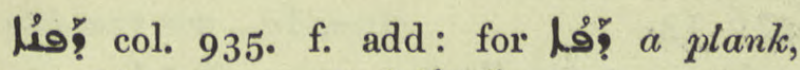

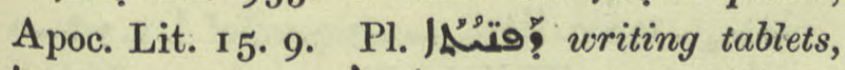

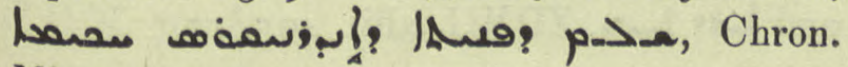
Min. 354. $2 \mathrm{I}$.

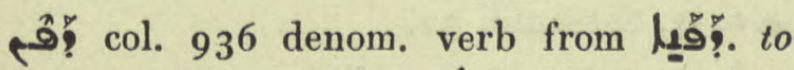
expend, spend, بنهم /, Sev. Ant. Lett. $217 \cdot 7$.

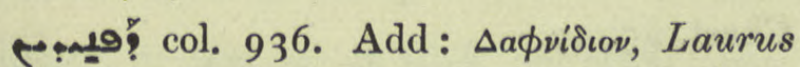
Nobilis, the laurel or bay-tree, bay leaves, Med. 40. I3, 103. 14, 107. I2, خيُح laurel berries, ib. 293. 14 and

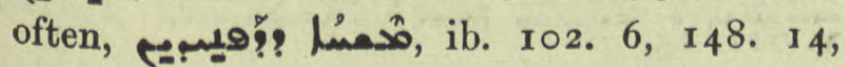

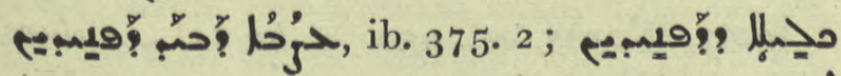
An. Syr. 185. 6 ; Jul. $247 \cdot 26$.

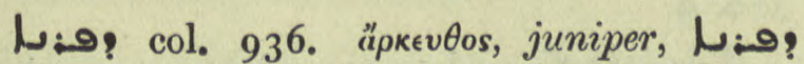

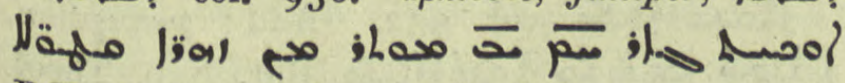
BH. de Pl. 28, حتN Q Galen. 252. 57.

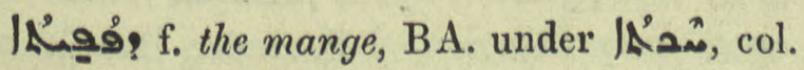
1265 .

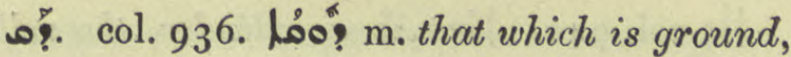
I 100 ? pearls reduced to powder, Chimie 84. 20.

| Chimie 22. $21,47.20$.

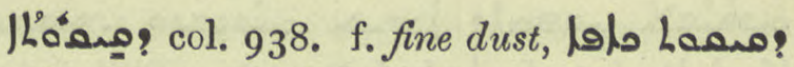
powdered stone, Chimie 56.20.

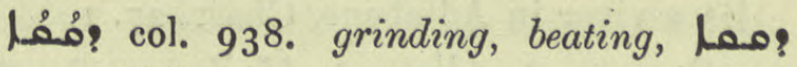
" 2 Chimie 23. I.

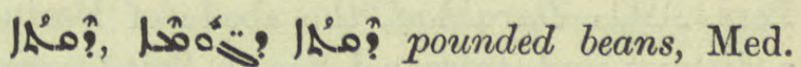
I81. 10, 22. dust, powder, A.M. B. v. 5IO. 8.

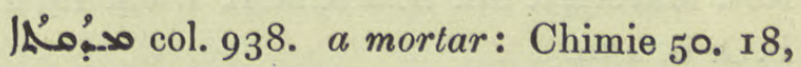
56. 9, Med. 242. I0, 320. I 7.

e. the ref. to Pat. Vit.=A.M.B. v. 198. 7 .

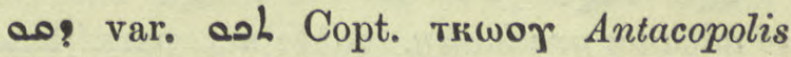
a bishopric in Egypt, held by Macarius, Diosc. ed. Nau 4r. 7, R.O.C. i. 189; name of a desert, A.M.B. iii. 6 I 4 . 16.

م. Kerkuk in Beit Garmai, Jab. 37, M.Z. 17. 96.

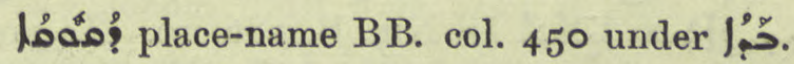

a.jog col. 939. I. a decurion, add ref. Anecd. Syr. iii. 201. 10/1 I.

Aso? col.939. 2. the tenth Indiction, Anecd. Syr. iii. 249. 13 .

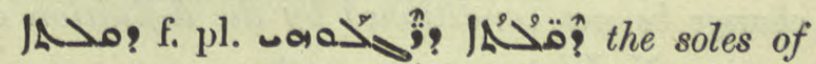
his feet, J.A. O.S. xv. 293. 3 .

ڤ้) col. 940. Add: to lance an abscess,

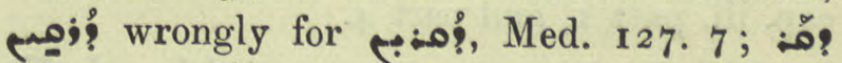

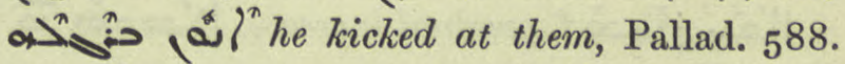
2 I. Pass. part. مئ thrust through, pierced, Jac. Syr. Hom. iii. 808 . I 8.

أم ? Bar. Sud. 85 .

|Lُفْ col. 941. stabbing pain, pleurisy, add refs. Hippoc. i. 11, iii. 22, 29, v. 7, 14,

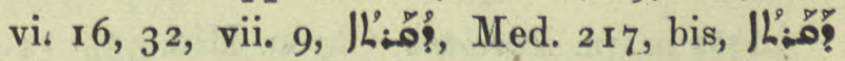
213. I, 14, pl. JL'مُّمُ ib. 202. 4, 227 , bis.

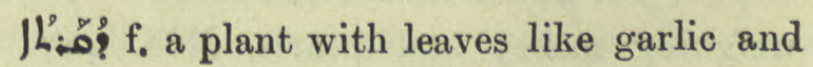
jellow blossoms, Med. 600. I r.

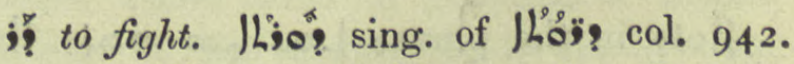
a combat, llig̣ Jac. Sar. Hom. ii. 57.7 . 


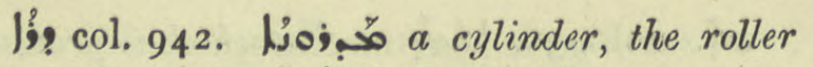

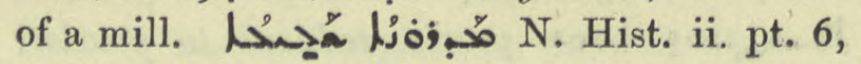
eight times.

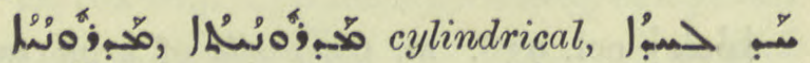

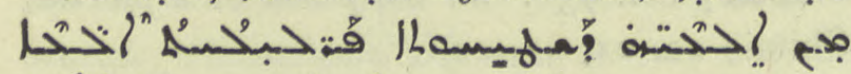

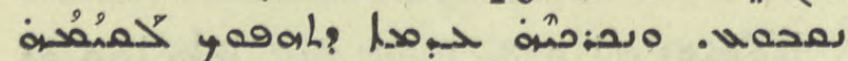

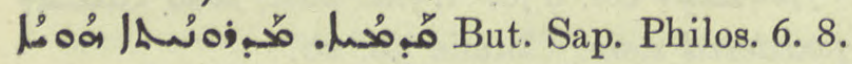

J'? a river in Adiabene, Chast. 27. I.

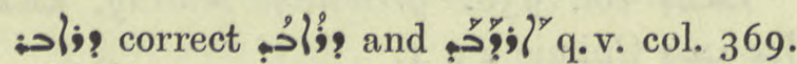
Pers. داراباد Darabhad, the abode of Darius; a district and episcopal See in Beit Garmai, Act. Maris 4r. ult. n.=A.M.B. i. 64. 14, I5, B. O. ii. 459 .

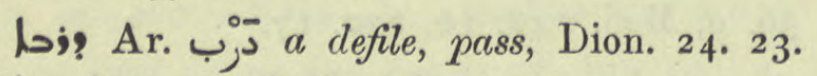
Nöld. in loc. WZKM. x. 166.

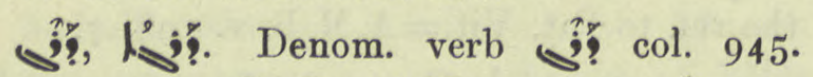
to advance gradually. Add : act. part. مبد

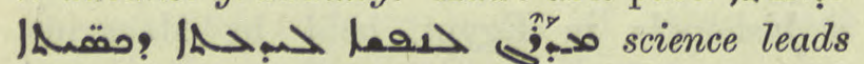
the soul on towards the knowledge of hidden things, Patr. Or. iii. i. I05. 5. To promote,

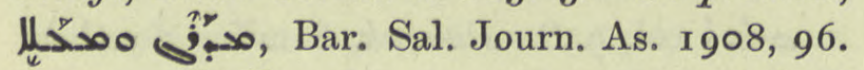
Ethpa.

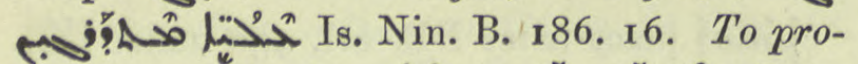

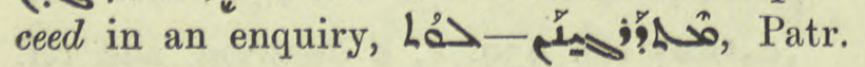
Or. iii. i. 103. 4.

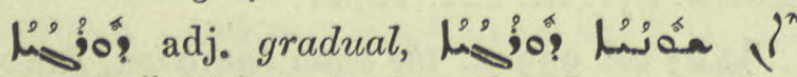

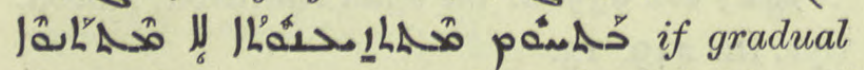
change be not conditioned by the definition of motion, N. Hist. cap. iii. part 4.

مدبّزفُ col. 946. gradually, N. Hist. cap. iii. part $\mathbf{r}$, and part 4 .

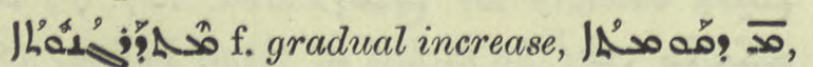
Nest. Hérac. 296. I3.

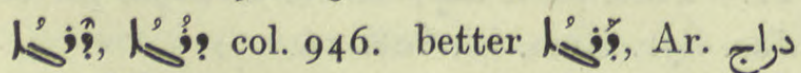
Praet. Miss. 45. 23. m. the francolin, p].

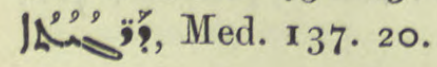

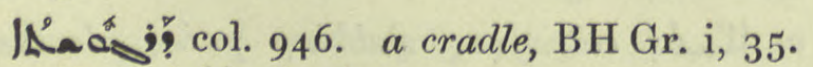

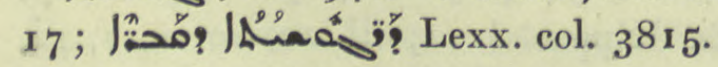

|?! m. pl. Dardans, Chron. Min. 355. I4. pă’: Ar. As a dirham, drachm. Used in weighing money and pearls, Med. 447. 3 .

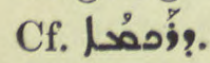

Hog! in the phrase for Hoj!

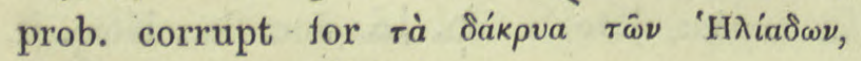
electrum, amber, Chimie $23^{6, n .} 4$. بơ Chald.

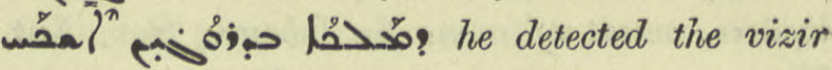
among the attendants, Ibn S. Thes. 22 a.

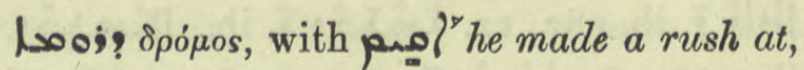
Anecd. Syr. iii. 100. 16 .

o;: Zend draono, a small round shewbread, Pers. درون, Jab. 440 ult., Pers. Mart. n. 860.

نั: Ar. درنج Doronicon, leopard's bane, Med. I4 I ult., 263. 12, 393. 10, 397. 16 and

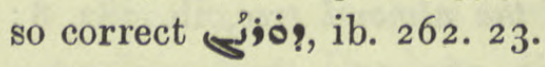

"ִ Pers. دروني a confidential servant, A. M. B. ii. 590.8 .

Loo'? col. 947 quoting Apoc. Act. i. 278.8.

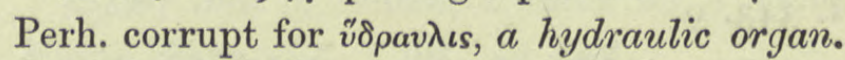
Usual form Noịa, S. Fraenkel ap. ZA. xvii. 86.

IAoo; see under ;o! Suppl.

مـ Tord, master. Transl. of àpxiarpos, Chief physician, Syn. Or. 562. Syr. 5, Pers. Mart. n. $97 \mathrm{I}$

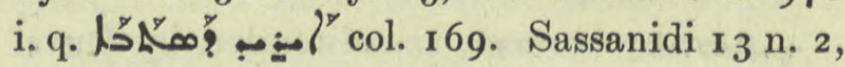
Jab. $5^{22}$, Or. Xt. i. 64 . I I.

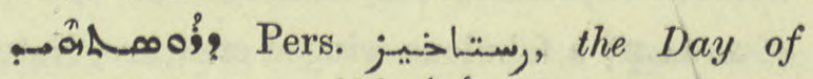

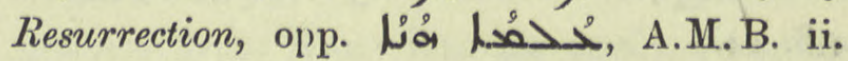
576. I3.

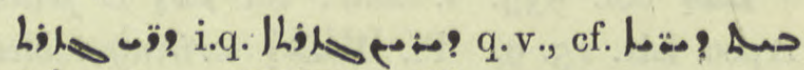

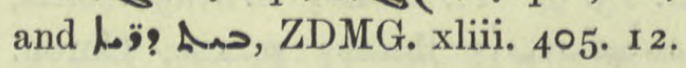

;'? name of a fosse at Shuster, from a c.;?, Sassanidi 32 .

; : col. 948 ult. Dele.

le;: Drisha, a place in Beit Nuhadra, Chast. 25 . 19.

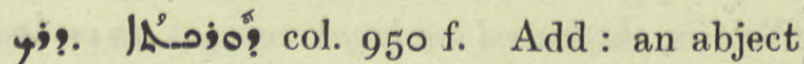
form of address, your footprints,

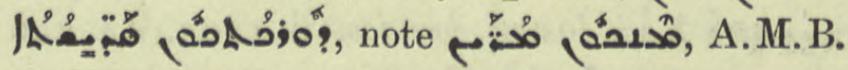
v. 6 II. I5.

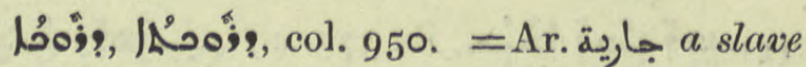
girl, girl, Nöld. G. G. A. r 884. 683, El. Nis. 77.42 ; Jolo Kal-w-Dim. 228. 9 .

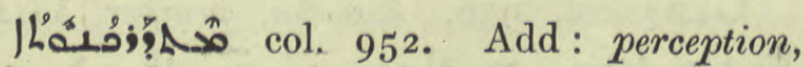

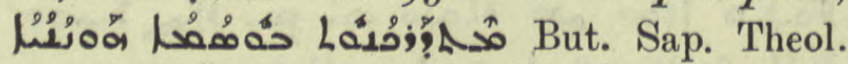

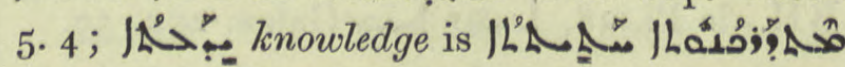
exact comprehension, BB. under IAt.- col. I 560 . 


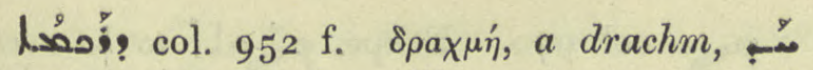
9. equals eighteen siliquae or carats, and four mo:i? equal fifteen denarii, or dinars, Chimie 69. I3/ I 4 .

درخت ؤْ prob. corruption of Pers. a tree? Kal-w-Dim. ed. Bick. 6r. 9.

pj? col. 952. Ethpe. pị!h $\left.\right|^{n}$ to use trickery,

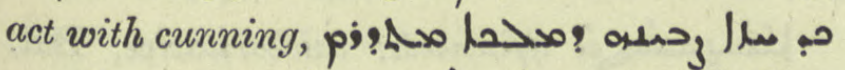
iii. 2 I 7 . II, 27 r. ult.

مهب: oraftily, Nest. Hérac. I7 ult. I77. 6 .

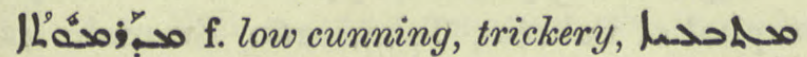
JLasoj, o profiteers and plunderers need trickery Philox. 1 23.8.

Jasoj? col. 952. dromonarii, boat-men, Anecd. Syr. ii. 18. 25 .

م the circus, A.M.B. v. $55^{8}$. I, 5 .

:50. pr. n.m. of a Persian, Jab. 522.

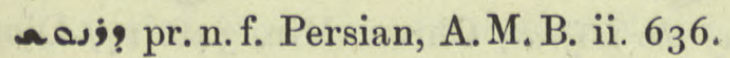

?'ס: כمس pr.n.m. Darsakis, bishop of the Armenian city Arzargan, BHChr. Eccl. 733. 3, called Sergius, B.O. ii. $378 b$ infra.

9.

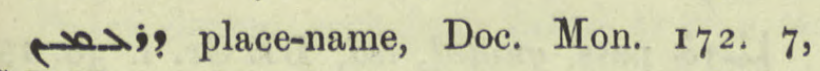
C. B.M. 707 b. anho; read asool? virarop, the highest, as M.S. Sachau quoted Schatzh 4. 14. But the third or lowest heaven, loc. cit., Fraenk. in loc. ZDMG. lvi. Ioo; Spec. Thesaur. I ult.

وُْمَّ

!' name of a fortress in Thrace, Anecd. Syr. ii. 22. 1, 178.18.

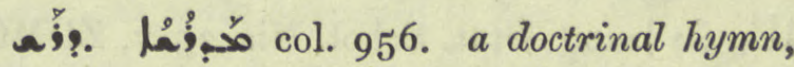
add refs.

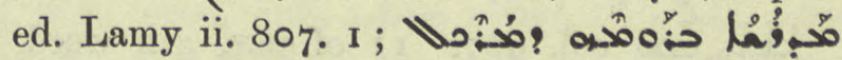
Brev. Chald. i. r29. I5; Qdham W. 123. 5 ;

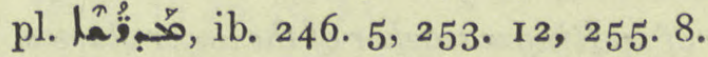

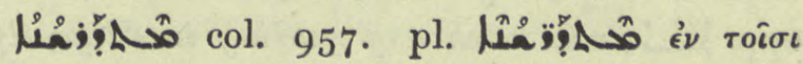

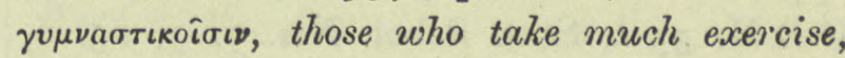
Hippoc. i. 3 .

12ำ? col. 956. a drug for spleen and liver complaints, ref. Med. 399. 3 .

Jan? Greg. Carm. ii. 49. 10 should be

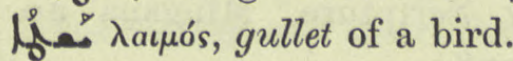

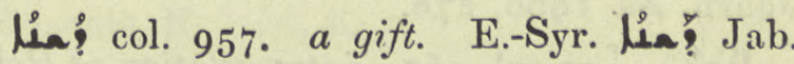
77. 4, 5 and passim.

مै denom. verb from to give gifts,

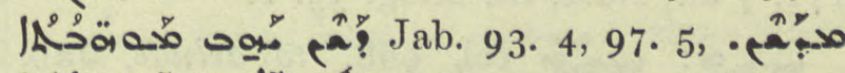

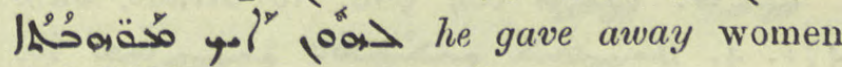
and children as presents, ib. 200. 4.

攺? col. 958. the desert, a certain plant

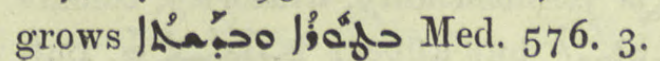

\section{$\therefore$ Q :}

Jäla i.q. Jöa pl. of LLoó an abyss, Jac. Edes. Hex. 32. 7 .

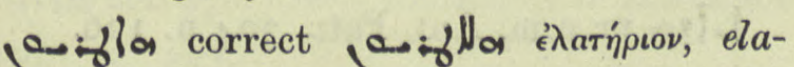

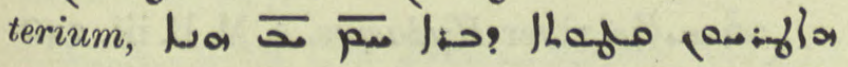
مخه مهار BH. de Pl. 73, Gottheil in loc. ZDMG. xliii. I25. Cf. Ar. PflnN. $33^{2}$.

\section{1}

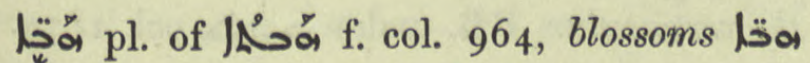
I. Jul. 93. 2 I ; metaph. ib. 1. 14, 100. 10, Is. Ant. i. I 74 . 1 I.

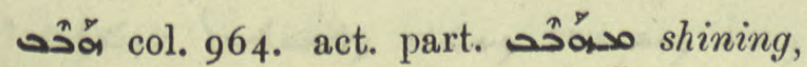

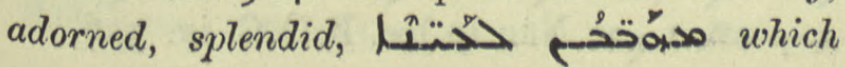

dazzle the eye, Is. Nin. B. 34. 6. Pass. part.

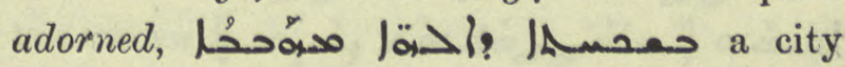
adorned with trumped-up gods, Jul. 2 I. Iо.

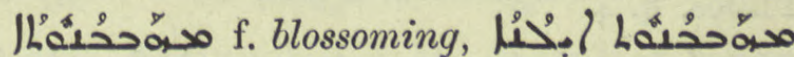

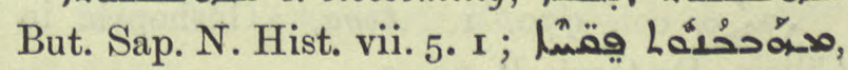
Theol. 3. 2.

นُ نُá col. 964. The enemy = Satan, Hormizd. 169, 1255, 1672, $2236,3066$.

lua extra name of Athanasius archimandrite of Karthamin, Laq eashl, Jac. Edes. Chron. Can. 575. ro. Fraenkel corrects hoof ZDMG. lii. I 53 . 
حصحá pr.n.m. Abib, Bishop of Tarsus, BHChr. Eccl, 387. 2.

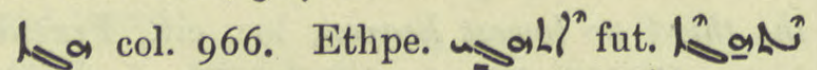
to spell, .

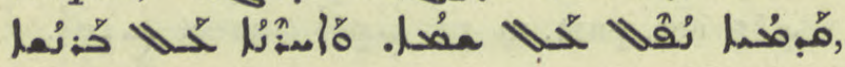
Op. Nest. 39. I.

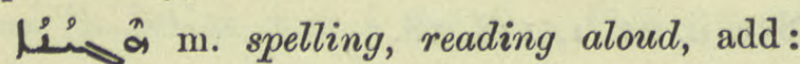

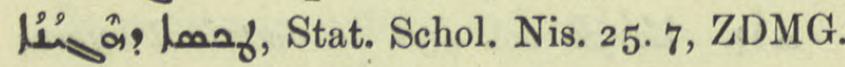
xxxvi. 132 , xlvi. 24.2.

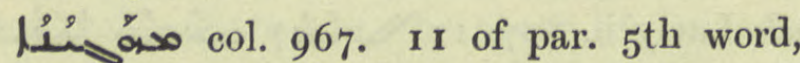
corr. 4 ald to pronounce, El. Tir. 49. Teacher of spelling = teacher of the lower classes, opp. مخمـن teacher of reading intelligently, C.B.M. 53 b, Stat. Schol. Nis. 7. 9, 25. 6, 27. 3. Pl. M.Z. 73. 15, Barhad 383. 12.

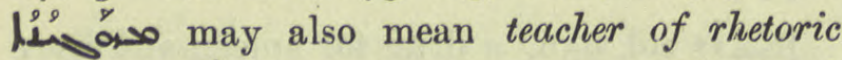

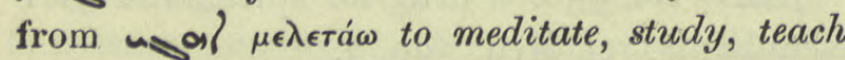
rhetoric, ib. 399 n. Teacher of the spiritual meaning of Holy Scripture, Mingana ap. Journ. As. 1906, 278.

JLán مُ مُ col. 967. interpretation of Holy Scriptures, Barhad ap. Journ. As. 1905, I60 n., I 7 1. 7 af., Nars. ed. Ming. 33. I.

অr:

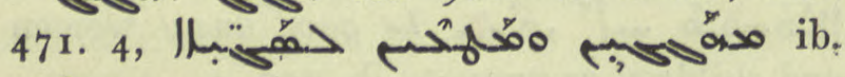
478. 14 .

يُ

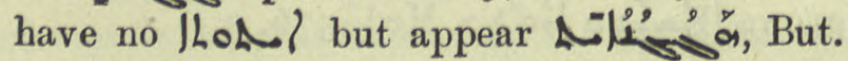
Sap. Philos. 6. 2.

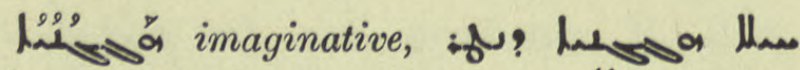

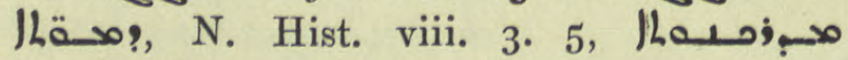

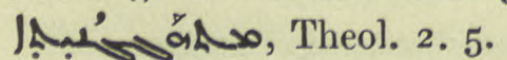

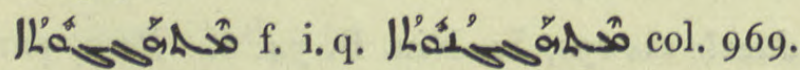
㪿 capable of limitless meditation, But, Sap. Philos. 5. 4.

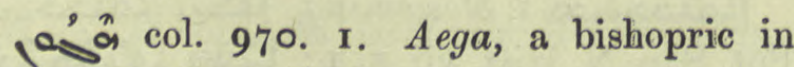
Cilicia, De Goeje B. 64. 3 .

mafoga pr. n.m. N. Hist. vii. 5. 3 .

(anga "̈plov, wool, Chimie 274, n. 3. Cf. ans, p. 3, and jasia below.

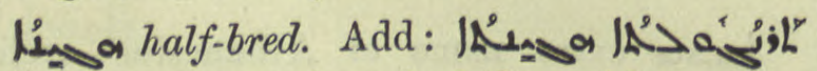
a common or barndoor fowl, Gest. Alex. 18. 7 .

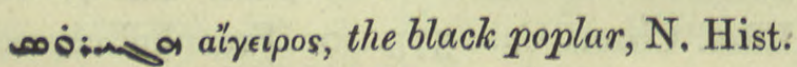
vi. 4. 1; Gottheil in BH. de Pl. 4. 10.
Pya col. 970. Ethpe. Ránl to be over-

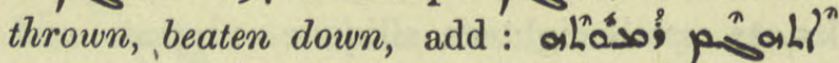
A. M. B. v. $5^{8}$ n. Ethpa. Ryăl(n I) to be

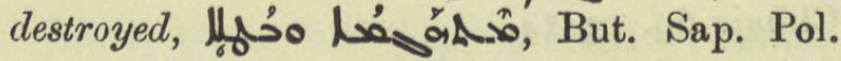
iii. 3 ; Poet. Syr. 8. 6. 2) to gather force, be vehement,

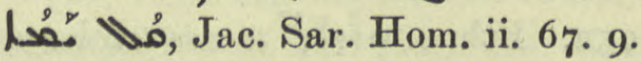

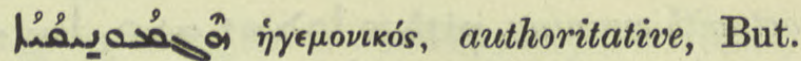
Sap. Philos. i. r. Eccles. provincial, Syn. Or. 69. I7, 24. See holow, and col. $3^{26} 5$.

- $\rightarrow$ Ar. capital of Bahrein, Tabari 260, Eranšahr 42 ; ZDMG. xliii. 404. 12, 407. 2 ; Syn. Or. 128, 2 I 6 ; Nöld. Syr. Chron. 47, n. I.

今á col. $97 \mathrm{r}$. Wal" to live as a Mohammedan, have intercourse with Mohammedans, 3 p. f. impf. uisah, Jac. Edes. Can. 13. 10 and $n$.

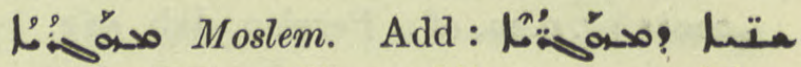
era of the Hijrat, Chron. Min. 348. 17, Dion. I. I3.

ما

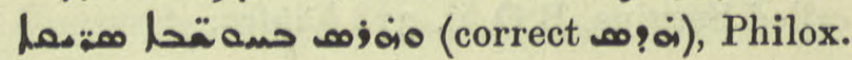
I60. 4 .

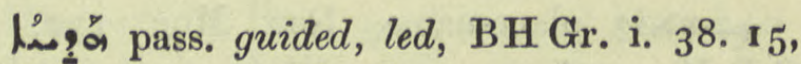

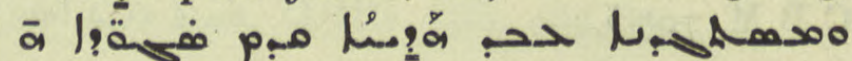
.

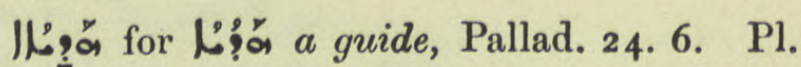
lïgá ib. 533. 2 ; Gest. Alex. 183. 5 cod. B. C. D. but Lُ?á in text ; Schatz. 240. 3

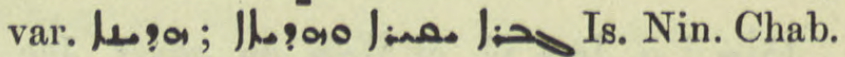
91. I, l.e?a ib. ro. I5.

K\}? a pr.n.m. Ant. Patr. 304 n. 126.

مْهُ ? ? a, Aram. Tָ Hadad, a storm god,Inscript. of Zenğirli, Lidzbarski 409, 44I, WZKM. vii. $130 . \mathbf{r}$.

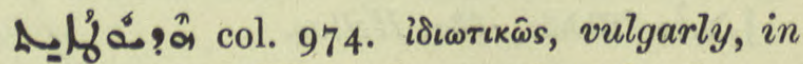
the vernacular, B B. under حصد col. 1879.

a a Edictales, Law students in their second year, Sev. Ant. Vit. 47. 4, marg.

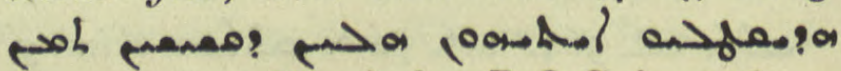
مب Nau in loc. R.O.C. iv. $55^{6}$. 
Lsُợá col. 975. a member. Add: a passage of a MS., DBB. I364. I, 2677 under /10.

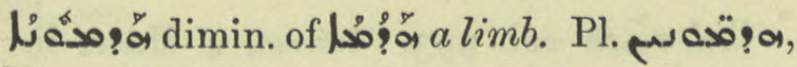
4 Macc. $33 \mathrm{I}$.

p!a denom. verb. Pass. part. m. pl.

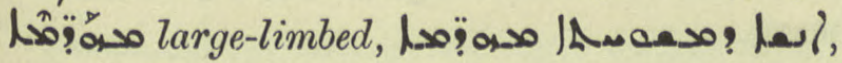
Anecd. Syr. iii. 337. 22 ; horses cannot carry them حم ? 20, ib. 23.

L'ơ ơ f. the being endowed with limbs, Is. Nin. B. 17. 14; manifestation in bodily form, ib. 50. 13.

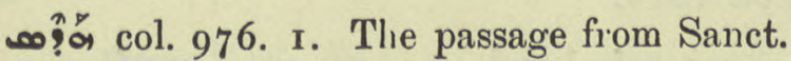
Vit.=A.M.B. v. 4 I 2.9 means: she occupied herself with the clothing of the nuns.

jo col. 976. Ethpa. j:̣̆all add: trans. to glorify, Sev. Lett. 230 . 18.

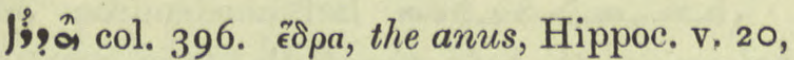
Hunt. clxx under joo col. 2727 .

م.joliga col. 977, the poppy. Cf. col. 1206 .

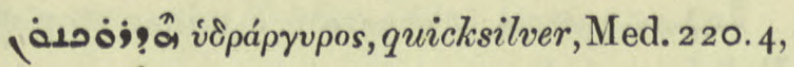

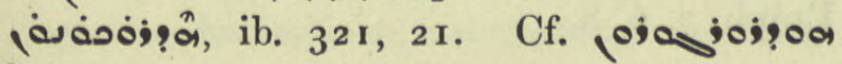
below.

لע. Ant. 294. 10, 296. 12.

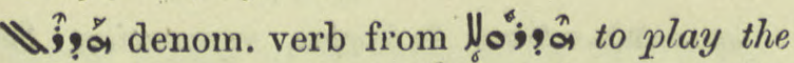
water-organ, to praise, a حن Hormizd. $230 \mathrm{I}$.

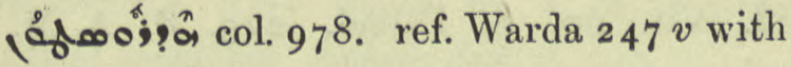
gloss. مavine and honey.

wasiga I) river Hedrinos = the Pishon, Chron. Min. 354. 2. 2) Hadrian(opolis), bishopric in Caria, De Goeje B. 65. 20.

j!a col. 978 . See a conjecture of Hoffmann in ZA. xi. 247 .

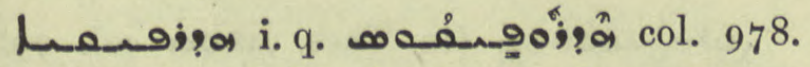
dropsical, Anecd. Syr. iv. 87. 9; pl. prọoa ib. 19 .

oa col. 980. 9a oa used impersonally, with a plural, in a corroborative sense, those very, those same, لـ لـa ọ, Ephr. Ref.

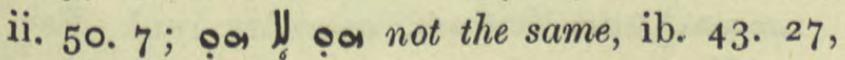
46. 40 .

K'ása col. 980. Add: state, eondition, BHCarm. I39. I I. 2716

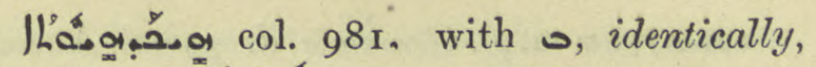
exactly, opp. حبَّمَ Nars. ed. Ming. $35^{8}$ note.

lóa col. 983 . Aorist passive infinitives in - Anvar formed with fóa are common;

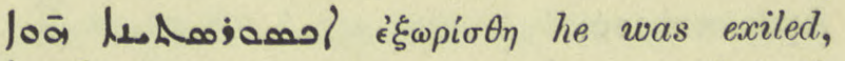

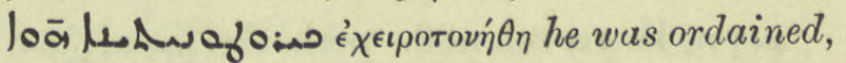
ZDMG. xxvi. 830 in BHGr. 1. 47. Add: yoa cso what happened to thee? Kal-w-Dim. ed. Bick. I 2. 8, 56. 7, Pallad. 69o. Imper, with pron. suff. uloa contr. of « N T ' soa, Amax thoa rloa be persuaded, Theod. Mops. in Jo. iv. $2 \mathrm{I}$.

Lawal correct JLaina state, likeness, Mar Mare. ${ }^{6} 6_{5}$.

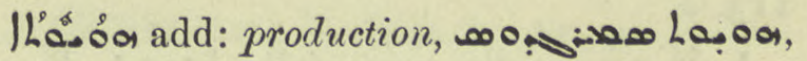
Chimie I 5.9.

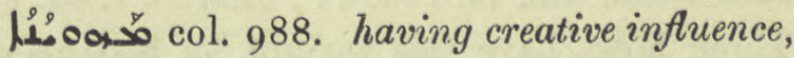

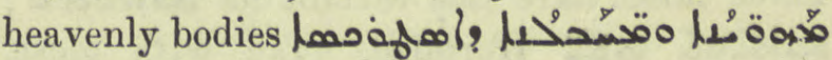
have creative and destructive influence over the elements, N. Hist. ii. v.

IL'ás.oas the act of creation, creativeness, add ref. But. Sap. Theol. 2. 3 ; Philos. 3. 4 .

Joa perh. ṽa入os or кúavos, a stone which cures dropsy, Anecd. Syr. iv. 87. 8, Land. ib. 83 trans.

الابلّة , Ubulla, near Basra, province of P'rath d'Maisan, ZDMG. xlvi. $75^{6}$. 9 af.,

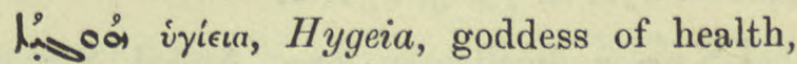
A.M.B. iv. 284 .

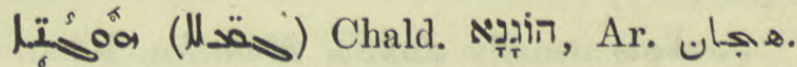
m. pl. swift dromedaries, so corr. h-goa, Kal-w-Dim. ed. Bick. 96. 23, 97. 19, 127 n. F. IA

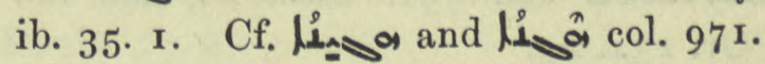

I!oa Ar. interj. Ho, Hist. Mon. i. 288 , ii. $5^{\mathrm{I}} 7 \mathrm{n}$.

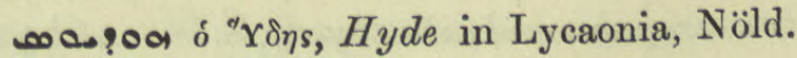
F. S. i. $473 \cdot 99$.

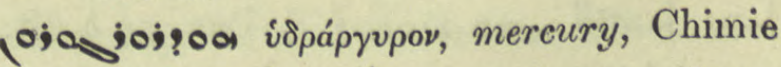

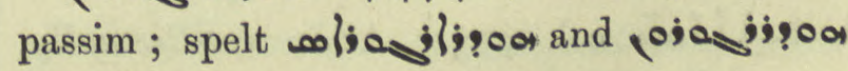
col. 989 .

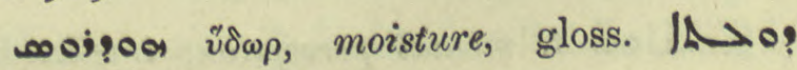
Chimie 7. ${ }_{3}$. 
Q. Syr.iv. 87. 19, hمبر col. 978 .

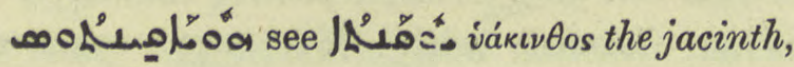
Apoc. xxi. 20.

اهـون ,هيب . cool, tranquil, wise (as BB. not strong as B.A. ib.) Nöld. and Schulthess emend clooa for

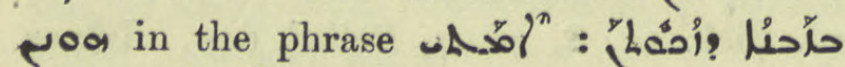

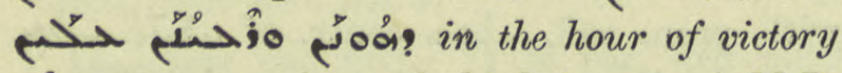
when we are wise and think it over, A.M.B. v. 15. 18; and again for ớ read roa lis o anyone with a troubled mind visiting S. Antony returned reasonable and calm, ib. II3. 2.

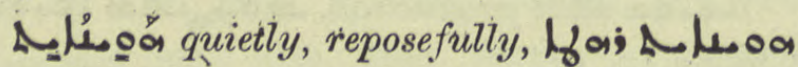
Loa, Stud. Sin. ix. 11 8. 2 1; A.M.B. v. 64. 20. Three MSS. have this variant for A fuow ;

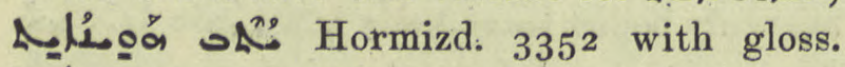
Atroar.

Loa a tambourine, Mt. Singar I I. 6.

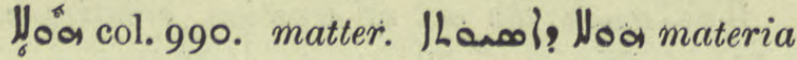
medica, Galen. 246.7 , 248. 4.

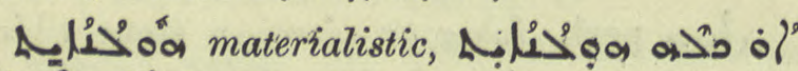

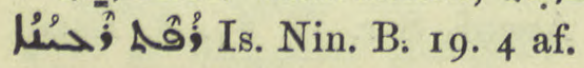

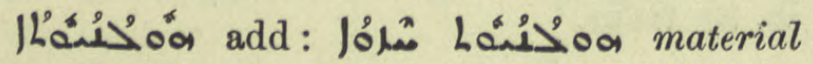
form, Is. Nin. B. 18 8 . Io.

Noo a village and monastery in Anzitene, Anecd. Syr. ii. 75. 5, 23 o. 2.

l’خْóa a dweller in lloa, Anecd. Syr. ii. 23․ 3 .

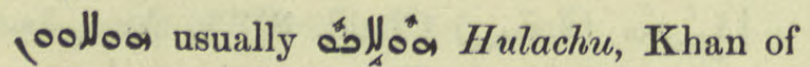
the Mongols, BHChr. Eccl. 733. 6, 755. 8, 759. 24 .

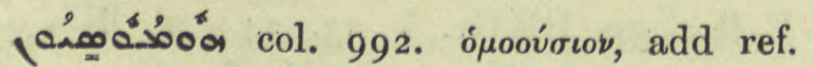
Syn. Or. 134. 23.

Dá denom. verb Pael conj. to compose one's

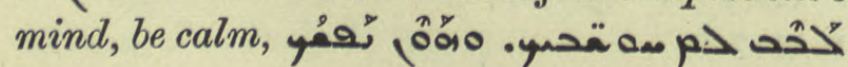
be courageous and calm thyself, S. Dan. $46 b_{3}$ af.

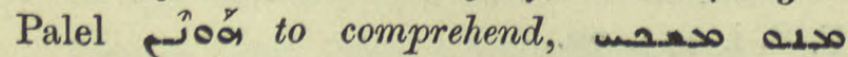
Sصl S. Dan. 57 ult.

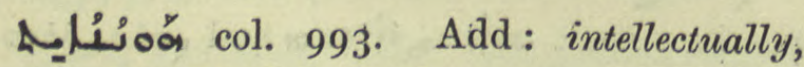
reasonably, Ebed J. Card. I9. 3.

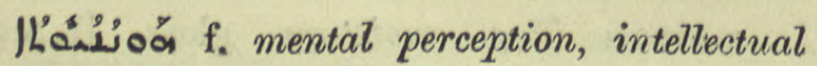
consideration, Hist. Mon. i. 3०7. 22. asifanm

Lioáso endowed with mind, But. Sap. Philos. 6. 5 ; pl. لöaso Warda $25 r$.

J'ásoả ocol. 993. Stávota, 4 Macc. ii. 2, reasonableness, ib. ed. Bens. v. 19 , vii. 3 ; |lewoax II à Jlogofă, Med. 5. II and marg.

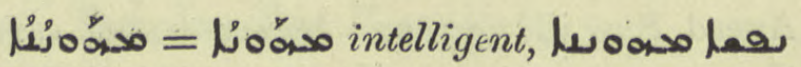
Nöld. F. S. i. 477.

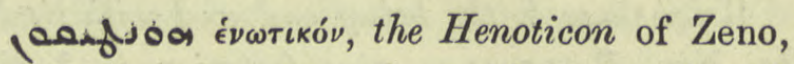

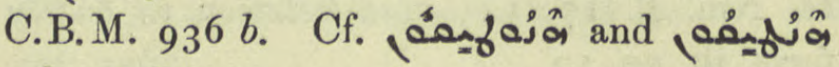
col. 1028 .

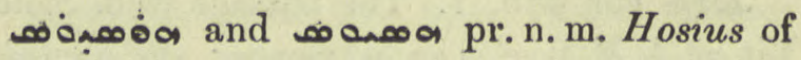
Cordova, Or. Xt. i. 84. 4.

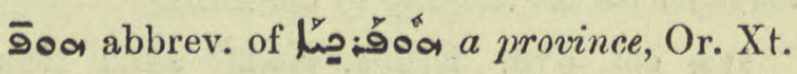
ii. $286.9,290$ pen.

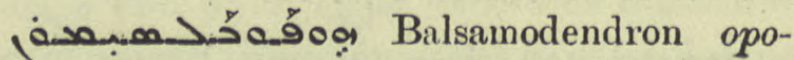
balsamum, the balm tree, Pallad. I23. 7 .

(of?0900 inoî́tis, under garment, Jac. Edes. ap. Manichéisme 100 n. I3.

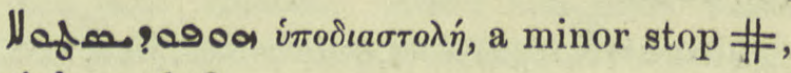
Epiph. 7. 6, 8.

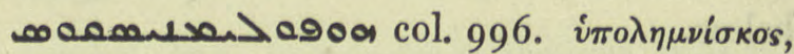
the critical mark $\div$, Epiph. I6. 10, pl. C.B.M. ${ }_{90}{ }_{5} a$, Lag. Vet. Test. 356.69 .

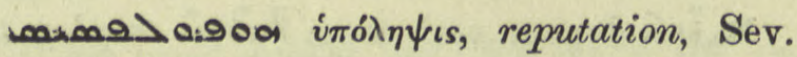
Lett. 148. 6, m man ح090a, ib. 378. 8. 14 , m 000 , ib. 347. 23 , 0000 , id. Vit. 6o. 8, Pallad. 225. 8, A. M. B. vii. 124 .

18) of a synod, Sev. Juett. 6 r. 6.

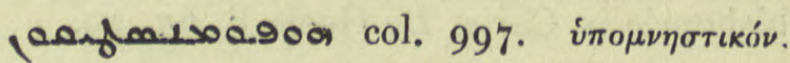
a memorandum, memorial, Sev. Lett. 107. 10; مanfonsogoa, Epiph. 6. 14.

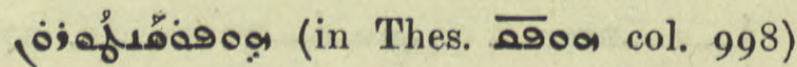

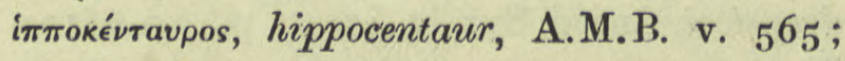

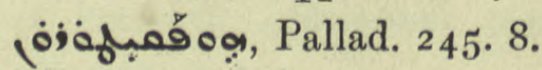

مaffooa virateia, largesse on the accession of an emperor, Jul. 23. 8. See lif̣oóa 2.

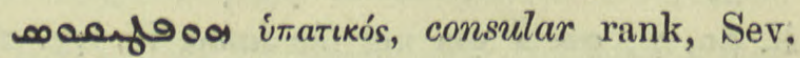
Ant. Vit. 104. 8.

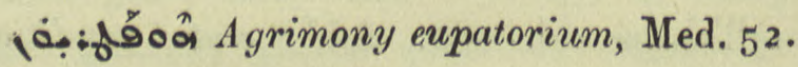
I 7, $198.12,296.5,603$. II. 


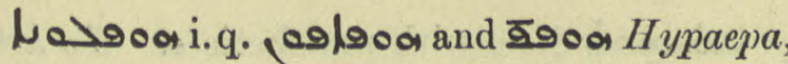
a town in Asia, Ebed J. 206, col. 2099 under סטגial.

م.0 í $\phi^{\prime} \nu$, hyphen, Epiph. 7. 6.

10.fhمo0a pr. n. m. A.M.B. iii. 276.

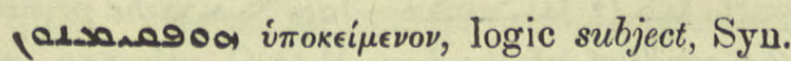
Or. 25 o. 2.

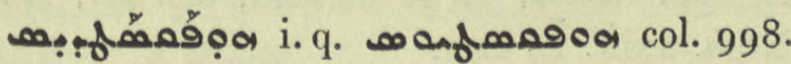
iлокьбтis, Cytinus hypocistis, a parasite of the cistus, Med. 64. 10, 159. 9, 160 bis, 208, 21 , 235. 16.

(90) 995 Hypericum.

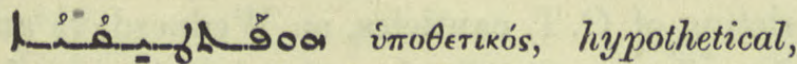
f. نa sat. Sap. Theol. 2. 2 ; Nat. Hist. ii. i. sect. 7 ;

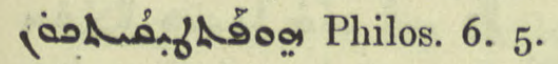

m Sev. Lett. I8. 10; pl.

h>ojoa the Heruti, Dion. Ined, 474. 2.

(a.joa ஸे

Pet. Ib. 106. 4, I1 2. 4.

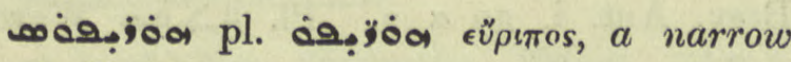
strait, N. Hist. iv. 5. 3 .

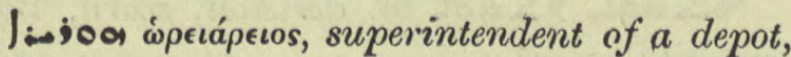
Pet. Ib. 106. 20.

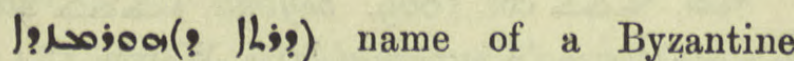
Imperial residence, Anecd. Syr. ii. 242. 2.

joa?hrojoa Hormizd + Ahura, name of a King of Mosul, A.M.B. i. 409.

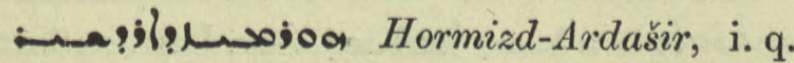
and again in our days Ahwaz on the Karun, Tabari I 3 n. 3, i 9 n. 5, Eranšahr I44 ; E.-Syr. bishopric Syn. Or. 673; 35, 44, and often; ZDMG. xliii. 4 ro. Later contracted to $-\infty .0 ; 00$, Hippoc. xxv. n. 3 . Intermediate forms see col. 1000 .

a- Dajoa Horus + Apollo, Horapollo, a grammarian of Alexandria, Sev. Ant. Vit. I4. 2, I5. 6, and often, (a) (900, ib. 23.4 ; R. O.C iv. 348 .

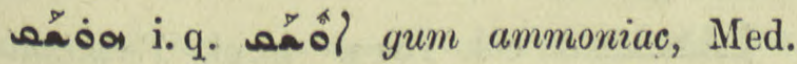
I $53.4,25$ O. 10. pla Ar. jo to put to flight, rout, Dion. 26. 6, 45. I2. Ethpe plall to be routed, defeated, Dion. $7.2,5$; c.c. مب ib. 45. 6, 7I. 1 I.

A A هout, defeat ; so correct IAw, Dion. 7I. 14.

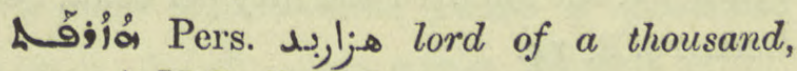
pr.n.m. A.M. B. ii. $3^{\text {I } 8 . ~ 5, ~ n . ~}$

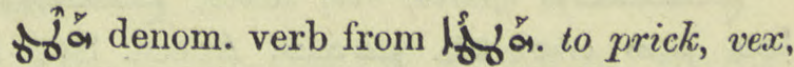
Poet. Syr. 98. 9. Ethpa. Söhl to become

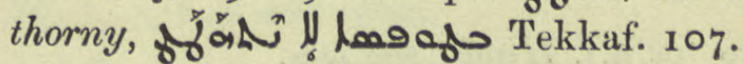

for a pr.n.m. of a bishop present at the Council of Dadišu, A. D. 430, ZDMG. xliii. 396. 17.

นาช์ Mt. Etna, N. Hist. iv. I I. 2.

1h้ófó col. 1002. pl. Luc. xxiii. 19 Curet.

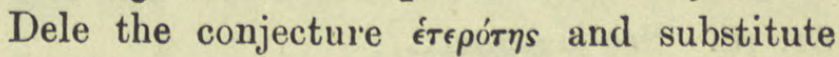
étaı ation, S. Fraenk. ZA. xvii. 86.

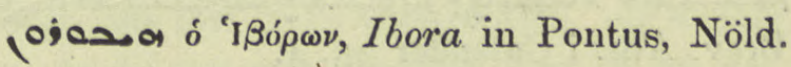
F. S. i. 475 . 140.

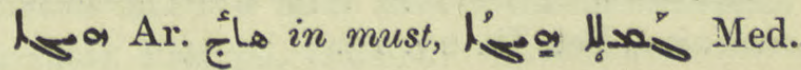
588. 23 . Cf. M oor.

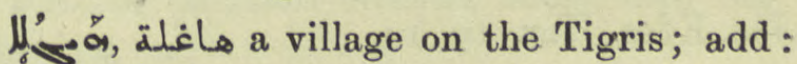
Hist. Mon. i. 53. 16, 199. 5, Pers. Mart. 238 ; M. Z. 202.8.

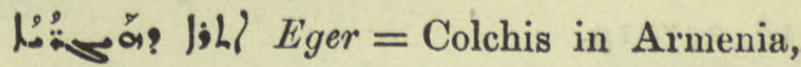
Pers. Mart. 736. $\mathrm{f}$.

es:a name of a village, A. M. B. iii. 474. Ne.a Arabic form of vi $\eta$ matter (for Noa), Pallad. 99. 5, Hist. Mon. I. 283. 20, Sassanidi 21. I I. Pl. hoa لخه مي Is. Nin. B. 9I. 4 af.

H h a name of a village near Nisibis, Chast. 57.8.

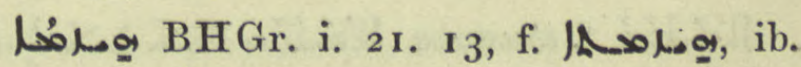
22. 5, pl. I 100 .0, ib. 33. 24. A thorny vetch on which the parasite dodder grows, Ar. PflnN, 146. Cf. Lool? col. $143 \mathrm{f}$.

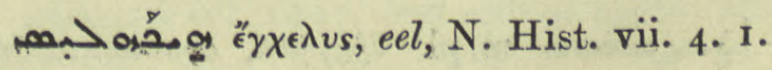

\מ̌ăhir denom. verb Ethpaial conj. from lla on to be enshrined, to be lodged in a temple,

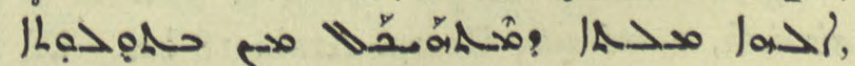
Warda $62 v$. 


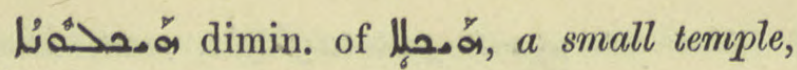
Chast. 3 I. 8.

ollor olla a cries uttered by demons, Pallad. 52 I. 9, quoted Hist. Mon. i. cliv. n. I.

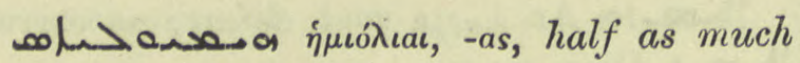
again, i.e. one hundred and fifty per cent., Sev. Lett. 29. 8, 14.

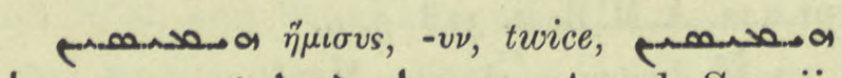
loa arodal anecd. Syr. ii. I 18 . 10.

t.a pr.n.f. Hind; see بa.

○ sar, opa Ar. هi. pr.n. Chwolson Grabinschriften I33; id. ap. ZDMG. xliv. 527.12.

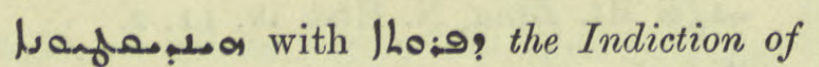
the $L a m b=849$ of the Greek era, Jo. Tell. 8x. 16. See Hanforat?.

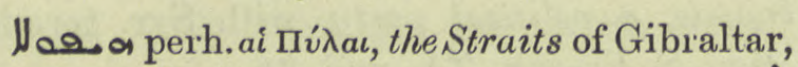

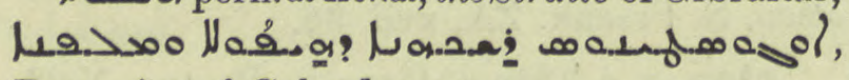
Brev. Ant. i Calendar 35. 4.

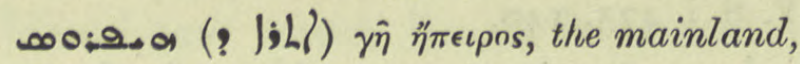
continent, Pet. Ib. 23 . 15.

J is a "H $\mathrm{H}$ as, one of the Isles of the Blest, Jac. Edes. Hex. 19. I 4 .

o: a place-name. Perh. the Hirran of

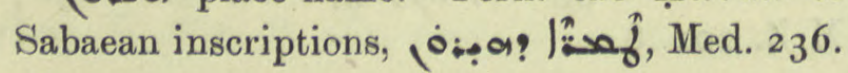
18, 247. 19 .

Aa Ar. هيت, Hit, on the Euphrates, Chast. 61. I5; 19.

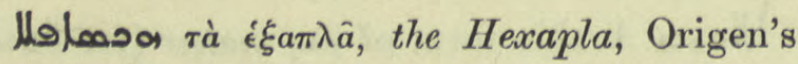
comparative edition of $\mathrm{Gk}$. versions of the O. T., Or. Xt. i. $300.4,8$, г. Byz. Zts. x. 300. 3 .

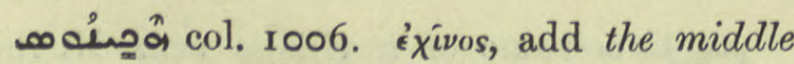
or true stomach of ruminants, N. Hist. vii. 6. 5 .

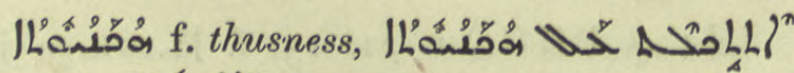

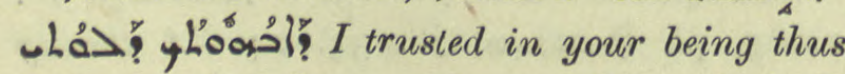
fatherly-minded toward me, Ishoyahb ed. Moncrieff 68. 5 af.

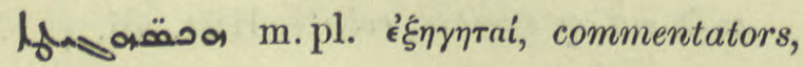
Hippoc. xxii. 9 .

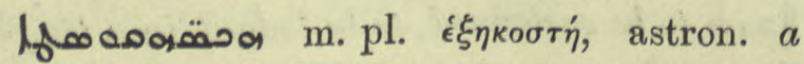
sixtieth, a minute, Georg. Arab. I 8 bis. Cf. Howareas? col. 187.

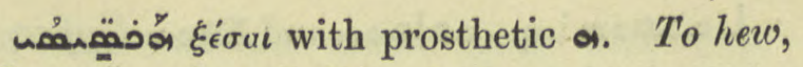

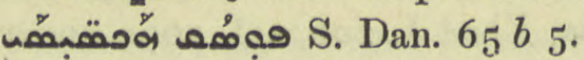

J from BHGr. i. 60. 21 and 23 .

Wa col. roro. Aph. "Mâl" to praise; act.

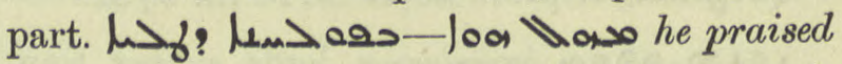
the conduct of the boy, R.O.C. vii. II6. 28. Ettaph. "arhLl" to be derided, scorned, Hex. Num. xxiii. I 9 Lag.

بحํำ 2) Rit. E.-Syr. each of twenty parts of the Psalter. Seven $\prod_{4 \sigma} \log _{0}$ comprising Ps. i.-lviii. are recited on Mondays and Thursdays; seven, Ps. lix.-ci. on Tuesdays and Fridays;

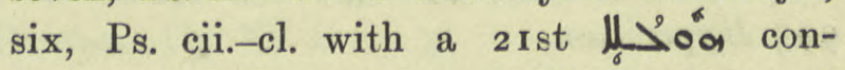
sisting of O.T. canticles, on Wednesdayss and Saturdays. On Sundays ten hulale are said, Maclean and Browne 227. f., Takhsa 84. r,

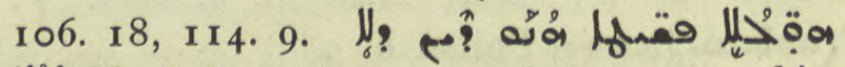

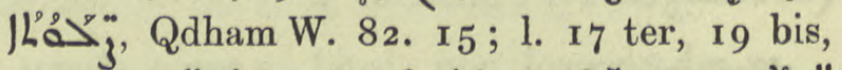

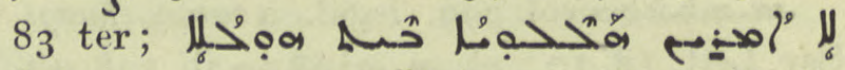

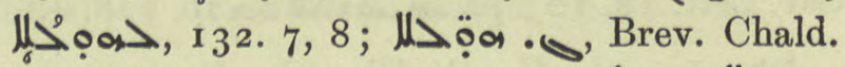

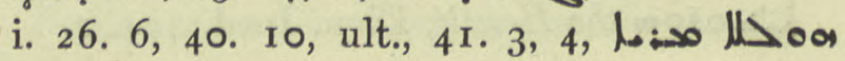
4) i, ib. 78.4 af. 3) Jac. the response

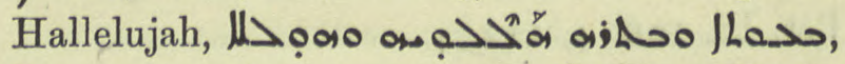
Brev. Ant. i. 59 a 3. Pl. لll I $48 v$.

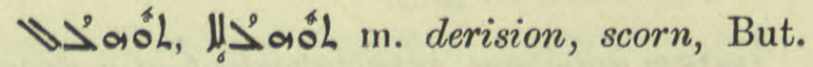
Sap. Eth. iv. 5 .

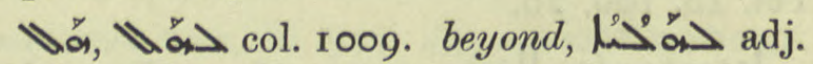

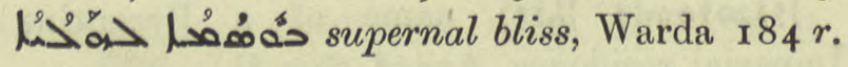

H. Ta a man from Aleppo, Protection 20. 6 .

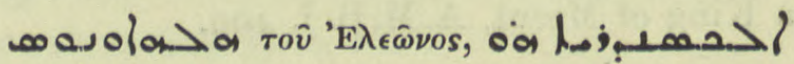

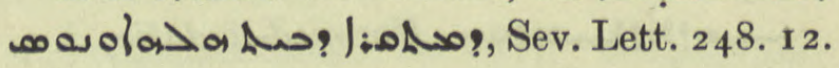

مa Egyptian name for Venus, under

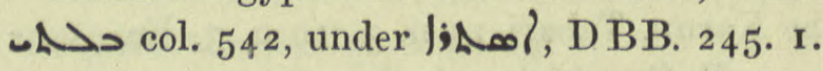

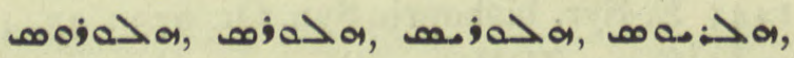
Haloris, near the source of the Tigris, Gest. Alex. 26o. ult., 26r. 2, id. ap. Z.A. vi. 374, ZDMG. 1x. 200.

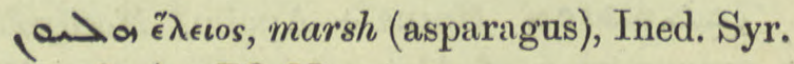
94. 5 af., Ar. PflnN. 52. 53.

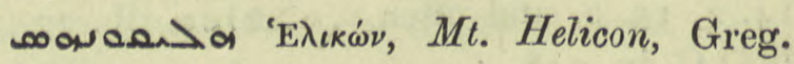
Carm. 32. 12.

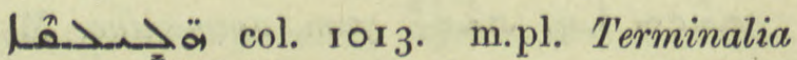

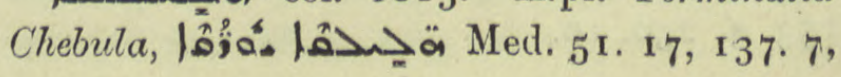




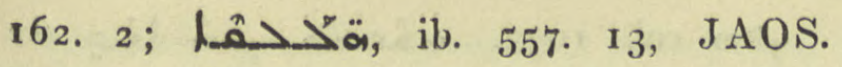
xx. I92. 16.

احمقa m.pl. Elissaei, a people named with Tyrians, Sidonians, and Phoenicians, Chron. Min. 356. 5. Cp. Vergil's Elissa.

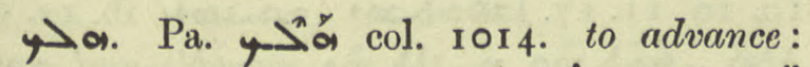
add: to flow on, sea waters Hol ll Ihojal.s! بa!, Jac. Edes. Hex. xvii. 14. Cf. Heb. of Eccles. I. 5 .

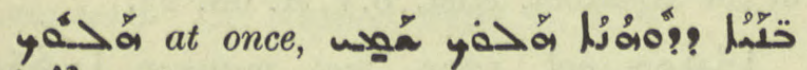

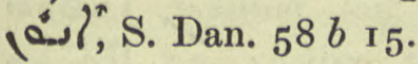

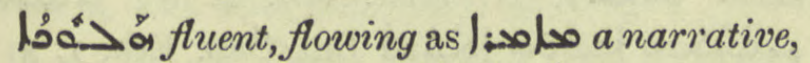

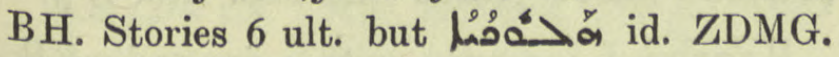
xl. 440.

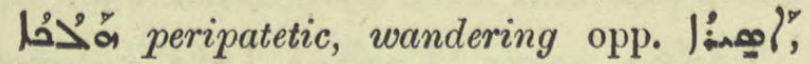
Is. Nin. B. $147 \cdot 17$.

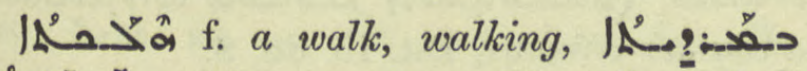

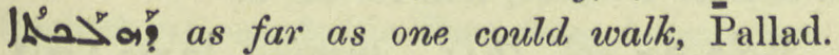
97. I. The power of walking, G. Warda 47. 17.

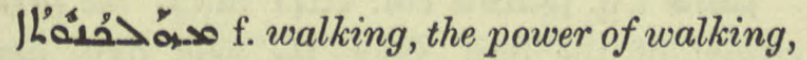
Hebraica iv. 211 , 100.

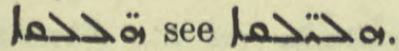

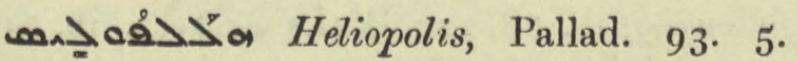
Cf. 1004.

\$0 $-0>$ a pr.n.f. a martyr under Shahpur, A.M.B. iv. 166.

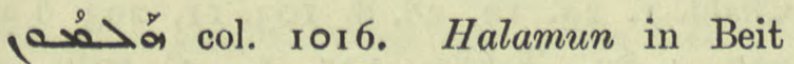
Nuhadra, add refs. Hist. Mon. i. 62. I, Pers. Mart. 2 I 5, Badger Nest. i. 394.

مهara B. Sal. in Lit. 2. 14. Corr.

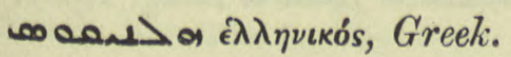

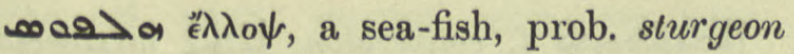
or sword-fish, Ar. FischN. $7 \mathrm{I}$.

بด्व pr.n.m. Elpidius, Nöld. F. S. i. 469.

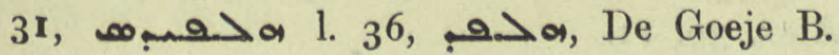
65. 19.

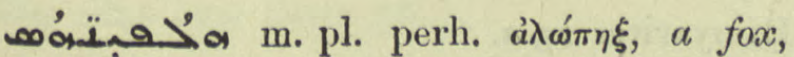
N. Hist. vii. 4. 6.

Lִนْó col. rorg. Dele "Forte sit" and add هـــ, Hamadan, Jab. 127 med., 178. 7 .

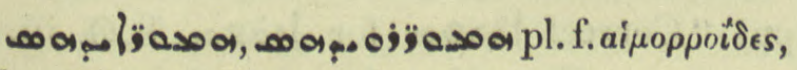
piles, Hippoc. iii. 29, vi. I I, 12, 2 I. andewor a Spanish bpric., perh. Numantium, Nöld. F. S. i. $475,145$.

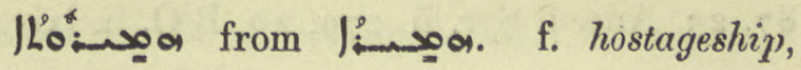
Dion. 63.4 .

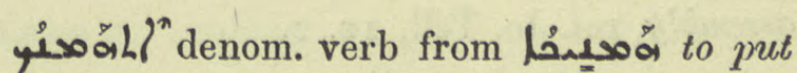
on a collar or neckchain, مَّ人 . clxx.

 vii. I. $7,6.2$ and often.

: شهار كير accountant, treasurer, Jab. 215 , 12 , 2 I6. 6.

معea and ussor i. q. Lasor the Land of Darkness i.e. the boreal regions with their long dark winter, Gest. Alex. I 70 notes 9 and 10 f. Id. ap. Z.A. vi. 368. 2, 3, 369 . 2, 10, ZDMG. lx. 190. 3, 192. 8.

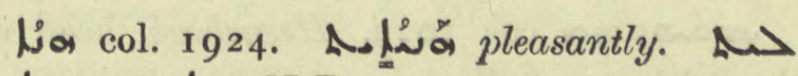

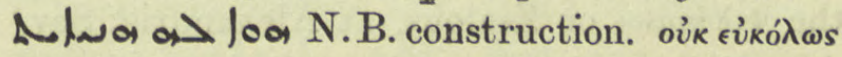
" $\sigma \chi \epsilon$, he did not enjo' his food, Pet. Ib. 98. 12 ;

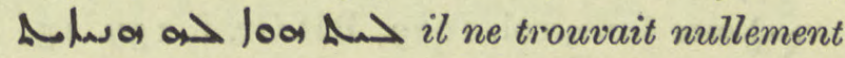
agréable, Pléroph. 16. 6.

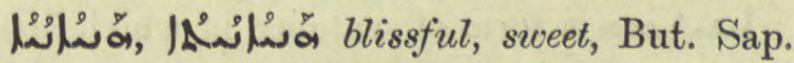
Theol. 5. I.

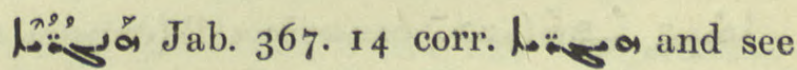
above.

بa Ar. هـ هـ A hundred camels. Hind, sister of Numan, a Christian Arab King, Sassanidi 9. I7; i.q. بـa daughter of Nu'man, BHChr. Eccl. ii 105.

Cichorium endivia, endive, Med. 553. 2 r. Cf. Ar. PflnN. 255, Lag. Sem. 61 .

लy name of a village, C.B.M. I I $30 \mathrm{a}$.

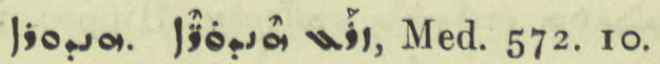

لمص name of a monastery near Edessa, Anecl. Syr. ii. 293. 12.

ف Med. 603. 10.

of arowa col. 1028. Add: évekótovv, Ps. liv. I I, Field Otium Norvic. 24.

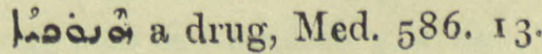


flua col. ro28. Anzete, a town of South Armenia, of 87. 23 , Anecd. Syr. ii. 279. 20, B.O. ii. lxiv.

مolua col. 1028. Pers. آنبْهَ Anjuman, assembly, ref. Jo. Tell, то. 2.

Hoa "̈varos, ninth, fosas loa? lo: the battle on the ninth day, C.B.M. $1059 a=$ Anecd. Syr. iii. 258. 9, ib. 249. 7 , 12.

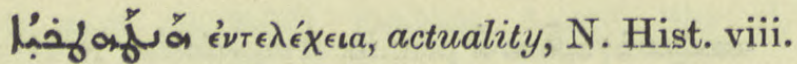
I. 2 gloss. بă

wa river Hani which flows past r.jls in 'Irak 'Arabi near the Euphrates, Dion. 26. 20; B.O. iii. ii. $7 \mathrm{r} 7$.

(حصب) (حم) (حم) BHGr. i. 47. 20.

. vii. $\mathrm{I}$.

sonsema col, ro29. Hesychius, head of the $\Phi$ Фло́тоуо or Zealous, at Alexandria, Sev. Ant. Vit. 32 ult.

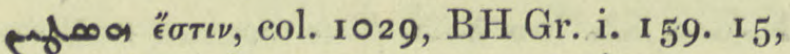
20. Cf. Hofl col. 298 and rofl col. 299.

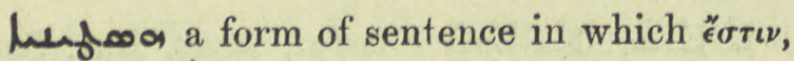
Syr. wad? is used, BH Gr. i. 159. 22.

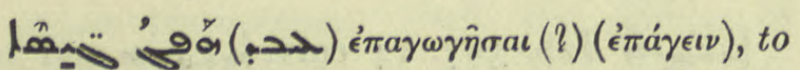
infer by deduction, But. Sap. Theol. 3. 5 infr., N. Hist. vii. 6.5 .

10. vessels, Ephr. Ref. i. 15. 43, 28. 6.

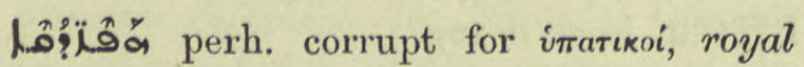
commissioners or officials, A.M.B. ii. I76. 2, أُ

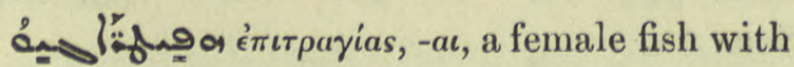
male form, N. Hist. vii. 2. 3 .

Һ Һ 45 , iii. I $5,19,2$ I 28.

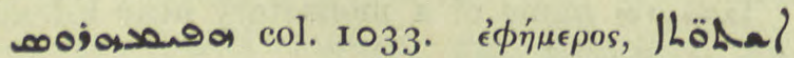

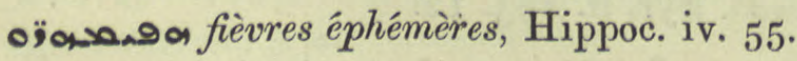

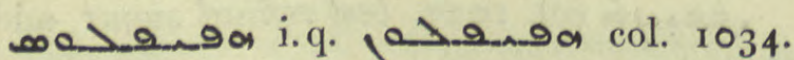
inimioov, the caul which encloses the bowels, N. Hist. vii. $1.3,6.5$, Hippoc. vi. 55 , vii. 5 r.

lo: 40. 10 .

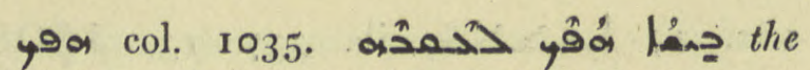
Evil One will be turned back, Pallad. 8r. pen.

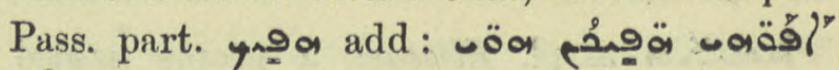
ه Io. Ethpe. chem. to be transformed, Chimie

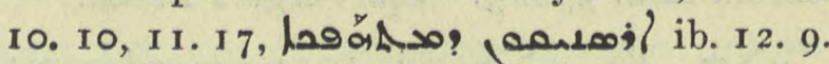

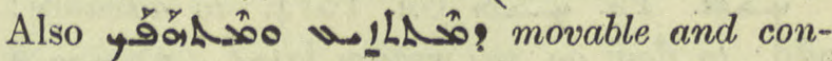
vertible property, Pallad. 193. 4. Aph. part. עด pl.m. of the alphabet, Hist. B.V.M. 68. 23.

léơóa col. ro39. act, manner, leø̊äa

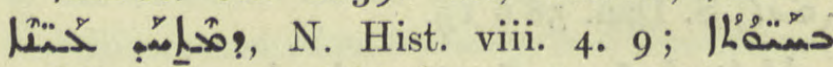
م. فُ But. Sap. Eth. I. 3.

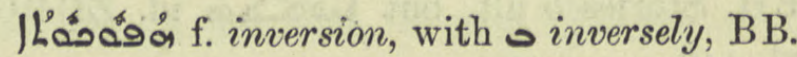
under

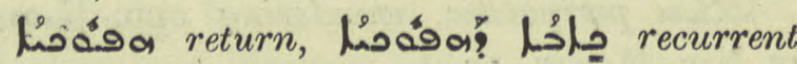
sickness, a counter-circular, Pet. Ib. 8०. 8.

lLánaga col. r64r. turning, lasodol? of the stomach, Med. 243. 18.

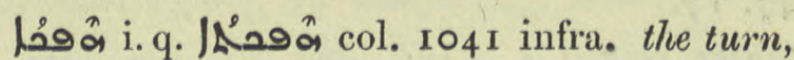
return of the day, year, the next day, next

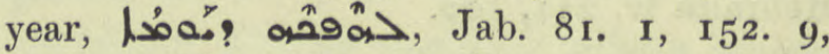

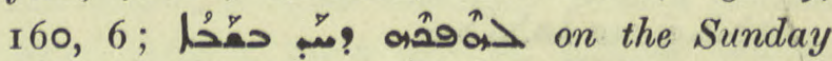

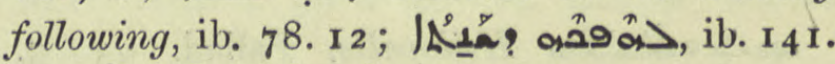

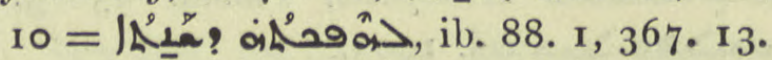

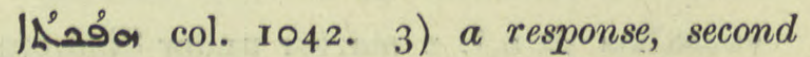
part of an anthem or canon, add: Brev. Chald. 1. $25.19,28.8$, 103. I1, 293. 6 af., 317. 4 \&c., Takhsa I35 I. 4) with

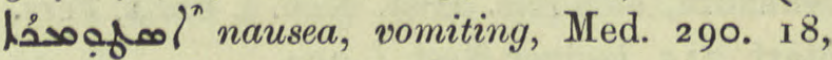
305. 19. 5) adverbial use, contradictorily,

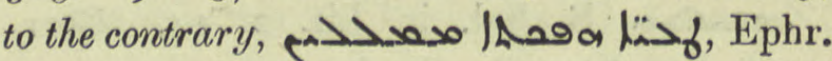
Ref. i. 78. I 2.

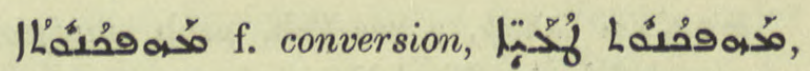
M. Z. $64 \cdot 7$.

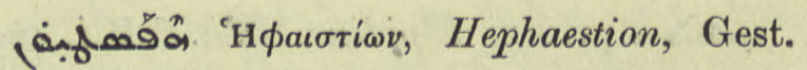
Alex. 39. I I.

p pa Hanfa'amm, a book of the Himyarites, Himyarites 25 a.

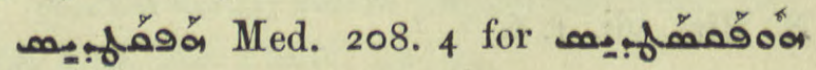
above.

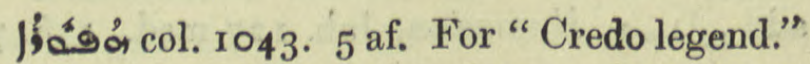

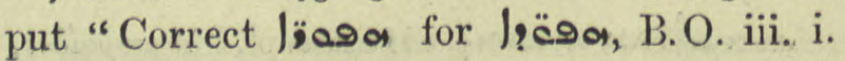
I 78. 12 as Hist. Mon. i. 143, 8." 


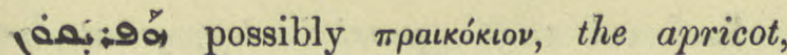
Med. 49. 5, but it may be for Hypericum, cf. l. I 5 .

| فำ occurs Natur 7 n. I synonym for

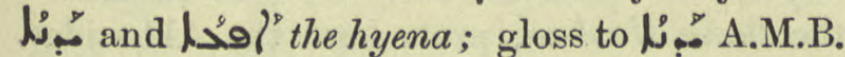
vii. 58 .

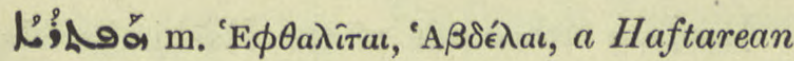
or Hephthalite, Jab. 269. I, pl. 1. 3, 267 , 1. 4 , white Huns in Bactria and on the Oxus (Labourt). Cf. Hippoc. xxvii. n. I.

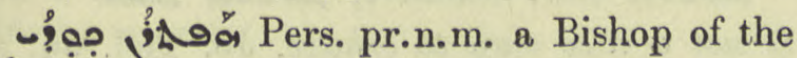
1

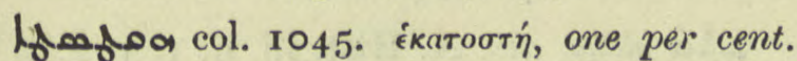
interest per month, Syn. Or. I5 I. 20, /flafloa Jhe ? so-called because this rate was regarded as authorized by the Church, ib. I80. $2 \mathrm{I}$, Stat. Schol. Nis. 22. I5. Pl. alfor, Sev. Lett. 29. 4. Cf. Sofofoa Syr. R. Rechtsb. 16, quoted in Thes. from Anecd. Syr.

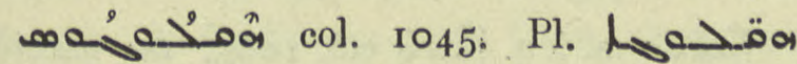

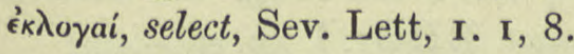

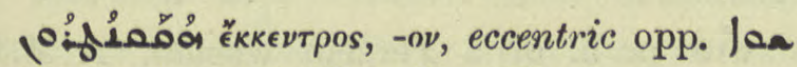
(0) مe concentric, But. Sap. Theol. 4. 5

ja col. I046. to dispute, act. part. i\}a : ;\}á? oa disputant, BHGr. i. 163. I3. Ethpalpal "ágól? to be excited, stirred up, jajas! arousing the Tree of Death, Manichéisme

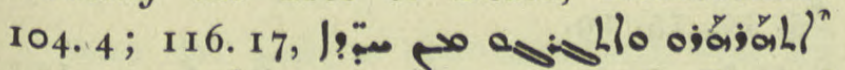

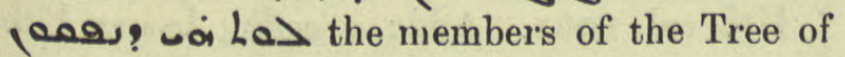
Death excited and instigated each other to issue forth, ib. 123.7 .

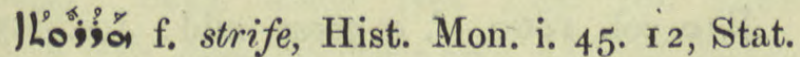
Schol. Nis. 21. 2, 183.2.

$\mathrm{L}^{\prime} \mathrm{o}_{\mathrm{a}}^{\mathrm{n}}$ col. 1048. reproof, with pers. pron. Y L'ion, Ephr. ed. Lamy iii. 39r bis.

I';ô perh. the spleen, Med. 593. 20. Cf.

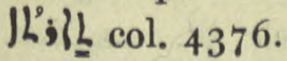

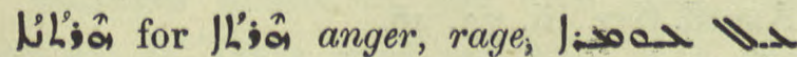
owliga oa Loa Dion. 56. 9.

sia col. I049. 4 of par. Correct Herat and see the passage quoted from Dion. in Chabot's edition, p. 100, II , I2, trans. 84 n. Cf. $\alpha^{\alpha} \dot{\sigma}_{\hat{a}}^{\pi}$ col. $10^{2}$ and Nöld.'s transliteration Harê, Harên, Tabari 42 n. Herat is the Arabic form.

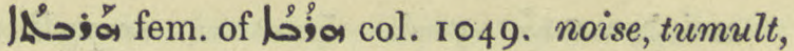
lo ملا مع مُحد, Greg. of Cyprus $49 b$.

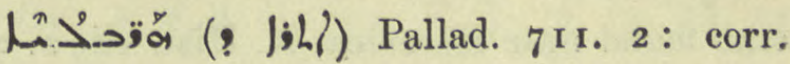
h q. qua q. subter.

ja col. ro40. Ethp. Soll? with o to consider, Jul. I 2 I 5 .

An ịió col. 1050. Ref. Anecd. Syr. i.

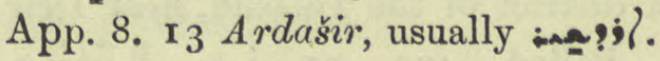

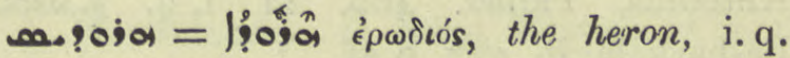

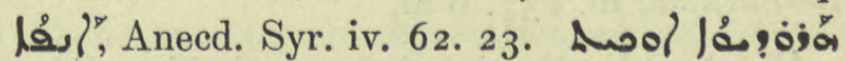
مa, N. Hist, vii. 6. 6 infra.

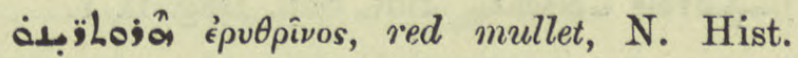
vii. 2.3 .

noja Chimie 38 . I3 mistake for nojo wars.

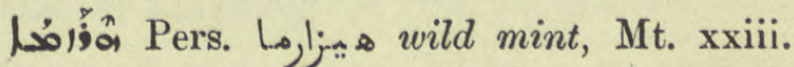
23, Lewis and Evang. da Mephar. ib. Cod. S. The parallel passage Luc. xi. 42 in both these versions has انُ2. Add: Med. I59. 23, 292.

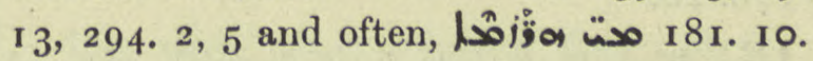

äa col. 1052. or hil 'A $\rho$ eía, a province of Central Africa where Heria, now Herat, was situated, Jac. Edes. Hex. 41. 9; a Metrop. See, Syn. Or. 53. 7 ; ZDMG. xliii. 396. I I.

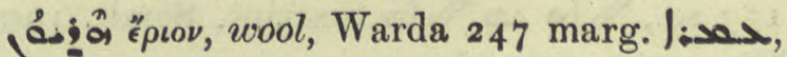
خذص ي-jid. ap. Hippoc. ii. vii. 7 .

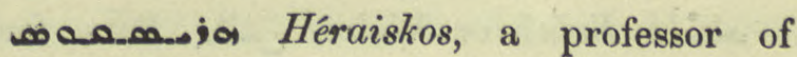
Alexandria, Sev. Ant. Vit. I6. 8, مسمه مa), ib. 22.13 .

ي

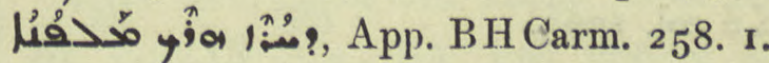

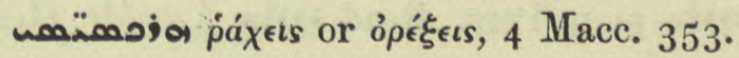

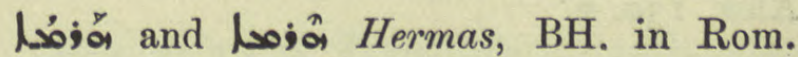
xvi. 14 , Bagst. |र्कें??

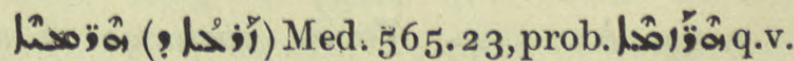
|j:حزa Pers. Buzurg framadhâr, (grand) Vizier, Tabari 1 I I, Syn. Or. 2 I. 2 I.

مa autumnale, i.q. لمه; مـ BH. de Pl. 8I, Gottheil ZDMG. xliii. I $_{5}$; Ar. PfnN. 174.

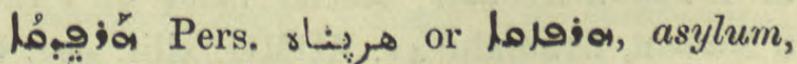
sanctuary, refuge, with >حَّ Jab. ${ }^{252,6}$ gloss.

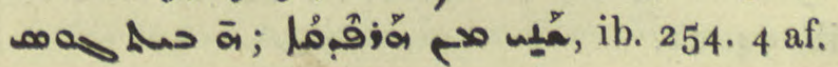




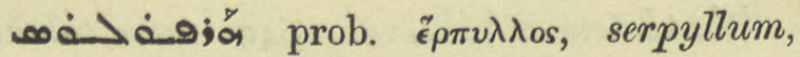
creeping thyme, N. Hist. vi. 4 . I.

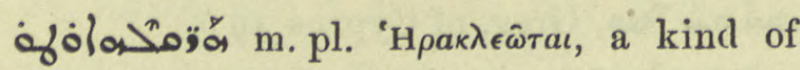
crab found near Heraclea, N. Hist. vii. 2. 2.

Mu佟a pl.m. Heracleonites, an Alexandrian sect of Gnostics, Coupes ii. I 17 .

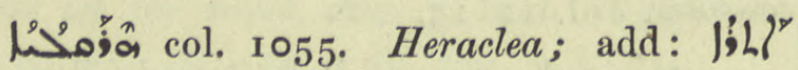

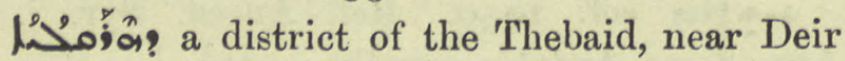
Antonius, Pallad. 400. II i. q. لِّهُ Be-harkala, ib. 237.6 af.

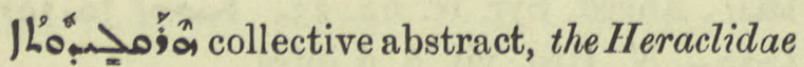
| But. Sap. Isag. ii. I. لlan â col. 1056. pearl barley, groats, porridge: see Aaoj col. $3^{87 \mathrm{I}}$ where all the synonyms are given.

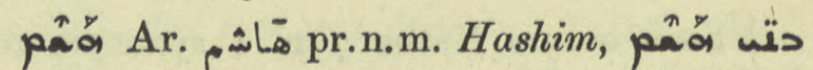
the Banu Hashem, prob. here the Abbasides, Bahira 205. I 2, 229 bis, \&c.

Hoplac either one of the Hashimite clan, or one from a village named $\mathrm{Hashem}, \mathrm{BHChr}$. Eccl. § ii. 265 .

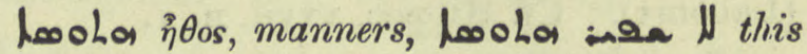
is not a nice way to behave, Jo. Tell. 69. 20 = Vit. Mon. 84. 26.

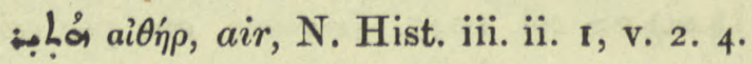

$\therefore$ a $\therefore$

alo col. 1058. quotation from Mark, BH Gr. i. 178.20.

Jóló Wah Wah, interj. of derision uttered by demons, Pallad. 52 I. ro.

ه/o ואל Wail, the commonest name in Sinaitic Inscriptions, Nöld. ZA. xxi.

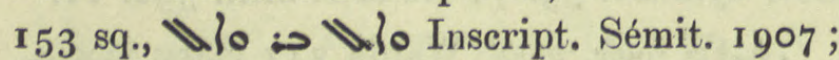
Ḥimyarites $25 a, b$.

صأ Balaš or Wales, بلاش Pers. form of the name Vologeses, Shah of Persia, A. D. $484-488$, Tabari 10 n. 2 , ZDMG. xxviii. 93, ff., xliii. 397. 12; Syn. Or. 53. 13. Cf.

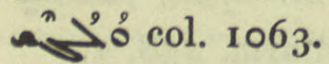

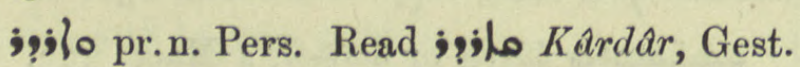
Alex. I30. 2.

fajlo: see a ojo below.

ang Ar. Ar. Vagus, surname of an Edessene, B HChr. Eccl. 667. 25.

مِ 10, 458.14.

بـ Wahman or Bahman-Ardašir, official name of P'rath d'Maisan, Eranšahr 4 I, ZDMG. xliii. 402. 6; Syn. Or. 89. 29 so correct for ib. $94.2 \mathrm{I}$.

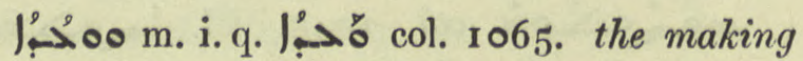
of an appointment, BHGr. i. 50. 5 .

ơ iv. 155 .

นُمْ ช้ Waziqites, El. Nis. Chron. I86. I6.

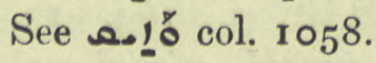

LLo:-1\% col. I061. vizier-ship, add refs. El. Nis. Chron. 202. 22, 203. 23, AKM. viii. $85.10,15,19$.

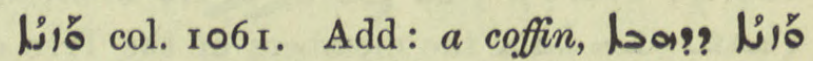
arst. Alex. 138. 16 .

1410 col. 1060. f. a goose, add: Jos. Styl. 66. 9 ;

ـó, fํㅇ col. ro6r. Pl. with pron. suff.

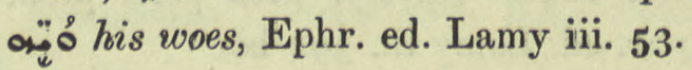

ل! 0 col. ro62 ult. of par. BHGr. i. 3 r. 9

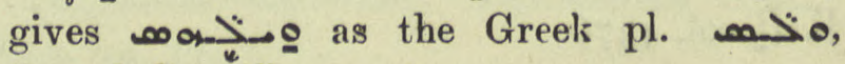
Anecd. Syr. iii. 75. 26.

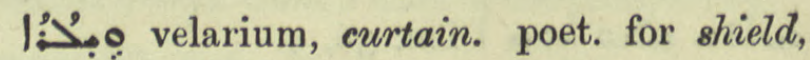

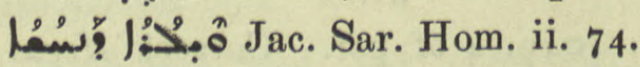

name of the Caspian Gate, Eranšahr 103, Gest. Alex. 140. 12. Nöld. identifies this with a fortress famous in Sassanian times,

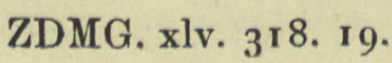




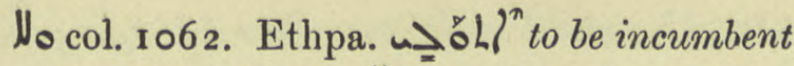
on, be fitting, ? ل 93. 2.

นُ corr. Al مه هo

جo col. 1064. The Gnostic Valentinus,

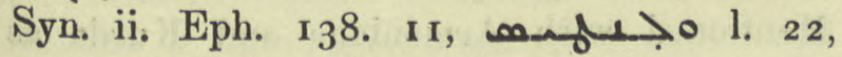
صald >, ib. I46. 2.

محر a place near Mardin, Mar. Benj. 64. 4 .

L._○ Valerianus, Bp. of Iconium, C.B.M. $720 a$.

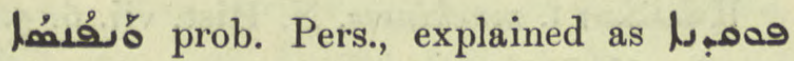

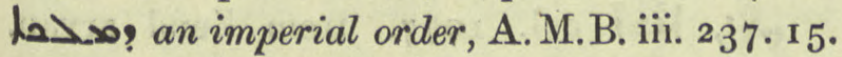

too Ar. واسط, Wasit, on the Tigris; the port of Kashkar, Mar Aba ii. 44.

مصم Ar. a place in Egypt, A.M.B. v. 23 . I3, Amélineau 51 ff., GGA. 1895, 690.

1000 or log, Pehlevi vāčak, a hlessing, grace before meals, Pers. Mart. 857 on Jab. 440. II. Col. 1064 a slave, Mar Bassus (colophon) 5 Syr.

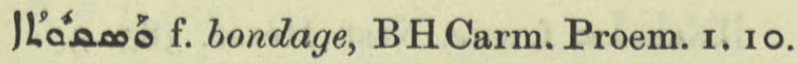

J:م00 m.pl. waspur, nobles, Tabari $501 \mathrm{n}$. Act. Apost. Apoc. 279.6.

00 Ar. وقف a charitable gift, Jab. 93. ult., the endowment of a monastery, ib. I39. 12.

14 corr. daisy; Lexx. under |'oL مب col. 2868.

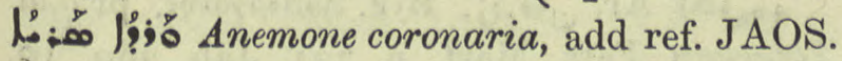
xx. 197. 6. Chem. a pellicule on the surface of a liquid, . ד >

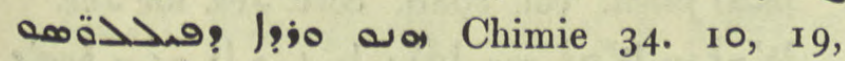
transl. $6 \mathrm{I} \mathrm{n}$. $\mathrm{I}$.

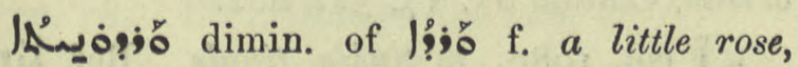

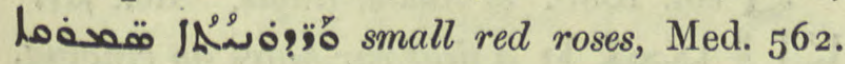

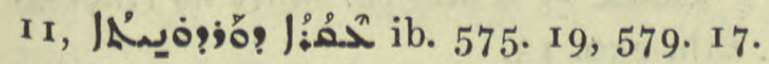

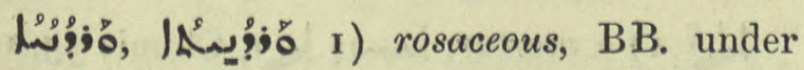
L!oj. 2) a village near Nisibis, A.M.B. iii. 552. 19.

joǧ Zend Verethragma, the Genius of Victory, usual forms on coins Vararanes, 2716

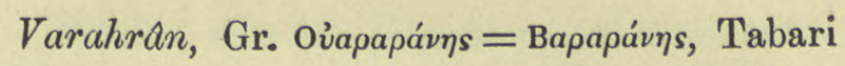
46 n. 3, 420 ; later form Bahram. Name of several Persian Kings, Sassanidi 7 quater, 8 ter; A.M.B. ii. 559, M.Z. $36.14,37.34$. Cf. plian.

a.0 Ar. ورزس wars, a dye, Indian saffron, Memecylon tinctorium, Chimie 38. 10, nojos ib. I3, lailo ib. 44. 6, 1.5o 1. 20, 49. 7, 50. 4 , transl. $67 n$.

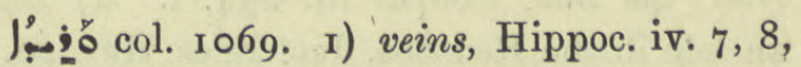
vi. I4, vii. $49,50.2)$ sinews, nerves, metaph. ocause you have stretched the sinews of your mind, Sev. Lett. I56. I I. 3) Dele "bestiola". I, bo is

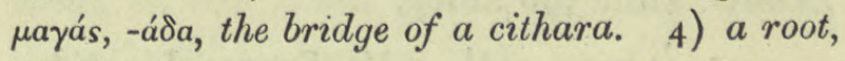

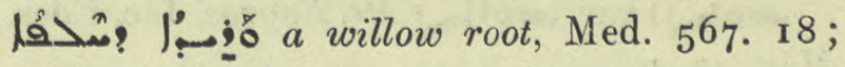

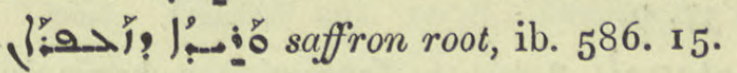

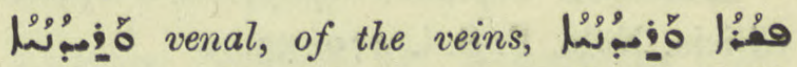
liquefaction of food in the veins, N. Hist. vii. 2. 1 , ib. 1.3 .

Jojo perh. єoprí, a festival, Jul. I 28.6.

jo i.q. jajo, jhio = pjas ZDMG. xliii. 395 ult., 396. 2, Pers. Mart. 43, 420.

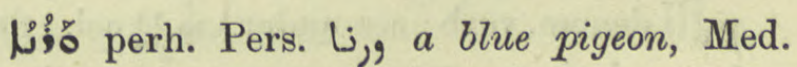
559. 8.

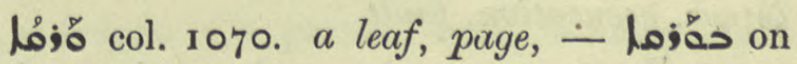
p. -, Brev. Ant. iii. $74 b$ I $8 ; 76 b$ six times.

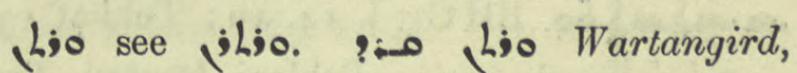
a village on the Euphrates, S. Maris 78. 2.

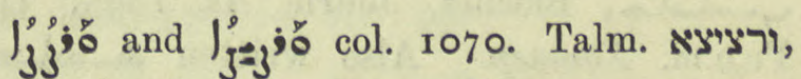
Ar. ورهـت ZA. xxii. 206, a newlyhatched chicken.

jliso col. 1070. Varathran=Verethragma, earlier form of jajo q.v. Nöld. ZDMG. xxvii. $196 n$, xxviii. ${ }_{5} 6$, C.B.M. $1093 b$ bis.

معم sandarac, gloss. to ammoniac, Chimie 5. 15 .

بـ هوش.شنك Hoshang, Gest. Alex. I30. I.

a Ano Pers. Vištasp i.q. 9.0 hacs. ZDMG. lvii. 563. 2.

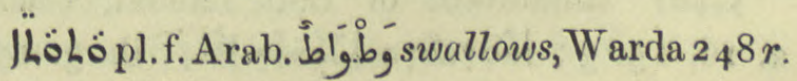


1)i name of a goddess identified with سُ. Gest. Alex. 204. 7, 206. 14. Cf. بيل col. $23^{87}$, Pers. Mart. 1 30 sqq. Budge corrects )

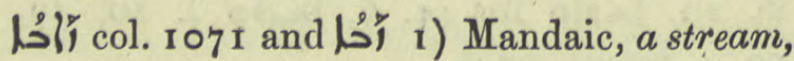
river, the Zab, Coupes iii. 234, f. 2) Záßa, an Isle in the Red Sea, Jac. Edes. Hex. 20. 8.

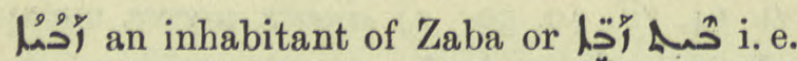
Lower Adiabene between the two rivers Zab, Syn. Or. 44.33 ; احصب/ Zabidas of Zaba, ZDMG. xliii. 396 antep.

nol li var. voj) Mt. Zagros, Jac. Edes. Hex. 37. 9 .

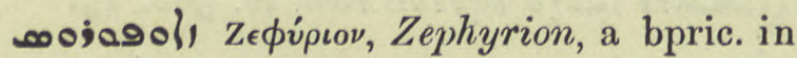
Cilicia, Nöld. F. S. i. 470.55 .

wh col. ro72. scurf, dandruff, Hippoc. iv. 77. ufi the mange, itch, Med. 48. 21 ,

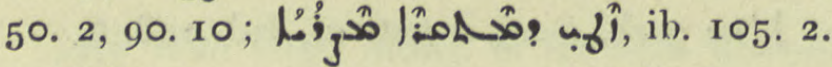

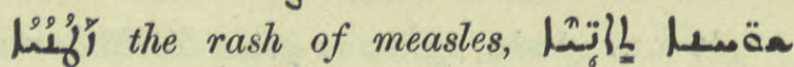

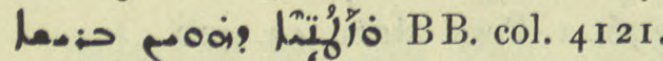

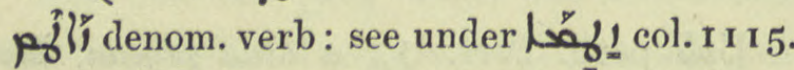

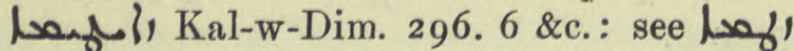

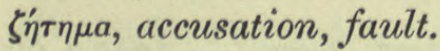

u-

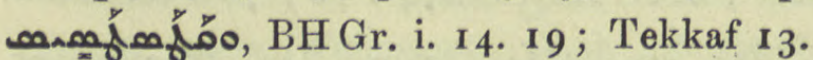

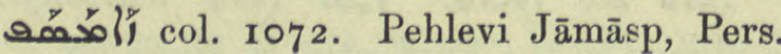
جـاoـ, Blochet, Journ. As. 1895, 347. Pr.n.m. Zamasp. Also written ה

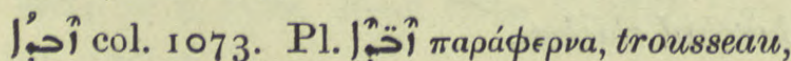
gifts from the bride's father, over and above

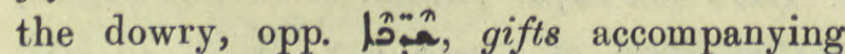
a proposal of marriage and Lis.so:" wedding gifts, Sar. Hom. iii. 104. 17.

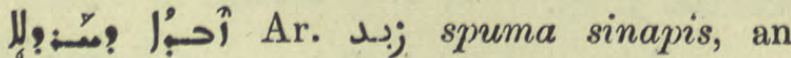
aromatic herb; sce under fُ

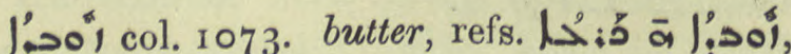
Med. 586, $15,59^{8}, 18$.

أ inhabitant of Beit Zabdai, Chast.

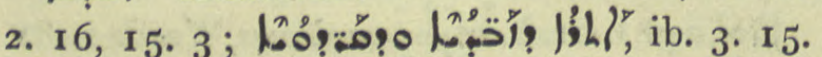

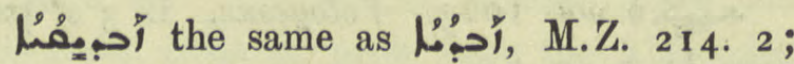
pl. Mar Aba II r69.

احبم.مسا احمبل m.pl. prob. same as Mentioned with Armenians and Kurds as children of Ashkenaz, Chron. Min. 355. 9.

الزبيرة : place-name, col. 1074. Add in the Hauran, Nöld.nn. on C.B.M. in ZDMG. xxix. 434 ; Lئl, Doc. Mon. 2 I6. 30.

إح col. 1074. 8 of par. corr. 色

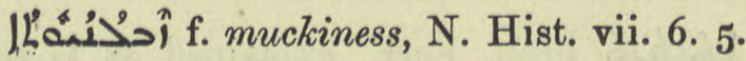

آحُّ col. 1077. from Zend zrvan, time, age, Pers, زمان (R. Duval). Cf. (هئ.

مخه particles for sale, trade,

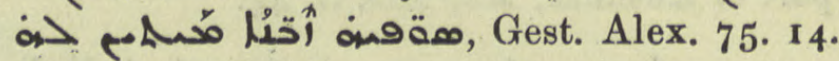
The goods bought, a purchase, ats), Syr. Rom. Rechtsb. I 2.19.

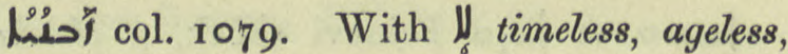

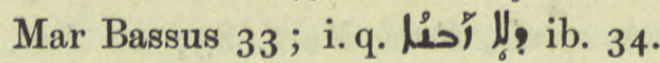

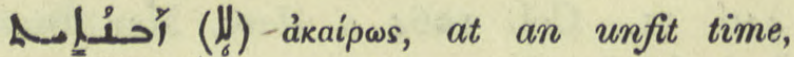
unseasonably, Sev. Lett. 5I 7. 6.

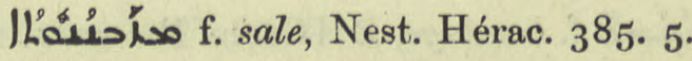

L'sási col. ro79. f. a dish, platter, Clem. Rom. Rec. 144. 5 quoting Mt. xxiii. 25, 26 ; in both verses the usual masc. form | is used.

J: البيلة a pitcher with a long spout, DBB. 678.1 .

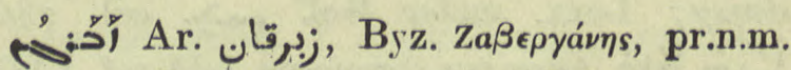
Zabergan, Pers. Mart. n. 731, Jab. 360. 10, 361.6.

1 أ pitch. col. ro8o. corr. Jes. for Jer.

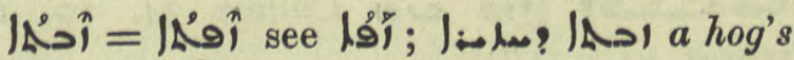
bristle, Chimie $85.17,322$ n. 2.

y col. 1080. to tinkle, tingle. Ref. Li? ? Lُ لإ BH. Eth. 250 . 13.

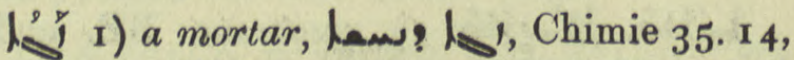
BB. under ILllo. 2) Pers. ز vitriol, Med. 583. I. Cf. kay .

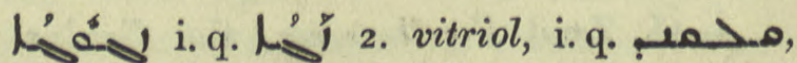
Chimie 5. 5 transl, 10. Cf. مخمس مג 
Lig 9. $\mathrm{I} 5,2 \mathrm{I}, \mathrm{I}, 5$.

1A. vessel, Chimie 55. 10, 19. The vitreous humour of the eye, Med. 68. 7,8 .

นُصُ (a furnace), Chimie roo. ult. The vitreous humour of the eye, N. Hist. vii. I. 2.

bُớ col. 108i from l’y a chicken. an

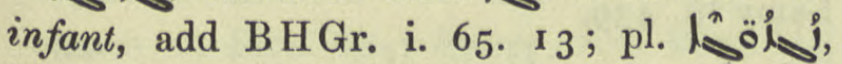
Tekkaf 22 r.

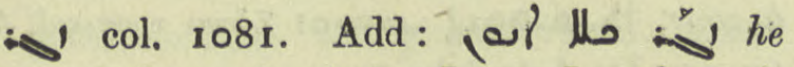
stemmed the rush of the Jews, Josephus vi. I5. 9. Pass. part. restrained as evil spirits,

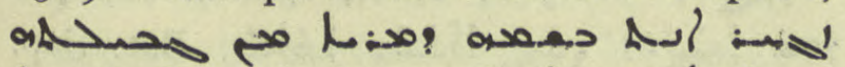
Anecd. Syr. ii. ro. I3 f. J forbidden intercourse, Isoyahb ed. Duval r. 6.

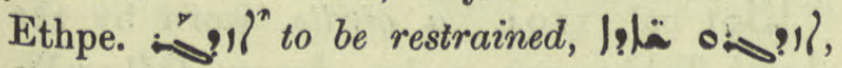
Coupes ii. 114.5 .

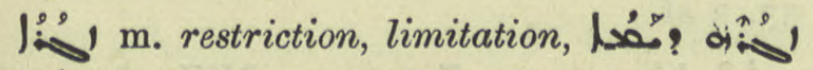

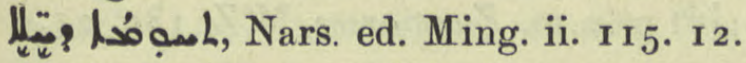

90. the throat, 288. I 5 , Hormizd 3240 glossed /jog.

o? name of a village near the Tigris, A.M.B. i. 455 .

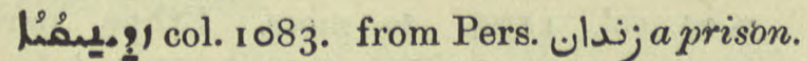
A hangman; gaoler, Aphr. 705.24.

م!) Pa. م?ึ̆ col. ro84. 2) to verify, confirm, al. El.

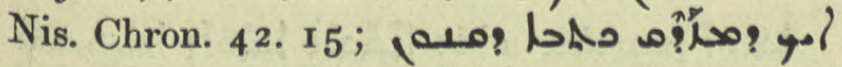
according to the Canon of Ptolemy, ib. I3. 3, 7 .

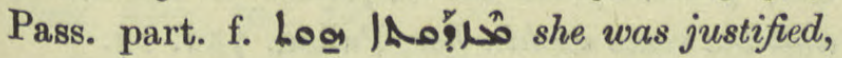
acted rightly, Pallad. 199. 8. 4) to bestow,

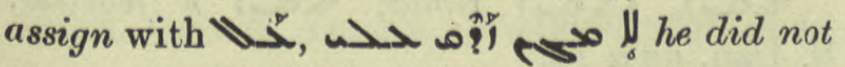
give me the books for nothing, Or. Xt.iii. 10 , 12.

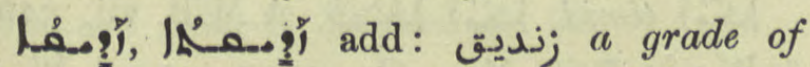

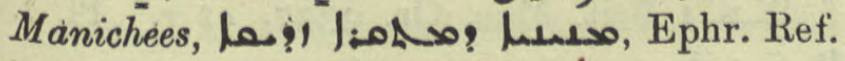
i. 30. 18. Pl. f. /Aم:!, ib. 128. 2 .

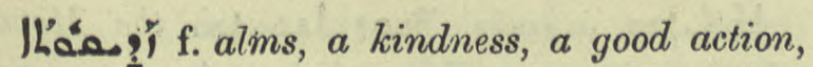

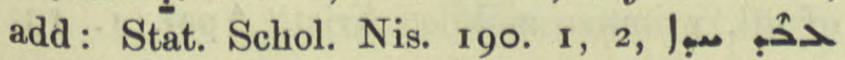

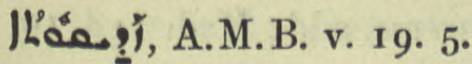

لح

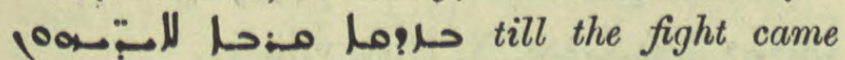
in normal course to hand to hand fighting, Josephus vi. 5. 3. Cf. I! 00a نم:حس ib. $24,12$.

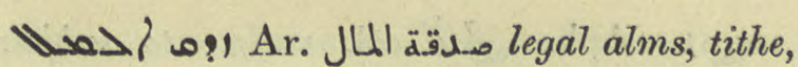
Dion. $127.22,155.4,7$ and often. Cf. uv).

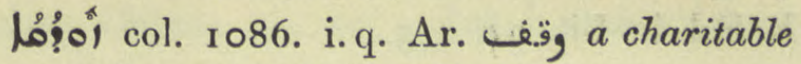
bequest, Jab. 93. ult., I39. I3.



L_o'!) col. ro86. Dele article and see أị below.

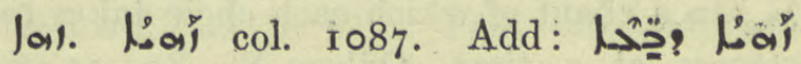
glair, white of egg, Med. 577. i 2.

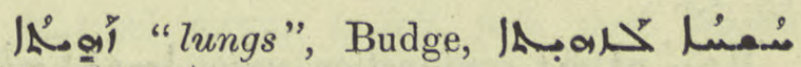

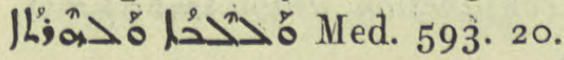

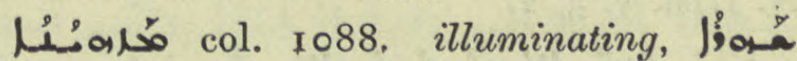

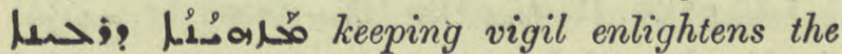
mind, Is. Nin. Chab. 95. I 1, 80. 6, 13; $\mathrm{LaO}_{3}$

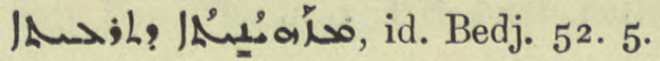

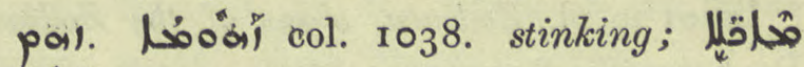

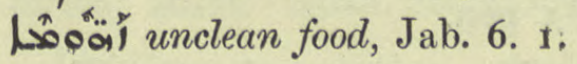

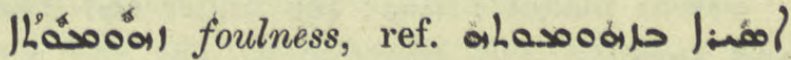

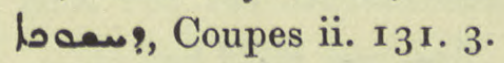

iăl col. 1088 to shine. Act. part. iăi,

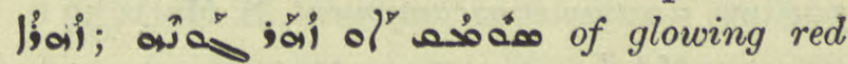
colour, Med. 289. 13.

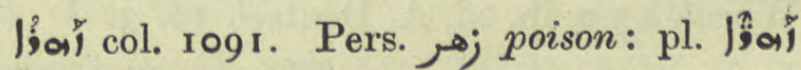
لِّمَ, BH de Pl. 207.

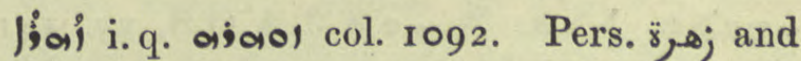

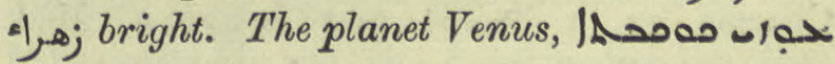

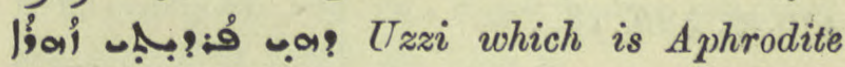
Zuhra, Bahira 203. $10 \mathrm{f}$.

ajaol col. 1092. the planet Venus, add refs. Med. $470.19,487,16$.

Jo1 col. 1092. to swell, become swollen,

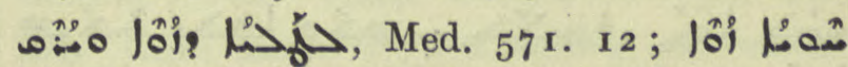
lóg, A. M.B. iii. 35.6 ; Aneed. Syr. ii. $3^{\text {I } 5}$. 19.

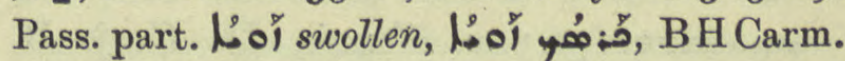

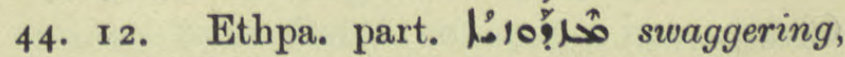
Mar Bassus 84. 1. Aph. uoll 3 of par. the ref. is to BH Carm. 162 . I5.

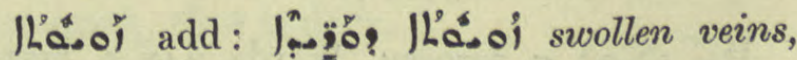
Med. 66. I $_{4}, 79 . \mathrm{I}$.

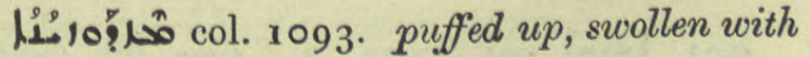
conceit, Tekkaf. I 10.

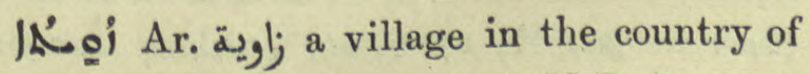
Kashkar, Syn. Or. 245. 25 ; A.M.B. iv. 215. 
0). Aph. Âl" col. 1095. to subjoin,

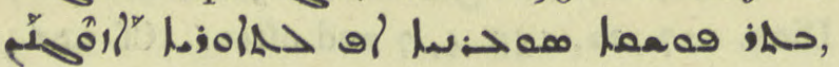
B. Sal. in Lit. $94.4 / 5$.

fُol col. rogo. 18 of par. Add: مب ol !ق a pair of trousers, Jos. Styl. 56. 14 ; مي Alex. 147. I3; ib. I6. 4) couplets, إم حلرّ a chant of which each choir takes two clauses at a time, Qdham W. 83. I5, 97. $\mathbf{I} 3, \mathbf{I} 5$.

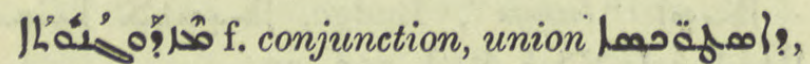
N. Hist. v. 5. 2.

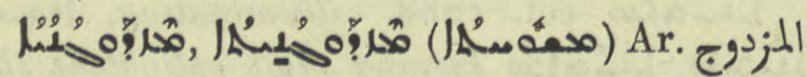
double-rhymed, having a rhyme at the end of each hemistich, Poet. Syr. 2. 3 .

10 ọol m.pl. Zodiaca, signs of the Zodiac, De Astrolabe 85. pen.

ajaol planet Venus: see under ;al Thes. and Suppl.

Shol col. rog8. Kurd. زوزان mountain pasture, a summer encampment, S. Maris $33 \mathrm{n}$.

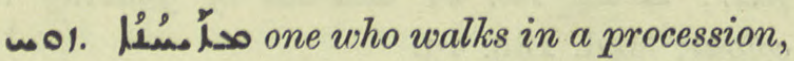
Warda ${ }_{5} v$, ib. ed. A. Deutsch I 2.3 .

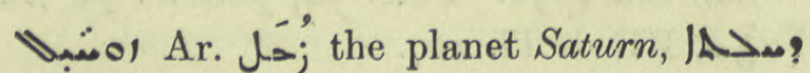

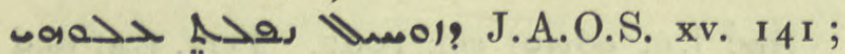

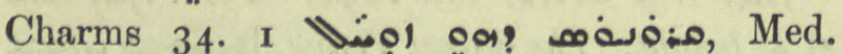
470. $7,486.18$.

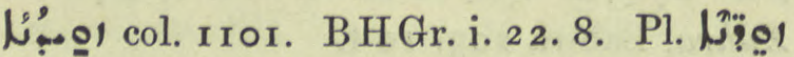

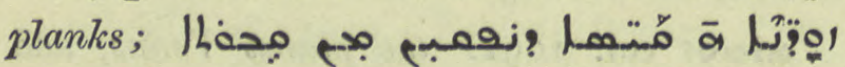

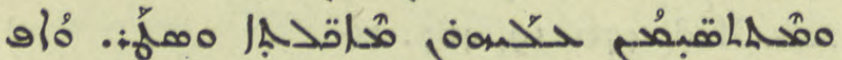

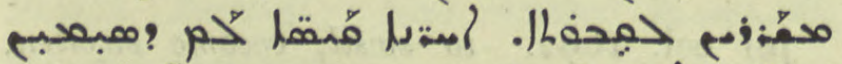
od ا, Op. Nest. 127.4 ff.

صـavol pr.n.m. Zeuxis, De Goeje B. 65.14.

Мor, Wi col. rгог. to ascend. Act. part. טli Loof's Nest. 372. 7.

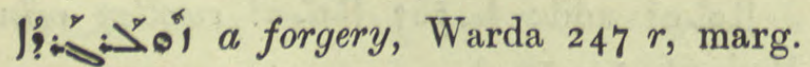
ملدما ?مع:

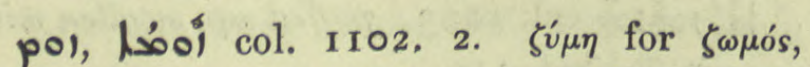

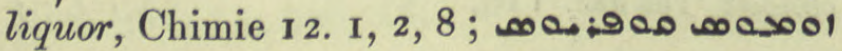

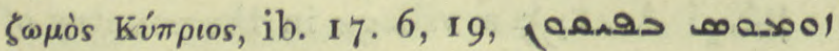
dye, ib. 57 . 17.

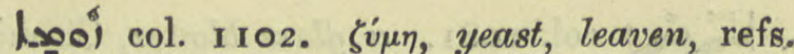

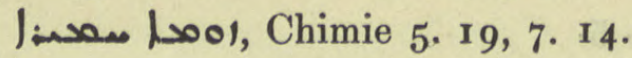

amsool see amsil. Zamasp, father of Mar Saba, Mar Bassus xii Syr. 4, סصח I ib. 118 .

(o) col. n102. Ethpe. 신) to be nourished,

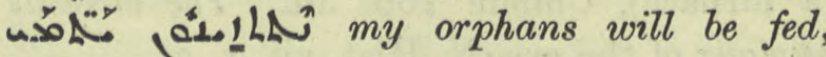

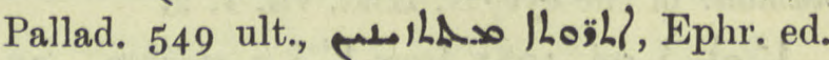
Lamy iii. 439.

Loi and other spellings, col. x 103. Geogr.

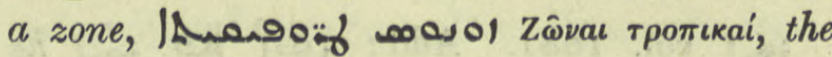
tropics, De Astrolabe 73.

I-

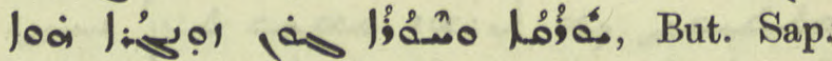

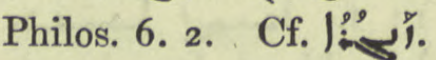

fofol col. rro4. a girdle, worn by the clergy, Takhsa I 1 9. 5 ; by a deacon, QdhamW, 33. 3 af.

|ผંษْỏ pr.n.m. Zwanarses, M.Z. 189. 21.

noi Zeus: 1) alchemist's name for tin, Chimie 3. 4, 6. 6; cf. I25. Earlier Hermes signified tin, transl. 4 n. 9. 2) astron. the planet Jupiter, Caus. Caus. 208. 2, 2 I6. 19.

nol col. Iro5. to move. Ethpe. part.

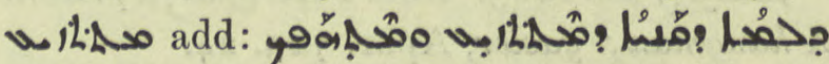
all her moveable and convertible property, Pallad. 193.4 .

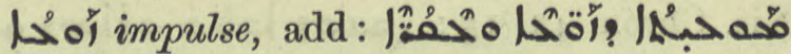
a'? growth from the impulses of the earth and the roots within her, Pallad. 752. 17 .

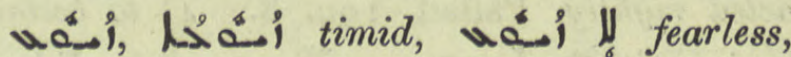
S. Dan. $55 a$ I 6 .

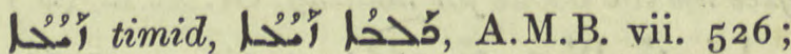
f. |ُحُْ Brit. Mus. Or. 3337. 42 r. Pl, f. ib.

مئم of all, primum mobile, Arist. Apol. I. 13.

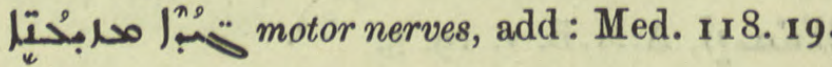

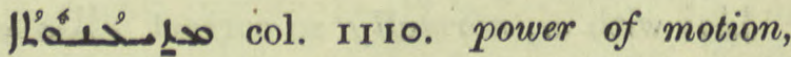

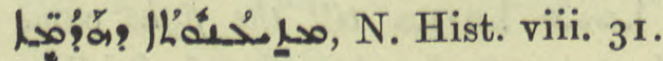

Ihós هـ f. shakiness, irregularity of handwriting, Sev. Lett. 469. I 5 . 


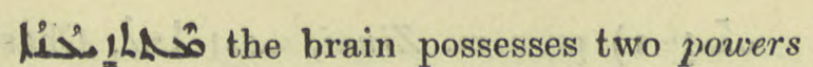

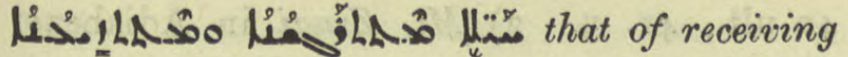
impressions and that of causing motion, Med. 129. 13 .

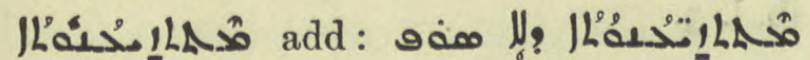
perpetual motion, But. Sap. Philos. 3. 5 tit. Metaph. emotion, |loes ال| |ل I I I was not roused to just indignation, Sev. Lett. 134 ult.

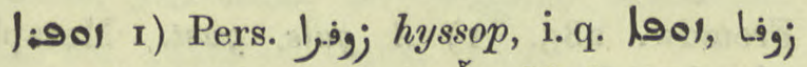

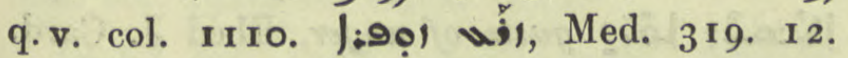
2) perh. for $\ddot{z}, \rightarrow$; the planet Venus, I:-901

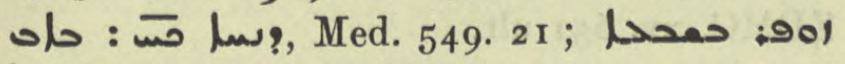
مa : Rylands 44, fol. 5 b.

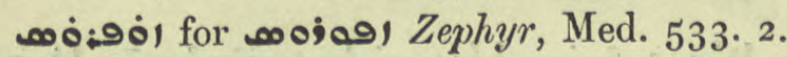

๑:901 prob. the egret, Kal-w-Dim. 40. 3 , 4I. 2, 42. 3. The word corresponds to Ar. عُلْجَج a white aquatic bird, n. ib.

Do1 col. I I 2. I) to rush or dart swiftly,

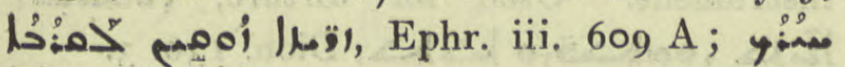

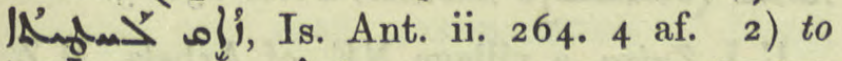
steep, grains of vetch in strong vinegar, Is. Nin. Chab. 29. Pa. مُ to blow violently.

loo, Zuqa, Bp. of Susa, ZDMG. xliii. 393, Syn. Or., 34. 6, 35. ult.; 6r 7. I. q. Loo? ib. 42.17 .

ió) col. II I 3. to grasp, grasping and then letting go a battering ram, Gest. Alex. ror. I 2.

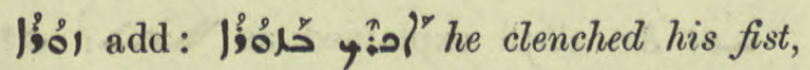
Anecd. Syr. ii. 15. 18. Metaph. with o in the grasp, in the power of Lóa I

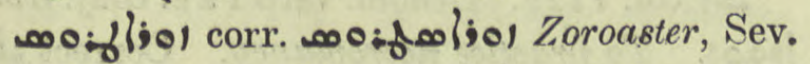
Ant. Vit. 62. 3. The usual form is Lao!j!.

ו ו col. I156. Amomum Zedoaria, Zedoary,

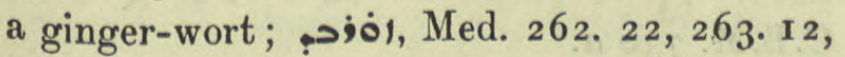
393. 10, 426. 6 ;

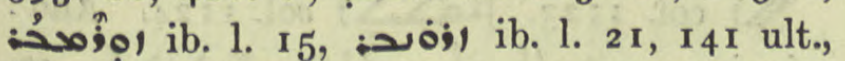
but corr. क'ó pr.n.m. King of a mountainous tract between Jilu and Gawar, A.M. B. I. 4 I 7 .
L'oól i. q. notor and extor col. I1 14 . beer,

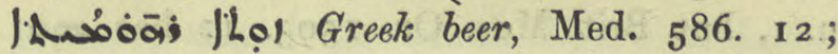

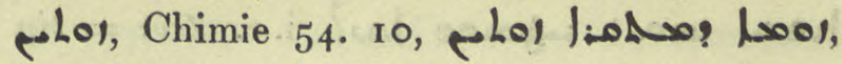
sirop de bière, ib. 86. 3.

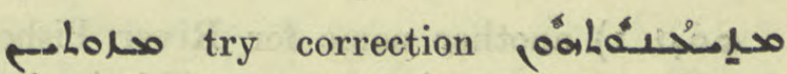

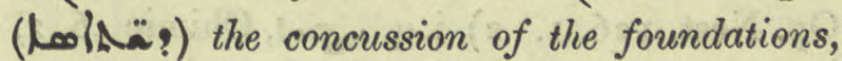
Josephus vi 5 . 13 .

أمسنهُ pr.n.m. Pers. Zachgushnas, A.M.B. ii. 649 . Cf. Pers. Mart. 70 n. 622.

(مڤّ) col. III5. to jolt, jerk, push, hustle with $>$ of the pers. Anecd, Syr. iii. 73. 21 ;

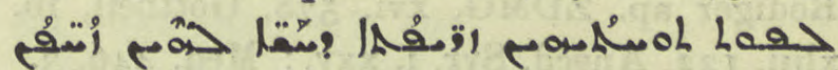
souls which are not firm in the knowledge of God rush headlong down deeps of passions, But. Sap. Theol. 4, 7 infra. Pa. Anecd. Syr. iii. 73. 8. Ethpe. ${ }^{n} ! 1 l^{n}$ to be thrust, כَمحَ: Hormizd 2880.

| Ant. Vit. 284 ult. $=$ Anecd. Syr. iii. 284.21 .

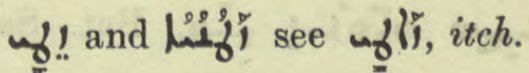

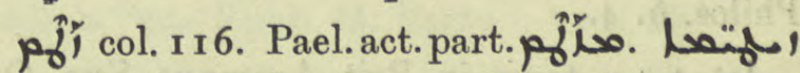
ح me, Kal-w-Dim. 147. 2; - - on fl, they accused me, ib. 299. 14. Ethpa. po? accused, Abog!l als?, ib. II4. II, part. 1

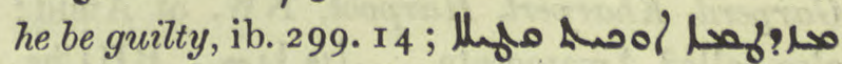
condemned to death, ib. 309 . 9 .

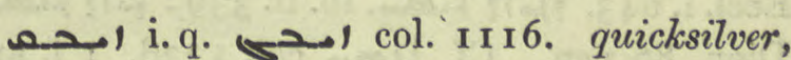
Chimie $39 .{ }_{15}, \mathrm{BH}$. Isag. $\mathrm{I}_{4}$.

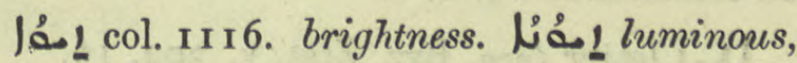
shining, Jasl was Coupes ii. I30. 23 ;

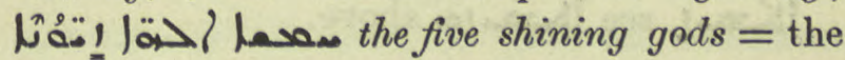
five pure elements, sons of Primal Man in Manichean teaching. ib. 127 infra, I28. 5 af.

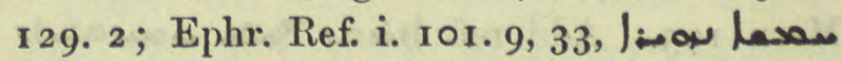

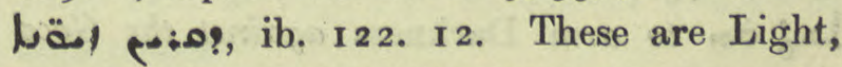
Wind, Water, Fire, the fifth is uncertain. Not earth, that is dark.

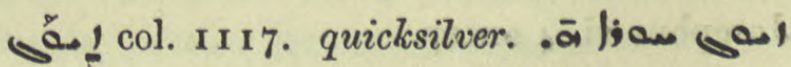

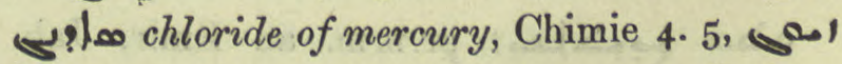
لم:0xide or sulphide of mercury, ib. 1. 3 .

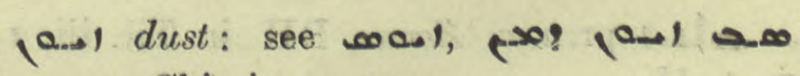

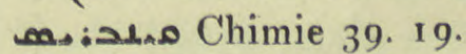


ஸó! col. I117. Zeus = tin, Chimie 256 n. I, but Brit. Mus. Or. I593. 12 has nol أرal i. e. Zevis = tin and $\zeta \iota s=d u s t$.

مبذا 1) another name for River Pishon, Op. Nest. 1 26. I. 2) i.q. (a), Chimie 46. 2, امa ib. 45. ult.

(b) a corruption of $\boldsymbol{Y l}$ three which belongs to an ancient Syriac system of notation of numerals derived from the Palmyrene, BB. under أزavel. 1 I 62 bis, Duval Gram. xv; 14 , Rödiger ap. ZDMG. xvi. 578, Gottheil, ib. xliii. 122 , Anecd. Syr. i. xxv.; Med. 446. 3.

إِ Chald. prob. projecting stone corbels which sustain the roof beams, Hist. Mon. i. 211 . 6. Hoffm. thinks it is

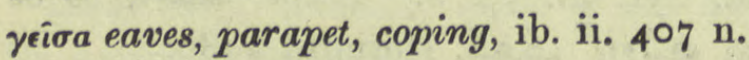

Hól.! cf. Heb. r. f. compactness, indissolubility by moisture, e.g. iron or brass,

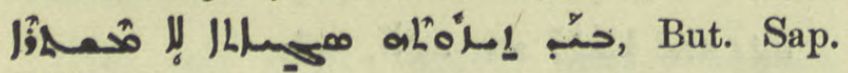
Philos. 6. 4.

Jiolsl a town in Beit Aramaye, Syn. Or. 70. 28.

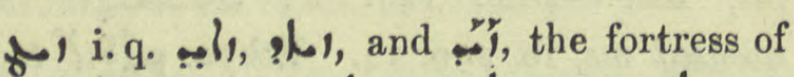
Ziyad, مaمعال Dion. Ined. 6r. 8. I. q. Arm. Junpunuspnpun, Garperd, Kharpert, Harpoot, NW. of Amid;

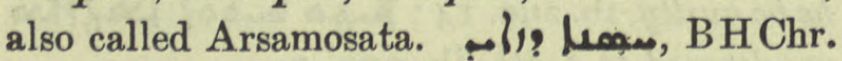

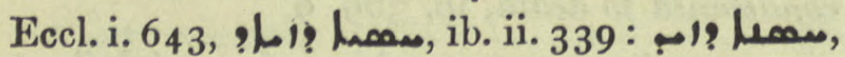
Dion. 86 pen. Cf. معel. 1338. I f. and cf. Ammianus 19. 6. I.

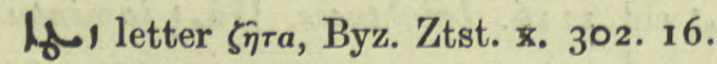

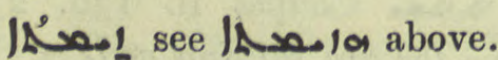

ế col. 1102. denom. verb from lís, Lag. Arm. Stud. 779, G. Abh. 43, Chrest. Targ.

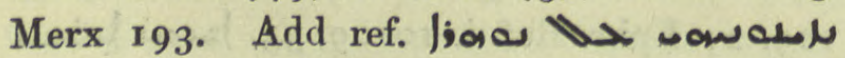
If oa to arm Darkness against the Good Light, Manichéisme 95. 10.

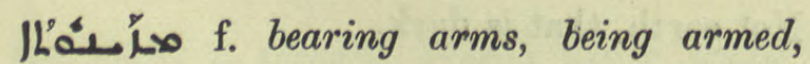
Josephus vi. 4 antep.

ملحبر pr.n.m. an Emir, Jab. 155. 1 .

el. pr.n.m. Zenas, BH. in Tit. iii. 13, Pesh. إِ إِ.
uLl 1) pr.n.m. Zinai, uLs lol, Chàst. 40 ult., 4I. 5. 2) Mt. Zinai in Adiabene; Hist. Mon. i. 6o. 13, 38r. 22, M.Z. 189. 1, 2, 210. 16, Chast. 33. II, 6r. 2. املس م:مA ib. 1.3 .

مـ I gentilic, a man from Mt. Zinai, Hist. Mon. i. II 3 . 20.

|مُ col. I118. m: Add: Assyr. ziqu, Ar. صيق , Hübschmann ZDMG. xlvi. 250, Fremdw. 285. 1) A gale, gust. Metaph.

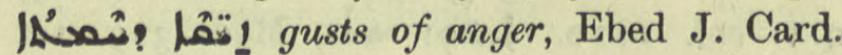
20. 4. 2) a shooting star, ZDMG, xv: 65 I ult.

مa. see under مoos.

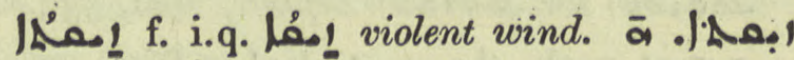

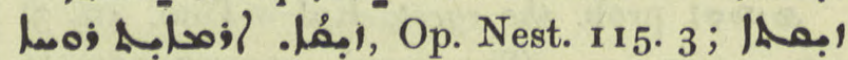

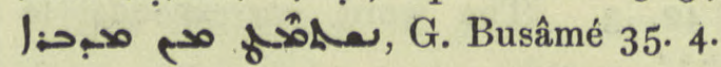

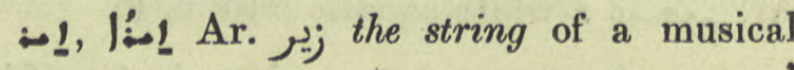
instrument. Used for torture,

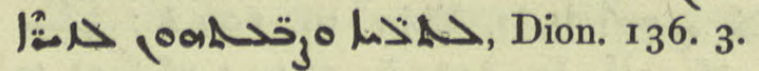

İ) Zaira, a village NE. of Arbela, M.Z. 19. 14 , Trans. 96, n. 3 .

(مئ) col. I I I9. The river near Zaita, on the Euphrates below Karkisiya is so-called, Dion. 26. 17 .

(b́) col. III9. Add: to beat at cards, a> ?

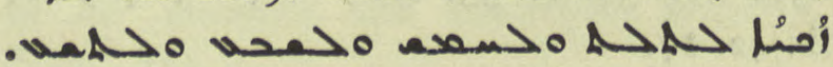

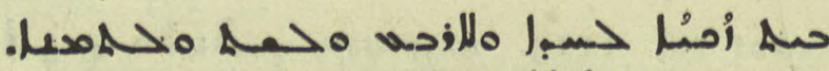

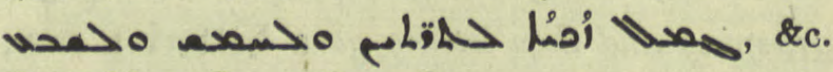
Brit. Mus. Or. 2084.487.

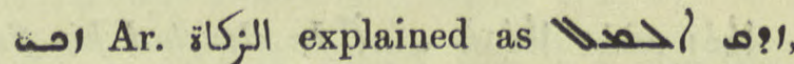
صـدقة المال enforced alms, tithe, income tax, Dion. 1 27.21 , Nöld. in loc.

iص col. I 122 . Denom. verb Pa'el conj. from أص̆: To practice necromancy. Correct and آحّ in the quotation from Ephr. Add:

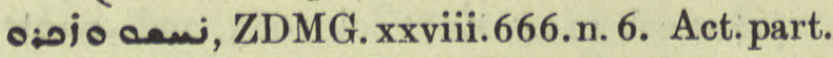

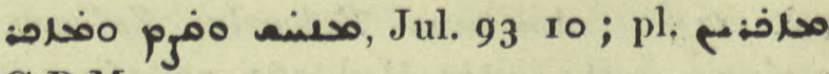
C.B.M. $730 \mathrm{a}$.

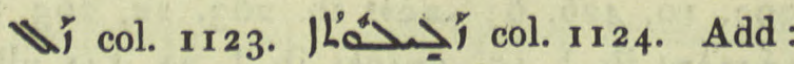

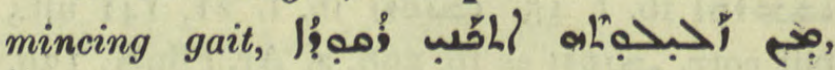
Hist. Mon. i. I $13 \cdot 3$.

ل⿳亠口冋. 


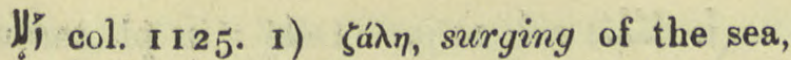
tempest, حرلا oحمسمم Sev. Ant. Vit. 22 I. 8,

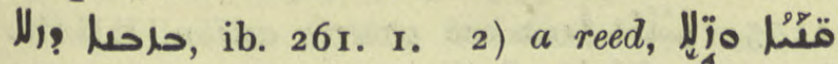

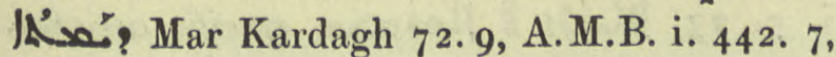
لู

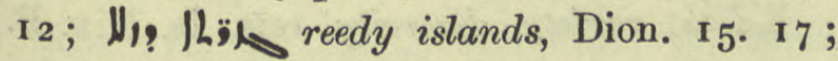
.

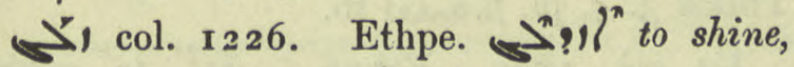

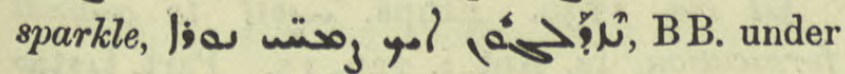
un col. 2348, ed. Duval 1240.

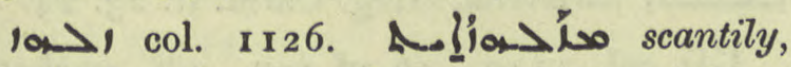
Anecd. Syr. ii. 114 , I, Isoyahb ed. Duval 8. 23.

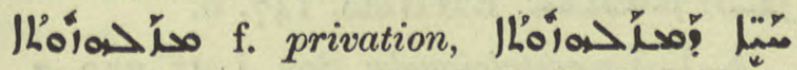
Is. Nin. B. 234. 5 ; BHChr. Eccl. i. 643; opp. JLasow, BH Sap. Pol. iii..

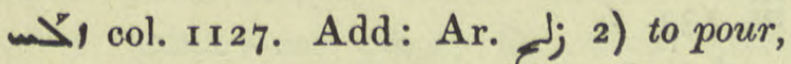
i they pour away water after use in baptism, Takhsa 75. I6. Past part. Jjad

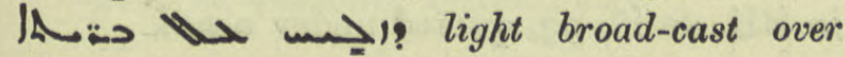
Creation, Jac. Sar. Hom. iii. 6r. 5 .

'حُصُما col. 1128. extravasation of blood,

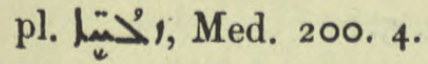

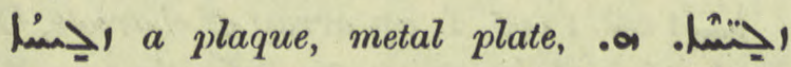

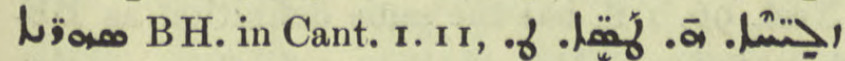
صفايح Op. Nest. 16. 2.

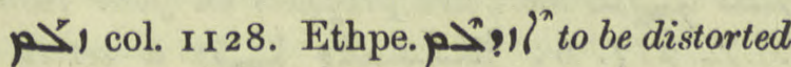

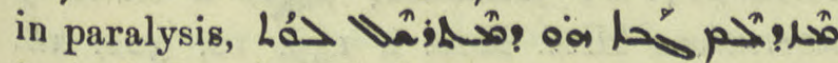

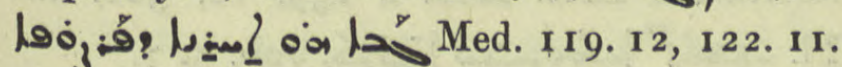

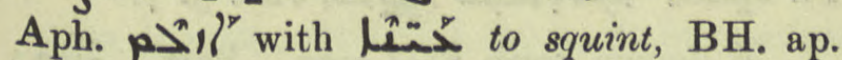
ZDMG. xl. 44 I.

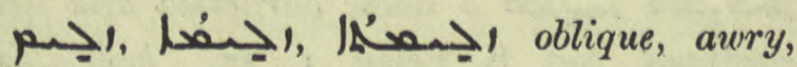
oblique sections, Med. 119. 2 ;

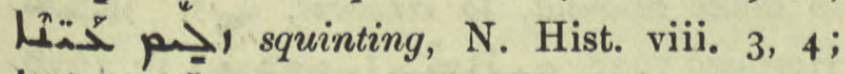

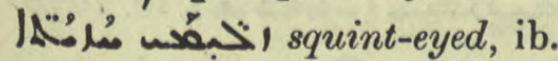

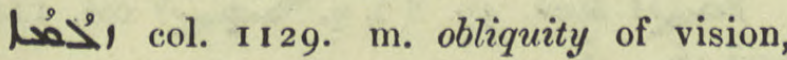
Med. 71. 2 I.

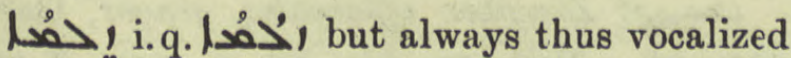
in E-Syr. fault, flaw;

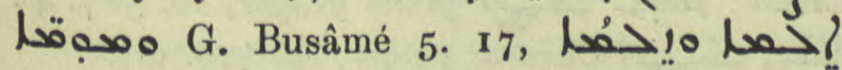
ill-will and envy; severe and spiteful flogging, S. Dan. $62 b$ r, $66 a$ antep.

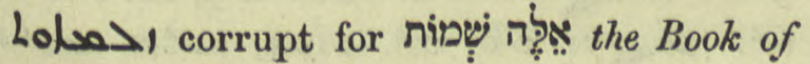
Exodus, Euseb. ed. McLean 4 I6. 2.

\section{0ixs}

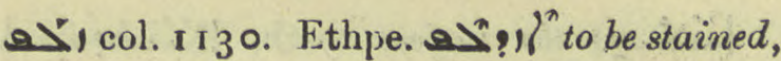
polluted, حهA ii. 377. 26. Pa. 2 ' to polish, embellish,

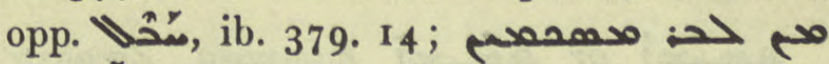
مهلا حهي adorned outwardly, Syn. Or. I42.

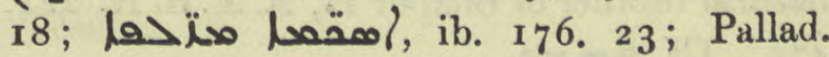

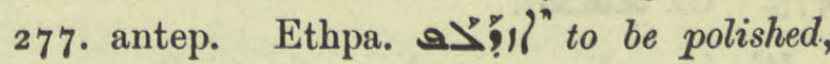

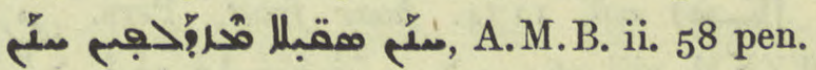

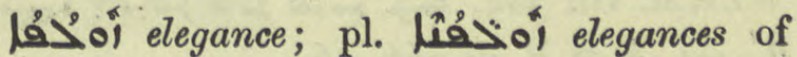
style, B H. Journ. As. 1898, 77. 29.

حصة|ر, حصا a small cake, BB. under col. 1878 and DBB. 937 .

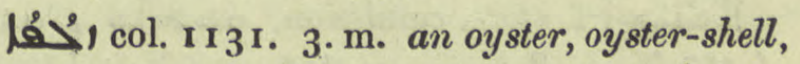
اخلما السملف

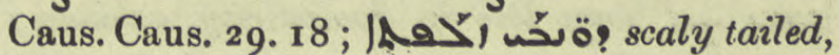

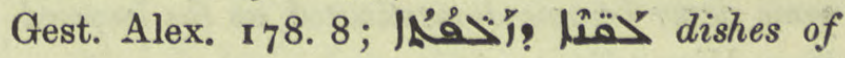
mother-of-pearl, ib. 190. 6.

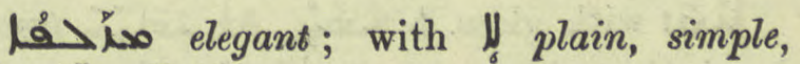

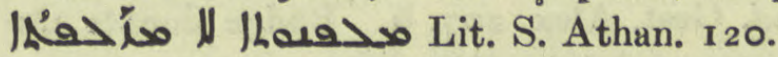

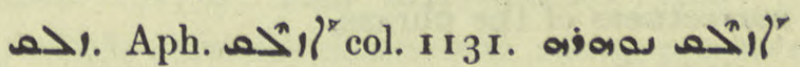

ال حلحها Ephr. ed. Lamy i. i 27. I6.

إِ a student of rays N. Hist, viii. 3. 4.

, m. brilliance, Jul. 49. 4.

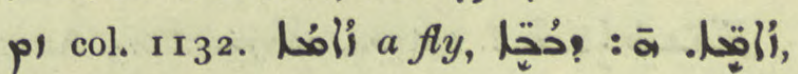
Med. 548. 19.

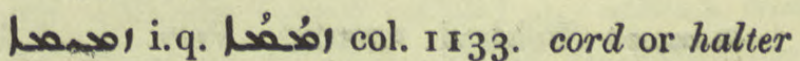
of a camel, Protection 84, 14.

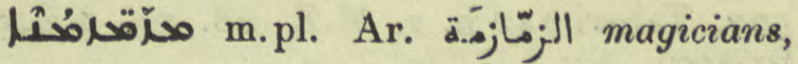
Kal-w-Dim. 375. 9.

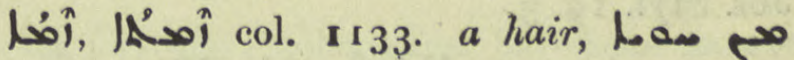
Iاقص פمص Is. Ant. ii. 318. 10 translates the lips of the serpent's mouth.

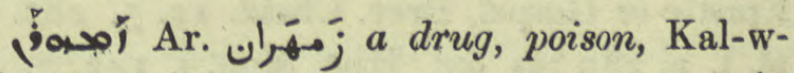
Dim. 142. 8, 143. 14; so correct jasoj in both places.

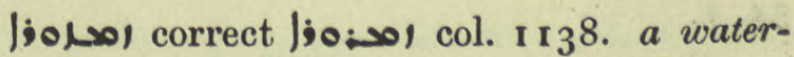

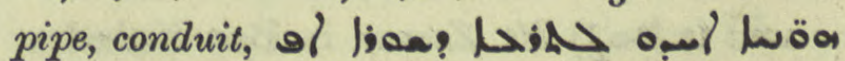
حرمس:3ion. Ined. 6r. I4. Nau suggests |jor bath-pipes.

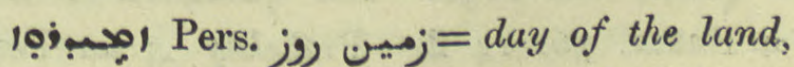
a feast day, Jab. 564. 3, lel >o Lbo: : a

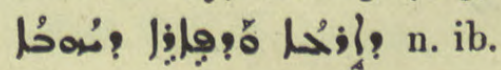




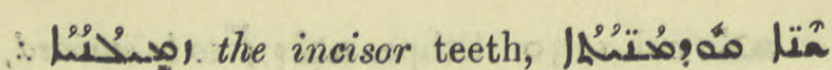

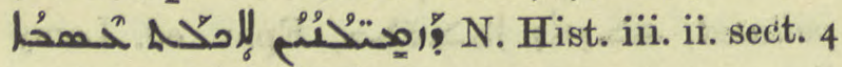

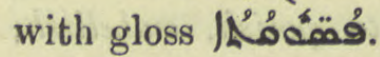

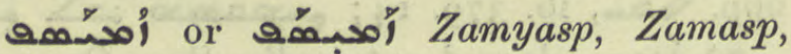
natural brother of Shapur II, A.M.B. iv. 224 , 475. Cf. $2 \times 0 \%$ \%

12. WZKM. viii 364 , ه جُ 10. A.M.B. iii 366. 16.

م.

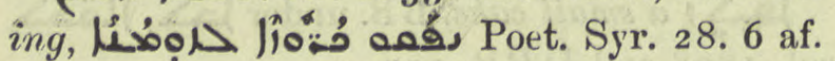

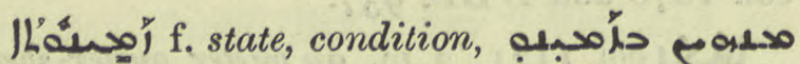

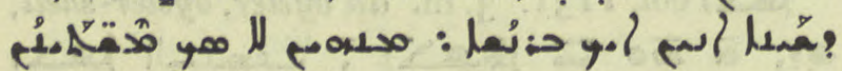
أمب some live in domesticity, as man; some never become domesticated, as the leopard, Nat. Hist. vii. I. I.

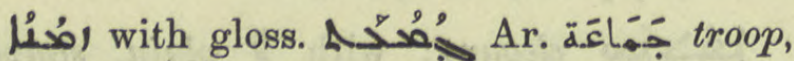
band, Gest. Alex. 207. 6. Budge doubts the correctness of the phrase.

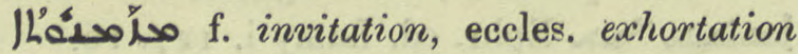
to catechumens before baptism, Nest. Chrest. ed. I. $82 \mathrm{n}$.

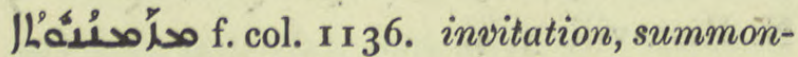

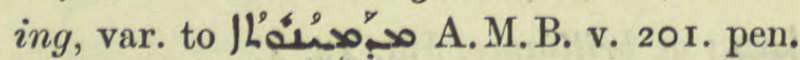

$1 L^{\prime}$ Syr. iv. 3. 22.

וספמאסן Pehlevi, Pers. Gamásp or Zamasp, King of Persia A.D. 496502, Tabari $1_{22}$ n. 2, M.Z. 68. 50, ZDMG. xliii. 399، 8. Cf. (סصمح' col. 1138 , Jos. Styl. 19. 3 .

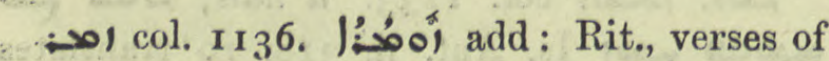
the Psalms sung before the reading of the Epistle or Gospel, Brev. Chald. I 2. 5, 282. 6, pl. 1. 2, 283 bis, \&c., Hist. Mon. i. 142. 7 .

أمذفز m. the singer, a fabled fish, Natur 54. I.

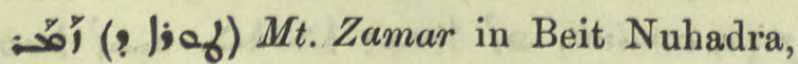
M.Z. 209. $1_{5}$, Chast. 33. 13 .

0 (0) col. $113^{8}$. The quotation in BB. $=$ Natur. $3^{6}$. 14 ff. Probably $j$ for Pers. روان runer, Hoffm. in loc., name of a bird.

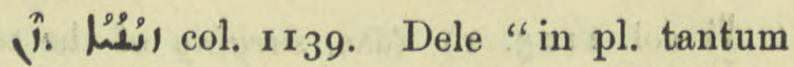
exstat, sc.” Ar. نوعي ispecific, special, B HNom.

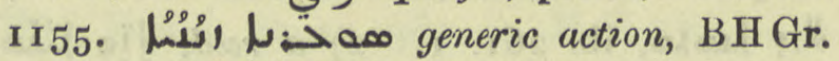

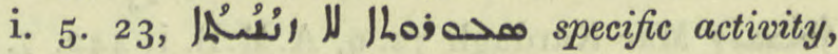
ib. 24 .

ILُưُ variety, diversity, refs. Is. Nin. B. 90. 4 af., I 76. ro, But. Sap. Theol. 4 . 3 infr.,

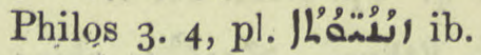

(i) col. II39. Ethpa. w?!l" to commit fornication, Is. Ant. i. 236 . I5. Part. Psا

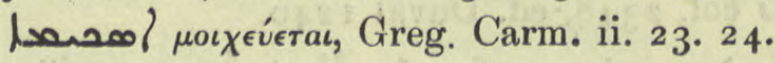

L'í col. II 40. I6 of par. adj. unchaste,

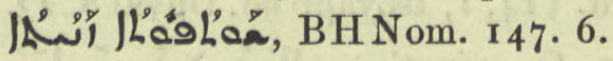

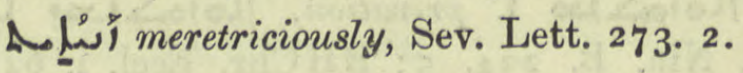

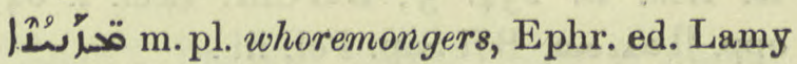
iii. 137.2.

ן col. Ir40. Dele the article; it is another spelling of |'s thyme and is found in BH. de Pl. 93, quoted under esool.

الحما Zenobia or Zelebi, on the W. bank of the Euphrates, a summer resort from Palmyra, Anecd. Syr. i. App. 16. 5 .

L'ícol. I140. f. an array of chariots, Op. Nest. 96. I ff. has the same definition as BB. but fuller. Metaph. a rank, line, series,

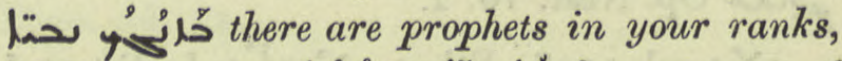

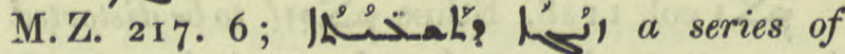
narratives, Hist. Mon. i. I39. I0, 302. 18.

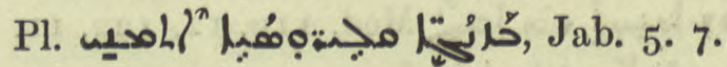

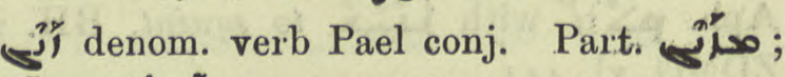

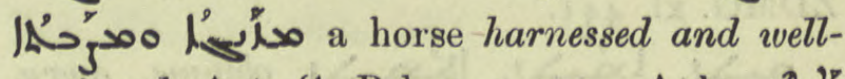
equipped, Act. S. Pelag. 1. 25. Aph. (से)"

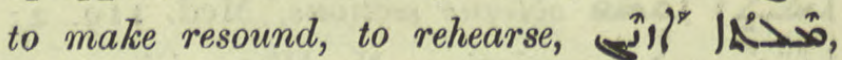

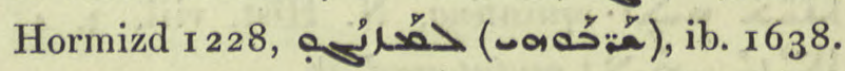

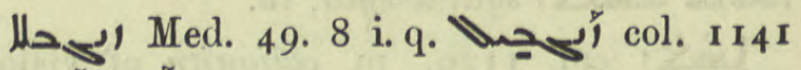
and

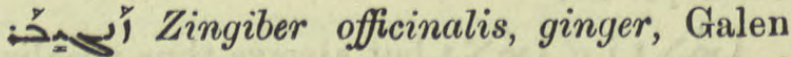
257. ult., Med. 59. bis, 88. 3, I37. 8 and

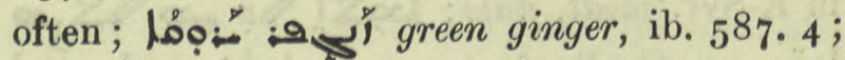

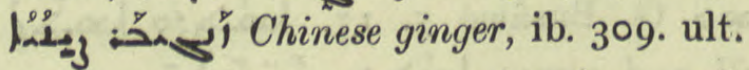

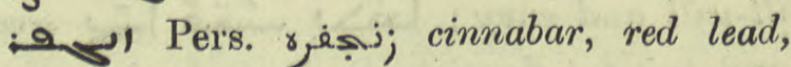
minium, Chimie 52. 20, : $\mathbf{S}^{\prime}$ : 3.8 ; 8 ' 62 ; مص: م ib. $365^{8}$. 
I: col. Ir 4 I and I: $\mathbf{S O}^{\circ}$ col. I 104, Ar.

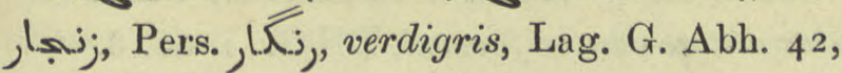
го6, El. Nis. 25. 6I ; Chimie 52. 9, I I.

ائ to deprive, pass. part. ايب: A ristobulus

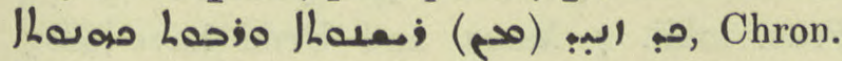
Min. I72. 6. Pa. I" col. I I 4 I. to deprive, لحه, Is. Ant. ii. 34. I3.

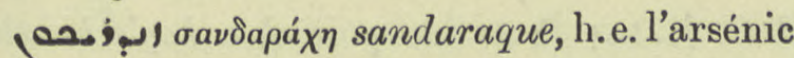
métallique sublimé, Chimie I 2. 7. 9 and $n$.

hoesl Zenobia, Queen of Palmyra, BHChr. Eccl. i. 57. 14.

م Zenodorus of Gaza, Sev. Ant. Vit. 56. 3 .

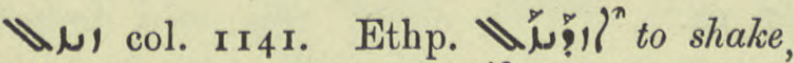

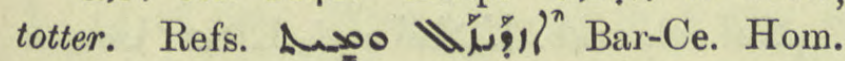

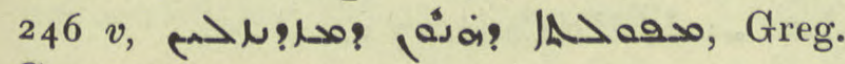
Carm. I. I00. 4.

لูุน้น์ m. quaking, Tekkaf. 102.

أُ col. I I 4 I ult. a) to cast, hurl along,

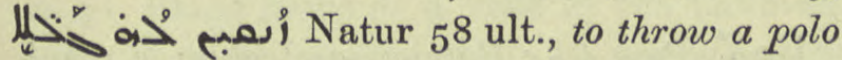
ball Mar. Kardagh. 9. I5. b) to reject a heresy, B. Sal. in Lit. 34. 14 ; Bar Penk. 141. 7. Ethpe. 'ịl to break

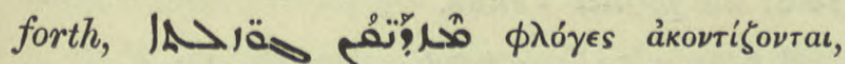
Aristot. ap. An. Syr. 138. 2, 145. 3 ; thunder and lightning are caused by wind compressed in a cloud

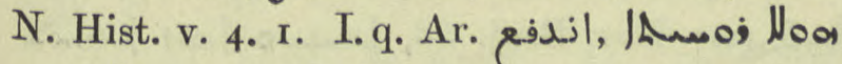
مسرو بما مسه, BHCand. 30v; to be repulsed, a fly

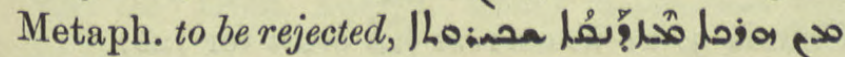
id. Cand. I I $r$.

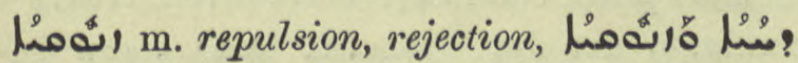
N. Hist. v. 4. 2 ; Ha la 42 I. I6.

synonymous with معنما tormented

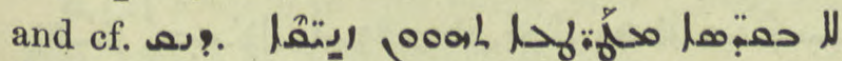
S. Dan. 62 a 5 af.

| forth of fire, An. Syr. 145. 4; a downpour of

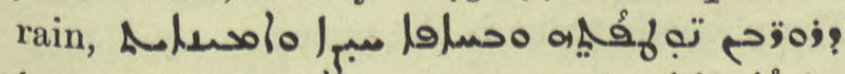

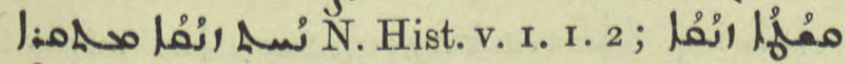

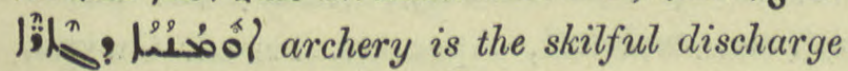
of arrows, Lexx. under مُ مُ 377 I.
|رُ col. I142. Add: 1) the gullet, Geop. 108. 28 ; 2) part of the chin,

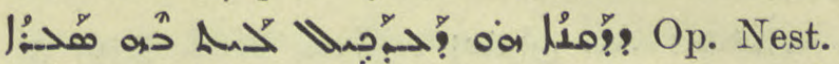
I5. I7. 3) Ar. الش. 1he cord or strap with which the neck of a wineskin is tied, Ibn S. Thes. c. 16.

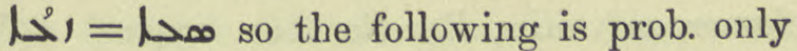
a mis-spelling. Act. part. Lî impudent, Sev. Rhet. $86 v$.

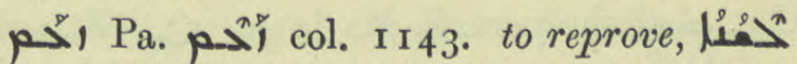
רص? I54. I5. Ethpa. p. p? $\}^{n}$ to be reproached, taunted, Ephr. ed. Lamy i. 505. 4 ; ل) لـ

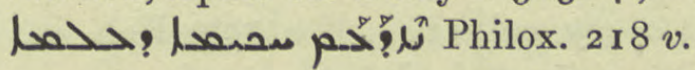

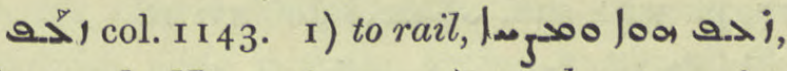
Diosc. ed. Nau 56. 9. 2) to thrust, resist,

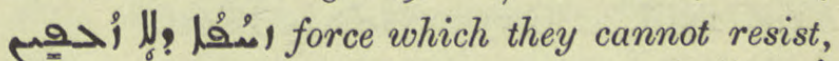
Ephr. ii. 107 F.; . أحس

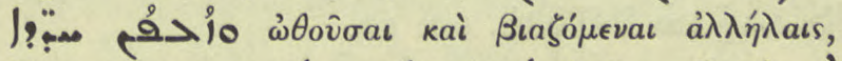

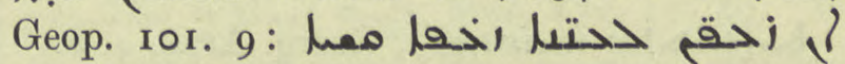
if winds drive the clouds with strong pressure.

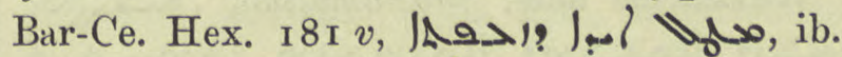

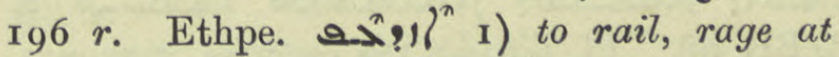
with $\triangle$ of the person, Jul. 42 . II. 2) to be pressed or driven forward, end? דal ص: Caus. Caus. 228.

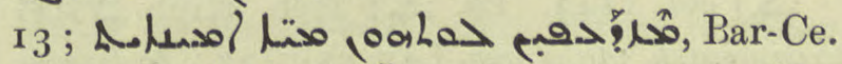

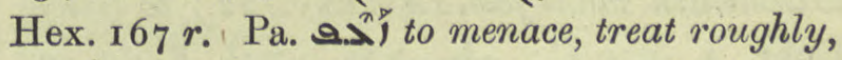

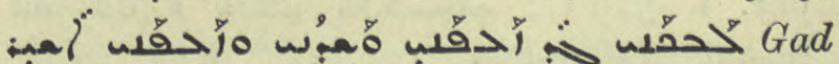
and Asher seized and knocked me about and cast me out, Jos. Narses 99. I3, I4, I01. I7.

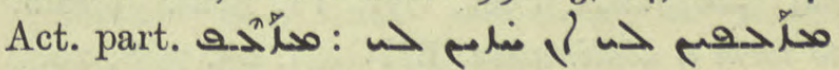
Jac. Sar. ${ }^{6} 6_{3} r$.

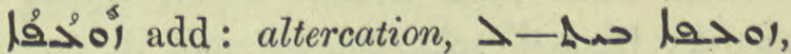

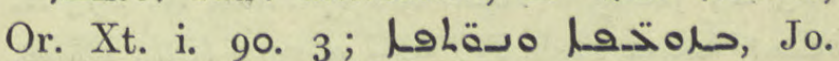
Eph. 8. 4.

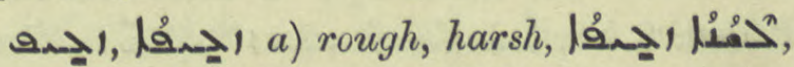

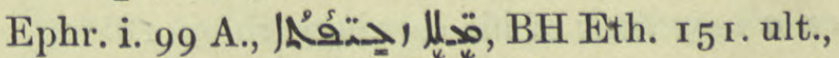

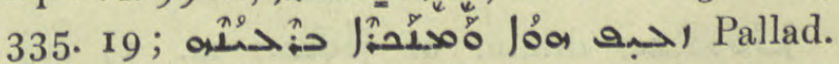

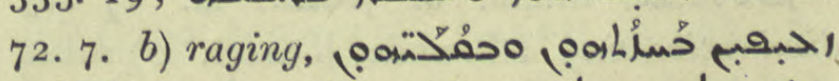
A.M.B. v. 20. c) acrid, sour lan l l loods an $-\infty$, BH. Terot. $268 r$. d) terrible, IL̈̈L?"

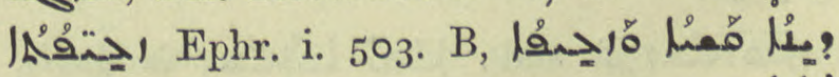

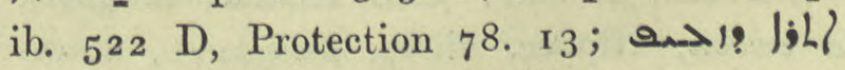
a Hist. B. V.M. I 22.5 . 
LLáanl f. raging of the sea, Pat. Vit. $319 v$.

أحصفํ. Ar. Aaffron, Med. 559. 5, 586. $15, \mathrm{BH}$. in Prov, vii. $\mathrm{I} 7$.

آح col. I 143 . to shout; to sound a trumpet.

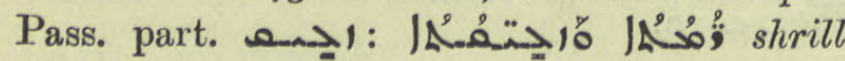
clamorous voices, Bar-Ce. Hex. I34v. Aph.  vv. 6, 7, and passim where Bagster has C. c. $\checkmark$ of the pers. S. Maris 40. 13. Ettaph.

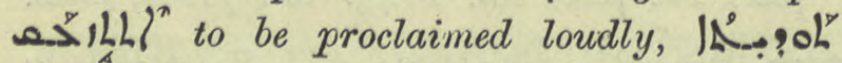

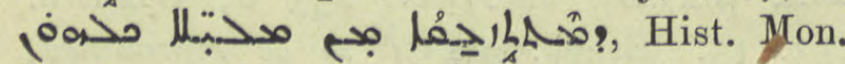
i. 379.5 ; Greg. Carm. i. 9o. 6 .

اעקה a koh. ix.

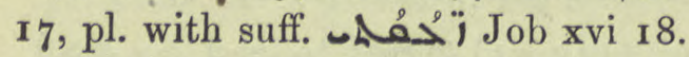

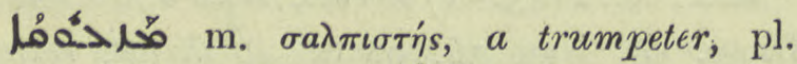

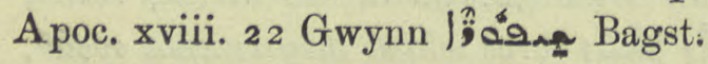

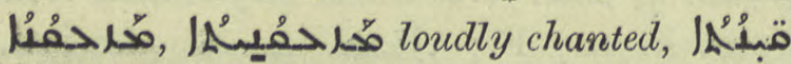

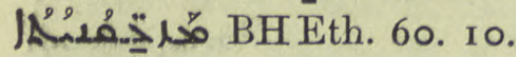

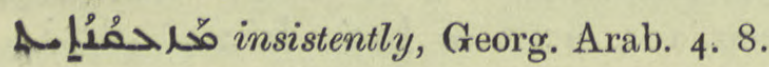

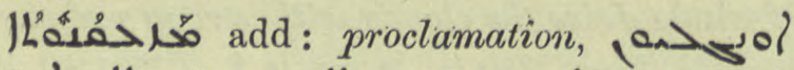

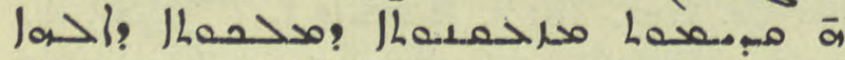

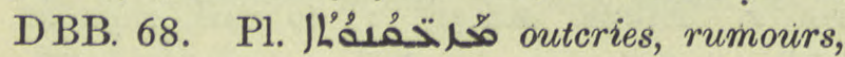

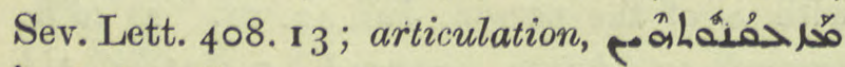
פمة خحما ib. 45 o. 6.

המעיט. col. Ir 44. Pa. Heb. Hiph to diminish, make few, Lev. xxvi. 22 ; to lessen,

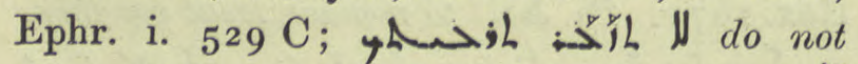

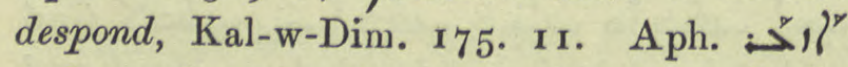

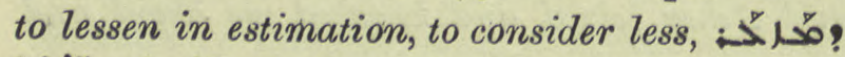

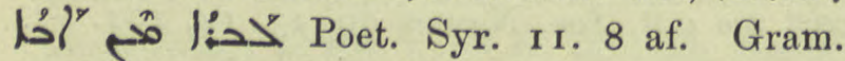
to form a diminutive, BHGr. i. $65.7,8,20$.

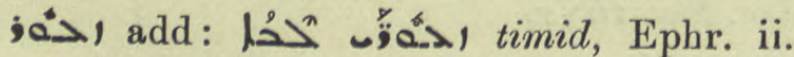

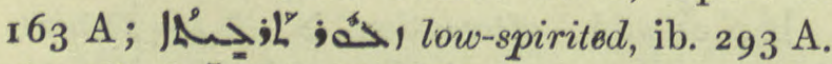
; Jes. xxyi. 20, xxix. $17 ;$ a short distance, Pallad. 8. 18, , on a very little of his earnings, ib. 9. 18.

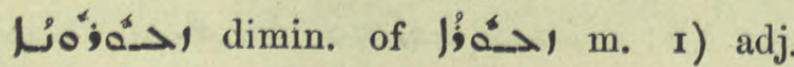
diminutive, tiny, BB. under JLer, j; 2) subst. a dwarf, pigmy, Pallad. 245. I6 = A.M.B. v. $5^{6} 5$.

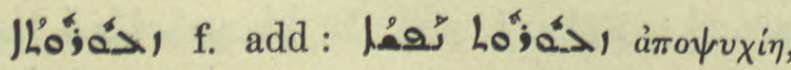
a swoon, Hippoc. vii $7, \mu \iota \kappa \rho o \psi v \chi i a$, mean-

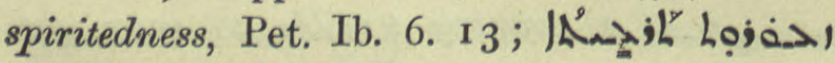
impatience, Pallad. 264. I5. 19.

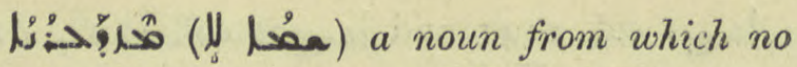
diminutive can be formed, BH Gr. i. 66. 8.

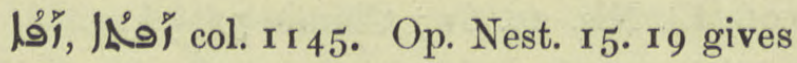

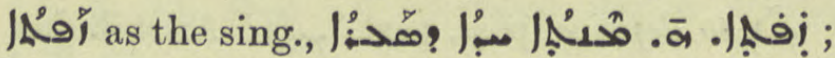
Prov. xxiii. 7 Maus. and Urmi have and so BH. in loc. Is .oLo: Caus. Caus. and BHEth. have pl. lôे.

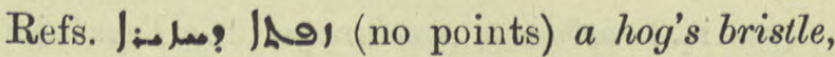
Chimie 322, n. 2 ; eyelash, مas! the eyelids and eyelashes, Caus. Caus.

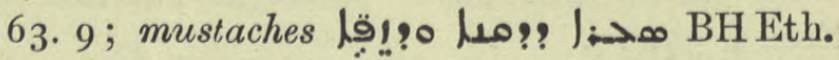
I6 I. 16 .

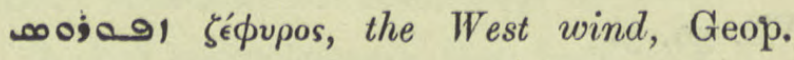
97. 14.

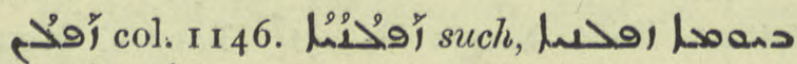

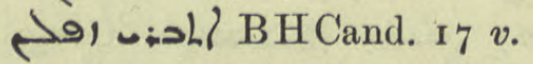

uد Ar. Ar. صir the fir resin, BHCand. 40 r.

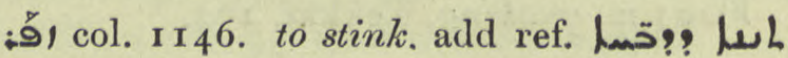
Liف, Jac. Edes. $48 v$.

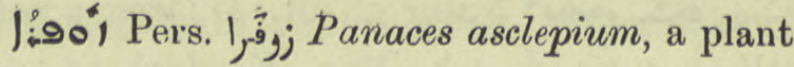
with leaves like fennel, Diosc. $547 b 2$, Dozy;

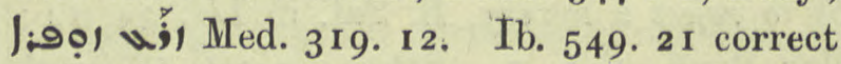
j;ol the planet Venus.

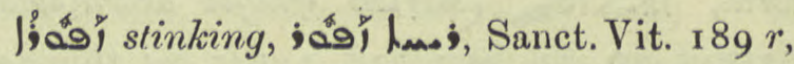

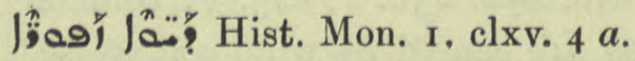

'פم: foul, abominable, add:

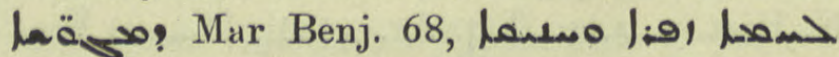
Anecd. Syr. iii. 24. 20, 26. 28 . A.M.B. iii.

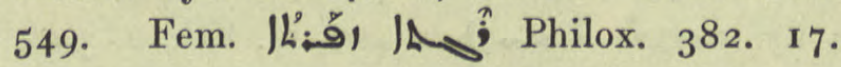

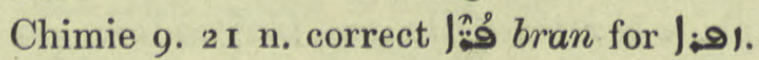

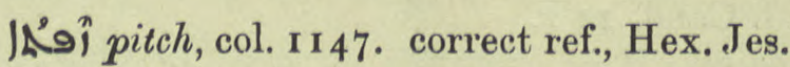


וهג| i iypà niova liquid pitch, ib. $46 v$, used as ointment, ib. $26 r$; إمُ

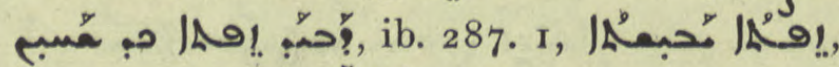

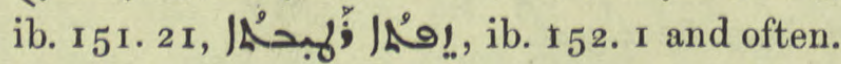

| ו col. I I 47. Dele "rescribe \&c." It is another writing of f'́l'ol col. I I 63 and Suppl. The phonetic value is the same.

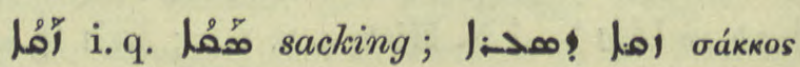
rpíxıvos, sackcloth, Apoc. vi. I 2 ed. Gwynn, 人مُ Bagst. 
ló col. I147. pl. أ̂ inflated bladders, Gest. Alex. 205. 8, 206. I. Bellows, B B. under jesy jol col. 91, D BB. 88. I 4 .

lo! m. pl. a kind of dropsy, Med. 339. I4, إممُ id. ib. 338. 2.

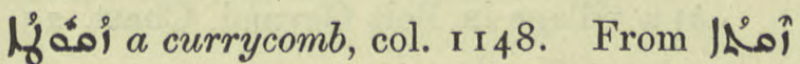
the $L$ being hardened to agree with o, Z.A. xvii. 86.

Los a number which can be divided without remainder, Med. 5I8. I, 3, 519. 7 ff. Cf. Ar. $15_{j}$.

مै) col. Ir48. Add: to hold oneself erect,

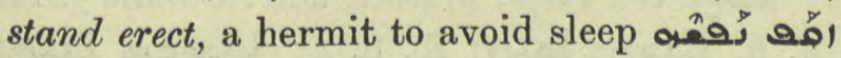
حân Pallad. 460. 23. L. I $4_{4}$ of par. With $\mathrm{l}_{\mathrm{C}_{3}}$ it is a way of

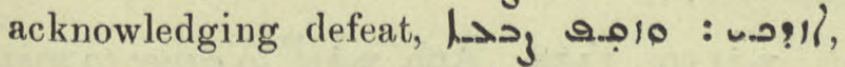

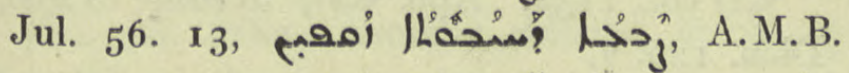

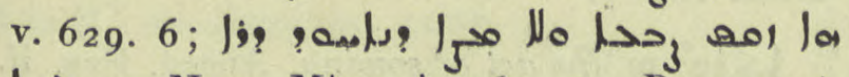
$\mu \gg$ Nars. Ming. i. 262. 7. Pass. part.

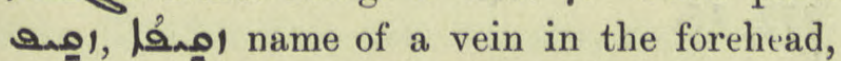

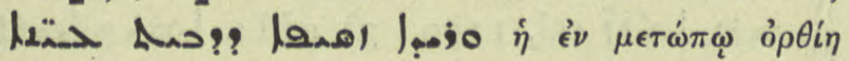
$\phi \lambda \epsilon ́ \psi$ la veine perpendiculaire du front, Hippoc. v. 64. Pael pass. part. محئمُ erect wings, Natur $5^{8}$. ${ }_{5} 5$.

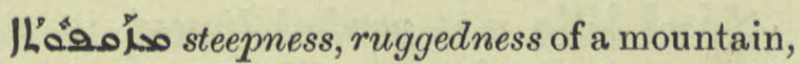
Mar Bassus 33. 453. Metaph. a rugged task,

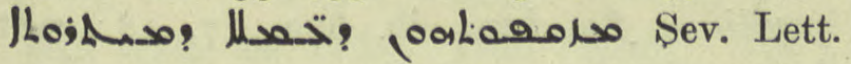
433. 2.

(ô) col. II 50. to weave. Act. part. fem. أم-ْí used of a bee constructing her cell, Bar-Ce. Hom. $198 v$, under Stista col. 4347 .

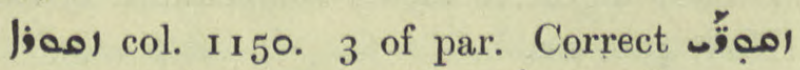

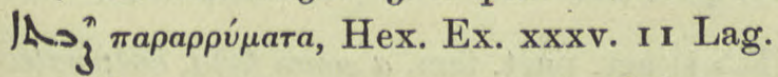

' col. I I I Sassan. 29. 2.

IL'ڤl) a town in Beit Balan, E. of Ctesiphon, Jab. 369. 3 af. Pers. Mart. n. 744.

مِادُ stabbing pains (of pleurisy), Med. 225. 6.

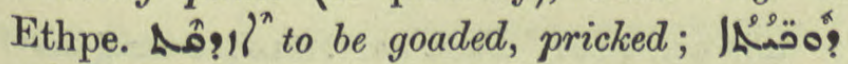

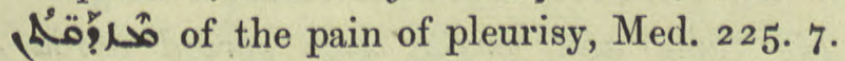

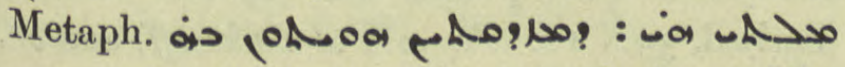
my words which pierced you to the heart, Vit. Mon. 84. 24.

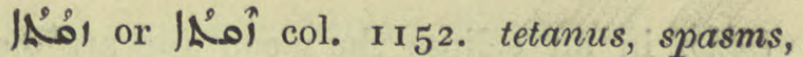

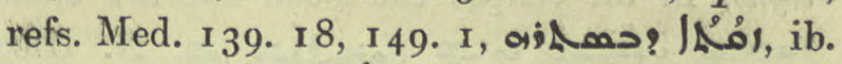

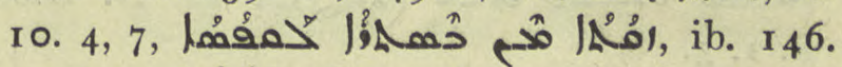

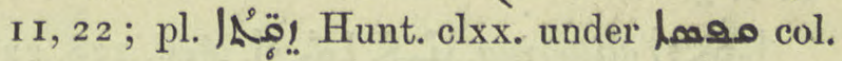
3697 .

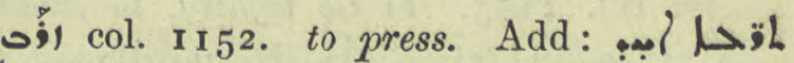
9 \% he made the doors fast, Pet. Ib. 53. 20,

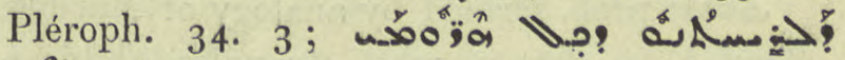
a حُشخص itself all the spices in the world, Hormizd I 190. Pass. part. ult. of par. correct having the

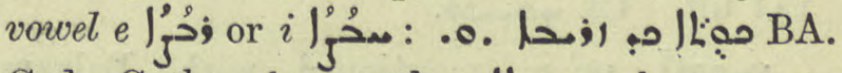
Cod. Goth 4679 under thas col. I7 $77=$

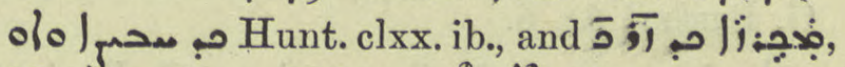

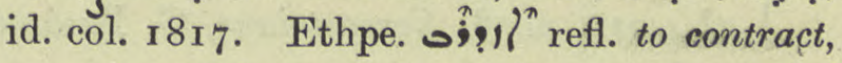
constrict, add: a snake trying to slough its

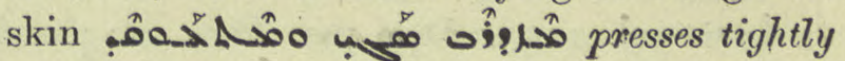
into a narrow crevice and writhes, Natur 38. 7 . To knot, become thick and firm; ;

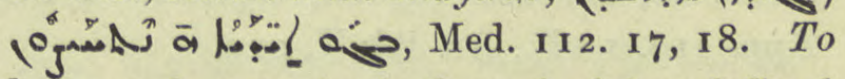
be coerced, restrained, the principles of Good

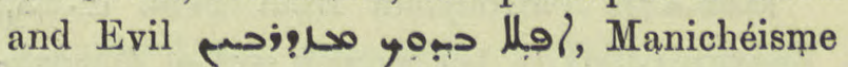
93. 2.

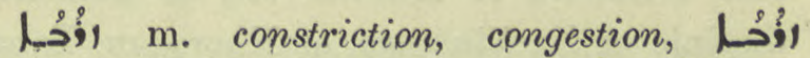

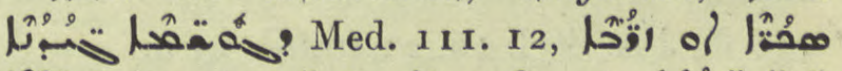

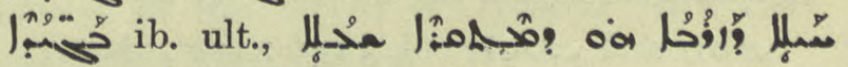
power of compression which causes coughing, ib. 224 . 19 .

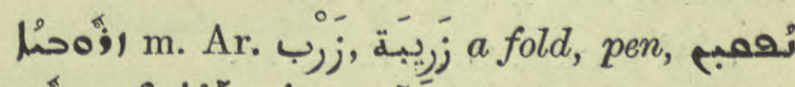

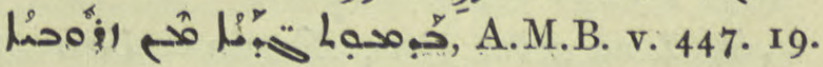

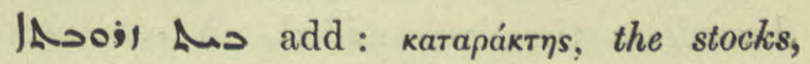
Schol. ad Hex. Jer. xxix. 26.

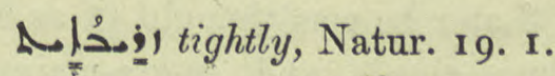

(j) Ethpa.

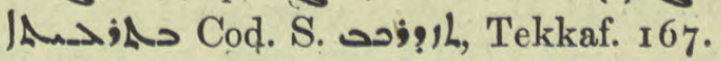

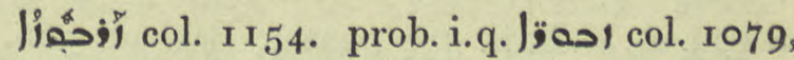

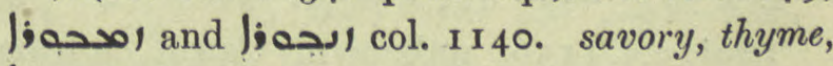
Lesïl Ar. PflnN. 18I, Med. 39. 19, 231. 3

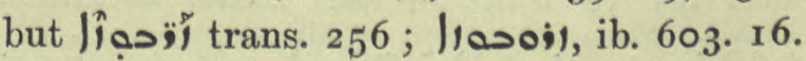

, hame of a tribe who eat dogs' flesh, Kal-w-Dim. ed. Bick. 83. I5.

í col. I I54. Pass. part. \$' glowing,

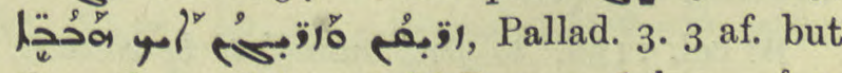
the other edition, A.M.B. v. 2. 6 has scattered. Palpal - $^{\prime}$ to shine with yellow 
light, in an eclipse the moon appears black , N. Hist. ii. 1.5 .

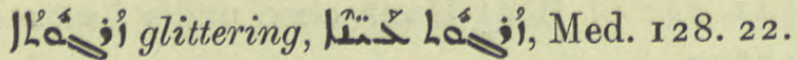
?’ col. Ir 55 . to tie tightly, to strangle.

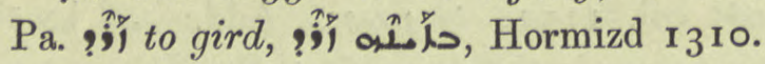

م أو prob. formed by analogy of Marduk. $Z$ arduk, a demon who strangles children, Charms 79. 13, I5, 81. 18, 84, Protection 34. $14,40.7$, 59. $14,64.20$, J.A.O.S. xv. I 4 I ult. Cf. your.

no!j: pr. n. mentioned with Balaam, G. Busâmé 62.6 .

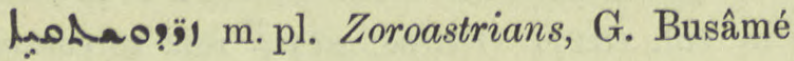
60. 4 af.

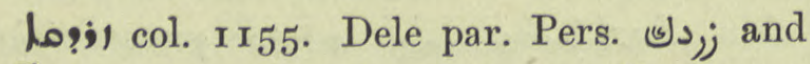
حس Carthamus tinctorius, Bastard saffron, safflower, DBB. App. 2 I9. I ult., Ar. PflnN. 2 I 9.

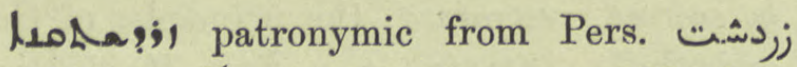
$Z$ arâdušt + T Tan, the magian sect of Mazdakites, followers of Mazdak son of Bâmdâdh, disciple of Zarâdušt son of Khoragan, Tabari 456 ff., Jos. Styl. 16. 2 I transl. I3 n.

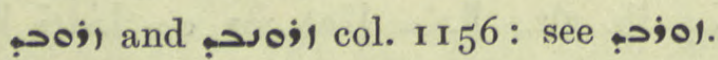

(j) Pers. ز ز

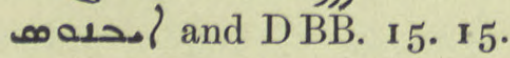

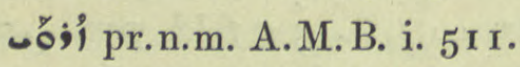

¿̊̊’ Zend zrvan time, Zôrvân or Zaruan boundless time personified. A creature of Ahura-mazda, Zad-sparam i. 24, Vendidad xix. 33. 44. Later he was thought of as the father of Ahura-mazda and Angra-mainyu,

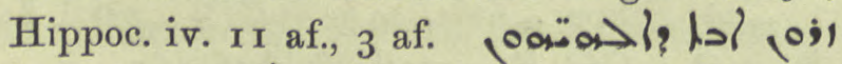

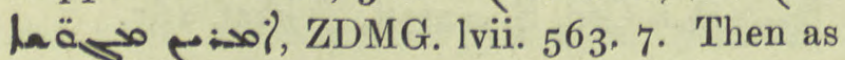
a god, A.M.B. ii. 577 quater, Barḥad. 366.3 .

500; col. I I 56. unknown. Can it be from Zend zar to unite + kar maker = Maker of Union? M. Addai Scher's note is ذريوخُن splendeur du soleil, Barhad 366.3 and n., men-

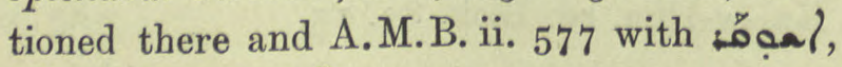

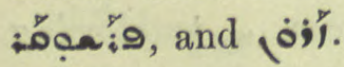

i) col. I I 56. to gird oneself, to be valiant,

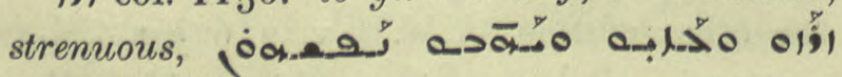
ILaso> Is. Nin. B. 270.3 af.

\section{Iוก}

") col. II 57. Pa. f"i to tear, lacerate,

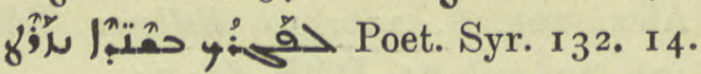

(j) or ii. I I I. I3, Syn. Or. 343, 344 .

4) a village in Beit Garmai, Chast. 23. 2.

:

|ํำ col. II 58 . a monastery near Melitene, add refs. BHChr. Eccl. i. 459. 8, ii. $3 \circ 5$, Chast. 2. $19,3.4$ af., 8. 19, A.M.B. iv. 216.

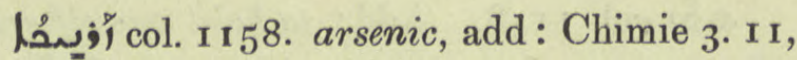

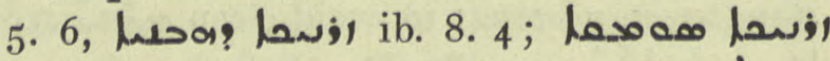
red sulphuret of arsenic. Lexx. under col. 2674 .

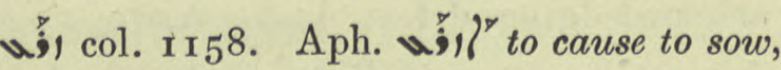
Dion. 126.7 .

 273. 15 .

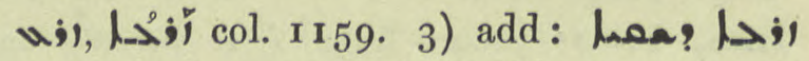
an irrigated crop, Book of Shem I14. I4. Many kinds of seed are mentioned in Med.

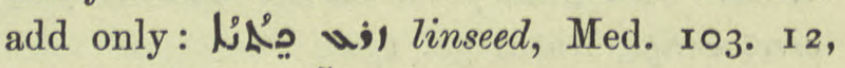
客 parched linseed, ib. $24 \mathrm{I}$.

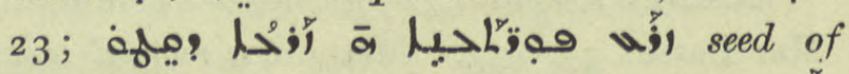
psyllium, flea bane, ib. 603. I7, 18; aî لمئز flea bane seed, ib. 415. 19, 603. I7; | 352. 8.

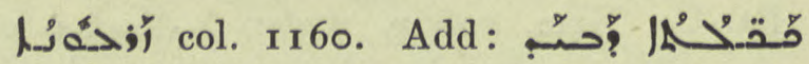
|' digestive tablets compounded of seeds, Med. 408. 2 2.

12. iُ act. part. fem. a sown field, cultivated

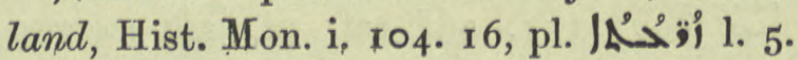

\\:| name of a village, BHChr. Eccl. § ii. 395 .

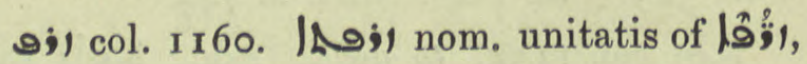
one mark or spot, Hebraica vii. 70.

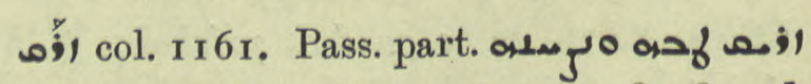
his fame and praise were spread abroad, Barhad. 392. I 2. Pa.

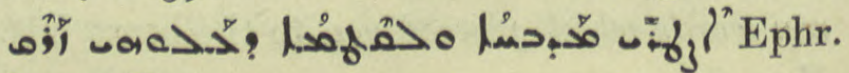
i. $477 \mathrm{~B}$. 


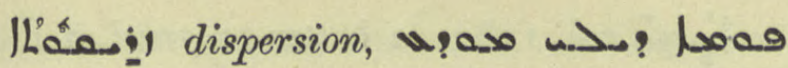
ut, /Loojl Epiph. de M. I6. I, 2.

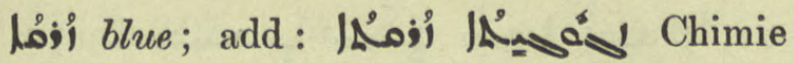
85. 20 ; درأ Josephus vi. 5 : see lo!).

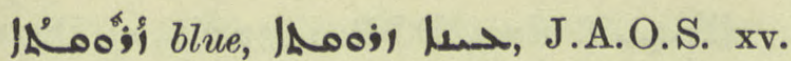
285. 5 .

IL'ópjl col. I I62. apparent colour of the sky, blue sect. 3 , cap. iii pt. 5 sexies.

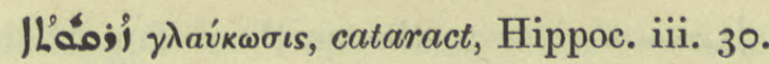

oj) Zark, village near Merv, Chast. 23. ult.

flíol col. I163. Arm. zoh, sacrifice, Sanscrit hotrá, Bactrian zaotra, Lag. G. A.

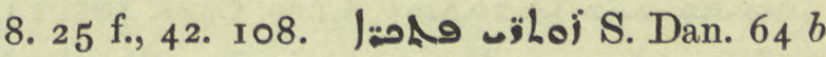
6 af., Hist. Mon. i. 201. 4, 396. I 7 ; JjLoi |ํํㄹ, Jab. 445. 3, Pers. Mart. n. 874. lihe col. ri66. wrongly referred to مa. It is lin an abyss, col. I 237 .

wh. col. Ir66. a fort in Tur Abdin. Add: BHChr. Eccl. 569. I3, 57 I. 2, R.O.C. xv. 229 bis.

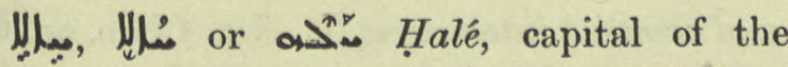
district Radan. Probably Ar. حولايا North of Baghdad, A.M.B. ii. $654=$ Pers. Mart. 71 ; من Jab. 2 I 1.7 , Anecd. Syr. ii. 24, 195.

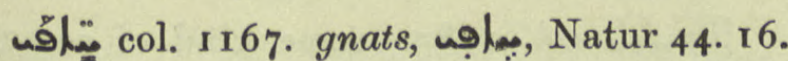

J) reeds, col. I 688 . Dele “forte." The passages quoted from Pat. Vit. are also in Pallad. 326. 1о, 13, 34I ult. = A.M.B. v. I53. 16, 19, I74. I3, I75. I4.

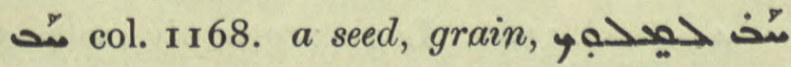

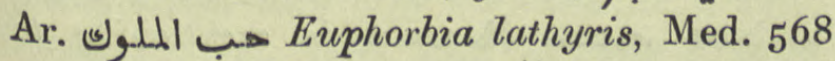
ult.; black cummin, ib. 583.5 .

مِّحُ col. II72. nom. unitatis of one grain, a quarter carat, Chimie 44. 3.

مد Pa. col. Ir69. to love, embrace, long for, rey embraced the opportunity of living with him, Hist. Mon. i. 45. 14. Aph. مै

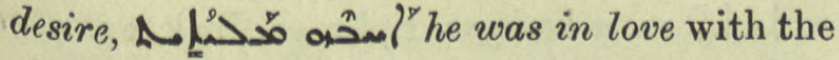
place, was entirely pleased with it, Pallad.

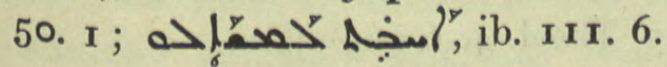

מs much as they wished, their fill, a

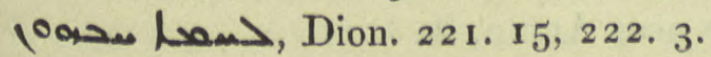

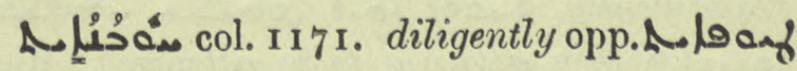
stightly, Syn. Or. I72. 29.

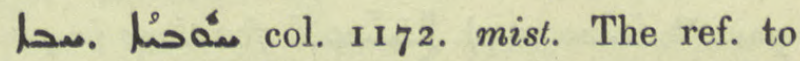
Zach. = Anecd. Syr. iii. 207. 12, 26 . Add : مَّة fogs lifting and blown away, Al Ihkham 65. 3.

محهزן Habura, formerly Circesium, at the junction of the Habur or Khabur with the Euphrates, Dion. 68. 22.

مح้ to beat down, batter down, col. 1173 . 4, 5 he beat doun the spike

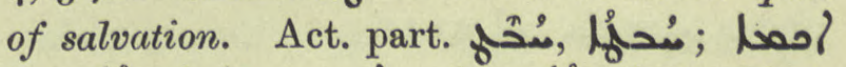

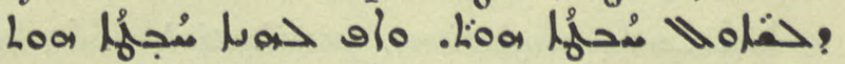
hoio in like manner as the Spirit beat down Saul and laid Balaam low, G. Busâmé $89 b$

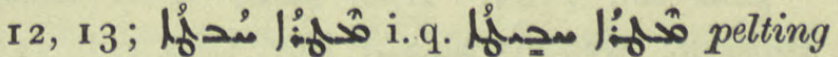
rain, Ephr. ed. Lamy i. 197. 3 ; محله المطر

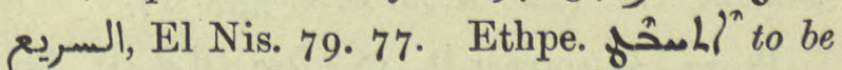

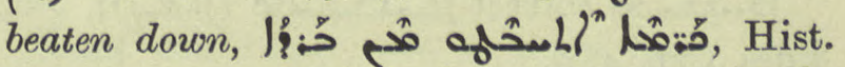

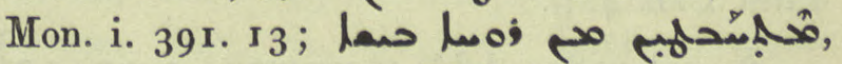
G. Busâmé $89 b$ 1 4, A.M.B. i. 197 .

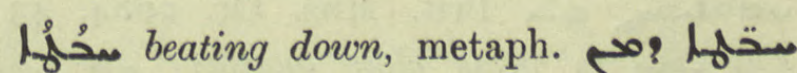
10o; the downfall of Simon Magus to the depths, Ishoyahb, Hist. Mon. ii. I69. 20.

a staff for beating off leaves, $\mathrm{cf}$.

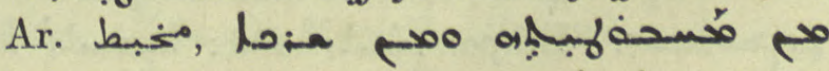

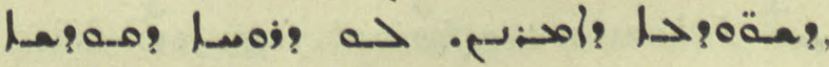
G. Busâmé $89 b$ I 6 . 
محسل a river to the north of Tur Abdin, C. B. M. I I 10 a.

col. 1 1 74. to mix, blend. Add: intrans. till the mixture is molten and tempered, Chimie 54. 8.

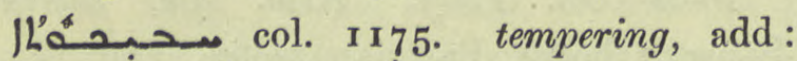

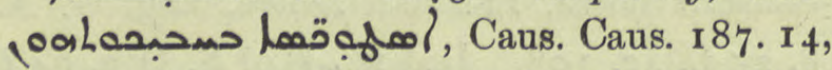
Galen 286. I2. Metaph. mingling, Bar Sud. I 10 n. $a$.

محُمُ m. combination of the four elements, Galen. 274. 3, مححم| 9مة' ib. 1.6; a mixture, Chimie 54. 14. Correct ib. 1.9 to

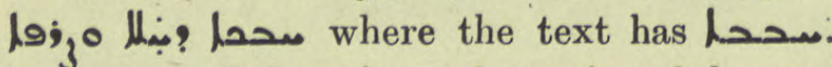
Connexion of the veins and arteries of the eye, Med. 74. 23 .

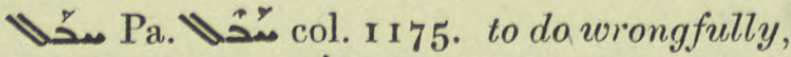

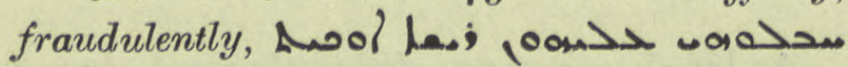
1 Syr. 492. 20, Chron. Min. 257. 15. Pass.

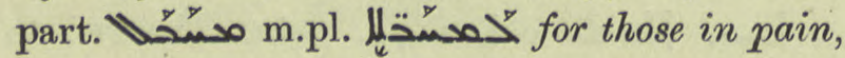
Med. 576. 9.

col. 1 I 77 . perverse,

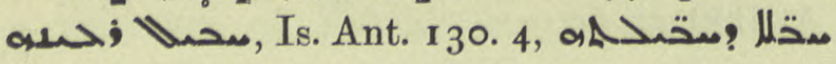
the snares of his perversity, 1. 5 .

محُل col. II 79 end of par. interj. Alas, add : frequent on tombstones, Lidz. Epigraph. 147 .

مخُحْن dangerous, fatal of diseases, BB. under > >0 col. I9II.

مَّة

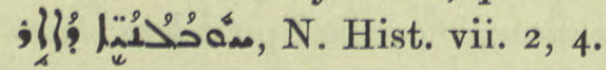

محم col. 1181. Aph. to be slow, negligent, فمحدى ?

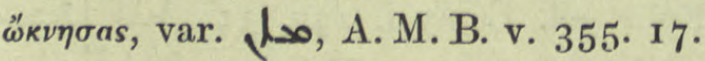

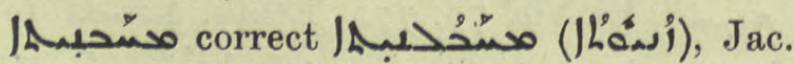
Edes. Can. 4. 5.

col. Ir8I. Add: to contract, טaicy a) Brit. Mus. Or. 2084. $43 v$ v.

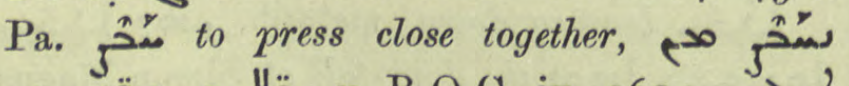
إخب م R.O.C. iv. 360; ? receive, Aphr. a 9 L. 7 another manuscript reads

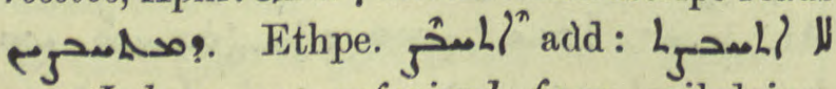
(⿻) I have not refrained from evil-doing, G. Warda, Folkm. 9. 4 .
造 col. I1 82. a batter or gruel of flour,

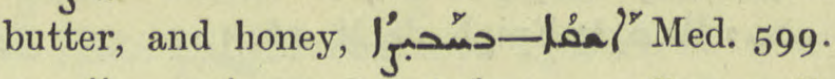
2I, Jaeng Coupes ii. 127 infra.

$1 /$ محيمّ f. alum, DBB. $133^{2}$ ult., cf. Duval's note Journ. As. 1893, 329 under 9 ig.

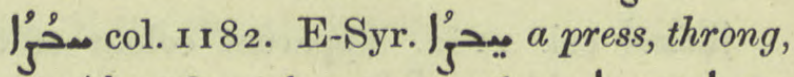

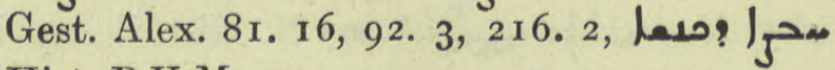
Hist. B.V.M. 220.20.

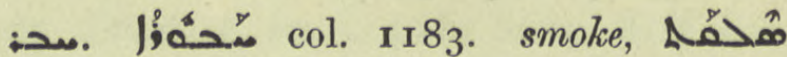

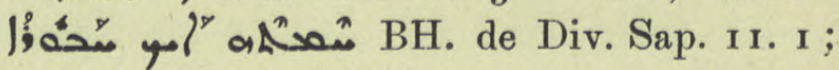
a funeral pyre, id. Carm. 129. 2. But the same passage quoted col. ${ }_{5}{ }_{7} 7$ has

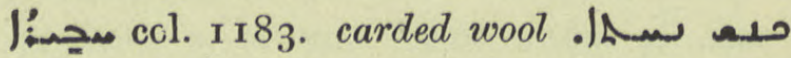

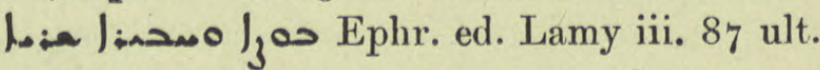

col. 1 I 83 . 3 af. 3 af. correct bustard for נحص:

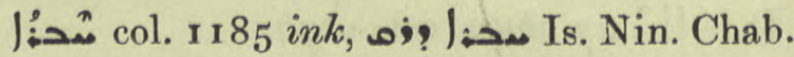
29 ; recipe for making ink, C.B.M. iii. Praef. x.

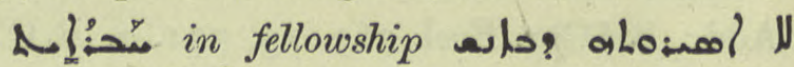
dissociation from friendly and cheerful intercourse, Is. Nin. Chab. 99. ult.

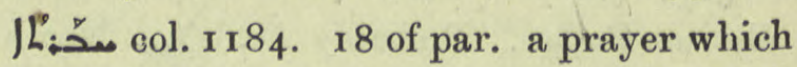
connects with the preceding, Brev. Chald. i. 29. I5, 39. I, Takhsa 74 bis.

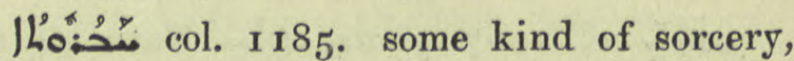
Jac. Edes, Can. 27. pen.

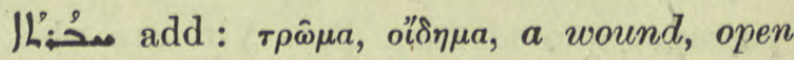
sore, Hippoc. v. 2, 6I, Med. I92. I I, Bar Sud. 44. 17.

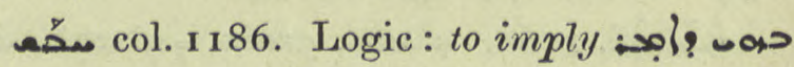
مخس particular he implies the distributive, Probus

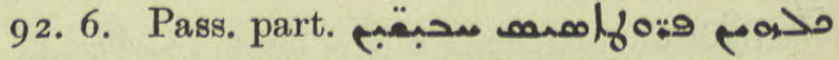
all the propositions are included, ib. 96. $\mathrm{r}$.

مُصحَ col. I1 87. Dele art. I), it may be a mistake for مaحد): see Ar. PfnN. 143. 2) the Persian apple, pl. مُّقب apples, Med.

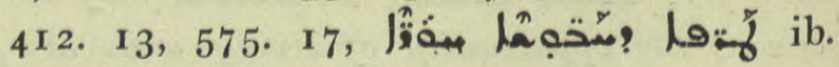
578. 10.

مَحْحُم m. a besieger, Ephr. ed. Bick. 22. 4, 5 .

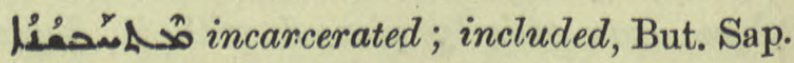
Isag. iii. 3 , Theol. 4. 6 and often.

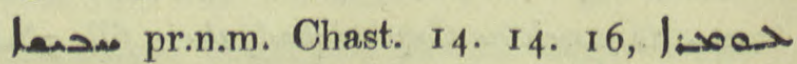
مar. near Arzun, ib. 1. 11. 


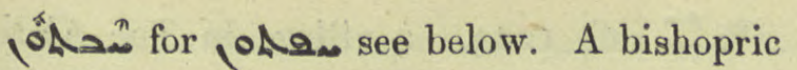
on the left bank of the Zab, Syn. Tim. i. 3 ro, Or. Xt. ji. 31o. I, 3ir. n. I. مّخ Mar Kardagh 4I. 2.n. II; olo id. ed. Abbeloos.

col. I189. a market day, hence selling. ب who instituted this evil and accursed custom of selling into slavery? Jos. Narses I5. 4. Noah by his cursing of Ham gave occasion to this evil custom of slavery, 1.9 .

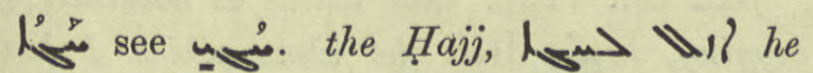
went on pilgrimage to Mecca, El. Nis. Chron. I $74.4,175.18$.

م col. I I go. Dele the article; $\left.\right|^{n-n}$ is pl. of $1 \stackrel{n}{\sim}$, El. Nis. 78.62, a hill shrine, high place, crag. Job xxx. 6 points Ephr. ii. I 3 B. in loc. has $\stackrel{\text { n...n }}{\sim}$ and BH Schol.

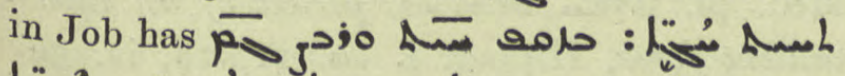

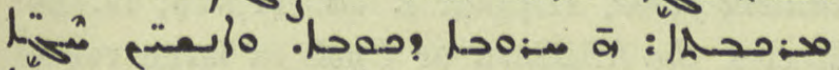

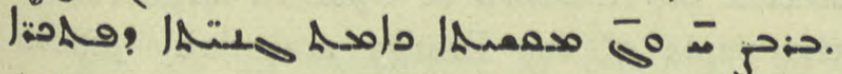
A.M.B. ii. 599 ult. مُ should probably be

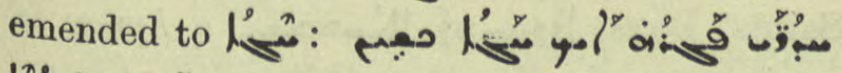
הـ heaped up round her body like a cairn. End of par. dele "Vox suspecta."

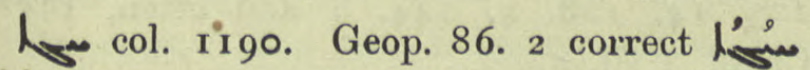
ไُ

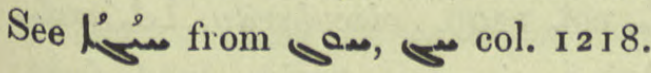

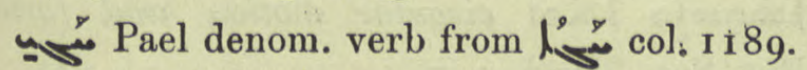
to make a funeral feast, to wake, a

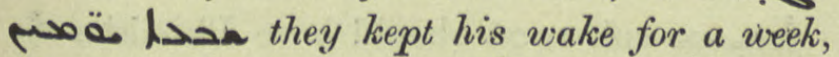

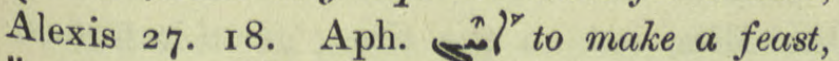
له Aphr. 74I. 20.

مـ Hajji, a pilgrim to

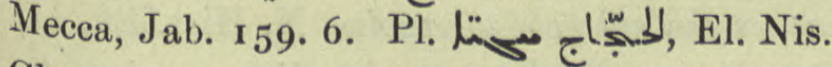
Chron. I 73. 24, I75. I1, I8 8 r. ult.

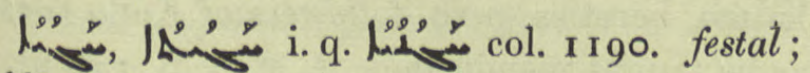
قدالا مـ S. Pelag. 4. I3.

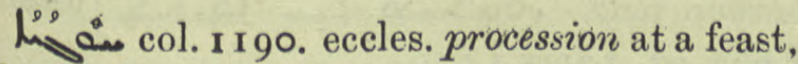
add Dion. Ined. ap. R.O.C. ii. 480. I I : Anam order of the procession of the Pax at Easter, Brev. Chald. ii. 4 af.

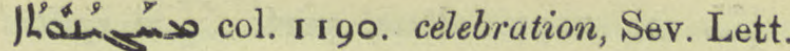
466 ult.

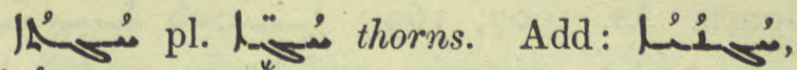

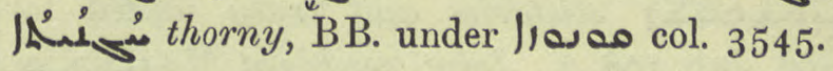

مי col. II9I. to surround,

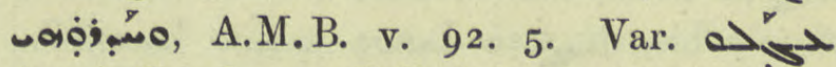
Ar. عبل to hasten, Pallad. in the like passage has محيح ; as it was for security the former reading is preferable.

\tortuous, winding, the cave of Machpelah

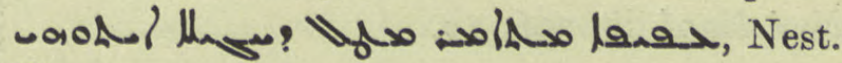
Chrest. 91. 106.

ل Hunt. clxx under مَمْمُ 2582, DBB. I 32 I.

مـ col. I193. lame, BA. under

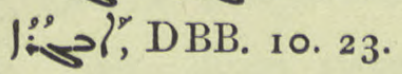

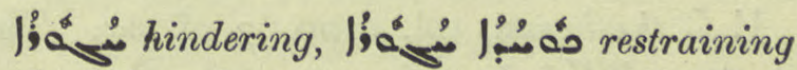
modesty is a human and animal trait, But. Sap. Philos. 6. 8.

ILo: مَّم f. haltingness, lameness, wal

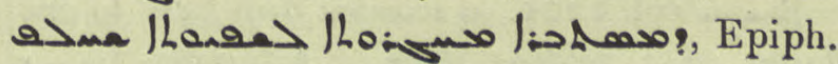
8. II.

col. Ir94. one. unique, Natur 28. 6.

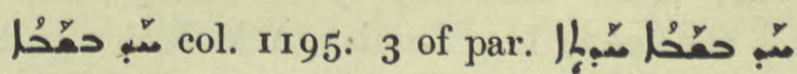
New Sunday $=$ Anglice Low Sunday, кvрเак'

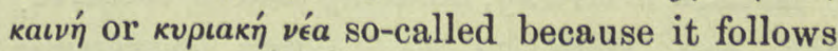

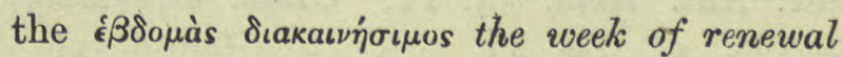
as the resurrection of Christ renews mankind each Easter Sunday, Hist. Mon. i. 2 I 5 . II, ii. $4 \mathrm{I} 3 \mathrm{n}$.

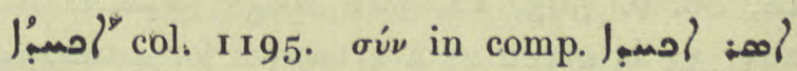
$\sigma v \nu \in \delta \delta \eta \sigma \epsilon$, Hex. Ex. xiv. 25. Logic : the coattribute, Probus 97. 5 .

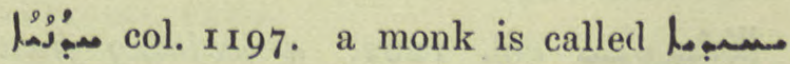

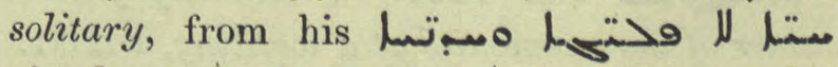
simple and single-minded life, BA. under

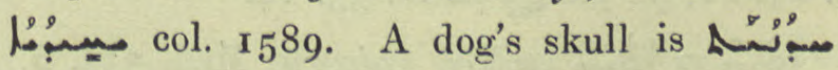

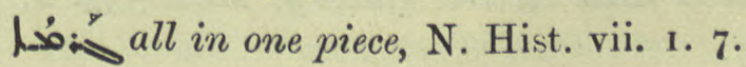

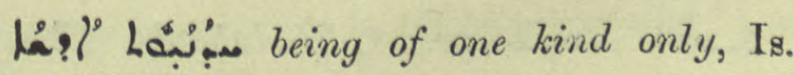
Nin. Bedj. 206. 9. 


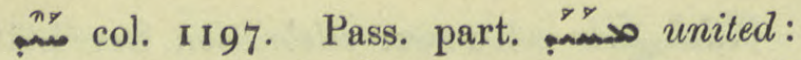

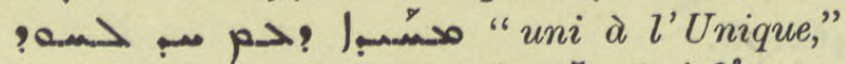

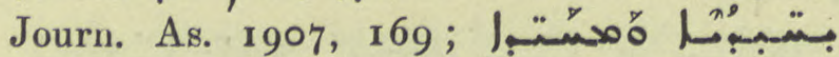
وجمبه solitaries and truly united to God, Jab. 508. 2. Joint, combined, Jle محصب

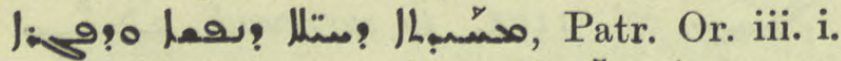

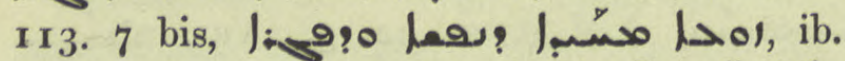

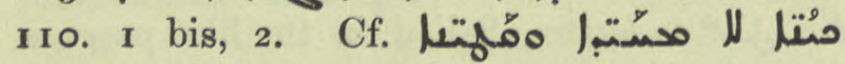
incorporeal beings $=$ i.e. not united to bodies, Is. Nin. Bedj. 195. 3 ; consistent, لע, هـ

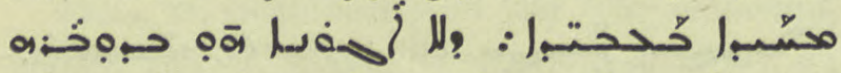
whoso is inconsistent in action, is undisciplined, ib. 206. 20.

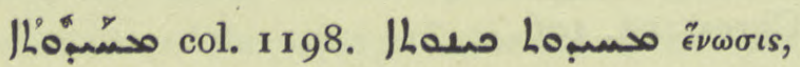
WZKM. ix. Io2.

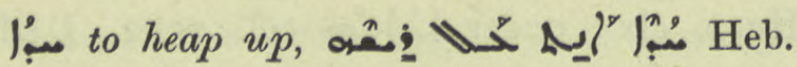
החוֹ, Prov. xxv. 22. Ethpe. heaped up, مع إمع with gloss Hormizd. 1960.

مبّ the breast, col. 1200. 3 of par. The

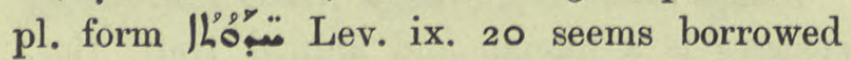
from the Targum, the Syriac form of pl. is . See Merx. Chrest. Syr. 195 ult.

I مُ col. 1 201. a wooden flap fixed to one flange of a folding door, BA, under low col. 234 o.

معبم col. 1200.

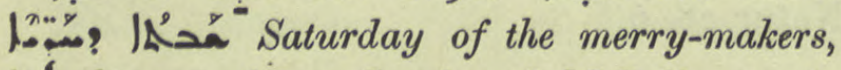
i.é. the eve of Low Sunday, A.M.B. ii. 24 r. 8.

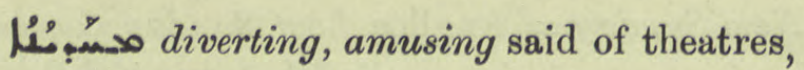
Sev. Ant. Hom. 54. II. Gram. expressing joy, Hebraica iv. 169. II.

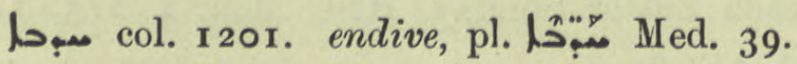

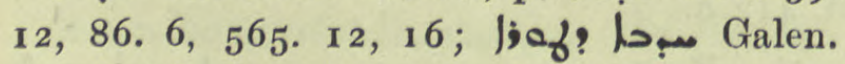
297. 33 is given as a synonym for Sonchus oleraceus. See col. 2568 and Ar. PflnN. 253, 255 .

L'á-_ م- col. I 202. a wedding feast; master of the ceremonies at a wedding, $\mathrm{BHGr}$.

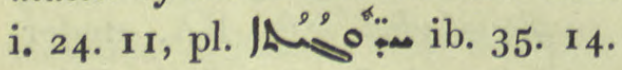

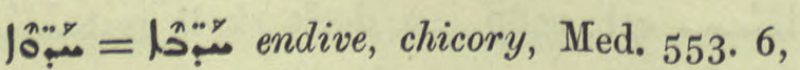
566. 7 .

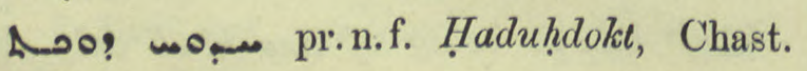
31. 18 . \ạc col. 1203. a village in Tur Abdin, A.M.B. iv. 499. I5; Mar Bassus 40 n., 55. 5 , 66. ult. ; טيم: ib. 48.670.

مبرّ col. 1203. Parts. a running sore of the head which spreads,

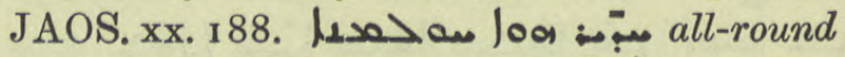

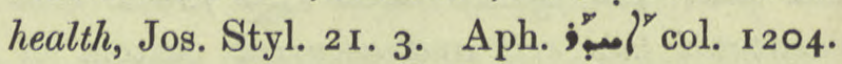

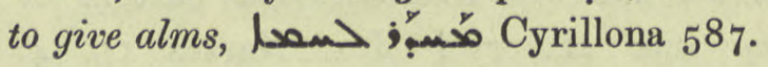

col. I 204. going round to beg, مبن:' begging for water, Cyrillona $5^{87}$.

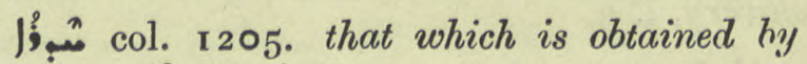

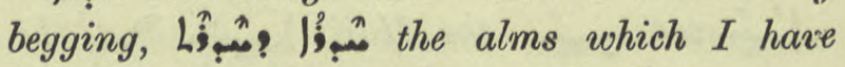
begged, Hist. Mon. i. 168. 18, Cyrillona 587.

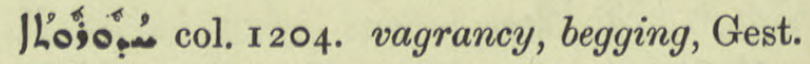
Alex. 20. 7 .

1205. odd jobs of housework, chores Loa مده معب! Pallad. 183.9.

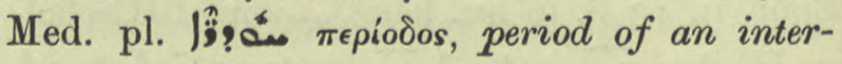
mittent fever, Hippoc. i. 10, II, 18, iv. 59. Eccles. the Khudhra or Cycle, a large vol. of the parts of the liturgy and daily offices proper to Sundays, Feasts of Our Lord, and

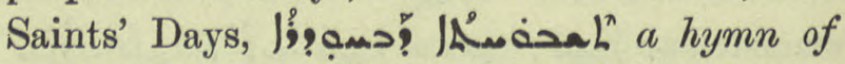
praise from the Khudhra, QdhamW. 16r. 7;

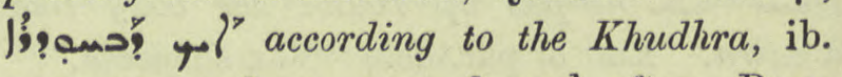
1 $23.14,128.4,144.8$ and often, Brev. Chald. I tit. 53. 2.

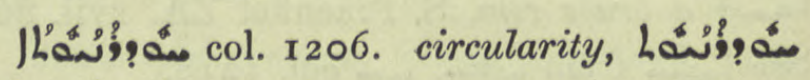

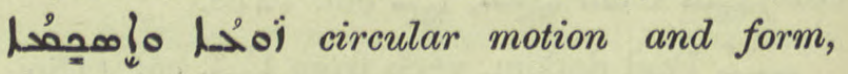
But. Sap. Theol. 4. 5.

مسب: col. I 206 should have P'thakha on the Mim, not on the Khet, as it is formed from the Aphel. revolving, causing to revolve,

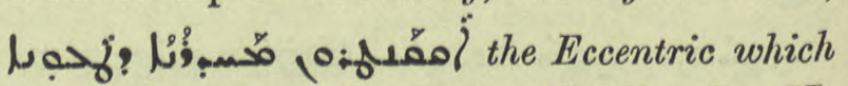
causes the epicycle to revolve, N. Hist. Bk. II, Cap. iv. 2. Pl. Pon onendicants, gloss to

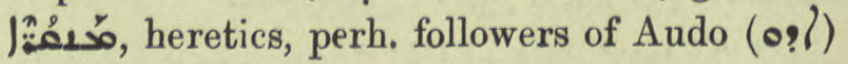
as he taught mendicancy, A.M.B. ii. ${ }^{5} 50$ ult.

col. 206 infra. Ar. مَّ to be or

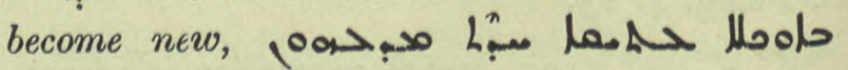
Ephr. ed. Lamy i 6r 9.

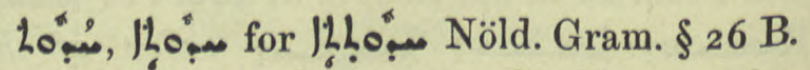
f. a newly married woman. Cf. Lexx. under مِّمُ col. 1200. 


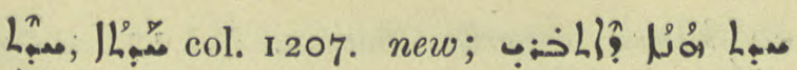
A. L he who is created anew is new, Nest. Hérac. 54.5 ; $1 L^{2}$ ?

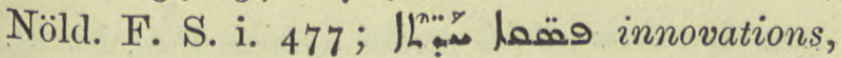
Sev. Ant. Vit. I I3. I.

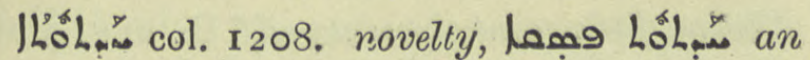
innovation, Pet. Ib. 79, Sev. Lett. I3. I 2 , I 4 bis, I 5 ter, id. Hymns 48. 3 ; pl. Lơ Lمبّ

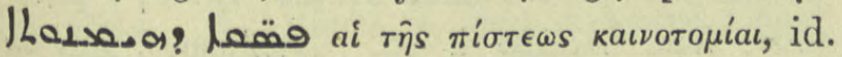
Vit. III. I3, II3. I2.

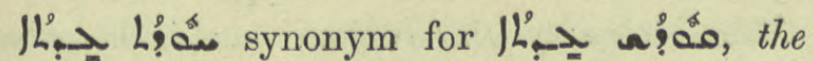
four weeks before Advent, Or. Xt. iv. 208, Ihe eighth Sunday before

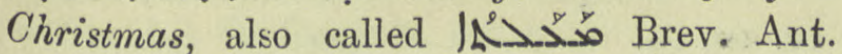
ii. 27 .

الم اللميثة. Ar-Haditha now Keshaf on the left bank of the Tigris about a farsakh below the mouth of the Great Zab, Hist. Mon. i. $95.21,13$ o. 16 and often, Pers. Mart. I78, I 90, 234, a bishopric Syn. Or. 619, 6, Chast. 34. I 2, 57. I I etc.

L'مُ an inhabitant of Haditha, M.Z. 212. 20 pl. مب: 2ib. 1. I9, 2 10. I3.

; a a city of Persia in ;anal, Chast. $5 \cdot 4$.

مa Pa. col. r208. With lowes to issue coin, Anecd. Syr. i. I 9. I. Chem. with lق) to act superficially / lasoas $>$ am to penetrate deeply, Chimie I. 5 and 6 af.

L.óåx col. I 2 10. Logic : demonstration opp. induction, Probus 101. I 7 .

1 a serpent, as the salamander, Anecd. Syr. iv. 75. 23 ; the lizard, B B. under |⿳⺈⿴囗十大 col. 2584 .

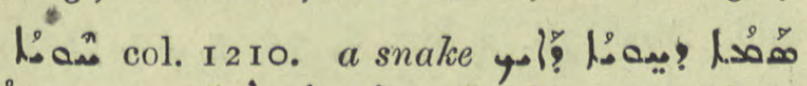
L is, in my opinion, gentian, Med. 264. I6.

pl.m. Ophites, serpent vorshippers, Coupes ii I I 7. Cf. مُّهُ.

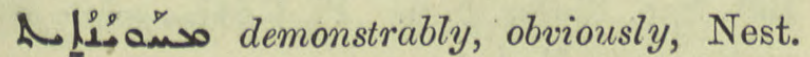
Hérac. 79. I4, 449. 19.

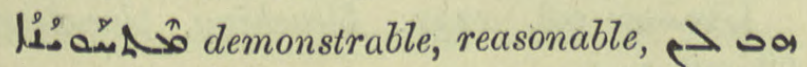
Sev. Ant. Hymns 293.

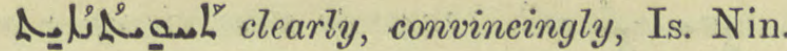
B. 8o. 6 ; BB. under / إب col. 1560 .

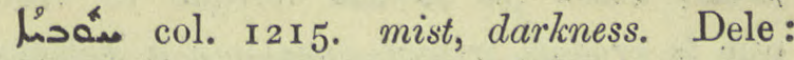
it is given col. I 172 .

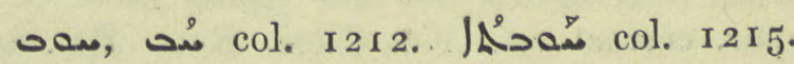
add : a defeat, rout, Josephus vi. S. Iо, I3. I 2.

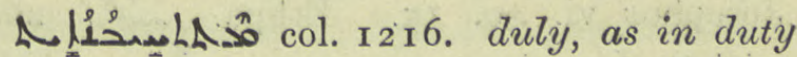

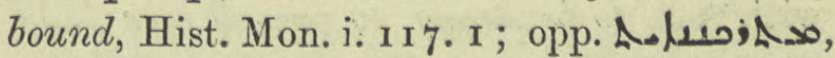
Syn. Or. 140. 9 ; ib. 13 o. 6.

9. col. 1218 . Correct: Rhamnus infectorius; it grows on the mountains of N. Arabia, has small black berries of a harsh flavour; in Khorasan they are eaten boiled:

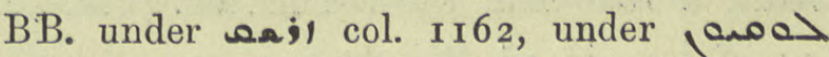

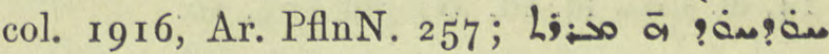

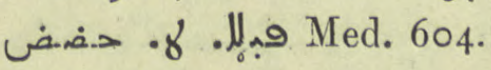

adinterj. stronger form of aol $\mathrm{Oh}, a h$,

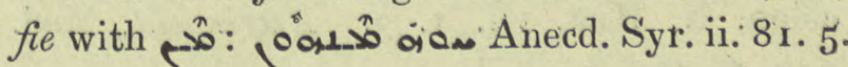

مa pr.n.m. Stat. Schol. Nis. I89. 3.

مده col. I 2 I9. sloe, blackthorn, add ref,

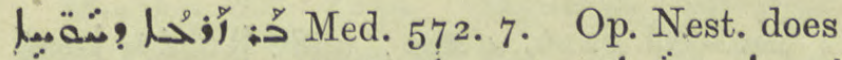

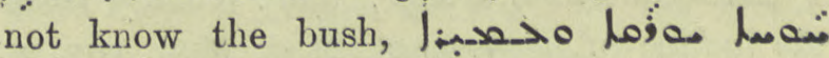
I38. 6 .

infor Hutir, a village, Chast. 2 I. I 9.

y articles.

L'áso f. weaving; texture, BA. under J Lo: 1 h col. $226 \mathrm{r}$.

مa col. I221. in the mountains N. of Damascus. Nöld. identifies this with Helbon הלבון Ezech. xxvii. 18, ZDMG. xxix. 436 .

مa col. 1222 . Dele: it is a mistake for col. 1219 .

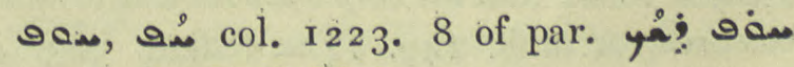
shampoo thy head, Med. 554. 10.

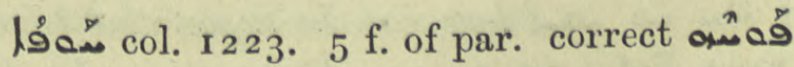
His breath; "even that which is born from our bosoms is not, save by the breath of God"; see Gen. ii. 7. 
jos, col. 1224. to close, tie up a pot, Chimie 36. 8; to become thick, to thicken 10 orsque tu verras que le produit trituré commence à s'épaisser sous le pilon, ib. 22. 21. Pass. part. 18 of par. correct ref. Not Clem. Rom. Rec. but Clem. Ep. 80. 8, add I76. 4. Pael مص pass.

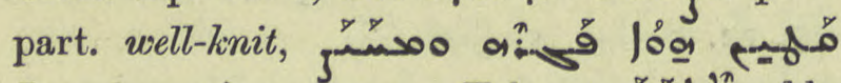
Josephus vi. Io. I5. Ethpa. with "خ to be strong, to prevail over

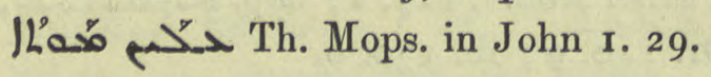

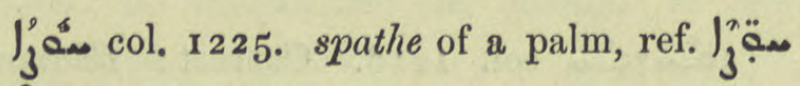

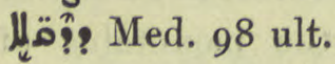

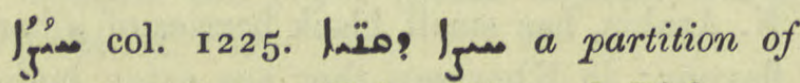
reeds, a reed mat, Kal-w-Dim. ed. Bick. 40. I 4, cf. J مo id. ed. Wright I 79. 10.

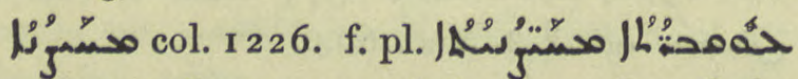
the contractile or constricting muscles of the colon, N. Hist. vii. 1. 3. Astringent, Med. 212. $6,293 \cdot 3$.

Lija a village of bano; tw Chast. 62. 13.

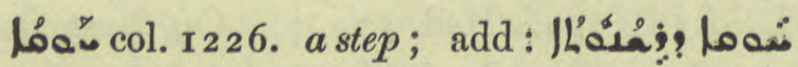
the ladder of preferment, Hist. Mon. I67. 3 ; ib. $174.9,270.20$.

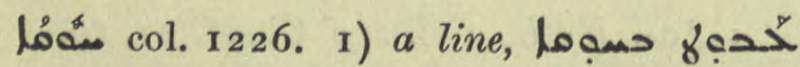

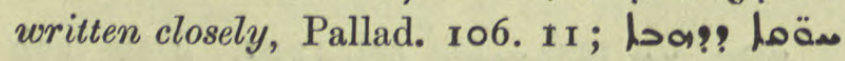
sentences illuminated in gold, Is. Ant. I 26.12 , مentences of Holy Scripture, ib. 1. 14 ; Nars. ed. Ming. i. ro n. 1. Metaph.

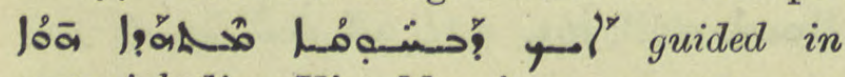
a straight line, Hist. Mon. i. I 74. 9; 270.20 ;

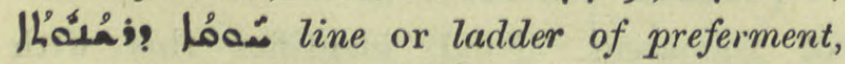
ib. 167.3 .2 2) for مó a pitcher, pot, the

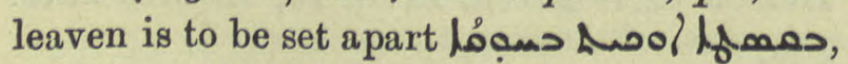
Takhsa I18. 2.

; مُ2 col. I 226. to look, to take a direction,

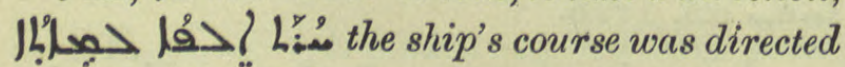
towards, A.M.B. 149. $14=$ Pallad. 323.4 ; Budge "was about to arrive". Act.part. add:

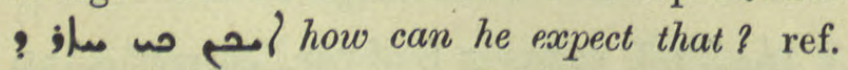
Ephr. Ref. i. 65. 23, 24. Perhaps i. q. Ar. حار

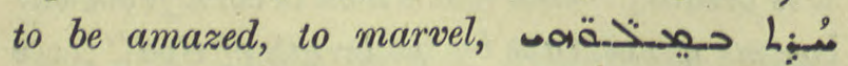

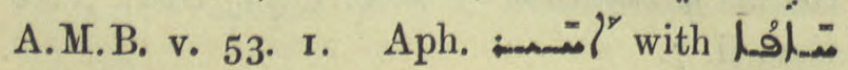

to hasten eagerly, Sassan. 7, I7, 7, 7 \%

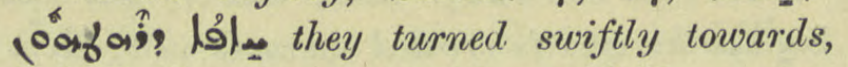

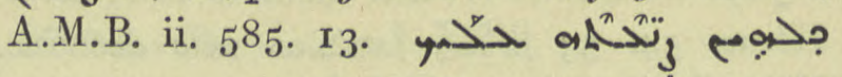
دب he turns all his guiles against thee, ib. iii. 222. 10,277 10; بـُ $I$ bring judgement upon myself, ib. 278. 5 af.

; مa Pa. معة col. r229. Chem. to blanch,

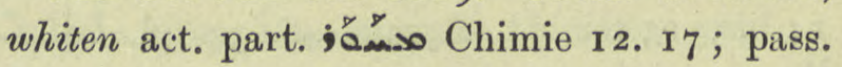

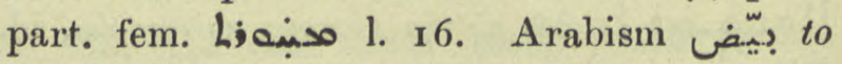
wear white in token of opposition to the Abbaside party whose colour was black, مa fin Dion. 51. 3 af., 52. 1, 3, Nöld. WZKM.

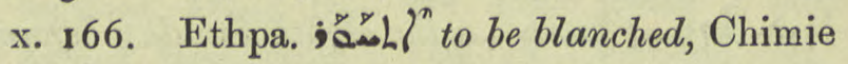
12. 10,14 .

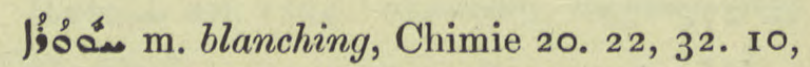
47. II,

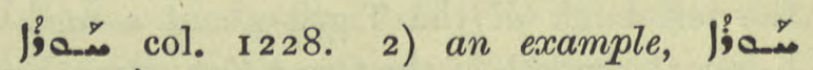

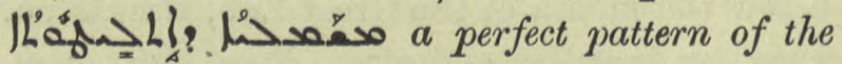
heroic life, A.M.B. v. 3. II ; archetype Jja. (2) BH. Hebraica iii. $25 \mathrm{I}$.

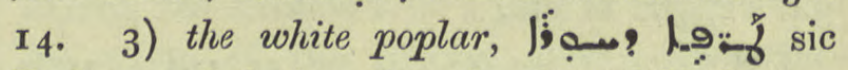
Budge, an infusion of poplar leaves, Med. 575 . I 7, 582.6 .

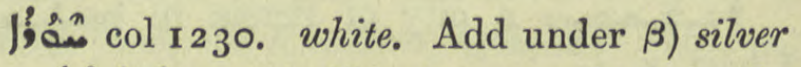
opp. لم gold, Chimie 12. 4 but tin ib.

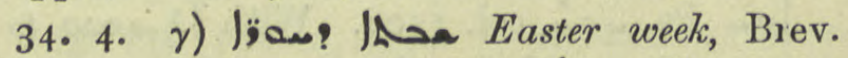
Ant. i. $3^{8} b$ pen., $48 a$ pen. جماُجُ Feast of the Mongols, Jab. $1_{42}$ ult. It is Feb. I, the Mongol New Year's Day, Bedj.

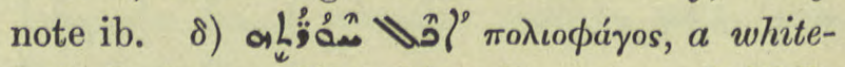
haired glutton, Hist. Mon. ii. 34. 27 , A.M.B. vii 86 \&c., Act. S. Pelag. 7. 27, 8. 6. M. pl.

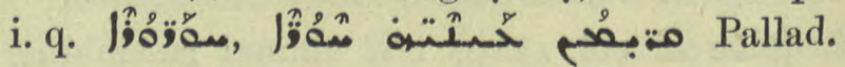
352. 5, Med. 559. r. See 3 ll. lower.

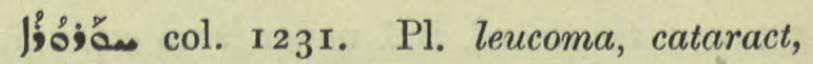

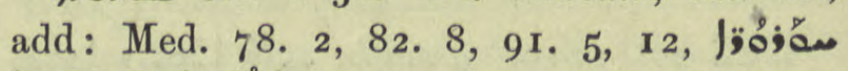

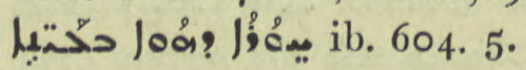

L'ớ: col. I231. a white cloud or mist

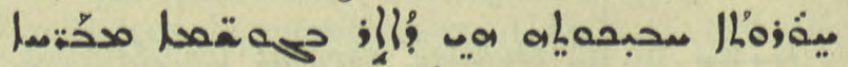

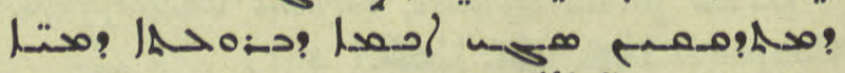

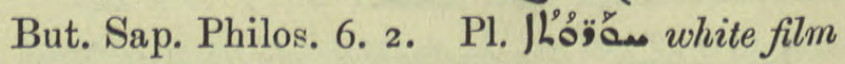
on the eyes, Med. 89. 9.

مَسْة col. 1232. bleaching, blanching, Chimie I. I.

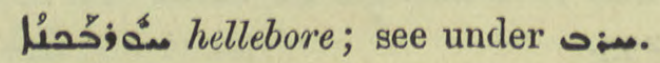


Rias

مُّم col. I 232. a district under the Bishop of Sarug, add refs. Brooke's Chron. 573. 8, Ant. Patr. 3 о r. Io.

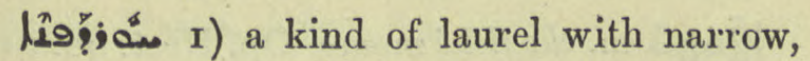
bitterleaves, note to Chabot'sYoussefBousnaya, R.O.C. iii. I73. 2) حردفنين ,حردفنيم perh. mountain hollow, Hordaphne village in the land of Amadia, C.B.M. ${ }^{\circ} 66_{7} a$, Pers. Mart. 1. I544. A village in Beit Arabaye, Chast. I5. I, 32. $2 \mathrm{I}$.

مـن an inhabitant of Hordaphne, C.B.M. ${ }^{1067} a$.

مarin (1. Haran = Carrha) De Goeje B. 65. I, 4 .

I of Amadia, Anecd. Syr. ii. 210. 6, 286. I4, I. 1 .

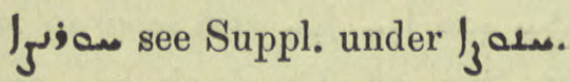

E-Syr. form of مدa Hashaba pr.n.m. BHChr. Eccl. 785 . 5 .

(? above.

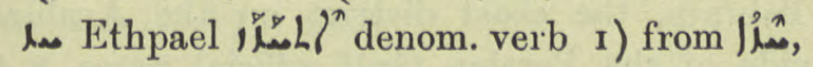
to penetrate, BH Carm. I37. 14. 2) from lile to be mangy, covered with scab, Poet. Syr. 58. 5 af.

מُ col. 1237 . infra, innermost recess, add :

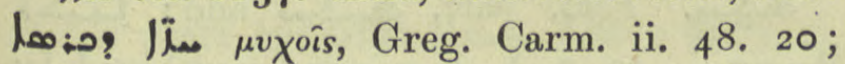

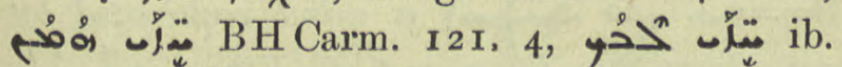

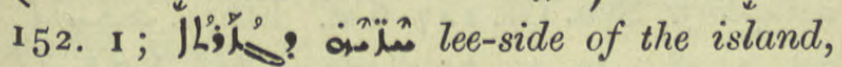
A.M.B. iii. $3^{8} 3$. 1 .

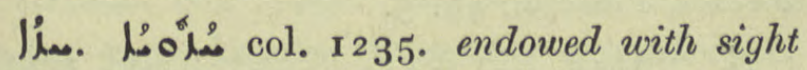

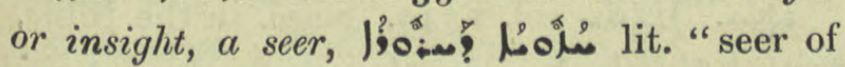
the opening", the retina, back of the pupil, Med. 7.x. 6, 6 . 6.

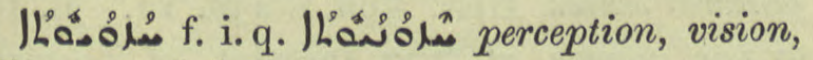
Is. Nin. B. 50. I5, 18 ,

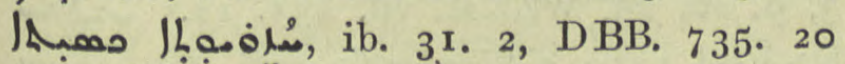

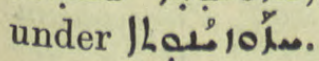

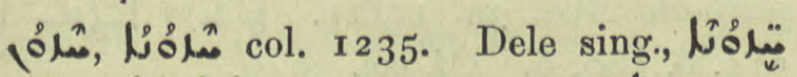

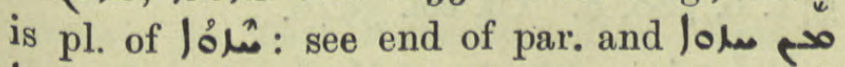
مدرة BH Gr. i. 31. I, Nöld. Gr. § 74 n.

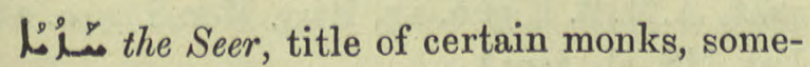
times considered a gentilic from lin Hazza.

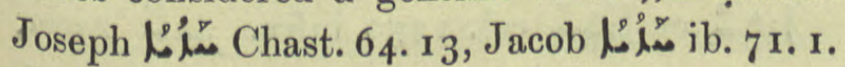
See Chabot, Journ. As. 1906, 265.

Lيُ col. 1236 . Metaph. perception, mental vision, Is. Nin. B. 128. 3,4 ; spiritual vision, 1. 6.

LLás col. 1236. a) act. vision. b) pass.

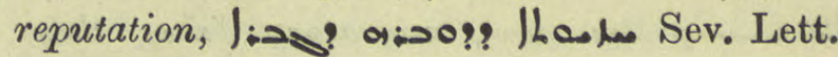
377. 16.

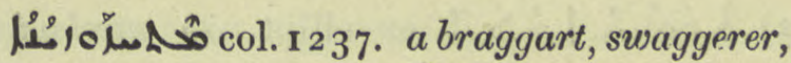

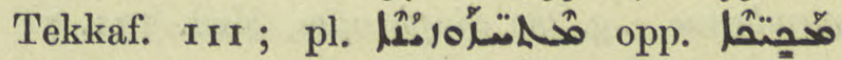
Is. Nin. B. I 89 n. I ; Syn. Or. 147.6 af.

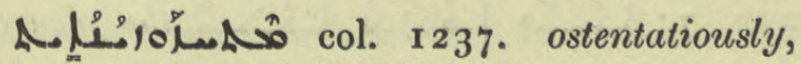
Syn. Or. 137. 29 ; R.O.C. iv. 262 . I.

| ö $\pi \iota \theta 0 \phi a \nu \hat{\omega} s, a$ backward look, A.M.B. v. 355. 11 .

L $\hat{L}^{\wedge} \mathrm{m}$. perh. prow of a boat, Gest. Alex. 205. $x$.

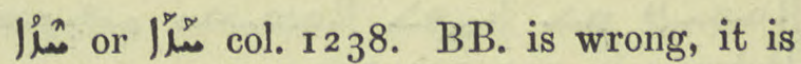

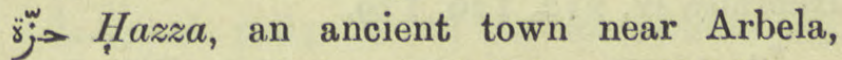
sometime capital of Adiabene, Nöld. ap. ZDMG. xxxii. 401 f., Tabari 20 n. 4, Syn. Or. 6r9. 5, Jesus-Sabran 490 n. I, 5 I6. 2 I, 519. 28 , A.M.B. iv. I34. 12.

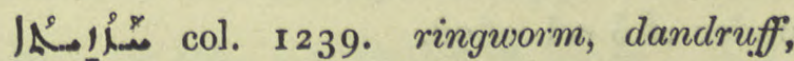

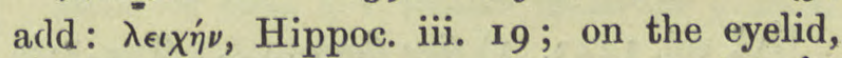

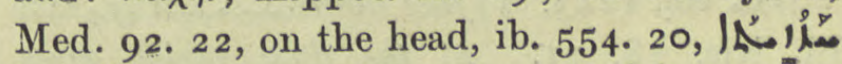

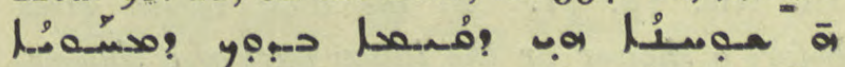

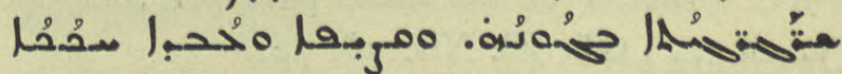
ringworm is a sore which may occur in any part, it is of the colour of sparks, it is irritable and itches, ib. $604.8 \mathrm{ff}$., Fet. Ib. 40. 1 I.

e. name of a village, Anecd. Syr. ii. 199. $11,144.24 ; 203.23$.

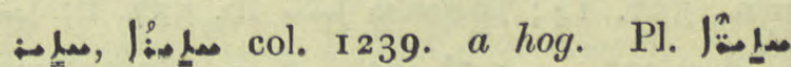
scrofula, Med. 5o. I i.q. 12 ميُ2 ib. 594. 6,

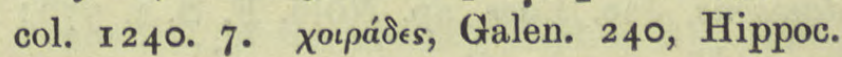
iii. 25 .

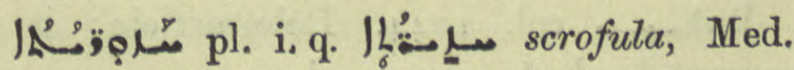
$583 \cdot 5$.

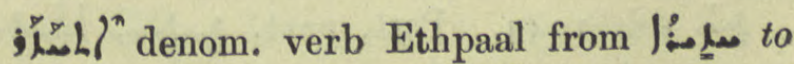

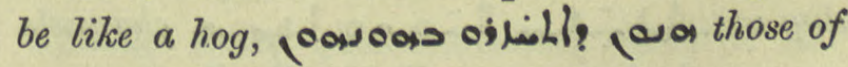
swinish minds, Manichéisme 98. 7 .

ماُ col. 1240. Arab. حزمام belt, girth, Beit Assyr. iii. 81. Cf. Fremdw. 103 n., El. Nis. 40.5 . 
o col. 1240. to cause a commotion, to incite, مnhac, Jac. Edes. ap. Ephr. ed. Lamy iv. $3^{63} .5$.

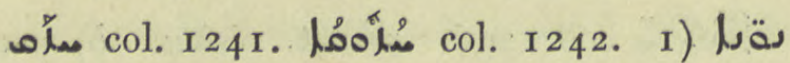

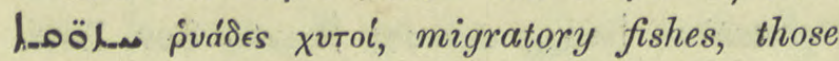
which go about in shoals, Ar. FischN. 89. 2) مo or ellipt. an enveloping membrane e.g. the diaphragm, Med. I31. 7 ; إحخا

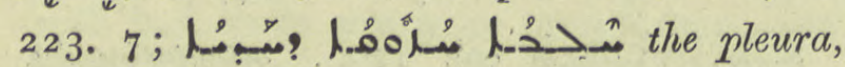
ib. $223.2,225 \cdot 4$.

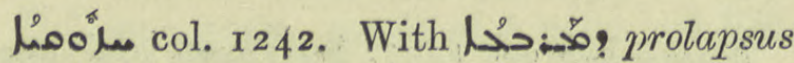
uteri, Med. 50. 22.

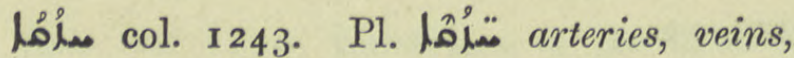
معبدهم: Bم. B. under مدرقيk

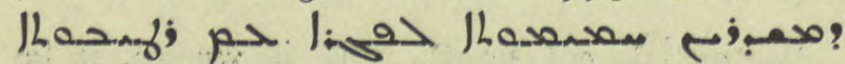
Hebraica iv, $212,120,129$.

iُ the Khaser, a tributary of the Zab, Bar Penk. I57. 12.

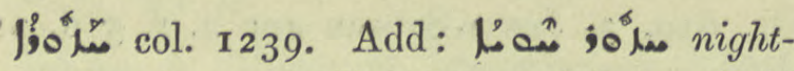
shade berries, prob. Strychnos colubrina, BA. under مै col. 3698.

الدِقيـق السهيد col. 1243. I) fine flour, El. Nis. 78. 71. 2) the dust which rises before a shower or the latter part of a shower, القف.و مـ السيل El. Nis. 78. 72.

ojh Hazru, a village on the road from Maipherkat to Amid, Dion. 54. Io.

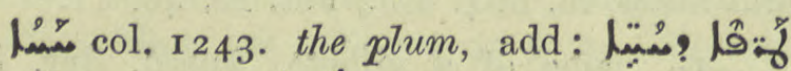

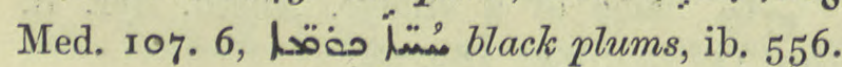

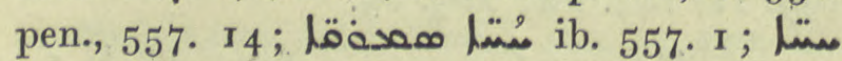
, بـa damascenes, damsons, Jac. Edes. ap. Nöld. F. S. i. 576 ; منتم פم: مeر peaches, ib.

j;0Acol. 1244. Dele article; it is corrupt for J;0/ s. v. col. 3347.

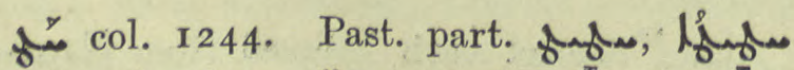
dug out, 每 channels excavated for water, Nöld, F. S. ii. 894 . Pa. ${ }^{2}$ to engrave, Poet. Syr. 98. 7

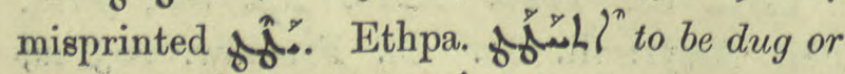

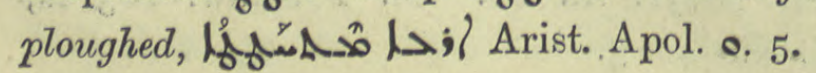

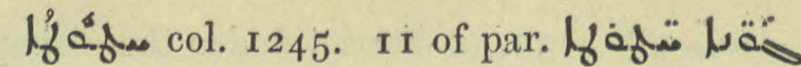
لمقئ striped, rainbow-coloured, A.M.B. v. 188. 9 .

Ifof m. the field mouse, Op. Nest. 90. 2 I.

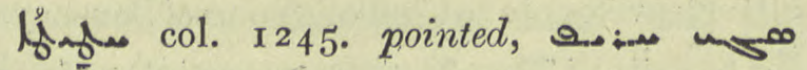

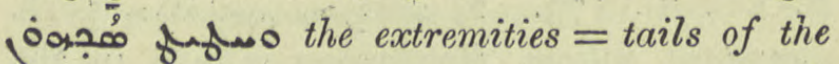
roach and scorpion are sharp and pointed, Med, $25.2 \mathrm{I}$.

L'of st. pointedness, stiff sharpmess of the tip of a palm-branch, Pallad. 666. 19.

fo col. 1 245. Ethpe. undolin pass. to be sinned, a> nopli? lask the sin committed by him, Sev. Lett. 295. 9, 296. 5, 8; pl.

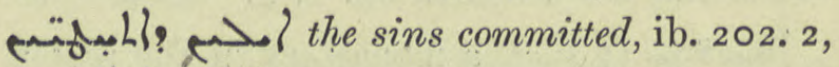
203. 5 \&c.; Cyr. 35. I3.

مدمُ col. 1247 . 3. correct "caret pl." to

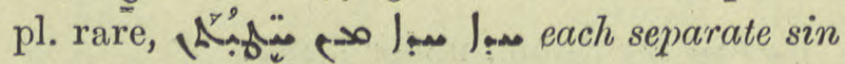
of ours, Ephr. Syr. ed. Overbeck 329 pen. $=$ Ephr. Jos. 260.5 ; ZDMG. lii. I 32 .

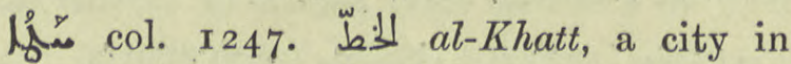
Bahrain, the coast district on the Arabian side of the Persian Gulf, official name : Syn. Or. 216. 23; 679. Add:

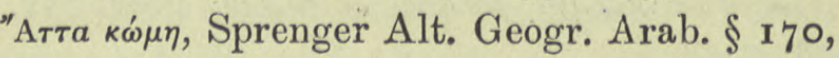
Sassanidi 36.9 .

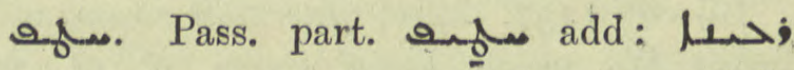

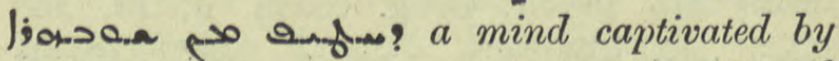
vainglory, Syn. Or. x 47.6 af. Ethpe. col. 1 248. to go headlong; y'? Nafohl? to come, Pléroph. 46.3 .

مـ مa col. r 249. masc. in the Bible, fem. A.jl? lea in the vernacular, Apis. م- ult. and note, $\varsigma$ note. Pl. $\int_{6}$ pastoral staves, Hist. Mon. i. 236 . 12 . L. 18 of par. sceptre, cf. חטר Inscript. of Zengîrli $3,9,20,25$, WZKM. vii. I3I. I2. مع Lُخ' col. I 250.3 Shepherd's staff, Polygonum

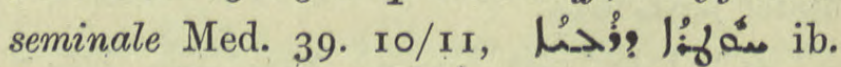
572. $6,580.6$. Cf. col. 3946 .

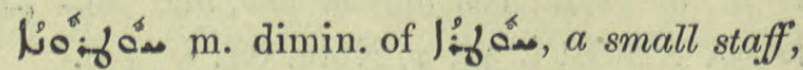
stick, A.M.B. i. 506. I 5, Hist. Mon. i. 340. 2 I,

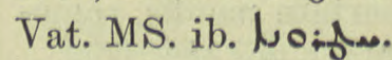

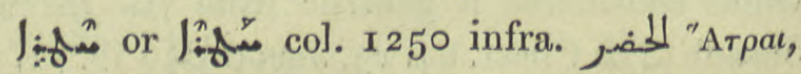
"A $\tau \iota$, "A $\rho a$, Hatra, a city in Tirhan, NW. 
from Tekrit, Coupes ii. I I I f., WZKM. xii. 36r, Pers. Mart. n. 1440, Hist. Mon. i. I48, I73. 7 , ii $3 \circ 5$ n. 5. I.q. I:fمه. Other Ḥatras were in Marga, Athor, \&c.

مُهُ col. I 250 ult. Dele.

hin col. $125^{2} .2$ to be reviving, refreshing,

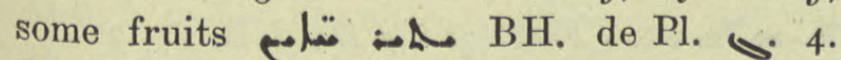
Imper. used as a salutation, مin Hail, Pallad. 495. I2, 13.

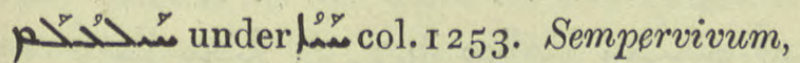
add: i.q. Gr. ol-il col. 3 and Pers.

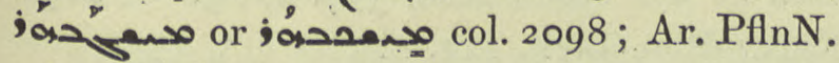
I61. Med. 139. 16, 563. I5, 598. 20.

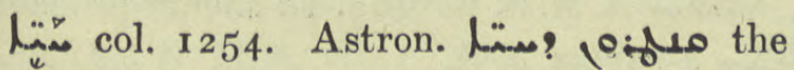
first of four spaces on the outer circle of the astrolabe, it is from the Eastern horizon to the 3 oth degree of the lower meridian. "In hoc loco vita hominum et spiritus continetur", Firmicus Maternus ii. xvii. 2, De

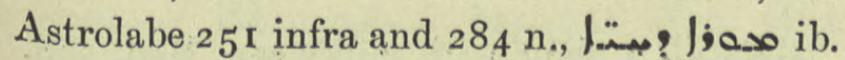

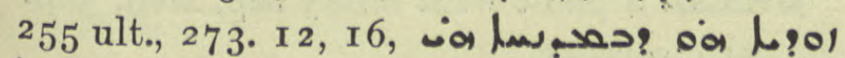

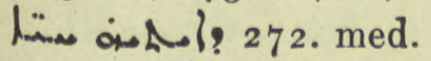

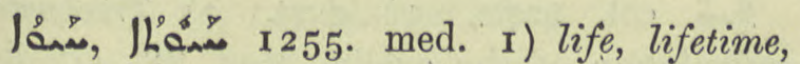
raosi? JLain, Syr. Rom. Rechtsb. 27. 3,

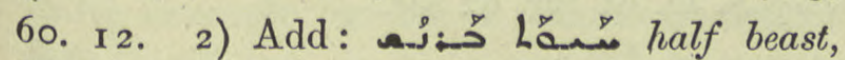
half man, Gest. Alex. 177. 5. A sheath, case, a pearl has Jij|g̣ / ILan, Natur 65. 3, 5.

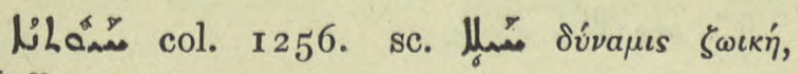
Hoffm. Herm. I73.

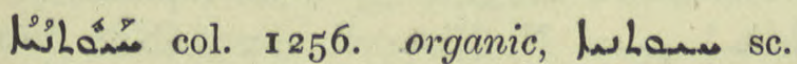

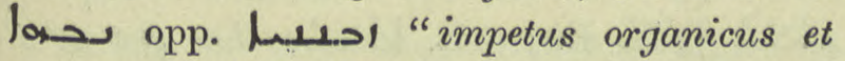
anorganicus", Hoffm. Herm. I73.

مسا ו"م م what measures should be taken to avoid lack of victuals, Ined.

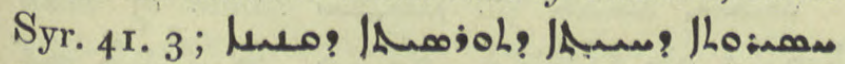
lack of fodder for the cattle, Dion. 193. 5

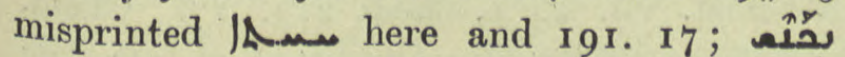

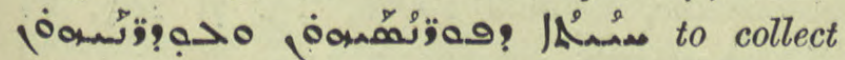
victuals for their sustenance, Hist. Mon. i. 186. 18. But Budge and Fraenkel think

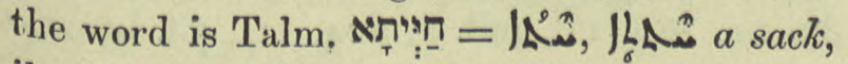
ib. ii. 368 n., ZA. xvii. 87 .

Whis a fortress in Mt. Singar, BHChr. Eccl. 633. 1 .

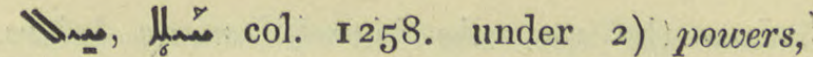

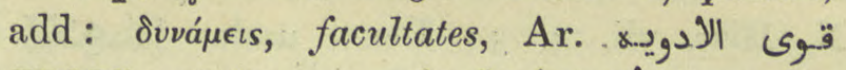
ZDMG. xxxiv. $475=$ =

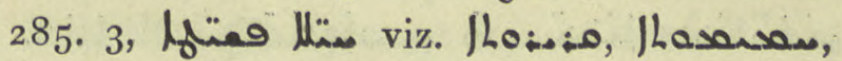
Jhara, Jlos.j; ib. 287. 2, 4, Gottheil in BH, de Pl. ib. xliii. I 24 .

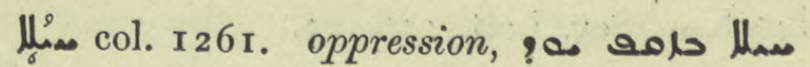
BH. in Koh. iv. $\mathbf{r}$.

"

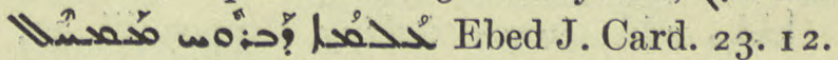

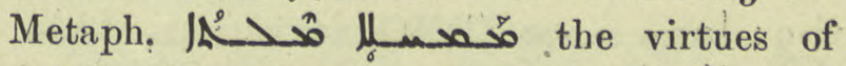
Aphthonia surpass speech, R. O.C. vii. I I 4. 26.

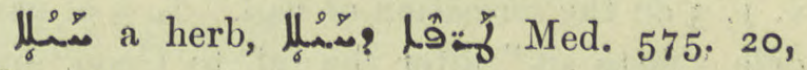
$5^{84} \cdot 2 \mathrm{I}$.

חילפסא Chald, willow, Med. 575. I7, 576. I. Cf. col, I 288 and col. 1289 .

14u col. I 264. Add : the spot where the

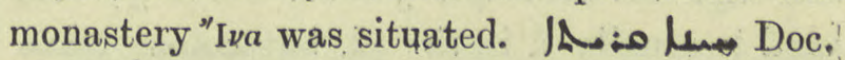
Mon. 215. 4, 219 quater, 220. Ir.

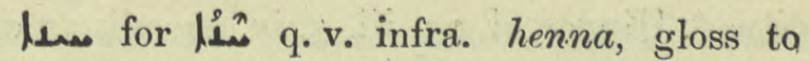
J:90 BH. in Cant. Cant. I. I3.

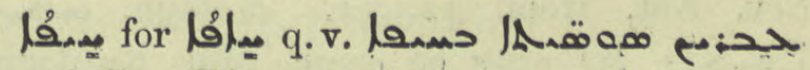
at a gallop, Dion. 57. 6.

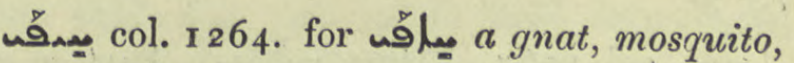
Mar Aba II 343 .

param King of Tyre, Jac. Edes. Hex. 2 I. 4.

مبشiran, eighth bishop of Adiabene, M.Z. 27 infra.

إي col. I 264 . I) a camp, sheepfold,

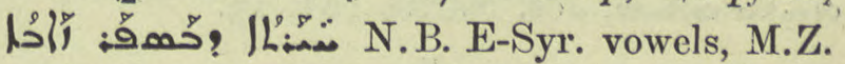
175. 18. 2) Hirta, capital of the Lakhmite Arabs SE. of the modern Meshed Ali, Hist. Mon. ii. 5 I n., Pers. Mart. 863 ; Sassanidi 9. $\mathrm{I}_{4}$, Chast. $43.6,20$.

A. معد.pl. people of Hirta, Chast. 44.

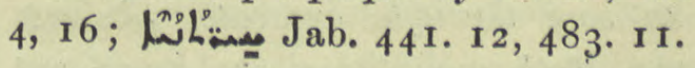

brass, Alexanderlied, ZDMG. 1x. I 80.86 .

مّ col, 1265. to scratch, rub. Act. part.

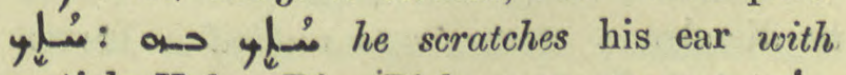
a stick, Kal-w-Dim. Bick. 5. 9 ; aranay 
دمقد N. Hist. vii. 4. 3 ; Lexx. under 12 을 col. 3641. Ethpe.

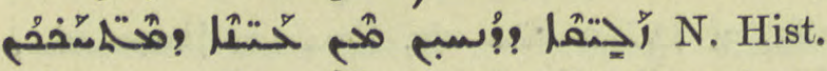
viii. 3. 3. Pa. مै to rub off. Metaph. to

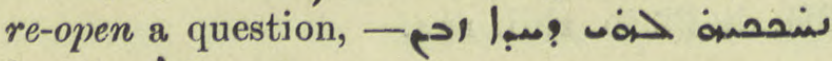

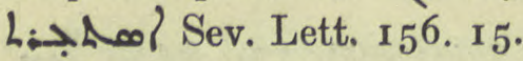

مُمْحمْ mange, itch, Rylands MS. 44 fol. $3 b$.

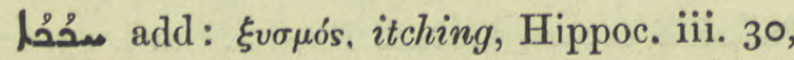

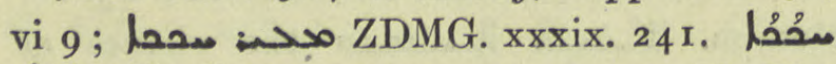
iإن slow friction in the air, N. Hist v. I. 3 on the formation of hail. L. 3 of par. dele "forte sit" after the ref. to Geop. and add: Urtica, the nettle, Ar. PfinN. 162.

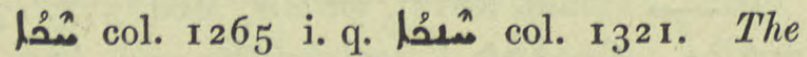
palate. Gen. com.ZDMG. xliv. 53 I n. I, e.g. مُ2ٌم Kardagh ed. $\mathrm{Abb}$ 48. 3 = ed. Feige 37. 12.

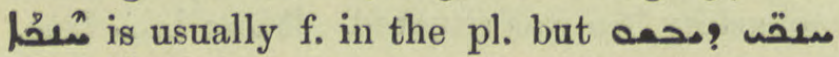
a 4 llo Is. Ant, i. 54. 17. The throat, gullet,

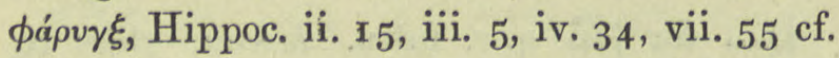
ib. 16 n. I. محني محمه open-mouthed, gaping, Sev. Ant. Hom. 42. I I. Metaph. يمحَف K'ais?: the jaws of error, Mar Kardagh. 75. 9 .

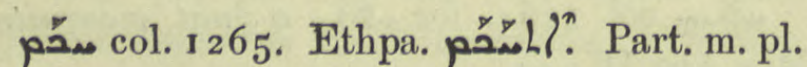

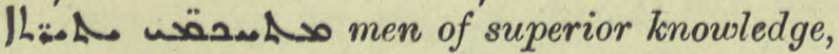
Georg. Arab. 9. 23.

|

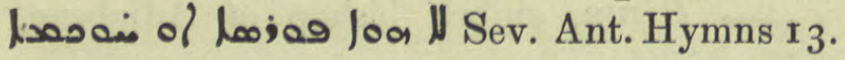

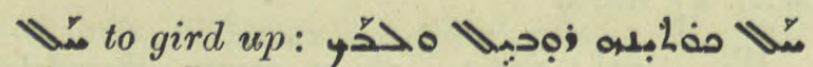

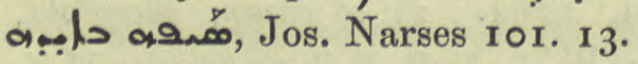

čcol. I 268. Ar. Al to mine, dig a mine, مosephus vi. I3. 7. Pa. waof ochey made a way through the throng and surrounded him, Pallad. 70. 7. Palpel همبحم to bore through, excavate, مon mountains are hewn out and dug through by gold-diggers, Is. Ant. ii. 82 pen. Ethpalpal "añ in to be loosened,

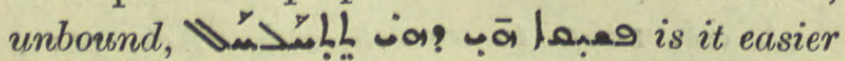
to dissolve a stone or to unbind a barren womb ? G. Busâmé $7 \cdot 3$ af.

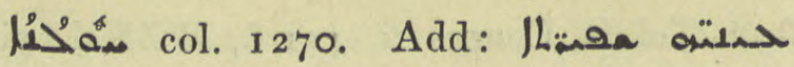

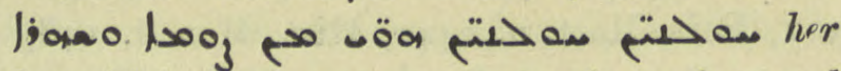
beautiful eyes were cavernous from fast and vigil, Brit. Mus. Or. 3337. $17 r$.

مخa M col. I270, a banquet, the Feast of Water, Coupes ii. 157. 12, 16, Feast of the Dead, 11. I 7, 2 I.

مفحم col. I 27 I. subst. a cavity, ventricle of the spider, N. Hist. vii. 3. I, of molluscs

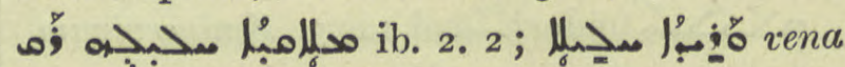
cava of the heart, Med. 337. 2, I 5. A ship, Kal-w-Dim. Bick. I 12.2.

مخديل A.M.B. iii. 603. 10 should probably a ravine, oleft.

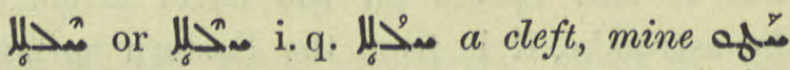
مُخدل لl Warda $5^{2} r$ but Aم: concealed mines, Isoyahb ed. Duval, 23. 2.

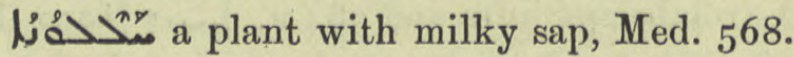
23 , perh. corrupt.

لـ col. I 272. I) dust. 2) Trichoma, granulation of the eyelid, Med. 88. 21 ; ح ib. 91 ter, 92 ter.

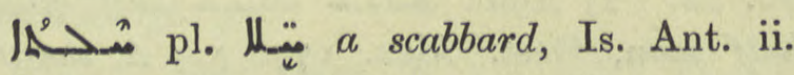
278 pen.

مّ see Hale.

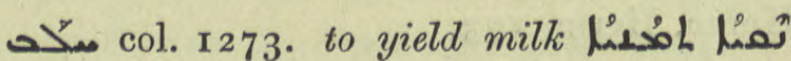

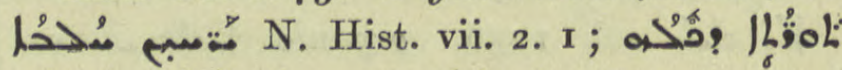

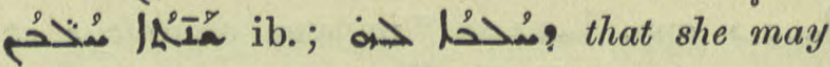
allow herself to be milked, Protection 17. 3 . Ethpe. C.B.M. $1008 a$.

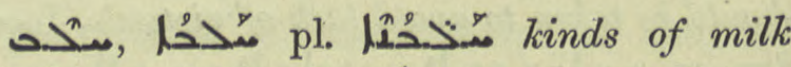

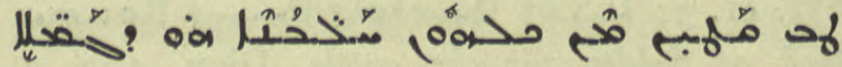
N. Hist. vii. 2. I, Med. 211 . 17. Chem. quicksilver, Chimie 1 2. 6, 13. 7, 267. I ;

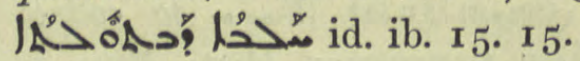

J0: مמخد م: opopanax juice, Med. 49. 6 , 87. II, I86. II, 398. 8, 604. II. Cf. 1000:0. col. 1627 and $/ ; 0^{\circ} ;$ s. col. 1630 .

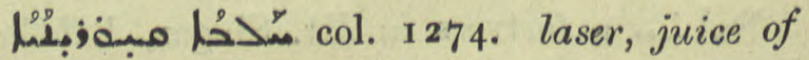
Laserpitium, Med. 99. 8, 166. 4. 


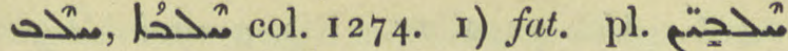

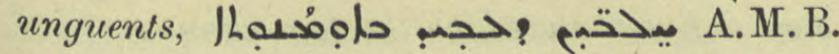
iii. 378 . Iо. 2) membrane, add: Hippoc.

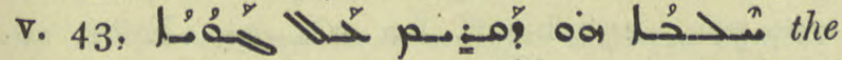

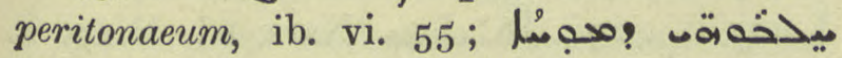

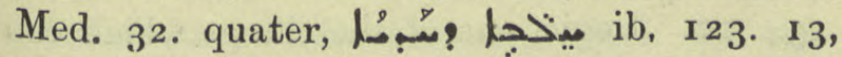

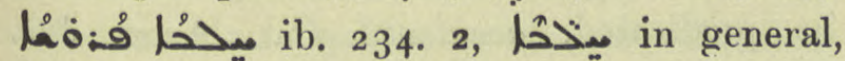

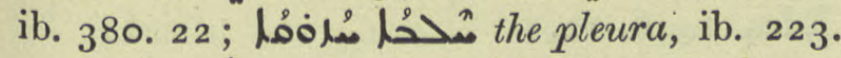

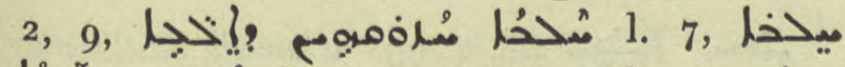

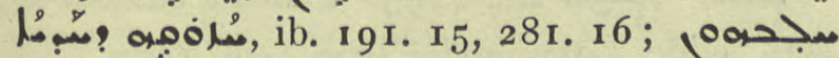
membranes of a bat, Anecd. Syr. iv. 69. I6; drum of a cicada, N. Hist. 4. 2.

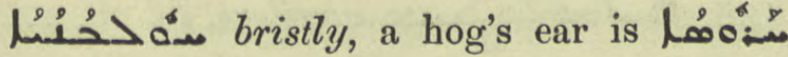

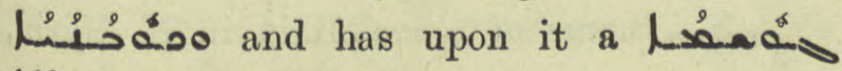
م. Hist. vii. I. 4.

هقا Anecd. Syr. iv. 69. 6, I 5 but Natur. 35. I5.

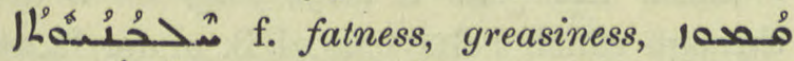

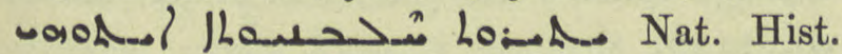
vi. $3 \cdot 3$.

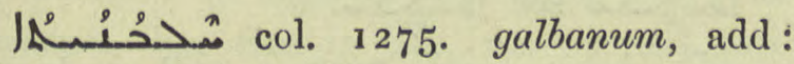
Med. I०5. I $5, \mathrm{I}_{47}$ bis and often.

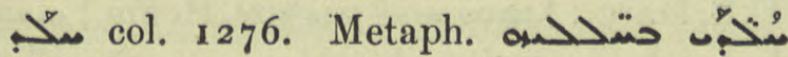
| $\boldsymbol{A}_{\mathbf{j}}$ ? those who burrow in holes = anchorites,

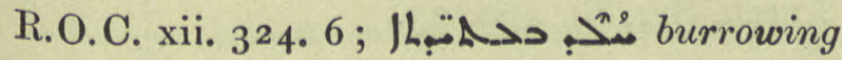
into the future, Hist. Mon. i. 245. Pa. מصح

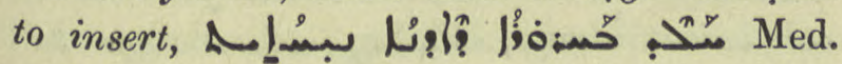
I08. 2.

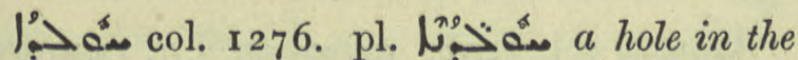
earth, ZDMG. xlix. 6 I 5 n. r.

$a-0$ a city near Babylon built by Nebuchädnezzar, Jab. I42. I2.

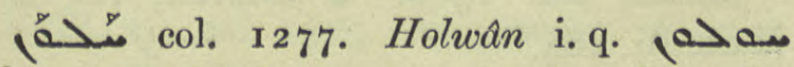
col. I22I. Modern name of מحس Syn. Or. 673; го9, 164, 21 4, ZDMG. xliii. 403. 6, 405. I, Journ. Sac. Lit. new series viii. I 6.

مخحْْْن coll 1277 . 1. 3 correct ref. An. Syr.

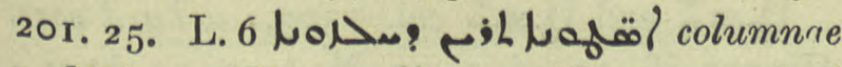
cochlides, cf. Fr. escaliers en limaçon; spiral columns i. e. those of Trajan and Antoninus, Zach. Rhetor's description of Rome, ed. Guidi 220 n. 2, 222 . 13.

مدجس col. 1277. Halah, pointed مدّجس 2 Reg. xvii. 6 \&c. Lee and Mausil. L. 6 of par. add: Syn. Or. 6 r 9 n. 5 .
Halhalah, a monastery, Chast. 2. 18 , 5I. 10.

col. 1277. 8 of par. To unite the Two Natures, Ephr. ed. Lamy ii. 607 n. and often. To infuse, مخله حم متمعه ib. 609. 3. Pass. part. مختمئ opp. monks living in community, R.O.C. vii. II5. I. Fem. محلمُ col. I280. a mixture? a drink like barley-water made of wheaten flour, Med.

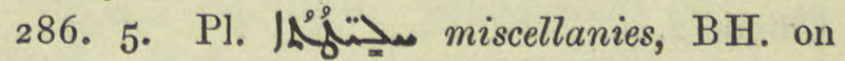
Prov. 25. 1 .

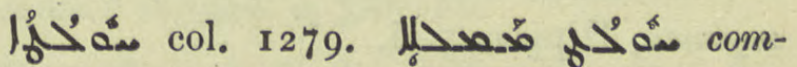
plicated or intricate discourse, Sev. Gr. under ملر col. 3578.

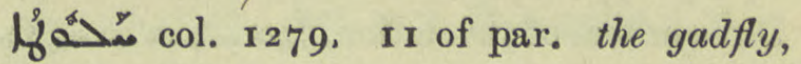
Dion. $59 \cdot 7$.

مُحْر Lyso:0 A $\mathrm{LOg}_{3}$ :0 Order of mixing and preparing Eucharistic bread, Takhsa I05. I, 8.

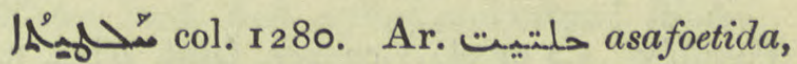
i. e. gum of Ferula Persica, Laserpitium, Med. 318. 20, 22, /20. sweet laserpitium, ib. 143. 13. Written ib. $172.10,562.7,566.16$.

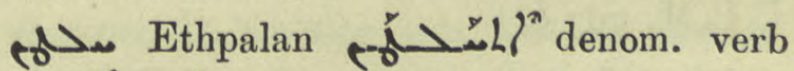
from مدئم: cc. o to hold intercourse, have dealings with, BH Carm. I I 4 pen.

م. col. 1280. Pa. u- correct the

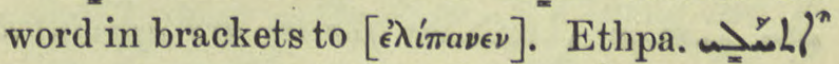
1. 2 of par. corr. to Laneos? BHGr. i.

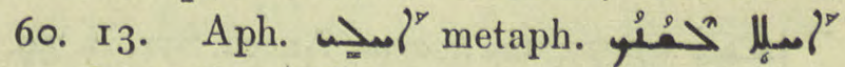
speak sweetly, Ahikar $\infty 5$.

مئم col, I280. must, Med. 59. 7, 295. 24 , 296. 12.

حمس اللا

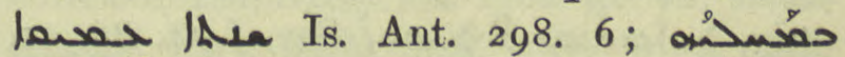

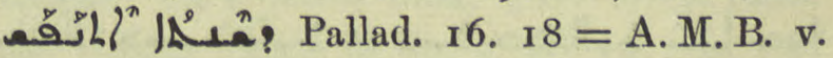
19. I.

مجلمA name of a mountain, Chast. 30. 9.

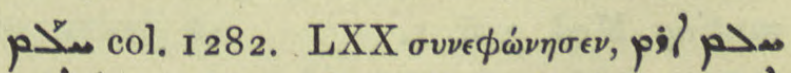
pigl Lal Hex. Jes. vii. 2 Psch. woha?.

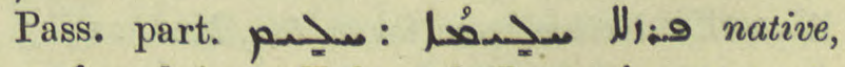
unforged iron, Ephr. ed. Lamy iv. 505. 2 I,

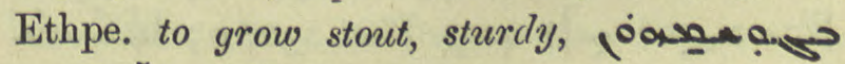
مكAم Natur 17.3 . 


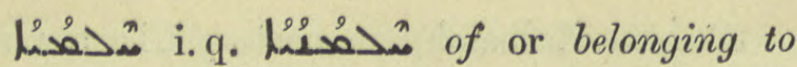

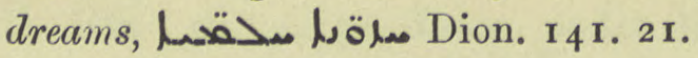

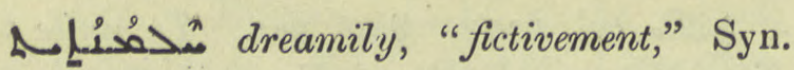
Or. 180.31 .

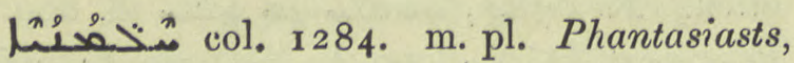
followers of Julian of Halicarnassus, C.B.M. $929 b$.

ميخحمُ col. 1284: f. anchusa, alkanet, Chimie 2, 9 .

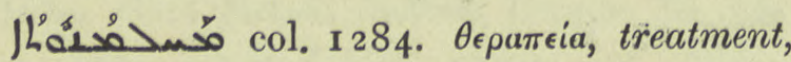
regimen, Hippoc. I. 6.

 2. 4. 2) made know'n in a dream, there are three orders of revelation, مئ مهلمخمد G. Busâmé 2. 9.

brine, white vinegar filtered, Chimie 4. I, 22, 2 I, 44. I 2.

فرز-وج plome kind of stone, Pers.

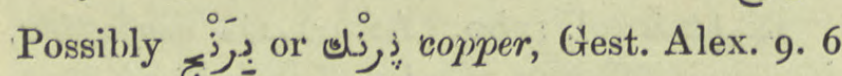
and note; ZDMG. xlv. 314. 3 af.

مخحص col. 1288. a blade, knife;

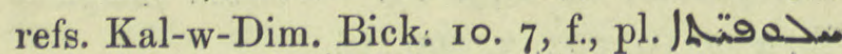
C.B.M. 993 b.

خليفة Ar. deputy, lieutenant, Dion. 208. 9 .

مُحه "סحم/ for we are appointed to the succession of the Empire, Jul. 16: 14.

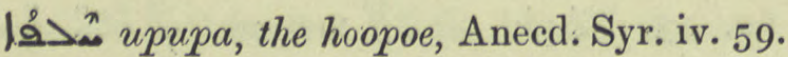

مُحُْ col. 1289. Corr. Salix Aegyptiaca, the Egyptian willow, Med. 579. 5, 584. I 5, BB. under us of col. 2597.

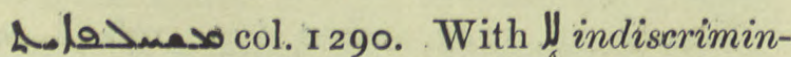

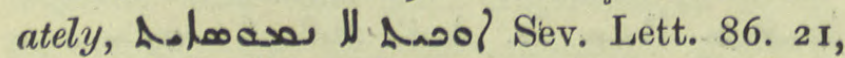
Joa لا معسخقامي إن ib. 195. 3 .

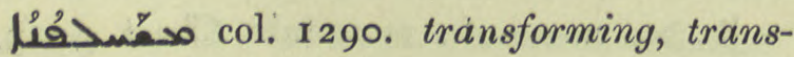
muting, Med. 346. 1.

مَّمْحص : col. 1290 . change. Add

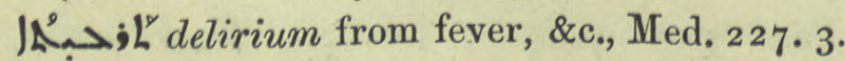
Conversion, Ephr.iii.334 a; axostasy اaم مه مa

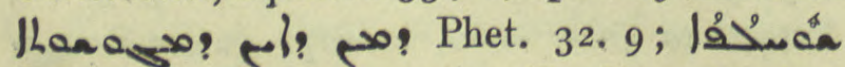

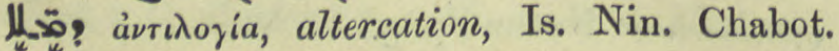

14. 6. Col. 1291. I7. Rit. a variable part; also a hymn in which the tone changes, Brev. Chald. I. 82. 1 2, 96. 8, 97. I, 98 pen., 107 pen., 298 bis ff. لمه محف مه Lamy ii. 429 ; la مars. ed. Ming. ii. 134. ro. Logic, one of the things which can be predicated, accident? Cat. Arist. Jac. Edes. 24. 6, I 2.

مخد col. r293. a small bladder or skin, add : Lexx. under fos col. 1607 .

$1 L_{0}^{\circ}{ }_{5}$ col. 1293. spoliation, Sev. Lett.

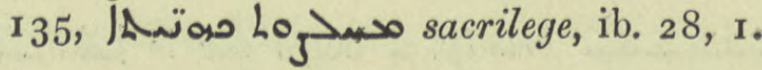

م. Dame of a mountain in N. Syria, C. B.M. $756 b$.

مُخحم col. 1294. Ethp.

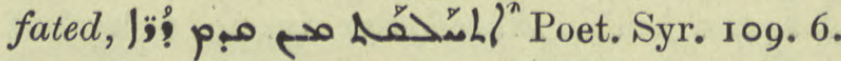

arge ring on a door, through which bolts pass, BB. under col. 1147 .

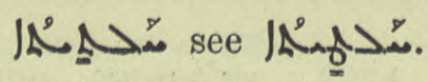

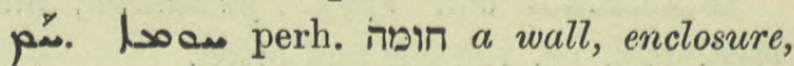

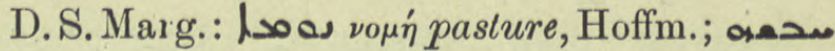
حسه owl they shut him up in the pigsty, Anecd. Syr. iii. 230. 23 .

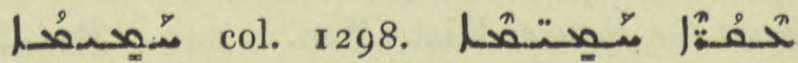
calefacient drugs, Philox. ed. Budge $2 \mathbf{I}$; abbrev. $\overline{\text { a }}$ BH. de Pl. a. 5. 6. and passim.

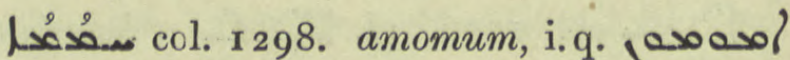
Merx ap. ZDMG. xxxix. $25 \mathrm{I}$, Lag. Agathang. 154, ef. Mitth. ii. 356; Med. 49. 8, 51. 6.

| Mart. iii. 264.

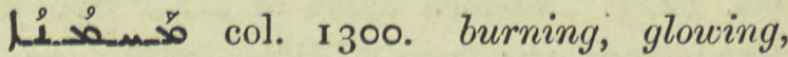

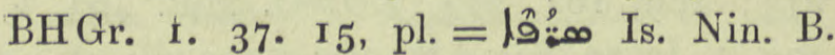
187.7 .

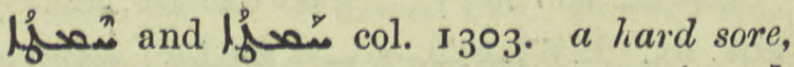
Hippoc. iii. 19, vi. 1, 32, Med. 77. 2 1, مُ0ّر إلإ همب: ib. 127.4 , Galen. 304, Jos. Styl. 21.12.

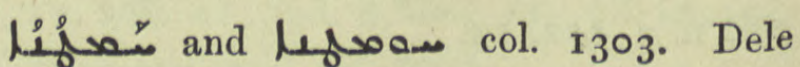
propugnaculum murorum. It is Talm. חומטון saltpetre, Fraenk. ZDMG. lii. 296, ef. xlvi. 743 . 
\مَ col. 1303. to collect, pile up close. L. I5 of par. N Nowo Shen Sheol gaped wide and piled up, Act. Mart. i. I3 ef. هon a city overthrown by earthquake heaped up all her inhabitants within her, Jos. Styl. 28. 19. To draw close together, to contract,

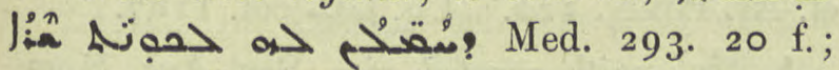

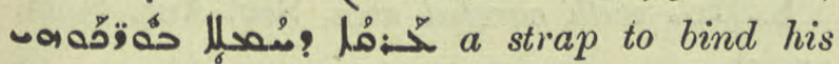
knees together, Hist. Mon. i. 218. 2 I. To be in close formation, form close, o'

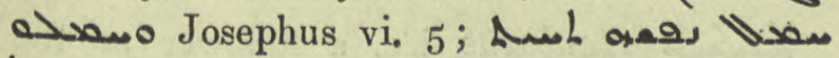
L.of: saint's beard, Chron. Min. 80 ult. $=$ Jo. Tell.

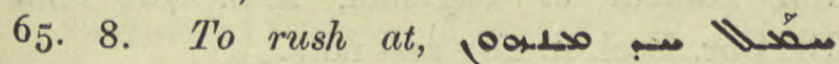
" إ) "il s'élance sur elle d'un bond," Chimie 245 n. I. Pass. part. "محئ some letters of the alphabet are معيم- compact, squat, opp. 22.

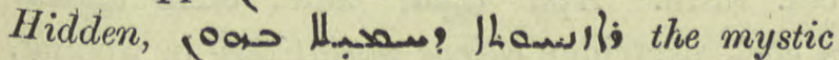
signification in signing the Cross over the

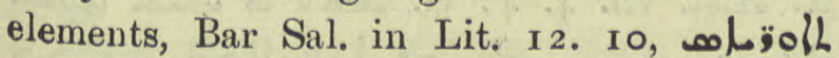

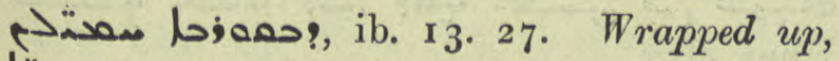

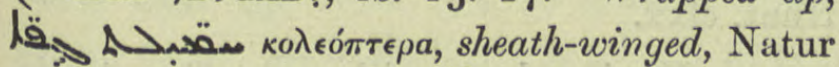

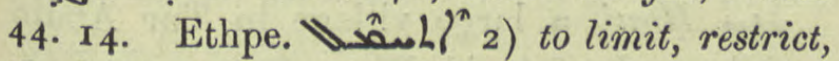
ل1. $\mathrm{Pa}$. Wh to draw close, draw up, a.u? Msains wad Manichéisme III. 6 ; the bear sُ squats close in his den, Natur. 8. 3. Aph.

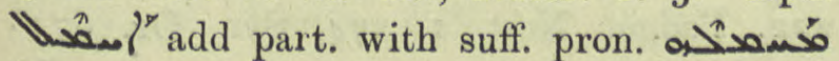
that which he has amassed, his store, $\mathrm{BH}$. Stories 18.73 .

Ihas col. 1305. compression of air in the ear, N. Hist. vii. r. 2 ; see l>a>f in Suppl.

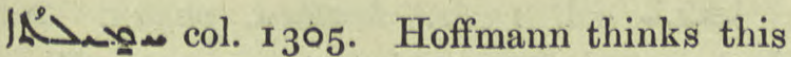
word North-Aramaic and Edessene while

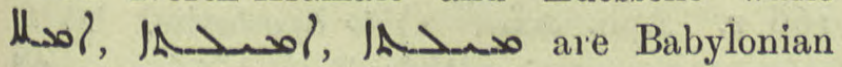
vernacular, Pers. Mart. n. 206, Ar. Fremdw. 93. Vullers thiuks Pers. 5 s. original, ZDMG. 1. 630. For western forms, $\kappa a \mu \eta \lambda \omega \tau \dot{\eta}$, Span. camelote, Engl. camlet see Lane, Dozy, and Vullers. Fringed rugs or material with soft, long hair, Hist. Mon. i. I $35 . \mathrm{I}$, ii. $280 \mathrm{n} .3$. 2716 مanم Chimie 25. $18,20$.

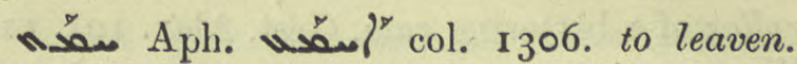

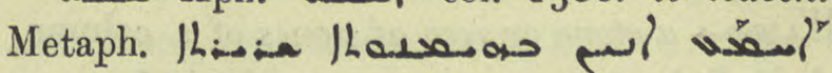
Bar Sal. in Lit. 27. 10.

1R

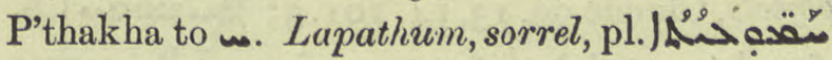
Med. $175.7,23$ 1. 4, 388. I I, 390 pen.

مَّم

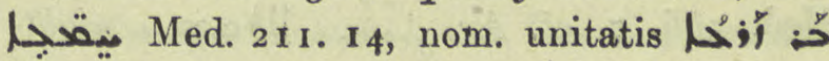

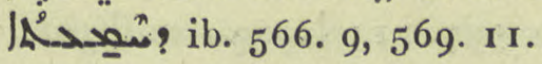

1R. Chimie ror. I.

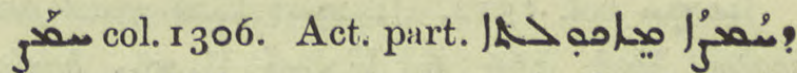
a disease which turns food sour in the stomach,

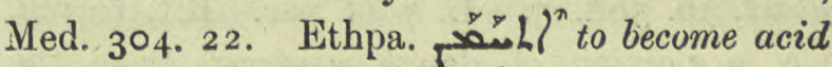
L

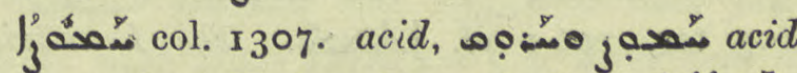

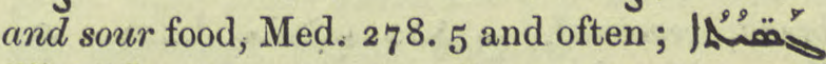

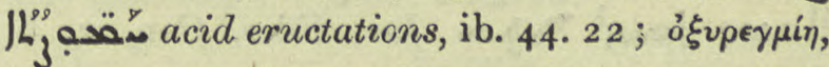
Hippoc. vi. 1. $3^{2}$. Bitter, briny, springs of

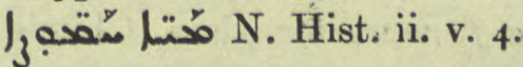

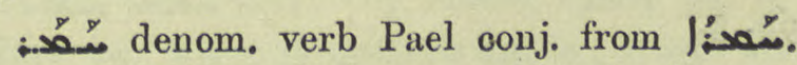
to ferment, Hochfeld Fabel 46. But the Brit. Mus. copy

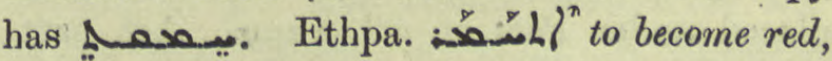
litharge and sand are to be left to cool in

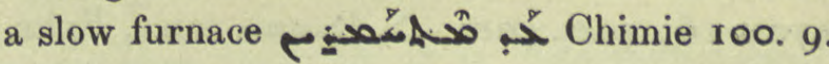

مُ pl. pines, BHGr. i. 30. 4 and trs. from ${ }^{2}$ on col. I 309 deleting the singular. Add : م مَّ pure wine, Hippoc.

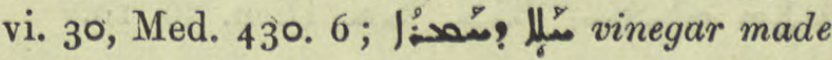
from wine, ib. $583 \cdot 3$.

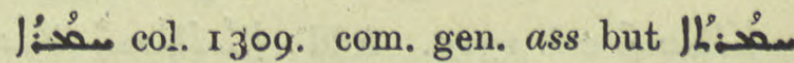
a she-ass, Kal-w-Dim. ed. Bick. 52. 18, مُخحُ

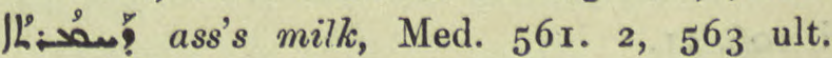
but ib. par. forms of pl., see BHGr. i. 28. 3, Duval, Gram. 62 quoting Ephrem's Comment. on Gen. xxxvi. 24.

Ar. Ar.sumen, Jew's pitch, DBB. 88I. 3 under J:ه

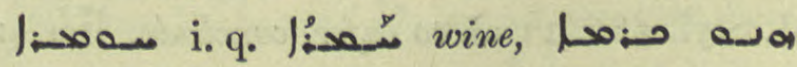
? Cyrillona in John xv, $58 \mathrm{r}$. 1 . 
Risas

130

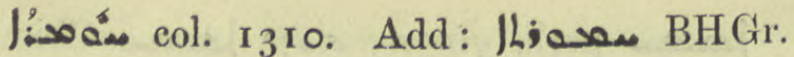

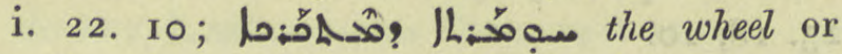
roller of a battering ram, Gest. Alex. IOI. II ; Ihison a stone or row of stones of a column, A.M. B. iv. 624. 15, pl. 1. 10, 625. 15 . Pl. J:- مـas glass beads or similar round objects, Chimie 99. I3, I 4, IOI. 5 ; IA 1.6.

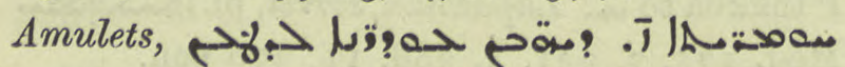

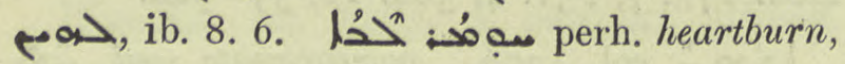
Med. 566. 7 , ef. Ar. مight-

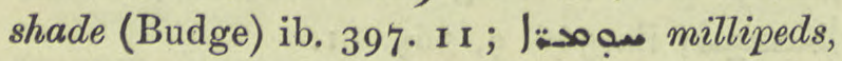
wood-lice, ib. 197. 8; links of a chain, Phet. I I.

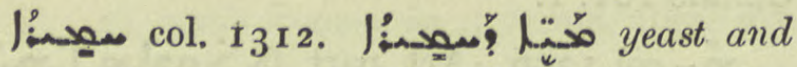
water, Med. 566. 6, مarley yeast, ib. 568. 5. L. 7 of par. Rit. leaven, i. e. a portion of dough reserved from the special baking for the Eucharist, Takhsa 105 ter, 106: ef. bread, reserved,

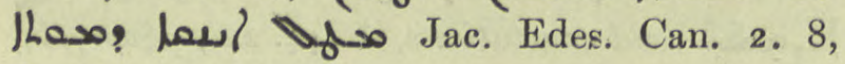
8.25 .

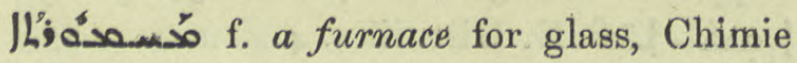
99. 3 .

مُ to be angry, impers. with $>$ and pers. pron. $>$ L מ placed under Pael on col. I 299. Act. part.

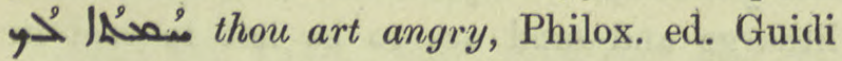

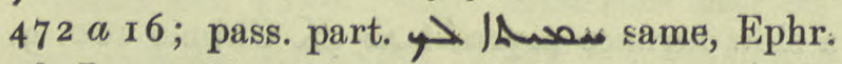
ed. Lamy iii. 485,575 , ef. col. r 299. Add :

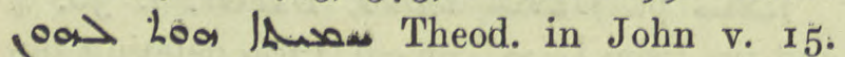
Aph. Aمa (i) to arouse wrath, excite fury, 10. 10.

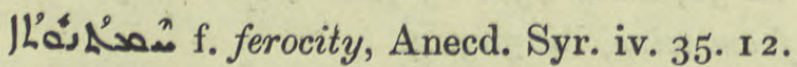

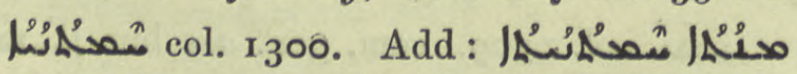
the irascible part of the nature, Patr. Or. iii. i. 106. 1 I.

مـ col. I3I7. 5. to suit the aim, to meet the need with $>$, the pool of Siloam aste ll

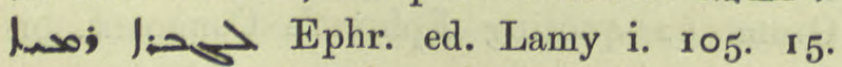

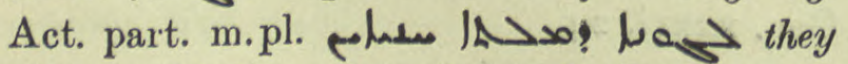
aim at highly coloured speech, Is. Ant. ii. 206. 2. Add : to conjecture, Jac. Edes. on J. Styl. 586. I I af. to infer, conclude, lîa es :Med. 132. 12 ; from certain symptoms

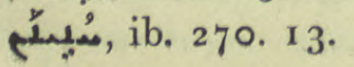

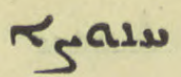

مئ col. I317. 5 af. the bosom, lap. Probably Heb. ZDMG. xxxii. 753 . Cf. Lag. Mitth. ii. $36 \mathrm{fff}$.

מُ Ar. Li Lenna, Med. 553. I3, 557. 12 \&c., 583.5 .

متر : unripe dates, D BB. 1334 under ef. Ar. PfinN. r 20. I.q. let col. Ir 67 .

Aُ with careful aim, Sev. Ant. Hom. 25. 10.

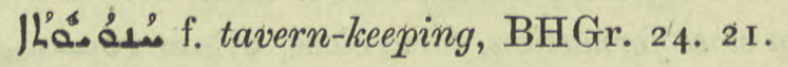

مُدحُخحُر Hanbalite i.e. a follower of the fourth orthodox school of Islam, named after Ibn Hanbal of Baghdad, A. D. 780-855, $\mathrm{BH}$ Chr. Eccl. ii. 263 and note.

col. 1319. to sigh for, long for. Part.

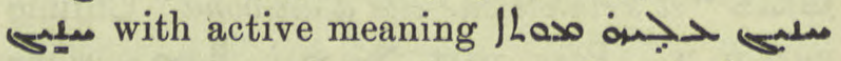
solt treatment of the body makes death long for it, Is. Nin. B. 285. 9. But cf. (2. same

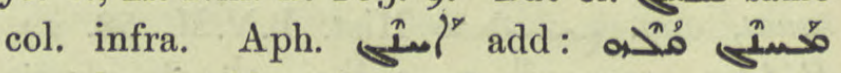
a cock partridge chirps plaintively in wooing, N. Hist. vii. 4. 4: ef. حختـو Hunt. clxx.

w Hani, a large village in Tur Abdin 50 kil. north of Amid, Dion. 103. 10, $\mathbf{1}_{42}$, 17.

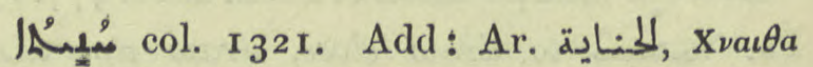

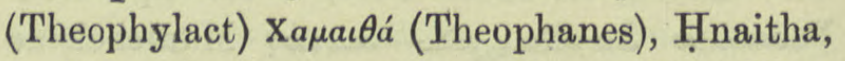
a city and diocese on the Upper Zab, Hist. Mon. i. to8. I, ii. 239 n., Pers. Mart. 2 16222, Badger Nest. I. 174, Syn. Or. 672 : 608 n. $3,619.6$, Jab. $162 . t$ and n., Or. Xt. ii. 3 10. I, 3 II n. I, Chast. 45,69 .

col. 1322. Now called Bavian, 25 miles N. of Mosul. See Layard's Nineveh and Babylon $208 \mathrm{ff}$.

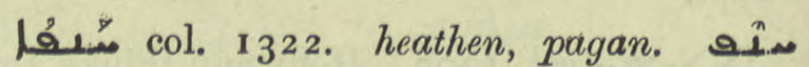
denom. verb Peal conj. to turn pagan, Warda

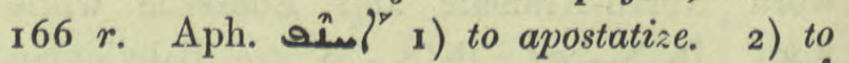

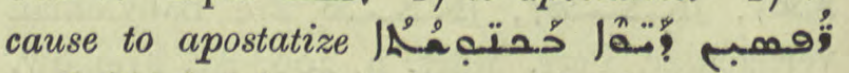

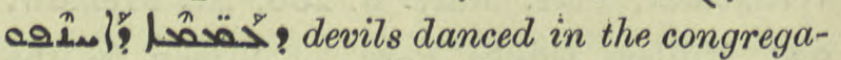
tions of the heathen whom they had made impious, Jac. Sar. Hom. iii. 8or. 18.

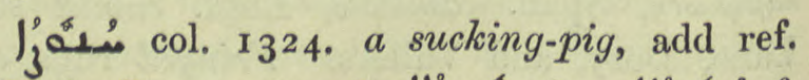

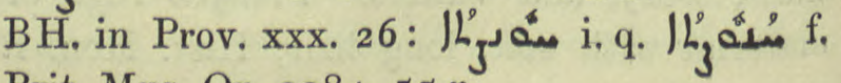
Brit, Mus, Or. $208455 r$. 


$$
\text { rstass }
$$

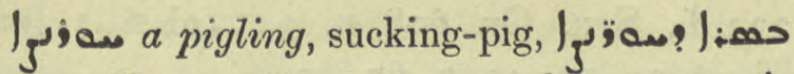
!مارئ Chron. Min. 378. 25 , the passage is quoted Or. Xt. i. 96. i9: cf. ZDMG. Ix. 678 in loc.

Ihiga col. I 324 . place-name, Anasartha, Sev. Lett. ror. r 5. Cf. Mansi vi. rogo.

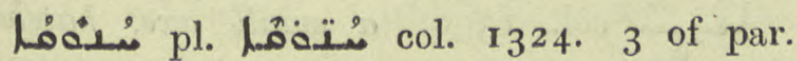

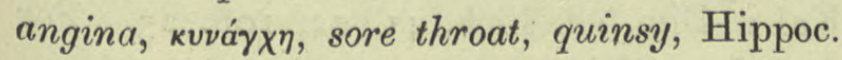
iii. I1, 19. 2 I, v. 9, vi. 36 , vii. 45 ; مُنةقَم ILיy

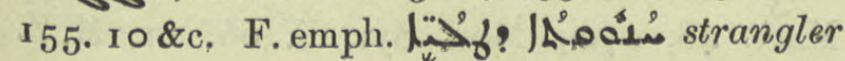
of children, name of a demon, JAOS. x. 289. 4, Protection 34. ${ }_{5}$ \&c.

Hangman of robbers, nickname of Cometes, Jo. Tell. 48. 5. Perh.

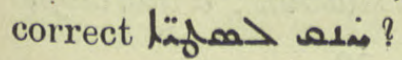

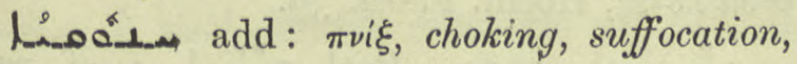
Hippoc. iv. 34 , vii. 55 , Med. 50. 2.

مe col, 1324. Add: narrow neck of a vessel, Chimie 50. I 5. معُم suicide by hanging, Med. 78. 6.

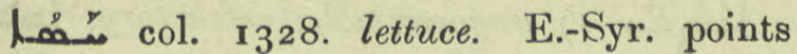

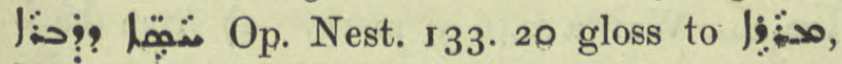

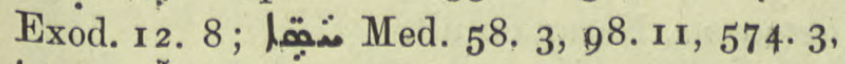

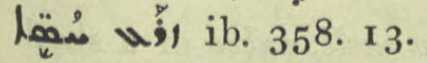

מمْمب: col. 1329. I) disgrace, dishonour,

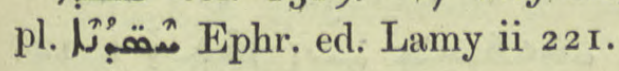

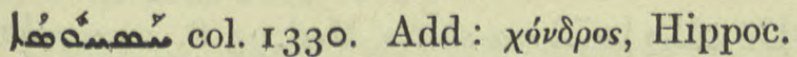

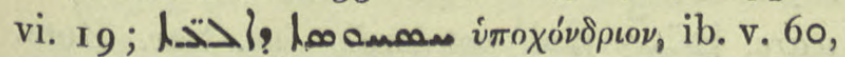

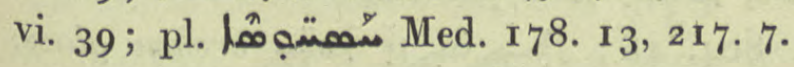

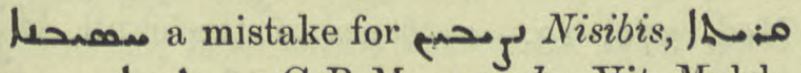

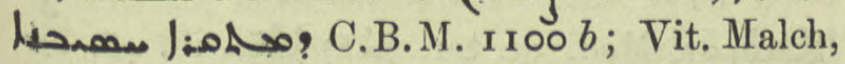
Van den Ven. 104, Kugener in loc. gives

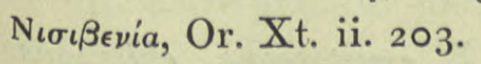

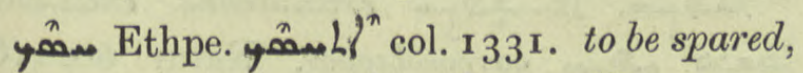
preserved from, , o Coupes iii. I09. 17 ,

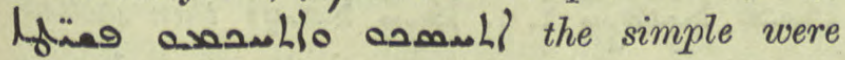
saved from error and enlightened, Sev. Ant.

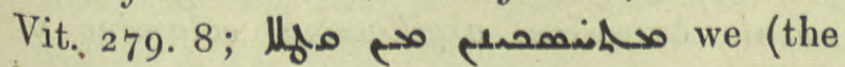
brethren of Joseph) shall be saved from killing him, Ephr. ed. Lamy iii. 289.

นُمْمِ col. I33I. abstinence, Is. Nin. B. 25. 4 af.

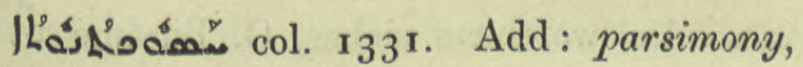
stinginess, Gest. Alex. $3^{8}$. I. iens

Losonin f. abstention from sending the usual Easter greeting, Sev. Lett. 68. I4.

مـفمـ abstinence, deprivation, Poet. Syr. 101. I3.

معهم col. 1333 curious, ref. Arist. Apol. 9. 9.

م- enviable, distinguished, لV]

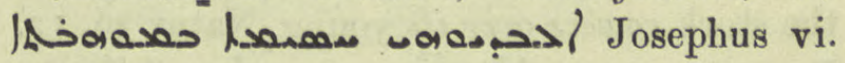
Iо. 6 .

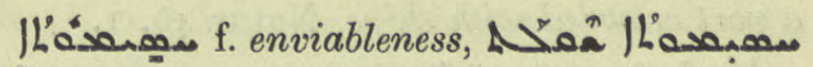
مب: the city was to be envied, Hist. Mon. i. $3 \circ 3.2$.

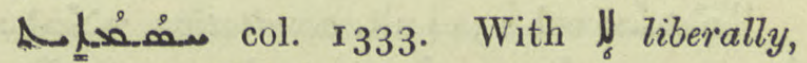
unsparingly, Epiph. 9, I 2.

|l 1إ that brotherhood which is never attacked by envy, Hist. Mon. i. 378. i 7 .

col. 1335. to hold fast.

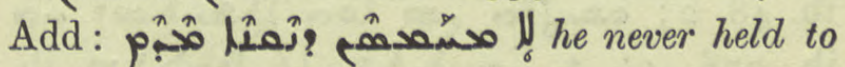
any possession, Pallad. 209. 16.

col.

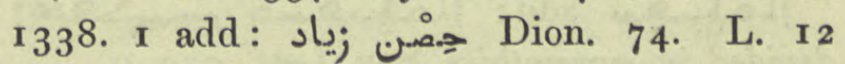

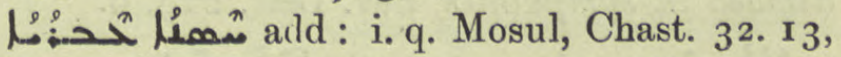
Nars. ed. Ming. ii. 408. 10 and n., Mosul and its environs, ib. ii. 41о, M.Z. I r. 3, transl. 87 and n. 1. But Hoffm. says it is اليهود Mear Mosul, cf. Nöld. in Sassanidi 22 n. 9.

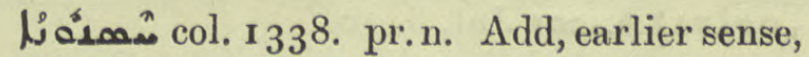
only a small fort, Chast. 32. 14.

1338. misprinted and add refs. Jos. Styl. 10. I5, Jul. 23. 21 , cf. ib. $37 \cdot{ }^{2} 5$.

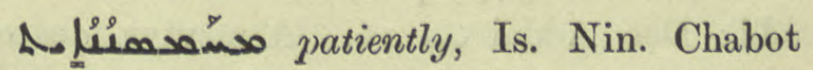
48. lxiv.

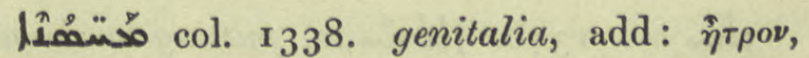
$\pi \epsilon \rho i \nu \epsilon o \nu, \kappa \tau \epsilon i s$, Hippoc. ii. 35 , iii. ter, iv. 80 , vi. 44 , vii. 36 .

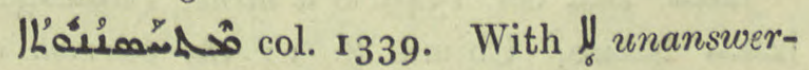
ableness, irrefutability, Jab. 260. 12.

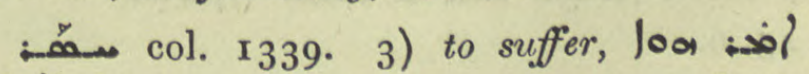
ahilh in the said his conscience was hurt,

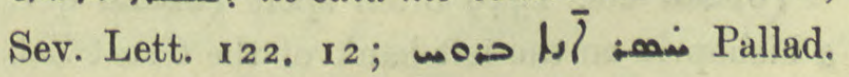
537. II, I 6. 
خسروان fuller form خسرو, Pers. Khosran or Khosruwan, a noble of Edessa, Doc. Addai r I7 : see Moses of Chorene ii. 237 \&c. Marquart, Öst. Syr. Züge. 296.

مallow? Duval translates mauve: synonym for lensor ol anchusa, Chimie 2. I2.

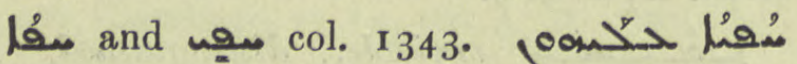
the stork cowers over its young, Natur 36 . 12.

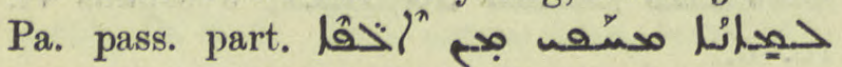
a port crowded with ships, Natur 46 . I.

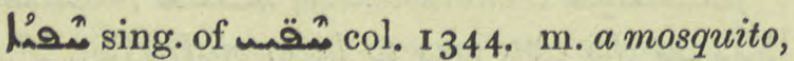
Dion. 59. 6.

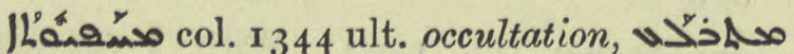
La S. Dan. $57 b 6$, Hist. Mon. i. 327.4 .

معَم. Ethpa. sense to be desired, oa > object was, Sev. Lett. 3 penult, ,

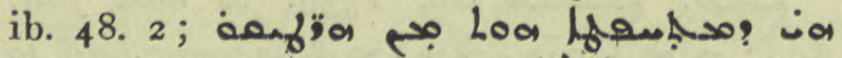

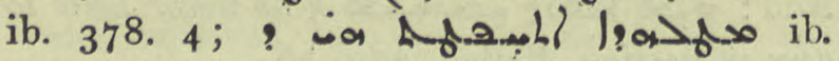
438. 3 .

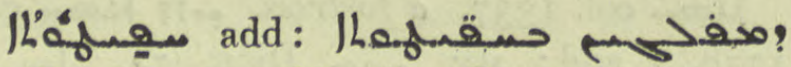
divided in sympathies, Sev, Lett. 229. 9.

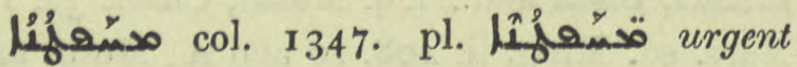
messengers, Sev. Ant. Vit. 268. 8.

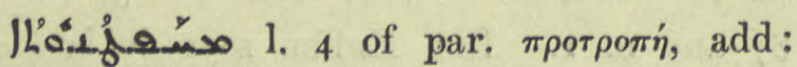
Epiph. ed. Lag. 617, cf. Symm. I52. 7. Diligence, Dion. Ined. 479. 12 ; أخحا , sosfom Jul. 8. 10.

مaئ col. 1347. Add: a measure, a handful, Med. 143. 14, مع 144 ult.

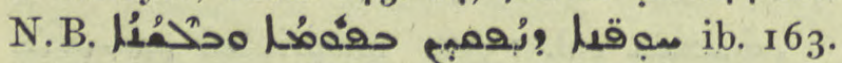
22, sores; corr.

nam col. 1347. pr.n.m. a noble of Edessa, add: Doc. Addai r r 6 . A martyr, deacon of Adiabene, A.M.B. iv. 133 ff., M.Z, 6o. 84 .

معَ: nutting to shame,

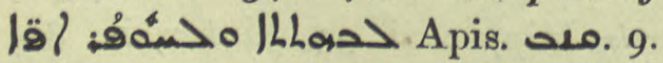

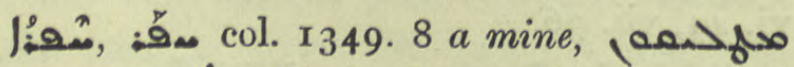
| مa Chimie 8. I; the burrow of a mole, مa حب) Satur 45. 6 .

مُ col. 1349 often spelt محفي q.v. a bishopric on the left bank of the Great Zab and in the diocese of Arbela, Pers. Mart.
233 f., Syn. Or. 608 n. 3 ; ohan? Jof Chast. 29. 10, 49. 7, 67. 20.

col. 1350.22 of par. the back, add: constr. state. N.B. E.-Syr. points, BHGr. i. 53. 20. For مَّ a double-

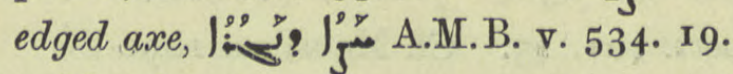

لـ col. 1350. a small axe, hatchet, Kal-w-Dim. ed. Bick. 41. 22, 106. 23. For deriv. see ZDMG. xlvi. 24 I, Fremdw. 86.

مُ Hassa, a hamlet near Kerkuk, Pers. Mart. 48, 270 ; Mon. Syr. ii. 66. II, M. Z. 199. 22 .

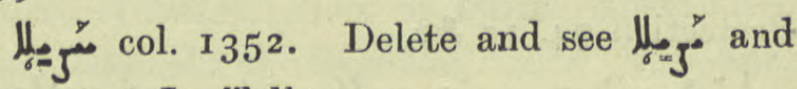
so correct Jo. Tell, 50. 5 .

Arab. حمد a stallion; a thoroughbred horse, Hochf. Fabel. 39.

$\theta_{\tilde{y}}^{y}$ col. I352. Pael $\theta_{j}^{n y} 1.4$ of par. correct ref. Ephr. iii. 328 B , C. to $9_{5}^{n \nu} 0$ is careful. So Can. Jac. Edes. I. I 2 should be مومثم as in the variant.

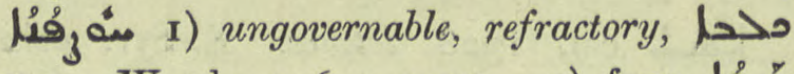

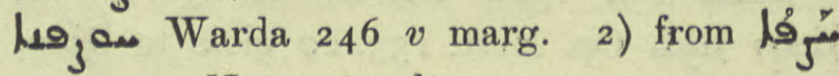
testaceous, Natur 60 ult.

مُ col. I353. I) a shell, valve of a shell.

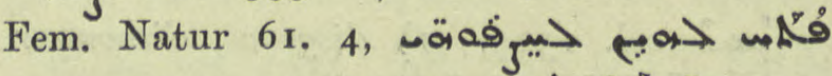
11. 6 and 8, , 1. I2; sea-shells, Med.93.8. 2 ) earthen-

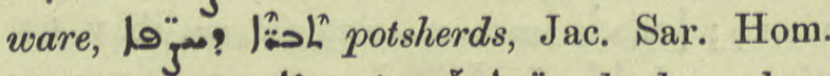

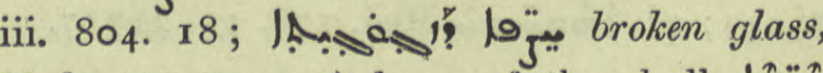
Med. 570. 13. 2) bones of the skull,

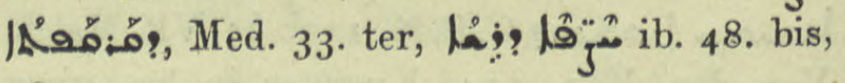
78. $19,79.5$.

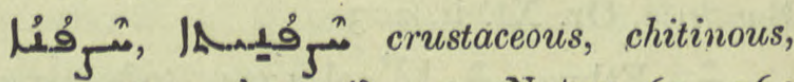

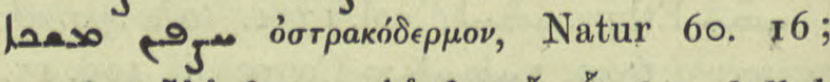

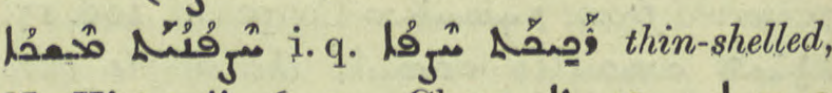

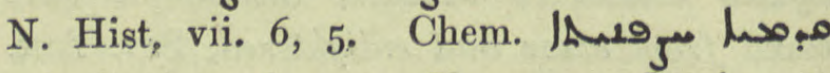

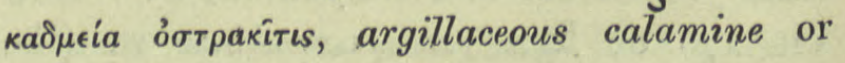
cadmium, Chimie 44. 17.

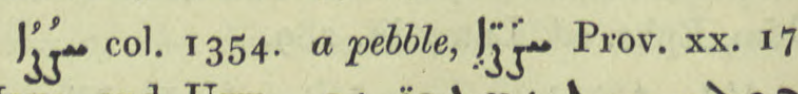
Maus. and Urm., حمخ مs

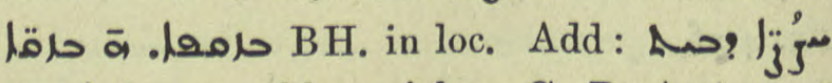
خلم i.e. worthless riches, G. Busâmé I3 $v$ 5 af., cf. Num. xxiv, 13. 


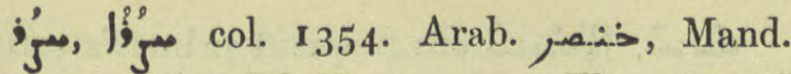
חיצרא see Ar. PflnN. 9 note. The mid-riff

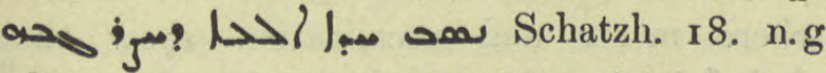
adding the word from Brit. Mus. Add. 25875 .

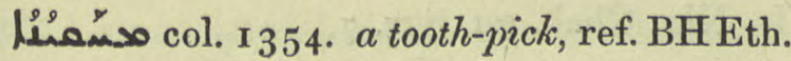
B. $125.14,160.9$.

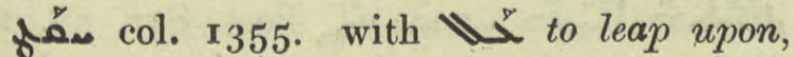
مart. Luc. 166. 2 = R. O.C. iii. 166. 2 .

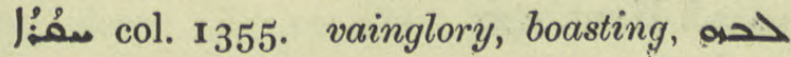

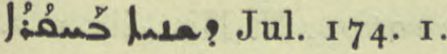

o col. 1355 .

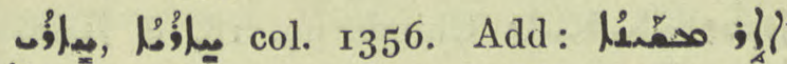
Lُؤم o serene and open air, Is. Nin. B. 92.3 af. ; مة حهـ the liberal arts, belles-lettres, Sev. Ant. Vit. 9. I2. With لl ignoble, mean, L م. لل 3०2. 9 .

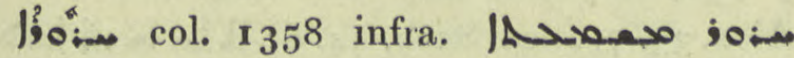
orifice of hearing, which birds have though no

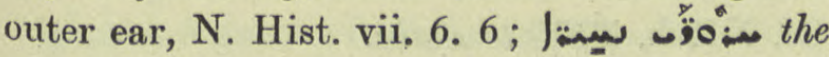
nostrils, But. Sap. Econ. iii. 2 infra, BA. col. 3559 under nasoes; the opening in the nerves of the eye, the pupil Med. 69. 8, 70.

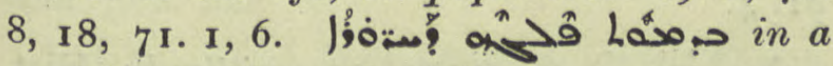
semicircular form, ib. $\mathbf{1 7}_{7} 8$. 14 .

مهلمسنةرل Syr. Rom. Rechtsb. 47. 10.

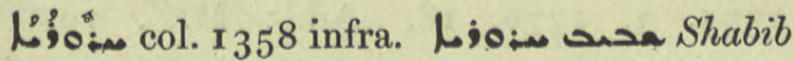
the Harurite, Brooke's Chron. 573. I 5 .

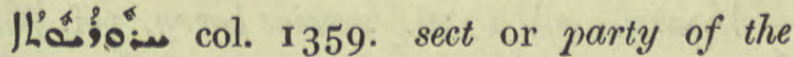
Harurites, later called Kharijites, Dion. 47. I, 52. 6, Jhaside id. ib. 30. I3, see note p. 27.

مئl col. 1359. to mute as birds, c.c. varal-w-Dim. ed. Bick. 77. 12.

مُم: col. r359. dung, droppings, BH. ZDMG. xl. $44^{2}$; مسنُ

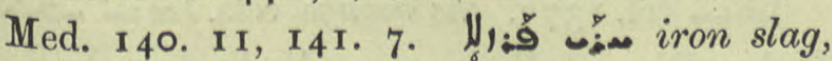
Chimie 5. 2 I, Med. I04. 3.

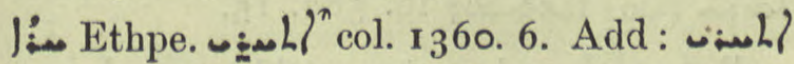
has pos ahins a fishbone stuck in his throat, Anecd. Syr. iii. 48. I 4. Part. contentious, contradictory, Pallad.6r. I ; fem. elamouring to take the first place, R.O.C. vii, I 13.18 .
Jho's col. I360. ioxiov, the haunches, Hippoc. iii. 22 , vi. $44,5 \circ$, vii. 56,57 . OA ahois squatting, Kal-w-Dim. ed. Bick. 26. 4, I2, the suggestion of ah n. ib. seems superfluous: / L'ö ماه sciatica, Med. 50. 22, 52. 8, / إlo ib. 6o. 17.

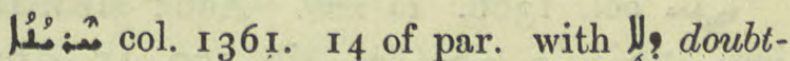
less,

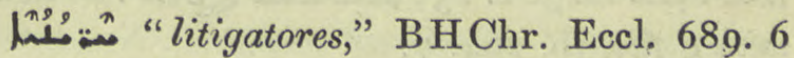
should be مَّ men of Harran. Cf. ib. 687 . 24 and Thes. col. 1376 .

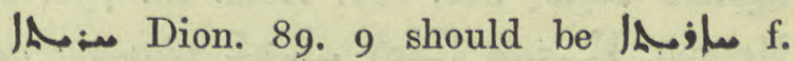
a drain, gutter. See under مال col. I 168.

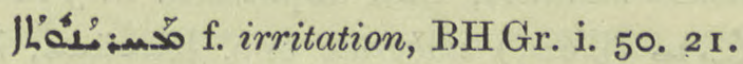

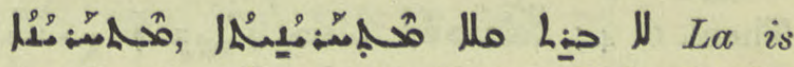
a word expressing contradiction, Hebraica iv.

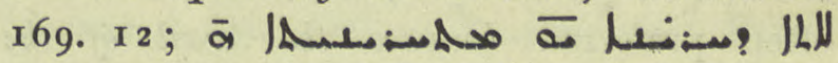
م BH. on Luke II. 34 .

هيخ col, I362. I) to decay, be spoiled, هار) rotten fruit, Ephr. Ref. i. 143. 35 : cf. vulg. Arab. 2) to lay waste.

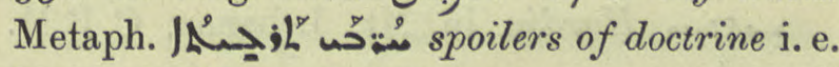
heretics, Pallad. 99. I 5 .

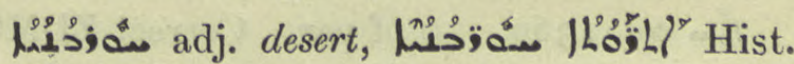
Mon. i. 274. 2.

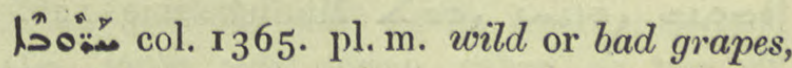

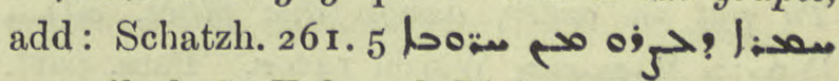
50. ib. 1. 6; Ephr. ed. Bick. 8r ult.

من من col. 1365. destruction of the host of Sennacherib, Ephr. Syr. ii. 59 F.

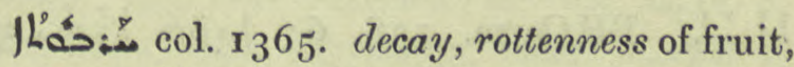

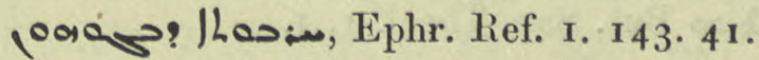

A- name of a town on the Tigris, Chast. 5. 2.



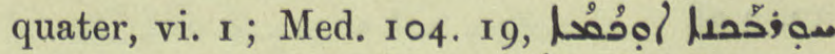

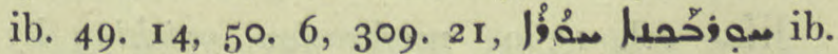
53. I 5, 56. I 7, 309. 21 and often. L. 5 of

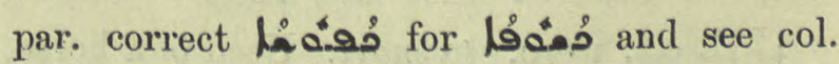
1803 .

خَرْبَث جَ Harbathgelal, E.-Syr. bishopric, probably on the Lower Zab, Pers, Mart. 26 I, S. Maris 44. 2 
and nn., Hist. Mon, i. 66. I 7, A.M.B. ii. 2. 5 .

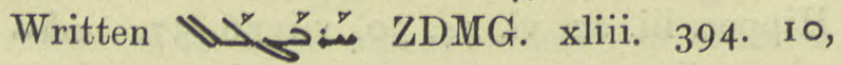
396. 10; Syn. Or. 33, 34, 43 and often.

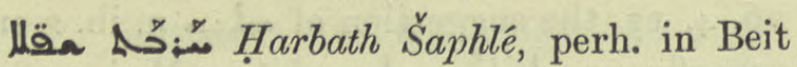
Nuhadra, M.Z. 202. IO, I 2.

col. I366. to rub. Add: Med. I64

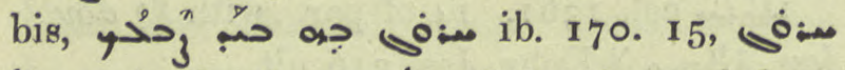

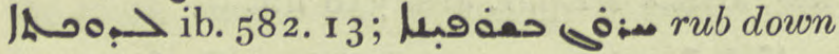
with a file, ib. 264. 7 .

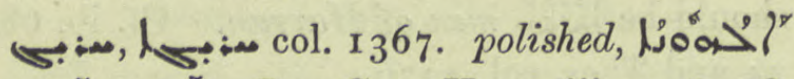

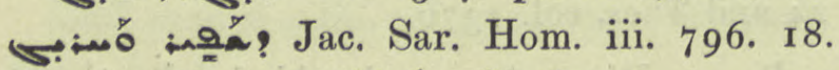
Metaph. potished manners, But. Sap. Econ. ii. 3 infr.,

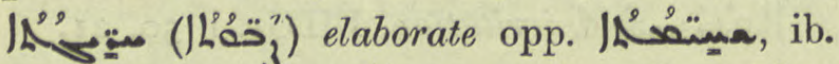

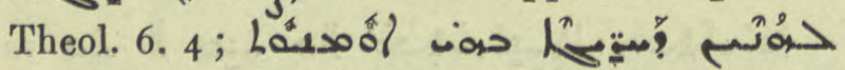

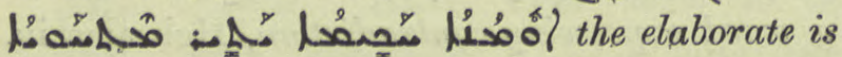
often preferred because the craft of the workman is therein better displayed, ib.

L'ás col. 1367. Add: agility, Anecd. Syr. iv. 38. 12 ; elegance, A.M.B. iii. 123. I.

ه. a village near Mosul, M. Z. I I. 2.

م- Hardin in Mt, Lebanon, C. B.M. $1145 b$.

مecl. 1369. ro of par. Correct ZDMG. not xxix. but xxiv. To pierce, transfix,

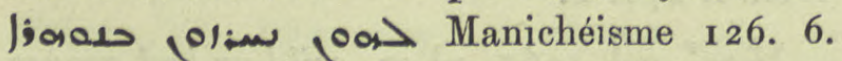
To put in order, assign due place, a! حمبm: Warda I40 r. Pass. part.

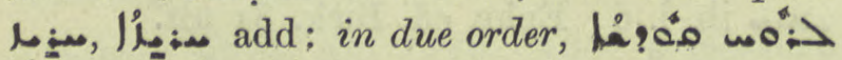
هو S. Dan. 5i 5 r 8. Ethpe.

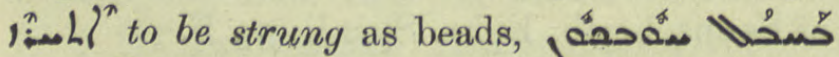

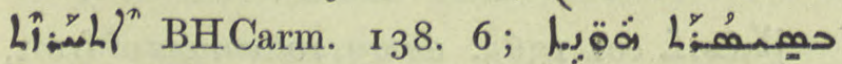
مهلمم:| But. Sap. Theol. r. r.

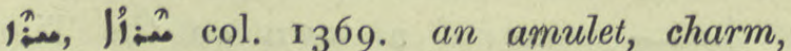

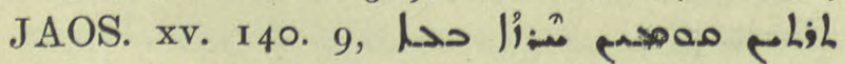
he requires a charm two cubits long, Protection 33. 3 af.

lin. col. 1369. perforation, pricking by hairs inside the eyelids, Med. 93. 4.

col. 1370. to snarl. Used of a horse,

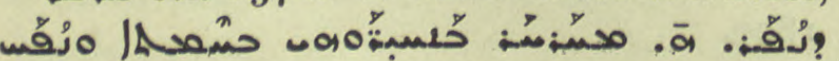
Op. Nest. 113. 12 in Nahum iii, 2. Ethpa.

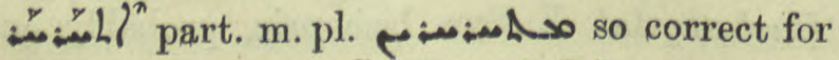
olith with to snarl, Dion, 205. 18. are frequent printer's errors

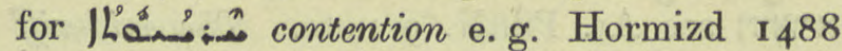
where metre and vocalization show that

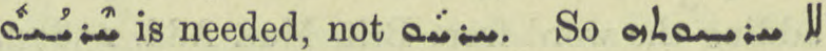
Is. Nin. Chab. 99. Syr. 7.

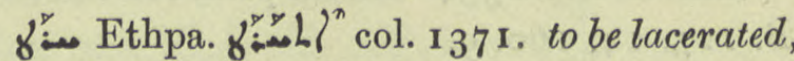
.

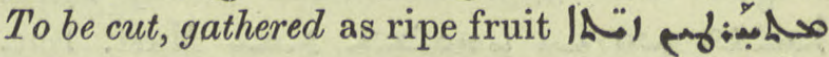
ماتا مهازئ. الشبهر Hunt. clxx.

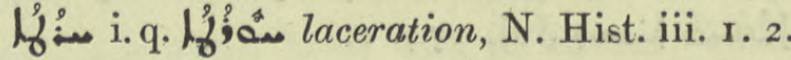

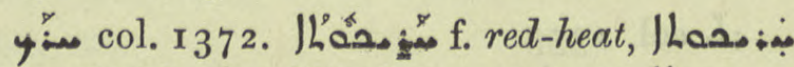

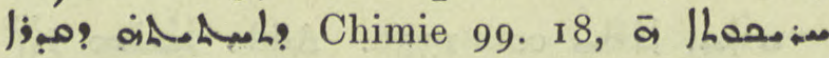
مبم ib. I9.

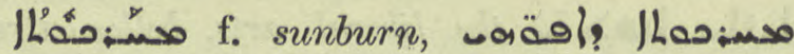
Is, Nin. Chab. 100. 9.

مِّi col. r373. Parel conj. Duval Gram. § 196, to fawn, wag the tail, add: Gest.

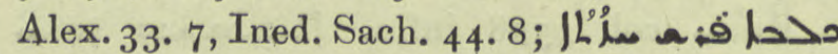

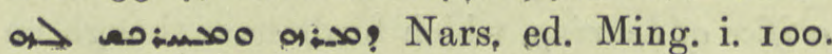
Cf. NHeb.

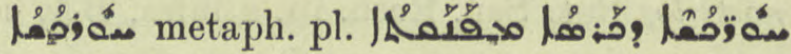
the seductions of a pampered body, Išoyahb 9.1. 4 .

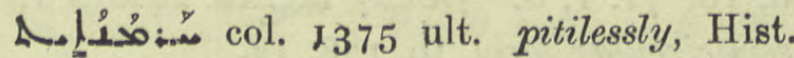
Mon. i. 180.1 .

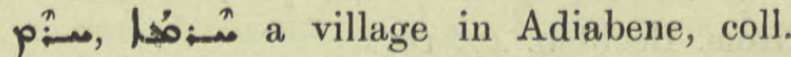
1375. 3, 1376. 18. Add: M.Z. 190. 5, 203. 17.

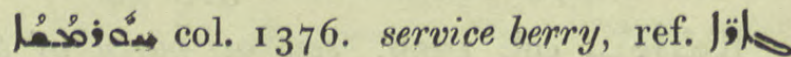

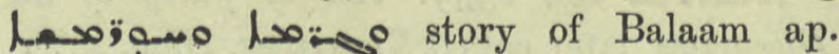
G. Busâmé I3. 2.

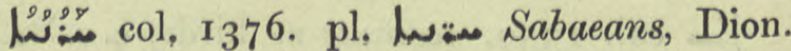

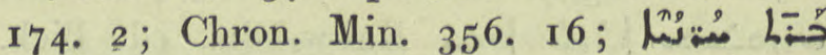
Jac. Sar. Hom. iii. 203.3 .

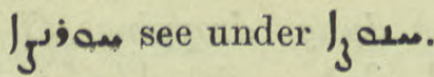

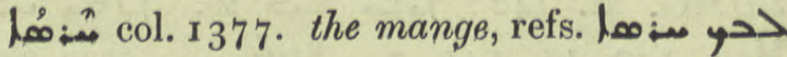
Kal-w-Dim. èd. Bick. 52, 4; Med.48.2 I, ţ

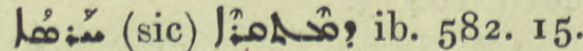

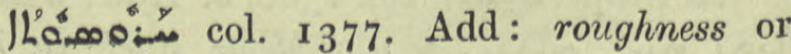
granulation of the eyelids, Med. 78. I7;

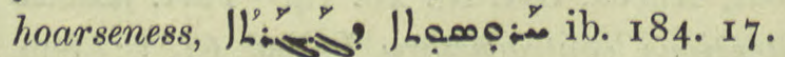

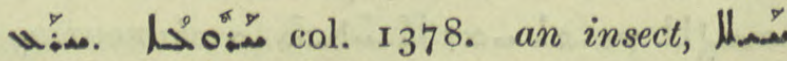

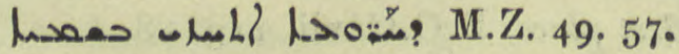




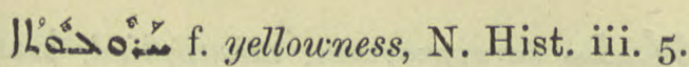

Lحُ col. I378. 3) Carthamus tinctorius,

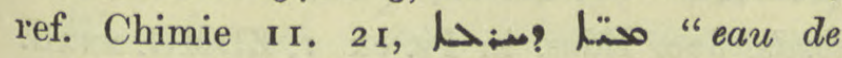
saffrron," an infusion of the above, ib. I4. I2,

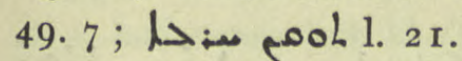

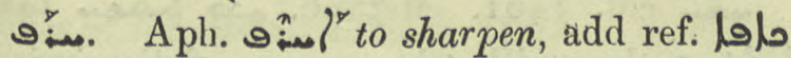

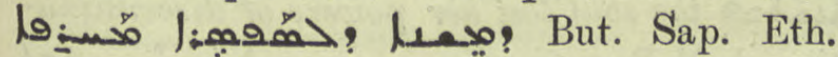
iv. 2 infra.

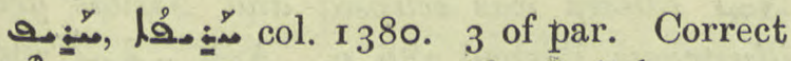

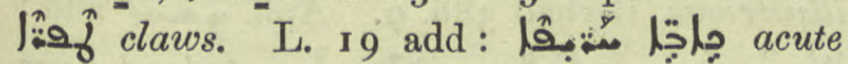
illnesses, opp. J Med. 46 . I5 bis, Hippoc. I quater; $\mathrm{BHChr}$. Eccl. $\S$ ii. 427. 9 af.

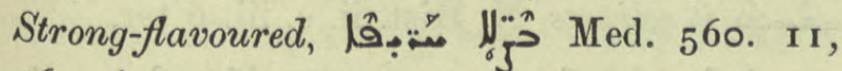
56 I. 6.

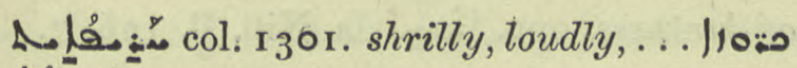

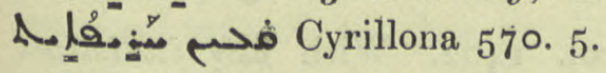

/Lớ col. I38r. shrillness. Add :

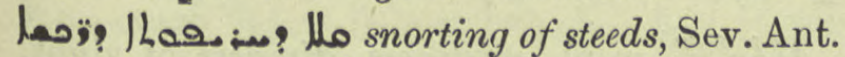
Hymns I 53, I 57 and note. Cf. Hex. Jer. viii. 16 .

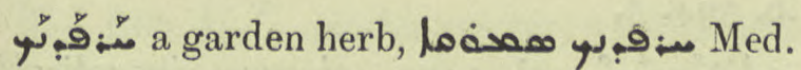
575. II.

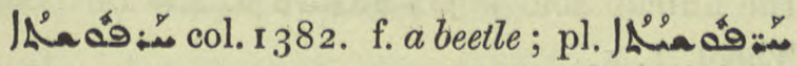
Med. 578. 22.

من:هل m. pl. Herpathanāye, sons of Shelah of the family of Aram, Chron. Min. 356, 22.

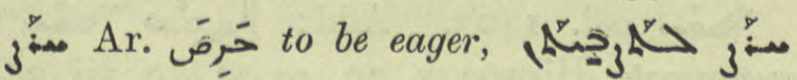
Poet. Syr. 98, 6.

مُ3: Ornithogalum, Star of Bethlehem,

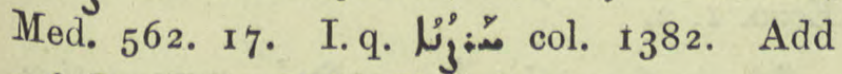
ref. Jac. Edes. Nöld. F. S. i. 575 .

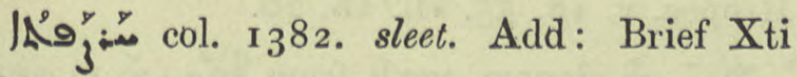
102. 3.

مُّم col. 1382. to gnash, grind the teeth,

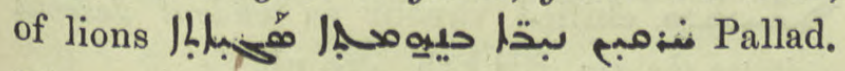
250. 7 .

م 2 m.pl. bands or stripes of colour, Chimie 8. 16.

ص col. I $3^{8} 5$. to be silent. Metaph.

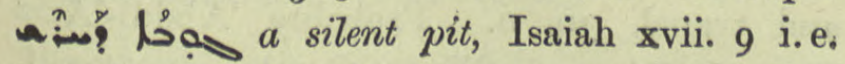
waterless so that it returns no sound when a pebble is thrown down, $\mathrm{BH}$. in loc. The

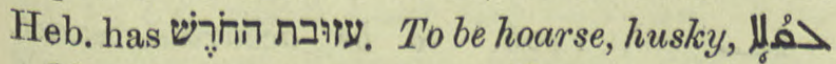
a

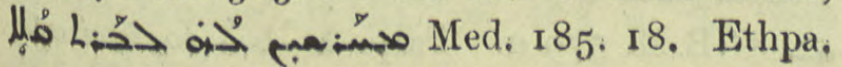

a .

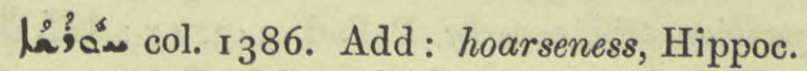
ii. 40 , iii. ter.

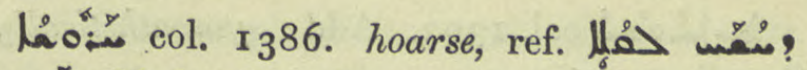
פمّن: Med. I82. 16.

1Lْón col. I386. BHGr. i. 195. 10 and

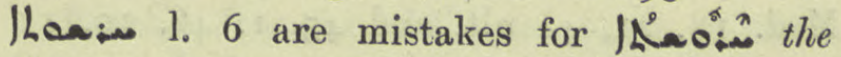
throat. Hoarseness, huskiness, Med. 183. I1, I 86 bis, 238 . I 7, 239. I, 246. ult.

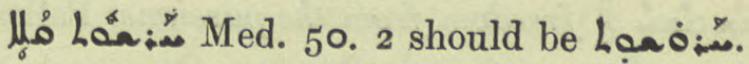

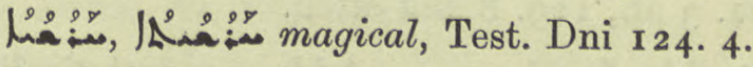

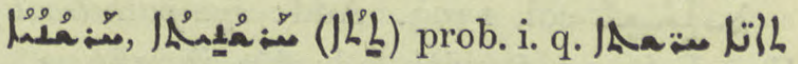
${ }^{\circ} \lambda v \nu \theta o s$, Lat. grossus, small winter figs which do not ripen, Med. 576 . 17, 582. 1 2.

L col. I388. to plough up, divide, ref.

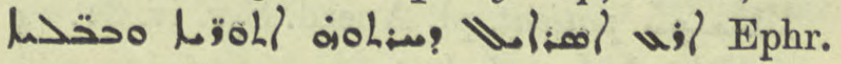
ed. Lamy ii. 275 pen. on Hab. iii. 9. Ethpa.

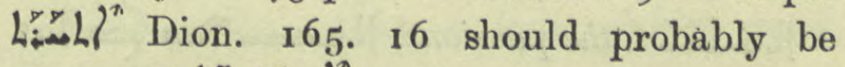
corrected Lin IA Lim holl there hardly remained enough for the poll-tax.

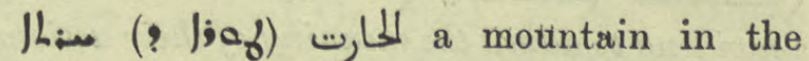
Gaulonitis, ZDMG. xxix. 430, Doc. Mon. 213. 19.

مr. Ar.

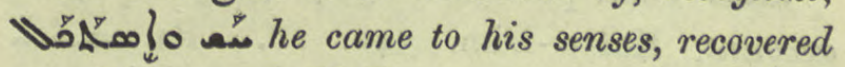
consciousness, A. M. B. v. 2 i. Io, var and?

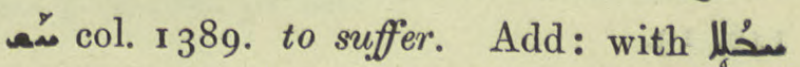

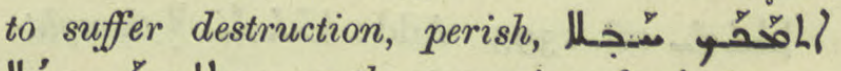
on the succession of priests was brought low and perished in the days of Aristobulus and Hyrcanus, marg.

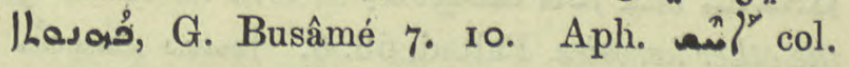
I390. to pound, work a mixture to the consistency of dough, Chimie 45. I4.

Anecd. Syr. iii. 49. 2 I should probably be מח he had suffered.

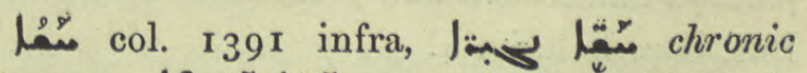

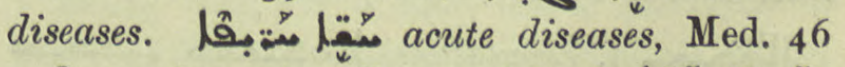

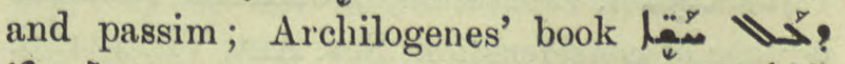

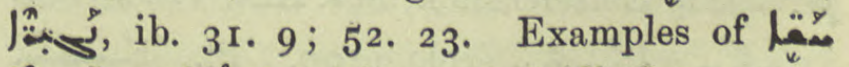
定 dementia, epilepsy and vertigo, ib, 46 ; مُ the falling sickness i. e. epilepsy, Med, 4. I, 


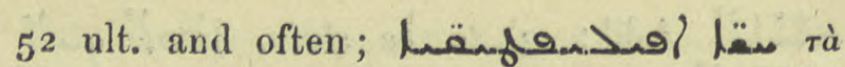
فَّ

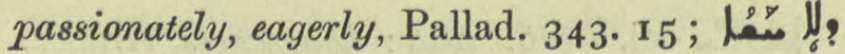
unfeeling, unsympathizing, A.M.B. v. 546 .

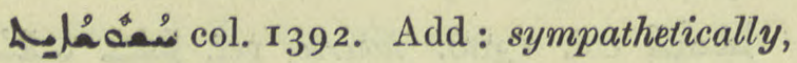
Sev. Lett. 363.10.

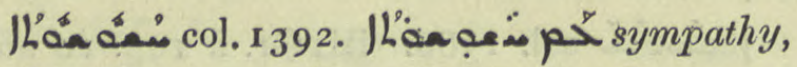
Med. I 8. 2 I, 44 ult. and 45. I, 46. Io, 1 I

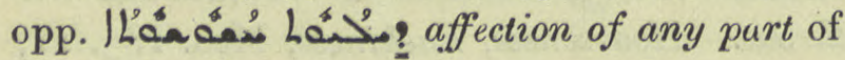
the body, 1l. I1, I4; ib. 3 I. $7,8,34$. I4; IL'án freedom from the passions; Pallad. 102. 3 af., 129.8 and often.

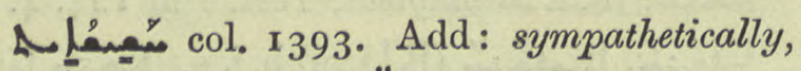

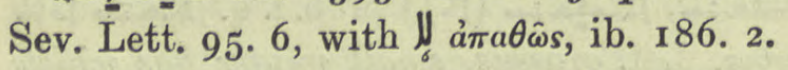

IL'col. 1393. compunction, a monastic virtue, Sev. Ant. Vit. 109. 12.

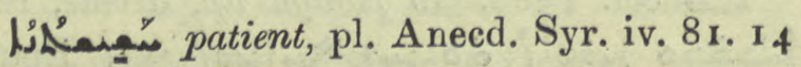

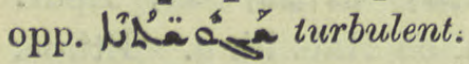

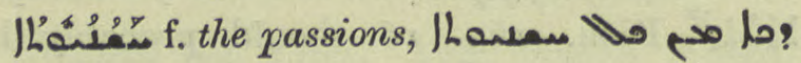
Sev. Lett. 208. 3 .

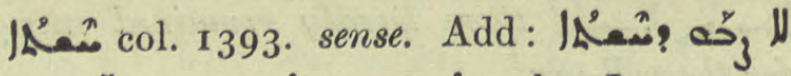

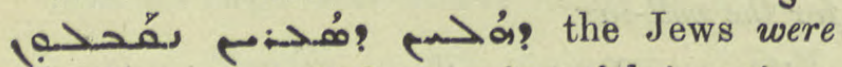
unwilling to admit the meaning of their actions, Bar Penk. 54. ro. Or they felt no compunction

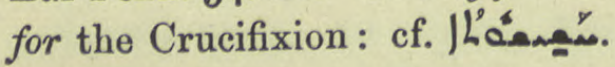

معِّ Pass. part. col. 1394. معِم of no account, Kal-w-Dim. 342. 18. Pa. معند

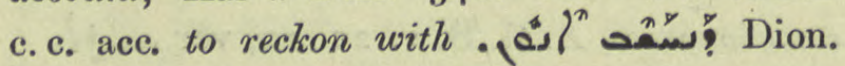
146. 13 .

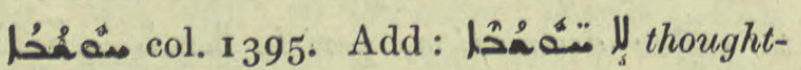
lessness, Pallad. 280. 6.

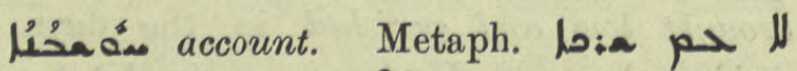

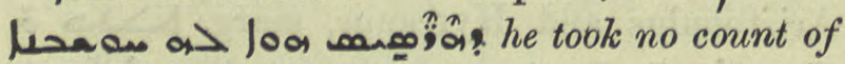

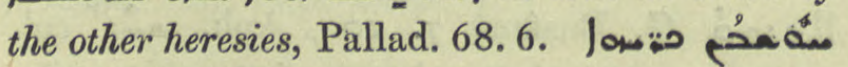
diagnosis, JAOS. xv. 138 . I.

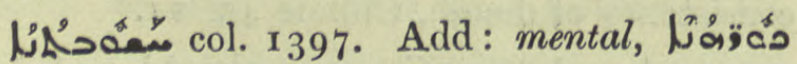

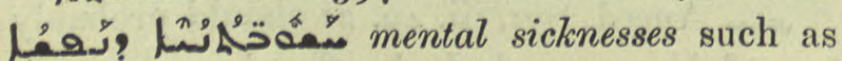

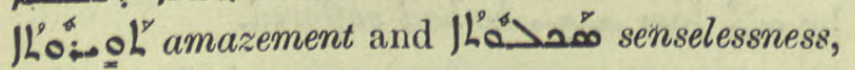
But. Sap. Eth. iv. 4.

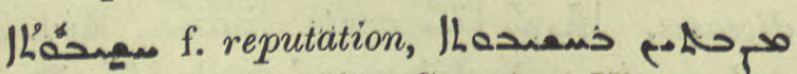
persons of consideration, Sev. Ant. Vit. 231. 6.

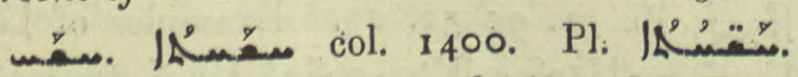
2) uses. I. q. Gr. $\chi \rho \eta^{\prime} \sigma \epsilon i s$ and $\lambda \epsilon^{\prime} \xi \epsilon i s$, Op. Nest. xiii. and $120,3,145$ ult. Use, meaning, locution, Nest. Hérac. 44. 5, pl. مُّقُمُ ib. ult.

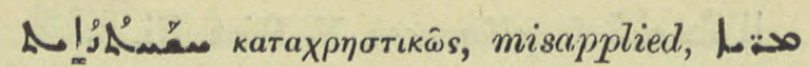

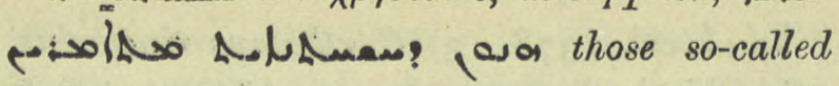
lords, Jac. Edes. in Ps. cx. I, ZDMG. xxxii. 477 ; ib. 479.1 .

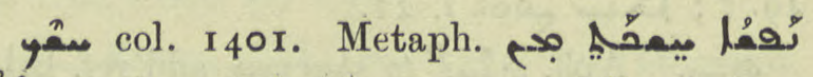

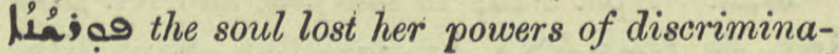
tion, A.M.B. v. 62 I. 9 ; محمَ Lion Edessa was eclipsed and Nisibis grew illustrious, Barḥad 386. 9. Aph. 4. مَّ" add : diخَحُ : do not cloud your mind with sleep, Is. Nin. B. I38. 5, 6. Part. مَسمُ

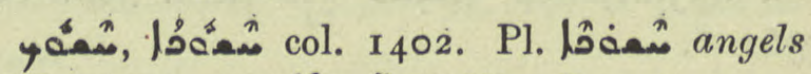

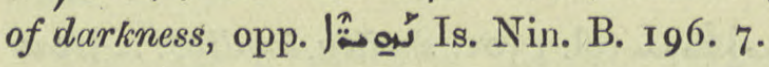

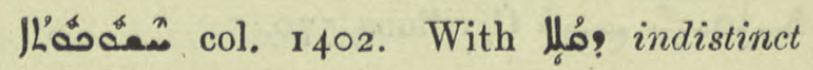
speech, Med. 22 I. 19.

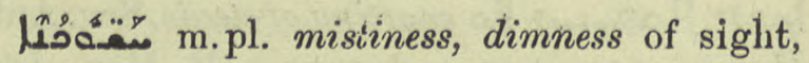

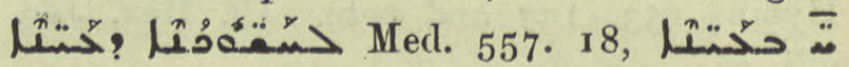
ib. $594 \cdot 5$.

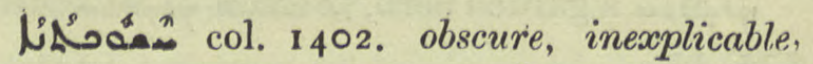

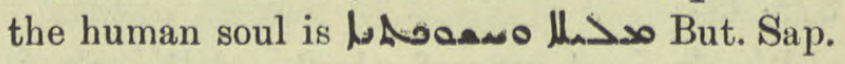
Theol. I. I.

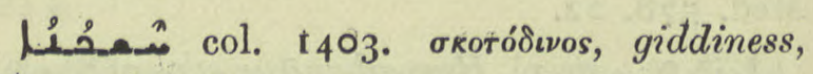
Hippoc. iv. 18.

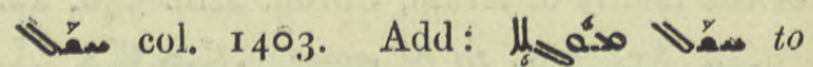
suppurate, Hippoc. v. 20,44 , vi. 20 , vii. 35 .

J'á. $>$ f. the goldsmith's art, But. Sap. Econ. 1.2.

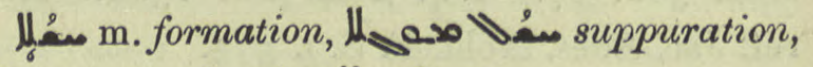
Hippoc. v. 18,55 , vii. $18,20$.

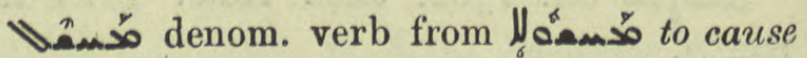

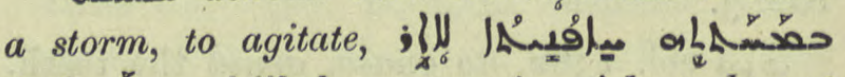

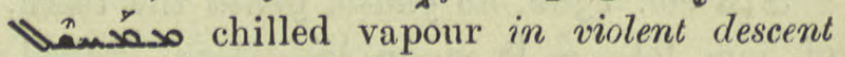
agitates the air, BH Eth. $45^{\circ}$ ult.'

مڤ col. 1405. مuests at supper, Journ. As. 1906, 278 pen., 279. I.

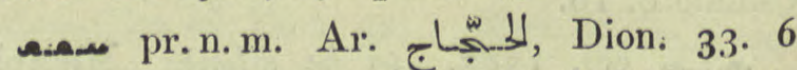
and transl. 30. n. Probably 'Abdul-Aziz Ibn Hajjäj : ef. Weil, Gesch. d. Chalif. i. 673 .

N. col. 1406. to be accurate, exact.

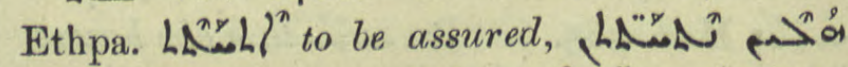
y C.B.M. 579 a. Palp. מع. to irrilate, 


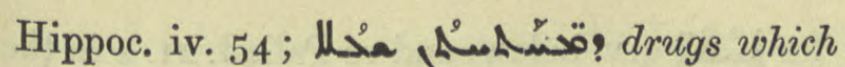
provoke coughing, Med. 23 I. I I.

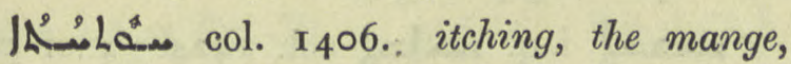
Hippoc. i. 19, iii. 24. Used chiefly metaph.

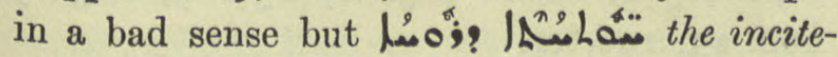
ments of the Spirit, C. B.M. $573 b$.

IL'L'as. f. accurate definition, exactitude, But. Sap. Philos. r. 2.

مَّ人 col. 1407. I) and 2) careful,

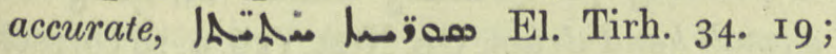
AمA this is correct, BB. under مص: col. I 729 and often; - >م

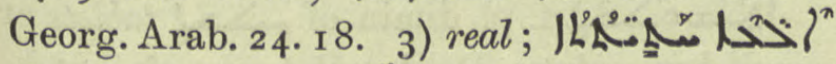
the true ribs opp. 12 gä N. Hist. vii. x. 7;

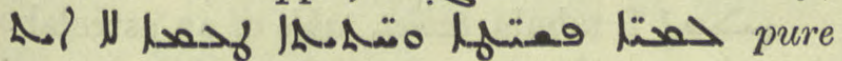
unmixed water is tasteless, ib. ii. v. 4 .

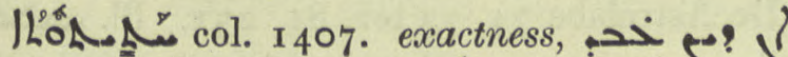

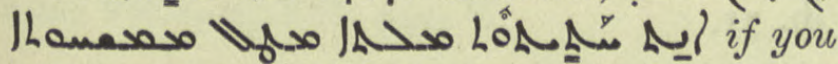
make a strict rule, if you stickle for correct behaviour, Sev. Lett. 484 ult.

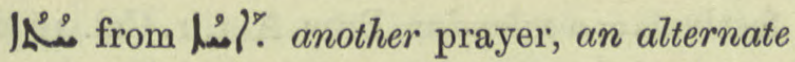
prayer, ơ مُ QdhamW. 36. I 2, I33. 2, Takhsa 80. 5,90 pen., 91. 6 and often.

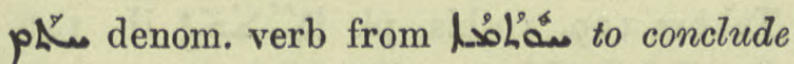
the service, to say the concluding prayer, Brev. Chald. i. 17. 5 bis, م' مُ0مُ ib. $5^{2}$ ult., QdhamW. I80. 4.

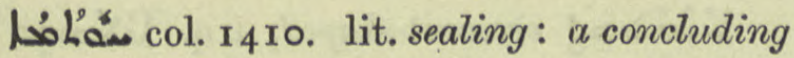
verse, prayer or benediction, add refs. مخيمه
Lsolo مroceeds with the concluding ascription of praise, QdhamW. 40. 8 ; Takhsa

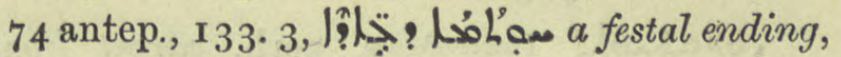

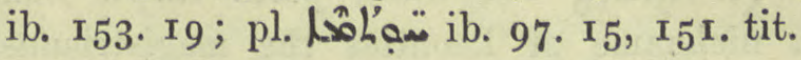

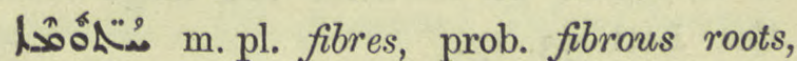
BB. under a sasoo col. 3626 , ed. Duval I 786 .

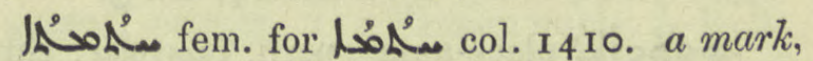

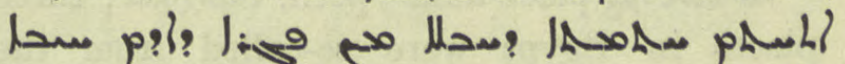
la marque de la perdition a été abolie, Bénédiction des Raisins, R. O.C. iv. 360. Cf. Journ. As. r898, 44I ff.

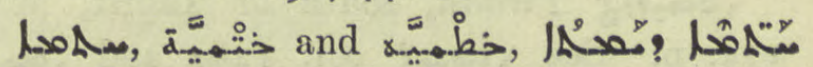
Althaea officinalis, marsh-mallow, Med. 208. 20, 214. 7, 297. I3.

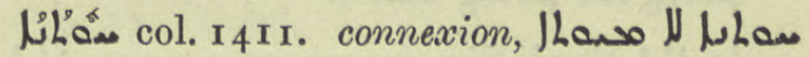
Junano immortal and heavenly connexion,

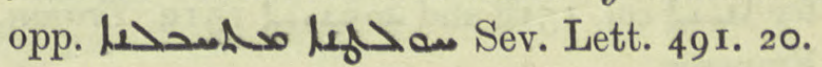

L'ớÁ i.q. OLl p a lady of high rank, Protection 4. 14.

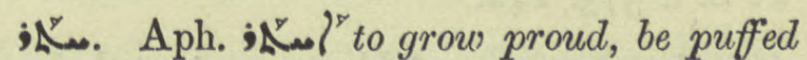

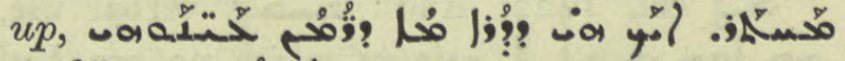

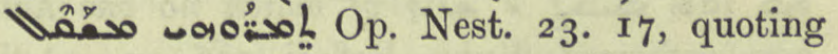
Prov. xxx. I3. F. emph. part. ILis o Jul. 24. 6 .

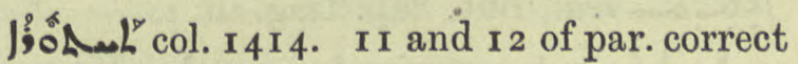
Aمأز and I of as B B. and Hunt. above. Refs. JLan " Syr. 3. 3, Jab. 9x ult.

\section{$\therefore \rightarrow$}

8 col. 1415. The number nine, a. - $\gamma$ * 8 (a) divide by nine, JAOS. xv. I38. 2 .

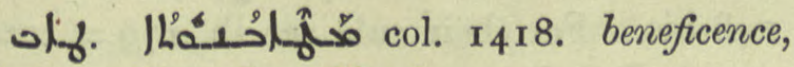
kindness, But. Sap. Theol. 4. I.

Derivs. in $\overline{\text { ב }}$ see under $\overline{-\gamma}$.

noflelf i.q. panely col. I418 pen. Tabenna in the Thebaid, Diosc. ed. Nau 92. 9,

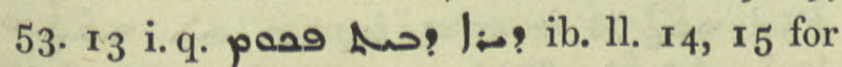
the monks of Tabenna followed the Rule of Pachomius, R.O.C. v. 403 n. 2. See Ifaiolef. m. a monastery in Seleucia of Isauria, R.O.C. iii. 258 infra; Pléroph. Introd. $9 ; 54.6$.

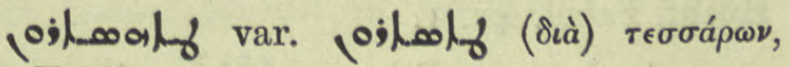
Or. Xt. iii. ro5. 2.

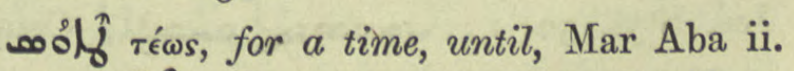
$3 \circ 5 ; \infty$ o أُ and R.O.C. iii. 240.5 af. Cf. sốla ̂̉ col. I433. I. q. uـ emphatic particle, M/خ;?

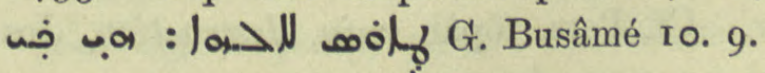

coioly r) Tyre, Or. Xt. i. 88. 19. 2) taurus, $a$ bull, marg. JjoL Warda $246 v$. 
لling m.pl. Taphile, descendants of Noah, Chron. Min. 355. 8.

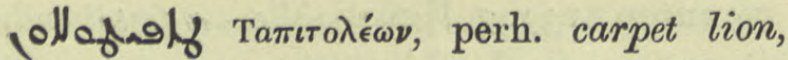
a nickname of Leontius the magistrianus, Sev. Lett. 93. I3.

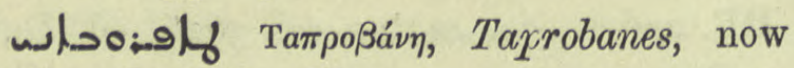
Ceylon, Jac. Edes. Hex. xv. I 4, xx. 3.

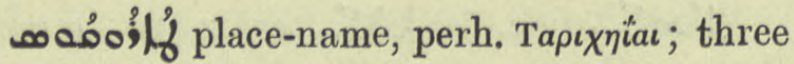
places in Egypt were so-called from the number of mummies preserved there, Med. 5०4. 10.

joajly Tirhan, south of Tagrit, M.Z. 174. 6. See jainf col, $1_{464}$ and arid col. 1517 .

pilf see poing below.

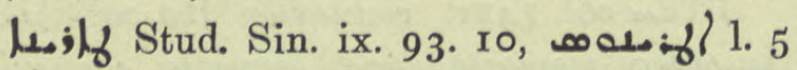
for les if col. I 518 and os of i 5 I9, Trajan, Emperor.

conflijly pr. n. Tepévicos, Terence, Nöld. F. S. i. 573 . 142.

of col. 1422. Add: :

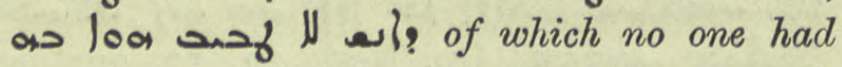
been informed, Ephr. Ref. ii. I 6. 13. Adj.

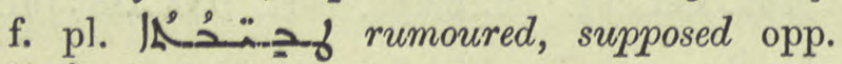

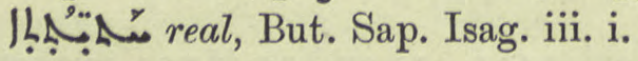

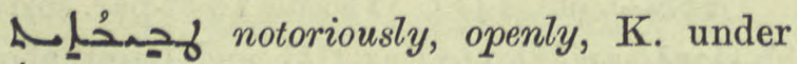
col. I559.

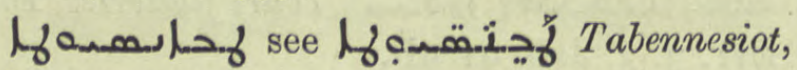
infra.

นُ made from sour milk, K. col. I424, El. Nis. 35. 93 , and so correct Ibn S. Thes. under

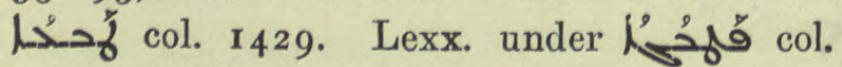
3087 and DBB. I534 have the same gloss viz.

lood of i.q. looof, loof the peacock, Hochf. Fabel. 4I. I.

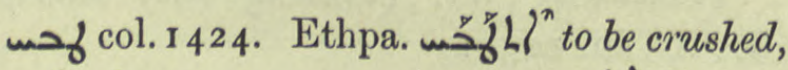
Jos. Styl. 50. I ; wasors ii. II 4 .

lüsof the crusher, smasher, Syrians understood thus the Persian name for an engine of war. Cf. Pers. tôpâh ruin, destruction, Jos. Styl. 49. 20 and trans. 42. n.

㘶 blows, A.M.B. v. 24. I5, n. variant

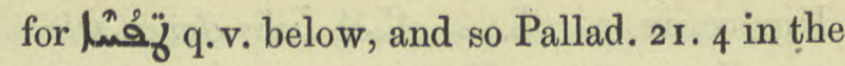
corresponding passage.

i.q. cane sugar, Med. 165. 2, 237. 9, 265. 9, 266. 15,296 ter.

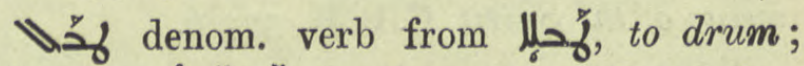
- ọa Warda $3 v$.

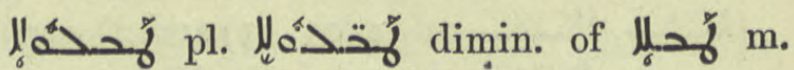
a small hand-drum, Op. Nest. I r6.9, so correct لٕlọ under $\log _{3}$.

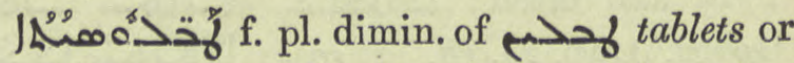
small brass plates, De Astrolabe 75 ult., 76.

مد f. tabula, tablet, table of an astrolabe i. e. the two circular planes, IA is De Astrolabe 74, 75 ter, 8I, 25 I. Pl. ib. $7 \mathrm{r}$.

12. opp. J;ode Gest. Alex. 51. I3. Rit. a table of festivals $=a$ calendar, Brev. Ant. I. Cal. 16 tit., I 8. 1. A moveable altar slab, (no longer in use)

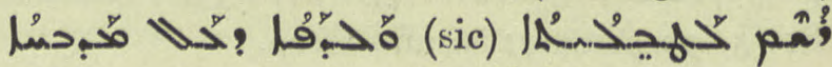

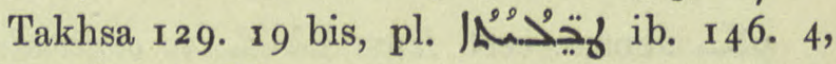
Bar-Sal. in Lit. 25. 6, 7 .

设ح tympanites, a flatulent form of drupsy, Med. 338. 2, 339. 9 .

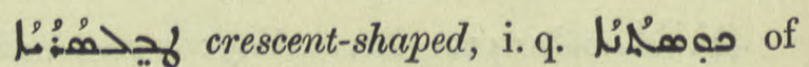
the moon when receding from the sun, N. Hist. Bk. ii. III. 3 ; مها مصن: حمب: un orifice bouché à moitié, Chimie ror. I4.

لُح

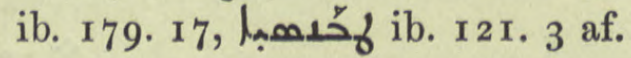

18 144 pen., 30r ; f for Sev. Ant. Vit.

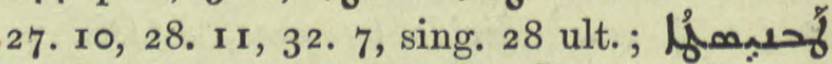
A.M.B. iii. 488 ; مُ 148 bis. For their rule see ib. $179=$ trans. 2 I 4 f., R. O.C. v. 403 n. 2.

טבै col. 1427. 4) astron. to set, sink,

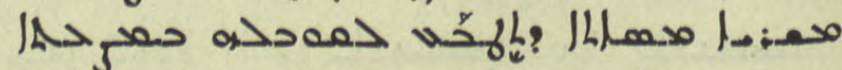
"مح- Rylands 44. fol. 4 sixteen times. Pael טح้ to immerse, baptize, a

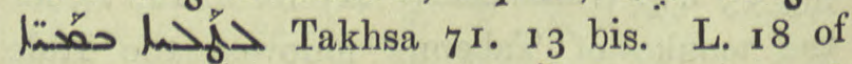
par. denom. verb from Кำรี to affix a mark or token, Dion. 167. 14 . 


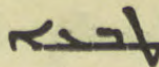

1حำ col. 1420. a passage of a book, text

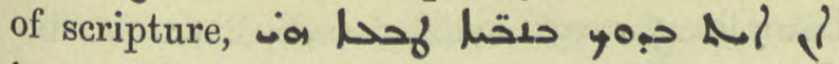
פو Or. Xt. r. 306. 6. Bot.

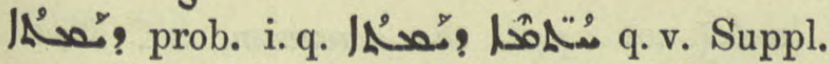
marsh-mallow, Med. 207. 8, 2 I1. 7, 265. 8.

人ُح $\mathrm{m}$. an official who placed tokens on everyone for taxing, Dion. I24. 7, I48. II, 167. 13 .

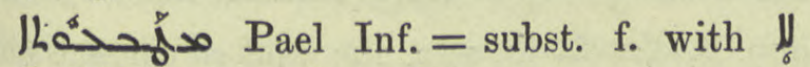
wakefulness, Sev. Ant. Hymns 60.

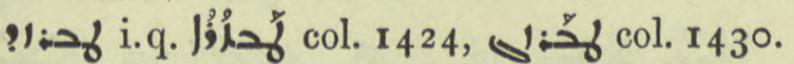
Pers. طبرز sugar candy, "sucre candi blanc", Chimie 39. 18 but "espèce d'alun ou de sel clivable", Duval's notes in Journ. As. both in the Syr. and Arab. lists.

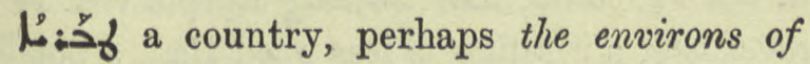
Lake Tiberias, Med. 444. 8.

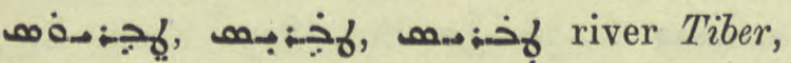
A.M.B. v. 510.

Nó col. 1431. to fry, parch, wanto fais chauffer dans un poêle, Chimie 29 pen. Act. part. ala ii. 373 .

1.:of m.pl. fried delicacies, Warda $247 v$.

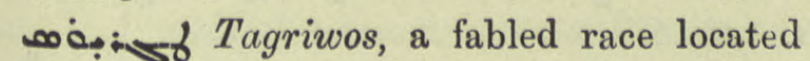
south of the Pishon i. e. the Adriatic, Chron. Min. 354. 4.

ol:ooof Teucrium, col. I433. Correct مححم for a for

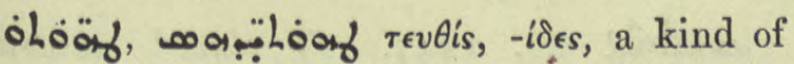

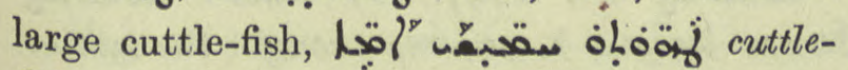
fish five cubits long, N. Hist. vii. 2. 2, 3. I, 6. 6 .

nolafjlfar col. 1433. тетартаios, quartan

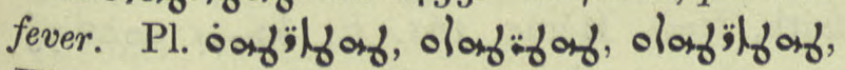
Hippoc. ii. 25 , iii. 21 , v. 66 .

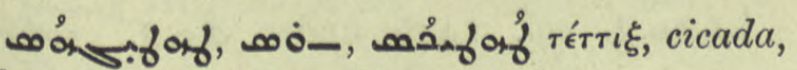
col. 1 433. N. Hist. vii. 2. 2 infra, 2. 3, 4. 2.

parf Pers. . $^{3}$ race, stock. This word prefixed to the name of the reigning monarch, was granted as a title of high honour, Pers.

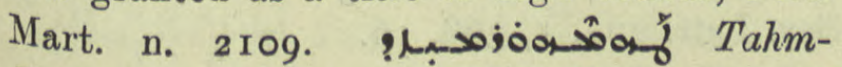
Hormizd, a marzban, A.M.B. ii. $620=$ pod ? lojoa Pers. Mart.n. 565 ; ' joof TahmJazdegerd, QdhamW. 236. ז3, Mon. Syr. ii.
68. I3, ? : is of Il. $2 \mathrm{I}, 28,3 \mathrm{I}$, ? is hoof ib. 69.14, in woof ib. 29 ; the name in the corresponding passage A.M.B. ii. $5^{20}$ pen., $5^{21}$. 10, I 4, $5^{22}$. I $5,5^{23}$. I 8 is spelt uniformly

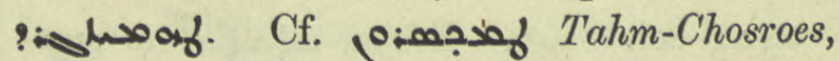
col. 1485 and josaf Tahm-Shapur, col. I 488. Nöld. Tabari 443.n. explains otherwise.

(م) مै pr.n.m. I) a Persian noble, A.M.B. ii. $584.9,585.3,588.5 .2$ 2) Bishop of Siarzur, ZDMG. xliii. 403.4 .

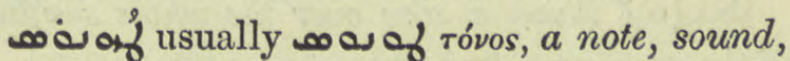

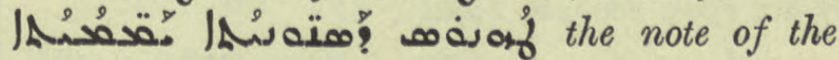
sea swallow, N. Hist. vii. 2. 3 .

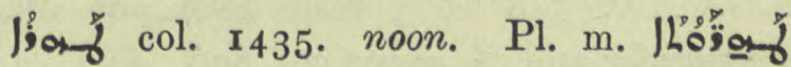
midday heat, Jesus-Sabran 522. 12, A. M. B. ii. $533 . \mathrm{I} 3$, vii. 68 .

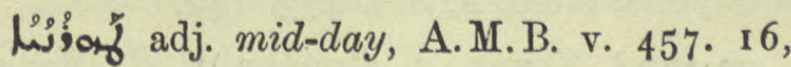
19, 20.

lof Ethpa. usofh? to be roasted, l:-so? on loa lẩ

fُof col. $\mathrm{x}_{43} 6$. a roast, roast meat, lẩ

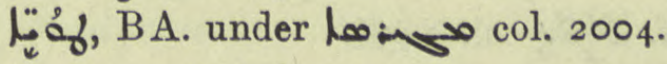

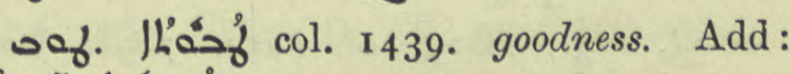
حصحخ (0a) דasion Chron. Min. 47. 14.

rlasof, rooh or isisioh, place-name, quoted from Ptolemy's Geography, Anecd. Syr. iii. 330. 1 .

u of and us of l, a red pigment used to stain glass, w of l loh ? Chimie 99. I2.

lnsof so Duval corrects he of (م00), Chimie 99. 13 "un rouge toubi", coloured with toubi.

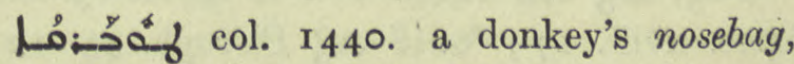
gloss. مه; G. Warda ap. Hippoc. vii. n. 22.

لز gate of Arbela, Jab. 175 ult. It is the name of some bird of prey, note ib.

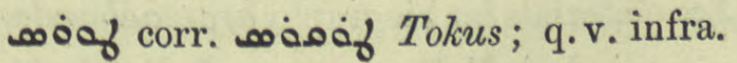

LR_of f. pomp, pride; a Sabaean or Nabataean word, Lexx. under l;oh col. I4I 4 . 
Liof col. I44r. a space of time. Add:

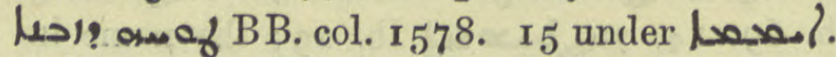
With pron. suff. 2 pl.

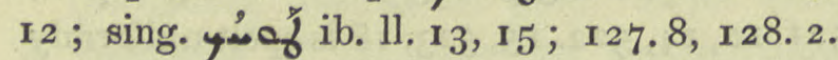

of of col. I44I. Ethpa. ufof l $^{n}$ to sup-

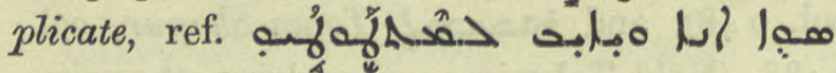

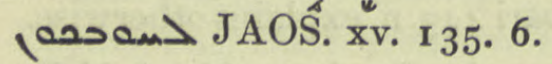

l.fof A rab.توتيLantimony, Chimie ror. I 7 , Med. 604. 21. Cf. NLLoL.

IAfof (Di) a sore over the whote surface of the eye; see $;$, ح: $\mathrm{L}$.

nobof Sev. Ant. Vit. 33. I3, Nau in loc. R.O.C. iv. 548 n. 5 read col_oo! dux, commander and ef.

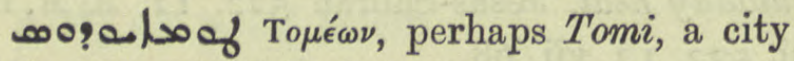
of Scythia, Nöld. F. S. i. 473. 142.

masoof Tomis, a disciple of S. Maris, S. Maris 32. 2 .

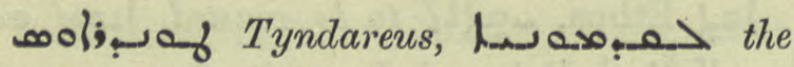
Lacedemonian, Arist. Apol. ب. I I .

er of prob. Tyana of Cappadocia, Dion. 15. 5. Cf. lit áf col. 1436 .

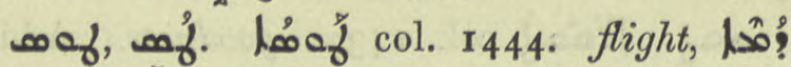

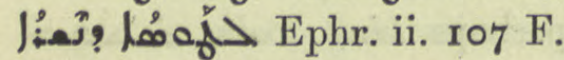

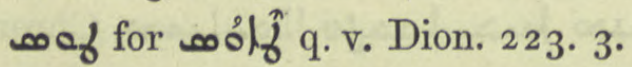

๓ớt col. I444. Tus, capital of Khorasan. Pefs. BHChr. Eccl. ii. 449; its ruins are 22 kilom. NW. of Meshed, Syn. Or. $3^{1} 1,316$, Jab. 20. 4 .

hخمof see un ä col. 1444. m.pl. a tribe of Christian Arabs nomad in the plain bordering on Tur Abdin, Syn. Or. 526. 26, 527. 8, $53^{2}$ n. 4, Patr. Or. iii. i. 28. r1. Mentioned with Jac. Edes. Hex. 8. n. 2.

oof, of col. 1444. ult. to travel by sea, Apoc. xviii. I7; \ilô of Pléroph. 45. 12 ;

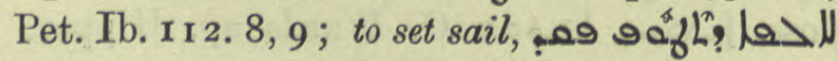
Sev. Ant. Vit. 74. 8, I I ; to travel, حصم Jillo oofl? BHChr. Eccl. ii. 557. Aph. a.fl to flood, submerge, $\mathbf{I} 4$ of par. add: act.

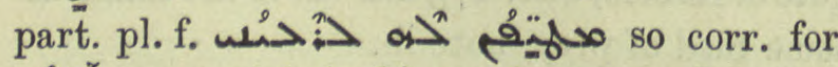
مدئهُ materials for biography are tooabundant for my mind, A.M.B. v. 4. $\mathrm{I}=$ Pallad. 5. 6.

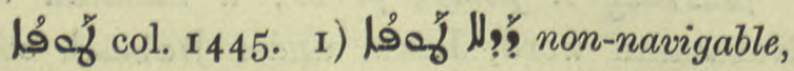
Sev. Ant. Vit. 253. 2. 2) a raft, pl. lö
Hist. Mon. i. 205. I 8, described ii. 397 ult. ff., פو loof a raft made of reeds, Patr. Or. iii. i. 49. 3 ; Dion. 84. I I ; a ship, Pet. Ib. 23.14. 1ต์ if col. 1446. the omentum, ref. Med. 291. 1 .

A.

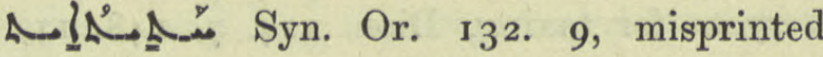

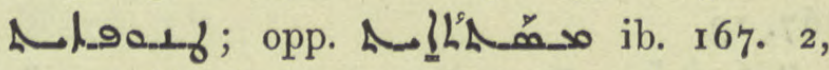
opp. مثٔهحُ ib. I72.29; and so correct the same passage in R.O:C. iv. 258 . 10 10 مه



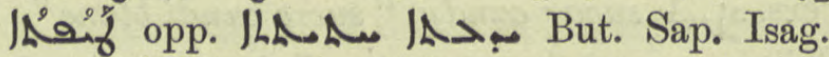
iv. 2, 年

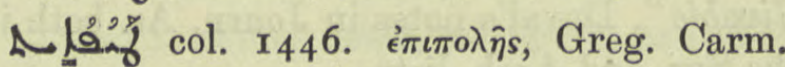
ii. 19. I4, obiter, : $\infty$ / Ala op Eph. viii. I7; carelessly, a hireling is less good than a bondslave because he reckons himself an outsider

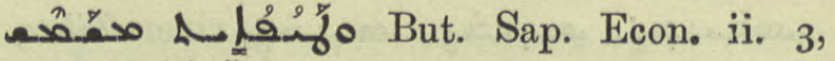

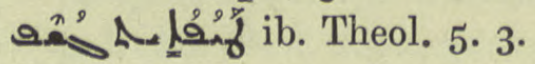

lenf $\rightarrow$ with $\prod_{6}$ col. I 447. so correct. non-navigable, Jac. Edes. Hex. xix. 5.

oof prob. Taif, mentioned with Ihirta, Sassanidi 36. I 4.

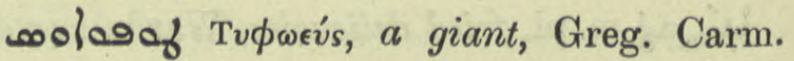
1. 100. 6 .

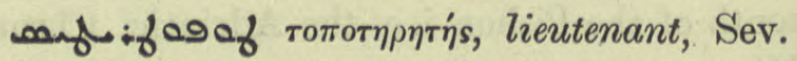
Ant. Hymns 721 tit.

م م0 of col. I447. a typhoon, Išoyahb ed. Duval 102. 7 .

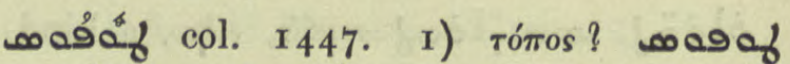

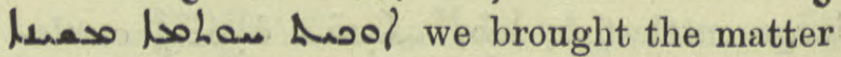
to a peaceful settlement or conclusion, Sev. Lett. 59. 18, 175. 3. 2) тúnos, a form, draft,

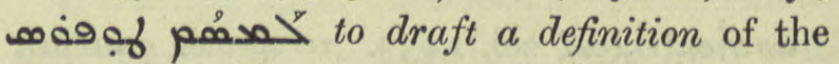

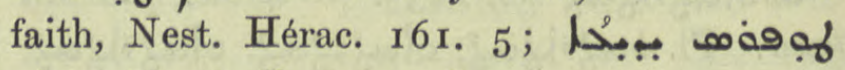

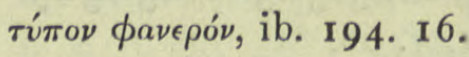

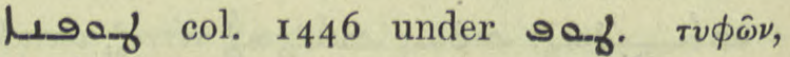
a cataclysm in general, whether caused by fire, wind, or water, Chabot, Journ. As. I 908 . I 42.

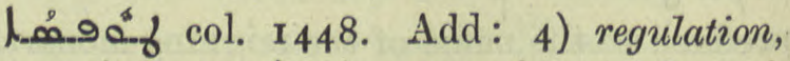

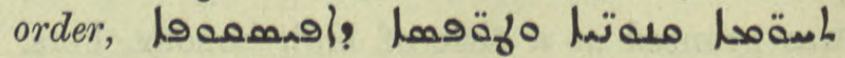
Sev. Lett. 204. I, 368. 16.

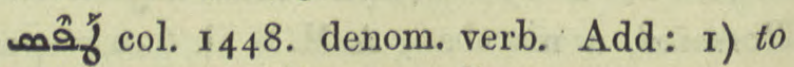

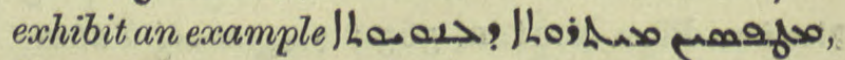
Pet. Ib. 32. 20, 2) to regulate, mark out 


\section{Kiareats}

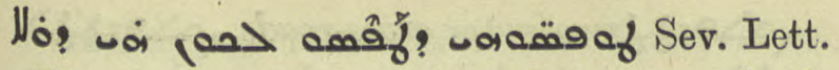
368. 16, ib. 153. 7. 3) to impress, make the impression of a seal, ib. 262. 3 ; cf. ib. 27 I infra, 272.

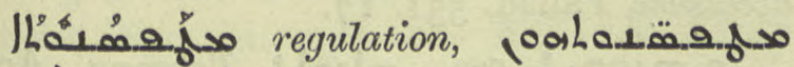
(:Sev. Lett. 400. 9.

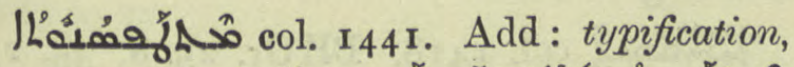
inner meaning, J Lenn

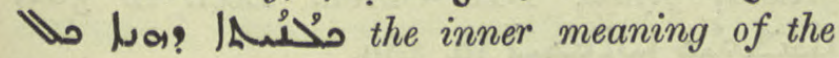
entire order of the universe, But. Sap. Theol. 4. I infr.

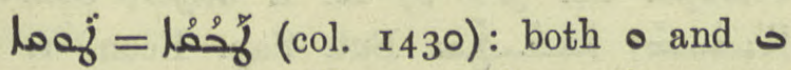
are pronounced alike and form the same diphthongs. A pan for frying or baking, Op. Nest. I I 5. 6, Nöld. in loc. ZDMG. xxxv. 499.

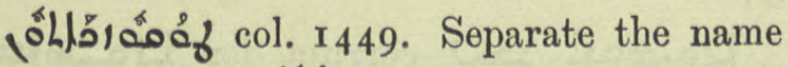
from the title (oLlb q.v. col. I 669. . Tukōs or ló? Docouz, first and Christian wife of Hulagu, Jab. II4. I. Misprinted ๓oof ib. 9r. I.

;of, fُ col. I449. to fly, move swiftly,

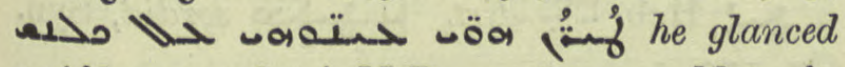
swiftly round, A.M.B. v. 88. 9. Metaph. $-y$ J did medical science turns swiftly to any means of relief, Is. Ant. ii. r10. 4. Aph. محف| ماله

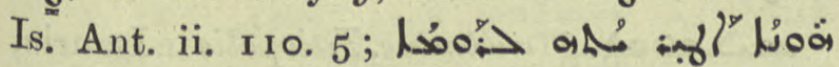
the mind takes upward flight. Jac. Sar. Hom. iii. 3 ult.

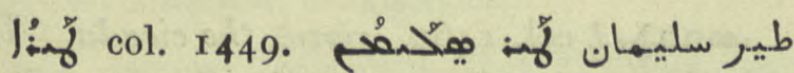
Solomon's bird, the hoopoe, Med. 593. I.

حــــ Melitene but prob. legendary, Patr. Or. v. 5 . 707 ult.

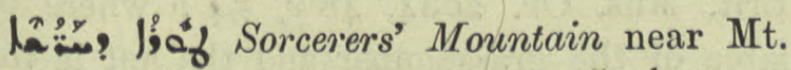

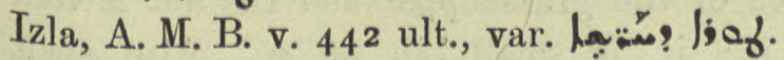

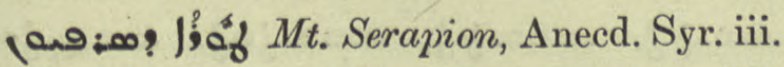
52. 4, a better reading is 10.0\% $M t$. Syraphtim, ib. 53. 6, г 3 .

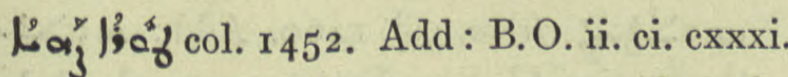
rog, 127,218 ; Dion. 55. 20. Also the southern slopes of Mt. Taurus, note ib. and Dion. Ined. = R. O. C. ii. 6r. I.

|’o col. r 449. Add: I) a space of time, إم all the summer, Mar Bassus 23. 306; the liof a long time, Ephr. Ref. i. 1 I 6.23 , ii. 33. 39. 2) distance, M 1 ) jos for some distance, A.M.B. v.

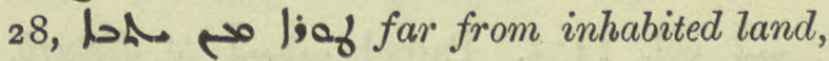

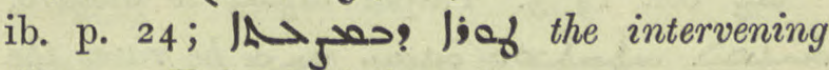
distance, Ephr. Ref. ii. $3^{2}$ ter, 33 ter.

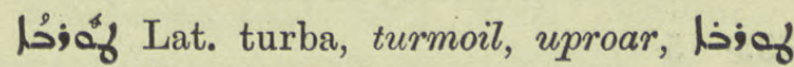

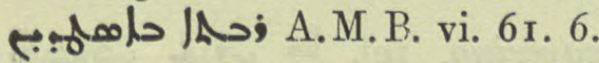

.

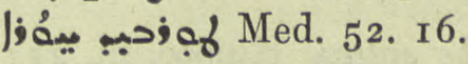

enjof Turgas, a monastery near Apamea, Sev. Ant. Vit. Ir I, 2, Nau in loc. R.O.C. v. 95 .

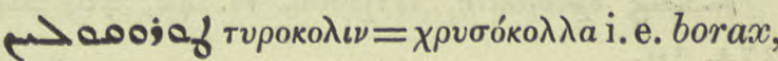
Chimie I4. II.

(Fiof col. 1453. a jackal. Add: Pehlevi Tūurek, Pers. تور s, Kal-w-Dim. 72. I3, 284. I I, 286. 22.

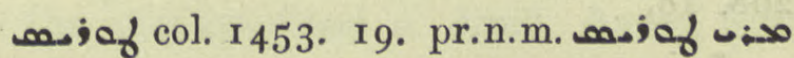
Martyrius i.q. Sahdona, Bishop of Mahoze d' Ariwân, Chast. 14. 16, 49: 10, 67. 12.

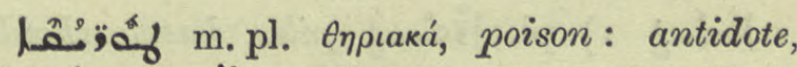

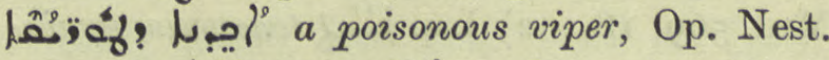

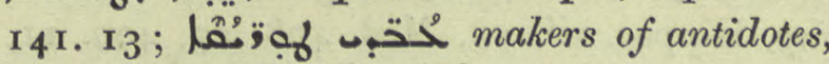

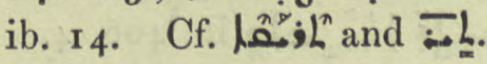

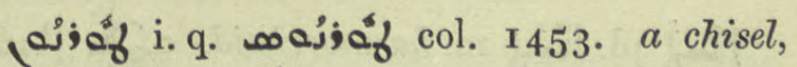

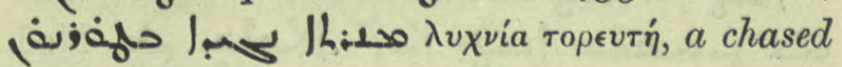
candlestick, Hex. Ex. xxv. 3I, 36; דمفي صoridon. Syr. ii. 24. 23 in Cant. v. 14 , Chimie 8. $\mathbf{6}$, N. Hist. iii. 2. $\mathrm{r}$.

masiof var. nosujof the Tyrrhenian Sea, Jac. Edes. Hex. xi. ro.

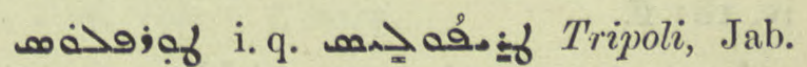
37. 15.

a of of col. 1453. Metaph. to superimpose, حصد Bar Penk. 59. 6. Pass. part. He.

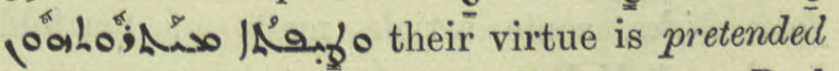
and superficial, But. Sap. Eth. 3. 4. Pael act. part. حبم a) oney smear it with honey, Kal-w-Dim. 93. 15 : Wr. suggests خטمير. 
Lif col. 1454. anointing; liniment,

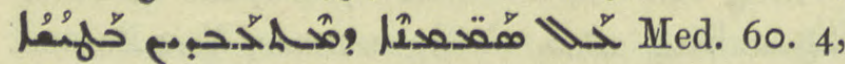

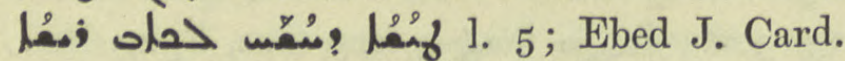

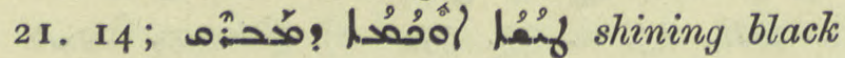
paste or liquid, N. Hist. viii. 4, 9.

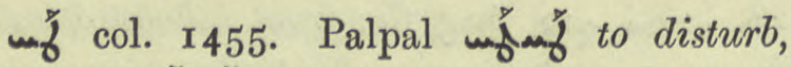

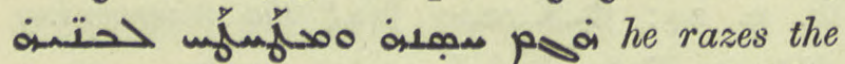
fortress and drives away its inhabitants, Bahira 233. 15 .

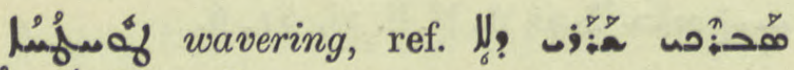
س

lling col. I455. the spleen, Hippoc. iii. 21 ;

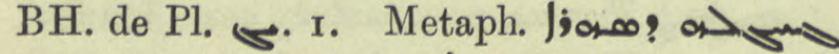
| Caus. Caus. 220. 10.

然 splenetic, Hippoc. vi. 42, 46, Med. 274. 19.

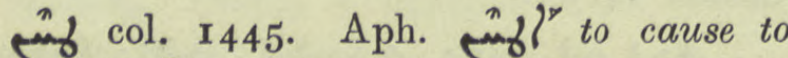

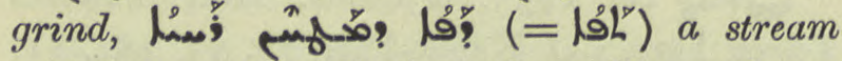
which turns a mill-wheel, Gest. Alex. 26r. I, 268. 18.

أم

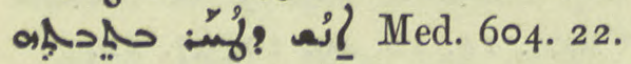

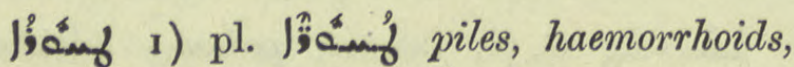
Med. 569. 18. 2) I. q. J ing, Hippoc. vii. 25, Med. 408. 23, 409. II,

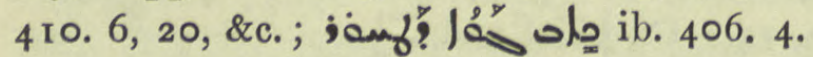

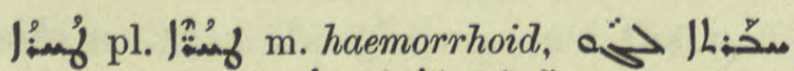
a) 407.15.

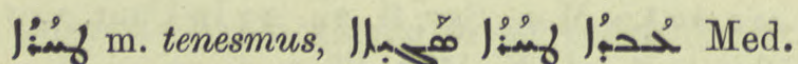

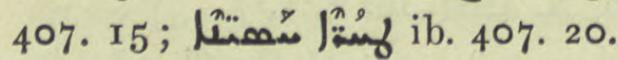

คُ iv. $18 \mathrm{I} \mathrm{ff}$.

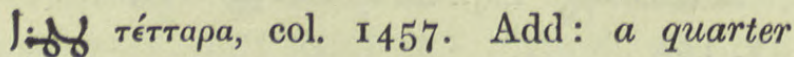
(drachm), BB. under (- Thes. 887 infra, DBB. $567 \cdot 4$.

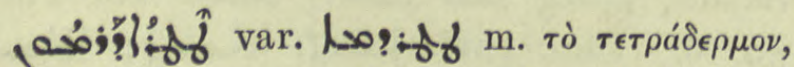
four hides, some article made of fourfold leather, A.M.B. v. 555. 8, 556. 2, 557. 14.

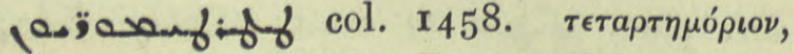
quadrant of an astrolabe, De Astrolabe 74 ult.,

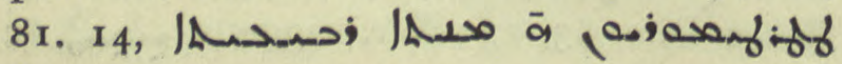
ib. 239.7 .

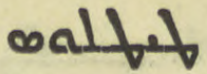

(a) بم: bring to the nearest crossroad, public places, Dion. 39.3 ; فحُ ? thou art open on all four sides $=$ too accessible, Pallad. 531. I7.

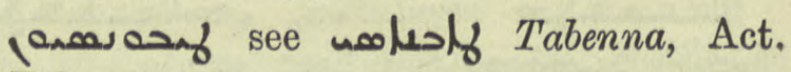
S. Pelag. I. I r.

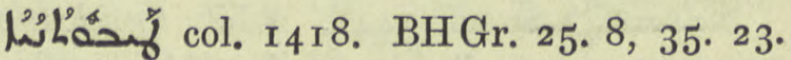
graced, gifted, BH. Chron. Eccl. ii. $3^{81}$, .

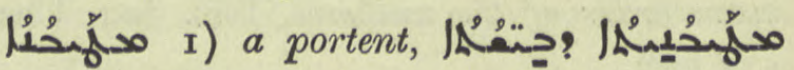
Pléroph. 26. 5. 2) m. a buyer, caterer, مut. Sap. Econ. I. I. 3) contraction of $\}^{2}$ ? 1 am ready, Kal-w-Dim. ed. Bick. 78. 22.

配 one ready, prepared, BHCarm. 34. 2 .

Hanesal saf corrupt for Hamolef a Tabennesiot monk, Anecd. Syr. iii. I76. I3.

מ.

whing Jac. Edes. Hex. xix. Ir. See wloter.

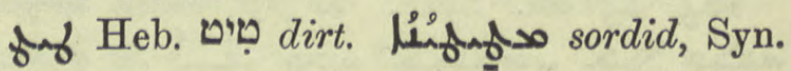
Or. $\mathbf{1}^{2}$. $\mathrm{I}$.

firf col. 1459. white spots on the eyes,

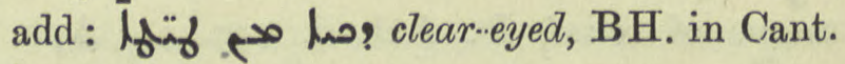
iv. 9 .

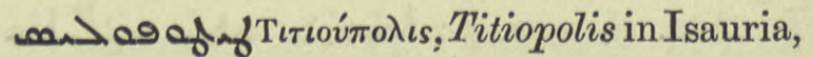
Pléroph. 43. II ; مolland Nöld. F. S. i. 47 r. 74 .

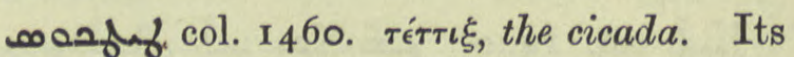
chirping was a pleasure to the Greeks and it was customary to keep it in a cage whence the Syrians imagined it to be a bird, Hochf. Fabel I5, Natur 28. I6; 1:Brit. Mus. Or. 2084. $48 v, 54 v$ where the fable of the grasshopper and the ant is told of it; it is small, ib $56 v$. Also spelt

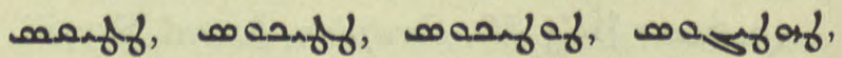
nasorstor, \&c. The Lexx. confuse it with the parrot and the nightingale.

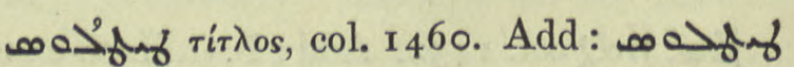

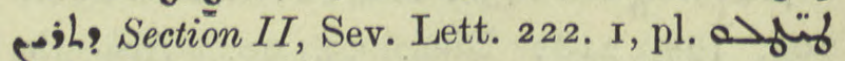

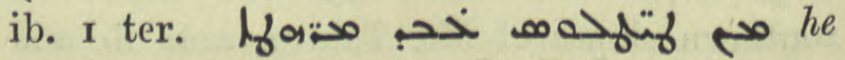
puts in a claim to riches, Jo. Tell, 60, 16. 


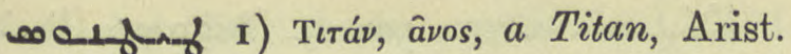
Apol. a. 7. 2) тітӑvos, lime, Chimie 6. I. Cf. oofy infra.

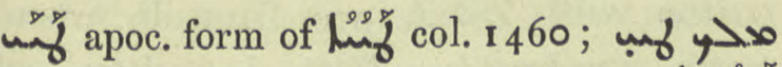
Anecd. Syr. iii. 247. 8. Add: pl. אר

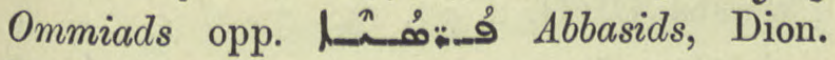
Préface xx.

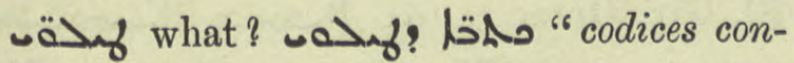
fessionis", these were certificates of nonNestorian opinion, BH Chr. Eccl. ii. 85 .

pasarf col. r46r. See parary Thes. and Suppl.

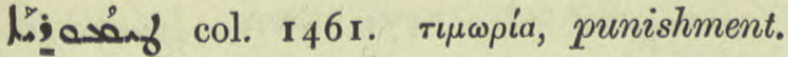
实 a penalty hath been decreed, Pallad. $73, \mathrm{I} 4=$ A.M.B. v. 96. II, theso kjaxan Jul. 160. 7 .

lasenf rauteion, the exchequer, the state, treasury, Syr. Rom. Rechtsb. 30 ; I w J Jow (ansand his house shall be confiscated,

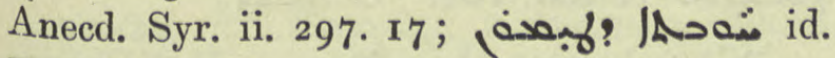
Pallad. 40I bis.

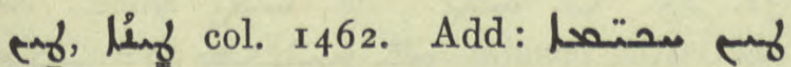
lutum sapientiae, philosopher's clay for sealing bottles, \&c., hermetically, Chimie 20. 9;

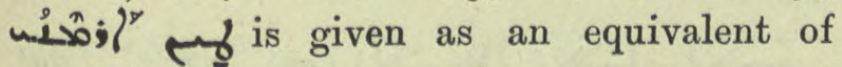
vermilion from Sinope, also as pafex

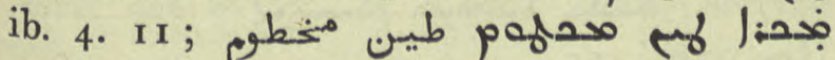
vermillon, verre scellée (de Lemnos) c'est à dire hématite, ib. 1. 4 , transl. 8 , n. 2. It was officially stamped, cf.

กु้ denom. verb from ling to daub, coat with clay, Aif? Uls the vessel thou hast

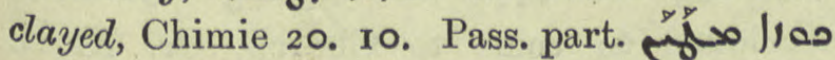
a phial coated with clay, ib. 38. 4; 96 ult.

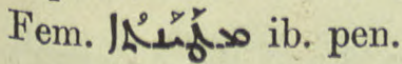

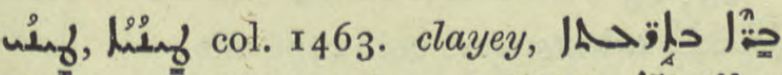

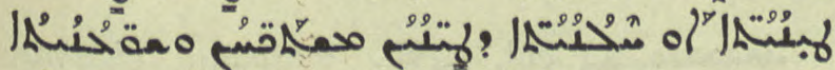

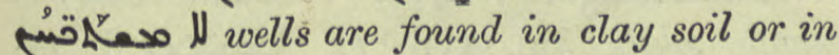
loam but not in rocky land, N. Hist. iv. I, 3 . Metaph. I f pastilles made of musk with a viscid base, Med. 605 with gloss. الراملك والسـدك.

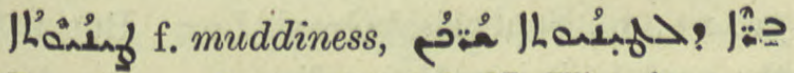
wells where only mud is left, N. Hist. iv. I. 3 .

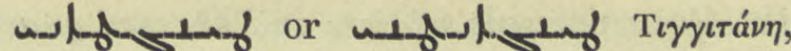
Tingitana, a province of Mauretania, Jac. Edes. Hex. xxxiv. 15, w/fhrof ib. xix. I1.

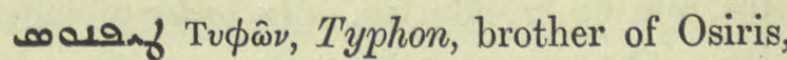
Arist. Apol. me ter, maarf 1. I 2.

forsian title, Captain of Archers, Phet. I I. Io, Pers. Mart. 61 n. 535 in loc.

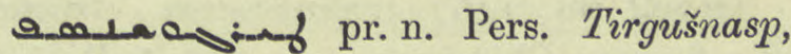
Jesus-Sabran 5 I I. I 8, Nöld. WZKM. xi. 188.

वैing col. 1464. Tirhan, a diocese on the Tigris, south of the Lesser Zab, Pers. Mart. I88-r91, Hist. Mon. i. I41. 17, Lag. Arm. Stud. No. 2245 p. I $5^{2}$.

axoind Pers. slofing the first month of summer answering to par syn. Or. 69. 26 .

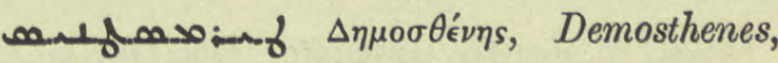
Gest. Alex. I27. 4, ZDMG. xlv. 318. 3.

| in the neighbourhood of Amid, Anecd. Syr. ii. I19. I0.

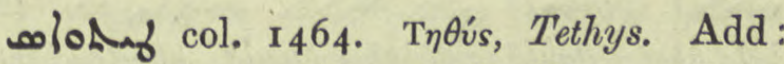
BH. ap. Hebraica iii. 25 o, Or. Xt. v. I6, n. 2.

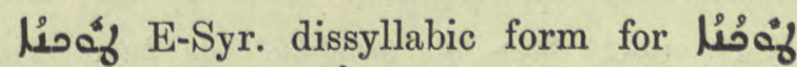
col. 1465. fraud lipof Op. Nest. 18. 13; Ebed. J. Card. 26. I0, I 26 pen.

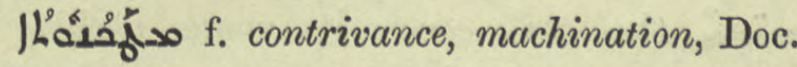
Mon. 36.8 .

Khة.

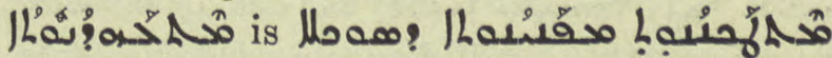

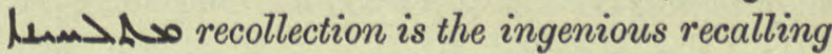
of a thought which might have been effaced, N. Hist. viii. 3.5 infra.

1ُ

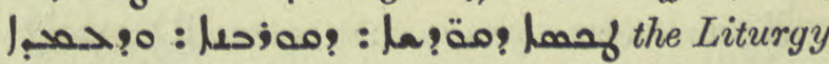
and Baptismal Service, Takhsa title page.

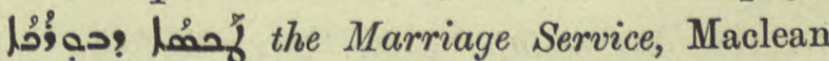
and Browne 233 ; بـ

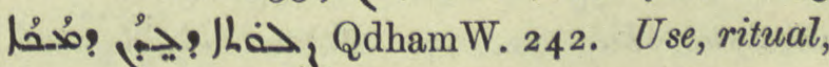
إم fمal according to the Chaldaean use, Brev. Chald. r. I.

ח

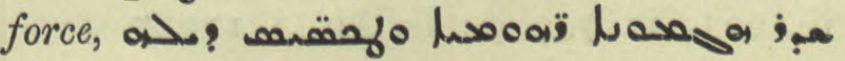


the Governor sent soldiers and his own guard, Hist. B.V.M. 181. 9; 202. 21， 208. I9; lo:90a! menef the Prefect's Guard, Sev. Ant. Vit. 60. 2, 283. I 2. An order of angels,

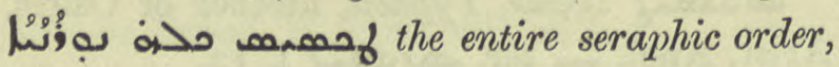

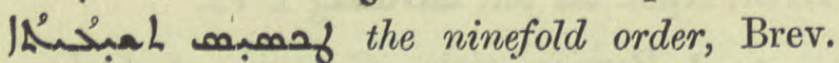
Chald. « Maclean.

lمُْْớ col. 1467. organization, structure

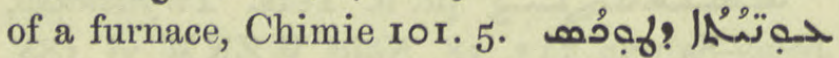
م. the eve of each month, they begin tri: order the month and bless it, QdhamW.183. 5. lïil nof the consumption of the Eucharistic species remaining after Communion, Maclean.

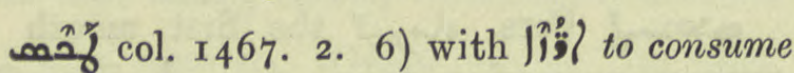
what remains over of the Eucharistic species when the communicants have received, Maclean.

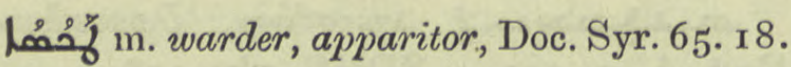

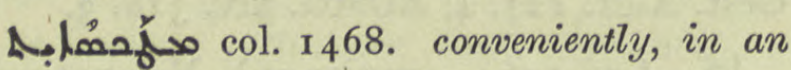
orderly manner, QdhamW. 33 pen.

If $\mathrm{Pa}$. W

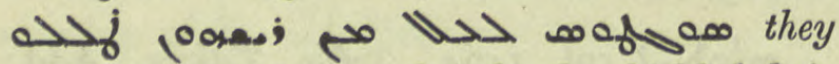
held their shields over their heads, shielded their heads, Josephus vi. 5 pen. Aph. r) "रfं" add: all las his buckler, Josephus vi. ro pen. To make

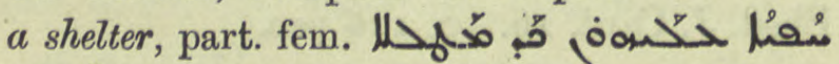
$\alpha{ }^{n}{ }^{n}$ the stork covers its young making $a$ shelter with its wings, Natur 36.12 .2 ) denom.

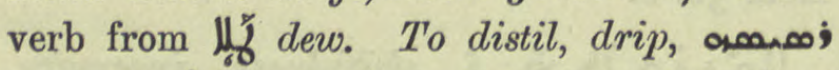
דحu the dew of the Lord in quietness He distilled upon me, Ode Sol. 35. x.

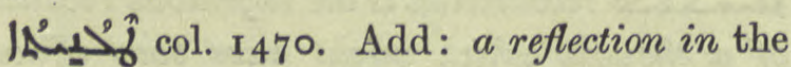
water, Hochf. Fabel $3^{6}$ f., shadowy apparitions, Pallad. I7. I5.

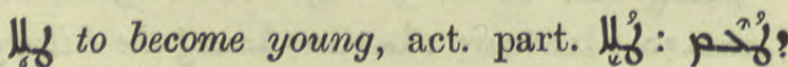
لy recovers his youth, Ephr. Syr. iii. $5^{84} \mathrm{~F}$.

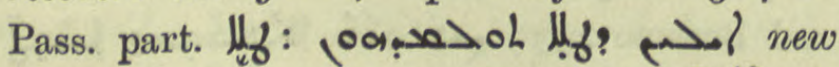
disciples, Jul. I2. I7. Ethpa. u1473. 3 of par. to receive fresh youth, Jhen

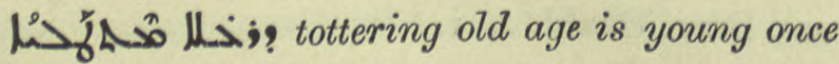
more, Sev. Ant. Hymns 45. 7 ; Ephr. ed. Lamy iii. 605 ; iv. 713.9 ; Is. Ant. i. I10. 7 .

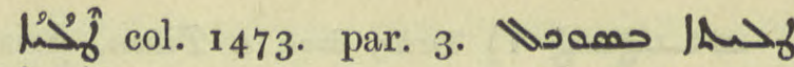

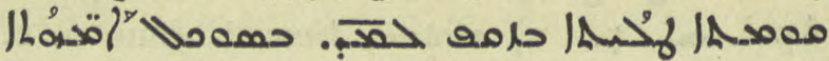
I in the sense of stature, youthful is written with Zekafa over Lamadh, when it means handmaidens Teth is written with P'thakha, BH Gr. i. 33. I 5 .

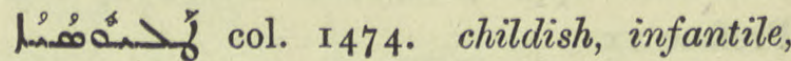
El. Tir. Gram. 3. 6 ; 1

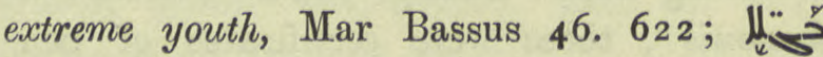
|

cólly pr.n. a country or city near Kashgar, Jab. I9. I I.

ترنجبين ترنكبين a kind of manna, Med. 296. II,

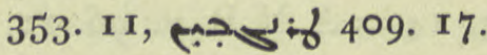

long col. 1475. from $\sigma \tau \lambda \epsilon \gamma \gamma i \delta(a)$ or $\sigma \tau \in \lambda \gamma^{\prime} \delta(a)$ a curry comb, Ar. Fremdwörter I I 3 .

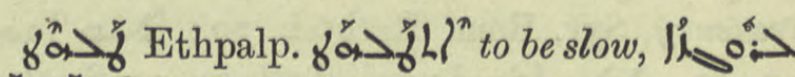
fa्

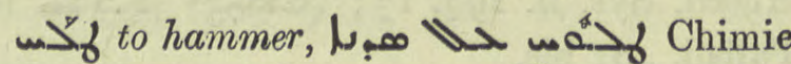

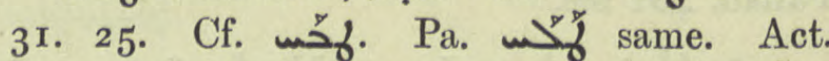
part. with pronoun a ㄴ) when you have beaten it out thin, ib. 27. 6 .

س narrow, لحم Jac. Edes. Hex. xlviii. I5.

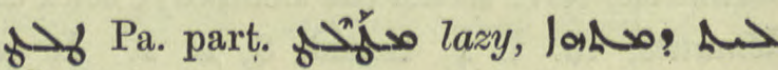
o

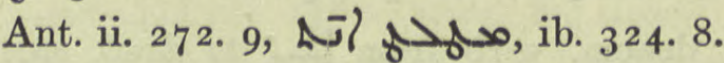

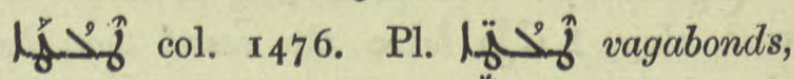
forbidden to come to Chürch, Or. Xt. i. 94. 6 .

A 1 Gest. Alex. 200. 8 and BA. Correct 贷

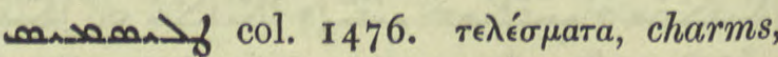
magic spells, Jul. xvii. 12, 1.

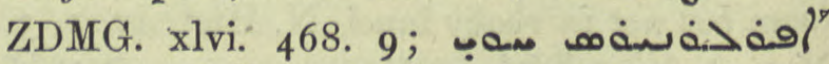

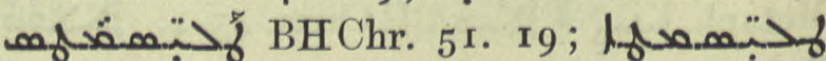
amulets, charms against the evil eye, $\mathrm{N}$. Hist. viii. 4. Rites, ceremonies of the Church, Jab. 52. 12 ; cf. Sophocles' Greek Lex. But from the context it is rather relics. 
طالشفر Pers.Arab. طمالسمق: bark of a tree of Malabar, Diosc. i. III, ii. p. 392, Ar. PflnN. 182; Lag. G. A. 50. 132. Med. 306 ult., 307 bis, 36 r. $4,369.5$.

p>d Pass. part. pad col. 1477. F. pl. IA Doc. Mon. 33. 9.

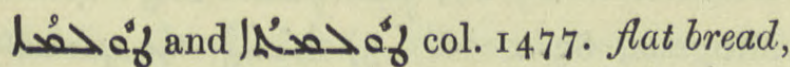
Fremdw. 35, BA. and Hunt. clxx. under col. 1693.

A Mon. i. 102. 18.

L., $>_{\text {f }}$ col. 1478 . Dalmatia, Nau in Mart. Pauli, R.O.C. iii. 51. 3, Guidi Trad. 52 ined.

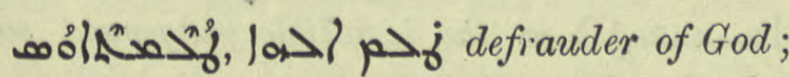
nickname given to the Catholicos Timothy, by his successor and opponent, Joshua, BHChr. Eccl. ii. I8I.

for for a talisman, charm, Protection 97. med.

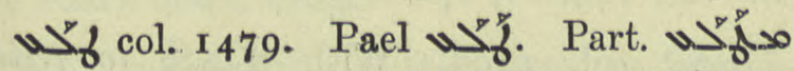
narcotic, causing torpor, BH. de Pl. 106; |

1حُْ

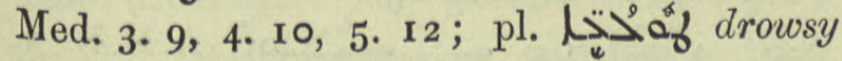
fits or torpor, ib. 3. 5, 193. 19.

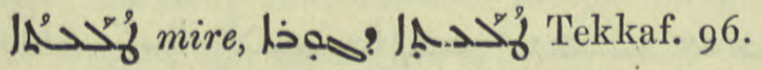

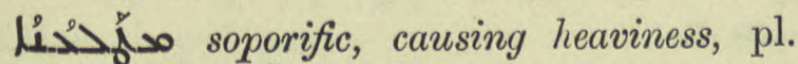

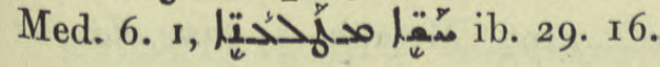

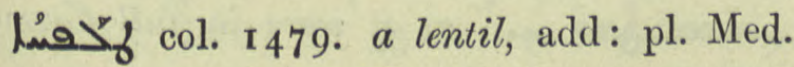

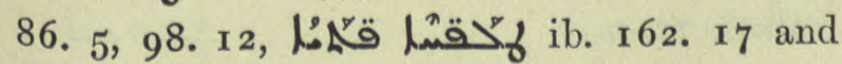
often. As a measure 23; a freckle, 585. 8.

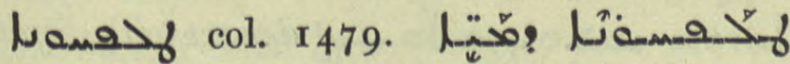
Lenticula stagnina, pond weed, Med. 39. I2.

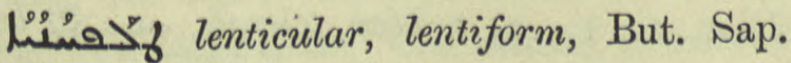
Philos. 6. 8.

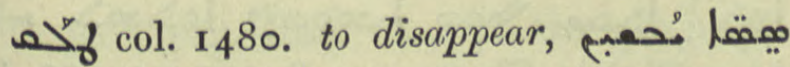
ب0 50 the warts will dry up and disappear, Med. 584. 9. Pa. Pa (๘), Ephr. Ref. i. I42. 2 ; to rout, put to 2716 ainast

flight, Patr. Or. V. 5. 722. 5. 2) Ar. طلق ii. to repudiate, divorce, oه مالخعم تقد Dion. 30. I 5. Ethpa. Q

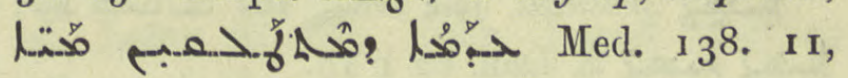
I 42.5 .

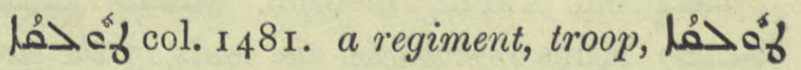
as 00 Patr. Or. v. 5. 722.3.

IAم of flat bread, BA. and K. i.q.hod of, IA $x$ of q. v. under bo col. 1693 .

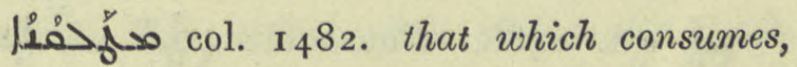
oه the earth consumes the life of all those buried in it, Caus. Caus. 207. 19; Doc. Mon. 4 I. s.

م talc, selenite, Chimie 2. 6. Various

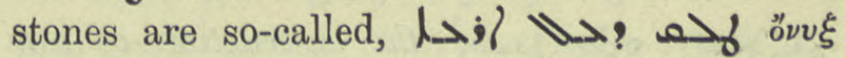

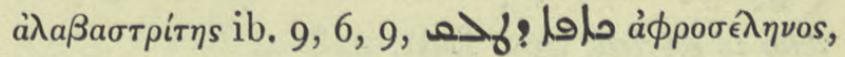
1. 7 , ảpíavтos, ib. 1. 9 .

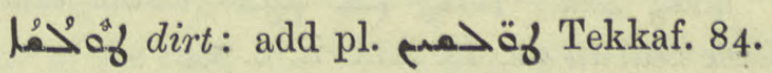

L.a $_{\text {f col. }}$ 1483. a mantle, BB. under col. I748.

p 1

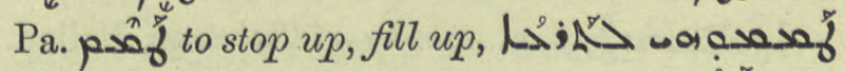

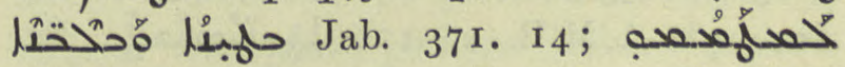
to fill up marshy ground with earth, Gest. Alex. 208. 14. Aph. pîे see p. I3 b. Ethpa. progh n $^{n}$ to be blocked up as rivers, Gest. Alex. 69. $14,16$.

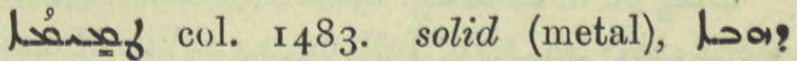

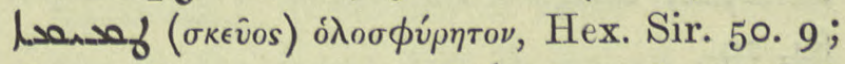
Gest. Alex. 233. 10; pl. إمتحم 1. 7; pl. f. /Aمتم ib. 2 I1. 8.

p.d Persian prefix to proper names: see par.

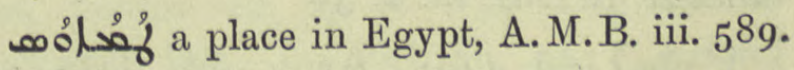

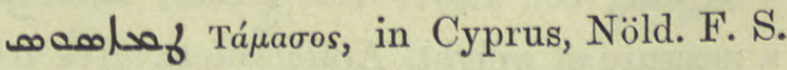
i. $47^{2} .80$.

人’

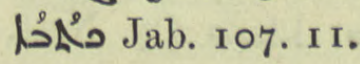

0:-0_2sof col. 1485. Tahm-Chosioes, a Persian general, Jo. Eph. 403. 2 I, 404. 8 : see ponf. 
Wš col. 1485. Infin. E1. Nis. 68. 79 .

? لll Sall San. 62 b 17 .

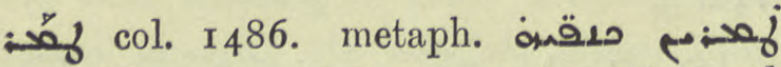
IA like a flood, Ephr. ed. Lamy iii 199. Act.

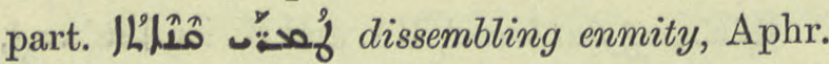
673. 16.

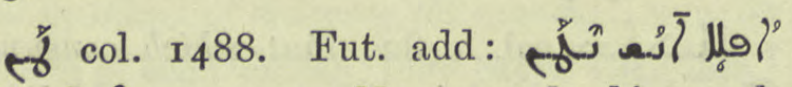

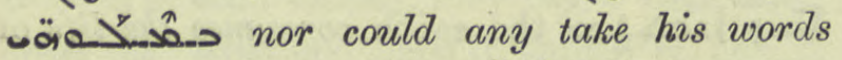

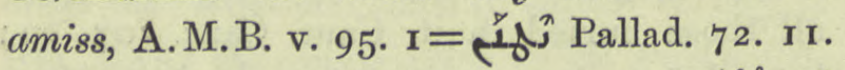
To be zealous, emulous, act. part. f. lif

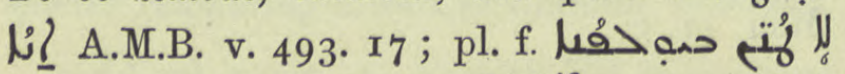
Natur 2. 5. Ethpe. "ishl to be envied, स: who is envied, Sev. Lett. 228. I.

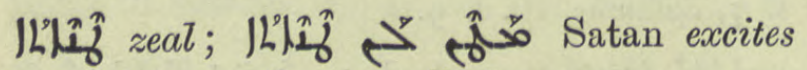
false zeal in us, Is. Ant. ii. 8. I4.

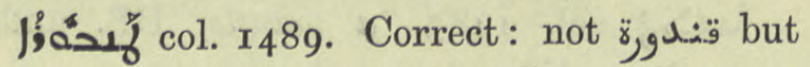
ف فندورة фávoovpa, Ital. pandura, French mandore, Span. bandurria, Dozy Suppl. ii. 284.

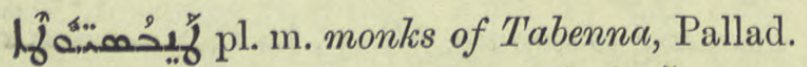
100. $7=$ A.M.B. v. 122 |م for, \&c.

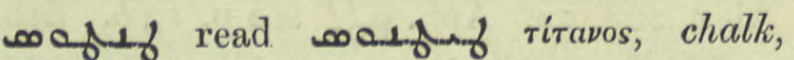
Chimie 6. I.

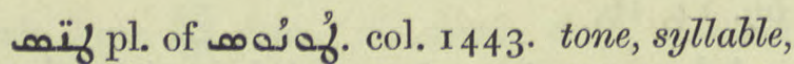

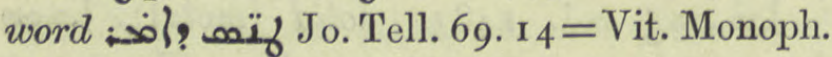
84. 20.

ल की col. I49r. to beat out metal, act. part. ميلّ BH. in Jer. xli. 7.

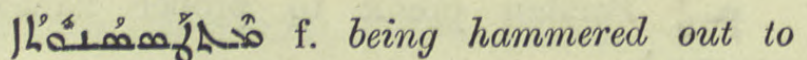
thinness, N. Hist. iii. 2. 3 .

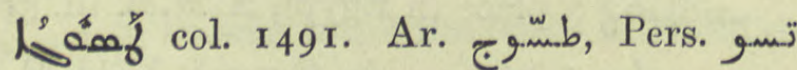
a measure of weight, Arabic dicts. give it as $\frac{1}{2}$ the weight of a carob or carat, $\frac{1}{4}$ of a tasg. But a ${ }^{2}=4$ carats, not 8 . Chimie 69 ,

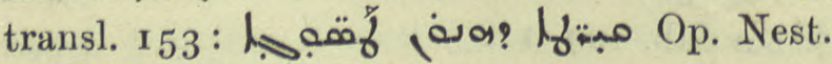
149. 19 in Mark xii. 4 2.

ر Indiction, Sev. Ant. Vit. 281. I ; Anecd. Syr.
146

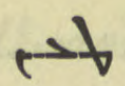

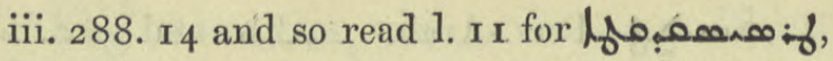
ib. 289. 24 and 254. 16 where Land has \}هم: مص مبمار

|حit has not escaped us, Jul. 21. 15;

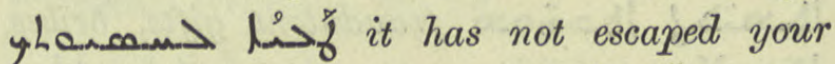

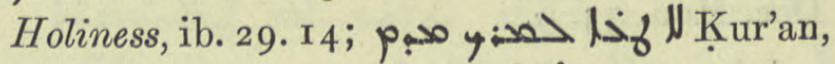
Mingana, 83 a. 18. 3) 人 add: the four planets are Kronos, Zeus, Ares,

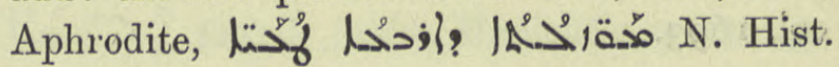
Bk. ii. cap. iv.

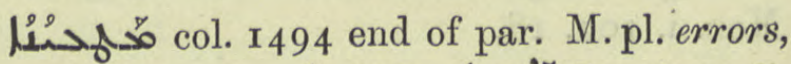
(2) Is. Ant. ii. 76. 18.

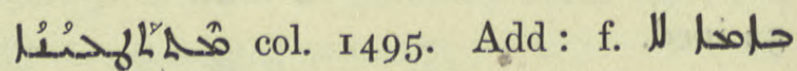
IA Sev. Lett. 278.3 .

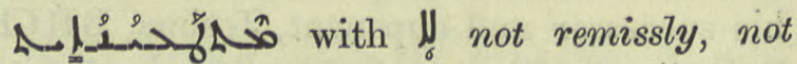
unmindfully, Bar Sal. in Lit. 64 .

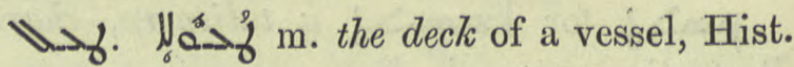
B.V.M. I 49. I I, I50. I 7 .

ค่⿱ appreciate a remark, Pallad. 7x. 10. Cf. |

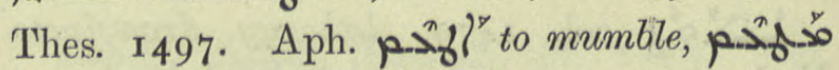

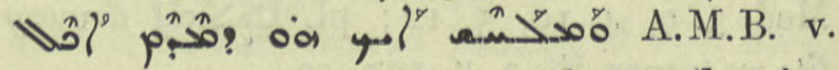
458. I. Metaph. to speak with taste, be witty עo 5 . 5 .

1.sด้ col. 1497. Metaph. I) taste, flavour

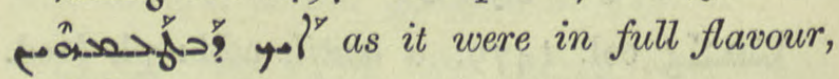
Pallad. 72. 9.

1 a passive (معم) but no example.

คै col. 1498. to take away, carry off, مـ and go on thy way, "va t'en honorablement", Dion. 22. I. 2) to bear, tolerate, سب f bear with me, Pallad. 320 ult. 3) to bring tribute 6 J.'? El. Nis. Chron. I50. 8, 201. 24, 209. I6, 213. 20, 216. 21. Part.

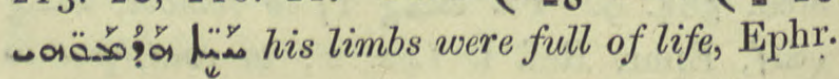


Jos. 5. I3; ; لحبَ full of encouragement, ib. 125. r. J ba a bodied, Med. 287. 2.

Lُลُ $\mathrm{m}$. a weighty or important matter,

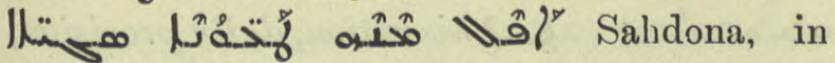
writing, let important matters slip, Hist. Mon. i. 47.14 .

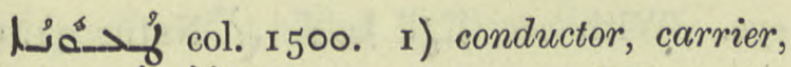
compressed air in the ear conducts sound, N. Hist. vii. I, 2. Astron. (Ptolemaic) the epicycle as carrier of a circle or of a planet, N. Hist. Bk. ii. iv. sect. 2 ; the eccentric as carrier of the epicycle, ib.

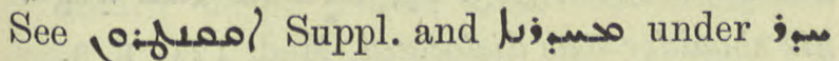
Suppl. 2) Ar. Lexx. under $\mu \infty$ col. 2676 and DBB. 1362 .

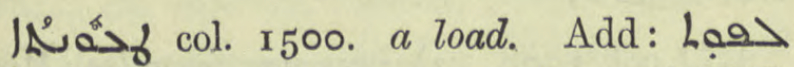
น iii. 592. I ; مصaح a load of money = payment, tribute, El. Nis. Chron. 2 I 6. II.

Lloar Syn. Or. I32.9 correct Aoloar: see above under $\curvearrowright \propto f$.

af col. I 5 ог to trip, halt in speech, زلح أ.

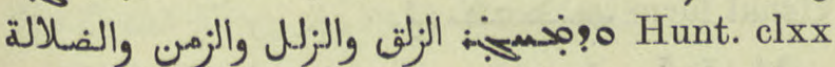
under JLe. مهe. Pael part. halting, limping,

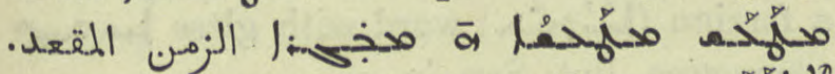

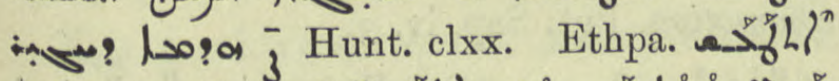

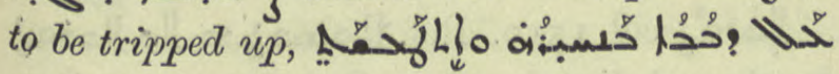
A.M.B. ii. 578 pen.

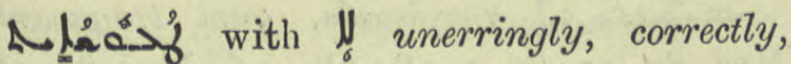
Ephr, ed. Lamy ii. 55. 8; so correct for Alearay.

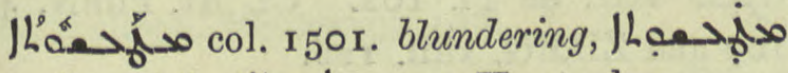

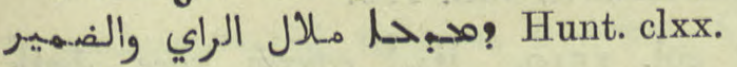

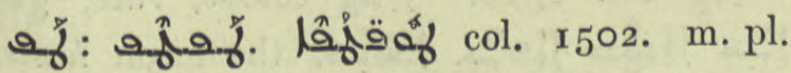
fickerings, Tekkaf. 59 .

laf col. I502. to press close, to cleave,

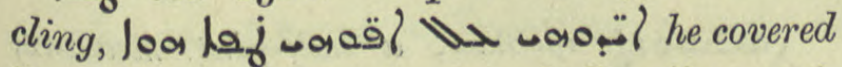
his face with his hands, Anecd. Syr. ii. 20. 6 .

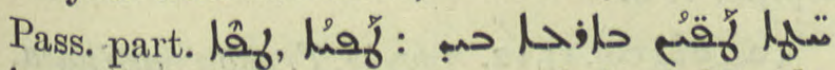
Hoon wheat clave to the ground from drought,

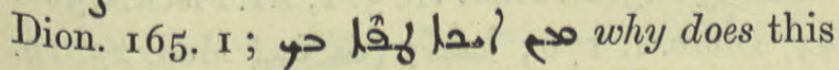
snake cling to you? Hist. B.V.M. 73 pen.,

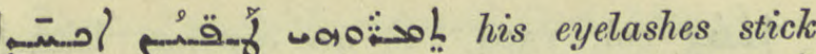
together, But. Sap. Econ. iii. 1. Pa. u글

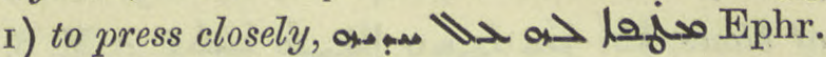
ed. Lamy iii 495, Ephr. Joseph. 308. 4;

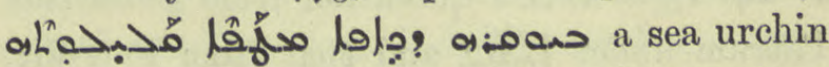
presses its light body against a heavy stone,

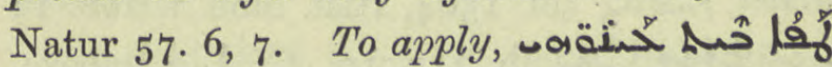
apply (salve) on the forehead, Med. 559. 9;

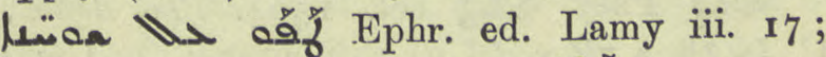

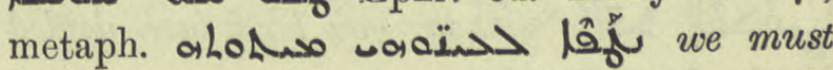
force on his notice the fact of Joseph's death,

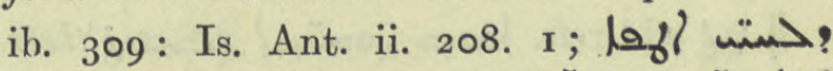

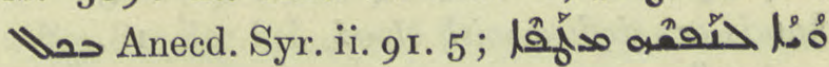
Jo̊ā A.M.B. iv. 537. r.

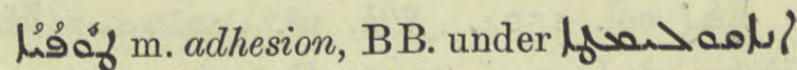
col. 253 ; مص incoherent words, id. under la? la col. $33^{\circ} 5$, DBB. 796.4 .

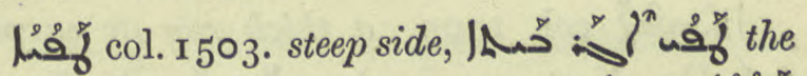

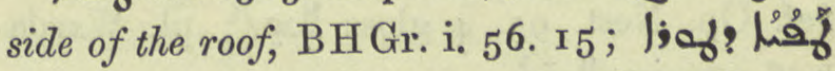
steep ascent of the mountain, Mar Bassus 32. $43^{6}$.

liْ a application, bandage, Med. 47. 19.

L̊ำ f. gram. a point, mark, Hist. Ant.

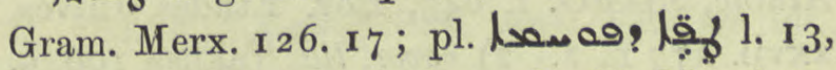
r 24. 6. Of a plant, perhaps Orchis Maculata, lö it has marks, is spotted, Med. 600. I.

19. a small place in the neighbourhood of Nisibis, A.M.B. i. 485 .

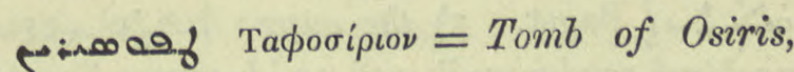
a fort in the desert, Pet. Ib. 67. 18, see note to transl.

2) to strike, smite, سوقُ he strikes his face with his hands, beats his

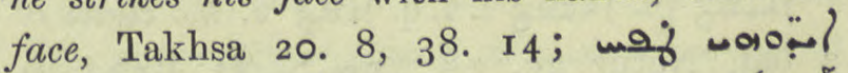

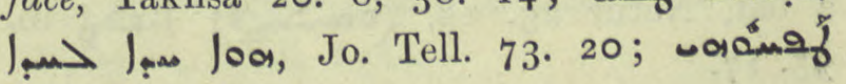
Hist. Mon. i. 103. 4.

مشُصس احفخف

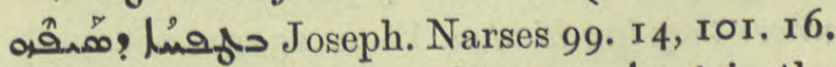
Three manuscripts have lïg for lo: following passage: 1. م م 0 they tremble at the sound of a leaf or at the rustling of trees, Is. Nin. B. 280. 6. 


\section{ruaf}

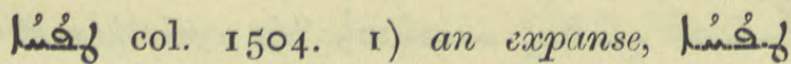
hea?, Protection 84.14. 2) beating, sound

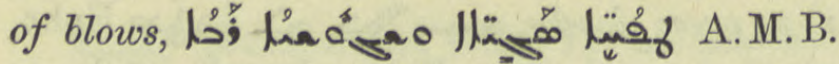
v. 24 . $15=$ B B.'s quotation from Paradisus Patrum under fín and that from Pat. Vit.

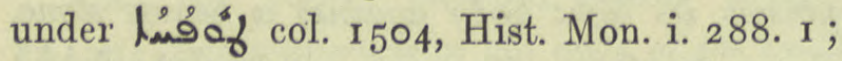

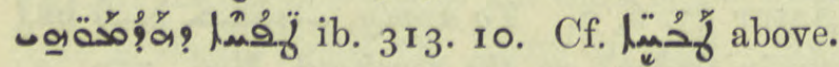

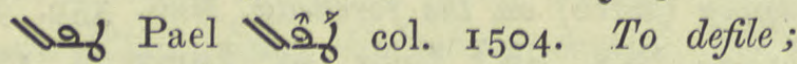
管 by the Devil, Hist. Mon. i. 226. 9 .

لllo을 add refs. hands are defiled with blood, Jul. 98. 18. See under llasaf above.

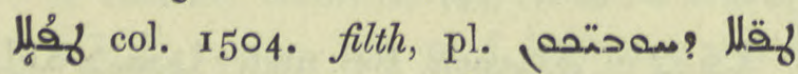
Ephr. ed. Lamy i. 81. 6 ; Syn. Or. 39. 1 7.

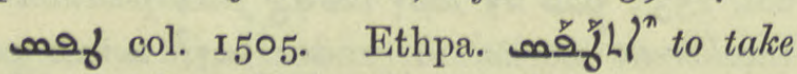
refuge, with 0, Ephr. iii. 41 3 B.

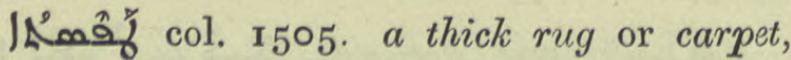
ref. Op. Nest. 95.4 gloss $\|_{6}$ ox '; pl. Warda $246 r$ marg.

ํํำ col. 1505. I) a pancake; usually written טפקא : Neo-Heb. Levy, G. A. Lag. 49. 126, طابـ modern Arabic, ZDMG. 1. 645, Rev. Études Juives, xxvii. 277 n. 2. 2) cover, lid, Takhsa ro6. 5 .

Jiी $\hat{f}$ col. ${ }^{1505 . ~ f i n g e r ~ o r ~ t o e n a i l . ~} 7$ of par. स: Mich.Syr. 282. I I Hist. Mon.i. I69. 2) claws, talons, add: "ح> o

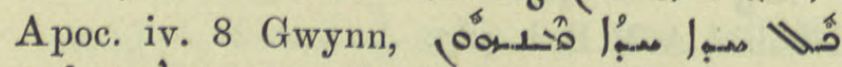

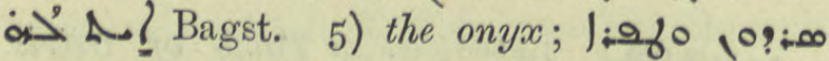

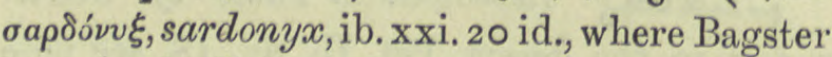

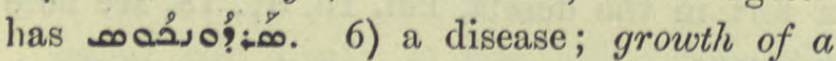
membrane over the eye, Med. 79. 7, liag

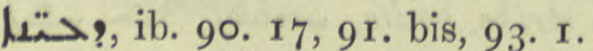

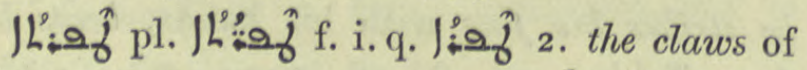

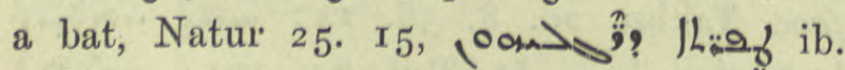
35. 17 .

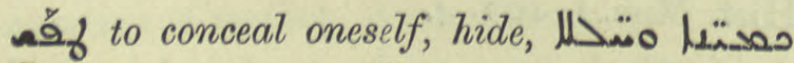
an foas? the lurking-places and hollows where they lay hidden, Dion. $\mathbf{5}_{5} .21$, act. part.

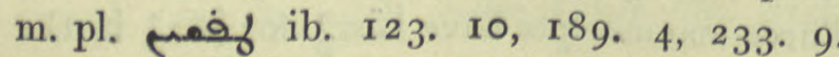

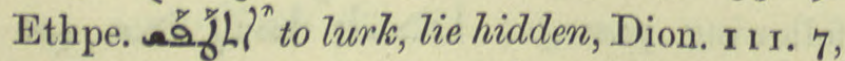
233. 12. But to take refuge, ib. 130. 23 : ef. ชăf. Nöld. on Dion. WZKM. x. 166.
مُ l.L... lost.

oflof takróv, a fixed sum or quantity,

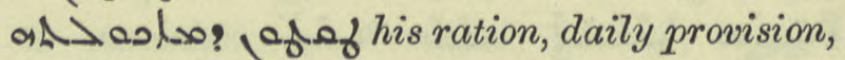
Alexis 8. 4 .

Jif and $u$ is to knock against, to put out an

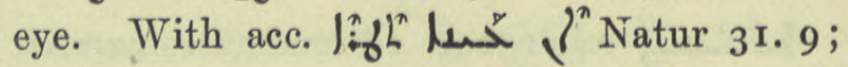

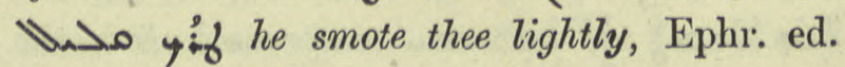
L:my iii. 67. To leave, let, ; ف ف let it cool, Chimie 20. 12, مَ - if ib. 50. I. Ethpa. -

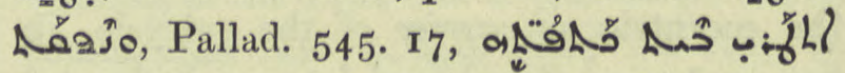
his shoulder was dislocated, Med. I 2 I. I, 3 .

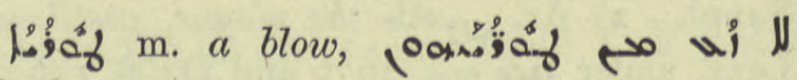
Warda $5^{2} r$.

Lُ:-6 col. 1508. 2) pressure, impulse, Med. 178.23 .

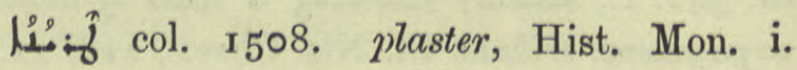
122. 16.

1حُ f. daubing, log

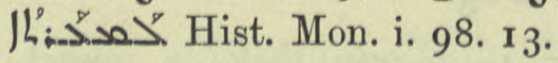

m>lol: Tripoli, R.O.C iii. $33^{8}$ n. 3 . Usual form

年 col. I510. mentioned by BH. as

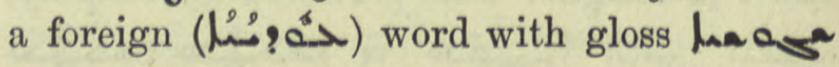
commotion, But. Sap. iv. 3 .

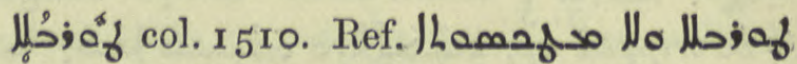
bustle and disorder, Syn. Or. 222. 25.

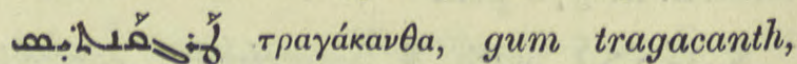
Med. $83.13,86.2,183$ ult., I 64 bis, I 81 r. I 8 ; | ك S BH. de Pl. го2. Cf. Ar. PflnN. 49, ro9 and ZDMG. xliii. 126.

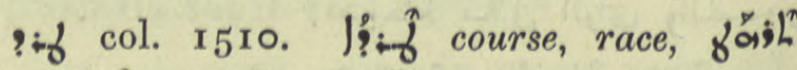

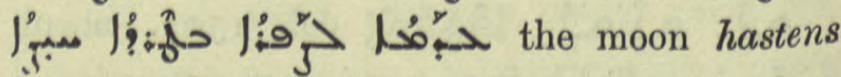
towards the dawn in swift course, Jac. Sar. Hom. iii. 75. 19.

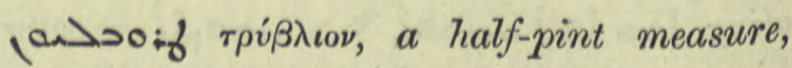
Epiph. v. 9.

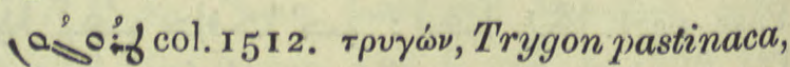
the sting-ray, N. Hist. vii. 3. I, Ar. FischN. 568 .

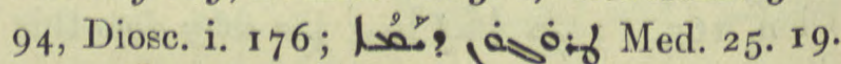
Cf. , d- d- 


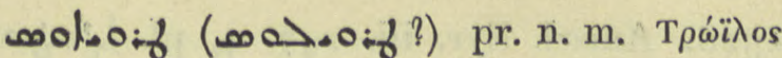
in Pamphylia, Nöld. F. S. i. 472.

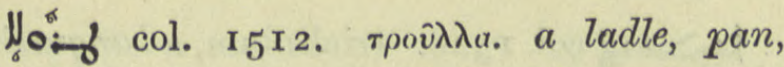
Chimie 32. 2 I, 84. 20; Med. 566. 3, 567. 7 .

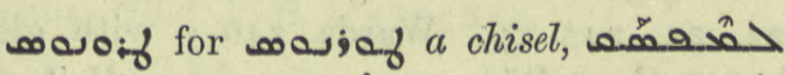

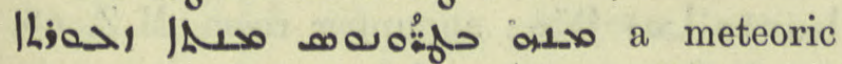
stone so hard that it was hardly possible to cut a chip off with a chisel, N. Hist. iii. 2. I.

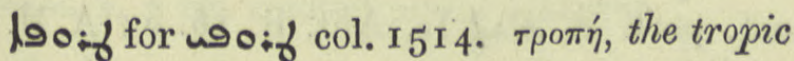
or solstice, De Astrolabe 80. 6, n90:f 1. 3, lojof l. II, loo: $84.3,6,245$. I5, pl. ल00:f ib. l. I3.

hoo:f m. pl. Turks, Ephr. ed. Lamy iii. 197. 2.

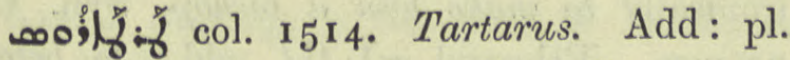
oilfy the infernal regions, Greg. Carm. I2 I,

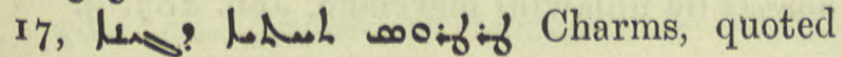
from the M.S.

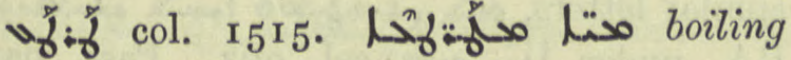
water, Brief Xti ror. 3 af.

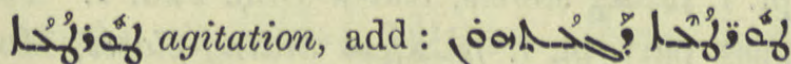
the vehemence of their distress, Jab. 1०3. 8.

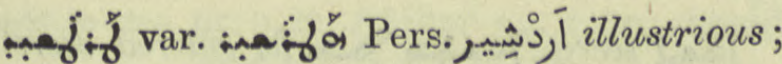
the Great King, A.M.B. ii. 576 ult.

iो. of the sting-ray, Natur 55. 8, See (a) of col. I5 I2.

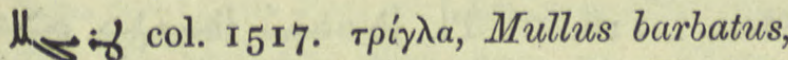
the red mullet, N. Hist. vii. 3. I, Ar. FischN. 568. 92 .

moafor if col. г5 I 7. tertian fever, Hippoc. iii. 20 , iv. 59 .

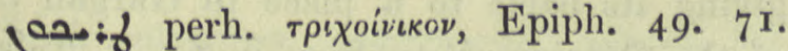
See Hultsch, Metrologie ro6.

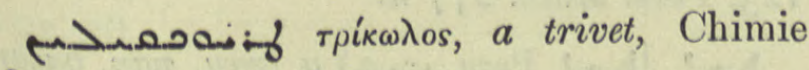
285 n. $\mathrm{r}$.

T róopxos, a kind of falcon or kite, N. Hist. vii. 4 . 5 .

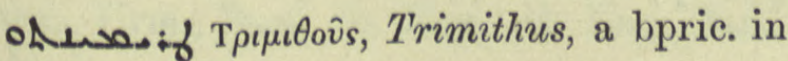
Cyprus, Nöld. F. S. i. 472. 79.

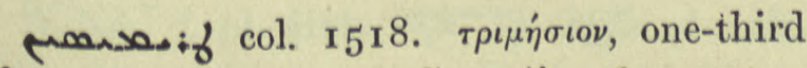
of an aureus, Anecd. Syr. ii. 26I quater, लmsoif Jos. Styl. 79. 2 ; one-third of a

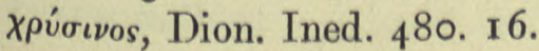

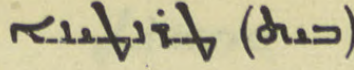

لـ

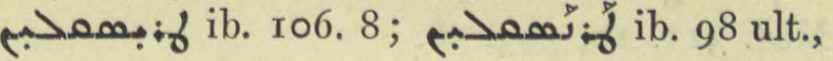
99. 3 .

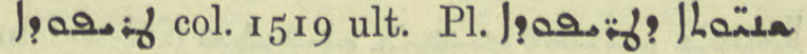
tripod ravings, i.e. Delphic oracles, Greg. Carm. 32. I 2.

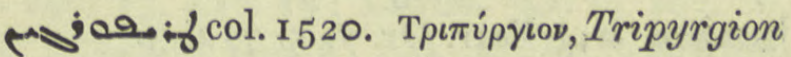
part of the city of Amid, Mich. Syr. 258. I4, 6 and 4 af., Anecd. Syr. iii. 206. 26, 207. 8.

10ز0فif col. I520. ashes, Warda $246 v$,

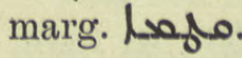

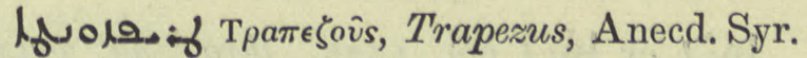
ii. 247.3 ; / 100 - Sev. Ant. Vit. 301 . 10. See $\overline{19}$ of col. I $5^{2} 6$.

لهُ

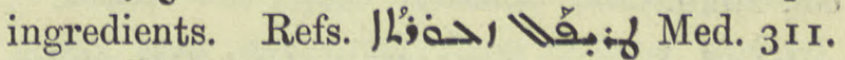

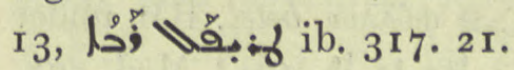

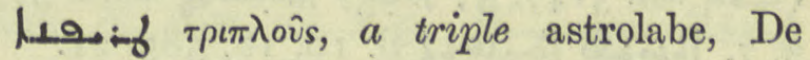
Astrolabe 74.

年 col. I52r. bono sensu expert in war,

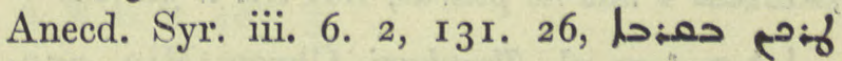
ib. 23 o. 15 .

صa $>$ if Tralles, a city of Asia, Anecd. Syr. ii. I84. 5 ; صollif Sev. Ant. Vit. 57 ult., 66. 4 .

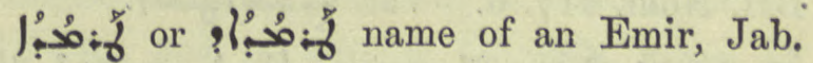
I 25.13 .

Msoif a village in the diocese of Amid, Dion. 22. $21,23.4$.

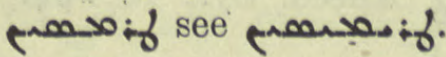

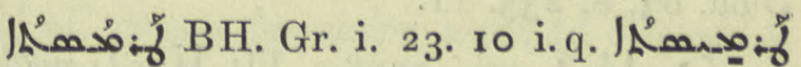
a sandal, col. I522. 7 of par., /Rُ |

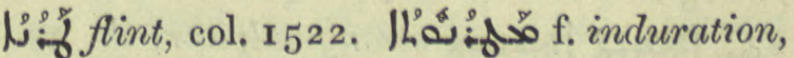

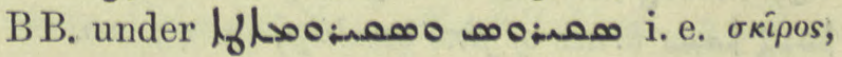
бкір

هله

I T Taranton, a fortress in Cappadocia, Brooks Chron. 574. 7, 582. 7, Patr. Or. v. 5. 743. II,

name of a prison in Damascus, A.M. B. iii. 340 . 
pa f a prescription for internal chills, Med. 311. ro, 393. 16.

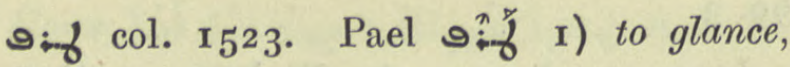
مsal-w-Dim. 70. 8. Cf. Arab. a...., a a look, glance. 2) denom. from ló IAjis o. Patr. Or. v. 5. 7I2. 3.

18. لُ the lobes of the liver, Med. 352. 6, 19. ปُ مخحفئl the edges or fringes of a cloak, Hist. Mon. i. 187. 13.

1ُ์ beats, Med. 224 . 12.

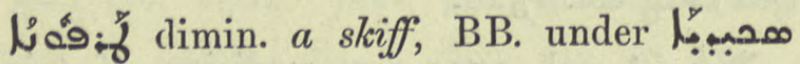
col. 2626 .

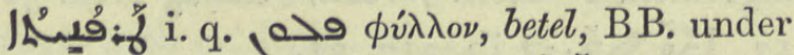

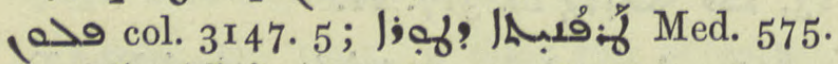

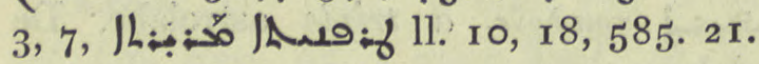

IRُ .

LAO.j m. pl. Terphaei, Chron. Min. 355 . 19, 20. Cf. فح

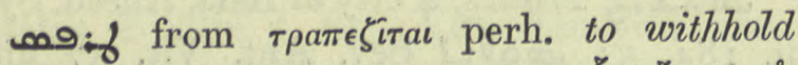

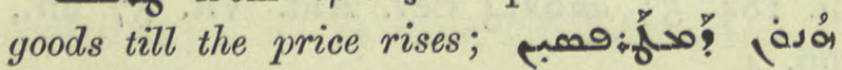
BH. Nom, 217.6 . Var.

लaid Lat. Tarpeius, Jjof Tarpeian rock, Anecd. Syr. iii. 50. 22.

م:- col. I527. to drive out, chase away,

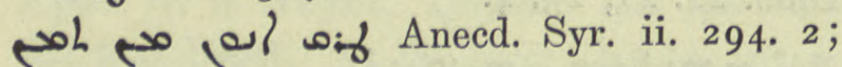
Dion. $63.8,235$. $1 \mathrm{r}$.

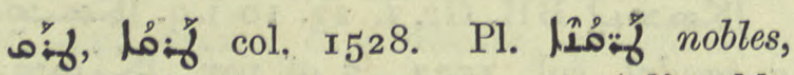
notables, Dion. 128. 18, 166. 24. Adj. add:

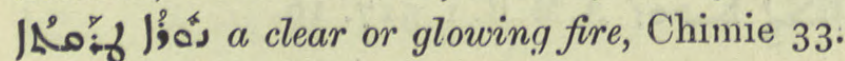

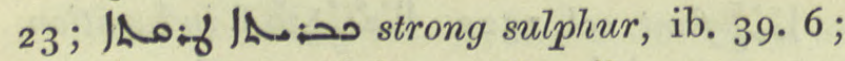

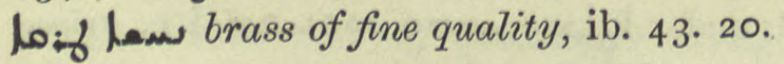

مof/fo:- трактатор, a treaty, Syn. Or.

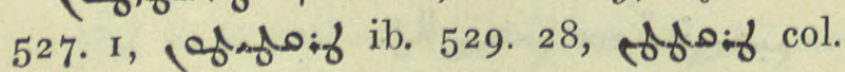

I528. Anecd. Syr. iii. 247. 4, pl. /o fford ib. $23^{2} .21,246.21$.

1. hall, 242. 22, 24, 245. 3; Warda $246 r$ with gloss

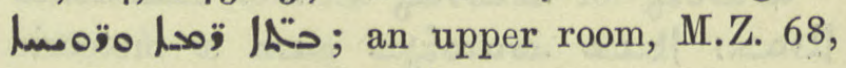
63 , S. George I $_{9} b 7$ af.

a. col. 1529. to daub, taint, with 0 , act. part. o $a$ if Is. Ant. ii. 16. 2 ; A.M.B. 445. ${ }^{3} 3$.

อ. R. Duval derive this from $\sim a_{-\gamma}$, Beit. Assyr. iii. 76, Duval gram. par. 176. Inf. ahenfind $>$ to besmear, bedaub him = ironically to make him a bishop, Syn. Or. 73 pen. Ethparel a f.jhl' add: to befoul oneself by unlawful marriage, Jab. 287.3 .

leg col. I529. Gram. to elide one of two

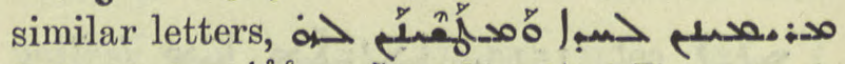
BA. under ling a col. 967. Pass. part. pl. f. |مُّم secrets, Kal-w-Dim. 226. I. Pael

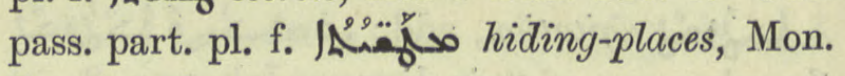
Syr. i. 92. 9 .

laef secret, Jand on he entered secretly, Ephr. ed. Lamy iii. 76 r. 12 af. Cf. N.-Syr. col. $\mathbf{I}_{532}$.

1 1 f.

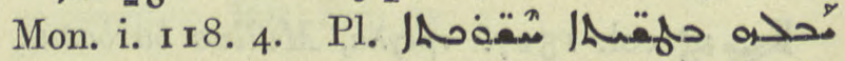
he led him by dark and hidden ways, ib. clxvi Syr. 5. Trs. hither two refs. following "latebrae", col. r530. 5 .

\eef Tšol or Șūl, pr.n. probably a tribe giving its name to a place in Gurgan SE. of the Caspian, Mon. Syr. ii. 68. 5, Tabari I 23 , Pers. Mart. 277 ff.

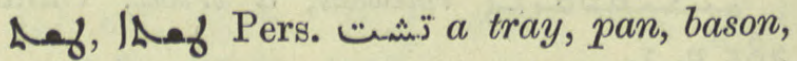

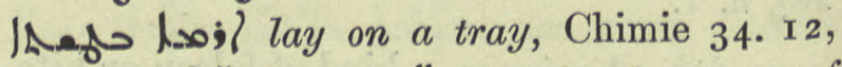

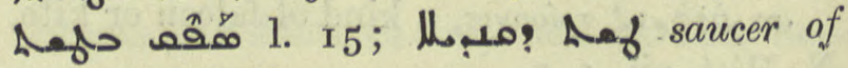
a $\operatorname{lamp}$, C.B.M. $49 \mathrm{I} a$. 
o. Pisidia, Nöld. F. S. i. 473. II 5 .

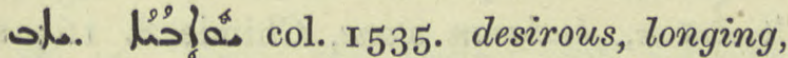
one of the forces of the animal soul is the مُ م. Hist. viii. 3. I. Pl. Theol. 4. 3 tit. quoted under lُ

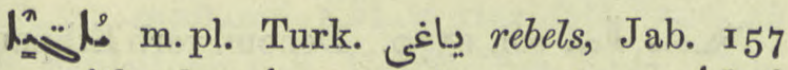
pen. with gloss ib. 193.1 .

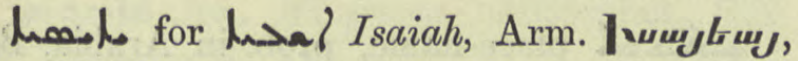
Eus. ed. McLean 4i6. Io.

L Jamnia, a sea port of Palestine,

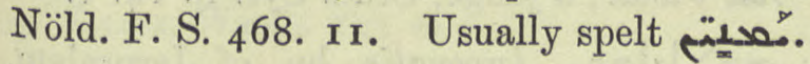

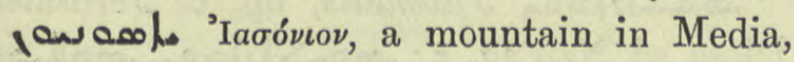
Jac. Edes. Hex. xxxvii. 9.

h إمه i.q. Iconium, Nöld. F. S. 472.92.

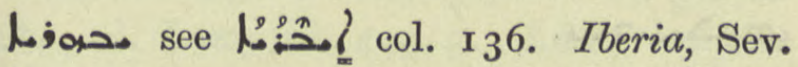
Lett. I I $4.1_{4}, 252.8$.

محه مסمى pr.n.m. Ahikar 52. I \&c. should

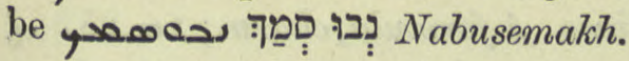

"خدبم col. I538. 9. the ibis, ref. so corr.

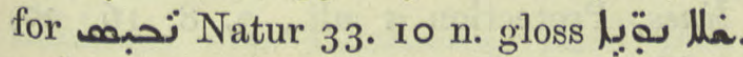

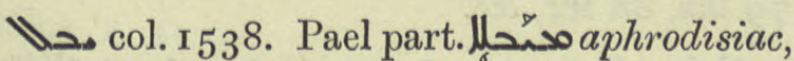

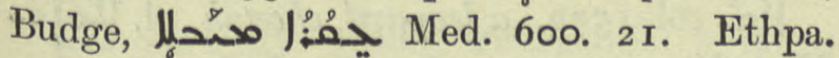
" her life was led = she led her life, S. Pelag. 2. 2 I. Aph. "जo ol" to conduct. The bladder امع Hebraica iv. 2 I 5. 253 .

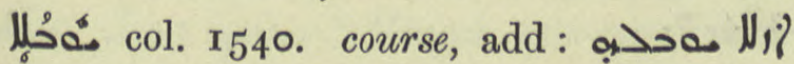
the sun goes on its uniform course, Ephr. Ref. i. $22.3^{8}$.

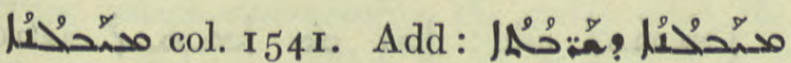
a genealogist, BH. on Luke iii. 23.

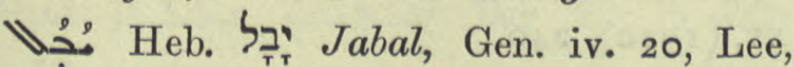

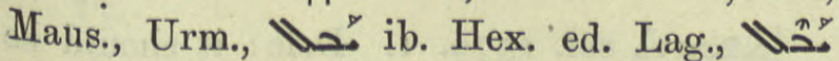
Ephr. i. 47.

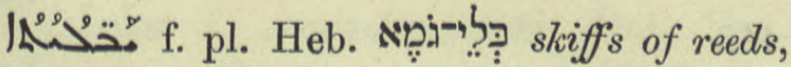
Hex. Jes. xviii. 2 where Pesch, and Maus.

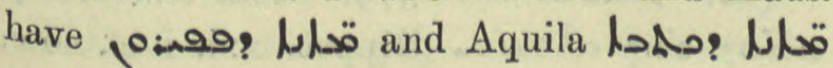

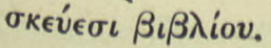

1... col. I542. the mandrake: the badenjan, aubergine or black egg-plant, Med.

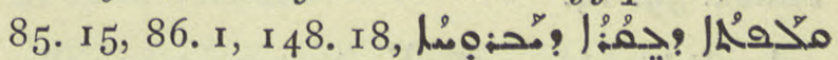

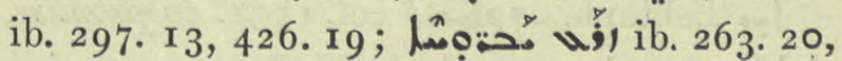

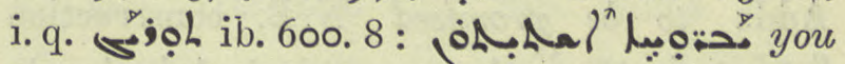
have drunk mandragora, i.e. have stupefied, benumbed the senses, Nest. Hérac. 263.14 .

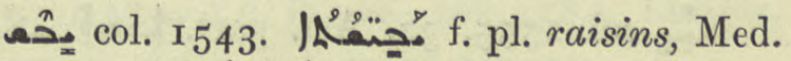

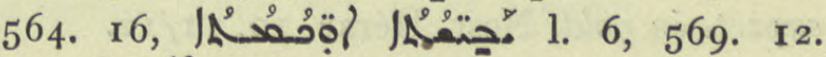

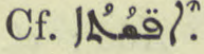

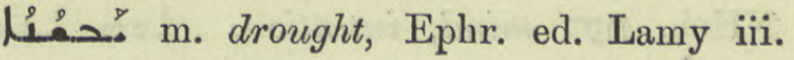
95 ult.

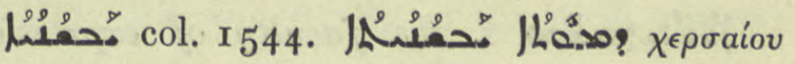
$\mu о \rho \phi \hat{\eta}$ any terrestrial form $=$ land animal,

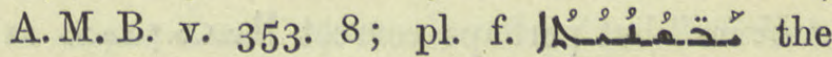

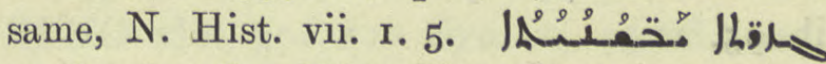
peninsulas, Jac. Edes. Hex. xii. 6 .

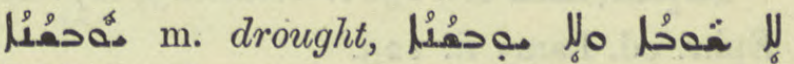
A.M.B. v. $467 \cdot 5$ :

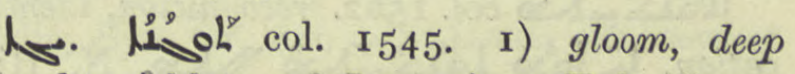
thought. Add to ref. Jes. xvi. 3; Ephr. ii. 46.

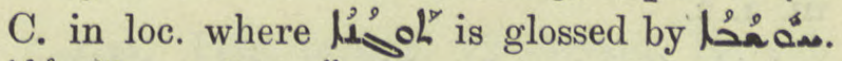

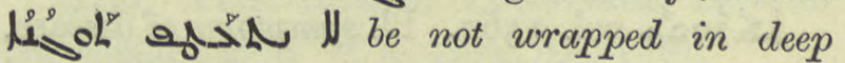
thought, Tekkaf. I6r. 2) a terror, horror,

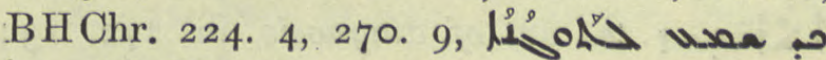
loo? id. Chr. Eccl. 683. I2. A synonym for to.' bent of the mind, K. col. 2328 ; for

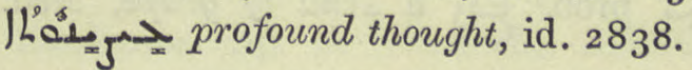

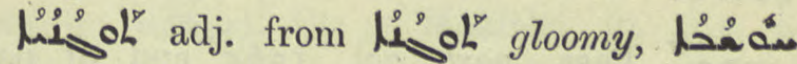
นُนُ ـ the soul, But. Sap. Eth. iv. 3.

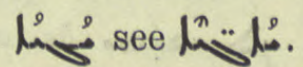

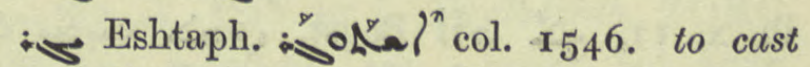

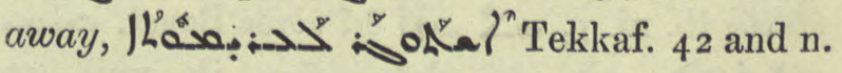

, إ_ col. 1546. the hand. Add: I) a

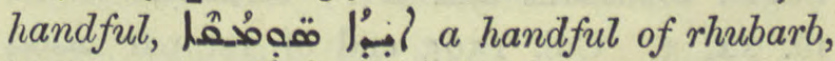

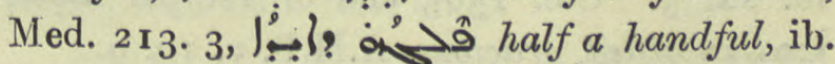

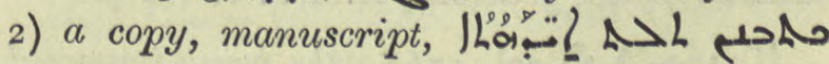
we had three copies made, Or. Xt. i. 300. I4.

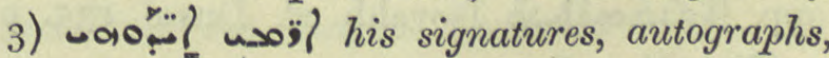
Sev. Lett. 19. 19. 4) pl. arm-cloths, the long strips of cotton which Orientals wear twisted 


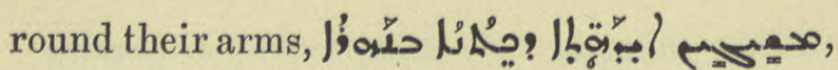
Pallad. 517. 4 af. Cf. col. $1_{547} \theta$ ).

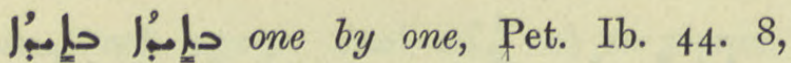
109. I9, A.M.B. iii. 428 ; one after another, Philox. 59. 14.

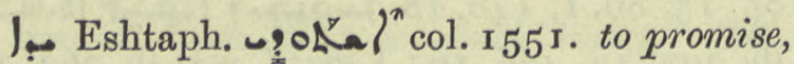
Add: to be promised N.B. construction حَّ Kal-w-Dim. Bick. 74. 20.

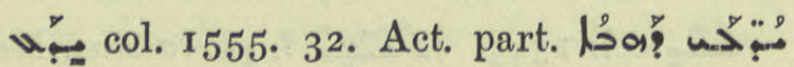
experts in gold, Nest. Hérac. I0. I I/I 2 .

1ُ2: col. 1560. 2) mind. Add: cf. NHeb. דעת mind, intention. Loo floa |al la la Philox. 92.3.

Ihá

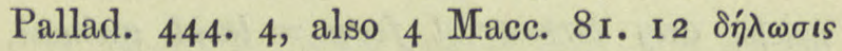
"Urim" but perhaps correct /has مa as ib. $95 \cdot 22$.

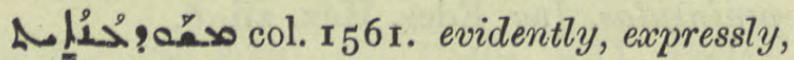
Ephr. ed. Lamy ii. I 25.

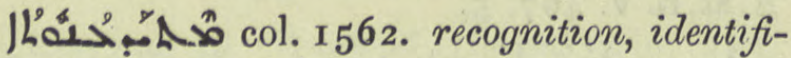

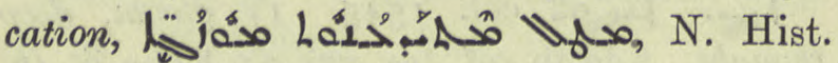
iii. 2.2 .

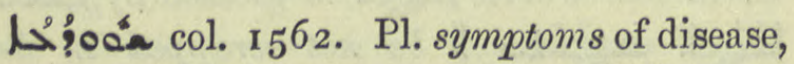

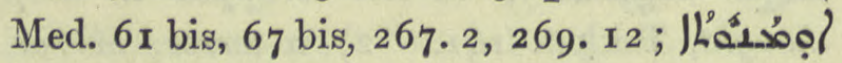

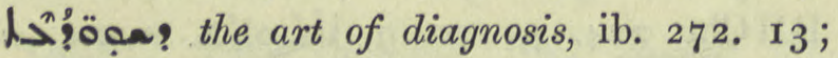

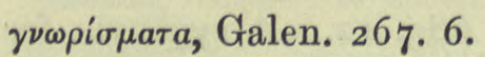

prob. وَّبَ a kind of grape, Med. 468. 2 .

so. Pass. part. col. ${ }^{5} 565$ end of par. With verb loo or with $>$ and pers. pron.

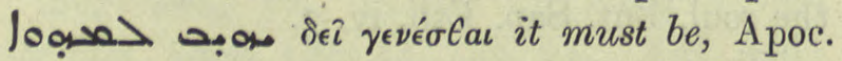

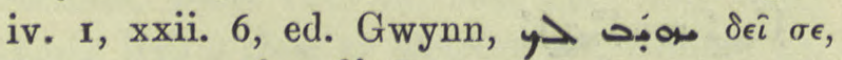

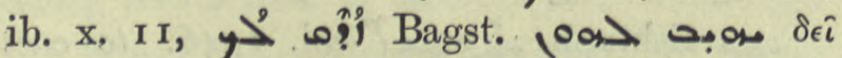
aủroús ib. xi. 5, a

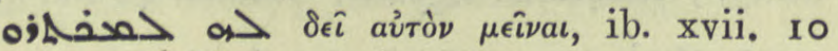

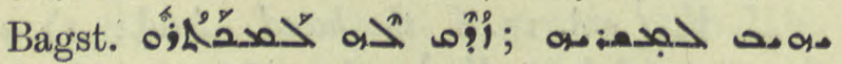
ib. xx. 3. Col. $1_{5}^{6} 5$ end of 1 st par par gifted, gracious, A.M.B. v. 10. 9.

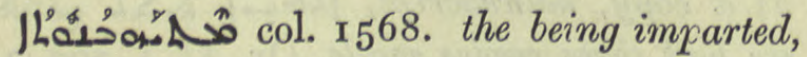

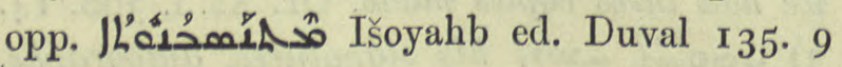
= Hist. Mon. ii. 139. 9 .

?oar col. 1568. ?ơn'L? denom. verb
Ethpalpal conj. to turn Jew, be proselytized, BH. Schol. in Act. xiii. 43 .

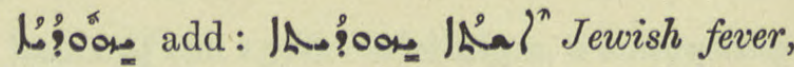
Charms 18. 27, 28; ellipt. $3.7,18.6$ af.

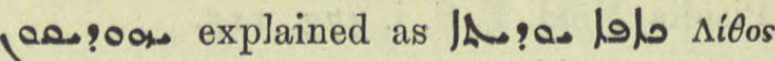
'Iovóaïkós, Judaean stone, Chimie 8. 16; see below under blo.

نُ col. I570. interj. iá, iá, Théodoret. Nestle Z.A.T.W. I90I, 329.

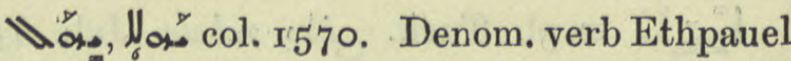

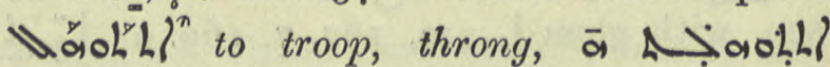

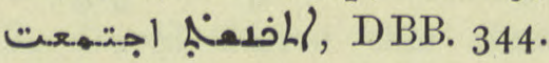

مَّ place-name, prob. in Arabia, Bar Penk. I 42. I 2.

;ai Jahar, a judge, Stud. Syr. ii. I4. I.

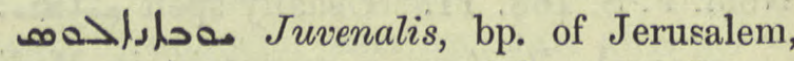

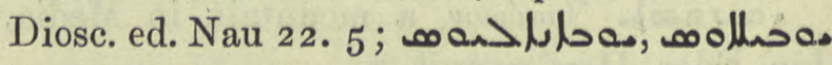
ib. 58. I ult., Chron. Min. 21. 8, مه حسالll, Anecd. Syr. iii. 341. 8, Pléroph. I3. 5 and n., I 5. 8, I0, II. Vars. مa مhل/o, م

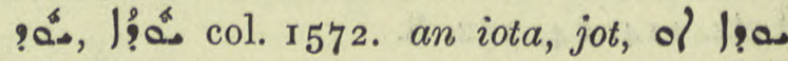

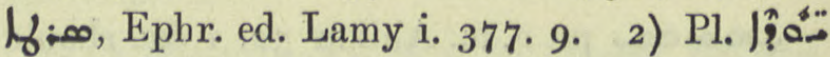
jots, writs, lines. A formula, charm; : a : 1 o

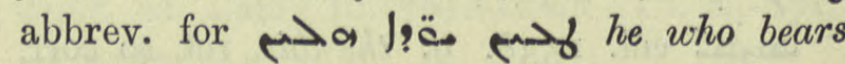
these lines, Protection I ult., 3. $8,16,20$, 79 bis, 88 ter, \&c.

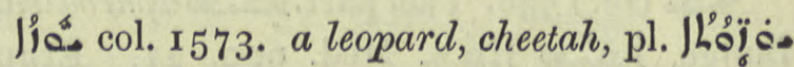
Hist. Mon. i. 247. 4, Lag. G. A. 83. 139.

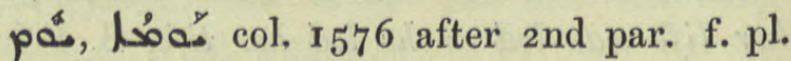
constr. for ever, in perpetuity, Journ. As. 1906. 2. 284,7 .

مُ day after day, day by day, Pallad. 80. I5.

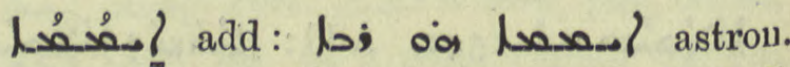
"jour maximum" the longest day? R.O.C. xv. (1910), 240.

Jäaso. col. 1579. r. Dele: it should be J 4I3.

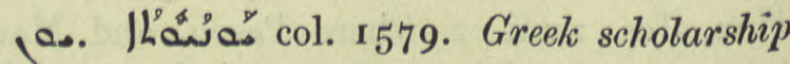

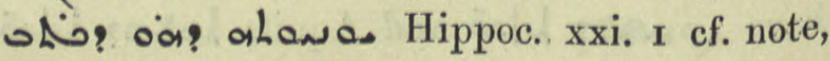
Or. Xt. i. 302. 12. 
We Armenian form of the name John, Ant. Patr. 306. I.

مف مonaton, son of Noah, born after the Deluge, Apis ${ }^{-}$. Schatzh. ${ }^{2} 3^{8 .} 7$ and n. 115 .

olua 'I Adriatic Sea, Jac. Edes. Hex. xi. I 4.

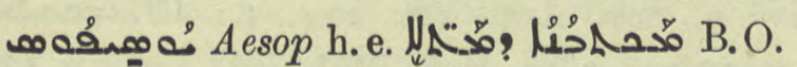

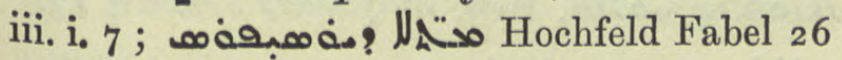
and Einleitung 5-7, Kal-w-Dim. x. I. Confused with Flavius Josephus, col. I 582 .

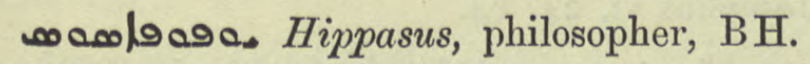
ap. Hebraica iii. 25 o. 7 .

 of Joppa, Warda 22 I $r$.

. 16. 9, I16. I2, I91. 9 and often. Cf. engl col. 165 .

معنسم col. I582. image, figure. Pl Ephr. ed. Lamy iii. 445. 3. Add : a figure of speech, trope, Sev. Ant. Vit. I3. 8. مبم "ר. in a list of Church furniture, Takhsa I46. 6 .



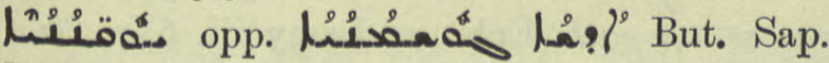
Philos. 4. 4.

مُ col. I $5^{8} 3$. denom. from form,

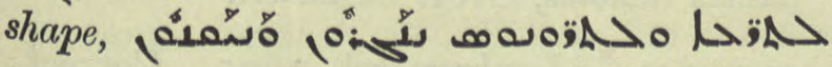
to carpenter and make doors and seats, Tim. I. ed. Braun 26 . With lioo to form an image in the mind, Ebed J. Card. 25. 2, 30. 4 ; "Hénoch que caractérise la grace" Sev. Ant. Vit. 21 2. $5=$ Sir. xliv.

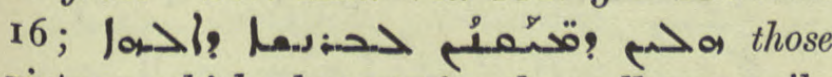
virtues which characterize the godly man, ib.

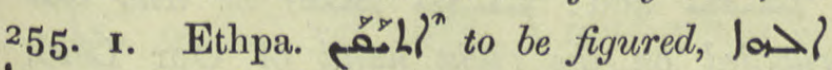
مئم Bar Sal. in Lit. 94. 17.

ممץ. See p. $156 a 2$.

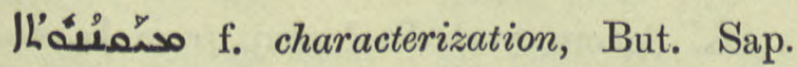
Periherm. I. 4.

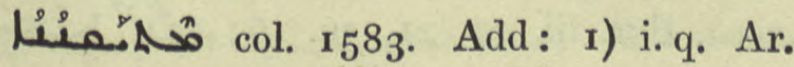

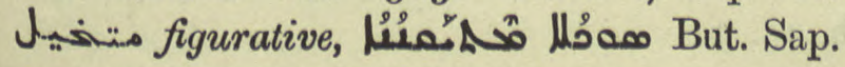
2716
Tress

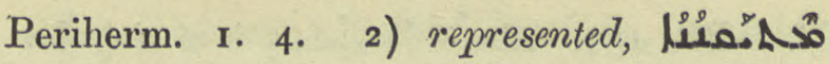

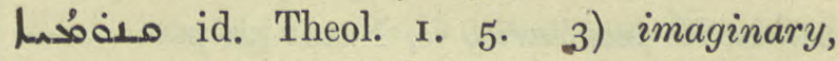

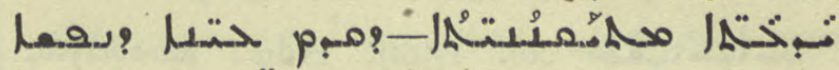

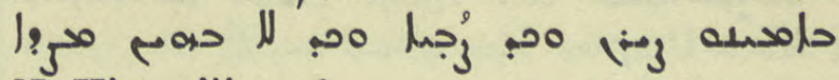
N. Hist. viii. 4. 6.

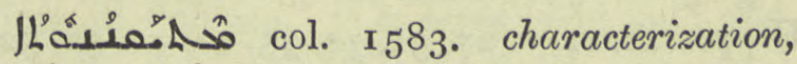
| Pl. mental concepts, / مَفْ id. Isag. 1. 2 ; Philos. 2.4 ter.

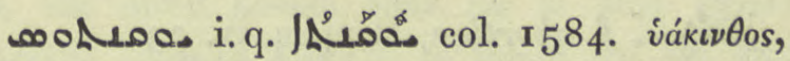
a jacinth, Apoc. xxi. 20 ed. Gwynn.

Pers. Yazdaidad, Bp., ZDMG. xliii. 395. 2.

9? Pers. Yazdad, Bp. of Rêw-Ardašir, ZDMG. xliii. 396. 6 ; Bp. of Herat, ib. 400. 6 ; Syn. Or. 43. I I, 64. 29: 62 I.

ZDMG. xliii. 396. I I ; Syn. Or. 43. I8; 62 I. 2) f. martyr of Beit Slok, A.M.B. iv. 201,207 .

مـ Pers. pr. n. Yazdlkhwast, Bp. of Media, Syn. Or. $479=214$. I9. So correct 1000/ ZDMG. xliii. 406. I5.

م Pers. pr.n.m. Yazdinnan, Išoyahb ed. Duval 76.3. I. q. e. col. I $5^{8} 5$.

Pers. pr.n.m. Yazdsapor, Išoyahb ed. Duval 36. I4.

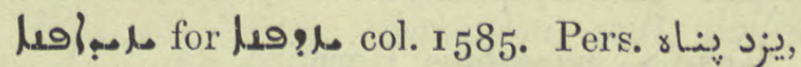
Yazdpana, Bp. of Nineveh, ZDMG. xliii.

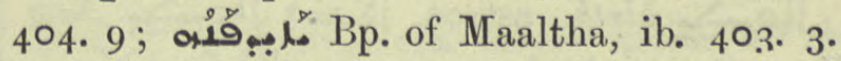
Mart. under Chosroes I, Jab. 394. 6 ff. ;

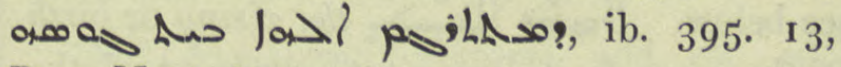
Pers. Mart. 87 n. 785 .

مامبدمامب Pers. pr.n. Yazdbozedh, ZDMG. xliii. $397 . \mathrm{I}$ and n. I. q. بر col. $\mathrm{I}_{5}^{8} 5$. Mart. under Chosroes, Tabari 287 n. I.

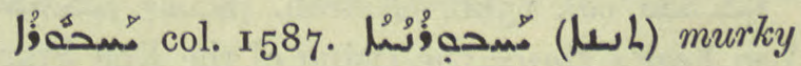
fumes, But. Sap. Eth. iv. 5 .

col. I $5^{88}$ pen. and $15^{89}$ mid.

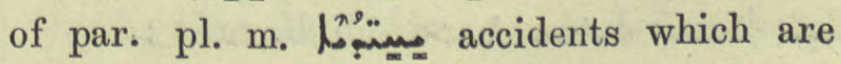
peculiar to one thing as blackness to ink, one of six sorts of accident, BB. under $\ln _{\rightarrow}$ col. 66I, where the part about ستب:م is incomplete. 
Cf. DBB. 454. a in this, its peculiarity is, Natur $5^{2}$ pen.

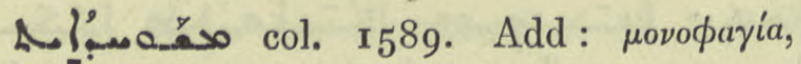
4 Macc. i. 27.

يم col. I 590, to mis-carry, be abortive, add: Hippoc.iii. I 2, v. 27,3 I, 34, 35, 4I , 42, 50, $5^{2}$, vii. 25. Of goats and sheep, N. Hist. vii. 4. 4 . Used of wheat not forming ears, تصل

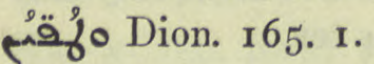

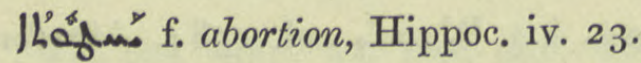

עn . Sap. Eth. iv. 5 bis.

| Nest. 97. 22, both on I Reg. iv. 23. Conder, Tent-work, i. I 72 , identifies it with our roe-buck and says that a great valley S. of Carmel is named from it, Yahmûr. Also Lag. G. A. 52, Arm. Stud. § I 546, Praet. Miss. 42-64.

سمe uncertain. Perh. the freight of a caravan, Jos. Styl. 54. I where Martin bas سمد.

ثذه I- Begetter of the Warrior, name of a plant, its blossoms are shaped like a hood and shine at night, Med. 598. I.

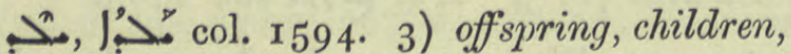

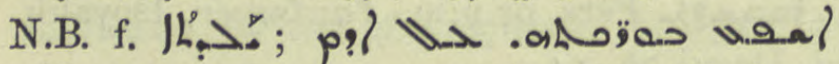

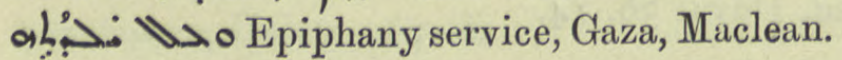

$1 L 20$ ?ُحْ parentage, Hormizd. 53.

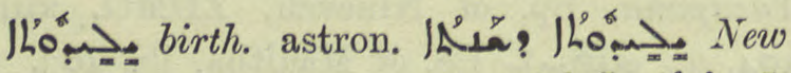
Year, Georg. Arab. 27. 12 i.q.

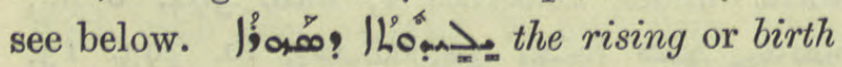
of the moon is its transit to the west of the

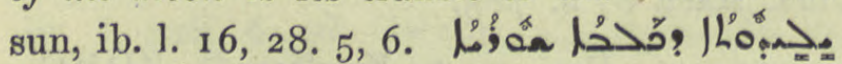

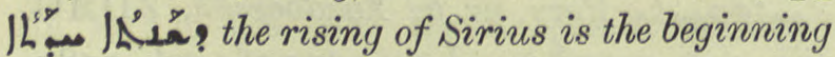
of the New Year, ib. 1l. 7,8 .

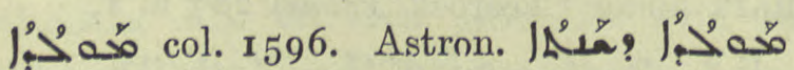
New Year's Day=the rising of the Dog Star, Georg. Arab. 24. 5, 10, 25. 11 , 27. 9 ; ب >

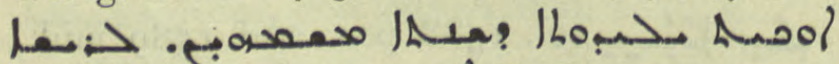

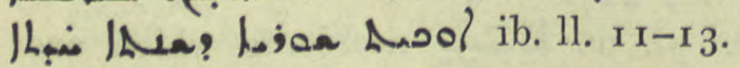

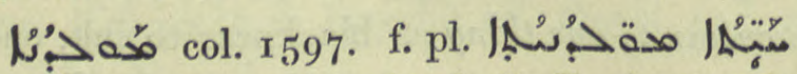
midwives who assist at birth, G. Busâmé 78 b,

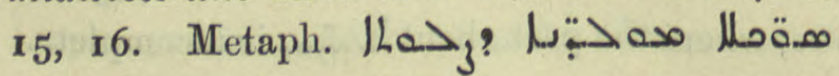

thoughts which beget prayer, Is. Nin. $8 r, 5$ af.; أمة ib ib.

مخهة| |Anecd. Syr. iii. 258. 3. Corr. "Eגovpot, Herulians, so Brooks in loc. p. 225. Cf. Procop. Bell. Pers. r. I3.

a) Pa. past. part. col. I 599 infra. Add

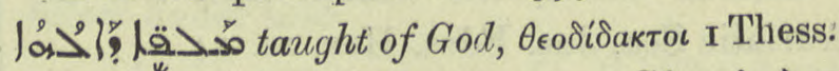
iv. 9, Š้ev. Ant. Vit. 244. I. Cf. فحذ

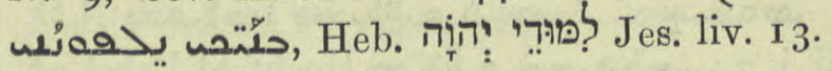

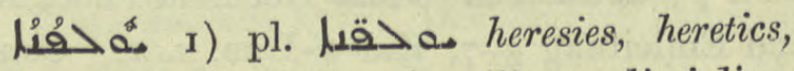
Ephr. Ref. ii. 5० ff. 2) military discipline, Josephus vi. 24. 14.

1 Sea, M.Z. $33.43,5^{8} .3^{\circ}$.

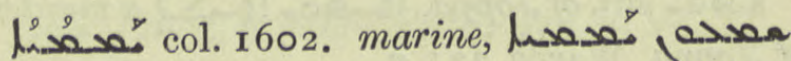

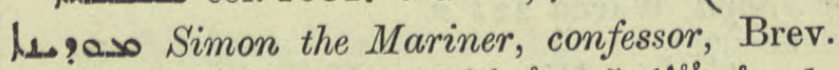

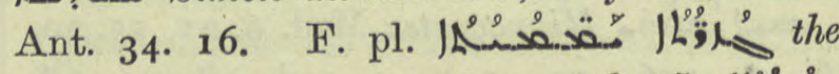

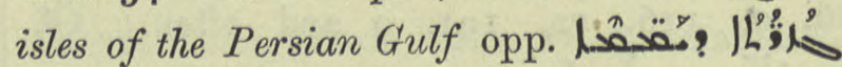
isles of the Indian Ocean, Syn. Or. 6r9 sqq.

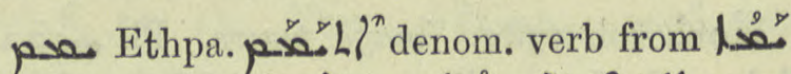

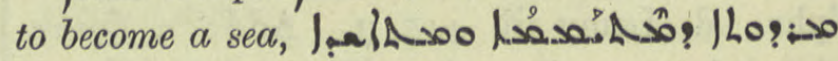
Ihesa $>$ Sev. Ant. Hymns r9 1 n., f.

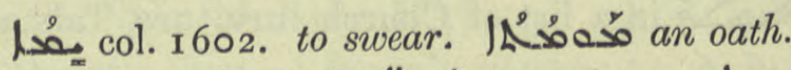

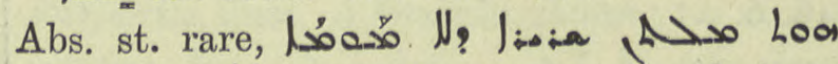

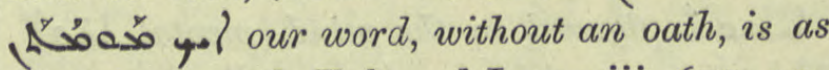
true as our oath, Ephr. ed Lamy iii. 657. 10.

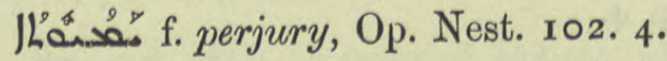

مقحه iambics, Greg. Carm. tit.

. Sambres, col. I605. Correct BHChr. $\mathbf{1}_{32}=$ ed. Bedj. 128.18.

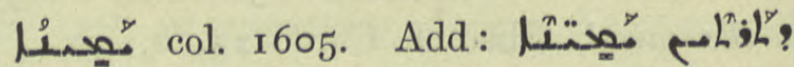

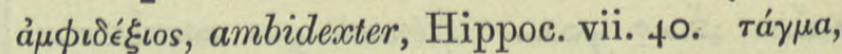
. I2. 15 .

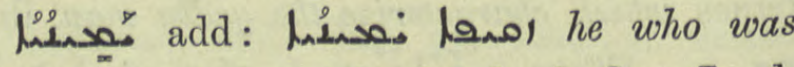
crucified on the right hand of Our Lord, Warda roz $r$. End of par. J which I have said, is right, Sev. Ant. Vit. 33․ $\mathrm{I}$.

مص denom. verb Pael conj. from

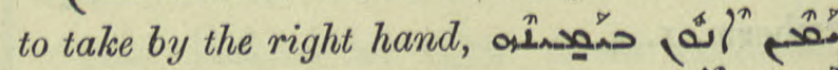

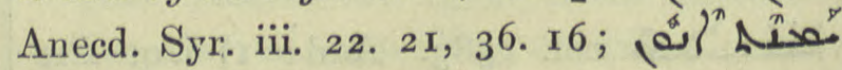
ib. $45 \cdot 9$. 
lise L a bishopric of the province of Assyria, probably Temanûn or Temanîm, E.of Amadiya, Or. Xt. i. 3 Ir. 2 and n. 3 , Syn. Tim. i. 3 ro, Syn. Or. 608 n. 3, 6 г 9 . 6.

اليهاoـ Yemama, a province of Arabia, Sassanidi $3^{6}$. I3.

axase place-name, Imameh, Chast. 70. 6.

an alembic, Chimie 33. 2 I, 34. 4 \&c. Usually انبيتق qr.v. Suppl.

- م- Jannes, Hist. Mon. i. I76. 13 .

مهם $I s u$ : this transliteration of the Greek 'I $\eta \sigma o v$ s must have been used by the Syriacspeaking Marcionites. It is not elsewhere preserved in extant Syr. literature, Ephr. Ref. i. 45.15 , ii. 6 r. $42,62.29,70$ four times, and often. Burkitt Trans. xxix.

Jeshuabnun, title of the Book of Joshua, Eus. ed. McLean 4I6. 5 .

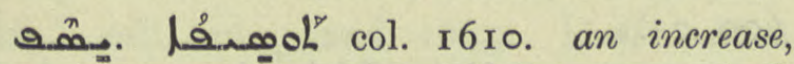

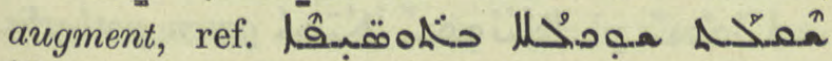

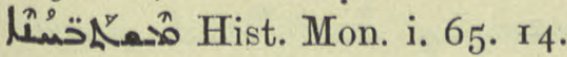

190ما Pers. Perse. a swift rider, Jab. 224 . I 2 .

A Aph. a

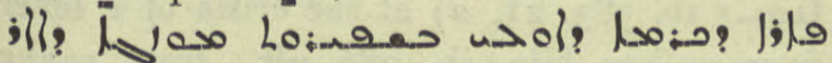
that the fruit of the vineyard may grow by fair temperate weather, Bénédiction des Raisins, R.O.C. iv. 359 .

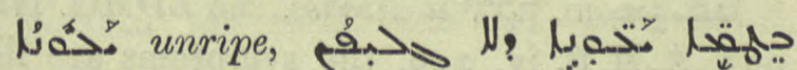
unripe and unpeeled terebinth berries, Med. 586. 5 .

متئ

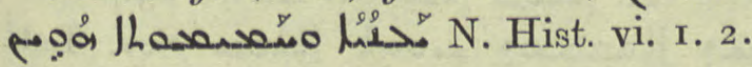

1. or Aُ col. I612. a sand-grouse but

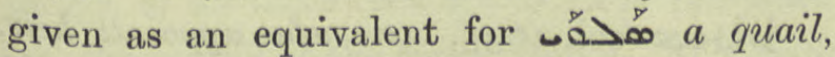
Lexx. and Op. Nest. quoted col. 264 r sq. N.B. E-Syr. voc. With gloss oطّ Nars. ed. Ming. i. $3^{8}$ note. Pl. f. مُحِيْم Med. 554. I6.

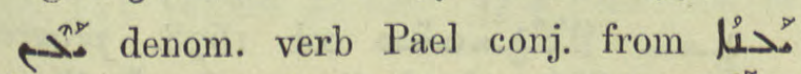
col. I6r3. to cause to covet. pas pal. لle pas pal-w-Dim. I87. 2 .

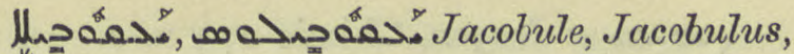
BHCarm. I 53. 4, 9 both times opp. Nestorius.

(ㅅ. Aph. ugol" col. I615. 2) to end, pass away, oa 1 our Lord came to an end as if He were dead, Jac. Sar. Hom. iii. 808 pen. 3) to bring to an end; add :

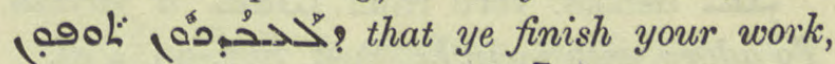

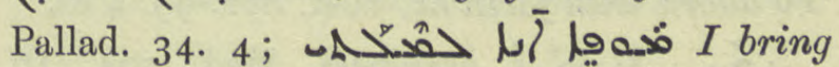
my remark to an end, ib. 40. 6. To put an end to, the devil boasts that he is able is on to finish the sea and do away with the land, ib. 28. Io. N.B. to

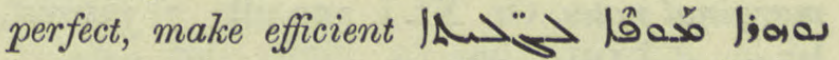
"lux perfecit manifesta" opp. مئ Ephr. ed. Lamy ii. 79 r.

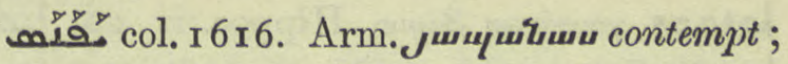
pertinacity, Aphr. ed. Par. $60_{5}$ ult., 677. 5 ; 

$\theta_{j}^{n}=$ col. r6r6. Fut. I pers. $\left.\theta_{3}^{y}\right\}^{n}$ to care

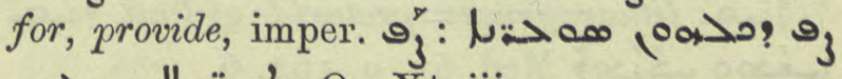

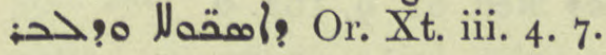

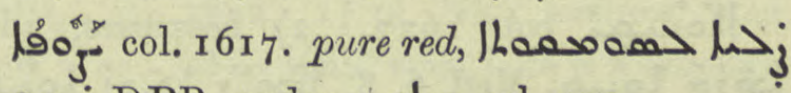

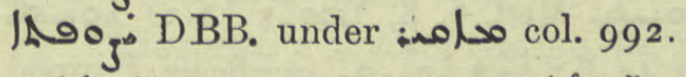

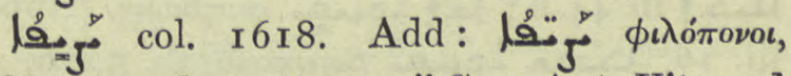
diligent students, "mugs," Sev. Ant. Vit. and $\mathrm{Nau}$ in loc, R.O.C. iv. 543 .

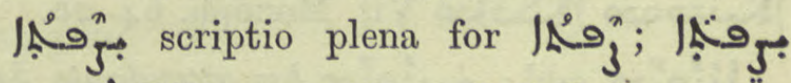
IA a / Divine providence, Pallad. 331. I6."

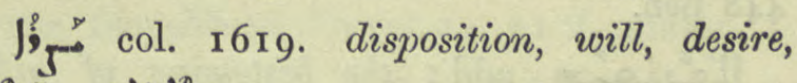
बis $5 \int^{n}\left\{l^{n}\right.$ he was thwarted, BHChr. Eccl.

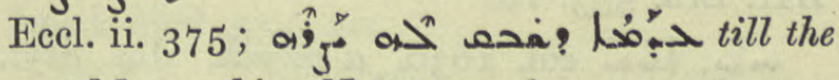
mood leaves him, Natur 55.6 .

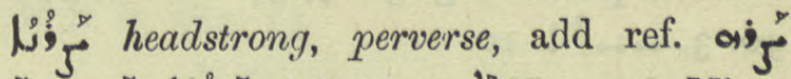

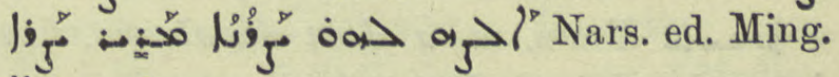
ii. 94.6 .

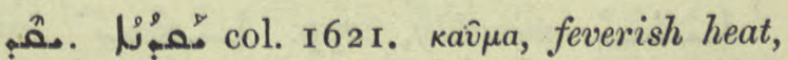

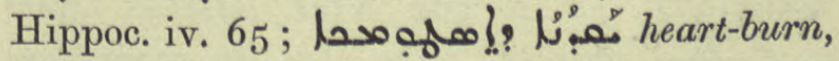
Med. 295. 17 .

โ⿳⺈冂: col. 1622. hot, burning, name of a hot medicine i.e. Syrup of poppies,

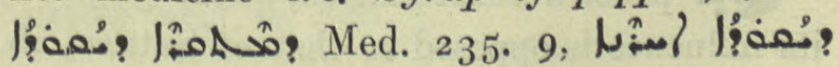
l. $20 ; 247.15$.

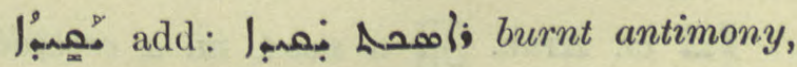
Chimie 99. 6, 8, I2. 
(م.) a dragon, Chimie 273 ก. 2.

مُمبم to congest, be inflamed, pass. part. inflamed, كمبم Hist. B.V.M. 73. 2.

مै. see under معه.

مُ denom. verb from To adorn with jacinths, Poet. Syr. 99. 4 af.

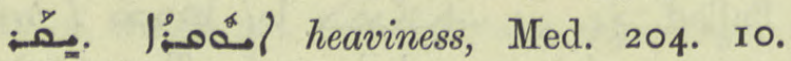

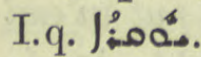

إ_ col. I624. 5) margin of a book, add: Ion: many are the marginal notes, Or. Xt. i. 300 ult. 7) interest, |إممث: Dion. I84. 9.

مع مع: مهم:ا

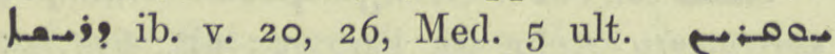
| ختخه|, oppression, nightmares, Charms 285. II , 286. 4 .

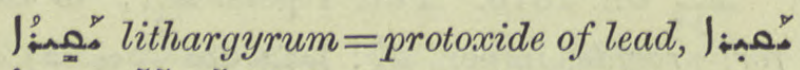
ชִ Med. 61. 9, I62. 6.

1L'o: col. 1627. 2) heaviness, add:

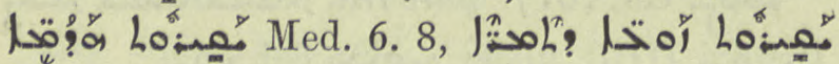

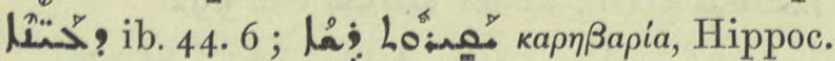

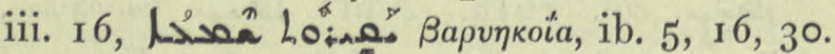

أهـ col. 1627. heavy, burdensome, 20.

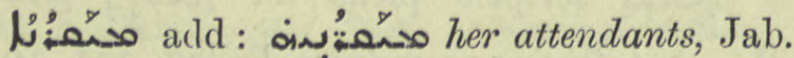
448 pen.

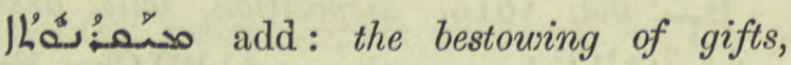
BH. Eth. 553. 18.

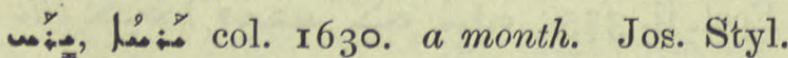
has is? passim e.g. 31. 7, 37. 10, I I, 39.23,

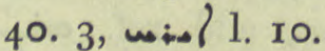

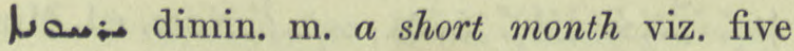
intercalated days, Georg. Arab. 5. I3.

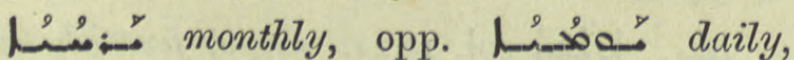
N. Hist. $5 \cdot 3$.

L م. m.pl. Yartutāye, a people descended from Aram, Chron. Min. 356. 19.

$\checkmark$ prob. a constellation, Med. 505. 22, its influence is opposed to that of Saturn, ib. 506. 3, to that of Jupiter, 1. 4 .

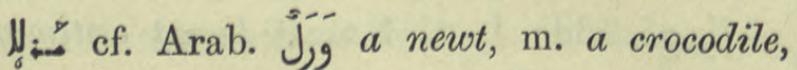
Anecd. Syr. iv. 42 sq.
مئهم Mongol injunction, charge, Jab. 48.

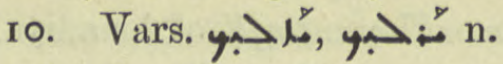

1 BHChr. ror. 5 .

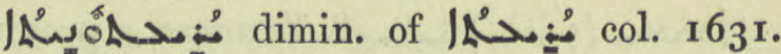
f. a small rug, a coverlet, Anecd. Syr. ii. I I 5. 9.

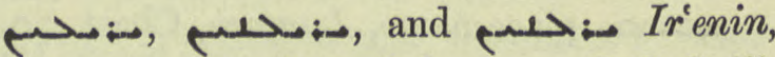
a village in the dist. of Apamea, R.O.C. iii. 337 n. 2.

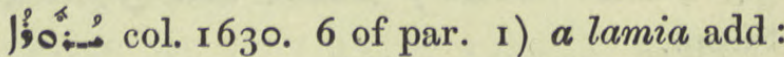

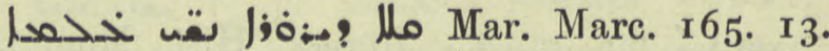
2) a plant, panaces heracleum, add ref. Med. 232. 2 .

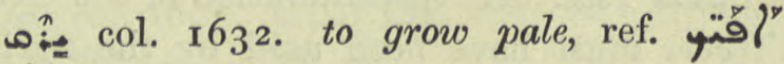
يِقِمْ Anecd. Syr. i. 68. 6.

人ُ add: yolk of an egg, Med. 6r. 10, 86. I1, DBB. under لحم: 423.

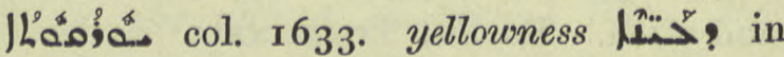
jaundice, Med. 557. 17 .

10ْ- col. I633. pale green? leaves, Med. 600. $2 \mathrm{I}$.

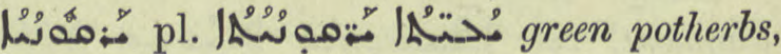
N. Hist. vi. 2. 2.

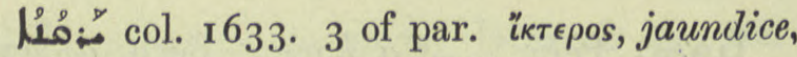
add Hippoc. iv. 62 , pl. 64 , v. 68 , vi. $4 \mathrm{I}$; jaundice affecting the eyes, Med. 53. 22, 54 .

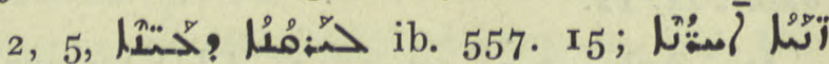

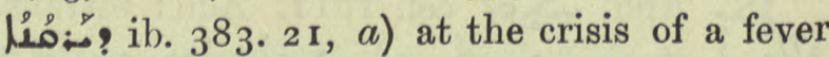
from yellow bile, ib. $3^{86}$ ult., from snake bite, 387 . I, from the liver, 1. 4, from the spleen, 1.5 , from the gall bladder, 1. 8.

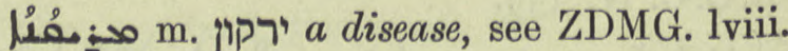
953, A.M.B. iv. 249, the symptoms are like dropsy

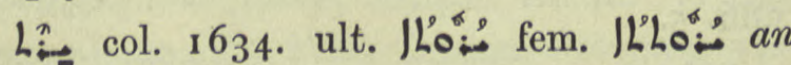
heiress, ref. JLLois Ihis Or. Xt. vi. 24. 6, 16;

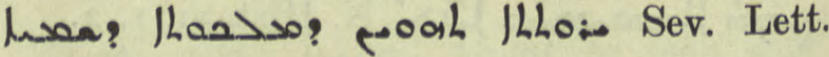
509. 8.

Uhios col. 1635 . one who appoints heir,

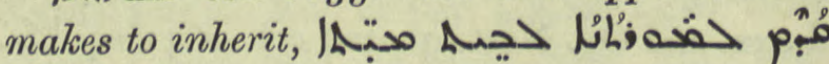
the heir precedes the testator to the grave, Hist. Mon. i. 222 . I 4 .

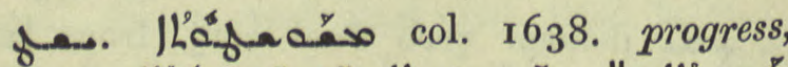

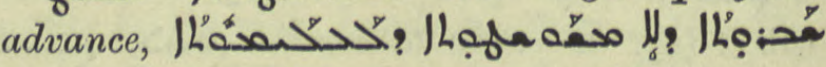
childishness which does not make progress, Hist. Mon. i. 65. 6. 


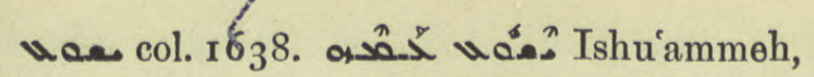
Master of Maran'ammeh, M.Z. 209. I6, 22.

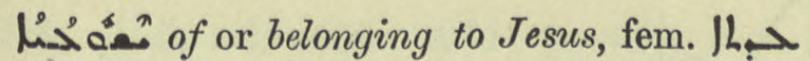

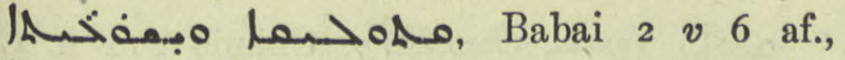

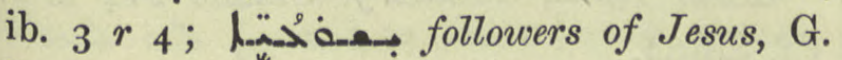
Busâmé colophon.

|مُ corr. lana a serpent, A.M.B. ii. ${ }^{26} 5^{-1} 3=$ Act. Mart. I. 7 I see note col. 2477.

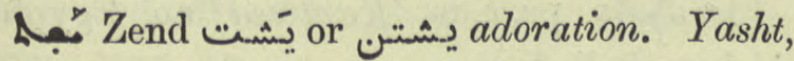
a hymn, A.M.B. ii. 589. I 5, Jab. 355. 7 .

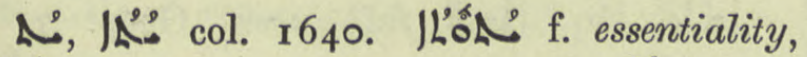

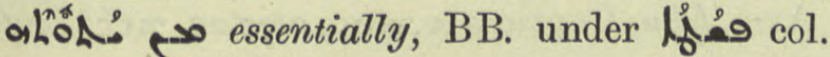
$3320=$ DBB. 164 r.

LA $\mathcal{A}^{\text {ny }}$ denom. verb Pael conj. to give existence, to constitute, Poet. Syr. 68. 5 .

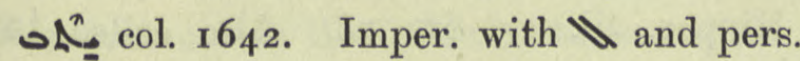
pron. ${ }^{\prime} ح$ sit still, Mar Kardag ed. Feige

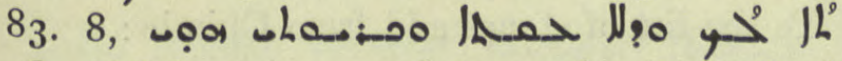

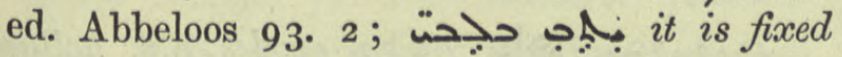
in my mind, Or. Xt. i. 96. I5. Act. part. .

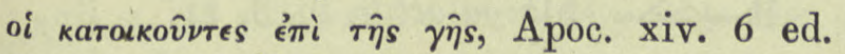

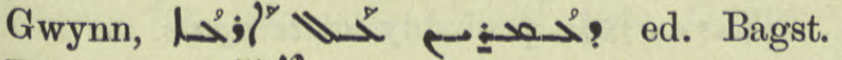

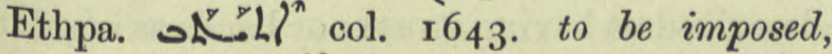

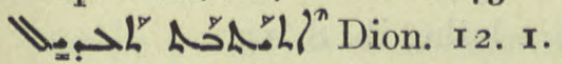

ـُـ col. I645. occupation, habitation,

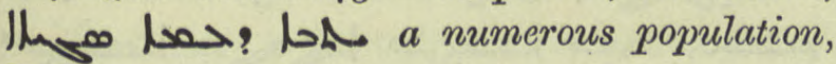
Dion. $13 \cdot 7$.

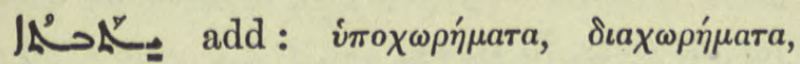

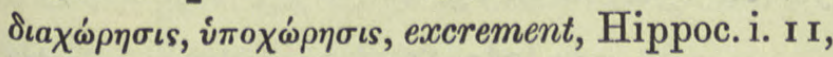
ii. I 4 , iv. 2 I, $28.47,83$, v. 60 , vii 5,2 I, 27 .

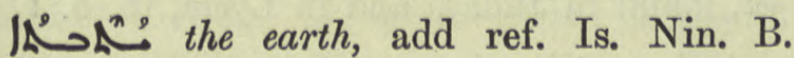
I 24. II.

L'Leš col. 1646. I5. Rit. Psalms or prayers originally said sitting. add : QdhamW.

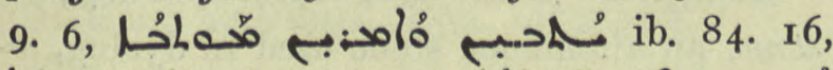

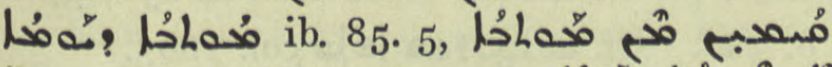

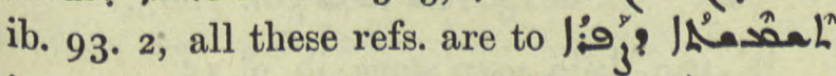
i. e. the second of the seven Hours. 2) a part of Nocturns, said sitting, محّا Brev. Chald. i. 60 ult., 6r. 2 I and often, I5 $5^{2}$. I2, I53. I 2 ; Takhsa 90 ter. 3) a collection of anthems in the Burial Service, usually said while walking in procession, Brev. Chald. 82. 33 .

IL'astoL col. 1647. 5 of par. diocese,

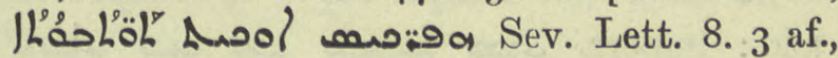
33. 4 af. 42 pen., 43.5 , I 79. 5 \&c.

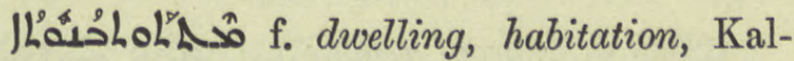
w-Dim. I 5. 20.

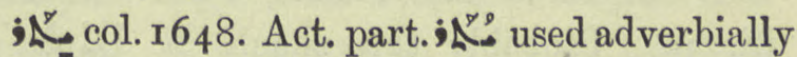

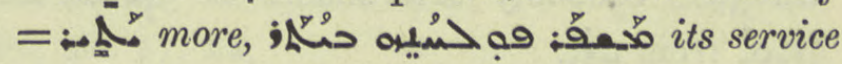
is yet more pleasing, Išoyahb ed. Duval 103. 27 .

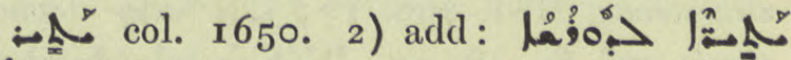
| I53. 19.

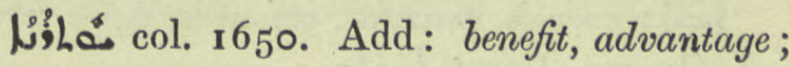
Peter the Iberian was ordained by God to be Jikeso foo? Pet. Ib. ro3. 7.

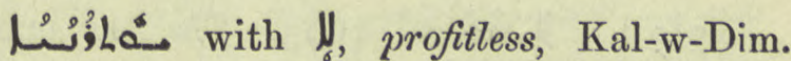
396. 20.

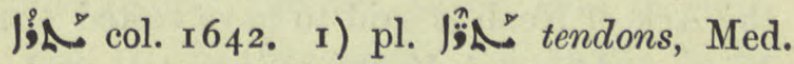

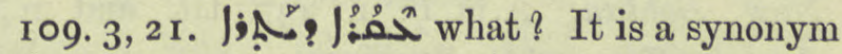
for Tithomalus a kind of spurge, ib. I 7 r. 20 .

Aـ

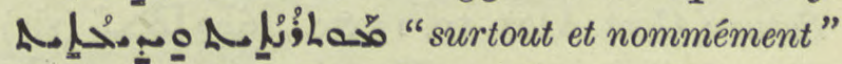
Letter to Jac. Baradaeus, Congr. Or. I897, I 9 pen.

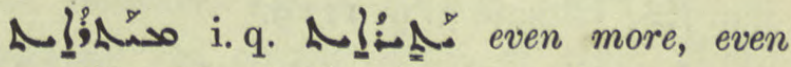
better, Išoyahb ed. Duval 24. 2 I ff. quater.

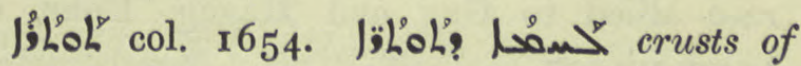
bread, Med. 554. 20.

J marg. J Uathre, a plain in Belešfar, A.M. B. ii. $5^{82}$. I 3 .

يثرب :

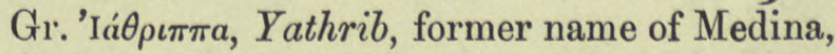
BHChr. 97. 9, ror. I 5, B. O. iii. i. 443 , iii. ii. $75_{5}$, ZDMG. xxiv. 263 n. 2. The other references on col. 1654 concern monastery near Aleppo. See Suppl. under \. Add: : 202. I3, 203. I8,

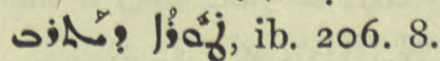




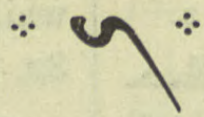

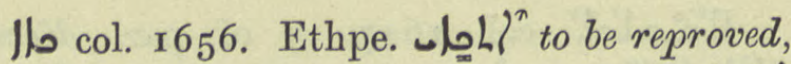

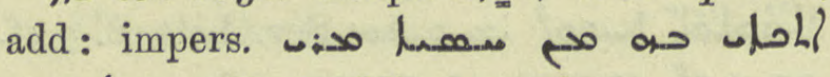
Chron. Min. 254 ult.

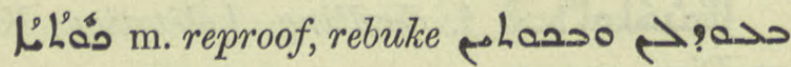
with our blame and rebuke, Ephr. ed. Lamy iii. 39. Probably this should be $\int_{6}^{\circ} \cdot a^{5}$, cf. 1/20\% from $\mathrm{l}_{3}$.

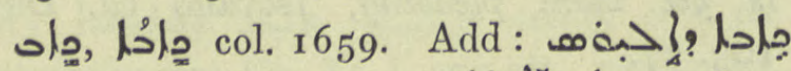

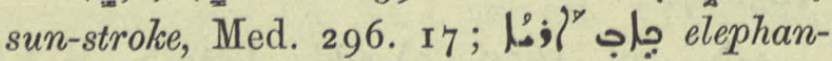
tiasis, ref. ib. 48. 20 ; ib. 5 o. 5 .

مovnpós, painful, grievous, Apoc. xvi. 2 ed. Gwynn, Bagst. لمُ2.

ماحمُ f. pain, grief, BHCarm. I57. 3 .

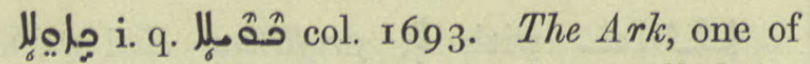
the Qardu chain of mountains is so-called for the Ark of Noah is said to have rested there. Now Bohtan, A.M.B. i. 445 tit. and n., 449 ult. ff.

مجاة col. 1 659. Add: Assyrian Ka-ai-ma-nu,

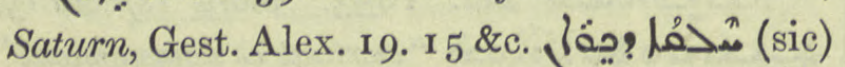
ill-luck, ib. 40. Ir. The planet Saturn ol

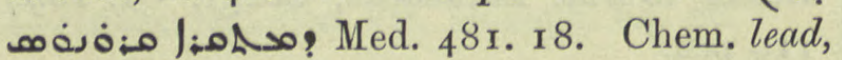

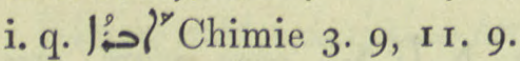

olo pr.n. Chaeon, olo a Scythian race allied to Gog and Magog, Ephr. ed. Lamy iii 197.

นُئم فำ Chaonea, a district of Epirus, N. Hist. iv. 5. 2.

u $\mathrm{X} a \lambda a ́ v \eta$, a monastery, Sev. Lett. 5०5. 22.

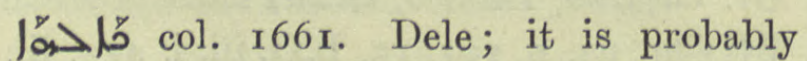

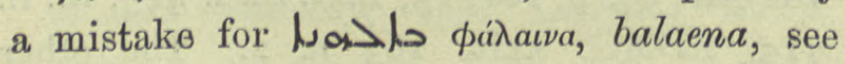
col. 435 .



م found in Cappadocia, N. Hist. vii. 4. 2.

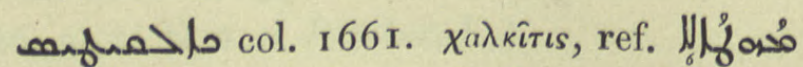
بط copper mine in Cyprus, N. Hist. vii. 3 . $\mathrm{I}$.

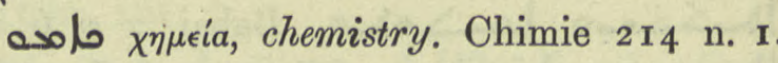

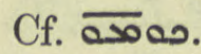

Lar. مارئ Gennesareth, Jac. Edes.

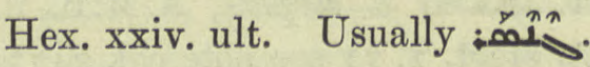

فارئم pr.n.m. Kantirsa, an Egyptian bishop, Pallad. $5^{\circ} 5 \cdot 5$.

| Arm. 45u, Coptic бsc, бос, orбoc, хec, Lag. Symm. 93 .

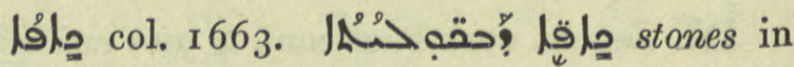
the bladder, Med. 244. 5, 245. I, 544. 7, Hippoc. iii. 25 , iv. 78 . Also called hooga? as causing vomiting, A.M.B. iii. 522 . To the list of stones add from Chimie :

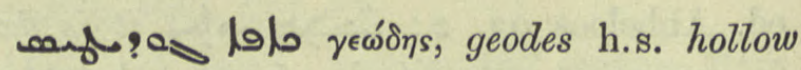
nodules, 9. 16.

glalactite, ib. 8. 1 I.

IA described as having streaks or bands as regular as if turned on a lathe, ib. 8. I5.

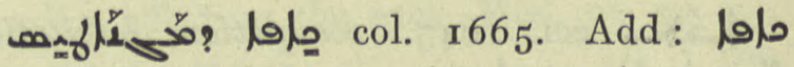

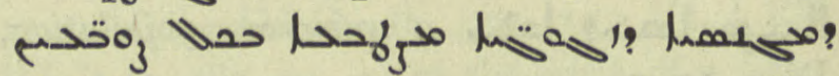
glass-blower's magnesia; it is used as a base for all dyes, ib. 9. $\mathbf{5} 5$.

ماهر "مض: St. John's stone, gagates, jet, found in Judaea and in Lycia, ib. 9. I.

J:d? व a fint, ib. 9. 20.

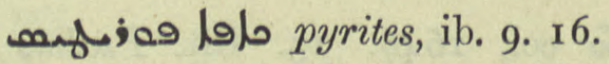

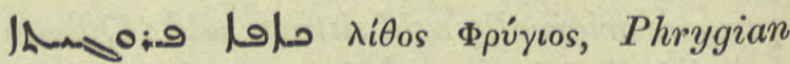
stone, an aluminous stone used in dyeing, ib. 8. 3 af. Cf. Con col. I665.

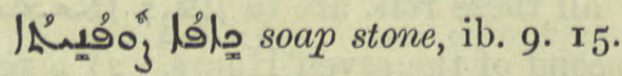

"light stone", kovфóiteos,

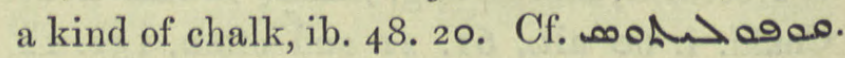

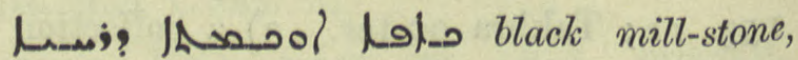
ib. 9 ult.

อ stony, prob. name of a plant, Med. 265.9 . 
'gُ camphor, N. Hist. iii. i. sect. 2. Usually مa q.v.

;

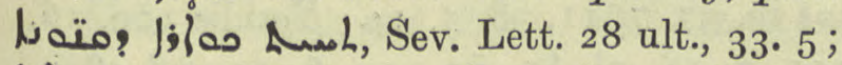

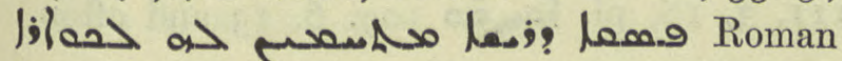
laws fix beheading as the penalty, ib. 77. 10; osas, ib. II6. I0, I23. 3 \&c. With $>$ liable to shame or

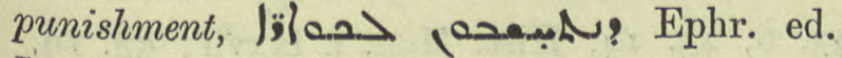
Lamy i. I73. I 7 .

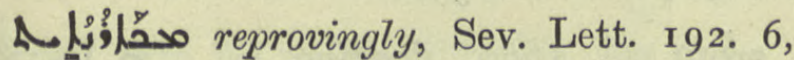
276. 16.



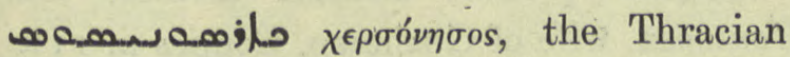

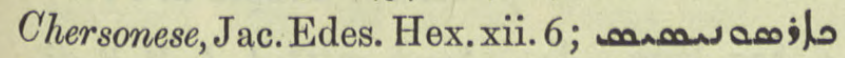
and the Golden Chersonese, ib. xv. 16.

مخصA (oh) م.B. synonyms, BHChr. Eccl. ii. 46I ult., Jab. I04, 7 .

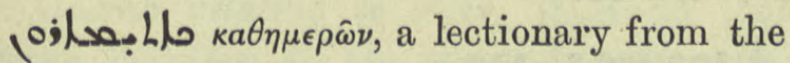
Gospels for each day of the year, C.S.B. I.I 4, I 28.

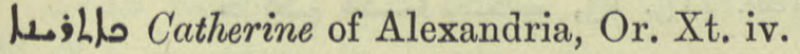
II 8. II I.

ปร้ Kabab, a Monophysite, Išoyahb 84. 8.

محّب angry. Impers. with $\lambda$ of the pers. An. Syr. 194. 24 ; masc. 26.

อُ col. 1669. the liver. Add: Hippoc. v. 55 , vi. $\mathrm{I} 8,4 \mathrm{I}$, vii. $\mathrm{I} 5,42,48.5 \mathrm{I}$, Med. I6. $20,65.17,330$ quater, $33^{1}$ sexies and

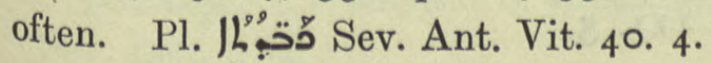

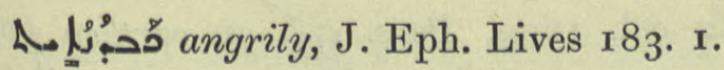

محب w w670. a round loaf,

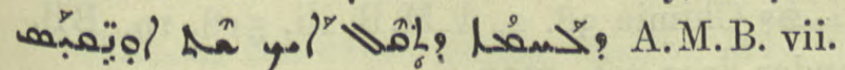

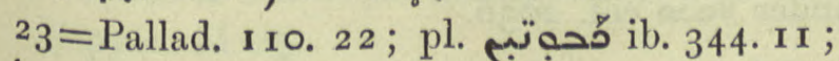
Sدة S. Dan. 398. 19; Pet. Ib. I03. I5.

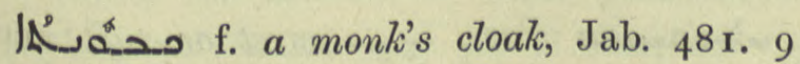
=Pers. Mart. n. 915. I. q. محس a col. I67I.

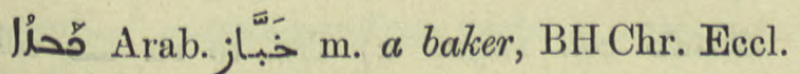
ii $26_{3}$ bis.
حِ Nars. Ming. ii. 4 I. 9 and $n$.

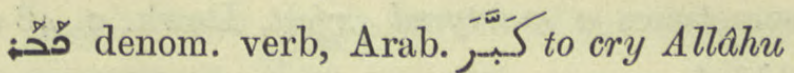
akbar, "God is great", مجي معب0, Dion. 89. I5.

لَّ col. 1672. great, abundant,

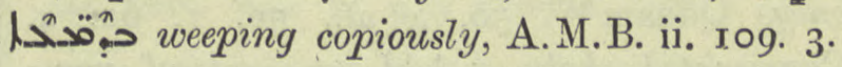

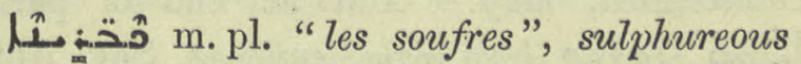
substances, Chimie 214 n. 4 ; i.q. ib., 48. 2 I, 22.

مح:م פר sulphur i.e. oil of eggs, Chimie 34. 23 .

محتم/ correct, sulphuretted springs,

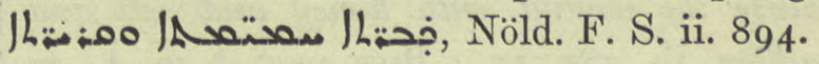

محص col. 1674. 2) to master a subject,

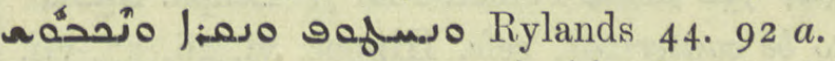

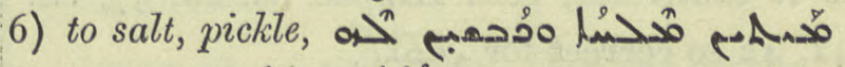

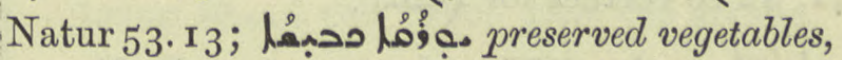

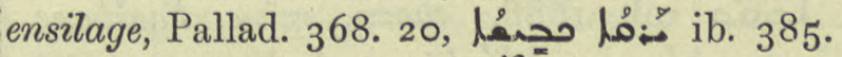

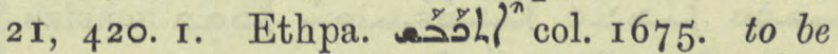

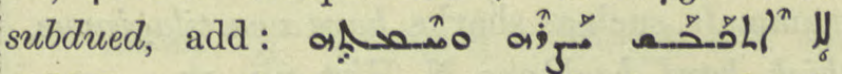
A.M.B. v. 458. I8. Astron. to be intercalated, IAsoe ophr. Ref. i. 24. 22 ; م. 1.24.

ححُمُ I) siege, add: Josephus vi. 26. 7 .

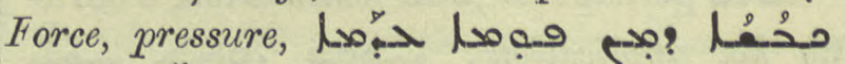
لإمبd the forcing down of food, Med. 269. 3 .

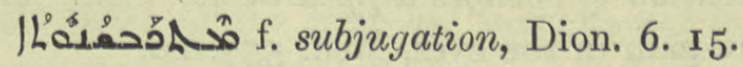

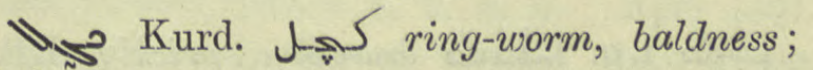
Med. 554. I5.

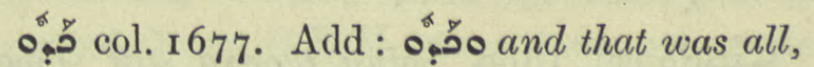
Jab. $165.12,15$.

condiment, sharp sauce from Pers. كuttermilk, r. Löw Ar. PflnN. 373; id. in loc. Z. A. xxii. 80. 8r.

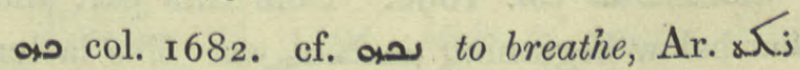
to have foul breath, col. 2370.

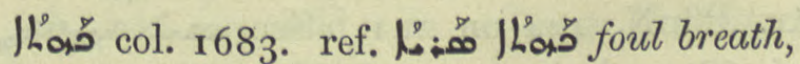
But. Sap. Eth. iv. 7 .

م. مa Pael col. 1684. Add: to supply or

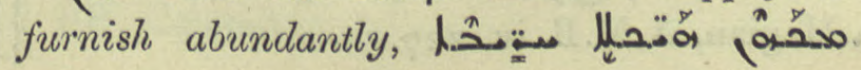




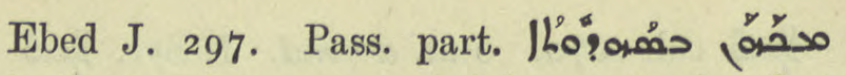

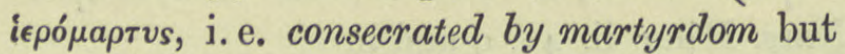
sometimes a martyred priest, Dawk. 2. $48 v$, $222 r$.

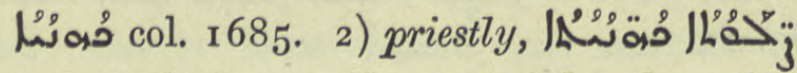
prayers which may be recited by the priest only, Qdham W. 50. 4 af., 59. I, 62. 4 . Consecrated, holy. Add at end of par.:

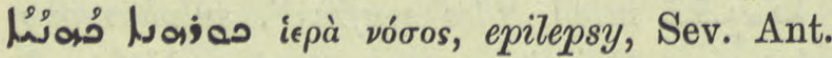
Vit. $85 \cdot 5$.

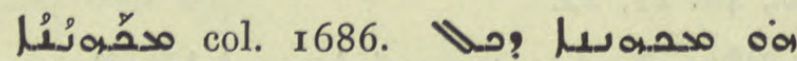
Consecrator omnium, Bar Sal. in Lit. 24. 6.

Já col, 1687. to cauterize, ref. Med. I76.

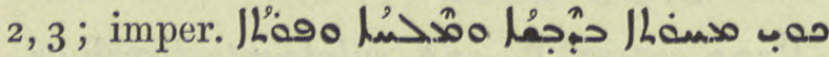
cleanse the wound with honey and salt and

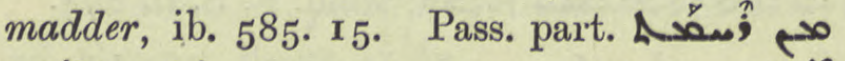
ئَ to be cauterized, refs. Hippoc. vi. 26, 57, vii. 4I, 42 so correct for (ื้อ้)?

مَّ col. 1688. thorn, spine, backbone,

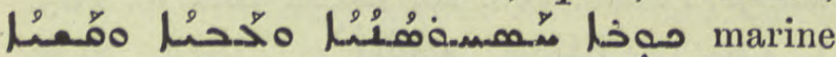
mammals, such as sharks, have a cartilaginous, thick, hard, backbone, N. Hist. vii. I 7 .

然 Acacia Nilotica, ref. Med. 209. 5 .

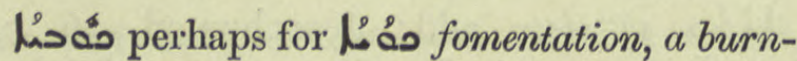

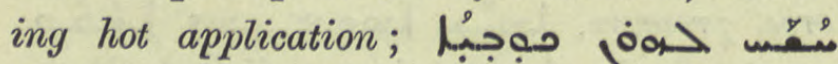

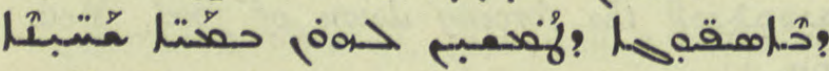
application of sponges dipped in hot water for severe pain in the eyes, Med. 557. 10.

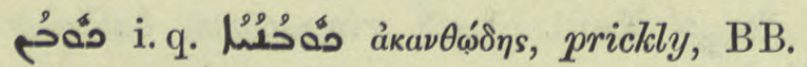
under sodule col. 1783 and so DBB.

นُนُحْ col. 169o. spiny, thorny, stiff, some thorns are not filf for want of

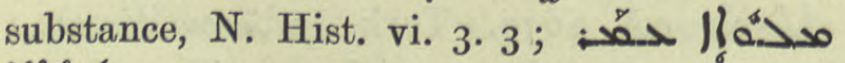


col. r690. Dele this par. and that below beginning is the better form, it is royrudis turnip, Löw Ar. PflnN. 424 on 24I. See col. 683 and $u$ a 30 DBB. 468.7 .

خوبى beauty, elegance, name of a Magian, A.M.B. iv. 227.
Y orders of priests with the Yezidis, attendants on sanjaks, Mt. Singar 24 ult., 36. 4, se b; ib. 37. 7, I I, 38. 5, 44. Yezidis 109. 16,

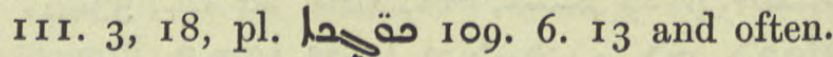

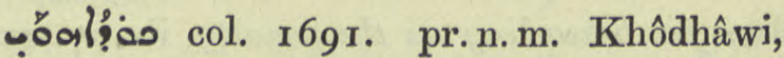
Hist. Hormizd. 79. 5, Hormizd. 1973, 2309 ; founder of the monastery of 43. 6, 45. 14, Hist. Mon. i. lxxvii ; 58. 19, 86. 2, QdhamW. 239. I.

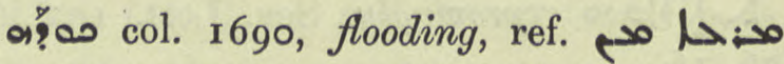
ه?

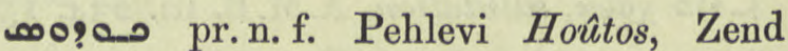
Houtaosa, wife of Gushtasp, Coupes ii. I I I.

خداى Lord or God, A.M.B. ii. 576 ult., 577.2 , Hippoc. xxvii. n. I.

خد God, Khodidad God given, Bp. of Harbath Gelal, ZDMG. xliii. 400. 4. Misspelt 1 o 1 ib. 398. I3 and 1. 18.

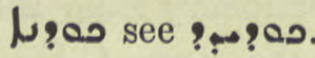

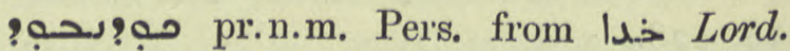

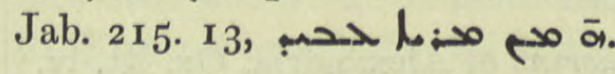

10 col. r691. to be timid, act. part. 1å

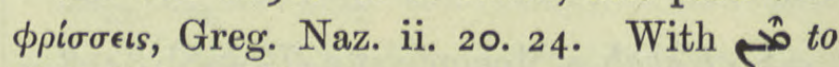

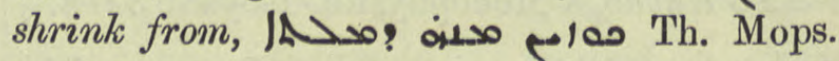
in Joh. vi. 52. Pass. part. مهي bashful, مهبر Hormizd. 1713, I731. Aph. حُُّ

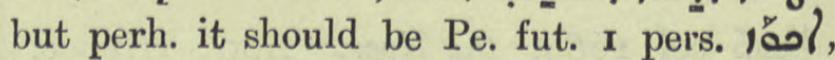
Ebed J. Card. 23. 9. עم: (corr. (?) they will be gathered together, Book of Shem III. 5 .

liós col. 1692. Pers. with a long narrow neck, Fremdw. 73, fí

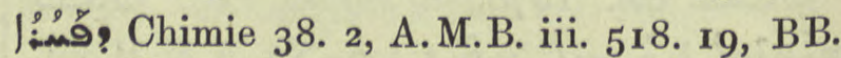
under ل Va col. 2036 .

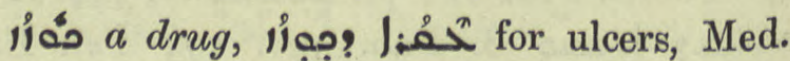
575. 19.

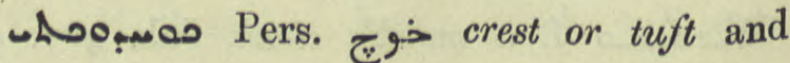
دوخت 3 virgin, pr.n.f. Chast. $55 \cdot$

مَّمَ col. 1692. 2) Trs. to rt. I7 78. 
anlojefor correct antogafon Zend Kayadha, unchastity, incest, A.M.B. ii. 578. 9, Nöld. Festgr. an Roth $3^{6}$ n. 8.

مُّ Gest. Alex. I7 I. 7 .

bo pl. مق col. r693. 6 af. Arm. Amep cudgel, مسمبل they smote him with cudgels, Act. Apost. Apoc. $\varsigma_{5}$; A.M.B. iii. I09. Iо.

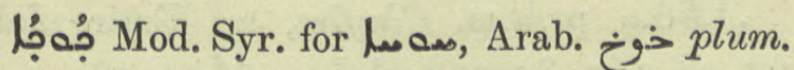
for ulcers, Med. 575. 20.

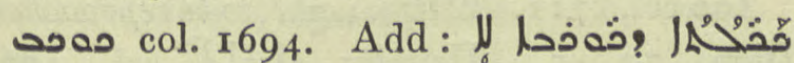
䠉 tablets of the Invincible Star, name of

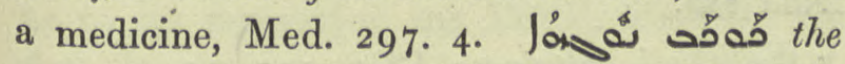
morning star, the planet Venus add: col. 350 under H-2. œ earth fم: مَ' chalk, Chimie 7. 20.

ธِ starred, marked with an asterisk, Epiph. 9. 57.

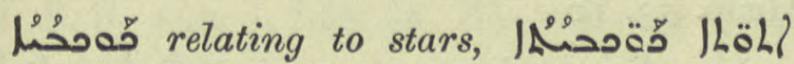
signs manifest in the stars, Bod. Or. 467 . $247 r$.

๑ว้ ๑้ name $a$ ) of a village, M.Z. 2 II. 9. b) of a mountain ib. 1. I6. For see $\rightarrow$.

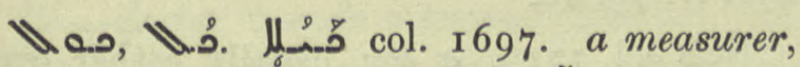

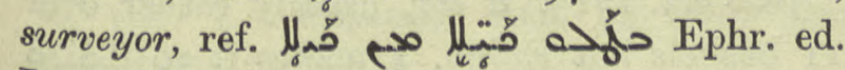
Lamy iii. 447 .

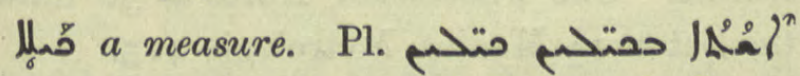
fever of varying intensity, Ephr. ed. Lamy iii. 23 .

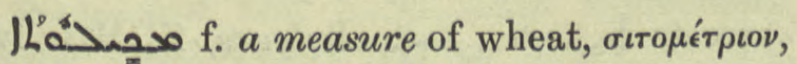
Sev. Lett. 182. 7 .

Х Jur. 87. Ir.

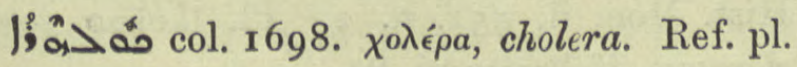
Hippoc. iii 29.

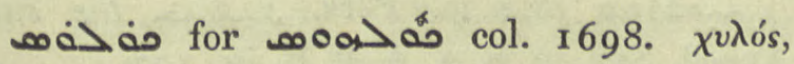
I) chyle, ref. mesenteric veins م م. Hist. vii. 6.5.

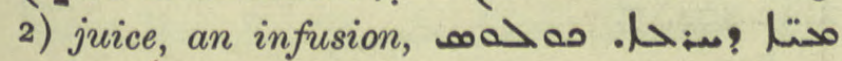
wod? onos? saffron water is an infusion of the blossom, Chimie 14. 12 ; liquid formed 2716 of pounded stone and water ib. 8. 9, го, م مح Il. II, I2.

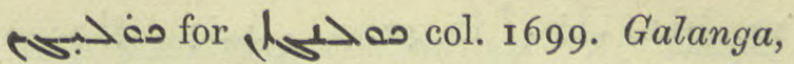
a gingerwort, Med. 309. 20, $363.9,319.4$ but 1.5 . 1.

pow, pỏ col. r699. 3) to withhold, defraud.

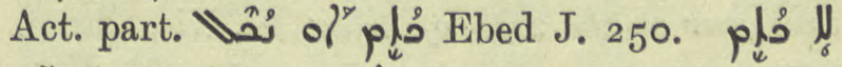

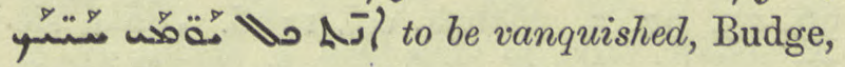
Med. 589.3 , thou shalt not fail?

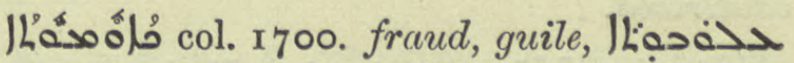

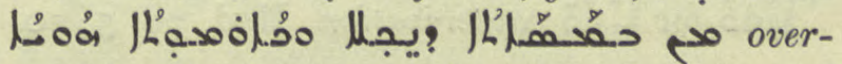
reaching is the using of a false balance and results in fraud, But. Sap. Econ. I. 2.

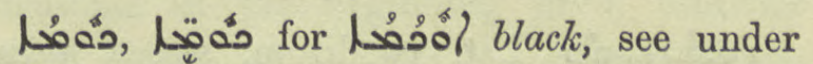
pol, hooo?.

pa Koum, a genius or legendary personage in the teaching of Mani, Coupes ii. I12, trad. $164 \mathrm{n}$.

م col. I700. Dele the article.

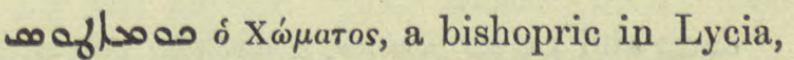
Nöld. F. S. ii. 474 . 12 I.

Wsos i. q. Wexas q.v. Suppl. and col. I7 53. name of a monastery.

م-1ace-name, near Tiari, A.M.B. i. $4 \mathrm{I} 6$.

(a), م. Pael مै col. I 702. to constitute,

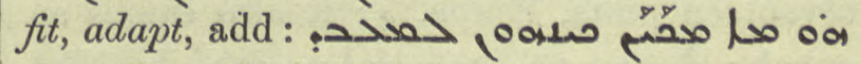
the effect which their nature is adapted to produce, Galen 274. 2 ; محَّنَ the limbs of the bear are not adapted to, not well-formed, Natur 8. 6, 9. 9. Aph. chem. to make set or harden, opp. or, في Chimie I. 8: see same passage quoted from C.B.M. II $90 b$ on col. I 702 . 22 af. and cf. lingl" ib. ult.

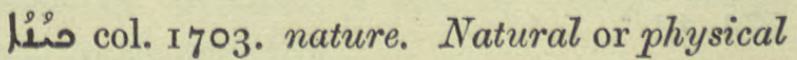
peculiarity, Natur 55. 13 and ult., Lـ naturally, by instinct, ib. 2. 6, 25. 3 .

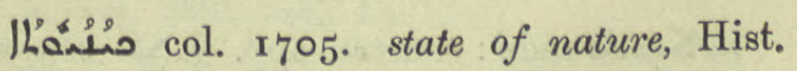
Mon. i. 18. 9.

مصِّـن add: in military formation, Josephus vi. 5. 3, 24. 12.

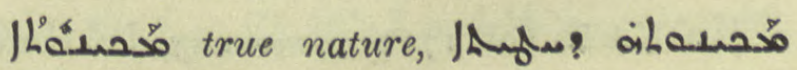
BH. Columb. $5 \cdot 3$. 
فذل حسب : col. r7or. to fall, add حمدا which falls on a Sunday, Brev. Ant. 1.37.

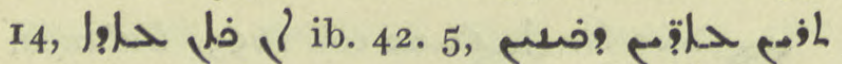

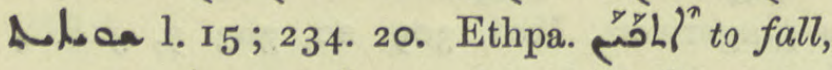

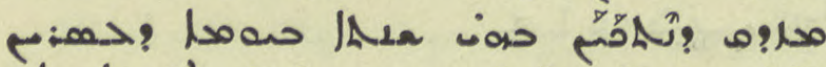
; this year on the 24 th of Adar, El. Nis. Chron.

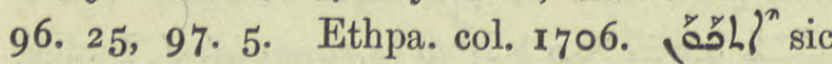
Hippoc. vi. 26, 57, vii. 4I, 42 for wảวhl to be cauterized.

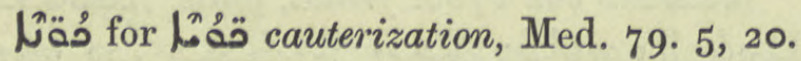
I. مفند.. Khonbogha,sun-worshipper from Turk. كون a sun and a bull, Jab. 15 5 5.

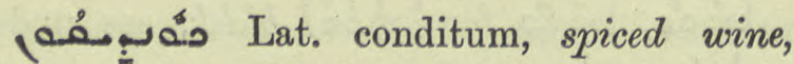

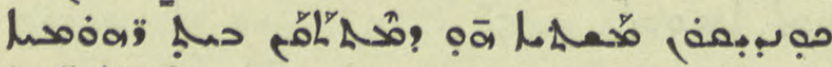

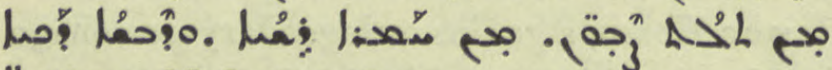

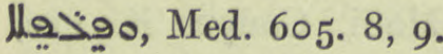

spelt from Damascus, Med. 586. 13. See sol. I707 infra.

س Sin. ix. 96. $21,97.2$, Chimie 17. 22, 53. 21 , 22. But 2 ib. 10, 14 is translated "litharge de Coptos", the latter word being obtained from the Greek.

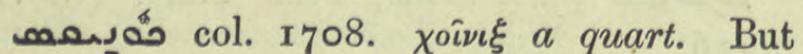
 seventeen pints, Epiph. 4 ; it also equals an

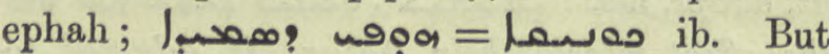
according to Josephus an ephah equals 48 Xoivikes.

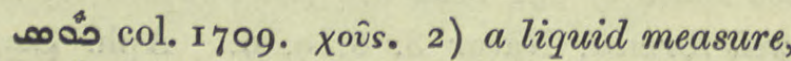

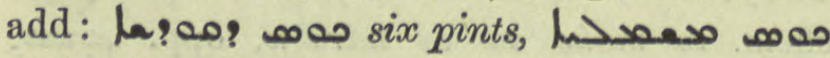
eight pints, Epiph. 5 ult.

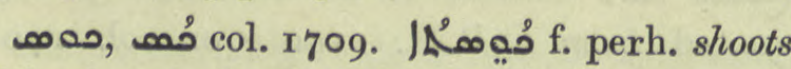
of barley, to be boiled with honey \&c. Med. 574. 13 .

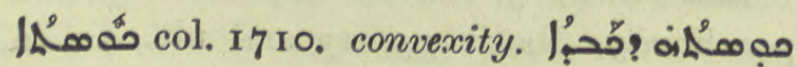
the convexity of the liver, Med. 352. II.

مخحم concave and $J_{5} \leq L$ plane, of the surface of the earth, N. Hist. ii. vo sect. 5 bis; of the air Ihangina

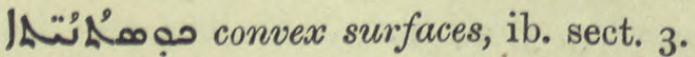

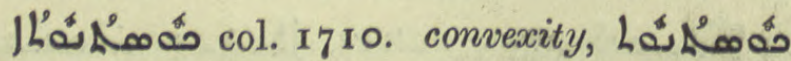
مَّتِّ (water being regarded as spherical),
N. Hist. ii. v. sect. 4 , ib. iv. 5. 4 : opp. | مدaم) ib. v. 2. I.

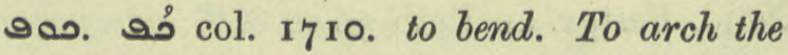
jaws to bite; mouths of evil beasts are bridled

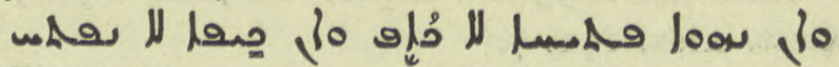
Protection 2 2. 12.

IRُ معبم No N. Hist. iii. ii. 4; رo 10.

لفة pr.n.m. Chufan, an Emir, Jab.

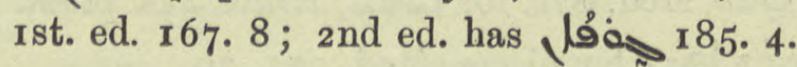

jo col. 17 II. Ethpe. ingll to be reproached, Dion. 70. 5. Usually $; /\left.h_{4}\right|^{n}$ col. 1668 from

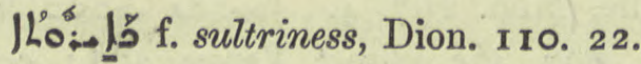

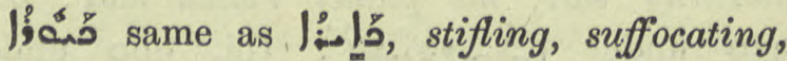
Dion. 74. 21.

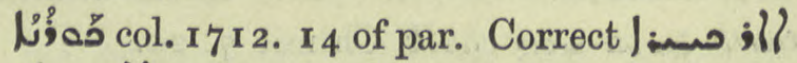
for li: ;il and so 1. 16.

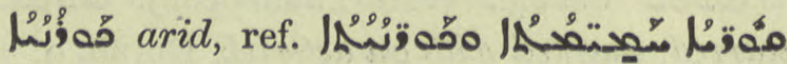
Geop. 53. 10.

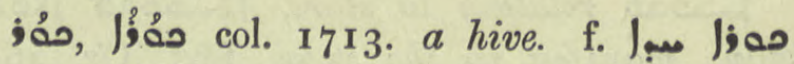

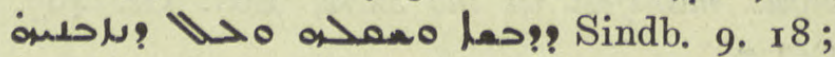

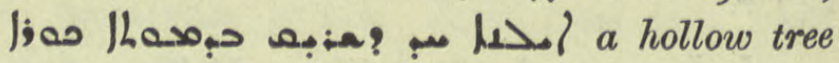
such as bees hive in, ib. I 8. 10, I1.

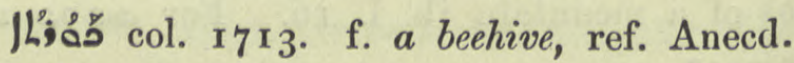
Syr. iv. 79. 18.

ן II bushels but Epiph. 4 makes it 301 a $^{2}$ bushels. One Phoenician Koros $=30$ Sata; the Saton $I_{2} \frac{1}{2}$ Modios, Metrologie $4^{\mathrm{I}} 5$.

hy a race, mentioned with Slavs and Sarmatians, Mich. Syr. 9. 18. Fraenkel corrects برجان حمق Bulgars, ZDMG. lvi. $9^{8}$ in loc.

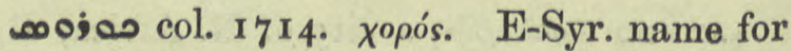
the part of a Church in front of the Bema,

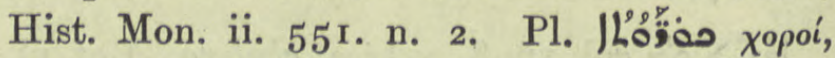
choruses, Hebraica vi. 84 .

خورشيد pr.n. Anecd. Syr. iv. 9. 2, A.M. B. iv. 137.

م0: Kurma, Bishop of Segestan, ZDMG. xliii. $404 \cdot 7$.

كُ كُور Pa country, region, Nöld. on A.M.B. ii. 577. I. 
Lia col. I 715. the quiet or monastic life, add: occupation, صعمل ?حم/ BH. in R.O.C. I9II, 272. 2.

1. 8 .

صهمب ; Cochin, R.O.C. 1912, 77.5

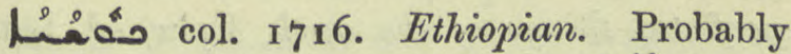
a black insect, Pallad. 377.5 .

صـ Kushnab, a village of Arbela, Syn. Or. 11.8 af.

مُ0َ Kao-ku-tscheng or Kaoscheng, a city in China, Jab. 9 ult. and note.

Lá col. I 7 I 7 prep. For different suggestions as to its derivation see Barth, ZDMG. xli. 628, Duval Gram. § 290, 294. 4; WZKM. vii. 130 .

J'مُ col. I7 I 7. Babyl.-Talm. כותא by elision

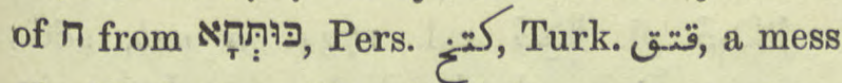
of milk, buttermilk, salt, and crumbs, $a$ sharp sauce, digestive relish, Fleischer ap. Levy NHeb. W. B. ii. 459 , I. Löw ap. ZA. xxii. 8o. But cf. حه ? above.

Vion Khotan in Chinese Turkistan, Jab. 18. $9 \mathrm{n}$. but in the text ف이.

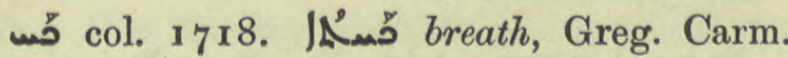
31. 3, a breeze, Jac. Edes. Hex. vii. 8, xlvi. 2.

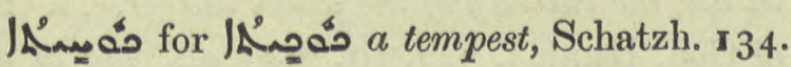
16, 136. 1. Cureton, Spic. 94 offers this correction.

مسّح Pass. part. col. I719 add: ond revered by them, Pet. Ib. 5I. 9 .

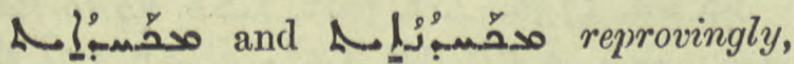
Theod. Mops. 176 in Joh. vii. 24 ; مصسبرلم סم of of Eli, Sev. Lett. 188. 2.

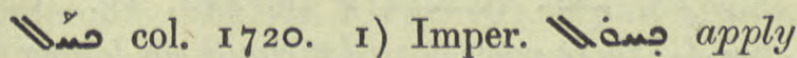
kohl, Med. $9 \mathrm{I}$ ter, $9^{2}$ ter, 555 . $\mathrm{I}_{4}$ bis, 556 ter.

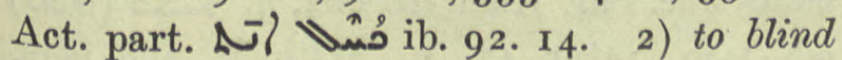

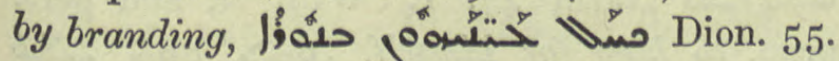
20. Ethpe. Vinhli?. 2) to be applied to the eyes with a kohl-stick

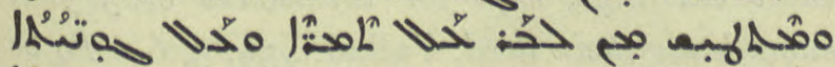

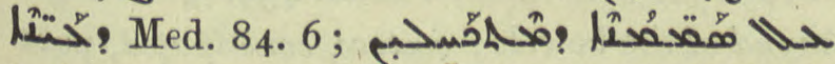

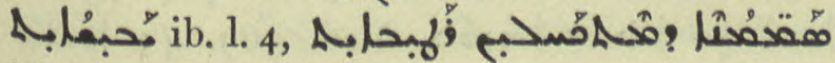
ib. $86.20,87.19$.
مئ antimony, add: Chimie I I. I, 27.5, 43. $8,47.5,58.3$.

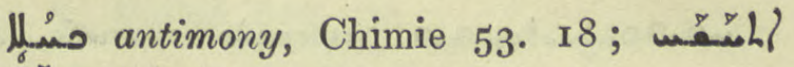

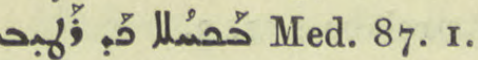

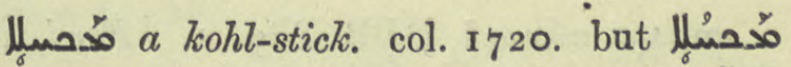

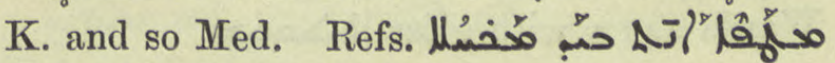
Med. 63. I 7, 91. I I, 107 ult., I75. 23,

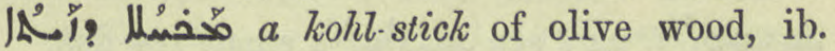
557. 5 .

ón m. a smearing or application,

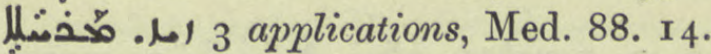

u้ col. I 72 I. interrog. particle. N.B. construction at the beginning of a sentence

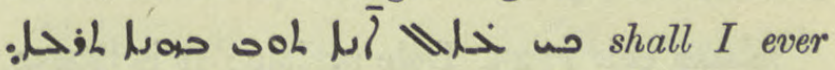
pass through this door again? Pet. Ib. 129 ter.

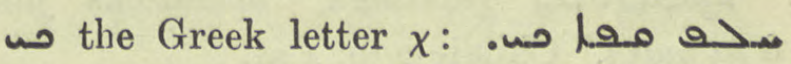

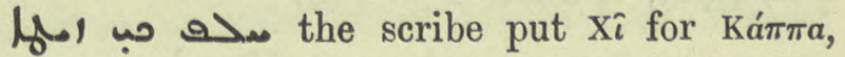
Z $\hat{\eta} \tau a$ for Xî, Or. Xt. i. 302. 16; Pallad. I80. 22.

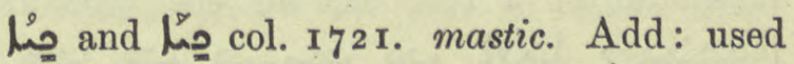

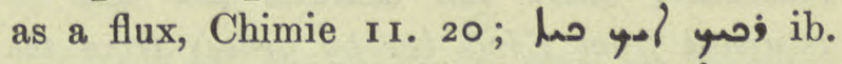

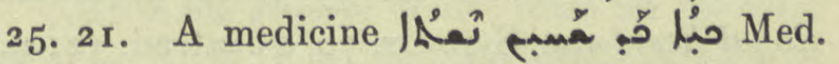
40. 17,5 O. I3, 5 I. 3, II, $.24,59.3$.

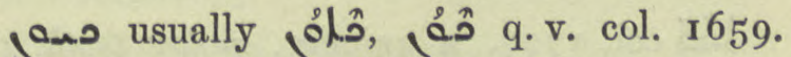
Saturn =lead, Chimie 47. 19; مق مت صa mercury, ib. 46. 7.

خيززران Chaizarana, wife of the Khalifa Mahdi, BHChr. Eccl. ii. I65.

هب pr.n.m. Kizo, M.Z. 9. 85 .

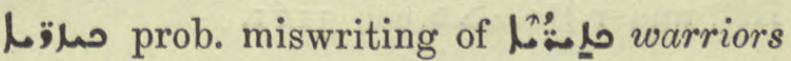
of darkness, the Beni Hashem are so-called, Bahira 230 . $\mathbf{~}$. In the corresponding passage in another manuscript $13 ْ$ قو carnal men.

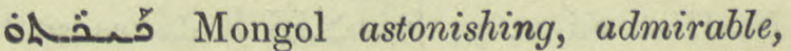
Chagatay Il-Khan, a son of Gengis Khan, Jab. 89 tit. and pen.

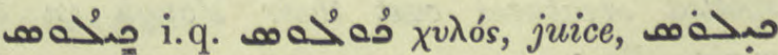
'ح

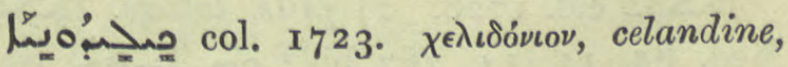
a clear yellow dye, Chimie 22. 24.

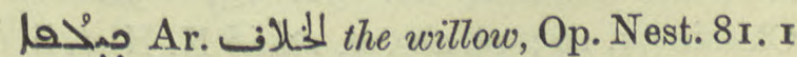

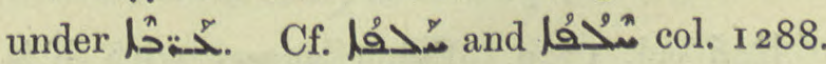

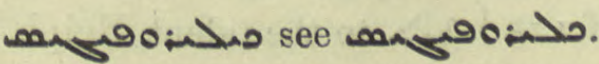

Y 2 


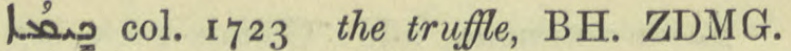
xl. 443 .

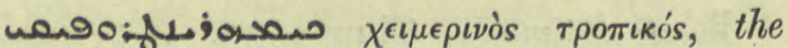
winter tropic, De Astrolabe 27 I. 3.

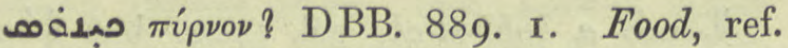
Warda $247 v$ with gloss مدمدئ.

م. Anecd. Syr. iv. 96. 19, 22 and م 1. 24 : see

مِمُ م Chios, island, Jac. Edes. Hex. xii. 7 .

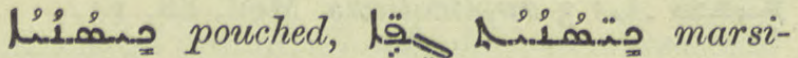
pobranchii, purse-gills, an order of fishes, N. Hist. vii. 4. 2.

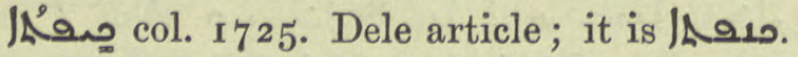

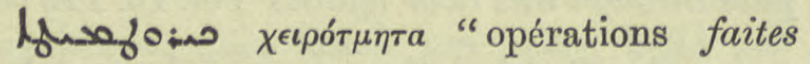
à la main," Chimie 239. 4 af.

unknown. If a place-name it should be a residence of the Sassanids,

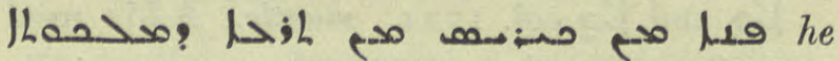
returned from Kiris (or by grace) from the Royal Residence, Syn. Or. 70. $2 \mathrm{I}=$ trad. $3^{2 \mathrm{I}} \mathrm{n}$. Chabot prefers $\chi a ́ \rho \iota s$, grace.

年 col. 1727. a grinder, molar tooth, ref. for throbbing teeth, Med. 56r. I7. A tusk, مُ فِ walrus tusks each a cubit long, Gest. Alex. 190. 3, 5; opp. 触 Natur 56. I3.

تَخت .

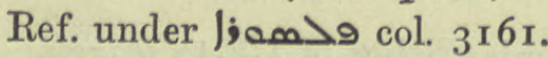

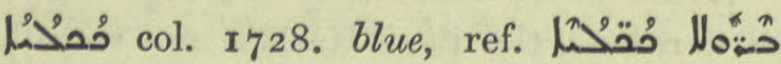
blue beryl, Hist. Mon. i. clxiv.

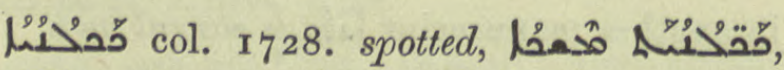

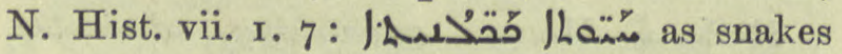
and efts, ib. 4. I, m. pl. ib. 4. 2,

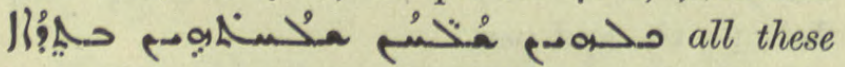
spotted creatures cast their slough in the spring, ib.

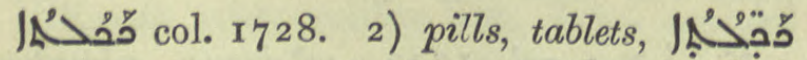

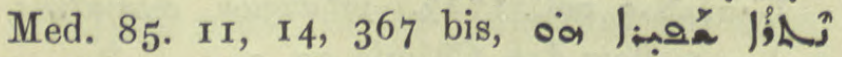

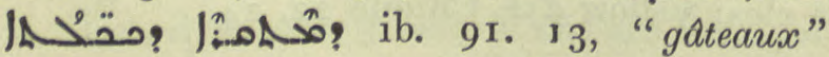

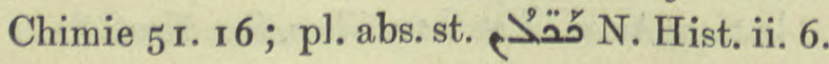

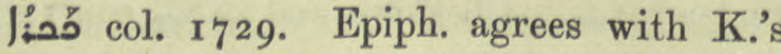
estimate of 125 רard, to the talent, Eph. 2.

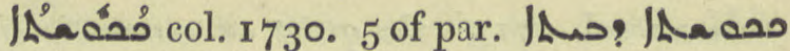
house cat, N. Hist. viii. 3. 3. Prob. a weazel, Takhsa I I 9. 7 .

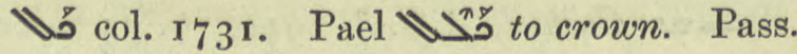

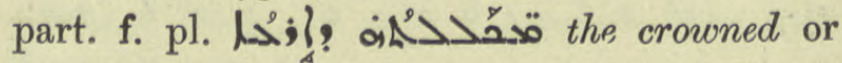
perfected things of the earth, Ephr. ed. Lamy

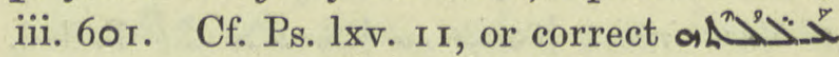
crops? Aph. Wôे col. 1731. Trs. this par.,

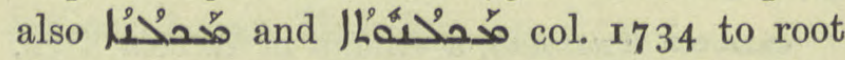
va col. $2372 \mathrm{f}$. Dele the Latin of first line of par. and compare quotation from the same MS.on col.2372. To imagine, think mistakenly. The second quotation, from C.B.M. 593, should

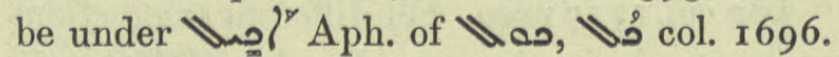

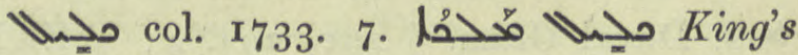
Clover, Melilotus officinalis, refs. Med. 8I ter, 94. 4, 148. 10, 32 I quater and often.

的 unas? قلا 629. 21 .

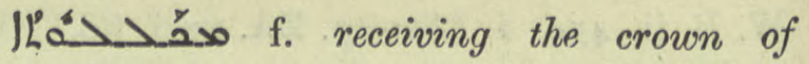
martyrdom, Mar Bassus. 38. 524 ; Jab. 455. I I.

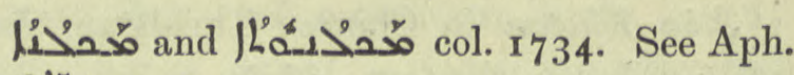
$\left.\forall \hat{\imath}\right|^{\nabla}$ just above.

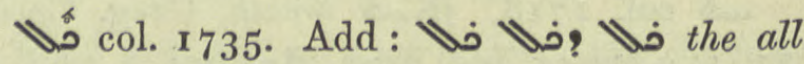
in all, the entirety of the whole universe, a title of God, Hunt. i. 347. tit. " خه Loof's Nest. 371. I3. With pron. aff. فحي

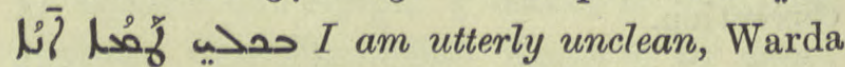
$33 r$; قحصم all of you, Kal-w-Dim. 77. I $5,18$.

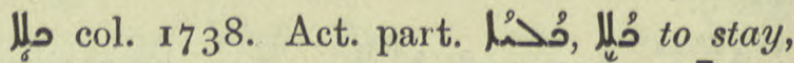

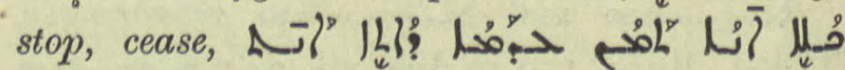
$I$ will stay there till you come, A.M.B. v. 433. I5, مخه

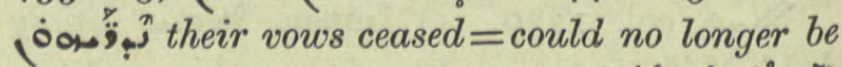

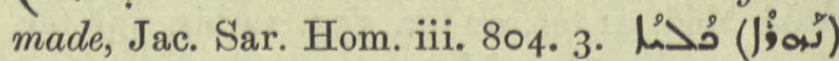

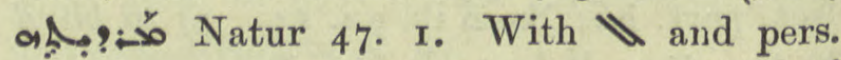
pron. to keep for himself, فُجْ

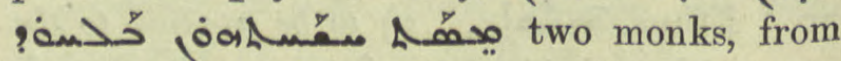
their earnings, kept for themselves only just enough for their need, Pallad. 459. 5, 6. To be bound of the bowels; costive, constipated, part.

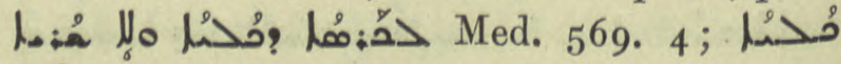
ص: diarrhoea will stop; 11. 6, 7, 13, $15,17$. 
Pass. part. بذاتهن which cannot be, opp. ' |'>l' necessary, But. Sap. Philos. 2. 6. Ethpe. uحم) 2) add: to

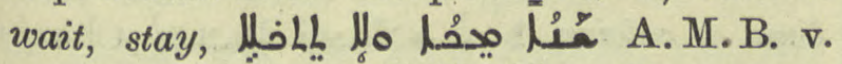

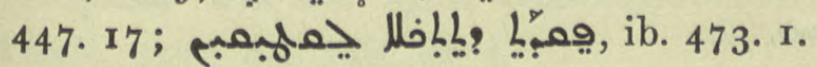

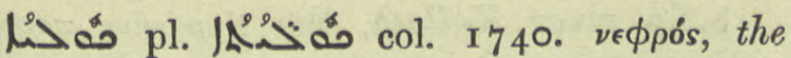
kidneys, Hippoc. iv. $75,76,78$, v. 55 , vi. 6 , vii. $3 \mathbf{I} \mathrm{ff}$.

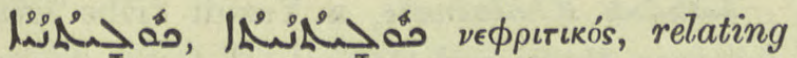

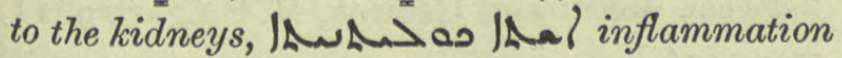
of the kidneys, Hippoc. iii. 30 ; the loins,

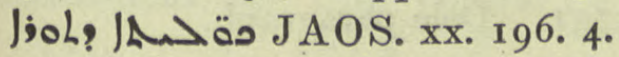

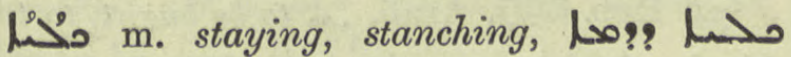
JAOS. xx. 196.8.

اLْفَ col. 1740. ref. prohibition, opp. IL'خ compulsion, But. Sap. Periherm. iii. 4 .

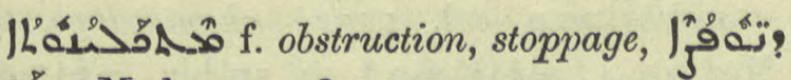

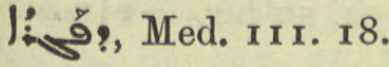

مُحِّ col. 1743. An old error for col. I748, a bladder supporting a raft, R. Duval.

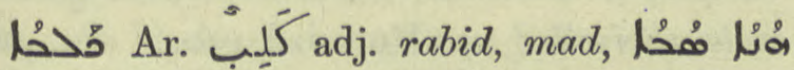
قُ Mar Kardag ed. Abb. 38. 4, ed. Feige 27. I2. Nöld. ZDMG. xliv. 534. 2 I f.

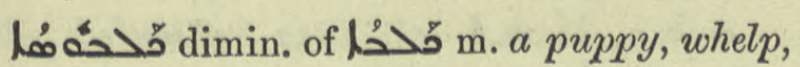
But. Sap. Isag. iii. $\mathbf{r}$.

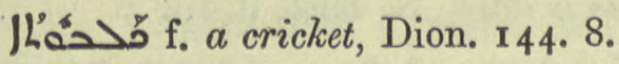

Lُشْ f. canine nature, Warda $230 r$;

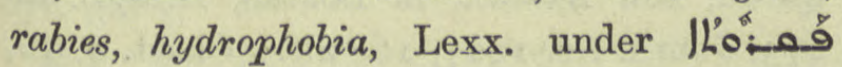
col. 3225 .

قُخدُم an inflated bladder: Meissner zur Ḥaikârgeschichte, ZDMG. xlviii. I82. But Ahikar ed. Rendel Harris 58. 12 has

مُحْ col. 1743. Galbanum, refs. Med.

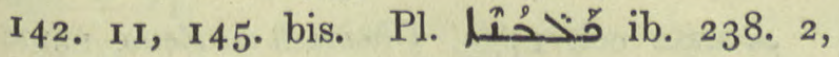
239. 2, 359. 4 .

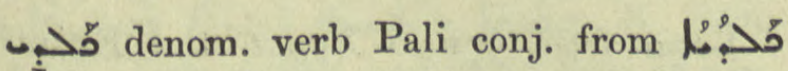
to pīay the Chaldaean, to divine, بـ Ephr. ed. Lamy iii. 403; Ephr. Jos. I21. 7. Aph.

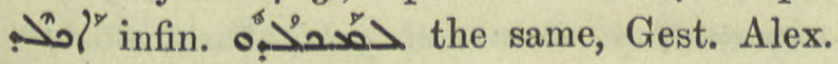
I09. 7 .

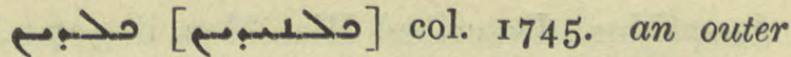

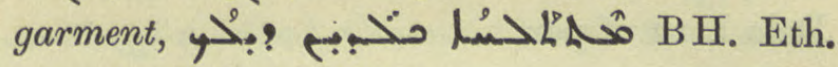
353. 8 ; Pallad. 600. $\mathbf{5}$.

 Takhsa 7 I. 16.

محه col. I 746. mil. $\chi € \lambda \omega \dot{\omega} \eta$, testudo, Jos. Styl. ed. Wright 48, 6.

مخه for col. I698 and Suppl. a white liquid like milk, Chimie 8. I r.

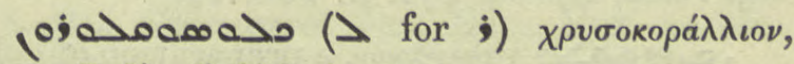
"corail d'or" Chimie ro. 9.

مخهم a kind of red earth, Chimie 86. 2.

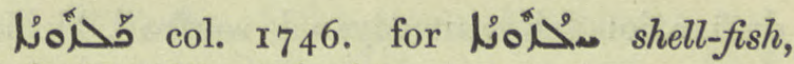

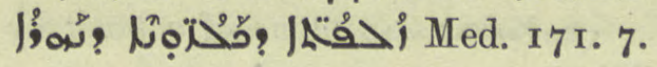

مخس I) col. I 746 pen. and ult. Dele par. it is حدب worn away, Jos. Styl. ed. Wright

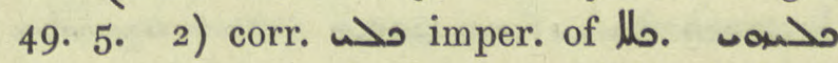
for wam stanch the flow of blood, Med. I8. 19.

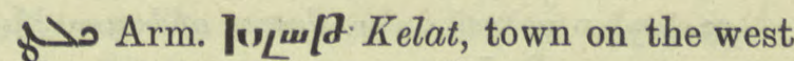
bank of Lake Van, Syn. Or. 619; مد فل Mar Bassus 243.

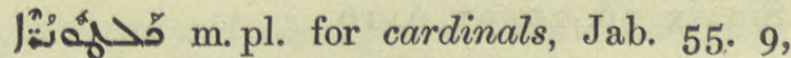
56. I, 78. I 5 with gloss

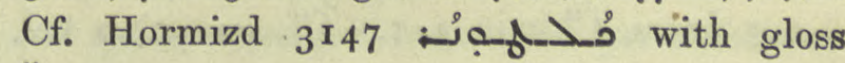
(1)

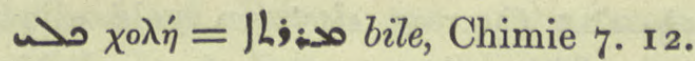

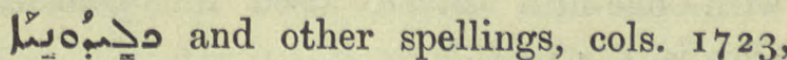

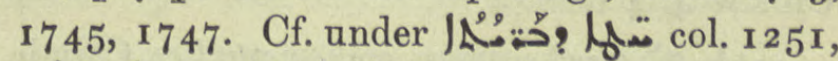

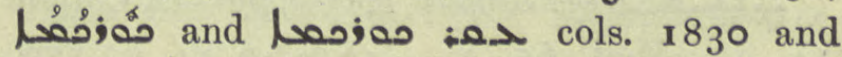
297 I, مَّم col. 2150 and L Le col. 3569, $\chi € \lambda \iota \delta o ́ v ı \nu$. Löw says that Syrians and Arabs take the larger variety of this to mean Curcuma longa, not Chelidonium majus, Ar. PflnN. 220.

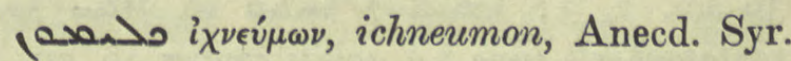
iv. $42.10 \mathrm{ff}$.

مخمه وزمع see.

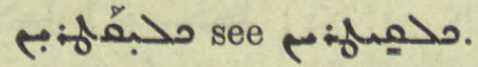

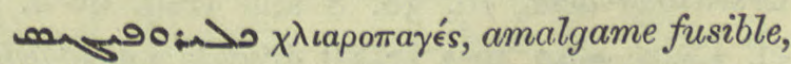
Chimie I6. I1, صحخ ib. I8. 7, I2.

مُح col. 1748. Nöld. prefers the form محّفا Gram. § I 21, a kelek = raft on inflated 
sheepskins. Described Hist. Mon. ii. 397 n. 2. Dele 1. I 2 of par.

Lُ0 Chalcedon, Bar Sal. in Lit. 72. 5, 79. 10.

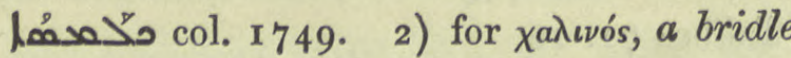
or bit, ref. A.M.B. vii. 63 .

وخيني.

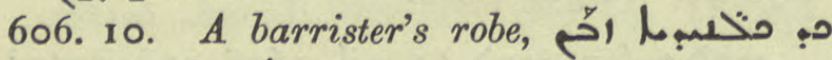
| 93. 3 ; محتبما تمزهم/ ?llowing silk robes, Anecd. Syr. ii. 268. I 2.

(ด) Chimie 48. 4; ib. 46. 20, "l'or fabriqué et rouillé par les manipulations de fixation, faites au moyen de soufre", Alchim. Grecs. I6. مخمعهم:50) name of a book by Zosimus, Chimie 243 n. 2.

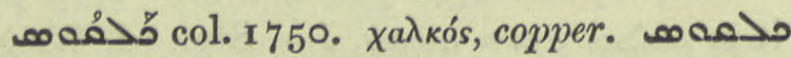

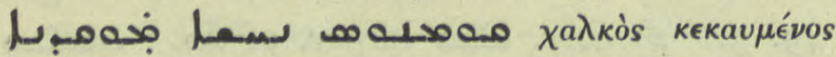
"cuivre brule" perh. protoxide of copper, Chimie 5. 12.

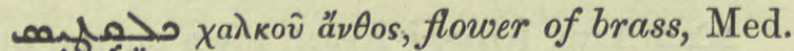
90. 14, , 1.20.

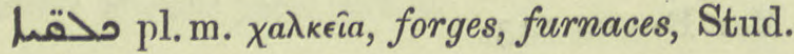
Sin. ix. 96. 20, 97. 6, 16.

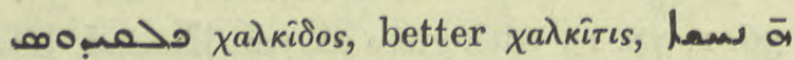
صحمبرم col. I 750 .

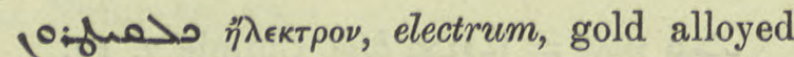
with one-fifth silver, used for polishing, Chimie 4.4 trad. 8.

仿 14. I 7, Med. 65. 3, محبمُّه: ib. 63. 20,

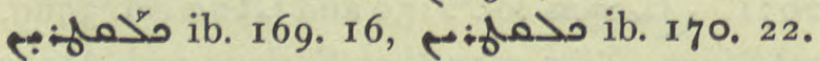

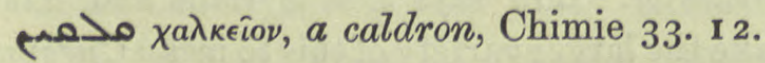

فُ col. I75r. chalk, lime, Chimie trad.

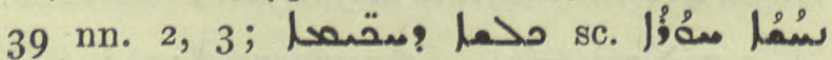
philosopher's lime i.e. white copper, ib. 20 . I5 but the recipe for making it, p. 34. 8,18 shows it to be pure lime from calcined eggshells and

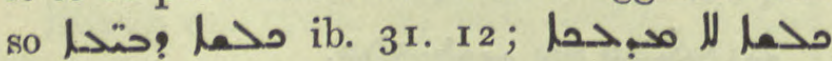
arsenious acid, ib. 38. 8 and trad. 66 n. 5 ; cf. إ) arsenic i.e. eggshell

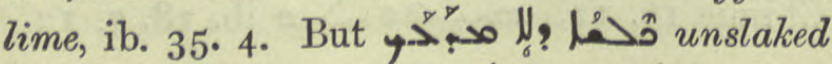

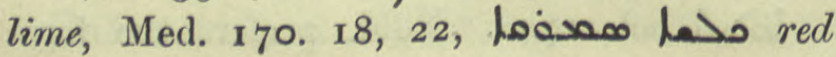
lime, ib. 593. 4 .
טمَ

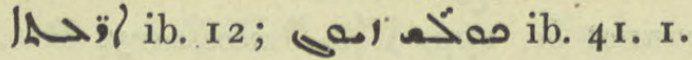

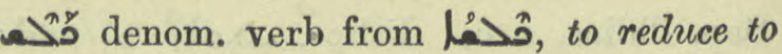

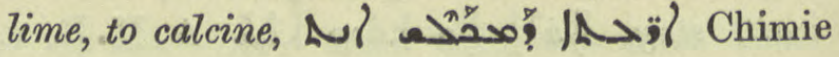
20. 12.

N now the Batman-sû, Jos. Styl. ${ }^{6} 5.6$ and n. $5^{6}$ of transl. Pointed فحد Jo. Eph. 4I6. I4.

مخدتم Khalednaïe, a Yezidi tribe living between Mosul and Gezirat, M. Singar I9. I1, trans. 26.

pǒ col. 1751. Dele paragraph.

p Heb. HD, Arab. black, Act. part. Chimie 95 ult. Cf. pól col. 182 and col. 1296.

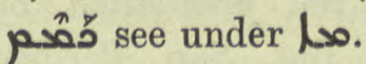

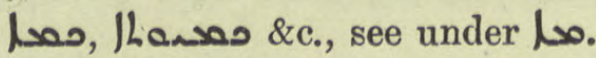

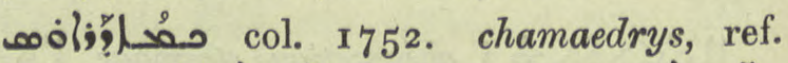

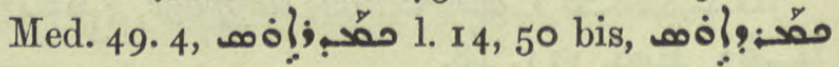
359. 19.

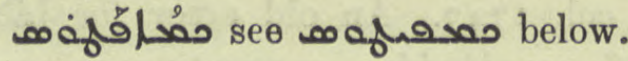

col. I 752. Ethpe. مصّم

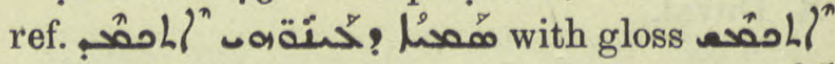
to be shrivelled up, Hormizd 1304. Pa. صُ

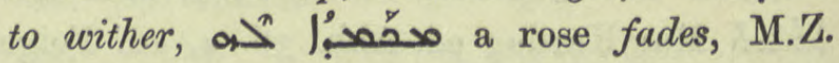
I7. 107 .

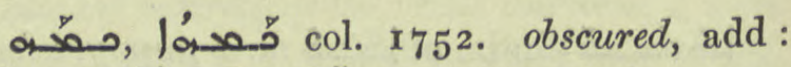

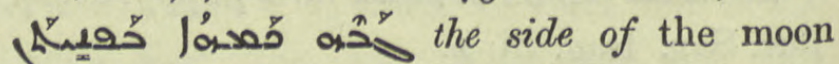
towards us is dark, N. Hist. ii. iii. 3 .

\ه์ Qardu, now Akmûn, in Bohtan, A.M.B. iii. 473, Journ. As. 1907, 162 ult.; Chast. 8. 16, I5. 12, "loos ib. 4. 4 .

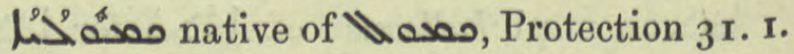
مaruasachomene, (basil?), Chimie II. 2 I.

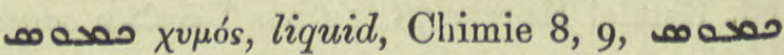
KäL? mulberry juice, ib. 83. 14.

a aso col. I 759. Chemosh $=$ Zeus, name for tin, Chimie roo. 3 .

صمي from хнег Egypt, the Egyptian science i. e. transmutation of metals पैă

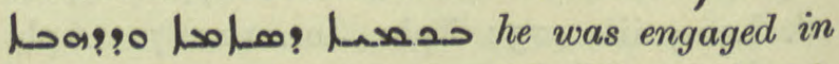
transmuting silver and gold, Dion. 66. 16.

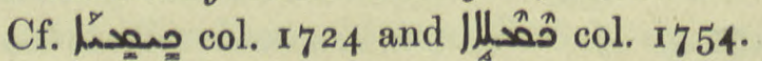




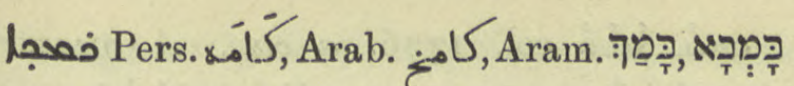
sharp sauce made with vinegar or with sour

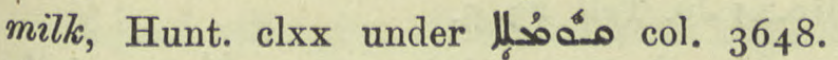
Cf. / PflnN. 373 ; ZA. xxii. 8o, f.

فِ Ar. PflnN. 247 , Med. 366 . I5, i.q. كَازبف ib. 367.13 .

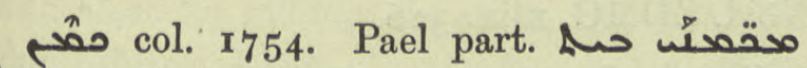

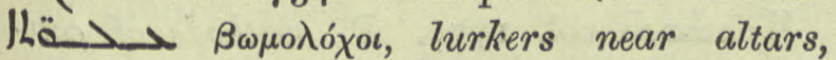
sacrilegious, Sev. Ant. Vit. 70. 10. Cf. صمس.

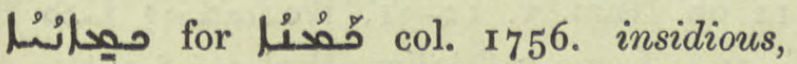
.

مصل i.q. I723. wintry or stormy weather, Dion. 57. 10.

asel. I756. Dele par. and wherever

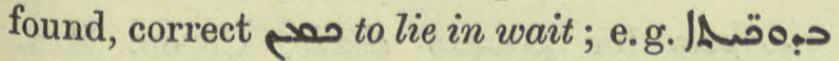

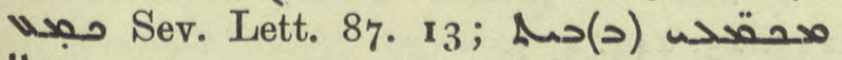
حلة ib. 225. I7, Sev. Ant. Vit. 235. I3 and marginal reading, ib. 70 . 10 where the text has محقصس.

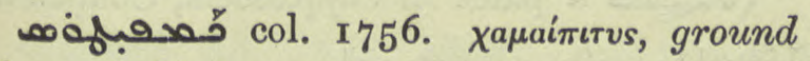
pine, refs. Med.354. 18, 355. 9, 359. I 8, 370. 4, مفحافㅇ ib. 49. 16.

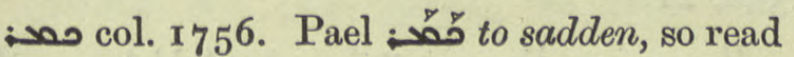
for a Pallad. 24 I. I5.

فمع col. I759. to shrivel, that tree withers from drought, Is. Nin. $3 r$

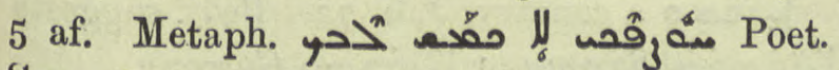
Syr. 58. 5 .

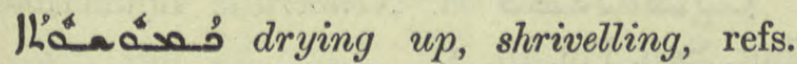

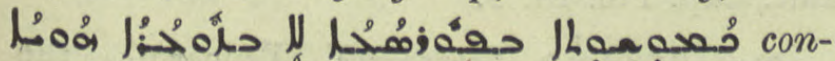
traction occurs by rarefaction, not by diminution, N. Hist. vi. r. 2 ; opp. $1 \operatorname{Ln}^{2}$;L' increase,

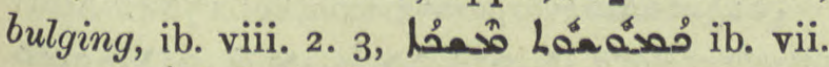

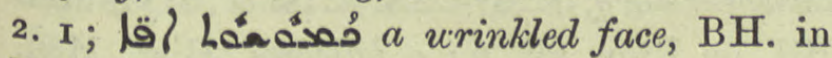
Eph. v. 27.

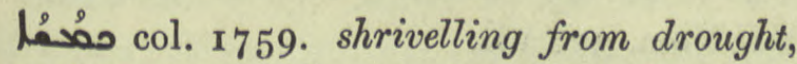

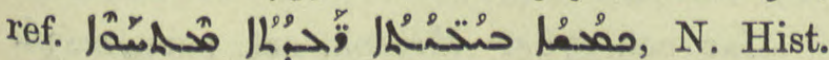
vi. I. 2. Dele second and third lines of par. a mistake for oمحم.

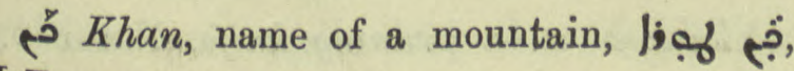
M.Z. 205. 5, 2 I 2 ult.

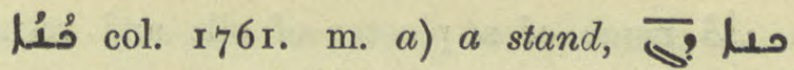
-a0 3 a tripod, Chimie I8. 15. b) a stalk,

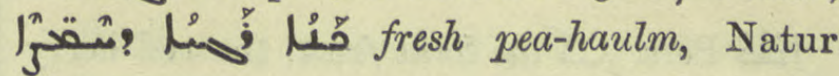
38. 15 .

مـ col. I76r. name of two months.

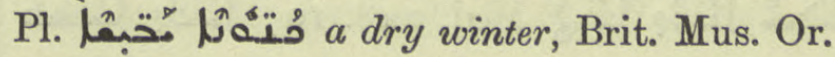

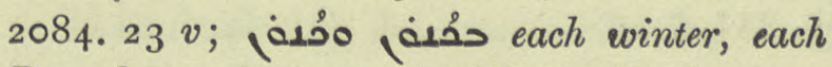
December and January, Pallad. I68. 8.

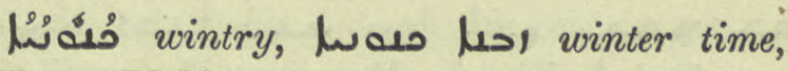
BH. ap. ZDMG. xl. 443 .

D.jen Gennesaret, Jac. Edes. Hex. 24 ult. Var.

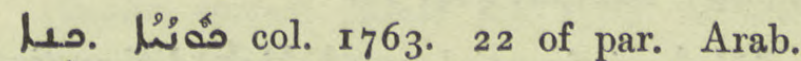
كunyahs of the Gods, Azazail Ir. II. A naming, appellation, للا 2019, Ephr. Ref. ii. 31. 9, 12, 22 ; id. ed. Lamy i. $253 \cdot 4$.

כ้ a race living in N. Armenia and Arzanene, Jab. 22 7. 3 af.

col. 1764. the juice of Cynara

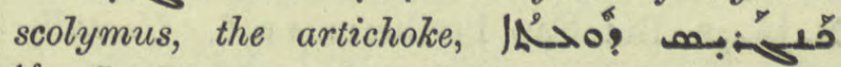

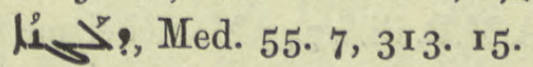

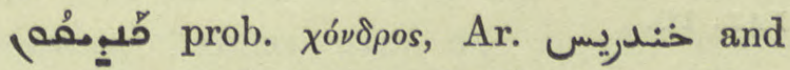
هـ fermented wheat gruel. Or a mis-writing

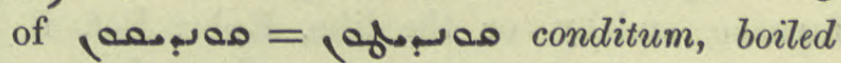
and spiced must, a hermit when persuaded to drink wine and water thought it was r $^{>}$ مَ', Pallad. 456. I, Med. 198. I6,

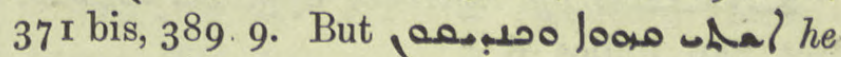
drank coffee and loaf sugar (?) M. Singar 6r. 8.

مبل Pael

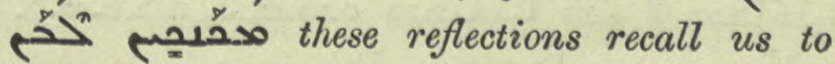
seriousness, Is. Nin. Io $r, 5$.

مُْْ m. modesty, modest behaviour, Ephr. ed. Lamy ii. 349. I3.

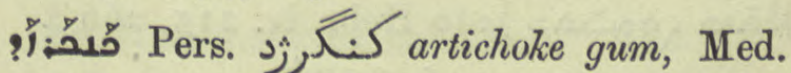
6०5. 10.

Q1 Mand. to gather together, assemble, Theod. Bar-Choni Coupes ii. I53. 26. Ethpa. อ

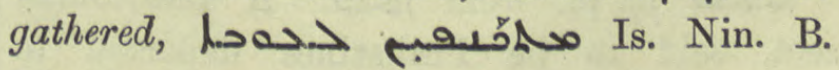
257. 14 .

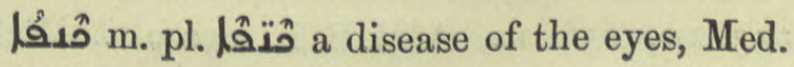
78. $18,89.8$. 
今. name of a plaster of oils and resin, Med. 25 I. 6.

 of Zizyphus lotus. So correct for Kal-w-Dim. 67. 15 and gloss, Ar. PflnN. 283;

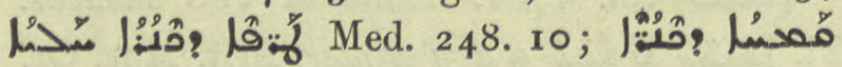
ib. 3 II. 7 .

صै col. I769. intrans. to gather together,

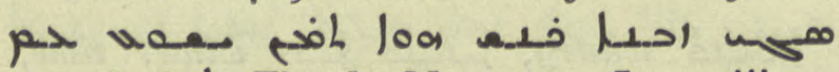

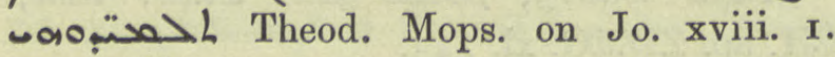

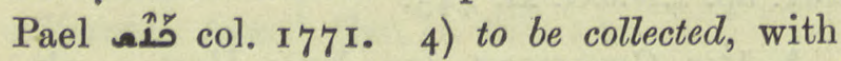
$>$ and pers. pron. $a \lambda^{n}$ oa himself, BHChr. 404. 16.

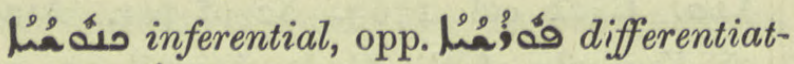
ing, مبr| But. Sap. Philos. 6. 5 .

مئم Nasol? the College of Nisibis, Stat. Schol. Nis. I72. 10, 17 .

ميمُ col. I773. Add: collectively opp.

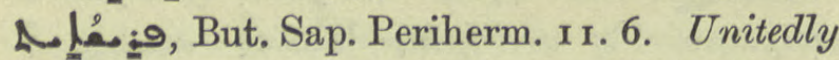

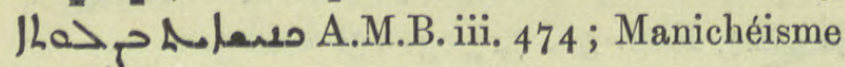
I42. 10.

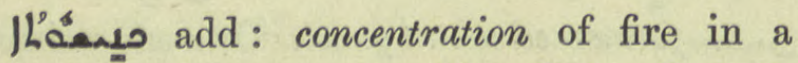
furnace, smithy, or the like, Ephr. Ref. ii. 36. 3 ; of sound in a trumpet, 1. 20 .

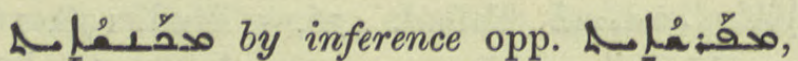
But. Sap. Philos. 6. 5 .

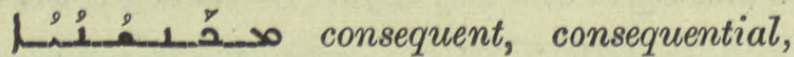

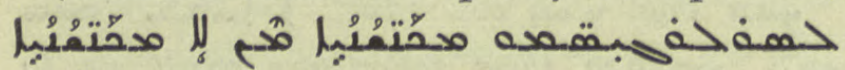
a But. Sap. Eth. iv. 4.

IL'c o col. I776. add ref. association,

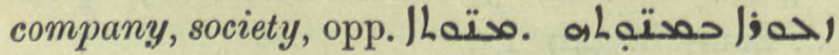

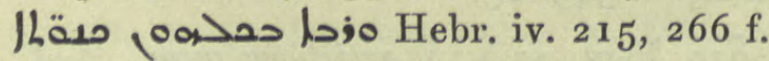

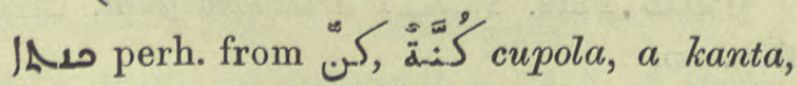
temple, shrine, Coupes ii. 156. 12, 13, trans. 228 n. 2.

o m.pl. from IAN. A Babylonian sect, said to be Philistines deported to Babylonia, Coupes i. 12 n. I, ii. 151. Med. 153. 10, 156. 12, A. M.B. ii. I50 ult., Mich. Syr. $255 a 5$.
๓อ, |

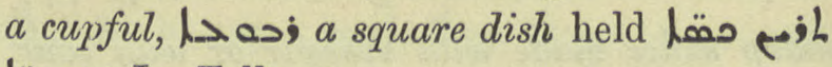
Jaحo. Tell. 23. 5.

col. 1777. Act. part. crunch. Add : to reduce by boiling, ả $\phi \epsilon \psi \eta \theta \hat{\eta} \nu a$, Geop. 47. I.

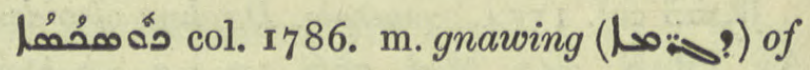
bones. Cf. Arab. כסכם Sis Nöld. ZDMG. xlix. 326.

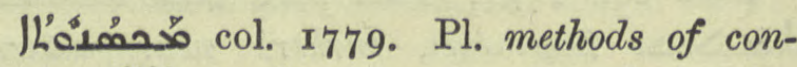

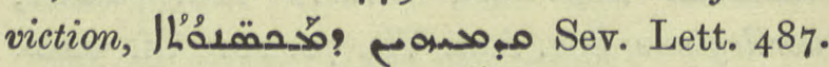

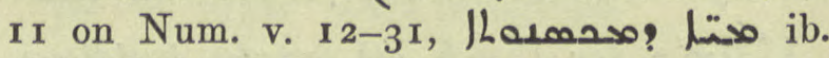
1. 19. Logic refutation, Probus 98 antep.

مُ Pa pol I780. to conceal. N.B.

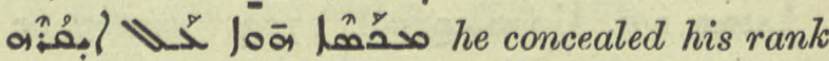

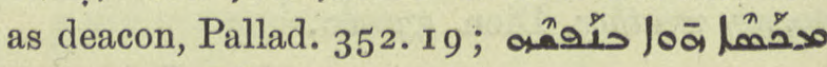
he concealed his virtues, ib. 372. I,

م כo for elixir, Chron. Min. 243. $23,244.4,7$.

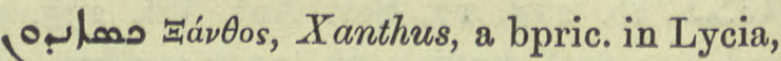
Nöld. F. S. 474. 125.

כחק a place in Cappadocia, Coupes ii. I56. 15 .

כ้ Casdians = Chaldaeans, Chron. Min. 356. 21 .

Ja or Jois, a) Salcha, on the Sebennitic mouth of the Nile, Sev. Ant. Vit. 300 ult., Nau in loc. Journ. As. 1905.375.

صمفَبُم a country, Jab. 72. Bedj. suggests

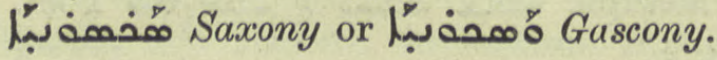

م. م. Xenocratic injunctions, But. Sap. Pol. iii. 2.

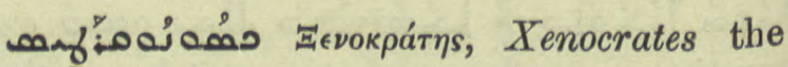
philosopher, But. Sap. Pol. iii. i. I.

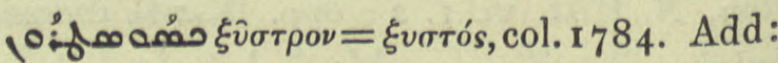

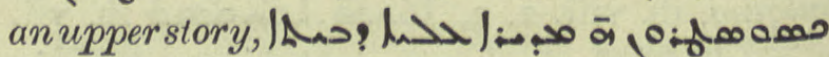
BH. on Prov. vii. 6 ; a balcony, Hist. Mon.

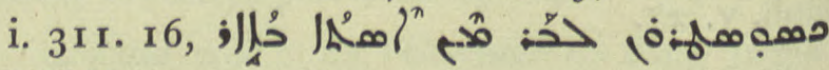
BH Nom. 328 ult.

حمُ col. 1784. Metaph. act. part. m. pl. IAْ pruning away sin, Syn. Or. 132. 2 .

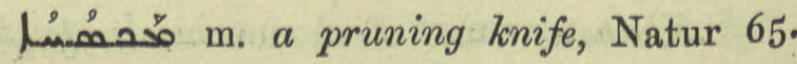
9, 17. 
صمهos Ctesias (?) a philosopher, Chimie 3I3. I.

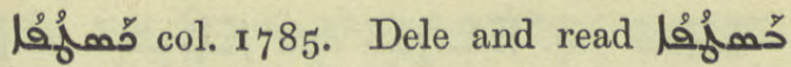
by scarification, R. Duval.

un the letter $\xi \hat{\imath}$, Pallad. I80, 22.

م powder, صمسنمe, Chimie 21. I6, I9; elixir of eggs pounded with yellow dye stuffs and alum for gilding, ib. 22. 9, 23. IO, I I, I 2, for gilding silver, 1. 13, 24. 5 and often.

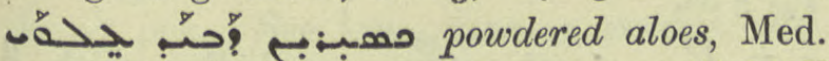

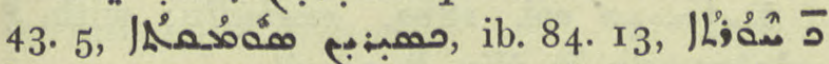
l. 20 ; BHChr. I30. I2, Chr. Eccl. 315. I8.

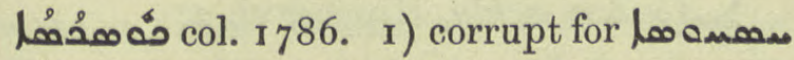
cartilage, R. Duval. 2) See under مصع.

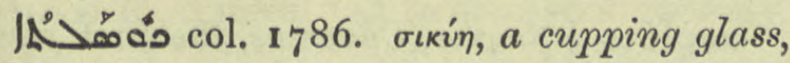

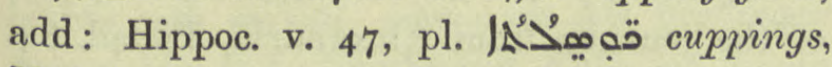
Med. 44. 9, 47. 19, BH. in ZDM̈G. xl. 443 .

פمخد f. clay, argillaceous earth, Chimie 300. 5 .

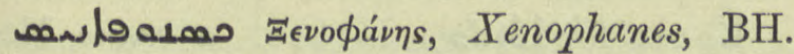
ap. Hebraica iii. 250.9 .

مـــــ 4oas l? a kind of alloy, Chimie 54. 20.

بموف Ar. Ar. the Iris, Med. 605. 12.

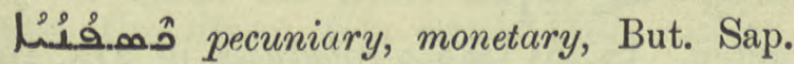
Eth. iv. 3 infr.

פـח Talm, בסיתא coral, Jac. Edes. Nöld. F. S. i. 574 wiṭ gloss مع. Cf. heoral, BB. and others col. 1787 .

قهم

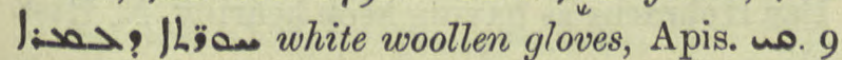
but it may mean a hank of wool.

KLْå col. I 793. convexity, convex surface opp. مُعُ concave, inner part of an arch, Jos. Styl. 32. 14, I5.

مُُمْ col. I793. Add: r) the centre, arch of a bow, Dion. 132. I 4. 2) bowing the body opp. لُمُ straightening, N. Hist. vii. 6. 6.

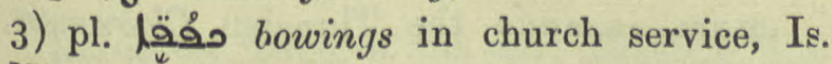
Nin. B. 284.2.

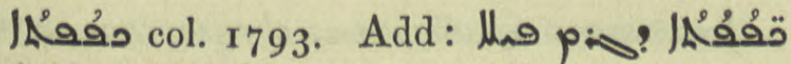
curved or arched oljects of ivory, may be tusks of ivory or ivory boxes, Gest. Alex. 2 II. I 2.

1ำล col. I793. Add: the curve, arch of a bow, Dion. 132. I6; the peacock م- forms an arch above his head with his tail, Natur 20. Ir.

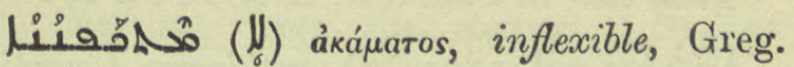
Carm. ii. 16, I 7 .

مَ col. 1796. to hunger. Act. part. m. pl. lis's the famine-stricken, Pallad. I18. I7.

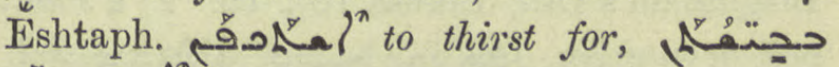

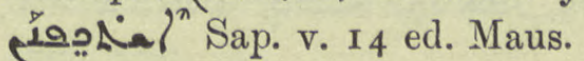

ل Hebraica iv. $215,256$.

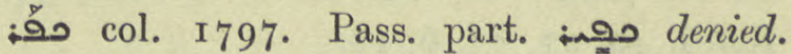

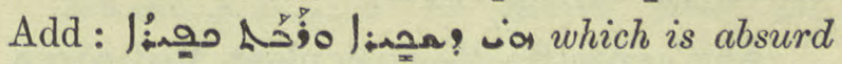
and altogether to be rejected, But. Sap. Theol.

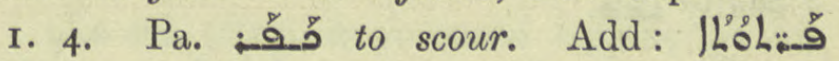

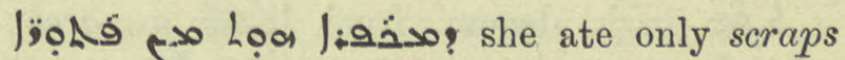
left on the table, lit. scraps which she cleaned off the tables, Brit. Mus. Or. 3337. 37 .

J Bar Sal. in Lit. 88 ult., 89. 2, 4; towels, napkins, Takhsa I40. 6 ; m. pl. scraps,

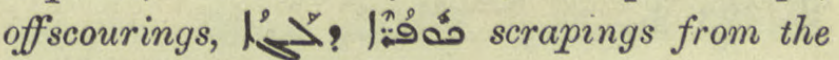
dishes, A.M.B. vii. 118.

'فْ col. I800. camphor, add ref. Med. I74. 8. Usualiy written

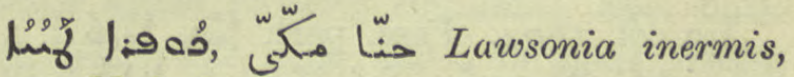
henna, Med. 605. 5 .

|

أiow litch, bitumen, Med. 605.6.

| the Asphalt Lake, Jac. Edes. Hex. xxiv. I6.

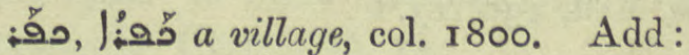

دِّة إهـ prob. in Samaria. Birthplace of the heretic Menander, Coupes ii. I I 4.

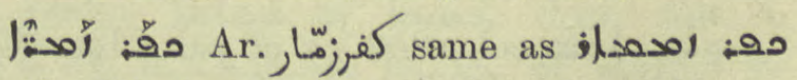
on the Tigris near Mosul, Syn. Or. 608 n. 3 , Tim. i. 308.

محِّ: Kefar Seorta, I 5 m. from Gaza, Pet. Ib. 50. 2, Pléroph. 20. 8, R.O.C. iii. 24 I. 8 af. 


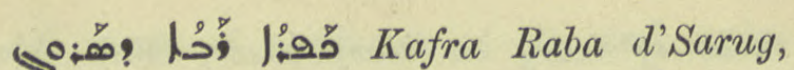
Patr. Or. v. 5. 703 pen., 7 I I. 8.

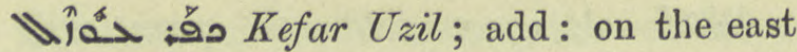
side of the Great Zab, Hist. Mon. i. I42 ult.; Pers. Mart. 236, 296, Chast. 38, 2, 6.

.

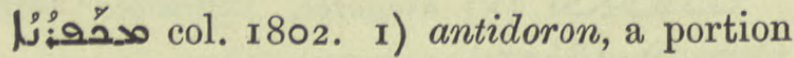
of the bread made for the Eucharist but put aside before consecration, Daily Offices, gloss; Brightman's Lit., Takhsa 105. 10. 2) a small

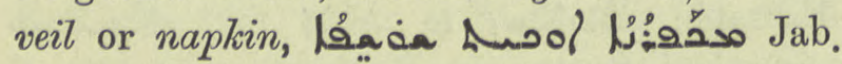
IOI. 5 .

190 Nabataean a subterranean passage. Or for lo burrow of a mole, Ephr. Ref. i. 40. $27,73.6$, 12 .

‡ِ Capparissa, the caper plant, Med. 3 ro. 9. Usually مُ.

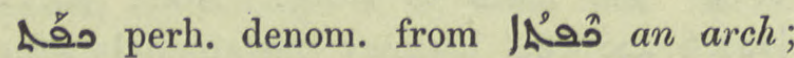

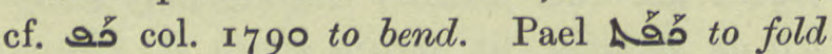
back, لب they proposed to fold back his feet because the grave was too short, Sev. Ant. Vit. 262 ult. Ethpa. Аด้

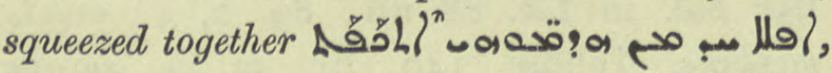
ib. 263.3 .

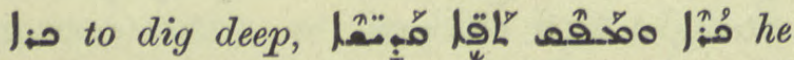
digs deep and lets loose holy streams, Greg. of Cyprus $40 b$.

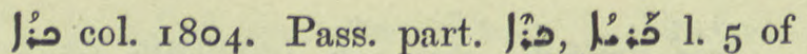

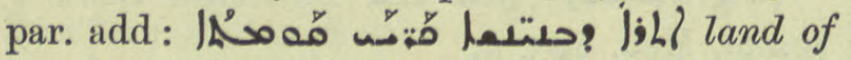
the Pigmies, N. Hist. vii. 4.

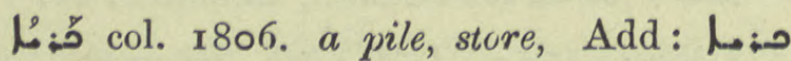
IA 1 a store of pearls, Ephr. Ref. i. 4r.

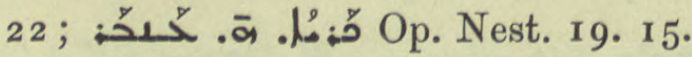

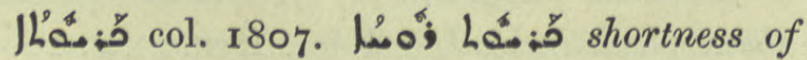
breath, Med. 130. I3.

12. 98. 6 .

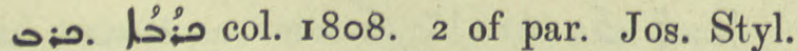

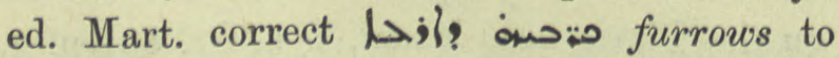

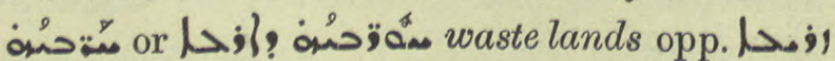
cultivated ground.

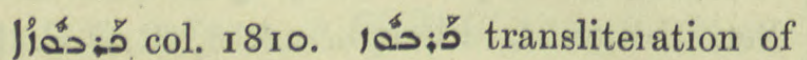
Pers. Cucurbita citrullus, musk melon,
Gest. Alex. 2 I I. 8 where the Syr. gloss

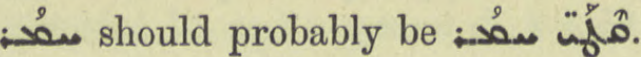

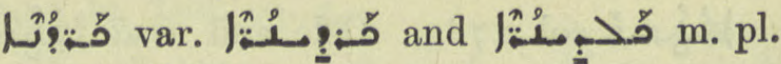
cardinals, Jab. 55. 12 n., 56 n., 75 n.

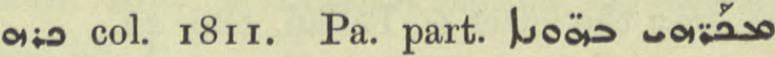
sick in mind, morbid, Warda 2 I $5 v$.

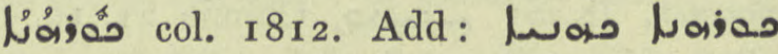

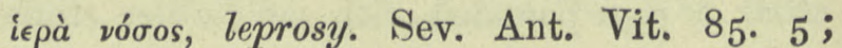

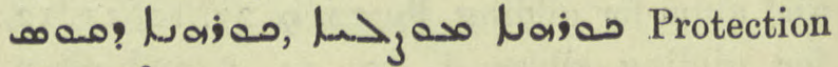
I7. 5 af.

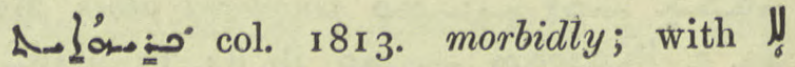
wholesomely, Is. Nin. B. 19 ult.

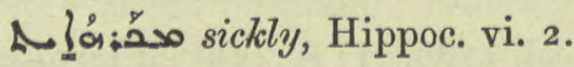

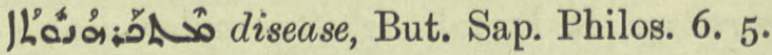

م of the journey from Alexandria, Pallad. 84. I $7=$ A.M.B. v. III. 5 .

Lo: col. 1813. caraway seed, Med. 264. 18, 305. 15, 307. 3, 12, 319. 10.

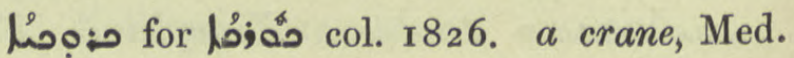
138. 19.

م: col. 1813. a singer, Warda $247 v$,

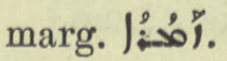

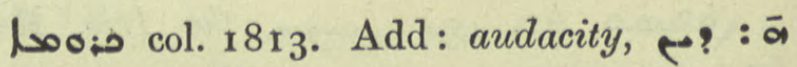
ILبم: مa Nars. ed. Ming. ii. $3^{29}$ antep.: :

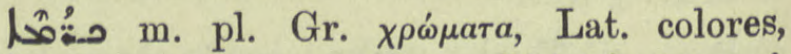
ornaments of style,

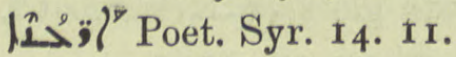

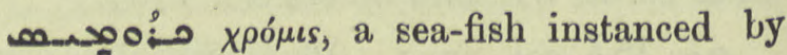
Bar Hebraeus as having keen hearing, N. Hist. vii. 2. 3 .

Q efflorescence, Chimie 16. 9. See حمن Suppl. above.

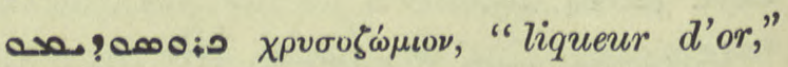
Chimie II. 4, (2000/0.00: ib. 55. 14, 56. $13, \mathrm{r} 5$.

ص the Thracian Chersonese, Nöld. F. S. 475 . 143.

1

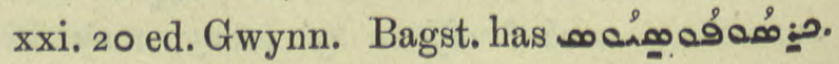


Nمعos: صo and other spellings: col. I8I6. $\chi \rho v \sigma o ́ к о \lambda \lambda a$, refs. Chimie 4. 10, 5. 2, 57. 22 ,

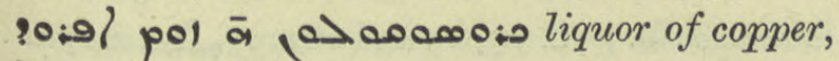
ib. 58.6 ; ib. 2. 8, 46. 7 ; "alliage d'or", ib. ıо. 10.

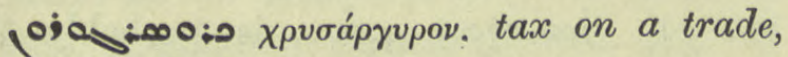
Syr. Rom. Rechtsb. 32.

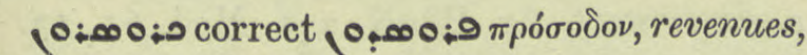
Anecd. Syr. ii. '267. I I.

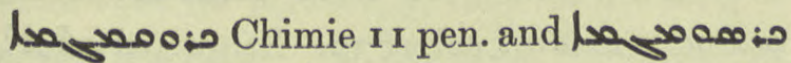
ib. 47 pen. See low extraction of saffron oil.

: Aph. A Lasex Takhsa 24. 4 af., to repeat

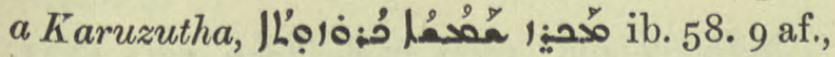
مُّم:ابي ib. 8 infra. 4) to disinherit publicly,

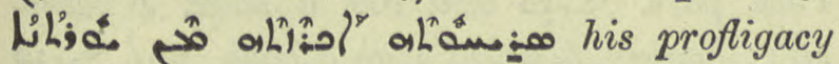
caused him to be disinherited, Ephr. ed. Lamy

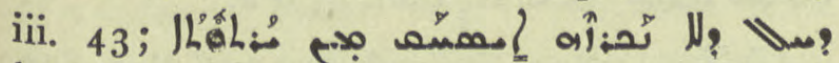

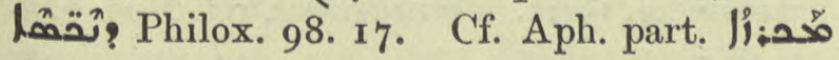

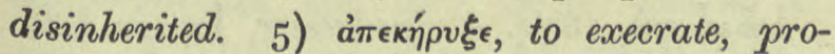
nounce accursed, 348. I7, 397 variant $n$. I5 where the text

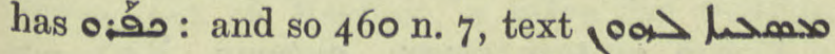

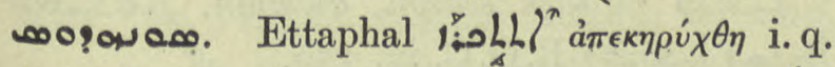
Aph. 5) Sev. Lett. 397 n. 6, text. a

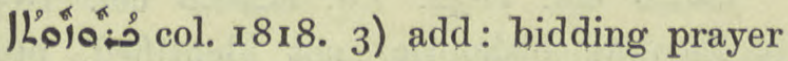

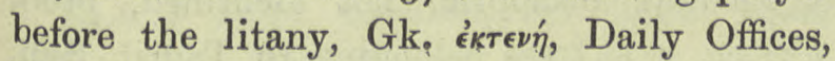
Maclean. Refs. Takhșa ro bis, Brev. Chald. i. 9. $13,19,29.2$ and often, Qdham W.

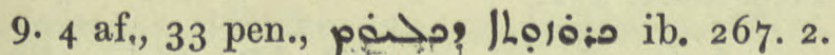
Pl. JLُơرٌْ ib. tit.

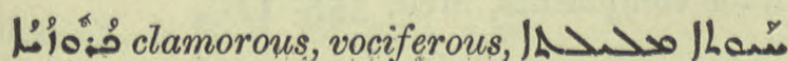
مut. Bup. Pol. iii. 3 .

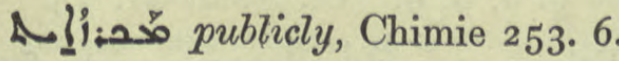

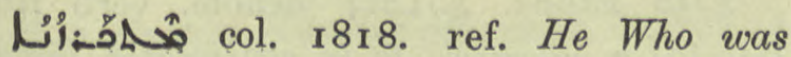
heralded, Ephr. ed. Lamy iv. 72 I, 16.

2: with gloss warmwood,

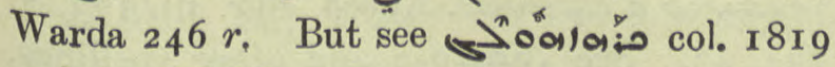
and Ar. PflnN. I 3 o.

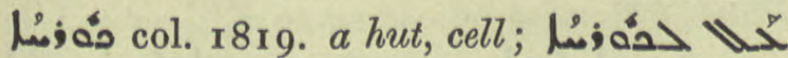

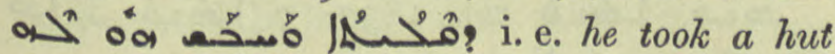
for his solitary cell and shut himself in, Mar Benj. 67.

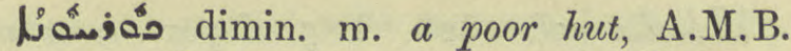

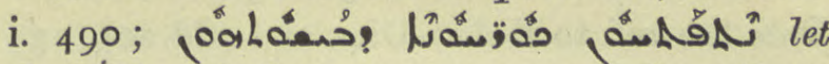
the cells of their poverty open, Sev. Lett. III. I3.

J.jo m. pl. perh. corrupt for Lat. creditor, Syn. Or. I 57. I 7 "créanciers"; Chabot infers this meaning from the context. Neither

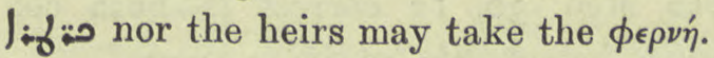

مै Chariton, Syr. Melchite saint, Or. Xt. iv. I 18.7 ; Brev. Ant. 36.5 .

م: m. i.q. جدريب A measure, Dion. I35. I I.

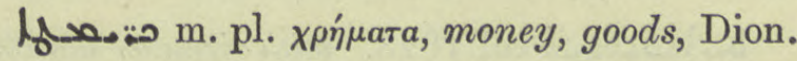
7. 15 .

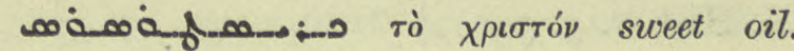
Perfumery, marg. Ihesams Warda $246 v$.

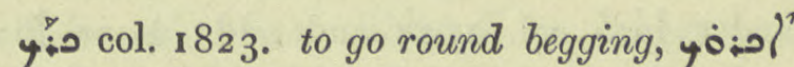
I will beg two darics, Pallad. 474. I4, I 5, 21, 22. To form a round mass, boil honey and asparagus مأل

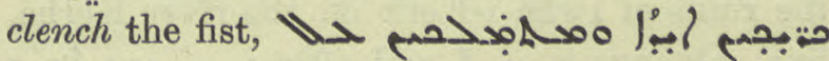
Comment. on Ps. Ixxxiii, WZKM. ix. 205 pen. Ethpe. y $\hat{\sim}^{\circ} h^{n} 1$. I3 of par. 3) trs. Ex. xii. 39 to following col. It is Ethpa.

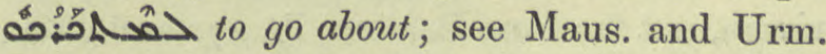
To be in circulation, y:م treatise which is being circulated, Sev. Lett. 504. I 5. Pa. 4 ${ }^{n}$. add: to twist round, Noآ he bored a hole spirally like a shell, Jac. Sar. Hom. iii.

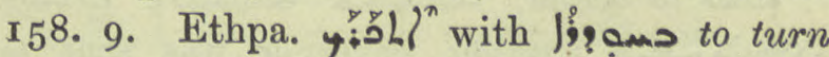
round and round, Med. 3o. 9. Rit. to walk round, form a procession, of lig Bar Sal. in Lit. 19. 24. Aph. y incessantly, Chimie I6. I4.

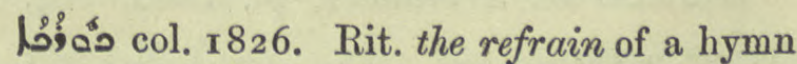

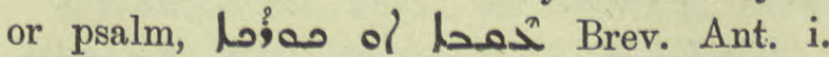

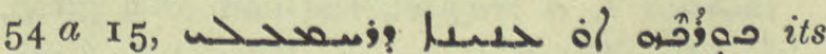
refrain or response Have mercy on me, ib.

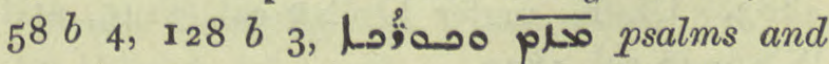
refrains, ib. 1 $25 a_{12}, 1_{6}, b 6,1_{7}$, iii. $360 b$ I 3 , Hist. Mon. i. $\mathrm{I}_{42}$, ii. $29 \mathrm{I}$ n. 3,446 n. 3 . Cf. Anglice rounds. 


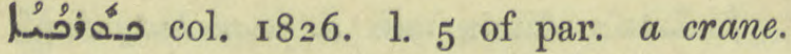
Dele the ref. to Kal-w-Dim. and add 205. 15 ; pl. Anecd. Syr. iv. 66. I5.

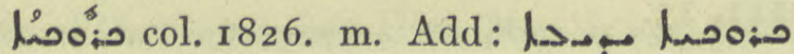
a) pachl / a certain term of days has been fixed for him, Sev. Lett. 24. I3.

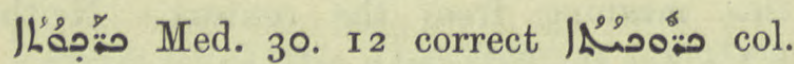
1826 infra. whirlpools, eddies in rivers.

مَّ | مَّ人 Sev. Ant. Vit. r 5. ult., marg., 98. 2.

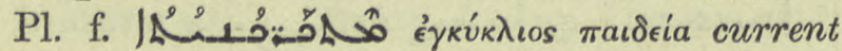
affairs, R.O.C. 115.14.

A. said of a whirlwind, N. Hist. v. 3. 3 .

|ว่: Fort as a place-name, col. I829. Cf.

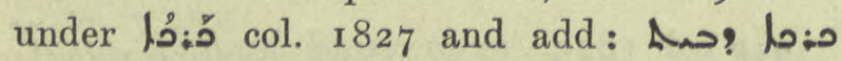
مخه م the capital of Beth Garmai now y Kerkuk, Syn. Or. 674; 53. 16, 91 etc. Hist. Mon. i. 68. 10, Pers. Mart. 267 ; م:م or or in Khuzistan, below Susa, near the ruins of Iwân-i-Kerk on the river Kerha, Syn. Or. 27 I n. 4, f. and often; م:0س ?

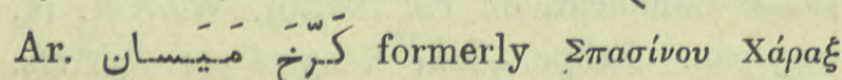
between the Tigris and the Eulaeus (Karun) rivers, not far from Bassora, ib. 272 and often, Guidi ZDMG. xliii. 4II； same place, Rahlfs on Kessler's Mani, G.G.A.

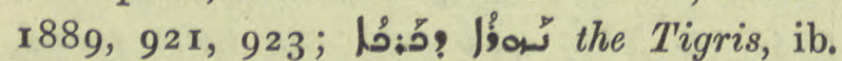
$97.4 \mathrm{f}$.

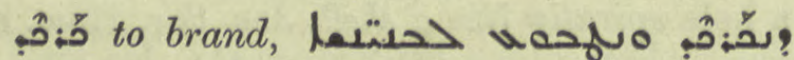
(0a) another governor was sent to brand and mark men on the neck like slaves, "stigmatiser et marquer", Dion. I24. 8; ט I 49. 4 .

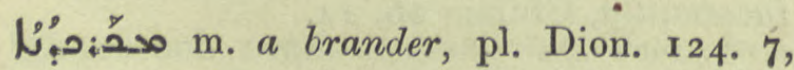
I48, 11,12 .

1 1 f. a ring at the end of a girth, El. Nis. 40. 7, BA. Cod. Goth. 4883. Cf. K. under /fُ |

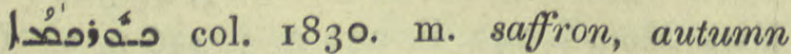

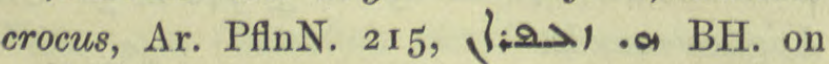

Prov. vii. I 7 , Med. 49 bis, $50.8,82$. 19, 83 bis,

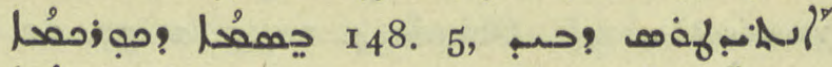

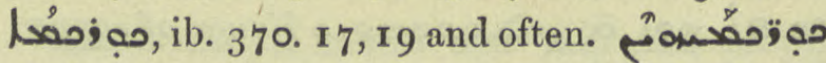
the blossoms or heads of lilies (?) ib. 302. 6 ;

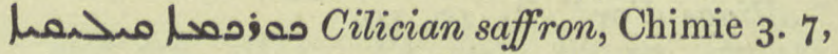

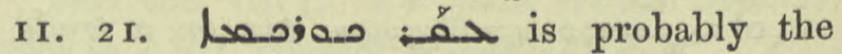
rhizome of Curcuma longa, the turmeric plant, a gingerwort, Ar. PflnN. 220, Med. 6r. r3.

مै denom. verb from yellow or reddish, col. 1830 . Ethpalpal pǒ:o้L?" to be made yellow or reddish, - wew

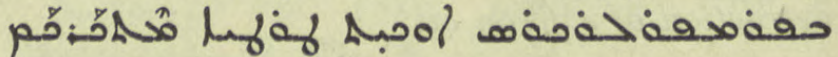
brass is turned yellow by pompholyx or antimony, N. Hist. iii. I1. 3 .

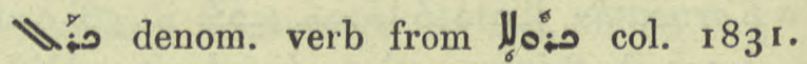
Pass. part. \ڤ bent like a hook, 4 Macc. 467; hooked, fastened with a hook, Ni \. Di

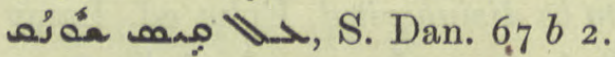

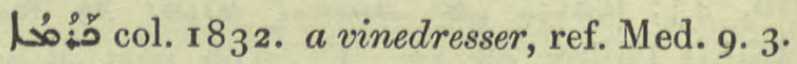

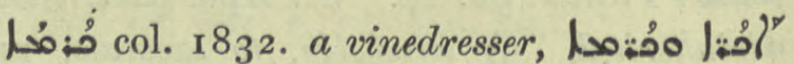

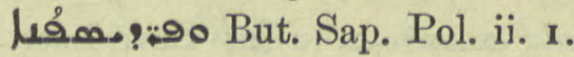

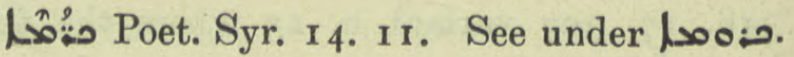

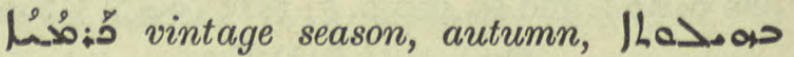
Ia: R.O.C. iv. 359.

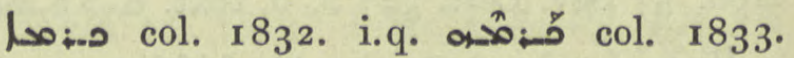
a Jacobite bishopric, not identified, prob. Karma, a fortress belonging to Tagrit, Syn. Or. 299 and often, Guidi ZDMG. xliii. 4 I I ; Pers. Mart. 23I.

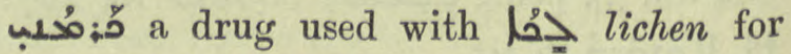
liver complaints, Med. 355. I I.

ضُ مهليم m. pl. abbrev. form from col. 1834. people of Carmelish, G. Warda ed. A. Deutsch 8. 8, 9. 9. Cf. Nöld. Gr. 75.

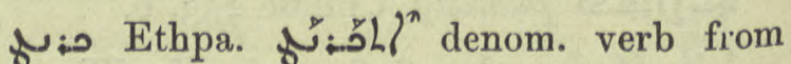

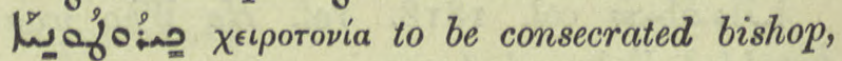
El. Tirhan 3, 4 .

مُْيمُّم a chronicle, Anecd. Syr. iii. I33. 7, 134. 23 . See صis and other spellings, col. 18 44 .

مِالم diarrhoea, ref. Med. 291. I7, 301. 20.

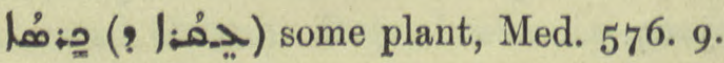


Konis

173 doreads

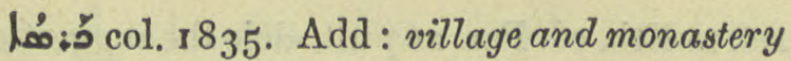
in Kashkar, Chast. 62. 8, 9 .

政 36. 10.

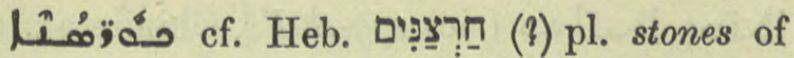

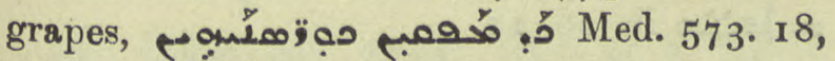

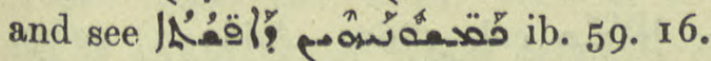

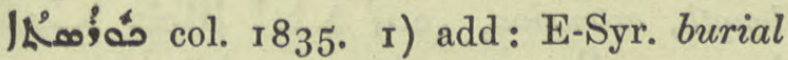
service-book for the clergy, that for the laity is

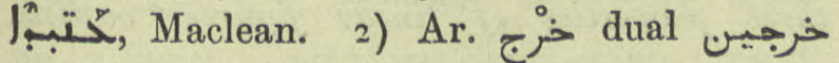
saddle-bags, Hübschmann, ZDMG. xxxvi I 30.

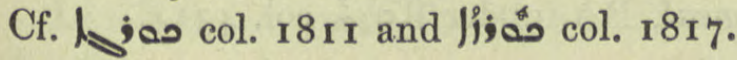

9ound Christian-Jewish, El. Tir. Gram. 4. 2.

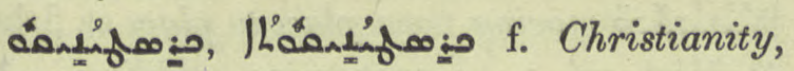
Syn. Or. 69. I 5 .

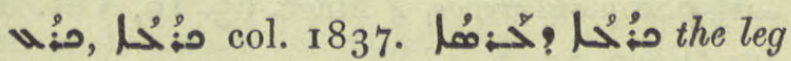
of a bedstead, Pallad. 604. 18 ; pl. Gest. Alex. 218. I3. L. I5 of par. dele ref. to , asooj;

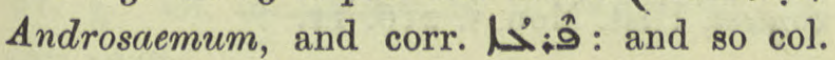
257 and DBB. 200. 3, see Ar. PflnN. 320.

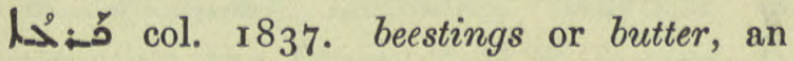
ingredient of Marham, a salve for wounds,

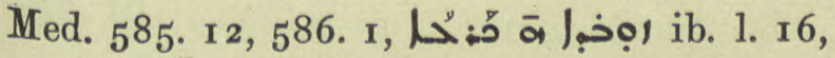

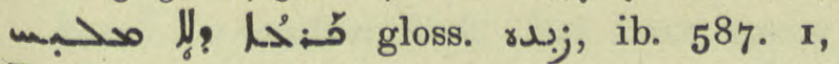
Protection 27. 3,6 .

|ر col. 24 II = DBB. I 263 .

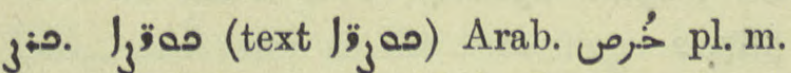
earrings, BH Chr. 324 ; id. in Jos. iv. 18.

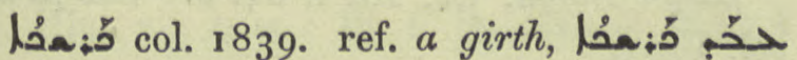

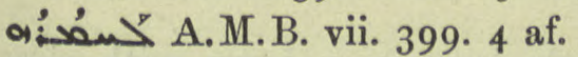

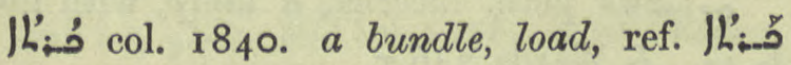
Hist. Mon. i. I 26. I6.

น’óhin col. 1840. a Kurd, Kurdish, joL

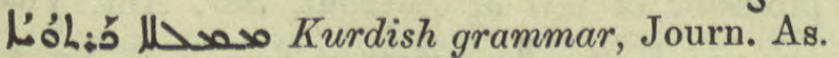

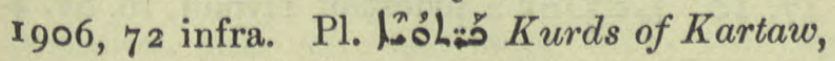
a region west of the Lower Zab, above Arbela, Pers. Mart. 207, Hist. Mon. i. 98 f., 294, 32 I, Jab. I 2 I. 6, I 23 med.

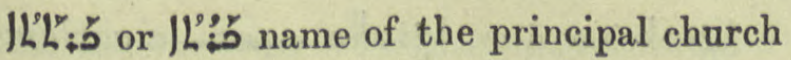
in Tagrit, B H Chr. Eccl. ii. $3 \circ 9$.

صمأهم

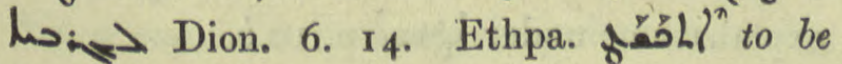

transfixed, pierced with arrows, Anecd. Syr. iii. $3 \circ 5 \cdot 9$.

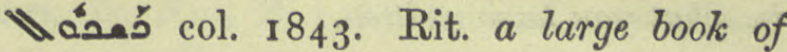
variable anthems for use at evening service,

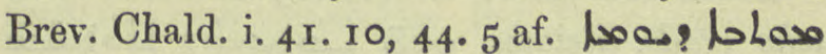

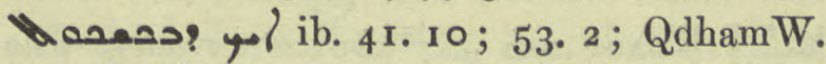
$84 \cdot 16,85 \cdot 5$.

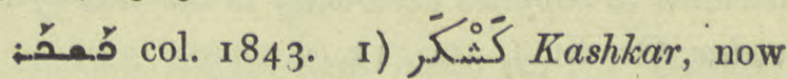
Wasit, the chief bpric. under the patriarchal See of Ctesiphon, Eranšahr 21 ; Syn. Or. 675 ; 33, 36 and often, ZDMG. xliii. 395. 7. 2) Kashgar also spelt on the borders of China and Turkistan, col. 1842 ; Jab. 19. 6 and $\mathrm{n}$.

Lمعْ a native of Kashkar; add: Syn. Or.

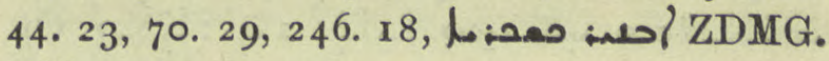
xliii. $39^{6}$ pen.

" مقا col. 1844. . scandalous, an offender, Ishoyahb Lett. 49 ult. Pl. Syn. Or. 174.29.

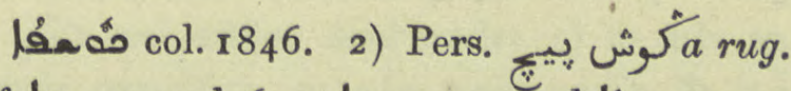
Cf. lan as col. 693, lowan and Joanas R. Duval.

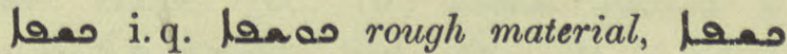
! 25. x. Cf. NHeb. ำ

בתר Hammurabi Code, Ar. to be energetic, lucky, S. Langdon, ZA. xxi. $283 \mathrm{ff}$.

D col. 1850. Aph. जी to dictate,

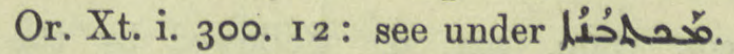

1ว́L’ó m. a register, S. Fraenk. ZDMG. lii.

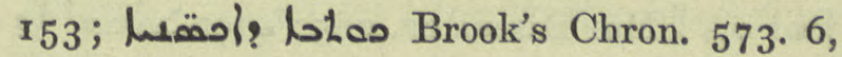
Chron. Min. 232. 14 .

Аـ فُمْ in writing, Hist. Mon. i. 65. 9 ; in the words of Scripture, Sev. Lett. I23. 6.

I'ósód col. 1852. the chancery, public office, BH. ZDMG. xl. 443 .

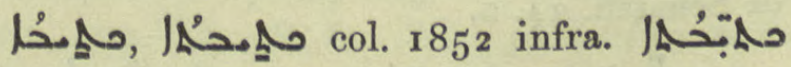
1:

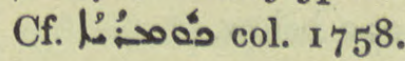

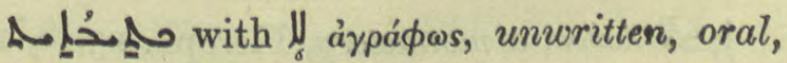
A.M.B. v. $384.17,18,20$. 


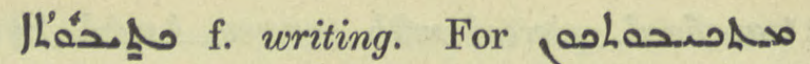
Anecd. Syr. iii. 275. 23 corr. in what you have written.

Кㅅำ add: a Writing Master. One who reads, dictates,

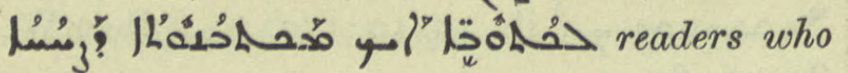
dictated to copyists according to the text of the codex, Or. Xt. i. 300.12.

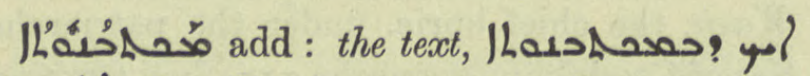
A 0 L Or. Xt. i. 362.6 and see under

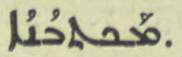

قط Cathay i.e. North China, Jab. 29. I.

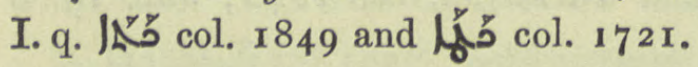

يُمآما col. 1849. Cypriotes, Greeks; add:

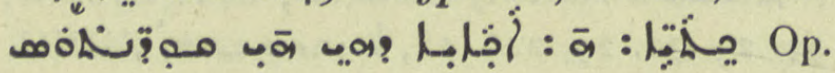

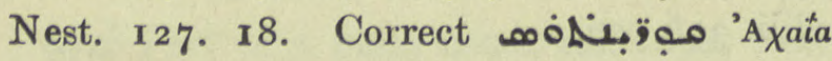

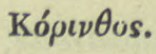

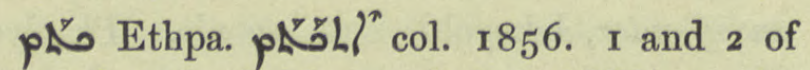
par. I) to be deeply marked, indelible, to the ref. to Eth. ed. Par. 267 ult. dele the suggestion

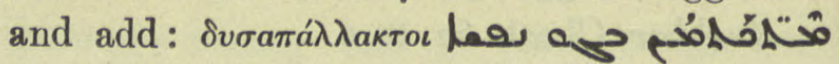
Stud. Sin. iv. 15. 8. Cf. An. Syr. 16. 9.

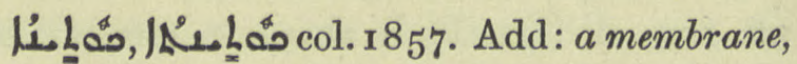

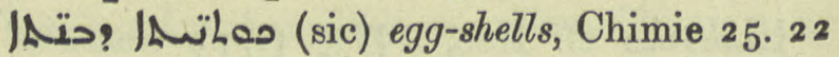
$=$ مخحا ib. 26. 1 I.

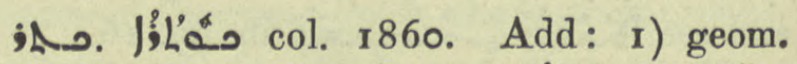

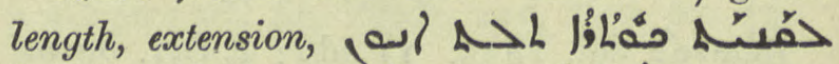

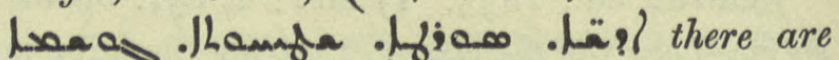
three properties of linear figures, outline, surface, solidity, But. Sap. ii. 2. 2. 2) a mid-

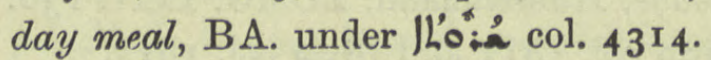

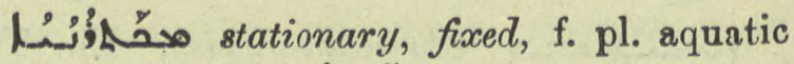

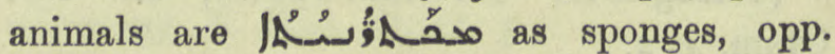

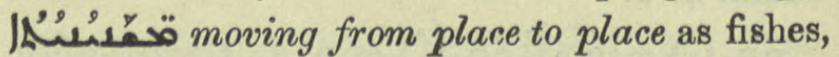
N. Hist. vii. I. I ; continuing, abiding, مئم

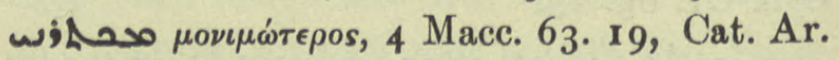
Jac. Edes. I 5. I.

م. مَ relating to contest, of contest, Hist. Mon, i. 216 . I I.

\section{$\therefore 1 \therefore$}

$>$ end of first par. add : often substituted for $r$ in Latin words e.g. Jofof and in Greek names especially those from Pehlevi or Persian sources. See confused mis-spellings of names in Gest. Alex.

ل col. 1869. par. 9. Followed by the Nom. in oaths, Verily, by, cf. Gr. ou róv,

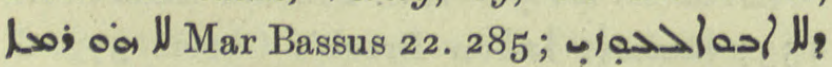
by al-Uzza, Bahira 203. 12, transl. 205 n. 4 .

col. r88r. pr. n. Laboda, twin sister of Abel, BHChr. 3, Apis مس 5 but called twin sister of Cain, trans. $25 \mathrm{n}$. I = Schatzh. 34. 2 .

1 $\lambda a ß$ os for $\lambda$ énos a scale, husk: see مelow.

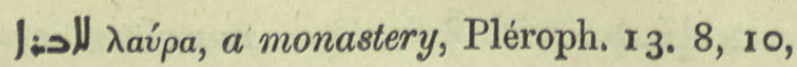
I7. 7, I I I. 7 .

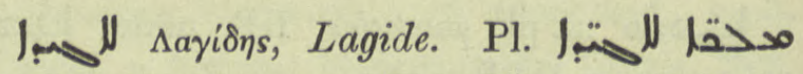
the Lagide dynasty, Jac. Edes. R.O.C. v. $5^{87} .2$.

hroll Libya, Or. Xt. i. 84.6. See lsa> col. 1903 .

a lلrh. lucerna, a lamp, with gloss لs:a .ä Warda $246 r$.

sanuoll Leontius, col. 1873. Add: professor of law at Beyrout, Sev. Ant. Vit. 47. 10. Lofuoll a priest of Tripoli, ib. 81. 4.

Looll col. 1873. Corr. a vice, screw, press,

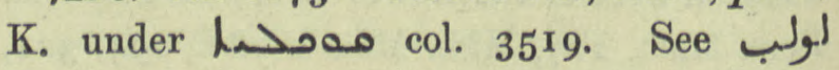
Dozy $35^{8}$ sq.

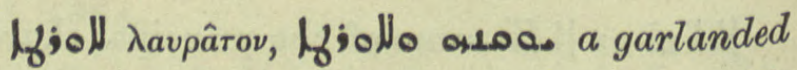
bust, Zach. Rhet. ii. 200 . $\mathrm{I}=$ Anecd. Syr. iii. 325. 4, Hamilton and Brooks. But see other explanations under $\mathrm{f} ; 0$ ? p. $10 b$. 
?;oll col. 1874. lapis lazuli, ref. Caus. Caus. ${ }^{26} 5$. I, Chimie 4. 6.

Laodíkєı, Laodicea, Apoc. iii. 14

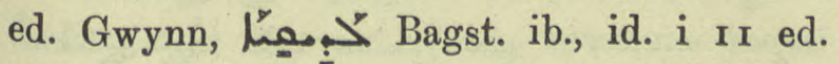

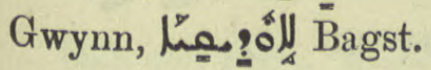

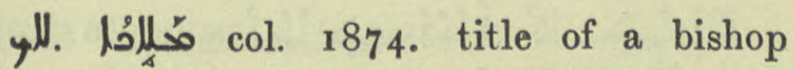
مe enquired after your Angelicalness, Jul. I 2. 2.

f. a company of angels, Apoc.

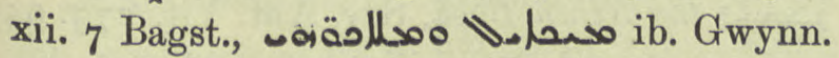

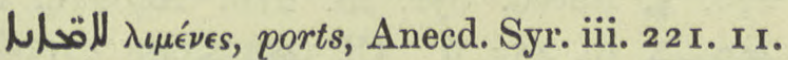
See

perh. Pontus Polymoniakos, Nöld. F. S. $475,146$.

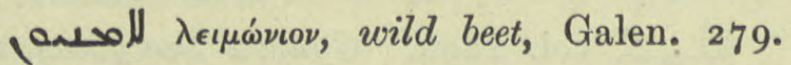
See mosar. 1940 pen.

م Lampetius, a heretic, Sev. Lett. 6I. 3. Cf. 1900 id. Vit. 106. 12, col. 1947 .

Lasanasis, daughter of Constantine the Great, A.M.B. v. 45 o. 6 , 453. 10.

الل f. a woollen cloak. Ref. Lexx. under حخده col. 2764.

الامهد Laconian, Lacedaemonian, Bod. Or. 467. $28 v$ quoted under مخدم col. I742.

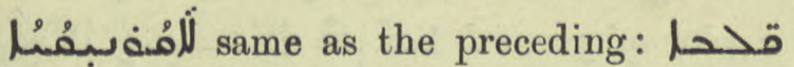
اللمفتبمُ hybrids from the dog and the vixen,

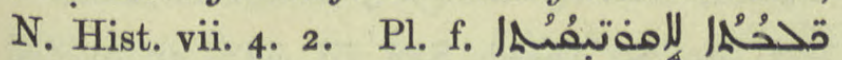
ib. $4 \cdot 3$.

مالم i.q.y ح, לֶת col. 1976. A half-homer,

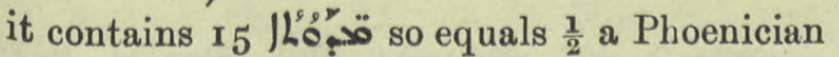

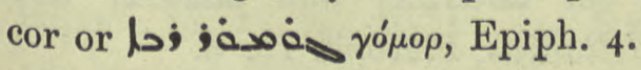

นُ

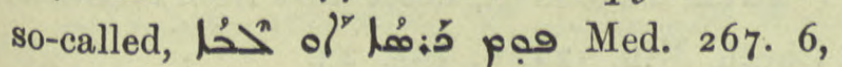
272. 10 ; مِّم a pain at the lower end of the stomach, ib. 280.9 ; from diarrhoea i.e. loosened pylorus, ib. 567.3 ;

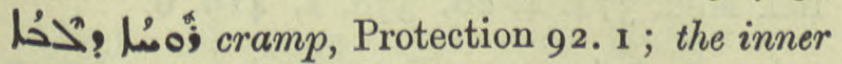
part $=$ palm of the hand, IAnă.

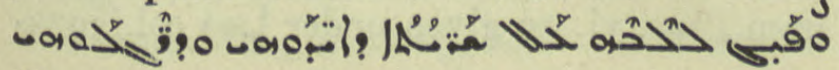
dip a rag in it and cool the palms and soles, ib. 580. 23. Metaph. the heart, affections,

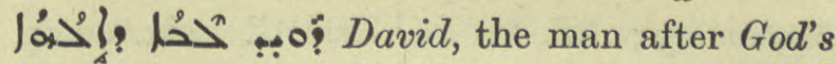

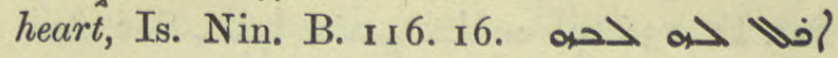

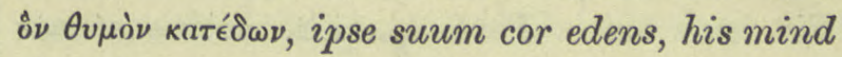
fretted $=$ reproached him, Pet. Ib. 54. 4 .

مكلّل

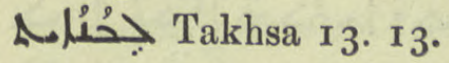

جُحُربه Libya, Gest. Alex. ro. I5. Usually >a col. 1903 or 1936.

ححار pr.n.m. Lebana, Patr. Or. v. 5. 729. I, 3,7 .

col. I880. congealed, frozen, Gest. Alex. I34. 8. Ethpe. ${ }^{n}>h^{n}$ a soft substance त्री can be crushed together, compressed, N. Hist. iii. i. sect. 2.

ححبـد prob. from Levi. a levitical garment, a dalmatic, A.M.B. v. I66. I3. I5. Cf. Du Cange 795 .

حح้ col. 1882. Add under I) to take to,

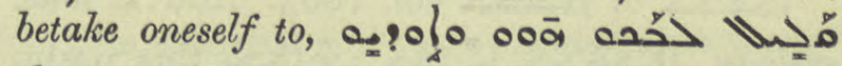
they nearly came to the point of confessing,

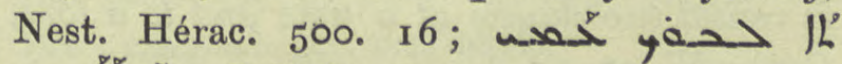
un

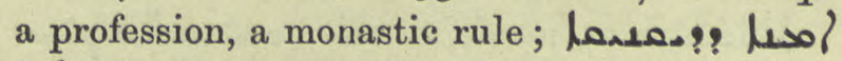

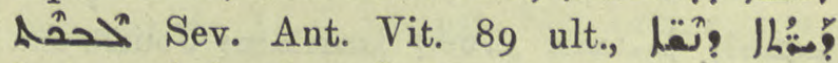

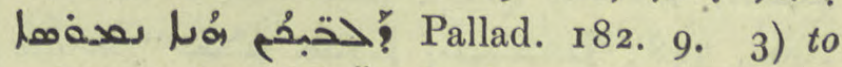

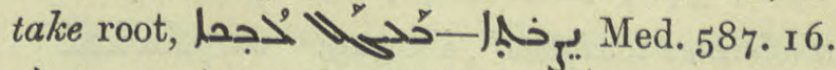
4) to persist, endure, "Ille tenebit", Our Lord holds on for evermore, Stud. Syr. ii. 14. With nouns : fol to be

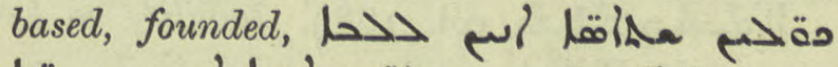

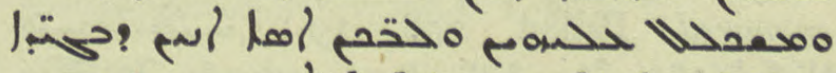

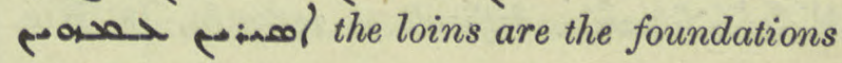
of the heart (or stomach) which is built upon them and the loins are based on the sinews which are bound up with them, Hebraica iv. 2I 5, $239 \mathrm{f}$. $\mathrm{L}^{\circ} \mathrm{g}^{y}$ to take refuge, Ephr. Jos.

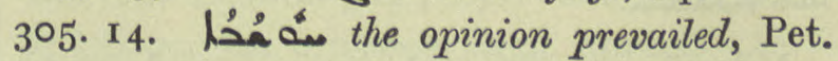
Ib. I31. I2. Sev. Ant. Vit. 58. 6. With heart, understand, A. M.B. v. 250.18 .

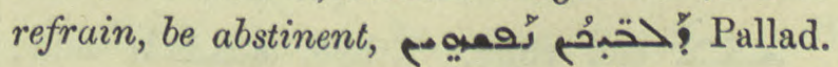

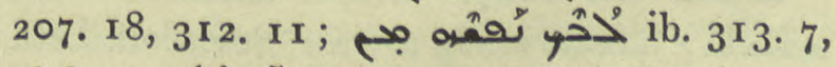

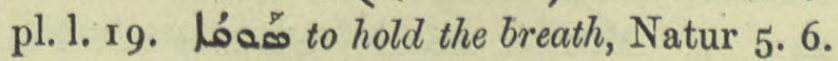


ل customary; Jul. 6r. 16, ZDMG. xxix. 90. 5,

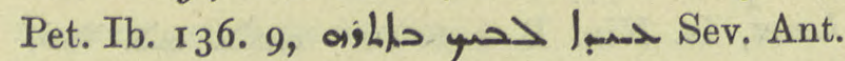
Vit. II. 10. Part.

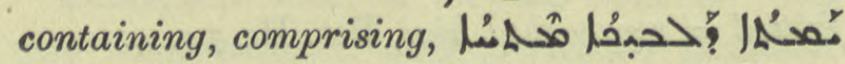
Pallad. I 19 ult. Astron. to hold,

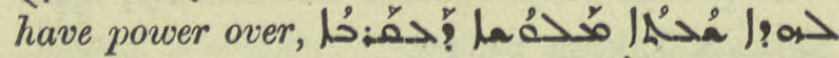

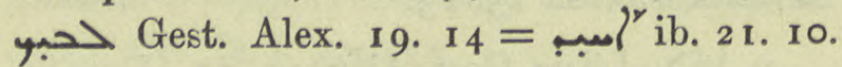
Chem. to seize, combine with, Chimie 47. 7 . Aph.

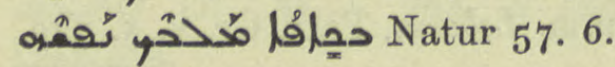

حُحكَهُمُ col. 1885. retentive, one of the four

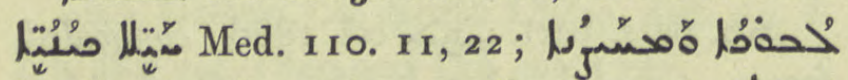
binding and astringent, ib. 212.6 ; 1.oj "l'esprit saisissant", Chimie I9. II.

>حسَّمُ col. 1885. Add: let, hindrance, obstacle, ;a!ll Sev. Lett. 194. 14. Restraint,

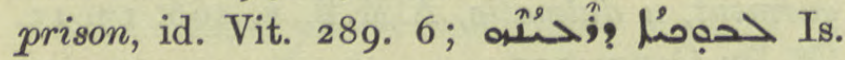
Nin. B. 253 ult.

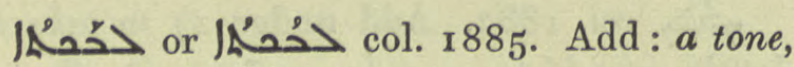

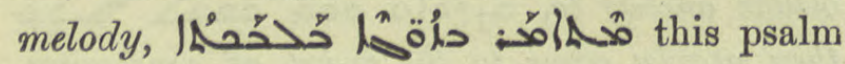
is recited two clauses at a time and to a tone, QdhamW. 97. I5.

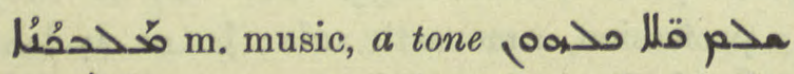

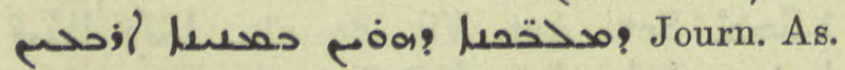

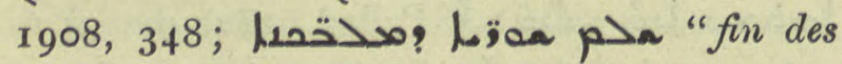

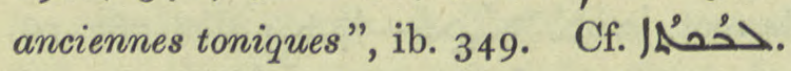

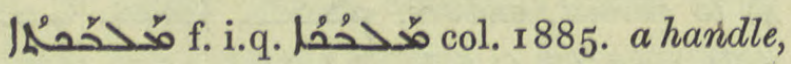
BA. under مُ مُ col. 3597.

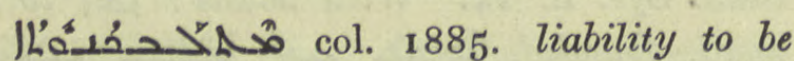

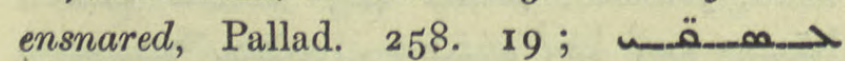
|lifficult to restrain, Is. Nin. $7 a \mathrm{II}$.

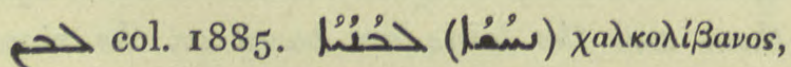
Apoc. i. I5, ii. 18 ed. Gwynn where Bagst. has ححُنُسُ.

مَححُ col. i 887. the frame of a doorway, Pallad. 130 ult.

ححَ:مُمه Labrax, sea-bass, Ar. FischN. 82.

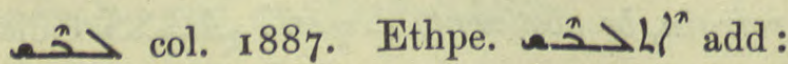

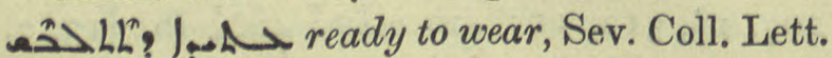
242. 1 2. Metaph. put on, assumed, viz. human nature, Nest. Hérac. 3०3. 6.

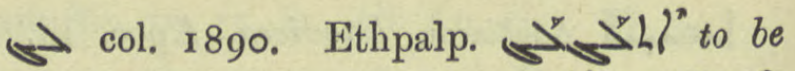

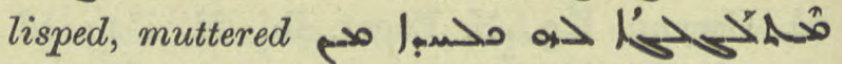
حمقصص Sev. Lett. 244.

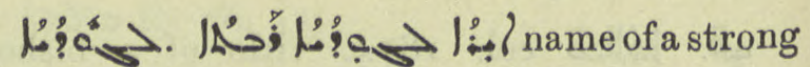
draught, Med. 48. 15, 49. 2 1, 136. I6.

Day $\lambda$ ónos, word, promise $=$ safe-conduct , Jo. Tell. 39. 4. Discourse, narrative, No Jah? the Ninth Book, Sev. Ant. Vit. 275. 9; 276. 4, Aneed. Syr. iii. 216. 20, 226. 23,

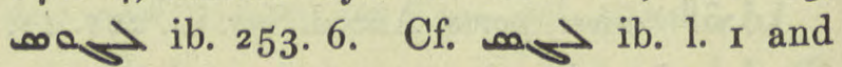
col. 1893 .

|

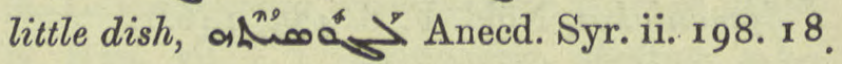

学 col. 1894. Cynara Scolymus, the

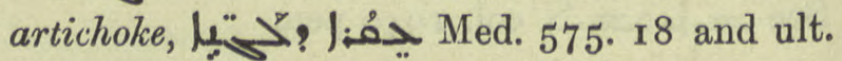

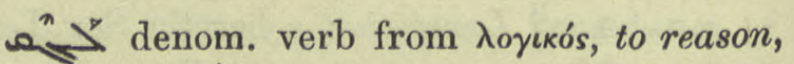
امب 9" S. Dan. 54. 19; the prophet

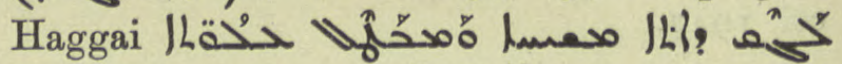

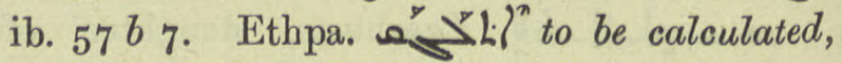
Poet. Syr. ro9. 6.

I col. r891. r. a basin. Dele the form

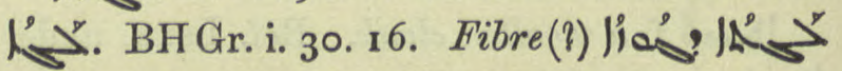
walnut fibre, Budge, Med. 55. 15, بن

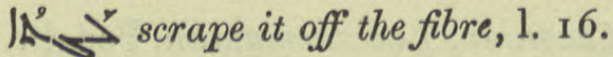

I2n col. 1891. a garden bed. Add: a plot of ground

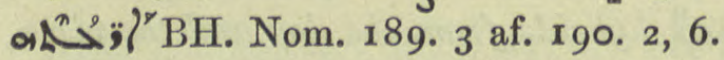

ו A Anecd. Syr. iii. 215.25 quoted col. 1813: audit, "statement of accounts," Hamilton and Brooks.

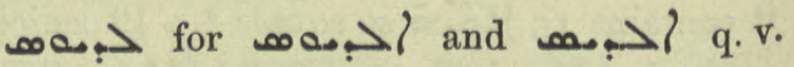
Pr.n.m. Helladius, Pléroph. 18. 8.

p. $\rightarrow$ the Ludim, descended from Mizraim, Chron. Min. 324. 7, 355. 23.

× col. 1894. ladanum, resin from gum cistus, see Lexx. under مسم col. 3 IIO. Ref. Med. 374. 4, 20, 378. I, 419. 6.

ح col. 1894. Dele. It is a quotation from Prov, vi. 19 given under /roj col. 3922. a) Ethpalpal a 


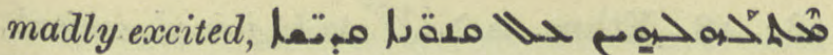
ILangho Lojano Sev. Lett. 82. I 2.

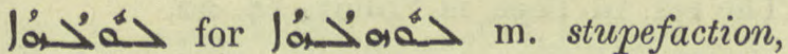
Tekkaf. 38 . 192 .

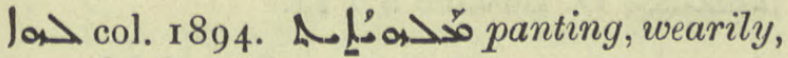

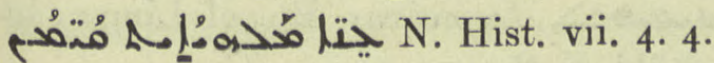

مُ stupid; a simpleton, BH. ZDMG. xl. 443 .

(a)/faragular Leontopetalon, so correct

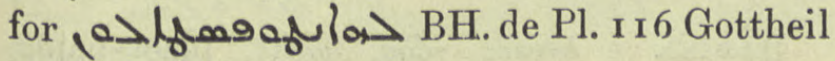
ZDMG. xliii. 126.

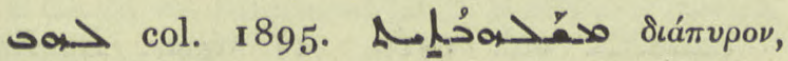
ardently, A. M. B. v. 348. 10.

| tion, Hippoc. v. 2 I.

fan Ethpa. fá kindled, to glow, Poet. Syr. 58. I 3. Metaph.

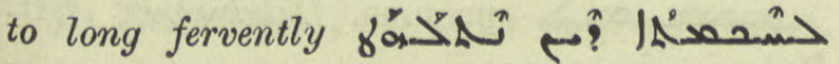
Tekkaf. 105.

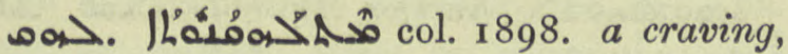

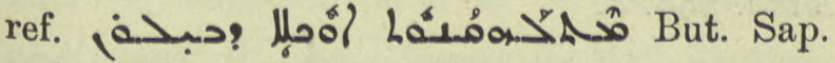
Eth. 4. 3.

Lat denom. verb from $\left./ h_{0}\right\rangle^{n}$ col. 1898 .

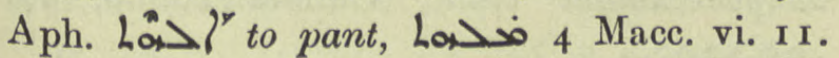

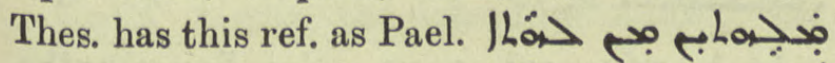

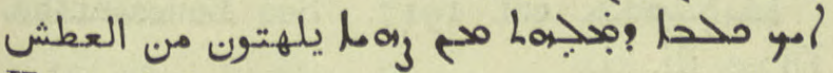
Hunt. clxx

I' Sev. Ant. Hom. 15. 12 ; the floor of the den will break asunder at the sound of thy shout, Jac. Sar. Hom. iii. 746. I. 2) asthma. Refs. BH. de Pl. Nos. 108, 216 , Hippoc. iii. 2 I, 25,29 , vi. 44 , Med. 186. I8, 189 bis and often.

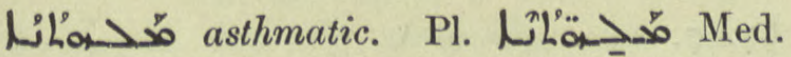
194. 4.

" col. 1898. double negative ل $\|$ not at all, by no means, BH Gr. i. 49. 7 .

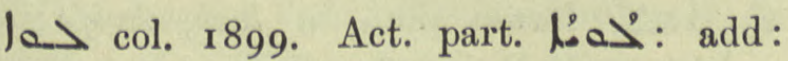
حت حم: this befalls thieves, this is a consequence of thieving, Ephr. ed. Lamy iii. 551. Pael كalm. Talo to accompany. Esp. 2716 used of a funeral procession: مخدة פتمدا

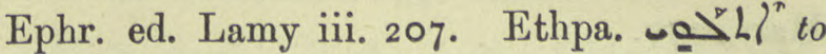

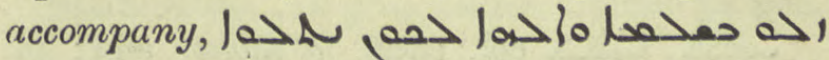
go in peace and God be with you, Ephr. ed. Lamy iii. 193 sexies.

ذحسم col. rgor. Add: company

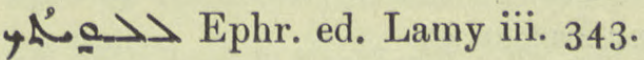

م 6 f. the companion? Name of a demon, Charms 84. 8.

حص corrupt: probably meant for

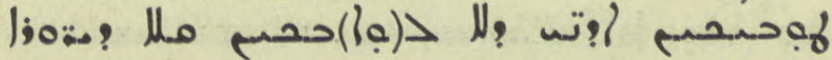
blessed are ye, my ears, for the voice of jackals has not taken hold of you, Mar. Marc. I65. 13 .

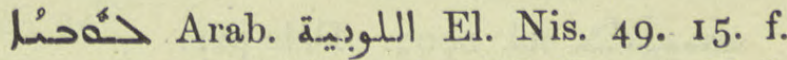
Phaseolus, haricot bean, Ar. PflnN. 234, Med. 597. 8.

حفحبr a village near Khantour, M.Z. 213.1 .

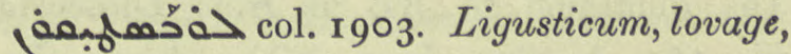
ref. Med. 99. 4, 196. 22, 299. 8, 308 ult. See רِكُ col. $193^{8}$.

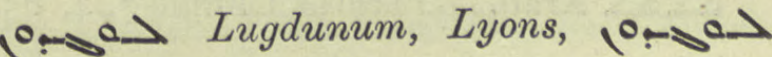
W Or. Xt. ii. 354 ult., Sev. Lett. r 93.2 r.

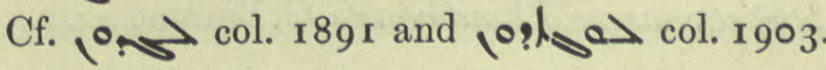

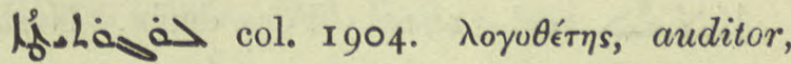
chancellor. Add : A.M.B.iv. 320, Sev. Lett. $3^{2} 4$ pen.

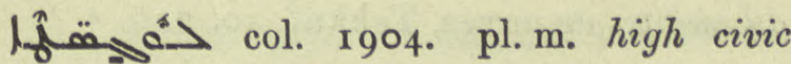
functionaries, ref. A.M.B. i. 25 r. r.

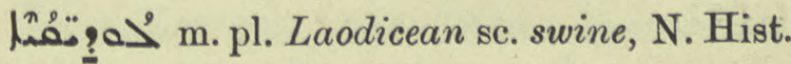
vii. I. 4 .

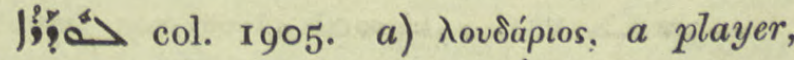

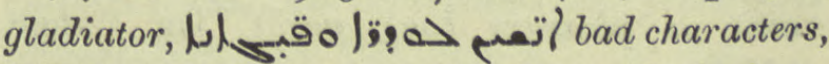
BHChr. Eccl. ii. 385 ; Syr.-Rom. Rechtsb. 7 .

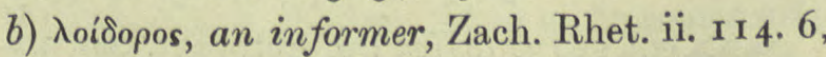
1 28. 7 = Anecd. Syr. iii. 272. 5 where Land has Jịa ; ib. 282. II, 283. 2. So Ahrens

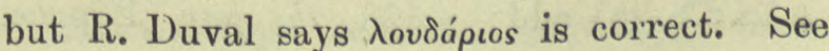
Nöld. Lit. Central Bl. 1899, p. I364. Pl. |jo calumniators; so Severus calls his gainsayers, Sev. Ant. Vit. 283. 5, 284. I.

A a 
10. col. 1906. 2 af. $L u z$, a village on the Zab, add: Hist. Mon. i. 36 r. 18, Pers. Mart. 277.

س > > > > col. 1906. a tablet. add: pl.

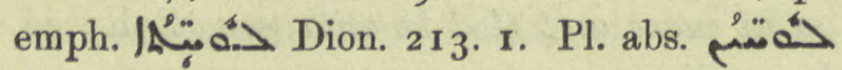
paradigms, But. Sap. Periherm. iii. 4 .

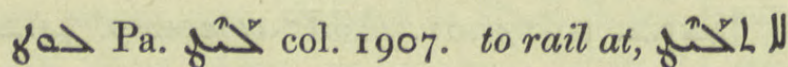
Aa مar Bassus 45.

I'os-d- f. accursed thing, abomination, Is. Ant. ii. 234. 15 .

(के ) name of a country. Perh. a mistake

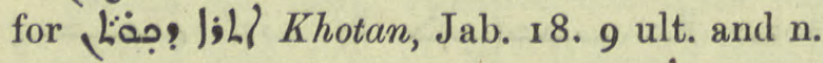

Lufo $>$ seems to be the usual spelling for litany. See hifh $>$ col. 1939 and add B. Sal. in Lit. 2 1. 8.

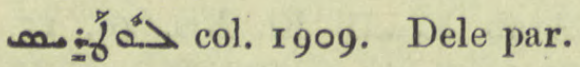

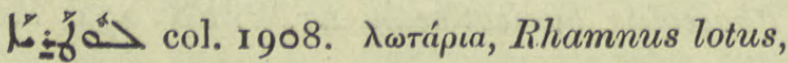
the lote or jujube-tree, Ar. PfinN. 283 , السدر El. Nis. 50.57 .

andweal col. 1909. Corr. Lonchitis. The quotation in BB. is from Dioscorides

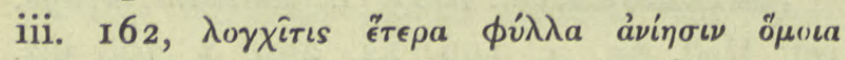

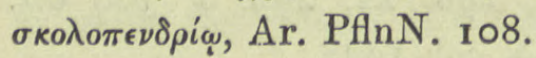

حه Arab. لو لو pearls, Chimie 7. 4, 85. 9.

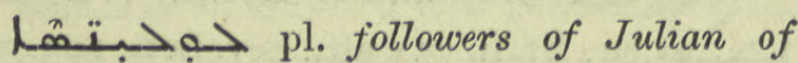
Haticarnassus, Phantasiasts, G. Busâmé 42. 24.

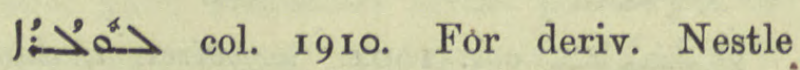
proposes lorarius, a harness-maker.

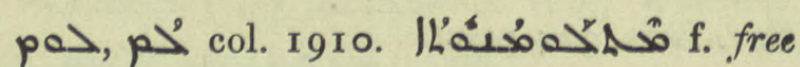
admission or access, Tekkaf. 40. 229.

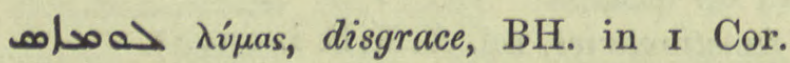
iv. $2 \mathrm{I}$.

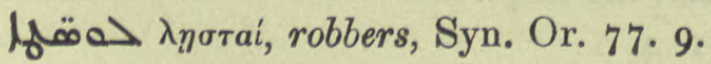

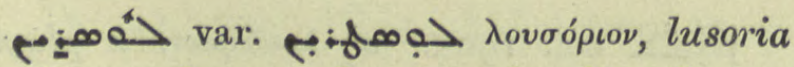
navis, a pleasure boat, yacht, Du Cange, A.M.B. v. 539. 16 n., 540. 2.

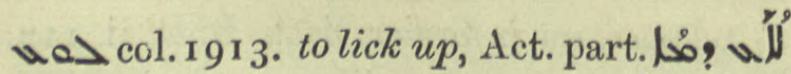
S. Dan. 66. 7 .

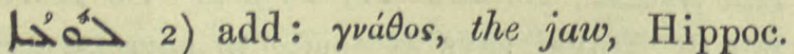
iv. 3 I, vi. 19 .

פQ col. I9I4. pass. part. ${ }_{2}>$ joined,

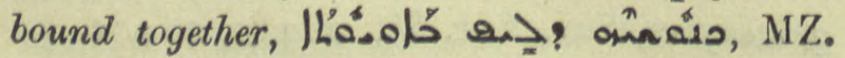

183. 18. Ethpe. $\left.a_{n} \backslash L L\right|^{n}$ to be joined, united,

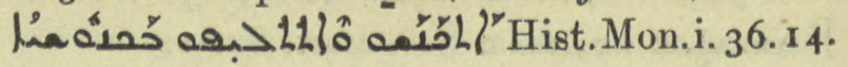
The ref. in Thes. is Philox. 19. 22.

يمعُ وقلا |Lب̣ạ o G. Busâmé $8 b$ I 4 .

صahمr9a Lupicinus, Bp. of Limyra in Lycia, Nöld. F. S. 474. 124.

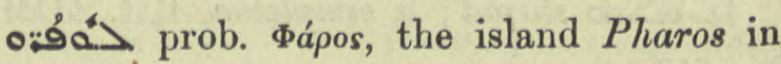
the bay of Alexandria, A.M.B. v. 558. 13 . Var. lolod.

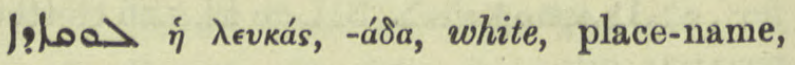
A.M.B. v. 558. n. 8.

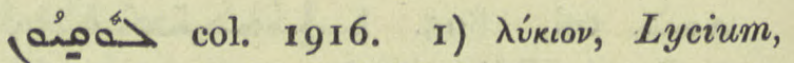
Ar. PfluN. 259. Ref. Med. 81. 7, حبمبف

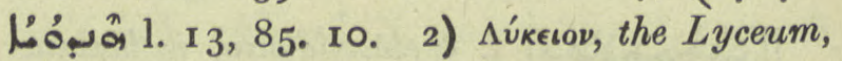
כلم Jمa حمه Jac. Edes. in Cat. Arist. 24. 3) Xєvкúv, chemist's paste, Chimie 29. 20. See 1.00.

صa_aـoم pr.n.m. Leukippus, BH. Hebraica iii. 25 o. 16.

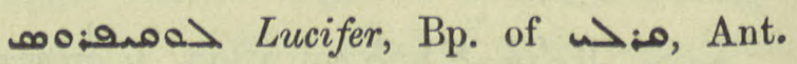
Patr. 298. 8.

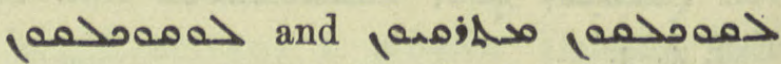

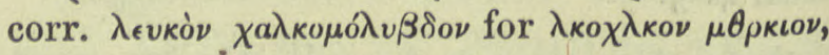
molybdochalque blanc, Chimie 18. 6, 17 , ( 1 . 3 .

col. rgr7. See Leucacantha, Diosc. iii. xix.

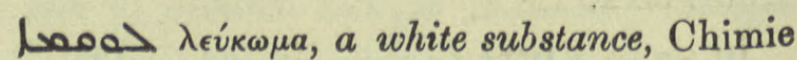
237 n. 6.

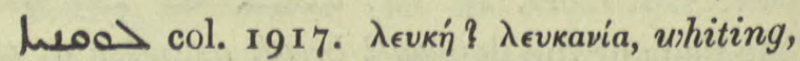
cement, paste, o Chimie 29. 18, 1.20.

>همتسا m.pl. encaustic, Chimie 294 n. I.

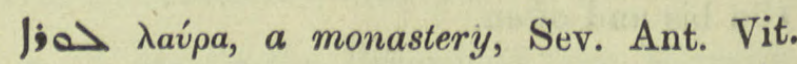
97. 7 .

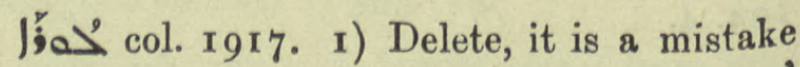

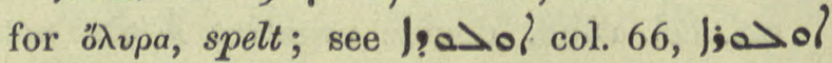
col. 67, i $\rightarrow$ ol col. 68, Ar. PflnN. 104. 2) خ lorum, lora, a lash, stripe; $\mathrm{pl}$ l. A.M.B. iv. 282 ult.

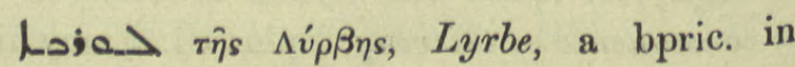
Pamphylia, Nold. F. S. i. 472.83 . 
ه حهaj pr.n. a martyr, brother of Brev. Ant. i. 35. I I.

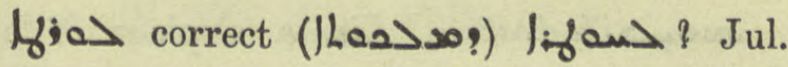
I28. 5 .

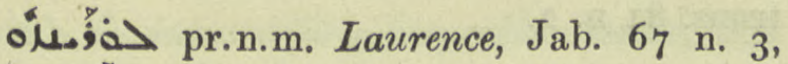

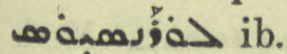

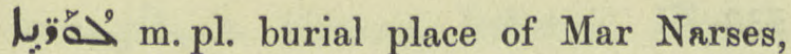
Mart. in Persia, A.M.B. iv. 180.

a d a col. igr7. to knead; to weld,

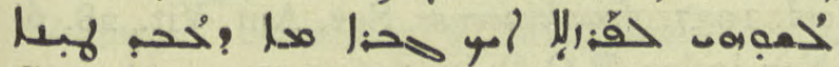
Gest. Alex. 267. 8, 268. 9.

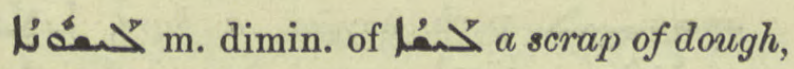
Chimie 85.18 .

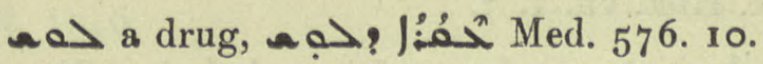

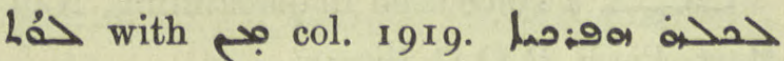
iha) province inside out, Sev. Lett. 25 pen.

入 Ethpe. $\hat{\imath} \lambda$ 亿 col. 1922. to be compressed, air is rarefied in a vessel filled with water خ 10

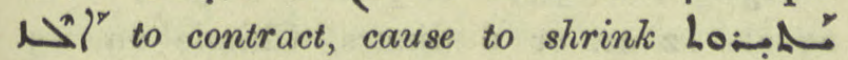
N. Hist. iv. ii. 3 .

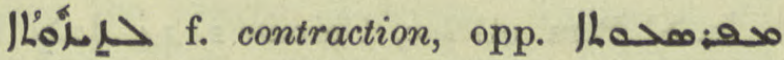
But. Sap. Philos. v. 4.

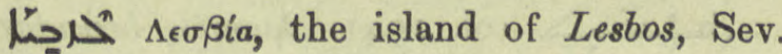
Ant. Vit. 26. 1 .

خرحُ a Lesbian, ib. 24. 2.

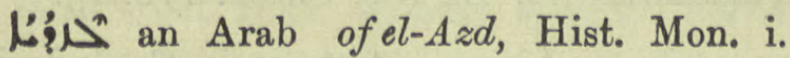
386. 8.

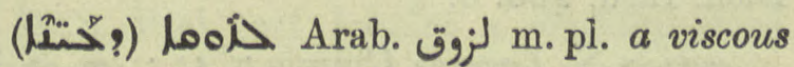
discharge of the eyes, Med. 557. 4.

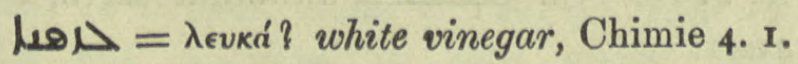

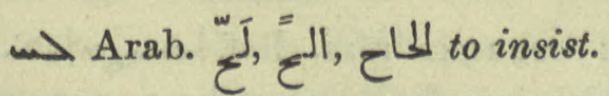

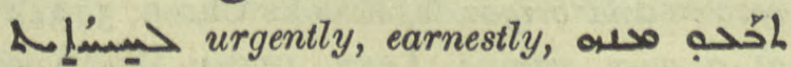

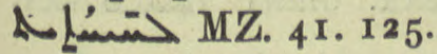

|حمَ col. 1924. effacing, the meeting of two lines at an angle J'ác'í, does not forbid them to continue to be two, But. Sap. Philos. 6. 8.

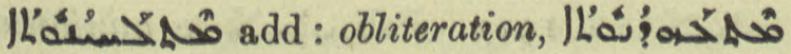

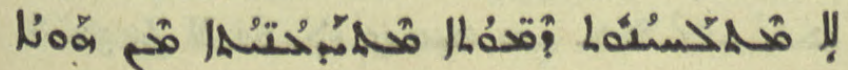

memory is the non-effacing of intellectual forms from the mind, But. Sap. Eth. 2. 2.

حarab. 1 glue, solder, Chimie 54 pen.

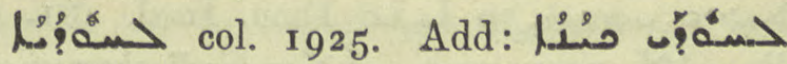

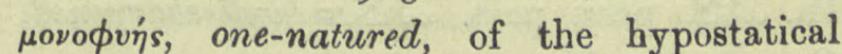
union /Loa)/ Lo: حon? Loof's Nest. 369. 6;

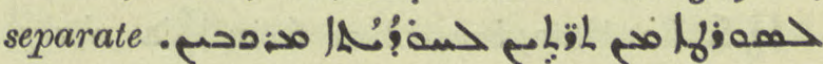

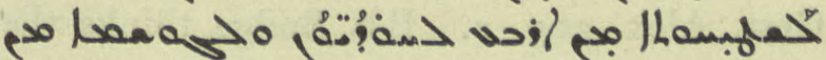
lisol a line consists of two separate points, a plane of four separate lines, a solid of eight, But. Sap. Philos. 8. 3 .

خ' Med. I70. I 9.

col. 1926. enticing,

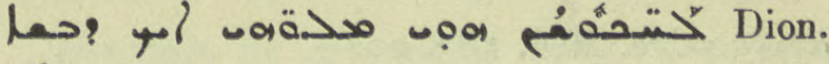
I6I. 20 .

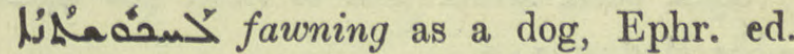
Lamy iv. 9I. 7 af. кє́ $\kappa \kappa \omega \psi$, Greg. Carm. ii. 26. Io.

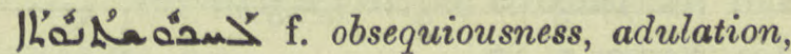
I' But. Sap. Pol. ii. 3 infra ; ib. iii. 4.

حشم pass. part.

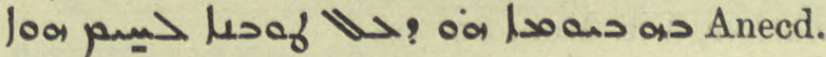
Syr. ii. 19. 18. Pa. Pn col. 1928. to articulate, to compose a dead body,

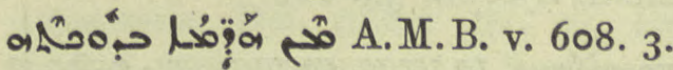

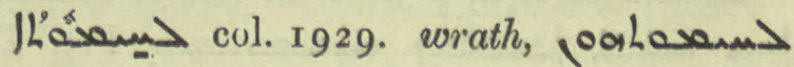

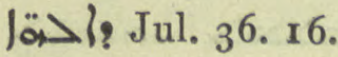

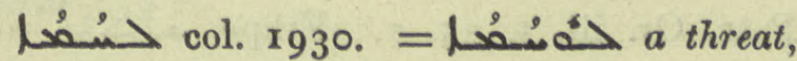
Dion. 13 \%. 2.

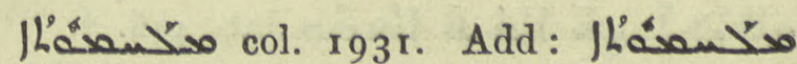
$L_{-}>$? the harmony of the universe, حتى قللا مدث مخه expressions torn from their context, Sev. Ant. Vit. 106. 2.

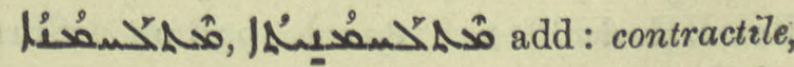
the contractile membrane of the eye sc. the iris, N. Hist. vii. 1. 2.

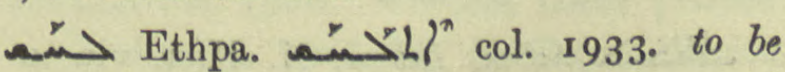

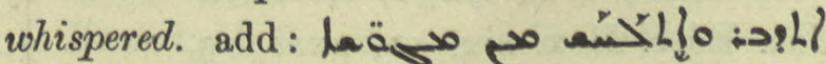
he was directed and influenced by the Magians, M.Z. 7. 25 . 
J'مَ' f. incantation, Schatzh. I34. 9, a variant is إتمعي

If col. 1934. to shave, sharpen, of متسر 0 : Dion. Ined. 46r. 6.

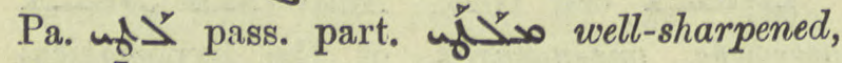
Geop. 1 2. 19 bis.

كئ Lat. lata, broad? or from lif sharp, Gest. Alex. 205. 2. But Budge thinks the word corrupt.

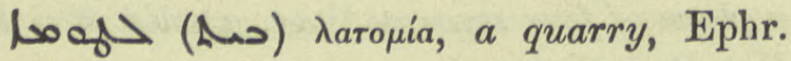
Ref. i. 94. 40.

maf pr.n.m. Laetus? colophon to a late MS. Mar Bassus x. 5 Syr.

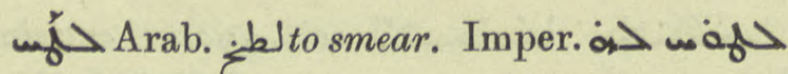
apply salve, Med. 583. 20.

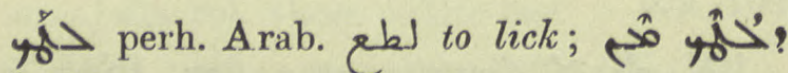
axo? Med. 591. 3 .

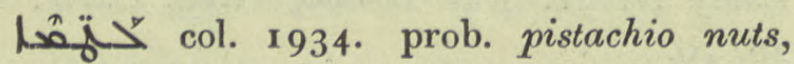

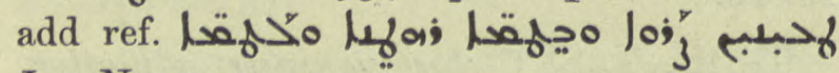
Jos. Narses 9. 17 .

af. Fraenkel identifies with N.Heb. לווטם a common

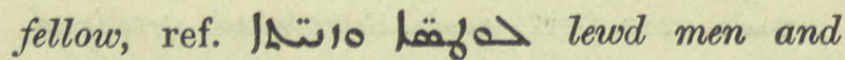
harlots, Vit. Mon. 89. 10.

The Lateran Palace in Rome, Anecd. Syr. iii. 55. 16.

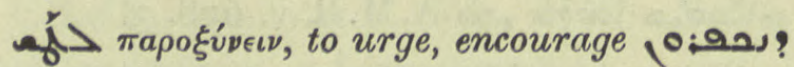
loa حet. Ib.

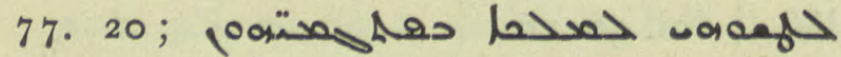
Patr. Or. iii, i. 35. 7. Ethpe. of $\left\langle L^{n}\right.$ col. 1936. Add: to be keen, addicted to, âf $\left\langle h^{n}\right.$

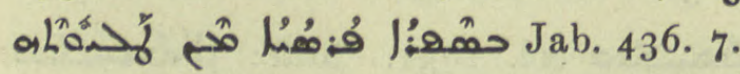

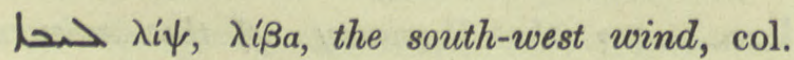
1936. Cf. حدم col. 1887, حمa col. 1966, col. 1942.

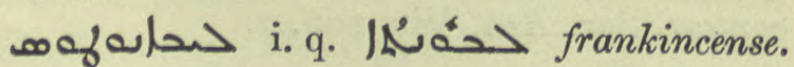
Ref. BH. de Pl. \& I I8, col. 1936 ult. Corr. ق a a decoction. Col. 1937. 3 delete the sentence beginning "Post".

حıßún, Libya, N. Hist. vii. 4. 2, Jac. Edes. Hex. xix. II, xxxi. I5, xxxii. 9. Var. Col. 1937 and uد > above, p. $20 a$.

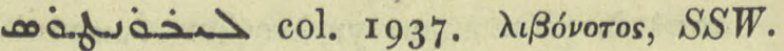
wind, N. Hist. v. 3. 2.

入uliás, Livias, a place of hot springs beyond the Jordan, Pet. Ib. 83. 16 trans. 81 n. 2.

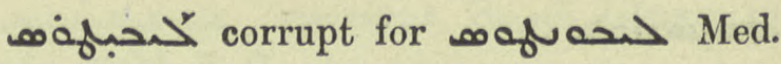
533. 8 .

>بـد col. 1938. a salve made from

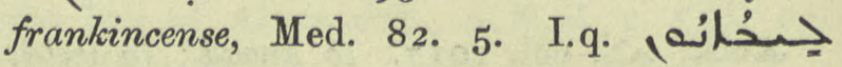
col. 1937. frankincense, Sev. Ant. Vit. 28. 6, $\mathrm{Nau}$ in loc. R.O.C. iv. 546, n. 2.

مِ

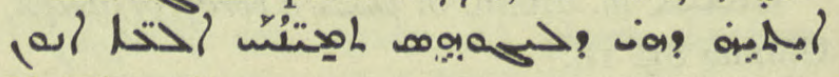
N. Hist. vii. I. 7 .

La a spice used in embalming, Nau in Mart. Petri, R.O.C. iii. 47.

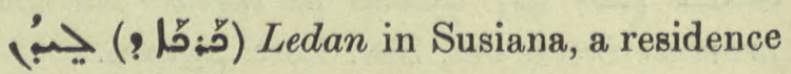
of Sassanian kings, A.M.B. iv. 160, 6, Jab. 378. 2. See ${ }^{n}$ col. 1893 .

רֵن col. 1939. a pound weight; I lb. equals $12 \mathrm{oz}$. or 6 dinars, Chimie 69.13 . $=20$ staters i.e. 100 drachms, Med. 446. I1.

حما حما ib. 5०. 6, 252. 9 af., Med. 3०9. 20.

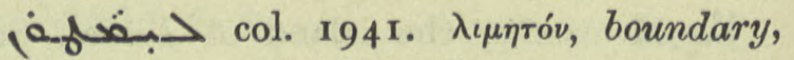
border country, A.M.B. iii. 299; خصمبر Anecd. Syr. iii. 314. 8, حمفه ib. 315. 2 ;

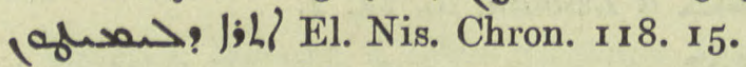

خnames, a fortress in Cappadocia, Dion. Ined. 460. 6.

Dast Lemnos, Chimie 7. 17 ; ib. 4. 10. Cf. $194 \mathrm{I}$.

حصa: robbers and brigands, Brooks Chron. 573. 14. 2) a cave, ravine, Lexx. under 1 I $^{2}$ col. 3086.

olágl|an the Liparian islands, N. Hist. iv. ii. 2. Cf. I حصف col. 1943.

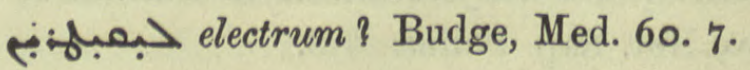

to annihilate, M.Z. 32.8. 


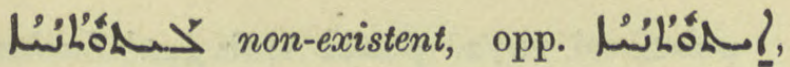
But. Sap. Philos. 2. 5, N. Hist. ii. 5, iv. I

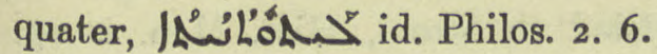

حسمُ livó مُ incurability, incurable disease, But. Sap. Philos. 2. 2 bis. iii. 29.

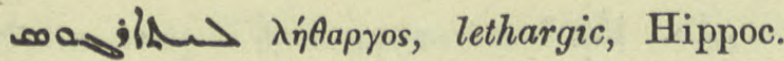

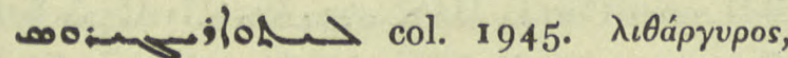

"litharge," Chimie 5. 3, O- jloh D ib. 58. 1, jog jlogh 1.4 .

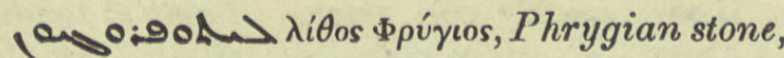
Chimie 219 n. 6. Cf. Lexx. under مـ col. 1946 .

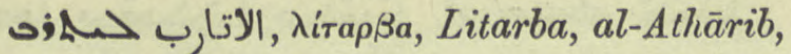
near Aleppo, Brooks Chron. 576. I $_{4}=$ C.B.M.

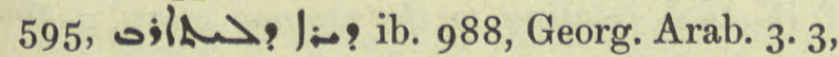

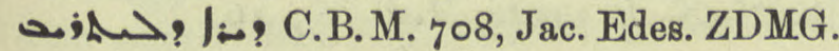
xxiv. $261,263$.

حما

To Thee, Lord of all, an anthem of praise beginning thus. Also called the Prayer of Adam. Takhsa 3. II, prayer before the Lakhumara, l. 3, 63. 8, 77 antep., QdbamW. 7, 10, 1 2. 2 and often, Brev. Chald. i. 49. 7, Ir.

حفُ حم:

19 col. 1951. a lamia, spectre, Charms 84. 7 , JAOS. xv. 288 f., $289 \cdot 3$.

>م Pael part. مخحمُ a doubtful word. To jeer at, insult, Anecd. Syr. iii. I 9. I9, I23. I $_{2}=$ Zach. Rhet. i. I48. 7 ; I53. I5.

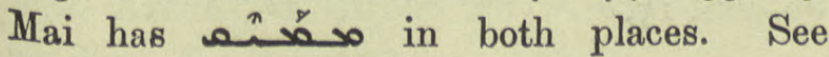
Script. Vet. Nova Collectio $33^{2}$ f. Brooks suggests محدالl Anecd. Syr. iii. I77. 10.

IA the pleura pulmonalis, Hebraica iv. 213.182 .

م connecting,

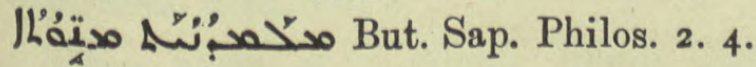

I' of col. 1955. the doctorate, teaching, Jab. 209. 5 . مैal

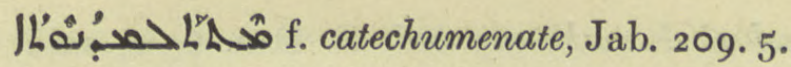

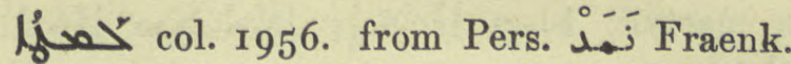
Fremd. W. 102. felt, of a tent is a house made of curtains or of silk or of felt, G. Busâmé $27 b$; حمصنّ هo a felt coat wadded with cotton, Op. Nest. 96. 13 .

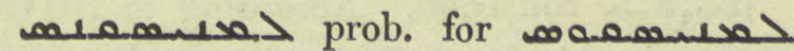
lemniscus, the sign $\div$ Epiph. I $5.3 ._{3}$.



m.pl. followers of Lampetius, a Cappadocian, anti-monk, antichastity and poverty, Coupes ii. I4I.

D Dambrus, n. of a

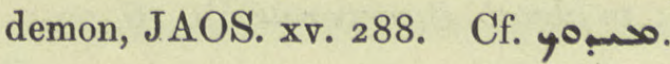

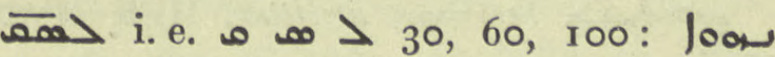
פח them thirtyfold, sixtyfold, an hundredfold, Mar Bassus 62. 9 .

$a$ i.q. ua> col. 1959. to lap, lick, لححه :Jul, 18 ult.

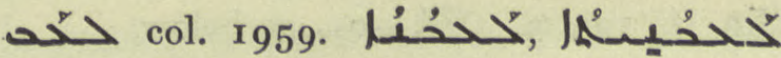
voracious, Natur 58.3 .

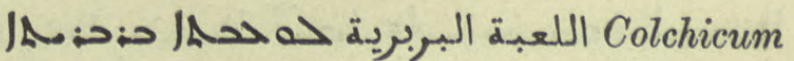
autumnale, BB. under l??, ed. Duval 33;

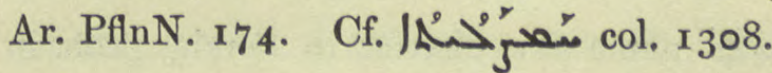

حهـ m. an orator, Sev. Rhet. $133 r$.

eat, Med. 557.4 .

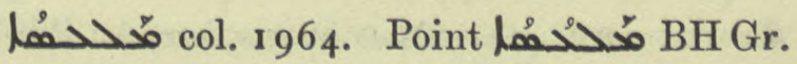

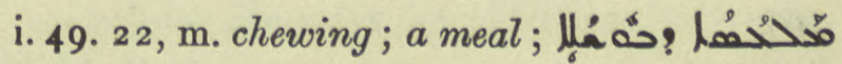

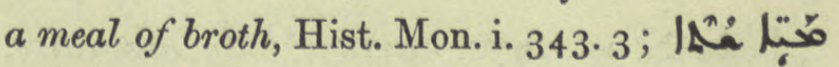

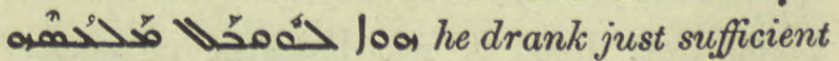
water to enable him to swallow the bread, Pallad. I 45.12.

|îق > col. 1966. Correct |îقớ? [Ezech.

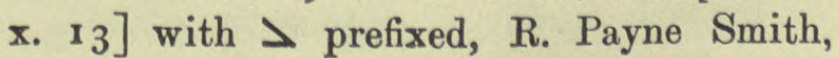
R. Duval, R. L. Bensley. Probably an old mistake, for the supposed word is found in

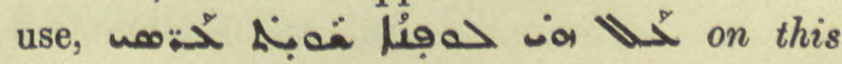


wheel I make my bed, S. Dan. $65 r$, said of

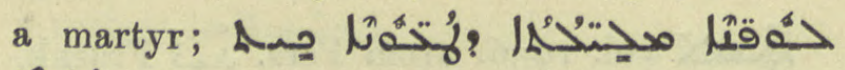

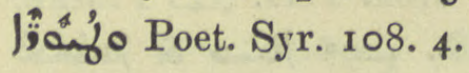

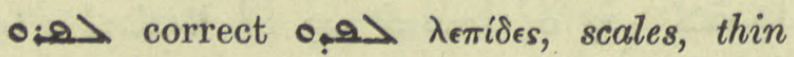
plates, Chimie 212 n. 2.

حس․ see Suppl. above.

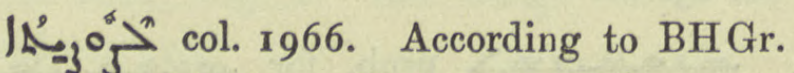
i. 65.15 this is a diminutive of $/ \mathrm{A}_{-5} \mathrm{f}$.

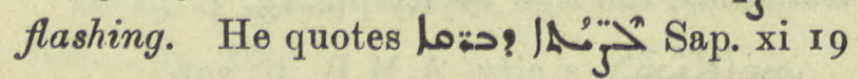
where $\mathrm{BH}$. ed. Rahlfs also has this reading; Lag. has $\left|A_{3}\right\rangle_{5}$ : see col. 546 .

م้ > col. 1968. to attract, the magnet خما Chimie 9. I4, amber Jُ 1.21 ; to absorb, incorporate, ib. 16. 23.

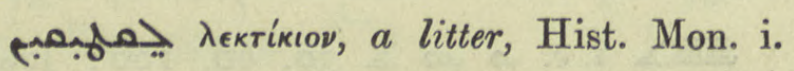
3II, II.

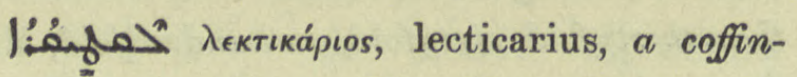
bearer, grave-digger, Vit. Monoph. 88. 24, Jo. Tell. 74. 20.

حمسعه Licinius, King of Magedo, Stud. Sin. ix. 123. 3. Cf. no

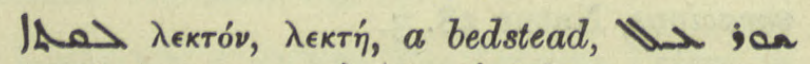

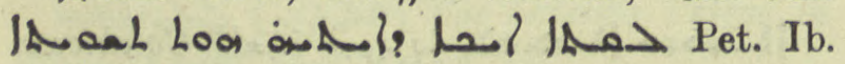
96. 2 and $n$. to transl.

- name of a district in Persia, prob. in Beit Garmai, A.M.B. ii. 2. 3.

م:> Lardin, a village, prob. legendary, Patr. Or. v. 5. 728 pen., $73^{\circ}$ pen.

(C): col. 1972. A mistake in Geop.

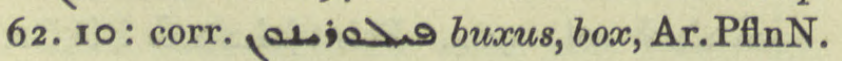
316.

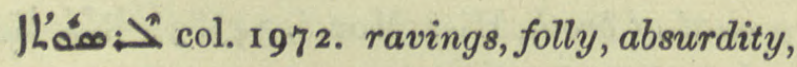

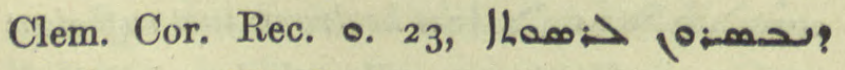
volowa? Tit. Bostr. x 24.30.

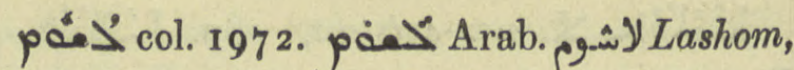
a bpric., now the village Lasim on the highroad from Baghdad to Mosul, Hist. Mon. i. 25 , ii. 44 n., Pers. Mart. 274, Syn. Or. 675; 33. 29 and often, A.M. B. ii. 663 .

حب. an Indian salve, Med. I 43. 7 .

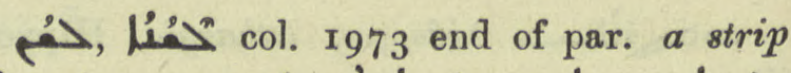
of water, רعبل

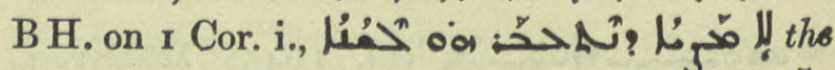

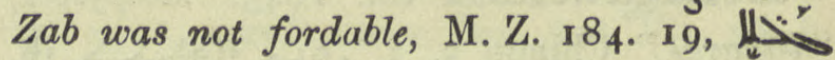
أُخمُ 1.20.

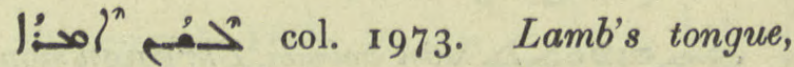
a kind of plaintain, refs. Med. 168. 15, 207. II, 208. 5, 399. 15 and often.

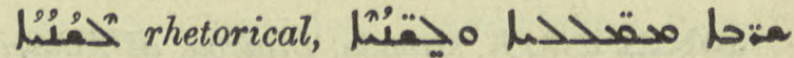
matters of logic and rhetoric, El. Tir. Gram. 3;

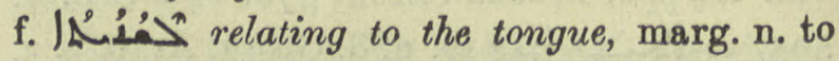

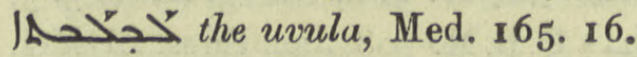

cóls ${ }^{n}$ and disease), A. M. B. iii. $5^{22} .9$.

Y col. 1975. Act. part. add: $\sim 20 L^{y}$ bN', befitting gratitude, DBB. 204 I. 5 af.,

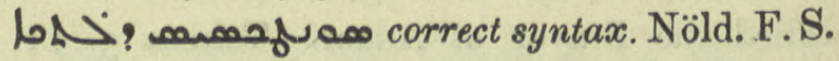
i. 482 .

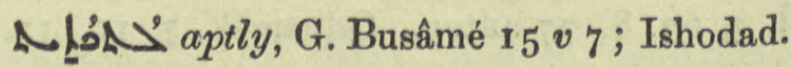
31. 19.

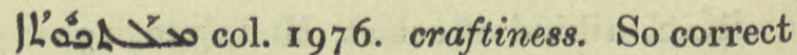
Anecd. Syr. iii. 132. 10 where Land has Ihen>o.

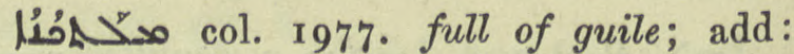

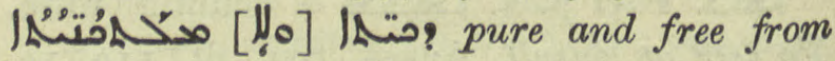
guile (so correct) Theod. Mops. 408. 14 in Jo. xxi. 17 .

pL col. 1977. Two roots: I. dialectic form of plis to murmur, Arab. ر. II. Arab. all to heap up, to impute, Fraenk. Z.A. xvii. 87 . 
Lo col. 1980. 4) how much. of log? however much, although indeed, Ephr. Ref. i. 45.19 .

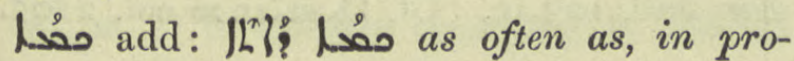
portion as, Pallad. 77 bis; o. 0 ? مصٌ to some

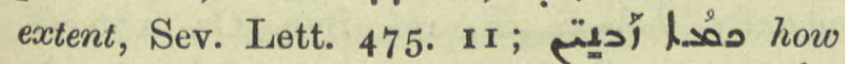
many times / too often, Pallad. I II. I4; مُّال

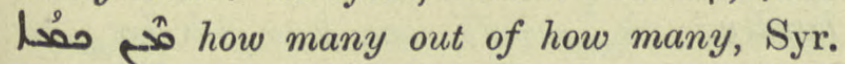
Rom. Rechtsb. 5.

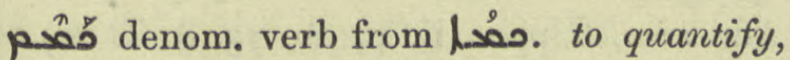
determine the quantity, | 1 Bat. Sap. Philos. 7. 4.

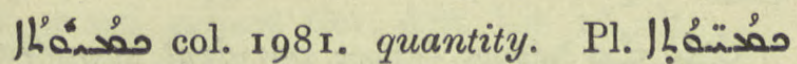

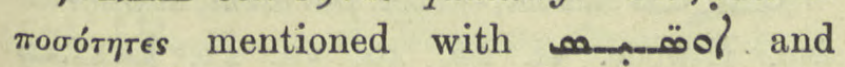
ف요 as subjects of consideration apart from matter, But. Sap. Philos. i. I.

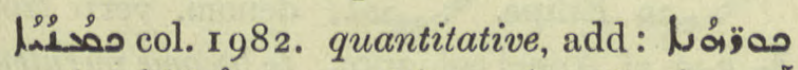

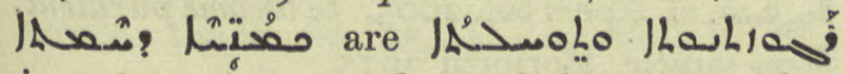
irascibility i.e. overplus and despondency i.e. lack, But. Sap. Philos. iv. 5; Jhes. sho

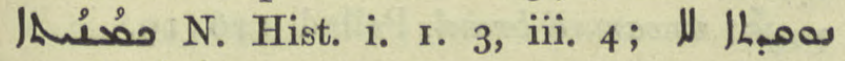

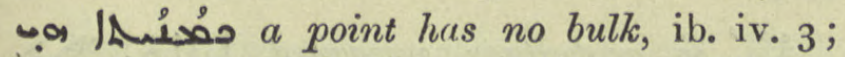
ōa Philos. 8. 2 infra;

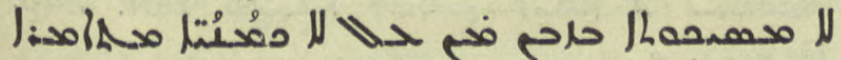

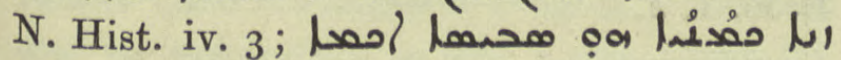

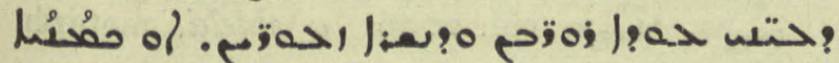

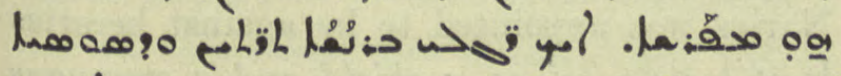
usil quantity relates to bulk as, the eyes of the owl are large and of the eagle small, or it defines number, ib. vii. I. I I.

م $M a^{\prime} \hat{e}$ êdos, an island, Natur. 45. I3, perh. Arados, cf. Strabo xvi. 2. $x_{3}$, ib. n. to No. 8o. See col. 370.

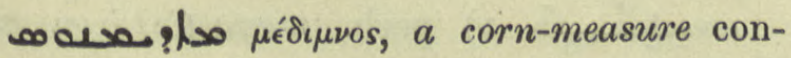
taining $4 \frac{1}{2}$ or 5 pecks, Epiph. 6. 6 .

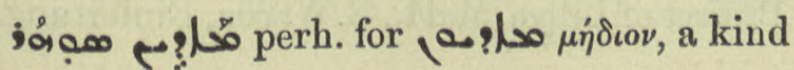
of clover, Med. 599. 15 .

andolso var. sonfolso Lake Maeotis, Jac. Edes. Hex. xiii 2.

jols name of a village where Ezekiel was buried in the sepulchre of Shem and Arpachshad, Nest. Chrest. 9I.
Wulfis;ols Mauritania, Sev. Lett. 332. I 4 .

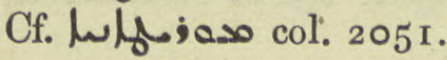

مار|زف Mazarp, an Indian city, Kal-w-Dim.

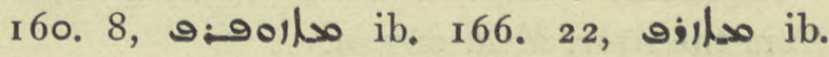
177. I7; these are corruptions of a Sanskrit name Mahilaropya as is also ed. Bick. lxi, lxiii.

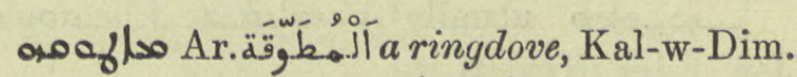

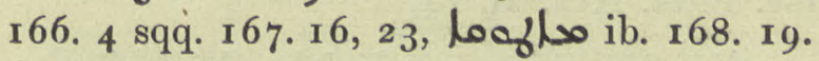

مارمفع م pr.n.m. Maxôs, Pléroph. 24 . II.

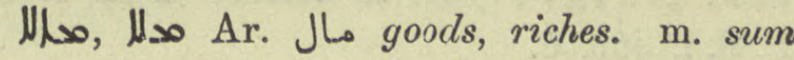

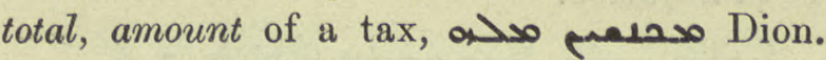

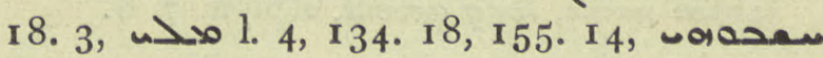
لll o ib. 1. 20, 162. 3, 170. 14; Nöld. on Dion. WZKM. x. 165 .

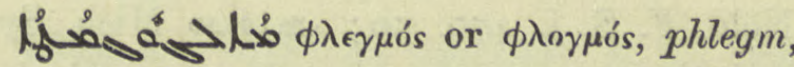
blood, A.M.B. vii. $5^{\mathrm{I}} 7$.

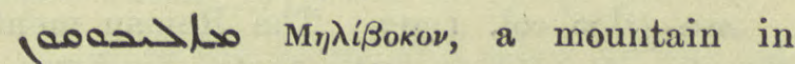
Germany, Jac. Edes. Hex. xxxiv. I r.

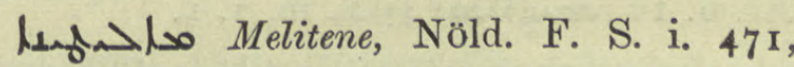

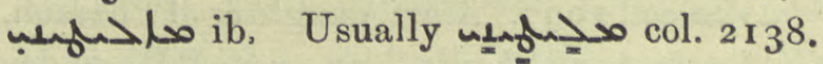

(?) (-) Journ. As. 1908. 92.

ol pr. n.f. Melania, Pet. Ib. 27. 18 ,

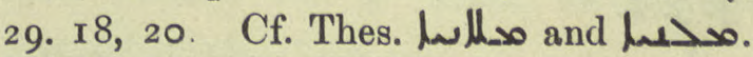

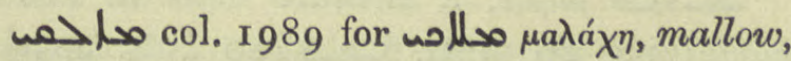
ZA. xvii. 88. Cf. Löw bei Krauss 34I.

$\left.J_{3}: \infty\right)_{1} 0$ pr.n.f. Mémarsa, Journ. As. 1907 , 419.

onemius, bp. of Citium in Cyprus, Nöld. F. S. i. 472.8 r.

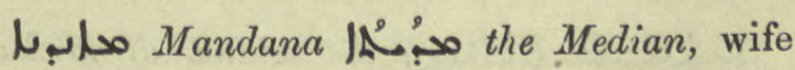
of Cambyses and mother of Cyrus, Journ. As. $1905,282.26$.

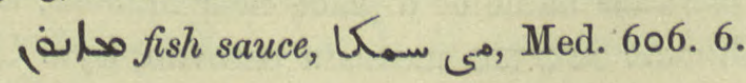

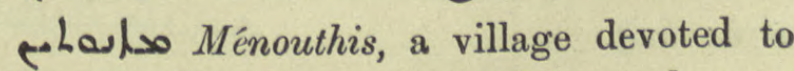
Isis 14 miles from Alexandria and near Canopus, Sev. Ant. Vit. 17. 10, 18. I, 19. 2, R. O.C. iv. in loc. $35^{\circ}$. 


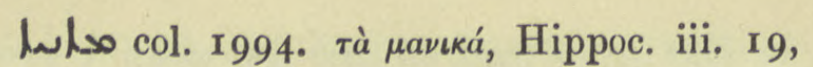
$2 \mathrm{I}$, occurs in spring and autumn, ib.

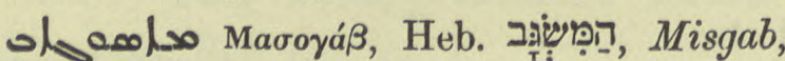
a town of Moab, Hex. Jer. xlviii. I.

مasonius, bp. of Kalenderis, Nöld. F. S. i. 47 I. 7 I.

مُمامتبمُ mesenteric veins which proceed from the liver, Med. 267. 9.

مامب Macedonius bp. of Xanthus, Nöld. F. S. i. 474 . 125 .

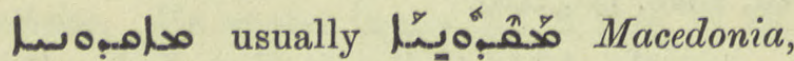
Jac. Edes. Hex. xi. I 4.

مامي Macer, bp. of Jericho, Mich. Syr. I, Fasc. I I. I $58 c 7$ af. so correct ox/ Nöld. F. S. i. 4 68. 6.

مهام:ا

مامئ

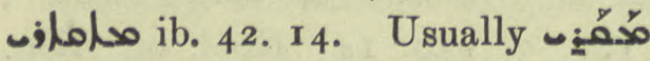

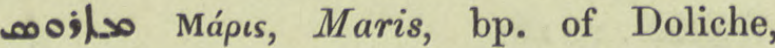
Nöld. F. S. i. 470. 50; مازן. 50 Mich. Syr. i. ii. $159 c 37$.

sand col. 1997. The Roman month Martius, March, Georg.

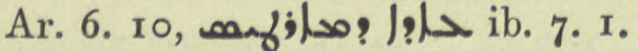

-jo col. x 997. Mari, daughter of Pharaoh, G. Busâmé $78 b$ ult., 79. I ; BB. under ( 594 col.

مدازيل Marinus, bp. of Beyrouth, Sev. Ant. Vit. 325.2.

مازן $\mu a ́ \rho \eta s$, a measure used in Pontus containing 20 pints, hands

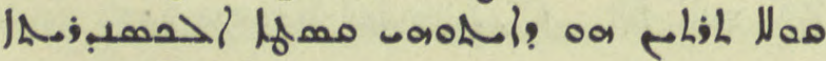
حسم: Epiph. 6. 2-4.

col. r999. Hieropolitans, inhabs. of Mabug or Hierapolis, Sev. Lett. 20. 3 af. correct ref. to Jos. Styl. 74. 8.

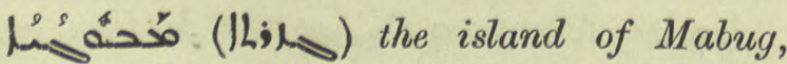
Nars. ed. Ming. $6 \mathrm{n}$.

סح:مA name of a place near Mahoze, the Talmudic means a caravan, Syn. Or. $676: 625$.

'० col. 2002. Megiddo, Apoc. xvi. 16 ed. Gwynn for Bagst. See Gwynn's note.
- 0. i.q. birthplace of the princess and martyr Irene, Stud. Sin. ix. 123. 3.

M. Magdal, daughter of Abdallah, Inscript. Sém. No. 46, Nöld. ZA. xxi. I 55 .

of

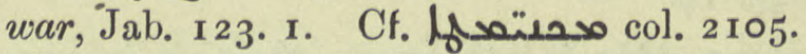

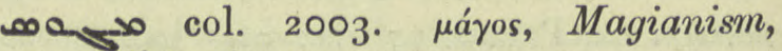

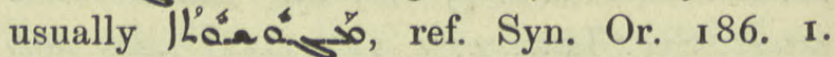
حمس hardly.

川 مَ col. 2004. infra. I) pus, matter. Add $\pi \dot{v} o s$, Hippoc. ii. 47 , iv. 75,8 I f. 2) adj.

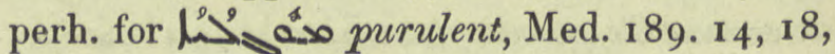
峞 ib. 202. 2.

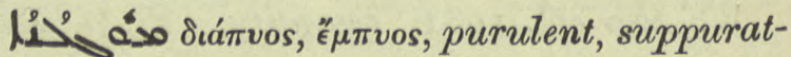

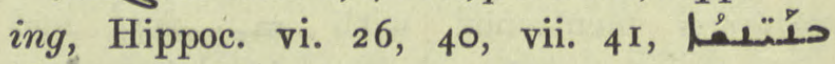

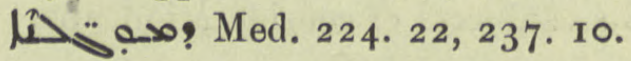

Wo Ethpe. " $30 h l^{n}$ denom. verb from U form matter, Hormizd 3075 .

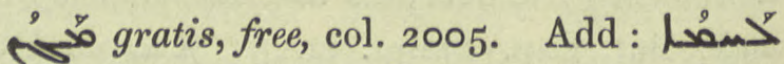
सै

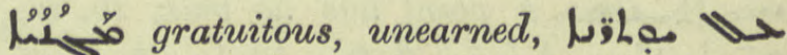

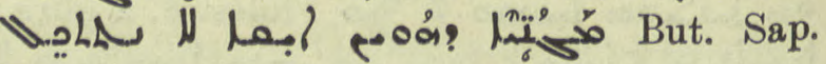
Econ. I. 2.

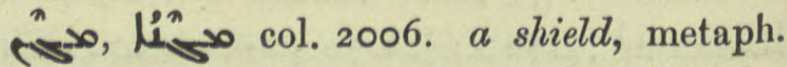
Narses was appointed to be against heretics

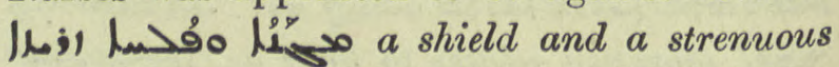
warrior, Nars. ed. Ming. 35. I. N.B. E-Syr. vowels.

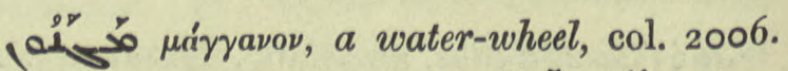
stakes round a wheel S. George $17 v 3$ af.

(I) perh. magnetic iron ore, Med. 594. 17. 2) pr.n. prob. Magnentius, Prefect of Libya, Mart., G. Warda, Einleitung 8.

her 5 magnesia, the drug, col. 2007.

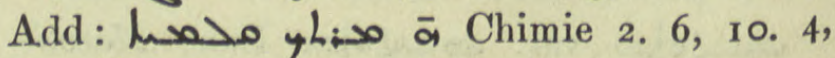

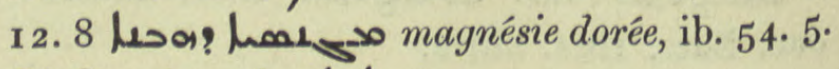
Cf. Suppl. under مإم).

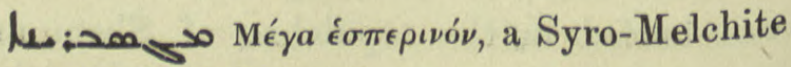
Office, R. O.C. iv. ${ }^{5} 5^{6}$. 


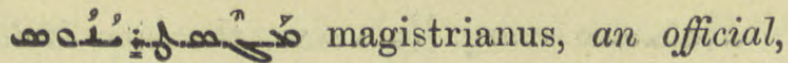

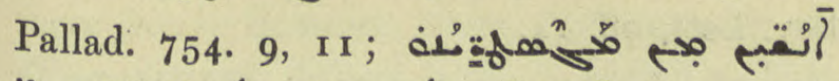
ib. 186. 6 ; Leifleng (xo Sev. Lett. 93, 17 .

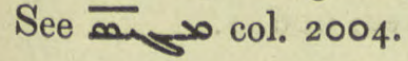

jऍ Ar. مغص to feel pain, $\mathrm{j}^{n}$ Med. 6or. 2.

( 1 ) to worship according to Magian

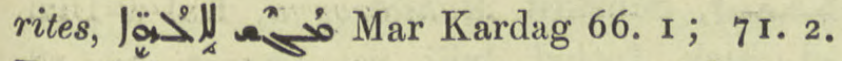
Esp. before food, pass. part. food blessed according to Magian rites,

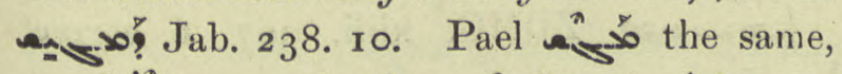

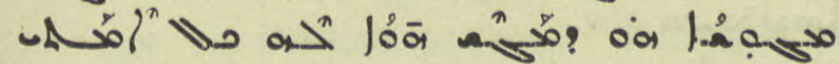
lóā Anecd. Syr. iii. 50. 24 ; BB. under col. 2009 ult. 2) To use magic arts

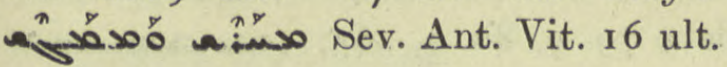

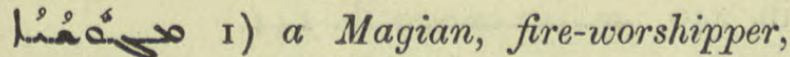
(م) Chast. 4. 5. 2) magian,

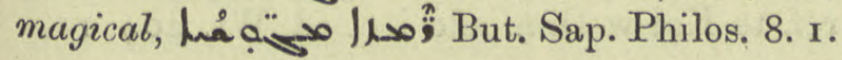

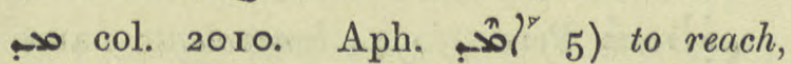

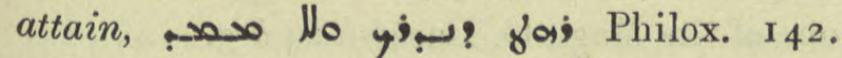

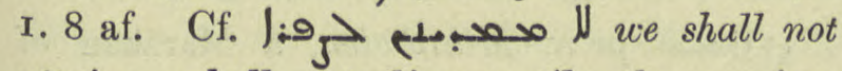
attain = shall not live until, the evening, Pallad. 24. I I.

lo to take refuge, escape, go free. Part.

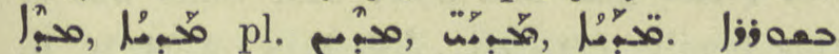
الم الl osuly he is steadfast and he seeks refuge in God, Lamy, Cong. Or.

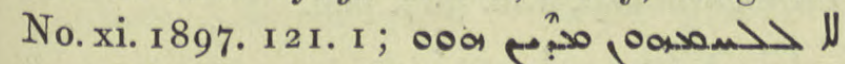
nor did they escape their threats, Dion. I30. 2. Pl. m. emph. قدم: blowing about, loose as

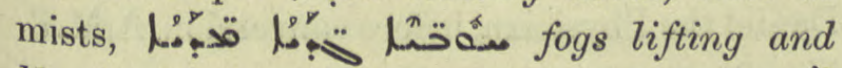
blowing about, Al'ybkam 65. 3. Aph. usol"

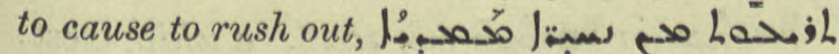
N. Hist. vii. 2. I.

Jifo, $-\infty$ col. 20I2. Lat. mediator, gobetween, Al'yhkam 70. 8.

IL' Econ. I. 2.

ô col. 2012. Media = the bpric. of Hulwan; it stretches from Hulwan to Hamadan inclusive, Eranšahr I 8, Pers. Mart. 259, Syn. Or. 6o. I and often, ZDMG. xliii. 399. 2. See Syn. Or. 53. II and above under مuبs.

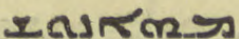

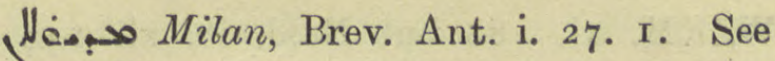
other spellings coll. 2012,2013 .

هِ. perh. Lat. mantica, ZA. xvii. 87 . Trs. from col. 849 to col. 2014. a wallet, hand-bag, مist. Mon. i. 102.17 .

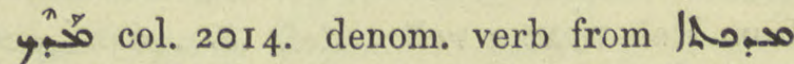
a mortar, R. Duval, to pound spice. In Syr. works on alchemy the form more used than مصميد.

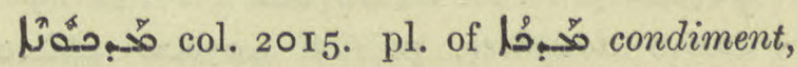
Nöld. Gram. 47 n. 2 :

 preparations, "made dishes", Sev. Ant. Vit. 243. 2.

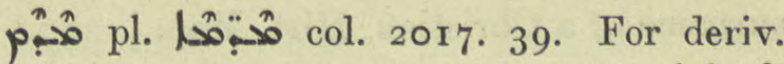
see ZA. ix I37. Something, anything |x内:هُ your churches and shrines, Jab. 73; 1.

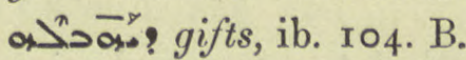

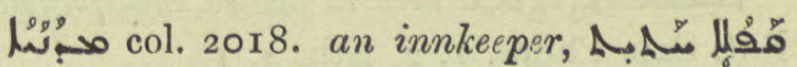

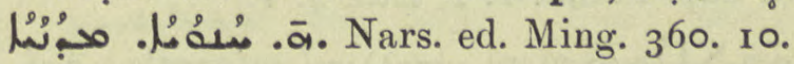

o- ingredient of a salve for wounds, Med. 586. I I.

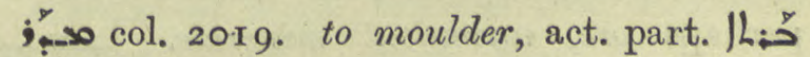

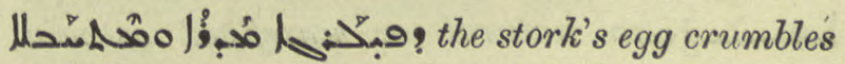
and decomposes, Natur 36.8.

| those formed out of earth, earthly beings, Hormizd 376, 1639.

10 col. 2022. I. Delete this line. It is

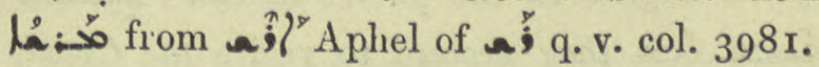

Jas col. 2022 . to reach boiting point, pass.

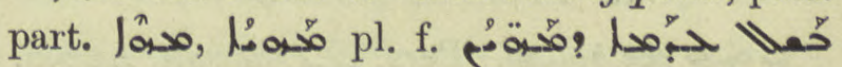
bring to boiling point, boil thoroughty, Med. 196. 19. Ethpe. wasol? ${ }^{n}$ to run down, become

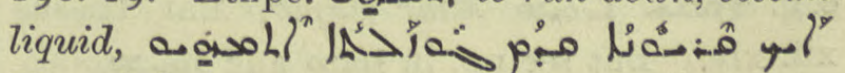
Hormizd. 2423.

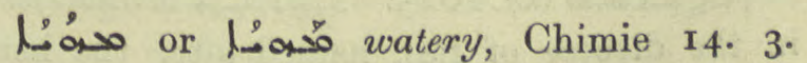
Weak, insipid, haso l上; thou feeble Avian, Pallad. 24I. I 2.

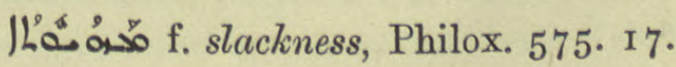

nolas perh. Pers. شهo The Great Immortal, Chabot, Jesus-Sabran 488 n. 2 and B b 
WZKM. xi. 188 but Nöld. ib. xii I 44 on Coupes de Khouabir says the name is Mandaic מהנוֹש, Mahanosh, Magian name of JesusSabran ref. 5०9. 22.

20ำ Sophocles (Greek Lex.) derives the Greek

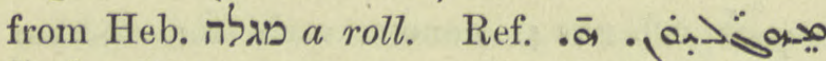

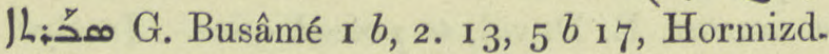
1138.

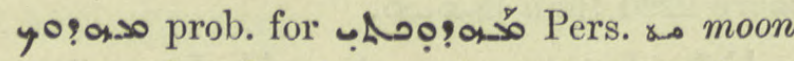
+ورخت Mart. A.M.B. ii. I tit.

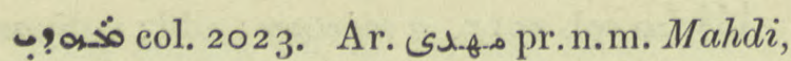

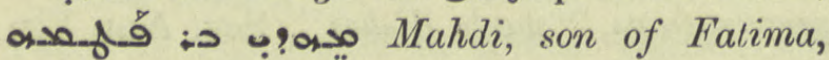

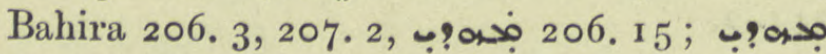
surname of a Christian, Hist. Mon. i. 237.

1 N. Hist. v I with gloss La> قِقة

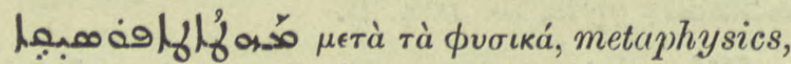
But. Sap. Philos. r. 2, Eth. 4. I.

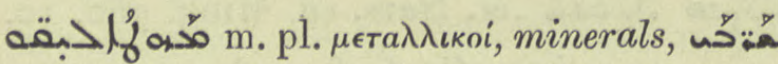
م. Hist.

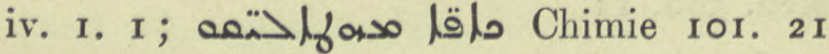
م- م-ســ

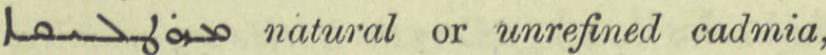
Chimie 16. 25.

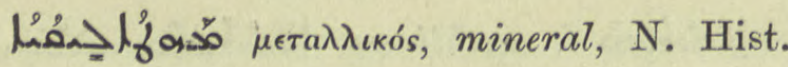
iv. I. $\mathrm{I}$.

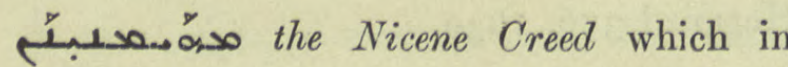
Syriac begins in the pl. We believe. مخمصجبم

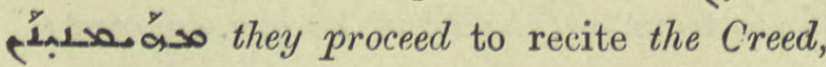
QdhamW. 43. 4; Brev. Ant. i 58 a 16.

;s a so see jouso. Pers. fos the sun, Anecd. Syr. iv. 9. 2. Often used as part of names, e. g. ;or was Mihr Sapor, Mar Bassus xii. xiv. But see nn. in ;aso and gaso.

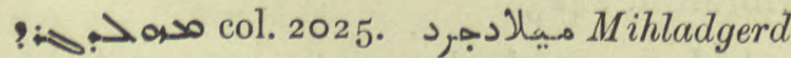
in the diocese of Rai, named after the hero Mīād, ancestor of the noble House of Mihran, ZDMG. xlix. 633, f. ; xxxiii. I48, Pers. Mart. n. $205 \mathrm{I}$.

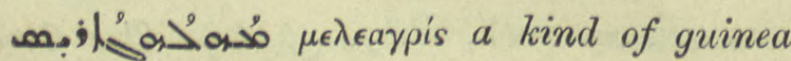
fowl, N. Hist. vii. 3. 2. ua >aso marg. Ka >aso, the city Meloe, Sev. Lett. 98. 14.

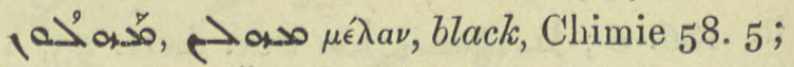

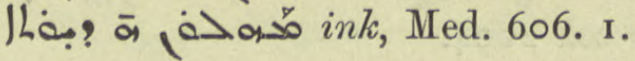

ט_إِ Hormizd. 2302.

Da ?معد, Sanskrit Mahilaropya, Kal-w-Dim. ed. Bick. Introd. lxiii. refs. $34.7,39$. I 8 . Cf. مها\}).

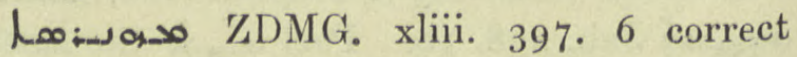

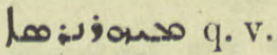

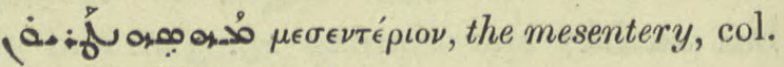
2027. N. Hist. vii. 6. 5 .

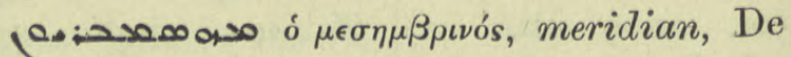
Astrolabe 79. 9, 80. 2, 265, oce ib. 240 , م.

;ax Ethpa. ;ásoll to be expert, ready, ref. to Kal-w-Dim. add i. q. ed. Wr. 204. II.

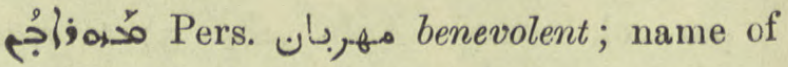
one of the Magi, G. Busâmé $64 b 5$.

?:9ax pr.n. Mihrdaden, a Marzban of Nisibis, Jo. Tell. 16.

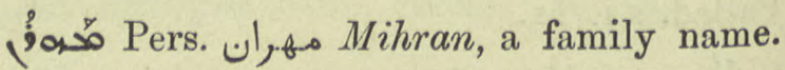
See jas o sar and add: Mar Saba was of

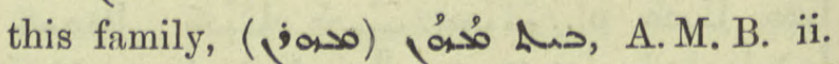
636. i $\mathrm{I}=$ Pers. Mart. 68. A cousin of Pirangashnasp, Jab. $3^{67}$. pen.

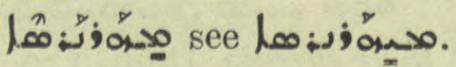

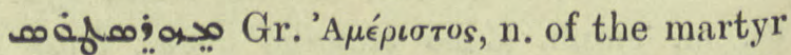
Daniel the Physician, before conversion, A.M.B. iii. 484 .

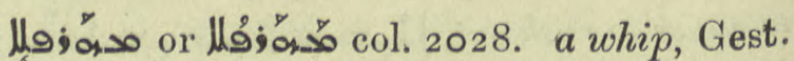
Alex. 15. 7, 59. 3. Cf. ZDMG. xlv. 321. 4 .

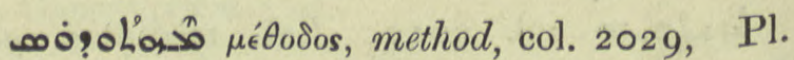
مـ Georg. Ar. 24. II ; قدم:0h BHGr. i. I4. 23.

sos col. 2029. Meum athamanticum, refs. Med. 245. 13, 370. 19.

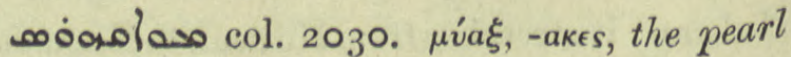
oyster, N. Hist. vii. 2. 2.

معers. Pediaeval and modern word equal to Pehlevi magupat, Zend magnu-

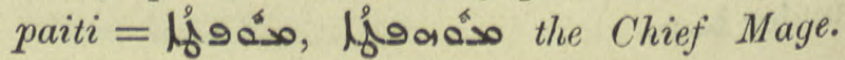




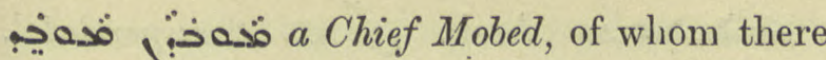
were five in Sasanian times, "Oberpriester der Oberpriester," Tabari 9 n. 3, 450 f. They were Ministers of State for Religion and Worship and often filled the office of Judge, Mar Kardag Introd. n. I4 ; 57. I 4, Jab. 226. I I, and often. Cf. froeso col. 2045 and Suppl. below.

l'ses col. 2030. I) winter station of

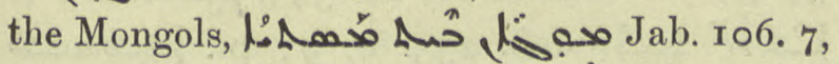
I 10. 9. 2) Persian pl. of 2:- Mogâns or

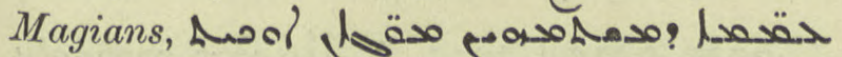
lä̋ G. Busâmé 6o. pen. The name of a city gate: see lor.

Allos of in the language of the Mongols, Jab. I $55^{2}$. I 2 .

Hoaco see ffocos.

os os os col. 2033. Refs. I) marrow,

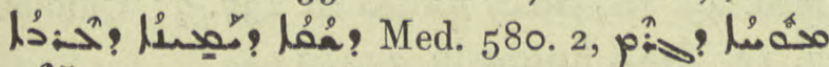
व

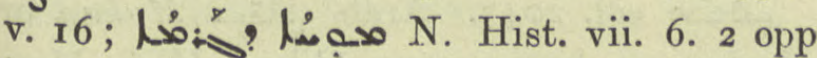

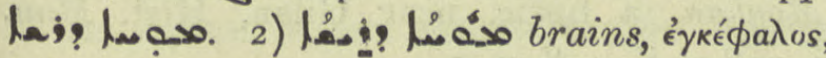
Hippoc. v. 16 , vi. 18,48 , vii. $46,47,53$.

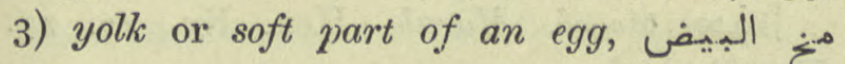

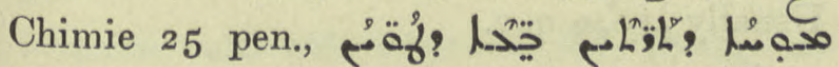
Med. 85. 22.

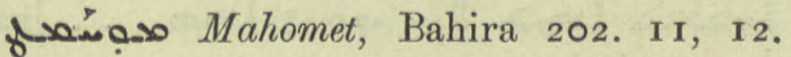

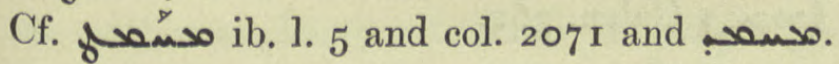

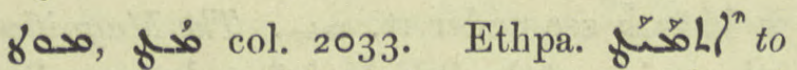

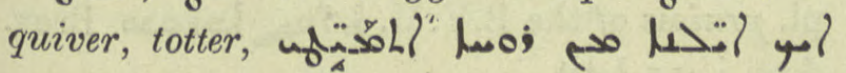

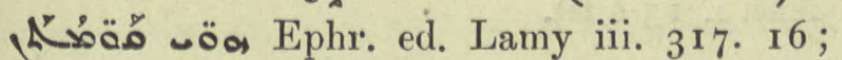

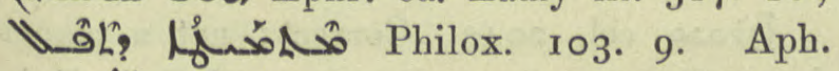
susol" to make to rock or reel, to shake,

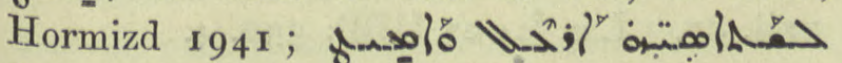
ib. 1943 .

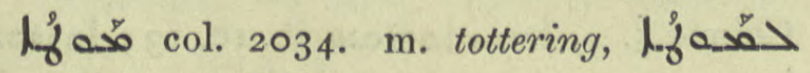

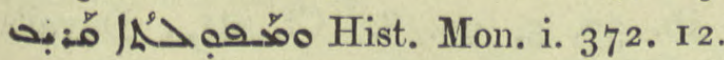

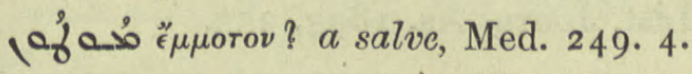

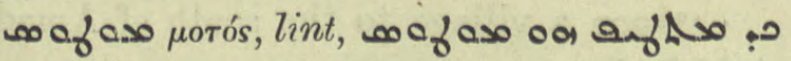

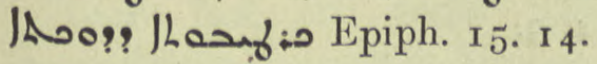

a م ماوية wife of Numan, King of the Arabs, Sassanidi I3. II.

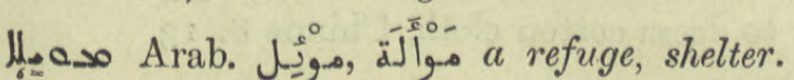
A khan, Hist. Mon. i. 104. 9, 10, ii. 232. nn. 3 and 6. Cf. ll ب.

\section{+tesas}

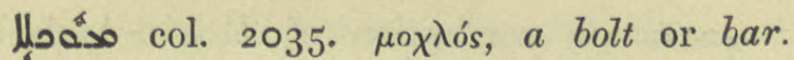

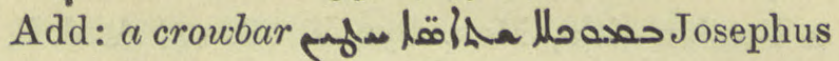
vi. 5 ult.

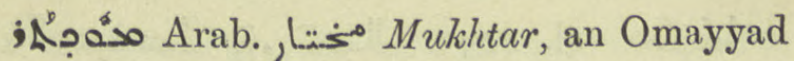
prince, Bar Penk. I 56. 2, I57. 2.

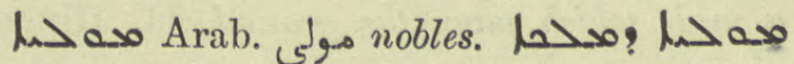
"la Plénitude Royale", Chabot, as if from verb محلا Name given to various armies, Dion. 85. 9, Nöld. WZKM. x. 195.

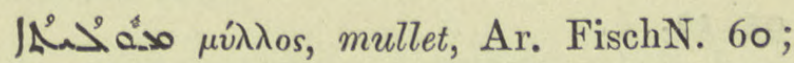
م مه DBB. 103 I ult.

ر مهبر silver furnaces, الرماص Alchim. Grecs. 265 infr., a remedy for wounds, Al'yhkam 70. II . Written مفحبم: Med. I73. 8.

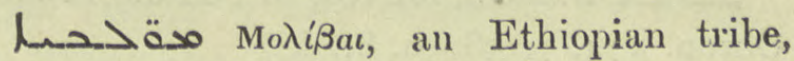
Anecd. Syr. iii. 330. 3 .

Leol. 2037. a pander, ref. Greg. Carm. i. II3. 22.



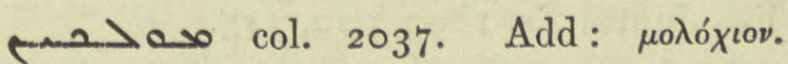
Corchorus olitorius, a potherb, Jac. Edes. Nöld. F. S. i. 575 ; Ar. PflnN. 190.

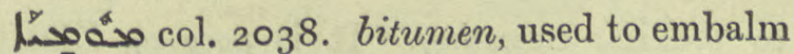

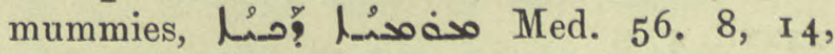
559. 17 .

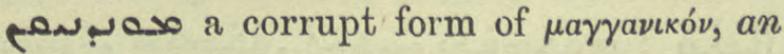
engine of war, Dion. 87.2, 4 ; مد ب. ib. 89. 5 .

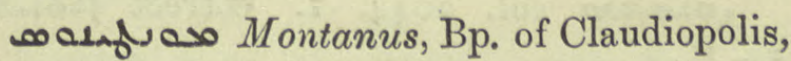
Nöld. F. S. i. 47 I. 68.

Wow منية a common Egyptian placename, prob. meaning garden or valley, haso wol? A.M.B. iii. 620.

fuses or sofuas name of a bishop present at the Council of Constantinople, Nöld. F. S. i. 482.

offues Montanus, Bp. of Neo-Caesaria, Nöld. F. S. i. 47 I. 76.

und os col. 2040. coin, die. Metaph.

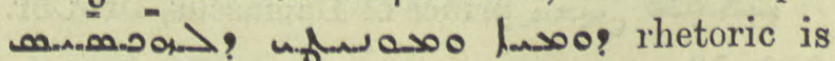
the figure and mould of locutions, Ant. Tagrit.

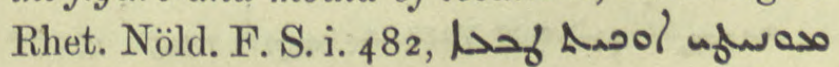
حصחمח, ib.

B $b 2$ 
محه لم:إص corruption of Mogân-Arzbedh, a Persian title, Mon. Syr. ii. 68. 14, A.M.B.

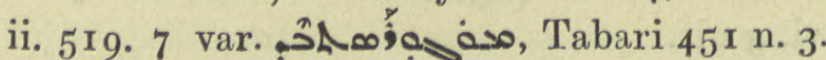

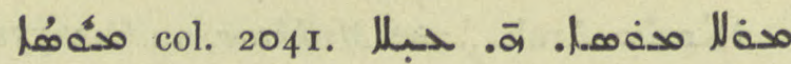

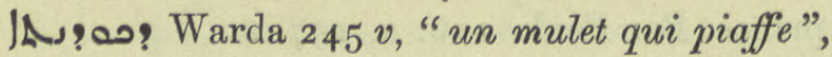
a mule which paws the ground, id. Hippoc. vi. 4 .

م the Muses, Arist. Apol. مص 20, Gest. Alex. 95. II.

uf 000 col. 204 I. f. $\mu$ iv $\sigma \tau \iota s$, the sac of the cuttlefish, N. Hist. vii. 2. 2.

$\mathrm{L}_{-}$- مasthia, a city of Lycaonia, Brook's Chron. 574. 7, $5^{82}$ n. 9.

Hoos Amasia, fortress in Pontus, Brook's Chron. 574. $7,5^{82}$ n. 8.

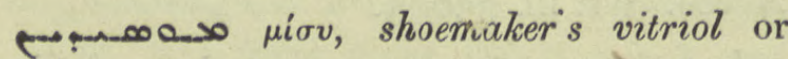
copperas, Chimie 3. 5, 4. 2, 11 . 14, 18,230 n. 3, 次

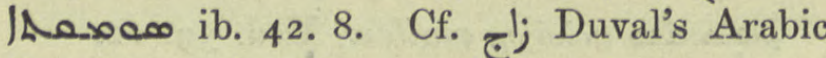
list, Journ. As. 1893, 320, 345 .

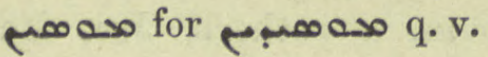

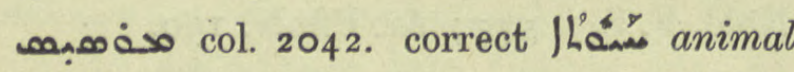

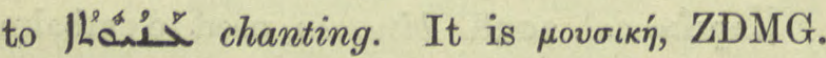
xxxvii. $47 \mathrm{I}$.

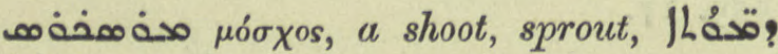

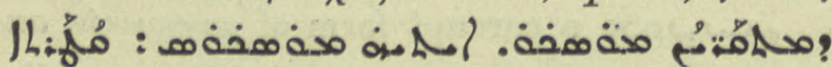

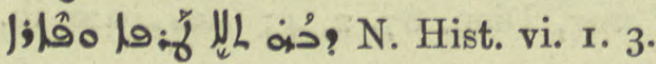

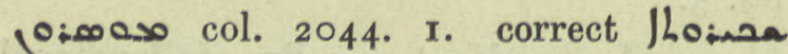
for Jlin: مجن:

1A o col. 2044. 8. Dele "felicitas", it = Jams a scented sedge, col. 2 I 77 .

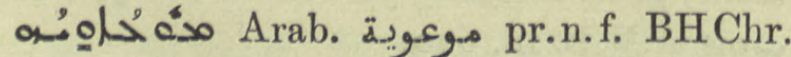
65 ult.

م. pr.n.m. a Buwaihid prince, BH Chr. 18r. 3 .

معز اليدين مبحر إخَب: prince of Melitene, son of Qilij Arslan, BHChr. 381. r3, 389. 19.

م.عين محب ג-بر prince of Damascus, BH Chr. 313. 12.

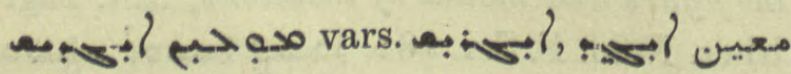
. للإيش pr.n.m. BHChr. 500, 12.

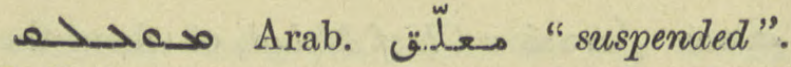
Moallak, a monastery mentioned with Balad on the Tigris, BHChr. Eccl. ii $3^{6} 3$.

מאופט מאט Abh. 189, f. I.q. مهمد Suppl. q.v. Add refs. Jesus-Sabran 564. 6. Oftener written

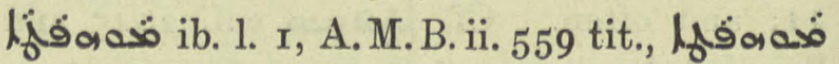
مol loa of

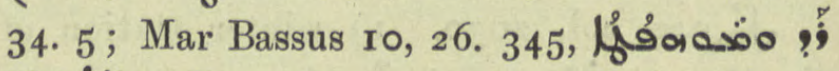

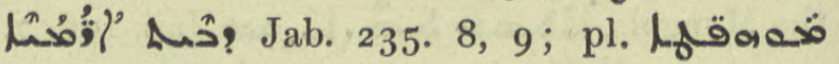
Mar Kardag 65. I, 66. 5 .

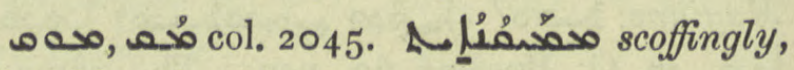
Hist. Mon. i. r66. I.

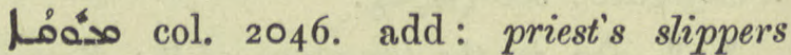
worn while ministering, opp. مقفئم Jab. 83 ult.

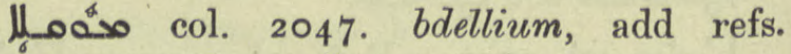

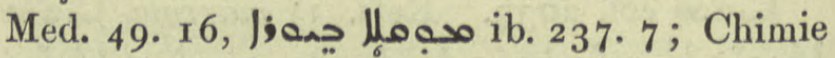
7. 12 ; ;a. oم ib. 252 . 11.

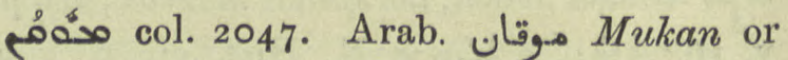
Moghan, a bpric. near Gilan, Hist. Mon. i. $23^{8}=$ ii. $44^{8}$. n. $\mathbf{x}$.

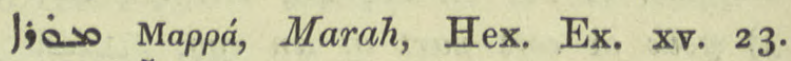

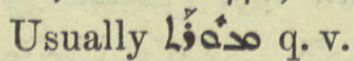

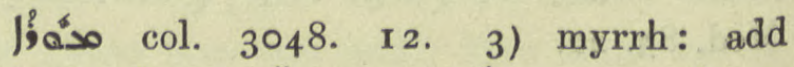

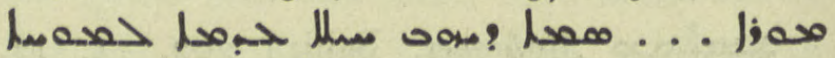
bo:s? Galen. $3^{62}$.

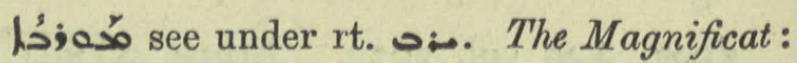
pl. praises of the B.V.M. مa مa Brev. Ant. i. ${ }_{5} b$ tit.

مa col. 2049. Correct: not a planet but a bird : prob. a wild pigeon, I. Löw, Z.A. xxii. 87. I. q. J: 09 DBB. $55^{\circ} .4$.

مه col. 2049. litharge, dross of silver,

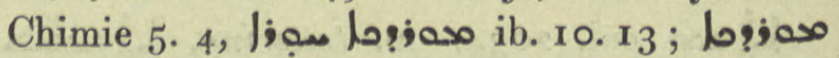

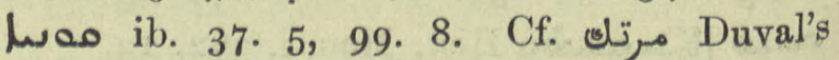
2nd List, Journ. As. 1893,355 .

معز!) a village to the south of Amadia, A.M.B. i. 4 I 4.3 .

, 2049.

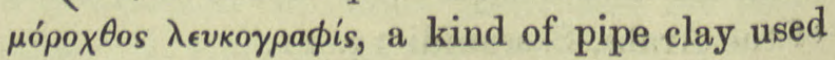
to dress cotton cloth, Chimie 8. I3.

husö0x col. 2050 . m. pl. signs made with consecrated oil, Jac. Edes. Can. 8. 8. 
مa col. 2050 infra. L. I of par. delete الآس مرسيین, these words belong to $\mu \nu \rho \sigma i v \eta$, the myrtle, col. 2052. Ll. 2 and 3, delete the words between فيلون and "legend." L. 5 and half 1.6 seem to belong to col. 2 r82. L. 6 after woon to end of par. delete. I) "wild myrtle", Budge, Med. 389.6

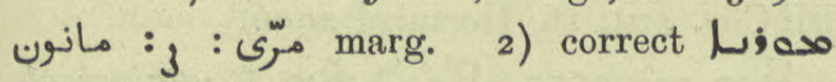
murena, Natur 39. 7, 40. 2.

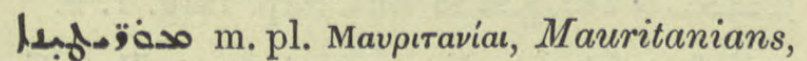
Pallad. I 20. 3 .

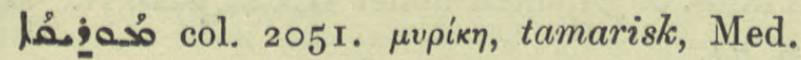
93. 23. For deriv. cf. Nöld. ZDMG. xxix. 649 .

H;ox púpaıva, murena, the lamprey, Anecd. Syr. iv. 7 1. 8, Natur 39 marg.; ib. pen. and 40. 2 f مax.

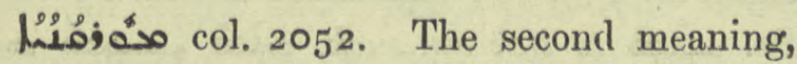
yolk of egg and the words

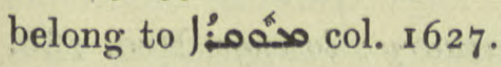

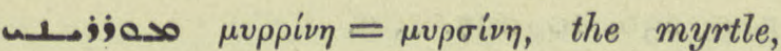

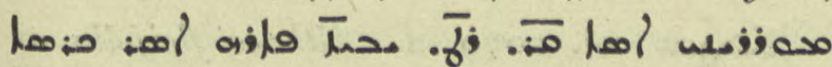
.

مa or col. 2052. to feel, INay alda! he felt his pulse, BH. Stories 337,

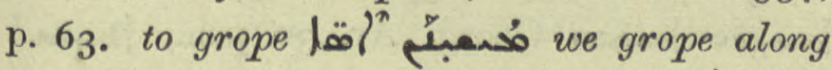

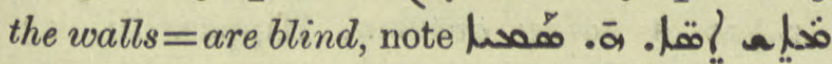

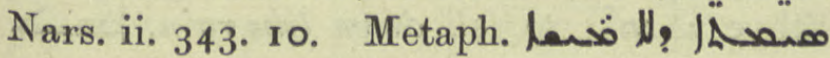
inscrutable treasure, Warda $32 r$.

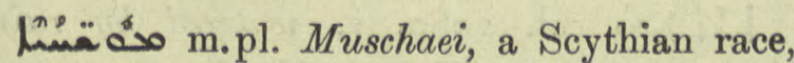
Ephr. ed. Lamy iii. 198. 3 .

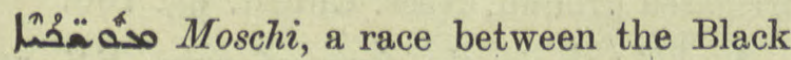
and Caspian Seas, Ephr. ed. Lamy iii. 97 ult.

فㅇำ مبح col. 2055 . musk or moschatin, ref. Med. 4IO. I, 3 .

 add ref. Med. 470. I.

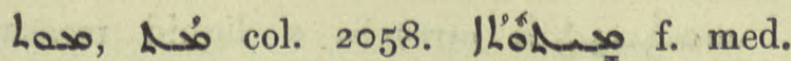
mortificntion, gangrene, Hippoc. v. 20.

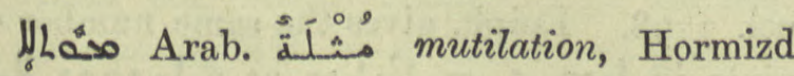
2453.

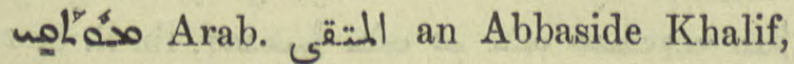
BHChr. 179. I2.

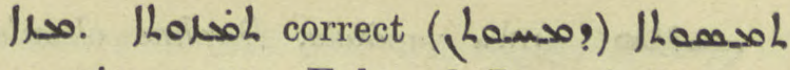
corruption, matter, Ephr. ed. Lamy i. 83. 9.

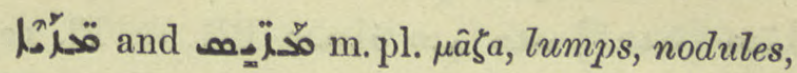
Chimie 240 n. 3, فمعم حه مانم ib. 245 n 2.

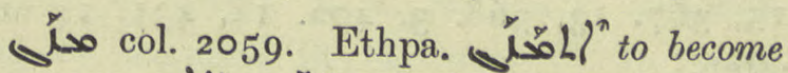

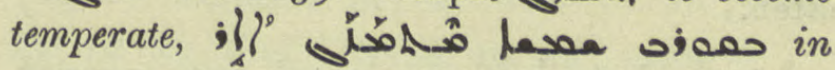
springtime the air waxes temperate, N. Hist. iii. i. Ethpalpal Hénol/ to be mixed, Hormizd. 1242.

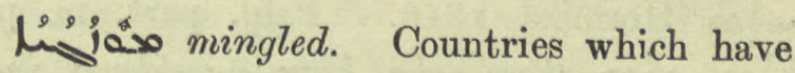
little rain and are far from the sea

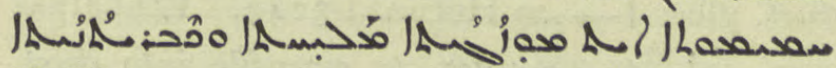
N. Hist. v. I. 2.

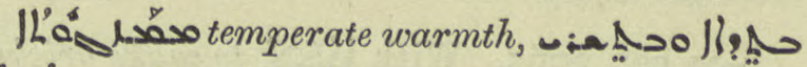

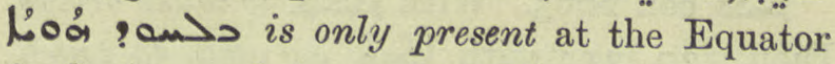
in Spring and Autumn, N. Hist. iv. 4. 2.

IRُ

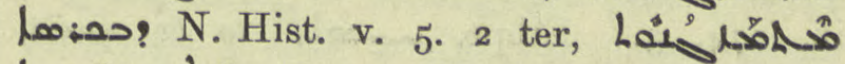
lenväfol ib. vi. 4. 2, Theol. 4. 8.

فํ. Part of a bird, perh. the crop, l: |

مهام Arab.-Pers. المَز Mazôn i.q. Oman on the Arabian shore of the Persian Gulf, Syn. Or. 676: 43 pen., 76. 29 so restore for (0;- $\infty$ and so 1 ro ult. ZDMG. xliii. 402. 4 , 404. Io ; rightly (ol o ib. 396. I6 ; Eranšahr 43 f., (olo? ) jh/ Sassanidi 36.11 I $=$ Chron. Min. $3^{8} .3$ af.

مدرة Syn. Or. 216. 2 I inhabitants of Mazôn, Omanites.

Lolso col. 206r. Amazon. Add: name of

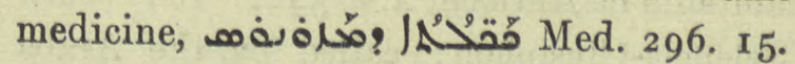

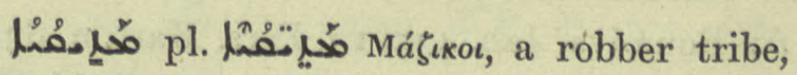
Pet. Ib. 85. 21 ; مارحمه Pléroph. 82. 8.

مسرمب ما مصم Anecd. Syr. ii. 106. 20 read

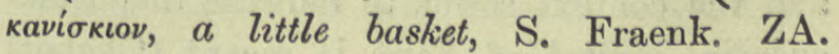
xvii. 87 .

مِار a village to the E. of the Upper Tigris, A. M.B. i. 453 .

مَّ人 Mazra, Bp. of Beit Zabde, M. Z. 3 ult., 4. 2. 


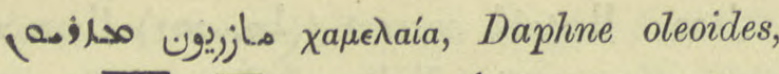

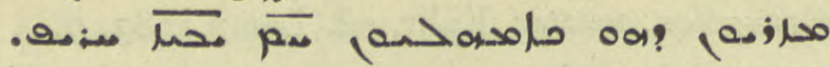
م- مسali BH. de Pl. I26 Ar. PflnN. 247, ZDMG. xliii. I 25, Med. 366. I $5,367 . \mathbf{1}_{3}, 368,3,402, \mathrm{x}_{4}, 43 \mathrm{x}, \mathrm{I}_{4}$ and often.

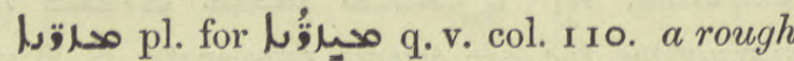
belt or cord, Dion. i6o. 8 quoting Jes. iii. 24.

col. 2064 . metaph. to desire, a مُ مُام Hormizd 1816. Palp. part. m. pl. oxose who have mouldered into dust, gloss othormizd 3249. Ethpalpal س. Hormizd I 799 .

مشُم col. 2065. With خحتُ to make bricks,

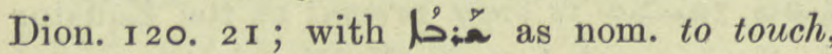

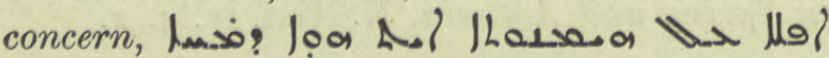
bin the affair had no concern with religion, ib. 174. 3. Ethpe. unsoli ? $^{n}$ to be spoilt, withered as a plant set in bad soil, part.

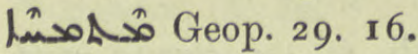

col. 2067. a parapet,

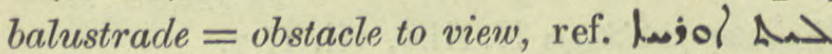
معشA| Odes od Sol. ii. 379 .

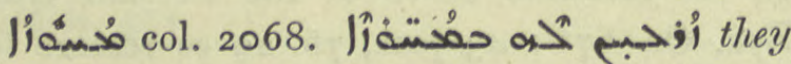
sow this plant on walls, Med. 598 ult. L. 8 af.

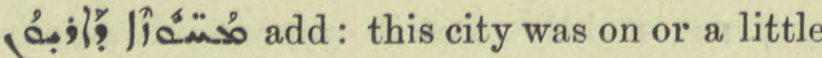
south of the Lesser Zab, in Beit Garmai, Syn. Or. 109. 3, 164. 23, 214.7 , Išoyahb I 23.22 ,

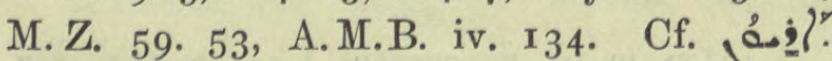

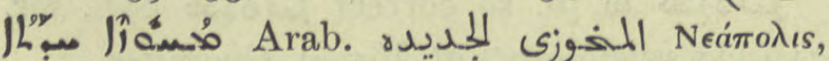
built by Chosroes Anushirwan for the deported inhabitants of Antioch, A. D. 540. It is south of Seleucia-Ctesiphon; called by the Arabs Ar-Roumiya, Jab. 435. I 3 , Pers. Mart, n. 834 , Tabari 239. But Syn. Or. $109=366$ the Bp. of Mahozê Hedata signs as Metropolitan and the context seems to indicate that Rewardašir, capital of Fars, is meant (Chabot) ib. 676 .

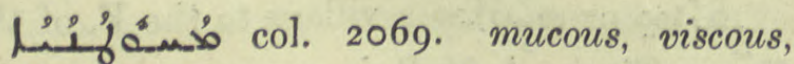
Hippoc. vi. $5^{6}$.

همفنُ Elisha, M.Z. 196. I5.
مسمةقـــ Mahunaeans, a Scythian race, Ephr. ed. Lamy iii. 197.

ou col. 207 I infra. to measure swords,

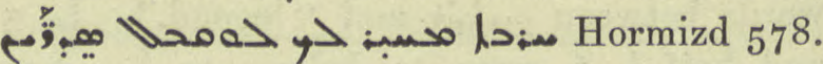

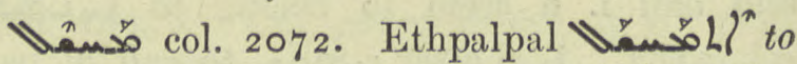

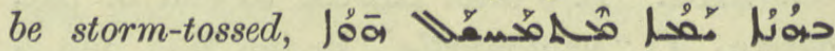
BH Chr. 424. 16, Hormizd 2996.

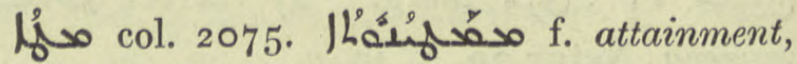

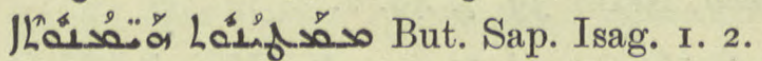

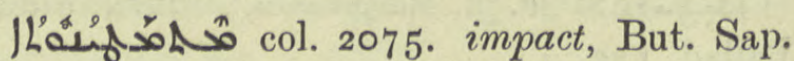

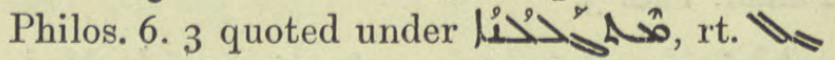
p. 74 .

Dastilene in Lesbos, Sev. Ant. Vit. 25 ult.

uñ o col. 2075. to lick or suck, add:

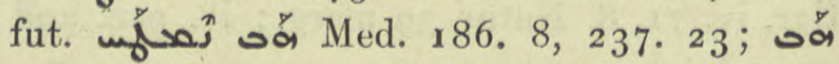

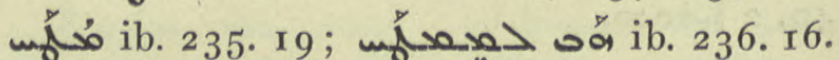
Pa. مَّل administer some as an electuary, Med. 184. 22.

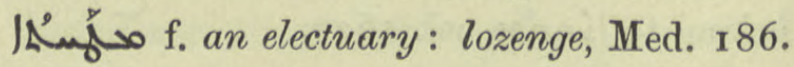
5, 9, 236. 10, 237. I, 9.

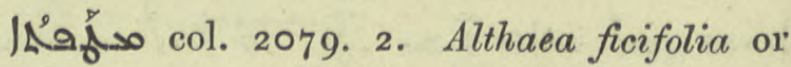

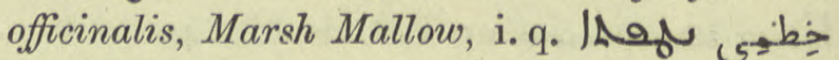
Ar.PflnN. 361 ; ib. I66; Med. 146. 5, 232 . 14.

مهلم Laمت ILindo a rainy autumn, Hippoc. iii. I3. Ethpe. - خسم: loa is of 4 Macc. 64. 24.

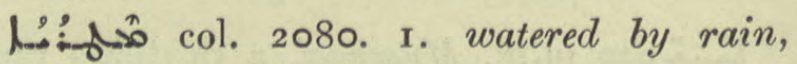
| irrigated ground, Nest. Chrest. 95. 186.

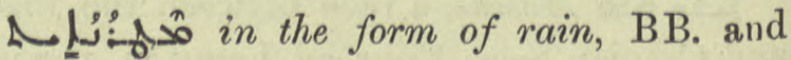
Hunt. clxx under lemi', col. 3939.

colloso of Metropolis, a bpric. in Pisidia, Nöld. F. S. i. 473. I 12.

l. Mt. Singar 24.4 af.

ع مَّ人 equalling seventy-two pints, BB. under فحخد col. 3798. Epiph, gives the same number of pints in measure of the sanctuary, 5. 7, endifin مصan, ib. 11. 8, 20.

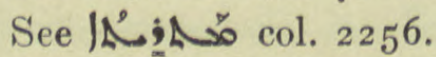




\section{rdits}

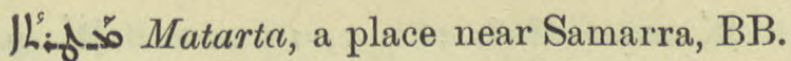

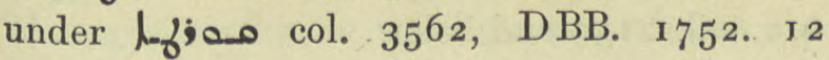
and $\mathrm{xxv}$.

人

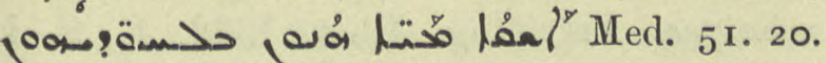

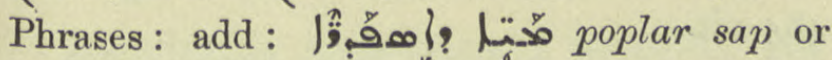
a solution of some part of the white poplar,

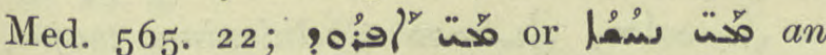
amalgam of mercury with brass or copper,

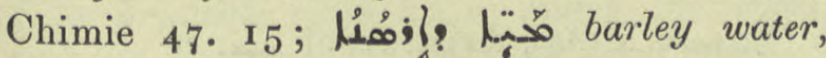

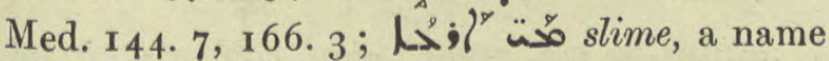
for leeches N. Hist.

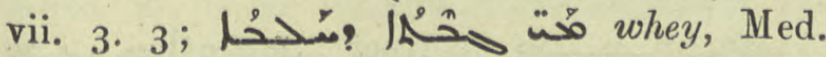

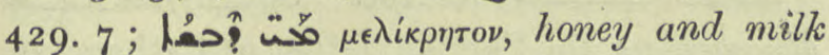
or honey water, Hippoc. v. 38 , Med. 45. 16, 2 I, 561. 3; ;

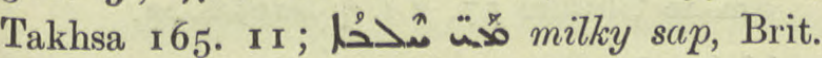

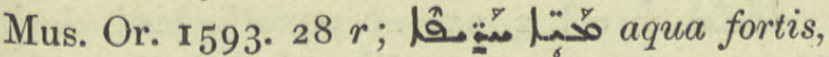

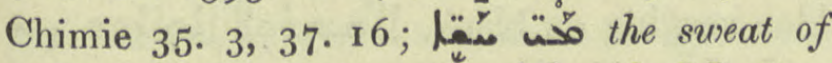
suffering, Hormizd 2362 ;

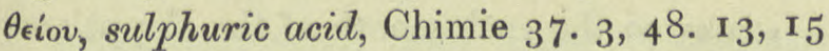

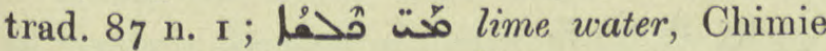

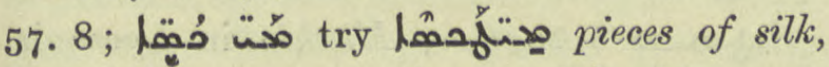

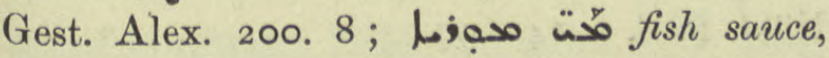
Med. 105. 13 ;

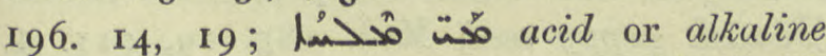

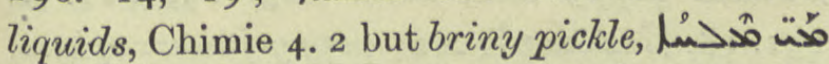
1. Med. I05. II and salt water

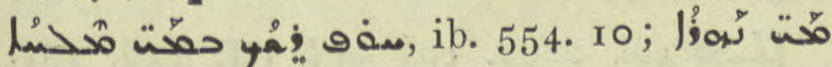
river water, name for an amalgam of mercury

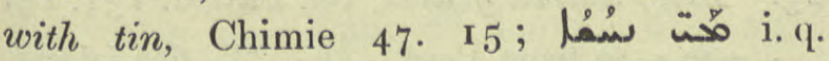

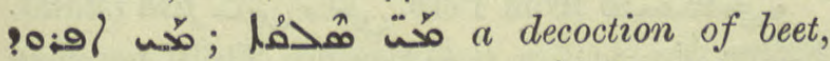

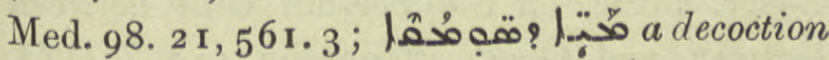

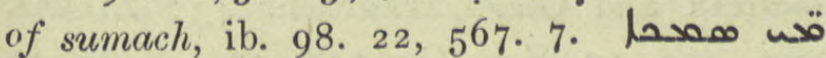

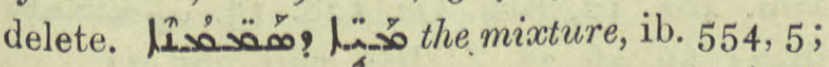

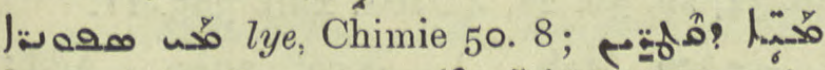

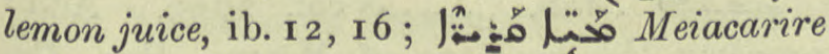
= Fontes Gelidi, Ammianus Marcellinus, a village between Mardin and Amid, Hist. Mon. i. I44. 2, I63. 20 ; محْمُ a a decoction, Med. 40. I $3,45.23,58.5,6,292.23,293.18$.

م o col. 2084. Arab. a race-course, $\mathrm{BH}$. on I Cor. ix 24 gloss to

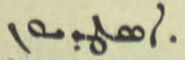

40 o 0 perh. from Assyr. utukku, Sumerian uduk. Name of a demon, which appears to women as a man and to men in the shape of a woman, Charms 40. 10, 59. 3, 62. 18 ; she has, various other names, ,

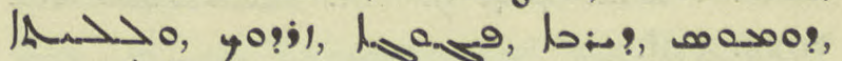

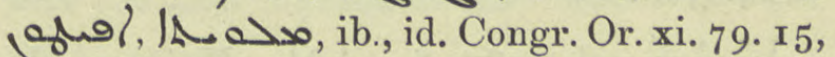
8 I. I8, 83.8 af., JAOS. xv. 287 infra.

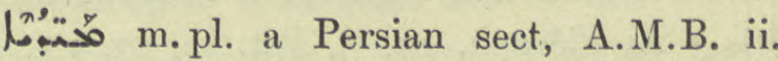

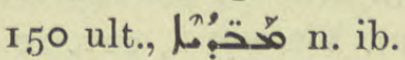

פou Pers. fo the sun for Zend Mithra, the Deity of Light; lasl

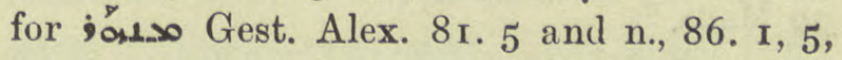
Nöld. ZDMG. xlv. 318. Often forms part of proper names; see the following and cf. - is o.

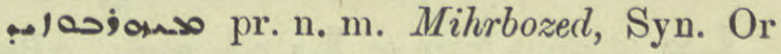
36. 5, ZDMG. xliii. $395 \cdot 5$.

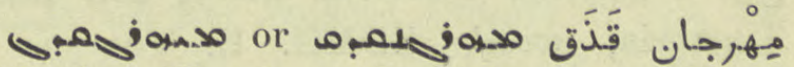
Mihrakan-K'atak, a Kurdish name "House of the Mihrak", a district between the rivers Shirwan and Choaspes in Khuzistan, Eranšahr 20 ; E-Syr. bishopric Syn. Or. г го. 22,

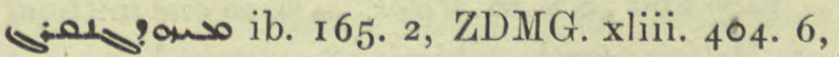
م: Trow ib. 405. 2 ; Phet. 42. $4=$ Pers. Mart. 67, col. 2084. Io af. Mihraganqadaq is the same ashöم حس q q.v.

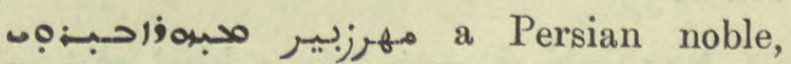
A. M. B. iv. 207.

o:-j;ans pr.n.m. one of the Magi, G. Busâmé $64 v 4$.

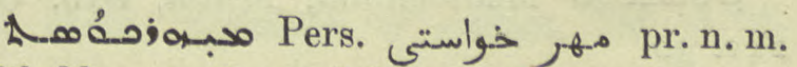
Mihrkhwast, Jesus-Sabran 569. 5, 570. 3, WZKM. xi. 188.

 gushnasp, Magian name of S. George, Jab. 436. $2,564.6$.

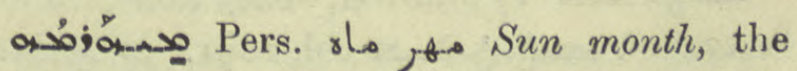
seventh month of the Persian year, Jab. $564 \cdot 3$.

هihrnarses, bp. of Zabé, Jab.

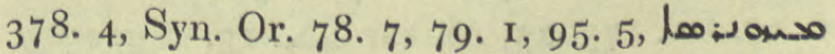
ib. 59. 23 ; ZDMG. xliii. 398. 5, , م-

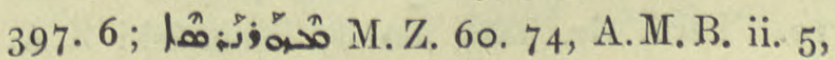

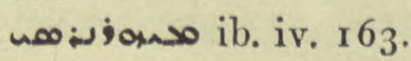


iso Mihrir, a Magian noble, A.M.B. ii. 560.7 .

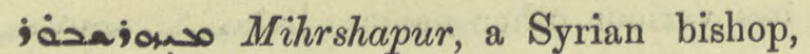

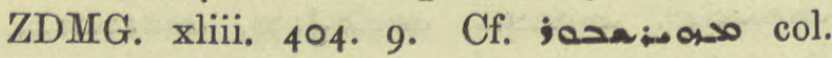
2024. 5 af.

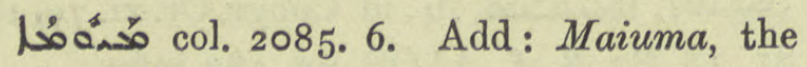
port of Gaza and name of a convent there, 从 : $\overline{\mathbf{s}}$ Pléroph. Ir. 5, Pet. Ib. 53. I5; ib. 44. I3 49. 13, Sev. Ant. Vit. 97. 8, 22 1. 10, 229. 12.

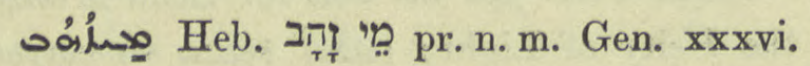
39 Maus.

مترك مببلهتبمُمِيا

مهص: 6 minium, Chimie 57. 6.

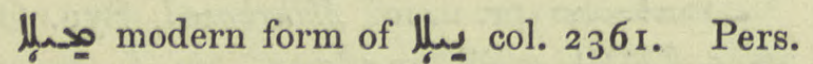
نيل Indigofera Tinctoria, indigo and Isatis Tinctoria, woad, ZDMG. li. 602 n. I,

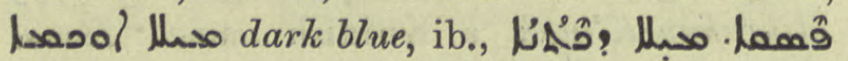
a strip of blue cotton, Med. 58 5. 18; 609. 7 cf. مُحْر.

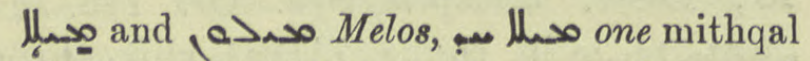
of Melian earth, Chimie 85. 20; مئ o. م مدخد soufre de Mélos, ib. 56. in, Trad. roo n. 4. Cf. مخه مص مد col. 1988 and col. 2 I 33 .

ڤ

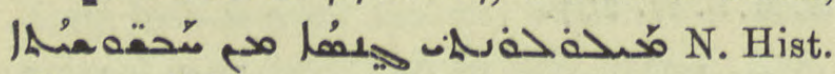
vii. $\mathbf{I} . \mathrm{I}$ infra.

مسخبمo Mediolanum, Milan, Syn. Or. 242. 30.

متحبر col. 2090. BH Chr. I30. 14. Bedjan

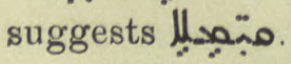

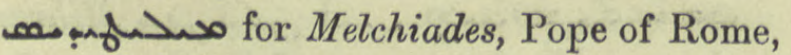
Anecd. Syr. iii. 48. 20, סصحلمب ib. I. 25.

I) $\mu \in \lambda$ itirns, honey stone, Chimie

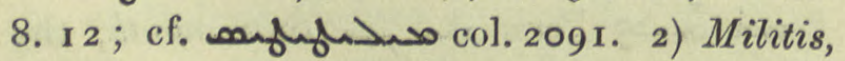
name of a prescription, corruption of م مكلمحه Melilotus, Med. 320 pen.

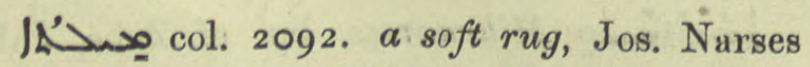
6. 19, Jul. 173. 25, Ahikar

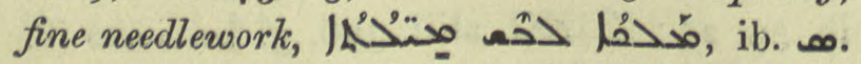
1 2. Perh. Gr. $\mu a \lambda \dot{n}, \mu a \lambda i o \nu$, Poznański Z.A. x. II9.

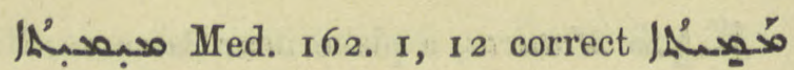
q. v. col, 2 I 50 and below.

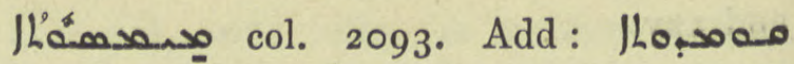

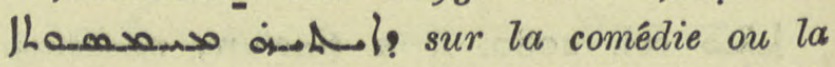
mimique, Ant. Tag. Rhet. Nöld. F. S. i. 484 ult.

مدبمد:ف $=\sqrt{ }$ ( celandine, swallow wort, coll. 1989, 2 I 56 .

Rauso Minos, Arist. Apol. \&. I,

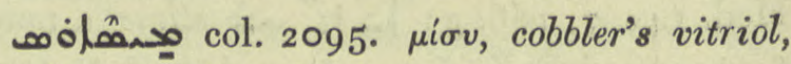

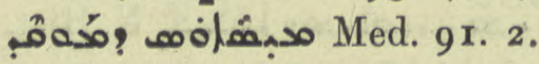

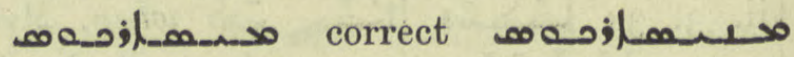
Mnesarchus, the Samian, father of Pythagoras, BH. Hebraica iii. 25 I. 5,

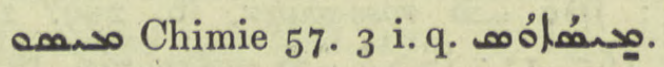

فم col. 2095. an aquatic bird, dark

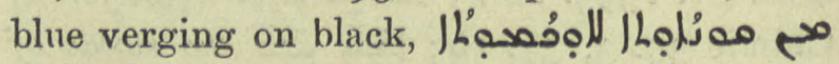
مصع ممخح N. Hist. vii. I 7 .

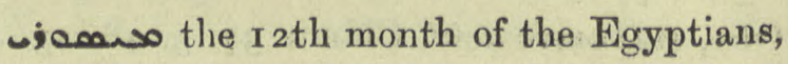
Georg. Arab. 5. 12.

I Nöld. F. S. i. 142.97.

flis col. 2096. the poppy, add:

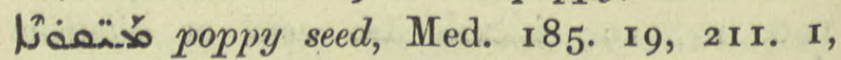

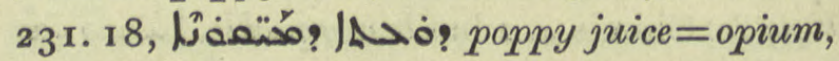
243. 22,244 . 16.

مِ col. 2098. oak gall, ref. Med. I81. 23. Long-shaped grapes, ref. Pallad. I3I. I5. Saran from Pers. E-Syr. bpric. on the Persian Gulf between Bahrein and Oman, Eranšahr 43; ZDMG. xliii. 404. I2, Syn. Or. I28. I5, एasaco ib. $34,26,36.28$, ZDMG. xliii. 395 . I I.

مسعم Arab. col. 2099. Add: properly the province of Mesene, Syn. Or. 62. 18, 66, 4, 8, 7 I ter, but used absolutely a) for I9. I2, 34. I I, 36. 6 ; scriptio plena, ib. 94. 3I so correct for כas ib. 213.28.

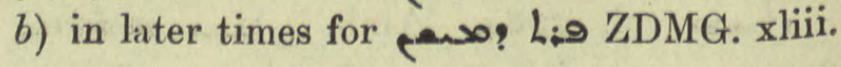
399. I2, 400. I4, I5.

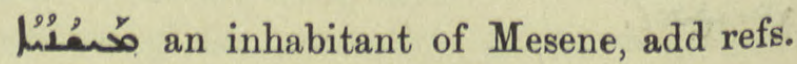
Syn. Or. 90. 8, 164. 15, ZDMG. xliii. 404. I 7 , Jab. 225.6. 


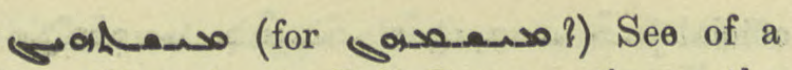
Metropolitan bp. in Beit Qatraye viz. on the W. of the Persian Gulf, Z.A. ix. 367 .

موتبين col. 2099. Add: Arab. a village of the Hauran, ZDMG. xxix. 431.

oof? ofin Mithridates, a Georgian, Pet. Ib. 29. 5 .

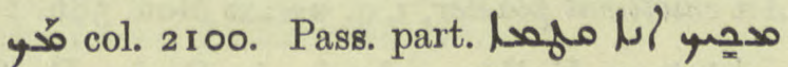
$I$ lie prostrate upon ashes, Journ. As. 1907 ,

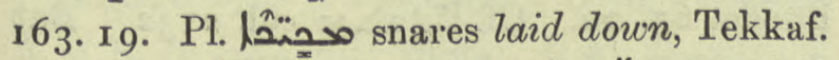

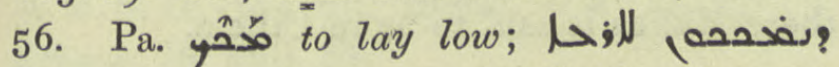
Apoc. xi. 6 ed. Gwynn,

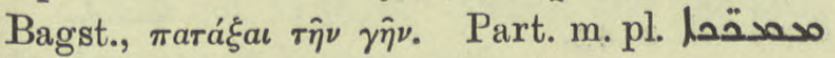
حمقهدلا ?:ح- prostrate under adversity, Isoyahb 22. 18.

مُ مكَمكوك col. 2 102. Add : a dry measure, $\frac{1}{12}$ of an loij\},

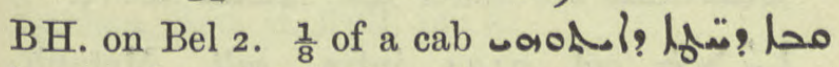
. 928.

ILْó col. 2ro2. Add under r) lowliness of plants in the scale of creation, opp. Jhard) But. Sap. Eth. i. 3. Under 4) a ship. Mandaic מאכותא Nöld. and Arab. Dozy Suppl. See notes S. Fraenk. ZA. iii. 54 and xvii. 254 .

|L' faintness of light, opp. aitoh intensity, Ephr. Ref. ii. 32. 9.

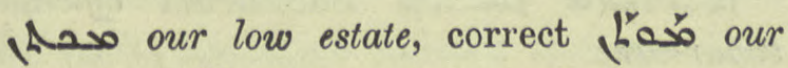
death opp. Eמيّت Ephr, ed. Lamy i, 277. 25.

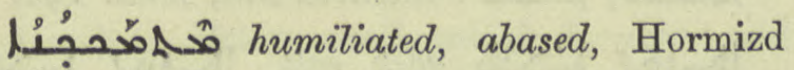

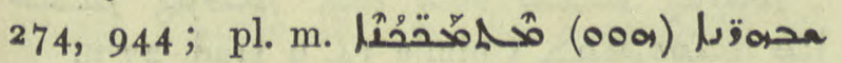
G. Warda ed. A. Deutsch 8. ro.

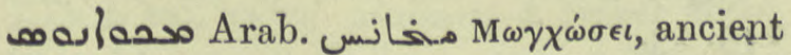
name Makhânis or Tmouschous i.e. Temple of Khousou, now Bakhânis in the province of Qéneh, Egypt, Amélineau 5 r 5 ff. A.M.B. v. 164. 16 .

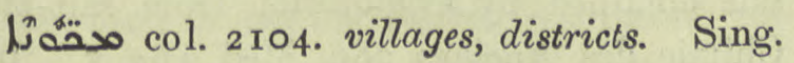

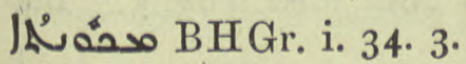

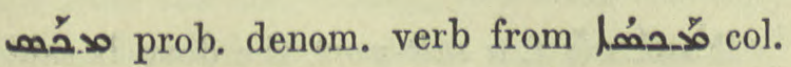
2105 impost, tax. To hire or let out, एव renaso those who hire sanjaks (Yezidi images), M. Singar 19. 4 af.

2716
مخمصُم m. the hiving, letting out of sanjaks,

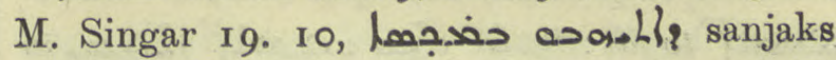

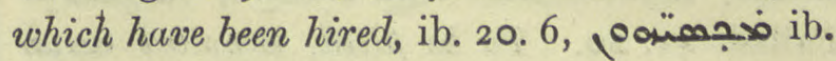

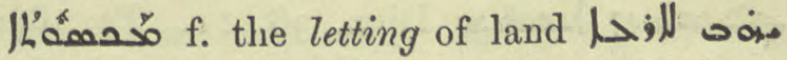
(Wo M. Singar $25.7,39.7$.

sounas Maxinos, one of the Seven Sleepers of Ephesus, Charms 96 infra.

مَحصن:

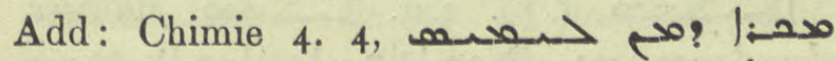

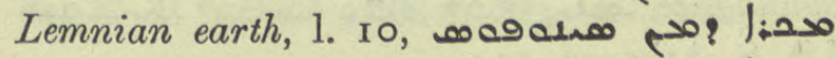
oarth of Sinope = red ochre, 1. I I ; مص:| ? carpenter's vermilion, ib. 7. I 8 .

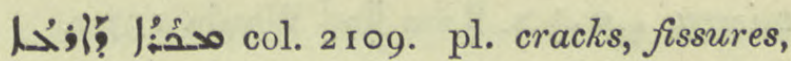
caused by frost, Dion. 194. I0.

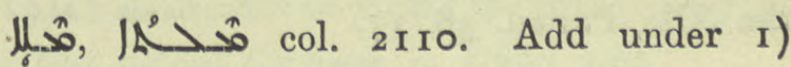

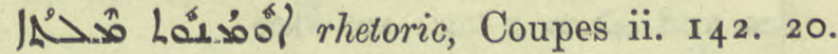
loa IN od on he was placed under an injunction by the clergy, Sev. Lett. 134. 10.

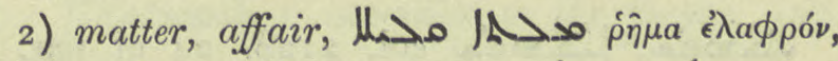
Hex. Ex. xviii. 26 ed. Lag. Pesh.; ? u. account I take of ..., Sev. Lett. 107. 13. 4) reason, pos (sic) Ihea 0:9 0 Pallad. r04. I9. I

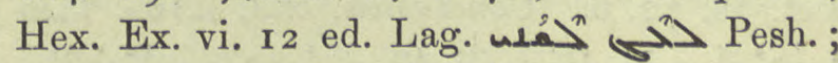
irrational, unreasonable, Sev. Lett. 240. I7

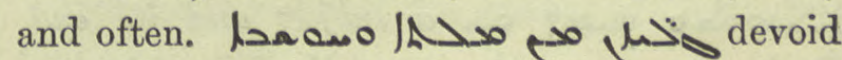
of reason and thought, Patr. Or. iii. i. ro4. 8. Under 5) end of par. Heool? IN

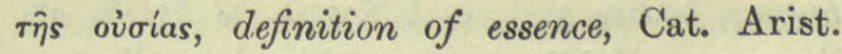
Jac. Edes. 2 I quater.

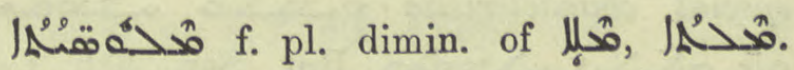
Vain speech, pretexts, Sev. Lett. 427. I7; إن falsehoods, calumnies, id. Vit. 9. 13.

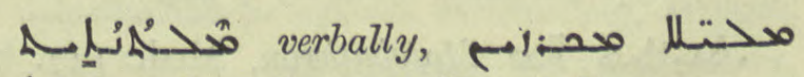
Syn. Or. I38, Hormizd $339^{2}$.

o col. 2117 to fill. To rise as the Nile,

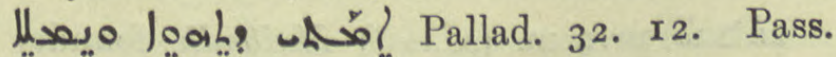

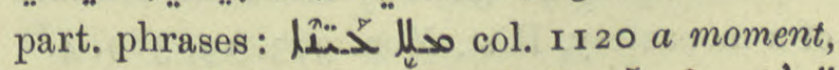

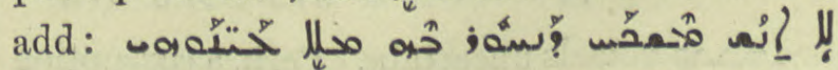
Pallad. 749. II ; Nars. ed. Ming. ii. I33. 8. c c 
1 10003 إl patched, Pléroph. 42.9. Ethpe.

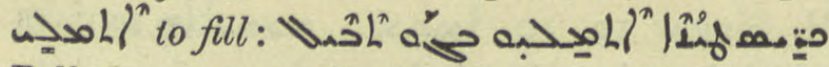
Pallad. 41. 20. To be filled, supplied منحه

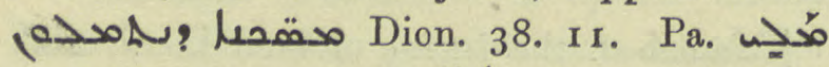

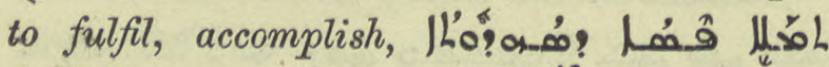

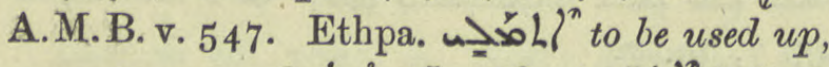

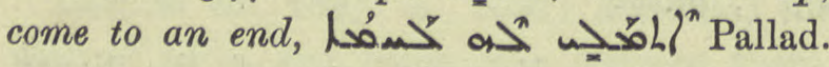
540. 2.

مصe name of an army: see under

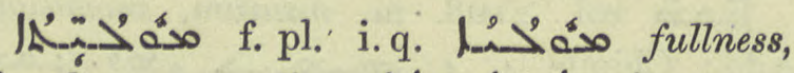
abundance, Lit. S. Athanas. I I 4 .

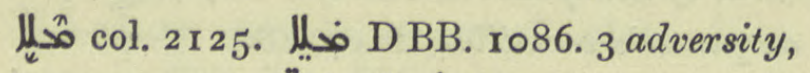

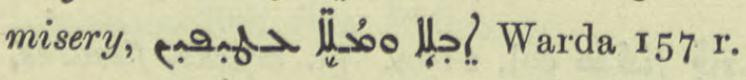

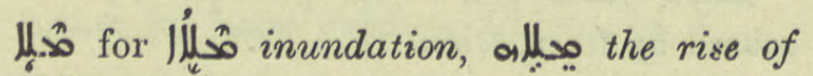
the Nile, Pallad. $3^{8} .17$.

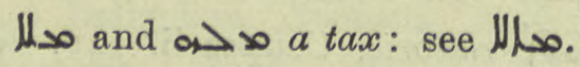

ס مْحُ vii. 116.

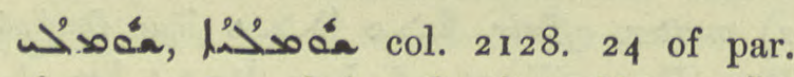

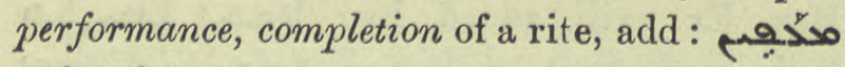
al they conclude or complete the funeral rites, Hebraica iv. 84 ; بر

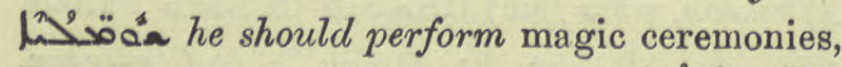
Pet. Ib. 72. 6. Conclusion, end, مُشَّا

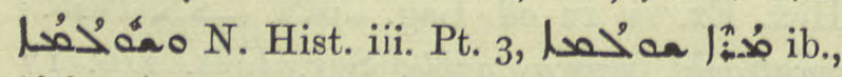

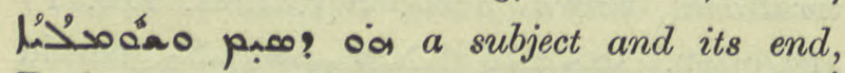
Probus 85.9 e.g. the art of Medicine a $>$ ? פor oa has for its subject

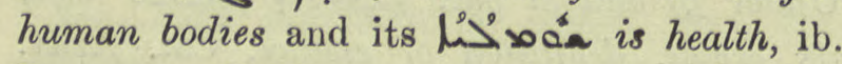
11. II, 12. Fulfilment, everything has a مخد ? special characteristic ōa aț sat. Sap. Eth. i. 4

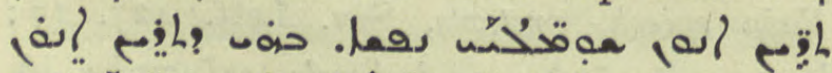

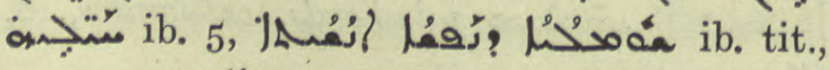
ib. 6 ; opp. Jho: luck, ib. 4 tit.

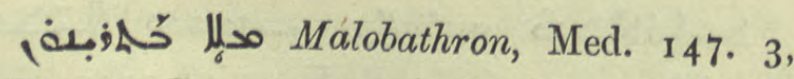

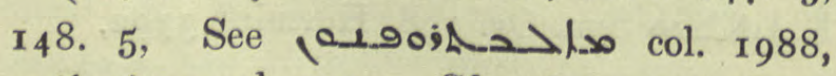

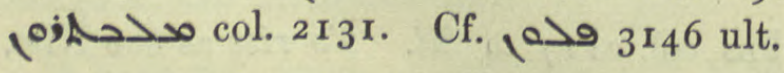

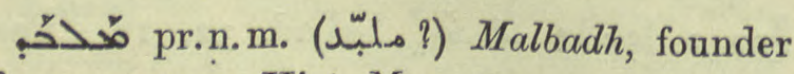
of a monastery, Hist. Mon. 373. 19, 389. 4.

هذح: vowels uncertain. Perh, a corruption of موبد mobed; see Hos. A Persian official

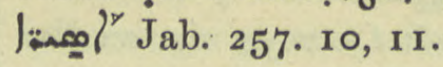

مكخ

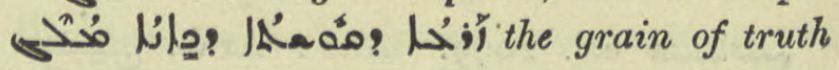
which the just man plucks out, Ebed J. Card. I 7 ult.

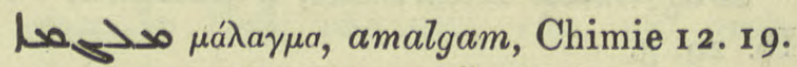
An emollient plaster, i. q. pă مَّ Med. 586. I 1 .

Jo $>$ o Mallus, a bpric. in Caria, De G. Bündel 65. 20. Cf. lö محص col. 2132. But there Mallus is said to be in Cilicia.

ho o col. 2132. 5 af. Delete par. it is done col. 2145 .

مه מحגa

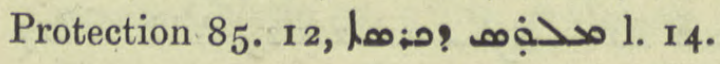

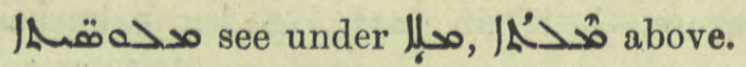

Linso col. 2134 infra, salt. Metaph.

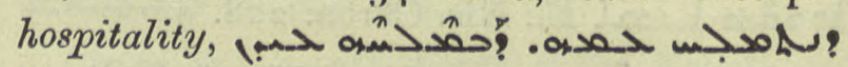
Waa shall we take his salt and let him become familiar with us by entertaining us? Ephr. ed. Lamy iii. $5^{\mathbf{I}} 3$. I.

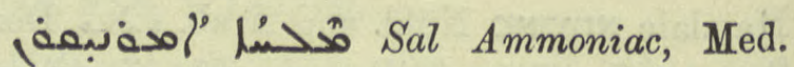
87. $4,91.5$.

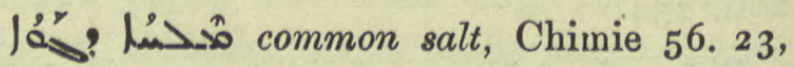
Med. 84. 1, 89. 20, 137. 10 \&c.

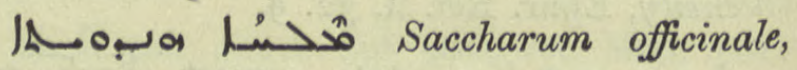
sugar-cane.

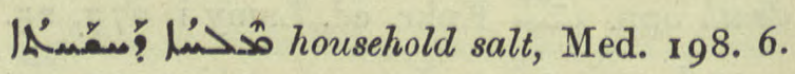

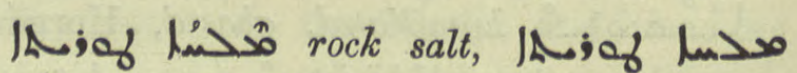
Chimie I4. 9 trad. 27 n.

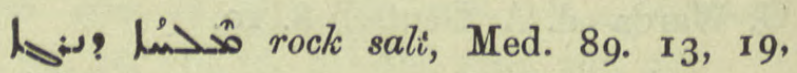
143. 13 .

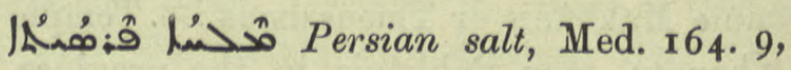
169. 2 .

14 Cappadocian salt viz. salt obtained by evaporation from salt-pits, Chimie des Anciens 266, Med. 165. 5. But

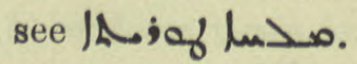

مَاك for. a salt-wort, the dove is said to

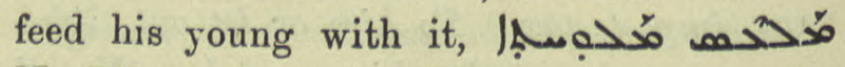
N. Hist. vii. 4. 4. Cf, Quatremère under

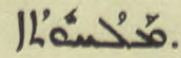


مَحُْشْ vii. 2. 2 .

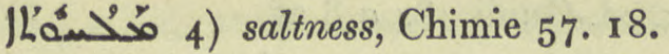

敌 adj. xii. 158.5 .

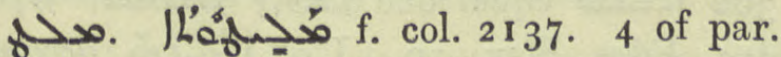

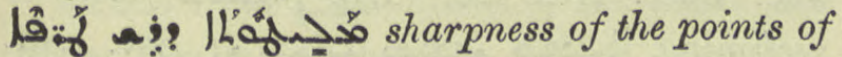
palm leaves, Pallad. 666. 20.

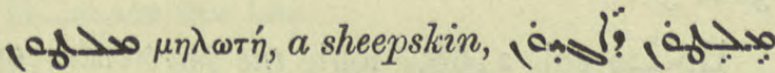
A.M.B. iv. $3^{1} 3$ ult. Hoffm. Z.A. xi. 237.5 , Fraenk. ib. xxii. 88.

of col. 2 ×38.8. Delete and see to sharpen; it is pass. part.

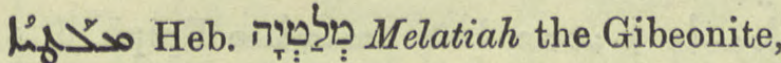
Neh. iii. 7 .

مخدم: Meletius, bp. of Larissa, Syn. ii. Eph. 10. 8.

of Meletius, bp. of Lycopolis, A.D. 306, مبحتبتُ

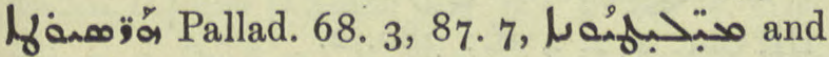

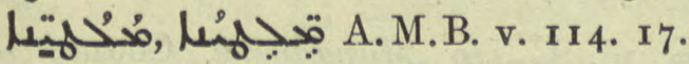

مَّ Mahlon, Ruth i. 2, iv. 10 , بحلمف BH. in loc.

مالك بـ Malik ibn Tawk, Dion. 94. I I, Nöld. in loc.

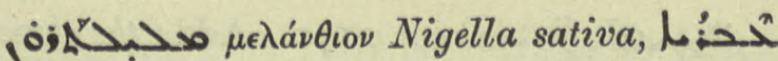

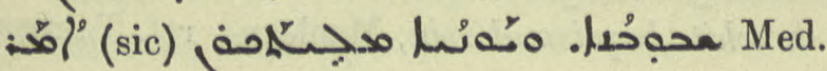
606. 10.

م همer Melian earth, which is white and

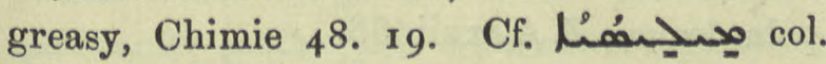
2091.

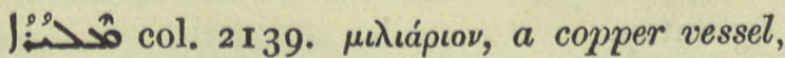


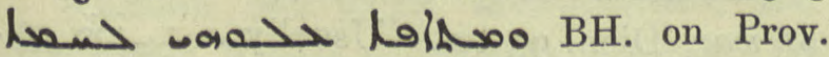
26. 21 .

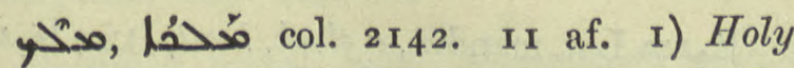
leaven used in making Eucharistic bread: the dough for this is doubly leavened, with J محمن kept from the last baking, and with this holy leaven, handed down from age to age and renewed yearly. 2) the priest's Eucharistic loaf; Catholicos 247 ff., Maclean's Daily Offices, Glossary; Brightman's Liturgies under
Malca, Hemira and leaven. Refs. Takhsa

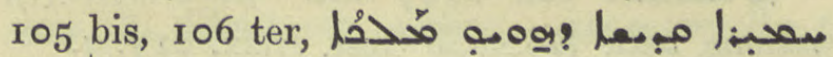
ib. II 4 . I, II 8 ult.

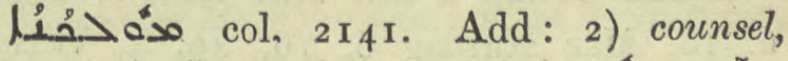

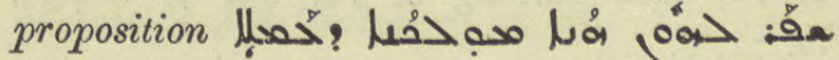

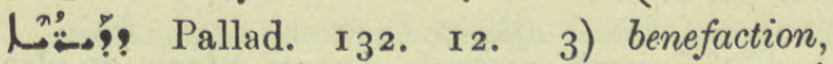
possession

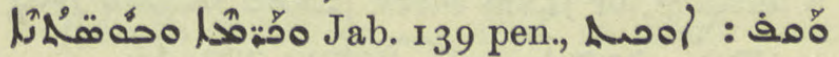

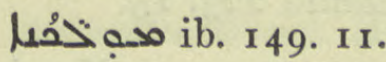

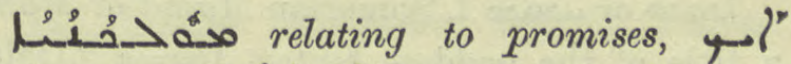
.

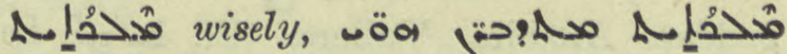
Sev. Ant. Vit. 2 I 9. 10.

the priest's portion, Takhsa 105 med., 106. 2 ; مُبْ IL'se places the priest's loaf on the east side of the oven, ll. 5 and 3 af.

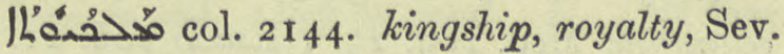
Ant. Hymns I I 3 .

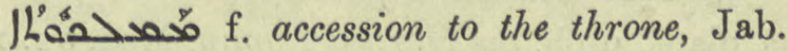
45. 6 .

مَّحمُ إذمف pr.n.m. an enemy of Kubla. Khan, Jab. I9. I.

Mn a name of Moses before he was circumcised, Lexx. under مأم col. 2054.

podathe of the mother of Nestorius, Or. Xt. i. 278.4 .

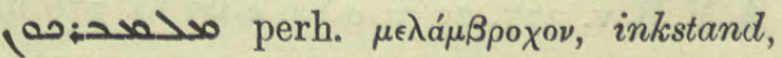

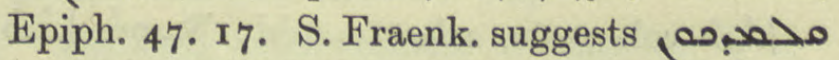

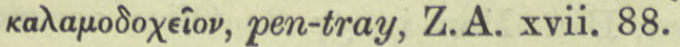

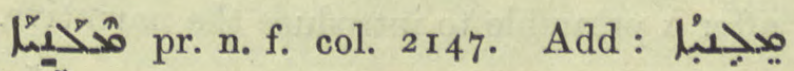
IS. Melania the Elder, Pallad, 192 ff., مجبنبا וحمillania the Younger, ib. 198 pen.

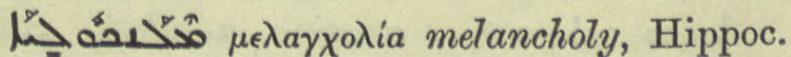

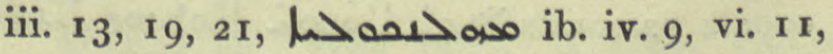
25,53 , vii. 37 .

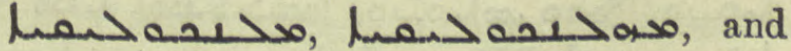

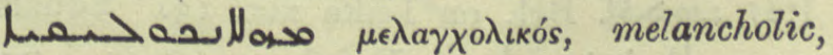
Hippoc. iv. 9, vi. 23,53 , vii. 37 .

col. 2147.8 af. Delete; it was treated under as col. 1961 . 


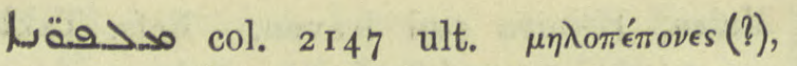

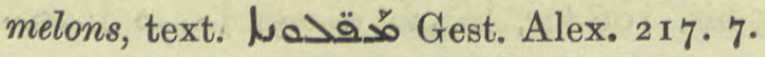

هم Melki, a hill near Arbela, Chast. 6. זо, Mar Kardag 6. 16, 49. 4, village, ib. 7 r. 8.

قs perh. metaph. barren places or else a mistake for $\left.\right|^{n}$ caves,

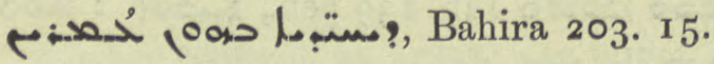

مa for Sumerian Mama or Mami, a title of the goddess Ishtar, Jensen ZDMG. xliii. 198. Pers. Lolo, wo title of the

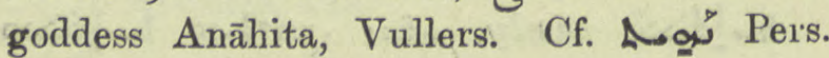
Mart. 74 n. 678 . Z.A. xxi. 255 .

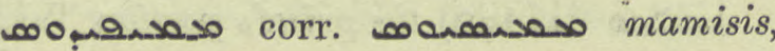
a sanctuary built at the side of a large temple, Sev. Ant. Vit. 29. 9, R. O.C. iv. 546 n. 4.

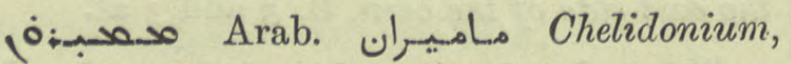

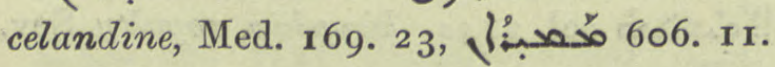

1 Lำ Glaucium phoeniceum, the juice of a plant, prob. the Horned Poppy, Med. 6 I. I3, 8I. I2, 84. I, 7, 85. I.

商 Gest. Alex. 76. 6.

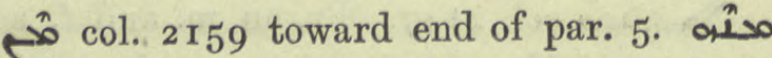
ه by Ephr. Syr., Nöld. Gram. §249; aùrópatos

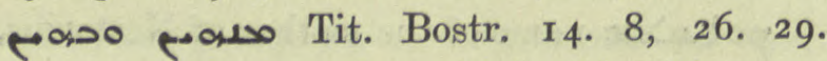

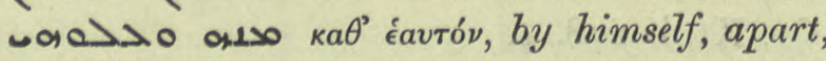
A.M.B. v. $377.20=a>0$ also ib. 376 ult.

مُنُ what then, Hist. Mon. i. r57. 8. Often occurs after a preamble to introduce the narrative.

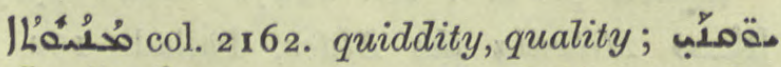

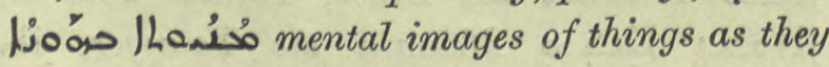
are $=$ concepts, ideas, But. Sap. Isag. i. I.

مس山 col. 2164. a mina, a talent. Add:

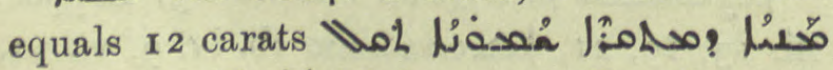
201 Med. 447.6. Equals I2 oz.

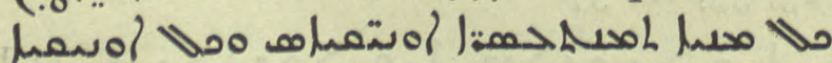

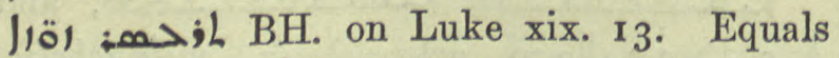
20 oz. IA مس

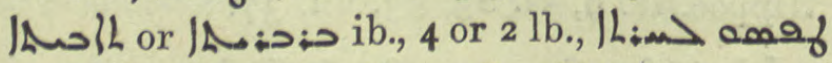
IAwilo ib. Equals 2 lb. Chimie 69. Ix. For deriv. see P. Haupt Z. A. ii. 265 n. .
นُนُนُ

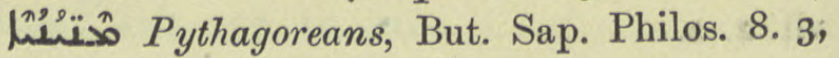
Philox. ed. Guidi $484 a$ af.

مــ col. 2 r68. 3) a degree, add: 700 stades equal one / مانُ



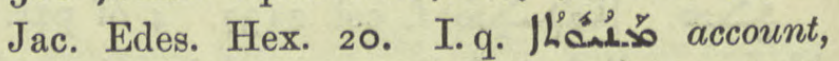
reckoning, Lett. 386. 3 .

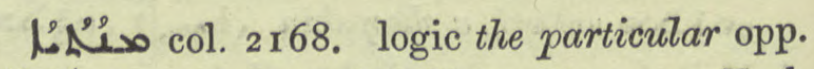
นُนْْ the universal, N. Hist. viii. 4. 2. F.pl. مهنتمُ

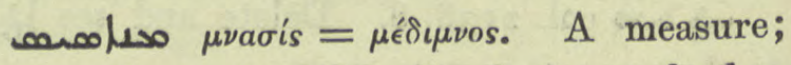
34 equal ro bushels of wheat or barley, مئم: Epiph. 6. 5 .

L1 Dame of an angel, Protection 79. 3.

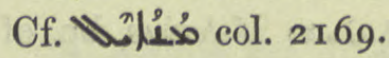

ohlew pr.n.m. Manethon, Sev. Ant. Vit. 62. 4 .

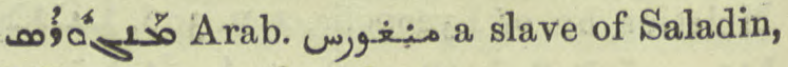
BH Chr. 272.4 af.; $3^{80 .} 2$.

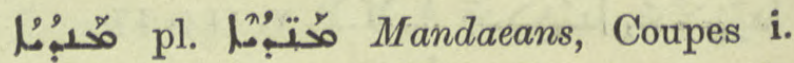
6, I 2 n. I, ii. I54. 16, Mich. Syr. ix. cap. 6 ; A.M.B. ii. I 50 ult.

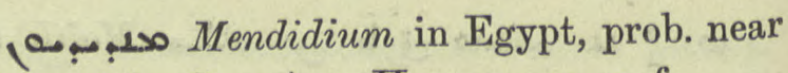
Alexandria, Sev. Ant. Hymns I 32. n. f.

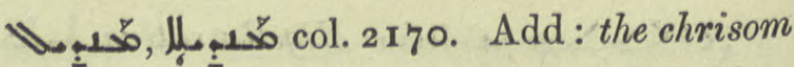
or white baptismal robe, Maclean; ref. Takhsa 7 x. 15 .

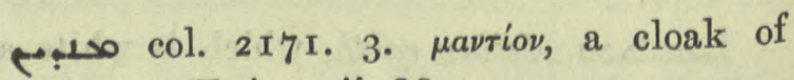
palm leaves, Z. A. xvii. 88.

محبر a bpric. in Asia Minor, De Goeje B. 65 antep.

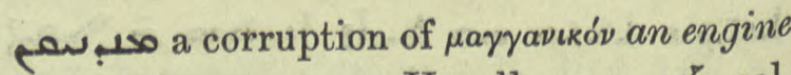
of war, Dion. 95 ter. Usually or col. 2 I7o.

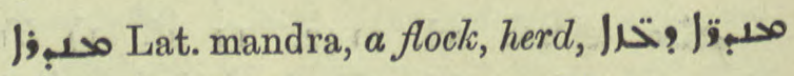
Dion. $1 \times 6.13$.

مدب:زمص the river Mcander, De Goeje B. 65. 13.

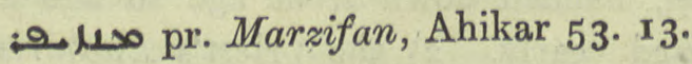

Afwo col. 217 2. coin, money, Anecd. Syr.

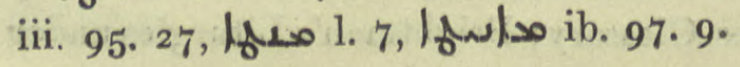


منتصصر pl. m. Manichaei, Manichaeans, Dion. Ined. $478.1,4$.

هـيمُل.

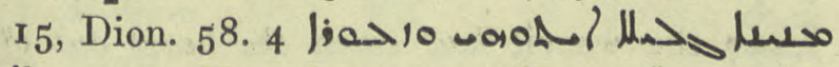
ib. 59. 5. Metaph. contemptible

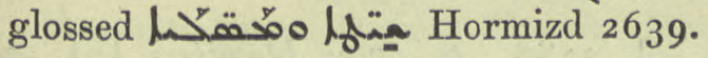

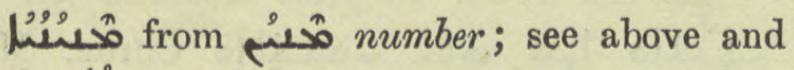
under مـ to reckon.

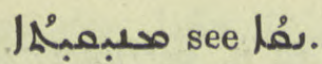

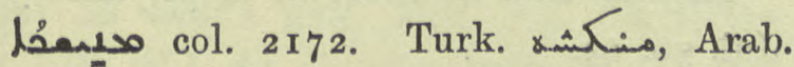

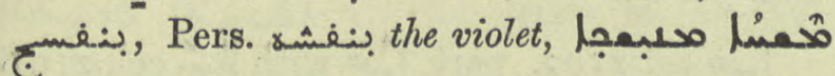
M̆ed. 39. I7, 54. 23, 57. 2, 58. 10. Cf. ZDMG. xlvi. 244 .

own pr.n.m. Manasses, N. Hist. iii. i. sect. 2 , Sist Jo. Tell. 73. ro, 77. 8.

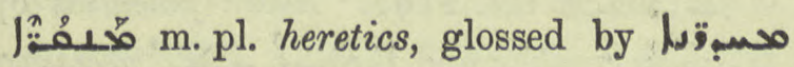
A.M.B. ii. I 50 ult.

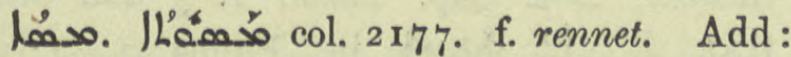
N. Hist.

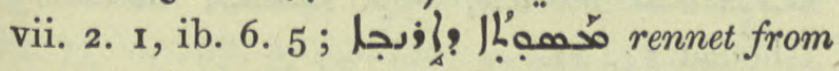
a hare, Med. 57. 6, 105. 23, 567. 16, 569. 22, from a sheep, ib., from a bear, ib. 590. 23 .

KLos col. 2177. 7 af. Delete the third

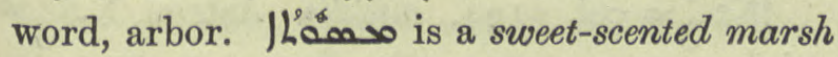
sedge, Diosc. i. I 3 , I 7 .

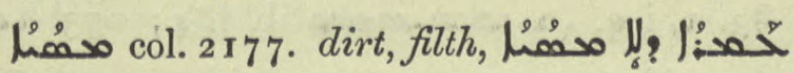
clean wool, Med. 559. 4.

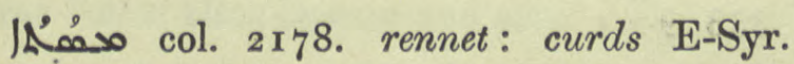
IŔn Med. 575. I4. Paste IAnsoo JLL

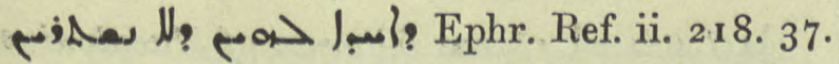

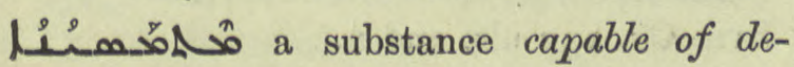
composition, Chimie 48. 14.

I'ómol col. 2178 . decomposition, add:

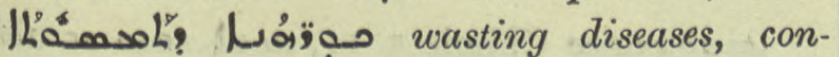
sumption, Med. 26r. Ir.

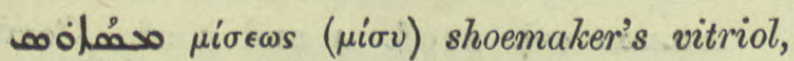

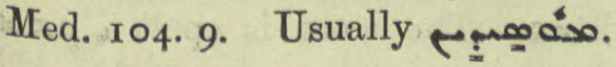

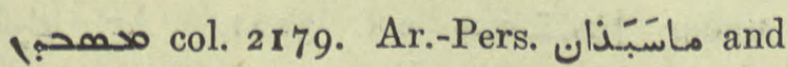

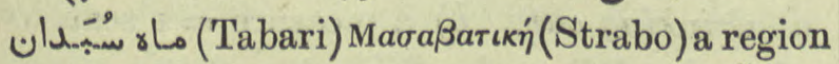
the capital of which was Sirwan now Sahri-Kailûn, Eranšahr 20, Syn. Or. 677: ib. I08, 29 , 1 1 0.25 so correct for see transl. $3^{68}$ n. 6 ; ZDMG. xliii. 403. 2 , 404. 8, Chast. 69 antep. ib. $46 . \mathrm{I}$.

ا مaمحلa Masablaha, one of the Arab buyers of Joseph, Jos. Weinberg 22. I6.

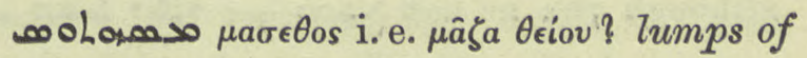
sulphur, Chimie 55. 8.

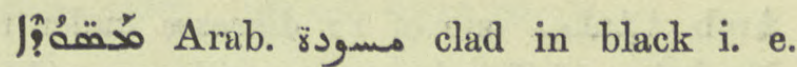
Abbasides, Chron. Min. 349. ro, Dion. 50. 3 .

مusonius, Bp. of Therme,

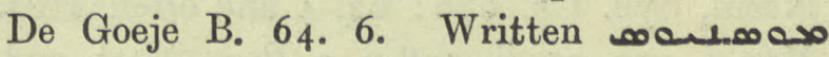
col. 2043 .

محسقمه col. 2095 a wild duck, N. Hist. vii. $x .7$ infra.

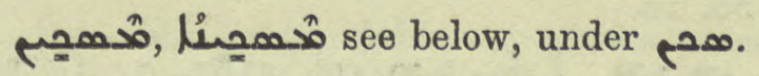

مكחת Dar. Masis, Gest. Alex. trans. 168 ult. $=$ Z.A. vi. $3^{6} 5$ l. 112 .

ل 2175 a goad. L. 4 of par. delete trutina, Act. Apost. Apoc. مح 7.

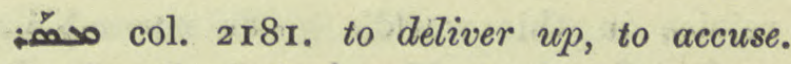

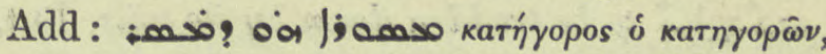

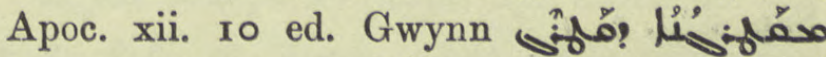

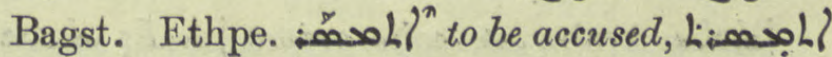

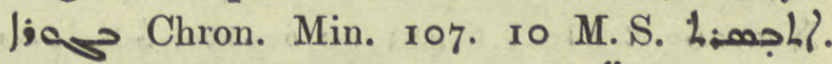
to be despised, I5. 19.

حل متبر| scomer, scoffer, ood S. Dan. 65 o 620 .

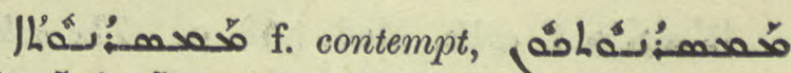

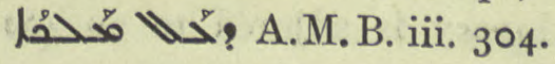

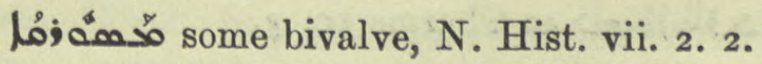

م 00 marg. Marookis, an Arab king under whom the Homerites suffered, Sev. Ant. Hymns 6r 3.2.

أُ

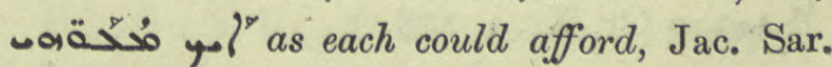
Hom. iii. 797. 5 ; A.M.B. vii. 808. 8, 10.

مهك col. 2185 infra. a place, region مححر محهe the Seven Limbos of Manichaeans,

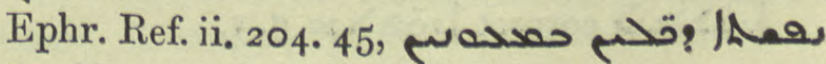
ib. $164 \cdot 36$.

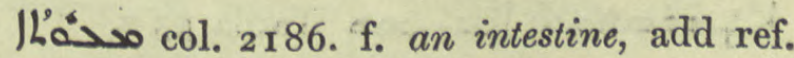
Chimie 54. 25, محس مس a sheep's gut, ib. $55 \cdot 3$. 
مدّد to pity, col. 2 186. Prob, a mistake

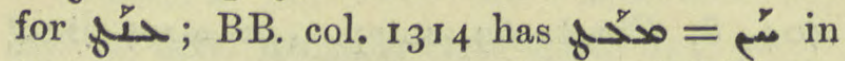
its second sense to be rancid, but DBB. 764 in the same passage has Duval BB. 1 447. I also has

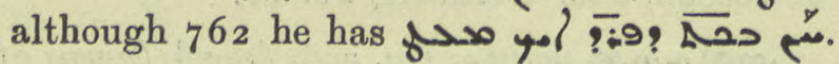

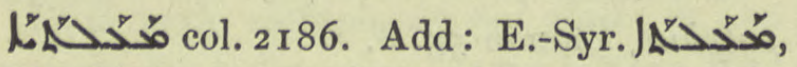
Arab. Ses one of 12 dioceses under the Maphrian of Tekrit, now a village an hour W. of Dehok and about 60 kilometers N. of Mosul, Syn. Or. 676: 62. 4 and often, Hist. Mon. i. 107. 2 I, ii. $23^{8}$ n. 3, Pers. Mart. 208, Layard's Nineveh $299 \mathrm{f}$.

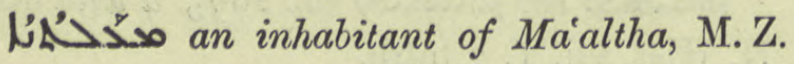
200. 10.

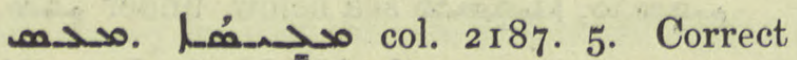

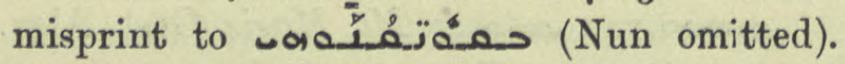

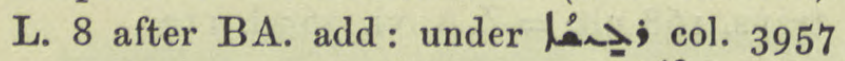

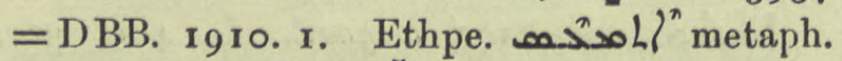

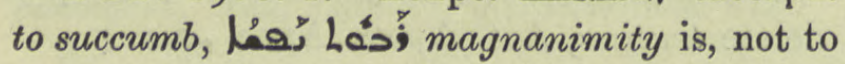
rejoice in this world's goods nor

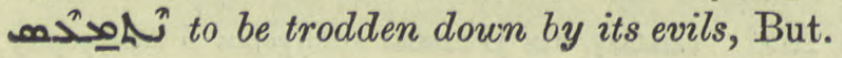
Sap. Eth. 2. 2.

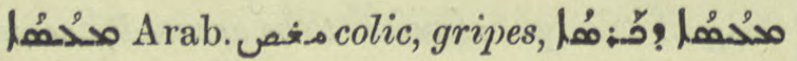
Med. 263 . 8, 4 I O. I , 6, 4 I I . 20, or pópos, à Joon

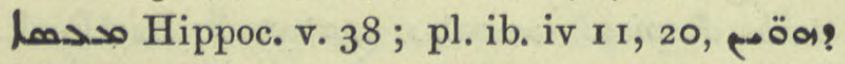

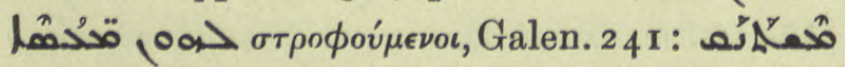

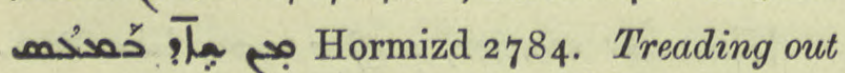
corn add: BA. and K. under مُحْئ col. 3622.

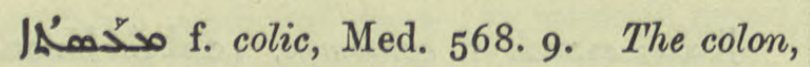
the spirit of lunacy is adjured to go

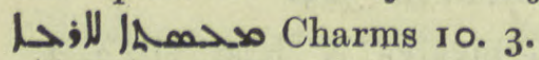

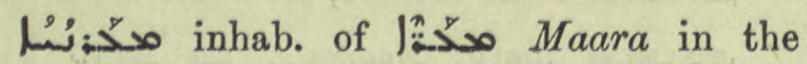

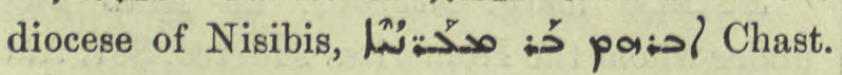
56. 14 .

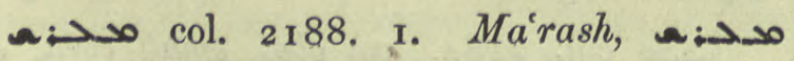
OحمدصA Or. Xt. i. 278.3.

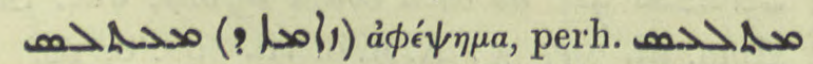
eatable; a decoction thick enough to be eaten, Galen. 240.

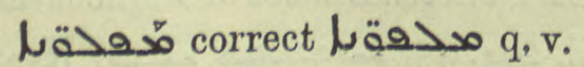

مَّم Mopsus, a demon worshipped

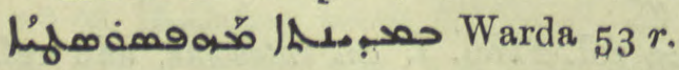

$5^{50}$ col. 2 188 to suck. Act. part. fem. حلمA| BH. on Prov. xxx. I5.
Ethpe. $35^{n}-0 h r^{n}$ to drain, empty, $\int_{35}{ }^{2}$

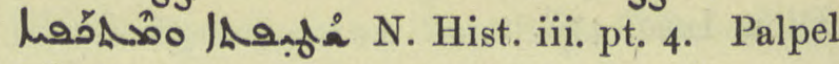

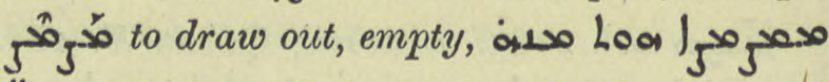
!n the weevil had eaten the heart out of the ear of corn, Dion. 59. 2.

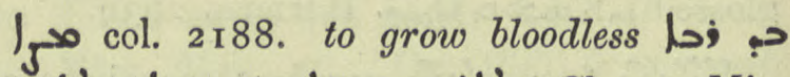
reblilo /5 ool wasilito Chron. Min. 217.20.

with no heart in it, perfunctorily, Jul. 36. I 3 .

آ col. 2 I 9 I.

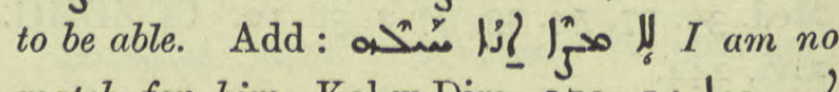
match for him, Kal-w-Dim. I79. I ; الم "م. to the best of our power, Jos. Styl. 43. 17.

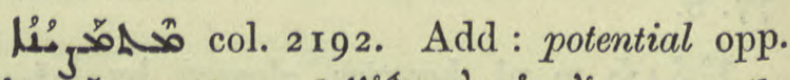

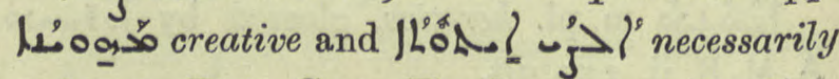
existent, But. Sap. Theol. I. 3 and often,

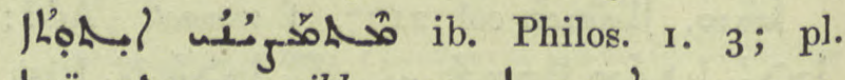

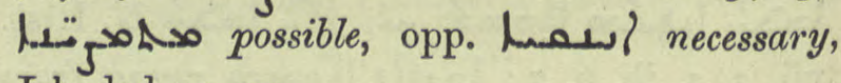
Ishodad I4. 2 I.

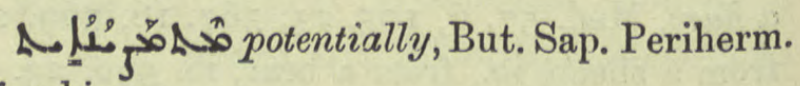
iii 2 bis.

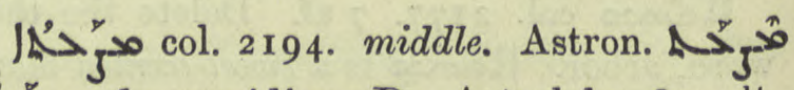

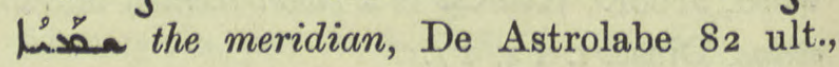

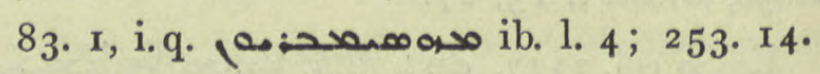

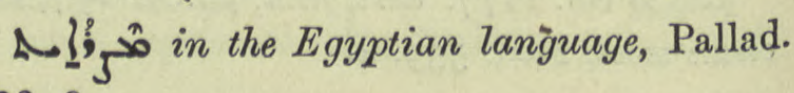
333. 9 .

مدما pr.n.f. Maka, Anecd. Syr. iii. 246. 17 .

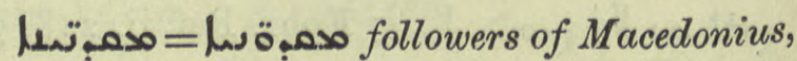
Syn. Or. 132 ult. and $n$.

ja col. 2198. 4. Correct misprint to م 0 q.v.

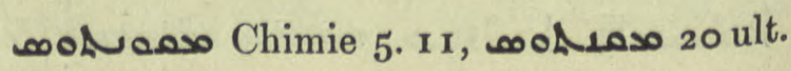

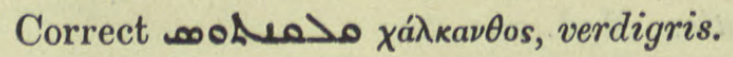

مصمع col. 2 198. 4 and 5 af. Correct

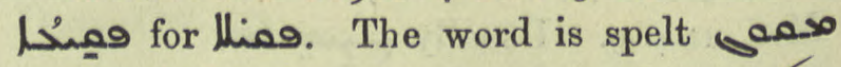

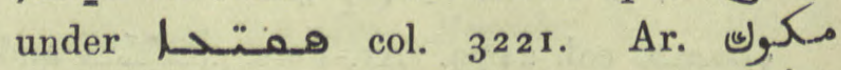
a) drinking cup. b) a measure $=\mathrm{I}$ seah i.e. $\Upsilon \frac{1}{2}$ pecks, BB. under /

L os the recipient or receiver of a still, Chimie 36. 9. 


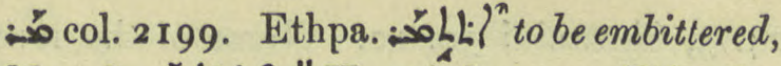

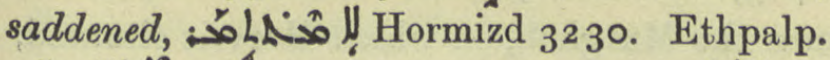
متحا / غ̇ं ed. Bagst.

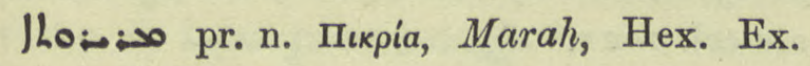
xv. 23 ed. Lag.

JL's: col. 2203. I) colocynth, bitter

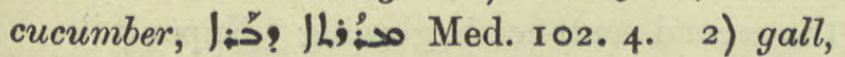

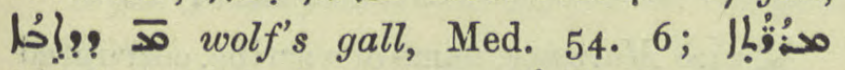

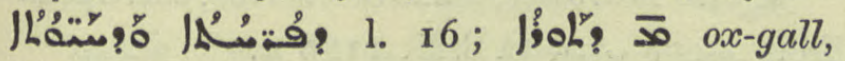
ib. 86. $21,87,88$.

1Li-so add: $\chi \omega \lambda \eta \dot{\eta}$, the bile, Hippoc. iv. $22 \mathrm{ff}$.,

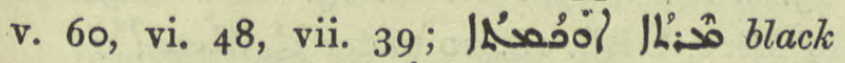

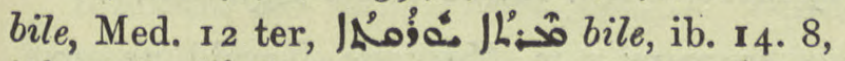

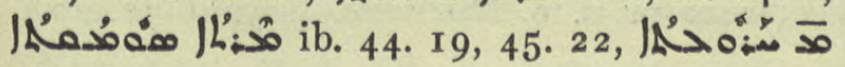
ib. $3^{82}$. 1. Venom of a scorpion, Med. 25. 18. 22 ; metaph. gall or venom of Nestorius, Jo. Eph. 122. 17.

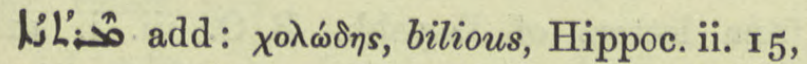
iv. 28,47 , vii. 29,56 , Med. 7.15, pl. ib. 15. 2, 291. 9,

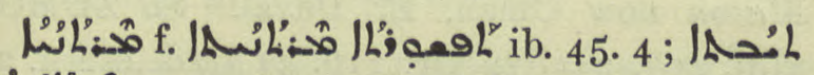
IRُ

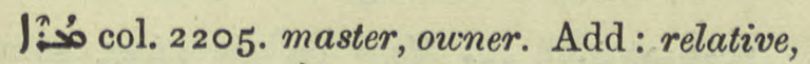

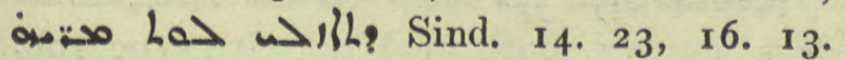
Cf. ZDMG. xxxv. 234 .

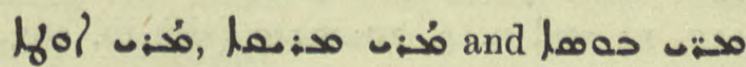
see below after of of and after 8 o

Phrases compounded with I: ó add :-

Iتِ i. 13, 16, Hormizd 227.

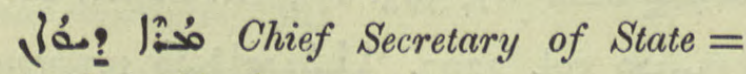
(ְْ Jab. 40. I.

مخبحه reason set over dumb creatures, Nars. ed. Ming. ii. ${ }_{2}^{3} 8.13$.

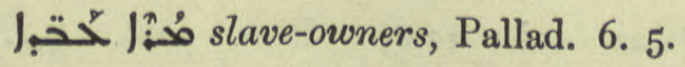

خالِ

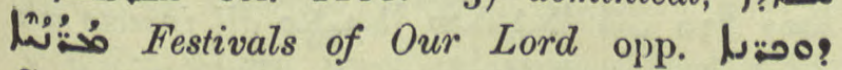
Saints' days, Takhsa 78 . I7.

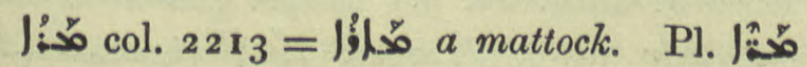
Ephr. ed. Lamy iv. 231. r9. L. 5 of par.

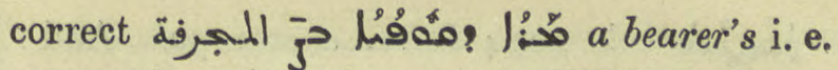
a gravedigger's pick or spade, D BB. 194. 5 .

|م: f. pl.iron picks or shovels (R. Duval)

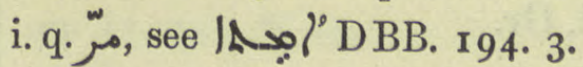

لم Maragha on the E. side of Lake Urmi, Jab. 43. I3, BHChr. Eccl. ii. 53 I var. as $1: 0$. See col. 2213 par. 8.

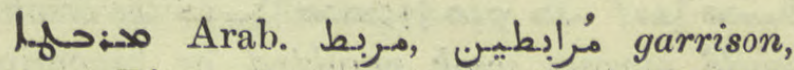
outpost, Dion. I65. I 7, 235. 9, Nöld. WZKM. x. 165 .

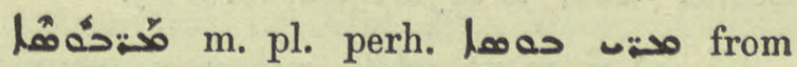
Arab. بوس evil, Hist. Mon. i. clxv. n. I, Hormizd 2445 .

S- col. 2213 . Add: to glance aside,

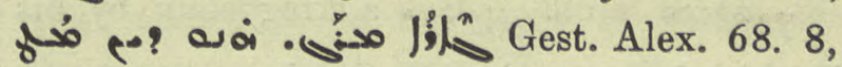
$\mathrm{BH}$. Moberg 60.

Lُ views as to derivation see Fremdw. 129, Hübschmann ZDMG. xlvi. 244. 75 .

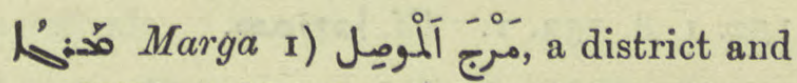
town NE. of Mosul. Col. 22I4. 4 of par. delete "Forte sit" and add: Pers. Mart. 222 sqq., Hist. Mon. ii. 43 n. 2 and often, bpric. ib. and Syn. Or. 608 n. 3, 619. 6, Chast. $5^{8}$ pen. 2) a monastery at the foot of Mt. Zinai in Adiabene, Chast. 60 antep., 65. 2 1. I. q. M o vib. 6r. 3, Hist.

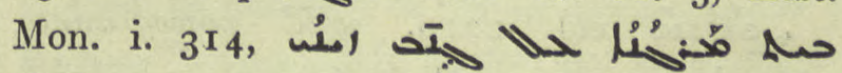

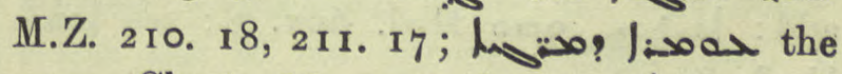

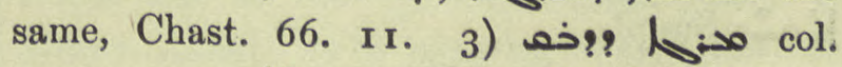
22 I 4, near Aleppo, add: Brook's Chron. 574 pen. 4) مرج الشها Fertile Meadow, El. Nis. Chron. I38. 12, I 59. 25 .

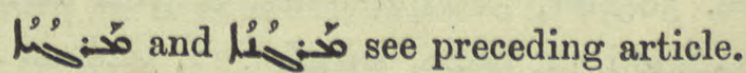

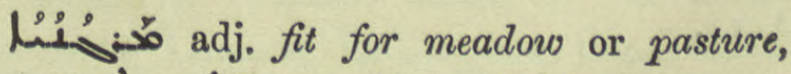
beneath snow mountains are moist meadow- or pasturelands, N. Hist. v. I. 2.

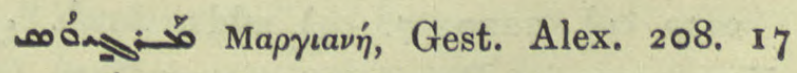
i. q. ثن the city of Merv, l. I 8.

م: مw suppl. 


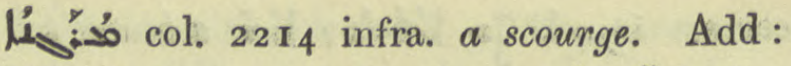

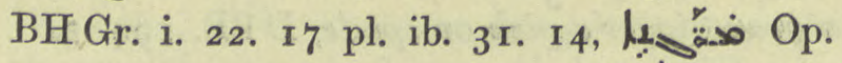
Nest. 98. 14. Nöld. on deriv. WZZKM. viii. $3^{6} 5$ and Mand. Gr.

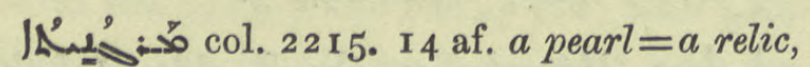
add: A.M.B. v. 613.3 af., 614.8 , 10.

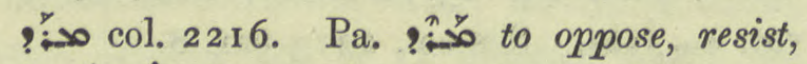

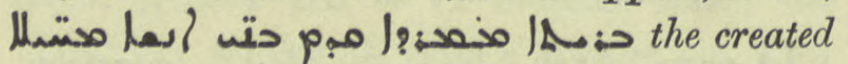
world opposes weak mankind by hindering seas and mountains, Ephr. Ref. i. 37. 12.

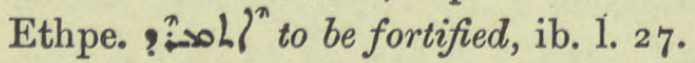

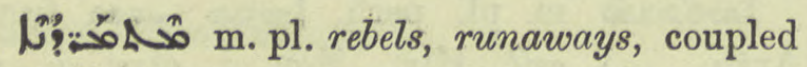
with /Lمته

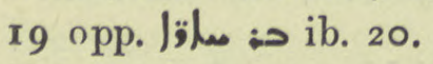

مد:وم: prob. مرداويج a king slain by his Turkish bodyguard, BH. Stories 23.106.

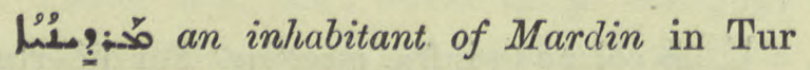
Abdin, Ant. Patr. 3०3, 3०4.

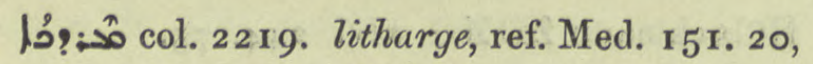

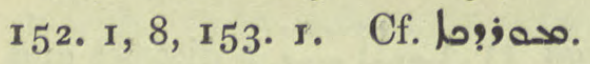

هas? م- مardinshah, a son of Chosroes II and Šrin, Sassanidi I 2. $8=$ Chron. Min. 19. 13 .

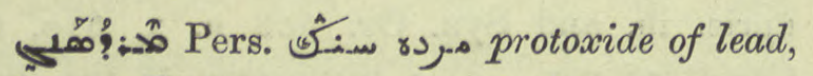
litharge, Med. 59. I3, 570 ult., 574. I3, 583. 16, 586. I2.

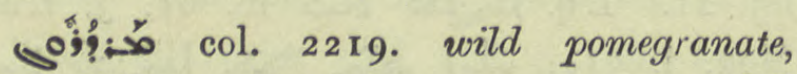

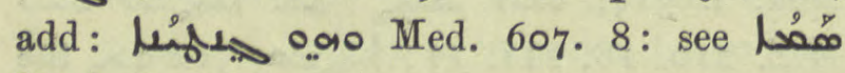

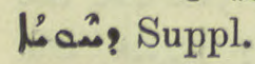

poa:- o perh. Ar. مَرِئ may it do you good, said before drinking, Ḿed. 587. I4.

pá: ڤ̌ Pers. pos plaster or salve for wounds,

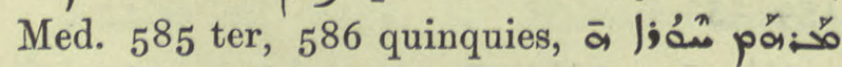

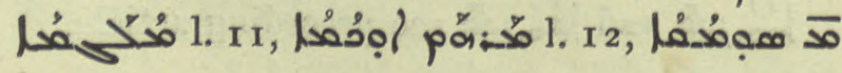
1. 14 .

axe;a:D Marehrahmeh, Bp. of Beit Dasen, Syn. Or. 62. 5, 68. I, ZDMG. xliii. 40I. II.

مـ Merv, now in Russian territory, Syn. Or. 677,299 n: $2 ; 43$. 18, 62. 5, ZDMG. xliii. 396. I 1, Hist. Mon. i. I98. 8, Chast. 22. 16,
24. 5, Sassanidi 30. ro. Name of the river, now the Murghab, on which Merv is situated, ib. 1.8 .

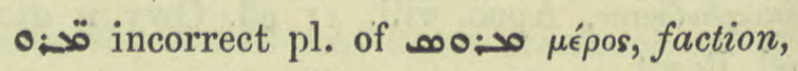
party, Anecd. Syr. iii. 272. 6, 8. For see col. $222 \mathrm{r}$.

J مَّ col. 2220 . Origanum Maru, wild marjoram. Refs. Med. 58. 6, 606. 5. Delete the second sentence beginning "Exponit".

$\mathrm{H}^{n} 0^{0}: \mathrm{S}^{\prime}$ Marauge, name of a scribe, corrupted from ed of C.B.M. II 78 a.

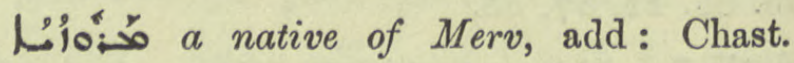
23. 9, 24. 3, Syn. Or. 66. 25. The language

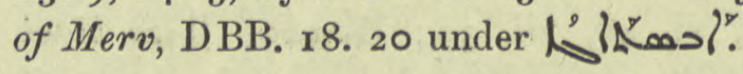

10:00 pr.n.m. Maruzan, Išoyahb 72. 20.

م: place-name: see polo.

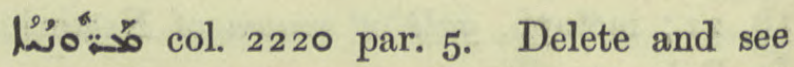
L/0:0 ib. par. II. In the passages quoted from letters of Išoyahb, B. O. iii. i. 127 $=$ Hist. Mon. ii. ${ }_{54}$ Syr. ult., B. O. iii. i.

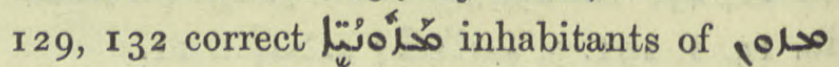
Mazon now Oman, R. Duval. So ZDMG. xliii. 407.2 =Syn. Or. 2 I6. 2 I trad. 482 n. 2 , Chabot, Sassanidi 26. I3.

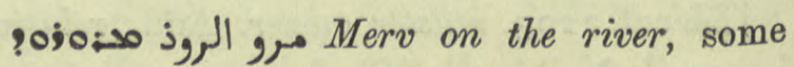
days journey south of Merv, Syn. Or. ro9: 23 and see trad. 366 n. $6=$ ZDMG. xliii. 412. I4 and n. 4, 90: 0, (sic) ib. 403. 12. Cf. AKGW. I901. 76, and Barthold Christ. in Mittel Asien $\mathbf{r} 6$.

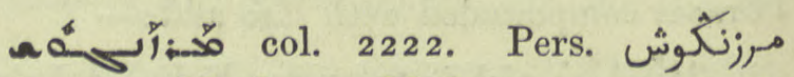
Origanum, Marjoram, Med. 57. 5, 59. 5, II, م 1. 7, 393. Ir ; Ar. PflnN. 41.

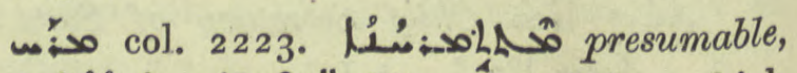

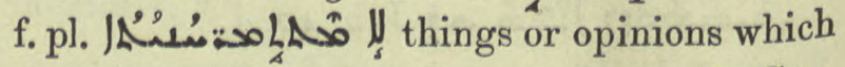
should not be assumed nor presumed, Sev. Lett. 480.8 .

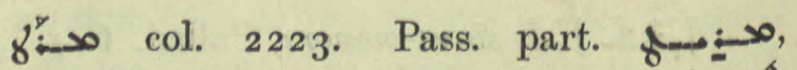

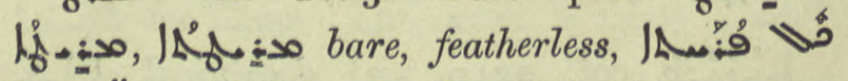

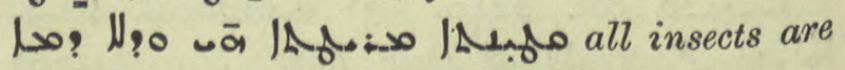
featherless and bloodless, N. Hist. vii. I. I, Ebed J. Card. 2 I. 9. Ethpe. finoL? to tear

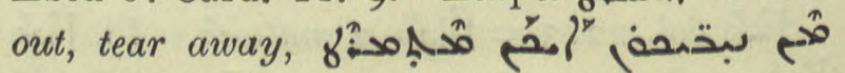
Hormizd 1896 . 


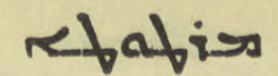

If of 0 col. 2224. a pallium worn by the Patriarch (obsolete), Maclean.

صof: $\infty$ Martus, name of a demon, JAOS.

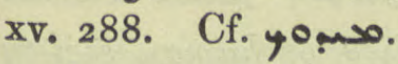

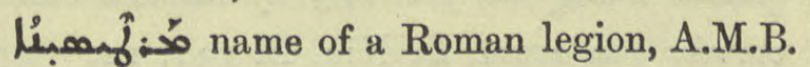
vi. 69. 6, / 1b. 80. 12.

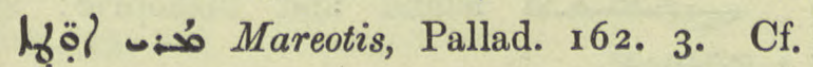
/fesis col. 2225 .

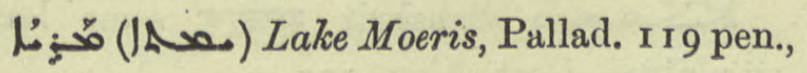
I34. 7 .

مريخ Pers. Planet Mars, Med. 470. 14,487 . 7 .

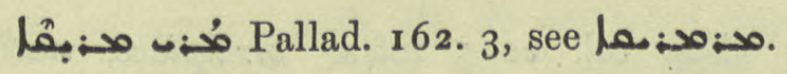

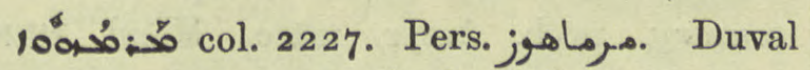
suggests مروخوش sweet herb. Origanum maru, Marjoram, Med. 88. 6, 142. 16, 162. I, 265. 14, Guigues on Serapion مرماحور vars. مرماحوز Journ. As. 1905, 57 ; Ar. PflnN. ${ }^{2} 5^{2}$.

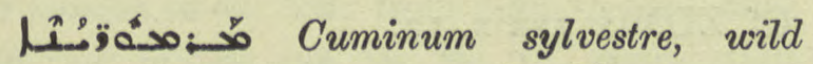

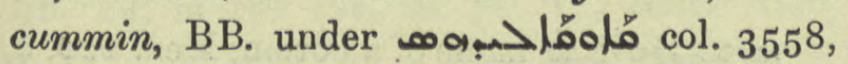
DBB. 689. 3, Ar. PflnN. 207.

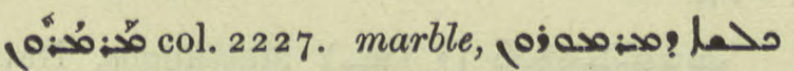
Chimie 13.9.

م:م:م:ممل Marmarica in Libya, A.M.B.

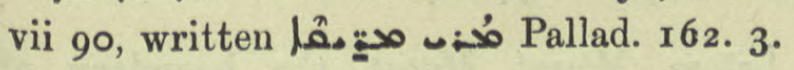

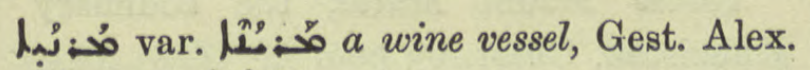

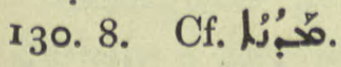

0 col. 2227 . Metaph. to repress, suppress thoughts, Anecd. Syr. iv. 51. 4, 18. 20. Ethpe. $\infty: \infty)^{n} ?^{n}$ to be plucked, torn

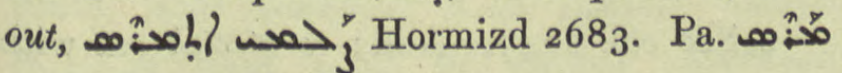
to tear down, ib. 2682.

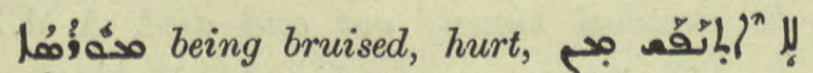
مهب Hormizd 2788.

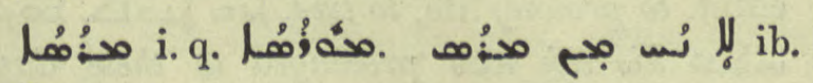
2785 .

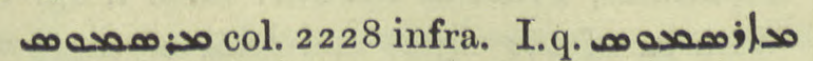

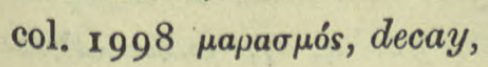

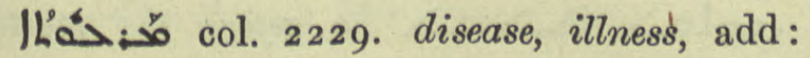

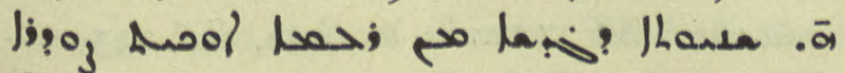
dizziness from a thunderstorm i.e. headache n. marg. to Cyr. 43 I. 9. 2716
مَّ the epigastrium, upper fore-part of the abdomen, A.M.B. iv. 155 .

مه:مُم col. 223r. potishing, add: Chimie 49. II, 25 I. 6, I5.

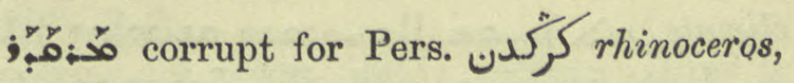
Gest. Alex. 2 I I ult.

corrupted from صَامُفْر . 16.

IA oforcassite, Chimie 3. 10, 4. 9.

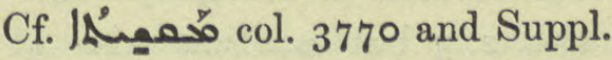

م- prob. i.q. Arab. Titharge, Chimie 2. 6 . Usually os: مه:

12́0 col. 2234. par. 5, 1. 3. Act. part. of

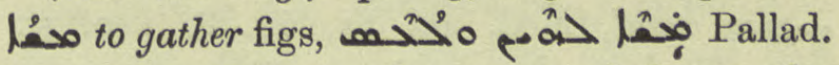
334. 20. This is the story of Jonan the Gardener to which BA. refers. Pa. collect with pains, to amass

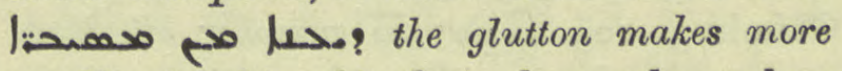
trouble for himself than those who endure hardships, Philox. 375-4. But the passage is difficult.

مَّمَ col. 2235. river Masius or Mygdonius, now the Jaghjagha, refs. Dion. 7 I. 8, A.M.B. i. $44^{2}$.

o col. 2235. I) to anoint. Add: to

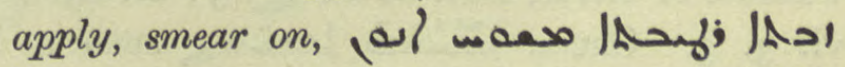
لـ Geop. 69. 8. 2) to measure. Add : to equal in measure, be commensurate, للإ 似 blame due to the offence, Philox. rog. I. Ethpe.

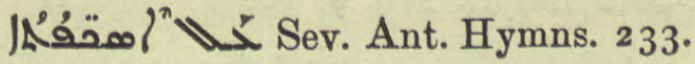

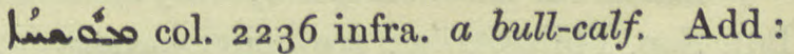

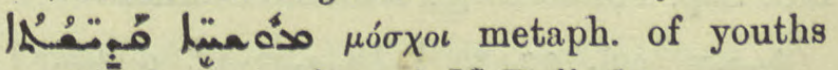
ready for martyrdom, A.M. B. ii. 85 .

مُ0. a measure, measurer, that which measures,

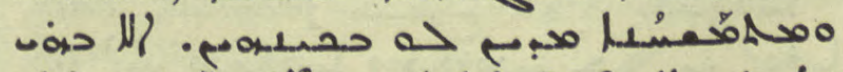

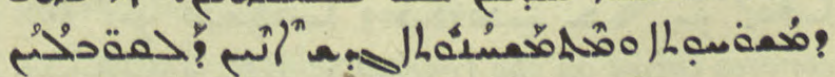
the singular and the plural are the measure and the measured, not however in their nature but in that measuring and being measured have happened to them in contrary fashion, But. Sap. Philos. 2. 5.

D d 


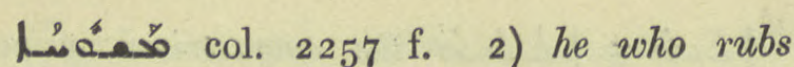
athletes with oil, metaph. Jab. ${ }_{4} 65$ ult., 492. 7 .

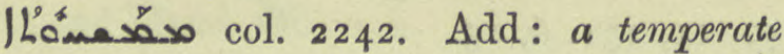

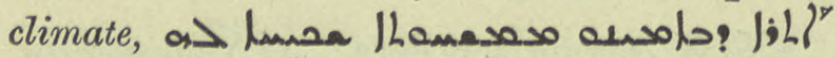

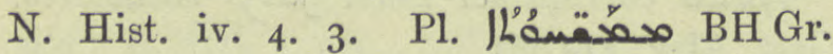

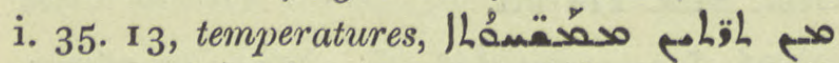

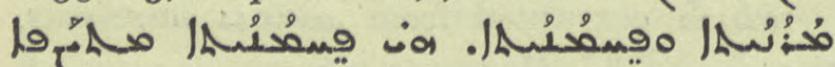
for healing the body more attention is to be paid to the relative than to the specific tem-

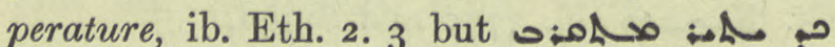
Ihemax sol ox when the temperature most nearly approaches the mean, ib. 1. 3 .

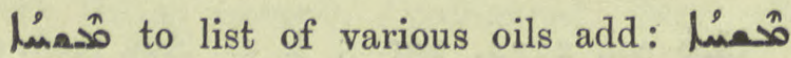
ب. nard, Med. 40. 14, 67. 8, 140. 5;

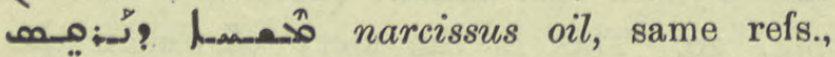

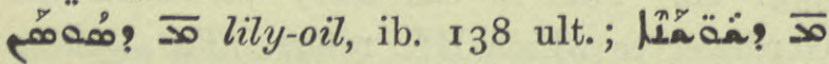

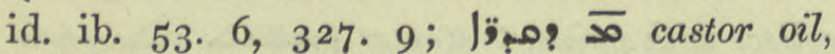

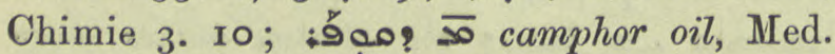

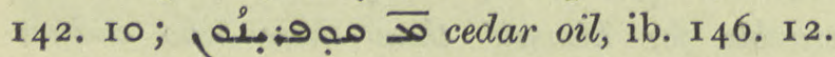

 Dion. 234. 18.

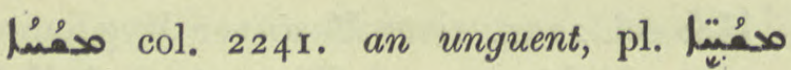
Med. 262. 18.

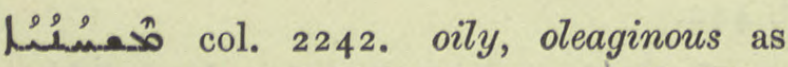
walnuts or olives, BH. de Pl. $\checkmark 3 ; \mathrm{N}$. Hist.

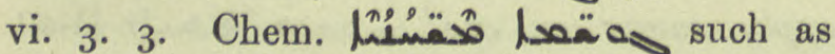
arsenic and sulphur, Z.A. xii. 158. 6. See Suppl. under مase.

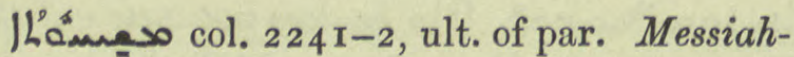
ship, Poet. Syr. 32. r. Belief in the Messiah

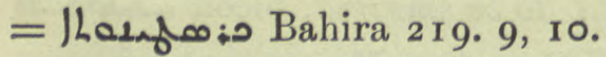

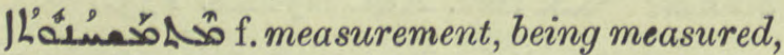
But. Sap. Philos. 2. 5 : see under مسع مa.

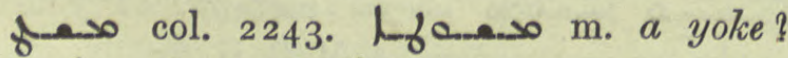

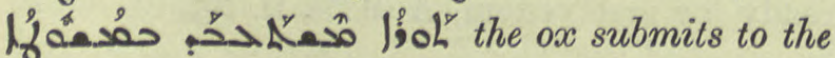
yoke, Pallad. 536. 2.

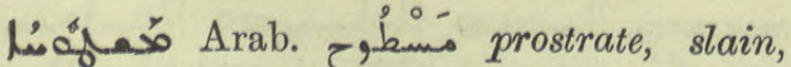
إم like corpses lying on the road-side, BH. Journ. As. 1878, 97. 822 .

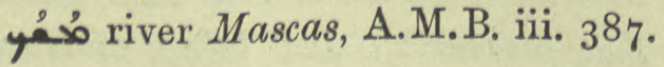

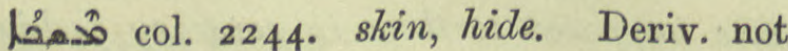
Indo-Germanic as Lag. G. A. 282, Arm. Stud.
I432 but Semitic: Assyr. mašku, Arab. مَFraenk. Z. A. iii. 54 .

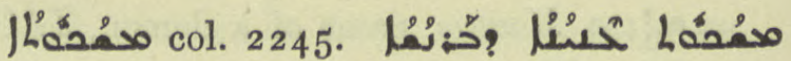
حעّ carries you away, Is. Nin. B. 13r. 12.

ک vasero.

مَّمـمـح Sanskrit मुष्कर some large amphibian, Gest. Alex. I75. 9.

ob. Armenian a 2 unt4 (?) i.q. lool hyssop, BA. under lool col. 1 I I 0 .

|مُ col. 2245 infra. birthplace, native country, prob. Assyr. mātu, Mand. מאתא town, Talm. also מאתא Z.A. xxi. 256 n. 3, Inscript. of Zenjirli, WZKM. vii. ${ }_{34} b$.

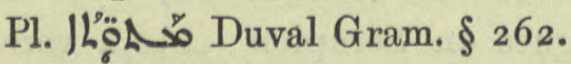

מתא מת מחסיא. Heb a Jewish village between Mahoze and Hirta, Sassanidi 27. 3 af. Cf. ZDMG. xxxix. I 2.

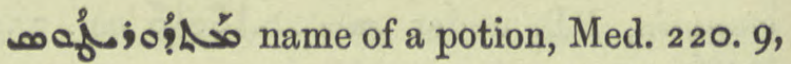
$244,20$.

مattūlīqōs, Natur.

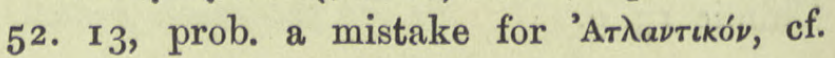
Anecd. Syr. iv. 97. 24.

Lod o Mount Matut, the boundary of Khuzistan, Chast. 63 pen.

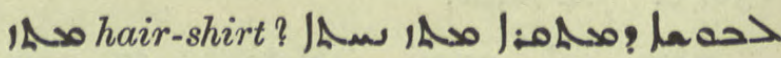
?مب مح: Sev. Ant. Hymns 20r, Chron. Min. 209. 12.

w o col. 2247. Add : to stretch out with

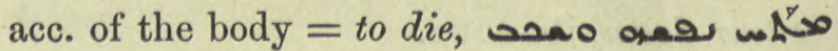
he stretched himself out and died, A.M.B.

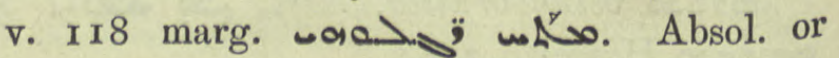

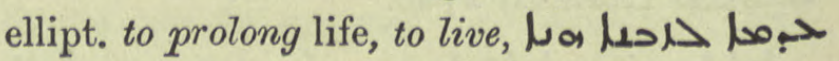
مسلمس his life was prolonged to these days, Schulthess, Probe = BHChr. Eccl. i. 85. 10. With $>$ of the object and Lé of the pers.

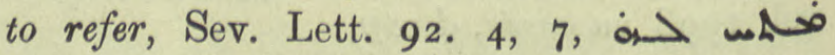
cale> IN $>$ ad he refers the matter, addresses his remarks to them, ib. 235. 10. With $\leftarrow_{3} \delta i \epsilon \epsilon \epsilon \iota \nu \epsilon$, he directed his steps, hastened,

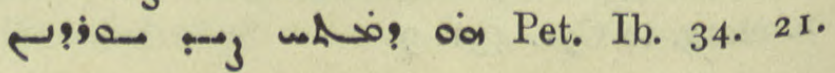




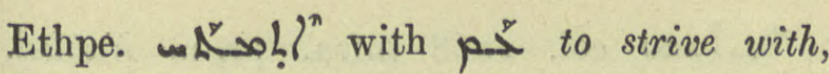
Ephr. ed. Lamy ii. 775. 21. Cf. Peal with $\mathrm{p}$ col. 2247.34.

مُهُ stretching, tense, headaches from wind are called مُتمَهمَ because there is a feeling of /Lanstension with them, Med. 33. 16.

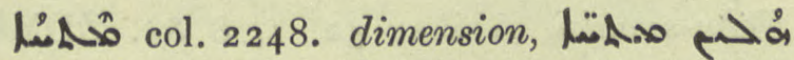

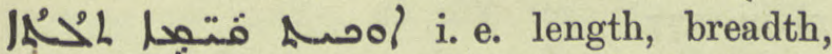

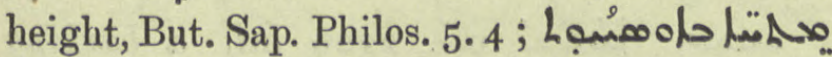

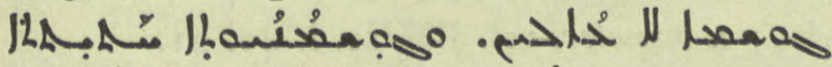
d dimensions have no concern with (lit. do not enter into) the essential body; and true corporeality is substance susceptible of dimension, ib. 4. 2. Tension, Med. 2 I. I 7.

A 1 مُ serried rank, Dion. I 5 . I3.

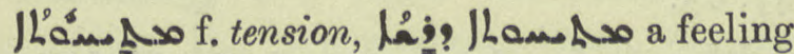
of tension in the head from a heavy cold, Med. 66. 14 , in the eye, ib. 78. I I, constriction,

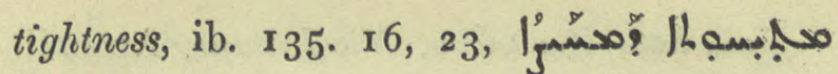
ib. I5 5 . 7 .

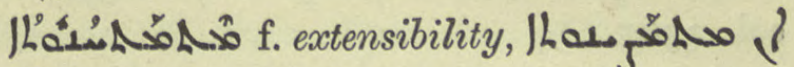

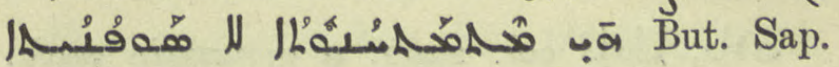
Philos. 5. 2.

póh o col. 225 I. Add: without

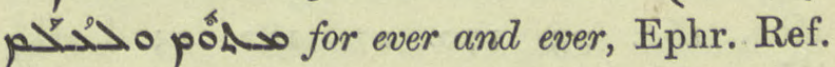

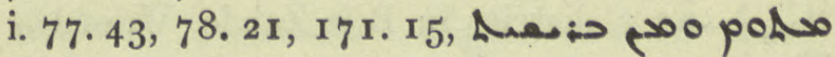
ib. 84. 4I.

مهلم col. 2252. third par. always pl. Entity, constituent parts, Mingana. L. 2 of

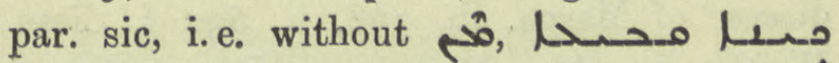

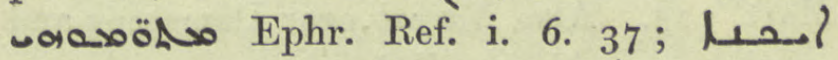

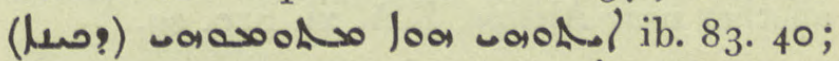

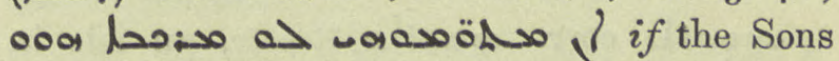
of Light from all eternity have not been

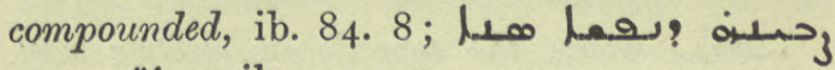

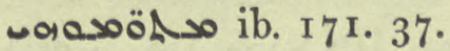

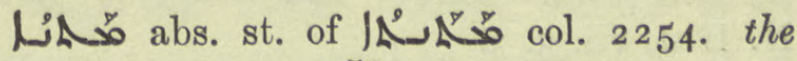

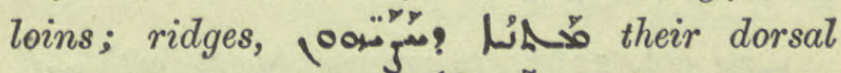

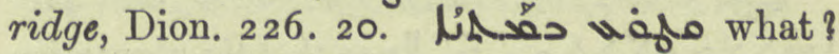
cut with a knife, Budge, Med. 584. 3 .
م pr.n.m. A.M.B. iv. 296. 3 . Perhaps a corruption of فذلمبد Photinus, cf. ib. pen., سحه l. I5.

" pints, IA Epiph. 4.

Nabarnugios, native name of Peter the Iberian, Diosc. 59. 6, R.O.C. iii. $25 x$ n., 368, Pléroph. 19 n. 2, תص:

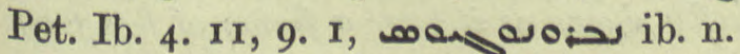

L'j col. 2259. Delete caminus \&c. it is

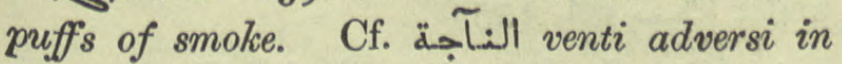
contrarium flantes, Freytag.

ol Neon, bp. of Selinus, Nöld. F. S. i. 47x. 75. Vars. ol. Ieon, Mich. Syr. 316 n. Leon text.
L.foj Noventianus, he opposed Eustathius, Basil of Ancyra \&c., at the Council of Rimini, Or. Xt. i. 90 . r 9.

i.مool Diocaesaria in Isauria, i.q. Prakana, Nöld. F. S. i. 47 r. 76. Neocaesarea, De Goeje B. 65 . 19 as Thes. col. 2259 infra and list in Mich. Syr. $3^{\mathrm{I} 6}$ No. 77.

pos for els Nain, Sev. Ant. Hymns 47.

S Nane voc. form of oost Nonnus col. 226. Inscript. Sém. No. I4, Nöld. ZA. xxi.

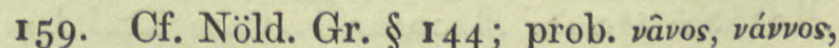
Heb. ננם , נעום.

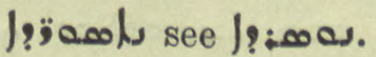

صaحfol Nestabus, bp. of Acre, Nöld. F. S. i. 468. 14, 0 , $3 \mathrm{r} 3 \mathrm{n.} \mathbf{5}$.

Log/ a crocodile, Nest. Chrest. 88. 47. D d 2 


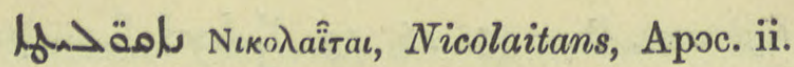
6. 15 ed. Gwynn يـ Bagst. and col. 2365 .

a col. 2262. a mountain peak; a glen or ravine. Often pr. $\mathrm{n}$. of monasteries and villages. . Mon. 163. 23, 170. 27, 182. 2 ; يل | Tigris, near Gezirat Ibn Omar, B.O. iii. i. 184, \&c. = Hist. Mon. 7. 14, 61. 16, 99. 18.

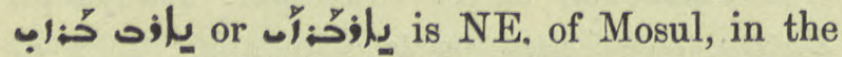
diocese of Marga, ib. 368 , ii. $63_{2}$ f., Pers. Mart. 225. Also Nairab near Damascus and another near Aleppo, Z. A. xi. 2 I0. sq.

مapkós, an isle in the Red Sea, Jac. Edes. Hex. xx. 7 .

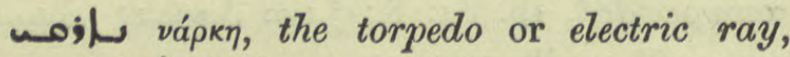

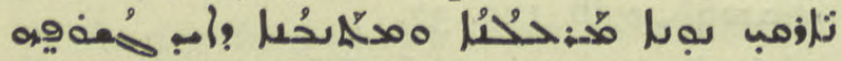
N. Hist. vii. $3 \cdot 3$.

טר col. 2265. to emerge, burst forth so that the plantation of the true vine should not flourish in the world, A.M.B. ii. 70. 9 ; Or. Xt. i. $34 \mathrm{I}$ n. Part. حلحصن ; sacramental power sprang up in their hearts, Hormizd I 290.

مُ correct بشكونغ a flower for بشقوقغ Fraenk. WZKM. $1889 \cdot 242$.

12. حمَ col. 2266 infra. Delete; it is ILئis emergence.

Aحph Ašly to set in motion. Part.

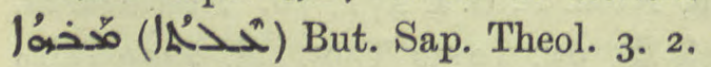

นُนُ 'ُ gram. active, of action. The long quotation from $\mathrm{K}$. is out of BHGr. chap. 9, p. 46. One limoj note is omitted: col. 2268.

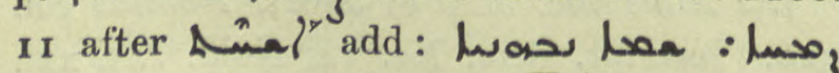

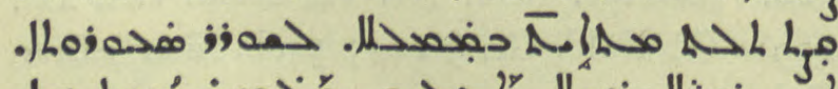

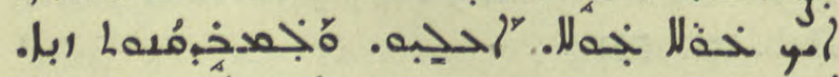

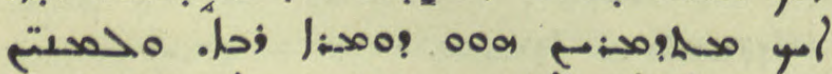

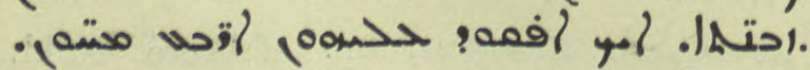

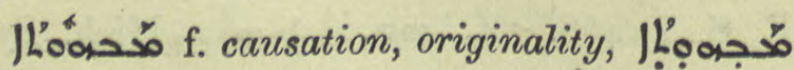
每 Theol. 3. 2, 4. 1 infra.
Whas col. 2268 infra. pr. n. Nabuel. Add : name of Nadan's warder, Ahikar 66. I5, ZDMG. xlviii. 177 .

נحم/:9r. n. Nebuzardan, Ahikar 49.3, 6.

مهب: Subian, Sev. Ant. Vit. 231. 9, but possibly محمصמم

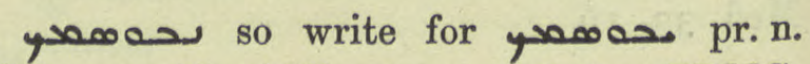
Nabu-semakh, Ahikar 52, r, 8 \&c. ZDMG. xlviii. 177 .

ـــ 2209 ult. a barking dog; a brawler, ref. BH Gr. i. 40. 2 I.

مaraf Neptune, col. 96 under ajol.

'حُحبم the ibis, Natur. 33. 10 bis.

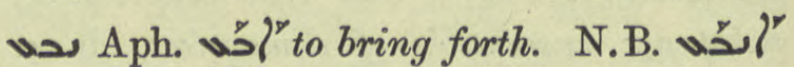
L Lod caused breasts to bud

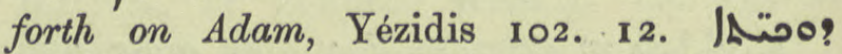

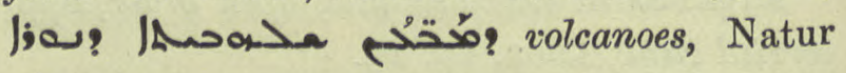
47. 15 .

| 2272 . spirtling, bubbling?

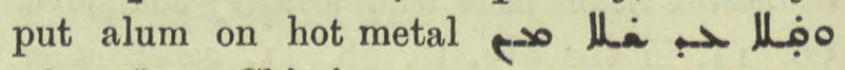

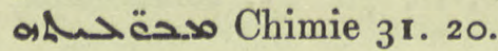

كُ col. 2273. 3) a narrow band or tie,

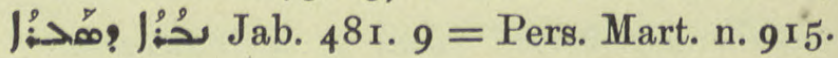

" הבה Nabröel or Nebroel, mother of Adam and Eve, Mich. Syr. 118 col. 3 trad. 200; Cosmog. Manichéenne $4^{2}$ n. 3. So correct \مص: Coupes ii. I30. 20. I Iberian name of Peter, Pet. Ib. 29. 5 .

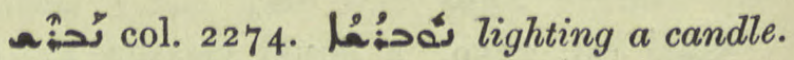
Metaph. fervency; add: BHGr. i. 5o. 9 ;

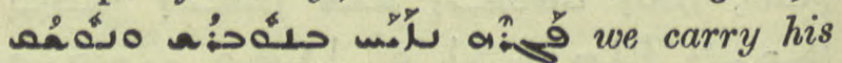
body in procession with lights and salutations, Hormizd 3096 .

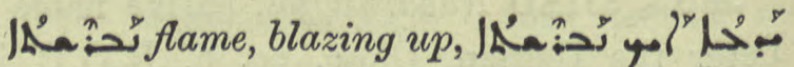

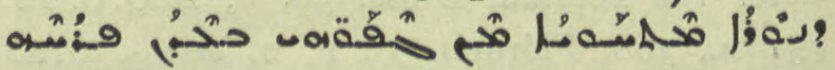
the firefly shows as it were a flame of fire from its wings at the moment of its fight, Lexx. under مبح col. 1559.

هotly, Pléroph. 30.4 .

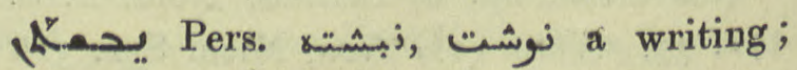

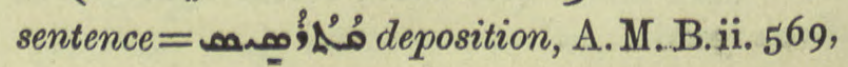


Mar Kardag 66. 6, 90. 10=ed. Abb. 77. 4, 99. 14 , Nöld. ZDMG. xliv. $53^{2}$.

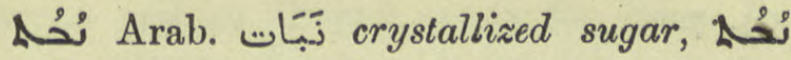
قِ gourd sugar, Med. 559. 6.

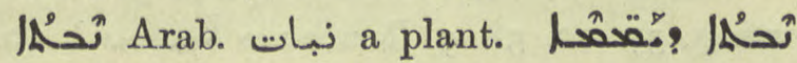
seaweed, Med. 559. 2.

\& col. 2275. 8. Delete and trs. ref. to $\checkmark \checkmark$ col. 2404, and see correction there.

y col. 2275. to dry up, become dry,

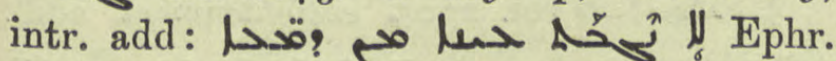
ed. Lamy iii. 319. 0 when it is dry, Chimie 16. 24. Pael 2 y to dry, trans.

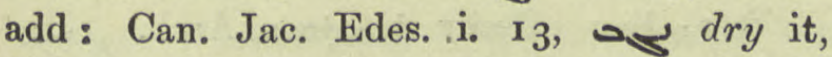

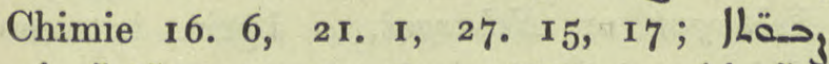
مَنِيحُ Loo' it dries up rheum, Med. 237. 10. Pass. part. fem. LRحny lin a cloud which has evaporated, Hunt. clxx, Hormizd 674 .

' col. 2276. an Indian fruit resembling the pomegranate, with fragrant flowers, refs. Med. 307. I5, 318. 3, 319. 7 . It is good for the liver. I. q. Nacest.

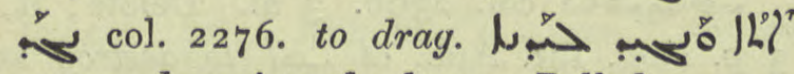
he came, dragging the hyena, Pallad. 494. I. فَّ |تمبهُ Ethpe. $N$ L ? $^{n}$ col. 2278. 18 of par. to be

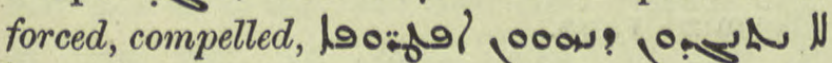
الحما they are not compelled to undertake guardianship, Syr. Rom. Rechtsb. 32. 9. Pa. ${ }^{2}$ to beat, scourge. Metaph. add: She came to reproach that Arian, Pallad. 24r. II. Aph. $\overbrace{}^{n}$ to draw, attract, the quotation from C.B.M. is found in Chimie 1. 8, 9, 11. Add : mercury avoids, flees from fire, ib. 13. 16,

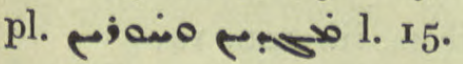

I's col. 2279. scourging. Metaph. add : (0) Jo. Tell. 74. I.

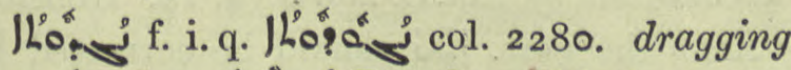
كut. Sap. Eth. i. x.

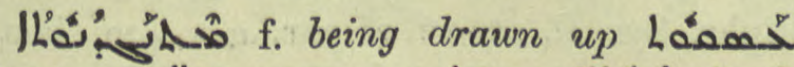

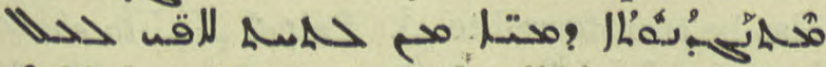
of birds with long necks lifting up their heads when they drink, N. Hist. vii. 4. I infra. a Aph. ay col. 2282. Ettaph. ay $\left.{ }^{y}\right\}^{n}$

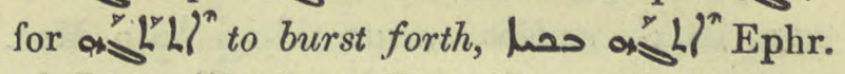
ed. Lamy iii. $589=$ Ephr. Jos. 268. 4 .

A Aoger f. i.q. laye m. twilight, Evang. Mephar. Mark xiii. 35 .

lo

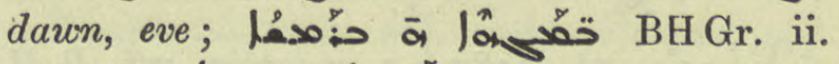
Ior ult. Monday =Sunday evening, Brev. Chald. ii. 64 this is the heading of a service preceding that of $ح$ the night service.

ló BHGr. i. 49. II given among examples of substantives with Zkapa on the second radical.

人. I נa m. pl. Hist. Mon. i. I42. 6. Budge translates accents and thinks it is the same as $\left.\right|_{: 0 .} ^{n}$ a kind of hymn, Thes. 2280.

Wy col. 2283. Ethpa. "ushin to be

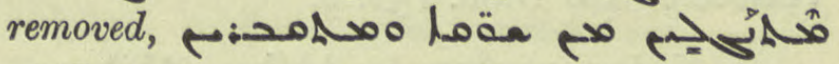
"entevés des rues et ensevelis", Dion. 2 I9. I 7.

m col. 2284 . impure, Arab. نبs, ref.

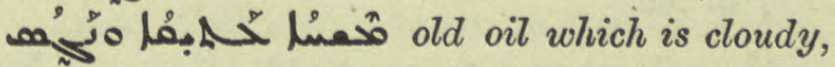
Med. 560.17 .

4 to be depressed or hollow of the earth's surface, في

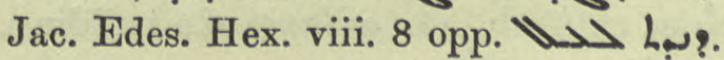

كخ of at the Creation the earth had no depressions nor protuberances, Jac. Edes. Hex. vi. 9 , viii. 5 .

$\therefore$ col. 2284. 2) to do carpenter's work. Metaph. o $>$ He framed us for heaven and earth, Sev. Ant. Hom. 89. Ix. Probably the Greek had a play on

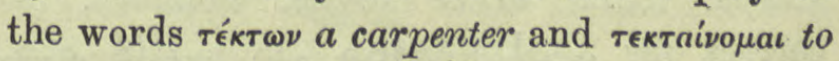
plan, devise. Aph.

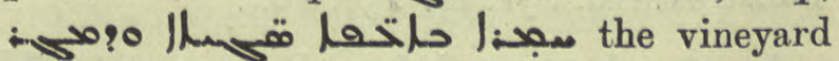
yielded manifold quantities of wine and of good keeping quality, Pet. Ib. ror. 2 I.

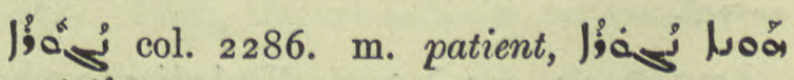

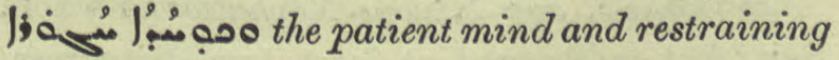
modesty are not possessed by dumb animals, But. Sap. Philos. 6.8 infra. BH Gr. i. I39. I 6 on Prov. xxv. I r has ljoy twice for loas chased silver. 
كُ lasting. Of diseases chronic

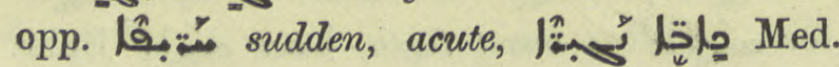

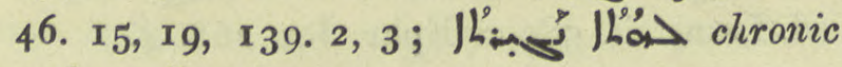
asthma, ib. 48. I9.

$1 L^{\circ}$ in f. duration, length of time. Add:

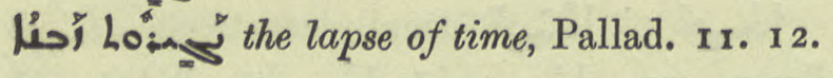

我舟 col. 2287. a sparrow, ref. B. H. Stories $73.3,5$.

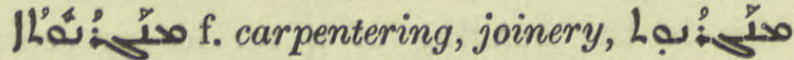
1ْ́: But. Sap. Theol. 3. 2.

*. IL 3 ' col. 2290. abomination, add: you are engaged in abominable traffic, A.M.B. v. 96 . 13.

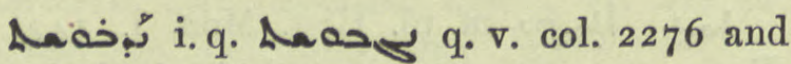
Suppl. Med. 36r. I 1, 369. 6.

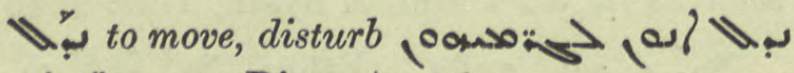

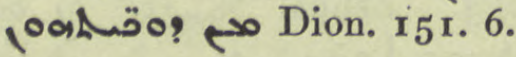

Nadan, nephew to Ahikar, Ahikar 33 ter, 38. 7. $๘$ 1. I I \&c. ZDMG. xlviii. $\mathbf{1 7 7}$ in loc.

; col. 2292. to vow, add : imper. jo:?; $\mathrm{ln}_{3}$ Loing vow a vow of purity of will, Chimie r. 5 .

ـ m. cf. Arab. collapse of a building,

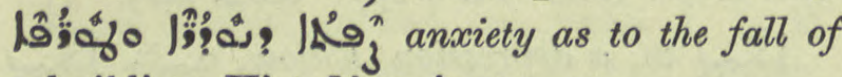
a building, Hist. Mon. i. 27 r. 17.

lou Ethpa. woull I) see lowhl. 2) write HaAt from tha to be refreshed, invigorated,

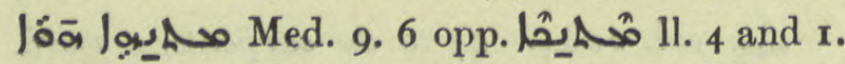

ia col. 2295. to disturb, agitate (so correct

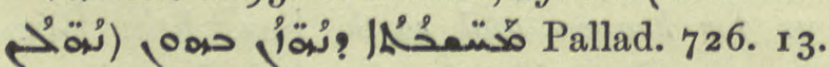
Ethpa. 'án'l to be struck, wounded, (so

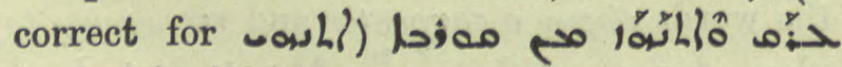
Noo Anecd. Syr. iii. 257. 10.

لئ col. 2295 infra. Pers. ناهيد Old Pers., Anahita, goddess of the waters. See بoul.

صollow, Nephatios to whom Sev. Ant. addressed at least two discourses, Journ. As. 1907, 344. Cf. col. 2363 and سوحنم Suppl.

Mas see las. pó col. 2295. Jl'ásoóár f. roaring, groaning, Ilsمُ 94. 7 .

لlasas $\nu \in \phi \epsilon \lambda \eta$, sublimed mercury, Chimie

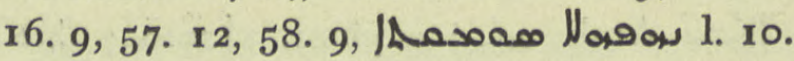

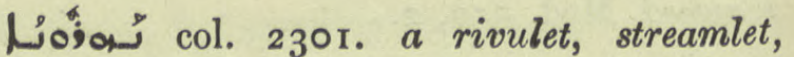
synonym for 1 a $\mathrm{DBB}$. 24. I3. Pl. BA. under llojpa col. 977.

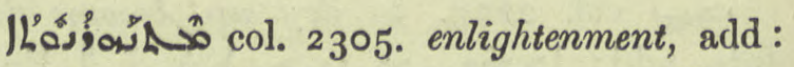
for $\epsilon^{\prime} \lambda \lambda a \mu \psi \iota s$, A.M.B. v. 345. 5, 10, Georg. Ar. I8. 1 .

Mayjar oftener jay jaw Arab. Nehargur or Nehargul, a bpric. between Maisan and Ahwaz, Yakut. The official name was ابزقباذ Eranšahr 4I, Syn. Or. 678; 33. 24 sqq.; Sassanidi 28. 16, 3०. 6, ZDMG. xliii. $394.5,396.8,397.8,4$ о . 8,4 06. 10.

م pl.m. Nomads? Name of a Christian people, Doc. Mon. $3^{2}$. $21,272.13$.

waspas col. 2306. 3 af. Delete; it is 3 p. sing. fut. Aph. of tol to destroy and occurs in a colophon warning against ill-use of the MS.

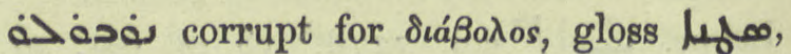
Hormizd 1492.

م Novinianus, Bp. of Jerusalem, Conc. Eph., Dion. Ined. 67. 14.

Nubkar, daughter of a Governor of Alexandria, Hist. B.V.M. I26. 21.

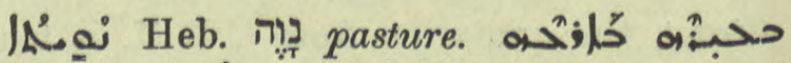
هis wild beasts find food on His earth and His flocks on His pastures, Jac. Sar. Hom. iii. roo. 14.

\section{مقدما פمسسم} I Loo the stains which $I$ abhor and am ashamed of, Act. S. Maris 74. 12. Abbeloos thinks the part. نُبْ should be corrected from to abhor; if so three passages from Ephr. Syr. 11. 20-22 of par, c.c. $\varsigma^{\infty}$ should

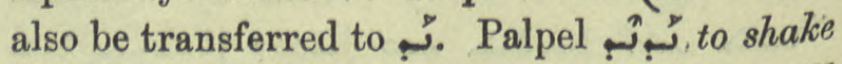

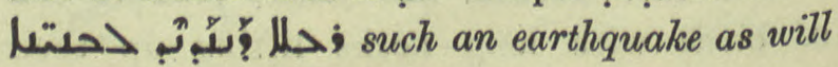
shake buildings, Sev. Lett. 383.22. 
تُصفُ col. 2310. a trembling, quaking.

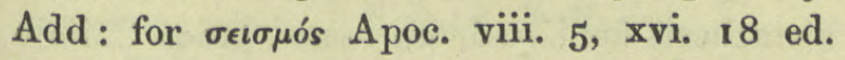
Gwynn and so Gwynn reads for joos ib.

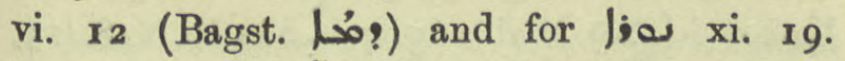
Bagster has Lُ0\% in these three places.

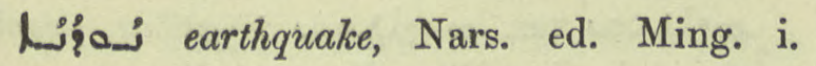
$221,19$.

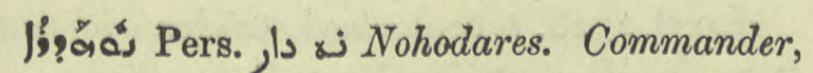

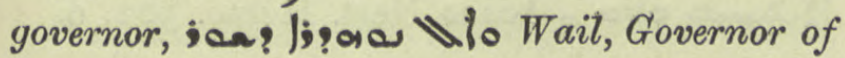
Sura, Nöld. on Pognon's Inscript. Sém. Z.A. xxi. 53 sq. In But. Sap. it must mean

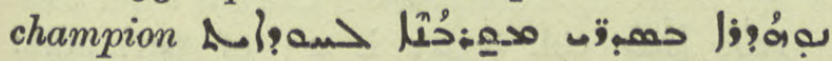

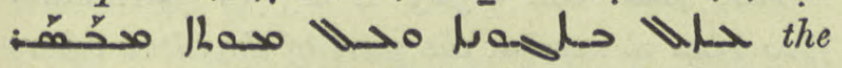
champion offers single combat to the warrior ranks and scorns death, Theol. 5. 1.

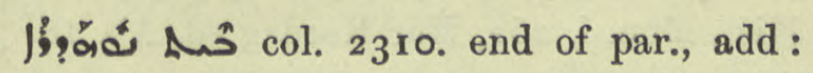
j?aes 0 Or. Xt. ii. 302. 2, 8. House of the Nohodar, Pers. Mart. 209.

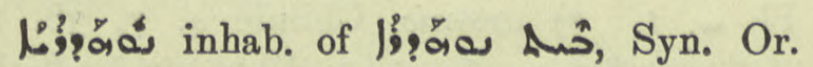
44. 24, ZDMG. xliii. 396 ult., pl. Išoyahb $145 \cdot 5$.

10 col. 23 ro. Aph. Iâs/" to spurn, Hormizd I 735 with gloss ححه

w w col. 2310 ff. Ethpe. wh/ for

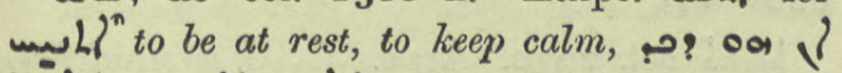
Ai (ي) Sev. Ant. Hymns 255,

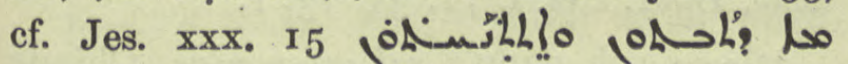

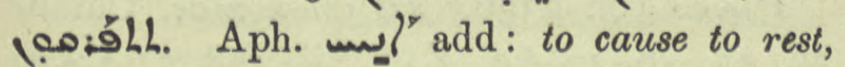
to cause to recline or sit down : مa (o) ל Thesus, after He had told them to sit down, took a towel, Ephr. ed. Lamy i. 391 r. 10 on John xiii. 4. معبس I have let my discourse find rest in the haven of silence, Pallad. 343. 14. To obtain a favour, to persuade, with fut.

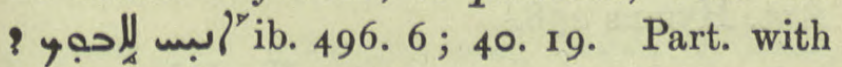
ميس خح, pleased with, Ephr. Ref. i. 178.7 .

لُ col. 23r5. rest, cessation, add: conclusion, end, for Ha this chapter now concludes, Bar Sal. in Lit. 45. 13.

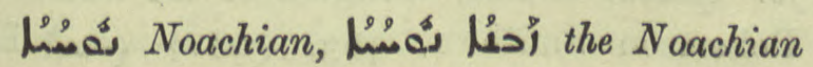
era, time of Noah, Warda $37 v$. col. 2315. gentle, quiet.

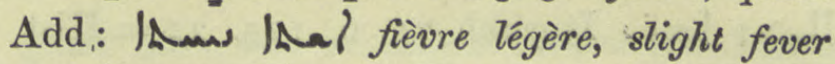
opp. مـ Hippoc. iv. 37.

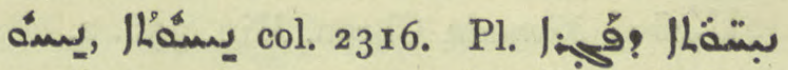
recreations, pleasures of the body, Pallad. 734. 3 .

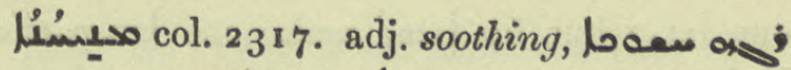
oa >aphr. Ref. i. 2. 23 .

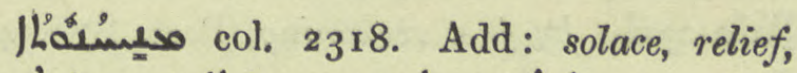

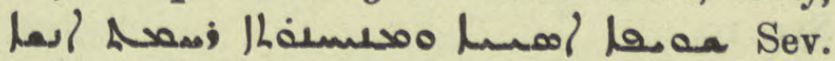

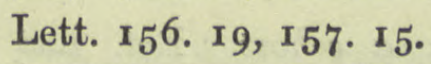

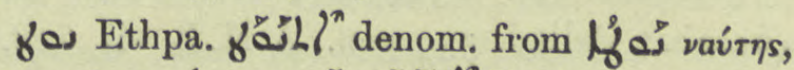
to navigate, hooi

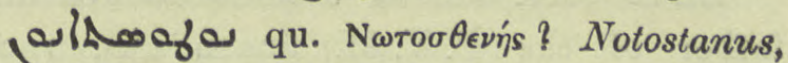
Bp. of Tamasus, Nöld. F. S. i. 472 . 80 but Mich. Syr. has Tichon, trad. 3 r6, supplied, nothing in the text, p. 160 .

تُقة

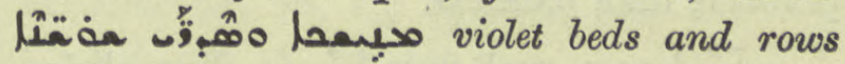
of lilies, Greg. Cyprus 42 a.

Nochanor, a bpric. in Asia or Caria, De Goeje B. 65 ult.

lenoser prob. corruption of some deriv.

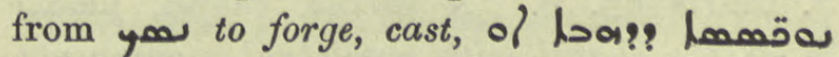
bolo? forged or molten images, Spic. Syr. 26. 24.

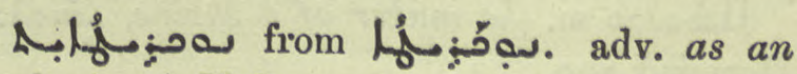
anchorite, A.M.B. i. 484.3 af.

Ihof بو col. 23 I 9. the life of an anchorite, Hist. Mon. i. 25. 8, 33 ult.

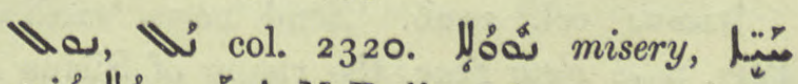

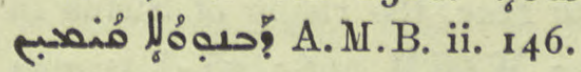

لإ. add: ugas: Vas a spider's web, cobweb, Anecd. Syr. iii. rog. 12.

دُ Ihe interweaving of the assumption of human nature by Our Lord, Nöld. F. S. i. 477. Perh. correct

l-o.. with final $r$, sas BH. in 2 Tim. ii. 17.

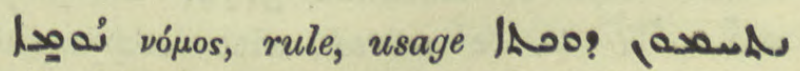
laod $>$ hose they should assign a place 
and rules for the combat, Dion. Ined. 64. 14; IST Išoyabh 75. 6, 125. $22=$ Hist. Mon. ii. 133. 7 af. Cf. תمax.

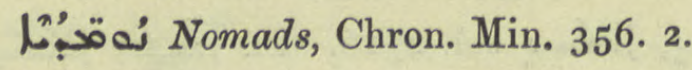

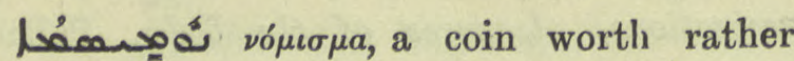
more than half a dinar, for roo f:

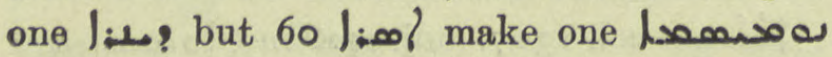
Epiph. 2.

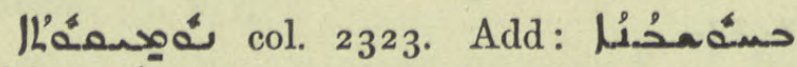
I' Syr. 10.

Oinas col. 2324 a cohort, Ref. La'

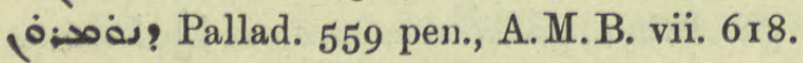

مa Sanachir = Joachim, father of the B. V. M. Journ. As. 1906, 283. 10.

๓or, sol. 2325. Delete and transpose quotation to sol. 2387. Kaols is more probable than Jleood.

๓ẩ denom. verb from lڤ́. To enshrine, Hormizd 278.

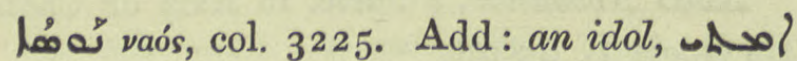

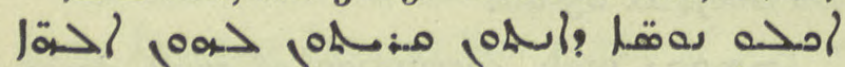
S. Maris 65. 9. A shrine, chapel,

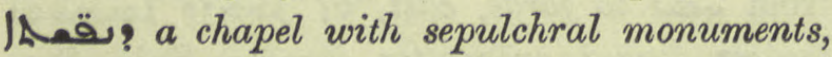
Jul. 222. 27.

منهم مَم Hormizd 290.

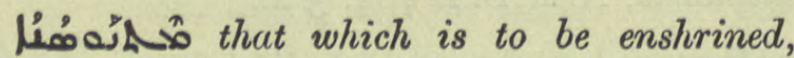
Hormizd 28 I, 290.

1901. 2326. Zend nawa sardi= Pers. نوروز New Year, the rising of Sirius in Jهily, F. Müller, WZKM. 366 ; El. Nis. 5r. 59 .

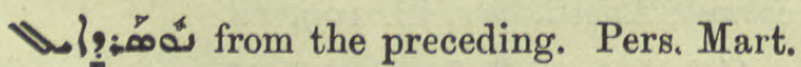
n. 523, Tabari 407 nn. 2, 3, Lag. Arm. Stud. iii. r6or. The first day of Summer, kept on the seventh Sunday after Whit-Sunday, the Festival of the Twelve Apostles is held on this day.

هor, to start, shy as a horse, !a note to Hormizd 3r74. Ethpalpal a cashin to be moved with compassion, Tekkaf 122.

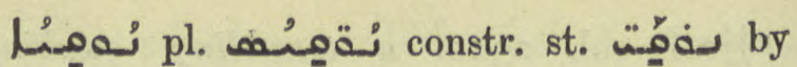

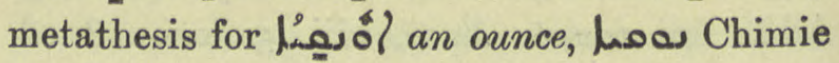
28. 8, 9, 29. I 2, 32. 9 ; pl. دقميم ib. 34. 21 ;

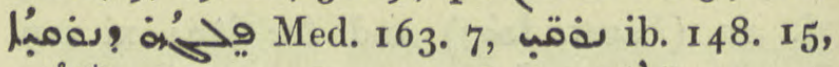

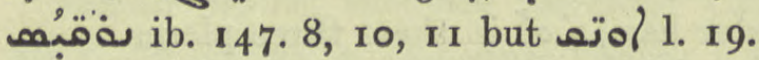

م of the night, Jac. Edes. Hex. xxxii. I I.

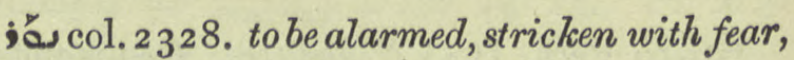

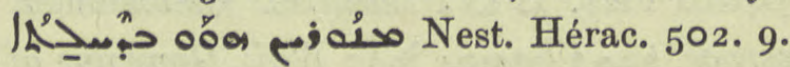

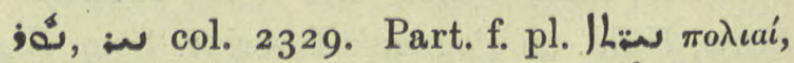

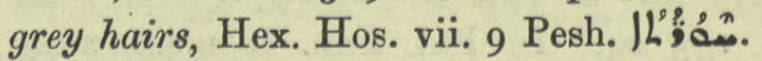

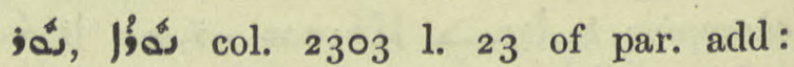
jos fire is made, Hex. Ex. xxvii. 3, Pesh.

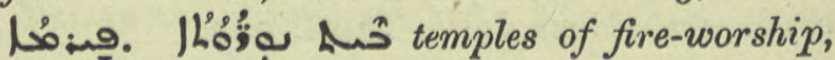
Hist. Mon. i. 262, Mar Kardag ed. Ab. 16. 3, 62. 7 .

IL'sis col. 2304. f. x) a blossom, BH. de Pl. 七. I. 2) arsenic, Chimie 4. 7, 38. 1, 42. 22. Synon. ßarpáx Łov ib. 7. I I h. Arab. $\ddot{z}$, -j a depilatory composed of quick-lime and arsenic, DBB. 298 pen. and ult.

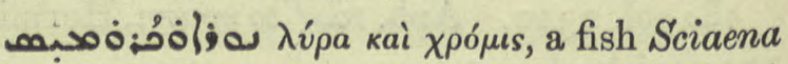
nigra, N. Hist. vii. 2. 3 , see Arist. Zoology $535 b \div 7$.

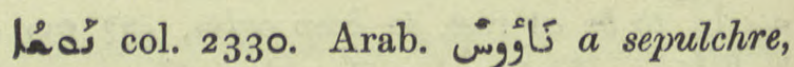
tomb, Ephr. Ref. i. 72. 35, 73. 1 .

; 36. I5, 40. 12 \&c., Z. A. xii. I58. 5 ;

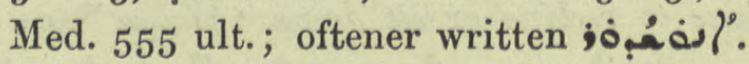

? $\rightarrow$ Pers. prob. Yazdgerd, King of the

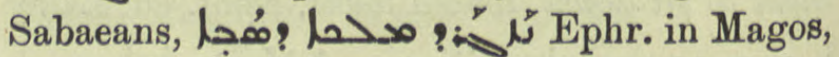
G. Busâmé $64 b$ ro.

Uu col. 2331. Ethpa. "

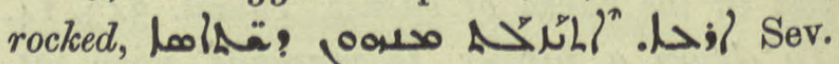
Ant. Hymns $25^{8}$.

;onk, jo?k var. po?h Yazdod, one of three messengers sent by Nimrod to Balaam, Schatzh. 178.13 .

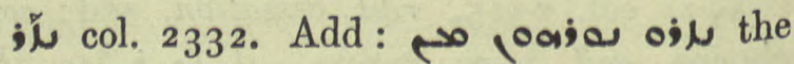
gods have preserved their altar-fire from pollution, Jul. 52. I5. Part. is sonsecrated for special service, Religion of the Semites,

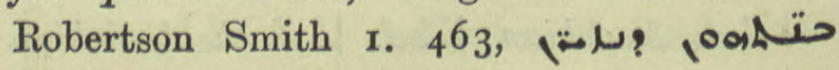
 
col. 2334. Pa. Uמص to render lean, to

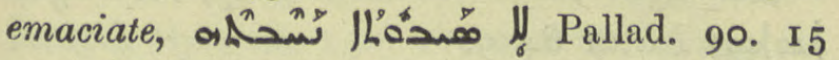
$=$ A.M. B. v. $119 \cdot 3$.

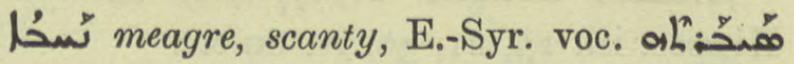
نسمًُا Pallad. I Io. 14.

مهبح: : meagre, barren, add منلا مac. Edes. Hex. xlii. ib. 14.

هـ

JLبحف two MSS. have this E.-Syr. voc., two have Jhe ; leanness, A.M.B. v. 63. 8, Pallad. 48. 3 .

\מ̂ col. 2335. Nechel, add: a village in Arzun 0\%\}, Chast. 13. 5, 7, a monastery near Nechel, ib. 8. 19, I3. I.

نسمْحُش surname, of the valley or of the

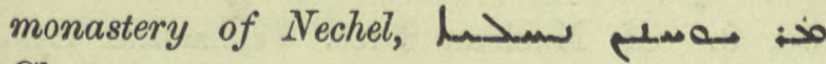
Charms 30. 20.

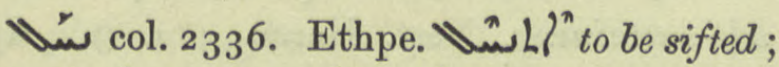

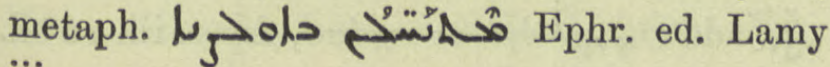
iii. 143 .

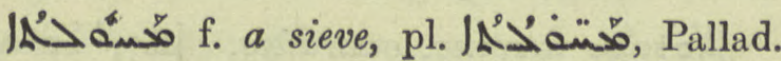
$63 \mathrm{I} .4, \mathrm{II}, \mathrm{I} 5$.

مa ل مañ a sieve, a tammy,

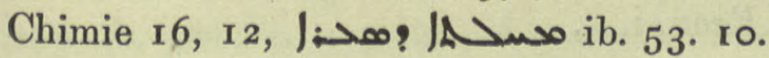

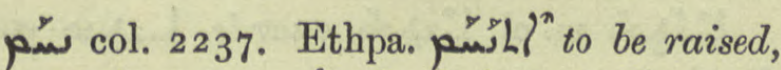

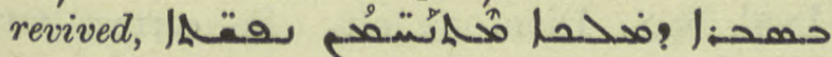
!متخمل Titus thought that the courage of the distressed fighters would be revived by expectation of the emperor, Josephus vi. $7 \cdot 3$.

12'

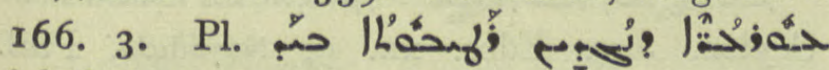

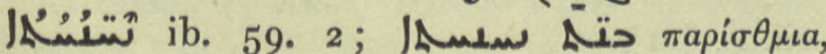
Hippoc. iii. 25 .

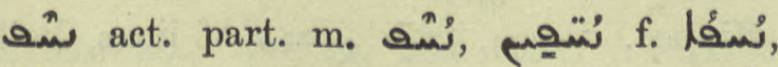

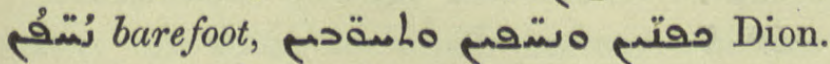

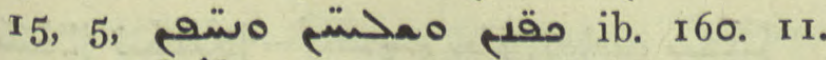

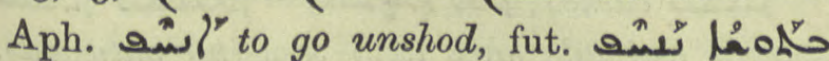
he shall wander barefoot, Jac. Sar. Hom. ii. 48 .

ú col. 2339 ult., pen. Delete. Correct

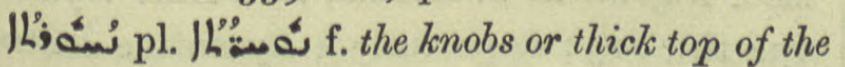

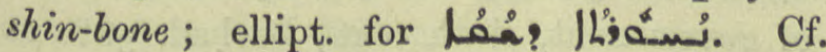
2716
Neo-Syr. سمسن: In Tripoli it is called

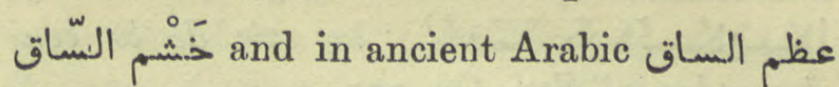

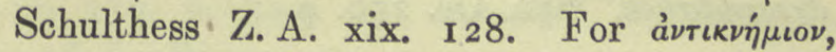
व

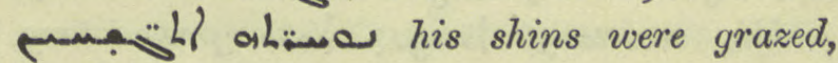
Jo. Eph. 2 10. 9.

Sسئlo the hollow of the shin-bone into which

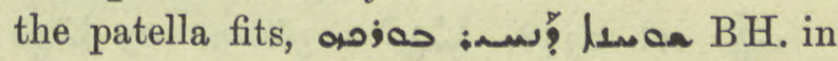
2 Cor. xii. 7. The head of the Nile flood,

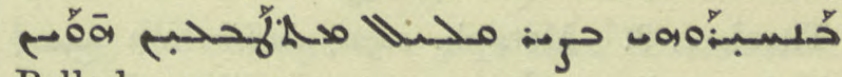
Pallad. 422. II.

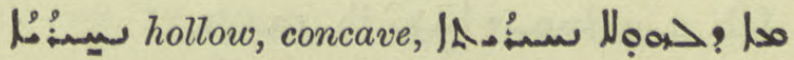

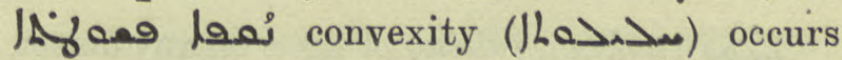
when an outer surface is contingent on concave matter, But. Sap. Philos. 8. 2.

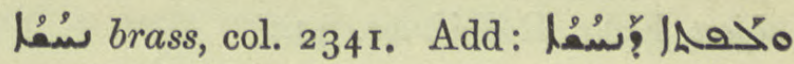
سمعا ?معب ;

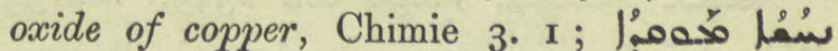

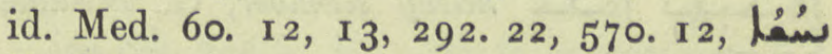
年 ib. 63 .

Nahash, name of the mother of David, Journ. As. 1906, 283.8.

תame of an angel who went before Primal Man in his combat with evil. Greek

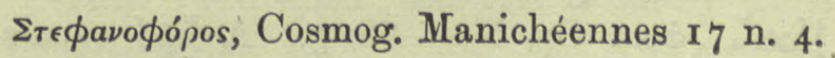

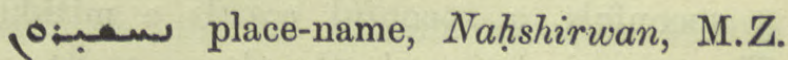
I 9 I. 9, 195. I7, Chast. 38 . I 5.

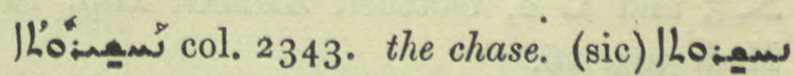

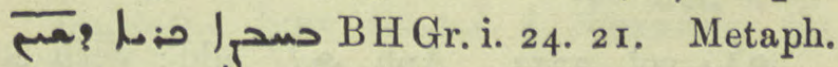

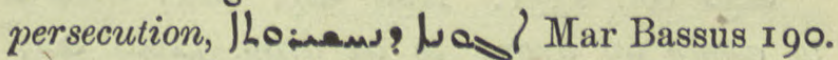

ش col. 2343. Add: to come forth from

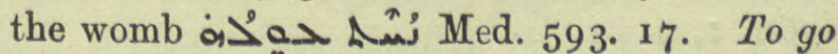
down a list, Philox. 59. 14.

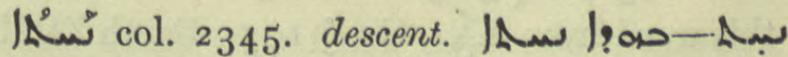
جا an' oppressor left the city and at his going down $=$ away, trouble ceased, Dion. I25. 2 ,

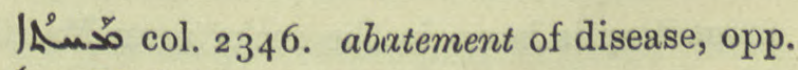
liaّ, Med. 8r. r.

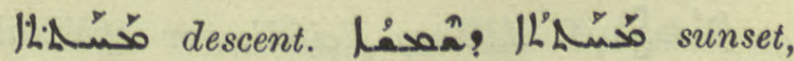
Georg. Ar. 19. 10, 20. 12; shrinking, subsidence of a swelling, Med. I6r. I4, 2 I.

E e 


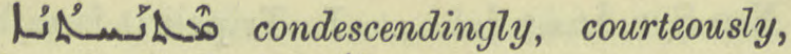
| descendance," Syn. Or. 25 \%. 9.

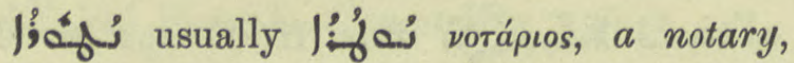
public secretary, Jaju! le; protonotary, Syn. ii. Eph. 1 2. 26. Apparently a popular etymology. Cf. R.O.C. vii. 19.

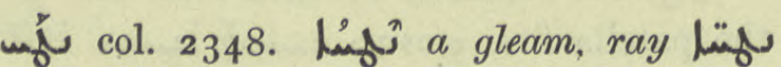

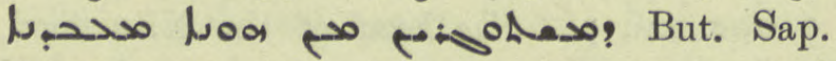
Theol. 4. 8. Metaph. sign, indication

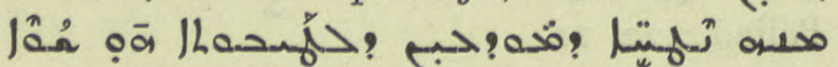

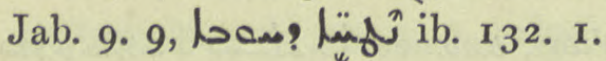

lif striking, falling of light on any object,

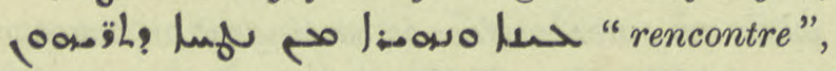
the glancing of the eye upon an illuminated object, Ephr. Ref. ii. 42. 33.

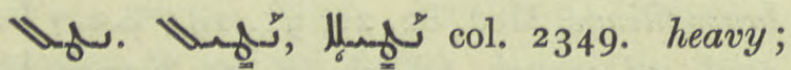

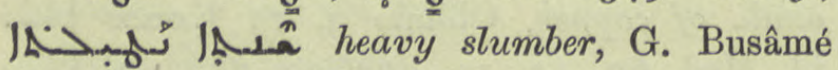
II. 14 .

نهي a) a weight $=2$ staters, 12 mithkals or $\mathbf{1} \frac{1}{2} \mathrm{oz}$. Nöld. sec. Gottheil. b) a measure, half-pint,

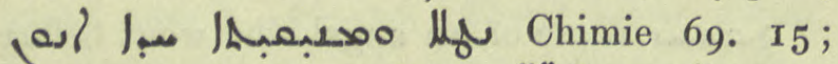
a wine cup, مبمبه|.

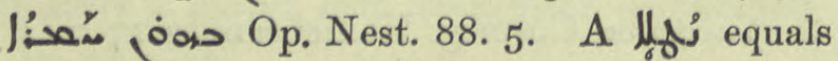
5 spoonfuls, a spoonful equals 2 mithkals. The numerical signs in the glosses to ly are $>5$ and 1 2, Gottheil ZDMG. xliii. 123; Med. 393. 16 .

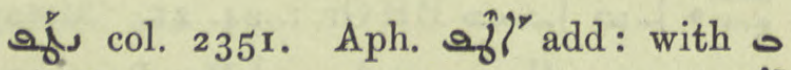
to apply by drops, to let drop, ras afl" JAOS. xx. 195. 4, I1, 13, Med. 101. 20, 560. $\mathrm{I}, 9$.

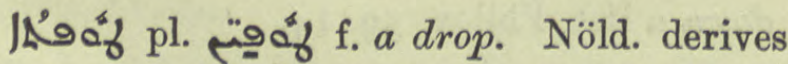
this word from قُ, Gr. § 105; Barth ZDMG. xli. $6 \mathrm{r}_{3}$.

نُ Add: Convent of the Drop i.e. where water drops from the roof of the cavern; this monastery is hewn in the rocks near Mardin, Mar Benj. 85. 6, R.O.C. ii. 263 n.

1Rำ f. Althaea ficifolia or officinalis, Ar. PflnN. ${ }_{360}$, Med. 58. 10, I39. 16, 3 I 5. 20,

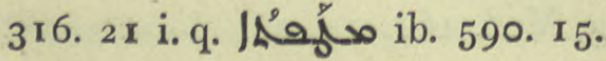

مَّم

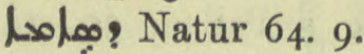

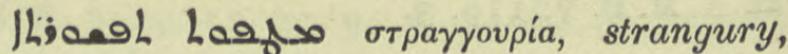
Hippoc. iii. I 5, 2 1, 30, iv. 80 \&c.

IRُ مه Delete art. It is done under $p$ col. 2079. I. q. مُّر.

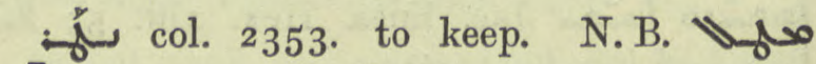

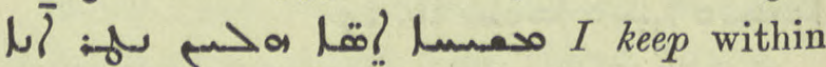
these walls for Christ's sake, Pallad, 154. 5. Add under 2) with J' faithful with $>$ of the pers., Pallad. 283. 17 .

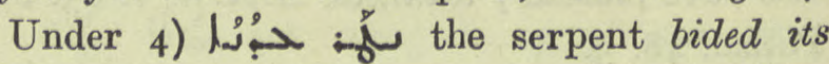
time, watched for an opportunity, Thes.

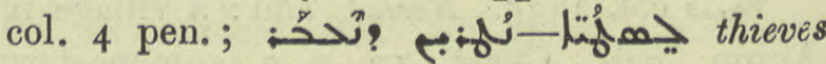
watched for his passing, Hist. Mon. i. 168. 9. Act. part. in composition, add : a bishop's successor, Journ. As. 1 906, 68 med., 69. 8. Cf. Maclean Dict. Vernacular Syr.

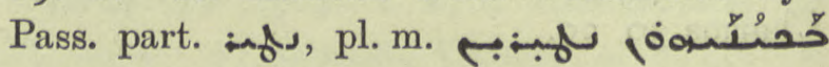
self-sustaining, Nest. Hérac. 4I 4. 3. Aph.

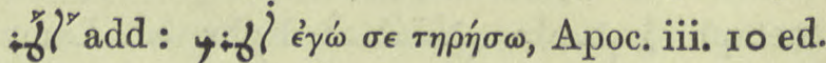

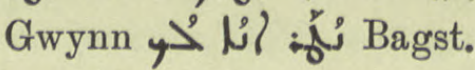

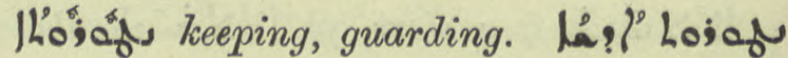
preservation of the species, But. Sap. Eth.

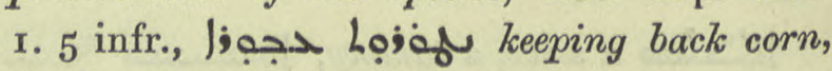
ib. Econ. i. 2.

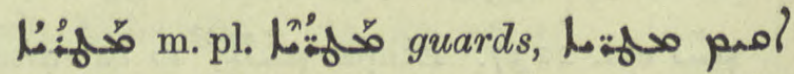
حion. $5^{2}$ ult. So correct for مَّه: Jab. 47 r. 8.

oid usually joid col. 2360. natron, اله Chimie II. 20.

N Nyádıßa, an island near Ceylon, Jac. Edes. xx. 9, Nayáo̊ßa, Ptol. vii. 4 , § I 3 .

يُ col. 2359. a canine tooth. kvvóoous, Hippoc. iii. 24, من:مبر تبّدم of lions, Pallad. 250.7 .

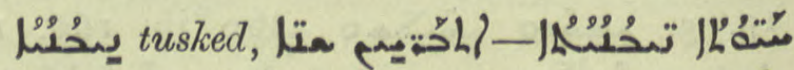
tusked animals have teeth showing outwards, N. Hist. vii. 6. 3 .

col. 2359 ult., i.q. بُ col. 2288 med. Najran in S. Arabia, Sev. Ant. Hymns I 54 tit., 6 I $3_{3}$; apparently a Byzantine centre of government, sixth cent., Barhạad. 388 . 10, 
Nars. ed. Ming. 36. ro. Spelt Anecd. Syr. iii. 232. 17, 236. 7 ; r ib. 235. 9 ; م-بة : Brev. Ant. Kal. $27 b$ I 2 .

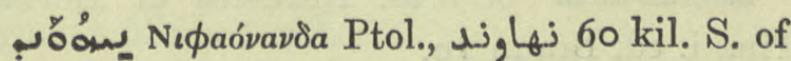
Hamadan, Syn. Or. 678 ; ref. ib. 603. 8.

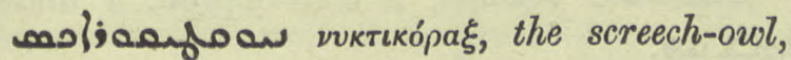
Löw ZDMG. xli. البوم (2030.

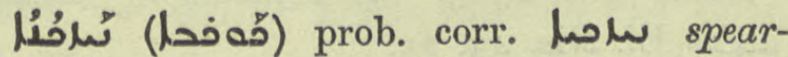
shaped, a comet, Med. 550. I9.

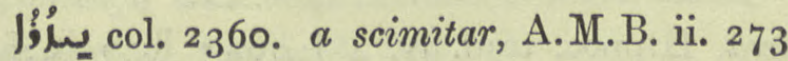
var. بـإن.

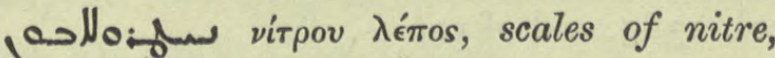

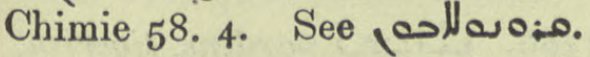

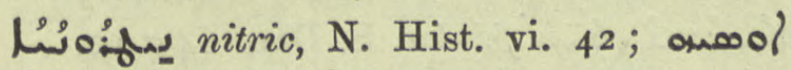
كitric substances are soluble in water, ib. vi. 4. 2.

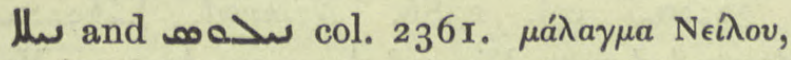
Nile plaster, شياف يعرف بنيلوس Galen. ed. Kühn xiii. ı8ı, Löw ZDMG. xli. 360 .

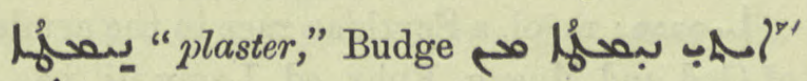
1 1 (.) Med. 574. 5.

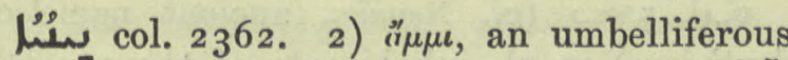
plant, Med. 99. 3, I6 1. 7, 19, 214.8 , 13 بُ ib. 36r. 4, 393. 9. A spice, Ar. PflnN. 260. 261 .

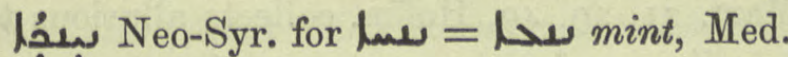
83 ult. It is the Arabic نانتخوان.

sas pr.n.m. N $\eta \phi a ́ \lambda \iota s$, Nephaleos = sober, Sev. Ant. Vit. 23r. 9.

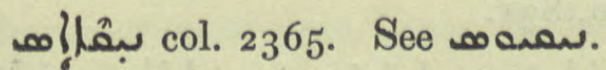

aL Nikatvóv, Nicene silver or copper, Chimie 233 n. 4, 236 n. 2, sar ib. 42. 5, (. 15.

صikvopos, that which conquers water. A stone to which curious qualities are attributed, Chimie 9. 3. I. q. Thracian stone trans. I 7. I n. r. Cf. Diosc. v. cxlvi.

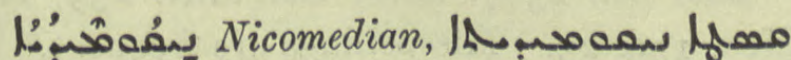
the pint of Nicomedia equalled 20 oz., Epiph. 5. I5.

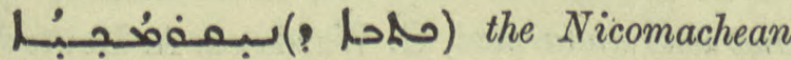
Ethics, But. Sap. Eth. 3. 4.
Low col. 2365. Nicias, Bp. of Laodicea, add: Sev. Lett. 42. 4, id. Vit. 325. 2, sمes ib. 319. 6, 320. 2 ; مader ib. 321. 6,

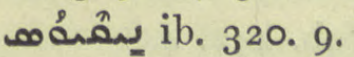

نقيوس.col.2365. place-name Arab, Nıkıồs or Peschati, now Ibschadj, about 4 kil. E. of the Canopic branch of the Nile, Amélineau $277 \mathrm{ff}$.

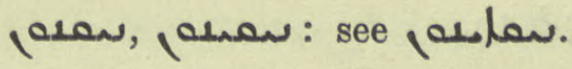

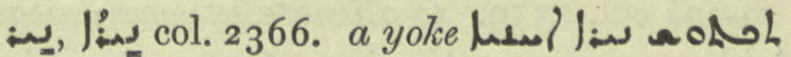
కvyouaxia, bickering between brothers, Sev. Ant. Vit. Iо3. I I, I2.

col. 2367. Add under 3) category,

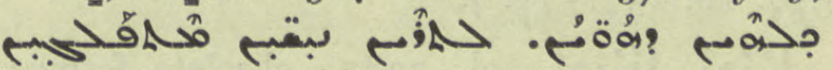

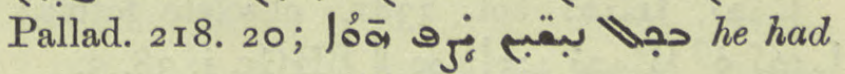
tried in every way, ib. 285 . 17 .

من Nishapurin Khorasan, Syn. Or. 6r9. ro. Cf. נתم/حم).

لا لمحس col. 2368. Pa. Pُ

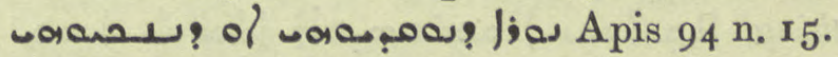

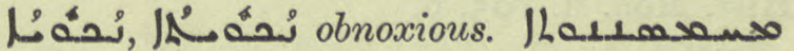
Ihan? wa ka perseverance is hateful to the young, BH. Stories 24. 145 .

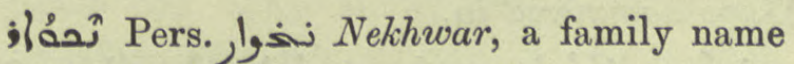
or title, Hist. Mon. i. 248. I8, M.Z. 2 I 2 . 12 transl. ii. $26 \mathrm{r}$ n. ancestor of the $\longrightarrow$, race, Marquart ZDMG. xlix. 644 med.

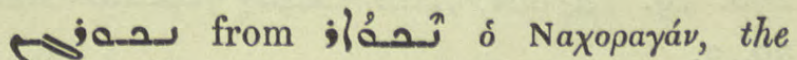
Nikhurgan or Nachwergan, a racial name $=$ title, Tabari ${ } 5^{2}$ n. 2, 347 ; Pers. Mart.

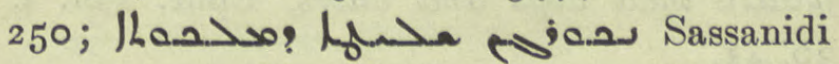
II. I9. 22; Mar Kardag 80. I 2, 8I. I, 8, Syn. Or. 526. 26, 529. 2 I.

Wa col. 237 I. Aph. \ôे to be of opinion,

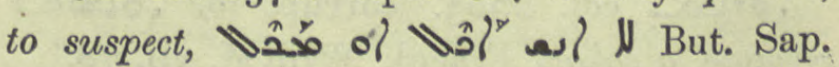

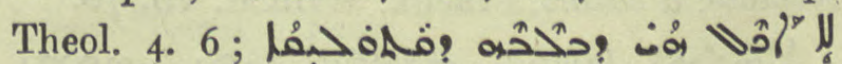
Hist. Mon. i. 102. 13.

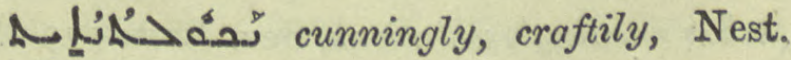
Hérac. 45I. 4.

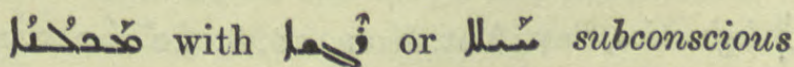
reasoning = instinct, given (wrongly) with ref. under $W^{\circ}$, col, I 734 . 


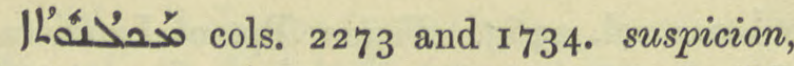
Jhal Sev. Lett. 22. 10; 64 ult.,

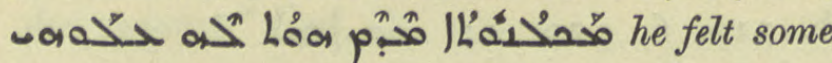
suspicion concerning him, Jab. 442. 4.

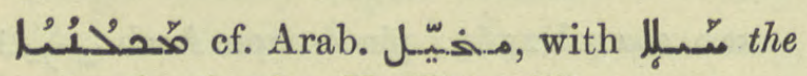
imaginative faculty, N. Hist. viii. 3. I, 5 .

تُ col. 2378. to repudiate. Ethpa. to be disowned, repudiated; محب: G. Busâmé $7 b$ I 9 .

كُصذّ col. 2379. to alienate, excommunicate.

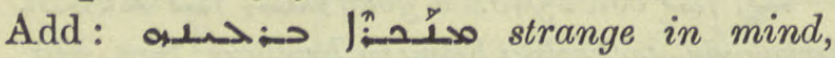
Pallad. 72. 8. To expel, expelled and excommunicated two monks, ib. $576.18,23 ; 577.7$.

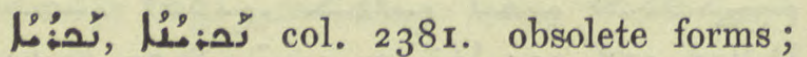

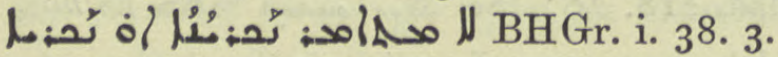

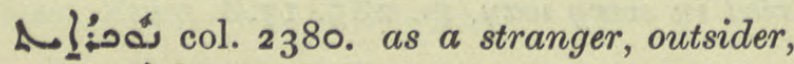
opp. حَـ intimately, Jac. Edes. on Arist. 27.

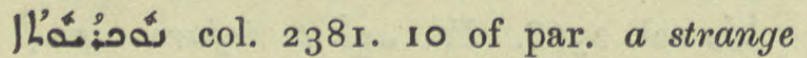

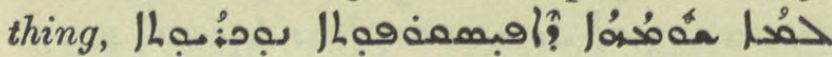
Hist. Mon. ii. I05. 6.

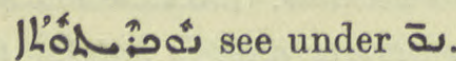

col. 2381. Delete par. It is תمِمُ hammered in.

ص้. 1 . Delete metaph. \&c. and add : gnawing pain,

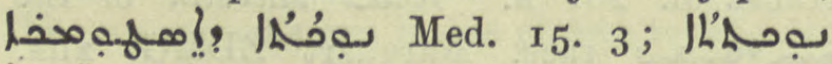
|

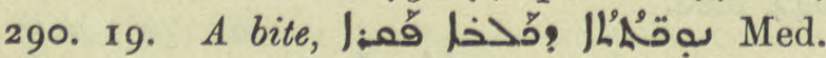
5․ 3 .

هم:ال

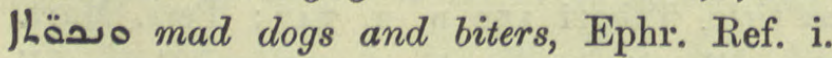
30. $4 \mathrm{I}$.

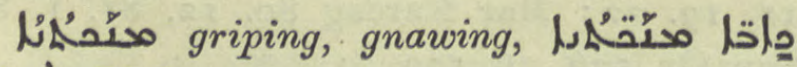
لإمة

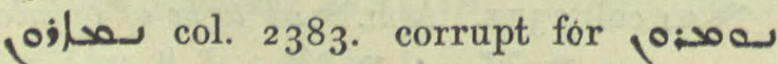

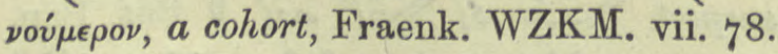

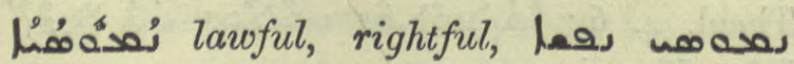

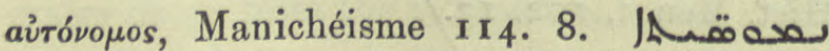
بقفم ensuing resolutions, Sev. Lett. 179 ult.

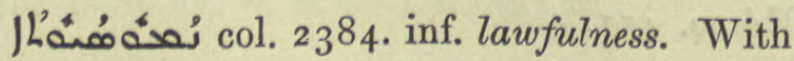

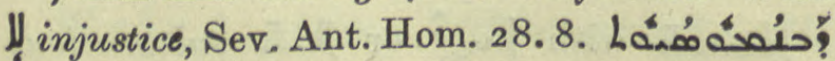

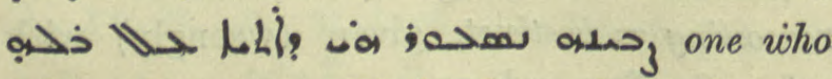
taking his own will for law, does whatever comes into his mind, Sev. Lett. 209. 2 ;

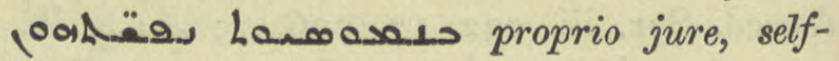
authorized, Bar Sal. in Lit. 49. 25.26.

nanerer pr.n. Nemesios of Emesa, Tim. i. ed. Braun 10.

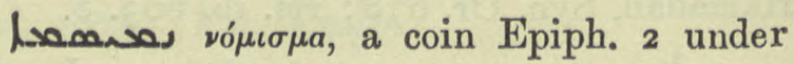
ln

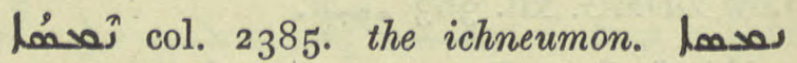
El. Nis. 43. 75.

كehlevi namak, Pers. xolj a firman,

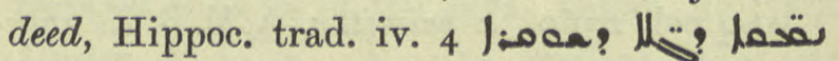
forged deeds, Syn. Or. 142. 24. Cf. تمِيمُما col. 2385 . end of par.

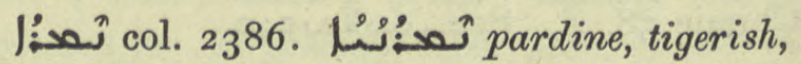

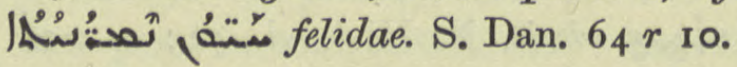

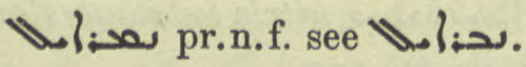

تُصن: descended from Nimrod, A $10: 0$ : Mar Kardag ed, Abb. 11 n., Hormizd 625.

رare m. pl. a Scythian race in the armies of God and Magog, Ephr. ed. Lamy iii. 19.

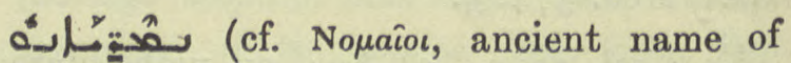
Scythians) m. pl. inhabitants of the seventh or Northern clime, Poet. Syr. 46.5 .

تُيس Mart. 21, 29, 49, Hoffm. collects allusions to

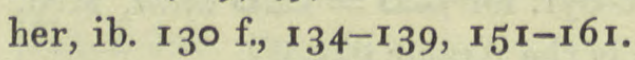

3يمع Nanestar, a district to the east of Babylon, birthplace of S. George, Pers. Mart. $9^{2}$, f. and n. 83 r.

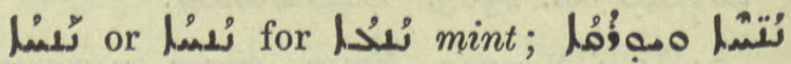
Hist. Mon. i. 159. 12, 338. 12 = ii. 322 n., Med. 568. I 9, 20, 575, 2 I ult. Nöld. Lit. Centralblatt, Dec. $1893, \mathbf{1} 75^{2}$.

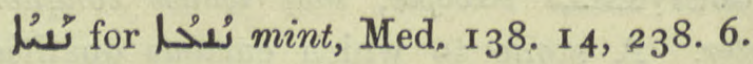

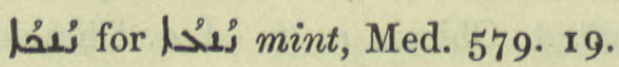

نُحُح col. 2387. mint, Ar.PflnN. 259, ref. Med. x59. 23. Misspellings are frequent.

'ُ col. 2387. Ethpe. $i l l$, S. George drank poison twice and was unharmed, G. Warda ed. A. Deutsch. $32 \mathrm{I}$. 


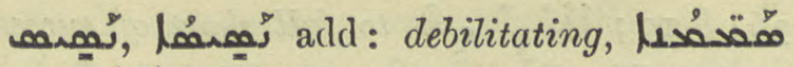

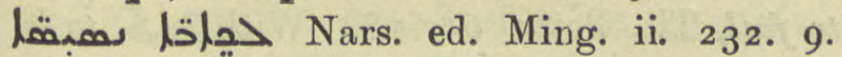

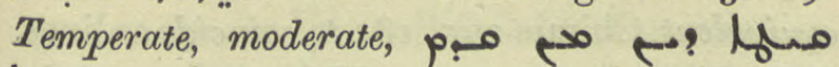

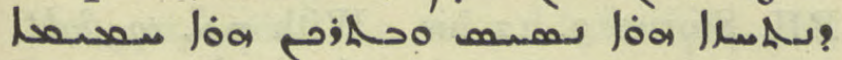
Rylands MS. $44.3 b$.

ا. Pa. Part.

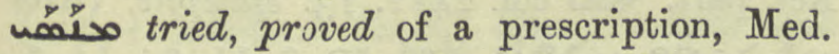

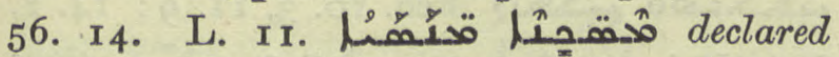
lepers, see Hallier Edess. Chronik and Ephr. ed. Overbeck 203. 25. Ethpa. uns'lin to experience, have experience of, Jescunodratil?, Sev. Lett. 293. I 8.

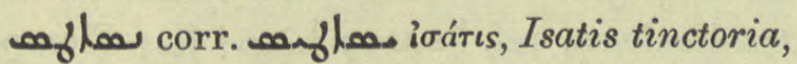
woad, with gloss مدحر, Chimie 7. I5.

مُ col. 2392. inf. v. 1 2, vi. 4 ed. Gwynn nouns: made observation, Jul. 2 I. I. With preps. In of ocide among yourselves, Bar Sud. 40. 14. Ethpe.

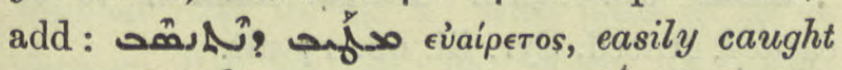

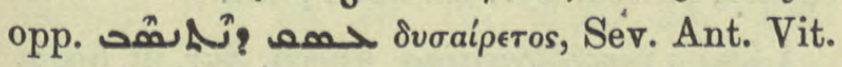
240. 6,7 .

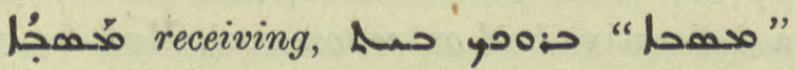
BH. on Philippians iv. I 5 .

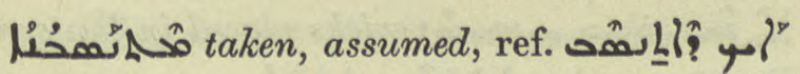

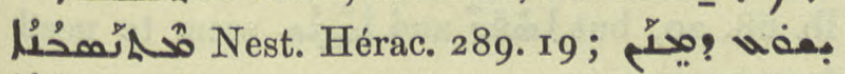

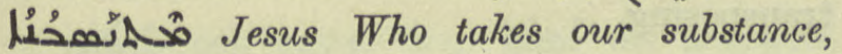
Hormizd 279.

| Išoyahb Hist. Mon. ii. I39. 9. Astron. determination of stars, hours, \&c. De Astrolabe 73 bis.

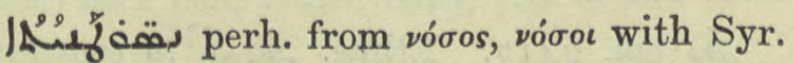
termination, illnesses prevalent in autumn. But more probably a mis-spelling of Med. $53^{8 .} 3$.

Nestabus, Archdeacon of Maiouma,

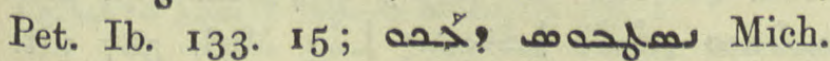
Syr. I59 a I. See صaحfol.

col. 2398. to pour out. Add: to inject,

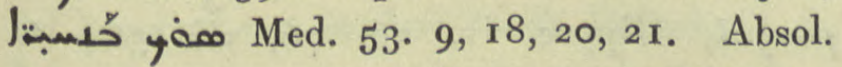
y y

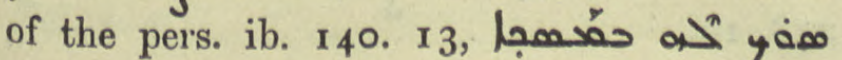

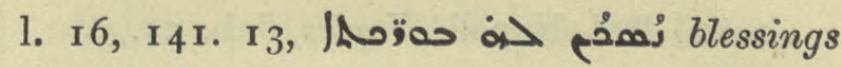
poured into Egypt as water into a haven, Ephr. ed. Lamy iii. 437. Ethpe. $\hat{n}^{n} L l^{n}$ to be

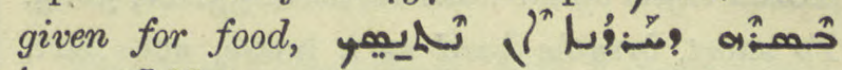
حصحبن: Natur 42 . 11.

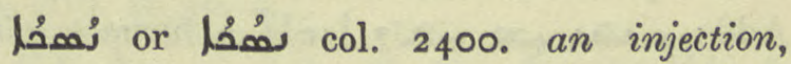

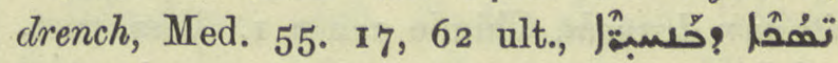
ib. 98.7 .

سُ the melting of glass, Chimie 53. 9. Pl. fluxes, ib. 97.2 .

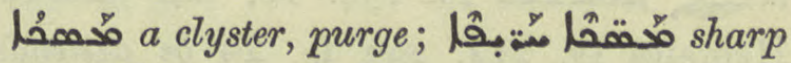
ciysters, Med. 47. 20, 65. 19, 80. 19, I35. 8 \&c.

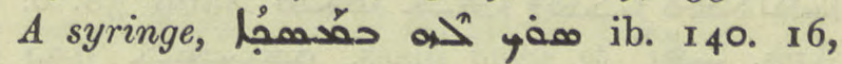
I4I. 13 .

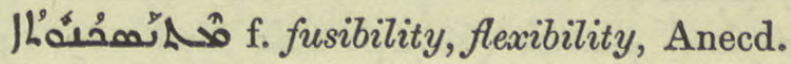
Syr. iv. 19. 22-25.

يمي i. q. 2400. Zend naçka prob. from نسخة , a nusk, treatise, مب بِ "let him recite a part of the Avesta, A.M. B. ii. $5^{80}$. 6.

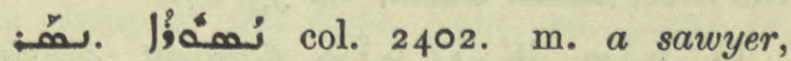

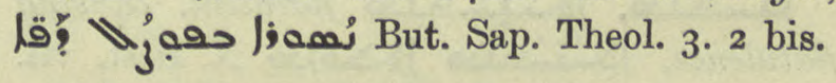

مَا col. 2403. 4. 1) Sierra, legendary mountains, Patr. Or. v. 74r. 8. 2) sawfish, Pristis, مavarur 7 and trans. $76 \mathrm{n}$. In Syr. glosses it is the dolphin and crocodile, Ar. FischN. 49.

لحد Arab. نَّنَ mint, Med. 554.

تححُمْ radjen raven, wings, Pallad. 752. 8.

col. 2404. to snatch, tear. Trs. from

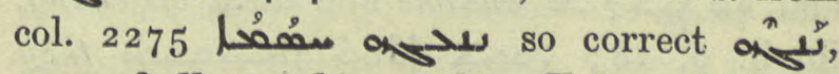
envy shall tear him to bits, Ephr. ed. Bick. xxxv. 287 .

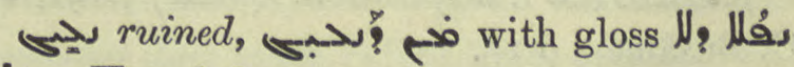
مع Hormizd I 295.

محّ. p? ? Chants of Adam, in praise of the B.V.M., Brev. Ant, i. $90 b$ × 3 .

$5^{5}$ col. 2406. to af. to grunt. Bedjan 'خ A.M.B. iv. 588. 5 . 
بهف Ital, Napoli, Naples, Jab. 54. 3.

هف w r col. 2408. to blow. Imper. כحسبت: inject by the nostrils, Med. 55. 2 I, 56.5. To snort, breathe hard as a horse, act. part. نُ Op. Nest. I1 3 . I 2 on Nahum iii. 2.

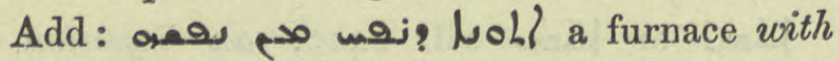
its own draught, Chimie 222 n. r. Pass. part. كوبس معبم his chest is inflated or expanded,

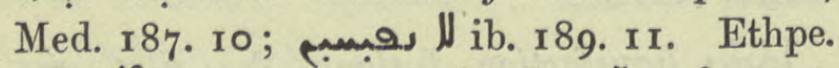

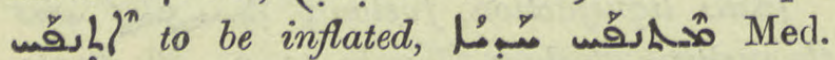
188. 22, I89. 4, I90. II ; to be swollen,

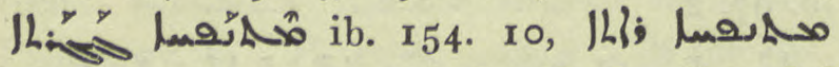
Hebraica iv. 214. 233 f. To be distended 6.

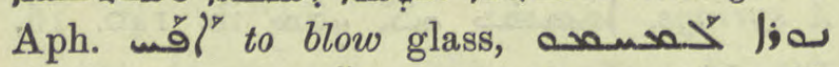
> Chimie roo ult. To

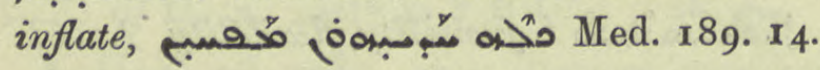

in m. a swelling in the chest, Med. 190. 18.

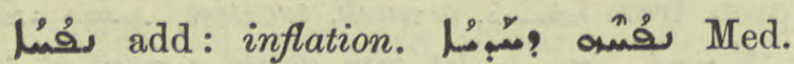
190. 20, 191. 2. 3. Snuffing or blowing up

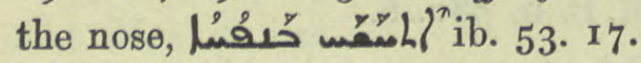

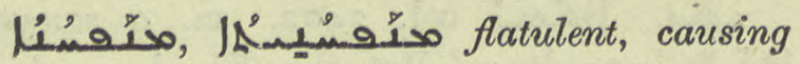

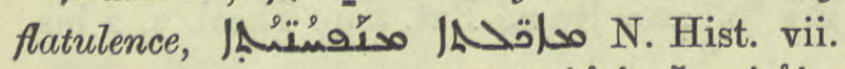

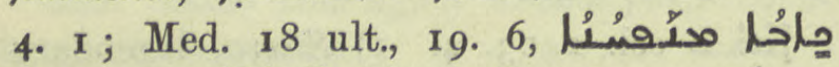

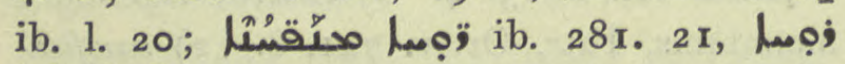

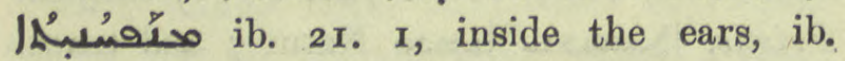
IOI. 2 , 19.

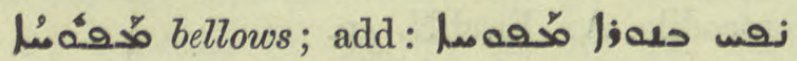

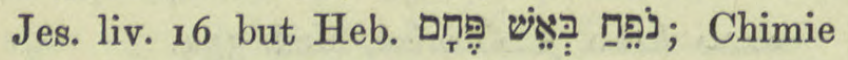
222 n. I.

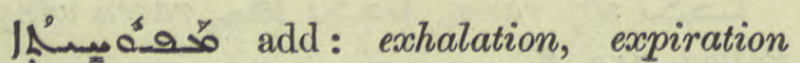

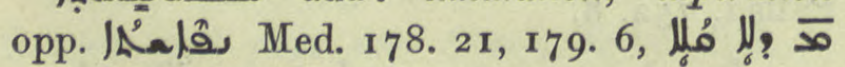
and

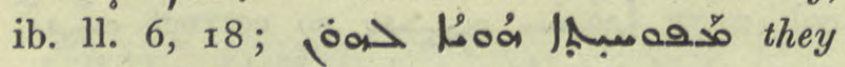
gasp, ib. 189.15 .

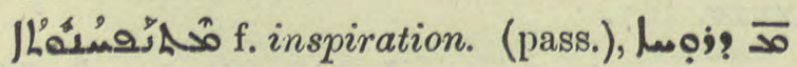
G. Busâmé 4 $b$ I 5 .

رa col. 24 II. naphtha. Add: :

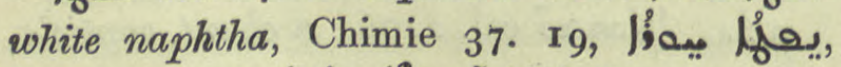

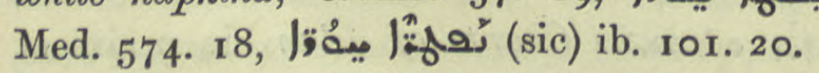

\. With preps. col. 24I3. With مـ:

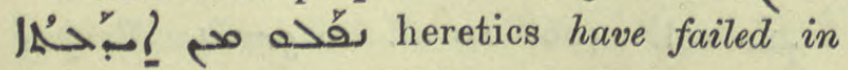
discernment, erred, Philox. de Trin. 78. $2 \mathrm{I}$;

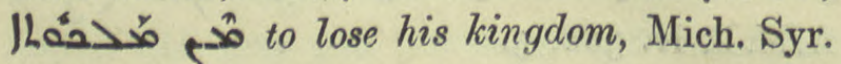

364 b 19; to fail, Tit. Bostr. 68. 18. With W. to be equivalent, Chimie 295. 18 ; to coincide as lines, BH. Stories 9. 12 bis. With $\mu^{\nu}$ to fight, struggle, in a contest with it, Ephr. Ref. i. 29. $3^{2}$. Act. part. \فُ col. 2412. I3. epileptic, فهُ  264. 12 ; مُّأ those who have fits at the beginning of each month, ib. ult. Pass. part. 스는 belonging to a case, DBB. I535 under

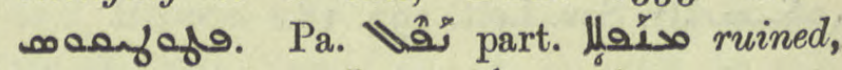

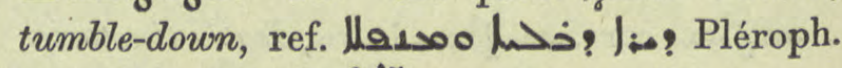
32. Iо. Aph. "ف̂े' to cause to fall away: with $\ln _{3}^{n}$ to turn from a purpose, Pallad. 137. 10.

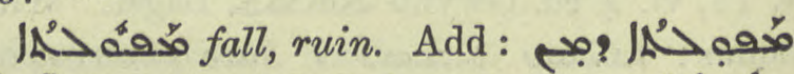
每 Pallad. 729. I5.

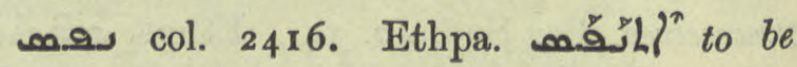
carded, Anecd. Syr. iii. 68. I 7 ult.

1ُ 1 ) wool-carding, Anecd. Syr. iii. 69. 2. 2) raw cotton, cotton-wool a desiccative laid on cotton-wool, Med. 64. 3; تُقْمَ

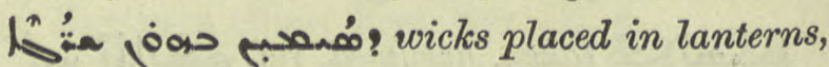

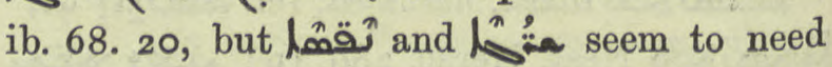
transposing.

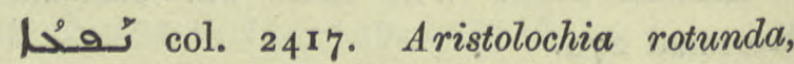
birthwort, Chimie 23. 18, 33. I ; يحُحم Med. 56r. 9, 563. 3, 587. $11,607.5$ where it is gloss to لمصحبه: JAOS. xx. 197.3, Ebed. J. Parad. ed. Cardahi 79. 8 ap. Nöld. ZDMG. xliii. 681 n. I.

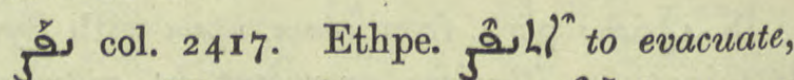
discharge, Hippoc. iv. 9 . $\mathrm{Pa} . \widehat{S}^{2}$ to purge. 1. 9 of par. after Galen, add: Hippoc. iv. I,

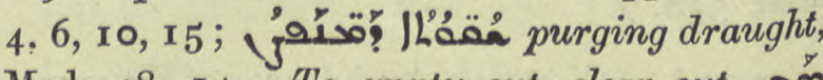
Med. 48. 14. To empty out, clear out,

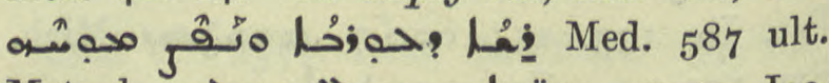

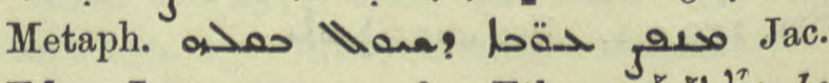

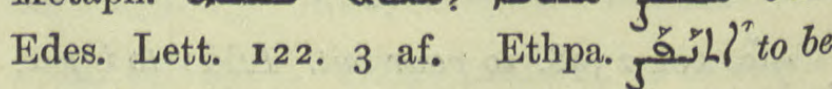
purged, Hippoc. iv. 26,76 , vii. $5^{6}$; to

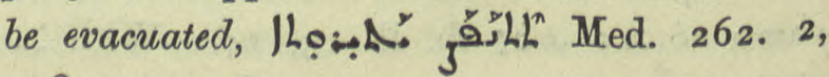
278. 10. 
l'َ̧s evacuation: excretions, Hippoc. ii. I $5,18,35$, iv. 5 and often, v. 33; Med.

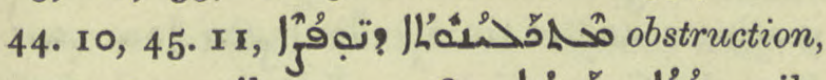

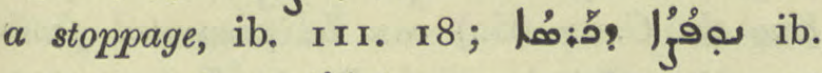
236. 2, 20; / م. of elephants, N. Hist. vii. 4. 2. Brandishing,

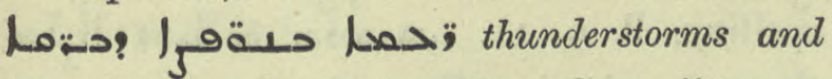
brandishing of lightning, Greg. Carm. ii. 32.4.

col. 2419 . to go forth, Ar.

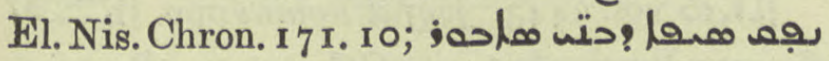
Jhin רah ib. 174. I5; I77. I5, I7. To utter, express, Sev. Lett. 30. 17, 31. 6,

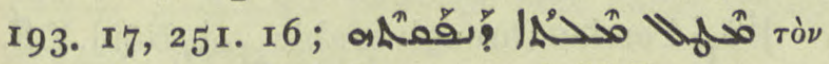

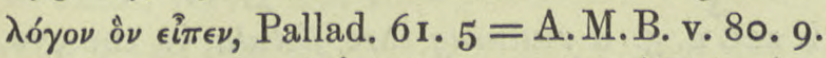

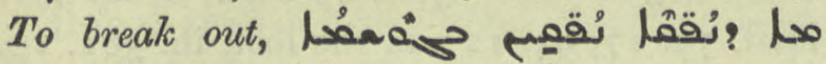

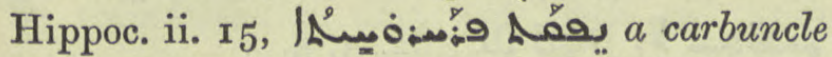
broke out, Pallad. 167. 14; Med. 583. 12;

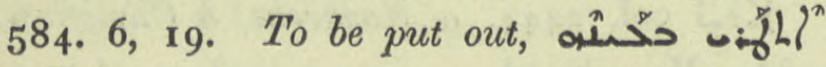
今مُه Pallad. 545. 17, 546. 2. To fast, go without food, ib. I5. 2; with /Lُ fasting, Pet. Ib. 46. 19. Aph. مीิ with nouns: ; ? $)^{\circ}$ to exhale, Pallad. 636 . 19 ; 'مُ to let blood, Journ. As. 1900, 89. 7 ;

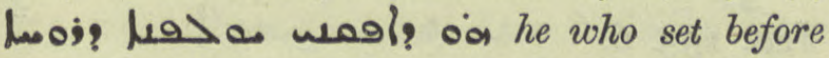
me spiritual teaching, Pallad. 110. 12;

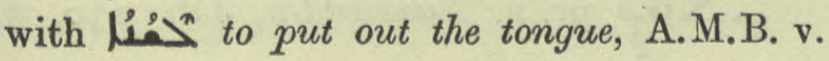
599. 9.

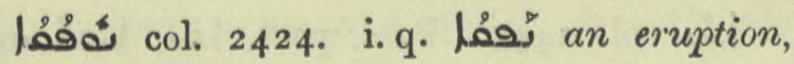
pustules on the eyes, Med. 84. I4.

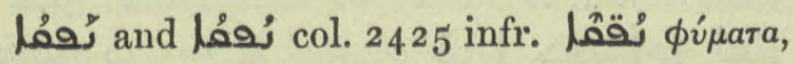
eruptions, boils, Hippoc. ii, I5, iv. 44, 45, 82 and glossary; it disperses a boil, D BB. 2012.7.

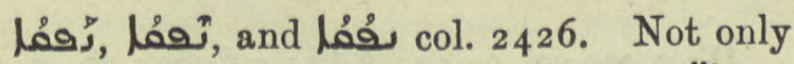
a mine but metal, mining products, 1.i) 149. I4 transl, 4. I4 and n. 2. But mines Ib. 59. I.

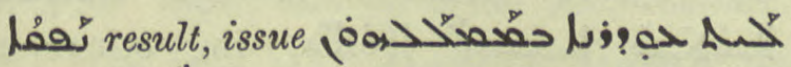
فمد of there is no advantage in discussions (of heresies) nor in their result, Pallad. 68. 8; lop But. Sap. Pol. x. 2.
A 1 consequently, Pallad. zor ult.

1Aمeas col. 2426. par. 7. Delete par. and see إمفي col. 2462.7 .

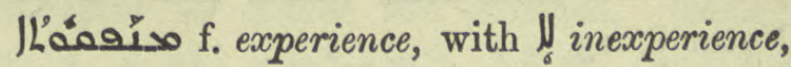
Is. Nin. B. 20. 2.

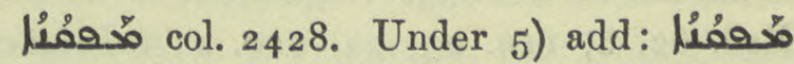

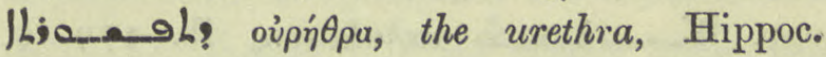
iv. 82 .

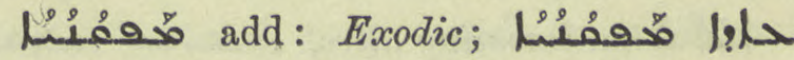
$\dot{\eta}$ єo I Cor. v. 8.

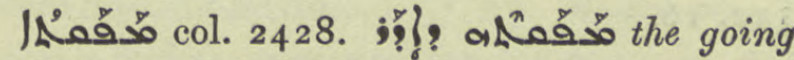
out of March, end of the month, Sev. Ant. Vit. 288. I I. ho: ? Hippoc. vii, $5^{8}$; $\phi \dot{v} \mu a, a b s c e s s$, ib. iii. 19, 25 , vii. 7. Cf. تُقْمَ.

كُّة col. 2429. to start, shy as a horse, with ר and pers. pron. Hormizd 3174. Ethpe.

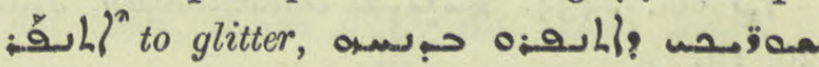
blessed be thy walls which shine brightly at the rising of His light, Ephr. ed. Lamy iv. 537. 23 .

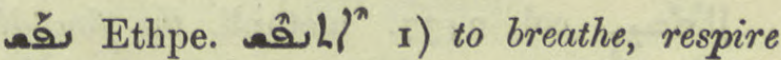
Budge points as if Ethpa.

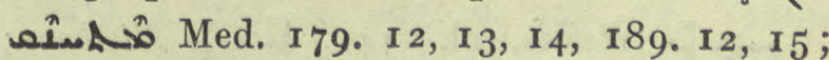

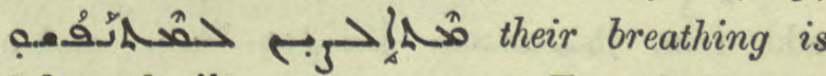
laboured, ib. 190. 2, 13. To exhale opp. كمب to inhale, ib. 193. 7 .

تُو

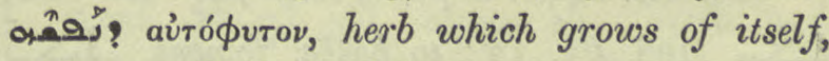

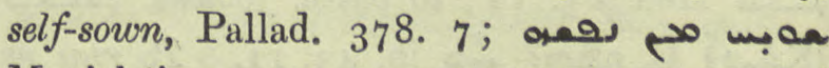
Manichéisme 109. 3 ; spontaneous, نهس ran récipient à digestion spontanée, Chimie 34 . I 4 , 39. I7; هُ0 ف0م" (monks) who despised the world and possessed their souls, Pallad. 85.7 , خa حبـ Jọ in what mood each monk went into church,

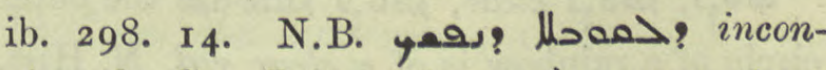
sistently, Sev. Lett. 348. I. 4) a grave stone, monument, add refs. Lidzb. Handb. I39. I, $3^{25}$, Müller Epigr. Denkmäler 67, Z. A. xix. I33 n. 3. Cf. Phoen. נפש. 
مُفِ col. 2432. breathing time, repose. Add: àvánavoıs, Apoc. xiv. I I ed. Gwynn,

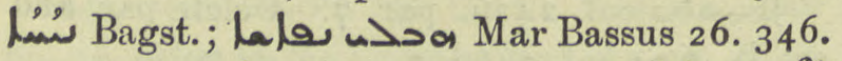
An air-hole Natur 8. I5.

12ำ sâ. col. 2433. respiration, breathing, Med. 62. 8, I19. 23, 24, I20. 2 ; inhalation opp. IR oxhalation, ib. 179. 6,

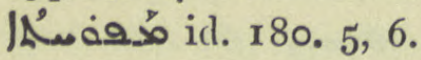

مح>ه. مع محسه. . becoming alive, lages the formation, constitution and animation of the Lord's body, Jac. Edes. Lett. I 22. 16 .

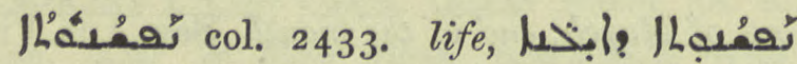
the vegetable life of trees, Natur 65. 16. Vivacity, courage, Josephus vi. 8. 2.

|Limate life, Mar Bassus 35. 483 , Probus 104. 8.

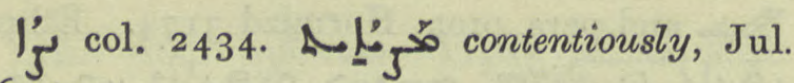
36,13 .

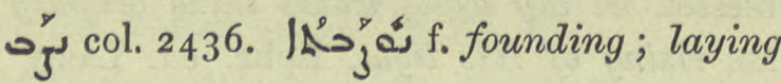
foundations, Philox. 4. 1 .

J's col. 2437. metaph. the founding of a school, Nars. ed. Ming. Pref. 42. 2.

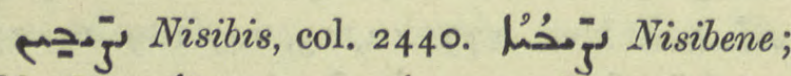

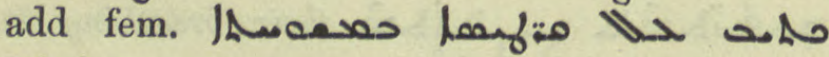
كَّك written on paper of the size used at Nisibis, Or. Xt. i. 300.10.

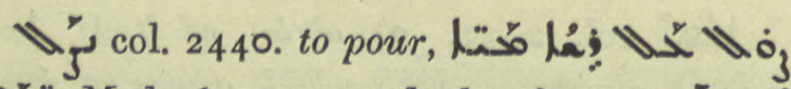

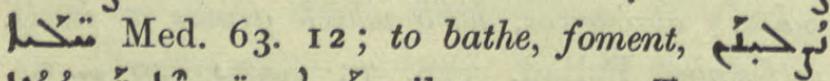

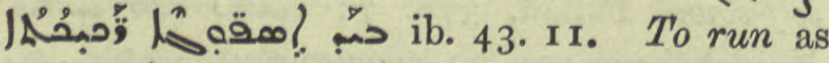

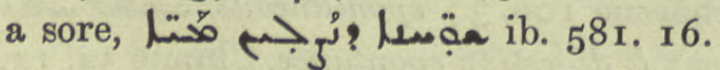

كُ لر

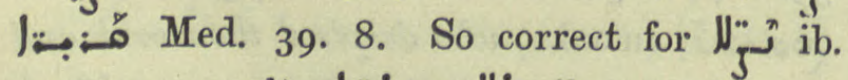

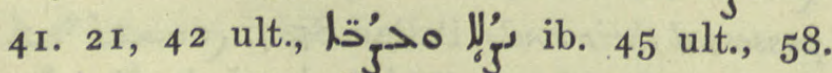
5, pl. ib. 20, 62 ult., 67. 7, 135. 9.

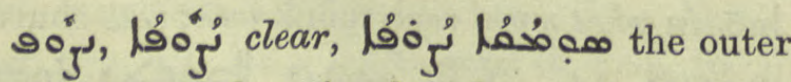
circle of a rainbow is of a clear red, N. Hist.

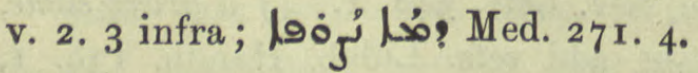

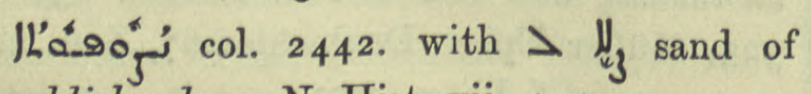
a reddish colour, N. Hist. vii. 4. 4.
'5 col. 2442. to make a harsh sound,

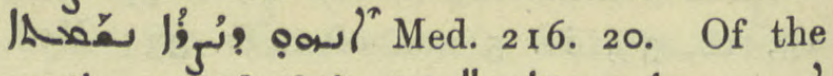

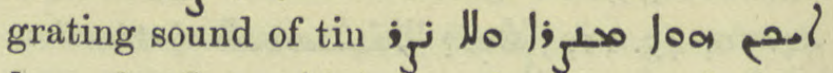
how the Crier (tin) can be prevented from

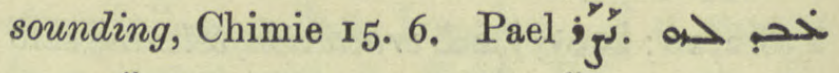
هis-

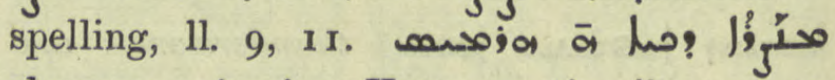
the pure crier i.e. Hermes = tin, ib. 53. 25 , I5. 6 .

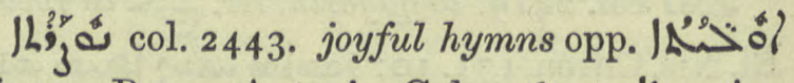
dirges, Brev. Ant. i. Cal. $5^{6} a$, Lng |ar ib. ii. $5^{2} 5$ a 2.

نُ ناسور Nöld.F.S. i. 220 n. 5 . a running sore, fistula, in the eye, Med. 79. 17, 94. 12 ; in the foot, ib. 58 r. 23 ,

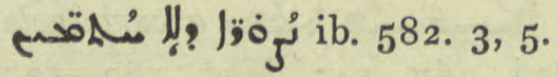

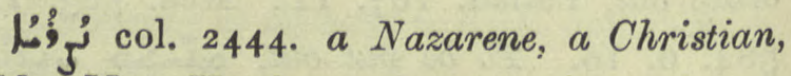
add: Mar Kardag 42. II; pl. m. 1. I4,

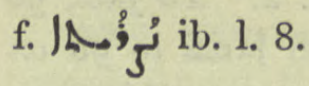

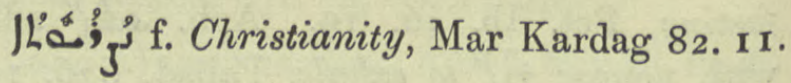

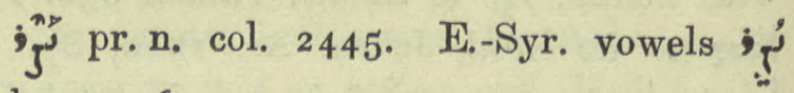
Jab. 155.6 .

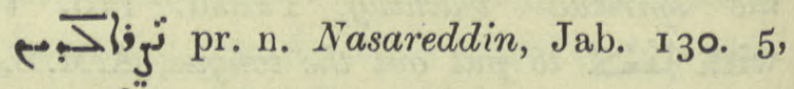
194. 3 af.

أُ col. 2445. Pass. part.

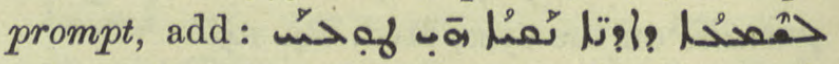
what we hear is readily forgotten, Pallad. 354. 6;

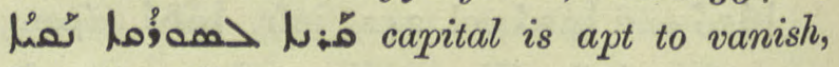
But. Sap. Econ. I. 2.

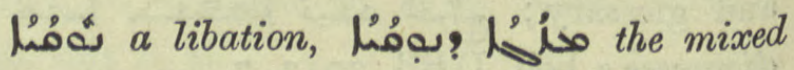

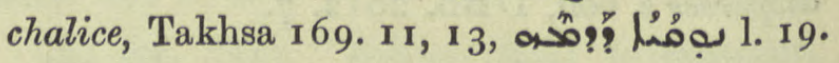

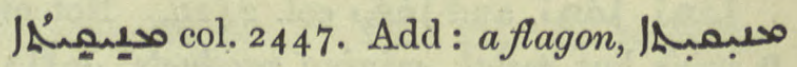

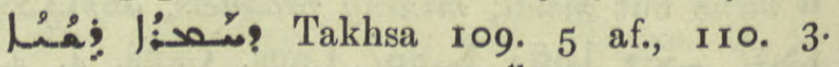

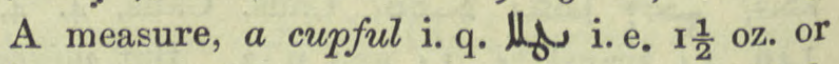

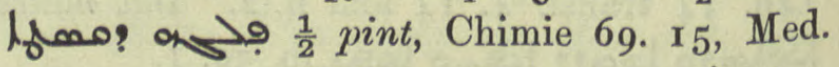
446. 13 , 14, ملم

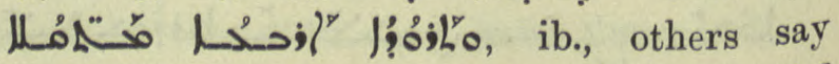
I menekitha $=$ I spoonful and I spoonful $=4$ mithqals, ib. Pl. abs. مهتمنُ ib. 55. 2,

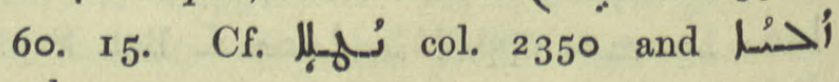
col. II 3 O. 
col. 2447. Pa. تُصح denom. verb from مُمُْم var. to م $^{2}$, Sev. Ant. Hymns I65 n.

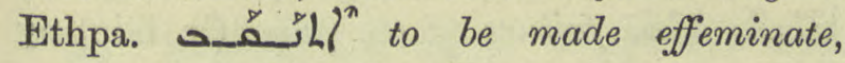

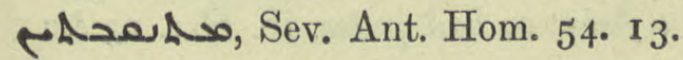

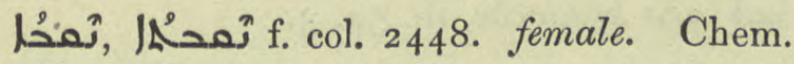
mercury, Chimie 19. 2.

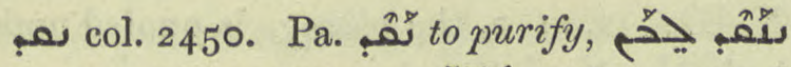
Pallad. 34. I r. Ethpa.

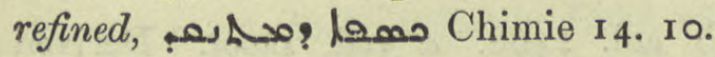

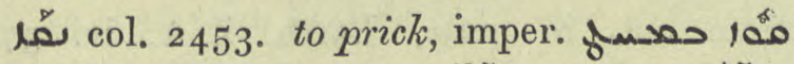

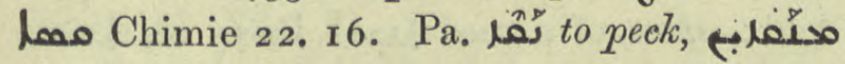
joal o pelicans peck their young to death, Natur 28. 1 .

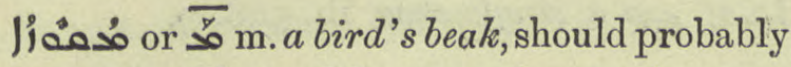
be transferred to this root, from col. $35^{1} 5$ under 10, Poznański Z.A. X. I 19 and cf. Nöld. Gr. § I 26. D.

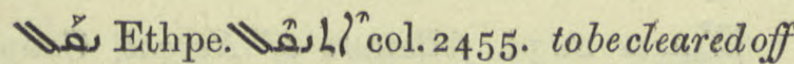

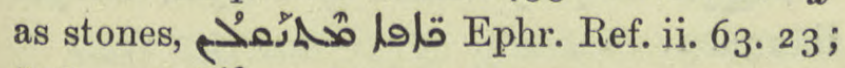

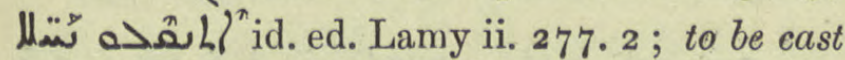

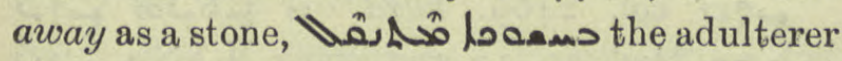
shall be thrown away into darkness, ib. iii.

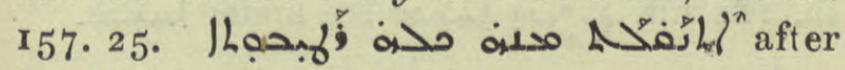
the Flood all moisture dried up, Jac. Sar. Hom. iii. 55. I.

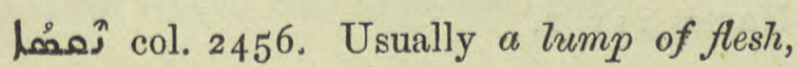
add: a lump or mass of molten glass, Chimie I00. $\mathrm{I}$.

คิ col. 2457. to adhere. Add: I) imper.

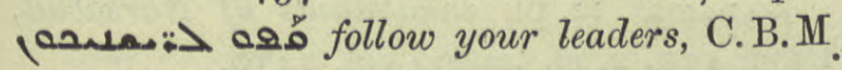
739 b, A.M.B. iii. 307 . 2) to follow, ensue, (jo) > symptoms follow, Med. 40. 5, Lay joall in consequence of their feeling cold do not suffer from thirst, ib. 1. 6; 291. 14 ; 10 مقصم lawful and proper resolutions, Sev. Lett. 179 ult. Ethpa.

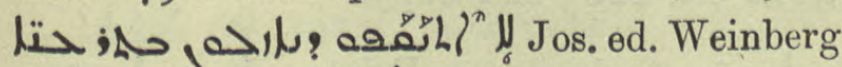

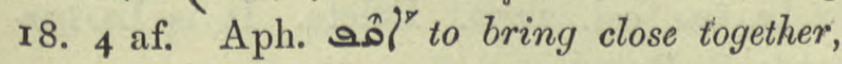

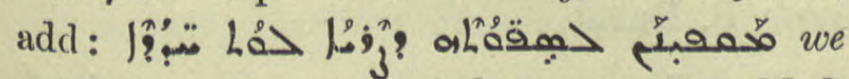
must bring the edges of the cut together, Med.

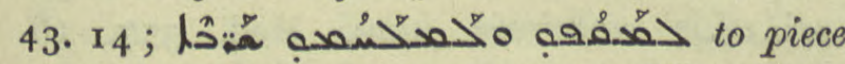
together and harmonize histories, Hist. Mon. i. 16. 12 ; س him whatever he was doing, Pallad. Ir. 6. To apply oneself with $>$ and a verb, a

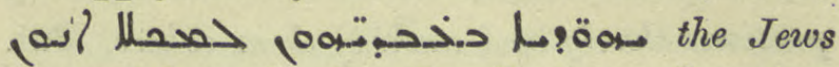
attempted to hinder the Romans, Josephus

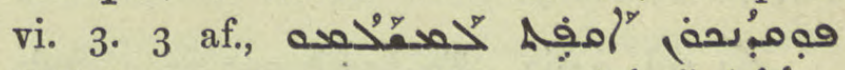

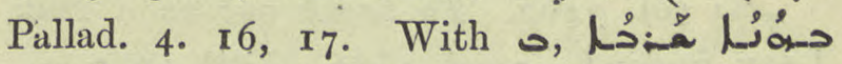

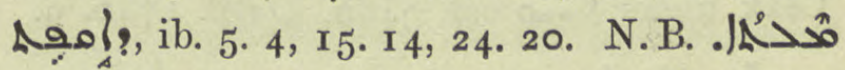

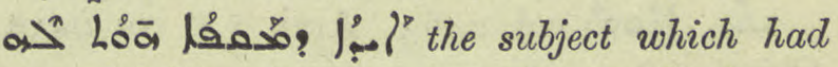
occupied him, ib. 80. 10. To give heed, pay

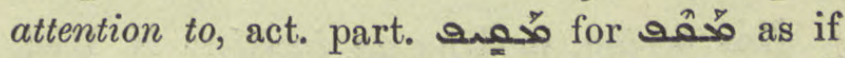
from a حaمe verb. Unless it should be q. v. col. 2460 and Suppl. BH. puts this word under قo Gram. i. 97. 23 . . even a fool is concerned for his body, Is. Ant. ii. 56. $11,204.7$. With مكمبه A.M.B. $25 \cdot 7$ = Pallad. 2 I. 16 which has

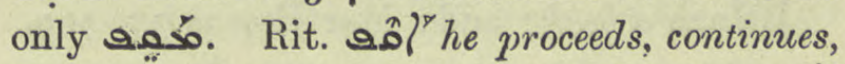

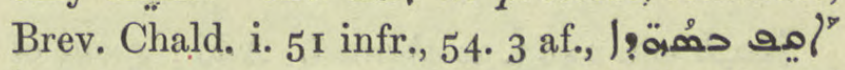
se goes on to the tune of the Blessed Martyrs, Takhsa I I 4. I 8, 23 , I I 5 bis. Usually

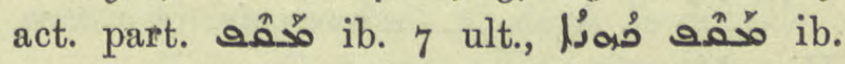
I 1 pen., I3 ter. Pl. مهم_a ib. 63. 8;

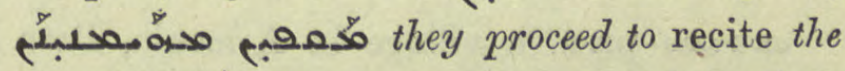
Creed, QdhamW. 43. 4 ; Brev. Chald.i. 26.8, 54. I 2, 59. I2, I3 and passim.

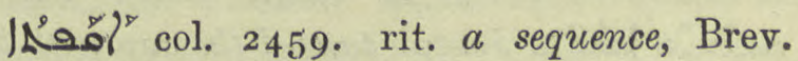
Chald. i. $238.3,295.6,308.22$, Takhsa 52. 16,53 quater, 54 bis.

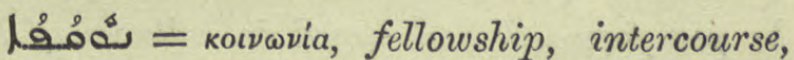

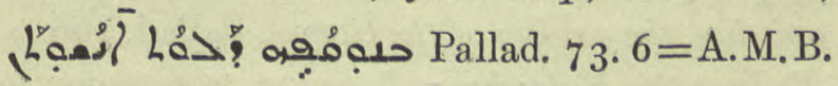
v. 96.3 , Schulthess n. G.G.A. 1895, 9. 679.

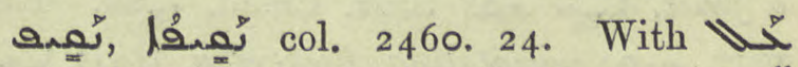
to stick to, pay attention, study, لـ لע |خ; a Ephr. ed. Lamy iv. 429. 7. m.pl. كُقبقَا adherents, “compagnons," Sev. Ant.

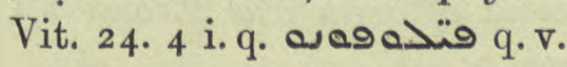

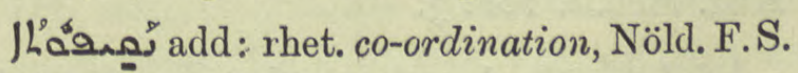
i. 485 .

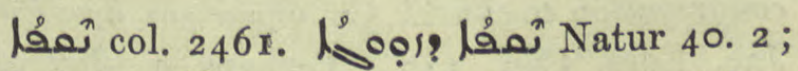

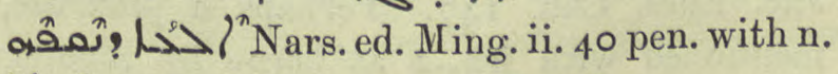
لُْ̊ conjugal intercourse; a wife.

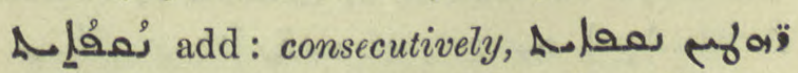
numerals run consecutively, Sev. Ant. Hymns 44. 2 ; in continuation, 1 Išoyahb ed. Duval i. 18, Nest. Hérac. 80. 2. 


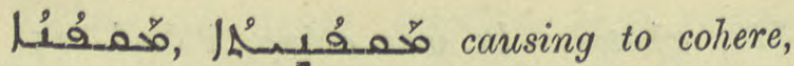

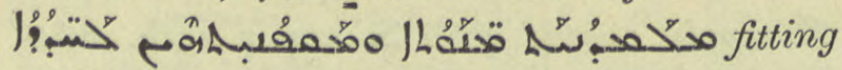
portions closely and making them cohere, But. Sap. Philos. 2. 4.

ـم้ 3462. To break out as a rash, Lexx. under مبمع col. 3486. D BB. I 7 I 10.9.

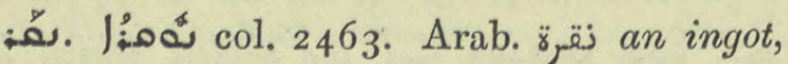
Chimie 38. 7, 40. 17, 52. 9, 10.

كُمْن boring, making an incision', N. Hist. iii. i. sect. 2 : cf.

تُمنُ

Jio 1 m. the beak of a vulture, Med. 589. Ir.

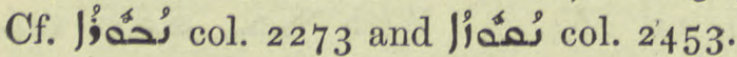

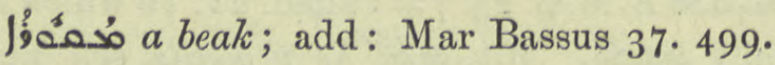

مَّمنُ 2463. a channel. Add : spout of

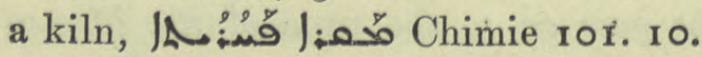

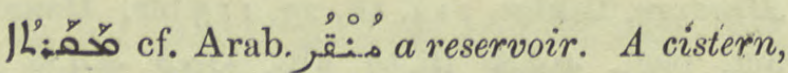
Hist. Mon. i. $225 \cdot 7$, ii. 426 n. 4 .

נم:0م pr.n. a village in Armenia, Patr. Or. v. 5. 730 , antep. and ult.

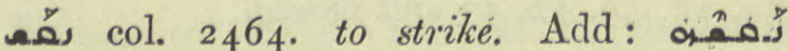
Joseph tapped the cup with his

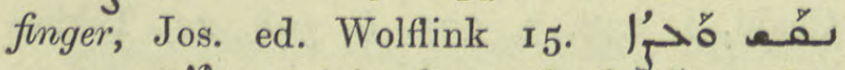

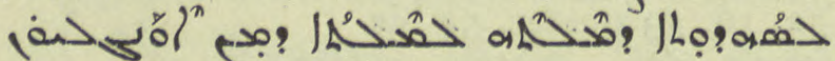
he put forward and urged in witness of his opinion, the saying of the Gospel, Pallad. r66.9. To beat the Nakusha = board = to summon to

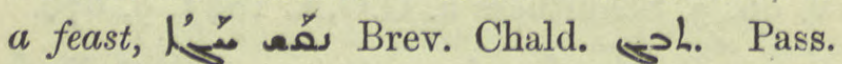

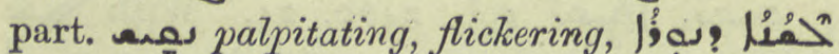

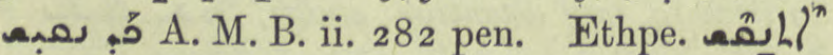

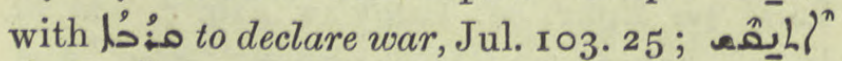

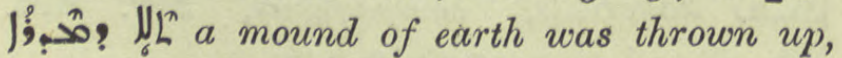
Hist. Mon. i. 206. 3 .

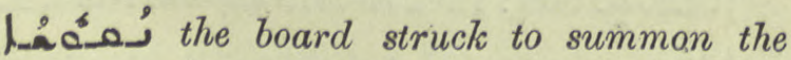
congregation to church, Gr. $\sigma \eta \dot{\eta} \mu a \nu \tau \rho o \nu, a ̈ \gamma ı a ~ \xi u ́ \lambda a$, Jac. Edes. Can. I 47. Perhaps a plectrum, mentioned with forne by Apollo,

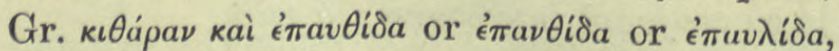

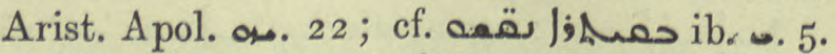

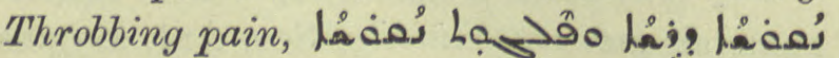
throbbing headache and migraine, Charms 79. I 2 ; J.A.O.S. xv. 28 5. 8. 9. sمُ sol. 2466.7 of par. the pulse; throbbing,

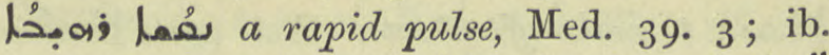

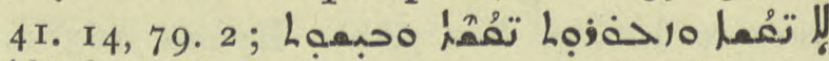

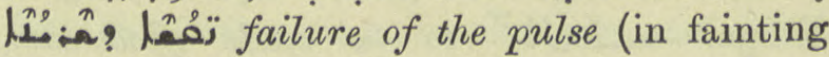
fits) and a faint or bad pulse, ib. 28r. r3 \&c. ; ل كعٌ headache, ib. 54. 3.

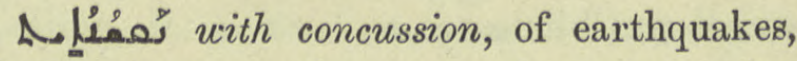
N. Hist. iv. II. 2, the passage is quoted under مَحمَ Suppl.

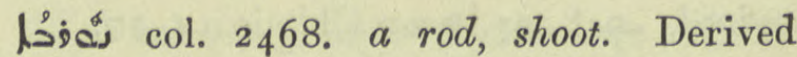
from sis to grow, Barth. Z. A. iv. 376 . Add: shoot of a reed

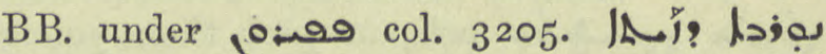
an olive shoot, Med. 561 r. 18 ; 人':il ب a single stem, ib. 598. 3 .

(ז) eol. 2469. i. q. Wis Nergal, Assyr. god of war, Coupes ii. r56. 8, 9. Mandaean ניריג, Mand. Relig. Brandt $5^{2}$.

A m. pl. followers of Nergal, Coupes ii. 156.1 .

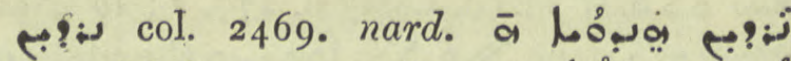

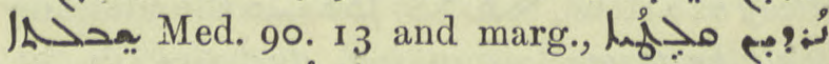

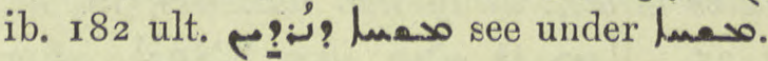

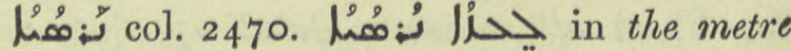

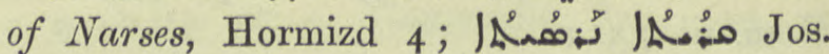
Narses I I I. 2.

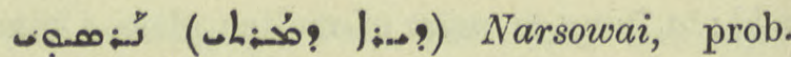
Jacobite saint, Jab. 483 . 5 .

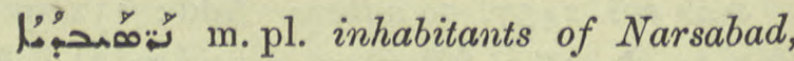
Dion. I10. 2, cf. لـ col. 2470.

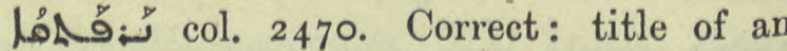
official, Act. Mart. i. 166.

ل Malapterus electricus, the torpedo or electric ray, Ar. FischN. 6r, Med. 11 r. 14. Cf. col. 2263 and Suppl.

aمةتمل col. 2470. Delete. It is pods, R. Duval.

مـ ." narcissus juice, Med. 557. I8,

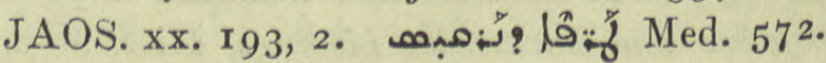
20. See under fore. 
ש col. 247 r. A village in Marga,

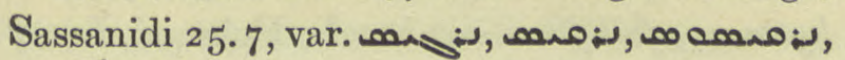
كَركيس Jab. 269.12.

col. 2472. sluggishly, Med. 539. 7 .

مُ col. 2472. to give over to oblivion, س.

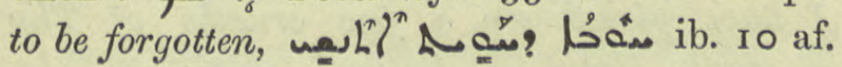
Aph. uevin' to make to forget. The quotationcol. 2473. I is to be found in Ephr. ed. Lamy

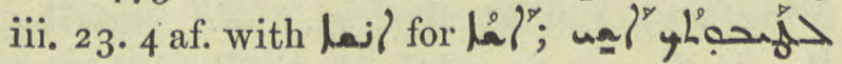
Hormizd $\mathbf{1 8 8}$.

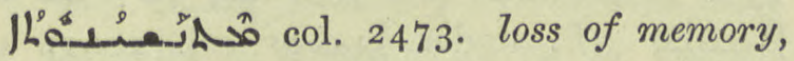
Hippoc. iii. 29, Med. 5. 12, 9. 7, 29. 9 ; a disease of the soul, But. Sap. Eth. iv. 3.

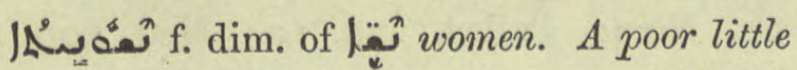

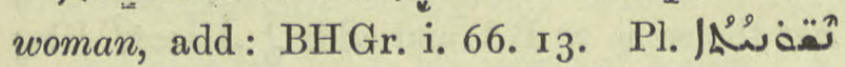
stupid women, But. Sap. Eth. iv. 5, BH. on 2 Tim. iii. 7 .

นُ

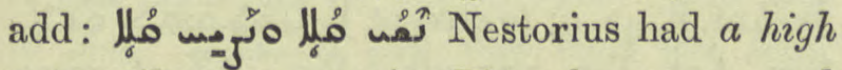
voice like a woman's, Pléroph. I2. 3 and

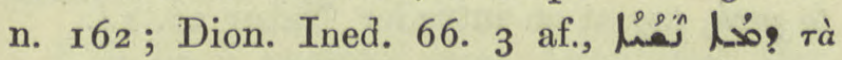

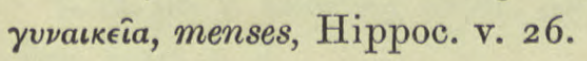

; Oftener ;

تُعَمُ

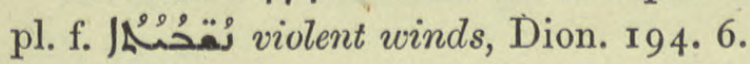

תمبر Chimie 59. 14 : see ses.

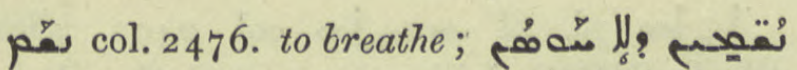
breathing out pitiless anger, Nest. Hérac.

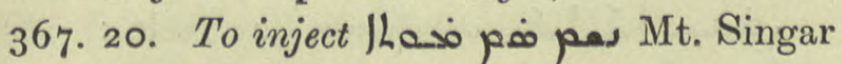
I5. 3 af.

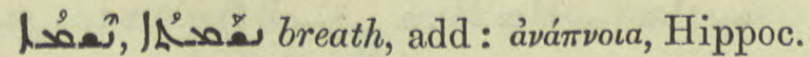

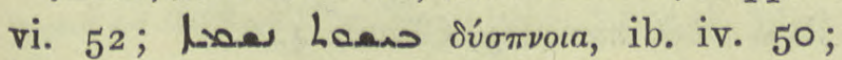

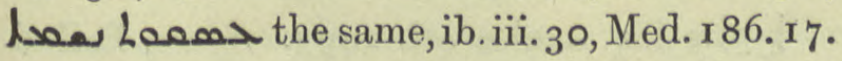

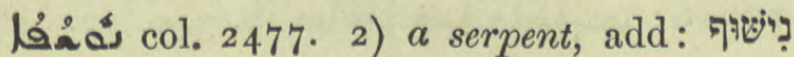
the hiss of a snake, Buxtorf; Pers. Mart. 77 n. 708 ; Harkavy Festschrift 40.

مas a snake. So Hoffm. corrects Yose> Pers. Mart. n. $708=$ A.M.B. ii. 678. 7 where Bedj. has foesa story is full of the casting out of serpents.
J m.i.q. J v. $535 \cdot 8, \mathbf{x} 2$.

"Lू' Ethpa. W L down, to bend beneath a weight, (0a) (Noa) Loa 14 I. 2 ;

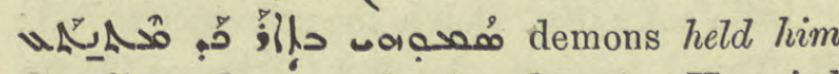
dangling downwards in the air, Hormizd 2834 .

ON col. 2483. to draw, add: لـ Natur II. I; agis a dra a fox his breath inwards when shamming death, ib.

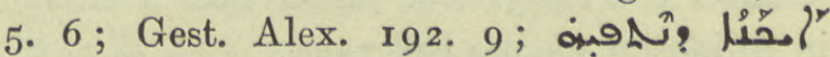
in order to draw away matter from the suffering member, Med. 42.20;

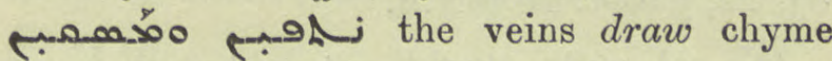
upwards, ib. 267. 10, ahُa $>$ A

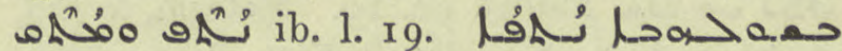
Awas the soul is ardently attracted to sacred history, Is. Nin. ed. Chab. 96 Syr. ult. L. I 9 of par. لل/ Pers. Lب آهـ Lag. Abh. 53. 20, B. N. I I. Pa. $0 \mathcal{L}^{\text {Av }} 7$ of par. add: : لإ ondistracted, unharassed, Syn. Or. 57 ult. Ethpa. $9 \mathbb{N}^{2} L^{n}$.

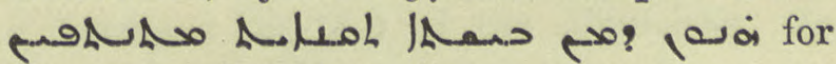

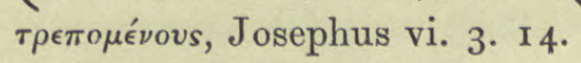

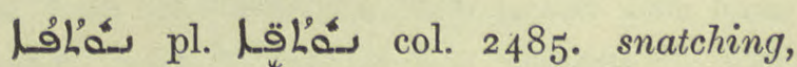
clutches of beggars, Pallad. 373. I6. Draggings away, Dion. 79. 18. Distractions, لV loles undistracted peace, Pet. Ib. 66. I7 ; ملإل بهم تبُْ Hist. Mon, i. $3^{8}$ I ult., Is. Nin. B. I. IO, I3O. I 4 .

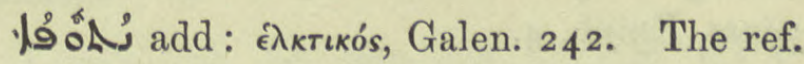
to BHEth. = ed. Bedj. 39. I2. Attraction, one of the four natural powers $\|^{\circ}$ of the body, Sut. Sap. Eth. I. 2, N. Hist. viii. 2. I, Med. I ro. I r. Adj. an alembic is tjonsoo looN draws upwards and whitens, "distille et blanchit," Chimie 19. 2, 1.901 "notre alembic pour distiller," 1.6.

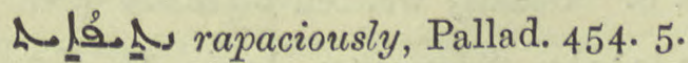

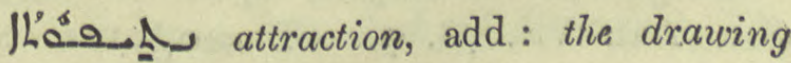
away of evil humours, Med. 47. 20, 65. 19, F $f_{2}$ 


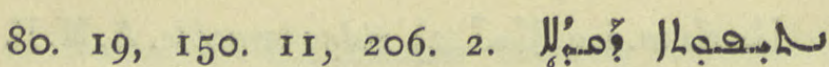
wryness of the neck, ib. I55. 16. Loa.d

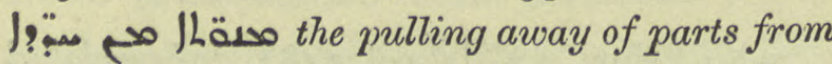
each other when a rent is made, N. Hist. iii. i. sect. 2 .

|

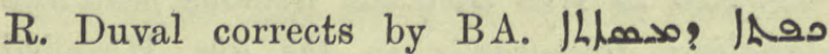

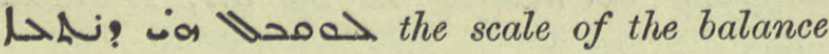
opposite to that which dips down, DBB. 69I n. r. Add: suction, drawing in the breath,

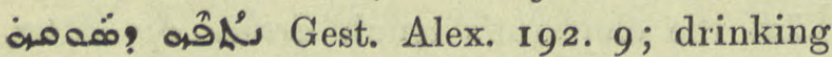

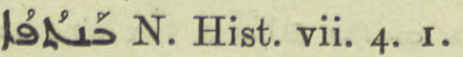

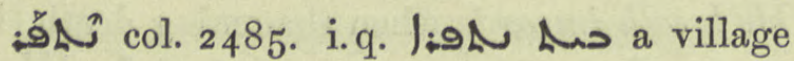
in Adiabene, cf. : col. $243^{\circ}$.

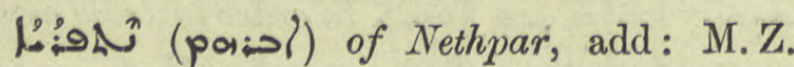
83. 2 I, Chast. 26 infr. f.

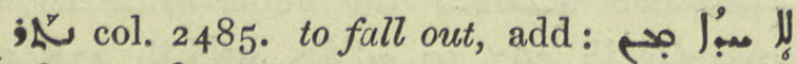

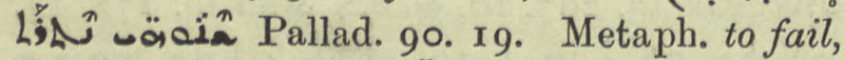
perish, ala> od

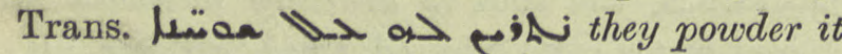
on sores, BB. under lin Al col. 287 , DBB. 216. Ix. Aph. jh/ to make to fall, to cast,

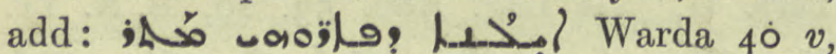

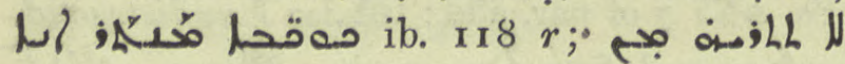

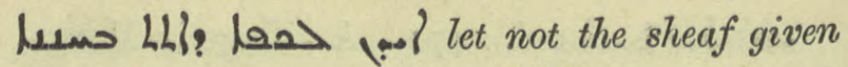
by mercy fall from our hands, Ephr. ed. Lamy iv. $425 \cdot 5 \cdot$. a depilatory, Med. 580. 13 .

引ُ 3 add: I) anything which falls; shavings, filings, يـئ| ?م- Gest.

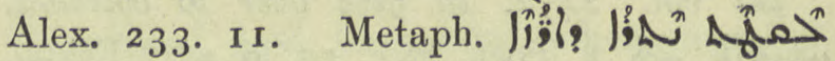
I have picked up a few crumbs of Thy mysteries, Ephr. ed. Lamy i. 705. 19;

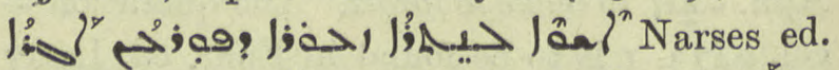
Ming. 233. I, Poet. Syr. 35.8 af. jow وقدبA the remains of the dead, Warda $118 r$. 2) a drip-board, oه oa

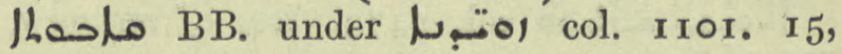
DBB. 679. 3) Nitre, Chimie 44. 6, Med.

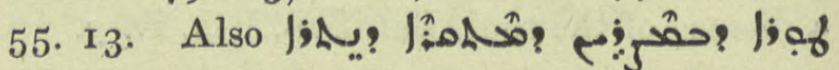
viz. Nitria, Pallad. 278. 10. Med. 606. I5 has the same as BA. and adds $\bar{\gamma}$.

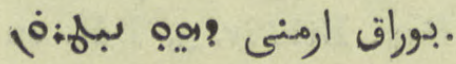

a col.2487. to tear. add: imper. an oL ol a $>$ oolo come, with eagerness seize and eat,

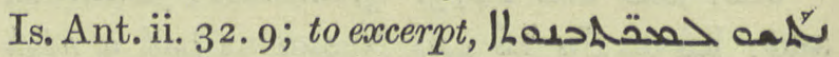
Sev. Ant. Vit. ro5 ult. Pa. $\mathrm{N}^{\text {ny }}$ of geese, to peck, tug at an intruder, Natur 22. 14.

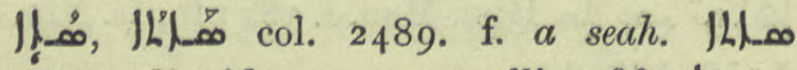
مقفي pints, Epiph. 4.

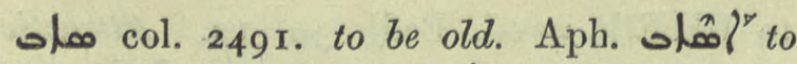
grow old. Add: loa oal Who was even then aged, Pléroph. 28. 3.

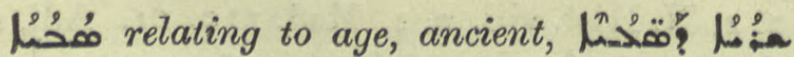
مُ مُ refutation of the conjectures of the elders, N. Hist. iv. 4.3 tit.

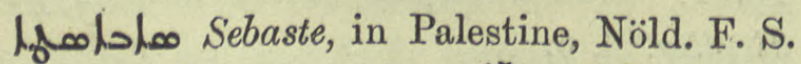

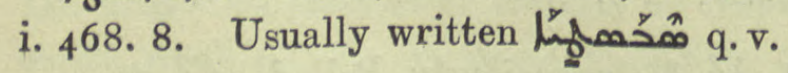

مof Sev. Ant. Vit. 78. 9. Cf. Nof far col. 2505 .

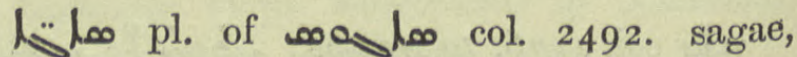
Sibyls, ZDMG. lviii. 497 on Stud. Syr. Rahm.

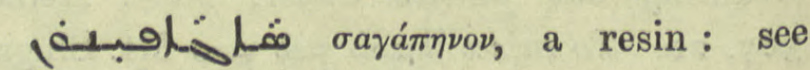

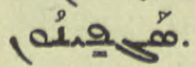

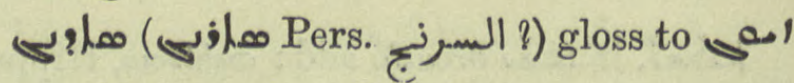
مarite mercury, Chimie 4. 6.



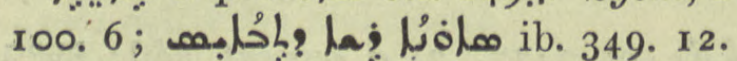

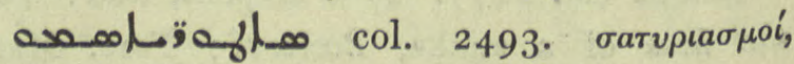
swellings, Hippoc. ii. 19 n. 4 , iii. 25.

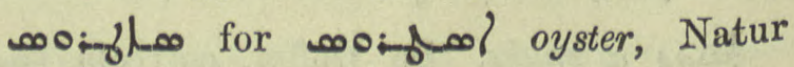
6r. $6, \mathrm{~s} 2$.

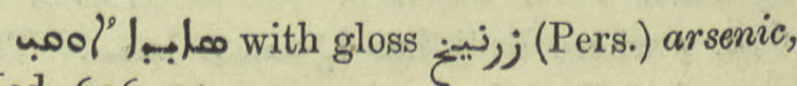
Med. 606. 20. 


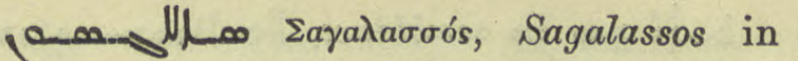
Pisidia, now Agulasun, Nöld. F. S. i. 473. I 1 o.

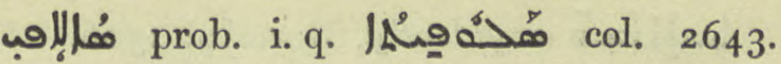
a turtle, N. Hist. vii. 2. 3 .

h:fo> 1 o see ho:9 Suppl.

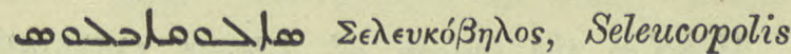
in Coele-Syria, Nöld. F. S. i. 469. 27.

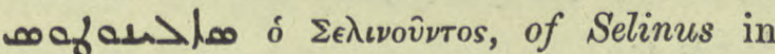
Isauria, Nöld. F. S. i. 47 . 75.

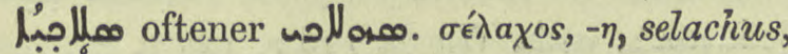
a shark, N. Hist. vii. r. 4 .

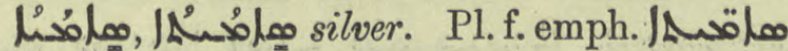
for IA or in a Comment. on the Psalms, WZKM. ix. 201 . 18.

on pierre argentée, argentiferous pyrites, Chimie 8. I7.

م 0 col. 2495. the island of Samos, 1. 2. corr. ناحية for for. Add ref. Jac. Edes. Hex. xii. 7 .

لم col. 2495. to put on shoes. Pael to shoe. مَّم shoe those who wear sandals, Poet. Syr. 99. 8.

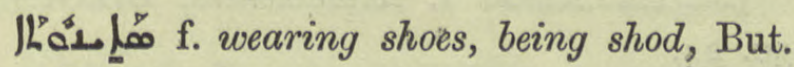
Sap. Isag. 2. 8.

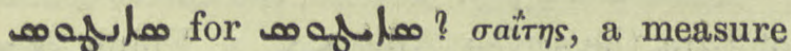

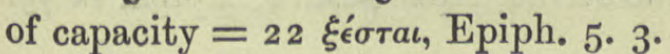

Saint Laurence, Jab. 67. 6 .

م Suppl.

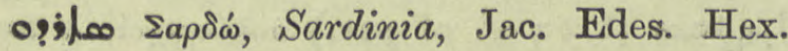
xii. 5. See 0 \%: $\infty$ \&c. col. 273 I.

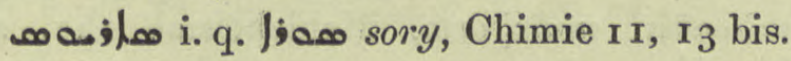

حمُ4 : مصل col. 2499. 5. Transpose

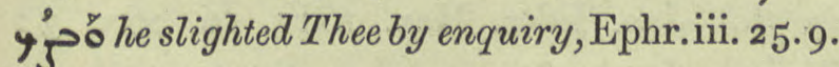

لم the savin, col. 2500.2 of par. add:

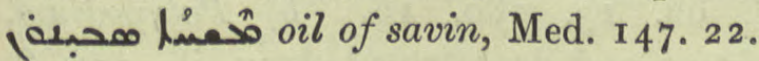

مُ col. 2500 . pr.n.m. Sabinus should be Olbinus, procurator of Tiberius Caesar, Hist. B.V.M. 97. I, xii. 2. See Duval Litt. Syr. 113 .

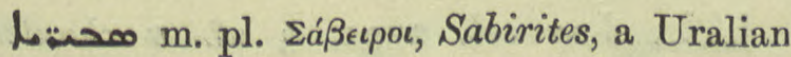
tribe related to the Huns, WZKM. ix. 92.
هُح a tree suffering from drought sucks up within it the strength of the water, Is. Nin. 3 pen. Pass. part.

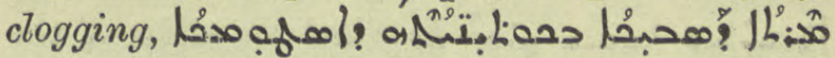

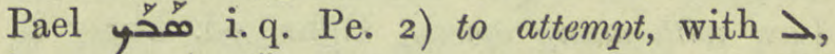
م- Dion. I3, 2,

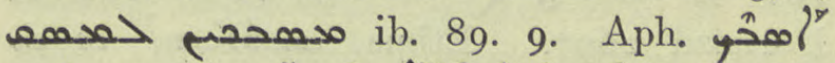

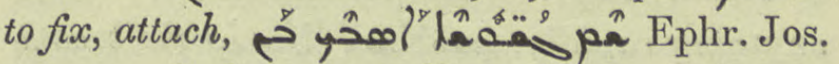
I 76. 4 ; هُ Satan attached mankind to the service of the creatures, Jac. Sar. Hom. iii. 8or. 4.

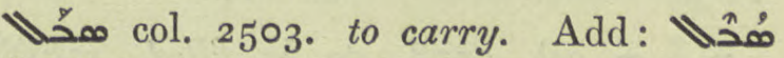

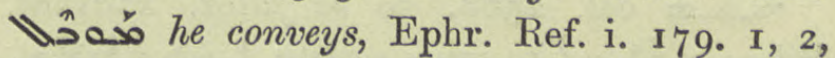

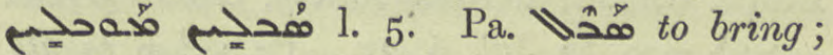
to inflict. Add : 1 of $>$ a

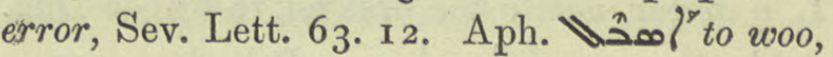
a) "

لِ corn to the threshing-floor,

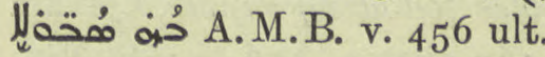

مُح part. yet points in the second manner for $\delta$ ' $\Omega \mu$ óópos (Epiph.) of the Manichees, the Supporter =Atlas, a giant who bears the earth on his shoulders, |A; معب/ Coupes ii. I 28. 5, 4, 3 af., Ephr. Ref. ii. 208. 39; Burkitt Introd. $\mathrm{cxxxv}$.

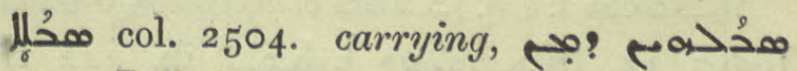
مهب:ح- Pallad. 48r. 3 of thieves carrying goods.

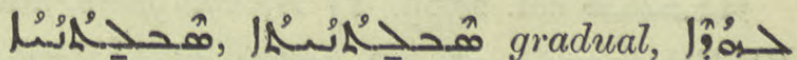

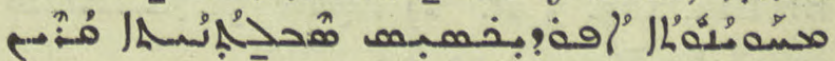
But. Sap. Philos. 5. 2.

لِّ

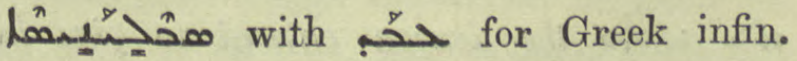

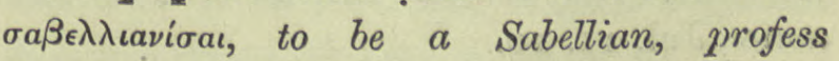
Sabellianism, BHGr. i. 47. I 7 .

1. Sibmah, col. 2505. 1. 3 of par. corr. the ref. to Jer. It should be Jos. xiii. 19.

u. ed. Maclean 4 I6. I3. 


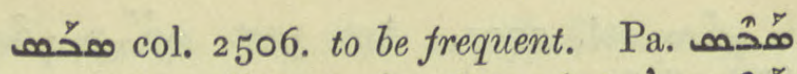
to multiply, Jiohes God strewed the sea thickly with islands, Jac.

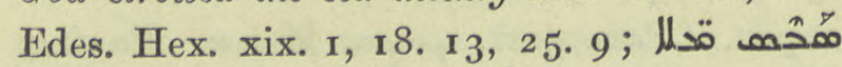

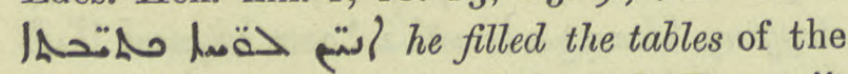
Law with many words, Ephr. ed. Lamy ii.

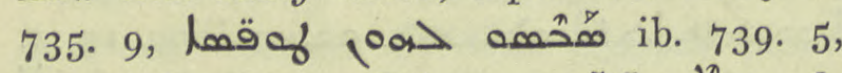
Hormizd 2697. Ethpa. ल anything rapidly, ing becomes rapid, Med. 229. 3 .

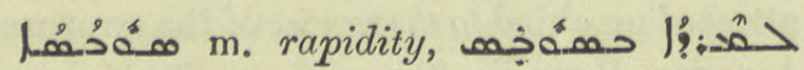
to travel at speed, Hormizd 2778.

טــ col. 2508 . to be filled, satisfied.

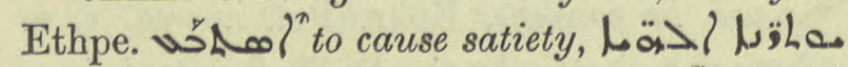

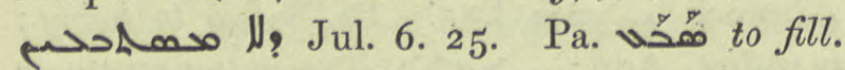
L. 9 of par. delete the first ref. to Kal-w-Dim.

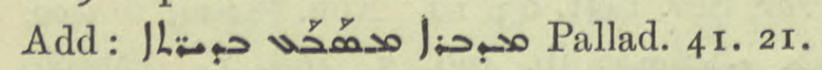

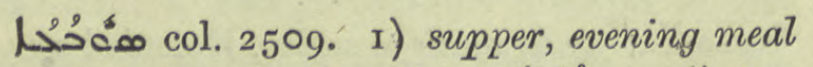

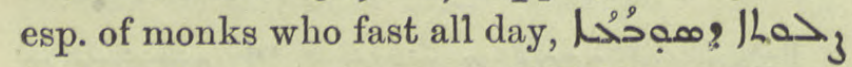
j;oL.9 grace after meat, Takhsa ror. 6.॰ 2) compline, service following supper, Qdham W. 128 quater, 167 quater, $176.2 ;$ B. Nin. 6. $\mathrm{I} 7 \mathrm{ff}, 7.2 .3)$ 3) Badger, Nest. ii 18. Also the place where compline is said. The latter is probably right

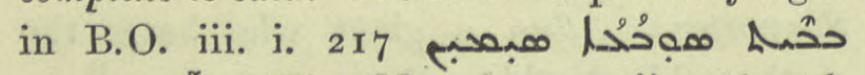
(ف)

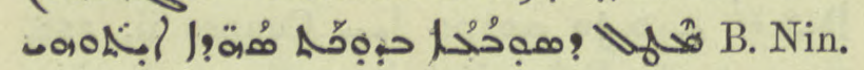
6 pen.

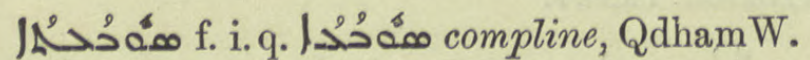
Index I 5 , 242 th. 266 ult.

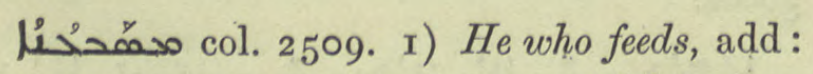
BHEth. 125.3 af. 2) replete, pl. مَّمَحْ opp. مقـ Išoyahb 52. 22.

مَح 2) to announce, inform, a o. a $>$ wolo the deacon entered to give him notice, Jul. I 1.20.

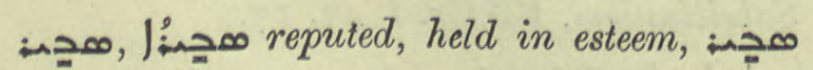
مa learning, A.M.B. ii. 565. Io; Joak'? لlal' , on nor can it be supposed that -, Jac. Sar. Hom. iii. 65. ${ }_{5}$.
A مصحتن: Sev. Lett. 5 I I ult.

入 1 confidently, Hormizd 2306.

IL' مـ col. 25 r 3 . news, tidings. Add: .

إهـ

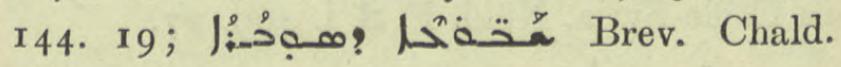
I. 27,7 .

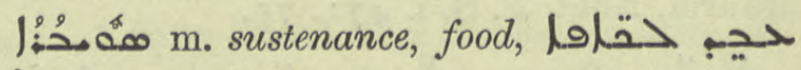

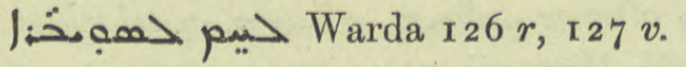

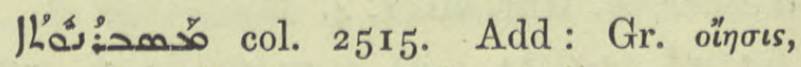

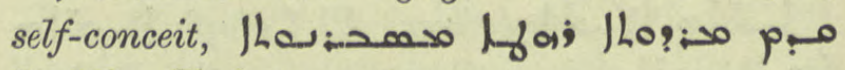
Is. Nin. Chabot I 2. 6.

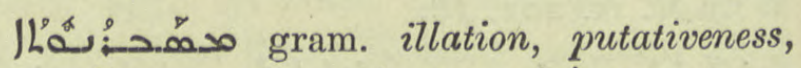

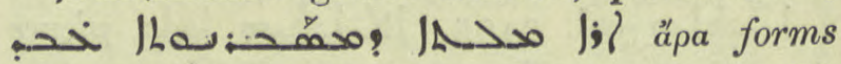
a putative sentence, Hebr. iv. 169. I4. Cf. BA. and BB. col. $172 \mathrm{r}$.

ox gram. expressing opinion, supposition, oo Tract. on Syr. conjunctions, Hebr. iv. 169. 9. Cf. BB. col. 1662.

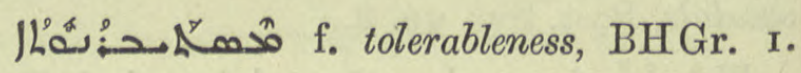
50. 24 .

120 col. 25 r 7 . Sabtha, the native country

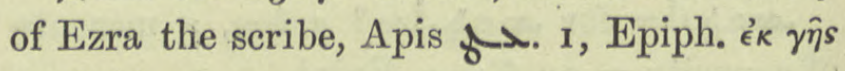

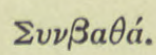

us col. 2519. Equivalents of Greek

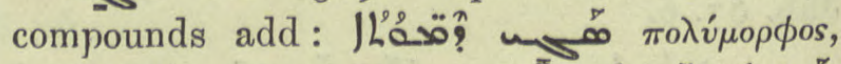
varied, Sev. Ant. Hom. 36. 9 ; полขто́коs, prolific, Natur 50 ult., Lلـ I.

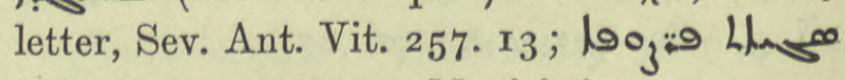

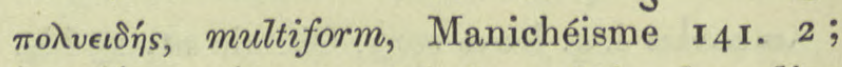
مبا in fish, Jac. Edes. Hex. xxiv. 16 , xxv. 3 ; 岁 a polypus, a growth in the nose, Med. 62. ×3, 180. х6. b) Polypodium, a solvent and deobstruent drug, ib. $35^{2}$. 8 , 365. 16. Cf. (0.00)

12. on col. $35^{2} \mathrm{x}$. Add: an anthem at morning service on certain festivals, Maclean.

مَفْ col. 252 I. 3 af. a chanter; chanting, Nöld. F. S. i. $4^{8} 5$. 
- col. 2523. Ethpa. Oَم prostrating himself, Hist. Mon. i. $340.2 \mathrm{r}$.

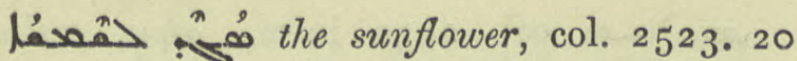

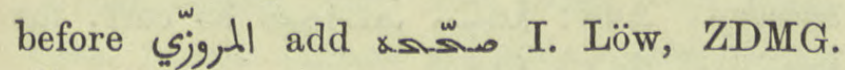
xli. $3^{6} 3$.

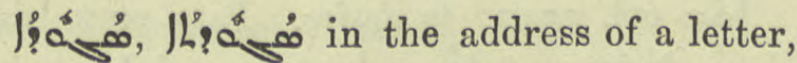
(פט, your Worship, John of Tella 3. 8.

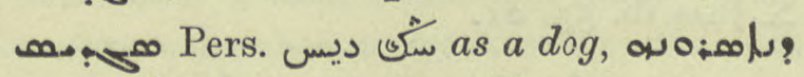
ס Phet. 29. 16, Pers. Mart. n. 562.

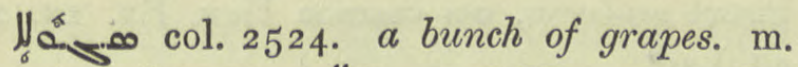
but fem. مهمة لال احمة DBB. 792 under IA of.

hefoos an inhabitant of Seistan: see

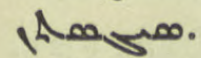

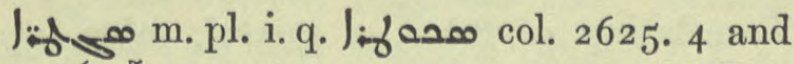
146. $9, \mathrm{I}_{3}, \mathrm{I}_{5}$.

$\prod_{6} \varsigma^{n}$ col. $2^{2} 5^{2} 5$. Delete end of par.

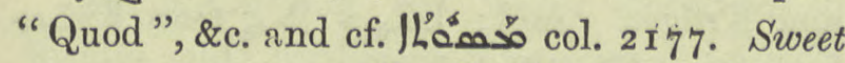

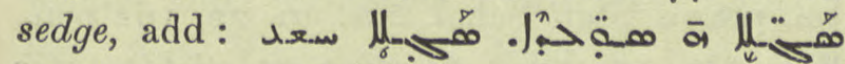
Med. 606. 20. Cf. J مَحبْ col. 2684 and Suppl.

J $\int_{-10}^{n} \mathrm{~m}$. Lat. sequester, a depositary, trustee, Dion. I28. I5. Cf. Iand I

20

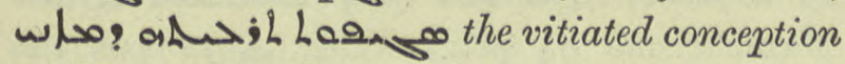
of Manes, Sev. Lett. 358. Io.

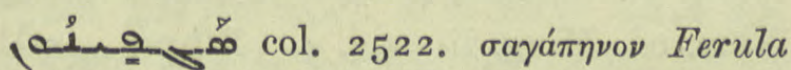
Persica, fennel and its resin, Med. 55. 6, 87. 9,

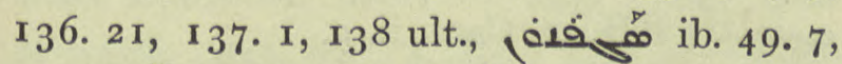

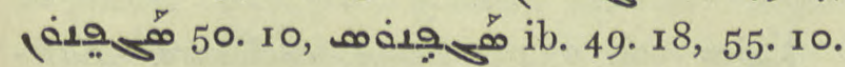

o to shut up, restrain, col. 2526.8 af.

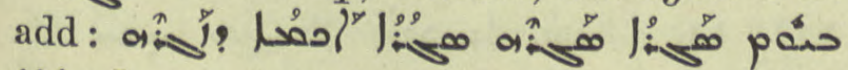

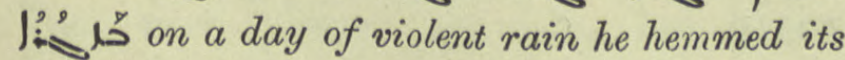
flood as he confined it by restraining it, $\mathrm{BH}$ Gr. ii. I I $3, \mathrm{I}_{3}^{24}$.

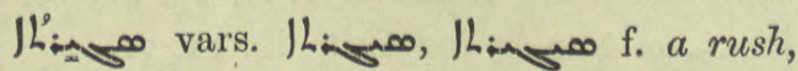
reed or sedge growing in stagnant water, the name may $=$ the impenetrable from its dense growth, Jac. Edes. Hex. ap. Nöld. F. S. i. 573 , f. حم grains of Sagira, J.A. O.S. xx. I88=Med. 554. 8.

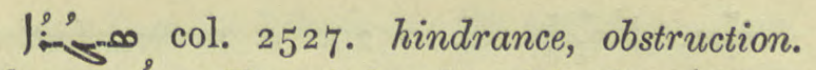

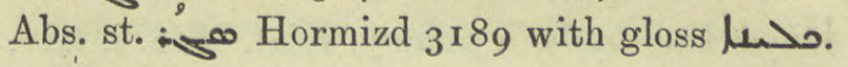

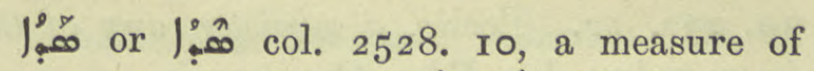
100 paces. Add: i. q. 200 cubits or the length of a furrow, Nars. ed. Ming. I.

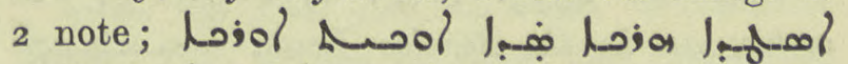

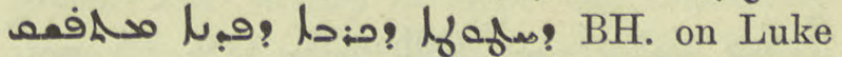
xxiv. 13. A Sabbath day's journey and so

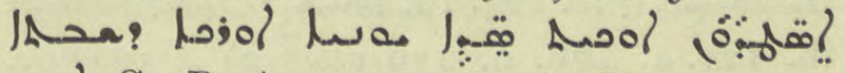
ळ) G. Busâmé $7 \mathrm{I} v 8$ af. Med. 531. 3 distinguishes between /

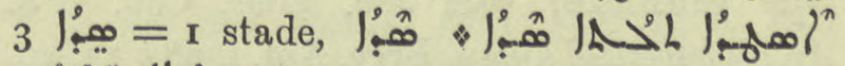
مُبار قمْحْم

هrab. Arue given as a gloss

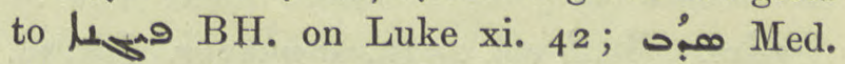
544. I2 $, 5^{81 .} 5,5^{82}$. I2.

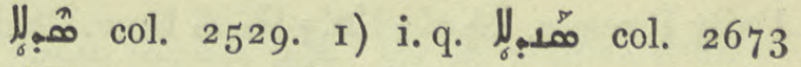

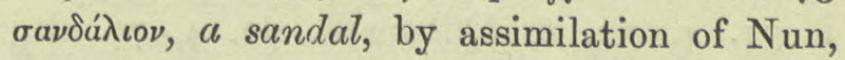
ZDMG. xlii. 392. 2) perh. Arab. Jِ a long string of pearls. Pl. Pr. levies or ranks, Hormizd 9 I I with gloss oيم: sing.

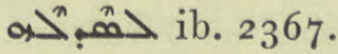

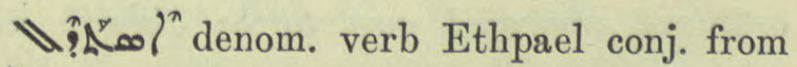
2) to be drawn up in a rank, to form a line, مormizd $324 \mathrm{r}$.

ڤึ. a block or flat piece of wood, A.M. B. v. 603. I3 with gloss 3 مُ

Ai) col. 2530. horehound, add : Med. 209. 21.

مُ0ْ col. 2530 . a sixth part. Add ref. $\mathrm{BH}$. on Prov. vi. 16 with var. مب:

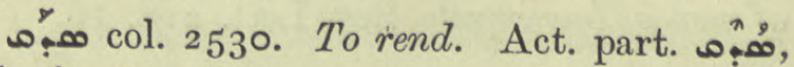

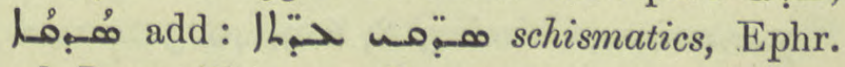
ed. Lamy iii. I37. Pass. part. همب

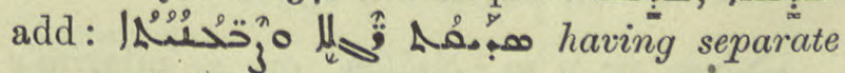
toes and fingers opp. webfooted, N. Hist. vii. I. 4, 6. 6. Pael part. لقِ

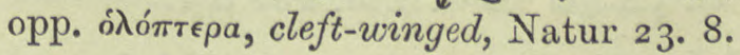

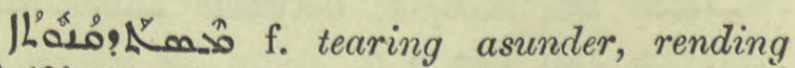

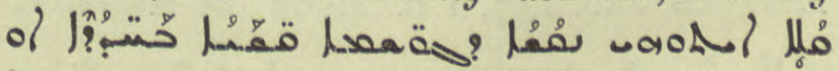
مut. Sap. Philos.

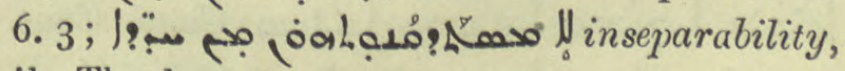
ib. Theol. 4. 6 . 


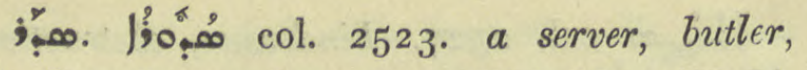
| 19, 232. 12. Eccles. a minister, any of the lesser orders, Jac. Edes. Can. I 10.

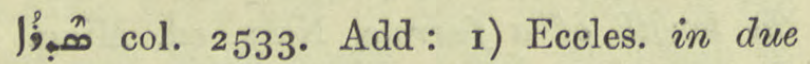
order, as in the Service Book, "أ| ز ib. 129. 4 , 178. 6, I79 ult. 2) A canticle,

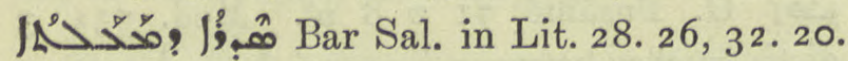
סمال Jac. Edes. Can. 7. 8. An alphabetical hymn, Brev. Ant. i. $5^{8} b$ 7, 20, ه-:

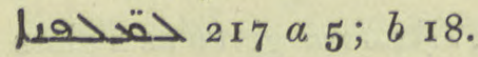

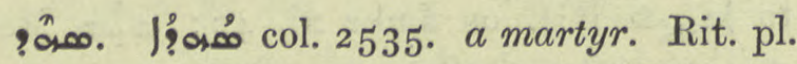
anthems commemorating martyrs said in the

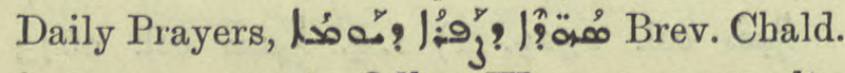
i. 51. $4,5^{2}$. 6, QdhamW. 72. 4,77 ult.,

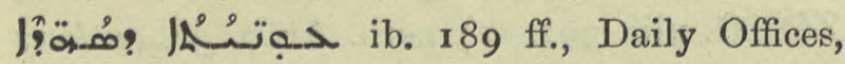
Maclean 12 and n. 2. N.B. witness, (a)

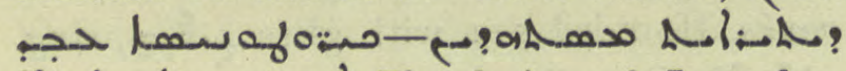

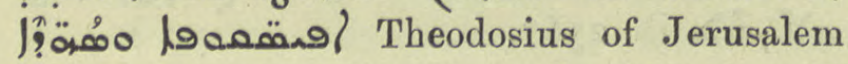
ordained approved men to be bishops and witnesses, Pet. Ib. 53. 4.

KLợan col. 2537. testimony. Hex. тò

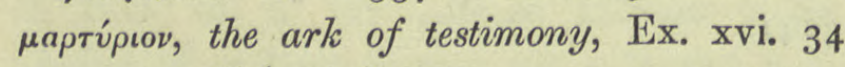

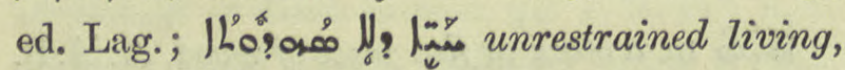
Sev. Ant. Vit. 108. 12.

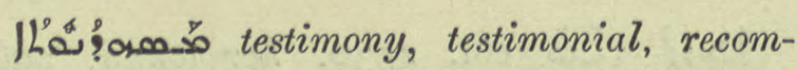
mendation, pl. Sev. Ant. Vit. I 5. 4, 24.8.

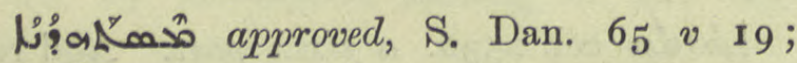

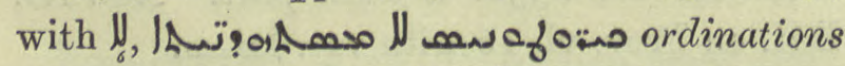
of men of low repute, Sev. Lett. 2 18. 16.

paro iron, Chimie $3.8,6.9,40.5$; poan ib. $221 \mathrm{n}$. Cf. poare col. $253^{8}$.

خan pr.n.m. Sahlon, on a pilgrim's inscription at Mt. Sinai, Journ. As. 1906, 292 ; cf. م000

بֵن Mt. Sahend, between Tabriz and Maragha, Jab. 147. I $\mathrm{n}$.

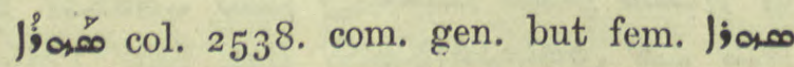
لمتر Caus. Caus.

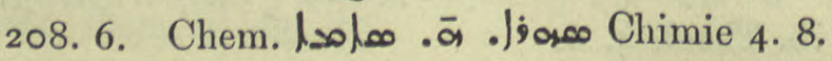

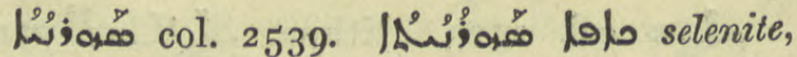
Anecd. Syr, iv. 100. 14. Cf. Chimie Trad. 3, n. II.

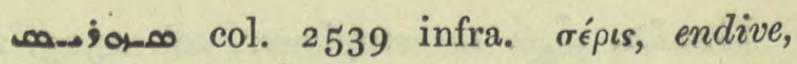
succory. Add: : مصف so so corr. for م. BH. de Pl. 148.

معُ col. 2540. to desire. Pass. part.

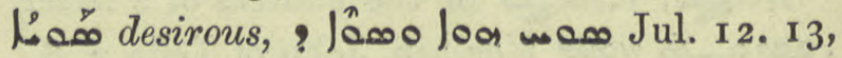
$>\int_{0}^{n}$ ib. $55.2 \mathrm{I}$.

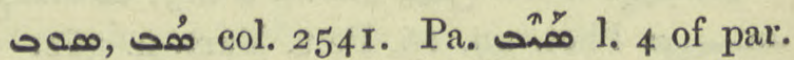

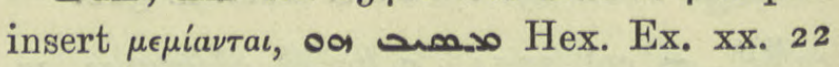
ed. Lag.

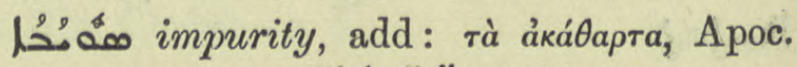
xvii. 4 ed, Gwynn /Ló.

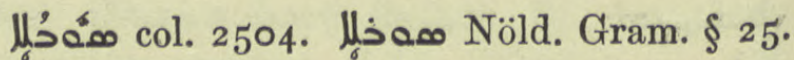
Translating $\sigma u ́ \mu \beta o \lambda o \nu, a$ symbol, token, sign, Sap. ii. 9 quoted Anecd. Syr. iii. 3. 15 but understood by Syrians to be $\sigma v \mu \beta \delta \lambda \dot{\eta}$, a clubfeast, Lexikalisches to Zach. Rhetor, Ahrens and Krüger. Add : مقحلا مهار o Sa: Stat. Schol. Nis. 30. 22, 192 pen., Syn. Or. 25. I7. A poker or stick to stir embers (Pogn.) Hippoc. Trad. iv. n. 2.

مe,

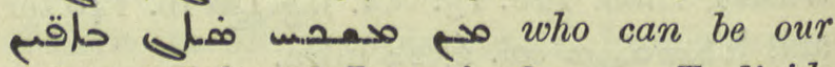
defence? Ephr. ed. Lamy iv. 829.9. To divide

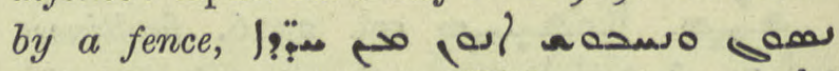
Manichéisme 94 pen. With o to put the

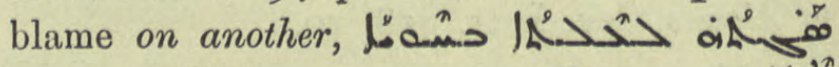

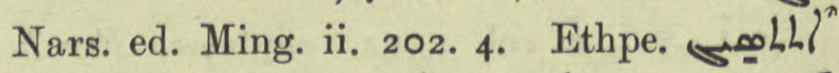

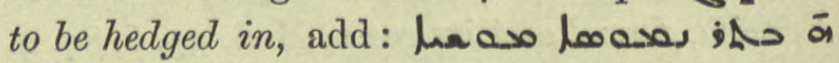
एolli? BH. on Cant. ii. 9.

Lُه col. 2543. hedge, enclosure. Safeconduct, Jab.

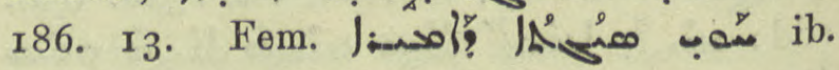
193. 9 .

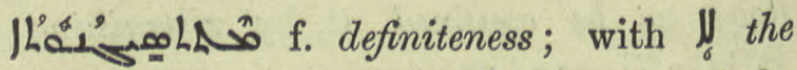
being indefinite, Anecd. Syr. iv. I5. 22, 16. 8 ; ther wold ol indefinite, ib. I2. 7.

ـof sat. scutum, a shield, Josephus vi. 5. 19 , mfors ib. 24 . II.

enf se $_{\text {? }}$ an ingredient of electrum i.e. of synthetic amber, Chimie 260 n. 4 . 


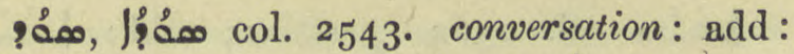
مسخصh : address, she changed her mode of address, Th. Mops. on Jo, iv. I I.

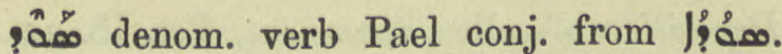

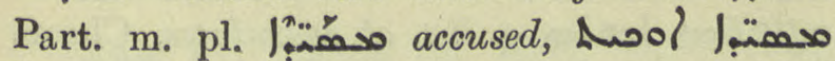
مهاتحلـ Kal-w-Dim. 300. I 2, Wr. thinks the word corrupt. م مصمن: Ming.

a a

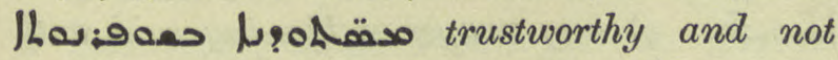
flattering speechifiers, Anecd. Syr. iii. 2 I 7 . I4.

مف col. 2545. Add ref. Sogdiana, Gest. Alex. 202. 4, 204. 16.

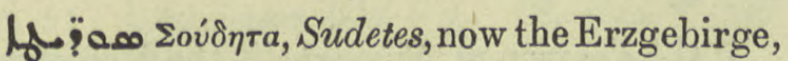
Jac. Edes. Hex. xxxiv. I.

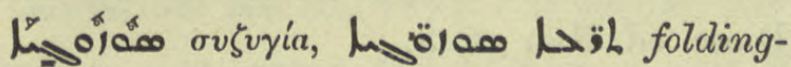
door's, portes à deux vantaux, Chimie Ior. 4 ; בـ as is paired, correspondingly, Hormizd 1964 .

col. 2546. Sozopolis in Pisidia, add : birthplace of Severus of Antioch, Sev. Ant. Vit. 2 I r. 5, Nöld. F. S. i. 473. I I 5, WZKM. ix. 97.

oمّ to long for, desire eagerly. Add:

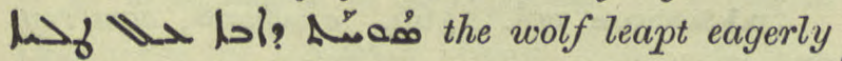
on the youth, Ephr. ed. Lamy iii. $3^{6 r}$.

J'ám. on col. 2547. desire. Add: BA. ed. Gottheil I 56 n. Io to /L'á.

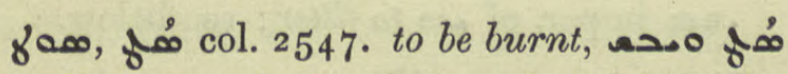
the olive tree is parched and dried up by frost, Ephr. ed. Lamy iii. 105. Pael صَّ

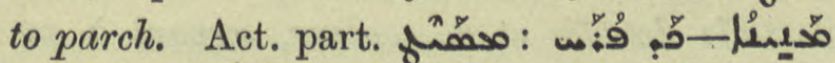

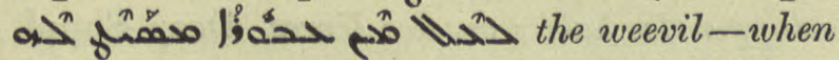
it flies over corn leaves it blackened as if burnt, BHGr. ii. ro7 quoted under مس مسار col. 2165 .

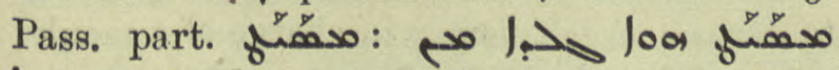
bon his skin was burnt by the heat of the sun, Vit. Mon. 93.22=Jo. Tell. 8r. 5. Ethpa.

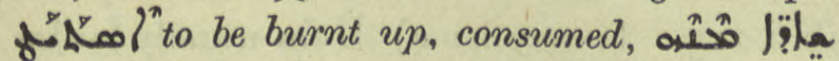
مormizd 1147.

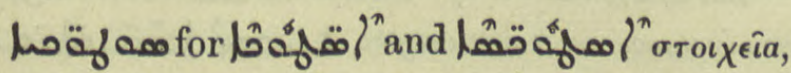
constituent parts, لـ $\frac{1}{12}$ th of the Zodiac, Rylands MS. 1917, I 6 6. I.

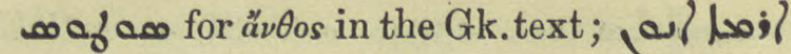
مarimie II. 20.

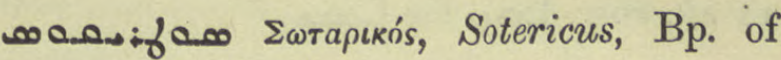
Caesarea, Sev. Lett. 61. I I, 291. 10, 387. 15.

4 finish. Rit. to say or chant the concluding

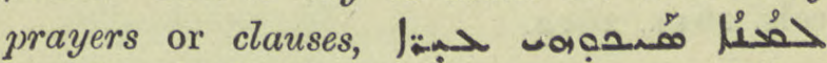

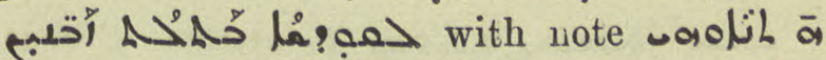
Nars. ed. Ming. ii. I34. I I ; محدم

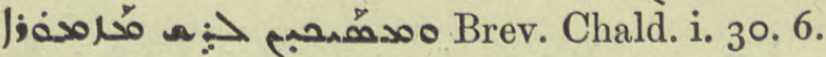

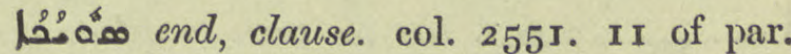
add: pl. قصه مُفْ concluding psalms, clauses or prayers, B.O. iii. i. $\mathbf{1} 78=$ Hist. Mon. i. I 42.7 , ii. 292 , Brev. Chald. i. $5^{8} .5$ af., Journ. As. 1907, 348. . Sev. Ant. Vit. 324. 9.

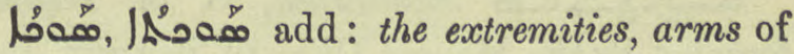
a shell fish, the nautilus

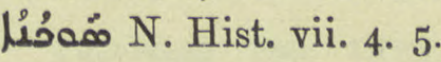

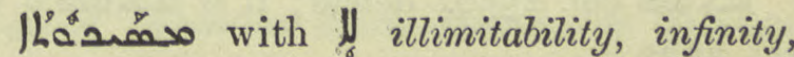
this is predicated of time, number, force (م) and measure (مُم) ل/حل N. Hist, iv. 3 tit. There cannot be JLana No two sides of a triangle, ib. Philos. 5. 2.

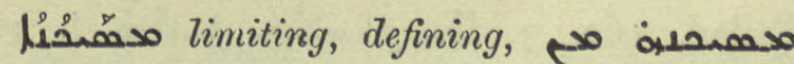

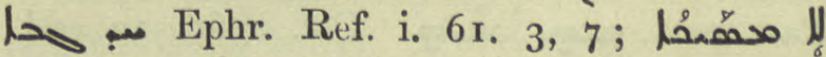

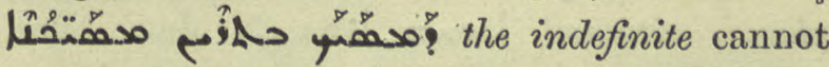
be defined (contained) within two limiting lines (two sides of a triangle cannot be indefinitely prolonged) But. Sap. Philos. 5. 2 ; Hormizd

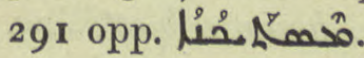

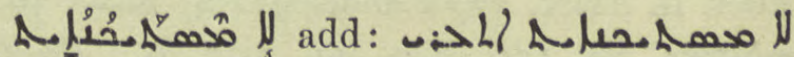
حمد sas the Word of God was contained in the womb without being confined, Sev. Ant. Hymns 4.

pـob. Socho, a village south of Jerusalem, Pet. Ib. 53 ult.

NoL. م مصُه نُم col. 2626 .

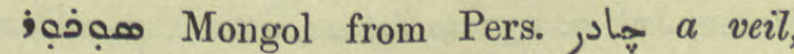
head-covering, Jab. 36. II, 97. 4, I3 1 ult. Cf. iA Suppl. 


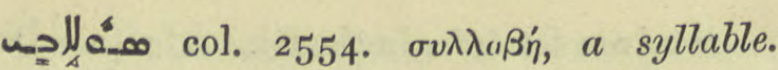
Add : مa Dحصـ Sev. Lett. 450. 6. Ll. 14 and II af., corr. hra for hasea.

مه حجمله: Silvester, bp. of Rome at the time of the Council of Nicaea, Or. Xt. i. 84 .

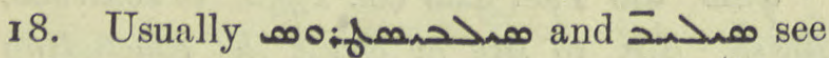
cols. 26 I I, 26I 2 .

حذب 92. 16.

" ow to syllogize: Len lest we turn to debate and reasoning, BH. Stories 35 . 187 .

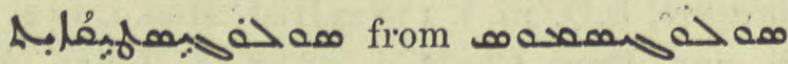
eol. 2555 and cf. 2 م \&c. col. 264 I. supported by reason, soundly, Tekkaf. $2 \mathbf{I}$.

Sultaniyeh, S. of Tehran, founded by the Mongol Uljai-tu, in $\mathrm{I}_{3}{ }^{\circ} 5$, Jab. I5I. 2, I78. II.

لمه col. 2556 . delete; it is lil q.v. col. 2639 .

(a)>a place in Egypt, Sev. Ant. Vit. 26. I.

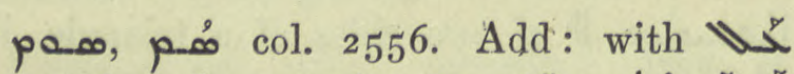

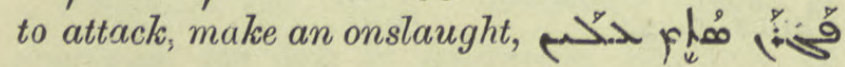

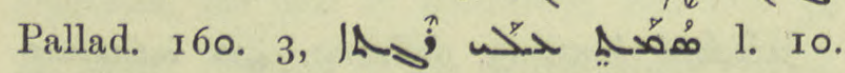

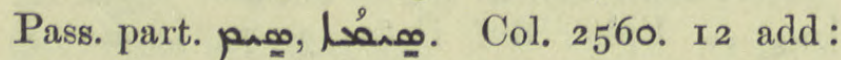
placed, situated

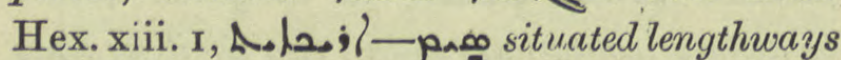
from west to east, ib. 1. 9. مal. Jul. 51. I. End of par. add: logic pro oa thesis, 'subject, Jac.

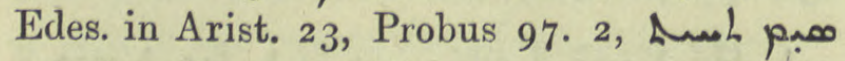
relative, ib. 106. 8/9, I1, 107. 9. Ready, prepared, avalus Jul. 45. I5, A.M.B. vi. 235.1 ; مصفحم معاتم able to see, Corp. Script. Xt. Or. xxvii. I I r. I2.

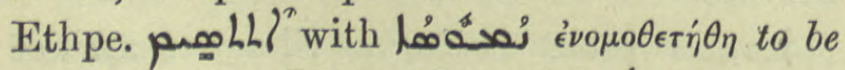
ordained, Sev. Lett. 48 I. 5 cf. $>$ م

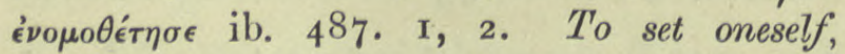
prepare, Swallian set himself with a great army to descend upon Rome, Jul. 7. 23 ; to set on, attack, prosh o

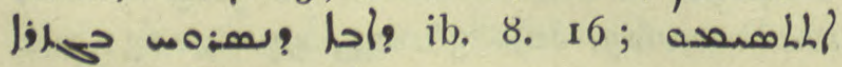
(oon حib. 24. 26.

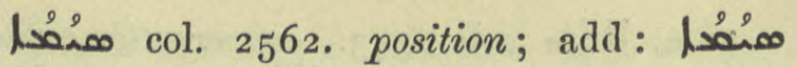
ISL being in a straight line, N. Hist. iii.

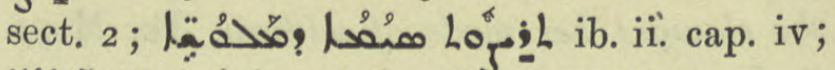
المُ Deposition of relics, interment, Pet. Ib. 33. 2. oflöa! L إم a list of consulates, Chron. Min. 269. 25 .

(2) ordination. 3) consecration of a church, also called

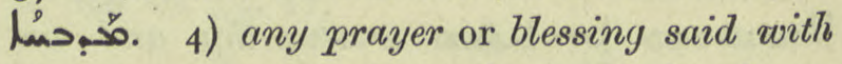
laying on or stretching forth the hands as Gk. $\chi \epsilon \iota \rho \circ \theta \epsilon \sigma i a$, Takhsa 65.4 af., $72.17,73.8$. 5) the Ordinal, Maclean. Cf. Brightman Lit. 578 .

$\left|L^{n} ;\right| s^{\prime} 0^{5} \infty$ pr.n.m. Sumarta(?) the Magistrian sent by Marcian to take Dioscorus into exile, Diosc. ed. $\mathrm{Nau}_{5} 6.8$.

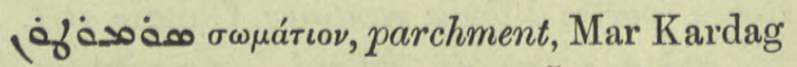

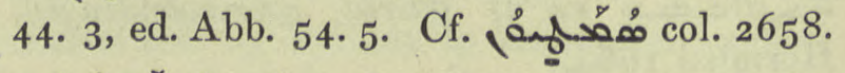

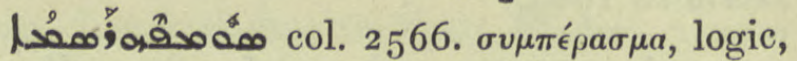
the conclusion. Ref. Probus 99. I 4, I 5, I6, I8,

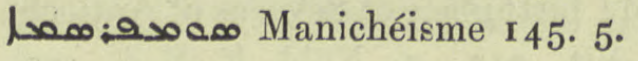

م Nöld. F. S. i. 47 1. 67.

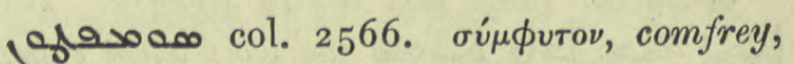
- Chimie 83. 15.

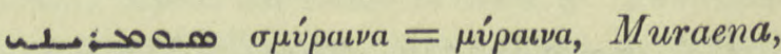
Ar. FischN. 553.

(م) imper. of to filter; see below.

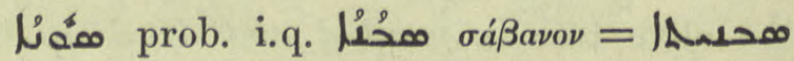

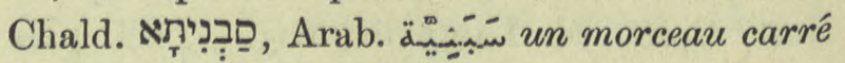
de toile doublée et de couleur, servant à envelopper des habits ou des livres, Dozy Suppl. i. $63 \mathrm{I}$, quoted by Budge, Hist. Mon. ii. +ro n. 3 ; eaves of a house, Ming. Two pilgrims to Jerusalem left money in charge of a monk ;

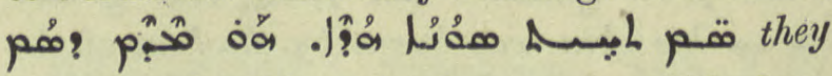
placed under this - ? whatever they did place, ib. i 2x3. It may be a) ficis usually foolo

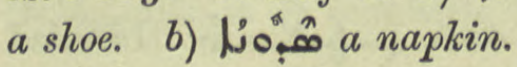

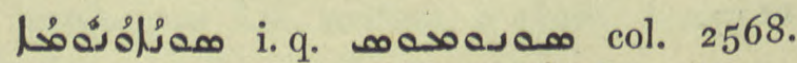

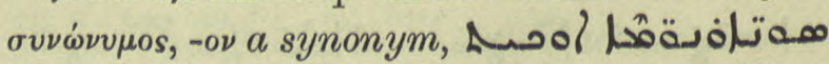
! But. Sap. Bk. ii. I. 2, Jac. Edes. in Cat. Arist. 2 I. 8, 30. 
manمe $\sigma u ́ \nu a \xi ı s$, an assembly, Sev. Ant. Hymns I 8 I tit. Cf. Cmenes col. 2570.

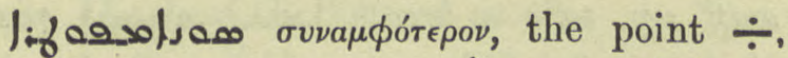
Epiph. I5. 20, p>n? of 0.f095/000 IA

J Phrygia Salutaris, Dion. 28. 2, 3, Weil Gesch: Chalifen i. 638, 0 مه 1 Ant. Patr. 302. I8.

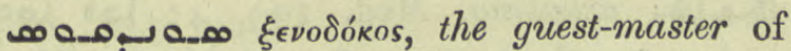
a monastery, R.O.C. iv. 340 n., 342 . I.

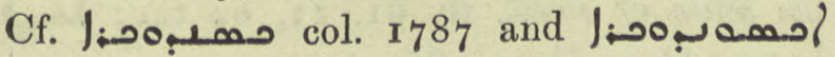
col. 185 .

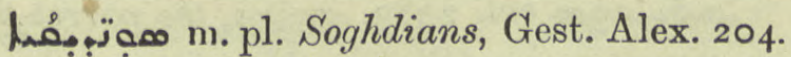

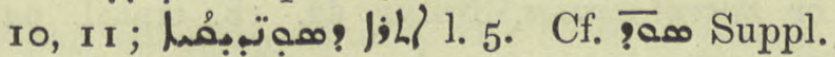
and ${ }_{\text {a }}$ col. 2543.

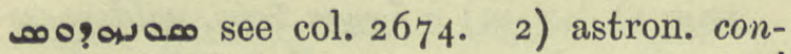

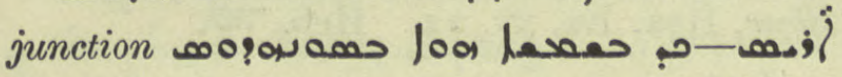
N. Hist. ii. cap. iv. sect. 3 ; conjunction of the moon, ib.

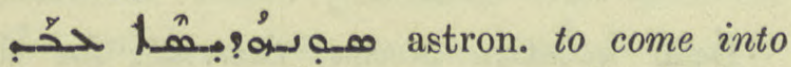

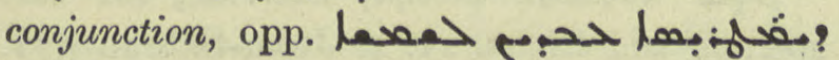
N. Hist. ii. iv. 3 .

مص col. 2675. astron. in conjunction,

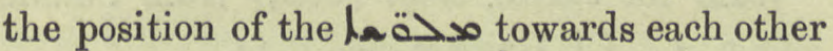
is of haslenas of has fard of Hasef , Georg. Arab. 26 infra,

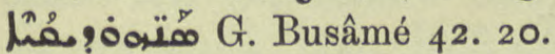

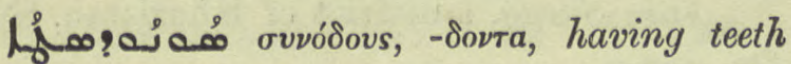
which meet evenly, as serpents and bulls, N. Hist. vii. 4. I.

|

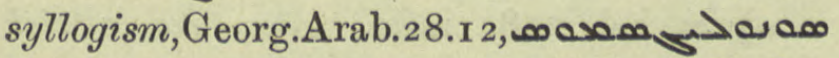
Išoyahb 37. I 4, مesacong 87 infra, 88. 1 .

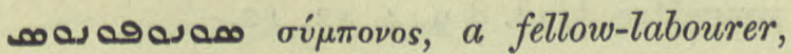
assistant magistrate, Sev. Ant. Vit. 25. 10.

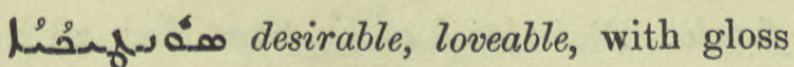

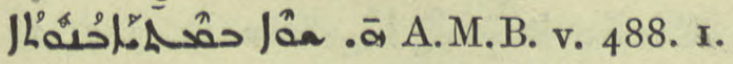

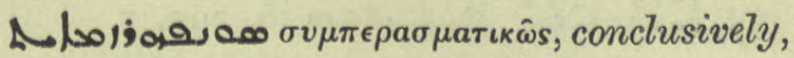
Syn. Or. 25 o. 3 .

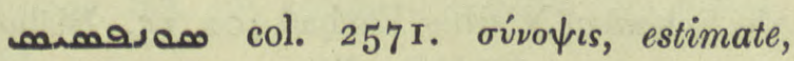
Anecd. Syr. iii. 2 I 4. 20.

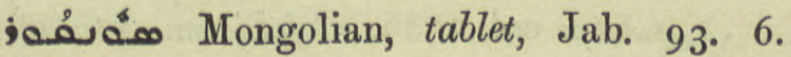
Cf. فِّرُ.

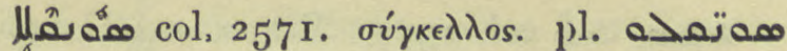
Pléroph. 28. I. A member of the Caliph's Court circle, Tim. i. Or. Xt. I 901, 300, I I af., cf. $149 \mathrm{n}$.

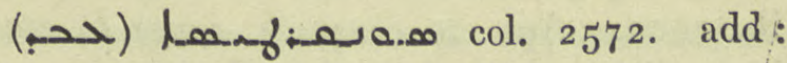

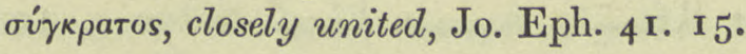

of of col. 2572. Delete "concilium, congregatio," and add: secret, i.e.private letters, the ref. to Sanct. Vit. $=$ A.M.B.iv. 320.14 n. 8.

col. 2572. an assessor, colleague,

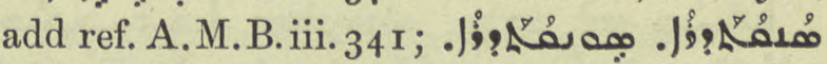

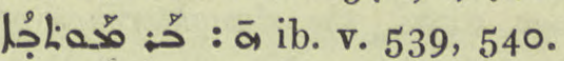

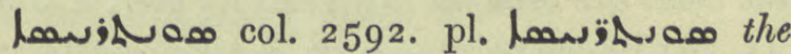
clergy assisting at the enthronement of a bishop, C.B.M. 9 I $4 b$.

l

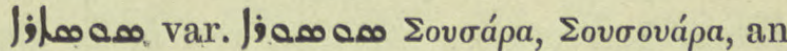
island in the Red Sea, Jac. Edes. Hex. xx. 9.

Q.

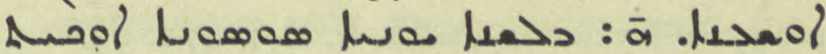
J 1 . Sal. in Lit. 54. 17.

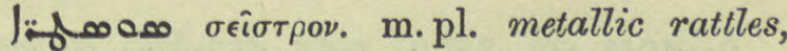
Apis. 29 n. $6=$ Schatzh. 60.6 .

مةقصم Dion. 93. 2 I, / ل 6 ib. 57. 6 .

col. 2574 pen. a centaur, add BHGr. i. 20. 7 .

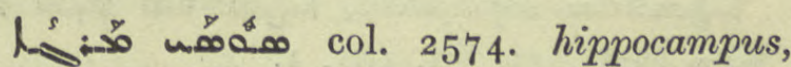
sea-horse, add: Nöld. F. S. i. 566. Cf.

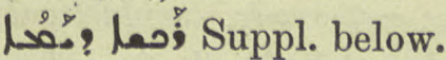

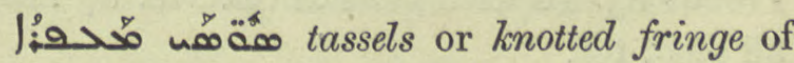
a Kefiyeh, Hist. Mon. i. I 68.

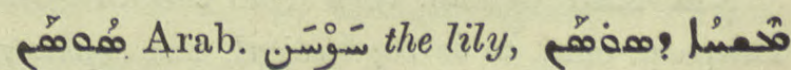
Med. $3^{8}$ ult.

a a I I add : a concerted piece of music إم pard on ovords of a chant each day and explained it, Barhad. $3^{8} 3$ Syr. ult., $3^{89} \cdot 3$.

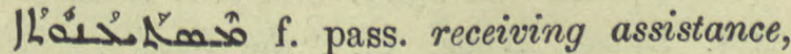

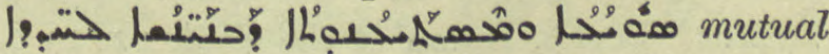
aid, co-operation, But. Sap. Pol. i. I, Eth. 3. 4, N. Hist. vii. I. I.

G $g$ 


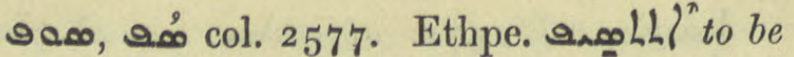
made an end of, destroyed, Hormizd 2872, 2877: :

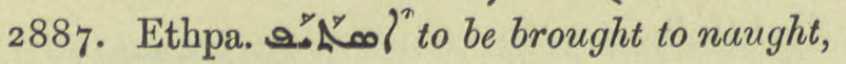
ib. 2909. Aph. to consume, cause to cease,

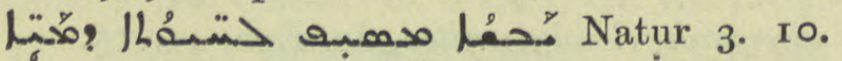
أl he made waters to flow away, put out the fire and spread darkness, Nars. ed. Ming. ii. I 74. 10;

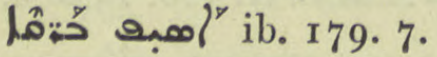

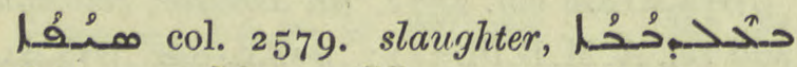

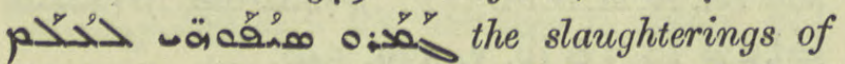
the devil are brought to an end for ever, Pallad. 41. 16; Budge points Bedjan in the parallel passage has his $\operatorname{swords,~A.M.B.~v.~} 54$ ult.

至

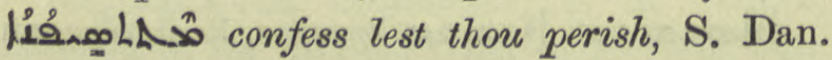
$63 r \times 9$.

०. a city of Lycaonia, Nöld. F. S. i. 472.94.

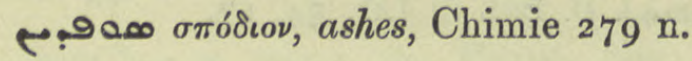

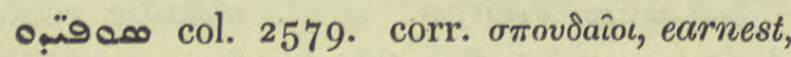
diligent. Cf. L!

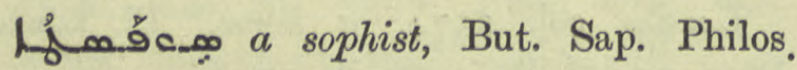
I. $1,2.3$.

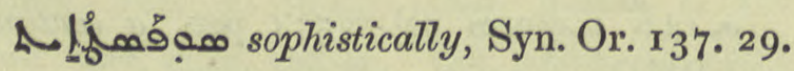

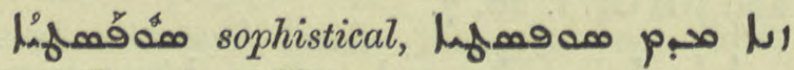
Var Sev. Ant. Vit. ror. r.

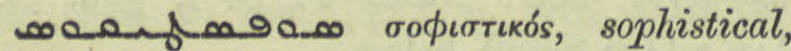
Probus 83. 10 with gloss المغ.الط, ib. 87 infra, 88 med. ter.

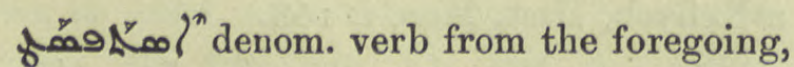
Ethpalp. conj. to be sophistical, use sophistry,

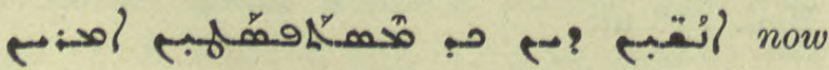
some say with sophistry, But. Sap. Periherm. r. 3 ; Philos. 2 .

: 900 $\Sigma \dot{\omega} \phi \rho \omega \nu$, King of Egypt, Hist. B.V.M. 127.13 .

مع مال مسمل Act. Apost. Apoc. cf. "the loud-breathing serpent," Bevan, Hymn of the Soul, ז 2. 13 $b$,

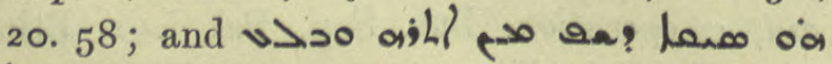
lrowoll that all-devouring one (Dragon) which crawled from its place and swallowed the innocent, Ephr. Ref. i. 121. I4. To inhale.

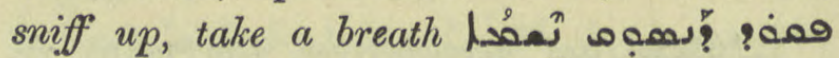
Med. 53. 8. Ethpe.

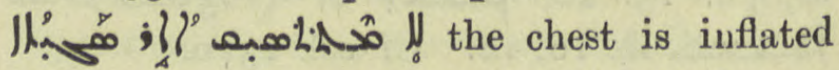
although much air is not inhaled, Med. 190. I, Iо, 12, I91. I \&c., Hormizd 3078.

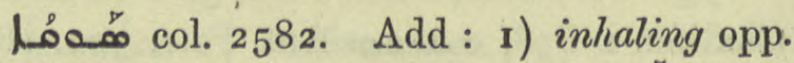

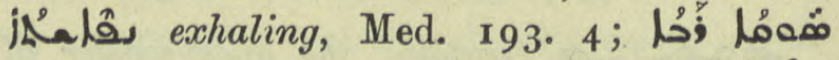
a deep breath, ib. 123. 16. 2) (2) the sense of smell, ib. 61. 21, 62 ter; lay لم: Caus. Caus. 186. 4.

م col. 2583. oxápos, the parrot-fish or parrot-wrasse, Natur $5^{2}$. 18, transl. 94 and see p. 25 n. 7 I. I. q. .

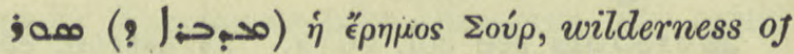

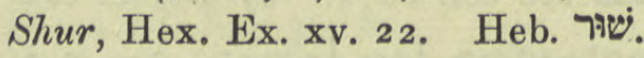

ן Natur 39. 7 آمه إ

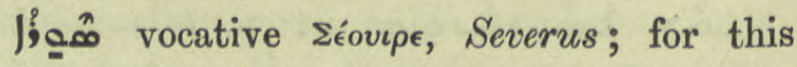
spelling add Chast. 21. 4, Jesus-Sabran 571 . I 7 , Sev. Ant. Vit. 277. 3 .

مع Chimie 282 n. 4. Cf. Kم: ممع col. 27 I 4.

p. مـ 2584. Samaria, Sev. Ant. Hom. 26. 9, 10.

50o Surag, a district of Khuzistan, Syn. Or. $323 \mathrm{n}$.

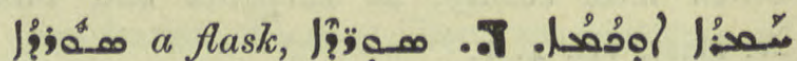
Med. 304. 6, 14 .

ڤó col. 2584 infra. $\sigma \hat{\omega} \rho v$, red vitriol, Chimie 3. 6 trad. 6 n. $6,4.5$ le sori $=$ un sel de fer basique, i.e. mêlé de peroxyde de fer rougeâtre, trad. 8 n. $4,42.12$.

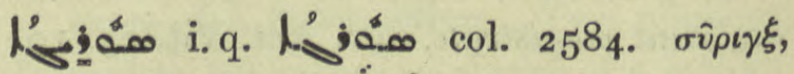

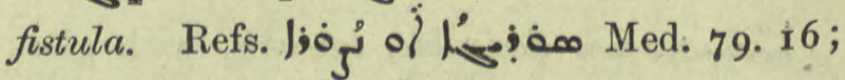

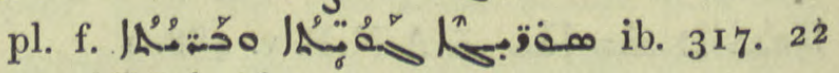
but مضة بـ ib. 393. 5 .

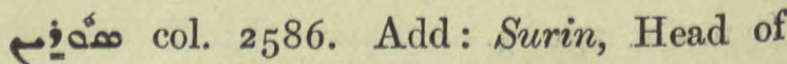
the School of Nisibis, Barhad 400. I 5. Bishops of that name, ZDMG. xliii. $403.6,8, \mathrm{I} 2$.

 ref. 20 Med. 245. 12. 


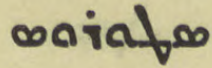

مبهز Severians, followers of Severus of

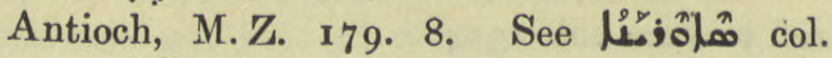
2549 ; لمَّمَ: Busâmé 42. 23.

مـ a strengthening eye lotion, . مذَبـة Med. 87. 2.

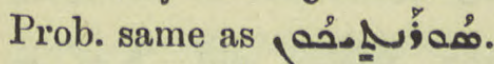

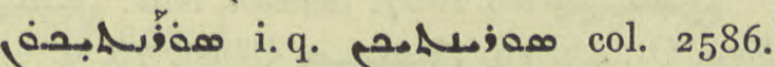
$\sigma \hat{\omega} \rho v$ à $\nu \theta_{\iota \kappa o ́ v}$, an astringent lotion, Med. I 7 I. I 8 , 176. 2, 215. 15 .

مـباب name of an Emir, Jab. I59. 4, I60. 10.

col. 2587. to make bare. Ethpa.

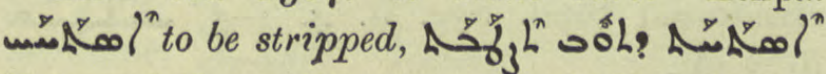
Ephr. ed. Lamy iii. 686. I6; of plane leaves, N. Hist. vi. 2. 3 see under afoo.

clear, pellucid, shining,

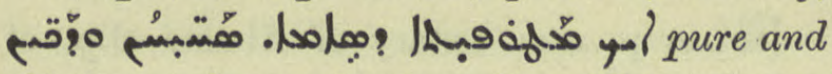
shining as drops of molten silver, Natur 64 . 10.

همחمسل Arab. level ground, a flat, plain, gloss to Ming. i. I9, course of a river, 罗 he wanted to turn the river water into that course or on to the flat land, BHChr. I ro. 9.

on col. 2588. 1) to bathe, 1a |

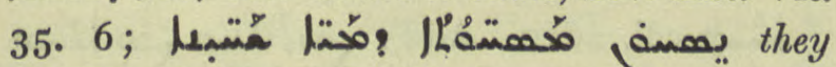
should take hot baths, Med. 2. 22. To spread as leprosy, lois Loa hin she was covered with running leprosy, Hist. B.V.M. 1 26. 22 ;

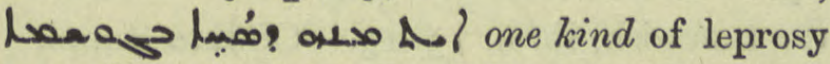
spreads over the body, BB. col. 77I. 5 af. under ${ }_{\mathrm{S}}=$ DBB. $5^{12}, 23$.

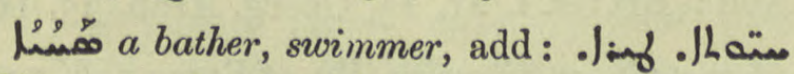

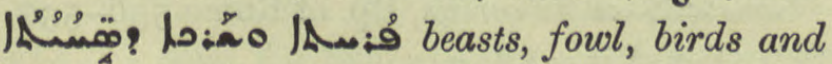
other swimming creatures, Warda $274 r$.

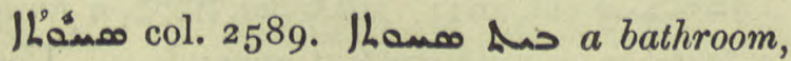

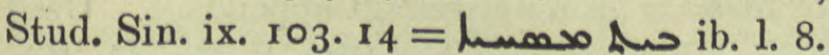

مسُم swimming, bathing. Georg. Arab.

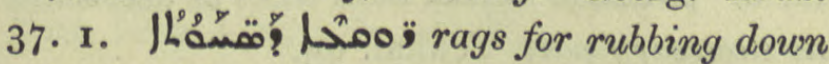
animals, BA. col. 3980. See Nöld. Syr. Gram. $\S 78$.

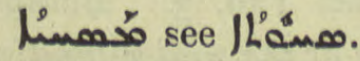

סמس Sahi river: see olow.

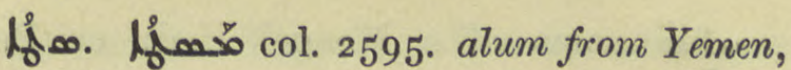
corr. يهاني. Delete the words "forte" and "species".

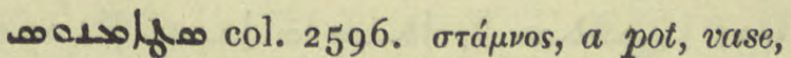
Mon? Loa and? wa loa?! sousafo

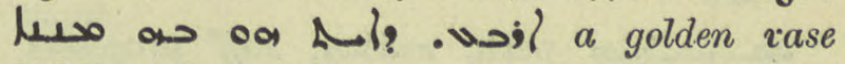
holding four pints, containing the manna, Epiph. 6. I on Heb. ix. 4 VHh.

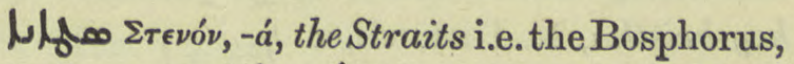
Pet. Ib. 237 ; ر/fool 1. 13.

(asto stadium, stade, Jac. Edes. Hex. 20 n. 1. 7 , pl. A. refs. to المهب: col. 2597. 7 .

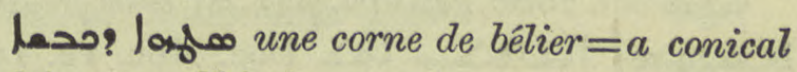
melting pot, Chimie 54. 16.

bofo $\sigma \tau o \iota \beta^{\prime}$, perhaps Poterium spinosum, an astringent plant, Galen 297.38 ; wo DBB. I329. 22 quoted Ar. PflnN r68. See Diosc. iv. 12.

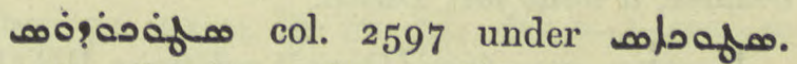
бтoıxás, -áoos, Lavender, Ar. PflnN. 272 ; Med. 245. 8.

صapofo col. 2597. collyrium, eye-paint, Kohl, لl Med. 607. I 4 .

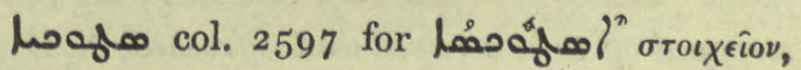
the elements of writing, م- م-

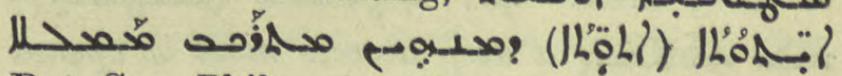
But. Sap. Philos. 6. 3, Isag. iii. 2.

م aright, the deacon's invocation at the beginning of the Anaphora, Jac. Ord. R.O.C. i. 6.

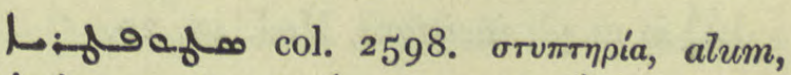
Chimie I2. 8, 16, Lifoafol 1. I3 bis, 个:-80fo/ l. 14.

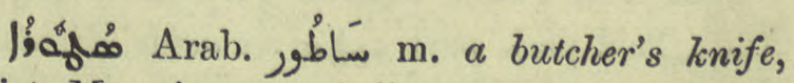
Hist. Mon. i. I5 5 . 16, ii. 308 n.

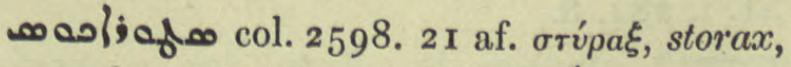

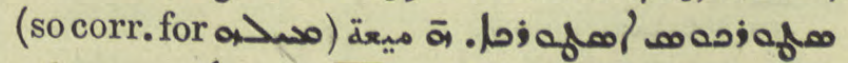
ufol مب BH. de PI. I59.

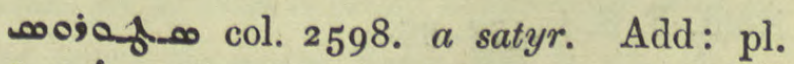
فُم ;

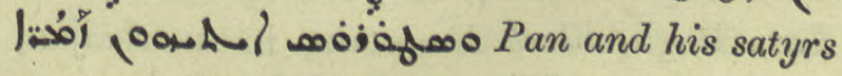
were musicians, Gest. Alex. I81. I3. 
uné name of a Mongol Emir or Beg,

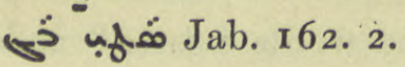

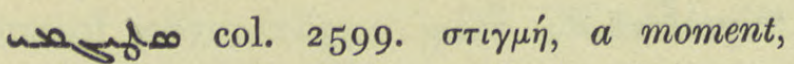

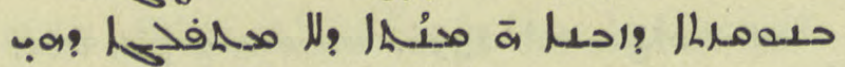
wa $\mathrm{BH}$. on Luke iv. 5 .

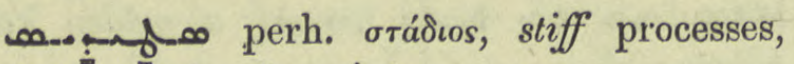

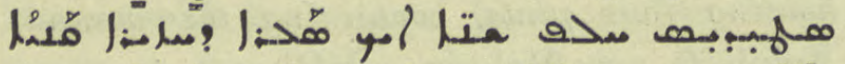
instead of teeth it has stiff processes like hog-bristles, N. Hist. vii. I. 7 .

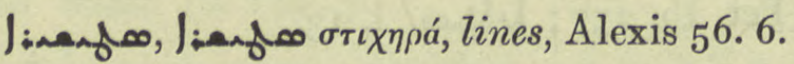
of. pr.n.m. Stilas, Governor of Alexandria, Anecd. Syr. iii. 144. 12.

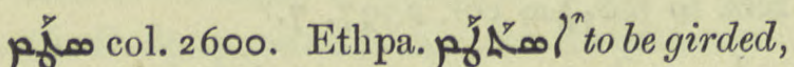

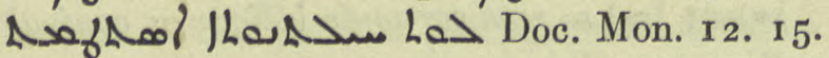

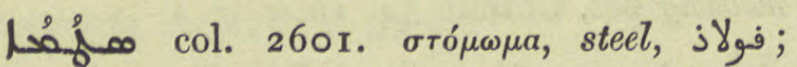

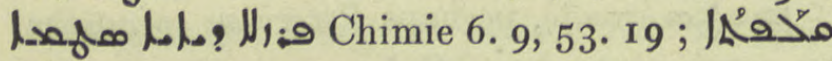

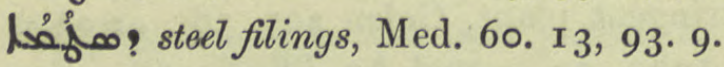

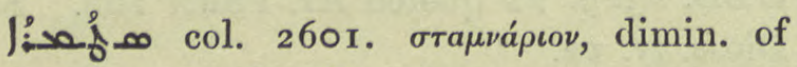

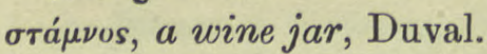

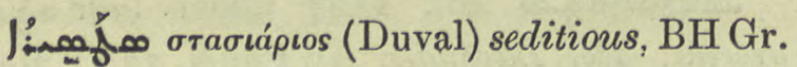
i. 18.24 , Sev. Lett. 203.9.

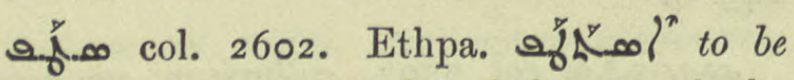
scarified, scraped, the broad leaves of the

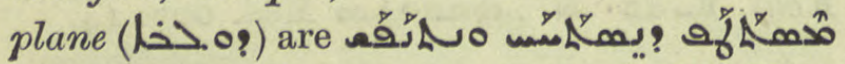
stripped off to be scraped and refreshed, N.Hist.

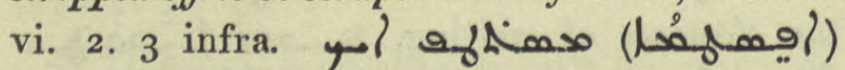

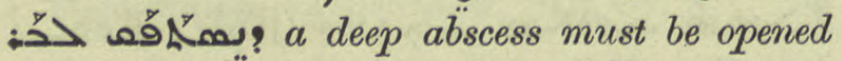
that it may discharge outwardly, Med.223. 23 ;

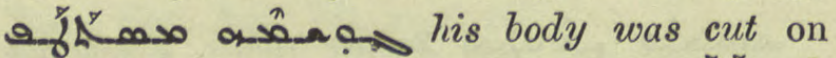
account of gangrene, so correct for Pallad. $162.19=$ A.M.B. vii. $9 \mathrm{x}$.

مهُ م. pl. incisions, Med. 47. 20.

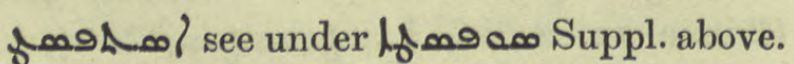

ما Add: Peal pass. part. Nestorius was lall Xt. i. 284.8.

Tा see below.

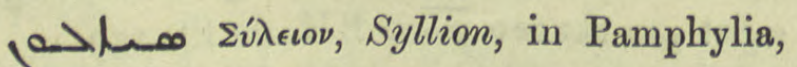
Nöld. F. S. i. 472.90.

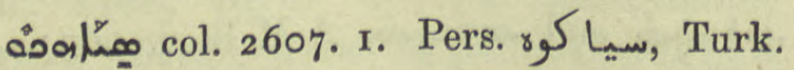
Kara Dagh, Black Mountain, in Azerbaijan, Jab. 35. 9, هب̣: 120.7 .
ل ref. to لla col. 2554 .

Sمد: Sibarius ? = Syrianos, Head of the third and last School of Neo-Platonism, BH. Hebraica iii. 25 I. 4 af.

هم Syn. Or. 43. 19, Aon Chast. 70. 9.

lafloos inhabitant of Seistan, Chron. Min. 355. 6; W W 5 o the language of Seistan, DBB. 18. 20, Coupes ii. I I I.

m.pl. Lat. signa i.e. statues (or

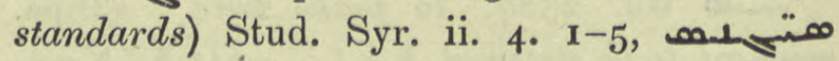
ib. 3 ult.

مَّب denom. verb from lime. Act. pl.m. مهمب:م Dion. 224. 9.

סسبْ: pr, n.m. notary of Abušta, Bp. of

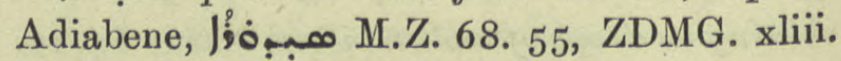
401.14.

مصلمفمل Epiph. 44. 31. Corr.

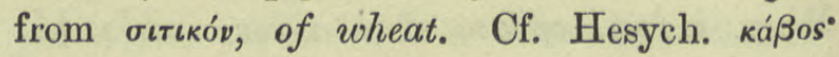

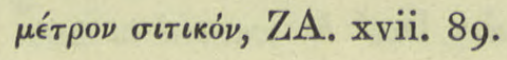

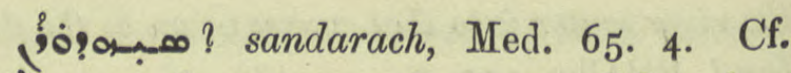
and col. 2674 and Suppl.

;olone see ;01. 2620 and Suppl.

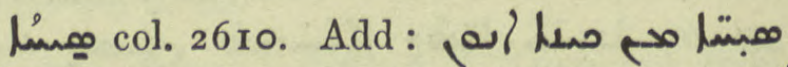
"مدّخدا: the tamarisk is of the nature of brushwood, G. Busâmé $57 b$ ro, given as an equivalent of حس and $\mathrm{VL}$, , other names of the tamarisk, ib. ll. 13-15. Cf. حس Suppl.

Mn col. 26rir. Lat. situla, a bucket. Add: BH Gr. i. 2 I. 13, Georg. Arab. 35. 16.

مמحح: مص Silverius, Bp. of Rome 536-537, Sev. Ant. Vit. 288.7 .

סלבון a fringe or tassel = an unimportant appendage. Used as an expression of humility, S. Fraenk. ZA. ix. 6. . no necessary part, Tekkaf. 95 .

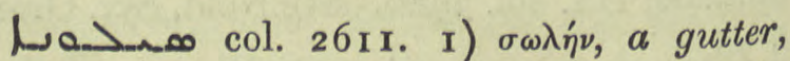
channel. Pl. drains, Jab. 136. 3. Metaph. متخه در إده channels of the understanding blocked by drink, Ephr. ed. Lamy iii. $5^{1} 7$ pen. 


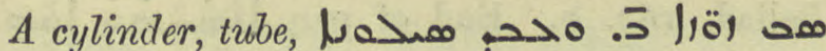
Chimie 44. I I. 2) Solen, the razor-fish, pl. متبحة N. Hist. vii. 2. 2.

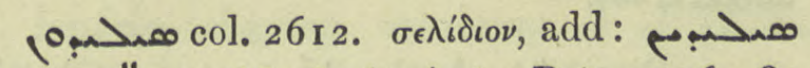
L.oll a title-deed, Ant. Patr. 306. 8; صمخبمـا 249 bis, 255 . $\mathrm{r}$.

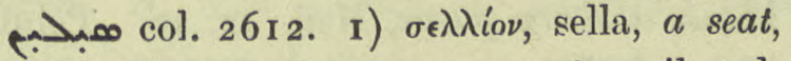
Mar Kardag ed. Feige 44. I, Abb. 54. 4 .

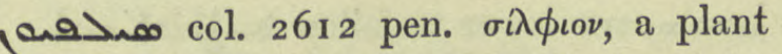
the juice of which was used for food and for

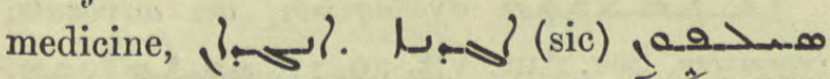

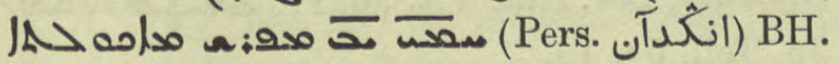
de Pl. I52, Silphium is Laserpitium, Ferula Asa foetida, carminative, desiccative, digestive;

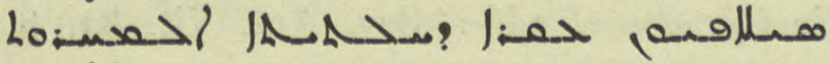

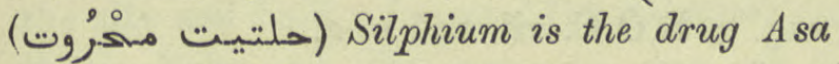
foetida and the root of the same plant, Galen 296. 16; مبحصبدف Med. 299. 6.

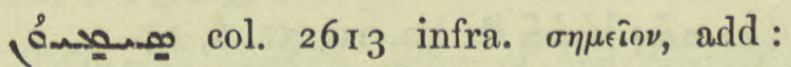
I) astron. a sign, a point,

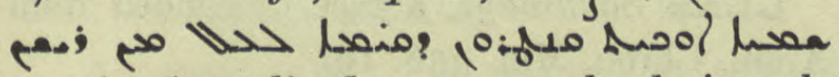
a point immediately over our heads i.e. the zenith, De Astrolabe 78 pen. م-

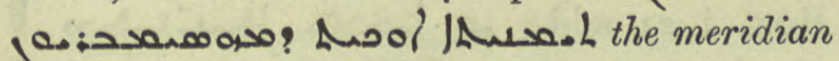
point, ib. $265.4,267.3,268.9,273.8$. The North Pole, ib. 270 . II, pond إمه

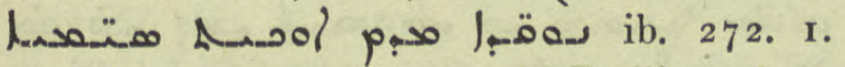
2) The mark of a scar, A.M.B. iii. 236 . 1 .

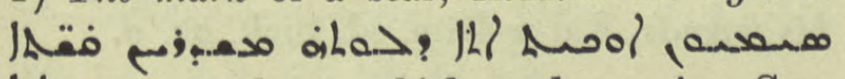
J) a mark at which archers aim, Sev. Ant. Hom. 25. 8. 3) a mile-stone, cf. Neo-Heb.

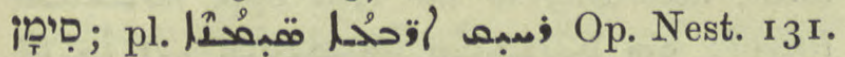
ro, Nöld. in loc. ZDMG. xxxv. 499. Col. 2614.4 corr. for Einladung, Ankündigung in BHChr. p. II. 4) shorthand, cypher, od Sمen in Geheimschrift, Anecd. Syr. iii. إحسة Sond in few words, as it were in shorthand, Hist. Mon. i. 2 I8. 6, 386. 5 .

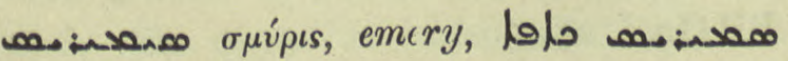
? Choimie 9. 5 .

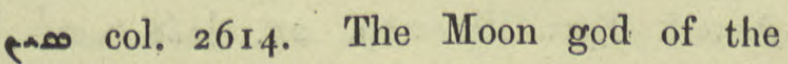
Chaldaeans, Sin. Chem. silver, Chimie 42. 6 unless asem = Egyptian silver is meant, see pp. $230,{ }^{2} 32$, and p. xx. Name of an idol, Lag. B. N. 46 , Jul. I46. I3, 22 ; B. O. i. $327 b$ Syr. 12 where the prefix prep. 0 is mistaken by the translator for part of the name.

مِّ Pael conj. of for to shoe, put on shoes. هuc Vit. Mon. I3. 4.

هبل A.M.B. ii. 65I. I I, see منب of a river.

متعبر children of Canaan, Chron. Min. 356. 8.

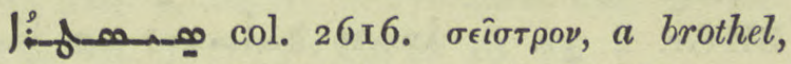
S. Fraenk. ZDMG. 1vi. 99. For Guidi's suggestions on this word see Nuovo MS. 63. 26 .

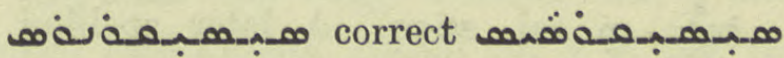

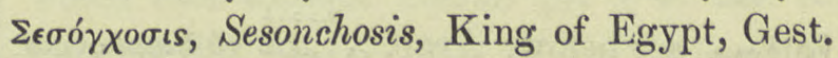

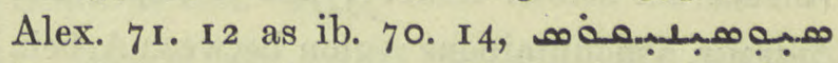

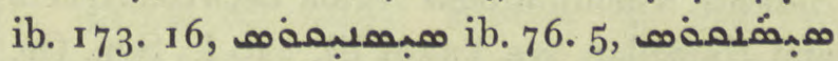
ib. $225.11,226.4,252.2$.

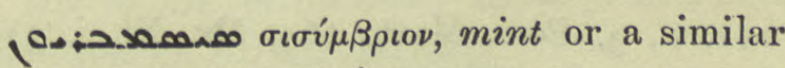
aromatic plant, 4. I infra; معبهم Med. 607.2 .

סחمڤ col. 26I 7. perh. participle of Pers. to hop, jump, some small bird. The passage quoted by BB. is given Natur 24. 16 , see transl. $49 \mathrm{n}$.

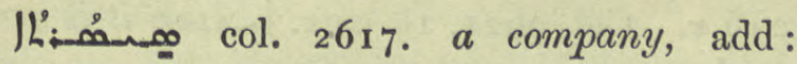

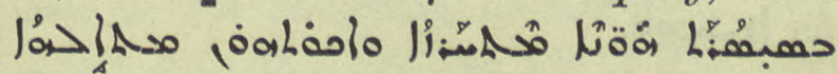
the soul freed from matter takes its place in the company of intelligences and becomes divine like them, But. Sap. Theol. I. I, 4. 8, a

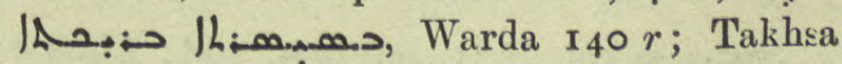
49. II.

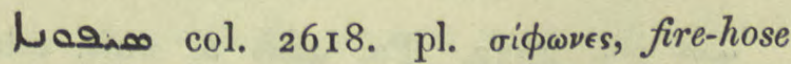

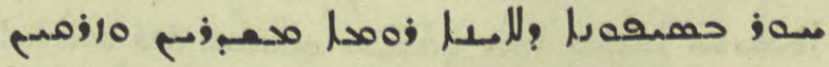
> $>$ see to what a height water-pipes send up and spray the water, Ephr. Ref. ii. 35. I6.

Jaمa col. 26r9. Lat. securis, an axe,

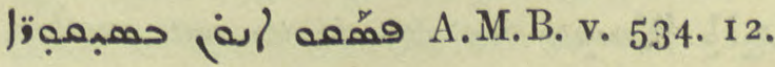




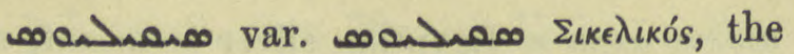
Sicilian gulf, Jac. Edes. Hex. xi. I I.

col. 26r9. anything secret or

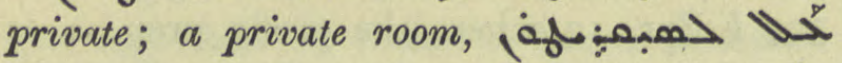

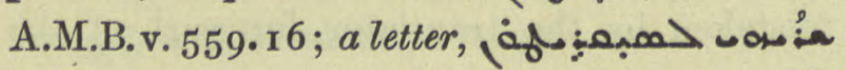
he opened the letter, ib. iv. 320 . 14. Cf. ف요 col. 2572.

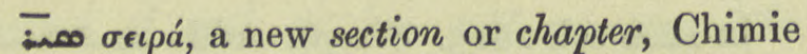

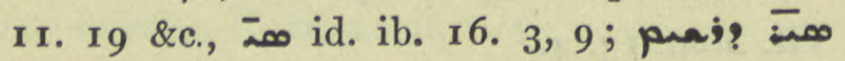
حection entitled Hidden Power, ib. 5 ०. $3 ; 233$ n. 3 .

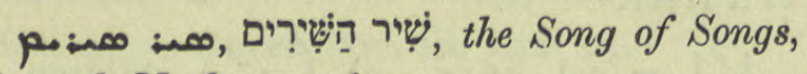
Eus. ed. Maclean 4 16. 10.

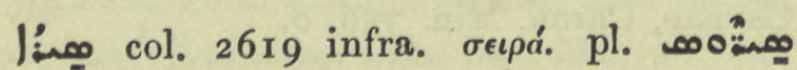

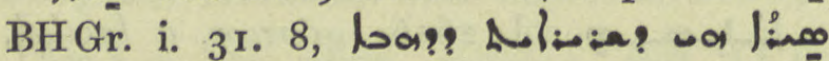

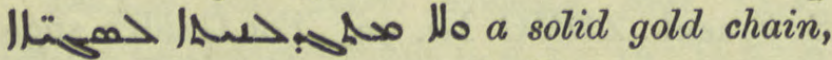
not a twist of many strands, ib. I 4. I9.

90\% of col. 2620. A later spelling is

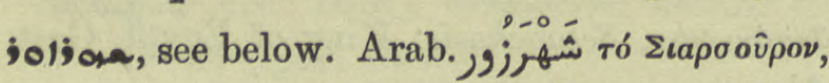
Siarzur, a mountainous region between Arbela and Hamadan, see Yakut iii. 340 f. Syn. Or. 683; 109. 5, 128. 19 \&c., Hist. Mon. ii. 2 I r. n. 3, Pers. Mart. 264, Or. Xt. i. 310; Jab. 288. 7 ; ZDMG. xliii. 403. 4, 405. 2, 406. I 5. jo/n Chast. 34. I2, j010 on city and bpric., ib. 39.6 af.

مصن:بمف Sirmium, on the Save, Or. Xt. i. 90.6 .

مبنتُنم col. 2620. a siren. add: pl Natur 26. I I, sing. مصبز ib. 1. I5, Warda 246 v. Col. $262 \mathrm{x}$. 18 corr. for $l ;>>0$.

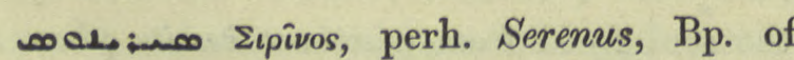
Augusta but Brooks translates the Syrian, Sev. Lett. I 28 . 1 .

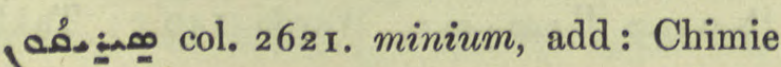

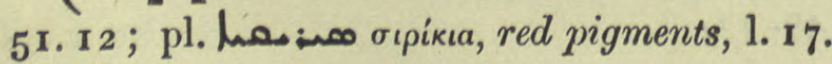

ס col. 2622. Pa.

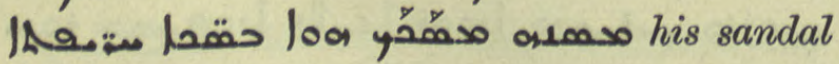
was studded with sharp nails, Josephus vi. I5. 1 \% .

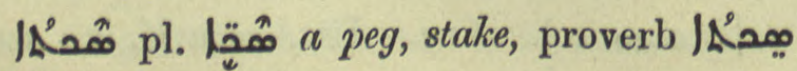
sمع one peg drives out another,

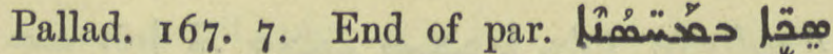
boils on the privy parts, Med. 105. I; B̈B. under woLol col. II I 4, DBB. 684. 23 under nopthtor.

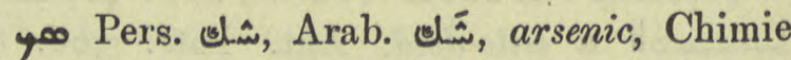
IOI. 20.

مَّم a sharp purge, Med. 42. 20. Pointed

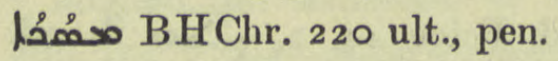

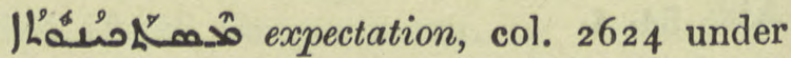

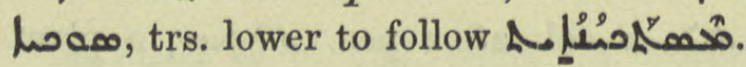

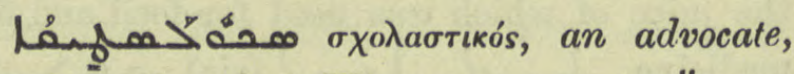
barrister, Sev. Ant. Vit. 39. I I, aمenfoll Jo. Tell. 63. I 4 .

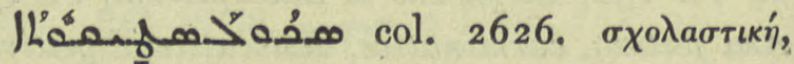
jurisprudence, add : Sev. Ant. Vit. 81. 8, 225. 3, R.O.C. vii. 106 n. 2 .

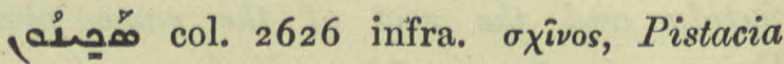
lentiscus, mastic tree, Med. 52. 1о, 145. 7,

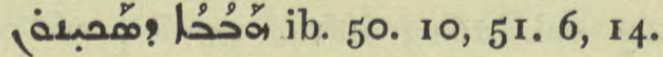

Sakhīnāye, a race descended from Aram, Chron. Min. 356. 19.

ه

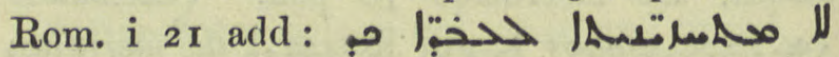
V- VHh. Rom. i. 20, and quoted BH. in loc. Aph. "

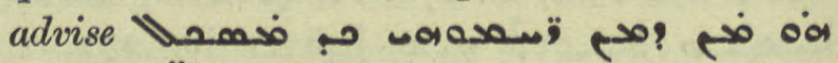
"الا مفح he who will not receive advice from his friends, Kal-w-Dim. ed. Bick. 24. 9.

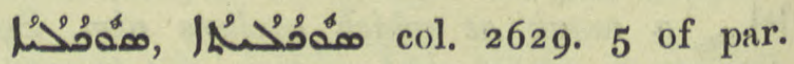
ideal, according to the idea opp.

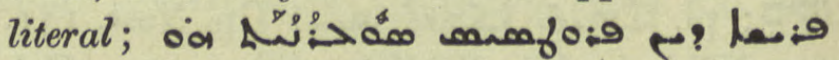
"סمبـ But. Sap. Periherm. I 1.4.

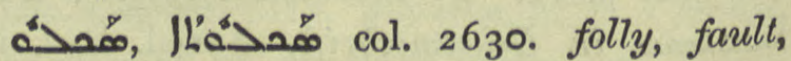

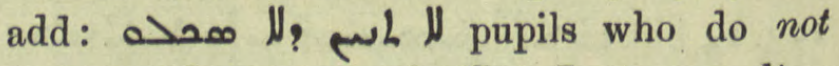
repeat without a mistake, Sev. Lett. 195 ult.

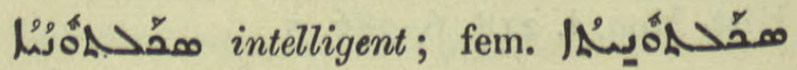
BHGr. i. 25. 5 .

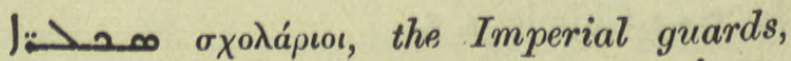

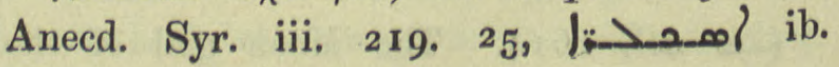
233. 20. 


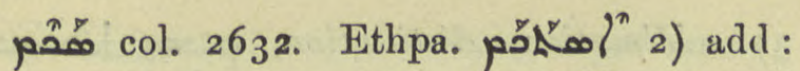
to be shaped or formed in a mould, col. 226 under tros?.

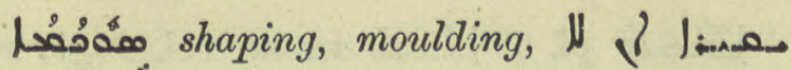

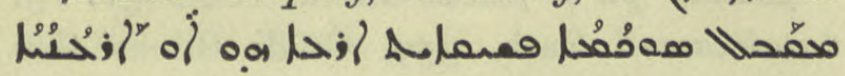
a heavy substance which does not readily admit of shaping is an earth or of earthy

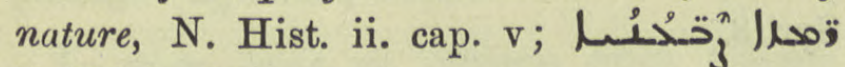
. 3 . 3 . 3 .

محسم pr.n.m. Aioxivns, Aeschines, Probus 98. $5, \mathrm{I}_{4} \mathrm{I} \mathrm{n}$.

مَم col. 2652 . delete the line.

مُ from Assyr. muškīnu, Littmann Z.A. xvii. $262 \mathrm{ff}$. A modern meaning is leprous; the cry of lepers in Jerusalem is Ana mäskin, ana mäskine. Rabbula cared especially for the مقتصع dwelling outside the city and built a hospital (lazar-house) for them, Ephr. ed. Or. 202. 19, 203. $25 \mathrm{ff}$. Add: ميهنب wretched is this time, Nars. ed. Ming. 2 I0. 3.

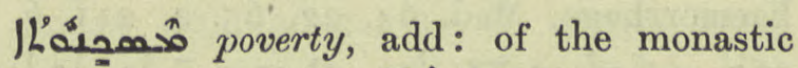

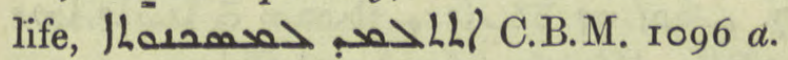

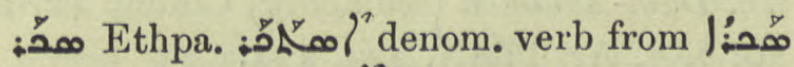

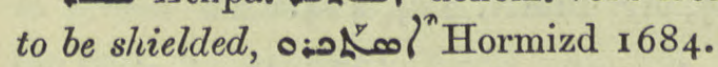

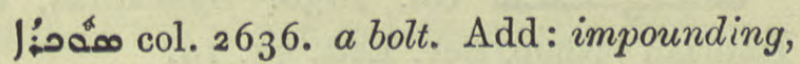
معص:| יمس1 Dion. 235. I7, 21.

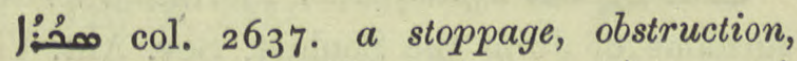

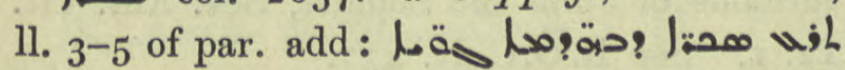

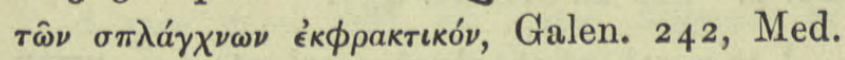
16. I $3,53.4,57.18,63$ ult.

وُ shield-shaped

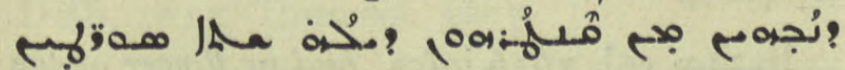
But. Sap. Philos. 5. 2 infra on triangles,

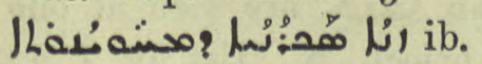

هم: col. 2637. '́⿴囗⿱一一)

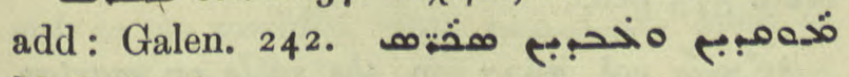
Med. 65.8.

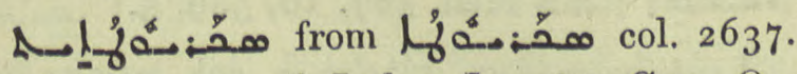
in the manner of Judas Iscariot, Syn. Or. 172. 8. See quotation under A مLلمال.

lloo col. 2627. Ethpe. un-oln to be depreciated in value, But. Sap. Econ. 1.2 infra.

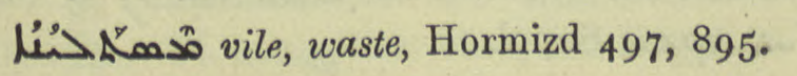
2810
ه مالم: Pléroph. 29. 3,

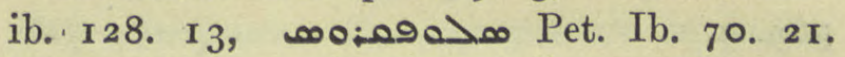
Vars. of $\infty$ e col. 2640 . Salofacialus, nickname of Timothy, Bp. of Alexandria.

L. حمه Fraenk. ZA. xvii. 89.

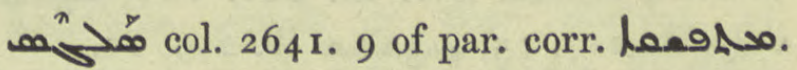
oـistake for waspar and صe (ل) Helladius, Nold. F. S. i. 473. 118.

محه م : 2643. pr. n.m. add p;ol? Silvanus of Urim, one of the twelve Bishops who consecrated Severus, Sev. Ant.

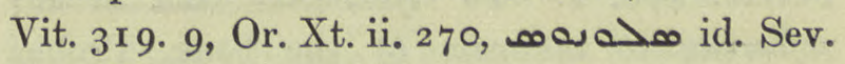
Ant. Vit. 32 I. 7 ; Charms 10. 19.

see محهمهم:0م above.

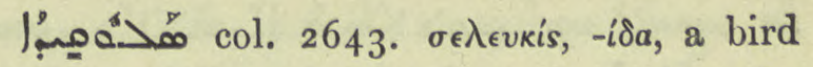
which eats locusts, Natur 37.3 ff. has the same as BB.

م>ه col. 2643. silurus, the shad-fish, Ar. FischN. 50, Arist. Apol. \&े. 9, perhaps a misreading of aỉ ${ }^{\prime}$ ovpos, Bensley 62. For the name cf. ZDMG. lxi. 637 .

of $\Sigma a \lambda \tau \dot{\omega} \nu$ or $\Sigma a \lambda \tau o ́ v$, Salton a district of southern Palestine, Pléroph. i. 7, I 77, R. O.C. iii. $36 \mathrm{r}$.

مسخب Suppl. above.

صَّقم col. 2642. Seleucia. Cf. col. 1693-4.

مجب Salakh, a Church province on either side of the Mountains of Kurdistan, $1 ; 2 / l^{2}$ פمحَy B. O. iii. i. 478 and $482=$ Hist. Mon. i. 108. 8, 109. 2, 3, مُح حَ: Outer Salakh, west of the mountains and round about Arbela, B.O. iii. i. $470=$ Hist. Mon. i. 109. 3 , ILُ $\Omega_{y}^{y}$ Inner Salakh, on the border of Azerbaijan, B. O. iii. i. $479=$ Hist. Mon. i. I 10. I6. See ib. ii. 240 n. 5, Pers. Mart. 243-245. Trs. hither the refs. under col. 2642 excepting the first of them.

مَّح col. 2644 . Salamia, a bpric. under the See of Antioch, add: Or. Xt. ii. 270, Sev. Ant. Vit. 3 I 9. I I, 320. 5, 32 I. 8, vars.

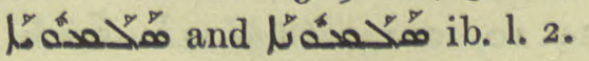

$$
\text { H } \mathrm{h}
$$




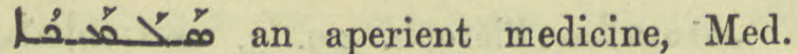
569. 2.

هُ col. 2645. the salamander, add: Natur 63.6.

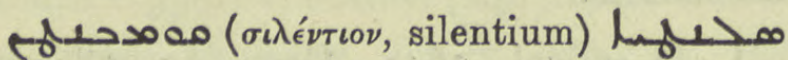

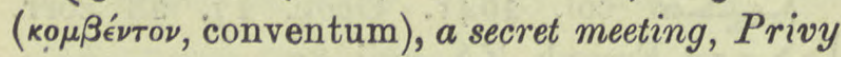
Council, Anecd. Syr. iii. 22 I. I8; 8 ; only, ib. 217. I5, ملهد ib. 219. Ir.

م above.

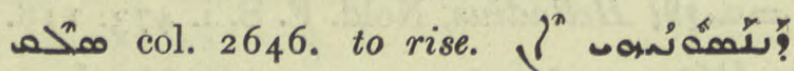
هـ would rise $=$ they tried to get a rise out of him, Pallad. 478. 4. To refer, fen La referring to the aim of the book, Probus 88 ult. Pass. part. distilled, Chimie 39. 7. Ethpe.

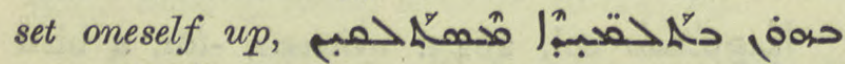
بحم with the disciples and rebulked them, Pallad.

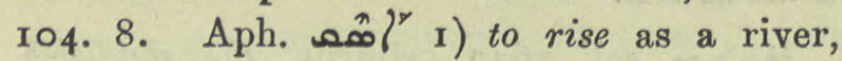
the Nile will rise high, Bk. of Shem iii. 5, I1 2. 18, II3. 9, II 5. I7. 2) to raise, add: to raise the voice in song, the siren همبت Natur 59. 8. 3) to lift up, place above, Takhsa

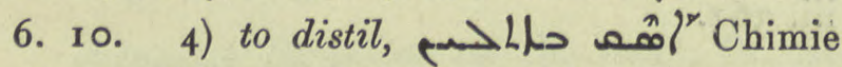
25. 3, 28. 16, 36. 10, 39. 18. Pass. part.

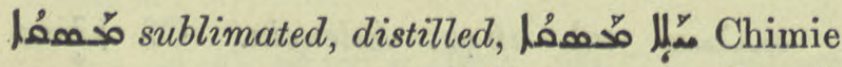

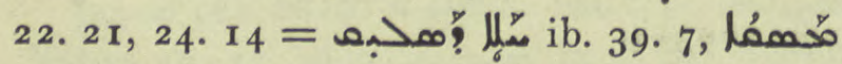
a.)! sublimate of mercury, ib. 1. I4. Pl.

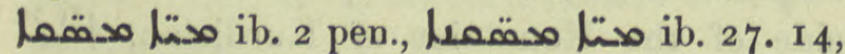

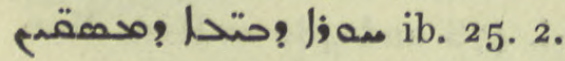

|لْ col. 2649. sublimation, Chimie 40 pen.

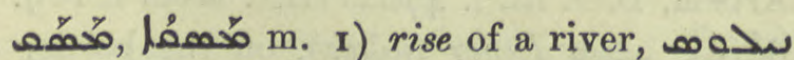

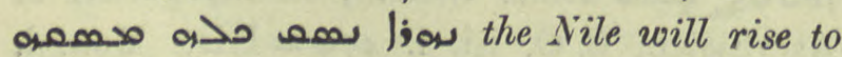
its full height, Bk. of Shem I 12. 18, 1 15. 17,

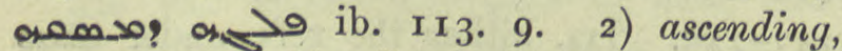

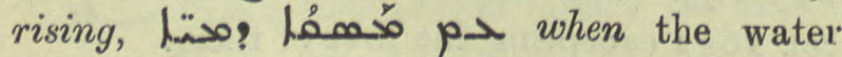
rises in vapour, Chimie 36. 9 .

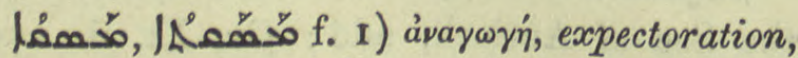

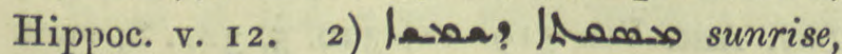

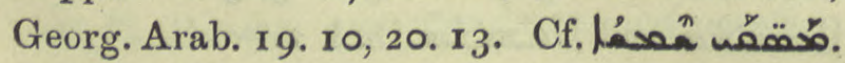

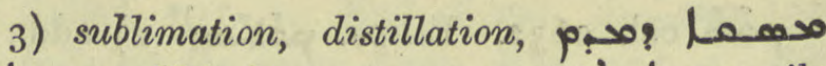

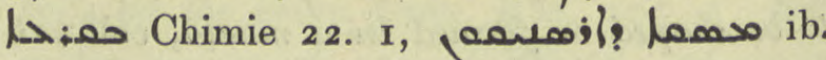
57. 18.

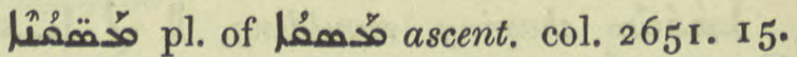
"staireases" Zach. 359, Guidi thinks this means coenacula, upper rooms to which ascent was by outside stairs, Zach. ed. Guidi 22 r. I Syr. 8 ,

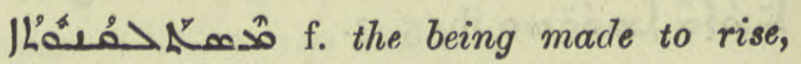

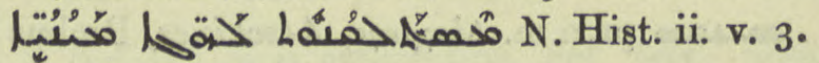

مخمل

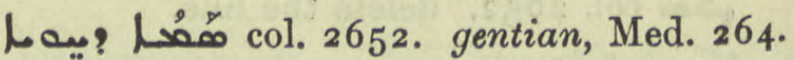
I6, $607 \cdot 7$.

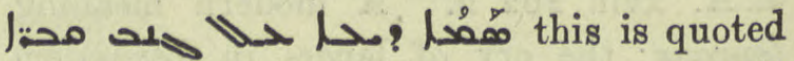
from Galen. ed. Kühn xiii. 35 , Ar. PflnN. 38 under V??. Correct (Lepidium) latifolium to sativum.

or of of tit. blood of two (brothers) دم الاخـويس. Dragon's blood, a dark red resin from Calamus Draco, stops haemorrhage, Med. 64. 22, 65. 2, 2 1 1. 6, 9, 667. محا ? ? i. e. Dam-Akhouên (see above) Chimie 42. I2, 50. 6, 7, Ar. PflnN. $274,424$.

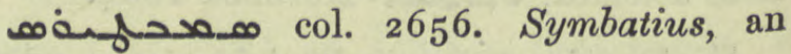
Isaurian bishop, Sev. Lett. 75. 19.

مه surname of John the Sophist, Sev. Ant. Vit. 12. I. See R.O.C. iv. 437 .

סמדא

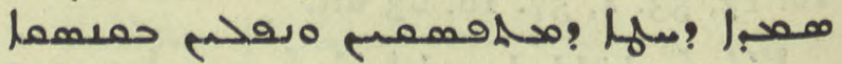
Epiph. 4 .

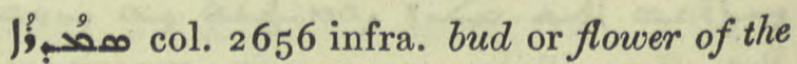

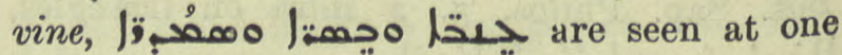
time on vines at Antioch, N. Hist. iv. 4. 3; Med. 573 pen.

;هُ

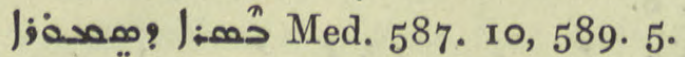

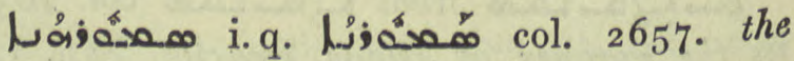
Muraena, sea-eel or lamprey, Laisa

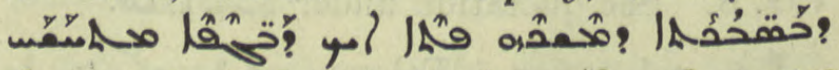
the sea-eel which uses for fins a broad process or tentacle of skin, N. Hist. vii. I. I. Cf. Suppl. 


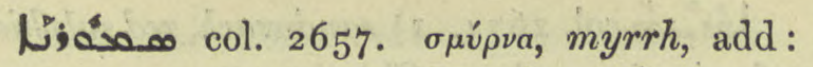

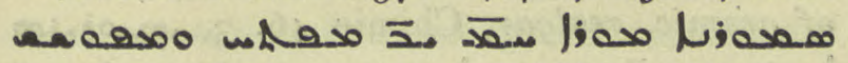
. . . . BH. de Pl. I55, ZDMG. xliii. I27, Galen. 297. 30, 31, Chimie 7. Ir, Med. 606 ult., Ar. PflnN. 246.

(a) passage

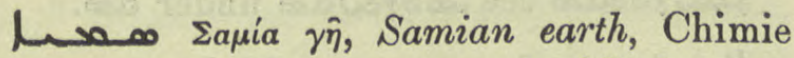

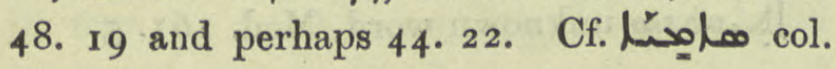
2495 and 0 owas.

औ/Wame of an angel, Protection 79. 13.

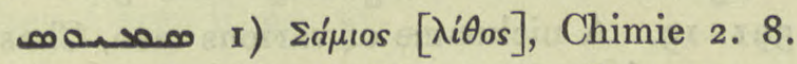
2) name of a demon, Charms 84 , Prot. 54 .

حسم col. 2684 , Duval 1427 .

col. 2658 . Corr. oof to to somoly taxus, the yew.

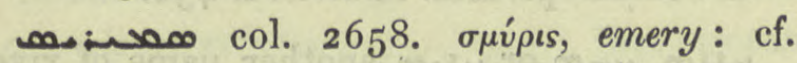
Suppl.

- col. 2659. 3) to arrive, reach a destination, with $>$ add: Jul. 13.27 , Mar Kardag 50. I I, 57.4, with o 6r. 5. Metaph. to attain (so corr. for هon) Pallad. 127. 18. Ethpa.

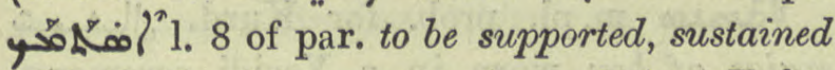

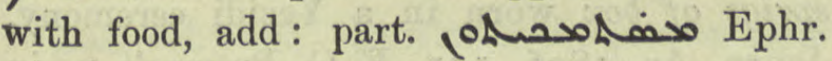
ed. Lamy iii. $4^{2} 5$.

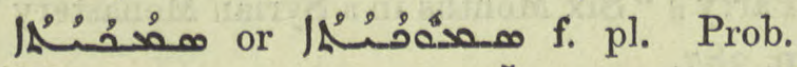

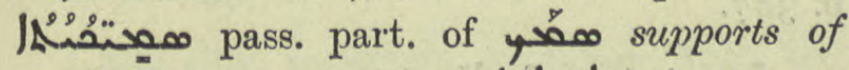

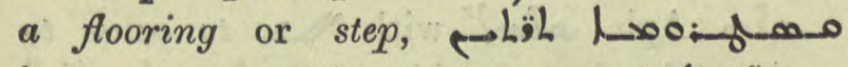

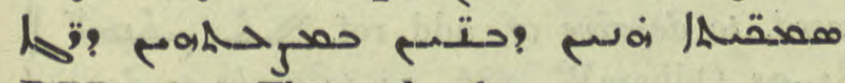
DBB. I 815 , Thes. col. 3677 .

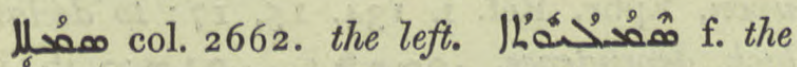
being on the left side, Apis. transl. I 9 n. I.

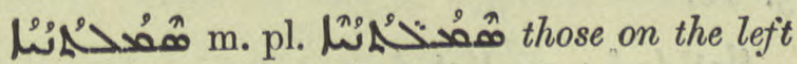

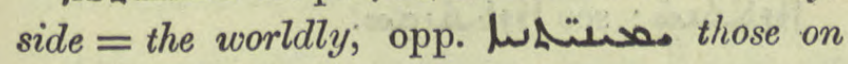
the right hand, Syn. Or. 166. 14, I5; opp.

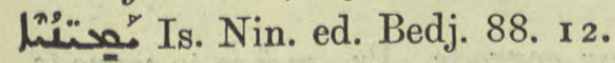

لم col. 2663. left-handed, unlucky, Tekkaf 140.

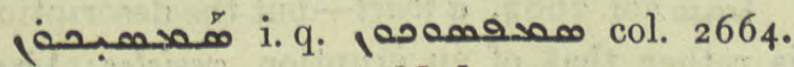
Origanum, marjoram, Med. 40. I5, 80. 21 , 266. 5, مِمحفْمبحف ib. I48. 4, 9, Ar. PflnN. 4I. I35. Cf. مص م: Thes. and Suppl.
W.> col. 2663. Arab. شa to read Holy Scripture. To say private or public prayers, Goldziher ZDMG. xlvi 44, Schwally ib. lii. I43, Fraenkel ib. p. 5II, Schulthess ZA. xix. 134. Ethpalpal Mخ s poverty; to renounce gain,

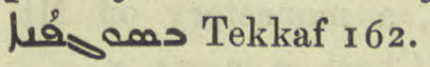

هـ

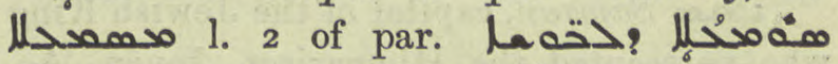

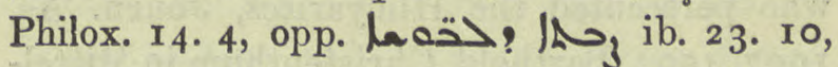
Ephr. i. 82 bis, ed. Lamy ii. 343 bis. Hence monastic garb, لر Hist. Mon. i. 24 . I7, Jul. 288.12.

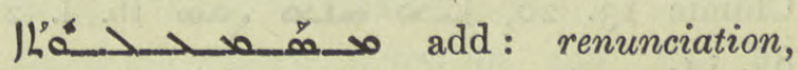

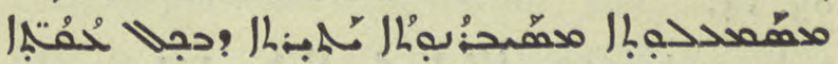
renunciation is extreme endurance in all adversity, Op. Nest. 24. I2. Wretchedness, عakovxia, فी A.M.B. vi. 403. 5.

مصن col. 2665. Pael

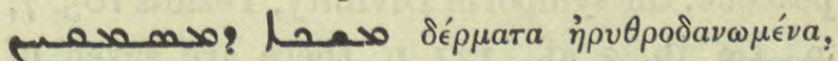
Hex. Ex. xxv. 5. To redden, to gild, Chimie 2. I.

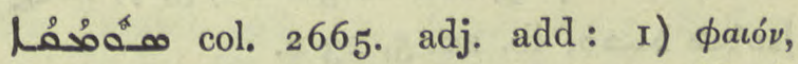
$d r a b$ i. e. a colour intermediate between black and white, Cat. Ar. Jac. Edes. I6. 4. 2) red, إ minium, Chimie 14. 9, = gold,

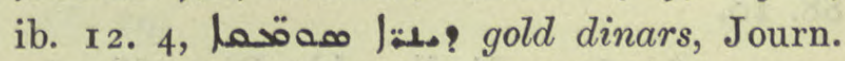

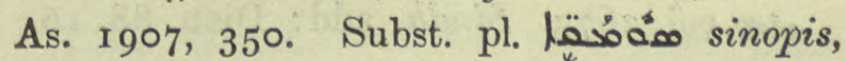
earth of Sinope $=$ red ochre, Chimie 7. 9 trad. I 3 n. 3 ; cornel berries, ib. 45.4 ; rlubarb; Med. 98. 23, 170. 14, 294. I0, so correct for مصحف JAOS. 192. 17, 196. 3. Sing. Med. 59. 8. A litter or palanquin covered with

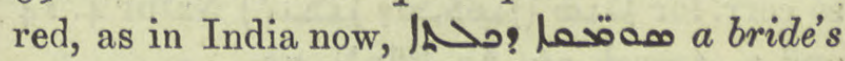
litter, Ephr. ed. Lamy iv. 627. 13.

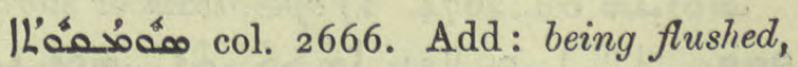
red in the face, Med. 44. 5, 234. 6 ; IL'مبه ? redness, inflammation of the eyes, ib. 9I. 2 I.

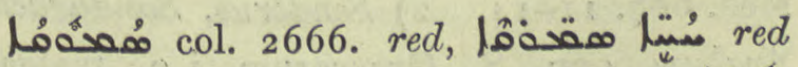

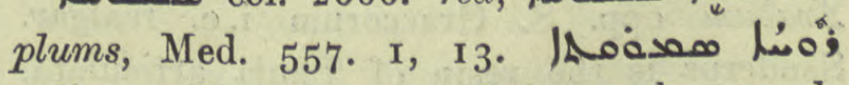
a disease, ib. 575.3 , I I. Subst. perh. sumach, pl. ib. $5^{60 .}$ I 1, $5^{62} .17,5^{65} .19,568.22$.

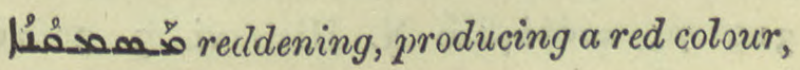
Chimie I. I, 42. 8.

H $\mathrm{h} 2$ 


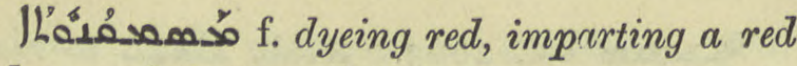
colour, rubefaction, Chimie 18. 5, 20. 25 .

ک flowers, Med. 599. I9.

;0مarten: see above, p. 234.

Lo:sam Samaruna, a monastery on Mt. Ephraim in Palestine, Chast. 9. 2, 24. 5.

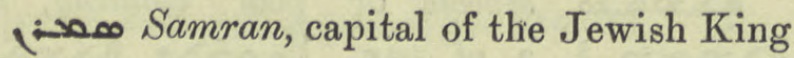
who persecuted the Himyarites, Journ. As. $1907,45^{\circ}$; Barthold, Christenthum in MittelAsien, v. 16.

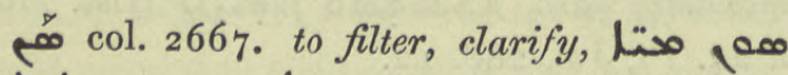

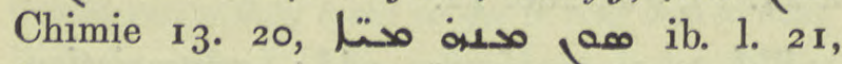
83.14 .

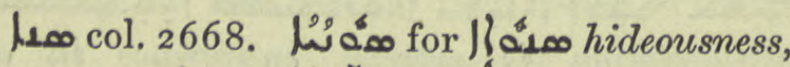

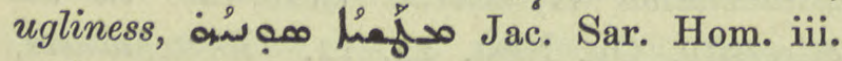
216. 12.

سنبهاق sanjak, a standard, the Yezidis give this name to a bronze peacock, the symbol of Divinity, Yezidis 105. 7 , م 3 ib. 3 af., I2I n. there are seven of these, ib. 107. 9, Mt. Singar 18, 5, 7, مترم)

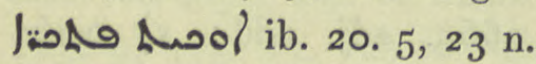

Ethpal. " or influence, "له Jab. 88. 10.

ب10 col. 2673 . Sindh, add: Dion. 88. 16.

مُ ميلl

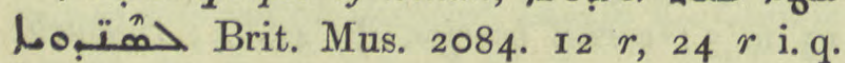
ر. col. 2673 , Dion. 85. 10, 196. 18.

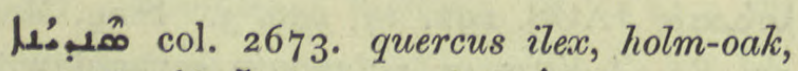

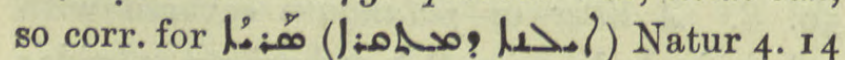
and n., transl. 36, Ar. PflnN. 72.

م col. 2674. I) opobalsam or storax

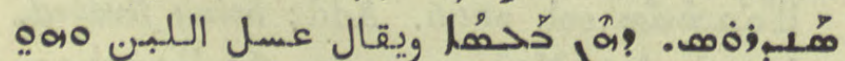

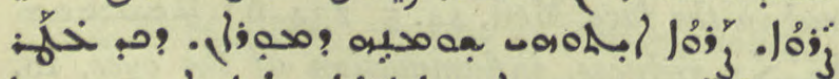

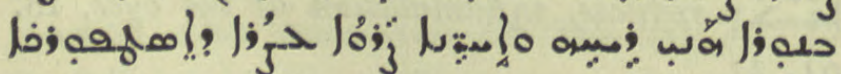
Med. 607. I1-14. 2) Senderus, Sandaracha Arabum opp. S. Graecorum i.e. realgar. Senderus is the resin of Thuja articulata, a Moroccan cypress, ZDMG. xliii. $3^{87}$.

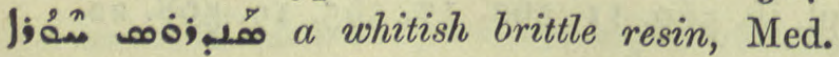

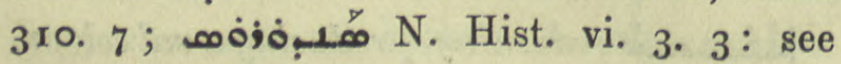
مavel.

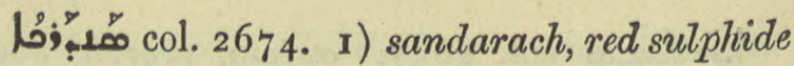
of arsenic, realgar, Chimie 46. 3 , مدب:כمس o olb. 3. 2, 2, 1. II,

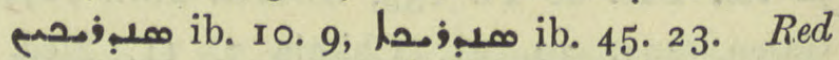
arsenic or saffron, of pao on ib. 5. 6. Med. I70. I7, 4I6. I4, \&c. 2) I. q. معب: q.v.

هصة

•

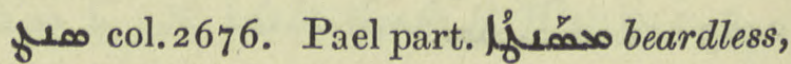

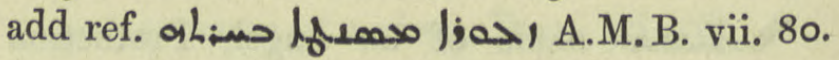

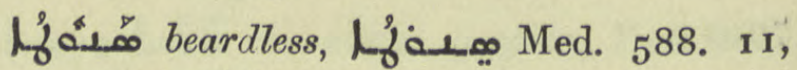
591. 19. A nickname of various men, Chast. 55. 17 ; لُّة

Do: معن Parthian pr.n. Sanatruq, Tabari I 8 n. 3, Pers. Mart. 185. Name of a Persian martyr, A. M. B. iv. 169.

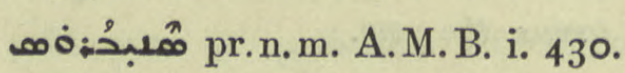

another name of river Gozan or Hulwan, which flows into the Tamara, a tributary of the Diala, lay jow

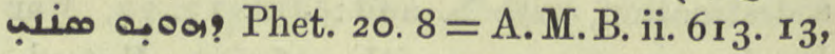
סس ; ib. 651. II, Pers. Mart. n. 544 هبب Sahi n. 626.

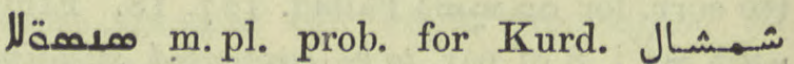
sprigs of box worn in a Yezidi ceremony, Journ. As. 1896, I I 5, E. G. Browne's n. in Parry's "Six Months in a Syrian Monastery", p. $3^{87}$.

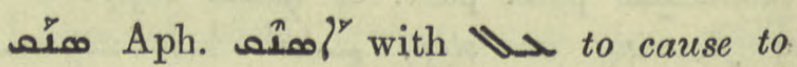

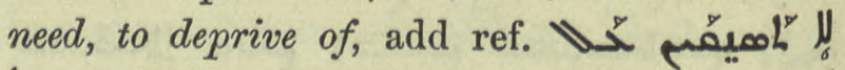
ل! QdhamW. 186. 3; to make to depend upon, Ephr. Ref. i. 134. 15, I7; to declare lack, dependence, para 3. 16 .

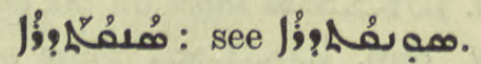

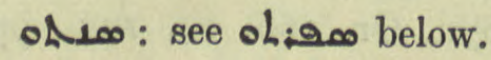

סח to gnaw, corrode. Pa. مص to corrode, fret, Poet. Syr. 99. 8.

人 is rather that of an eruption, vesicles;

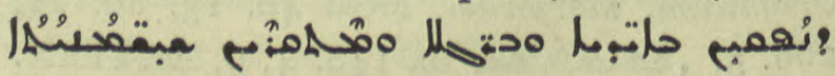
Med. $5^{8} 4.6,7,9$, ib. $5^{89}$. I 1 . 
on col. 2682. vowel-points uncertain. m. a measure; five sese go to one minute and ten to one foot, Med. 53r. Io; one onم equals twenty låต० ib.

oseseli officinale, meadow saxifrage, Med. 99. 4, 355 .

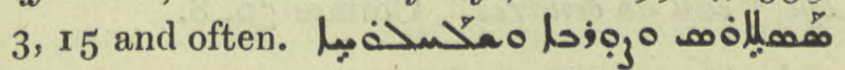
فر الانجبدان الرومى the blossom of Greek fennel,

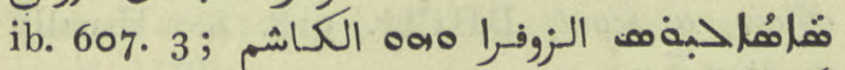

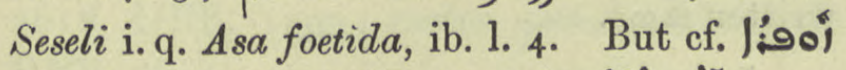
Suppl. and Ar. PflnN. $3^{6}$ under H?

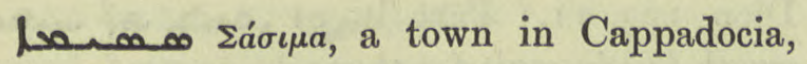
Greg. Carm. ii. 39. 17 .

مaسمe of an idol, corrupt for Dionysos, Hist. B.V.M. 200. 20.

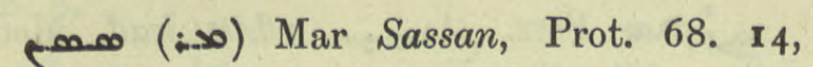
Charms 86 pen.

IRـ col. 2683. the medlar. Delete

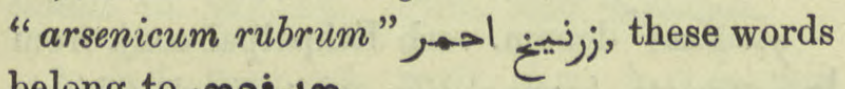
belong to معد:زסم.

مـحل col. 2683. Pael on attack.

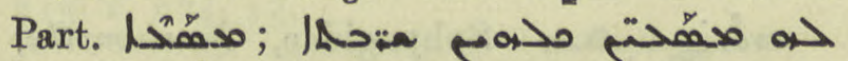
: Bahira 206. 16.

هحد unknown. Used to pulverize tin, هe: Chimie 36. 20.

محب حث مهر : col. 2684 . pr.n.m. add

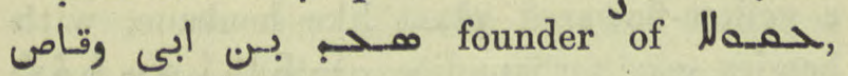
Sassanidi $33 \cdot 1$.

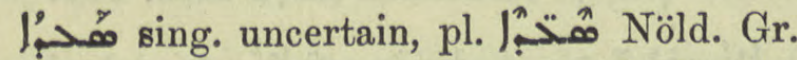

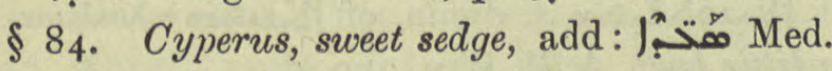

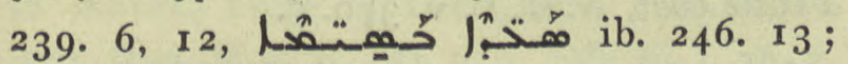
Ar, PflnN. 276.

هـ pl. Budge, perh. the root of Cyperus esculentus, Med. $57^{2}$ ult., $587.5,606.20$.

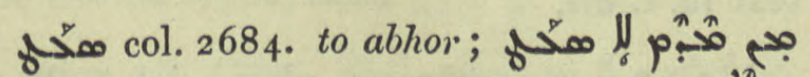

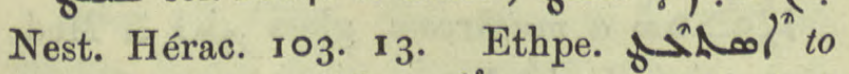
be abhorred, بمب G. Busâmé I 3 I Hormizd 1872 .

IL'ád abhorrence, pahad of of لمات the detestableness of pretended friends, But. Sap. Pol. iii. 4 .
Laso what? Ahrens and Krüger give excrement, Anecd. Syr. iii. 2 I 2 . 6.

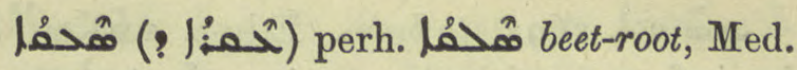
584. 6.

مَّ col. 2686. end of par. to visit = punish, Uחحت: Apoc. xvii. 16 ed. Gwynn, who suggests صمدر Gr. $\mu \iota \sigma \dot{\eta} \sigma o v \sigma \iota \nu$, Bagst.

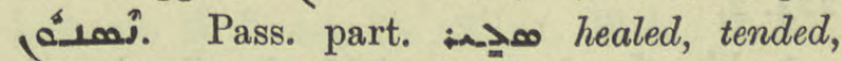

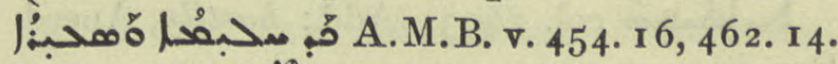
Ethpe. : $\mathrm{SLC}^{n}$ to be cared for, nursed, tended, Hippoc. vi. 37 .

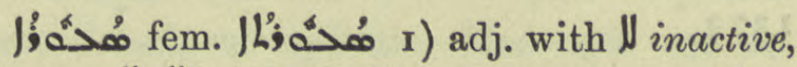

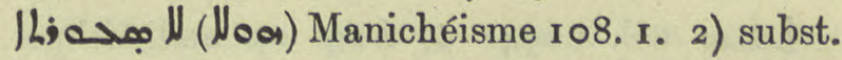
1. I 5 of par. caterer, purveyor, add: Pet. Ib. r 10. 3, I5. 3) a visitor of the sick, a healer, A.M.B. v. 462 . 4. Eccles. $a$ visitor i. q. chorepiscopus and periodeuta, a priest who visited villages and monasteries as representative of the bishop of the diocese, Takhsa I 28. 20.

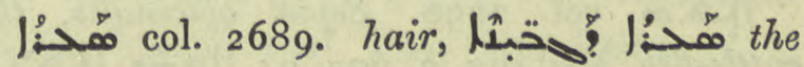
eyebrows, Med. 580. II. محت: ? palm fibre, Daniel 398. 13. J مَحة ferns, Budge, but perhaps صَّ Med. I62. 4, I4; or for مْحَ: 3 ib. 8 as l. 2. 8 add: тò ảoíavtov, Galen. ZDMG. xxxix. 268 ; Med. I98. 19.

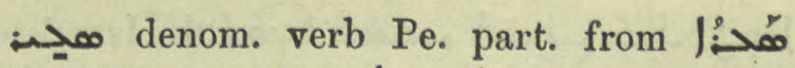

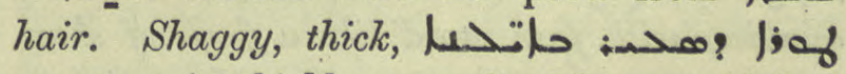
a mountain thickly covered with trees, Ahikar \$. 4. Aph. WDol" to have the hair grow, in Pallad. 15 I. 23.

oa hairy, 90a Jos. Wolflink 17 infra.

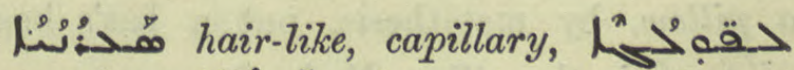

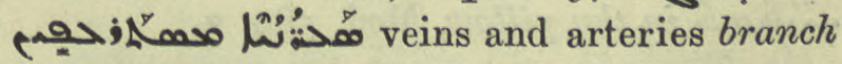
into capillaries, N. Hist. vii. I. 3 .

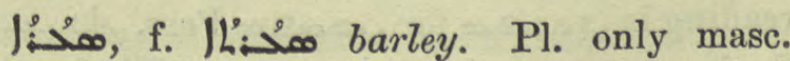

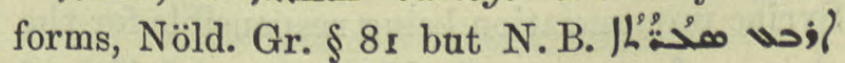
four barley corns, used as a measure, Med. 447. 7 but مُحن: 1l. 20. 2 I. A measure of time, ib. 53 r. 17, r 8 . The passage seems corrupt. 
مح: place-name col. 2692. Add: Séert, near Arzun, Chast. I 4.12.

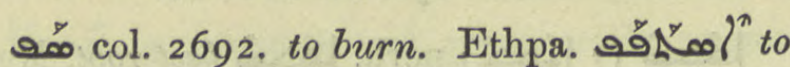
be inflamed with love, حل ا

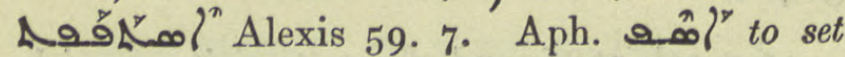

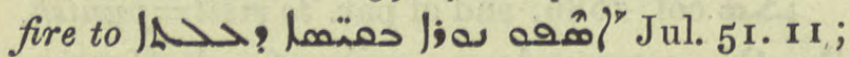

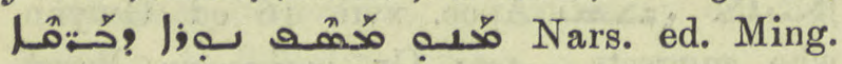
224. 2.

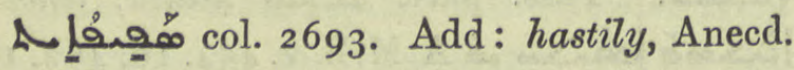
Syr. iii. 135. 17.

|فُ مْهَ col. 2693. conflagration, Hormizd 1273.

1ـ col. 2694. to heap up and Ethpe. ULAol to be heaped together: see refs. in Dion. under مصف Suppl.

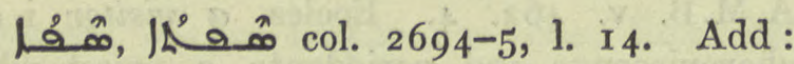
19 on filled to the brim, Chimie 59. I4.

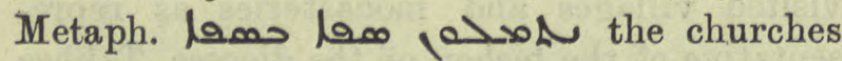
will be crowded, Dion. Ined. 488. $2 / 3$.

رلم col. 2696. Sepia officinalis, the

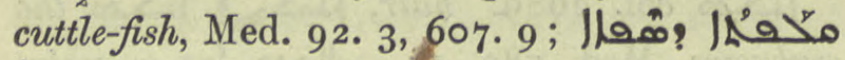
cuttle-bone, dentifrice, ib. 169. 21, I73. 22.

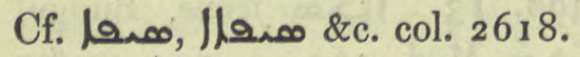

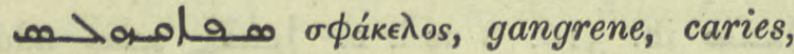
Hippoc. vii. 46.

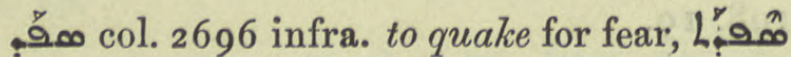

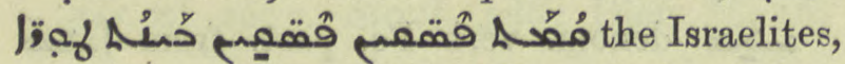
quaking with fear, stood like a flock of sheep among the mountains, Jac. Sar. Hom. ii. $3^{6}$. I3; مصف poa م. Jul. 79. 25 ; Ephr. ed. Lamy iv. 5II. I.

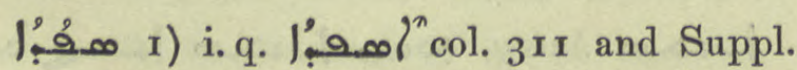
a wine measure, Dion. 107.21. 2) for مَّْ a pillow, by metathesis, put a bat's head

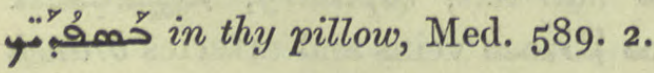

(0)ด - col. 2697. Correct; the best reading is 20000 for Syriac pronunciation being responsible for the change of $\triangle$ to 0 . Spahbedh, General, Tabari

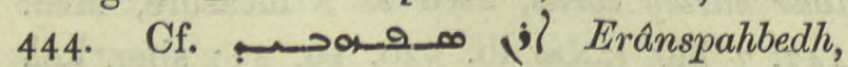
Commander-in-Chief, col. 393 .

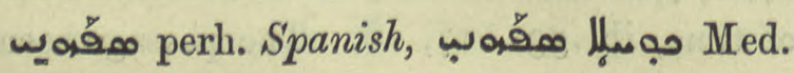
593. 22.

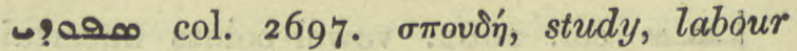

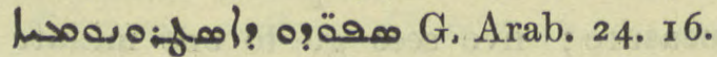

19 L B H. in loc., ZA. xvii. 88.

قu مaه ل: Saponaria, Soapwort, مaه lye, "eau de cendres", Chimie 50. 8.

lion on Lat. speculator, an Intelligence officer, a scout, BHChr. 518. 8. Usually

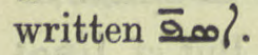

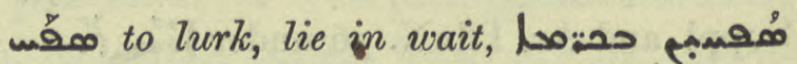

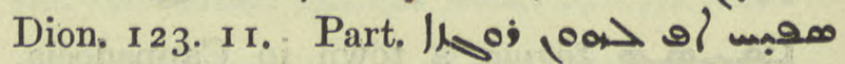
wrath lay in wait for them, ib. 135. 2.

aras for sosplat, bitumen, Chimie 8. 3 .

مaب: wers. Persite lead, Med. 586. I5; معبم0 Chimie 5. I3, 10. 2, 25. 10, 46. 19, 47. 5, (1. II.

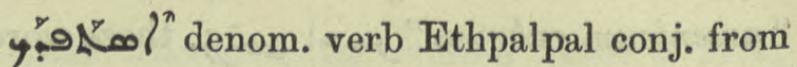
مفـب:ما I00. 15 .

oـ Sphyngidae, hawk-moths, N. Hist. vii. I. $\mathbf{~}$.

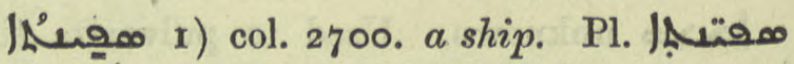

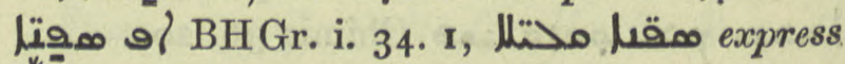
boats, mail service, Syn. Or. 19. 9. 2) f. a yellow-flowered plant like henbane, with

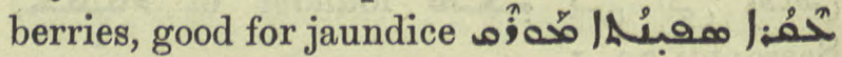
. 600.

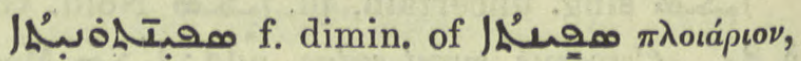
a little boat, A. M. B. v. 370. 5 .

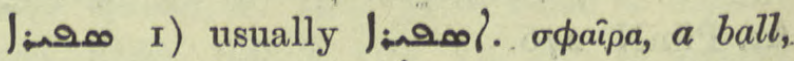
round mass 33. 19. 2) col. 2699. $\sigma \phi \hat{v} \rho a$, a heavy hammer,

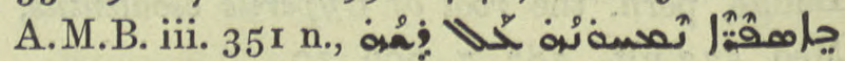
text ib. l. ult.

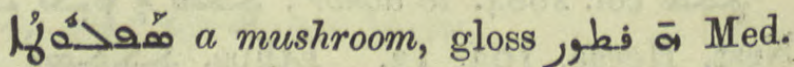

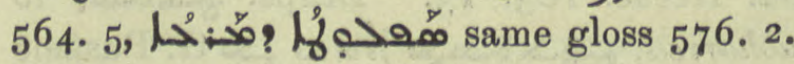

لإه BHGr. i. 65. 14; Med. 50. 10, 196. 10,

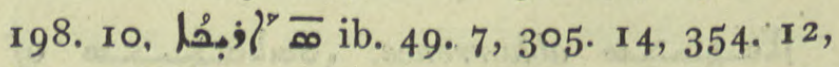

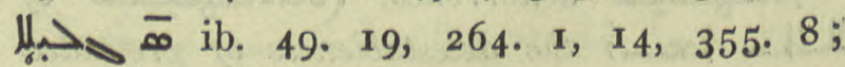

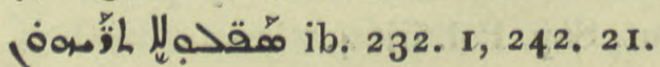




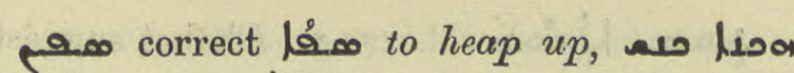

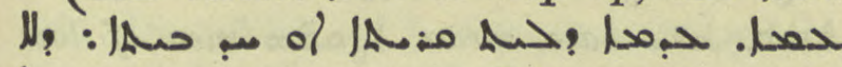

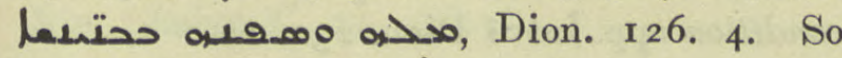
correct Ethpe. uी $\mathrm{A}$ / to be heaped up, over-

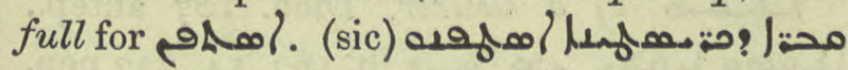
(oa) Dion. $3^{8 .} 19$.

مون Arab. any rough substance used for scouring, e.g. cuttlebone or fish skin; under Raan col. 26 r8, D BB. r346; Hala: o col. 26I8, DBB. I393; under قمعمب col. 3326 DBB. 1644 .

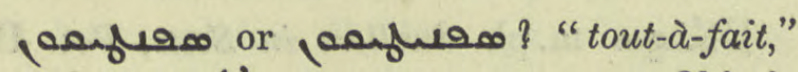

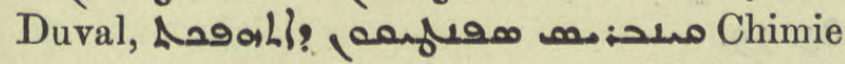
10. 10.

Protection 88. 8.

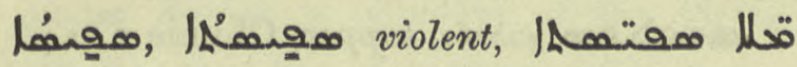

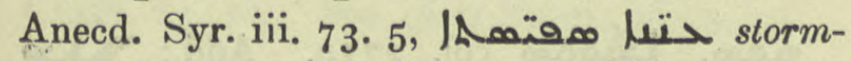
clouds, ib. 325. 23. Prob. read محتميل

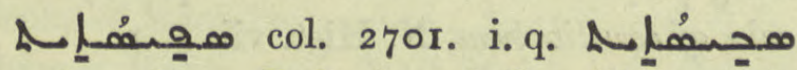
eagerly, often. Refs. Anecd. Syr. iii. 3. 5 af., 2 I 5. 2, $224, I_{3}$.

مaم مصمله denom. verb. See under

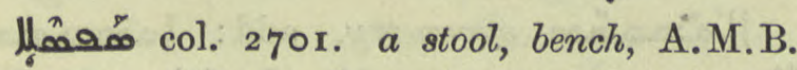
v. $577.4,608.3$. L. 4 of par. the presbyter's

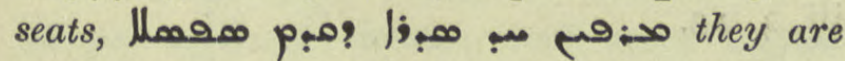
to leave one row of seats for the teachers, before those of the presbyters, Stat. Schol. Nis. 185. 5 .

|ดُمْْمَ col. 2704. Saphsapha, a district of Marga, north of Beit Abhe, Pers. Mart. 223 , Hist. Mon. ii. 150 n. 3 , refs. ib. i. 76.7 ,

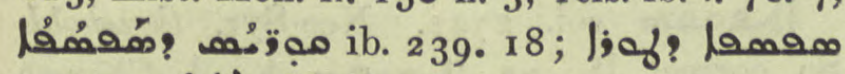

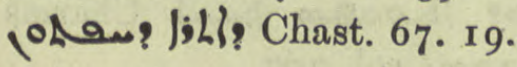

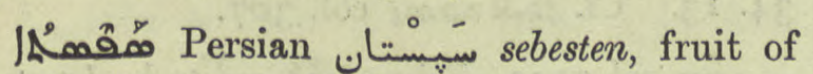
the Cordia Myxa, Med. 576. 5, 578. 19.

مaم col. 2704. Pael 20 2) to pour out,

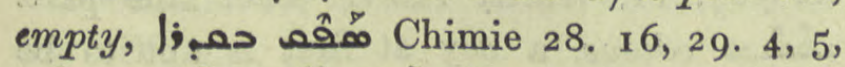

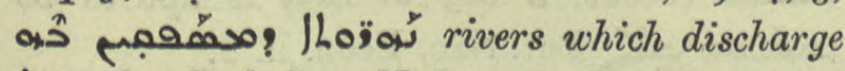
into the Red Sea, Natur 48. 6. To purge, evacuate, Hippoc. i. 22, 14, 17, with 50, ib. iv. 2, 20 clear out a vein, to bleed, ib. vii. 49. I.q. Aph. to grant,

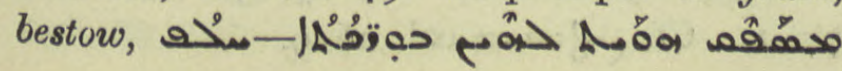

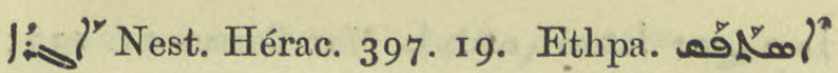
to be purged, Hippoc. i. 3, 2 ; to be evacuated, discharged, ib. i. 22 , iv. $2,25,47$, م

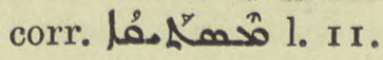

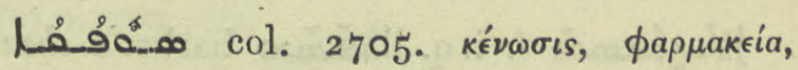
evacuation; the evacuations, add: Hippoc. i. 3,22 , ii. 8.22 , iv. $18,41, \operatorname{Med} .135 .19$,

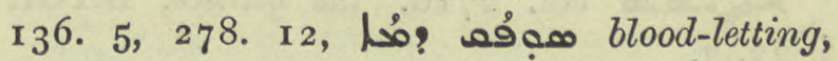
ib. 203. 2, 7, Hippoc. vii. 49 .

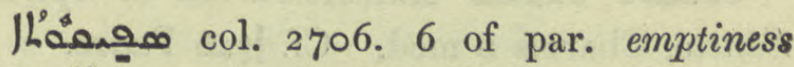
opp. Jlean o repletion, Med. 46. I9, 24 , I16. I3, I35. I9, 23, I36. 6 \&c. vacuity

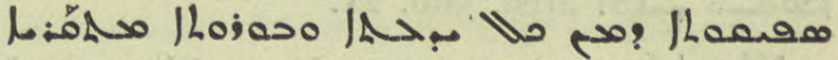
lack of knowledge is called boorishness, But. Sap. Theol. 5. 5, Periherm. ii. 5 ; Jheran مصan an infinite vacuum, Hebraica iii. 25 o. 19,25 I. 4 .

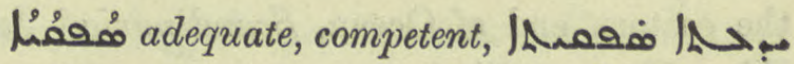

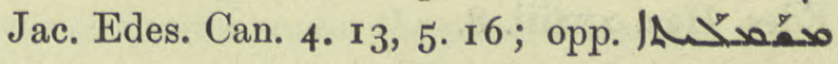
of the human soul, But. Sap. Philos. 7. 4 , حقمُـل مُهمْتِا ib. N. Hist. ii. cap. 2. 2.

. $\sigma \phi_{\epsilon} \epsilon^{\prime} \lambda \eta \dot{s}$, lye, Chimie 12. 18.

مصفّ: col. 2706 infra. Delete the ref. to I Esdras vii. 21 , that is the word la $^{\circ}$ repeated, مص:| مُهم: sase scribe of the law, S. Fraenk. ZA. xvii. 88. The quotation from Aphr. (=ed. Par. 700. 20) = Heb. ספר to recount; with his mouth he recounts the usual instruction.

م- م- on tonsured, a shaveling, حتي| ספمثرل moines, Chabot. Dion. 82. 16, I00. 22.

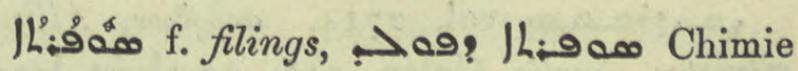
2I. $14,52.18$.

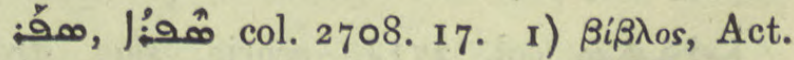
i. 20 ; add : Apoc. iii. 5 , xx. I 2 ter. ed. Gwynn

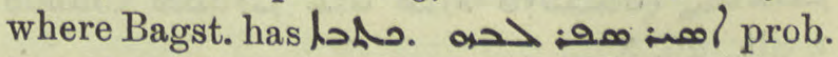
bound in the book of his heart, Charms roo. 5 . 2) the art of writing, script; Syrians have

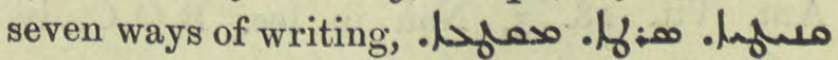

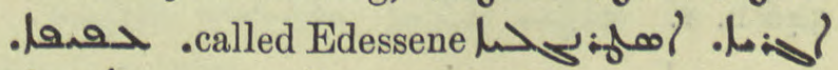
also JAw Ihe El. Nis. $95-73,96$. Cf. Thes. col. 2739 . 3) letters, learning, 
Rhaias

الر Mar Bassus II. I33; . 200fane learning, Pallad. 25. 4.

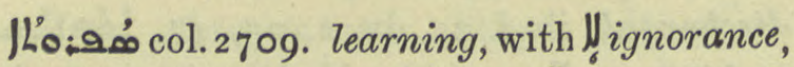

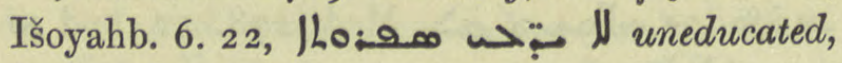
Georg. Ar. 30. 19.

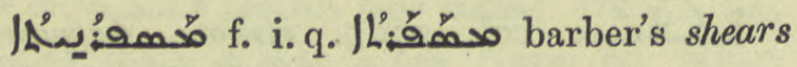
or scissors, Pallad. 128. 7.

Ho col. 27 ro. the quince, BHGr. i. 22.7 .

0Adeo o o 0 transliteration of Spiritu Sancto, Charms 6 med. Cf. I: Padre.

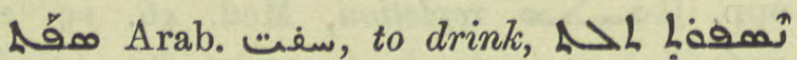

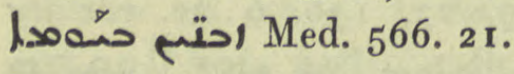

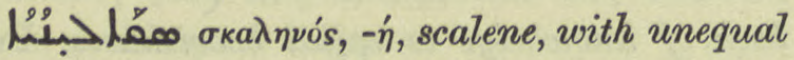
sides sc. مملف col. 2718.

(J) Great Scandia, an island at the eastern end of Ocean, Scandinavia? Jac. Edes. Hex, xxxiii. 8.

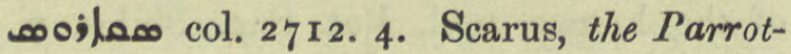
wrasse, the only ruminant among fishes, N. Hist. vii. 4. $\mathbf{x}, 6.3,5$.

col. 2713. scolopendra,

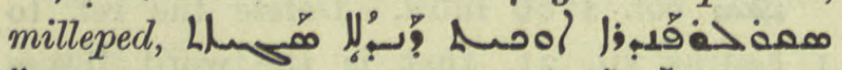

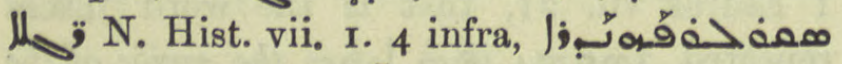
ib. 4, 5. م- scolopendrium vulgare, hart's tongue fern, Med. 398. I7.

ممعه:م.pl. Secundians, followers of

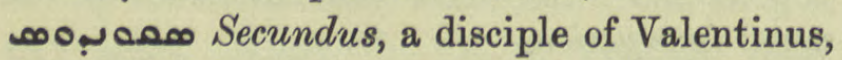
Coupes ii. 116 .

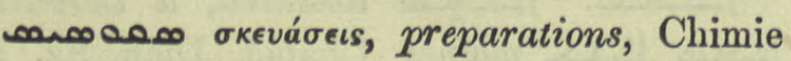
r3. 6 .

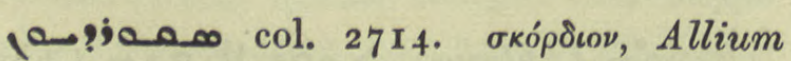

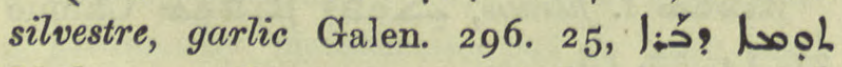

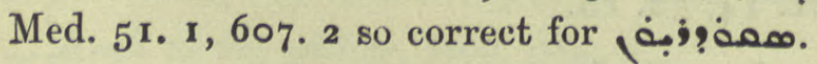

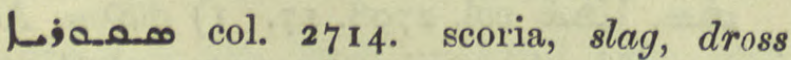

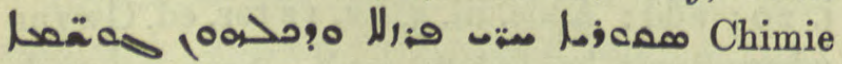
5 ult., 26.8.

هم:نuáptov, a utensil, vessel, Sev. Ant. Vit. 28. 5, Nau in loc. R.O.C. iv. 546 n. I.

Dochlaos pr. n. m. Ekoretuós, teacher of Bar Daisan, El. Nis. 96, 8r =ult.
معمك col. 27 I 5 . Chabot suggests Arab. سقط base, mean, Man of low condition opp. bio; Dion. 232. I 2.

بمـ 1) a usurer, Dion. 107 antep.,

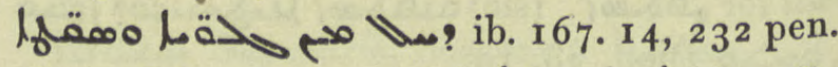

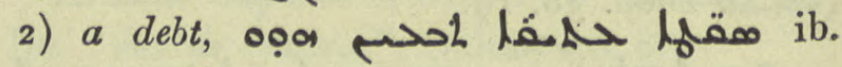
I62. 14 , ult., 199. 1 I.

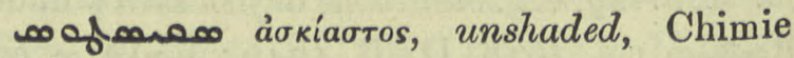
IO. II.

معيميمار Scete, Pet. Ib. 85. 20.

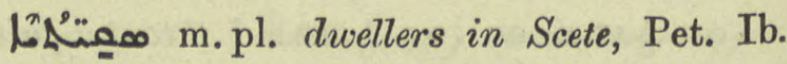
85. 19.

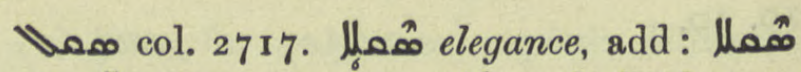

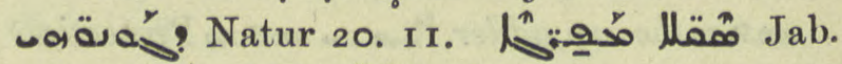
450. 10 should be قمُ

ص

مصمص

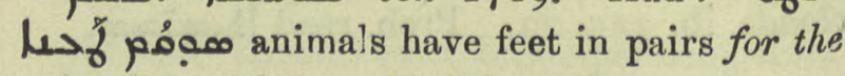
sake of equilibrium, N. Hist. vii. I. I.

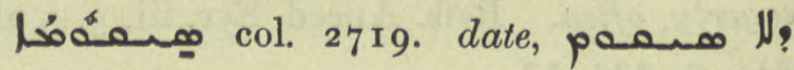
Vhas and andated MS. and the date of which is missing, Syn. Or. I I. 4 af.

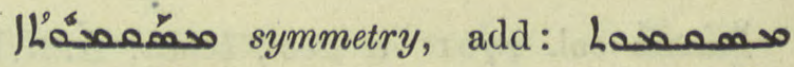
IAseso the evenness of handwriting, Or. Xt. iii. 4. 15 .

| 27 19. scammony, convolvulus,

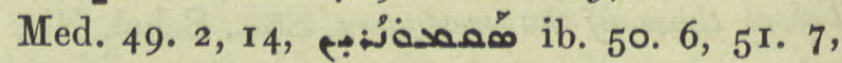
I5, 23, Chimie, Cambridge MS. $74 v 6$.

;

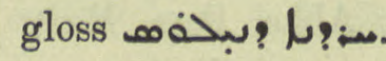

IAمa col. 2721. Neo-Syr. threshold. So correct In Charms 34. 13. Cf. /Aهaveol col. $3 \circ 7$.

معٌم: col. 272 21. Ethpe.

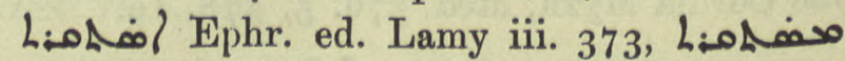
ib. 387. II, Warda $126 r$. Pael odious, a boy at first is apt to be But. Sap. Econ. ii. 2.

Aم malignantly, Sev. Lett. 444. 17.

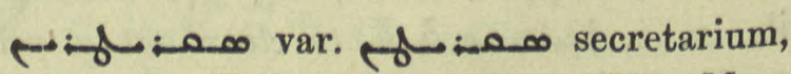
sacristy, Jo. Tell. 60. 10, 63. 5, Vit. Mon.

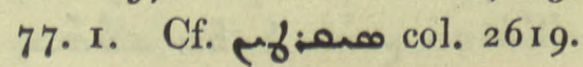




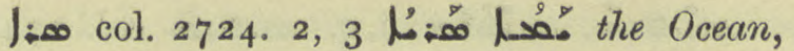

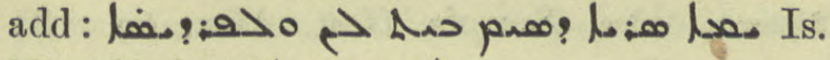
Nin. Chabot 8*, I4 10*.

ه: col. 2725. to deny an accusation, Ephr. ed. Lamy iii. 363. 9. Ethpe. Dishln to rave, Jos. Weinberg 24. 5 .

ه: Arab. شراب syrup, Chimie 36. 20.

هis col. 2726. (Hymn of the Soul)an unknown place. Delete ref. to Nöld. ZDMG. xxv. (so corr. for xxx.) 679 and add his later opinion, ib. lvi. 43I ; Bevan's n. op. cit. $19 b$.

o: the inner fortress of Beit Slok= Kerkuk, A.M.B. ii. 5I I ult., Eranšahr 2 I, Pers. Mart. 45 .

م Sarbat, a tributary of the Tigris, Chast. 13. 20. See (0); Suppl. p. 35.

ل.

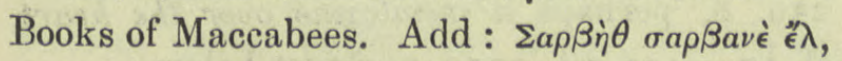
Grimm Makk. xvii, Keil Makk. 22, Geiger Urschrift 205 . Note by I. Löw, ZDMG. xli, 360. Cf. ed. Maclean 416. I3 and n.

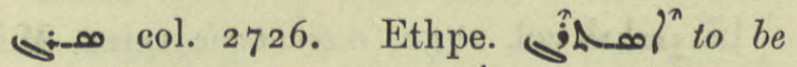
connected, knit together, ון

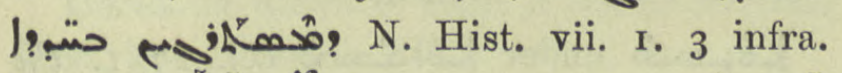
Ethpa.

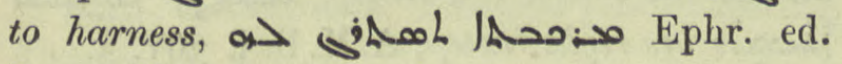
Lamy iii. 417. 10.

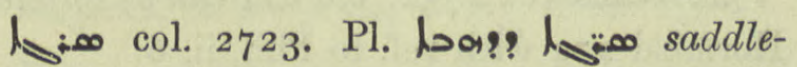
cloths, trappings worked with gold, Sassanidi 27. 3 .

$\because n$ : Hormizd 1326 .

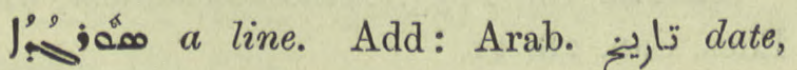

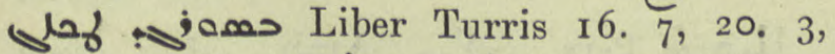
إمChronologie des S. Sanqlawaja ed. F. Müller 18, ZDMG. 1. 747 .

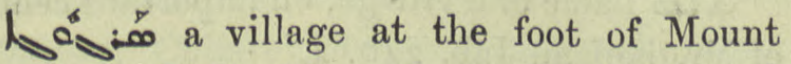
Kardo, A.M.B. iii. 446. 5, Hist. Mon. i. exxviii. $n$.

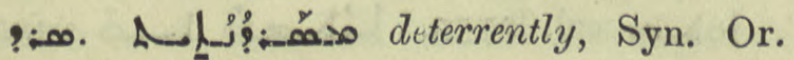
I38. $29,140.6$.

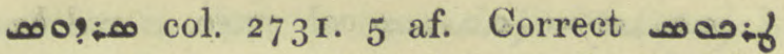
rápixos, dried or pickled fish, os ōa him بو A.M. B. v. 83. I, م: Pallad. 63. 6.

مِّ: col. 2722. Sardis, Brook's Chron. 574. 16 .

hy of a Scythian race, Ephr. ed. Lamy iii. 197 .

م:فistular, Med. 202. II. Cf. 1;00,

" سروش . "the Obedient" an angel, Sacred Books of the East, xxxvii. 2 I. 8 nn. 2, 3 . With alchemists a name for whitened copper, م:0 Chimie 3. 7, 6. 7, cuivre (saroch) ib. 260 n. 2,266 . 16.

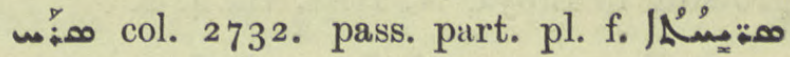
adulteresses, Ephr. ed. Lamy iii. I65. I4

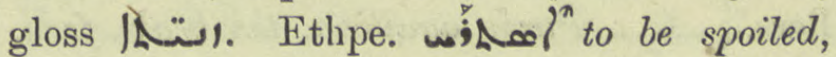

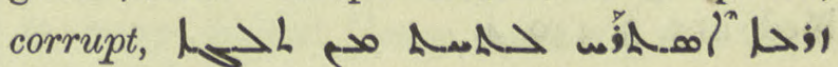
the seed rotted under the snow, Dion. 57. I6.

حـُ فُ viciously, Ephr. Ref. i. 67. I5.

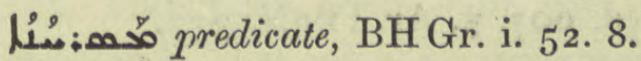

ช. to delineate, draw, IL'? ${ }_{1} \underset{\sim}{\longrightarrow}$ ? he drew the sign of the Cross on the wall, Mar Kardag 24. 7. Pass. part. هم: written about, mentioned, Ebed J. Card. 2 I. 6.

将: Jacobite, or Maronite script, Duval Gram. §9,

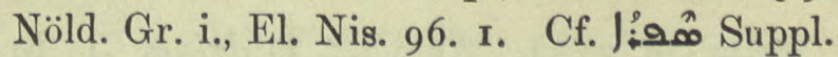

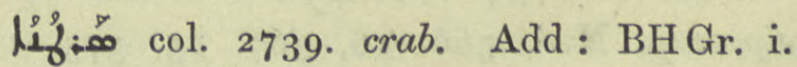
22. 20. Pl. A+f: kinds of crabs and crayfish, Chimie 6. 17. L. 4 of par. Cancer, a sign of the Zodiac, Med. 443-522, its colour is J' ib. 503. 19, its country i. e. that over which

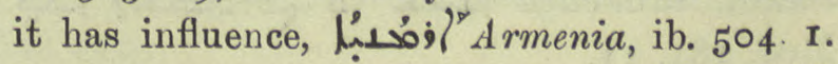

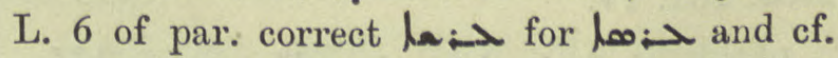
col. 3001 . L. 8 of par. the disease cancer,

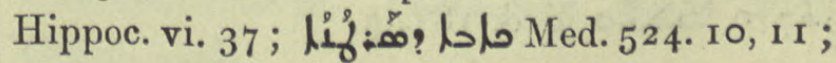
pl. ib. 48. 2 I, 50. I and often.

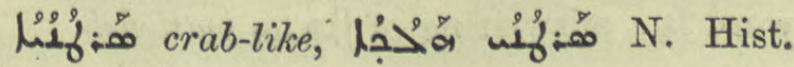
vii. 6.6 infra.

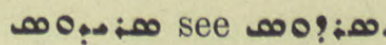


م salt, Chimie 5. 8 trad. ro n. 10. Pers. اسرنج or

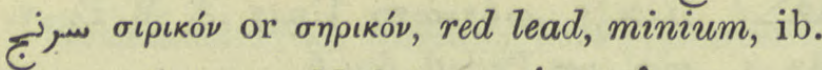

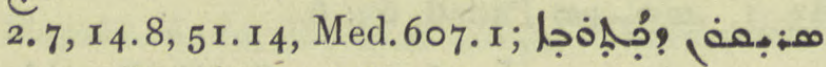

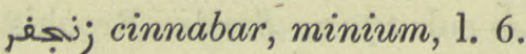

4 × col. 2740 . to adhere, stick of food,

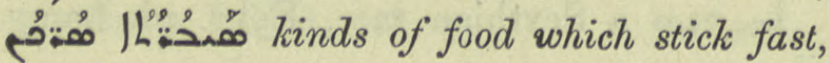
clog, Med. 269. I6.

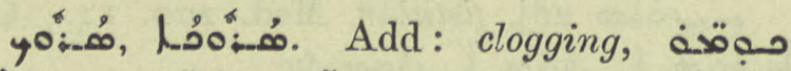

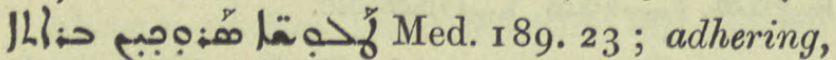

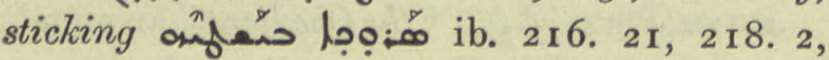
277.24.

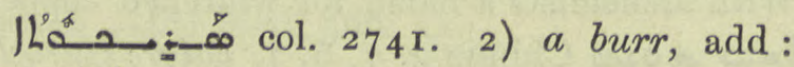

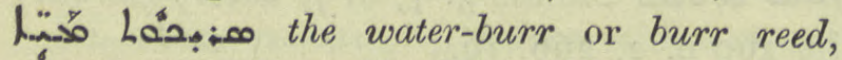
growing in Libya, N. Hist. vii. 4. 2.

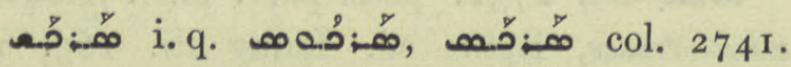
Pers. Pteris aquilina, the brake fern, Med. 2 I 5. 5, 430. 4 .

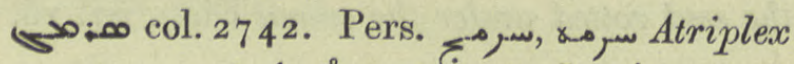

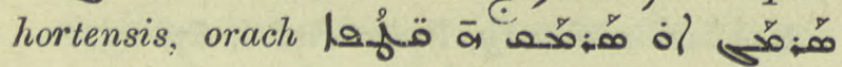
ق. Med. 607. 5, Ar. PflnN. 337.

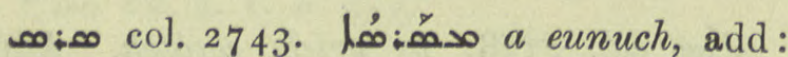
metaph. to abstain Wa.oh o. Gaza MS. 2 I $8 a$, Maclean.

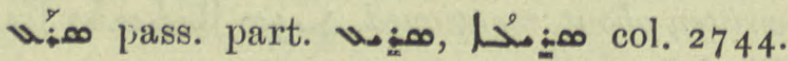
slit, fissile,

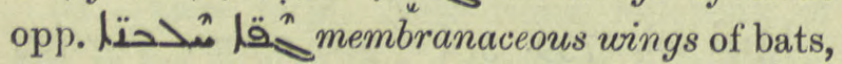
Natur 35. I 4 .

ق م: col. 2754. Ar. ش.رب to drink. I) to sip, sup, Med. 566. 16, 567. I5, 573. 1 о.

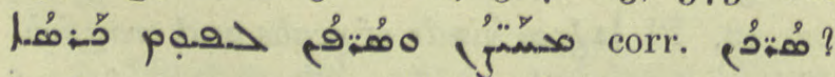
foods which contract and block the exit of the rectum, Med. 46. 6. Ethpe. 9iseol to be

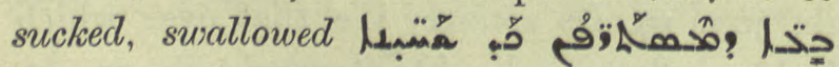
Med. I8r. I r.

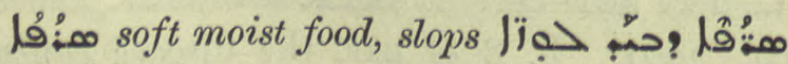

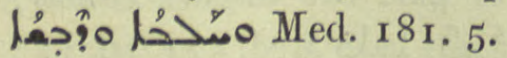

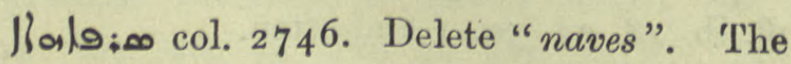
word is a miswriting of Jlane cuttlefish.

ג Arist. Apol. «. I.

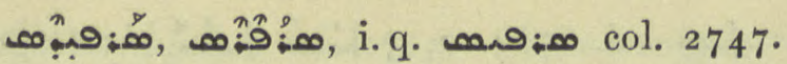

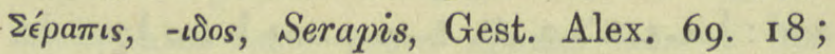

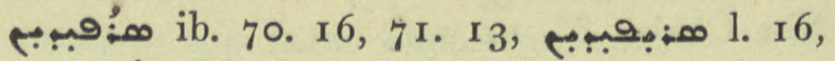

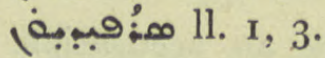

م- on the Serapeum of Alexandria, Epiph. 20 ult.

( ) and a names of a mountain, Anecd. Syr. iii. 52. 4, 53. 6, I3.

لا سَ- New Year, the $4^{\text {th }}$

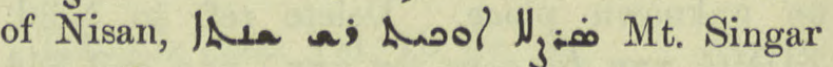
32. 2,6 .

م: م: col. 2750 . a cirrus, feathery cloud, Dion. I33. 5 .

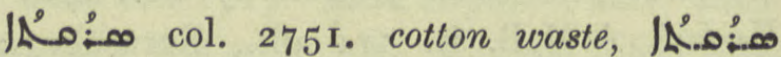

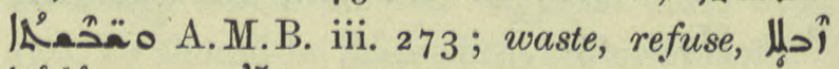

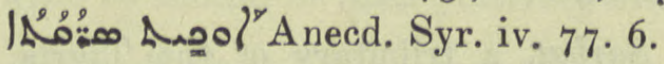

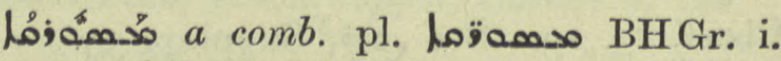

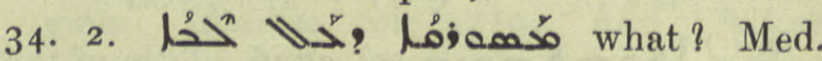
259. 8, perhaps a membrane over the heart, cf. لم ow col. 2752, and just below.

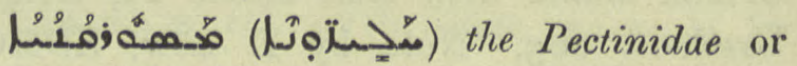
scallop shells, N. Hist, vii. 2. 2.

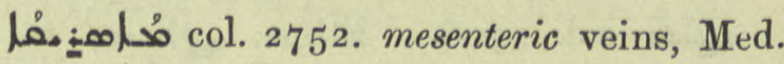

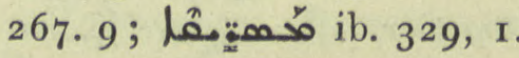

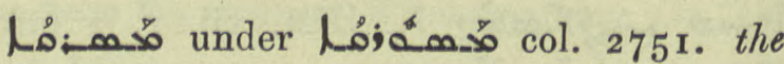

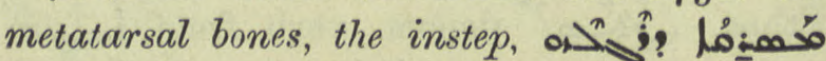

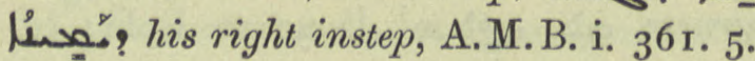

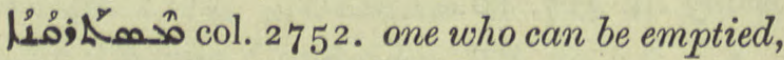
Hormizd 274 with ref. to Phil. ii. 6.

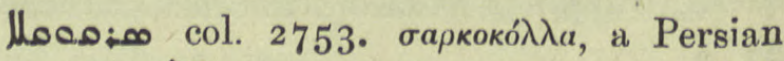

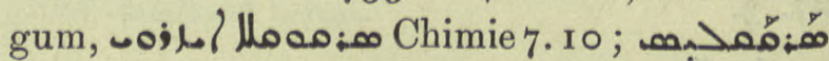
Med. 6r. I, 5, I5, 84. 1, 85. I, 3. Cf. Log从ג.

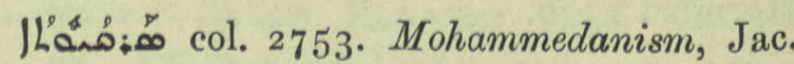
Edes. Can. 28. I r.

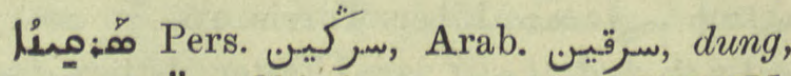
احلا هimie 41. 5, 79. 5, WZKM. viii. $3^{6} 5$.

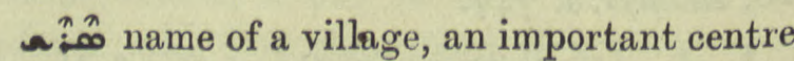
of Magianism, Jab. 240. 4 .

a $0: \infty$ see above.

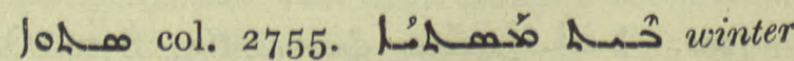
quarters, Jab. I $3^{\mathbf{I}}$ med. and often. 


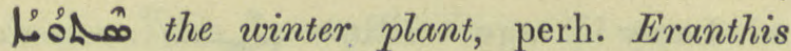

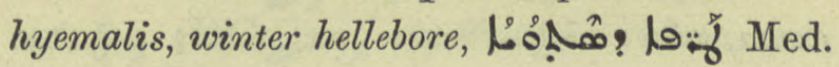
575. 20.

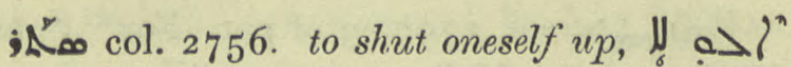
全 unless I had secluded myself entirely,

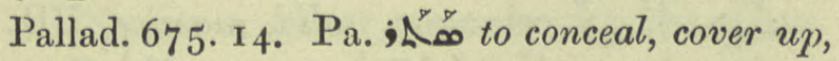

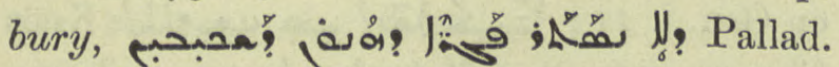

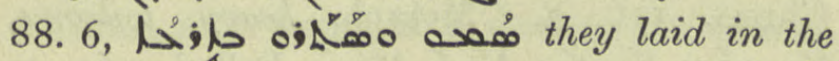
earth and covered over, 1. 7. Ethpa. iLئl' col. $275^{8}$ end of par. The quotation from BB. about oes?jol the heron is to be found in Natur 34. I3.

ן o col. 2758. The second canonical

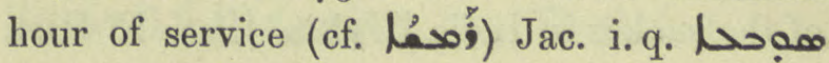
Nest. compline, Brev. Ant. i. Cal. 5 I a I I, $57^{b} 2,59 b 5$.

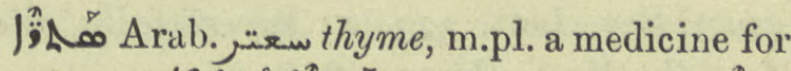

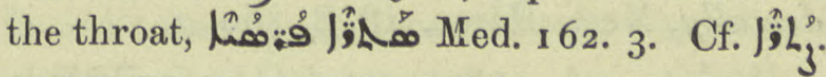

ILis_ o f. a shelter or hiding place, Dion I $5^{2} .5$.

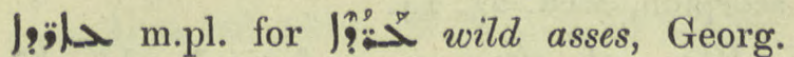
Arab. 31. 5 .

حـا thicken, lall it becomes rather stiffer than honey, JAOS. xx.

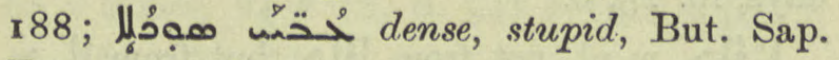
Econ. II. 3 infra.

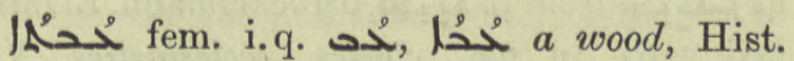
Mon. i. 339. 8.

حُحفنُ Hist. Mon. i. 159 .

حـ m. thickness. The solid part of arsenic, Chimie 42. 3 .

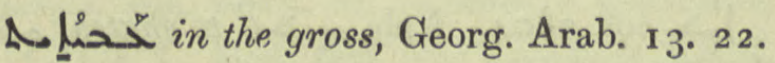

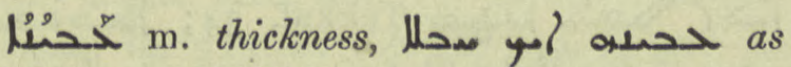
thick as a cord, Dion. I33. 2.

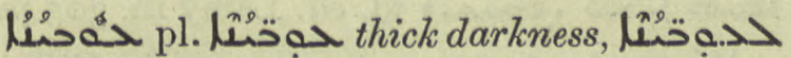
porhuih loah, Odes of Solomon, ii. 23 r.

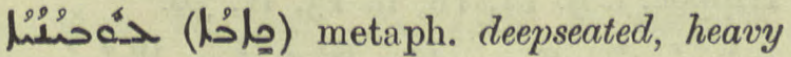
sorrow, Jab. 6. 4 .

حבِ col. 2766 infra. 3) to make, appoint to an office, add: with governor, Arab. ع., Dion. 26. 6. Act. part. هNoo? خصم it has taken place, the result is

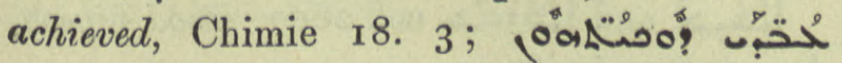
locum tenentes, representatives, Anecd. Syr. iii. 326. I I, I2. Col. 2767 supra. With $>$ to

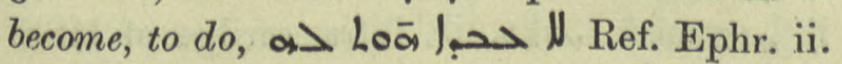

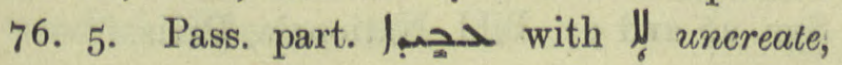

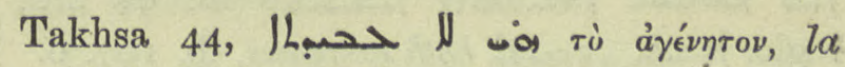
qualité de l'incréation, Manichéisme 98. 2, |lly add: to prompt, induce, Georg. Arab. 1 2. 7. Ettaph. حصدّ bono sensu Hormizd r355. Palp. خح to subjugate, wild elephants

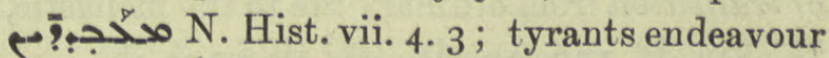

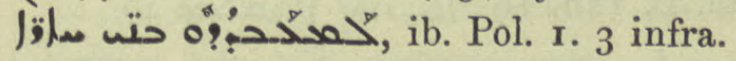

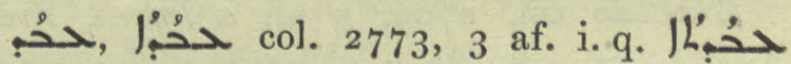
f. goods, property, ajha> ححبs, Chron. Min. 258 . 8, A.M.B. v. 434 . 2, Dion. 40. 16 , 52. I. Effect, proof, oa مح قعلا لح raho!an, Pallad. 75. I7, 21 ; pen. 76. 2.

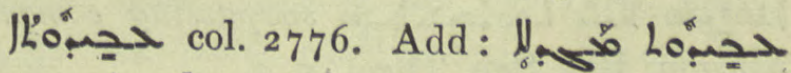

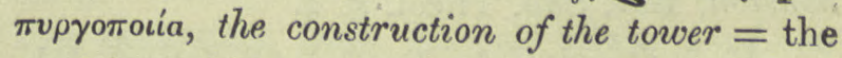
teaching of Nestorius, Sev. Ant. Vit. 330. I I. حصب ' $A b d S$ Suk, name on a mosaic tombstone, Flor. Vogüé 234. I0.

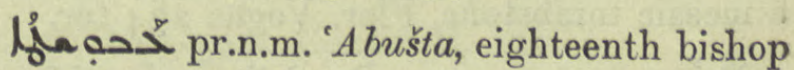
of Adiabene, M.Z. 65 pen., ZDMG. xliii. 4 or.

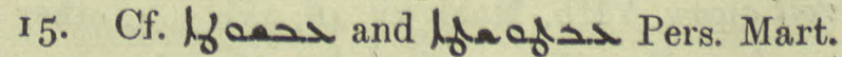
n. 8 iо.

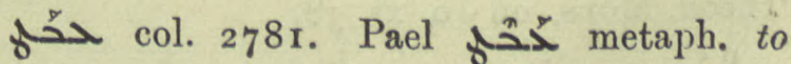

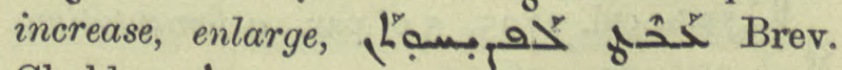
Chald.

ILafor denseness. Add: ohofod פיم:حا serried ranks of battle, Josephus vi. 5. 4.

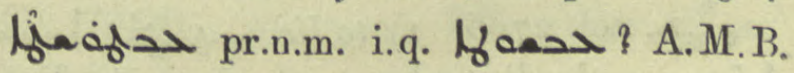
ii. 5 ㅇ. $\mathrm{I} \mathrm{I}, \mathrm{I} 3$. 


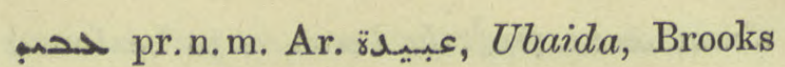
Chr. 574 ult., ححاحب ib. 875 . I.

حבّ col, 2783 . Add: to surpass; the size

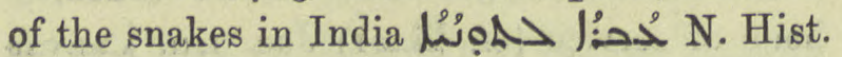
vii. $4 \cdot 2$.

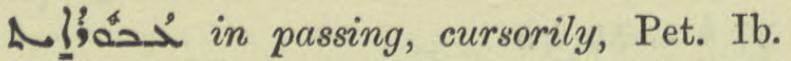
20. 3 , Sev. Lett. $3^{2} 3.2$; superficially, incidentally, Nest. Hérac. 1 8. 8, 169. 3 .

حصمَزئl col. 2787. evasion by quibbling, betrayal, Jlessasa! لح Pléroph. 14. 5, 16. 10.

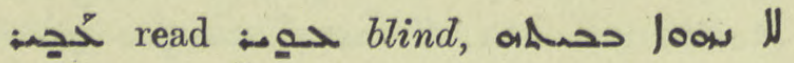
לֶّ S. George $24 a, 4$ af.

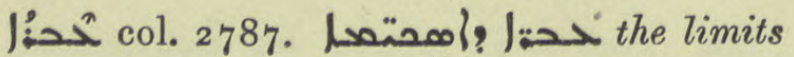
of figures, Probus 90. 15, 93 ult., o ib. 94 ult.,

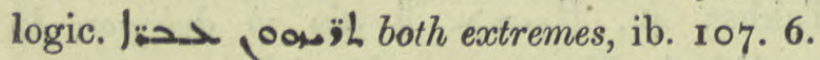

JLo: col. 2788. Spic. Syr. correct 1200

ơl col. 2789. Add: issue, conduit, a gutter for filth,

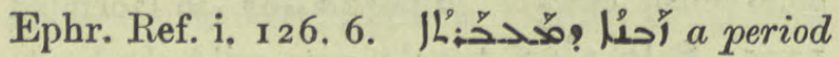
of transition from ordinary life to the monastic,

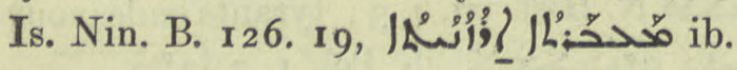

مخحد:ُ col. 2709. 5 af. a hymn, Brev.

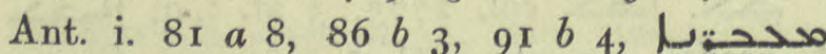

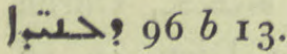

J'a์

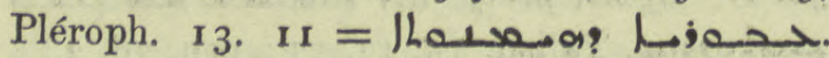
 hension, But. Sap. Theol. 5. 2.

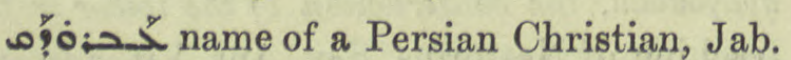

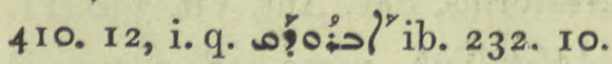

רan col. 2791. 'Abshai, pr.n.m. on a mosaic tombstone, Flor. Vogüé 234 ter.

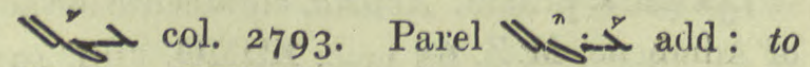
roll away, VHh. Mark xv. 46, xvi. 3, 4, other

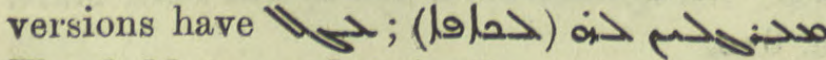
Theod. Mops. on Jo, xi. $3^{8}$.

لـ col. 2795. 5 of par. a fomentation, Med. 301. 22, 316. 7 .

Hón dimin. a little ball, Anecd. Syr. v. 77. 12.

"حم col. 2796 , 1) to lie, Ais as the dust wherein the dead lie, Ephr.

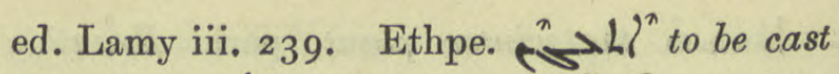

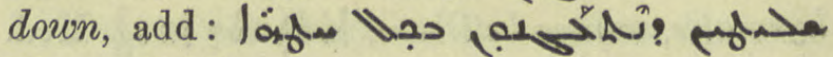
Nars. ed. Ming. 360 n. 2.

1L'ás col, 2797. 3 of par. For " Sanct. Vit." read "A.M.B. v. 269. I 4."

خ col. 2797 . solid, massive, $\mathrm{BH}$.

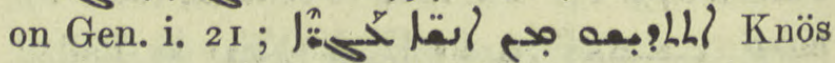
Chrest. I16. 5. Metaph. heavy, toilsome, 年 Pallad. I69. I, Anecd. Syr. ii. 6 r. 8.

ح An. Syr. 120, 2.

$1 h^{\circ}$ - خ thickness, bulkiness of fishes, Lo:

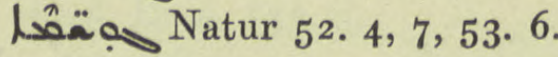

H.' m. col. 2789. m. the sting of a scorpion, Med. 25 . 14.

حِالُْ

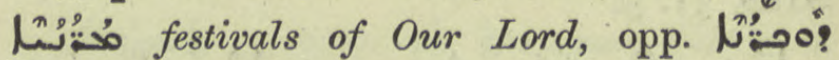
Saints' days, Takhsa 78. 17 ; Brev. Chald.

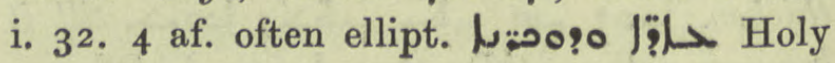
days, ib. 25.6 .

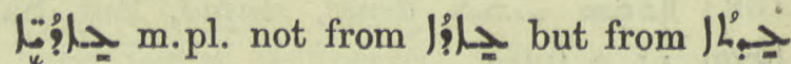

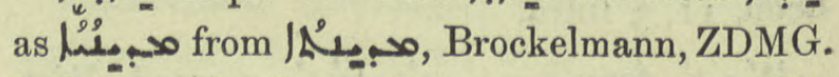
lxii. 39I in loc., clerics, ecclesiastics, Vit. Monoph. 90. I 7 .

حسَّ col. 2804. a pad or plug of cotton

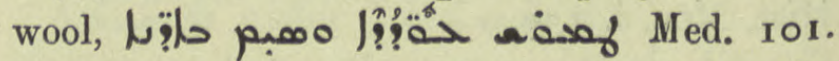
$21,102.11,103.20,104.7,22$, for the womb, 325. Io so corr. for Jị.

حمبّ 仿 Ephr. Ref. ii. I4I. 21 ; to slash off,

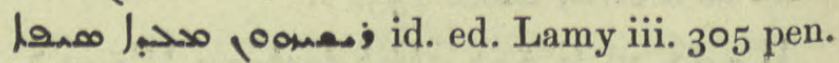

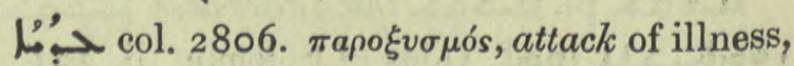
Hippoc. i. 9. 11,18 , ii. 13 , iv. 30.

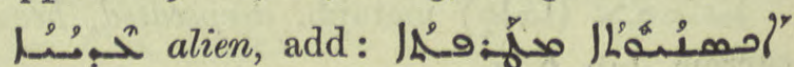

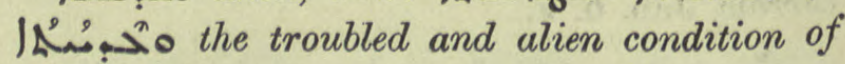
pilgrimage, Jab. 475. 6.

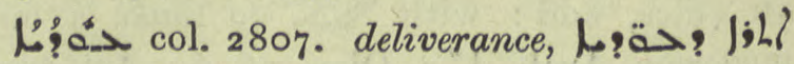
Ephr. ed. Lamy iii. 225.

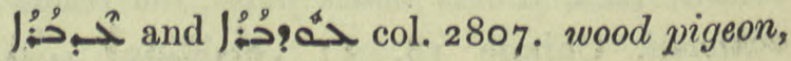
Anecd. Syr. iv. 83. 5.

col. 2808. pr.n.m. 'Adi, ref. Or. Xt. ii. 4I 5 med,, ح ib. infra, scribes of MSS. Syr. 26 and 27 , Bibl. Nationale, Paris. 
Arab. Jus to decide justly, apportion

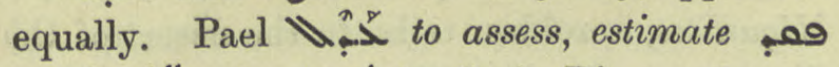
حion. I 48. 2, 9 .

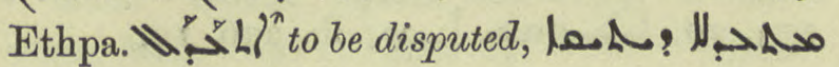
Syr, Rom. Rechtsb. 7. 7 .

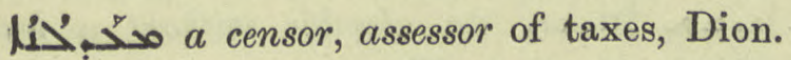
I $21.20,147.21,148$ ter.

مصب04 f. name of a demon, see Suppl. above.

لحب. 28 colo. census, WZKM. x. I65, refs. Dion. 10. 20, I I. 5, I 2 . I.

( 1 Ethpa. take delight in, Tekkaf 66.

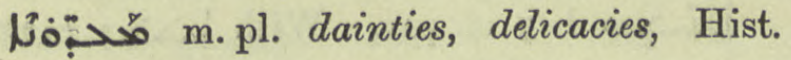
Mon, i. 373. $\mathrm{r}$.

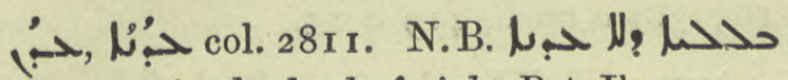
vì่ äkaipos, in the dead of night, Pet. Ib. 22 . I I, Sev. Ant. Vit. 24. I 2. Col. 281 2. I 5. Rit. the three night services, add ref. of خד: Brev. Ant. i. Kal. $59 b$ antep., $60 a$ sexies, $64 b$ I6, ii. 54 I $a$ 1 $2,13,564 b$ I 8, 58 I $a$ II, I3.

مدـ: :

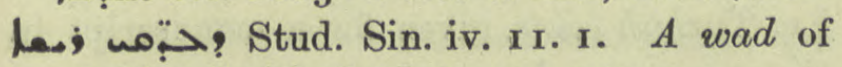

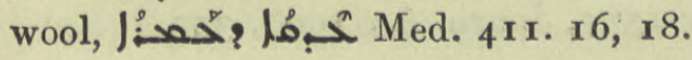

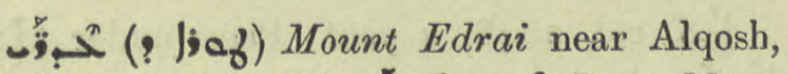
Hormizd r397. Cf. 21 and col. 2815 .

حَ col. 2815 . m. Saponaria officinalis,

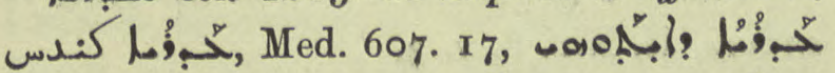
مبدبمة ib. $3^{1} 3.12$; it. ib. 390. I, 398. 9 , 429. 18 ; Ar. PflnN. 244, f.

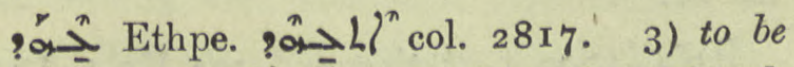
mentioned gaxhl? ba the (afore-)mentioned, Vit. Mon. ro. I9. Pa. ?

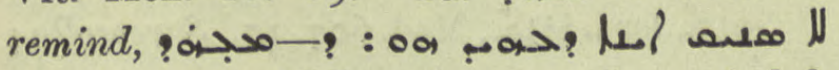
he who is mindful needs not to be remindad, Ephr. ed. Lamy iii. 4/3 af., ib. pen., 33. I.

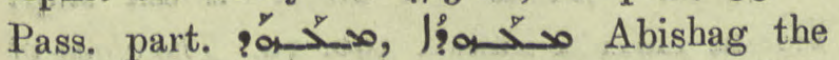
Shulammite ars (s) is mentioned in it, $\mathrm{BH}$. on Cant. Cant. Proem.

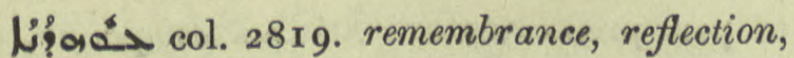
مجإ a little reflection has

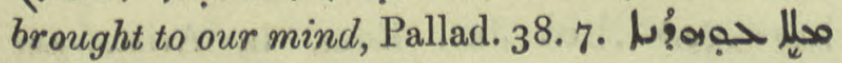
mindful, Natur I I. I 5 .

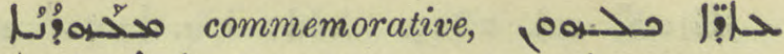

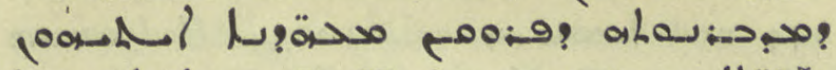

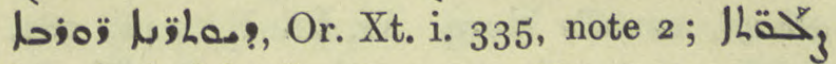

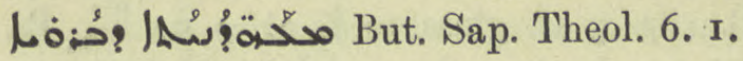

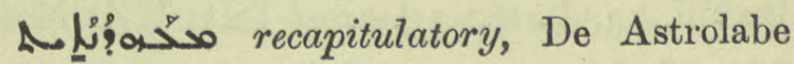
ed. Nau 86.

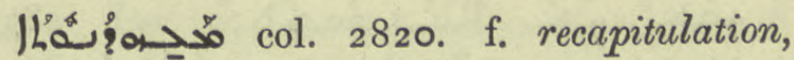

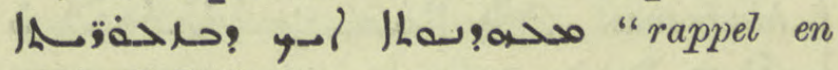
abrégé", R.O.C. xv. 24 I.

; ?רג'; those who argue madly, BH. Stories 30 . I 59 .

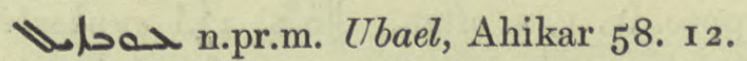

حه col. 2825 . Pael ${ }^{2} \stackrel{n}{\sim}$ pass. part. fem.

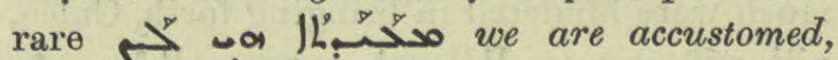
Sev. Lett. I 7 pen.

حُ حُبْ col. 2826 infra. m. custom. Add:

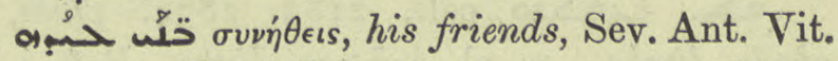

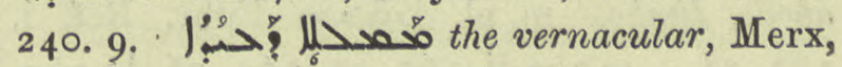
Hist. Gram. I 72 . 1.

حسُبْنُ El. Tirhan 3 ult.

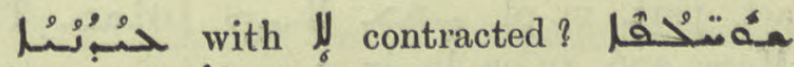

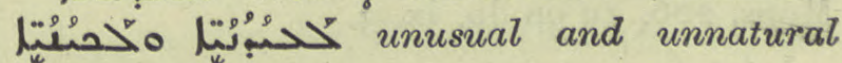
changes, Jab. 148 . 1 I.

!a col. 2827. particle again, indeed.

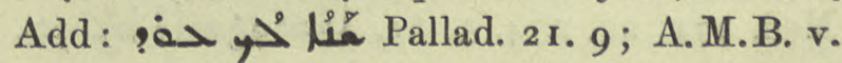
I3. 20, 34. I3, 55. 7, QdhamW. 185. 6. حهar Benk. 46. 5, 65. 3. Cf. Nöld. ZDMG. xxii. 485 , xl. 725 . The quotation from K. col. 2827.4 af. to col. 2828 . 6 ending مصم:l is from BHGr. i. $162.2 \mathrm{I}$.

?a col. 2828 . Audi, founder of the

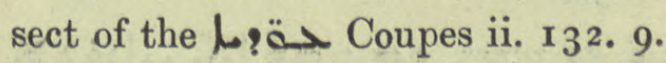

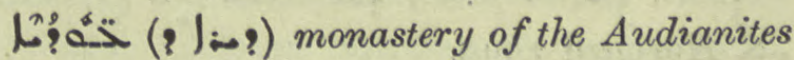
at Rome, A.M.B. v. 29I. I5, Bedj. corrects

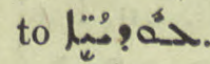

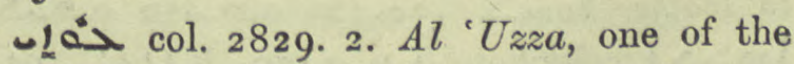
two chief goddesses of the Arabs; she was worshipped at Hira, Tabari 17 I, Anecd. Syr.

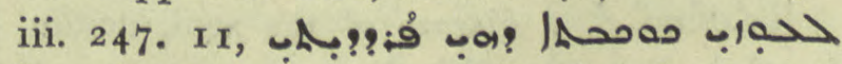
joal Bahira 203. 10, I1, Is. Ant. i. 2 Io. I4, Nöld. ZDMG. xli. 7 10; BB. under /3Ae? col. $3^{26}$. 


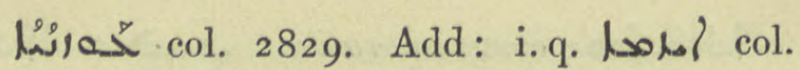
I44 and Lxol.a col. I003. Hedysarum alhagi, camel's thorn, Ar. PflnN. I46. 282. DBB. 483.5 af. gloss to مص

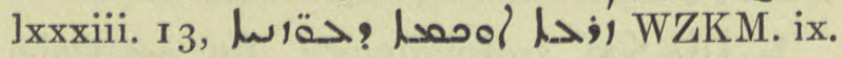
209. 20.

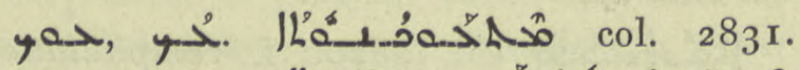
f. hindrance,

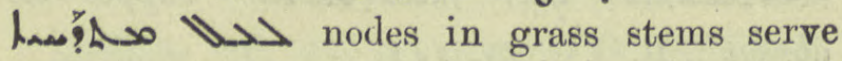
to hinder moisture from being pressed upward, N. Hist. vi. 2. 2.

$ل_{G}^{\alpha}$ col. $283^{2}$ ult. the foetus, $\ddot{\epsilon} \mu \beta \rho v o v$, add: Hippoc. iv. I, v. often. نُحْئه euphorbia, spurge, so-called because cattle eating too much were hindered in bringing forth, Galen.

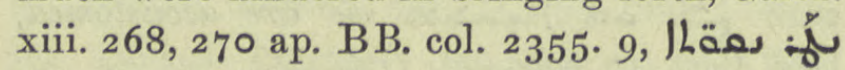
the same, col. 86 .

(a). Laح cols. 2185,2834 . place, abode, limbo. m.pl. محدة Ephr. Ref. i.

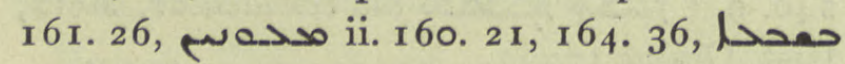
محتم ib. 204. 45.

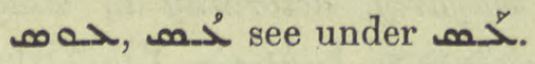

وه فح col. 2835. to double, to gain,

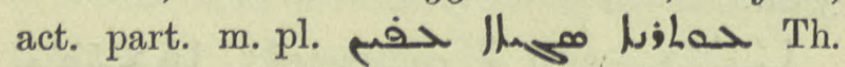
Mops. 206. 19 on Jo. ix. 3 .

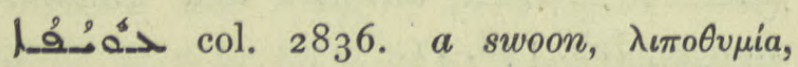

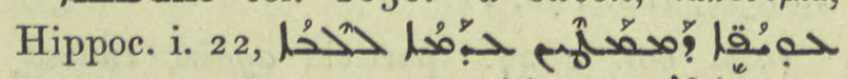

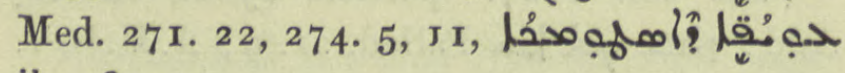
ib. $28 \mathrm{I}$. II.

|حَ collective twigs, sprigs, foliage, add :

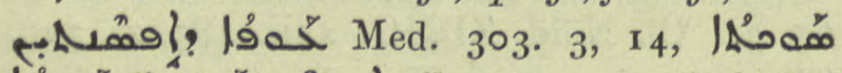

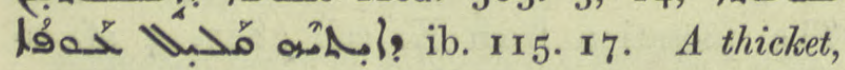

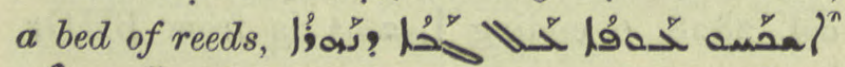
की ० cxxvi. gloss ل्रांi.

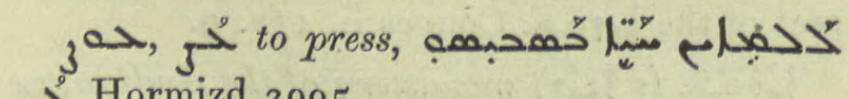
$5^{2}$ Hormizd 2995.

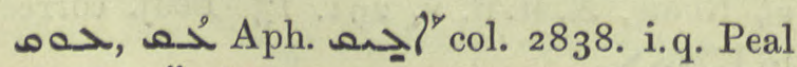

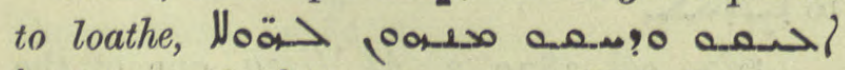

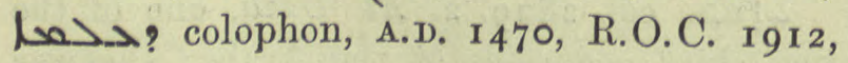
88 Syr. 3 .

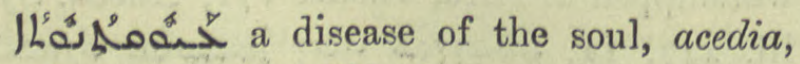
boredom, But. Sap. Eth. iv. 3 .

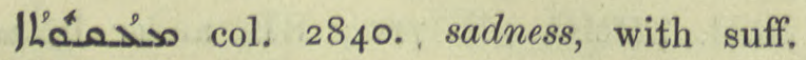

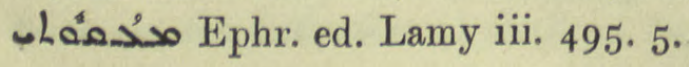

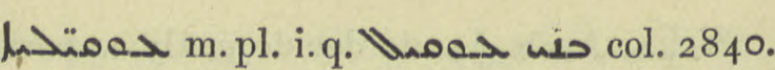
'Uqailites, a robber tribe in the desert of the Euphrates, El. Nis. Chron. 22 I, II, 222. 2 I, 233. 20, I ib. 226.19.

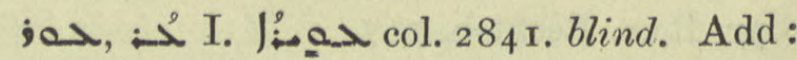
lisal an alembic without a head, Chimie 33. 20. The caecum, a part of the great intestine, Hebr. iv. $21_{5},{ }_{2} 59, \mathrm{~N}$. Hist. vii. 6. 5 .

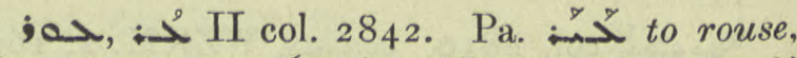

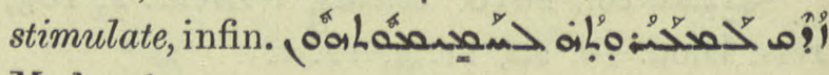
Med. 262. 10.

L'o col. 2845. insomnia, Med. 538. I5.

بح 1.5 of par. With 1 invitatorium, part of Nocturns, Brev. Ant. ii. $34^{2} b$ 16; 381 $a$ med., 4I $9 a$ I5.

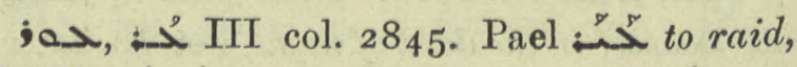

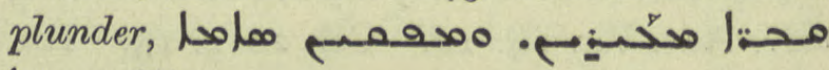
bag? Dion. 132.4.

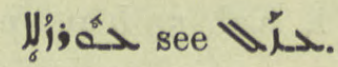

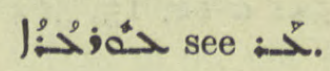

ح. m. ottomans i. e. Turkish coins, given for a manuscript in the year I555, Journ. As. 1907, 355 .

حُخ

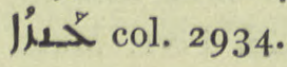

ار col. ${ }^{28} 8$ o. Ethpa. ref. الل let him not cease to endure, Is. Nin. 8. v. 3 .

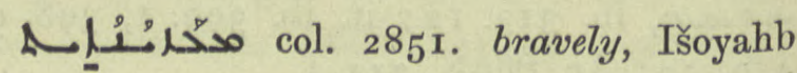
I0. 10 .

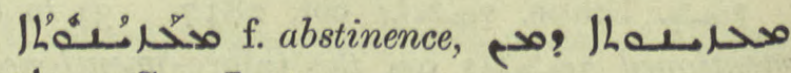
JLةقدa, Sev. Lett. 490. 9.

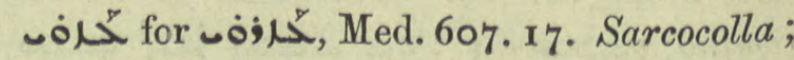
see 1 for other spellings.

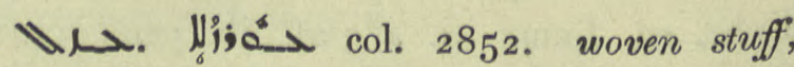
Pallad. 390. 19. The clash of battle, Sev. Lett. 500. 7; ه.M.B. v. 598. 9. 10 of par. predicates are $\mathrm{N} / \mathrm{N}_{\mathrm{j}} \mathrm{C}$ compound, as ح:

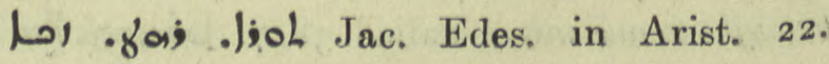
$3,4 \mathrm{ff}, 24.16 .19$. 


\section{(1)}

مـد פ Georg. Arab. 25. 3.

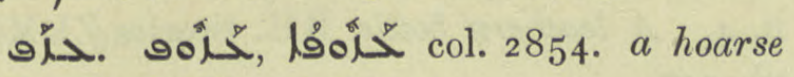
voice, Med. I82. 16, 607. I7.

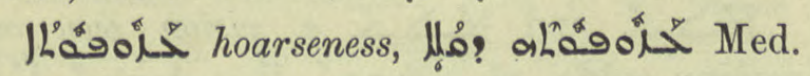
I8I. 12.

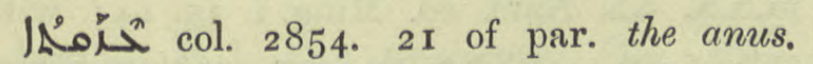
Refs. Hippoc. vii. 36 , Med. 570. 7 , 16.

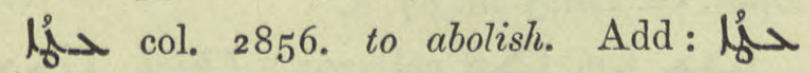
J'joh Pharaoh swept his heart clear of uprightness, Nars. ed. Ming. ii. 296. 8. Cf. Aph.

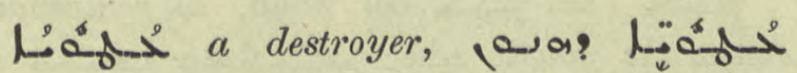

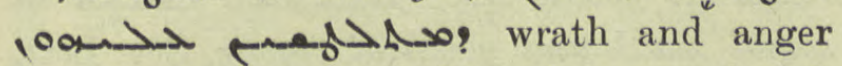
destroy those against whom they are whetted,

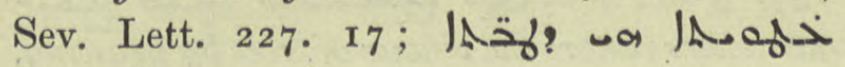
Greg. Carm. I. 4. 22.

\ר to find great difficulty, slave and master

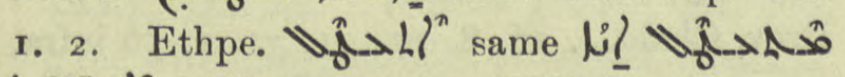

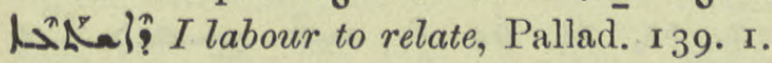

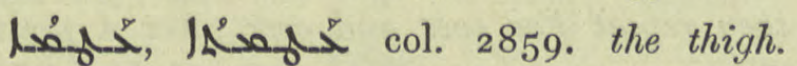
Original meaning, bone, Nöld. Z.A. xxi.

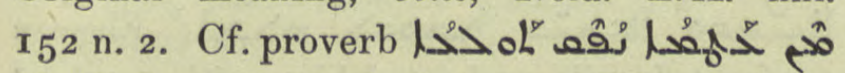
(dead) bones bring forth worms, Jab. I94. I3.

حر col. 2860. I) to return, of the tides, Natur 46.4. Rit. to repeat the responses 슴구, Brev. Chald. i. 3 r. $14,36.16,37.16,59$. $\mathbf{I} 3$ and often. 3) to put on. Imper. oog

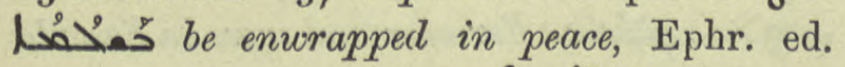
Lamy iii. 495. Aph. $\tilde{R}^{\hat{R}}$ ( I) to cover, clothe, and covered with blessings, Manichéisme 7o,

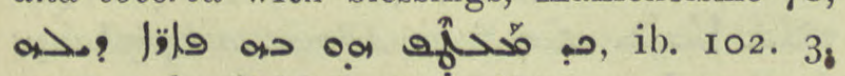

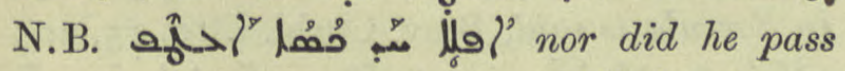
round a single cup of wine, Pallad. 46r. 4. 2) to return, "Wils? away, Chron. Min. 332. 26.

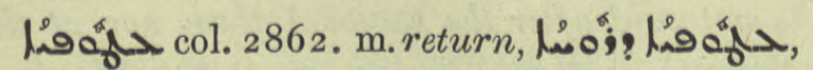

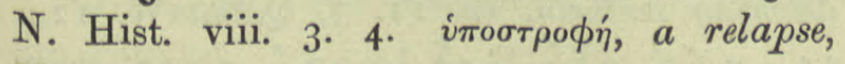
Hippoc. ii. 12.

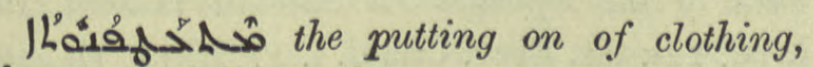

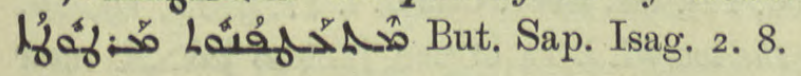

حـ col. 2863. to rise as fumes, add:

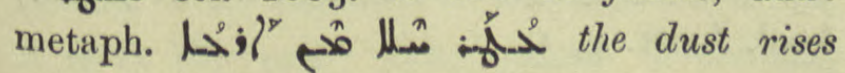

like vapour from the earth, Ephr. ed. Lamy

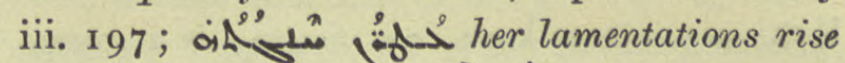

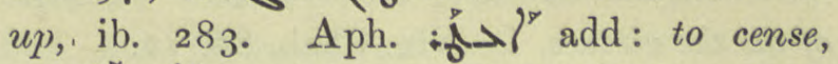
war.

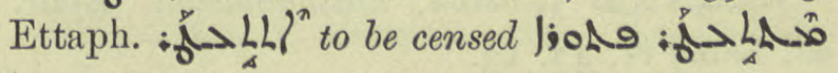
مبرم Brev. Ant. i. Kal. $5^{2}$ a 3 af., $b$ 3, 5 . To be burnt as incense,

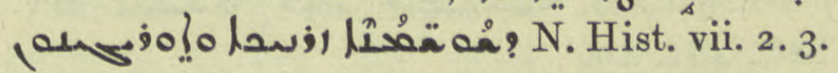

ـــ cedar oil, add: Med. 583. II, I8,

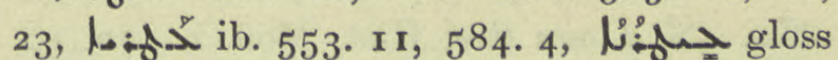

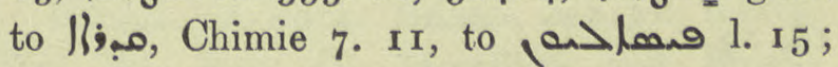
IOO. $2 \mathrm{I}$.

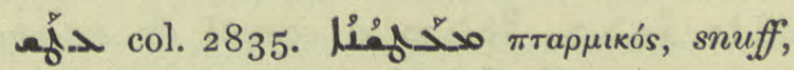

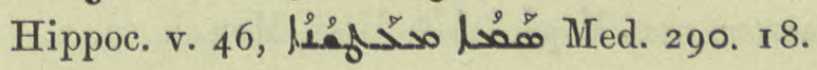

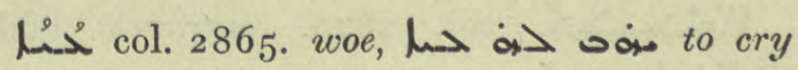
woe upon, Jul. 10. 6, I I, 30. I, 32. 4 and very often.

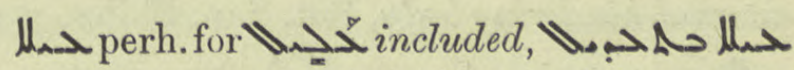
"comprise dans le t'adil," any shop not included in the census list was confiscated, Dion. 122. 10. Cf. $>$ Dh for entered, ib. 186. I3.

خ خ a spring, names compounded with: حسم A Ain Ger, Brook's Chr. 573. I4, 58 r n. 7. Ain Dulbe or Dilebb, a village near Maaltha, Narses, Pref. 4 I. لlم Dekla in Beit Garmai, Chast. 5 ult. حس مقدـ Ain Siphni, E.-Syr. bpric., I5 kil. north of Mosul, Syn. Or. $66_{5}$; 1 10 ult., Pers. Mart. 197; ZDMG. xliii. 404. 10;

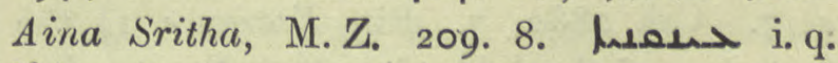

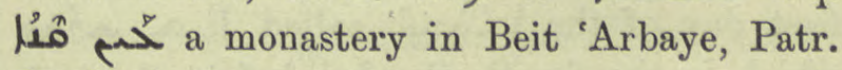
Or. iii. i. 29. 5, 30. $\mathrm{I}$.

'GoL a col. 2868. Aldebaran, the chief star in the constellation Taurus, م م.

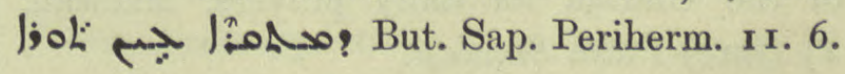

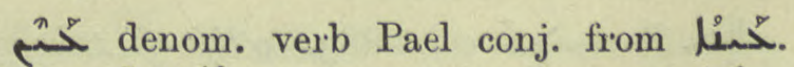

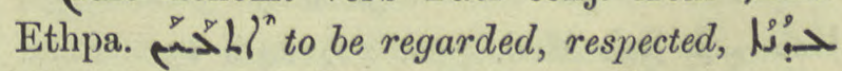

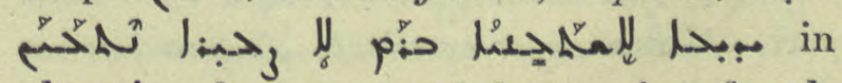
education let not a settled time for play be regarded as despicable, But. Sap. Econ, ii. 2.

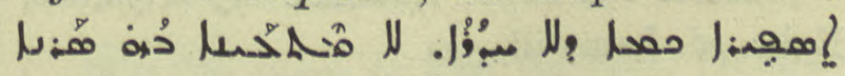
Philos. 4. 2. . respected, El. Nis. Chron. 7 I. 18. 
محسب

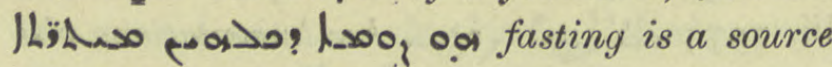
of all the virtues, Is. Nin. Chab. 80. 12.

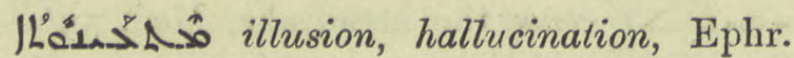
ed. Lamy iv. 47 . I3.

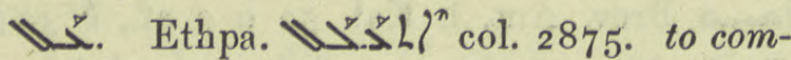
plain with o, his Lett. $48 \mathrm{I}$. 19.

إبح : col.2887. prep. end of par. I. add

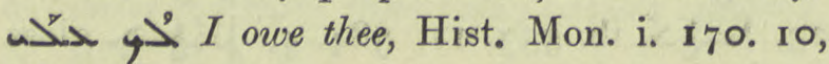
I6, : 9 .

لخده col. 2878 . 10 of par. accidental,

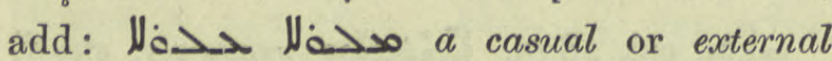

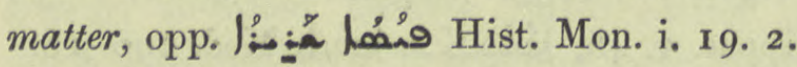

ILas col. 2879. entrance, add:

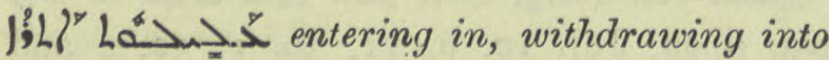

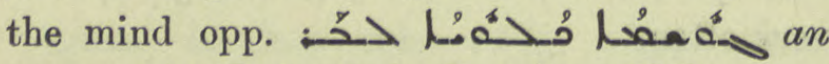
external hindering body, But. Sap. Philos. 5. 2.

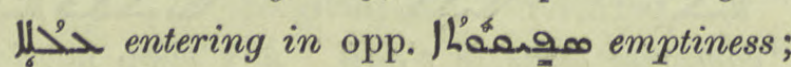

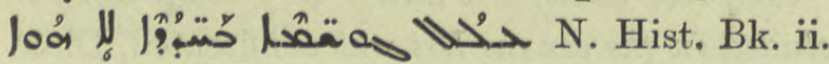
cap. I, sect. iv.

خح pastor. Add: leader of the choir, choragus Brev. Ant. i. 8.5 .

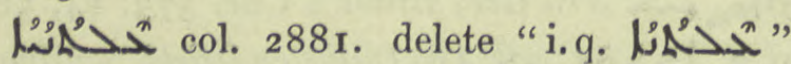
and add: airıarıkóv, causal, ธa fia Asol? a sound is causal of $=$ causes the vibration of the nerve of the ear, N. Hist. viii. 3.2.

مَحْ bringing up, vomiting, Med. 268. 4, 7. Col. 2882, 7. Rit. Entrance I) the first day of the مبإُم حبمار which is the fourth Sunday before Advent. On this day the congregation move from the summer chapel i.e. an open court, to the nave of the Church for daily prayers, Maclean.

2) (rare) Palm Sunday.

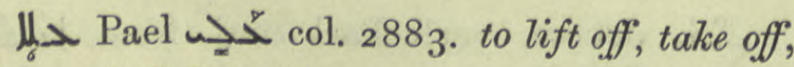

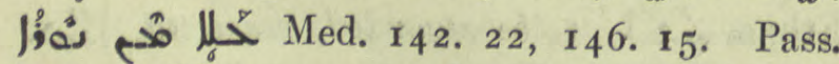

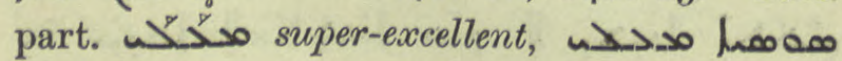
Sassanidi 1 2. 19.

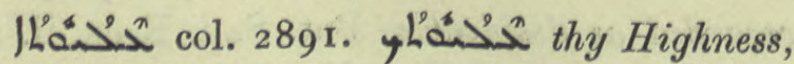
Ephr. ed. Lamy iii. $5^{2} \mathbf{I}$.

| of arguments, Išoyahb 7. 1.

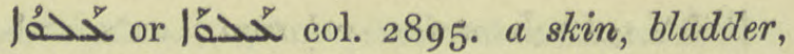
add: a bellows, la da Chinie 240 n. 4. A leathern bottle, BH. Stories ZDMG. xl. 447 .

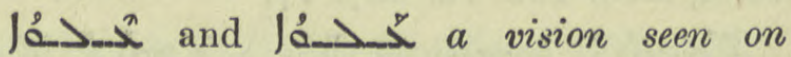

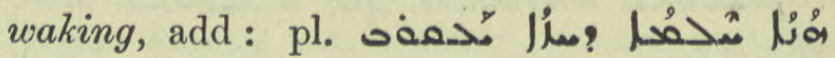
مars. ed. Ming. i. 35. I4; with suff. I pl. دخ دخه in our waking vision, Ephr. ed. Lamy iii. 87. 2.

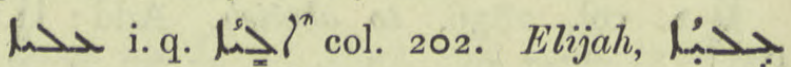
إخبُ Bahira 207. 14 pen. but? ib. $209.3,16$.

حمد Pael col. 2896. to rejuvenate,

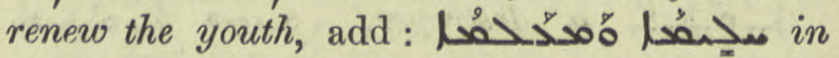
sound health and youthful vigour, Sev. Lett.

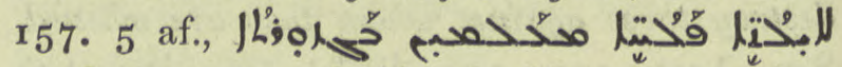
gardeners restore the vigour of trees by prining, G. Busâmé $2 b$ I 7 . Rit. $\mathrm{p}^{2} \overline{\mathrm{z}}$ to repeat the second half of the doxology, مخدخ they say, For ever and ever and continue, Brev. Chald. i, 26. 8, 27. 21, 31. 1, 56 infra,

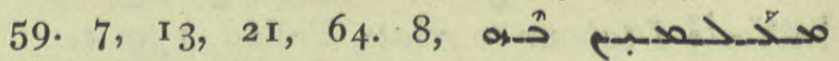
they repeat For ever and ever after a psalm, Takhsa 135. I 2. Ethpa. p I $^{2} h^{n}$ add : | Eleazar confronted evils and hardships with youthful vigour, Sev. Ant. Hom. I r. I4.

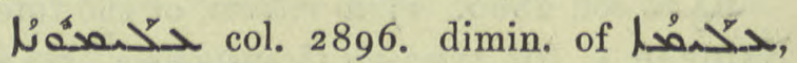
a youihful bachelor, Sev. Gr. $47 r$.

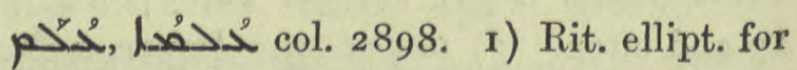

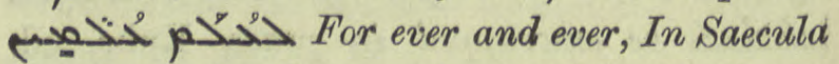
saeculorum i. e.the second part of the doxology;

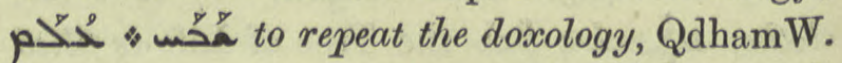
47. $7,17,48.4,20,49.16$;

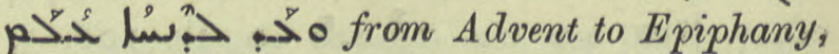
In Saecula is said, Brev. Chald. i. 10. I5, 20;

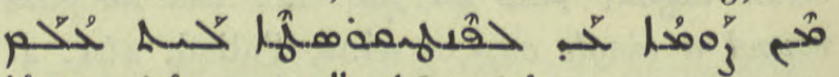
from Lent to Whit-Sunday the clause For ever and ever is not said after the Royal Anthem nor at the Evening Anthem, ib. I1. 3, QdhamW. 124.

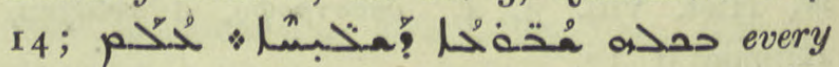
week of the Apostles In Saecula is said, ib. 1. I6, I 25 ter, Brev. Chald. i. I I. 5, I I, 19, 26.

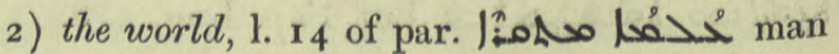
is called the microcosm, N. B. without |los', Apol. Arist. 8. 1 . 


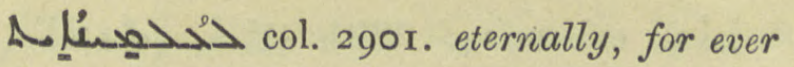
and ever, add refs. But. Sap. Theol. 5. 4 , Eth. I. 3 .

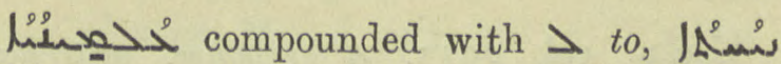

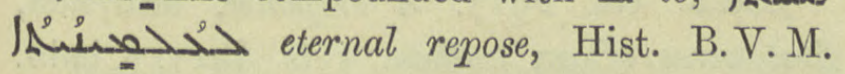
I00. 2 .

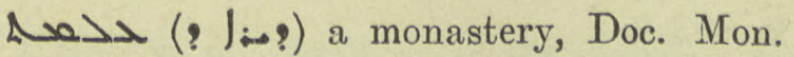
218 . 1 .

حلح Ar. Ar.

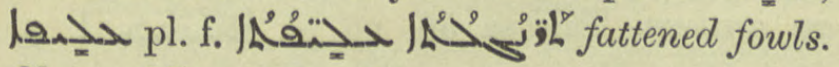
Chron. Min. 378. 26.

حلحم col. 2902. Delete the article and cf. the passage from Geop. in Anecd. Syr. iv. ror. 20 where oater is given for هذه.

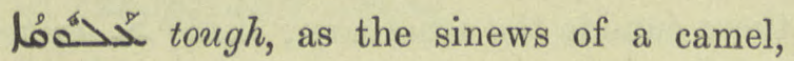
Natur I 8 ult.

f. morbid growth obstruct-

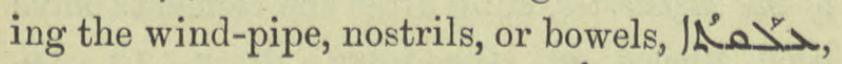

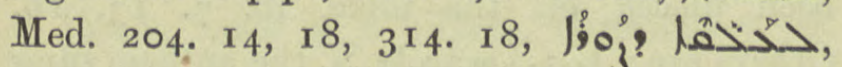

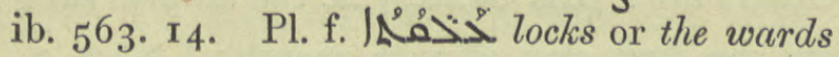

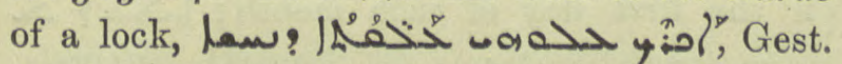
Alex. 268. I 2.

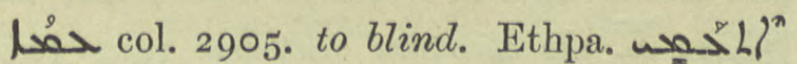

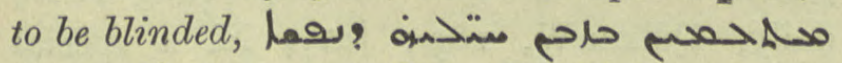

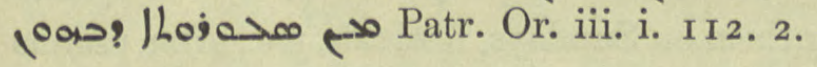

حمَّم: 2906.2 i of par. to penetrate,

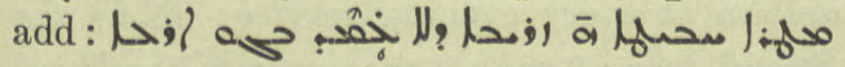
pelting rain i. e. violent rain which does not sink into the ground, BH. on Prov. 28. 3.

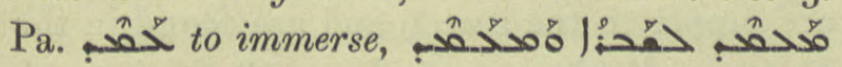
$a)^{n}$ the priest baptizes and immerses the child, A.M.B. v. $625 \cdot 9$.

حمب andergarment,

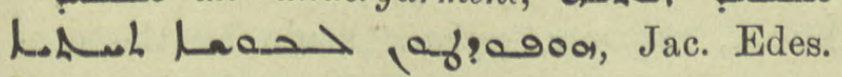
Manichéisme roo note $\mathbf{r}_{3}$.

لإل حمِنب: like unbaptized heathen, Syn. Or. 180.22.

ते col. 29I0. to grow dark; add:

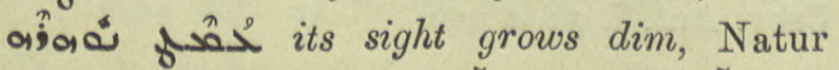

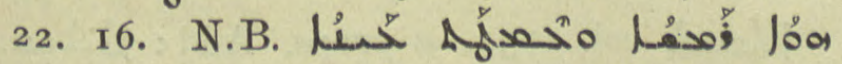
evening drew on, and it was difficult to see, A. M.B. iii. 306. Aph. 2716

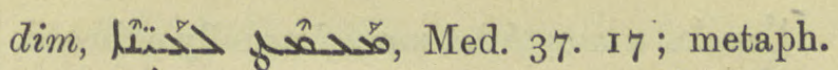
afsol time has not obscured the heroism, Sev.' Ant. Hom. Iо. 2.

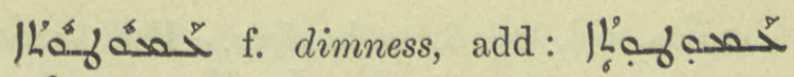
lan 3 ? blunting of the senses, Med. 29. II.

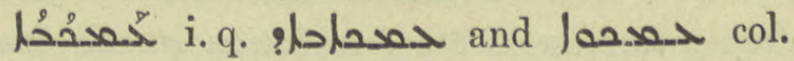
29r2. a village near Arbil, Jab. I92. 8, 195. 8. Now low ल.

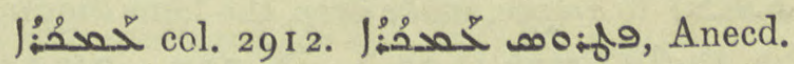
Syr. iii. 208. I2, Hamilton and Brooks I 57 . note 5 translate "a native of "Amkhoro" but say it should be an adjective; Ahrens and

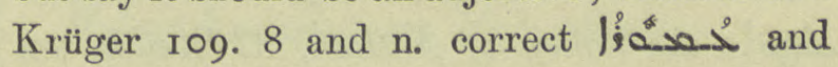
supply of Amid.

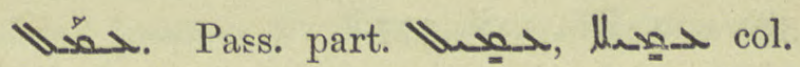
29 I3, l. 5 of par. "גatient,

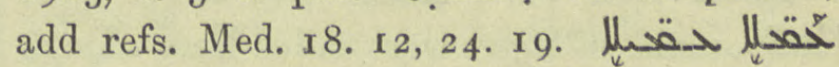

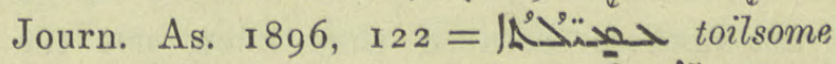
labours, Narses 6 n. Aph. ".sin 7 " to vex,

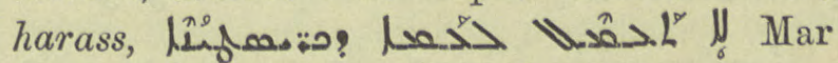

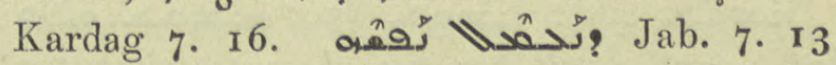

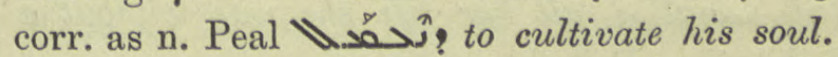

每 Mon. i. ror.

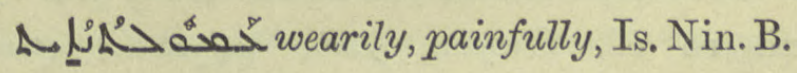
207.4 .

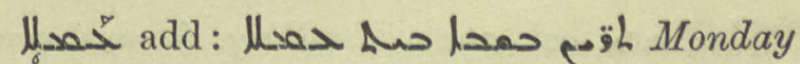
work-a-day, Lexx. under eiL col. 4469 .

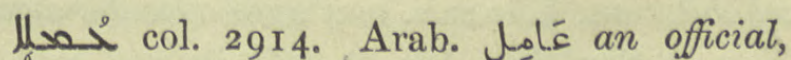

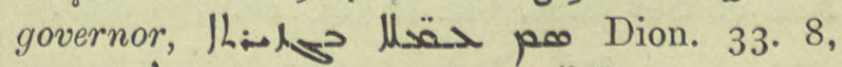

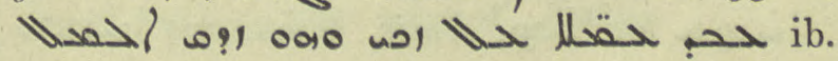
I 27. 2 I and often.

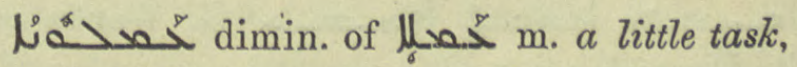
Georg. Arab. 37. 20.

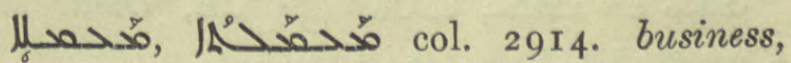

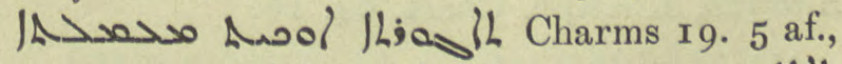

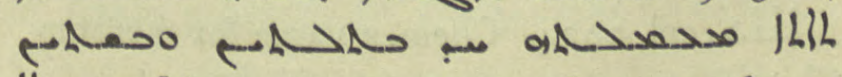
llos 0 his turnover may reach 30 or 60 or Ioo per cent, ib. 20.2.

حم้ col. 2915. to shut the eyes; metaph. to shut out, preclude, متح Or. Xt. v. 102. 69.

K $\mathrm{k}$ 


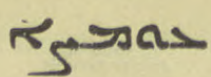

$\int_{5}^{\prime} \mathrm{s}^{\circ} \rightarrow$ a mouthful, morsel usually of bread. Add : a morsel, bolus of a medicine thickened with honey, Med. 306. 7 .

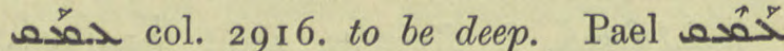

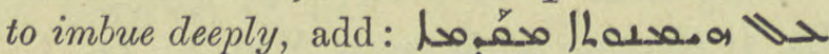

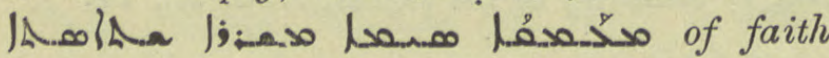
deeply implanted the foundation stands firm, Babai $2 b 7$ af. La入 اتجهA Is. Nin. Chab. 96. 9. Aph. |

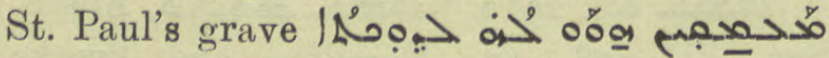


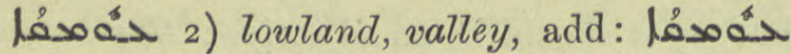
yiofu? the Valley of Antioch, Al Amak, ten miles from Marash, Brook's Chron. 572 pen., $5^{81}$ n. I, ZA. xxii. 377 .

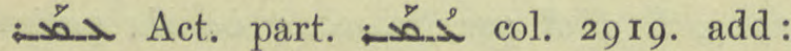

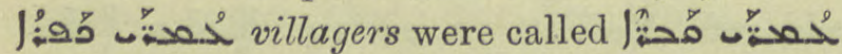
grave-dwellers because they knew not the pleasures of city life, But. Sap. Eth. 3. 3 .

: loa wookt? all her life she was without a habitation, Pallad. 203. $2 \mathrm{I}$.

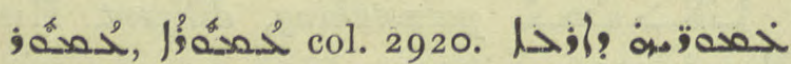

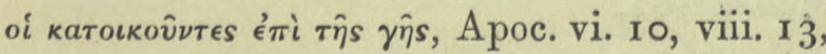

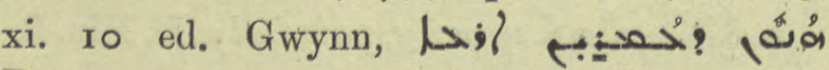
Bagst.

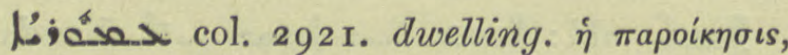
Hex. Ex. xii. 4 ed. Lag. End of par. cohabitation, fosariages concubinaires, Nau on Sev. Ant. Vit. R. O.C. iv. 568 med.

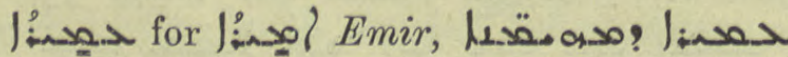
Dion. 138. 15. For l: I. T. Warda ed. A. Deutsch 27.5.

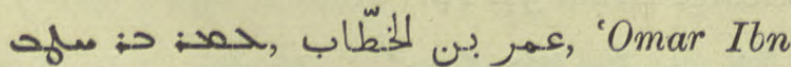
Khattāab, Khaliph, Chast. 64 . 16.

ل حiُ col. 2924. Chem. a name for mercury, Chimie 10. $4,15.4,25.6$. Cf. llow.

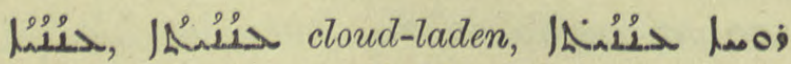
. N. Hist. v. 3. 3.

Lُนُ founder of the sect, Löw ZDMG. xlv. 702 .

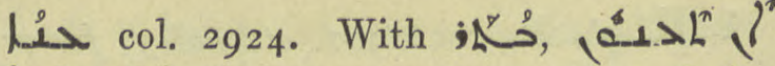
س. Išoyahb 51. I 2.

hiُád col. 2927. a response, antiphon, first verse of an anthem, refs. Brev. Chald. i. 58. 9, 315. 10. Pl. Hist. Mon. i. 142, ii. $293 \mathrm{n}$.

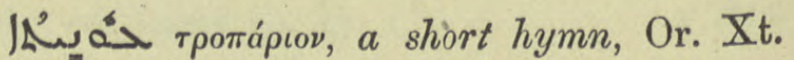
ii. 215 , iv. 206, 207. An anthem, stanza of

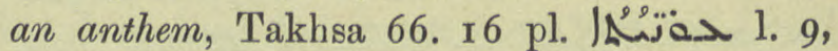
$\omega \delta a i$, Hist. Mon. i. $\mathbf{1}_{42}$, ii. 293 n. A response,

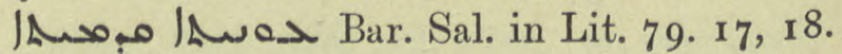
The anthems sung during the Liturgy are:a $>$ Sol? $\bar{\lambda}$ sung after the reading of the Gospel; plo: $\Sigma$ anthem of the Bema, q.v., sung during the communion of the laity by those outside the sanctuary; of the sanctuary, sung in the first part of the

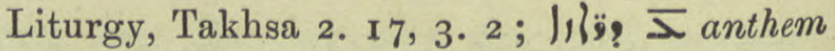
of the Mysteries, sung after the elements have been placed on the table, ib. 7. 13, 16.

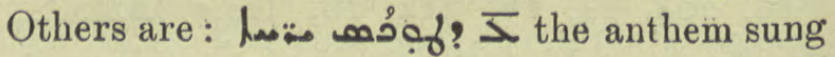
on the first day of each month; three special anthems on Sundays, festivals, and Saints' days at Nocturns after قaم ? آخ daily, QdhamW. 189 ; $: \Theta_{3}$ ? $\bar{\lambda}$ a special anthem on Sundays, festivals, \&c., after psalms

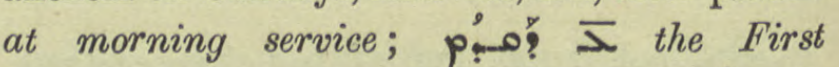
anthem at every evening service; $\mathbf{L}^{2}$ ?

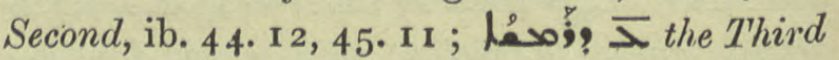
anthem at evening service on ferias; these are proper to the season and are found in the Mac, Maclean: refs. Brev. Chald. i. 9 I. 8 , 104. $18,105.4$ af.

冬 col. 2930. 3) Rit. Jac. = E.-Syr. hus, response, refrain made by the congregation in a prayer or hymn, Brightman's Lit.

2) Jac. an antiphon, a psalm or hymn recited antiphonally, Brightman's Lit., Brev. Ant. i. $5^{8} a$ pen.

صبا بنُ : 要 eye, Med. 68. 9, 77. 16, 17, 19. 


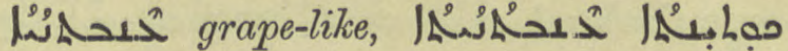
same as the aforegoing, N. Hist. vii. I. 2.

حصد व

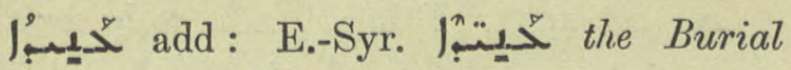
Service for the laity, Maclean.

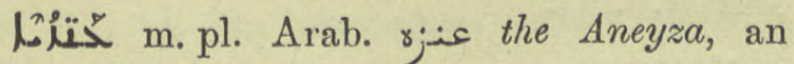
Arab tribe, M. Z. 205 .

Loو col. 2034. Arab. Acrom Pers. the gum of Penaea sarcocolla and P. mucronata, Med. 557. 9, 586 . I 4,

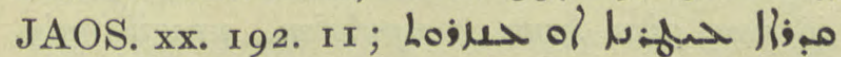
Kedria, résine de cèdre ou sarcocolle, Chimie 7. II. Cf. Mloco:- Miswritings in the

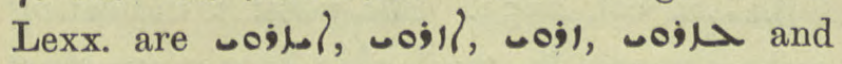
Med. (Budge) only woh.

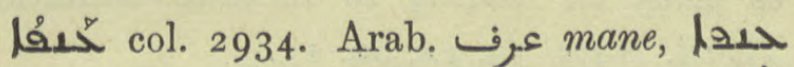

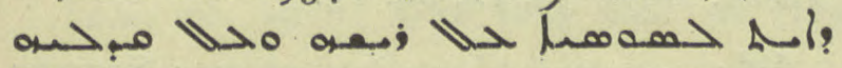
Dion. 49. I 2, 14.

اA name of a village, Sassanidi 7. I8. Prob. i.q. حلد on the Euphrates, col. 2936.

so correct for حُص , حهم col. 2936. Arab. in the quotation from Ephr. ed. Bick. A حُ

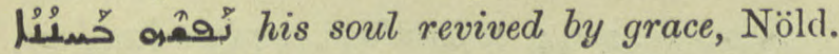
WZKM. xvii. 199. L. ult. of par. corr. بحم م-a مخ may the weak recover new life, Ephr. ed. Lamy iv. $3^{8} 3$. I3.

حَّ Arab. to patrol, go the rounds.

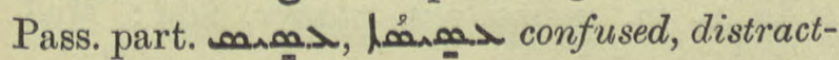

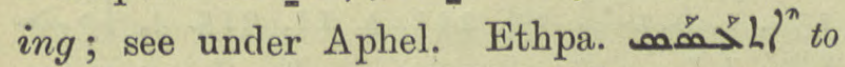

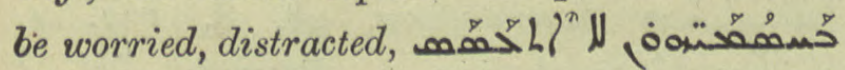
he was not distracted by their envy, Hormizd 2769. Aph. . $^{7}$. to worry, chafe, fret קet us not worry over their frantic tale of Marcion, Ephr.

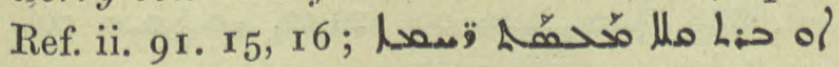
M Ant. ii. ${ }^{2} 5$. $1 \mathrm{r}$.

خمّ col. 2938. an army, add: Dion. 71. $17,97.3$.

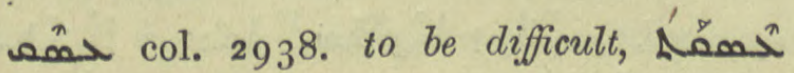

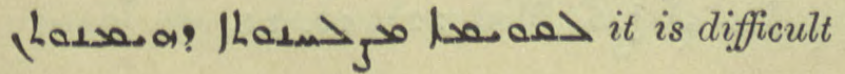

to establish the victory of our religion, Išoyahb 3. 2,3 .

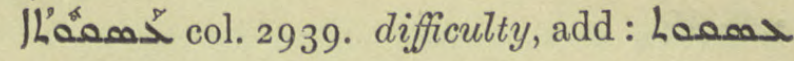
ل 22, /hay ' Laمan insensibility, But. Sap.

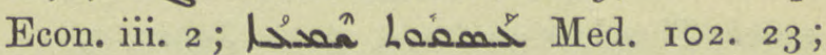

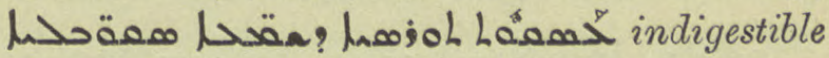
food of contrary rumours, Išoyahb 4. IO.

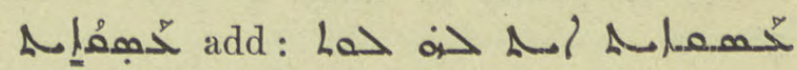
it was awkward for me, Pet. Ib. 7.4. 10.

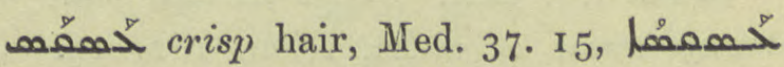
1. 22 .

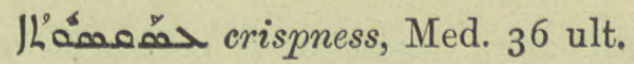

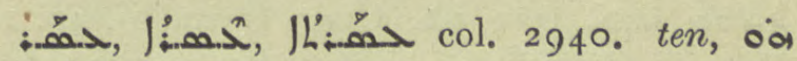
פ $ح ح م:$ He Who gave tenfold increase, R.O.C. I9I2, 70. Ir. End of par. Nöld. (WZKM. xvii. I99) thinks that three refs. to

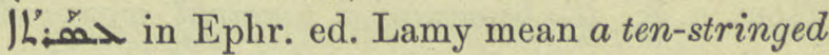
lyre as Bickell conjectures, on Cyrillona ZDMG. xxvii. 596. 4 af. All four places refer to the joy of harvest and it seems more likely that

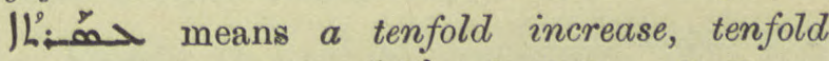

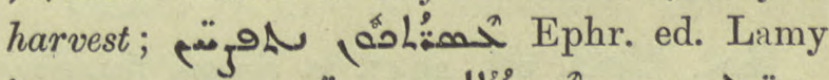
iv. 399. 13, ib. 4 I5 pen., الل الحمت: let not the songs of increase be changed to lamentations, ib. 423.5 .

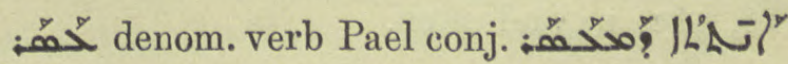
هُ a woman whose child is carried into the tenth month, Med. 593. I7. Ethpa. : a) to pay tithe. b) to receive tithe, with

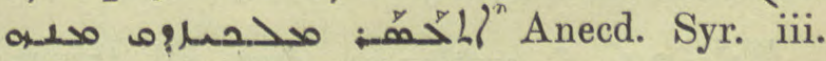
II. 25 .

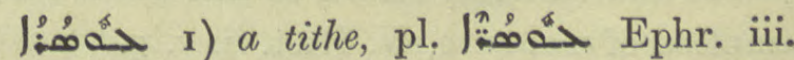
187. 13. 2) tithing, tithe collecting, Dion.

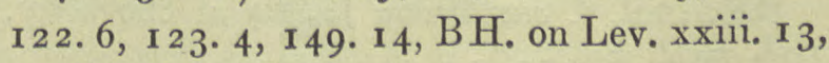
xxvii. $3^{2}$.

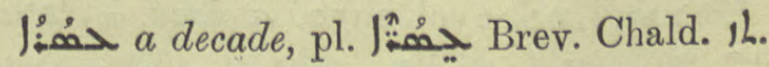

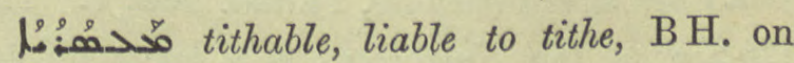
Lev. xix. I 9 ter.

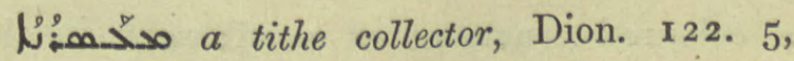

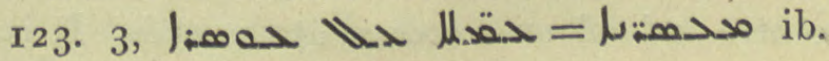
1 49. I3. 


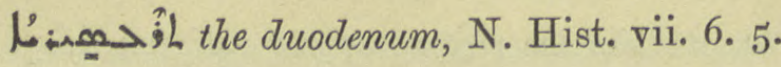

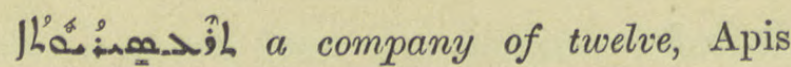
مب. 10.

خح col. 2942. to double, multiply. Act. part. m.pl. ب을 J H much, Theod. Mops. 206. I9 on Jo. ix. 3.

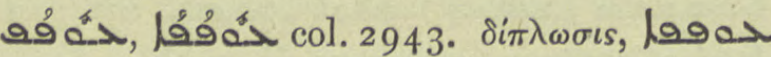
ba?! compound or alloy of gold, Chimie $3^{8}$ uit.

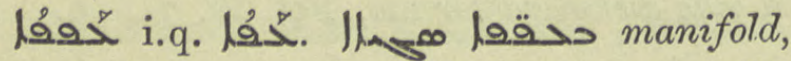
many times as much, Dion. 162. 16. Cf. 1. 1.3 of par.

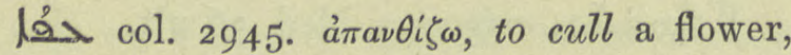
Aoa la خareg. Carm. ii. 40. II. Ethpe. enwrapped in love, Is. Nin. $6 v$ 9. Pa. Pح

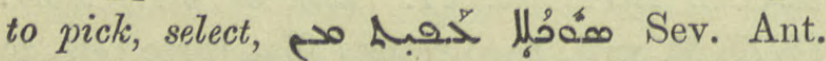
Hom, 9. 8. To be occupied, involved, wrapped

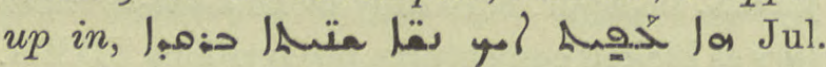
49. 18 .

مُهَ́ col. 2946. Add: hay, Chimie 277 not. 2.

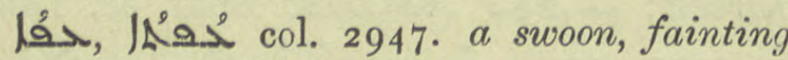

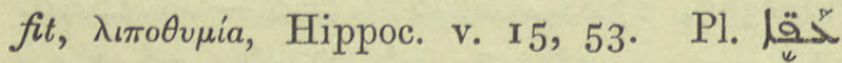
Med. 274. 22.

لخ خ خ col. 2948. m. an oak gall, Med. 237. 16, 560.13 and often.

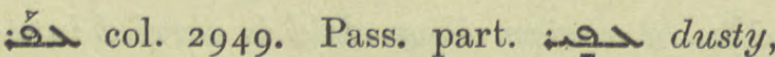

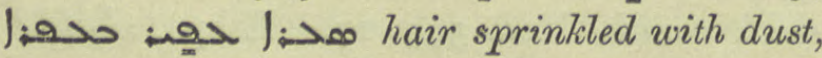
Anecd. Syr. iii. 89. 23 .

ILơ plants and animals, return to this state when emptied of moisture, N. Hist. ii. v. 5 .

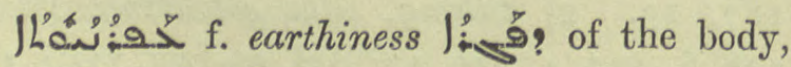
Hist. Mon. i. 379. 9.

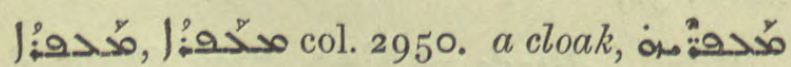
|अै|â? her silken mantles, Pallad. 200. $\mathbf{x}$. The

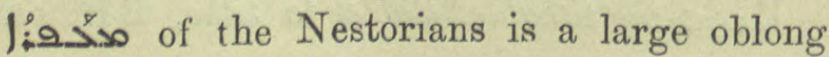
piece of white linen or cotton cloth with a coloured ornament of needlework in the centre and coloured crosses at the ends. It is worn over the shoulders and can also be put

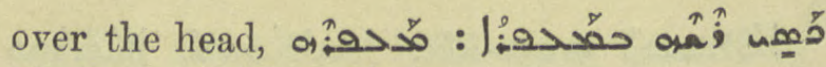

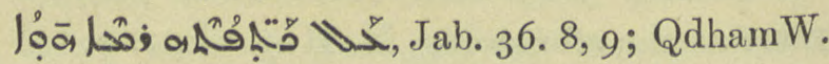
r०3. 4. Also a veil put on a child's head at Baptism, Maclean.

5. col. 2951. Ethpa. $35^{2} \mathrm{Ll}^{n}$ to be written

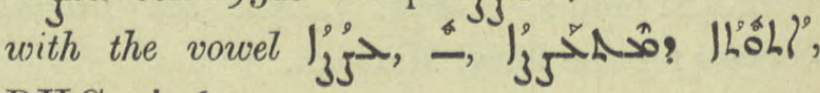
BHGr. i. 64 .

'S col. 2952. 16. Add: to put out the eye, Loa l. Min. $289.6,7$. Pa.

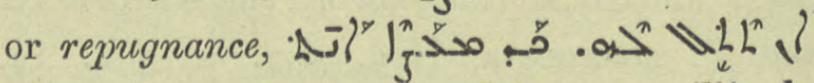
if you give from compulsion, unwillingly, Pallad. 528. 5 .

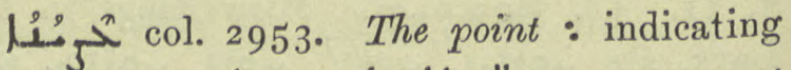

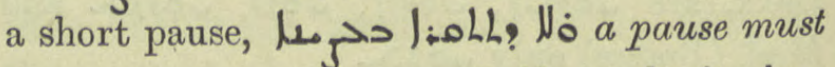
be made in reading to indicate hesitation, Theod. Mops. 55. 14, 60. 15.

$\mathrm{L}_{5}^{\prime \prime}>$ constraint, pressing entreaty, Severus came to Constantinople Oحص, Sev. Ant. Vit. 300. 6.

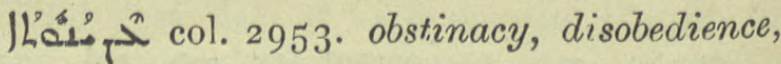

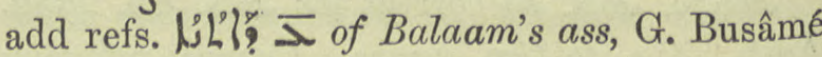
I5. 7 af. ; Stat. Schol. Nis. Io. I 7 .

حِ col. 2954. to bandage, apply a bandage, imper. 0 Med. $3^{\mathrm{I}} 5$. 10 and often. Ethpe. ${ }_{5}^{n}>L l^{n}$ to be bandaged, bound on,

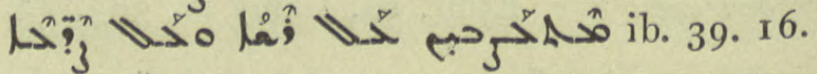

لح

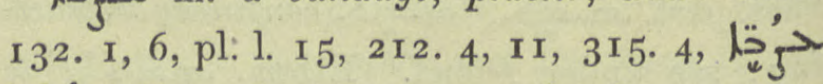

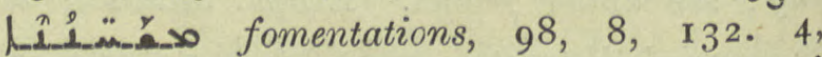

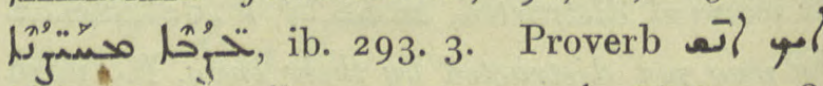
Philox. 98. 14. To the references at end of par. add:

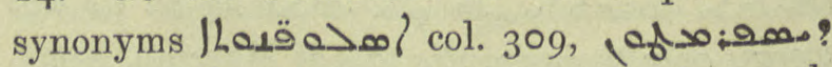

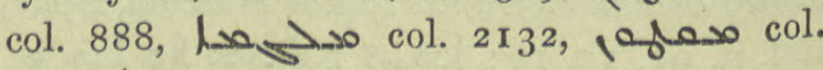
2198 , معه تمدار col. 256 r.

'j col. 2955. to squeeze, press, imper. w

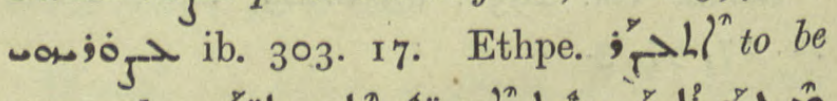

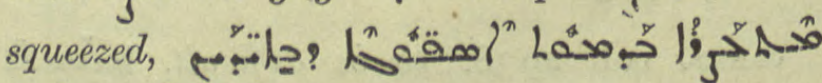

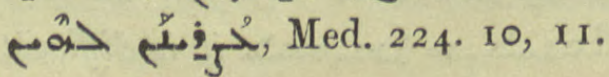




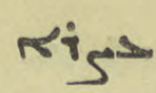

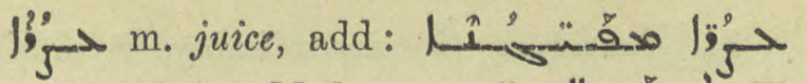

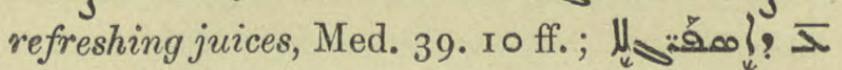

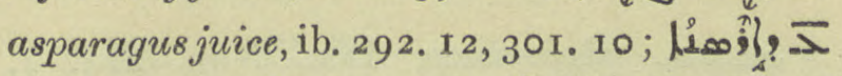
barley water, ib. 607. 18; / | sour grapes, ib. 39. 10, 292. I1, 301. I, 7 ; |

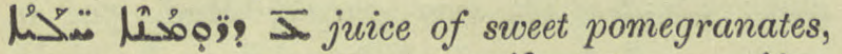

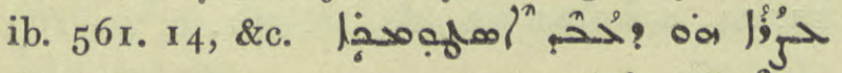

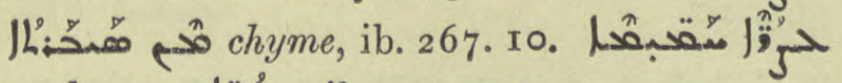
prob. corr. $\operatorname{läs}_{\text {خ }}$ ib. I33. I3.

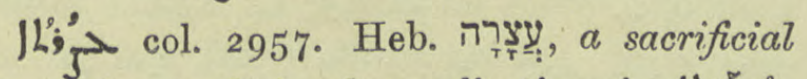
feast, the traditional vocalization is $1 L_{\text {i }}^{y}$, BH. on Amos v. 2x. Nöld. thinks should be corrected to $\mathrm{Lig}_{\mathrm{j}} \mathrm{C}$ in the following passage, submit to the slaughter, Ephr. ed. Lamy iii. xxvi. 3 af.

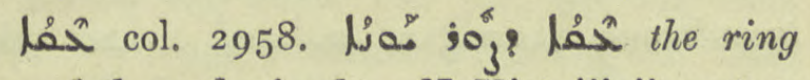
round the neck of a dove, N. Hist. iii. ii. sect. 4 . | a sparrow in its second year, ib. vii. 4 . 4 .

حمام pr.n.f. Acacia, cf. جمع Acacius ; حملم مسaم Nestorian tombstone from Séminietschie, now in Paris, Chabot, Journ. As. $1908,{ }_{42}$.

حمُـد

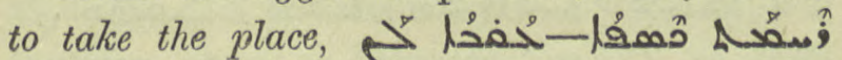
بـ love of money supplies to us the place of all Gods, Jac. Sar. Hom. iii. 81 4. 10.

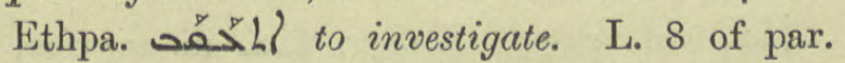
the passage from Bar Cephas is given by Bensley Arist. A pol. 53 and seems rather to mean "they, viz. the heavier parts of creation, unless supported, would be sought for according to the law of their being, viz. they would sink down.

|خم col. 2960.25 of par. the lowest part,

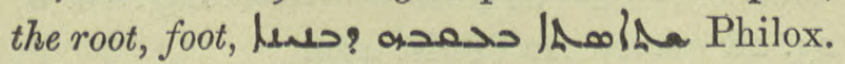
7. 19. Col. 2961. 3. Rit. Jac. a variable termination of a prayer, a short prayer at the conclusion of an office, lad of Jas s Brev. Ant. i. Kal. 60 b 3, 30 ib. 3 af., إمس to first to eighth termina-

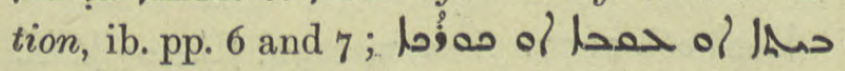
pari? the appointed verse or termination or

\section{خمeni}

refrain, ib. $54 a$ I 4 , ii. $3^{64} b 3$ af., iii. $8 a$ i2, $83 b 8,84 b$ 1 $2,90 b$ pen. E.-Syr. كّمهبץ

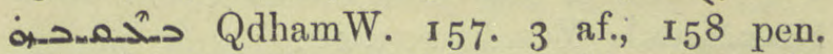

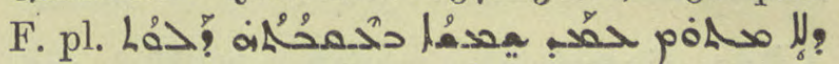
perh. corr. هُشمُ her resentment, Pallad. 205. I 5 .

1. 296r. good at tracking,

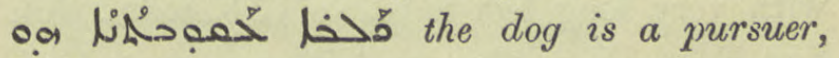
tracker, Natur I I. I3.

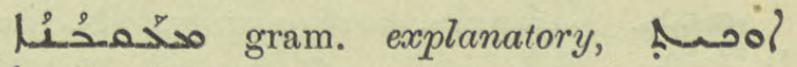

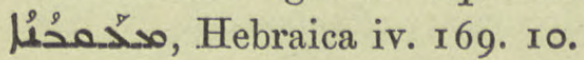

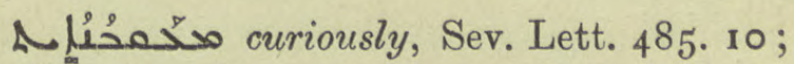

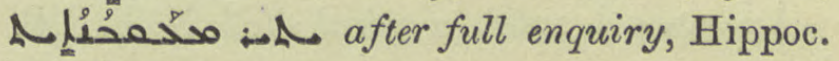
xxi. Syr. 4.

مoa 'Oqba b. Mohammed al Chuzâi, Wali of Mosul, A. D. 886, Hormizd x 696, I 7 I 7 , ZDMG. xlviii. $53^{2}$.

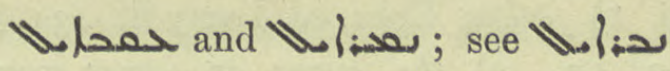

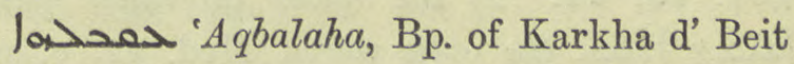
Slokh, Syn. Or. 34. 17, 35. 22, 45. 10, ZDMG. xliii. 394. 8; Bp. of Ramonin, ib. 394. 7, Syn. Or. 34 16.

at Kennesrin, Sev. Ant. Vit. 268. 10, $3 \circ 5$. 3, Sev. Lett. I $_{7} 7.4$, ZDMG. liv. 380.

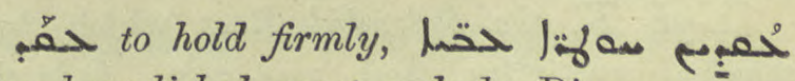
they brandished great cudgels, Dion. I 73. 3. To set up, to establish רت_as, G. Warda, ed. A. Deutsch I8. 24. Chem. to fix, become firm, خحم خ Chimie 96.3,

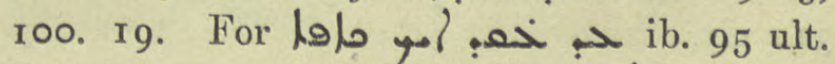

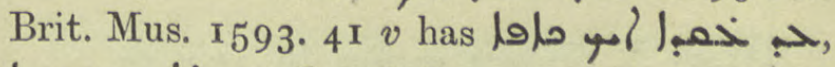
ib. 96. 8.

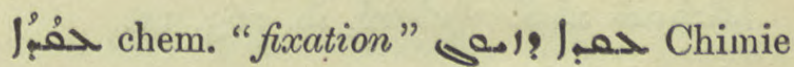
95. 20.

ل العاقول حمa Akula, ancient name of Kufa and 35 miles S. of Babylon, Hist. Mon. ii. 187 n. 4 , Dion. 49. 2, 62. 3 .

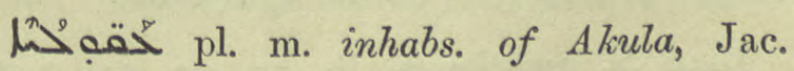
Edes. Hex. 8 n. 2, Bar Penk. 156. 14, 157, 3, Patr. Or. iii. i. 28 . II , Dion. 85. II I I55. 2 I.

"حمـ pr.n. col. 2963. 4 af. Delete "corruptum est", \&c. and see معـمس Suppl. 


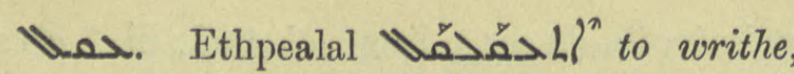

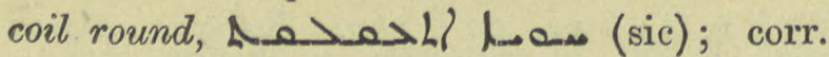
(1) 12.

حمُ the gripes, a griping pain, Med. 296. I $7,23,297.6$.

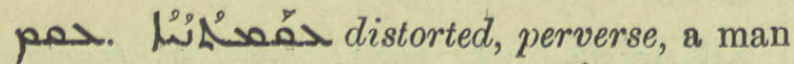

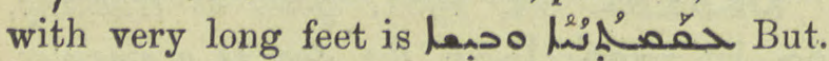

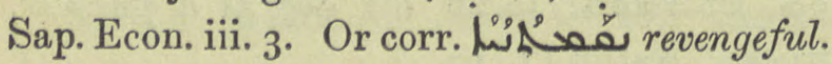

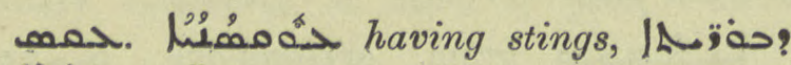

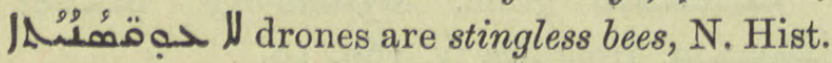
vii. $4 \cdot 5$.

حمِمفم col. 2968. a spiral shell, Nöld. F.S. i. $55 \mathrm{x}$.

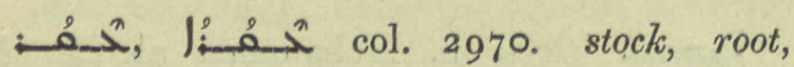

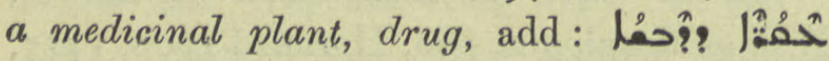
مَّ honey-bearing flowers, A.M. B. v. 9. 7 . Metaph. r) father, Ephr. ed, Lamy iii. 43I. 5, $507.10,12.2)$ the lower or inner part,

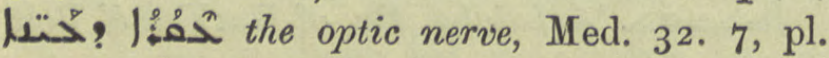

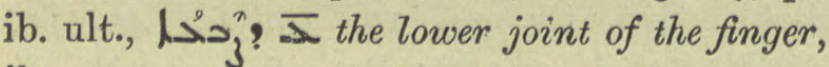
ib. 27.7 .

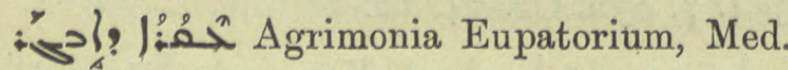
357. 2 .

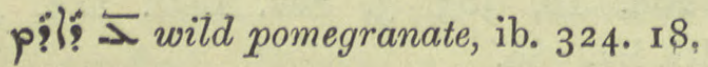

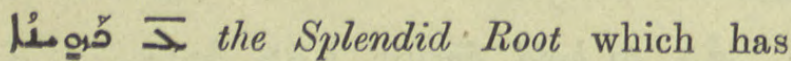
magical properties, Med. 594-597. It is the mandrake, Mandragora officinalis. Cf.Frazer's Folklore of the O.T. Vol. ii., pp. 372-397.

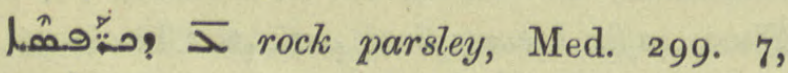
3 I 2 ult.

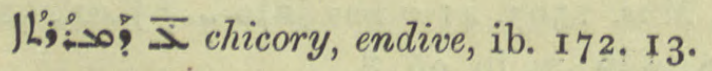

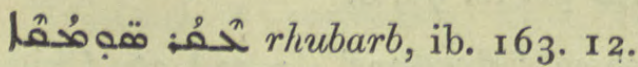

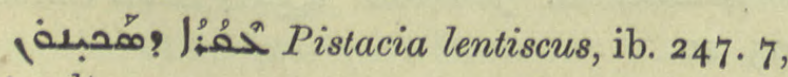
3 다.

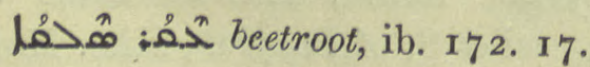

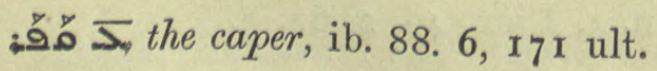

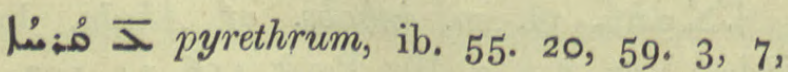
555. 2.

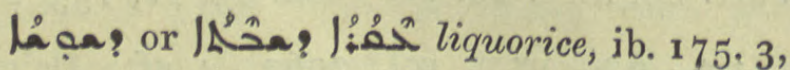
247. 7, 299. 15 .

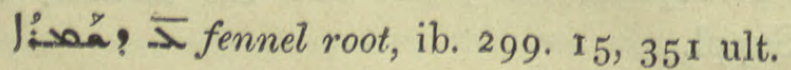

น I32. 18 .

حمن: place-name, Doc. Mon. 214. 4, $2 \mathrm{I} 5.2 \mathrm{I}$.

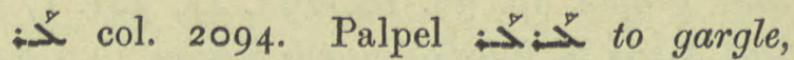
add :

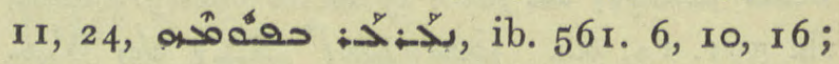
565.10.

إخمْ a gargle, Med. 59 ult., pl. ib. 1. $\mathrm{I} ; 6.2$.

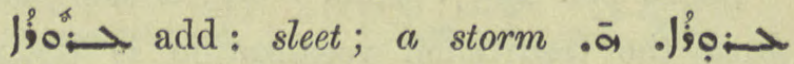

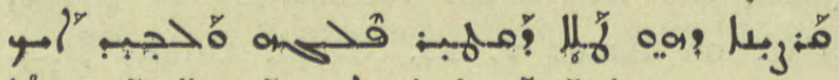

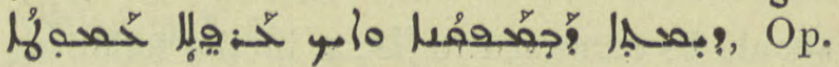
Nest. I1 2. 12 on Amos I-r 4. A tempest,

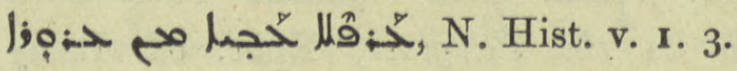

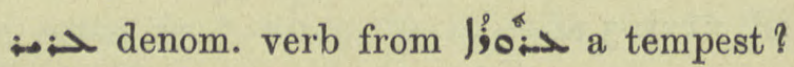
tumultuous, Jharfis antool - حis "the ocean was tumultuous with spray," (Brooks) Anecd. Syr, iii. 290. 2.

ح mast of a ship, iorós, Greg. Carm. ii. 32,5 .

إن col. 2975. to take. To take food, "

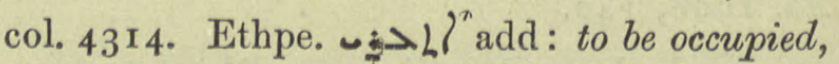

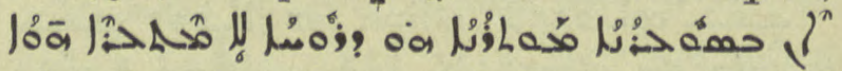
when he was not occupied in some spiritual work, he prayed continually, Jo. Tell. $25 \cdot 3$.

col. 2977. E.-Syr.

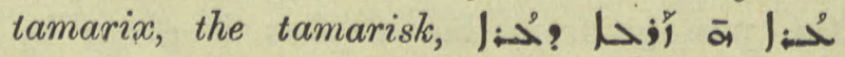

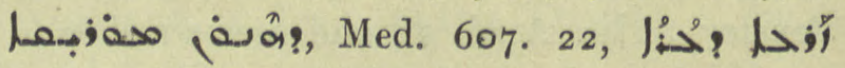
ت. تهر طرفا

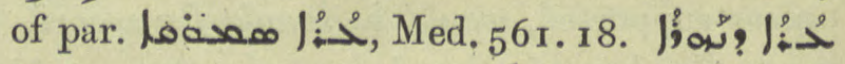
Agnus castus vitex, Med. 607. 23. 23. Praet. Miss. 50. 57 ; Onomasticon Lag. 16. 6, 23. 28 quoted Ar.PflnN. 419.

IRำ f. rigor, shivering fit of fever, add; ṕíos, Hippoc. iv. 29, 46, 58, 63 and

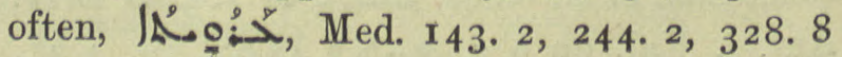
and often.

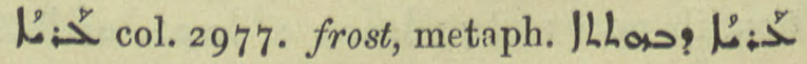
the chill or shiver of shame, Jul. 149. 4. 
حَح delete. The gloss belongs to and is rightly given col. 2993 .

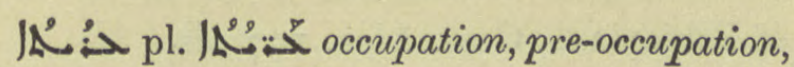

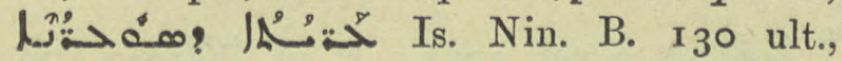
I3r. 5 bis.

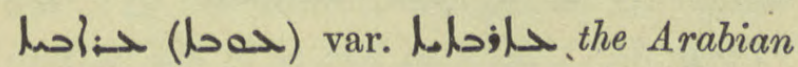
Gulf, Jac. Edes. Hex. I4. I0, I2. Usual form ho: خ.

放 col. 2929. 2) to give security, to pledge, 寉 I pledged myself for the lad, Jos. Wolflink 20 ult. 3) to mix,

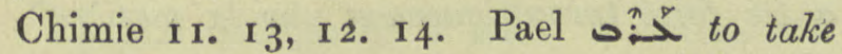

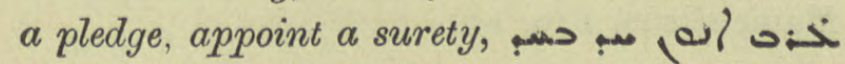
Dion. I I I. 22.

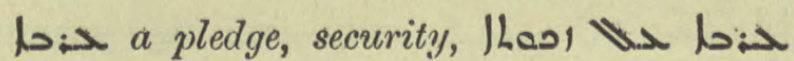
Josephus vi. 8 ult., Dion. I Ir. 2 I.

bias col. 2980. last par. but one, from Op. Nest. 95. 6 should be bias col. 690 . Delete the paragraph.

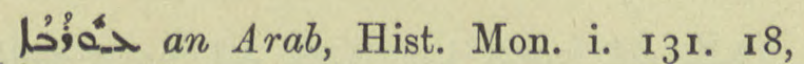

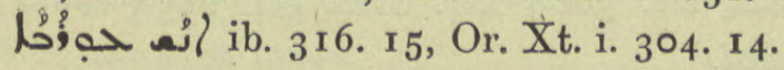

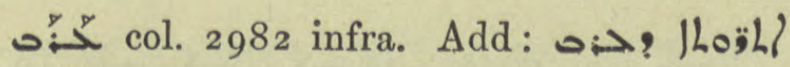
i.q. Beit 'Arabaye, the western part of Mesopotamia which formed the Metropolitan diocese of Nisibis, Syn. Or. 5 2. I 7 ; 6 17 , ZDMG. xliii. 399. 1 2, Eranšahr 25 , Z. A. xxi. ${ }_{53}$.

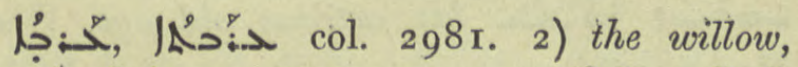

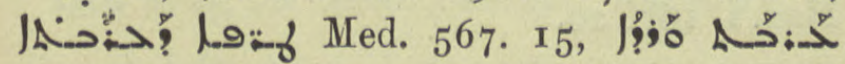
1. I3 af. add: شجبر من Mary's Tree, prob. the rose-laurel or oleander, Med. 607. 20, 2 I where the description is that given in Op. Nest. 105. 3, viz. a willow with blossoms like roses.

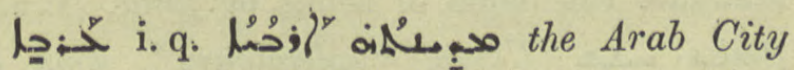
of Naaman, the Lakhmide Arab, i.e. Hira, Bahira 203. 3, 10. A place in Qardu, Chast. $65 . \times 5$.

1ح col. 2983 . a desert, a hollow waste, add : J Chast. 55. 18.

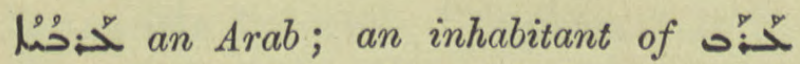
Oمُ2 Ho the Village of the Arabs in Adiabene, M.Z. 6o. 85 .

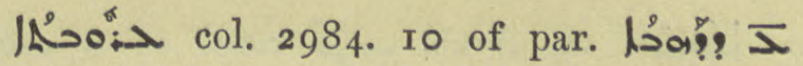
Friday in Whitsun week, add to refs. : Daily Offices, Maclean 276, Brev. Ant. i. Kal. 40.

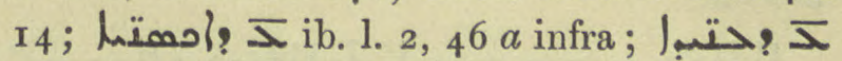
Friday of the Departed, ib., it is the Friday after the Eighth Sunday of the Epiphany, Daily Offices 268 ; these two Fridays and Nö口 $\overline{\boldsymbol{\lambda}}$ are mentioned together, Brev. Ant.

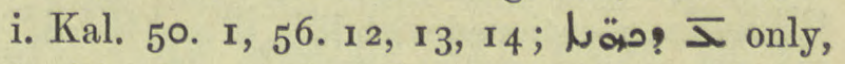
ib. iii. 182. 2 ; the other two Fridays, 184 .

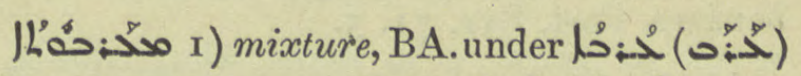
col. 3982 . 2) westerliness, lying to the west, opp. Jaemar, De Astrolabe 260 antep.

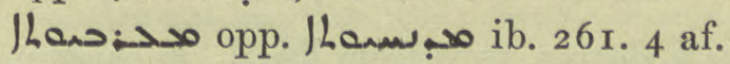

年 col. 2986. centaury, knapweed, PflnN. 302, Med. 140. 9, I4 I. 5 .

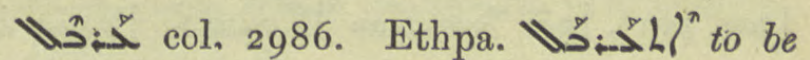
sifted, metaph. Stat. Schol. Nis. I 70. $\mathrm{I}, 2$.

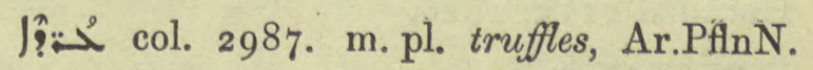
$3 \circ 3$, Med. r6r. 4 .

ح- Arab. حـ Cyrillona 600. 4 af.

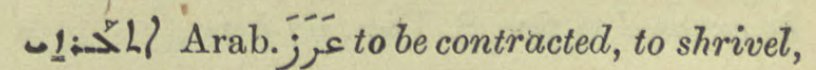

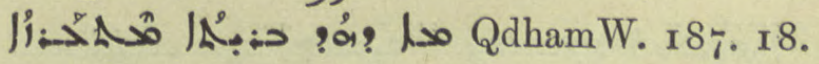

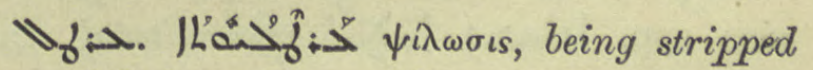
bare of flesh as a bone, Hippoc. vii. I 7 .

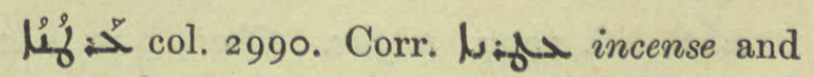
trs. to col. 2864 .

| Ar.PflnN. 304 f., ref. Med. 429. 18.

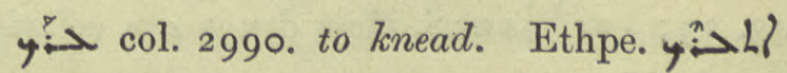
to be kneaded, pounded, Hormizd 2177.

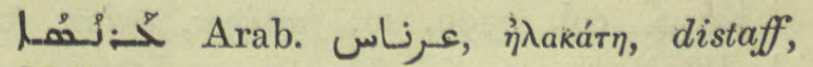
A.M.B. v. $3^{62}$. II

I perhaps Arab. 575 . 13 .

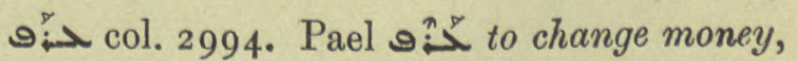
to commute, أحسّي sold her goods and realized her property, Pallad. 193. 6. Ethpa. 9:- S $^{2}$ to be changed as money; ref. under مappl. 


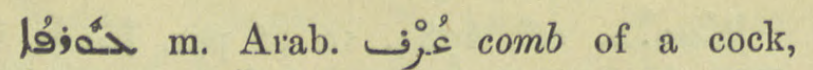

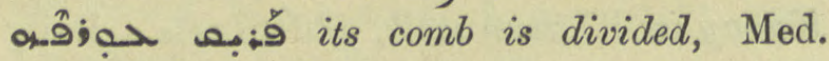
588. 3 .

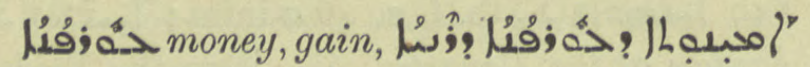
the constant profit of meditation, Pallad. 55. I 9 =A.M.B. v. 73. 10 .

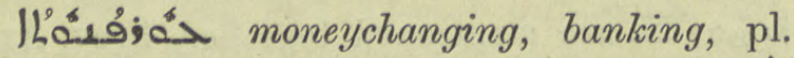

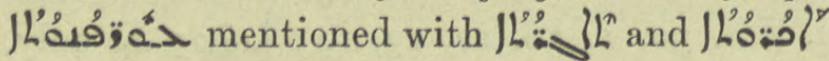
the labours of husbandmen, merchants, and bankers, Poet. Syr. 71. 5 .

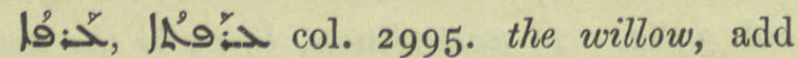

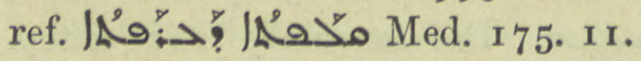

ได้:-خ denom. verb from

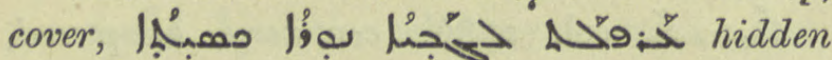
fire enveloped the elect one, Hormizd 460, 677 .

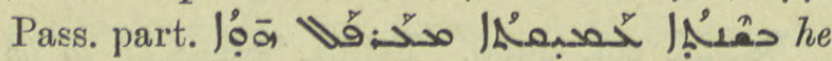
was enwrapped in deep slumber, ib. 409.

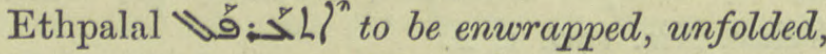
Hormizd I I I 5, I 828 .

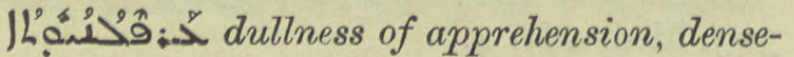
ness, Is. Nin. B. 2 I 8 ult.

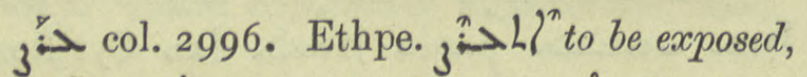

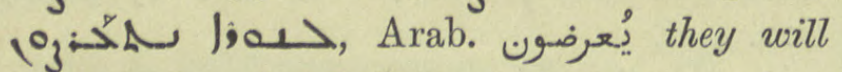
be exposed to fire, S. Dan. $67 b$ io.

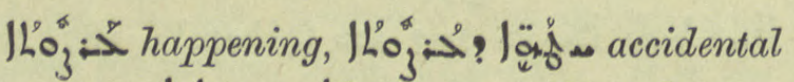
sins opp. l!Leح! lä premeditated sins, C. B.M. $570 b$.

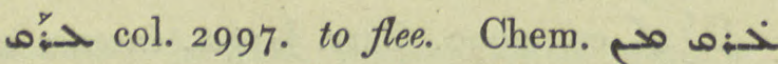
jos it is volatilized by fire, Chimie 40.17 .

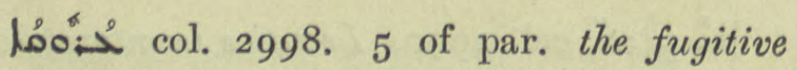
$=$ mercury as volatile, Chimie I5. I, 36. 7 , 43. 23 ; مas loo:خ these drugs are volatile, ib. 40.15 .

1َ' col. 2999. m. juniper, cedar, \&c. Ar.PflnN. 57, 63 f. The cypress, Cupressus sempervirens, Hjelt on Jac. Edes. Hex.

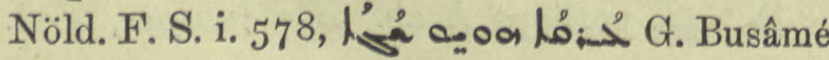
$78 b \times 7$.

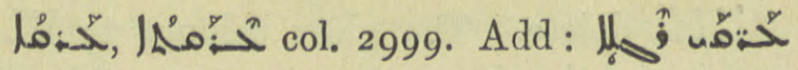

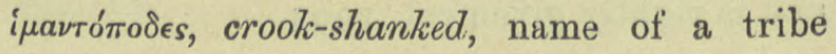
of Ethiopians, Gest. Alex. I 77 ult.

مَمِ actions to be avoided, shunned, Sev. Lett. 274. 5 .

(sulphur of Irak, Chimie 43. 14.

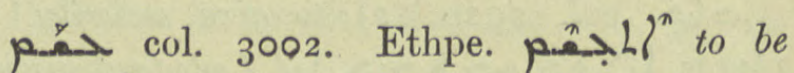

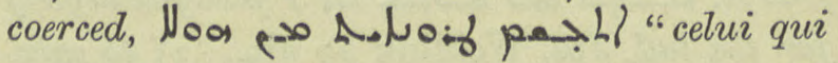
a été forcé tyranniquement par la matière", Manichéisme 149. 10.

حُو : col. 3003. to wax strong, add | branches grow strong, Ephr. Jos. 324. I 2.

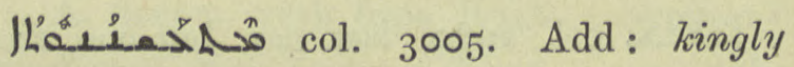
power opp. مest. Hérac. I $47.8 ; 488.4$.

col. 3008 i.q. حسل col. 2935 to be wicked.

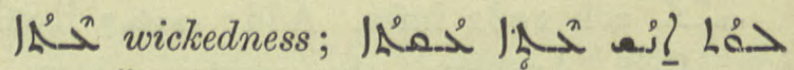
; man, Pallad. Io. 4 af.

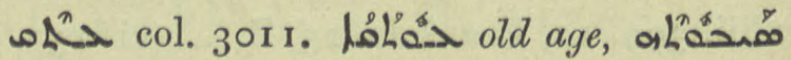

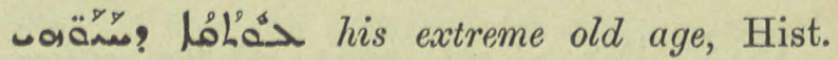
Mon. i. 167.20.

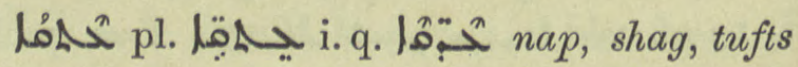
of 'wool, Op. Nest. 95. 4, ef. لحتم 1.22. סحقس סح: Is. Ant. i. I I8. 10.

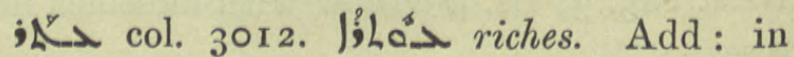
Mandaic the name of aeons or jinns, JïLeح Jias حتس "Uthré, "splendours", sons of Light, Bar Khoni, Coupes ii. 155. 4, 10; $24 \mathrm{I} \mathrm{n}$.

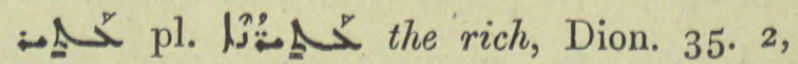
ז 89. 3, 204. 10. 
: so and so, son of so and so, Charms 96. 97.

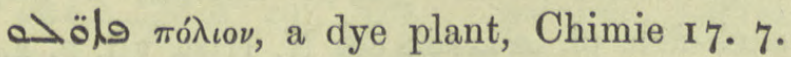
See par col. 3063, and Suppl.

jole Paor, an Egyptian martyr, Sev. Ant. Hymns 6ro. 2.

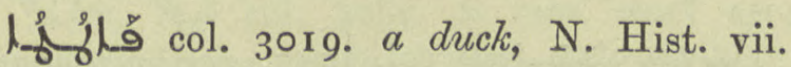
I. $5,3.2$.

- 1. Sev. Ant. Vit. 88. 3.

פllalaea, a village near Ascalon, Pet. Ib. 77. 15 .

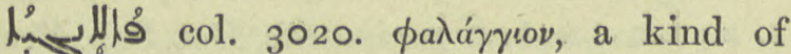
spider, N. Hist. vii. 3. I.

قاحل m.pl. Paltians, Sev. Lett. 5r. 6. See of below.

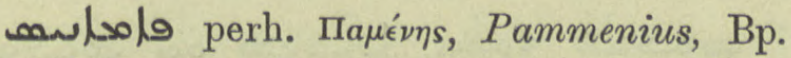
of Ariassos in Pamphylia, Nöld. F. S. i. 472. $9 \mathrm{I}$.

Who חaiva, an island off the coast of Mauritius, Jac. Edes. Hex. xix. I I.

小 copolule for oluslo col. 3022. Paneas (Caesarea Philippi), Nöld. F. S. i. 468. г6.

. vii. 2. 2 .

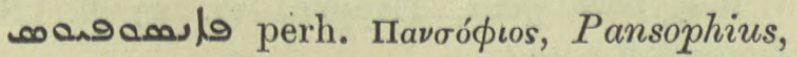
Bp. of Ibora in Pontus, Nöld. F. S. i. 475. I 4 O.

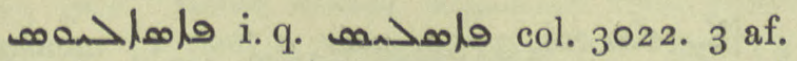
Phaselis in Lycia, Nöld. F. S. i. 474. 126.

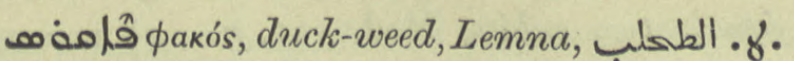
Med. 608. 2.

م in Cappadocia, Nöld. F. S. i. 47 r. 63.

Lọjlo for Philadelphia, De Goeje B. $65.2 \mathrm{x}$.

2716

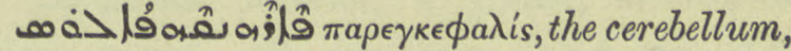
N. Hist. vii. I. 3. Cf. Cs حـ Suppl.

هاز هápos a) Paros, Brit. Mus. Or. I593.

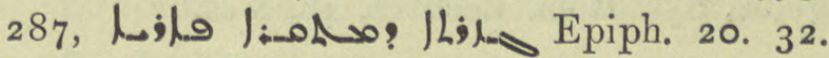
b) Parian earth, Chimie 48. I9.

صajorile name of a priest to whom S. Basil wrote, Journ. As. 1909, 280.

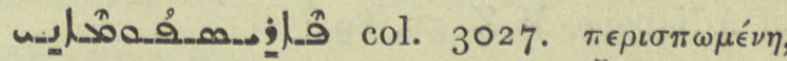
a circumflex accent called also Hebraica iv. I68, Syr. I, 2.

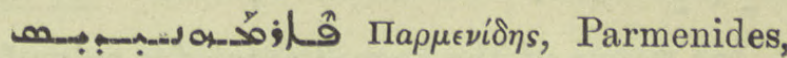
N. Hist. vii. 6. 2.

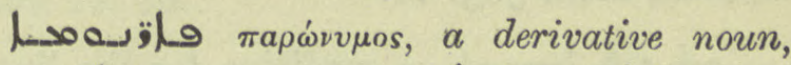

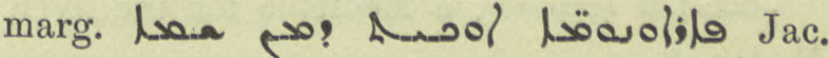
Edes. Arist. 21. I4. Cf. col. 3025 .

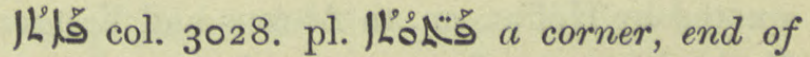

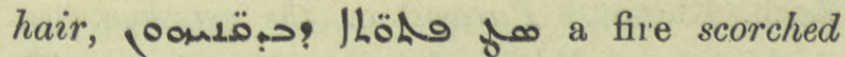
the ends of their beards, Warda $33 v$, Hist. B.V.M. i 6 . ult.

Lin ô col. 3029. Add: delectation, N,

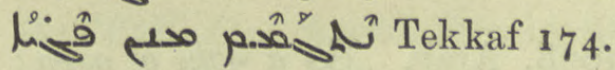

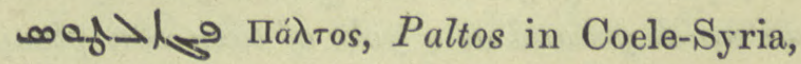
Nöld. F. S. i. 469. 29.

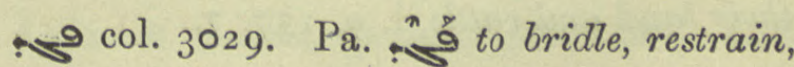

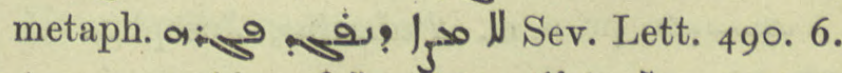

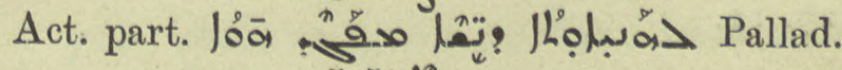
230. 3. Ethpa. 9 ? $^{n}$ to be yoked, harnessed,

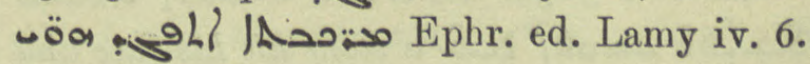

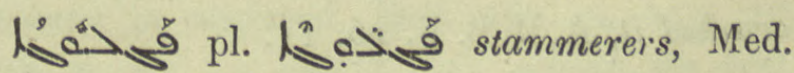
I 80 . I 2 .

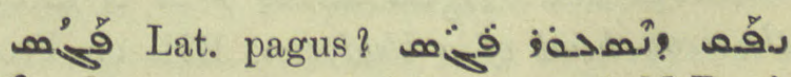

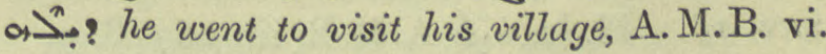
87. 9.

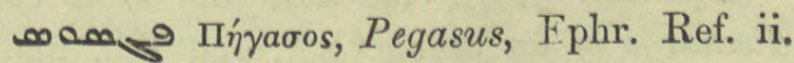
27. 1 .

นُนْ col. 3032. used in magic, prob. interpreting chance meetings, Ibn S. Thes. $22 v$.

L 1 
$\therefore$ col. 3033.5 af. correct the passage

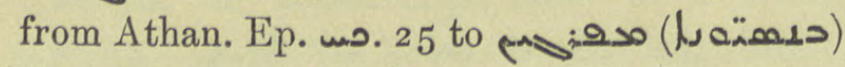
rejoicing in temptations.

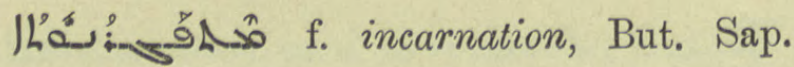
Isag. 2. 8.

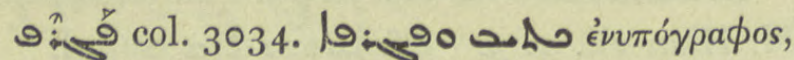
a husbandman inscribed as belonging to the glebe, Syr. Rom. Rechtsb. 48.8 af.

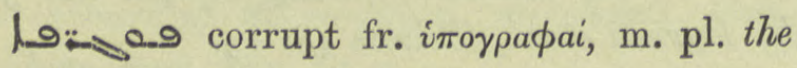
epistles of S. Paul, Hormizd 3 I97.

an: 9 pr.n.m. Persian, Pagrasp, mobed of Adiabene, M.Z. 50. 89 .

ق̋ col. 3०35. to wander, flee. Part. adj. Iُ Iflee, Test. Rahm. 64 med.

log? one of the ingredients of a salve for wounds, Med. $5^{8} 5$. Ir.

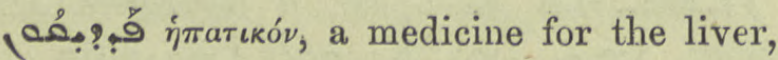
Med. 308.4 .

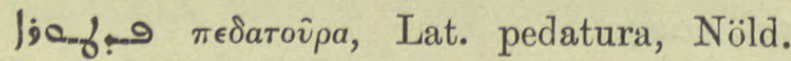
quoted by Hoffm. nn. to Ahrens and Krüger's Zach. Rhetor. Parapet or the passage just within the wall of a fortress, Anecd. Syr. iii. 207. 20, 208. I 2 .

فํ a Persian village, A.M. B. iv. 2 I 5 . I3.

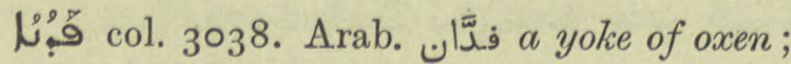
as much ground as a yoke of oxen can plough in a day, Dion. I55. I4, oهب their land and cattle, 1. 9, I57. 7.

ans pr.n. m. Pedanius, Josephus vi. 27. 12.

م-9: 3039. to crush, shatter,

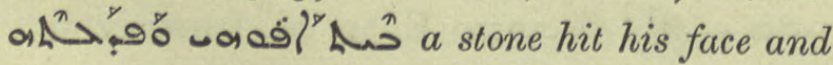
crushed it, A.M.B. ii. 26 . I I.

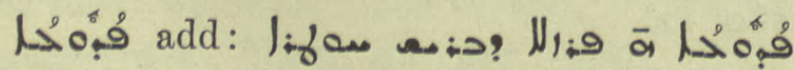
the iron head of a bludgeon, BH. on Prov.

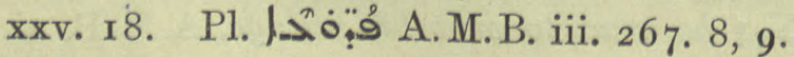

فํ. discharge of an arrow, BA. and BB. under

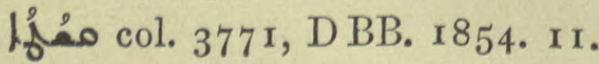

Jhể for JLA a piece of bread, Dion. 98. 18.

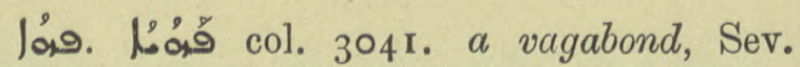

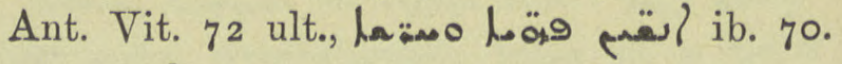
ro, 7 I ult.

قُ col. 3042. add: Arab. فهُ a cheetah, ounce, ZDMG. xlv. 703, xlvi. 468. 5, Dozy Suppl. ii. 285 .

5ja- col. 3044. $\pi \epsilon \lambda \omega p i s$, a large shell-fish, pl. قُ

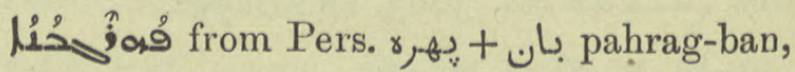
Lag. Arm. Stud. no. I796, I799, I793, the town guard, Pers. Mart. n. $866=$ Jab. 442. 3 .

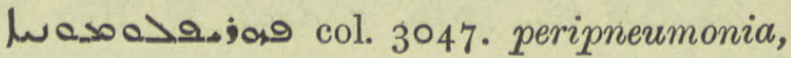
Hippoc. iii. 22. 29.

م/

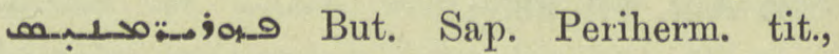
Isag. iii. I. See Sus col. 3262 .

كeripatetics, But. Sap.Periherm.

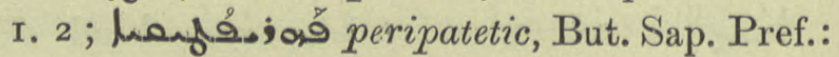

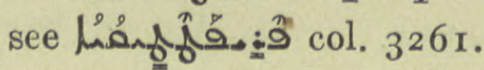

๑ col. 3047. фov, Valerian, refs. Med. 245. I 5, 354. I8, 359. 2, 370. I3.

هصa Phoebus Apollo, Sev. Ant. Vit. 246.7.

$\checkmark$ co col. 3049. to grow weak or cold; add :

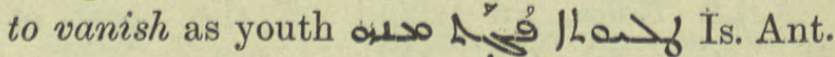
ii. 122. 2, ytand fُ ib. 124. 20. Pael 敌 cool with a moist rag, Med. $5^{80}$ pen.

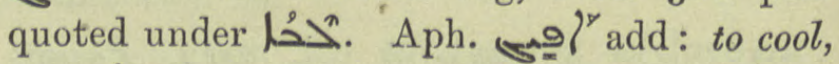

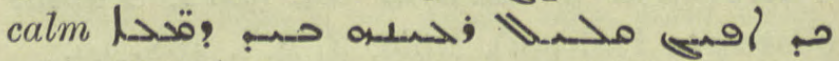
when his mind was somewhat relieved by tears, Jul. 8. 14 .

I. $\rightarrow$ l. 00 gout, Hippoc. vi. 27 ff. See lis 9 col. $3 \circ 37$.

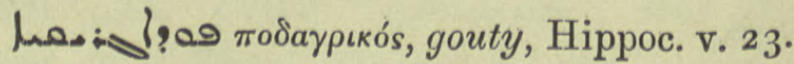

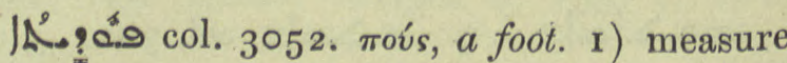

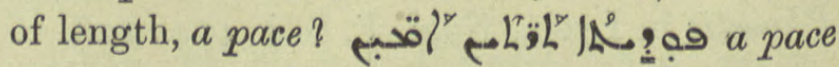
equals two cubits, Med. 53r. 7. 2) measure of time, equals ten sese (i. e. $\frac{1}{5}$ of a minute) ib. 1. 10.

Jåog้ col. 3052. a pustule, Med. 159. 5,

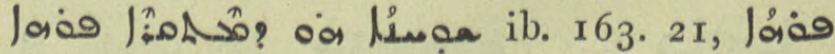

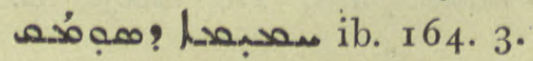




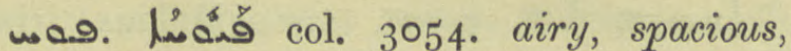
.

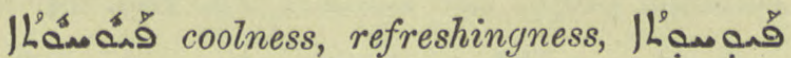
;\}? Is. Nin. B. $237 \cdot 4$.

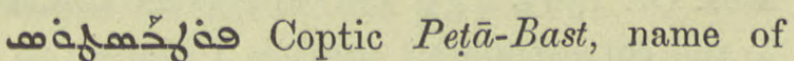
a monk, Pallad. I26. I r.

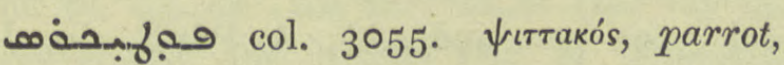
Natur 33. 5 .

|م->

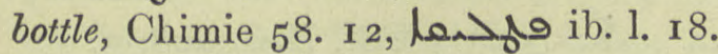

\es, \ف́ col. 3057. to mix, work up; to knead, imper. Ma์ Chimie 43. 10, Med. 164. $8,55^{8 .}$. 7 .

Neg col. 3058. 7 af." Delete "forum".

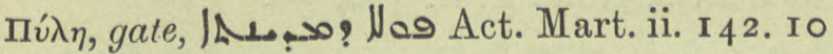
$=$ A.M.B. iv. I09. 18 .

لِ for

, لlas steel. See col. 3r43 and Suppl. under لق.

وف حةتشْ Paulonians, followers of Paulonas, a pupil of Ephrem Syrus, Hist. Mon. i. I 77 = ii. 354 n., I 4 I. I, Nest. Hérac. I 2. 3,6 ,

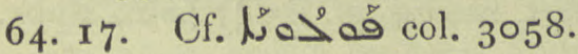

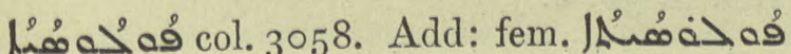
Pauline, Takhsa I34. 2 af.

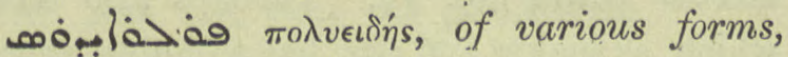
ธุ

1 state. lafa入 Civil Law, Sev. Ant. Vit. 52. 6, 56. г.

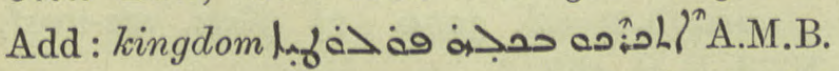
iii. 233. 1о. Metaph. mode of life, M ف حفة

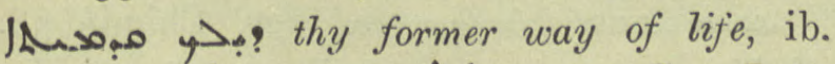
243 pen., lit $\rightarrow 0$ ? A.0ol hindes Sev. Lett.

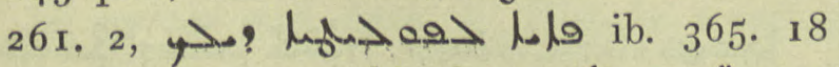
perhaps statesmanship here;

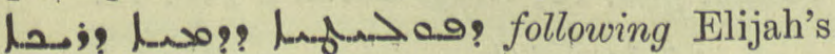

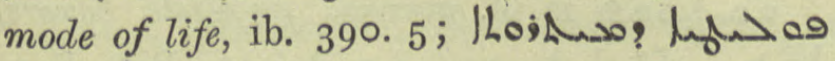
Anecd. Syr. iii. 66. I I.

حمل ?مبال : houd with le.s: 128. 10, with 10 1. I3,

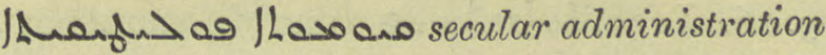
opp. IA.

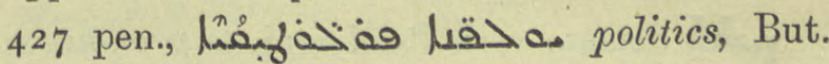
Sap. Philos. i. I, Pol. ii. tit.

مجه Nau 23 pen., 28. I5; see بْلِ infra.

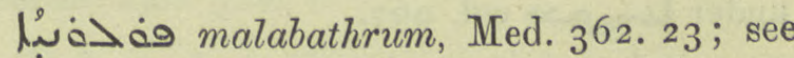
פ 9 col. $3{ }^{\mathrm{I}} 46$ ult.

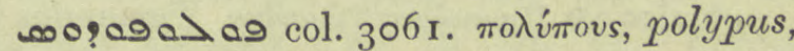
a zoophyte,

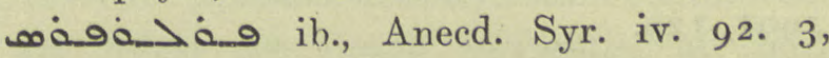
ल 1.5 .

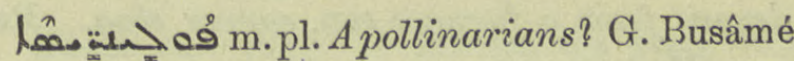
42. 22.

L. Nau 22. 10.

פa Palcarion, name of various

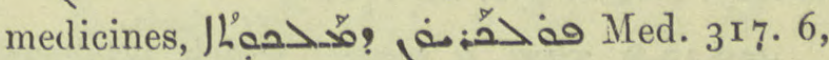

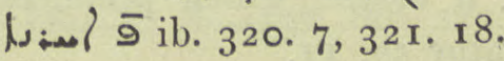

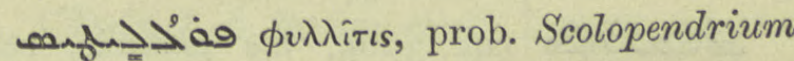
officinärum, hart's-tongue, Diosc. 3. I $2 \mathrm{I}$.

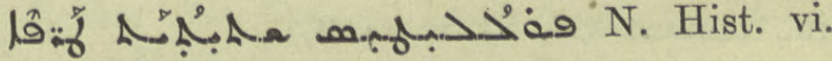
2. 3 infra.

. Ar.PflnN. 67; Anecd. Syr. iv, 38. 21.

فة polypus, Med. 62. I3.

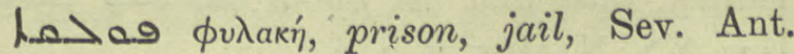
Hymns i 8I. n. ter.

p. i. $q . \underbrace{2}$ the middle part of the alimentary canal, viz. the stomach or the pylorus; the passage is confused, Med. 267. 5, 27 I. 20 ,

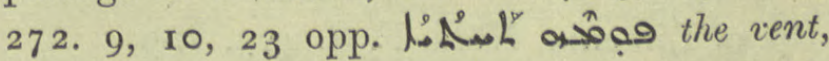
ib. $267.7,329.2$. lísoọ pọ what? "inside of pomegranates", Budge, Med. 2 I3. I4, 2 I 4 . 9 "pomegranate pulp", ib. 409. 22, 4II. 2.

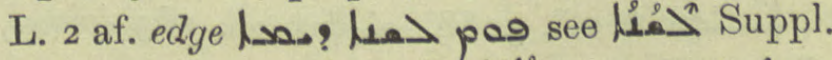
Col. 3064 . I 7 pos os

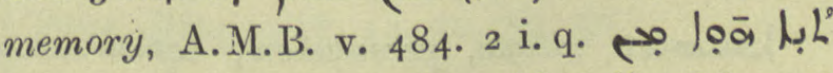
l = Pallad. 232. 2. Orally, add El. Tir. Gram. 
3. r6. L. 30. Hol? h hoog name of a monastery, Doc. Mon. 2 1 9.14.

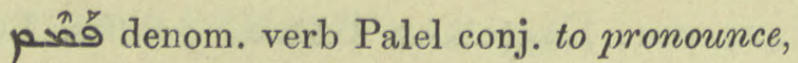
م.

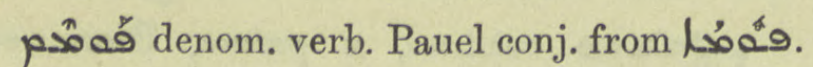

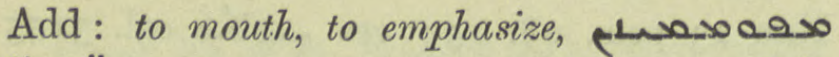
Jhin we lay stress on the second letter, BA. under lengaso col. 967 .

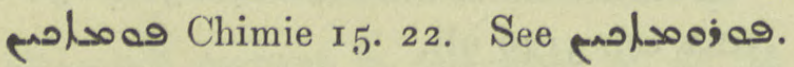

(ब.ب) Pisidia, Nöld. F. S. i, 473. I I I, Byz. Ztst. I901, 129.

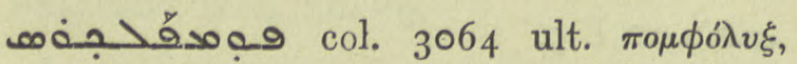
deposit from a furnace esp. copper slag. Ref. soot, Med. 8r ult.

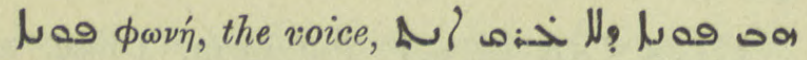
give your word not to escape, Vit. Monoph. 85. 27 ult. Pl. محه حفة they applauded loudly, Anecd. Syr. iii. I 70, 9.

هivpa, a mussel which produces golden threads, Natur 57 . I I,

ص Anecd. Syr. iv. 58. 6, Natur 28. 6, وفبجم ib., פم נתم Or. Xt. v. 24. 8.

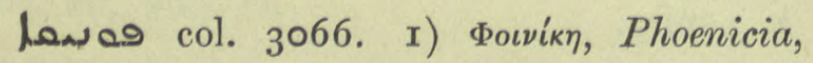
add: Hex. Ex. xvi. 35 ed. Lag. 2) a prescription for phthisis, Med. 242. 3 .

ص هolvikias, the Phoenician wind, so the Greeks called the wind between the

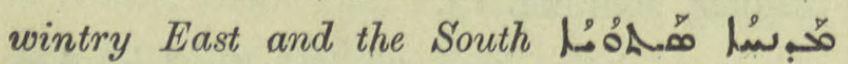
lisa. $>$ N. Hist. v. 3. 2.

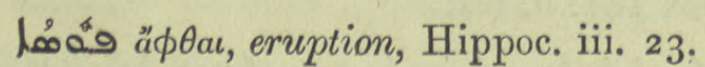

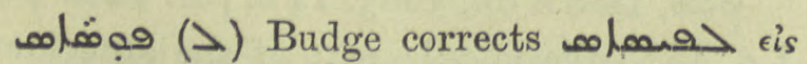
Míav, Pisa, Gest. Alex. $3^{8 .} 8$.

ooffltoø Фavoros, Faustus (Prostas?) Bp. of Limenia, Nöld. F. S. 473. ro9.

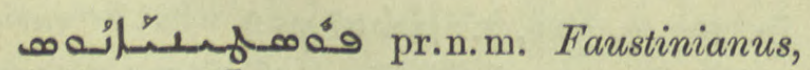
C.B.M. $1130 b$.

1 Coupes ii. III. I.

حـen " p. 43 . II. loos as Lat. posca, an acidulous drink, a beverage of water and beaten egg, used by common people and soldiers, Jo. Tell. 23. 8.

h pistachio green, Brit. Mus. Or. 1593. $44 r$ and so correct loL oos in the corresponding passage, Chimie roo. 2, وملما ib. 1. I4. See col. 3r99 pen. and add: Ar.PflnN. 69, Jac. Edes. Hex. Nöld. F. S. 575 ult.

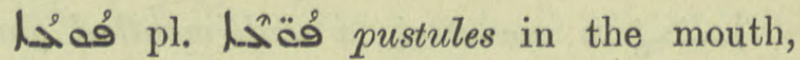

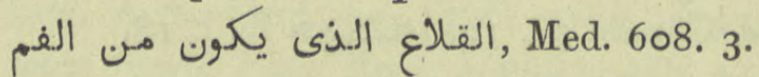

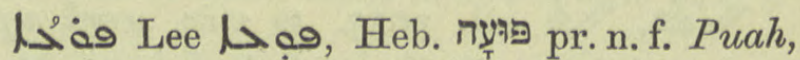

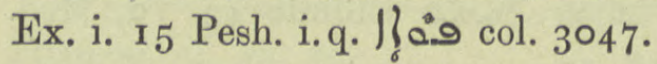

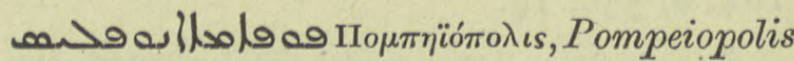
in Cilicia, Nöld. F. S. $4700^{6}$.

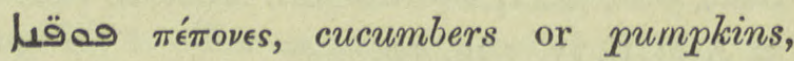
Sev. Ant. Vit. 28, 6, Nau in loc. R.O.C. iv.

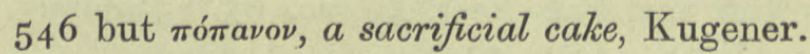

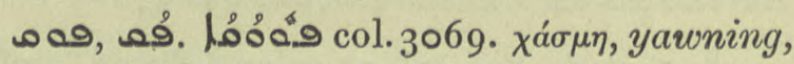
Hippoc. vii. $5^{2}$.

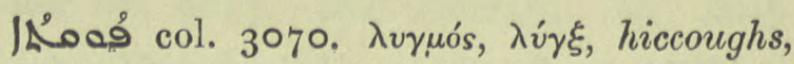
Hippoc. v. $3 \cdot 55$, vi. 1 3.38 , vii. quater, Med. 28. 10, 20, 289. 8, 290 ter and often.

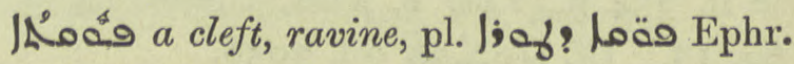
Ref. ii. $3^{6 .} 6$.

حتس هقمُف which, as snakes and efts, has a double tongue, N. Hist. vii. 6. 6, وفمب ib. I. 5.

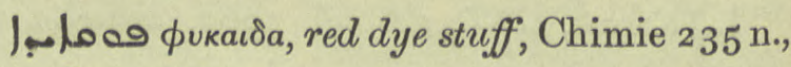

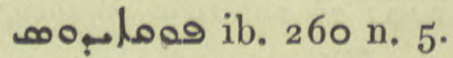

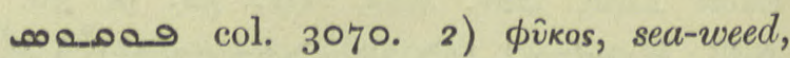
Nöld. F. S, 568 .

 23 bis, 59. I, 2, ,

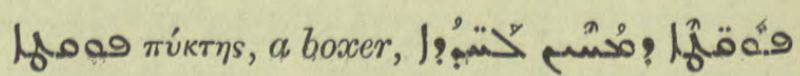
Pallad. 596, ıо, Greg. Carm. i. I го. I r.

. But. Sap. Isag. 2. 4 .

uم-0م0 3070. the art of boxing, But. Sap. 
Isag. 2. 4. A boxing match, a contest خح

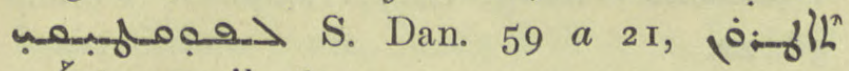

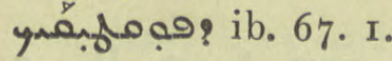

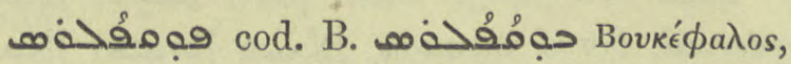
Bucephalus, Gest. Alex. 3I. I2.

jos, †ُ col. 307r. to be hot. Act. part.

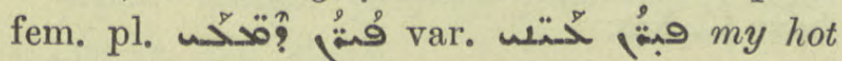
tears flowed, Jos. Narses 105. 2. Ethpaual

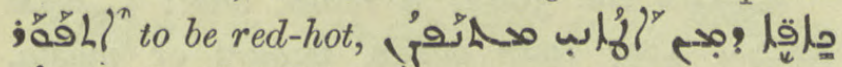
stones cast forth from Etna are burning hot, N. Hist. iv. I. I.

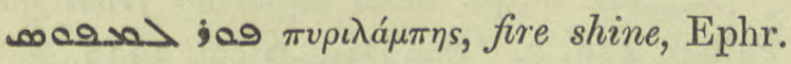
Ref. ii. 26.47 .

;ณ์ П П⿵人pos, Porus, an Indian King, Gest. Alex. 139. 17. See صojo col. 3073.

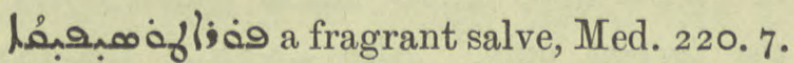

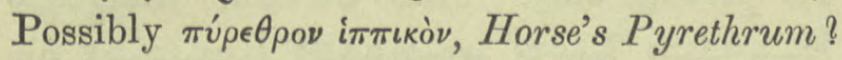
Cf. pilajes col. 3072 infra, two other spellings col. $3 \circ 73$.

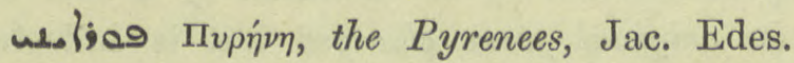
Hex. 34. 8.

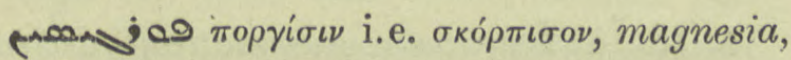
Chimie 54. 3 .

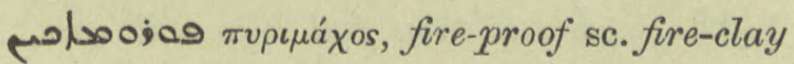
مan Chimie I6. 6, 294 n. 2 and so Duval corrects for 15. 22, 292. I. The corresponding passage Brit. Mus. Or. I 593 has polsoogeg.

andase Pyrrhonians, followers of the philosopher Pyrrhon, Probus io2, 6.

10\% pl. 1) Sev. Ant. Vit. 68. 4, Nau corrects 申óvou, ruffians, cut-throats, R.O.C.

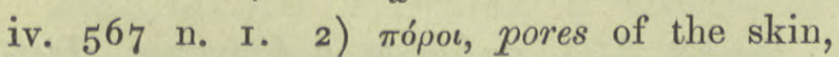

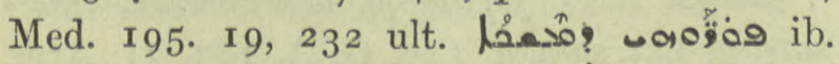

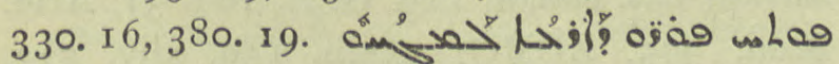

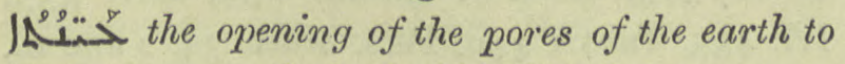
let springs gush forth, N. Hist. iv. I sect. 3 , II. 3 .

๓0;09 col. 3073. I) pr.n.m. Pyrrhus of Epirus, philosopher, Sev. Ant. Vit. 40. 10. 2) the island Pharos, A.M.B. v. $55^{8}$ nn. 8,9 , حمْ

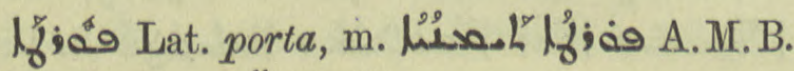
iii. 306.5 var. لู
.

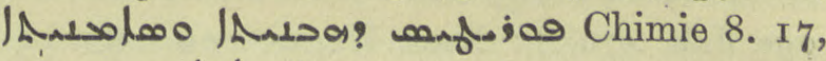
. 1 ib. 9. I3, 6 .

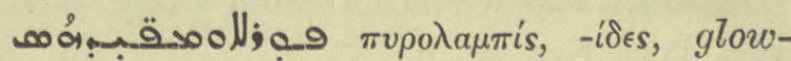
worms or possibly $\pi v \rho a \lambda i s$, pyralis, fireflies,

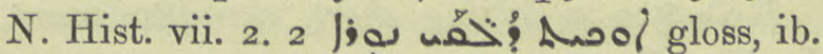

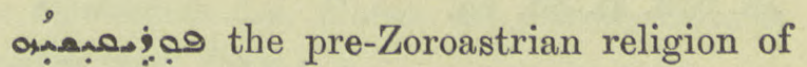
Persia, Jab. 526. I.

lsoges Lat. forma, a sealed letter, an

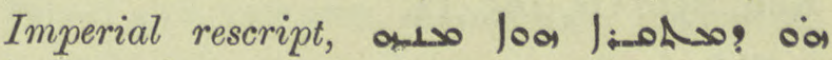
isoga Pet. Ib. 57. 19. Forma, Túnos vel Epistola, littera formata cui impressa est forma seu imago scribentis, in sigillo scilicet, Du Cange.

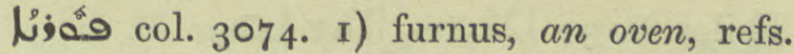
Natur 63. 5, 8, A.M.B. iii. 383 ; ب.

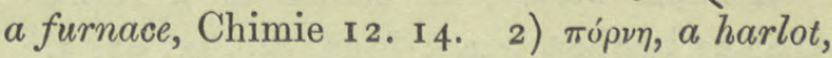

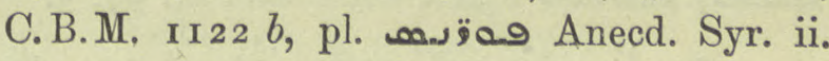
269. 27 .

Awiog col. 3074. a flat loaf, ref. pl.

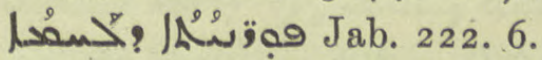

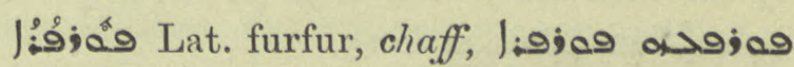
oa his delight is mere chaff compared with his former bliss, Ephr. ed. Lamy iii. 53, 26, iv. 423 . $2 \mathrm{I} ;$; as light as chaff before him, Hormizd 909, L L

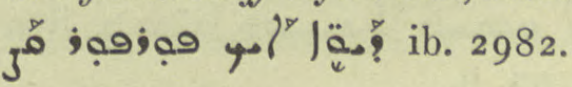

هors. Peritten accusation, Jab. 238.3 , 14.

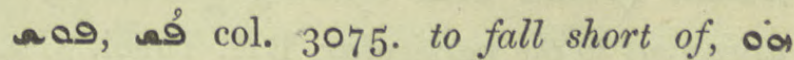
"حla la Aleshly substance fell short of being God, Nest. Hérac. 20. 8. Aph. engl" to leave off, he does not leave off throwing stones, Anecd.

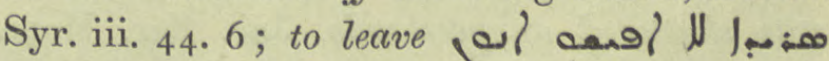
they left none remaining, G. Warda ed. A. Deutsch 8. I3.

مُ0ْ col. 3077. pause, rest from labour, Is. Ant. ii. 118. 15. L. 6 of par. intermission, pause between attacks of intermittent fever, Hippoc. iv. 43,5 , vii. $5^{6}$.

قويn add: Anecd. Syr. iii. 69. 24, Jقب 


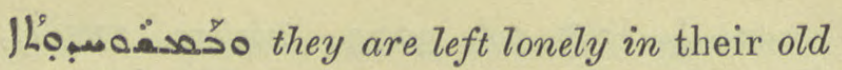
age. Jab. 476 . 7 ; Nest. Hérac. Iо6. I 8.

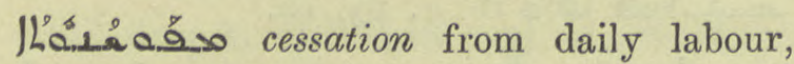
rest, Poet. Syr. 7 7 . 7.

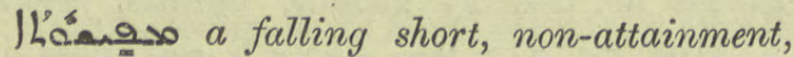
Is. Nin. B. 88, 19.

بوشن E.-Syr. bpric., now Gôriyan W. of Herat, on the Heri Rud, Syn. Or. I65. II.

J'å col. 3078. Rubia tinctorum, madder, add refs. Chimie 45, 4, I I, \&c., Med. 198. I2, 354. II, I4, 370. I9, Dion. 87. I4, I23. 12.

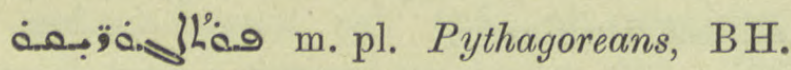

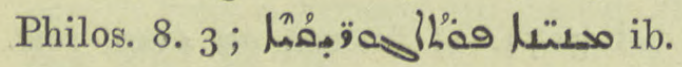

fisolos $\pi v \theta \mu \eta \dot{\eta} \nu$, the bottom of a furnace, حصب: Stud. Sin. ix. 97 . 5.

.9. powerful muscles on each side of the neck,

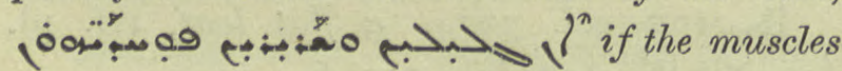
of the neck are full and firm, Med. 36. 13 ,

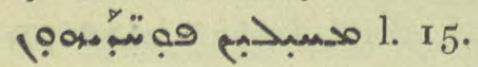

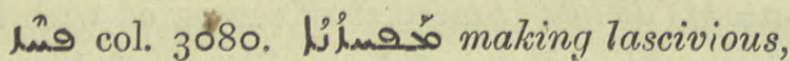

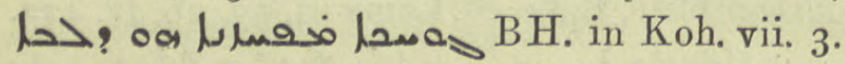

هُ $\mathrm{Pa}$. Syr. Rom. Rechtsb. 33. 6.

3) equality,

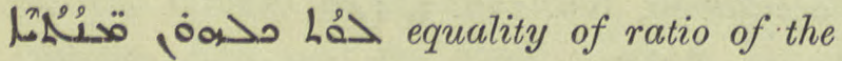
universal to the particular, BH. Theol. 4. 2.

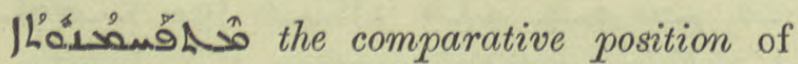
heavenly bodies, Georg. Arab. 25. 3 .

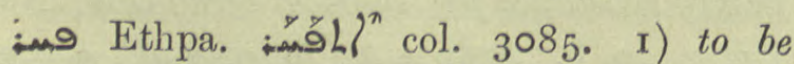

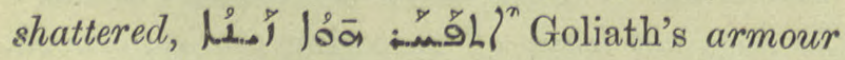
was shattered as potsherds, Jac. Sar. Hom.

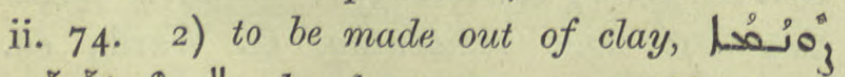

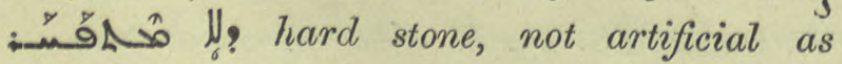
earthenware, ib. 65 .

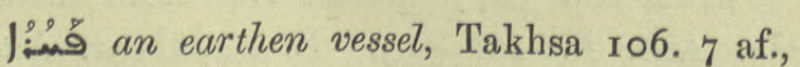
109. 8.

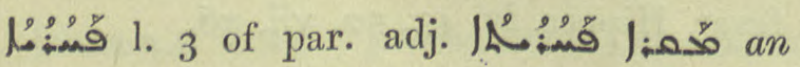
earthenware spout, Chimie ıог. Iо, I I. The ref, to Jac. Edes. ed. Mart. is ? not ।.

\section{rdarafs}

كبريت . marg sulphur, Chimie 36. 22.

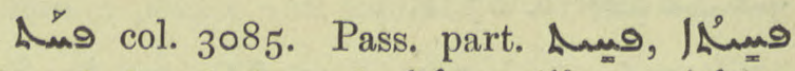

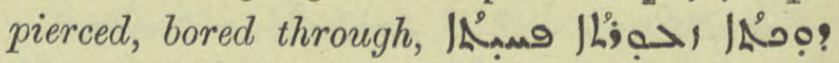
I the text and the parallel passage in Pallad. have وئin.

ف

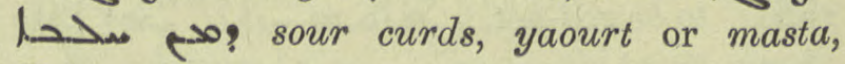
Z.A. xxii. 8 I.

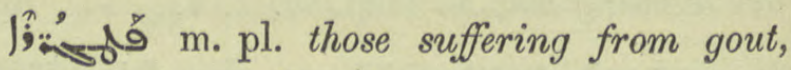
Pallad. 428. 7. See /; 1 . col. 3038 .

مهل |م 1 is defined as referring to time past

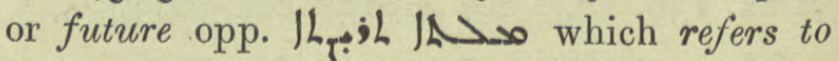

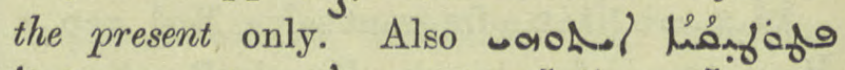
IA.>a s on of | (word) which by the addition of a case has its original form changed i.e. is inflected, BH. Periherm. i. 2.

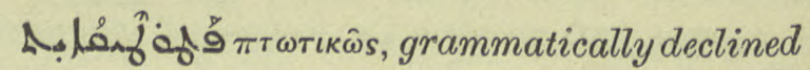
but here a play upon words, עـ

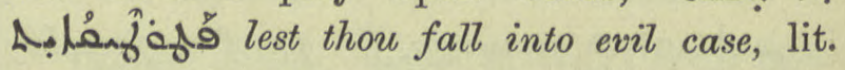
lest thou fall fallingly, 'Tekkaf. 22.

ल gives four letters which form cases $0>>$ ?,

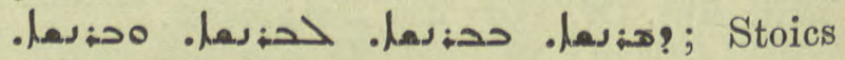
and grammarians add a fifth, the sign of the Vocative fas: of of Periherm. i. 2 infra 2000 ?

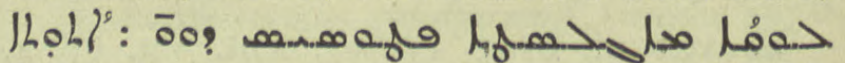
G. Busâmé 5I v 4; Jac. Edes. Arist. 2 I ult.

lanfo Arm. Pqluw2lu, a Governor, chiefsatrap, Lat. vitaxa, Lag. G. A. I87. 31, Arm. Stud. 375, ZDMG. xxxiii. I 59, xliv. 533, lvi. 435, Tabari 449, Pers. Mart. 34. 2 and n.,

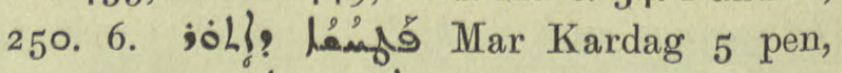
31. 10, 32. 4, lemflo? Anecd. Syr. iii. 259. I, I3, 25, 260. I; ; iii. 497,498 , iv. 22 I, ل

ไLْลَمَ governorship, vice-royalty,

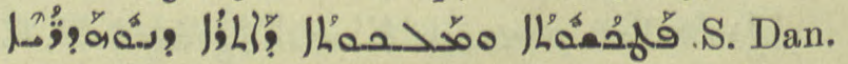
เо $b$ I9=A.M.B. iii. 497 med. 


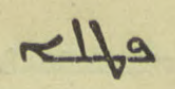

263

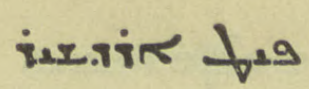

به

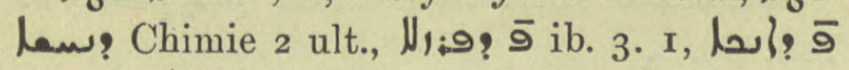
l. 6, J:ص? l. II and often. I. q. h 3089 .

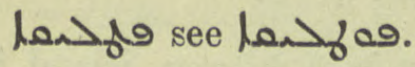

คิ. ๑. )

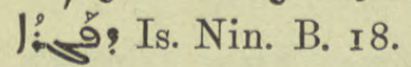

lafo i.q. aso.jte col. 30rg. Fatima, Bahira 238. 24, 234. I5 \&c. Lsaje Fatimites, ib. 242. 6.

बَّ col. 3०91. to perceive, refs. se complained that Severus did not recognize his dignity, Sev. Ant. Vit. 279. I ;

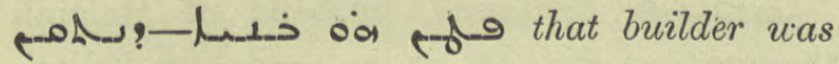
intelligent enough to frame, Ephr. Ref. i. 14. $4 \mathrm{I}$.

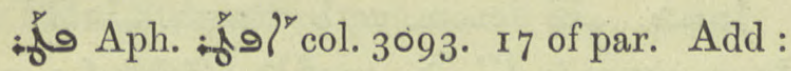

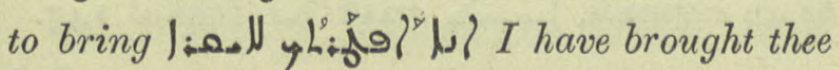
to honour, Ahikar 51. I3 possibly y hifiel.

أنققسم

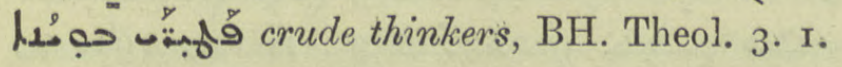

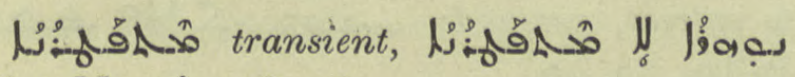
Hist. Mon. i. 378 . 16.

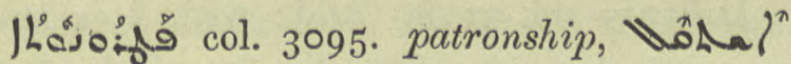

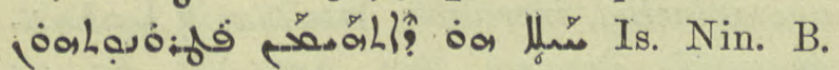

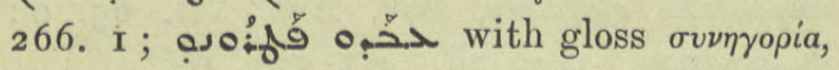
pleading, Hormizd I 6 I I.

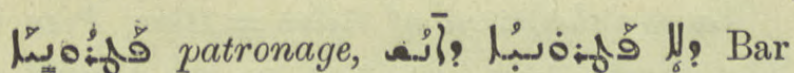
Penk. I3. I.

eawo-jo Petronius, a disciple of Pachomius, Diosc. ed. Nau 94. 2.

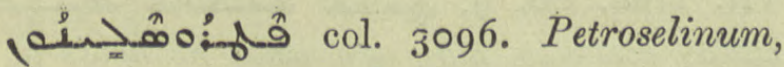
rock-parsley, قُّم: Med. 49. 7, 20, 5०. $8,608.5$.

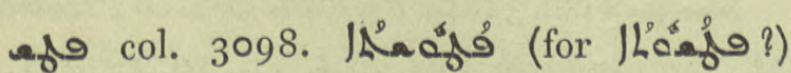
$\mathrm{BH}$. says this is a description of a natural

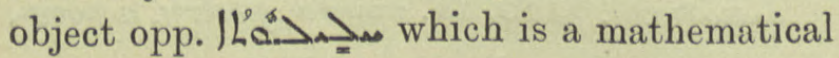

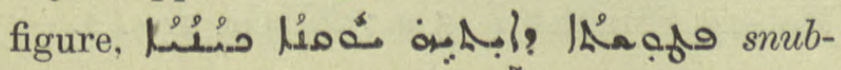

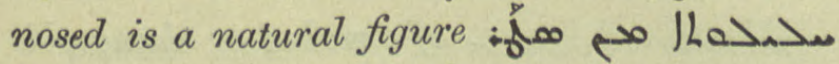
مهب LiLLe $\log _{\mathrm{H}} \mathrm{S}$ concavity is a concept apart from nostril; now it is nowise possible to conceive of snubness without a nose, Philos. 8. 2.

م 320. 18.

פr.n. of an alchemist, Pebichius sc. Epibichius i.e. Horus the falcon, Chimie 47. I 6, trad. $85 \mathrm{n}$.

مُ col: 3099. Add : guards, armed police, Tabari 448 , infantry, ib. and 442 , Lag. G. A. 74. 188. 1

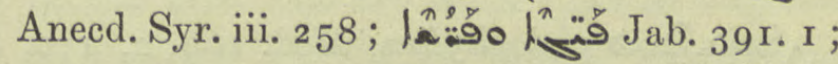
Gest. Alex. 63. I, A.M. B. iv. I29; Paigân sâlâr, chief of potice, Jab. 269. 10.

1.

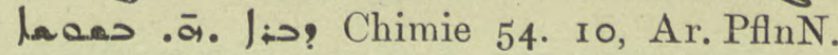
37 I f., Med. I35. 9, / 608. 2 and often, cf. Suppl.

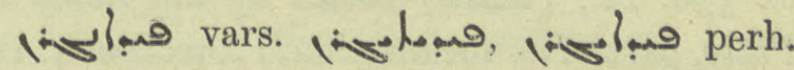
Arm. P'aitakaran, Paidangaran, the fourth region of Azerbaijan along the Araxes,

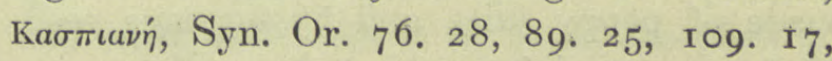

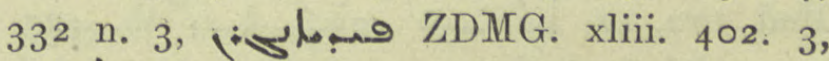
is 9 .

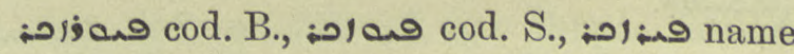
of one of three messengers sent by Nimrod to Balaam, Schatzh. I78: I 3 .

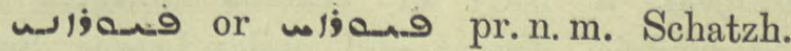
I 78.13 .

Jiـ a tablet or plaque of metal used as a symbol of authority with the Mogul Kings, Jab. 36. I 5, taken away from the Catholicos,

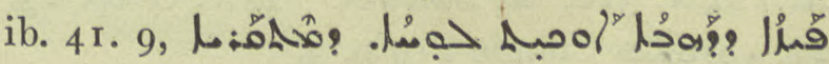
ib. 93. 5 .

1. hem, border, Du Cange gives oт́́ $\mu \mu a \tau a$ and

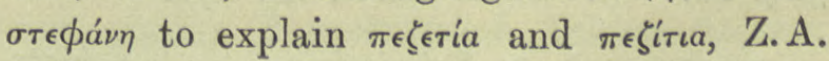
xvii. 90.

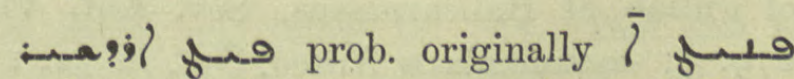
official name of مـنياذ اردشير al-Khatt. City on Persian Gulf, founded or more probably re-founded by Ardašir, Eranšahr 42, Tabari

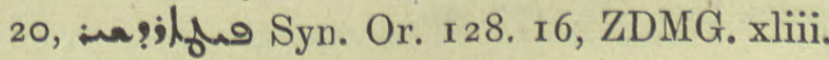
404. I 2 . 


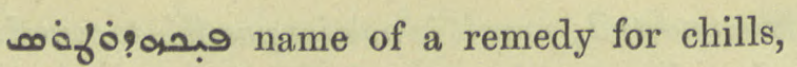
\&c., Med. 359. 22.

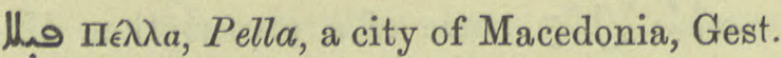

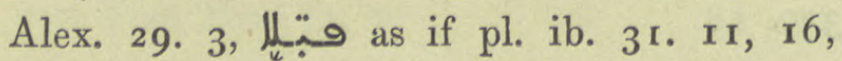

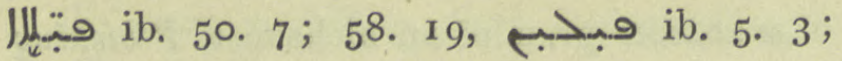
ولإف ib. 23. I4. Cf. ملإم col. 3134 and

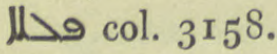

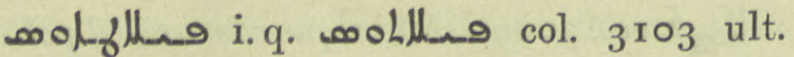
Philotheus, Bp. of Irenopolis, Nöld. F. S. 471.69 .

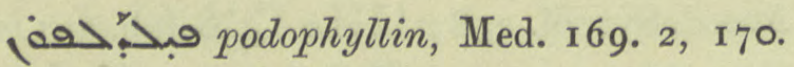
I6, 20, I7 1. I, I1, 18.

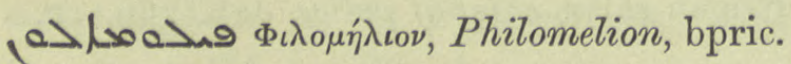
in Pisidia, Nöld. F. S. 473. I 9.

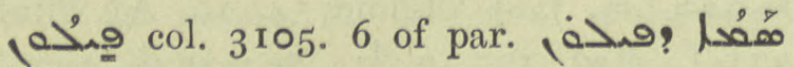

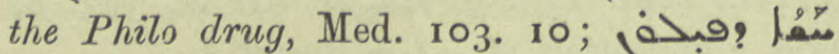

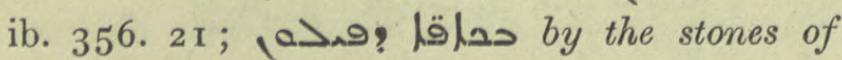
Philo, part of an incantation, Protection 4. 19.

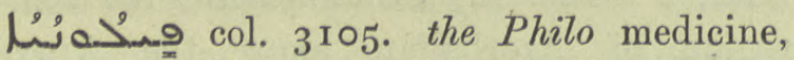
made of poppy juice and hyoscyamus seed,

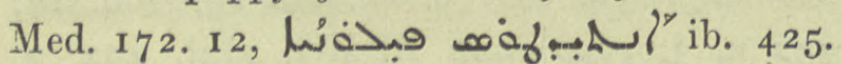

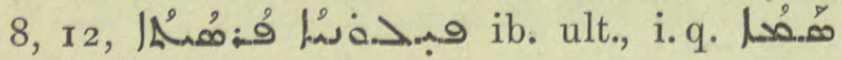
(ف)

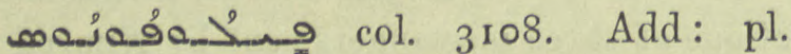

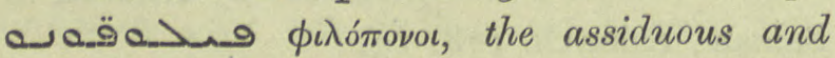
diligent, a body of zealous converts at Alexandria, devout and philanthropic, Sev. Ant. Vit. I 2. 6, 24. 3, 33. I, 2 I 4. I 2, R.O.C. iv. 347,543 .

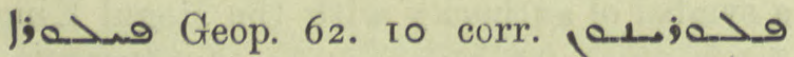
Ar.PflnN. 316, the limetree. Refs. Geop. 23. 21, piollag Chimie 219 n. 3 .

h. the restoration of a church, Anecd. Syr. iii. 324. 20. Cf. Jahrb. Theol, vii. 190.

פمخصمחمده Felicissimus, an associate of Julian of Halicarnassus, Sev. Ant. Vit. 279. 5 ; Antar. Anecd. Syi. 51. 17. Cf. 3 col. 31 IIO.

mamson Philomusus, Bp. of Pompeiopolis, Syn. Or. 470.56 .

وبلl see.
م corr. Tell. 6o. 6.

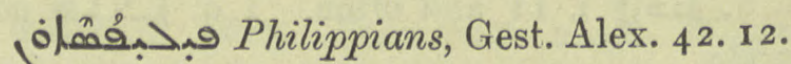
Usually

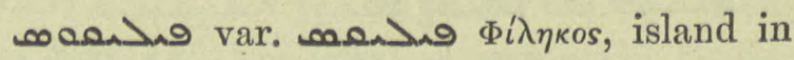
the Red Sea, Jac. Edes. Hex. xx. 7 .

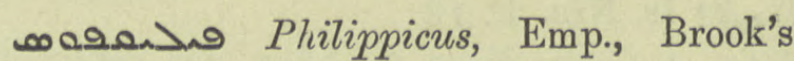
Chron. 572. 4.

Tivos, superficial colouring of silver, Chimie 213 n. I.

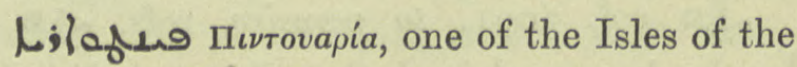
Blest, Jac. Edes. Hex. xix. I 5 .

م Pinianus, husband of Melania

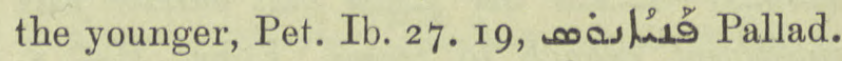

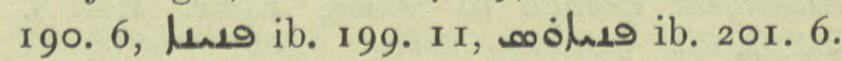

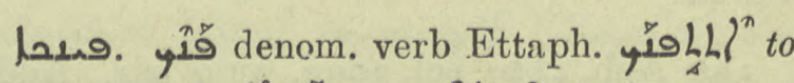

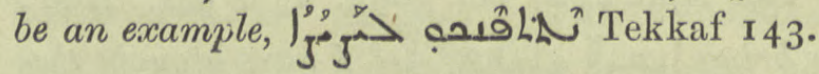

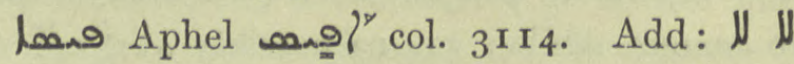

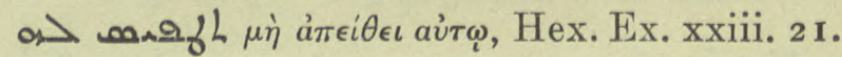

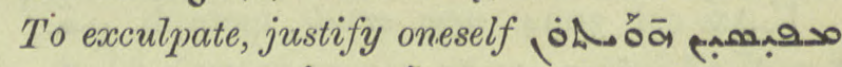

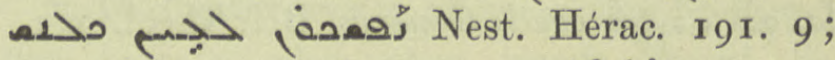
to entreat, persuade, Pallad. 83. 20.

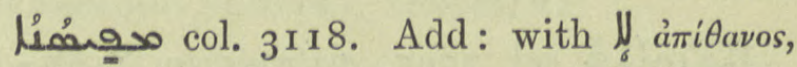

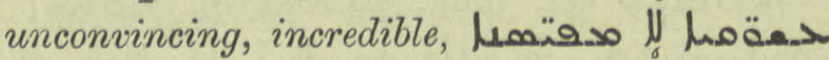
impossible slanders, Sev. Ant. Vit. 65. 9; . 6 id. Lett. I37. I6.

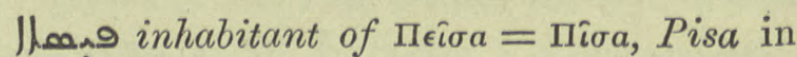

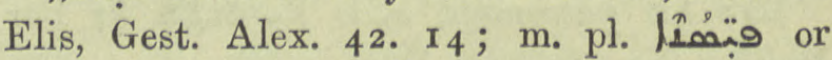

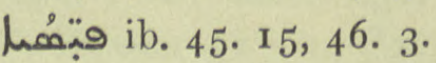

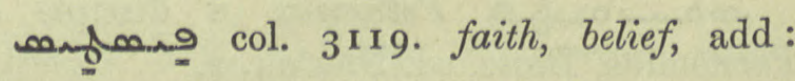

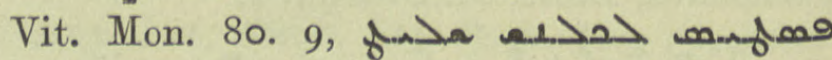
+olug everyone may say what he holds to be true, 1. 16.

1. of Pisidian birth, Sev. Ant. Vit. Iо. I2, 2 I r. 4.

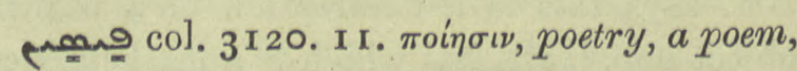
Moberg, Strahlen ii. 5 n.; BH Gr. I94. 2.

นُ 3 120. of streaked or changeable

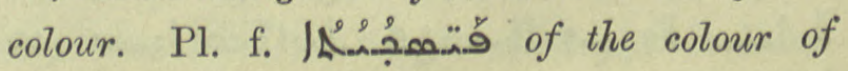
pigeon's plumage, Natur $3^{2}$. 5 . 


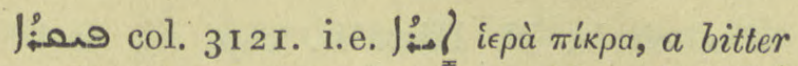

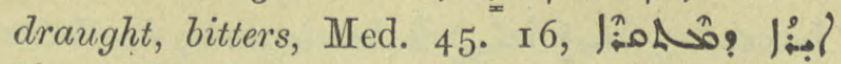
פبمنْ

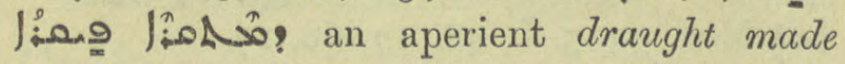
from aloes, ib. 74. 5, 273. 9, 291. 5.

9 a village in Tur Abdin, A.M.B. iv. 505. х3, 506. 10 = Mar Bassus 65. 5, 66. 7 . Probably i. q. Feer, Pers. Mart. 28.

- 20 col. 307x. Pers. يی an aged man, a minister of the Yezidis, in charge of fasts and feasts, Yezidis II 4. 5; وب: حبه Pir Bub, a great personage of the Yezidis, ib. I I6. 9.

andarying or achadyis q. v. Pirgushnasp, Magian name of Mar Sabha, Pers. Mart. n. $184=$ A.M. B. iv. 227 bis, ff. n.,

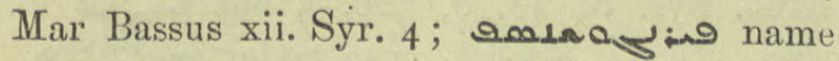
of Mar Grighor before baptism, Jab. 350. I 2 $=$ Pers. Mart. 78 n. 7 r6.

فيروز Vers. Victorious. pr.n.m. Feroz,

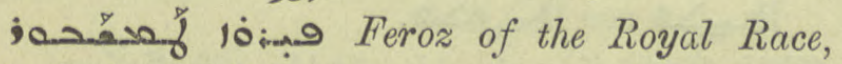
mobed of Adiabene, temp. Sapor ii, M.Z. 53 . 142. Cf. por Suppl. Name of a medicine,

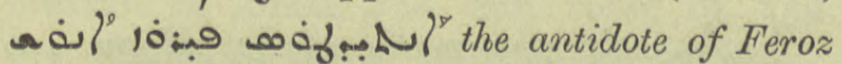
Anosh, Med. 426. 9.

lasojo: Tepipoou(?) the flowing around of humour underneath the cornea of the eye, Med. 75. 22 .

con Arab. 17. 2.

(음 I) Pirin = Perrhe, Anecd. Syr. iii. 316. 17. 2) Pirin, a fortress of Beit Zabdai, Mar Bassus 10. II9, 24. 315.

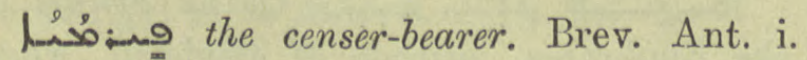
Cal. 29. 5 .

a forerunner, A.M.B. ii. $25 \cdot 9$.

moling Mútıs, the Pythian, Gest. Alex.

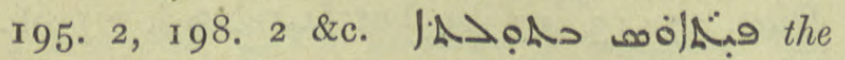
Pythian virgin, the Pythoness, ib. 3r. 8, I09. 9. Cf. פosh col. 3I24.

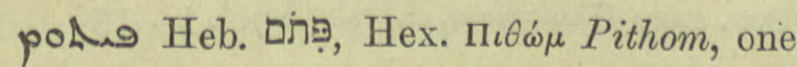
of Pharaoh's treasure-cities, Ex. i. II, ed. Lag.

2716

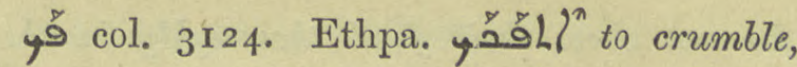

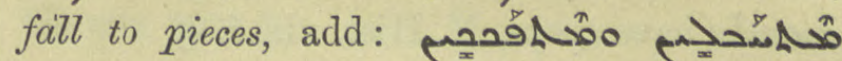
paper, wood, and stone rot and fall to pieces, Anecd. Syr. iii. 72. 27, 205. 22.

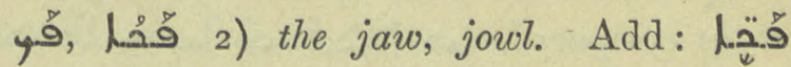
óóntes, the tusks of a wild boar, Gest. Alex.

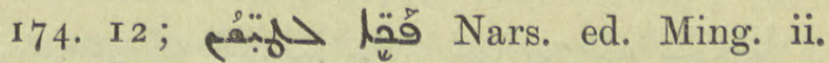
357.2.

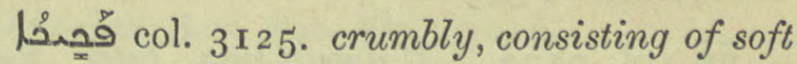
or loose particles, a substance which is

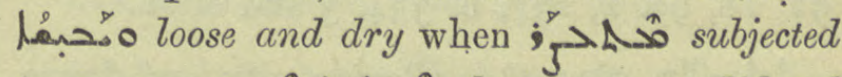
to pressure and if

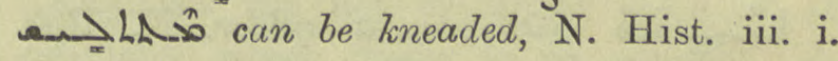
sect. 2 .

ดص col. 3125. to be or become tasteless. Add: ims فُ let its odour grow faint, Ephr. ed. Lamy iii. 2 I9 ult. Pa. aڤ้ add :

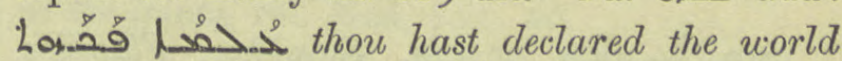
insipid, dull, Hormizd 343I ; Koj:- o-s

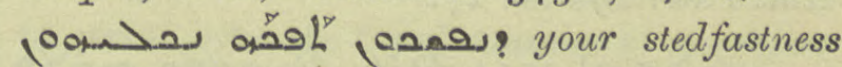
will make their guile of no avail, Josephus vi. 10. $\mathrm{I}$.

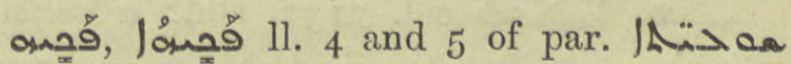

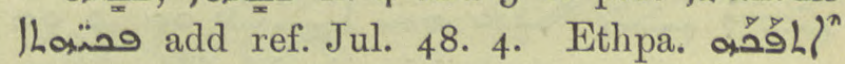
to fail, be in vain of an attack, Josephuis vi.

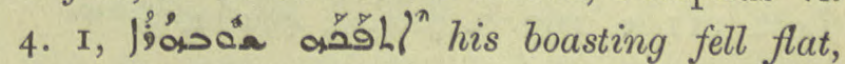
ib. 29 ult.

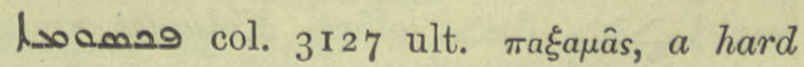
biscuit, Anecd. Syr, ii. 348. 22.

holsocosedimin. of the preceding word,

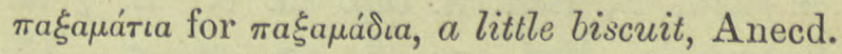
Syr. iii. 172.24 .

in col. 3I28. to bind. Metaph. (a) let them sit silent, Mar Aba ii. I 40 ; ( 1000 with clasped hands opp. outstretched, Takhsa 22. 12 ; w: net, Ephr. ed. Lamy iii. I37. Pael gُ to

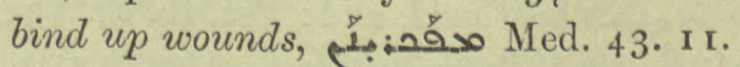

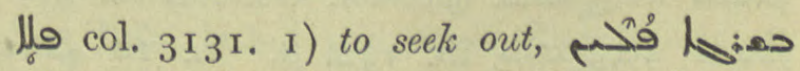
(oa) 00 a G. Warda ed. A. Deutsch 8. 5.

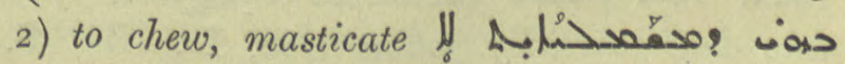

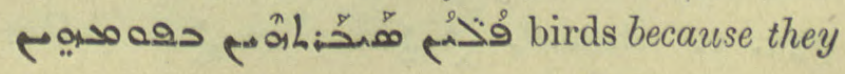
M $\mathrm{m}$ 
do not chew their food thoroughly in their mouths have crops, N. Hist. vii. 6. 5.

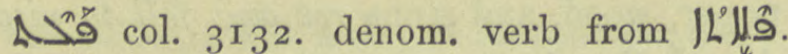
Ethpa. Аัธ้น/ to be spoken of in parable, to be compared, ar BH. on Prov. i. I.

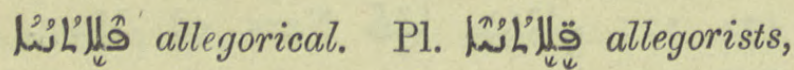
Hist. Mon. ii. 349 n.

ل⿺ 92. 18, 99. 2, 237. 6, 354. 3 and often.

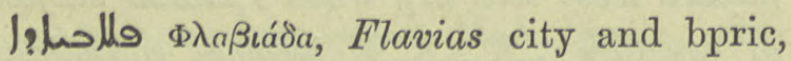
Sev. Lett. 59. 16.

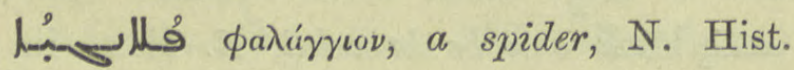
vii. 2. 2 .

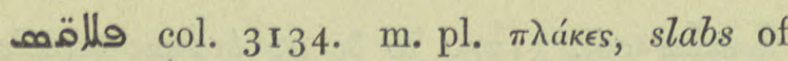

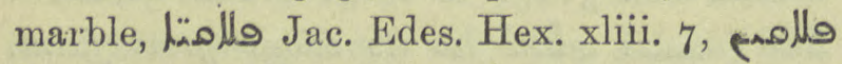
ib. 48. I6. Cf. Cقامق col. 3163.

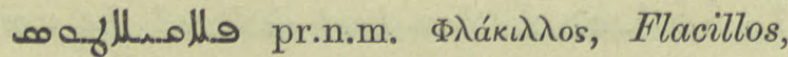
Ant. Patr. 298 no. 27.

هخخمص, Hebraica iv. 2 I 2. I 35 .

ง. مُ

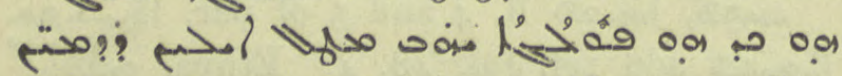
he gave the same decision in like cases, Sev. Lett. III. 9, I63. 3. 324. 5, 494. 4, 7.

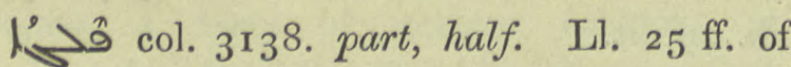

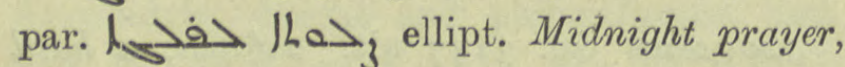
BH. R.O.C. I9II, xii. 273. 30. Geomet. م-faras uy $=$ radii, N. Hist. ii. cap. iii. 6. L. 7 af. hemiplegia, Med. 54. 8, rо, 96. 5. Cf. lasilog 79.

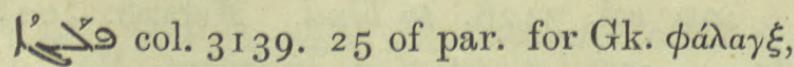
a phalanx, army, add: Gest. Alex. I34. 10, 206. 8, 207. 4 and often; Hist. Mon. i. xo8. 12, $228.21,302.18$.

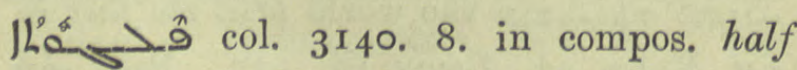

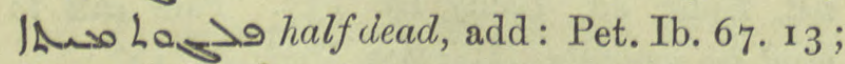
thes Los Lẩ

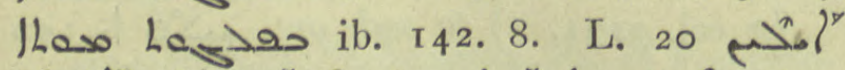

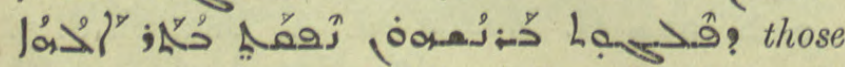
who half-heartedly follow God, Is. Nin. B.

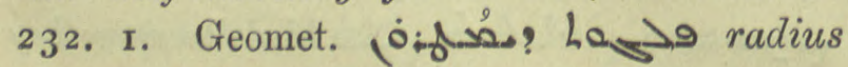

of a circle, N. Hist. ii. cap. iii. sect. 6 .

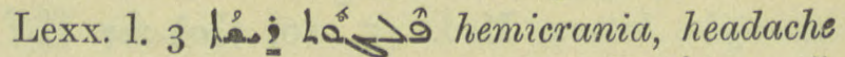

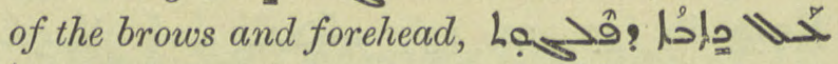
la: Med. 33. 5 ff., 48. I7, 60. I7.

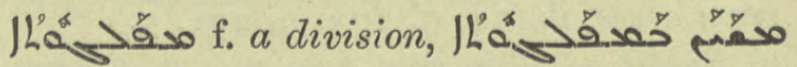
the Third Clime is tranquil in its divisions, Poet. Syr. 43.4 af.

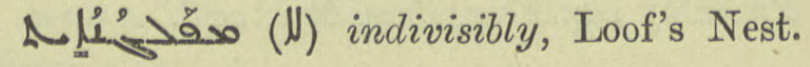
375. 10.

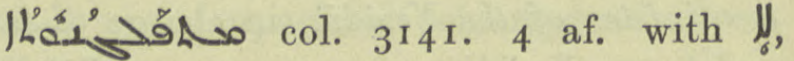
indivisibility, But. Sap. Philos. 2. 5 .

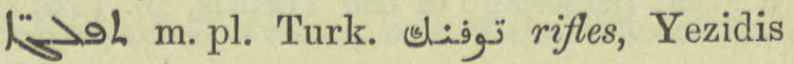
II 5 . 5 .

ف pr.n.f. Pelagia, Lewis, Gospels vii; Stud. Syr. ix. 48. 8, 306. 5 but h

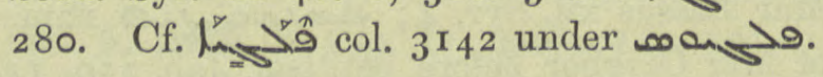

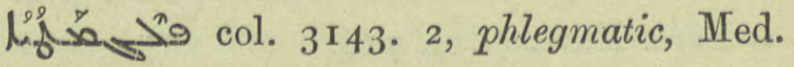
I9. $15,217.17$.

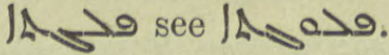

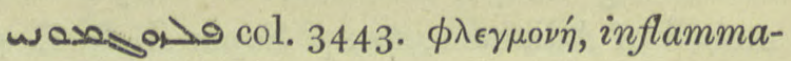
tion, Hippoc. v. 2 I, vi. 39,47 , vii. 15 .

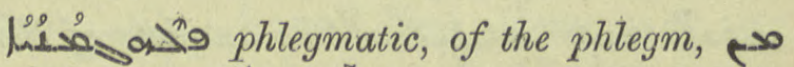

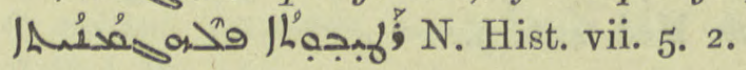

ه: oa? Chimie 21. I4, a steel filing, ib. 52. 18.

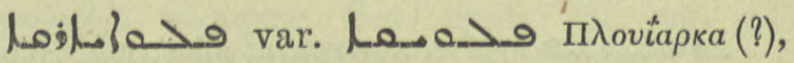

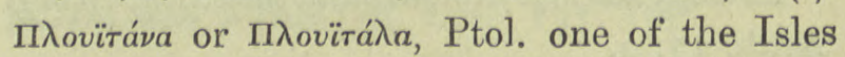
of the Blest, Jac. Edes. Hex. xix. I 4.

هخه|ز مام nalis s Anol Manichéisme I42. 4.

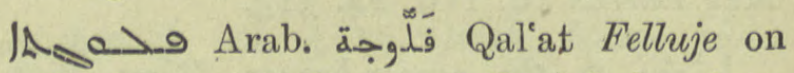
the Euphrates, latitude of Baghdad, where irrigation from that river begins,

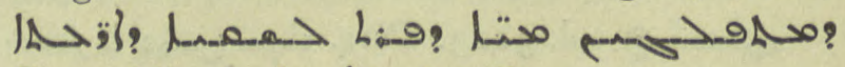
Sassanidi 28 ult., / L

פ Dion. 27. 13, 17, 19 but les/0 9 1. 7 prob. misprint.

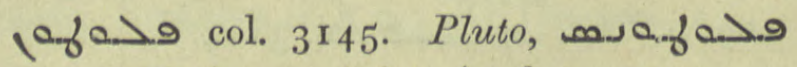
gen. case $\Pi \lambda$ oú $\tau \omega \nu o s$, Arist. Apol. R. I 5 .

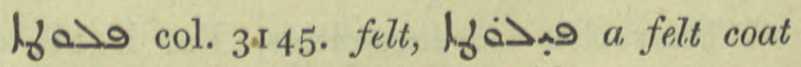
or cloak, cut to measure, Gest. Alex. 198. 3, 7, 
200. 5, 6. Assyrians of to-day wear felt coats.

L.II, I 2 .

فُ col. 3r46 ult. Malabathrum, betel or base cinnamon, Med. 49. 5, 87. I7, 16 r. I 8 \&c.

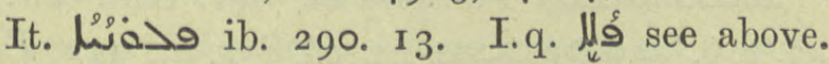

tua pr.n.f. Palona of Thessalonica, Hist. B.V.M. I 27.2.

Joreod $9 \mathrm{col}$ 3r47. a citizen of Pelusium,

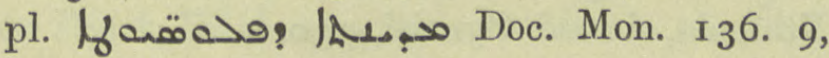
I 43.4 .

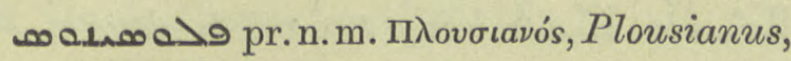
Sev. Ant. Vit. 89. 7 .

๓ Econ. iii. $\mathbf{~}$.

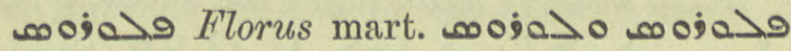

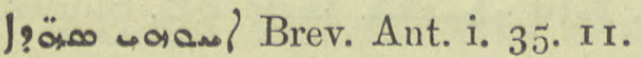

פע פ col. 3r49. I4 af. To work, form,

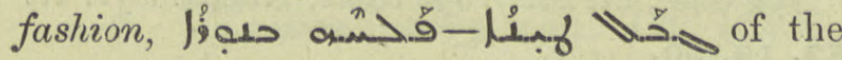
creation of Adam, Jac. Sar. Hom. iii. I59. 16 ; لحُ Sev. Ant. Vit. 208. 8, cf. 1 g $^{2}$ $\chi a \lambda \kappa \epsilon \dot{\omega} \omega \nu$, to be a smith, work in metal, Arist.

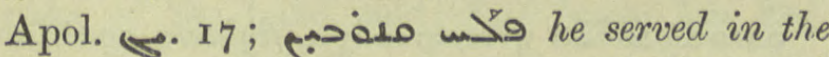

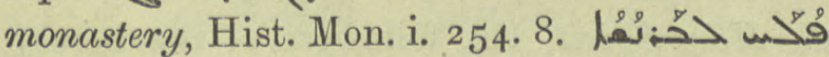
man-worshipper, epithet applied to Nestorius, Jo. Tell. 40 ult. Ethpe. mج A. Sar the monastic life which is perfectly led, R.O.C. iv. 341. 8.

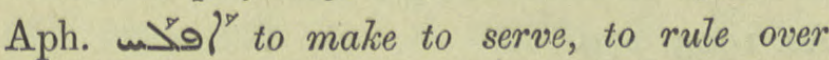

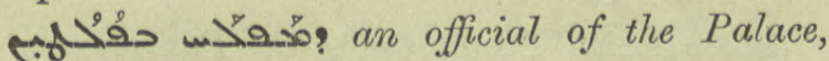
Pallad. 57. I $\mathrm{I}=$ A.M. B. v. 75 . 14 .

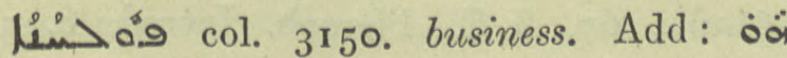

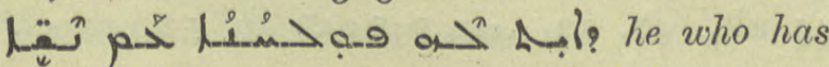
anything to do with women, Pallad. 759. 9. A cloth, מم: صapraped it in a clean cloth, Pet. Ib. 39. 10, 40. 6.

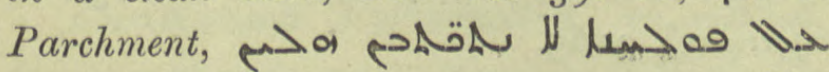
Stud. Syr. Rahm. 42. II, oa

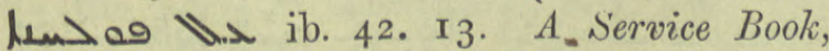
Hist. Mon. i. 47. 7, 9, many service books are

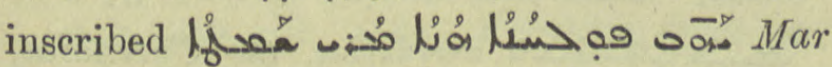
Shamta presented this Service Book, ib. l. I I.

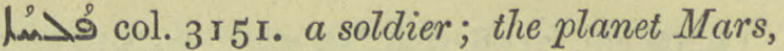
Chimie 29r. 6.

كـ فُ after the manner of a servant, opp.

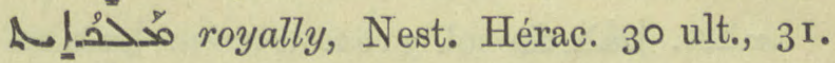
I 4.18 .

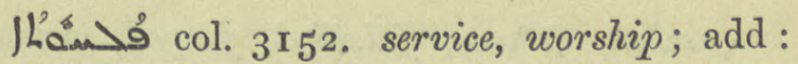

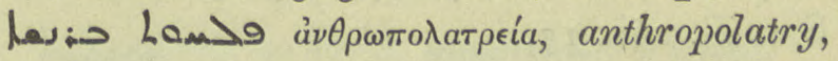
so his opponents called the opinion of Nestorius, Sev. Ant. Vit. 242. 6.

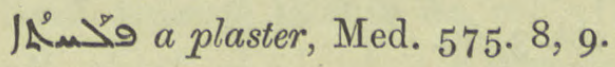

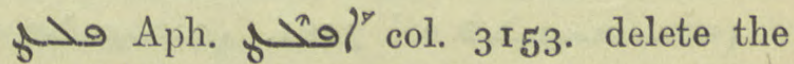
ref. to Kal-w-Dim, To free, loose

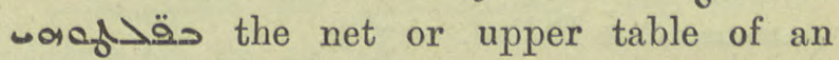
astrolabe moves freely in its grooves, De Astrolabe 74 .

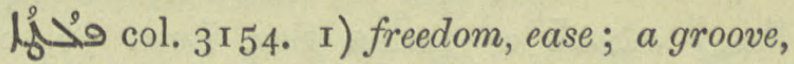

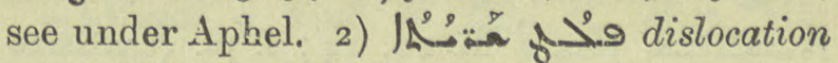
of the joints, ref. Med. II2. 20.

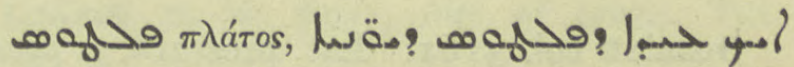
he wrote after the Greek manner of amplification, Anecd. Syr. iii. 200. 2.

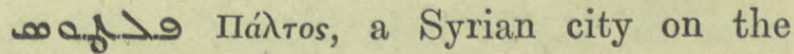
border of Phoenicia, Sev, Lett. 5I n. 4. Cf. og 9 above.

ص A.M.B. v. I48. $5=$ = Pallad.

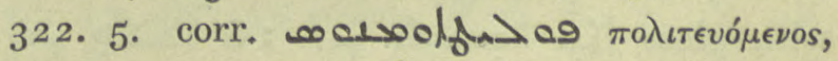
a magistrate; .

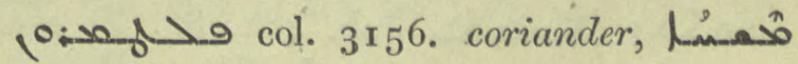

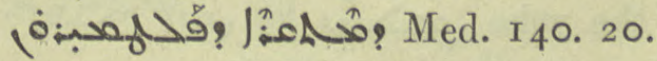

corr. Gest. Alex. I 25.8.

A transliteration of Filio: see l: Charms 6 med.

(2) Med. 266. Io, 425. 20 i.q. (

פcol. 3157. assurance, confirma-

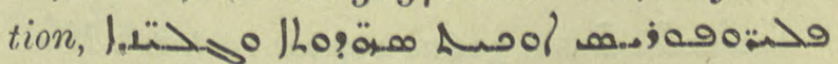

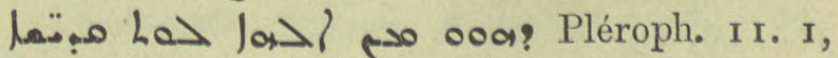

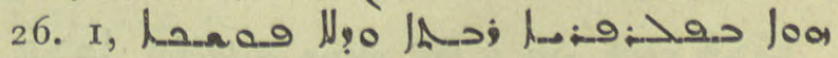
a great and undoubted assurance, ib. 45. I2, 52. 7 ; Sev. Ant. Vit. 108. 2. 


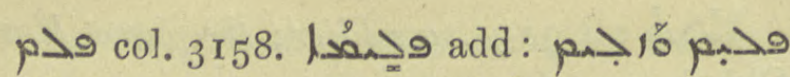
crooked and distorted, Hormizd 2429; ant وحتمصA of the moon, Prognostic. I8 r, $20 v$.

هم>مل :

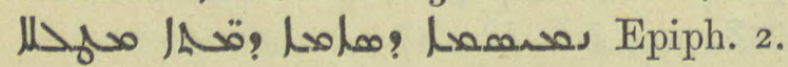

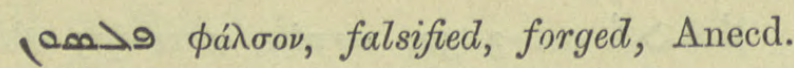
Syr. iii. 160.17 .

ملما هحقهر| aroia l:a? Anecd. Syr. iii. 221. 15. Cf. on 3160 .

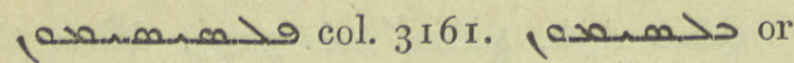

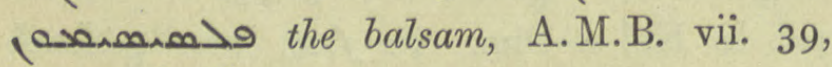

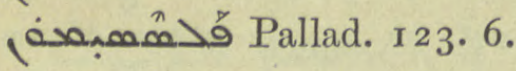

فِ denom. verb from Lat. falsarius, a forger. To forge, falsify, of Ihisll Sev. Lett. 76. 20,

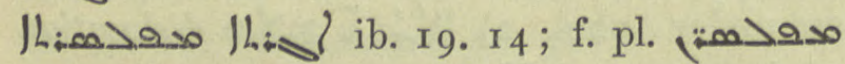
ib. 505 . 10.

| 3 I6r. Lat. pressura, a press,

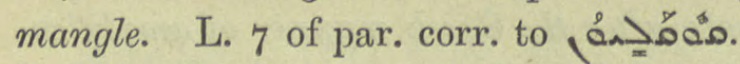

مa palaestra; Sap. Isag. I I. 4.

مب مَفْمد Hormizd 2846

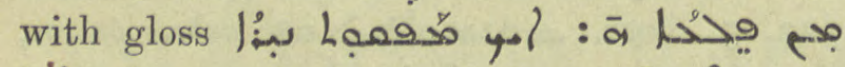

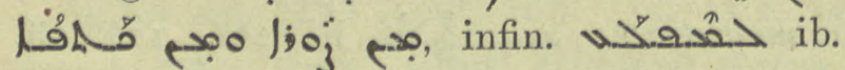

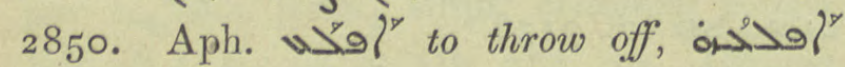

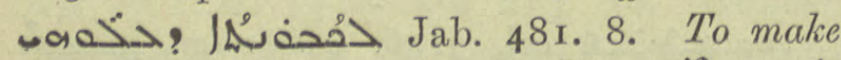

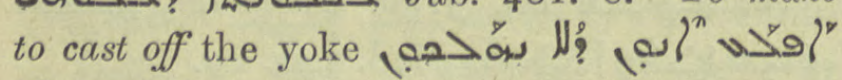

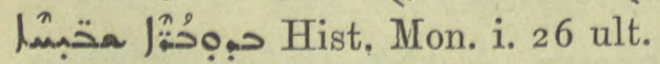

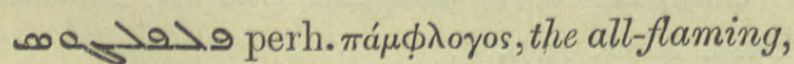
Ephr. Ref. i. 58. 4 .

น

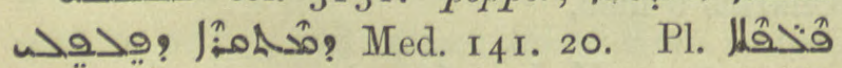

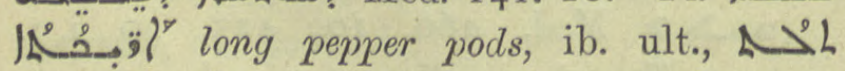

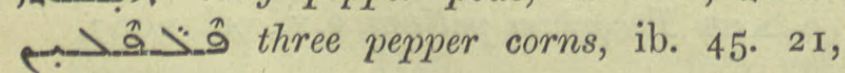
260. 9 .

;0.นด้ 3ㄷ. 16,19 .

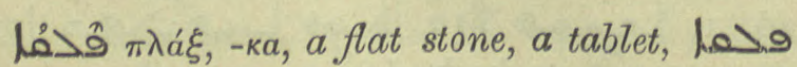
la with gloss Lion Hormizd 3235. The

\section{celagefia}

vowels are probably by confusion with a battle axe.

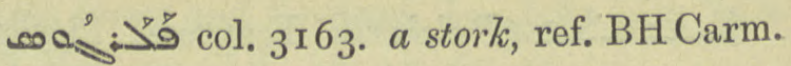
I04. I6.

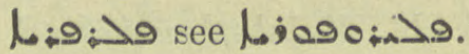

م. 9.900.9 Pamprepios, Sev. Ant. Vit.

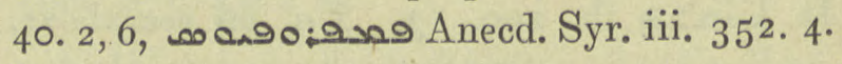
I. q. $00000 ; 901$ q. v.

ف Pan, Gest. Alex. 181. I3.

9 (? |jod) var. (9. Mt. Pan in Outer Ethiopia, Anecd. Syr. iii. 330. I.

Lُف

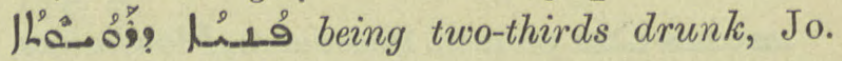
Tell. 73 ult.

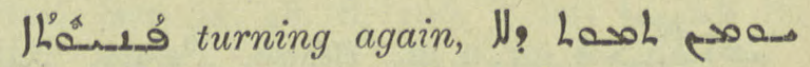
Lowe G. Warda ed. A. Deutsch 27. I7.

حـid y BH. Stories 70. $37 \mathrm{I}$.

فำ 53. $19,26$.



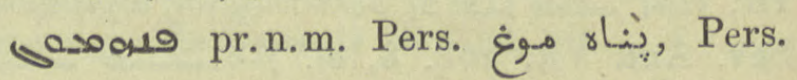
Mart. 70, nn. $620,62 \mathrm{I}=$ A.M.B. ii. 649 .

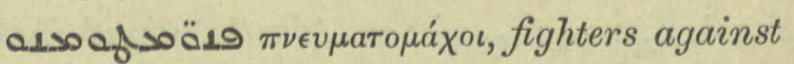
the Holy Spirit, viz. followers of the heresy

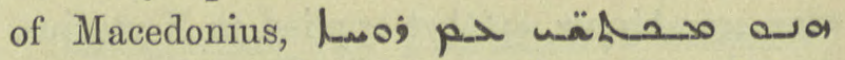
.

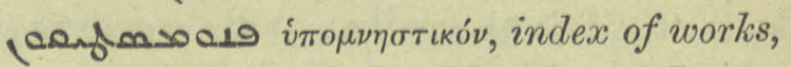
R. Duval on Labourt's De Timotheo I, Journ. As. 1905,179 .

๓ Bp. of Titiopolis in Isauria, Diosc. ed. Nau 66 ult., 67,8 ff., Pléroph. Introd, 8. 5 ; 43 ult., 47. 12, هـ 000\%900.

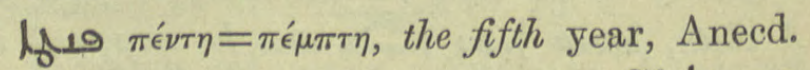
Syr. iii. 200. I7, 225. 4, 249,22. Cf. Msos

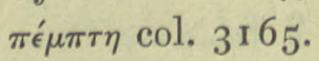

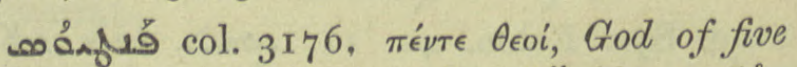
provinces "

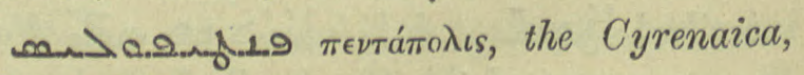
Diosc. ed. Nau ro5. 10. 
هب perh. Ar. فانيد a preparation of sugar and almond = marzipan ? Dozy Suppl. Used with various drugs for palsy, \&c. Med. 137. IO, $242 . \mathrm{I}, 245.2 \mathrm{I}$.

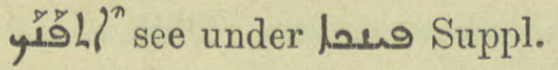

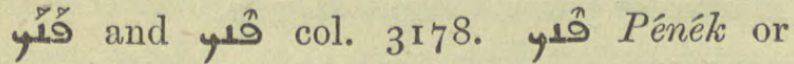
$P$ enk, a village in Beit Zabdé, near the Tigris, Chast. 3 , I 7, Bar Penk. i. n. 3; yî A.M.B. i. $45^{2} .9$, a Persian city? ib. iv. 2 I 5 pen.

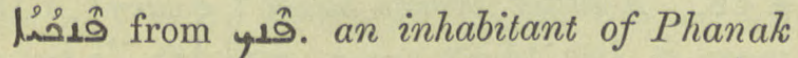

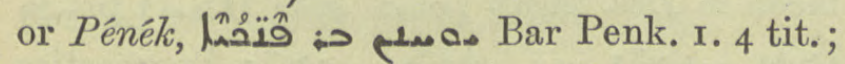

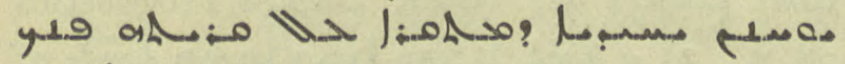
p) فne, Journ. As. 1897, I69. 6.

هa Anemosyne, Arist. Apol. 20. Cf. /neosars col. 2 I93.

فت col. 3 I 78 pen. Geop. 48. 22 corr. |

manals cognomen of a certain John, Vit. Mon. I I ult.

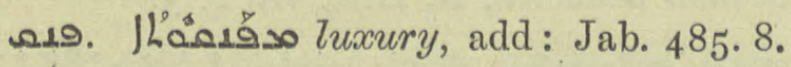

ص. col. 3I8I ult. Delete the line: it is a mistake for eng.

12ก์ col. 3182-3. I4 f. sole of the foot,

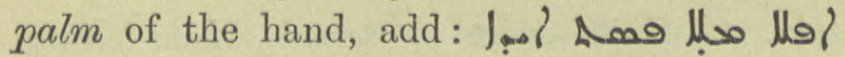
not a handbreadth, Josephus vi. I4. I7. Ll. 18 and 26 a linen or cotton cloth, a rag,

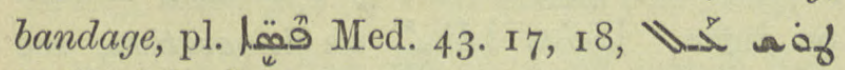


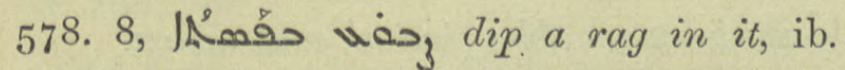
$5^{88}$ pen., 596. 2. A napkin, G. Warda Hippoc. Trad, vii. n. 2o. A head veil, BA. under 出 $\rightarrow$ ia col. $375^{2}$.

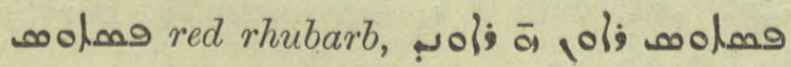

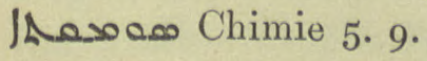

قُمباب place-name, near Seleucia-Ctesiphon, Jab. 262. 10.

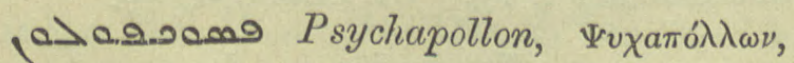
destroyer of souls, nickname given to Horapollo by the plebs of Alexandria, Sev. Ant. Vit. $3^{2}$ pen., Nau R.O.C. iv. $54^{8}$ n. 4.

(anang col. 3185. Plantago psyllium,

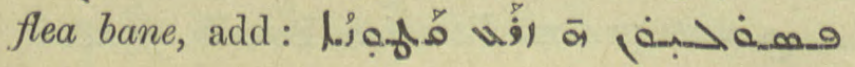

N. Hist. iii. ii. sect. 4, vi. 3. 2, Med. 93. II, I $4,297.2$.

paos P'som, an Egyptian martyr, Sev. Ant. Hymns 6ro. 2.

l Anecd, Syr. iii. 256. 22.

My

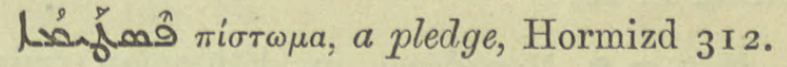

مُ

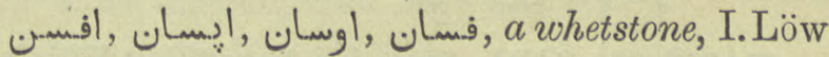
ZDMG. xlv. 699.

פor for see below. Chimie 50. 14, 5 I. I 2.

م ref. Ephr. ed. Lamy iv. 363. $2 / 3$.

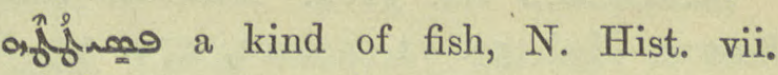
2. 3 infra.

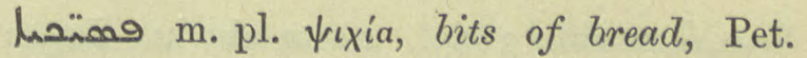
Ib. $84 \cdot 7$.

lheng the stop $\dot{x}$, Epiph. 7. 6 .

unen $\psi(\lambda \dot{\eta}$, a Greek accent, Hebraica iv. 168. Syr., 2.

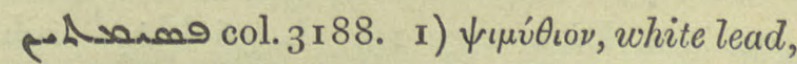
ה Chimie 5. I3, II. 5, I2. IO, 25. 10, for eye lotion, Med. 81. 7, 18, 22, 82. 6 .

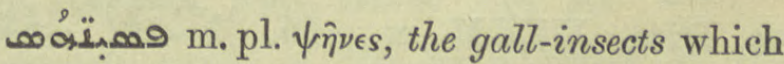
infest the fruit of the wild fig, N. Hist. vii. 3. I infra.

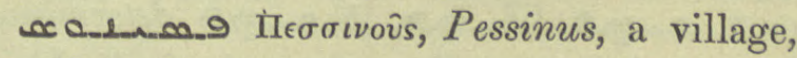
Sev. Lett. $95 \cdot 3$.

o

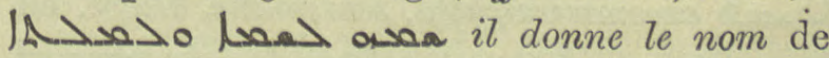
фáoss au nom et au verbe, Probus 9r. 8,

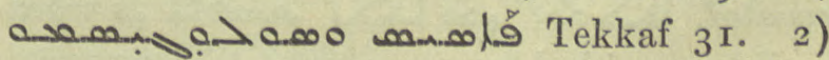
apparition of a star, De Astrolabe 266.

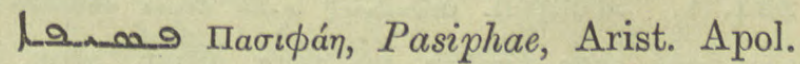
D. II.

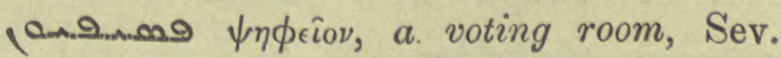
Lett. I 2 I. 6.

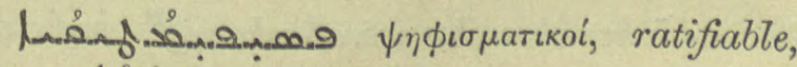

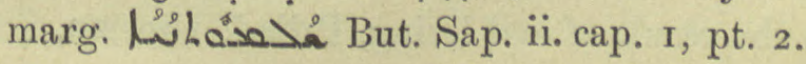


Wh col. 3189. to hew stone, to quarry,

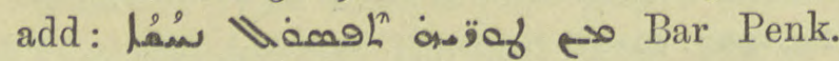

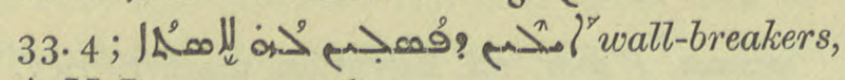
A.M.B. v. 55 I. 16.

هقa Psylli, an African tribe, snakecharmers, WZKM. ix. 97 n. ז.

مفهم for for Natur 8. 10.

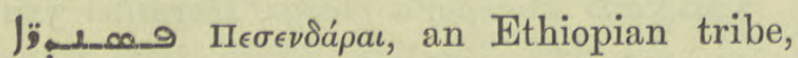
Anecd. Syr. iii. 33०. 5 .

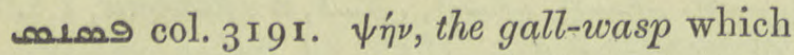
impregnates the female flower of the palm, I. Löw ZDMG. xlv. 700. Cf. רمشتים م.

|Lْعَم BH. Econ. r. 2.

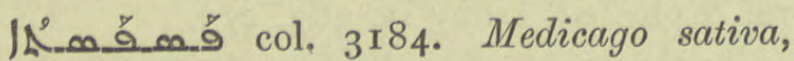
Tucerne, Med. 53. I1, 54. I2, I72. 18, Ar. PflnN. 95. 422.

อ. col. 3193. 2) to decree. Impers. a)

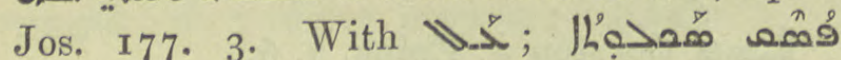

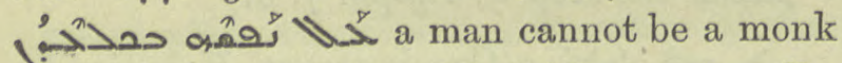
unless he convicts himself of folly continually, Pallad. 746. ro. To tax,

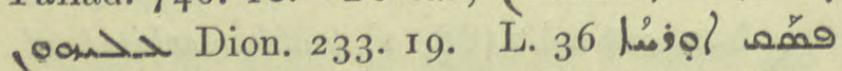

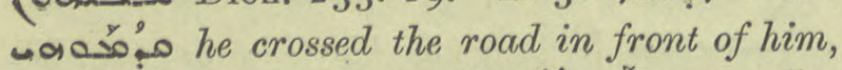

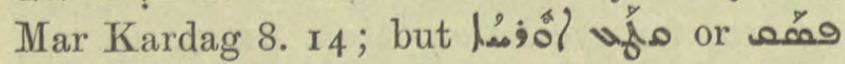
Ar. قطع الطريق to rob on the highway, Nöld.

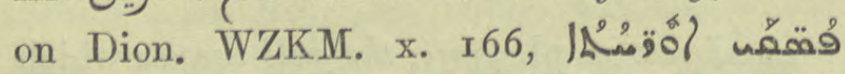
highwaymen, Dion. 198. г3, 199. 6. With $\int_{35}^{0}$ to take usury, Syn. Or. 180. 2 I. Pass.

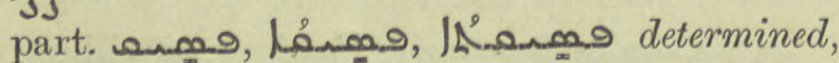
decreed, معin the end of all things

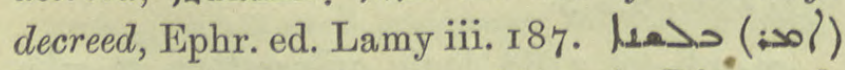
لمeng excommunicated, schismatic, Diosc. ed.

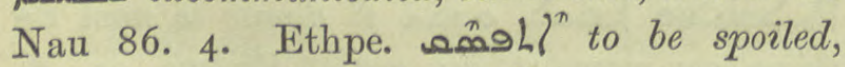

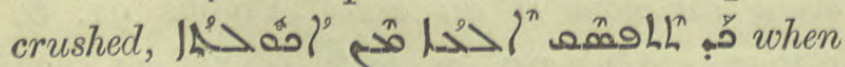
a rib has perished from gangrene, Med. 223 ult.,

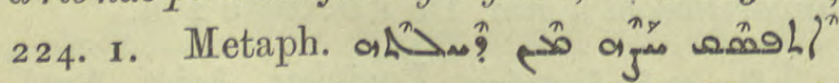
his back was broken by fear, Hist. Mon. i. 2II. 8. Ellipt. with onderstood, wa> > despaired of, Pet. Ib. 4r. 10; 0. ñoh/

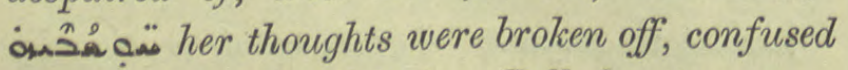
as the result of evil living, Pallad. 169. 20.

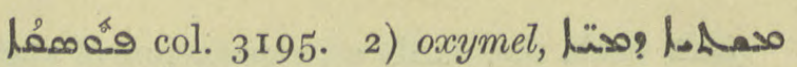
fمosa of Chron. Min. 46 pen. = Jo. Tell., Life of.

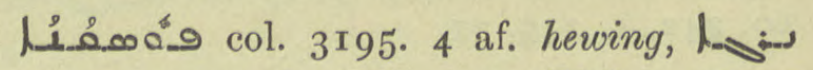
1. $A$ term of years, Coupes ii. II 2.

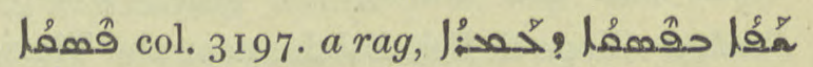
strain through a woollen tammy, Med. 587. I 5 ,

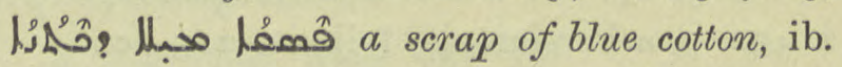

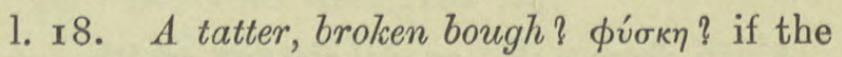

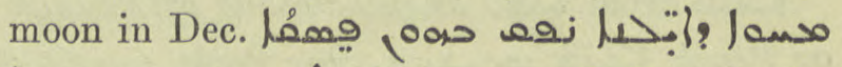

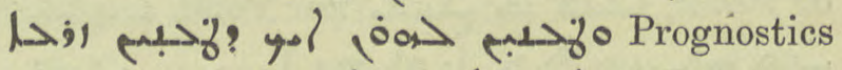

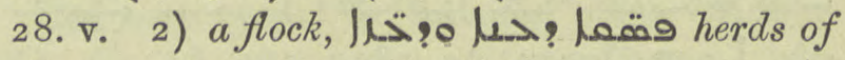
sheep and goats, Anecd. Syr. ii. 3II. IO;

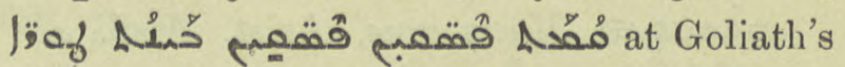
threats, the flock of Israel stood in huddled groups on the mountains, Jac. Sar. Hom. ii. 36. II, 3) the abdomen of an insect, حسدحا إن cicadas make a shrill noise and tune with the membrane at their abdomen, N. Hist. vii. 2. 3 .

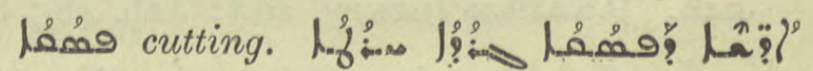

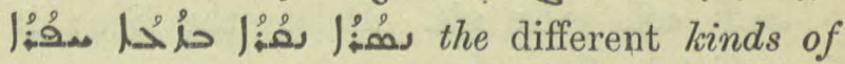
cutting are scraping, gashing, sawing, boring, piercing, and digging, N. Hist. iii. cap. i.

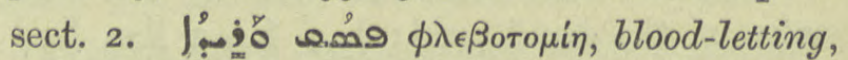

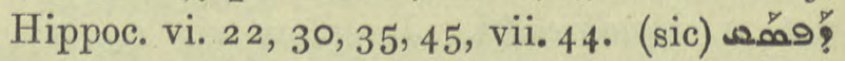
LSh os at the conclusion of a Motwa, Takhsa 90 infra. Koh Suppl. Gram. Sl.

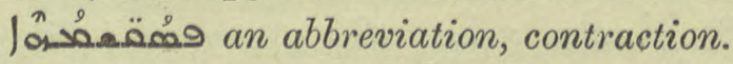

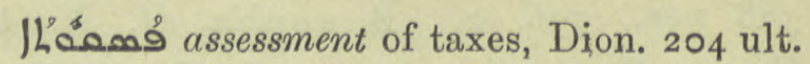

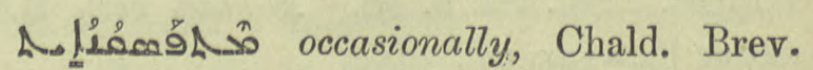
نे in Lit. 87. I3.

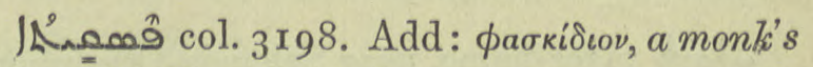
habit, Vit. Dan. R.O.C. v. $39^{8}$ n. 2 ; the text

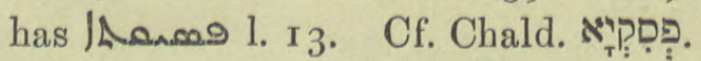

mrenمeng Paschasius, a deacon, Anecd. Syr. iii. $217 \cdot 23$.

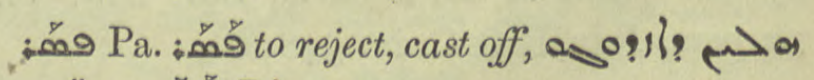

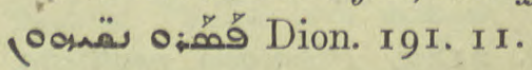

(a.s pr.n.m. Pesarion, Pet. Ib. 35. 8. 


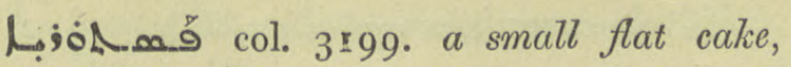

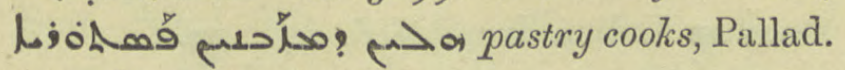
I 2 I. 6 .

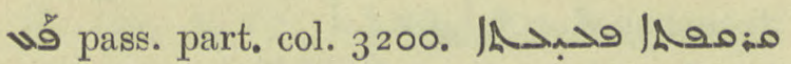

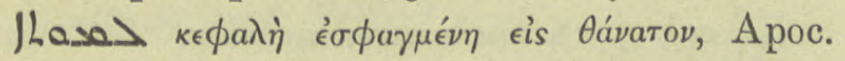
xiii. 3 Gwynn, JLard Bagst.

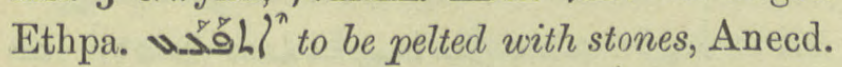
Syr. iii. 205,23 ; مخدم

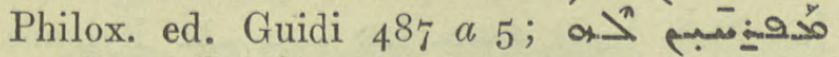

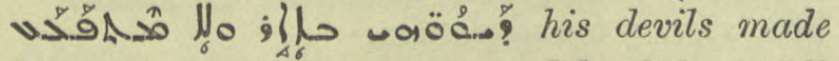
him able to fly in the air and he did not fall and get bruised, Hormizd 2790, 2795.

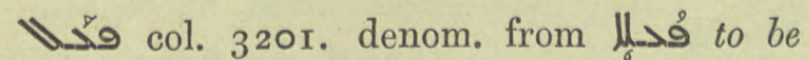
a labourer, to labour, WZKM. xvii. 199, xxii. 199. K.'s quotation from S. Ephr. حهف:

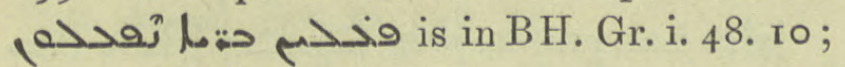
مـar company laboured fasting, Ephr. ed. Lamy iv. 387.17 ;

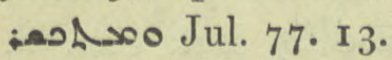

لـ and often.

:-59 col. 3202 . to open the mouth wide,

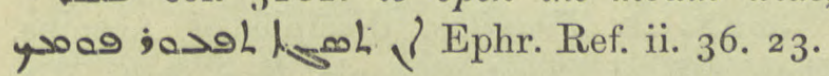

فاغ Xanthoxylon Avicennae, Ibn Bait éd. Leclerc no. I650 (from Duval's nn.

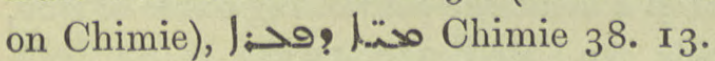

1.

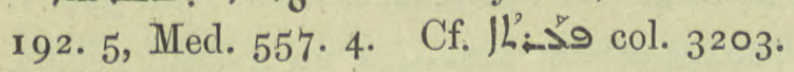

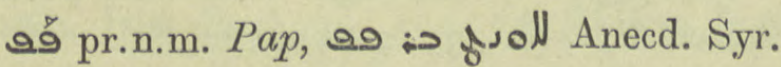
iii. 206.19.

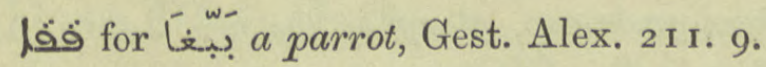

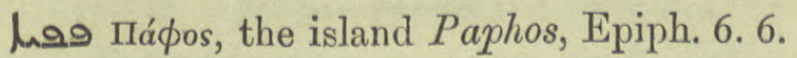

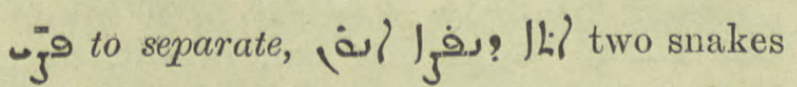
fought and another came to separate them, (uncertain) Assop's Fables, Brit. Mus. Or. 2084. 49 v, Hochfeld Fabeln 29 pen.

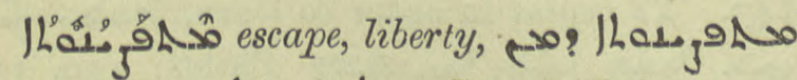
Can. J. Tell. 29. I3.

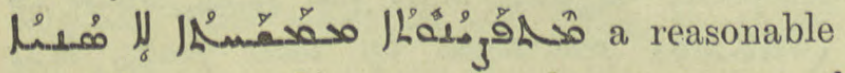
man does not object to a moderate amount of liberty, But. Sap. Eth. iv. 5.

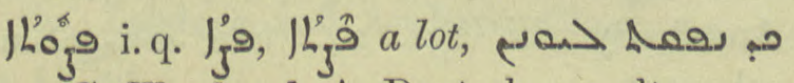
$\operatorname{llog}_{\mathrm{J}} \mathrm{G}$ G. Warda ed. A. Deutsch. $\boldsymbol{I}_{7}$ ult.

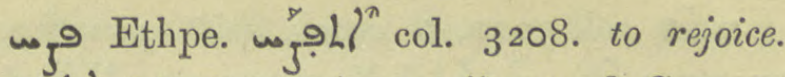
مu

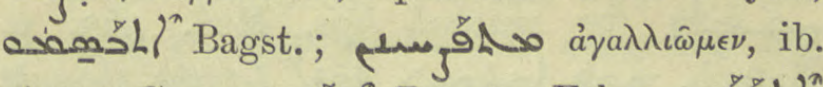

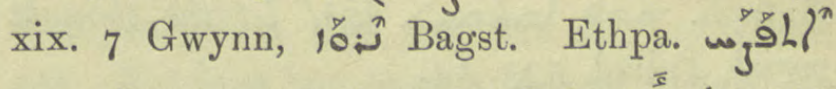
to suffer from dysury, see BA. حلمداً

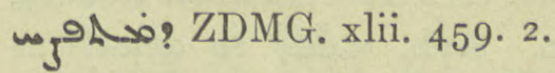

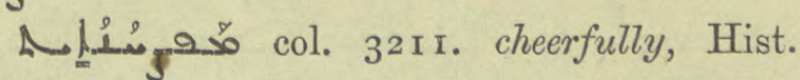
Mon. i. I 24.20.

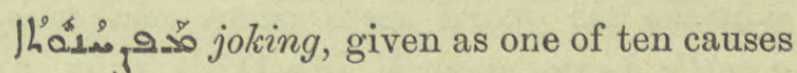
of exasperation, But. Sap. Eth. iv. 5 .

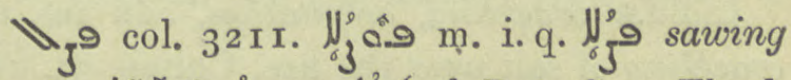

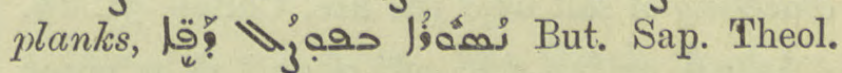
3. 2.

\. ol Arab. تفعيل particularization,

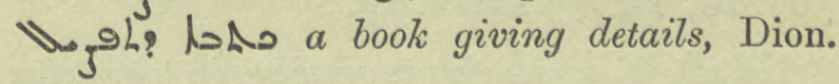
232. 9 .

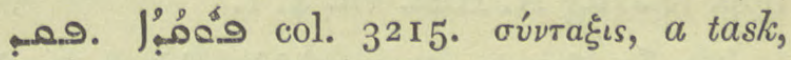
Hex. Ex. v. 14, 18 \&c. Lag.

همبْرا مهحتا : Add. A command. Imperial edicts, Sev. Ant. Vit. 9r. 10, Jab. I50. 5. Authority, he restored to the

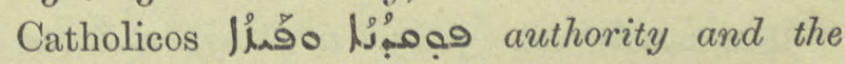
symbol of it, ib. 43. 9; orders, requisitions,

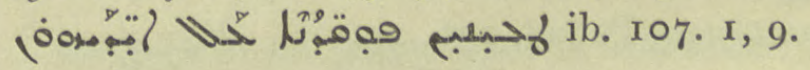

A 1 Q given orally of God's laws, opp.

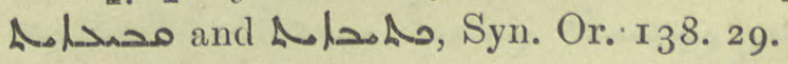

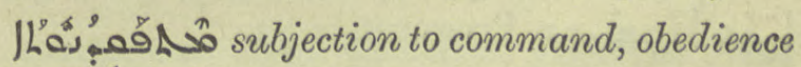

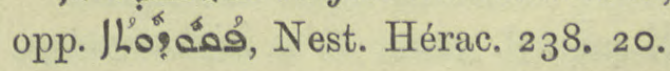

?a-a name of the grandfather of the B.V.M., Schatzh. 2 I2. 10, 226. 8.

Juم Pqidha, first bishop of Adiabene, M.Z. 2. 2.

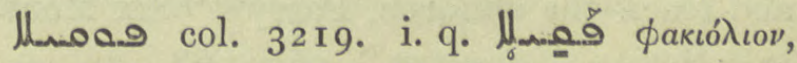
a kerchief, Anecd. Syr. iii. 324. 9.

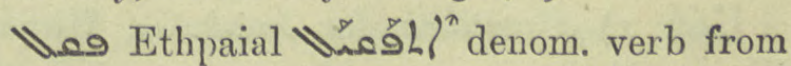
lla. Lexx. under \âดL?.

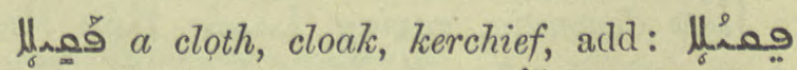

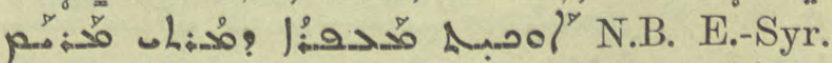

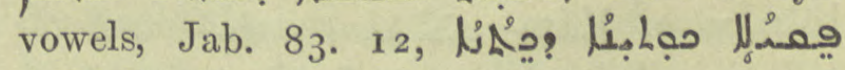
A.M. B. vii. 19.

Mng Lat. faecula, lees, sediment of vinegar or wine, وملا ib. 329 n. 3 . 
טم้ col. 3220. to crack, split open, frog's

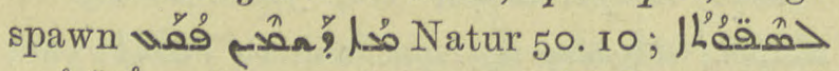

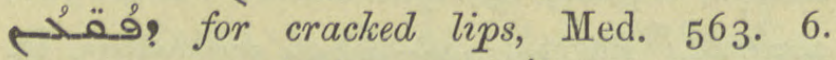

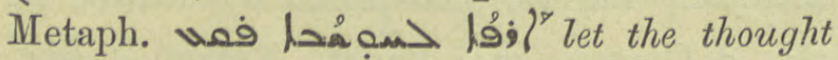
perish, Pallad. 533. 14.

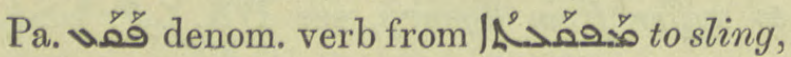

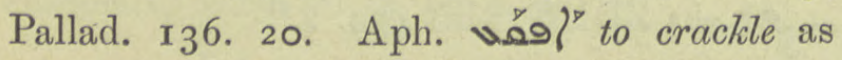

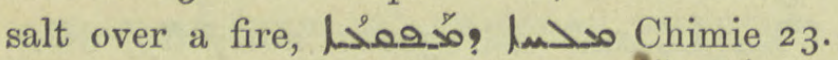

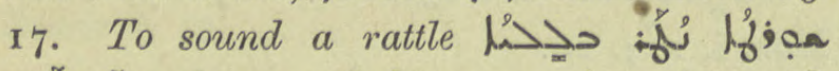
vâa

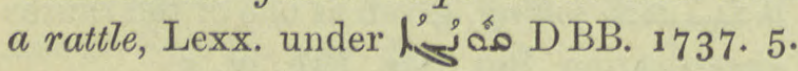

1حْمْơ crackling of salt over the fire, Chimie 3 I. I 9 .

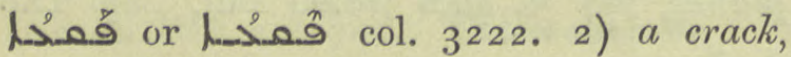

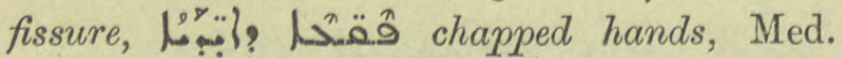

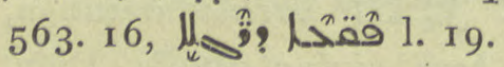

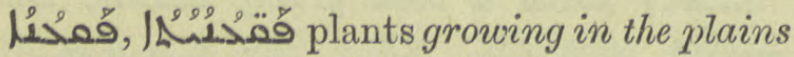
opp. / Lُ

$1 \Lambda^{2} \rightarrow$ و flat country, a field, add: a garden

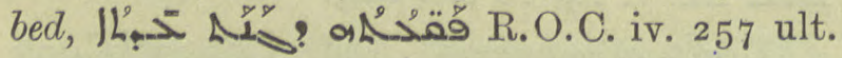

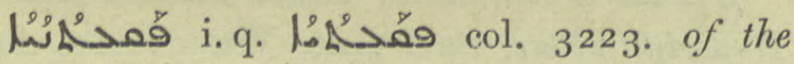

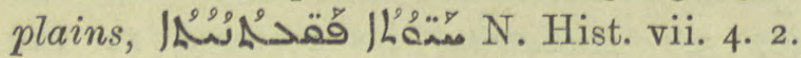

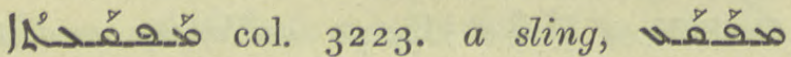

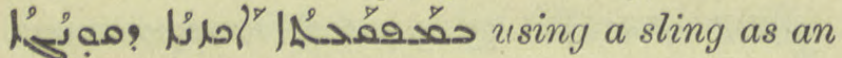
huntsman, Pallad. I36. I9.

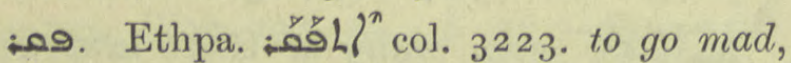

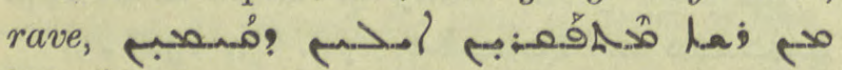

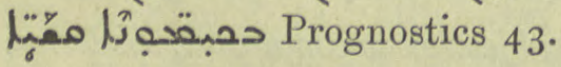

فِ Hunt. lii. under معدما col. $3^{6} 5^{8 .}$ 2) one of the seven eccles. orders of the Yezidis, i.q. J o a sacristan, Mt. Singar 25 ult., they perform menial duties, also they dance and teach the children, Parry's "Six Months in a Syrian monastery", 362,368 .

J iii. 25, Med. 113. 14, 18, 19, 21, pl. l. 12, I 4 4. 2. 2) demented, Med. го. 8.

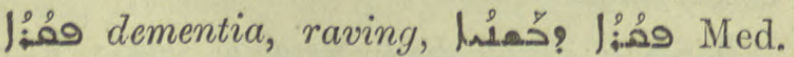

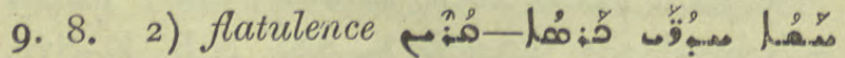

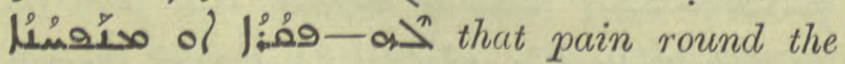
belly is called inflation or flatulence, ib. I9. 6.

ـص col. 3225. Ethpal. 3 . a snared bird مئilox. 293. 10.

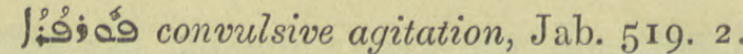
:9้ an Indian drug, Med. I 4 I. 23.

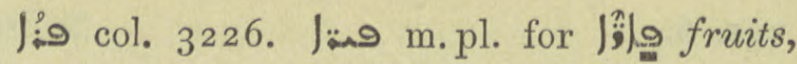
Josephus vi. 7.4 af.

J'ف col. 3228. an uneven number. Correct the first word quoted from B.O. to 1 คُ.

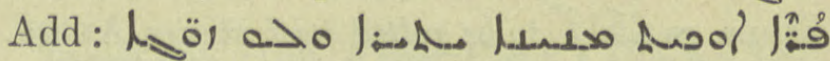
B. Sal. in Lit. 24. 14, 22 ; Rylands MS. xliv. $7 b$ and passim.

J10:9 col. 3229. f. a hide, pelt. add: JLo:

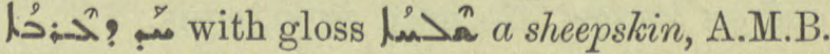
vii. 79 .

Jhợمhọ:9 f. peltry, dealing in hides, BH. Econ. I. 2.

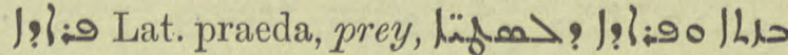
Journ. As. I 901,242 n. $5=$ Test. Dn̄i 8.

|soawol:s Lat. pronomen, a pronoun,

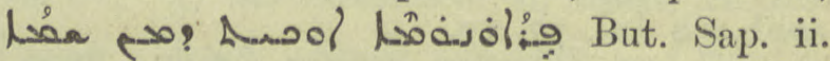
ch. i. pt. ii.

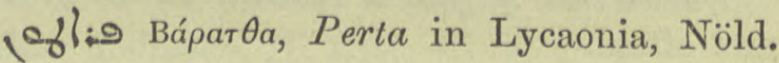
F. S. $473 \cdot 98$.

os 0 Fravitas, disciple of Acacius, Patriarch of Constantinople, Sev. Ant. Vit. IO2. I.

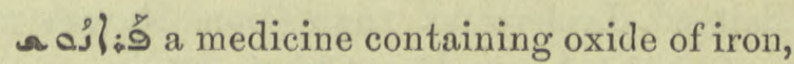
for fistula, \&c., Med. 3 1 1 . I 7 .

مص:9 Pers. pr.n, m. ZDMG. xliii. 397. I.

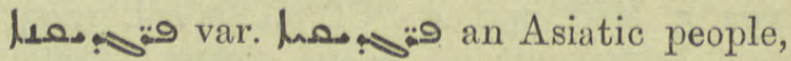
Anecd. Syr. iii. 334. 2 from Ptol.

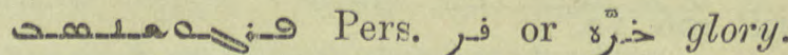
Farn-gushnasp, glory of the fire, Journ. As. I 895, 348. Cf. amana ang.

Wh: col. 3234. to charge, prescribe. add: . 9 Anool Wy:9 Bar Sal. in Lit. 62 ult.

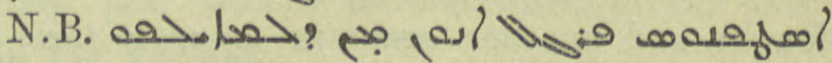
Stephen the slave prevented Bassus and Lily from learning Magian doctrine, Mar Bassus

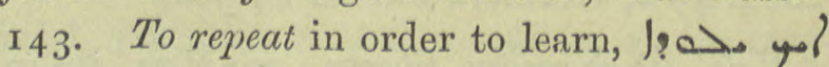

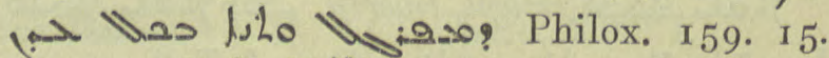
Ethparal "W. (oostor restrained by their fears, Mar Bassus 225 .

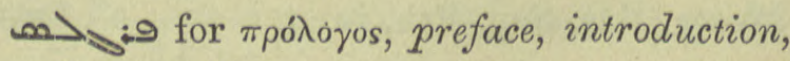
Anecd. Syr. iii. 232. 24. See $\rho 0 y 0>0: 0$ col. 3246 . 


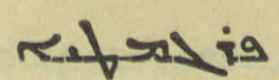

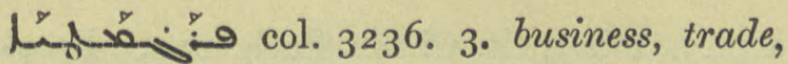

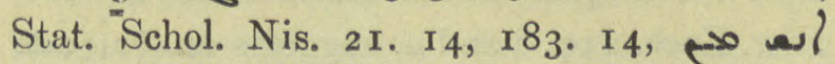
ma Canon v. ib. $184.3,17$; h. A stoutly to work, Tekkaf 119.

Hafos:9 col. $3^{2} 3^{6}$. a merchant, buyer, so correct لlodses 9 Ephr. ed. Lamy iv. 237 pen.

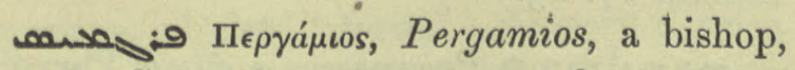
Anecd. Syr. iii. I69. 8; a hyparch of Alexandria, ib. 177. 27, 178. 20.

פִ 1 an Indian herb, Med. 324. I. See under

Hes var. Ho name of a country, Ptol. Anecd. Syr. iii. 328 . I I.

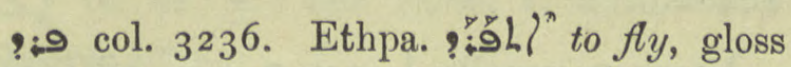

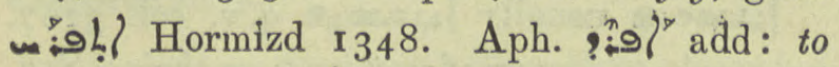
put to flight, صد Dion. 44. 22 ; هلم BH.

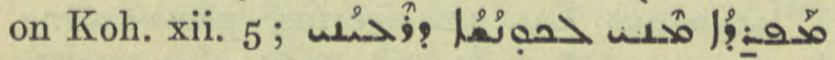
it scatters my recollectedness, Is. Nin. B.

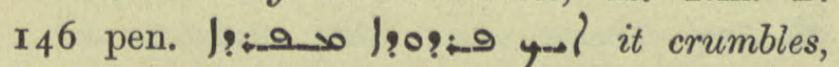
Chimie 299 n. I. This should probably be the Palel ?ी?

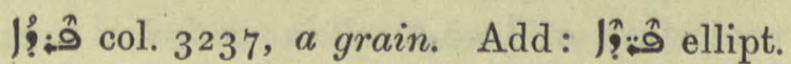

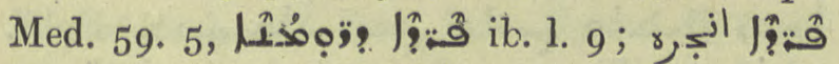
the nettle, ib. 608. 4. لl ! ! / grains of sand,

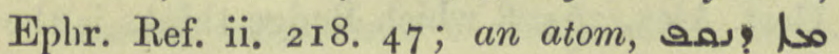

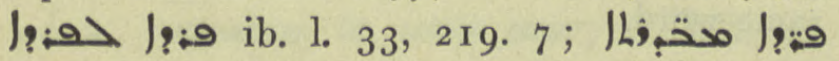

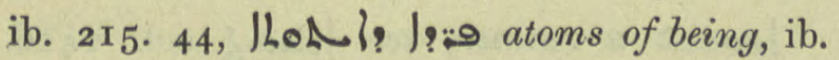
$217.44,218.7,18.29$.

J!०:: m. a particle, Chimie 299 n. $\mathbf{r}$.

9 9 m. pl. scattered fragments, particles, Ephr. Ref. ii. 67. 4I or /!०9:0 n. ib.

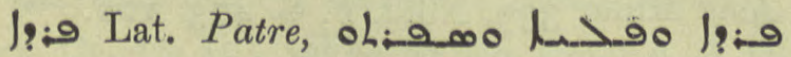

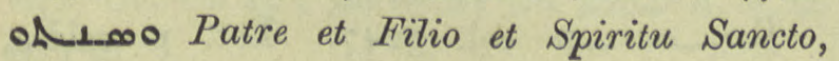
Charms 6 med.

.

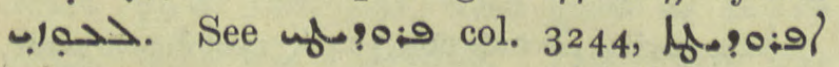
col. $35^{\circ}$.

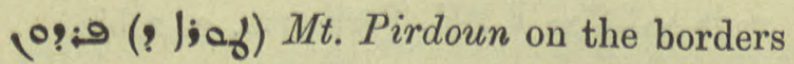
of the Zabdaye and Arabaye, Chast. I5. 3, 32. 19 ult.

2716 alasoia

คو tetter, dry scab, Dion. 2 I6. 2, 2 I 7. 3 .

L?:-9 col. 3239. BHChr. 144. $7=$ ed.

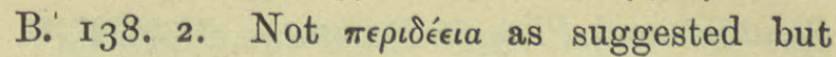
I: 9 fear, Z.A. xvii. 90, BB. col. $3^{2} 3^{8}$. 2nd

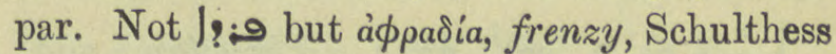
ib. 266 .

منص : seontodon pardelianches, Leopard's bane, a plant, Brit. Mus. Cod. Add. 25. 878. 10 $a$.

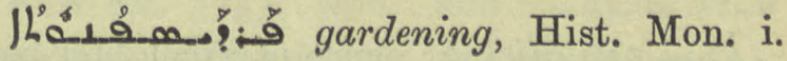
271. 9.

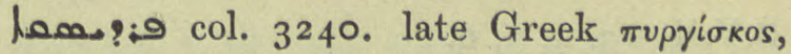
a case, closet, cupboard, S. Fraenk. Z.A. ix. 8, Du Cange i 276 . I.q. I. 3072 .

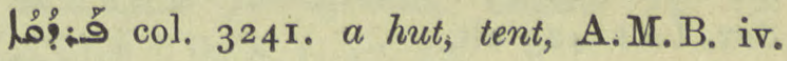
256. 18.

a:9 $\Phi \rho a ́, \Phi \rho a ́ \delta a$, Pharah, capital of one of the three provinces of Segestan, Syn. Or. 89. I ; $90: 0$ ib. 88. 26 .

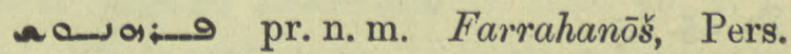

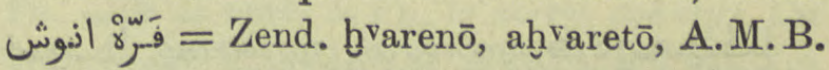
ii. $662 . \mathrm{I} \mathrm{I}=$ Pers. Mart. 72 n. 642 .

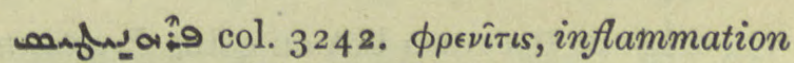
of the brain, Hippoc. iii. 29, vii. Io. See enof 9 col. $3^{269}$.

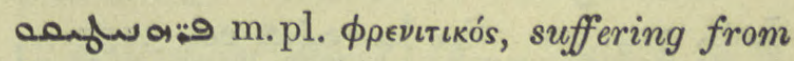
inflammation of the brain, Hippoc. iv. 72 .

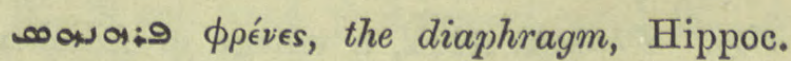
iv. 17 , vi. 18 .

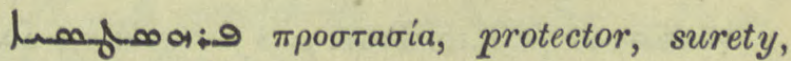
L.

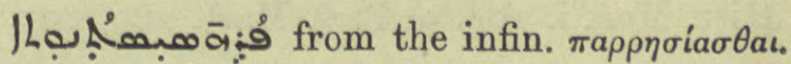
boldness of speech, confidence, Hist. Mon. i. 287. 5 .

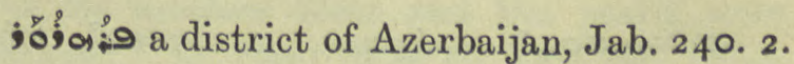

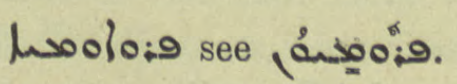

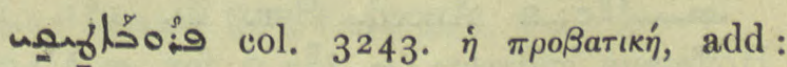
A A Charch of the Sheepgate and Pool, Jo. v. 2, Pléreph. 35. 3 .

(ف) N. Hist. vii. 6.5 .

N $\mathrm{n}$ 
L

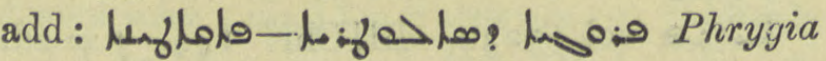
Salutaris and Phrygia Pacatiana, Nöld. F. S. 474.

Ly of-g col. 3243. Phrygian, add:

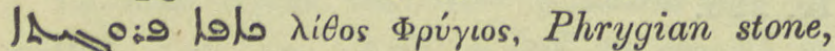
Chimie 8. I 9 .

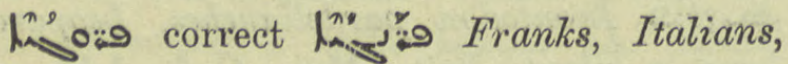

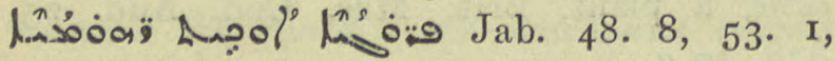
I 27 ult.

Any $0 \div 9$ chicken; name of a daughter born to Joachim and Anna, later than Mary, Hist. B.V.M. I $5 \cdot 4,5,6$.

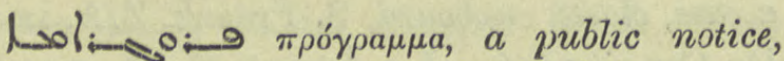

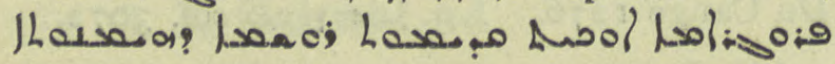
Sev. Lett. 423 ult.

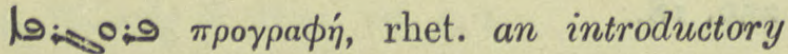
sentence, Nöld. F. S. 484.

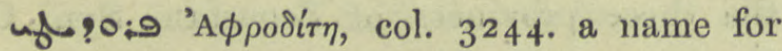
brass, Chimie $4.9 ; 90: 9$ abbrev. of the same, ib. $45 \cdot 15$.

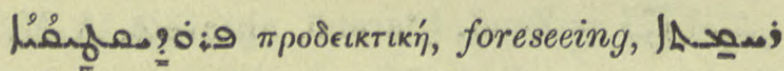

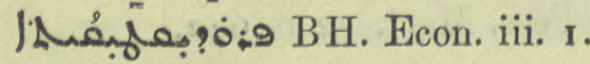

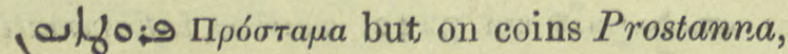
a city of Pisidia, Nöld. F. S. 473 . ro7.

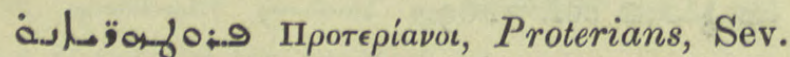
Lett. 314. 20. See le;of:9 col. 3259 .

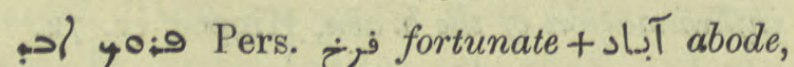
city. Pharolk Abad, a village of Adiabene, Chast. I8. I 2.

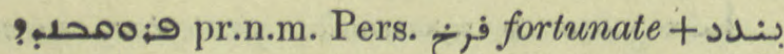
origin, stock. Farrukhbindad or Wandad, Hist. Mon. i. I09. 9, Pers. Mart. 289, 297.

فِ pr.n.m. Pers. Pharolkhdad, Jab. 253 . I.

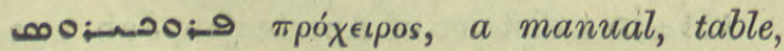
R.O.C. xv. 240.

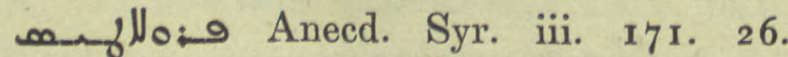
Hoffmann suggests and for and on

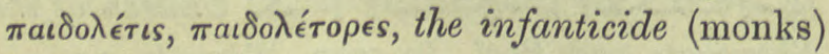
but Kugener, R.O.C. v. $46_{3}$ infra, rejects this as it would entail six further corrections. Hamilton and Brooks regard the word as a pr.n. mis-spelt, Chron. Zach. II I. Brooks, Hist. Zach. 2 I 7.8 and n. suggests enofol:0; or ons of

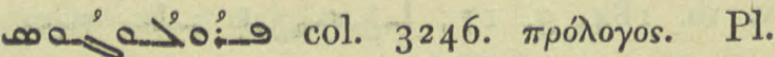
م Theol. 4. 6, 7 .

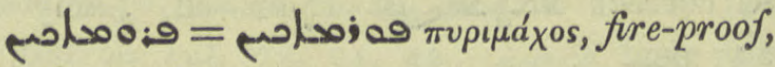
Chimie 17.23.

م Nöld. F. S. 474. I 29.

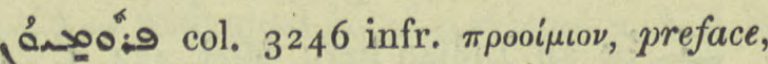
exordium, introduction, Awol ponolo:0 Ihis ? enfin h hoolo:9 ib. 357. 2; Nöld. F. S. 484. 2.

J:100:9 usually J: was:0 q.v. col. 3267 .

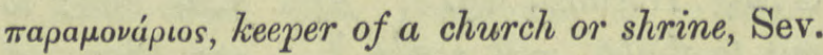
Ant. Vit. 63. I 2, R.O.C. iv. 344.

J:م-0:9 Lat. primicerius, proto-notary,

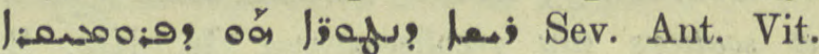
$25 \cdot 5$.

Ju:9 prob. a weapon of Northern barbarians, Jos.Styl.62. I 3 where Wright accepts Bensley's conjecture of /

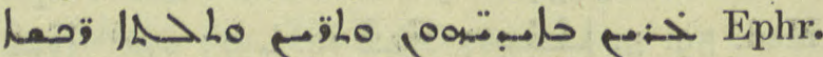

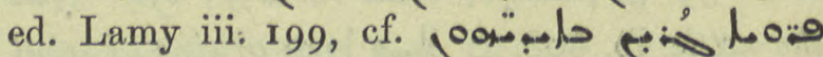
Gest. Alex. 264. 8 which Budge thinks is the same word.

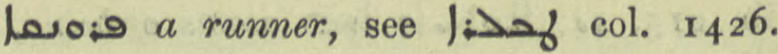
8 of par.

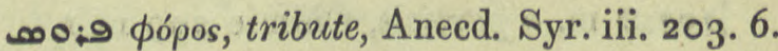

a.) Lycaonia, perh. Passala = Thebasa, Ramsay's Asia Minor 339, Nöld. F. S. 473. 102.

Lo, $-\infty 0$ - o col. 3247. Lat. praesidia, fortresses, fortified palaces, Anecd. Syr. ii. 329. I. Delete ll. 3 and 4 of par.

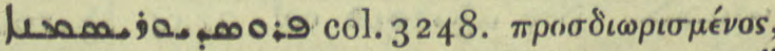
definite. Add fem. إل $=1$ BH. Periherm. 2. 2 bis.

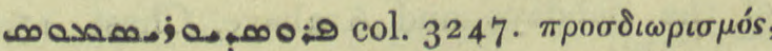
a corollary, BH. Periherm. 2. 2, Tekkaf. 33 . 
0000:9 Prusa, in Bithynia, now Broussa,

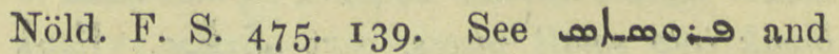
1000:9 col. 324.

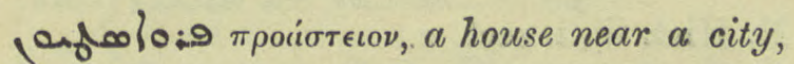
country house, Pet. Ib. I5. I 5, (a-d-0000

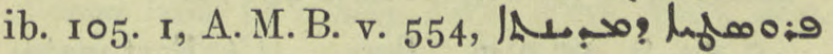
suburb of Alexandria, ib. 558. 14.

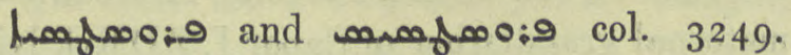

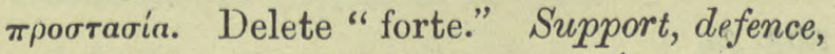

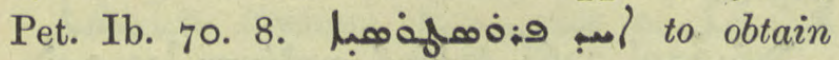
support, countenance, Jab. 279. 13, Syn. Or. 7.3 antep. but to protect, support, ib. $5^{6} 5$. I5.

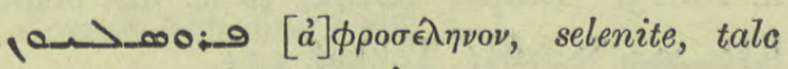


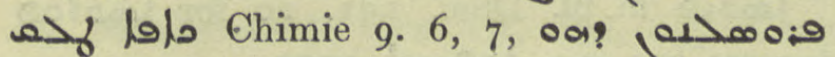
f ib. 86. 3. See Diose. v. clviii under

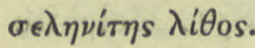

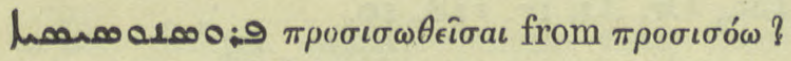
R. Duval equal, Chimie roo. 23.

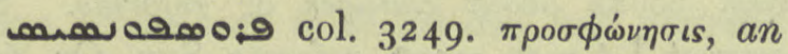
address, add: Sev, Ant. Vit. $3^{22} .4$.

1 addressed, dedicated, Sev. Ant. Vit. 105. I r.

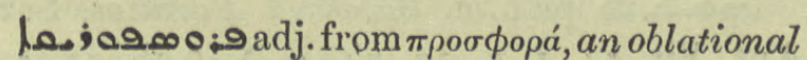

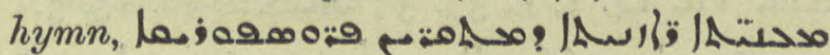

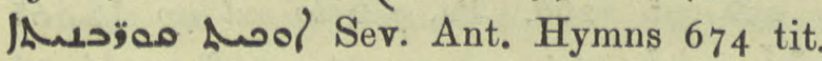
68r tit.

|

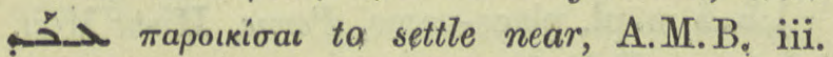
298 ult.

|jo:9 a thin paste, "une bouillie", Chimie iii. $3^{2} \cdot 2,3,52.2$.

jo:- an Indian race, Anecd. Syr. iii. 335. 3 .

| Q Pehlevi fravartak, a letter, writing,

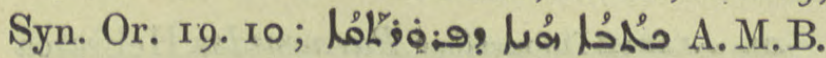
ii. $x_{3} 6.7$.

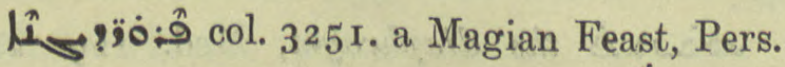
Mart. $79=$ Jab. $35^{\mathrm{I}}$ with gloss of lå:

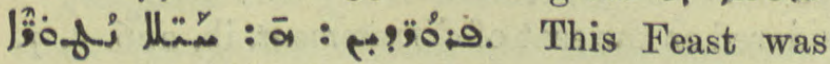
connected with the vernal equinox.

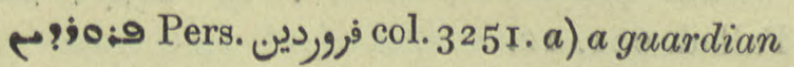
angel, b) name of the ist Persian month = March. $\operatorname{lå}_{0} \cos _{0}$ Pehlevi fravashi, guardian angels. See preceding paragraphs.

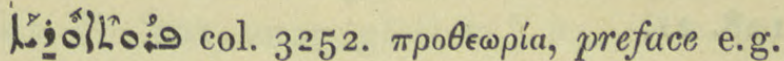
to books of Aristotle, in But. Sap. to Econ., Eth., Periherm \&c., Poet. Arist. I I 4.3 .

10\% $0_{0}^{\circ}: 9$ col. $3^{2} 5^{2}$. par. 4. a latrine. Corr.

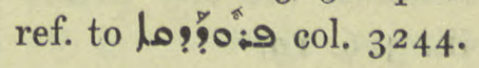

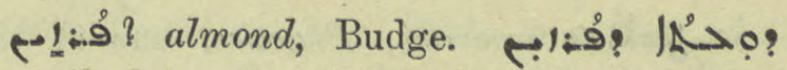
prescribed for pleurisy, Med. 576. 7 .

لإ: ? - $\rightarrow 0$ ib. 52, 18.

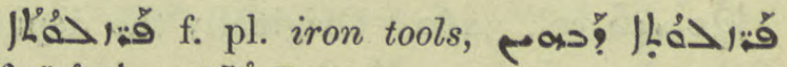

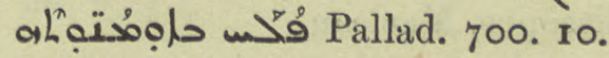

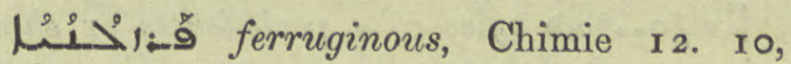

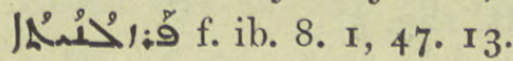

先 584. II.

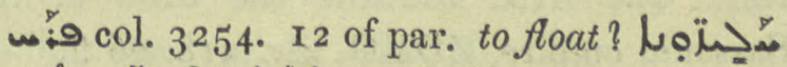
مَّمبه تُمُسُ Pa. w. w to make to fly, to carry, convey; the

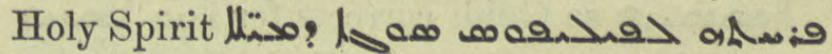
Ephr. ed. Lamy iii. 235 on Acts viii. 39 .

Khócos col. 3255. f. flight, add ref.

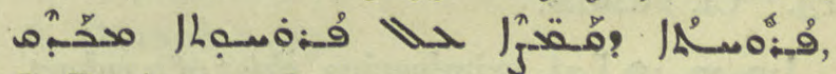
G. Busâmé $37 b$ Io, I I.

فํ. flight, power of flying, Natur 27.8 .

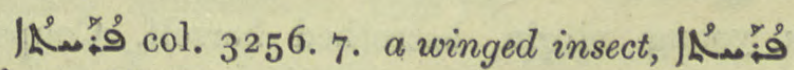
ملهبئ2. Hist. vii. I. I.

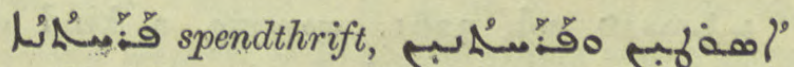
But. Sap. Eth. 3. 3.

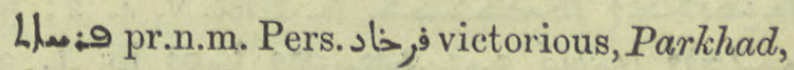
R.O.C. iv. 167 .

ف: فำ I:? the necklace broke and the pearls were scattered, Jac. Sar. Hom. iii. I57. 10. Aph.

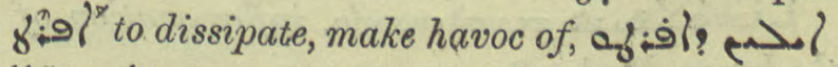

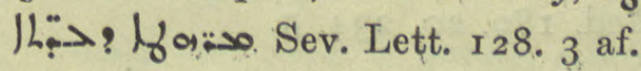

1 8 ช́: a section, half the pinion of a bird, -acfof. פै Med. 593. 7, 9.

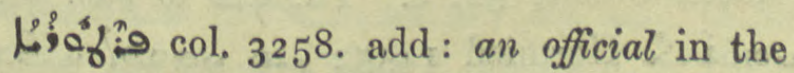
Praetor's office, L.E.S. I 59. 3 .

$\mathrm{N} \cap 2$ 


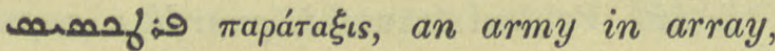
مoog" Anecd. Syr. iii. I 28.2 I .

Jodof:9 m.pl. guardian saints, add ref. A.M.B. vi. 33 ult., i.q. J̈g ib. 48 pen.

قصن:م Perrhe, Anecd. Syr. iii. 316. 9.

lafol: $\pi \rho a \kappa \tau i k \eta$, Practice, one of the books of Mar Aba, Syn. Or. 69. 22. See land 3298 .

(a) Syr. iii. 335. 19.

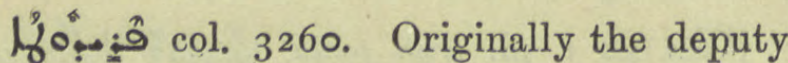
of the chorepiscopus, Maclean.

Kheforias office of a periadeutes or chorepiscopus, Sev. Lett. I23. 5 .

فو. pr. n.m. Gest. Alex: 207. 8, 208. 6, 8. Nöld. identifies him with a notorious robber chief who lived towards the close of the Sassanid empire, ZDMG. xlv. 31 $8.26 \mathrm{ff}$.

ف the city of Paris, Jab. 68. 6.

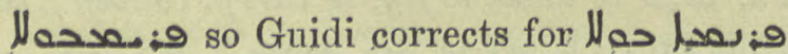
$\pi a \rho \epsilon \beta \beta_{0} \lambda \dot{\eta}$, camps, barracks, Zach. Brooks 197 , I I, Bull. Arch. Rom. xii. 222 ; Mich, Syr. 3 I I5. Cf. لla:or.

I Q Lat. primicerius, chief of a cohort, Pléroph, 68. I.

\$n:- P Parisag, to whom the Earth, a young virgin, betrothed herself, Coupes ii. II 2 .

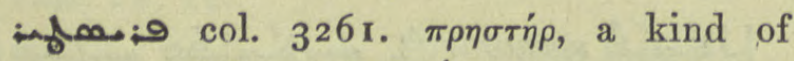

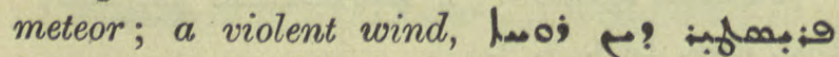
N. Hist. v. 4. 2; pl.

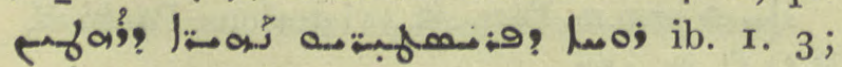
Q ib.

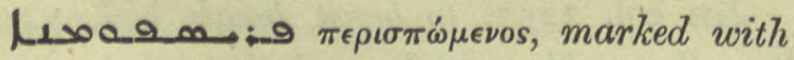
a circumflex, Epiph. 7. 6.

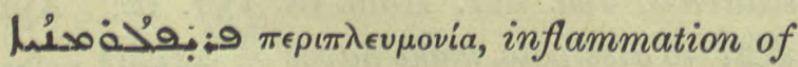
the lungs, Med. 189. 20, 234. 5 .

y:- col. $3^{262}$. to rub, crumble, imper. 40:9 Chimie 33. 20. Ethpe. y:92/? to be

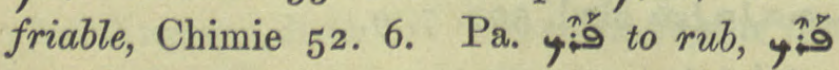

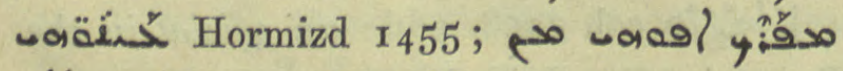
ahtas Nero rubbed his face in confusion,
Mart. Luc. ed. Nau r64 ult. Palel فै to

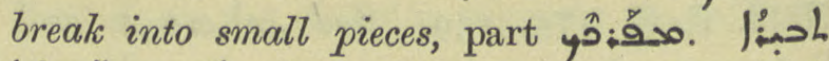

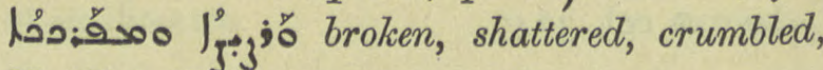
N. Hist. iii. i. sect. 2.

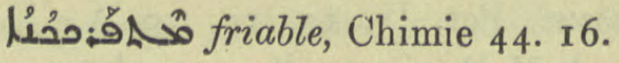

10.-9 pr.n.m. Pers. فرخ Fortunate, Farrukhan, son of the Parthian Artaban and maternal grandfather of Sapor I, ZDMG. lvii. 563.2 .

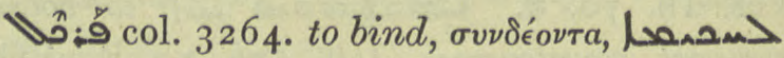
breg. Carm.

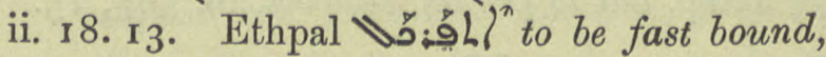

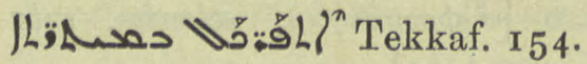

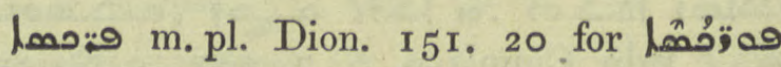
towers: see 0 a jos col. 3072 .

ف 2 (Eu)praxia, Jan. I2, Syr. Melchite Lit,, Or. Xt. iv. I 18 . 10.

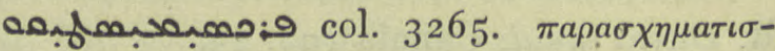
тikoi books about changes or reforms. So correct h Afonsamogeforms, Syn. Or. 69 .

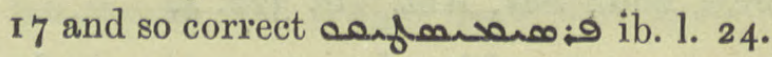

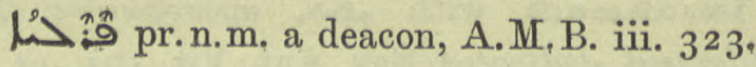

مaح:9 pr.n.m. Mápaìos? Paralios, Sev.

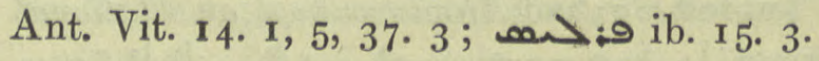

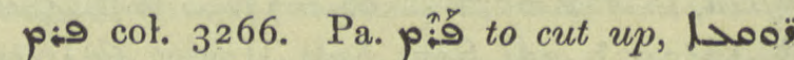
p. o she cut up rags to stuff cusbions, Anecd. Syr. ii. 199. 18.

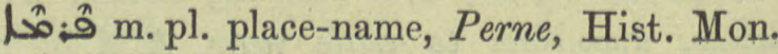
i. lxiv. n., Pallad. 472,603 .

لNox:9 $\pi \alpha \rho \epsilon \mu \beta о \lambda \dot{\eta}$, the Camp, in Nicopolis, now Ramleh, Amélineau 31, A.M.B. 555. 6.

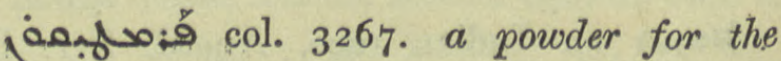
eyes, ref. Med. 84. 5, 10.

.

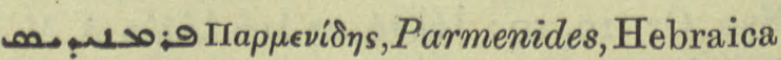
iii. 25 o. 10.

صفّ the diaphragm, Med. 129. 22,

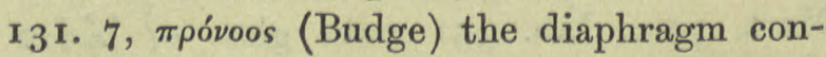
sidered as the seat of discernment, ib. 226. 5, 7 .

|خا فِ Pallad. 62x. 12.

فำ pr.n.m. Parniton, Pallad. 57. 12 $=$ Á.M.B. v. $75 \cdot 14$. 


\section{rodesia}

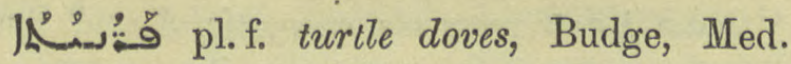
I37. 21 .

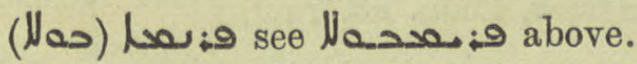

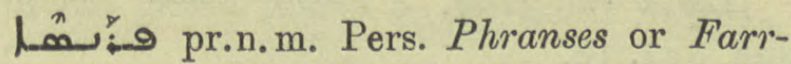
Narses from 1, a holy Fire. Pers. Mart. 289.

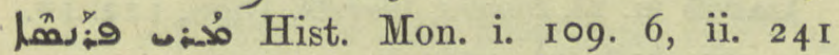
n. $3=$ B.O. iii. i. 479 col. 3272 . Badger, Nestorians ii. 307, M.Z. 203.16 .

هـ Jab. 68. 5 .

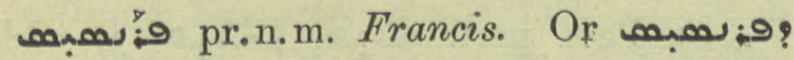
of France, Jab. 69. 3 and $\mathrm{n}$.

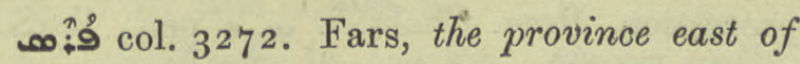
the Persian Gulf, Syn. Or. 679, 257 n. 2 ;

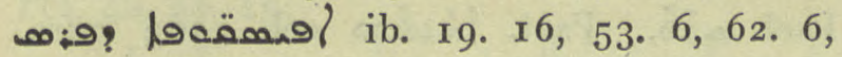

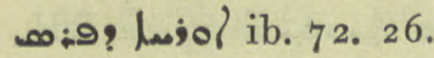

פ. Ethpa. . 10 upon allowance; to be divided into portions,

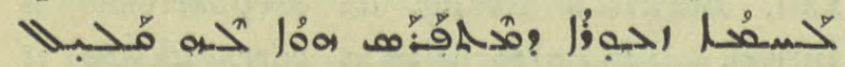
a little bread of which he allowed himself small portions, Hist. Mon. i. I26. 20. Probably Kal-w-Dim. 4. 6 should be transferred hither from Ethpe. col. 3276 .

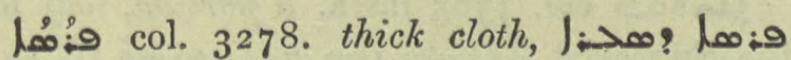
hair cloth, Patr. Or. v. 5. 725. I, 747 pen. A rug, carpet, 202. 6.

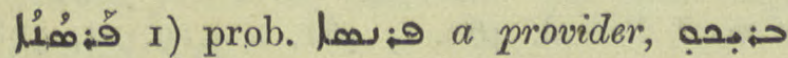

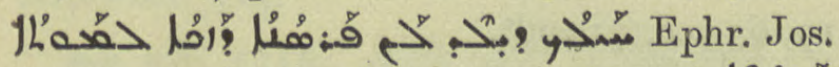
313. 12. 2) a hoof, see col. 3280. 3) فُ for $\hat{\Lambda}_{3}^{\hat{n}}: \hat{\theta}$ grape stones, Med. $569 \cdot 5$.

ه: 1 the peach tree, Chimie 48. 17.

رم:0 Perseus, Arist. Apol. بـ 18.

 add: A.M.B. iii. 233, Bar Penk. 118. 5, Jul. 66. I 7 .

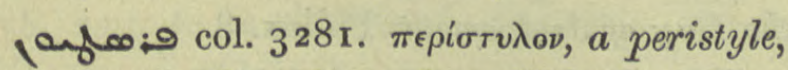
inner court with a colonnade, a pillared corridor, م حand Zach. Br.ii. 195 . 12, Guidi Bull. Arch. Rom. xii. 22 I and n.

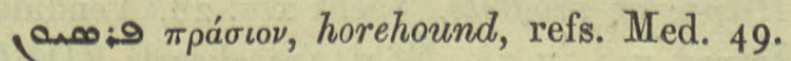
5, 50. 5, 209. I7, 21 and often. >ia

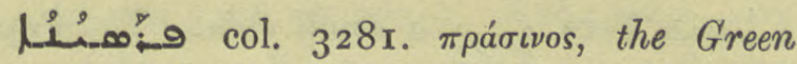
Faction at Constantinople, add: فم: Sev. Ant. Vit. IOI, marg. gloss to l. 6 .

ف: $\mathrm{m}$ m.pl. an Indian people, Anecd. Syr. iii. $335 \cdot 3$.

ف: 0 col. 3282. selenite, talc, Chimie 10. 5. Usually (a) 10 q.v.

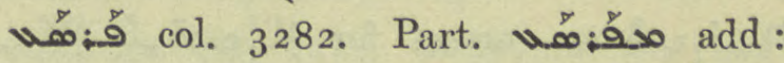
few, scattered, Hormizd 2793 with gloss

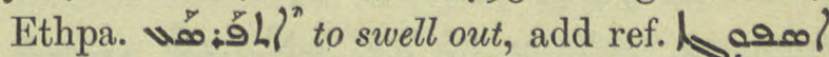

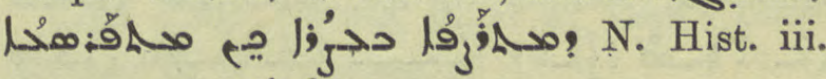
cap. 1 , sect. 2 infra.

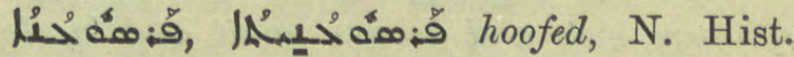
vii. 1 . 4 .

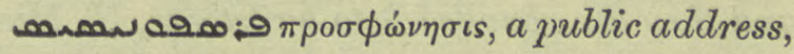
Anecd. Syr. iii. 1 22. 19, 189. 16.

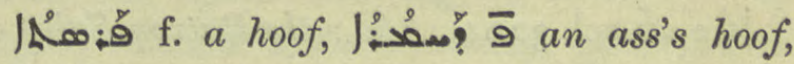
Med. I72. I5, 558. I3.

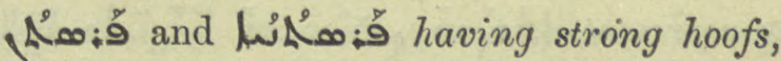
Ahikar هـ 15, N. Hist. vii. I. 4.

1. Shah, ref. Jesus-Sabran 522.4 af., 533. I7 transl. 493 n. 2.

U:

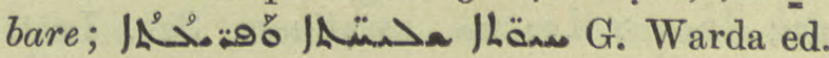
A. Deutseh 7. I3.

Lْó:- col. 3286. gram. relating to,

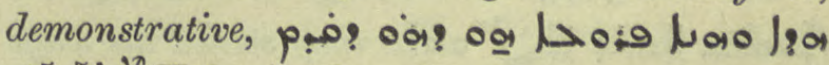

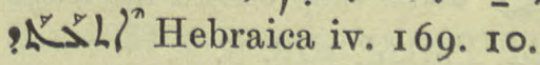

 But. Sap. Periherm. ii. 5, iii. 2. 2) fissured, localities which have fissured and porous depths have especially violent earthquakes, N. Hist. iv. I I. 3 .

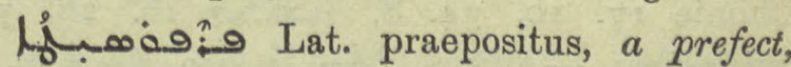
governor, A.M.B. v. $53^{6}$ with gloss : 宛 :

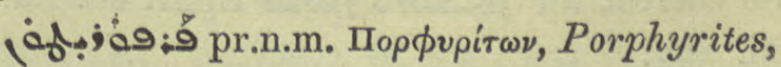
Pallad. 2 5 5. $\mathrm{I}_{3}$,

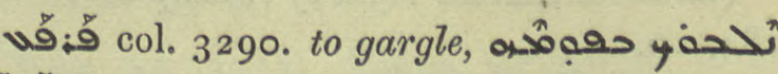

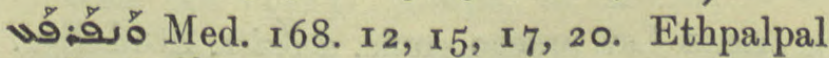

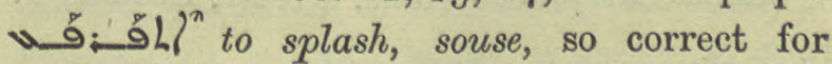

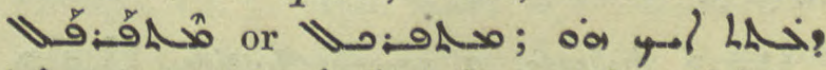

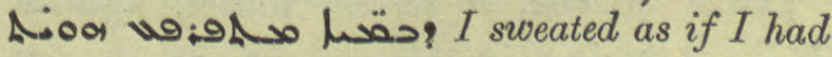
been doused with water, Mar Marc. I64. I 4. 


\section{Rigis}

1:9:9 Pers. 8ر, a small coin. The Pope

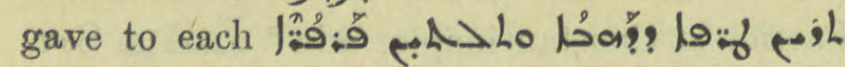
مol two sheets of gold and thirty silver coins, Jab. 80. 9 .

0:9:9 purple, Chimie 53. 17 .

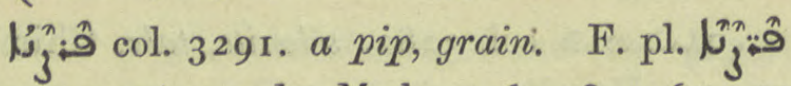
pomegranate seeds, Med. 556. 18, $5^{6} 5 \cdot 4$;

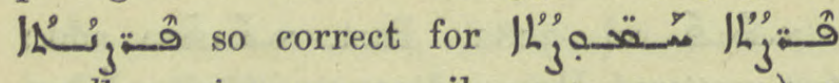
small unripe grapes, ib. 137. 22. 2) a

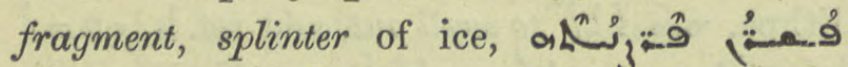

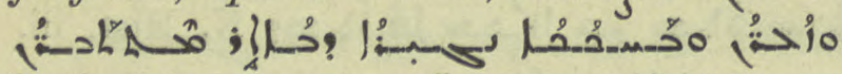

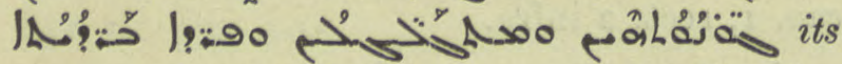
splinters melt and become small and by slow friction of the air their angles are broken off and they are rounded into hailstones, N. Hist. v. $x$. 3 .

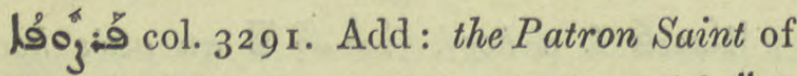
a Church, Maclean. A representative, with

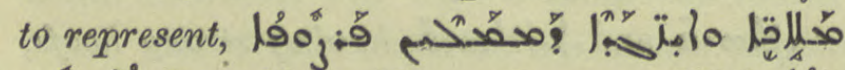

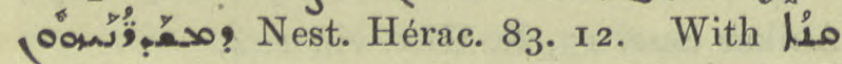
to be apparent, likely I:

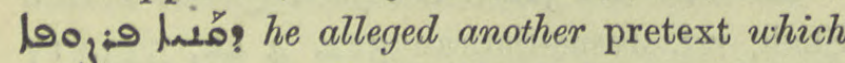
seemed to have some likelihood, Coupes ii. 132 .

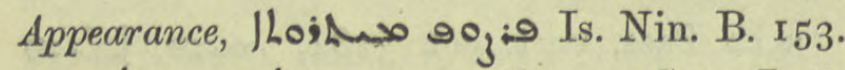
I $7 \mathrm{lgoj}_{\mathrm{9}}$ Loinan seemliness, Sev. Lett. 104. 18 .

م:9 col. 3293. With l'

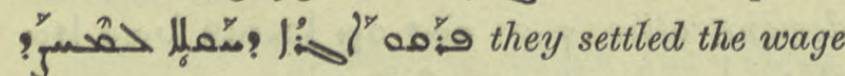
for reaping a field, Pallad. 548. 8.

9ه وما IA 0 \% Dion. 109. 20, 23, 103. I1, 184. I5, written 100:0 ib. 135. 5 .

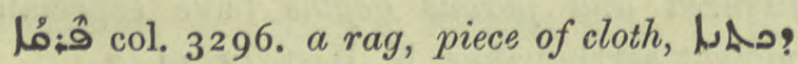
Chimie 17. 10, 34. 8, J:خ' ib, 28. 7; a bandage

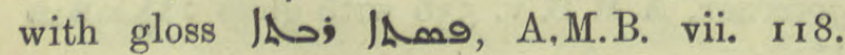
So correct for IA iii. 297 .

ف ه: for right of redemption, Dion. I84. 10.

פ Lat. praeco, -onis, a crier, Stud. Syr. ii. $3 \cdot 5$.

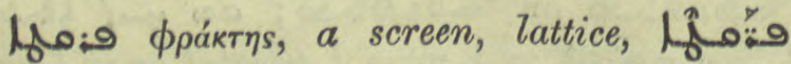
 ioaria

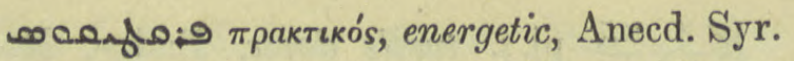
iii. $183.17,192.7$, 13 ib. 135.8.

ه: Africa, Dion. 88. I4.

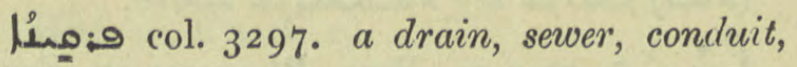
Jab. 254 . 18.

פ. 2 m. pl. towers, turrets, Dion. I22. I6. See 0 a jos col. 3072 .

L.:- Fr. frères, col. 3299. Correct as

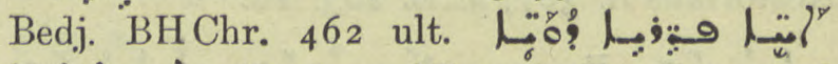

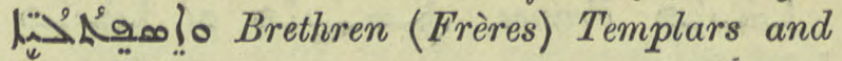
Ḧospitallers.

a 9 Pass. part. a special vestments worn for the procession, Brev. Chald. i. حص pan., then Bحمع BaA Brev. Ant. i. Cal. $62 b$ I5.

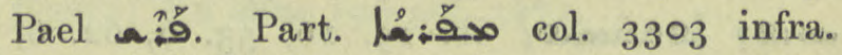

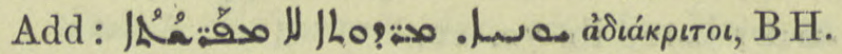
on Prov. 2 r. r. la:ax? paryof the separate Gospels opp. the Diatessaron or Harmony col. 3304 supra, add: Lewis's Four Gospels xxii, Burkitt's Evang. da Mepharreshe ii

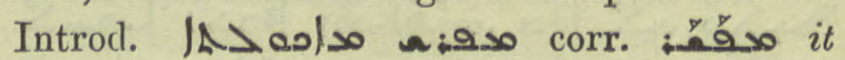
aids digestion, $\mathrm{BH}$. de $\mathrm{Pl}$. $5_{52}$.

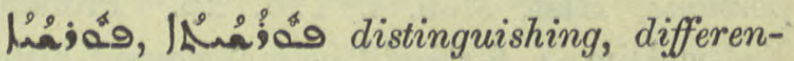

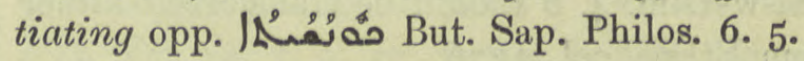

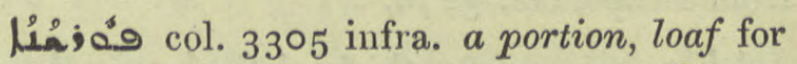

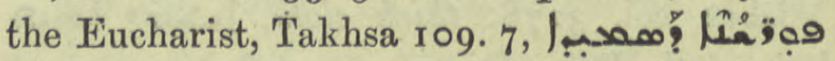
ib. 166. 22, 167. 2, 13, 21. Pl. oblations,

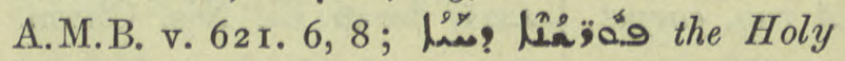
Eucharist, ib. 625. I7. A portion $=$ food, a meal, Pallad. 460. I, 461. 2, 519. 14, 568. x $9,635.6,7$ I3 ult.

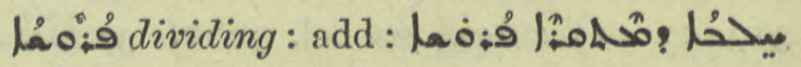
the diaphragm, Med. 223. 6, 225.4 .

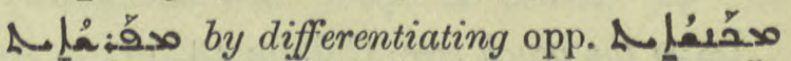
by inference, both adverbs used with But. Sap. Philos. 6. 5 .

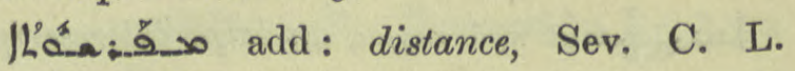
333. 11 .

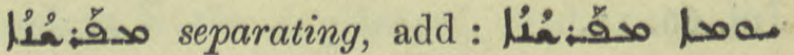
the Day of Judgement, Philox. 8.

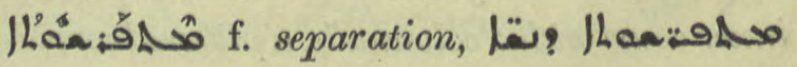
مه Dion. 79. 19.

فำ Zend Frashôkara, the Forwarder, Helper of progress, Yasht xiv. 28, Vendidad Introd. v. 8 , iv. xi. Nöld. Frašokereti, ZDMG. 
xliii. 676 note on Ebed J. Cumont suggests frashagar, the Renewer, "le rénovateur," Cosmog. Manichéenne i. 8, n. 2, A.M. B. ii.

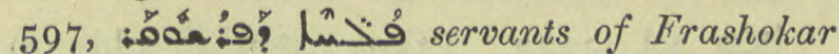
$=$ flatterers and haters, Ebed J. Card. Ior. I3. For further refs. and explanations see soea?.

L.9 col. 33 I I. to split, burst open, vipers at

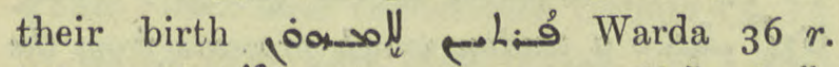

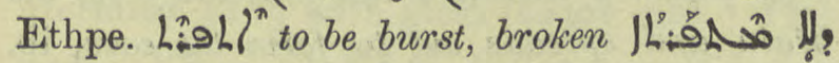
lest the leech break, Med. 214. 2 I.

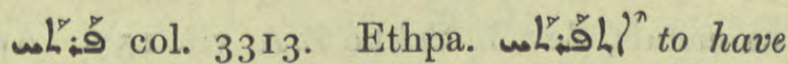

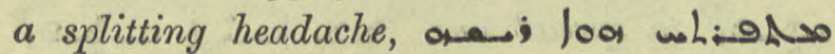
Dion. 126. 13. Metaph. to be crushed with grief, Hist. Mon. i. 206. 5 .

定 fibrous, fibrous stalks, Pet. Ib. 28. 19.

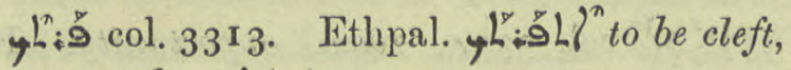

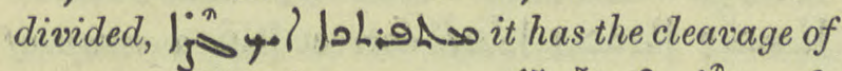

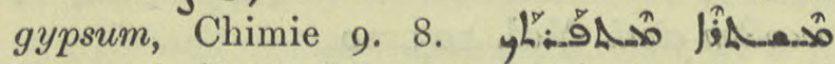

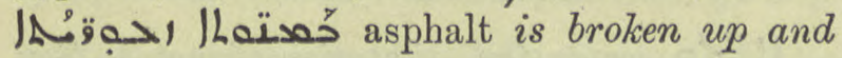
divided into small pieces, Natur 47. I0.

bliso so correct for ba!? loliges grains of gold, Chimie 49. ro see 25 I med. bohis fragments, ib. 233 n. 2.

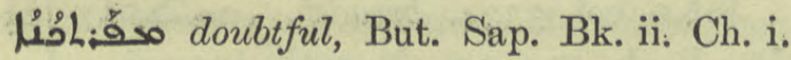
Pt. 2.

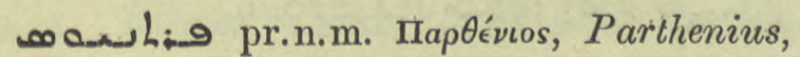
Jo. Tell. 82. 8 .

1uliog col. $33^{1} 5: 7$ of par. Plantago

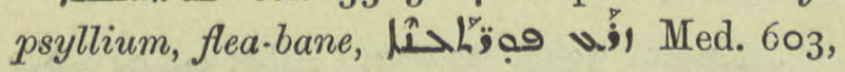
Ar.PflnN. $3{ }^{1} 4$.

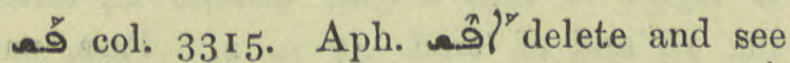

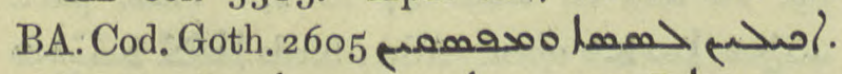

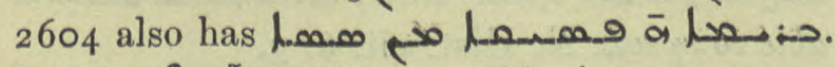
Pauel Lisá Med. 572. 5. Metaph. to dispel,

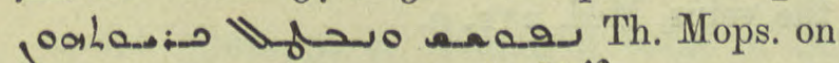

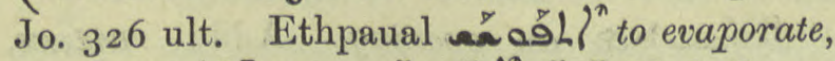

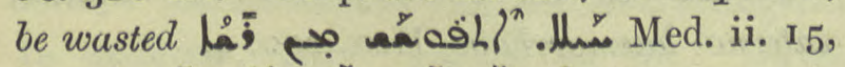

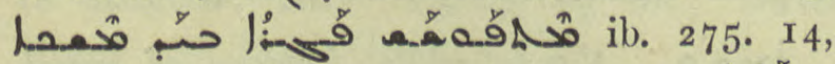

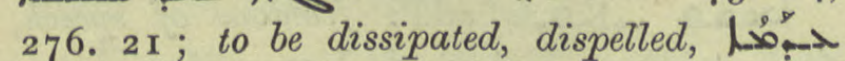

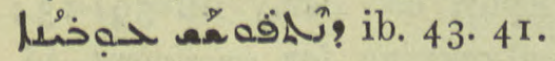

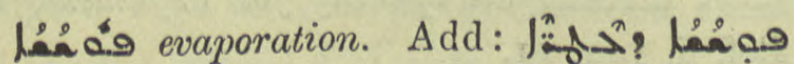
?ִ dissipation of the fumes of wine, Med. 4I. 23 ; waste lo 277. 10. $289.24 ; 290.4$.

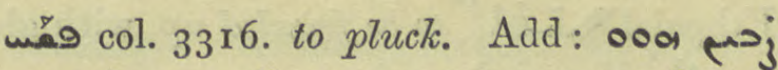
l wanted to crab the man's reputation, Sev. Lett. 377. 15, 378. 13. Pass. part.

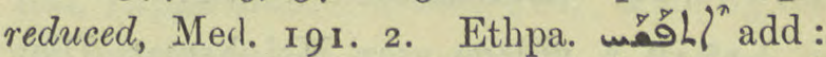
to take trouble, lلإمبه

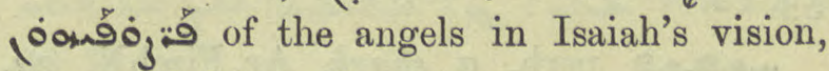
Narses ed. Ming. ii. ${ }_{3} 6$. $1_{4}$.

|นُนْفَف col. 3318. disappointment opp. |حَّ人َ, Ephr. ed. Lamy iv. 429. 7 af.

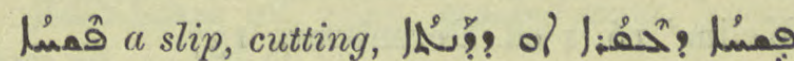
A

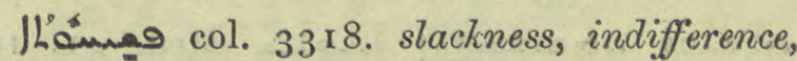
the ref. to Pat. Vit.=A.M.B. v. 29. I3.

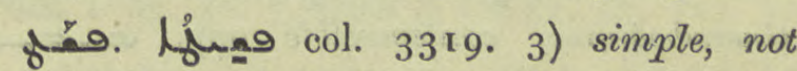
compound, the four elements, fire, air, water, earth, are fön lääas N. Hist. Bk. ii,

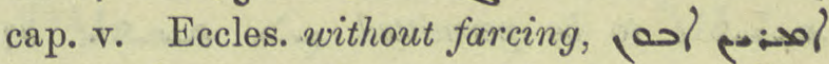

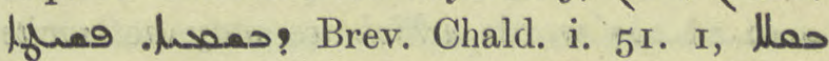
|ر ib. I3. 26, 40. 3. Absolute, IA

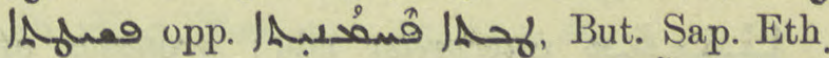

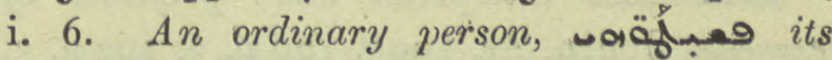
citizens, id. Pol. I. 3 .

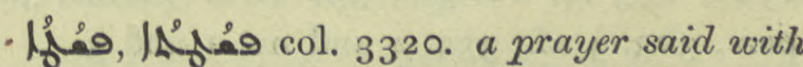

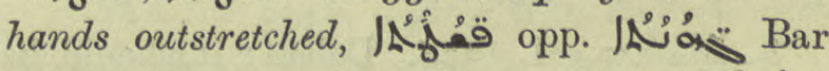
Sal. in Lit. 52. 10, 55. 6, 72. 6, 7 ;

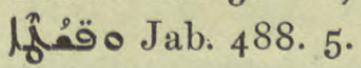

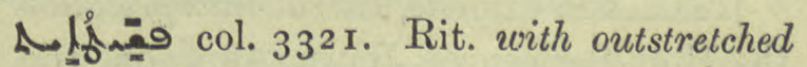
hands, Takhsa 134. ro. Simply, همبر

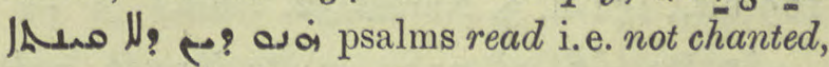
Brev. Ant. i. Cal. $53 b_{4}$ af.

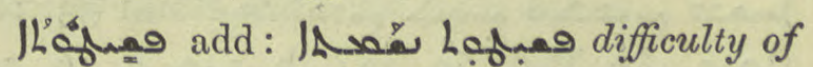
breathing, Med. I54. 6.

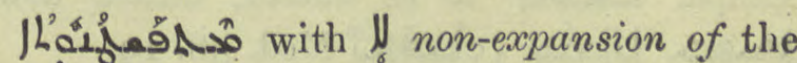

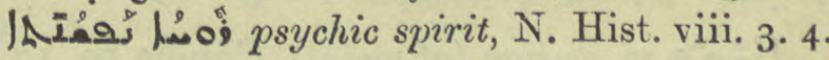

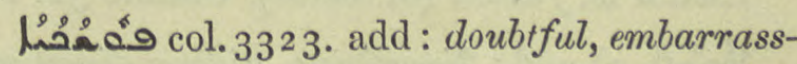
ing, Sev. Ant. Vit. I06. I I.

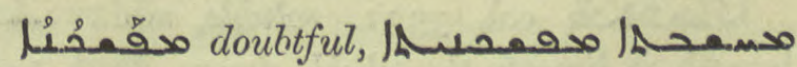
Sev. Lett. 95. 4.

बف urested, perverted, of Nestorius, Magh Pléroph. 1 2. 7. Aph. \فी

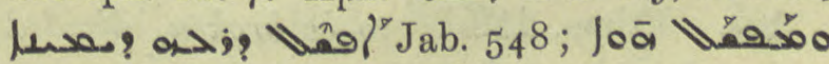
वट so correct for 
Ethpaiel \قُ. स्डकी? Hormizd 2348.

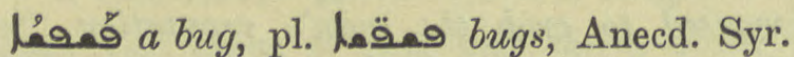

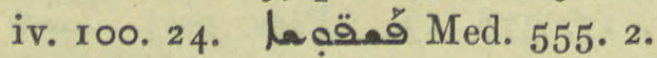

\$. 53. 18.

..9. 9 perh. a shark, Ar. FischN. 22, quoted from DBB. I644, I393. 9. col. 3326 . سفين Chagrin-roche, cf. Dozy Suppl. under and Maimonides 'Mischnah: Commentar zu Kelim' I6. I ed. Derenbourg I37.

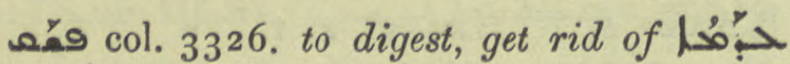

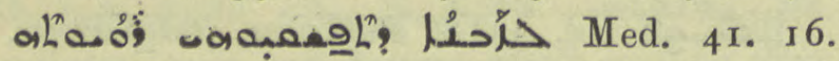

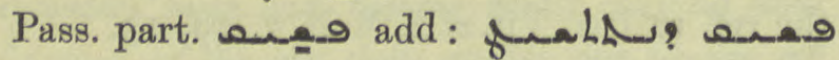

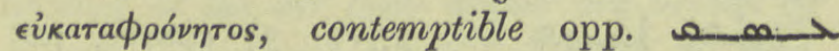

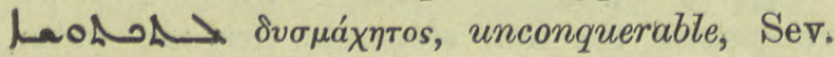
Ant. Vit. 240. 7 ; Pet. Ib. 28. 5. Ethpa.

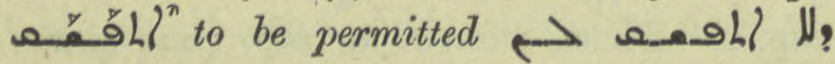
wandsh words which we may not write down, Vit. Mon, 87. Io.

مهـــــ interpreter add: professor of excegesis, Journ. As. 1905,160 n. $3,162 \mathrm{n} .3$, I 906,275 n., Barḥad. 398 n., Or. Xt. i. 322 pen., $3^{2} 3.4$ af., $33^{2 .} 4$ and $n$. $r$ and 3 .

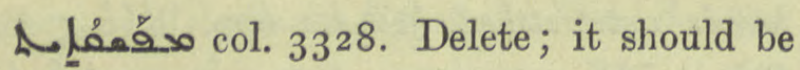

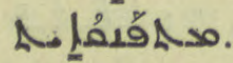

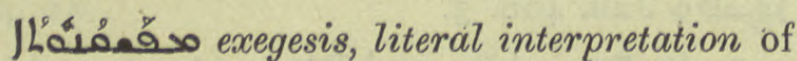
the Holy Scriptures, Journ. As. $1905,160.3$, I69 ult., I 71 n. 3, Narses 32.1 ult.

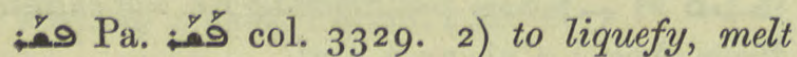
مدàn: Natur 53. 16 ;

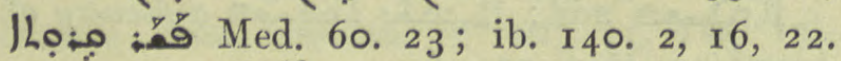
Ethpa. : מع to be melted, melted down .

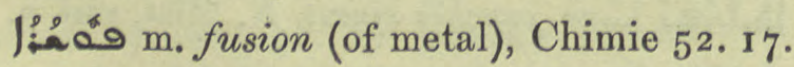

|3) add : liquefying, aiding digestion, .

قِّعْ: :

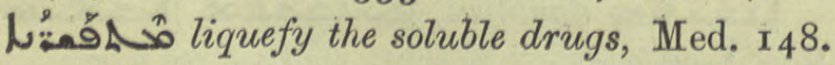
20, 149.8 .

بـ col. 3331. Black Bryony, Med. 264. I, بم: 1. I5; Ar. PflnN. 91 .

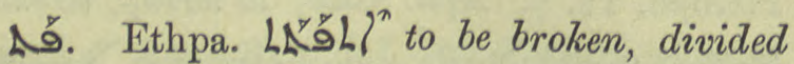
as bread, add ref. S. C. L. 87.8 .

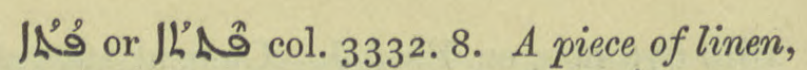
the priest covers the Mysteries Sمa دقد Bar Sal. in Lit. 88 ult., 89. I.

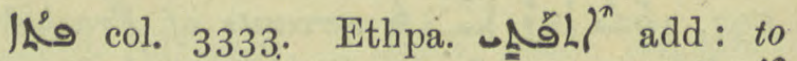

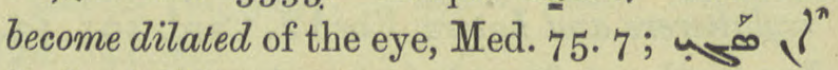

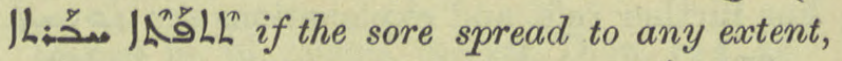

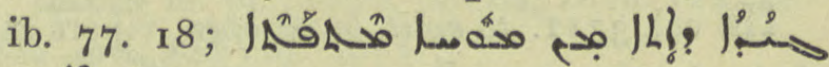

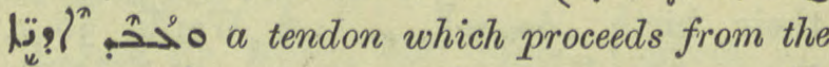
brain and spreads out to form the ears, ib. 99. 18. To be malleable, capable of extension as metals, Sev. b. Šlakko Z.A. vii. 157. Aph. أ oashl the Arabs spread over their lands,

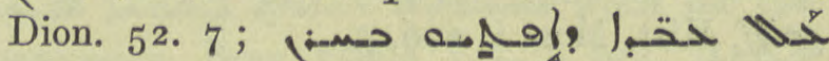
of slaves who overspread Harran i.e. who revolted, Chab. ib. I 77 pen.

| 1ำ col. 3334. room, opportunity. But

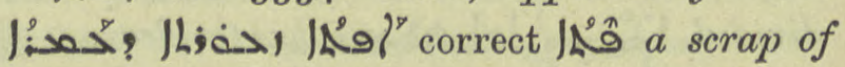
woollen cloth, Med. 103. 20.

fن́ág expansion, ditatation of metals, Sev.

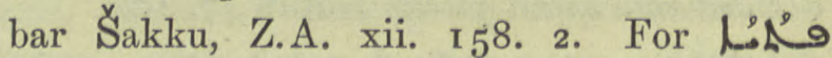
breadth, Lílẹo lógọl Jac. Sar. Hom. iii. I 43. $18,20,2$ I.

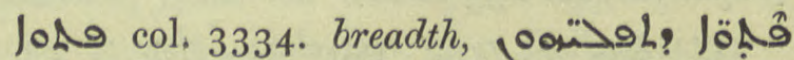
Bar Penk. 104. I 5. Metaph. superabundance, . 9 Jul. 2 20. 9.

NoLiف (. مattula (?) name of a scribe, in a colophon, Kal-w-Dim. ed. Bickell $\mathbf{5}_{52} \mathbf{a}$.

فنمس حسبه : col. 3337. to open. Add it begins to look bright, Chimie 24 antep., 99. 16 and cf. trad. 47 n. 2. Pass. part.

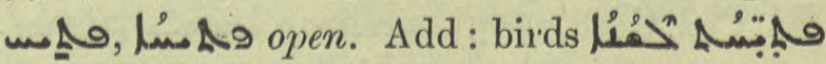
able to speak, N. Hist. vii. 6. 3 .

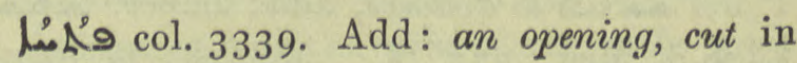
the veins, Med. 27 I. 9.

JLown an opening. Add: rupture, internal haemorrhage, Med. 200. 4.

foes or add: freedom of speech, free utterance, Ephr. ed. Lamy iii. 59. I3; محام Ref. ii. I 42. II.

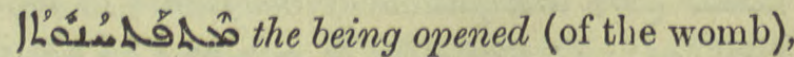
N. Hist. vii. $5 \cdot 3$. 


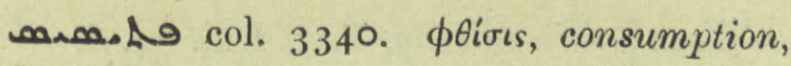
add: : Hippoc. iii. I3, I 5, 2I, 28 , v. often, vi. I 2, vii. I4, Med. 243. I 2, 249. 3, 608. 4 and often.

4 مै col. 3340. Pa. y ฝै i. q. Pe. to mix, imper. مب:? 4010? mix these ingredients, Chimie I 5 , I 5 ; Hormizd 2183 with gloss /Lass.

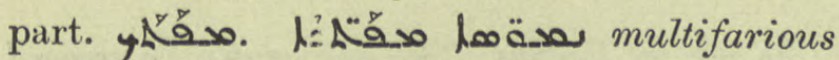

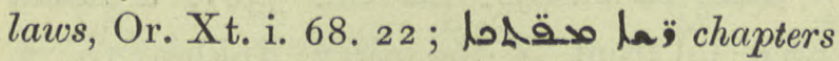
on various subjects, Journ. As. I906, 56. 2.

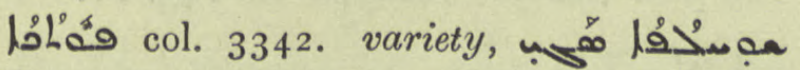
فِقَّ

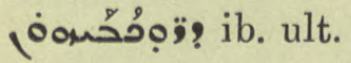

لف่A an embroidered or variegated garment, JLم he was stripped of the many-coloured garment of light and clothed in shame, S. Dan. $5^{\text {I }} 67$.

لمِ an col. 3343. an idol. Term of abuse, Dion. 22. 19.

ش

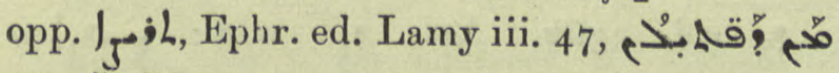

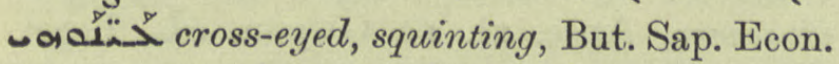

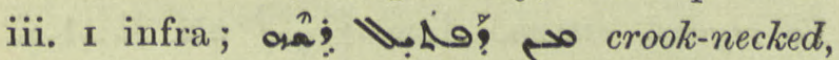
ib. 2. Ethpe. "MñLl to be crooked, awry of the spine, Med. I 24.8 , of separate vertebrae, 1. 22 .

لן Hist. B. V.M. 206. 19.
L'á>. crookedness, crooked gait of a crab, Ephr. ed. Lamy iii. 47.

|Å> lint, Hippoc. v. 44, a wad, plug, Med. 63 ter, 64 ter, I04. I4, 56r. I, 570. I, 6. A coil, هحب: Protection 33. 6.

لِ Med. I 24 . I 2, I 4 .

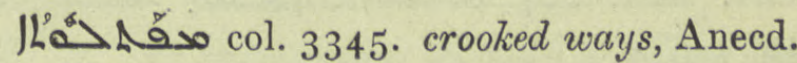
Syr. iii. 342.24 .

A Ar. فi, ii. Aph. Âे" to revolt, to excite sedition. So correct for a $\mathrm{A}$ ?

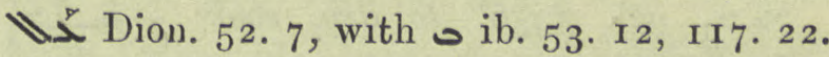

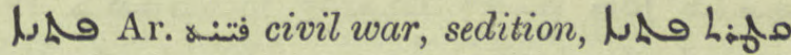
Dion. 9. I, I 8, го. I I, 56. I 5 , Harkavy F. S. 42 .

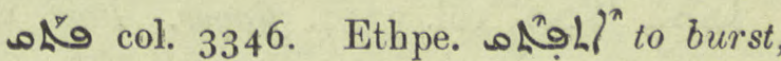
as an abscess, Hippoc. iv. 82, ib. vii. 7, Med. 231. I 5, 233. I 6 .

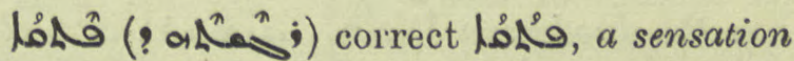
of retching, Med. 27 O. I5.

jớ

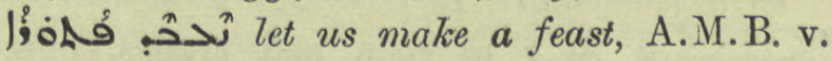

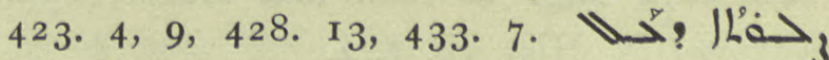

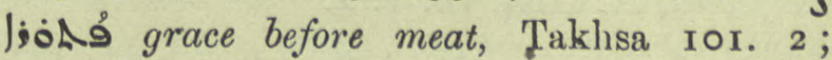

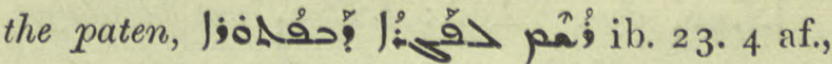

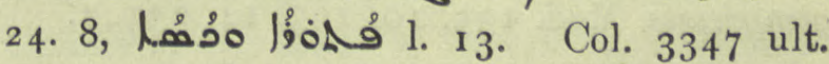

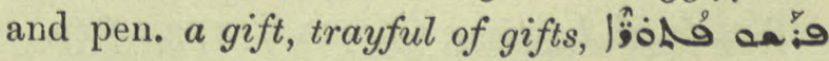
they set apart, dedicated gifts, Jab. 24. I.

\section{$\because \quad \because$}

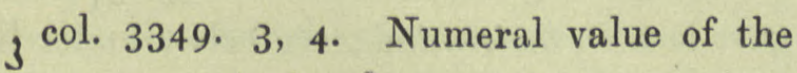
letter : corr. octoginta to nonaginta, octingenti to nongenti, Amir I3. 9, I5 ult., Duval Gram. xiii, Nöld. Gram. 2.

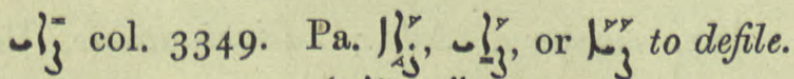
Imperf. 2 pl. m. iii. 145. Ethpa. $\mathrm{L}_{3} \mathrm{j}^{n}$ to be flecked with

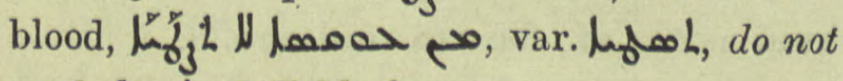
need the goad, Tekkaf I 20.

2716
$\mathrm{C}_{3}^{\mathrm{V}}$ a bpric. on the W. bank of the Tigris, stretching from Seleucia to Kashkar, Tim. i. Or. Xt. ii. 300. 4, Eranšahr 164. Hoffmann, Pers. Mart. n. 759 identifies this region with but between the two rivers Zab.

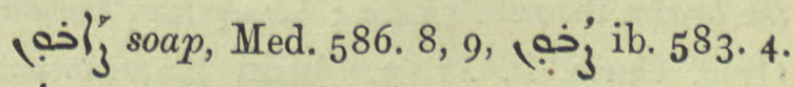
See $\operatorname{~og}_{3}$ col. 3430 . 
ls y col. $335^{2}$. to be willing. L. 23 of par.

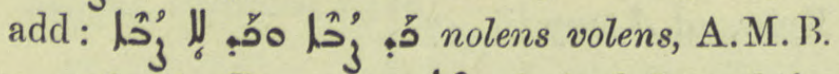
v. 456 . I6. Pass. part. l⿵ $_{3}$ desired, longed for unless you emend L',

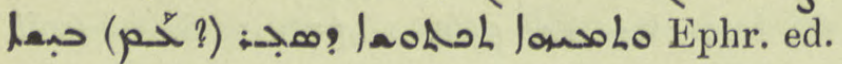
Lamy iv. 75. 9.

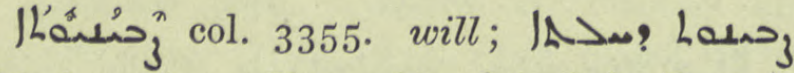

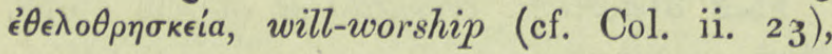
Sev. Lett. 207. 2. corr. IN Lentas as ib. $33^{6.7} 7$.

رُ Gest. Alex. 20r. I 7 .

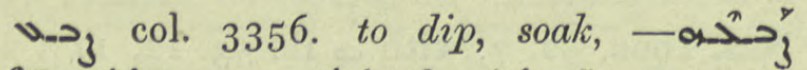

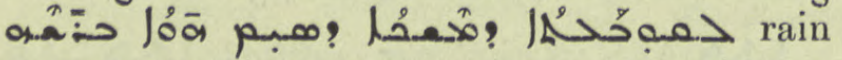
soaked the leather cap on his head, Med. I33. $2 \mathrm{I}$.

Lحُ

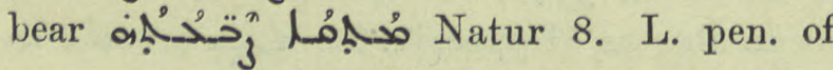
par. corr. to $\mathrm{IA}^{2} \mathrm{\Omega}_{3}^{n}$. The lesser intestines

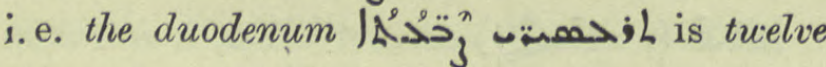
fingers long, N. Hist. viii. I. 3 .

مبّمعَ N

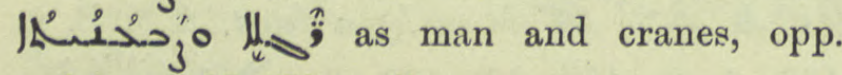
webfooted, N. Hist. vii. I. 4 .

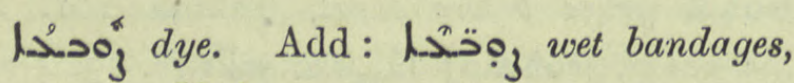
compresses, Med. 135. 10, 195. I 4, 235 . I.

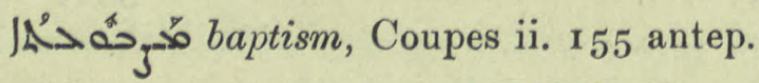

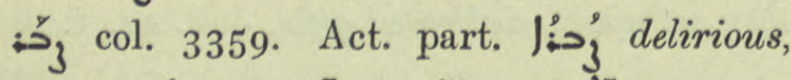

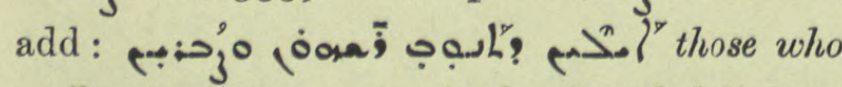
suffer from torpor in the head and delirium, Med. 55. 22. Ethpe. $\left.:-z_{3}\right\}^{n}$ to be said foolishly, Sev. Lett. 253 . I 4 .

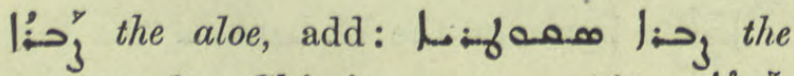
Socotrine aloe, Chimie 45. 4, , Med. 355. 18 .

lڤُ ii. 2 , iv. 50 , vi. $11,25,5$, vii. often, Med. 4. 9, 6. 6, I 5. 6. Pl.phrenitis, inflammation of

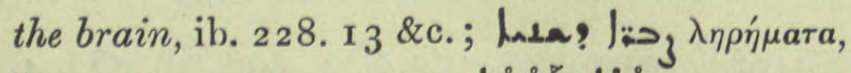
Greg. Carm. ii. 47. 3. . idle tales, A.M.B. iii. $3 \circ 9$ ult.

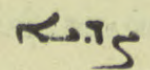

JLo:sy vain talking, Test. Dñi. 74. 6.

$\mathrm{A}_{3}$ col. 3360 . Pa. $\mathrm{AS}_{3}$ to prepare bread for the Eucharist, Takhsa 105 med., pُaْ

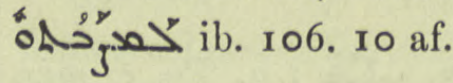

$\mathrm{ILS}^{2} \mathrm{~S}_{3}$ add: preparation of bread for the Eucharist, 1 Takhsa 105 tit. 8, 9, r 10 ult.

1 مُ pace, in front of the altar, Takhsa so. 7,

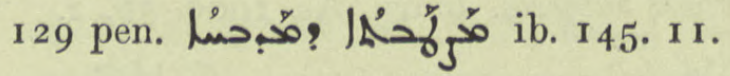

Lئُ a dandy, Probus 98. 6.

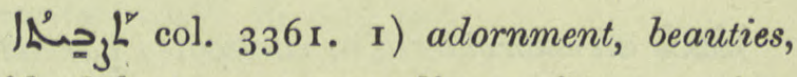
said of the trees surrounding a city, مفعمب aAjخ Josephus vi. 2. 9; the wisdom which adorned S. Antony, 3 af. 2) preparation, $1 L^{2}$ the stone on which Eucharistic bread is prepared, Takhsa I I 4. 5. 6.

ف col. 3362. Aph.

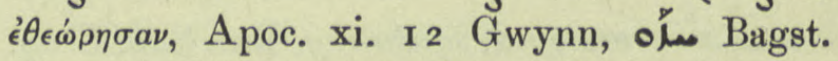

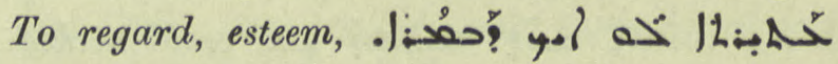
But. Sap. Econ. ii. I.

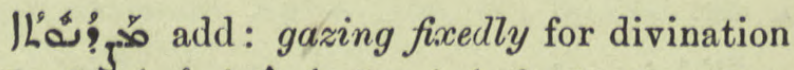

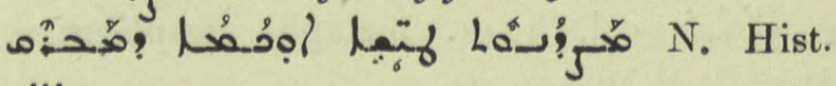
viii. $4 \cdot 9$.

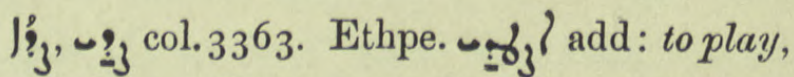
pretend, ing with the idea of leaving their home, $\mathrm{BH}$. Stories 34. I 8 I but Palladius has were torn in their mind; see below under $/ \xi_{3}$.

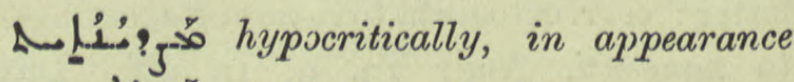

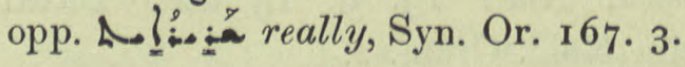

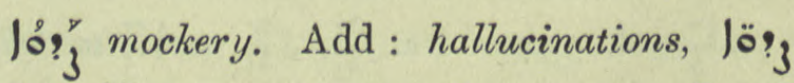

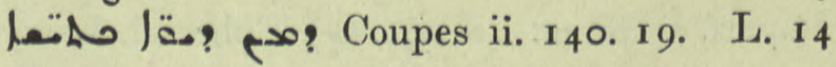
of par. correct refs. to Is. Ant. For 216.4 read 218.4 af., for 218 read 220 . 6 .

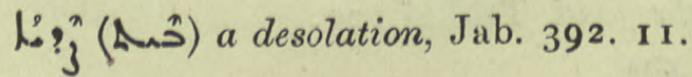


Li? col. 3366. the city Zorah, add:

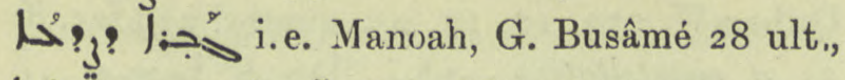
Lrit. Mus. Or. 2084. 60 v, 人? ib. $66 v$.

L'?3 the temple, 600. 17 .

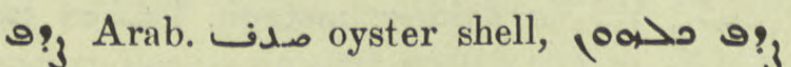
all kinds of shell-fish, Chimie 6. 17 ; mother-

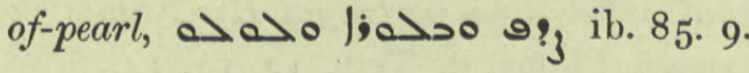

; Dion. 126 . 14.

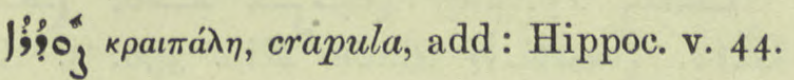

Lioay col. 3368. a drought opp. leaf,

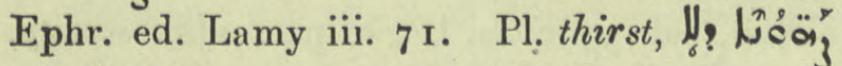

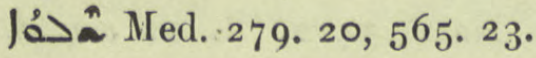

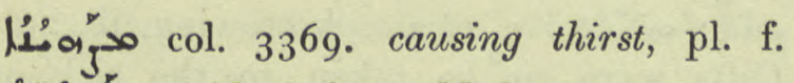
إم 22.

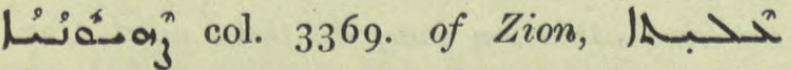
L'vion, the upper chamber in Jerusalem where the apostles assembled, Warda I $25 r$. $A$ native of Zion, Lebbaeus, Lُنُم, $222 v$.

\aj col. 3370. usually to neigh, whinny. To hiss or roar, of the Burning Bush, Nor لمa Narses ed. Ming. ii. 289. 13; of the ears of مajo أُخ Brit. Mus. Or. 2084. 44 v. Pael part.

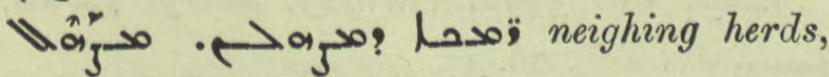
Ephr. ed. Lamy iv. 66, 18.

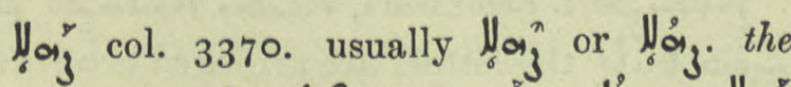

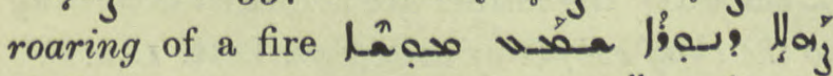

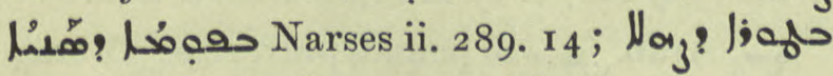
$\sim_{0.0} \rightarrow$ Protection 100. 4 af.

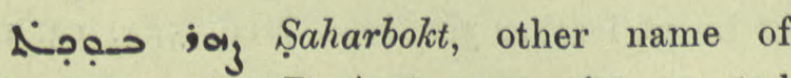
Ṣliwazeka, G. Busâmé $5 v$ tit., quoted Nöld. F. S. i. 49 I, 493.

Jółẩ col. 3370. Cicuta virosa, hemlock, Med. 232. 10, add : شوكرن حششيشة ib. 608. 8, Ar. PflnN. $3^{81}$.

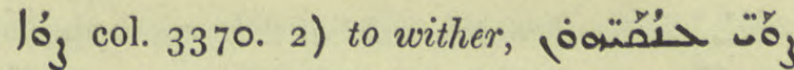
A.M.B. ii. 70. ro. 3) mimetic to twitter,

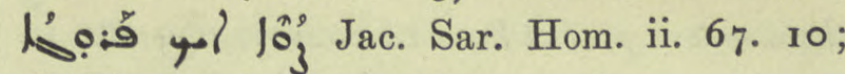
G. Warda ed. Hilgenfeld I3. 26 . I.

$\nu_{0}, \Omega_{3}$ col. $337 \mathrm{I}$. With $>$ to arrive at,

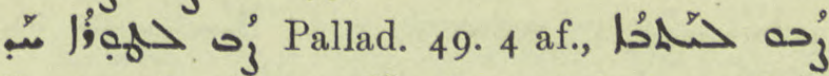
ib. 54. 6. Pa. 1 I) to bring, conduct,

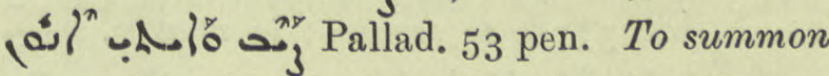
Jab.

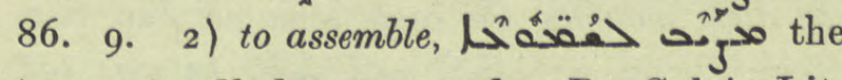
trumpet calls hearers together, Bar Sal. in Lit.

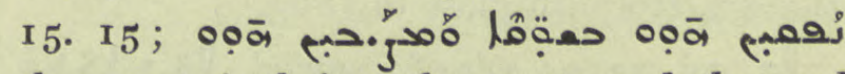
they went forth into the streets and thronged them, Pallad. 8I. I I, I 2. 3) to provide, procure Med. 43 ult., A.M.B. v. 2. I 4 .

1.oo add: a hut, lodge, Dion. 152. 5 , 1ว

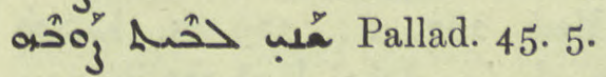

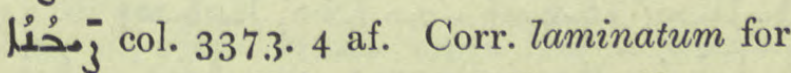
Jemanense. Cf. Talm. ציב and Buxtorf's note ;

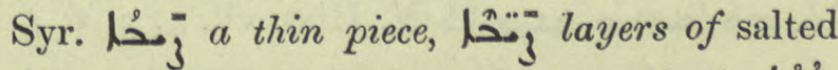
meat. Laminar, laminated, slaty, fissile, lơ بحُُ1ْ rock-alum, alum occurring in schistose formations, Med. I69. I5, 21 2. 13, 21 3. 13, 20, 214.7 \&c., ellipt. i.e. بحُُ

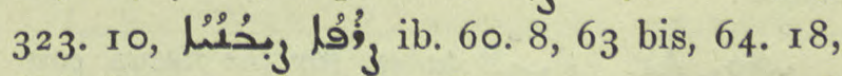

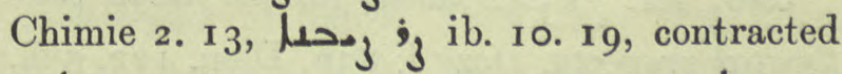

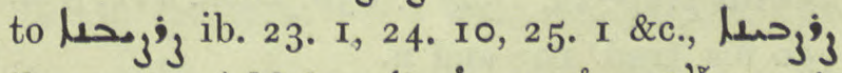

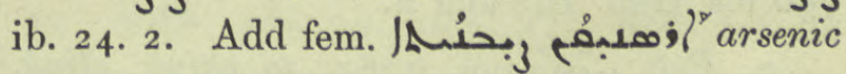
occurring in schistose rock, Med. 87. I0.

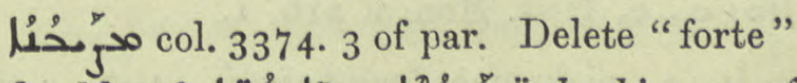

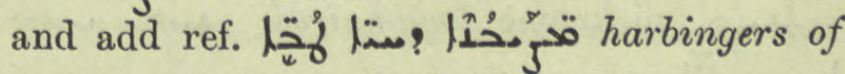
the blessed life, Bar Penk. I I 9. II.

Do imper. of verb šs to plant.

90 Pael $\stackrel{n}{n}$ col. 3375. to hunt out, seize,

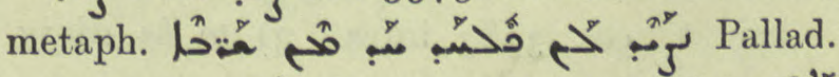
4. 6. Aph. $\div-\bar{j}$. Trs. the following ref. to ? ? Aph. of verb ? sane gaze intently on the shining sun, Jo. Eph. 235. 9.

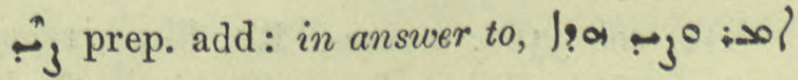
Vit. Monoph. 79. 27. 


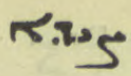

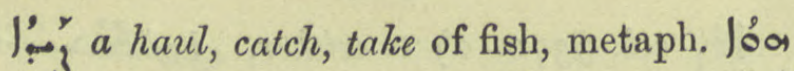

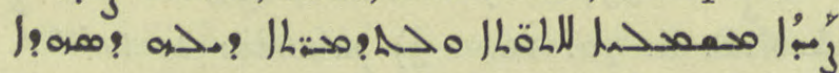
there was a perfect haul of conversions due to the signs and wonders worked by the martyr, Sev. Ant. Vit. 218.3 .

رُّ of network, add: IfLLos the retina, N. Hist. vii. 1. 2.

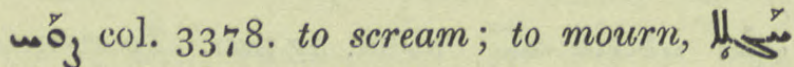
س the dove mour'ns, the eagle screams, Jac. Sar. Hom. iii. 90. 9 ; at heart, Hormizd gloss to $\mathrm{I} 803$.

لماهيخ bits of old shoeleather, Med. 554. 12.

लि $\mathrm{N}_{3} \mathrm{Ar}$. Sultan, part of a woman's name, A.M.B. ii. I tit., 5 .

boog col. 3379. the jejunum, a part of the small intestine between the duodenum and the ilium, Med. 267. 8; 329. 3, مُ

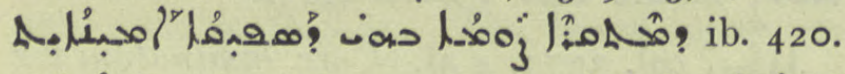

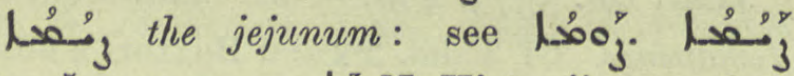

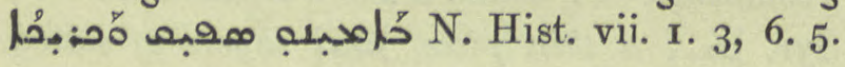

wo, Sauma, Bp. of ص: ZDMG. xliii. 395 infra.

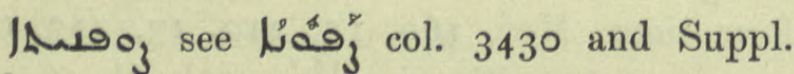
below.

u90, Ar. الصَّ.وَافي taken for the fiscus, confiscated property, Dozy i. 836 , Dion. 122. 5 ff., 150 bis, trad. 103 and $n$.

$30_{3}$ Pali $-\mathrm{jo}_{3}^{y}$ col. $33^{82}$. to chink, clink, |ị̂, $30_{3}^{y}$ Hist. Mon. i. 2 Io. 1.

م- م-ن $\log _{3} 0_{3}$ Charms 30 pen.

$\mathbb{L}_{-3} 0_{3}^{*}$ a separate flame or flake of fire,

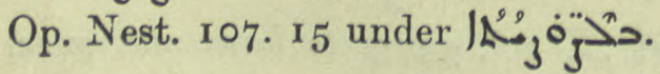

;o, , ; col. $33^{8} 3$ infra. 3) to be dizzy, add: : 30. 8, I3. 3 . 3 .

j;oj Heb. 'צ् balsam, for a sore mouth or for sores and scabs on the head, Med. 554. 20 , 563. $1,575.2,583 \cdot 5$.

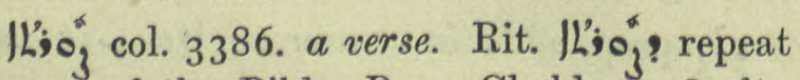
a verse of the Bible, Brev. Chald. r. $5^{8}$ ult., 6o. $12,65.4$ af., $85.5,90.4$ and often, QdhamW, 233. 10, I5, 234 quater, 235 ter, ff.

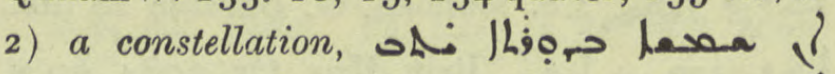
Brit. Mus. Or. 2084. 6 r, lligg g Jam ? od: ib. $7 v, 8 r$ and often.

A. $\mathrm{L}^{2} \mathbf{L O O}_{3}$ in formed letters i.e. in writing opp. Syn. Or. 138 ult., I 4 O. 6.

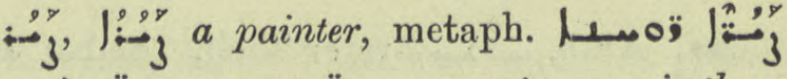

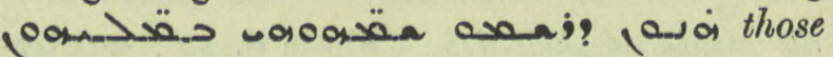
spiritual artists who portrayed His Names in their speech, Philox. de Trin. I 2. I3, 14.

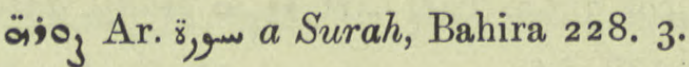

مـ $\mathrm{LLFO}_{3}$ pond?? Chron. Min. 356. 5. Cf.

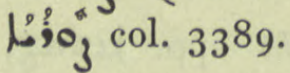

$\mathrm{LLoy}_{3}$ col. 3390 . m. l. 2 of par. add: society, ILoj pes' sociable, Natur 54. 2. L. I2. sound, add: Gifts were to be taken to the sick and afflicted Liga ح the kind attention may do them good, Can. J. Tell. $3^{2 \cdot} 5^{\text {. }}$

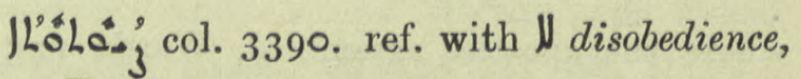
Test. Dñi. 74. I.

$\omega_{g}^{y}$ col. 3390 infra. to beat or strike as great heat, joanis lesa Coupes ii. 156. 24. Aph. $\mathrm{w}^{2}$ ' to write out, add: to draw up a will, Syr. Rom. Rechtsb. I $4.3,4$.

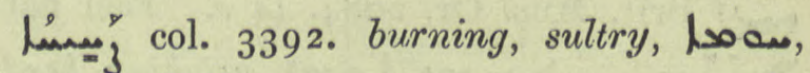
Jo. Toll. 26. I 5 .

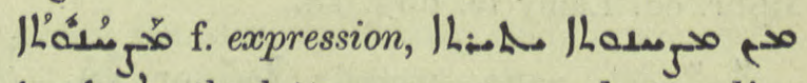

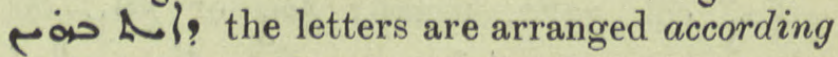
to the chief subject of each, Sev. Lett. I. 6.

مع to pound, pulverize. To bray or rub down, لlla on old scraps of burnt leather for kohl, Med. 554. 13, $1_{5}$, 570. ${ }^{2} 3,571.16$.

ح حتة حتهم a familiar fatuities and wandered from the point, Sev. Lett. 376.9 . 


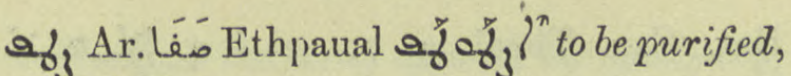

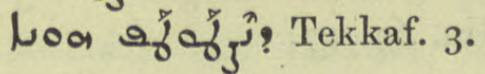

$\ln _{3}$ : see under $\Delta_{3}$.

$\checkmark{ }_{-1}$ col. 3395. Lepidium latifolium, pepperwort, cress, ref. Med. $3 \circ 7$ ult.

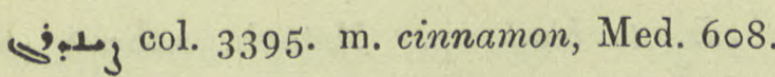

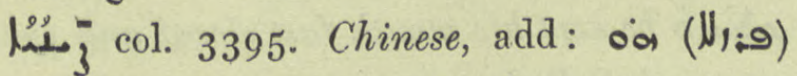
le $\mathrm{g}$ Chinese metal, mentioned as one of the seven malleable natural bodies, Sev. b. Škakku, Z.A. xii. I5 5. 2, I I. Cf. للإيد الم-يـني Chimie 66 ult. probably an alloy of tin.

53 Sis, 53 Jodxo! liof Ahikar 50. 7 .

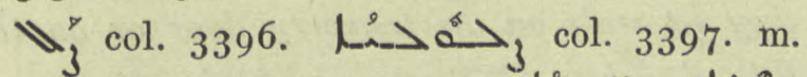
a mawkin, scarecrow,

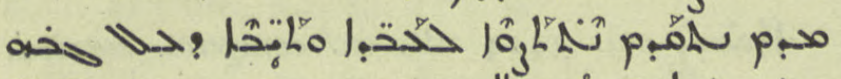

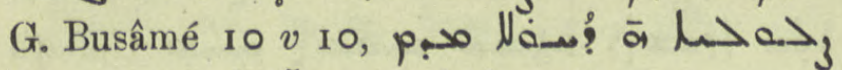
إم: ib. marg.

 Geop. 52. II, 14.

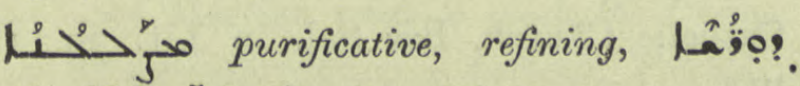

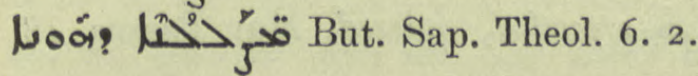

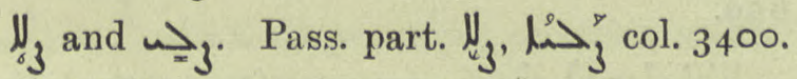

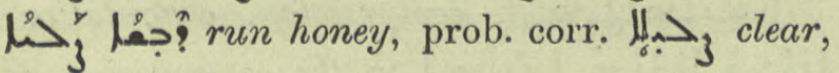
Med. 555.8.

${ }_{3}{ }_{3}, 1 L^{\circ} \rightarrow_{3}$ col. 340I. prayer. Add: IL' because only bishops and presbyters may say

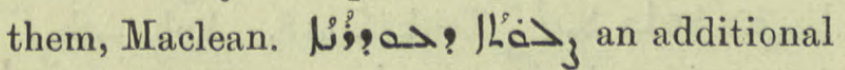
collect at daily offices, QdhamW. 106. 5 af.

$\mathrm{L}_{3}$ inclination, tendency, defined as inherent in hodies, that natural impulse whereby a body returns to its position, N. Hist. cap. v. sect. I, variations from sphericity of the earth's surface, Jac. Edes. Hex. xlviii. 10, xlix. 3.

$\mathrm{An}_{3}$ a stone on which spices are pounded,

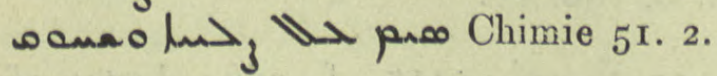

$1 L^{\prime}{ }_{3}>_{3}^{\prime}$ col. 3402. variation, deviation, Iha ¿heo: Jac. Edes. Hex. xlviii. 9, xlix. I, مب: ib. 1. 5 .

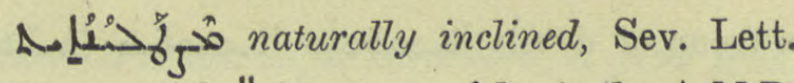
42 I. 19 ; with $ل_{\sigma} a \dot{k} \lambda \iota \nu \hat{\omega} s$, unfalteringly, A.M.B. v. 377 . 18, Hist. Mon. i. 2 I 6.

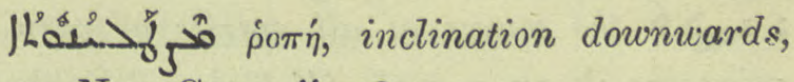
Greg. Naz. Carm. ii. 28. 14.

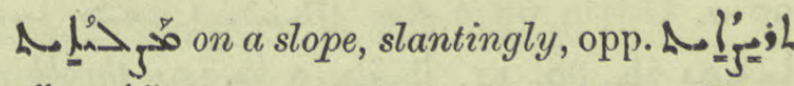
Non exactly level, Jac. Edes. Hex. xlviii. 3,5 .

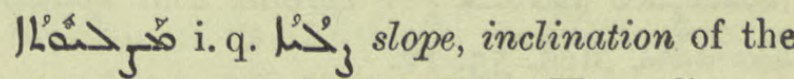
surface of the earth, Jac. Edes. Hex. xlix. 9.

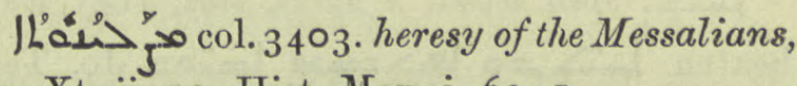
Or. Xt. ii. I3, Hist. Mon. i. 69. I.

سَلَبَة Ar. a bark-rope; a leash, ef. Dozy Suppl. i. 672 a. Hist. Mon. i. 229 . 18, ii. 433 n. A halter,

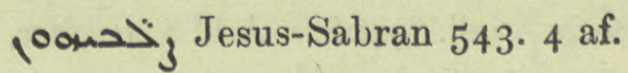

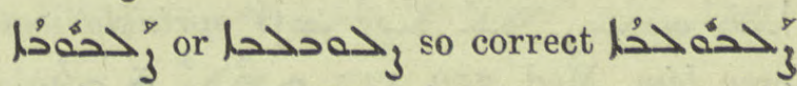
col. 3404. Palma Christi, Ricinus communis,

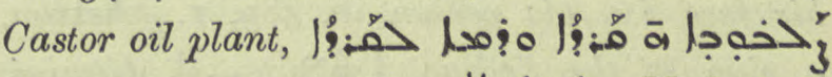

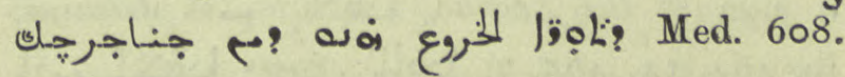
IO, II.

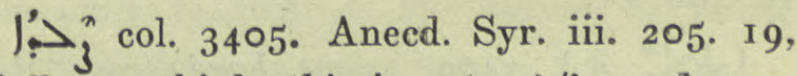
Hoffmann thinks this is not $\sigma \in \lambda i \delta \epsilon s$, columns, as Thes. but $\psi a \lambda i s, \psi a \lambda i \delta \epsilon s$, arches.

ا Assessment of taxes, Dion. 163. I5, ح-ح

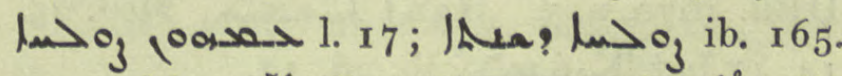

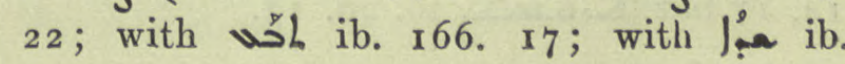
209. 2.

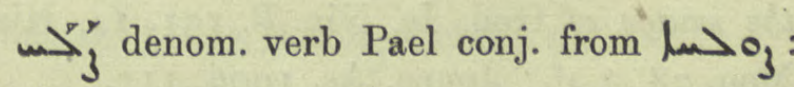
cf. Arab. لص to make a convention, to settle by agreement, to assess, with $\mathrm{p}^{\mathrm{\nu}}$, Dion. ${ }^{2} 3^{2}$. I5. $20,233.22$.

$\mathrm{w}_{3}$ col. 3405 . to split. See following article.

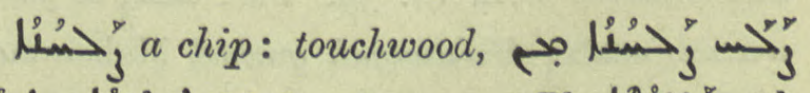

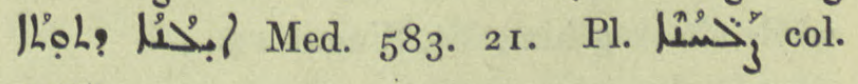
3407 .

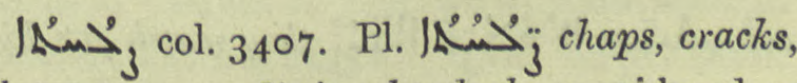
Charms 3.4 , splitting headache considered as 


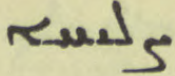

286

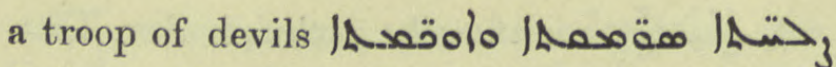

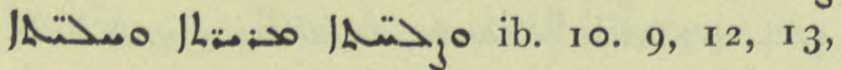

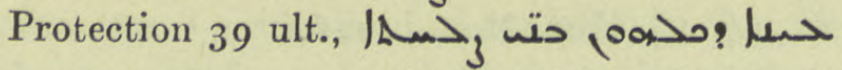
: 1 the source of all spirits of pain is migraine, ib. 77. 4, 3 af. Cf. Talm. T. Sabbath 90 a, Gitțin 68 b \&c. I I 1b. 80. Ix, كمع throbbing

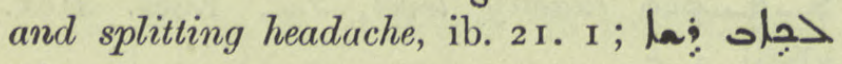

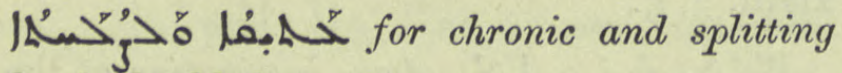
headache, Med. 55. 2.

لُ

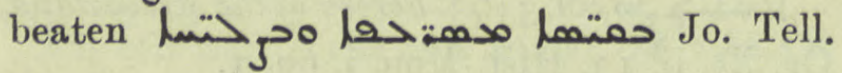
50. $\mathrm{I}$.

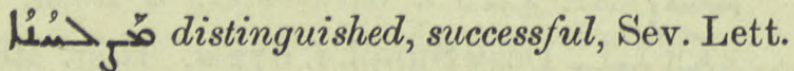
287. 9 .

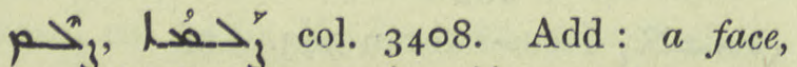
范

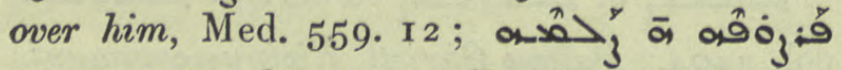
his face, i.e. his person, ib. 589 . 1. Astron.

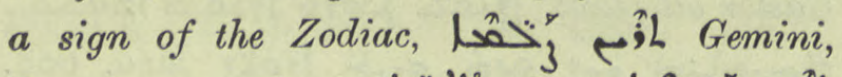

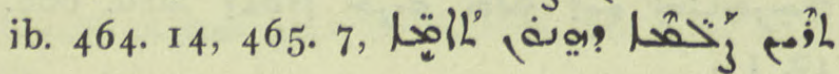
ib. 466 . $\mathrm{I}$.

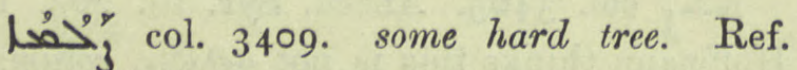
Med. 587 . 12 .

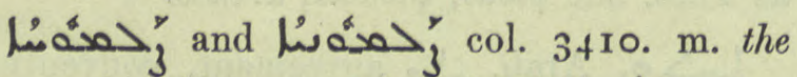
tarantula spider, one codex of BB. has he

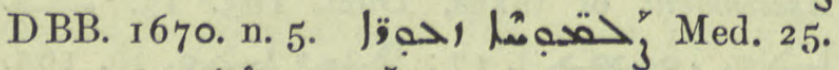

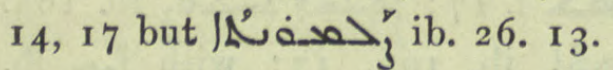

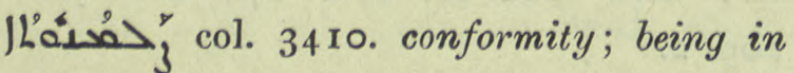
the image of God, Is. Nin. B. I51. I4, Hist. Mon. 28. 3 af.; Journ. As. 1906, 117.

$a_{3}$ col. 3410. to hurt, injure, ref. $\left.\left.\right|_{20}\right\rangle$ ? yor $\cup$ ? $\Omega_{j}$ lest thou injure thy father, $\mathrm{BH}$. Stories 9. I4. Ethpe. $\left.\left.a_{-}^{n}\right\}_{3}\right\}^{n}$ to be

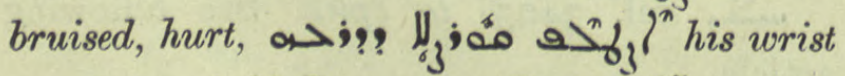
was injured, M.Z. I 4. 39. Pael $\boldsymbol{\Delta}_{3}^{n y}$ to gash, به while shaving him, Mt. Singar r 5. 4.

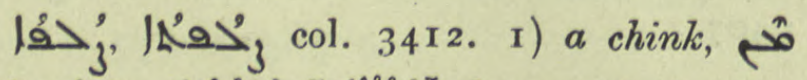
هَ through chinks and by the hinges of the door,

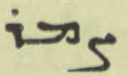

But. Sap. Theol. 4. 7. 2) i. q. $\mathbb{R}^{2} \mathrm{C}_{0}$ contusion, Chron. Min. 67. 17 .

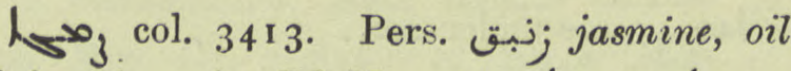

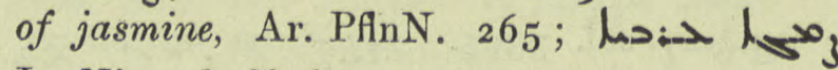
Is. Nin. ed. Chab. 29.

3ु to bind up. Metaph. مी مै he kept his mouth fast closed and spake no word, Pallad. 24 I. Io.

I: مه ه S. Dan. 62 a r. A coffer, casket, ajh ل

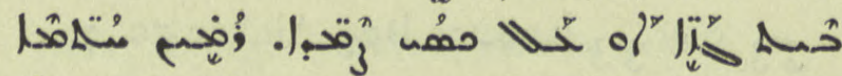
they set seals on the treasury door or on the lids of caskets, G. Busâmé 5.8 af.

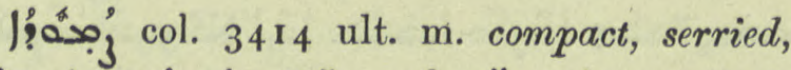

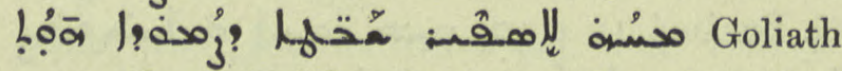
smote the united band of the tribes, Jac. Sar. Hom. ii. 37. 3 .

$1 L^{\circ}+0_{3}$ f. close union, said of the Holy Catholic Church, Bar Sal. in Lit. 39. 12 ; 1200 crowded, massed, Mar Bassus 48 659 .

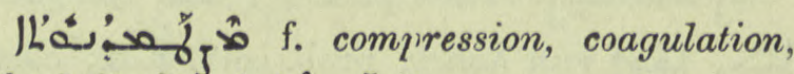

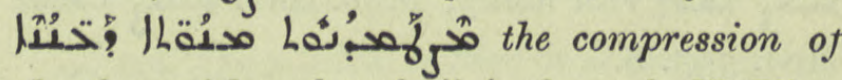
cloud-particles when hail is formed, N. Hist. v. I. 3 .

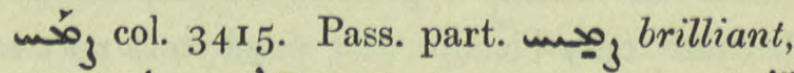

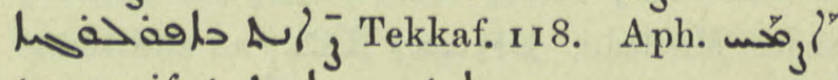

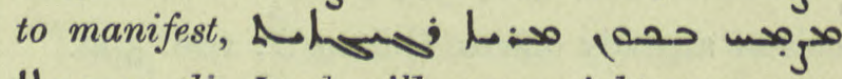
|haos! the Lord will cause righteousness to appear attractive in you, Sev. Ant. Hymns 96.

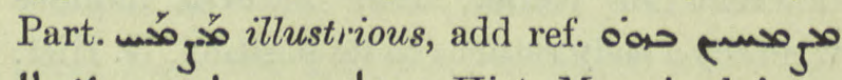

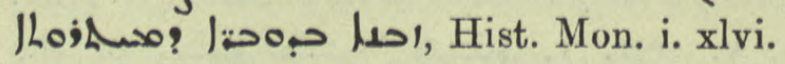

L.

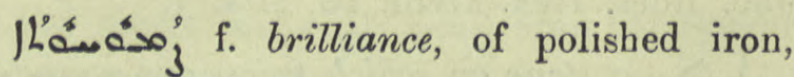
Is. Nin. B. I $90.1 \mathrm{r}$.

Ih's a pearl, Chimie 85.6 .

कू col. 3418. to suffer from strangury, - 


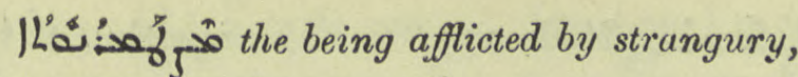

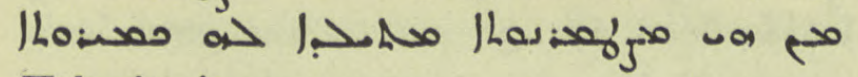
Hebraica iv. $214,233$.

$\mathrm{NO}_{3}$ col. 3419. to be silent concerning, ref. Sev. C. L. I40. II.

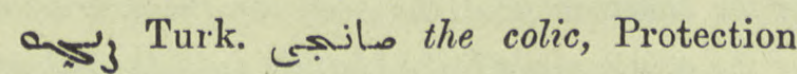
I 7. 6,9 , 1 о.

丩:album, sandal-wood, Med. 6I. 4.

(ب) حبزمةرا الحفة; Jab. $3^{6 .} 9$.

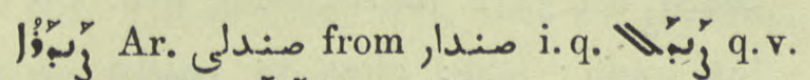
Med. $3^{2} 3$ ult., ${ }^{\prime}$ ib. 4 I 9.5 .

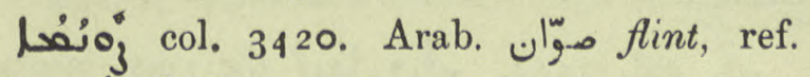
S. Dan. 65 o 7 .

$w_{3}$ Ethpa. $w_{3} ?^{n}$ col. 342 I. to defraud, cheat. Unusual uses: la Marcian tried to evade the Divine anger by misinterpreting a portent, Pléroph.

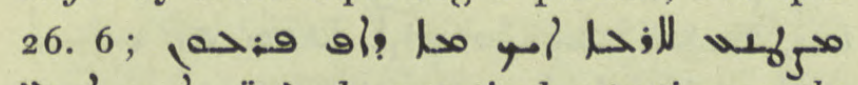
רe practised extortion on the land as Pharaoh did to the children of Israel, Dion. I48. I.

$\log _{3}$, Jank, unknown drug. Used to purify tin and marble, Chimie $3^{6}$ pen., $45 \cdot 3,95 \cdot 13$.

$u_{3}^{y}$ i. q. $\}_{3}$. to be filthy, $u_{5} \gamma_{5}$ o ool $a_{3}^{y}$ ?

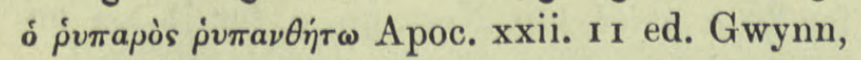

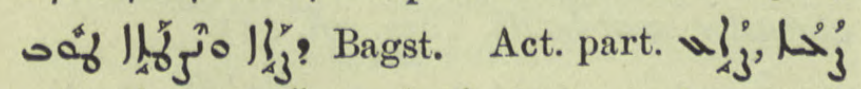

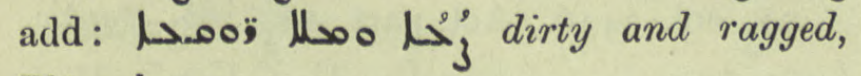
Pléroph. 42. 9.

"لا !م: Dion. 85. I5.

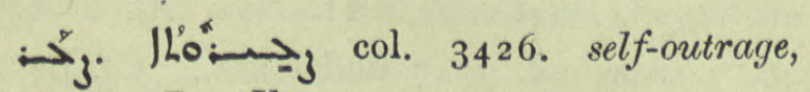
indignity, Pet. Ib. 34. $1 \mathrm{I}$.

16́ه์ sol. 3428. Pl. bonds, shackles,

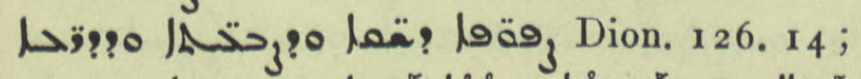

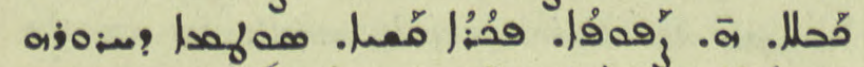

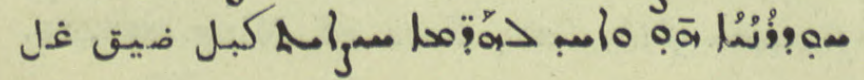

ras dis

a bond is a shackle, a hard cord, a fetter with a circular opening which holds the limbs fast,

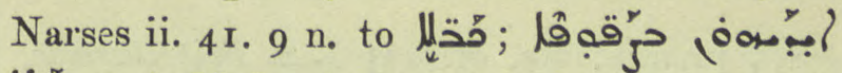
क人 a A. M. B. vi. 29.

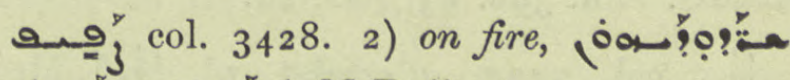

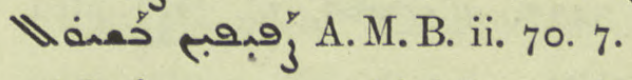

أُ prisons, but “Marterblöcke”, Tekkaf. roo.

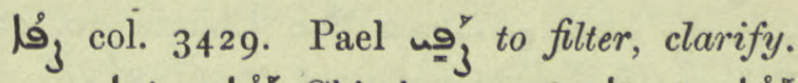

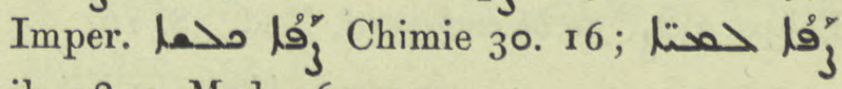
ib. $3^{8 .} 9$, Med. $5^{6} 5.13$.

$\mathrm{l}_{3}$ col. 3429. with ref. to Anecd. Syr. ii.

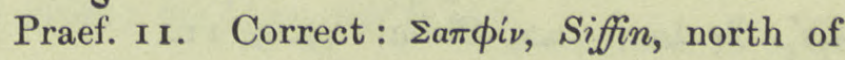
Palmyra, on the Euphrates, Dion. 9. I 3, Journ. As. $1900,286.2$.

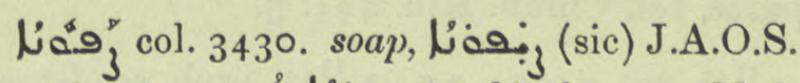
xx. I89. $8=$ = 554. 17 .

ملاما soapstone, Chimie 9. I5. Cf. $\mathrm{N}^{\circ} \mathrm{J}_{3}$ Suppl.

مُ مُلب :

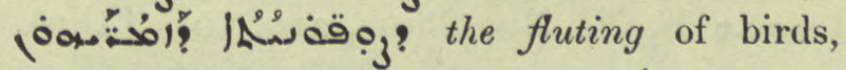
Jac. Sar. Hom. iii. $9^{2 .} 3$ var. n. I / 1 .

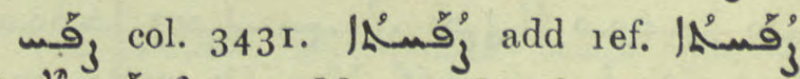
(ف) upon them, Bar Penk. 84. I3.

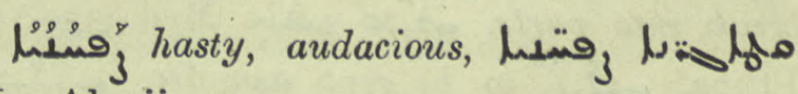
Mar Aba ii. 97.

صفئهـ Arab. a thin plate, a leaf of metal, /

${ }_{3}$ El. Nis. Chron. I 40. I, i.q. اق ${ }_{3}$ Siffin, q.v.

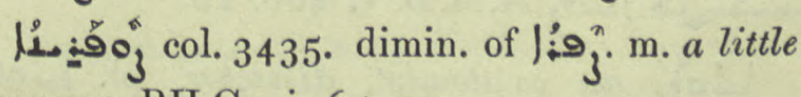
sparrow, $\mathrm{BH}$ Gr. i. 65 . 19.

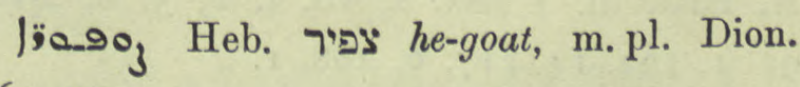
I64. 23 .

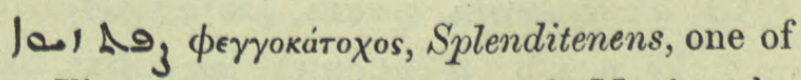
the Five Heavenly Powers of Mani, who support the world, Cosmog. Manichéenne 22 and n. I, Coupes ii. 128. 5 af., Ephr. Ref. ii. Burkitt's Introd. cxxxv. Perh. Assyr. șabit, holder, p. cxxxvi. n. I. 
$\int_{33}^{n}$ col. $343^{6}$. a nail. L. 10 of par. pl. $J_{3 j}^{n} n$ excrescences, warts, Med. 584. 19.

;3 pr.n.m. Bp. of tö? Lus Beit Dārāyé, ZDMG. xliii. 396. ז $3,408.24$.

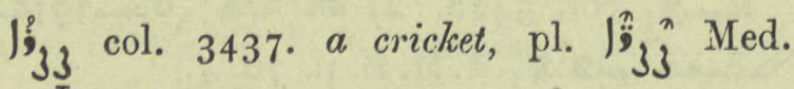
578 ult., 579 . r.

; col. 3437. to fix, condense, imper. ;o,

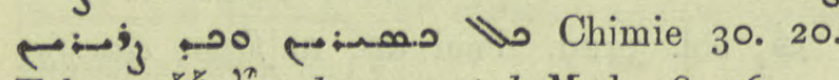
Ethpa.

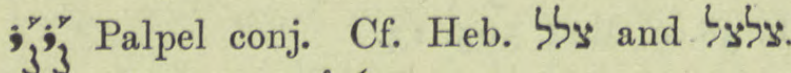

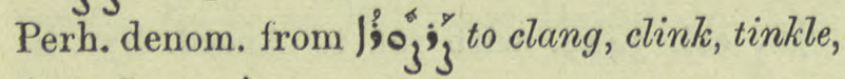
Chimie 40. 9, Ir.

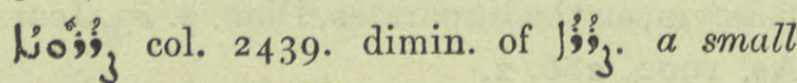
purse, ref. Chron. Min. 244. 5 .

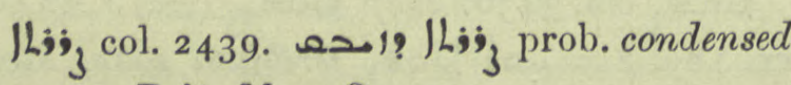
mercury, Brit. Mus. Or. 4I.

; 3 , name of a river near Beit-Slok, Chast. 40. 15 .

$\mathrm{I}_{3}$ col. 3439. to cleave, to shoot as a star, Law | $\}_{3}$, El. Nis. Chron. 220. 10. Ethpe. $\left.\because-\delta_{3}\right\}^{n}$ astron. to shoot, explode H,00a

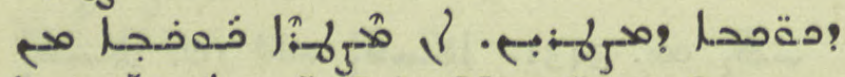

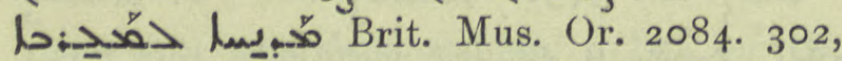

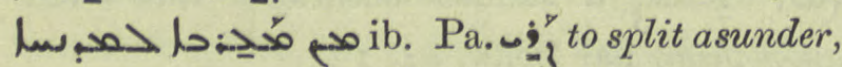
break into parts, - Med. 559. 6. Ethpa. - $=b_{3} 3^{n}$ to be rent asunder, r.

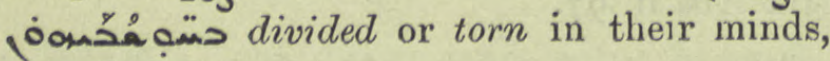
Pallad. 436. 21 .

of J Jo's $_{3}$ col. 3441. a scratch, cut, crack, حبن

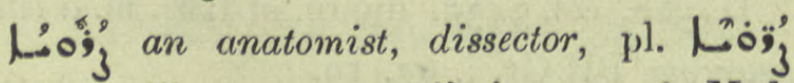
N. Hist. iii. I sect. 2, vii. i. 2, 3, 6, Med. 97. I, I34. 14, 217.6 , 268. I.

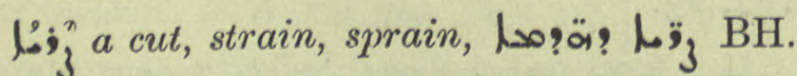
on Luke, p. 2. 9 ; a cut on the head, But. Sap.

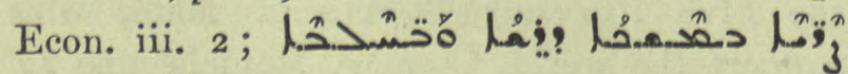
wolla Med. 43. 9.

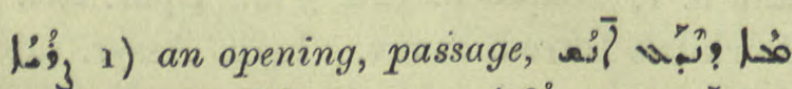

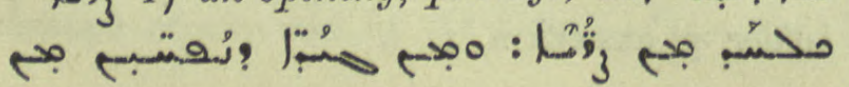

\section{$9 i^{2}$}

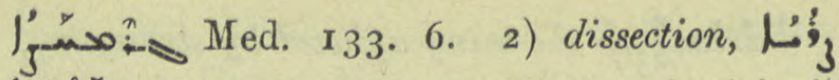

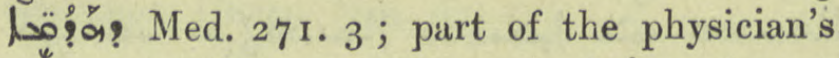
science, N. Hist. iii. ii. sect. 3 ; $\mathrm{L}^{\prime} \mathrm{H}_{3}$ is

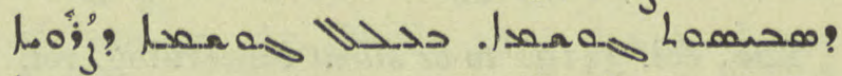
Leify so pany anatomy is the sundering of the continuity of the body by the intrusion of the anatomist's body [hand] into the subject for dissection, ib. iii. i. sect. 2.

15.' Sagittarius, the Bowman, a sign of the Zodiac, Med. 465. 9.

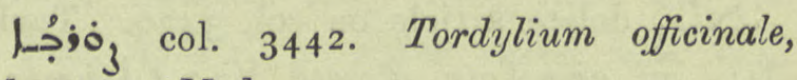
a hartwort, Med. 305. 14, 319. 12.

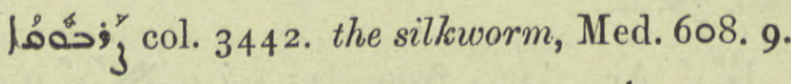
lo: ry resin; add: gloss to poulo kŵvos, Chimie 7. 13. Cf. under no suppl.

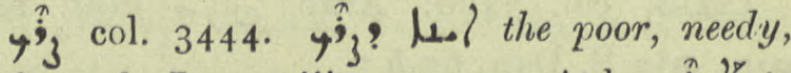
Ephr. ed. Lamy iii. 4I. I3. Aph. $y^{3} l^{2}$ to

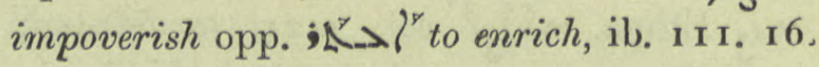

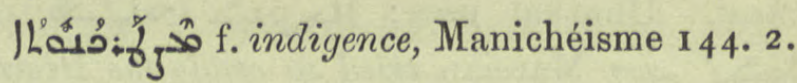
p)

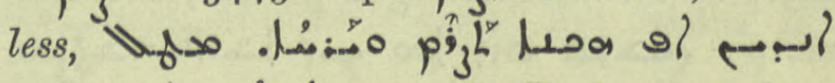
y to be wild, furious, a wolf hearing sheepdogs مatur I4. 1.

ILơosos f. audacious violence, Hist. Mon. i. 399.6 .

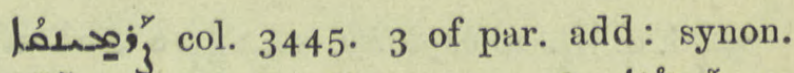

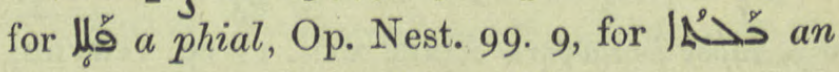
alabaster scent-boltle, Thes. col. $54 \mathrm{I}$.

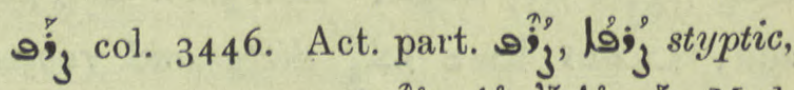

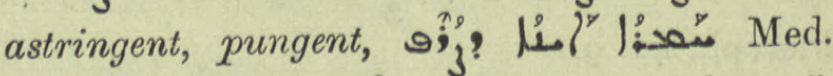

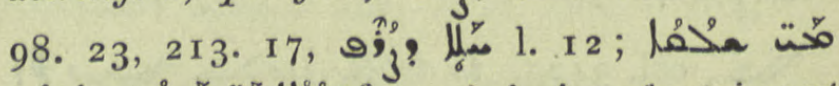
مُ and styptic substances, 1. $2 \mathrm{I}$. Trs. hither Geop. 46. 3 from col. 3347 under fơ on $\mathbf{y}_{-3}, \underline{2}_{-3}$. Ll. pen. and ult. of par.

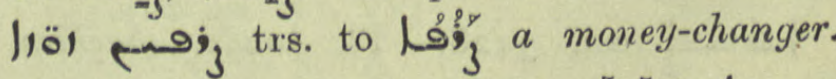
2) to refine, smelt, looper the Spirit rainly smelts us in the furnace of the Law, Nars. ed. Ming. $218,10$. 


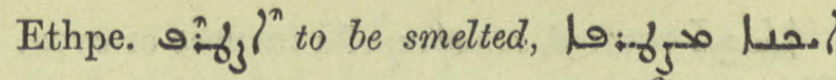

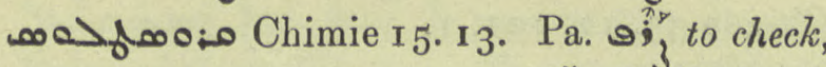
to contract the tissues,

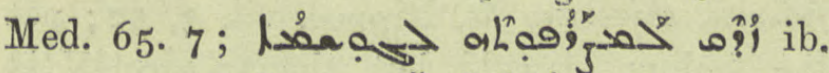
290. I, 1. 2. Aph. ف Part.

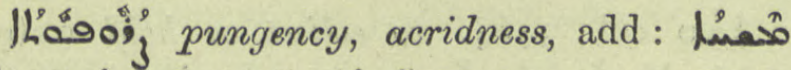
J' Med. 150. 2. Fruit

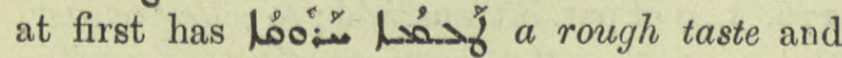
when mixed a little with the sun ILosoj; $>$ $\mu_{45}$ ô inclines to tartness, But. Sap. Philos: 6. 4 .

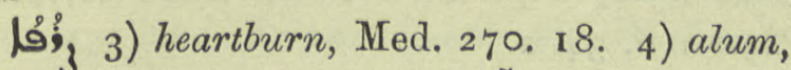

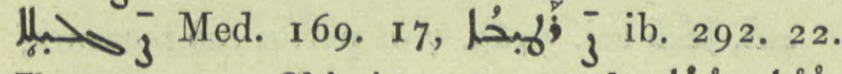

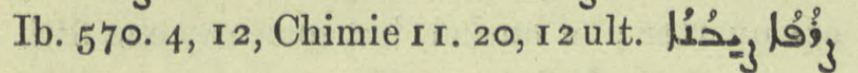

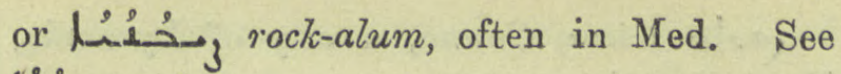
رِحُ

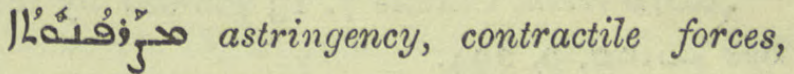

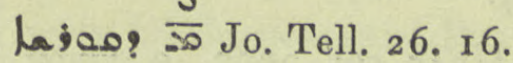

. Duval Gram. Syr. § 196 . Add ref.

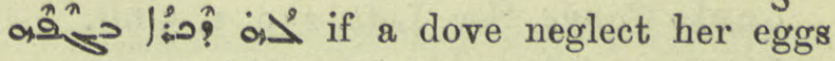
the male bird gives her a blow with his wing,

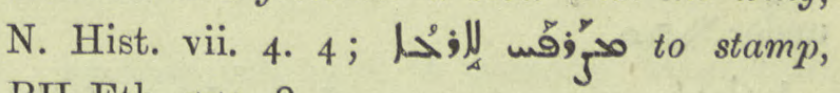
BH. Eth. 553. 8.

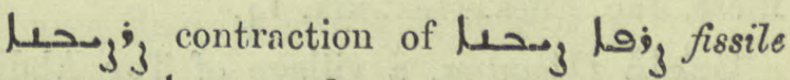
alum : see $\log _{\boldsymbol{\beta}}$ under $\boldsymbol{\Delta}_{\boldsymbol{j}}$.

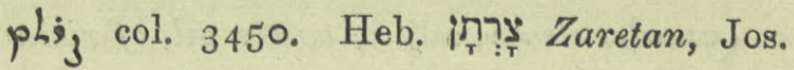
iii. 16 .
م, כa abbrev. I) for (andoefrain, farcing q.v. 2) for llo to the chant or tone, Brev. Ant. i. r $35.5, \mathrm{r}_{59} b 6, \mathbf{1} 64 b$ and often.

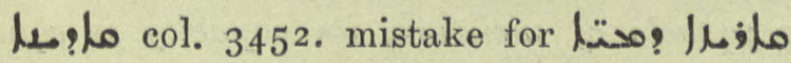
Pers. كاريز مارئ a subterranean canal, Nöld.'s n. on Sassan. 121 n. 3, Fraenkel on D BB. 24.17 in WZKM. iii. $24 \mathrm{I}$.

م مavoros, remittent fever, Hippoc. iii. 20,29 , iv. $5^{8}$, vi. 25 .

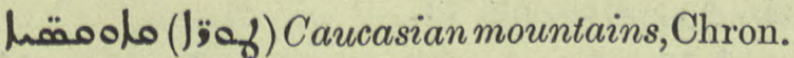

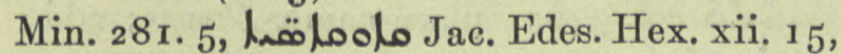
مامس Thes. 3453.

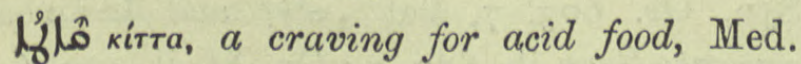
278. 4. I. q. As $_{\mathrm{b}} \mathrm{col}$. $360 \mathrm{I}$.

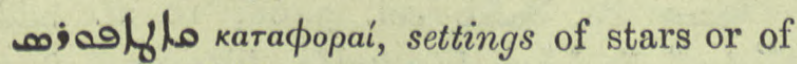
signs of the Zodiac, De Astrolabe ${ }^{2} 5^{\mathbf{I}}$ pen.

wholflo кarà кavinv, i.e. in the new part

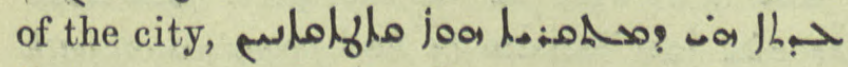
Ihema? era Sev. Ant. Hom. 3ri3. 8.

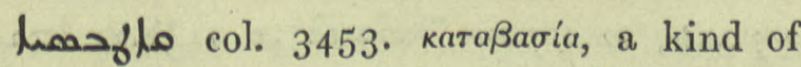
hymn, add: Dawk. 4, قاطباسيا R. O.C. iv. I6I bis.

2713

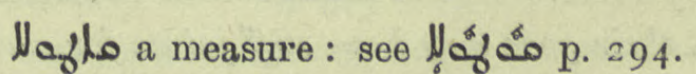

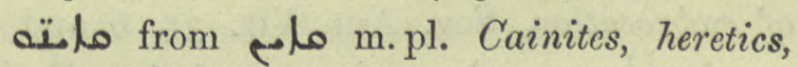
Coupes ii. II 7 .

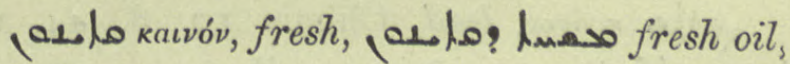
Chimie I6. I 7 .

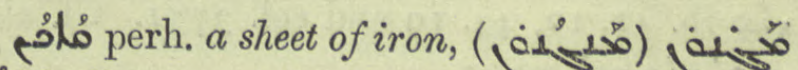
. A.M. Bii. 280.6.

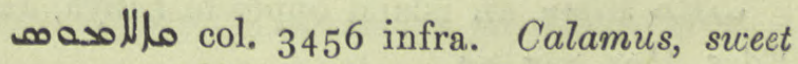

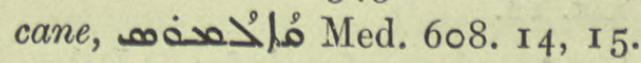

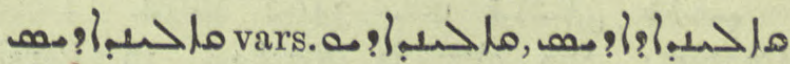

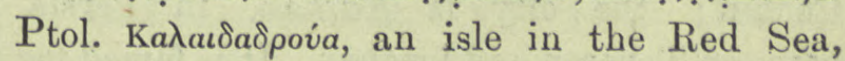
Jac. Edes. Hex, xx. 8.

ماخسماdه م pr.n.m. Callistus, Sev. Lett.

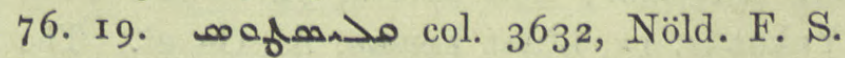
i. 473 .

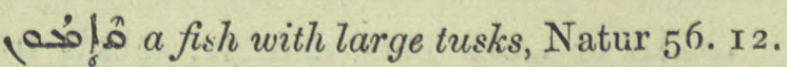

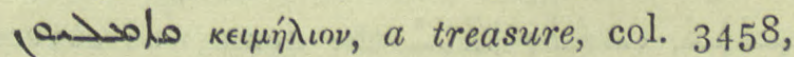
Pief. Diosc. ed. Nau 83. I I.

ماسنـ Kavapía, Canary Island, Jac. Edes. Hex. xix. I 5 .

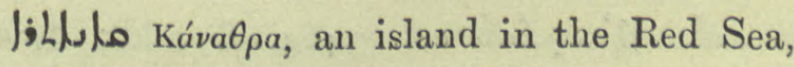
Jac. Edes. Hex. xx. 6.

P p 
مان Kávva, Canna, a city of Lycaonia, Nöld. F. S. i. 473 . 100.

(م) resin, Chimie 7. 13 glossed by log. See مصاس山م, p. 294.

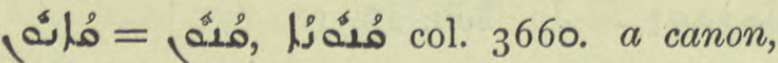
rule, Pallad. 28 ult.

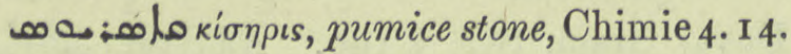
ه Capua, Sev. Lett. 25 o. I, 5 ult.

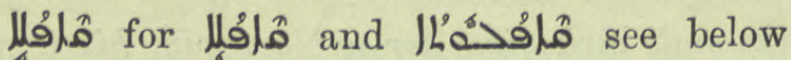
under

ماو:|\{ Karpapia, one of the Isles of the Blest, Jac. Edes. Hex. xix. I 4.

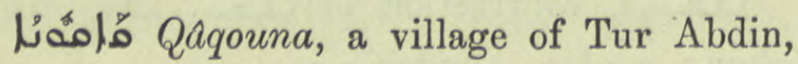
Mar Bassus 39. 540.

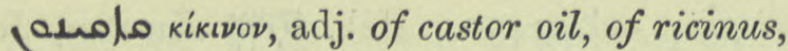

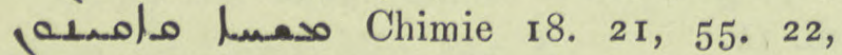
56. $7 \mathrm{ff}$.

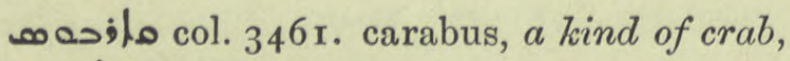

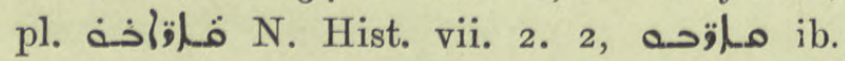
6. $3,6.6$.

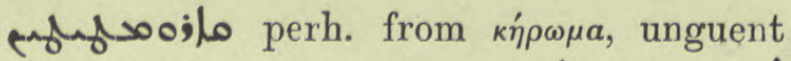

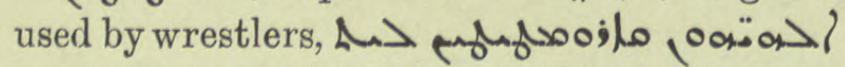
(oa) their gods they have stiff joints for want of embrocation, Sev. Ant. Vit. 35. 6 and n. wadel ما ib.

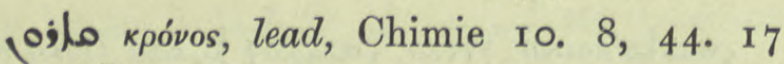
i. q. j卣 ib. 4 I. 18, م: مib. 44.3, I 5, I6, مُ ib. 42. I 2, 44. I 9 and col. 375r. Cf. also

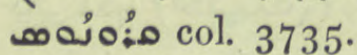

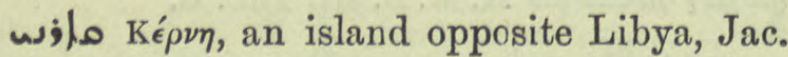
Edes. Hex. xix. I 2.

\section{قـاشـان مُامُمَ 3462 infra. Delete} Cochin; the city is Kashan, in Persia, between Teheran and Ispahan. It is famed for its pottery which is so fine it can be taken for porcelain; also for its enamelled tiles, Yakut iv. I 5, L'Art Persan, Al. Gayet. The ref. in Jab. 2nd ed. is 146.2.

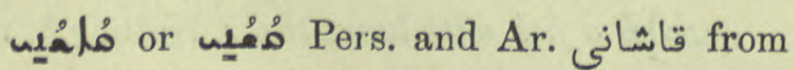

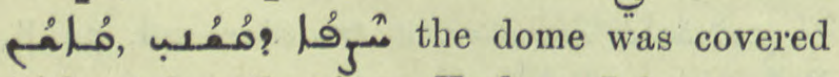
without by tiles from Kashan, Jab. I37. 6,

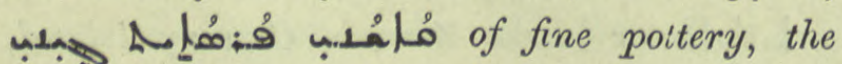
Persian name for china, ib. 145 ult.

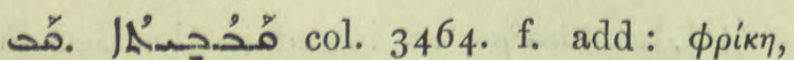

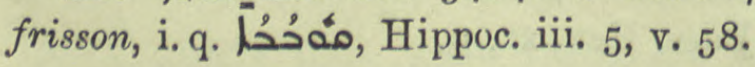

مُحمِف vaulted, concave, of a furnace or oven, Chimie 53. 12, ror. I.

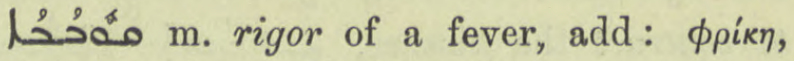
frisson, shivering fit, Hippoc. vii. $3,5^{2}$; not the feeling of cold but the hro\% / shivering and shaking of the whole body, Med. 4. $16 \mathrm{f}$., a symptom of quinsy, ib. I27. I I ;

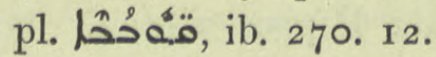

مُ col. 3464. to collect or hold water.

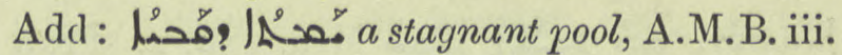
276. L. 9 of par. intrans. add : to collect matter,

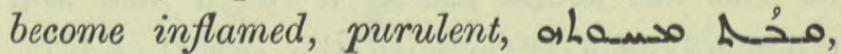

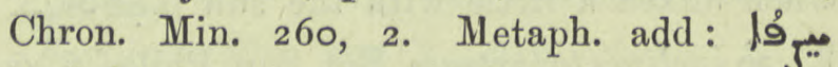

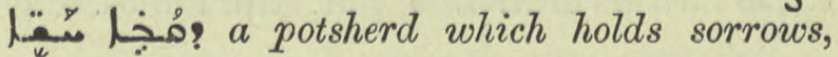
Narses ii. 34. 2.

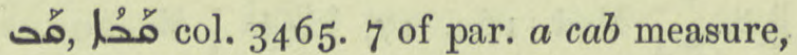
one quarter of a bushel, but some say one fifth or one sixth, Epiph. 4. For xoivı, Apoc. vi. 6 ed. Gwynn, pl. قـ ib. Bagst. has פסטمם م.

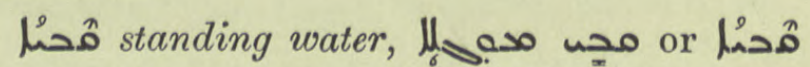

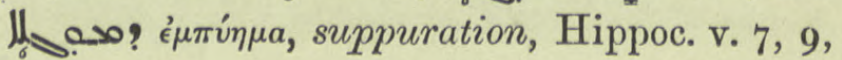
I 4,62 .

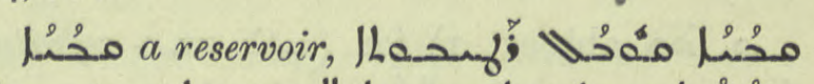

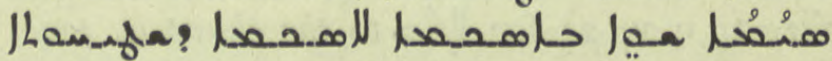

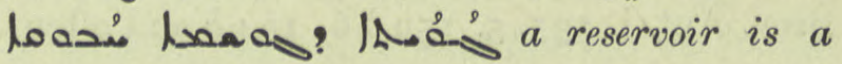
receptacle of moisture, its capacity is equal to the inner superficies of the containing body, N. Hist. iii. cap. i. sect. 2 .

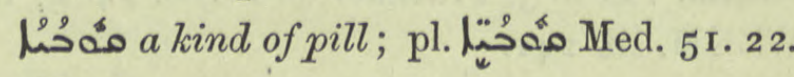

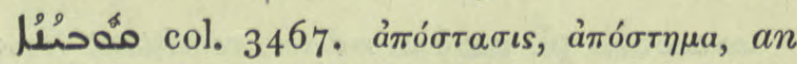
abscess, gathering, Hippoc. iv. 3I, 32, 5 I, 74, vii. 33 ; Med. 97. I 1.

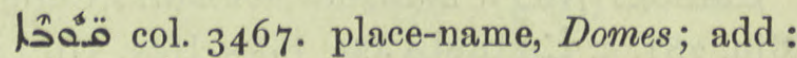

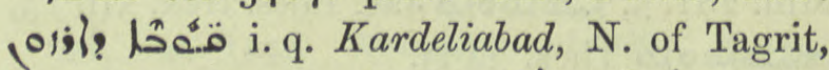
Syn. Or. 603. 7, but 101\%) and مقد as separate Sees, ib. 6r9. 3 ; Or. Xt. ii. 300. 6.

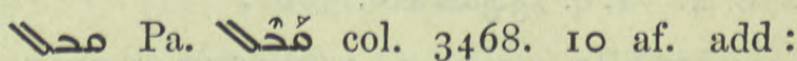

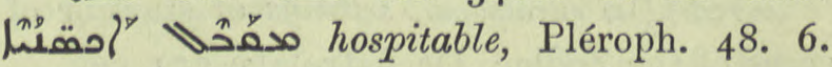
To promise, agree, col. 3469. זо. without حمدة

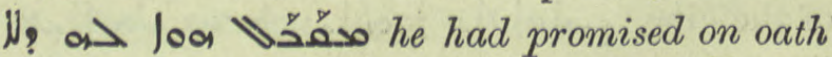

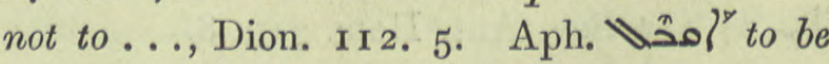
opposite, to oppose, "معد| oه joe a d darkness is driven away as light opposes it, Pléroph. 26. 10 =Mich. Syr. $205 b$ 
6 af. Saphel \مَّ to be present, part.

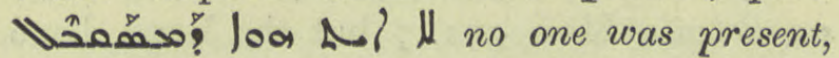
Ephr. ed. Lamy iii. 293. To appear suddenly,

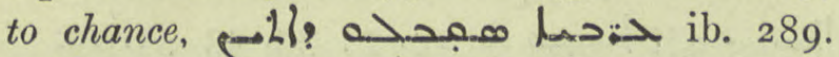
Cf. Midianites happened to

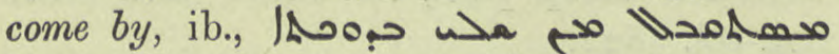
he arrived there unawares, Josephus vi. 2. I 2. Cf. Levy T. W. ii. 185 .

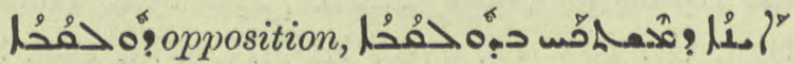

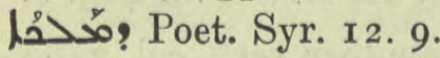

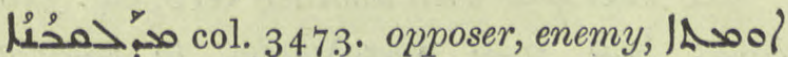
.

لم col. 3474. Matricaria chamomilla, مَّة chamomile tea, Med. 58. 5,

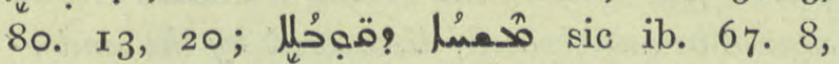

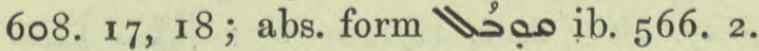

بـ for $\kappa a ́ \lambda v \xi$, the cup or bud of a flower, (Sap. 1 I. 8 Hex., Galen $72 v$ ) I. Löw would emend in both places /مقحح, ZDMG. xlvii. 519, Z.A. xvii. 90, WZKM. xii. 359. But add : inflorescence on a preparation of eggshell lime, Chimie 35. 10.

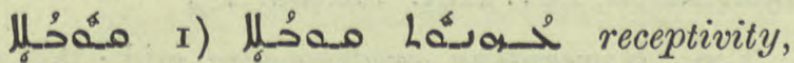
N. Hist, viii. 4. 6, 9 ; لمُ the being easily cut, Philos. 2. I. 2) Rit.

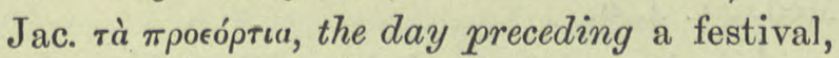
a vigil مrev. Ant.

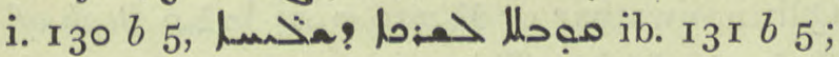
2 I6 $b$ I3 ; $217 a 8$. A hymn or psalm sung at Nocturns on vigits, lsoing ILa>, after the sedra a hymn and then the prayer of the censer, ib. $5^{8} b$ pen., مه hymn sung to the chant, The Two Worlds, ib. ${ }_{59} b 6, \mathbf{I}_{4} b$ I 3 , $1_{74} b_{3}$, ii. $467 a 3,264 a 4$ af., $474 a$ I, \&c.

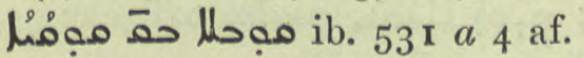

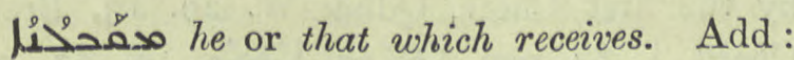

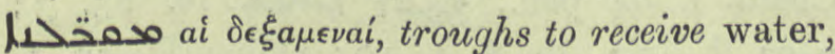
Hex. Ex. ii. I6. Capable, susceptible, the four

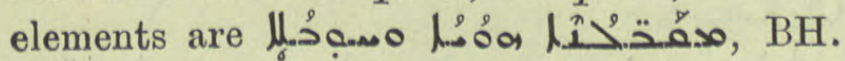

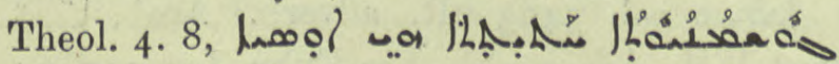

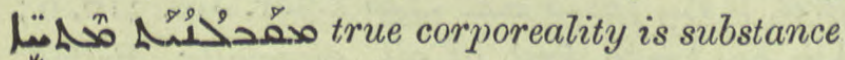
susceptible of measurement, ib. Philos. 4. 2 ;

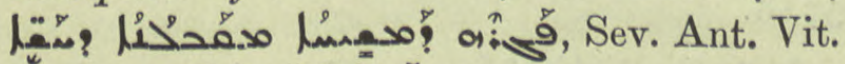

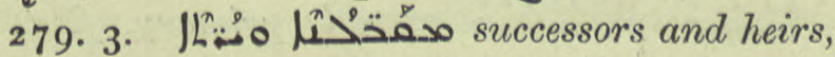

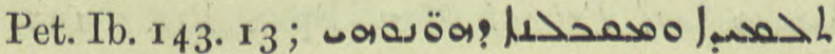

pupil and receiver of his ideas, Tim. I, ed. Braun I 6, but a guarantor, surety, ib. I 9.5 . Chem. the receiver of an alembic or still, Chimie I 9. I 8, 2 I pen. Rit. E.-Syr. a humeral worn by the deacon at the Communion of the people and on which he receives the paten, Maclean; مَّحَّ Takhsa 27 , I 46.6 , Hist. Mon. i. 263.7 .

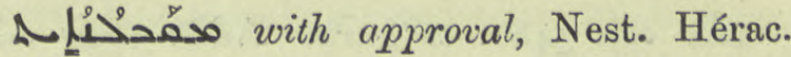
366. I 4 .

ل o- a ll la Bar Penk. I54 ult. Good

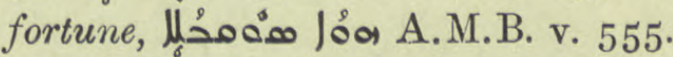

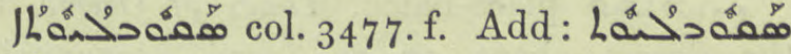

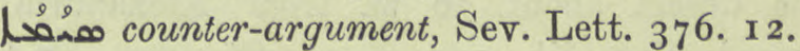

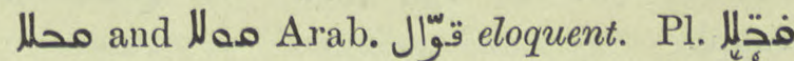
Qawwals, precentors and singers of the Yezidis,

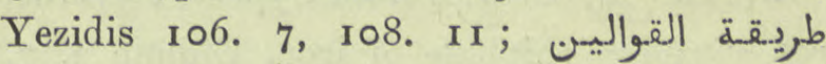
مخلl (2) the path of the Qawwals, ib. I 16.2 .

Cappadocia, Nöld. F. S. i. 47 I. 3 .

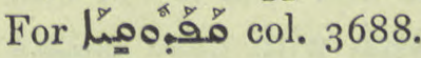

مخ.حم: mis-spelling of = م. م1ح+ cinnabar, Chimie I3. I6.

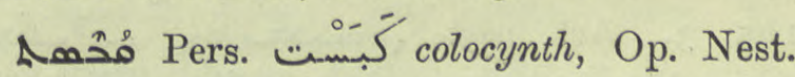
I I 2. I7 .

مدب م- مـ $>$ col. 3478.27 of par. Cf. هeither side got the better of the other but they st abilized their victory i. e. proceeded no further with it,

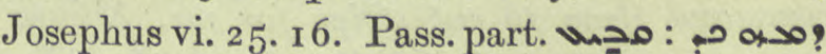
aíra $\pi \epsilon \pi \eta \gamma$ ós, his blood was clotted as hard as stone, Hist. B.V.M. $3^{8} .5$. Compact,

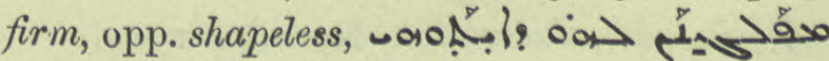

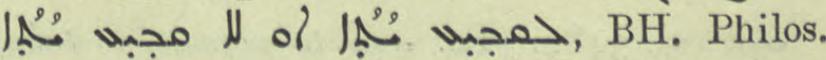

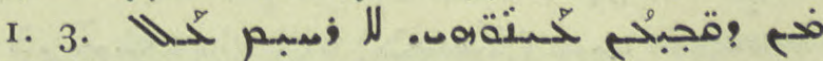
حلتّلُمُما he whose gaze is fixed, who stares at people, is not liked, id. Econ. iii. I. Inherent,

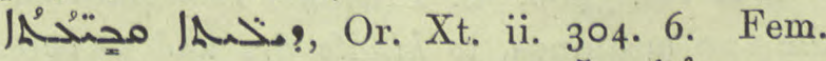

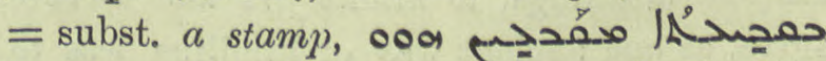
f foa with a stamp they affixed tokens on them $=$ branded them, Dion. $148 . x 6$. Chem. fixation, solidification, Chimie 329 note 4 .

A. محن infixed by instinct, implanted, of God's laws, Syn. Or. I38. 29.

$P$ p 2 


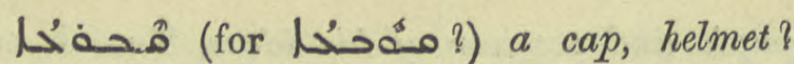
Pallad. 2I 5. $2 \mathrm{I}$.

 Med. 598. 5. Pl. معحل alids, substances, metals viz. silver and gold, Chimie $3{ }^{1} 4$ note 5 , Duval suggests مة حما.

محعملا Lat. cubiculum, a chamber, Stud. Sin. ix. 96. 6.

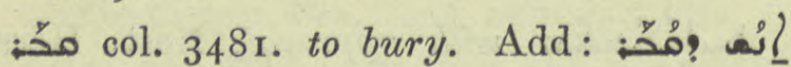

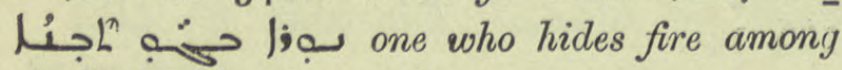
chaff, Pallad. 726. 3 af.

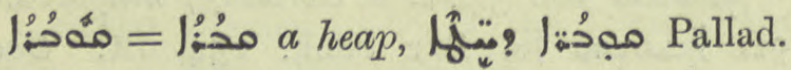
$553 \cdot 3$.

مص:م: place-name, Tomb of Osiris, in the desert about $30 \mathrm{~m}$. from Alexandria, Anecd. Syr. iii. I35 pen. Cf. مreo09 Suppl.

from Cyprus, copperas, red vitriol = sulphate of iron, Chimie 3. 7, 4. $5,63.16$; black vitriol (shoemaker's),

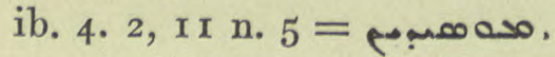

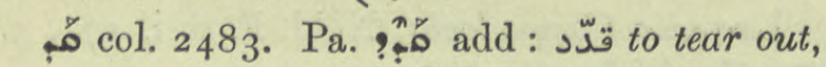

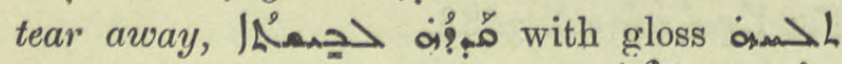

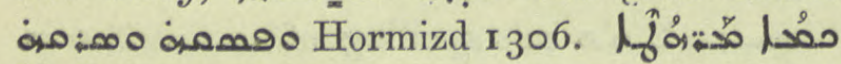
what riches did she strip off, Pallad.

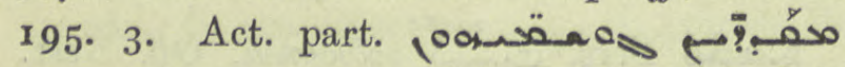
(0om 0 cats tore their bodies with their

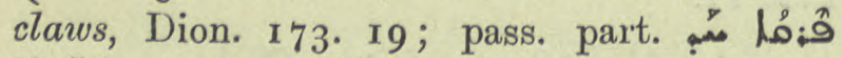

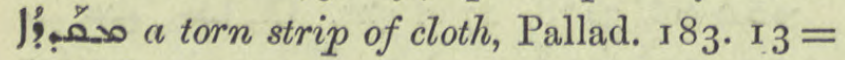
A.M.B. vii. 118.

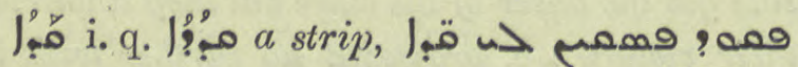
command them to cut strips for me, Ahikar

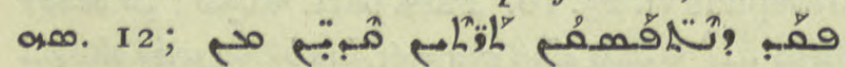
مب: A.M.B. iv. ${ }_{4} 6$.

مَٔ add : laceration, Hormizd $13 \mathbf{r} 9$.

col. 3484. Pa. مِّ to possess, to take

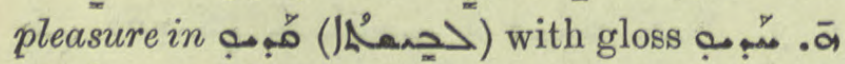

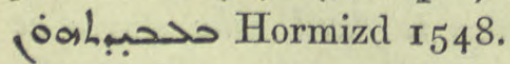

مبّْ col. 3486. S. Fraenkel remarks that the two meanings, to kindle fire and to bore agree, as one ancient way of kindling was to rub sticks; but that ou to tear out the hair is a mistake for סi Heb. Lev. xxi. 5, Jes. iii. 24, Jer. xvi. 6, Z.A. xix. ro8. But this requires further emendation of derivatives.
مُتِمَ small boils, Med. 591. 7 but the Lexx. have

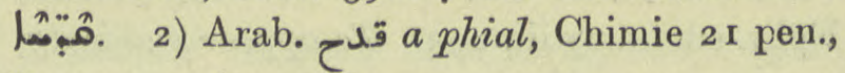
IAng مـ ib. 24. 7 ; 35. 23, 51. 19.

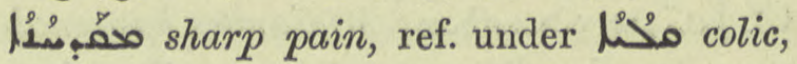
BA. col. 3622 .

مب معمله مبر the region of Qadis. Arab. قادِ near Herat, Syn. Or. I65. 12, 679; ZDMG. xliii. 405. 8.

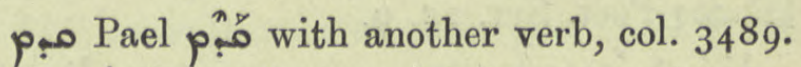

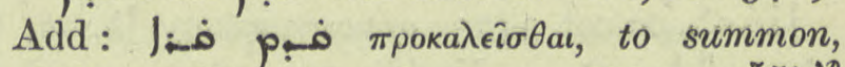

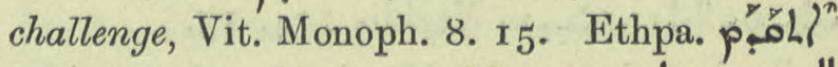
to anticipate, discover, add: للا محمصر

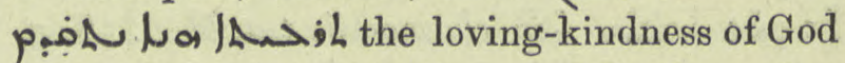
cannot be anticipated, discovered by the under-

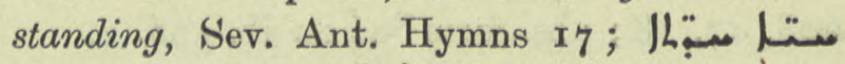

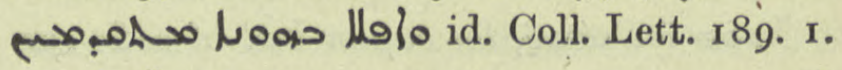

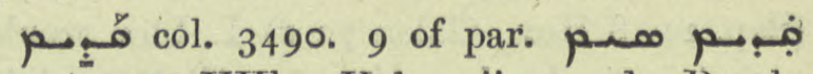

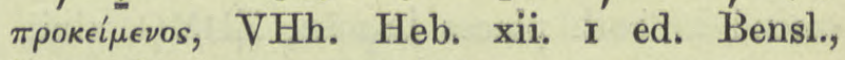

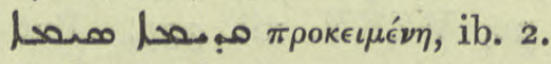

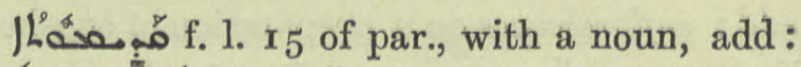

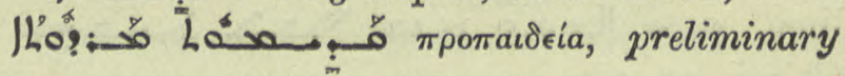
instruction, Sev. Änt. Vit. 66. I3.

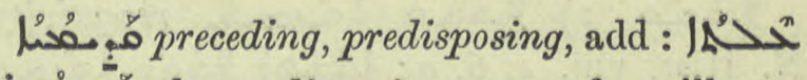

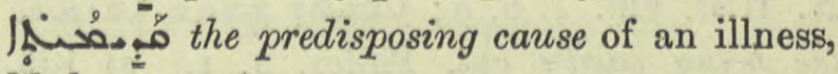
Med. 4I. $4,6$.

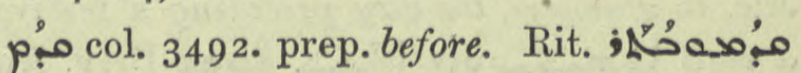

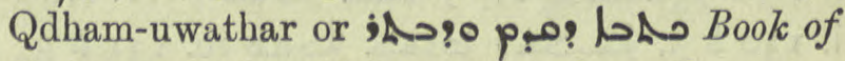
Before andAfter. This contains the anthems \&c. for feriae and festivals so far as they do not vary with the season. A choir is divided into those on the north side (our Cantoris) who are before or begin and the half on the south side (Decani) who follow $=$ are after, Daily Offices, Introd. and Glossary, Catholicos of

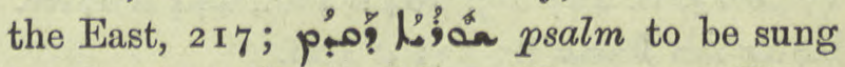
by the first Choir, QdhamW. 29. 13, Brev.

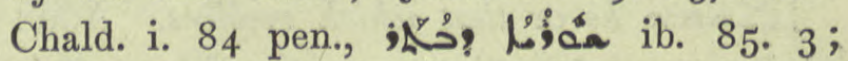

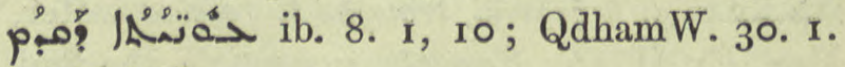

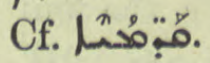

قدمَا col. 3493 ult. adverb. Arab

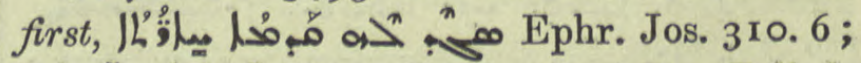

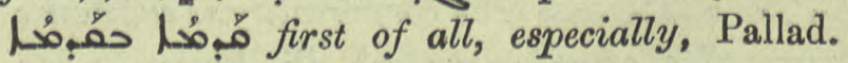
57.8.

ple primal, primary, first, إنما مبه 
27, 123.25 . Rit. opp. Lirst choir;

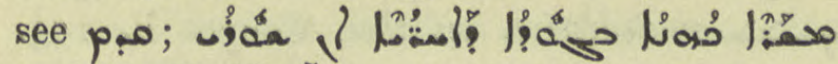

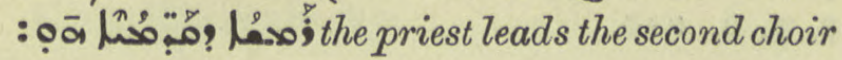
when the evening shuraya is sung by the first choir, Brev. Chald. i. 3. 20; :

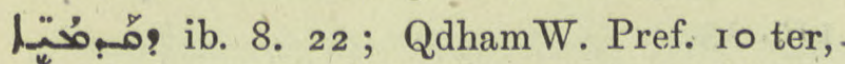
II pluries, and passim. week taken by the first choir, ib. 28 tit.

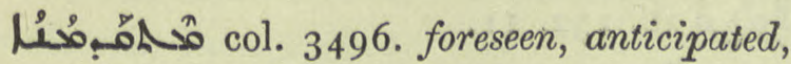
年 (the Virgin Birth) was unimaginable, Sev. Ant. Hymns 48. 7 .

مبهـ : Cadmus. Add 3497. Cad مبمه م Arist. Apol. $\longleftarrow$ I 3 .

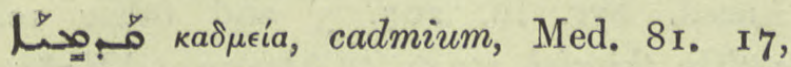
82. I 5, 20, 608. 19.

; col. $349^{8}$ ult. pr.n. Kedar from Heb. קד to be dark.

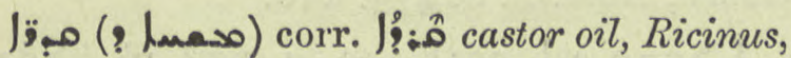
Chimie 3. I I.

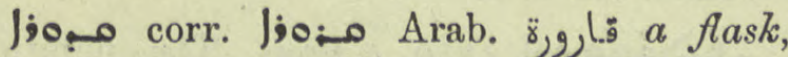

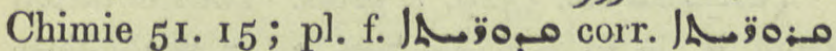
1. 10.

ן col. 3499. Anecd. Syr. iii 205. 8.

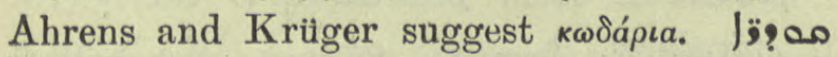
Lـح引\}? reed mats or mattresses, Zach. ed. Brooks ii. 23. $10=$ Mich. Syr. 258 .

مـ: Doc. Mon. 239. 8 corr. مـ, مـ Cordova, col. 373 I.

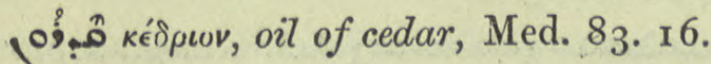

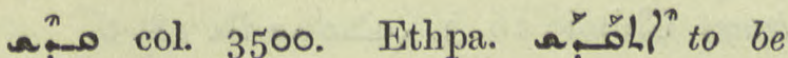

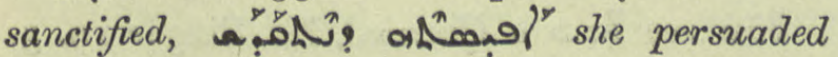
him to embrace the religious life i. e. to become a monk, Pallad. 196. 2.

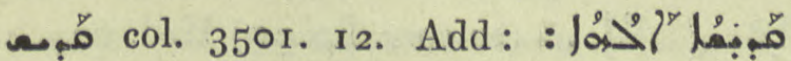

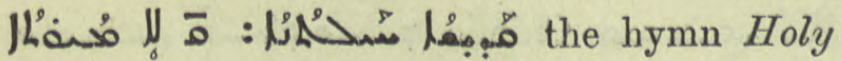
God, Holy Mighty One, Holy Immortal, QdhamW. 34. $5 \mathrm{ff}$; Brev. Ant. i. $134 b$ infra. This is the Trisagion, not the Ter Sanctus, see Brightman's Lit. $5^{89} \mathrm{f}$.

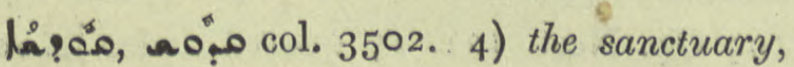
osually the space under the canopy, in front of the altar, Maclean. 5) the Eucharistic species, Maclean.
1 Ter Sanctus, Brev. Ant. i. I34 $b$ I6. 2) consecration, hallowing, Order of the Liturgy of the Apostles, Takhsa i. tit. Frequently an Anaphora opp. the whole مقومل Liturgy; the E.-Syrians have three

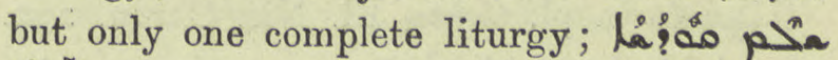

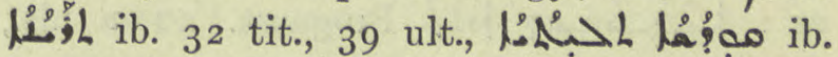

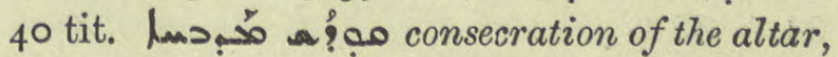

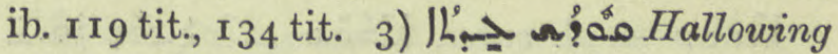
of the Church = the four weeks preceding Advent, Maclean, Brev. Ant. ii. i. tit.; heading pp. $\mathbf{I}-26$.

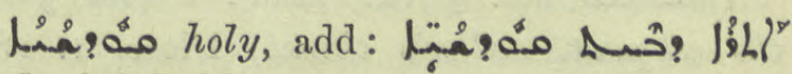
Palestine, Bahira 203. 23 .

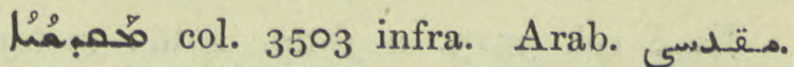
Not a native of Jerusalem but a pilgrim who had visited the holy city, Nöld. ZA. xxi. Ior ;

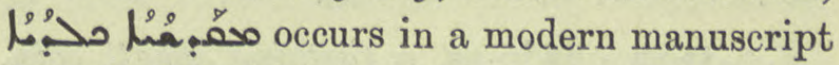
(г888), J.A.O.S. xx. I88.

مُُْمُ 3501. f. a nose ring, ref. Dion. 223. 10 .

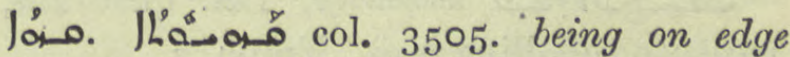
1" ? of the teeth, Med. I75. 17, 20.

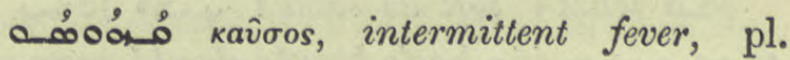

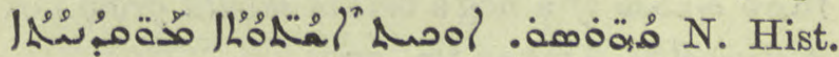
vi. 3,2 .

Mă col. 3506. Pa. Mâم to assemble, congregate, add: the Magi were commanded ! Anecd. Syr. iii. 187. I; \0,00 Mar Bassus 2. 22.

年 noisy, pertinacious, Hist. Mon. i. I58. I5.

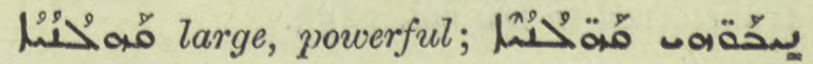

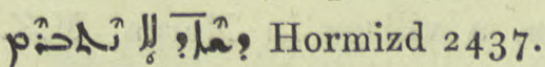

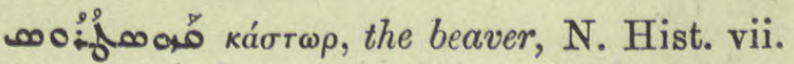
2. 3. Cf. jof مo col. $3^{675}$.

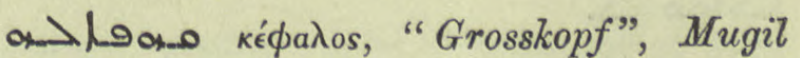
cephalos, a large-headed mullet found in the Mediterranean, FischN. $5^{8}$.

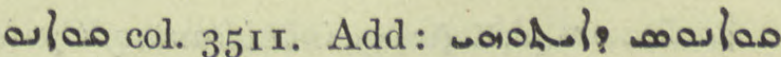

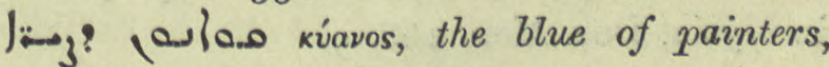

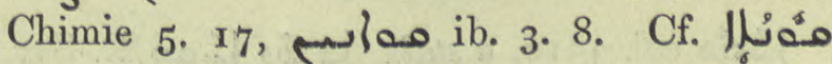

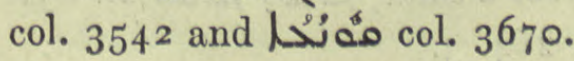




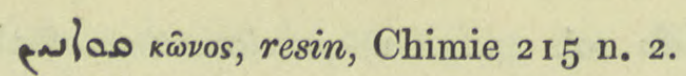

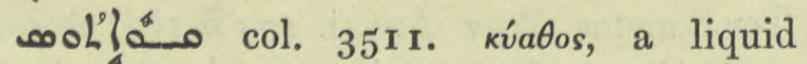

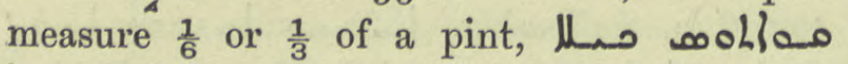

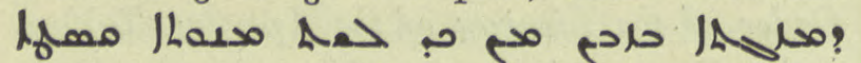
مال

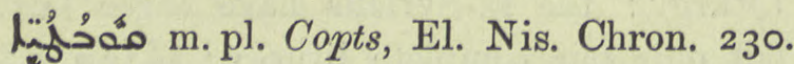

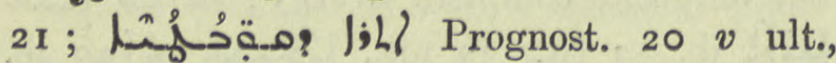
$34 v, 35 v$.

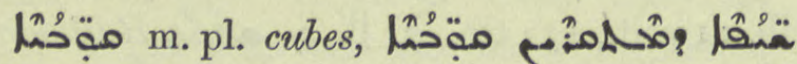
tablets called cubes, Med. 5I antep. Cf. مه a cube,

(2001. 3512. Add: Cottus gobio, the eet-pout, miller's thumb, Diosc. I. 18I, Ar. FischN. 8I.

مُ col. 3513. a fetter, Add: metaph.

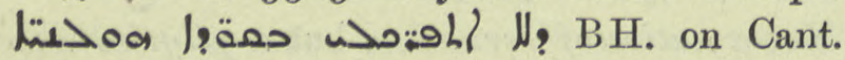

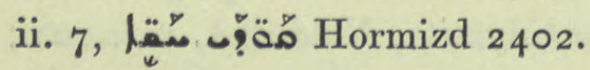

م, مa quadrans, a farthing, small coin.

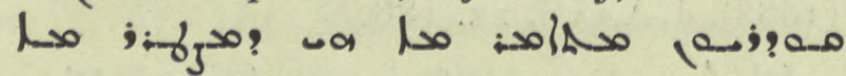

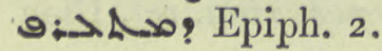

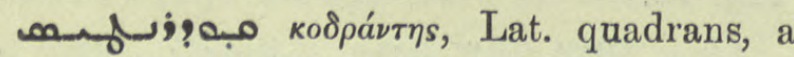
measure equalling $\frac{1}{4}$ oz., Epiph. 2 .

10 what? soft as sable? 1000 a: of if a hog's bristle should grow soft and woolly, Ahikar leo. 20.

10 imper. of verb كمل to peck, prick.

ا مـ col. 35ז5. a dance, a chorus.

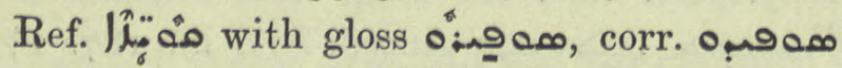

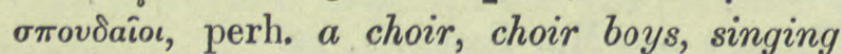
clerks, BH. ed. Gottheil ${ }_{5} 6$ n. 10.

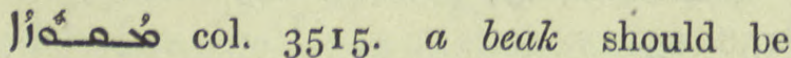
transferred to مُ col. 2453.

مَّ Kuzbu, a village in Marga, M.Z. 70. 19.

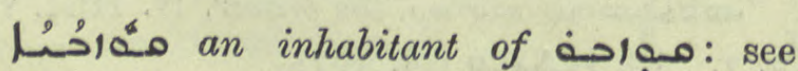

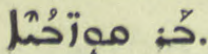

معر a village in Arzun, birthplace of the Patriarch Išoyahb I, Sassanidi 27. I 2.

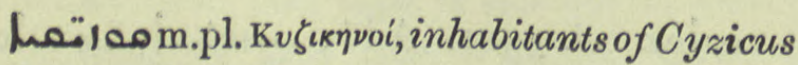
in Mysia, Doc. Mon. 268. 9.

 pointing, Med. 264. 3 . foo deriv. unknown. curse, low Jo حخد malediction on thee, Coupes ii. I55. $2,5,6,240$.

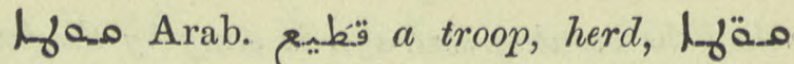

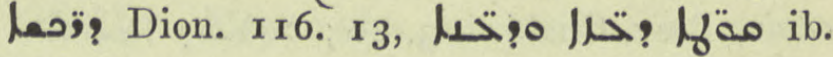
I17. I3.

مال

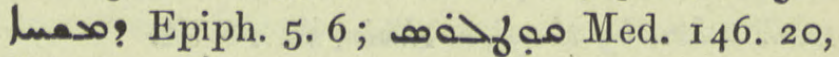
ل्ढه

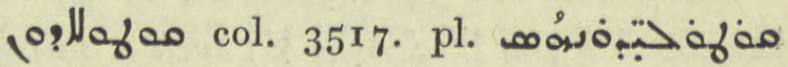

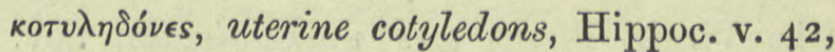
N. Hist. vii. $5 \cdot 3$.

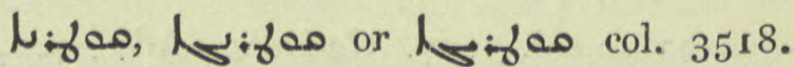

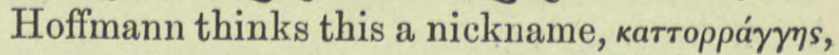
Anecd. Syr. iii. 207. 3 and other refs. catlike, thievish, see nn. to Ahrens and Krüger, 339 f. = Zach. ed. Br. 25. 24, 26. 2.

otwo Kováv, Konana in Pisidia, now Gönen, Nöld. F. S. 473. 118.

مبقخه col. 35 I 8 pen. a snail, pl. N. Hist. vii. 2. 2.

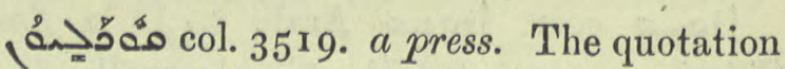
from K. is in BH. de Sap. 35.7 with gloss المبكبm.

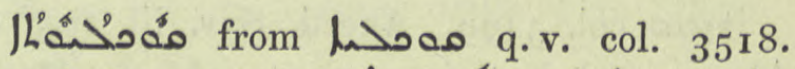

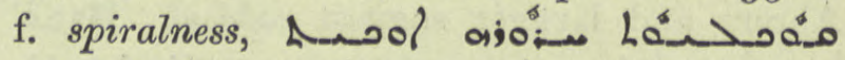

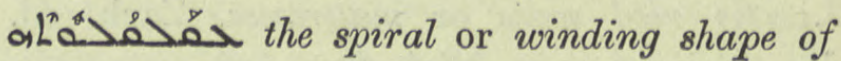
the orifice of the ear, N. Hist. vii. I. 2.

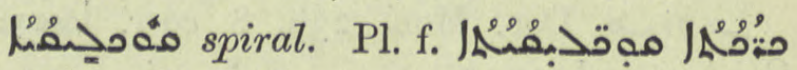
N. Hist. viii. 3. 2.

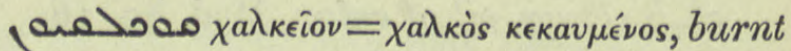
brass, Chimie 16. 3, مع محه ib. 18. 6.

فة a Q a dawal, Mt. Singar 19. 5, 25 n. 2.

مهُحلال :

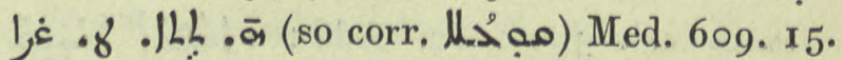
IL'n

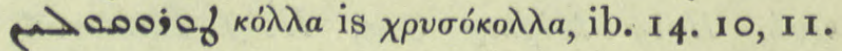

لمَّ a measure, 10 pints, Epiph. 6. 2-4; cf. مازظ Suppl. Itwo potfuls, Med. I43.

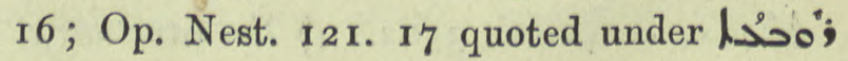
col. 3798 .

متل

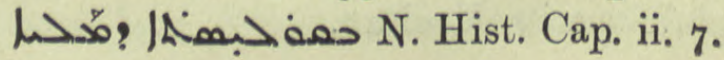


مه var. olace-name Koגón now Lake Tsana or Dembea in Abyssinia, Jac. Edes. Hex. xxiv. I 3 .

Claudiopolis in Isauria, Nöld. F. S. i. 47 r. 68.

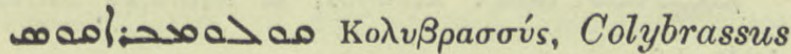
in Pamphylia, Nöld. F. S. i. 472.84.

رoه Lat. colonus, a husbandman, tiller of the soil, Dion. $23^{6}$. 2 .

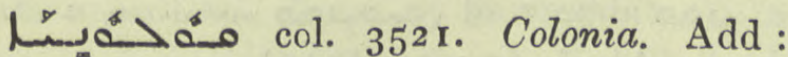
BH. Prooem. to Philippians.

מم col. 3522. кoi $\lambda \eta$, the vena cava,

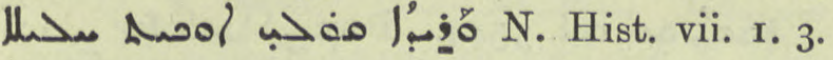
see لllue.

مه Colchis, Jac. Edes. Hex. xii. 14. Cf. محصدا a Colchian, col. 3634 . قولنج مه colic, Med. 57 I. I 5.

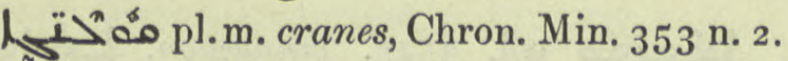
Marg. gloss to Jheogas.

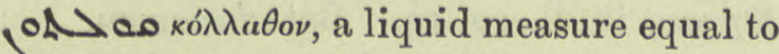

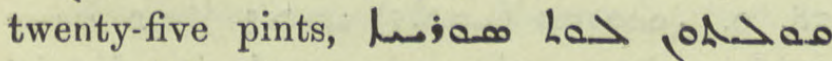

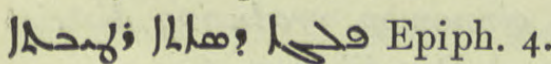

pao, مُ col. $35^{22}$ ff. Act. part, with suff. مre is still alive, Jos. ed. Wolflink I5. 6, r6 ult. Pl. f.

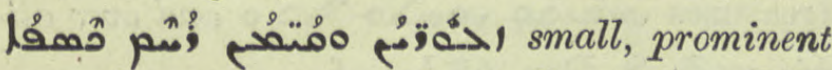
eyes denote an avaricious disposition, $\mathrm{BH}$. Econ. iii. r. With فلام to resist; Jare-proof, Chimie 33 pen.

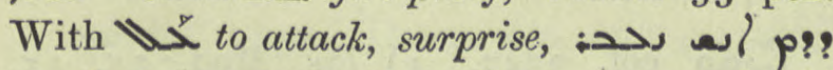
wa דar paso als lest anyone should pursue and surprise him, L. E. S. I30 ult.

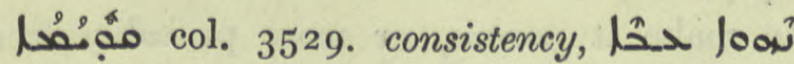

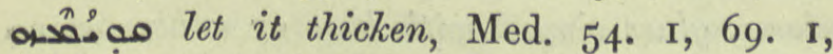

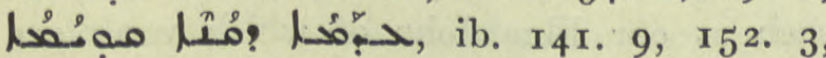

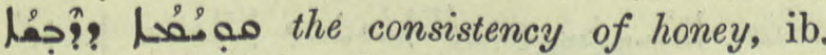

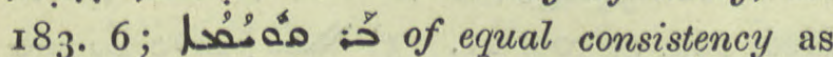

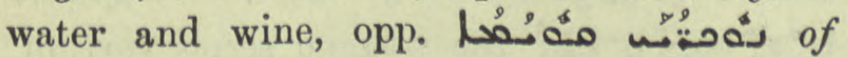
differing density as water and oil, N. Hist. iii. Cap. i. sect. 2.

مُ مُ col. 3531. 1. 5. a measure, the length of the outstretched arms, about a fathom, pl.f.

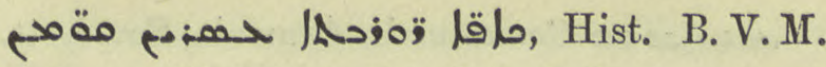

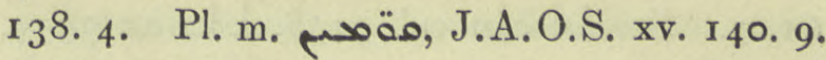

1 col. 3531. f. stature,

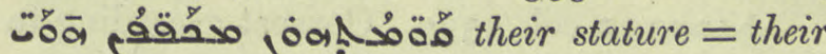
bodies were bent, A.M.B. ii. 345. I I. N.B.

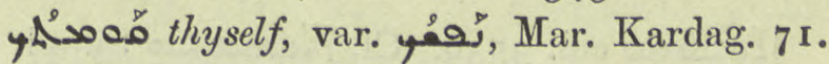

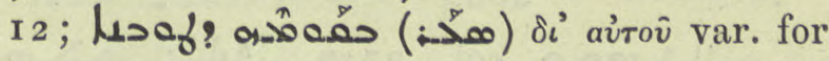
leof? a. 9 , A.M.B. v. 9 I. 19.

at a certain time, Pallad. 62. 5 .

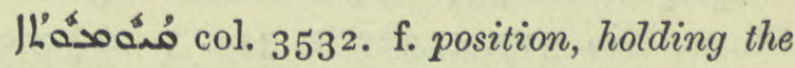

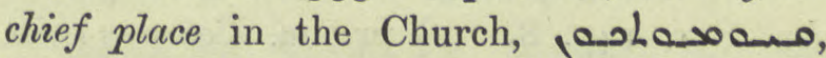
Išoyahb III. I5. I8. Pl, Jمهo stations opp. معرهقر journeyings, R. O.C. vii. I I 8. 5 af.

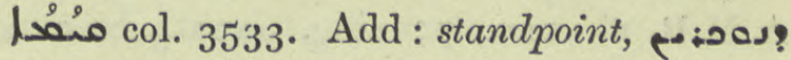
oasan opinions differing from their point of view, Ephr. Ref. ii. 7. 20. Dimension,

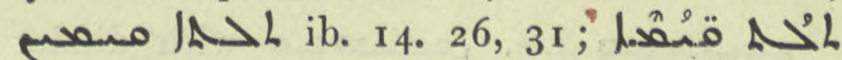
lson $0^{\text {s }}$ ? the three dimensions of a body, N. Hist. viii. 2. 3 .

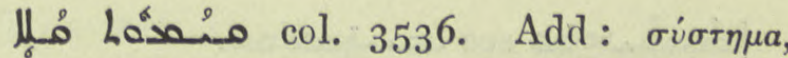
harmony, Hist. Mon. ii. I 49 note to i. 75. 8 and 293 note to $\mathbf{I}_{42}$. I 7 these refs. are the same as those of B.O. iii. i. given in Thes. Syr.

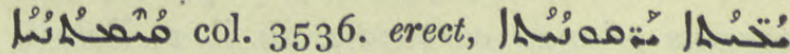

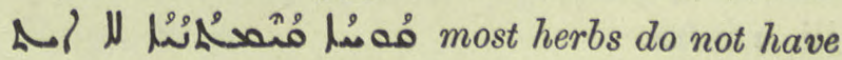
an erect stem, $\mathrm{BH} . \mathrm{N}$. Hist. vi. 2.2.

مَمْمُ col. 3537. m. metaph. persistence, resistance, evil and sin cannot stand against repentance, Hist. Mon. i. $39 \cdot 7$.

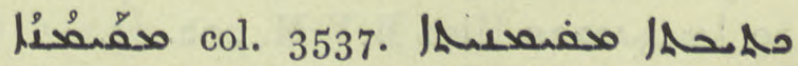
a letter of recommendation, Sev. Lett. 297. 3 .

pa col. 3538. Add: Kum, in Persia W. of Hamadan and 80 kilometers NNW. of Kashan, Syn. Or. 6 r 9.

مه

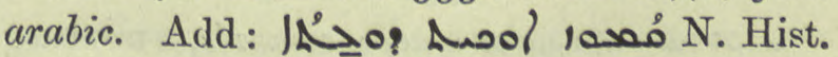
vi. 3. 3, مم: ib.,

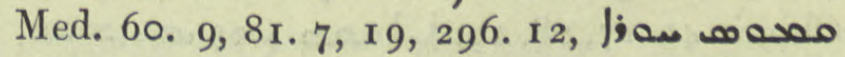
Chimie 48. 23, 5r. r6, jexa ib. 34. 6, 227 n. 3,23 ก 3 . 3

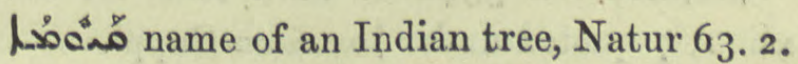


م

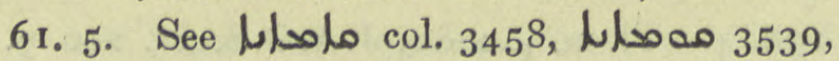
Leror.

3539. Correct according to M

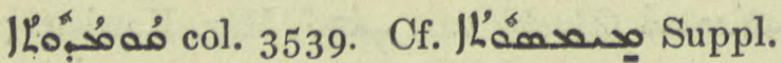

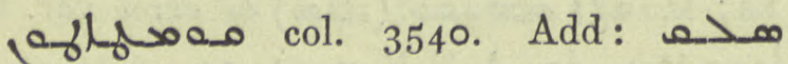
ofsroم he was going to Court, Pléroph. 4 I. 13.

مه مهلم Chron. Min. 317. 1, 320. 2, 322. 3, of An. Syr. 145. 8. 2) pr.n.m. Cometus? ib. Chron. Min. 66. 2 1, 67.2 ; cf. Sev. Lett. i. 45.

مع مسم comes, -itis, a count, A.M.B. vi, 120. 7 ; sing. ouflis Pléroph. 75. II, مع مسلمع 76.2 Chron. Min. $3{ }^{1} 7 b$ I, $320 b 2,322 b 3$.

م- i.q. م- مa with dimin. termination, cummin, Chimie 273 n. 6, 276 n. I; مذهمبف (sic Med. 609. 7.

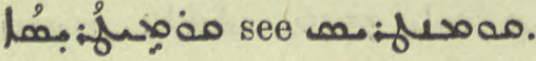

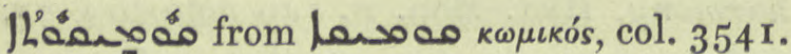
f. revelling, carousing, one of the foolish trades,

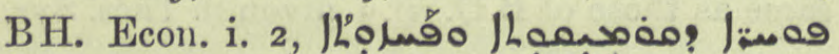
taverns of mad carousals, ib. ii. 2 , Pol. ii. I. iii. 2 infra.

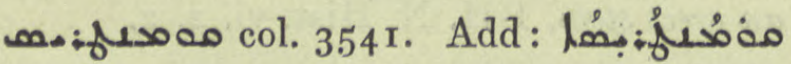
m prison, A.M.B. iii. 299. 10, 312, 350,

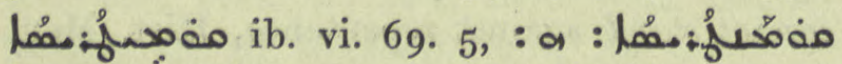

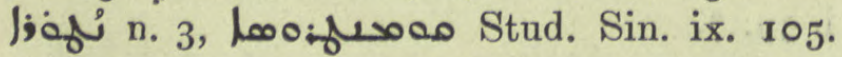

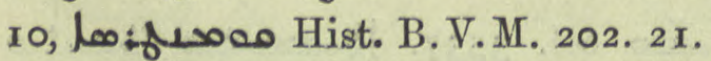

مه مع مه pr.n.m. Comnitos, corrupted from Kopqríavos, Budge, Hist. B. V.M. 95. 8.

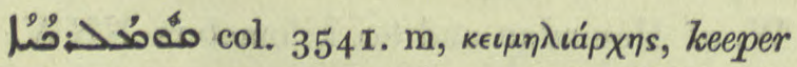
of the strong room, treasurer, ZDMG. liv. 382. I, S. Fraenkel note, ib. $5^{62}$.

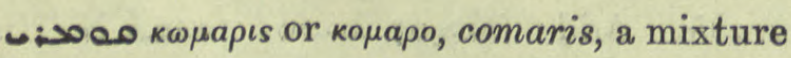
of lead filings, \&c., used to dye artificial gems,

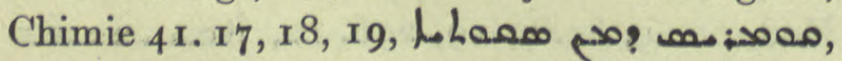
ib. 13.24 trans. 26 note 1 .

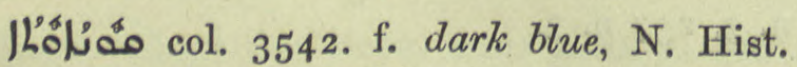

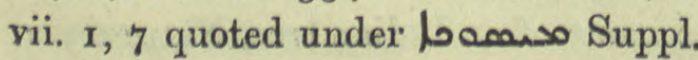

(20) 3543. Canopus, a suburb of Alexandria, Aneed. Syr. iii. 183. 2.

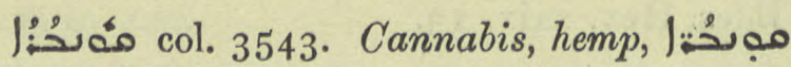
Ly. Med. I72. 17 .

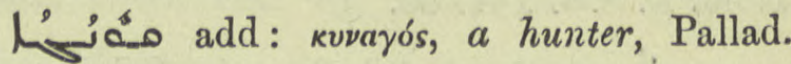
136. 19 .

a.jhy a congiarius, a measure containing six pints, Epiph. 6. 4.

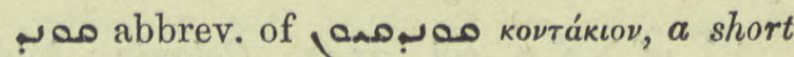
hymn, Alexis 65 ult., R.O.C. iv. ${ }_{54}$.

col. 3605 kivóvขos, peril.

كنداور fors. a governor, commander, S. Fraenkel ZDMG. xlv. 3I9 on Gest, Alex. 203. 10.

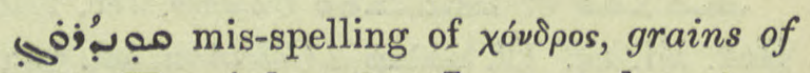
معبزo incense, Hormizd 1269 . Lexx. under col. 3659 .

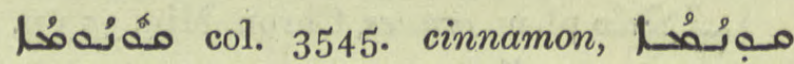

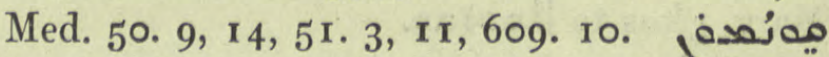
Styrax, ib. 1l, ro-r3. But cinnamon, Natur

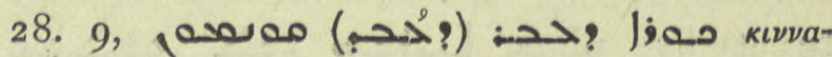

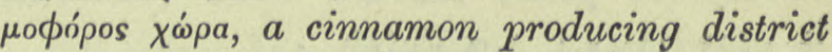
in Ethiopia, Anecd. Syr. iii. 330. 6.

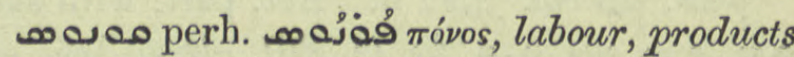
of labour, he gave them power over his treasures مخ مخدo and over all his goods, S. Dan. $68 b_{4}$ af.

مarso Lat. Quintilis, July, Georg. Ar. 7.18.

مَّمَ col. 3547. I) 7ye, Med. 55. I5, مفنسئl alkatine ointment, ib. 92. 7 . 2) pottery in which ashes (kovia) are mixed to make it porous, or more probably from kv́avos, glass tinged with blue or a blue stone such as our Blue John of which vases are

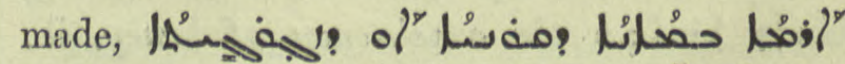

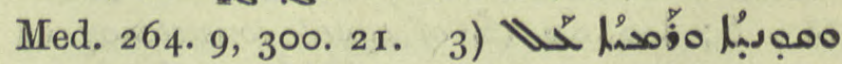

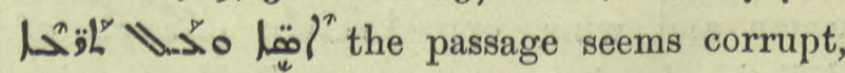
if it means she strewed ashes on the wall and the doors, the of 0 wants a point above instead of beneath and the o before fios: should be deleted, A.M.B. v. 441. 9 . 
مبن gentilic, of Qoni, Syn. Or. 44. 23 , ZDMG. xliii. 396 pen.

(20) col. 3548. 2) אóviov, lye, wood ashes, Med. 93. II, 609. I 4. Usually written مذّيَّا. مع for кvávєov, blue, Duval, Chimie I I. 8.

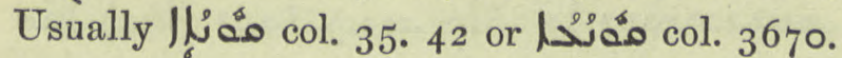
همبلمعهل مفذبمُر BH. Stories 10. 23, pl. مفببقا ib.

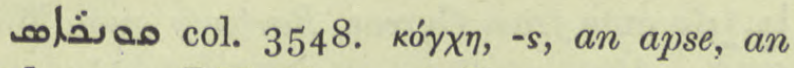
oval recess, BH. Nom. ed. Par. 15 ult., J معدا Pet. Ib. I I. 20. Usually q.v. col. 3666 .

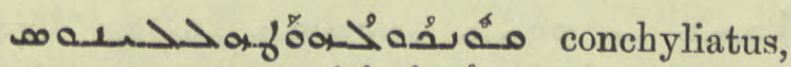

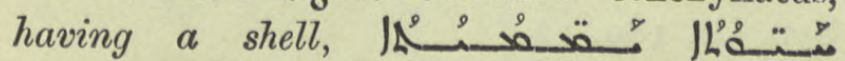
مف نصف sea shell-fish, N. Hist. iv. $5 \cdot 3$.

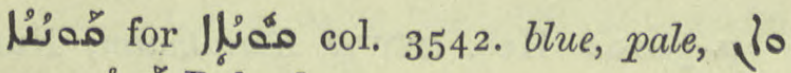
a) as Rylands 44. 22 a.

مع Kus, town on the r. bank of the Nile, م Pajotection 3. I7, xxvi. n. 2.

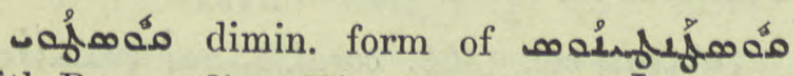
with Pers. ending, Hist. Mon. i. 225 , ii. 426 n.

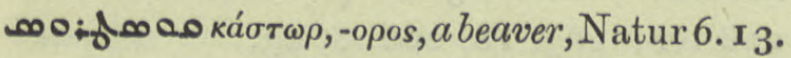

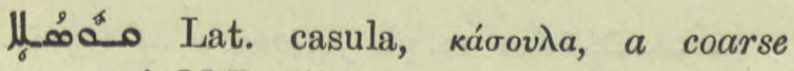
garment, A.M.B. vii. 4I 4 .

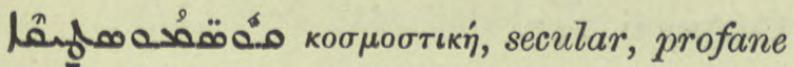
opp. to and formed after the analogy of

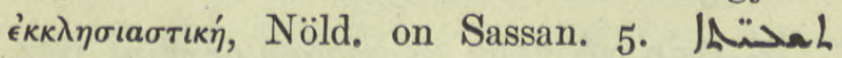

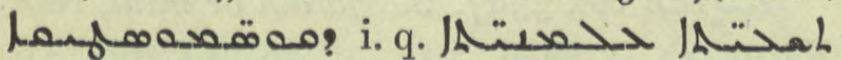
histories of worldly matters, Sassanidi 7. 2, =Syn. Or. 9. 4 .

مُ col. 3551. Delete par. and see

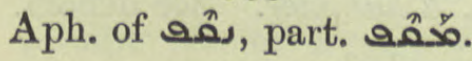

๑م์ col. 355 r. a village, add: in Marga, Chast. 49. 8.

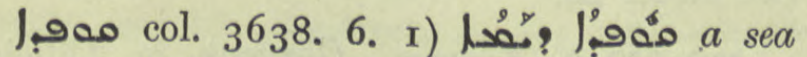
urchin. Add : a fish found in the Upper Zab, Brockelmann, ZA. xvii. ${ }^{2} 56$, Ar. FischN. 76 ; Hist. Mon. i. 394. I, 5. To refs. from Lexx.

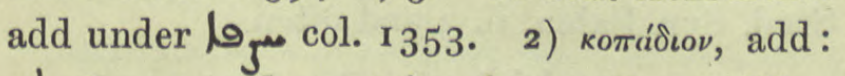
A long strip of meat, Praet. Miss. 83. 97 .

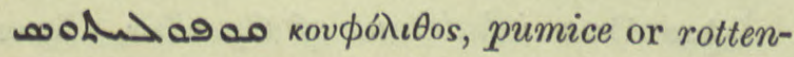
stone, "pierre légère", Chimie 234 n. 3. 2716

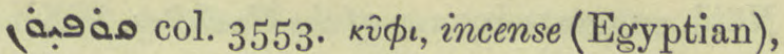
V. Loret's study of Kyphi, Journ. As. I887, Cyphus, Diosc. i. xxiv. 6 drachms of it are

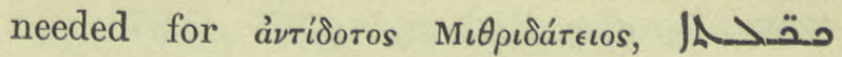
(مه I 16, I I 7, Med. 245. I3, mentioned among

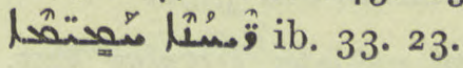

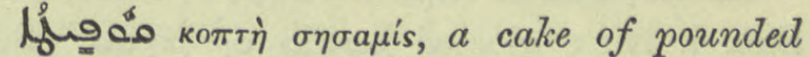
sesame, pl. مدفقبر Op. Nest. I 1 2. 3; Ar. FremdW. 37 f.

لla

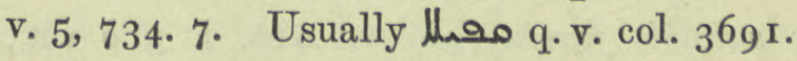

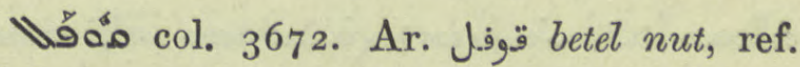
Med. I74. II, 430. 5, 609. BB. gives to la peach, ed. Duval I 497.20.

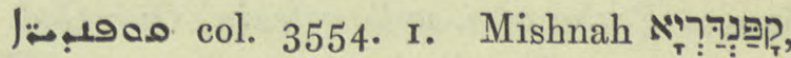
Lat. compendiaria, short cuts, I. Löw ZDMG. xlvii. 5 I 6 .

人مُهم on Sassanidi 26 n. 3. Hailstone, El. Nis. Chron. 2 I 7. 15, 23.

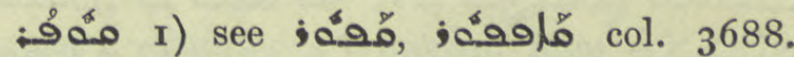
camphor, Med. I42. Iо. I3. 2) name of a medicine prepared from myrrh, ib. 356 . 10.

J: مهن kúpos, a measure equalling 2 bushels, Epiph. 6. 4.

(

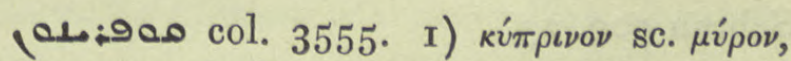
unguent made from the flower of the cyprus or gopher bush, Med. I48. I4, I49. 6, $3^{20}$. I3. 2) Cyprian copper, Chimie 54. 2 i. q. مa ib. 5 .

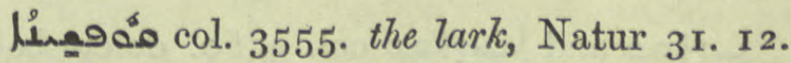

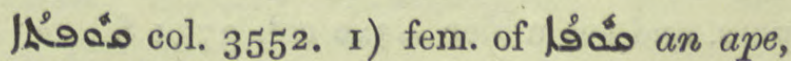

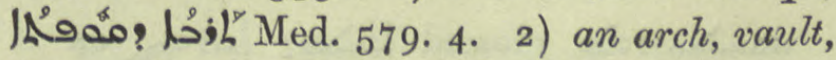

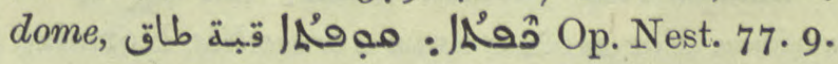

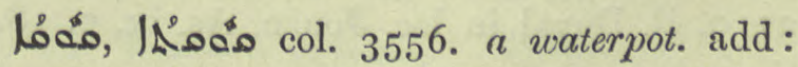
مفنمتا :حتدهو I 72 ult.

นُمْمَ col. 3557. The potter; surname of Simon Kukāya, composer of many hymns: see 1l. 5 and $\mathrm{I} 8$ of par., hence ellipt.

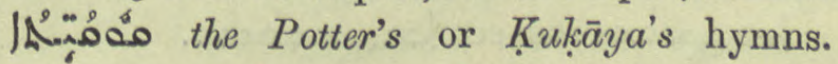


Nöld. suggests hymns of the Cuchites, WZKM. xii. 355. Add ref. Coupes ii. 144. 3. Infra placenta, add : مَّمُ "cakes" containing aloe, Med. 45. 19.

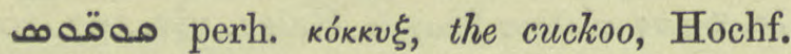

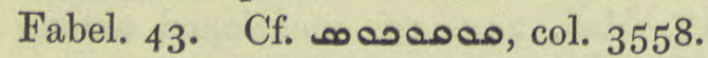

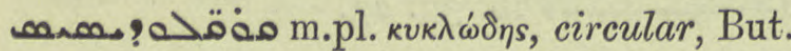
Sap. i. iv. 3 glossed مa of of of See ص مه col. 3558 infra.

(201. 3559. Add: Jac. refrain,

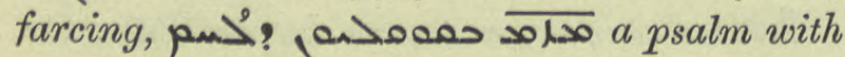
its suitable farcing, Brev. Ant. i. $5 b$ ult.,

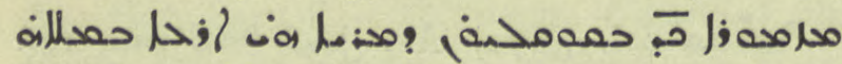
Psalm xxiv. with refrain, the earth is the Lord's and the fulness thereof, ib. $\mathrm{1} 25$ a

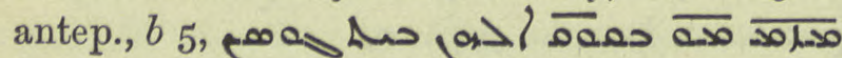
lha-> Psalm xlvi. with refrain, Our God is

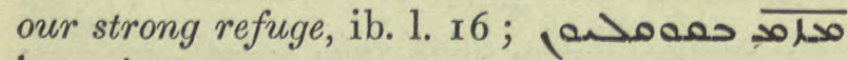
h 2 L Psalm to the eighth refrain, ib. $128 b$ I,

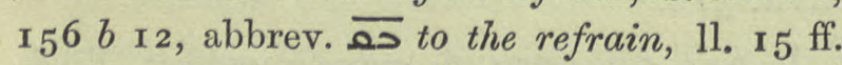
Psalms may be said L/fres simply i. e. read

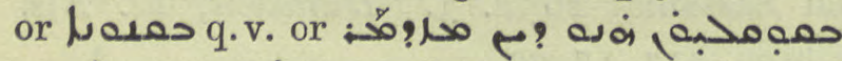

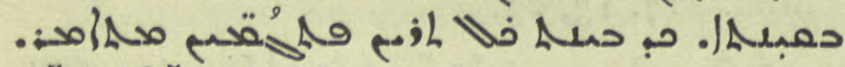
a Hallelujah being said between each couple of phrases, ib. Kal. $53 b$ infra, 54 . I ff.

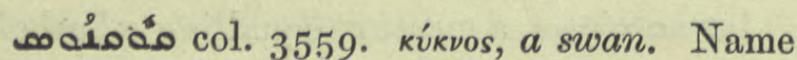
of a white eye-salve, Med. 82. 13.

مه Qur, a village in Adiabene, JesusSabran 509. 23.

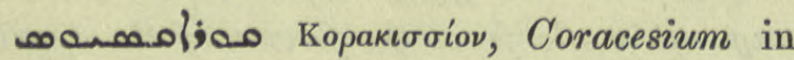
Pamphylia, Nöld. F. S. i. $47^{2} .85$.

مـ Kurds, part of place-name: see

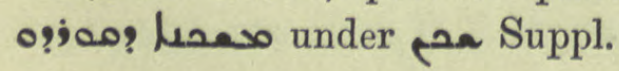

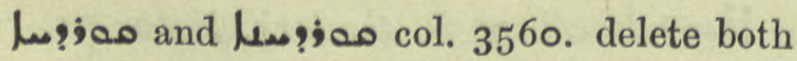
pars. and see under wi col. 373 I f.

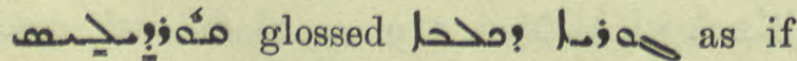
кvvísıov. For a crocodile, Hormizd 2710, R. Duval in loc. Journ. As. ix. 5. 184. See cols. $35^{61 .} 7$ and $373^{6}$.

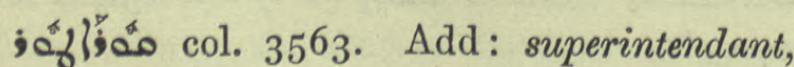
steward, Jo. Eph. 407 ult., Sev. Lett. 128. 5,

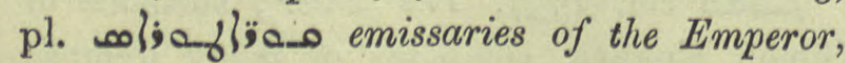
Jo. Tell. 39. 10.

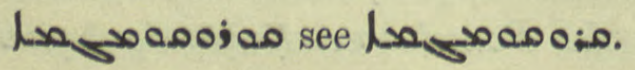

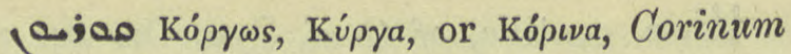
in Lycaonia, Nöld. F. S. i. 472.96.

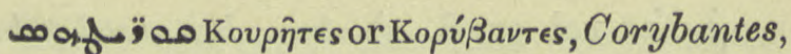
Greg. Naz. in BH. ed. Moberg 62. 24. Note

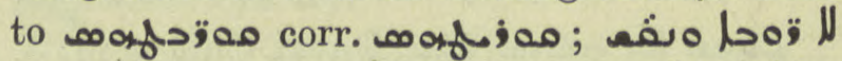

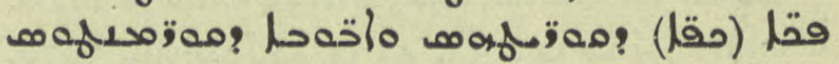
id. Gram. i. 27.2 I f.

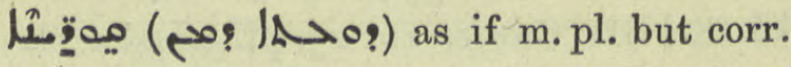
ئم gum from Cyrene, Barbary gum, Med. 87. 9, 160. 2, 184. 18. Asa foetida. Cf. مدهز ـuppl.

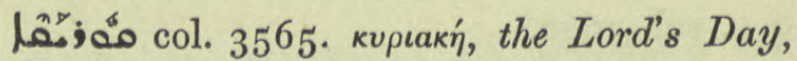

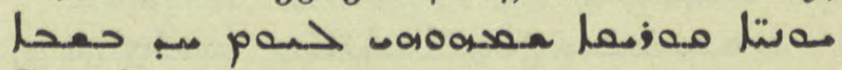
Syn. Or. 189. 12.

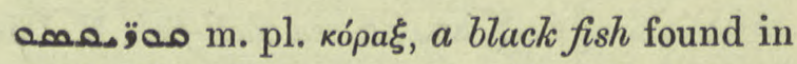
the Nile, Pet. Ib. х 28. 9, Ar. FischN. 80.

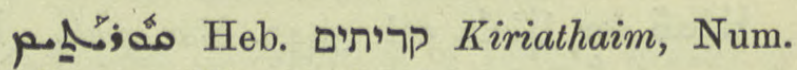
xxxii. $37, x$ Chron. vi. 76.

لأ col. 3566. Delete end of par from 2); a mistake for $ل_{3}$; مa see col. 3757 .

مبزלالحف

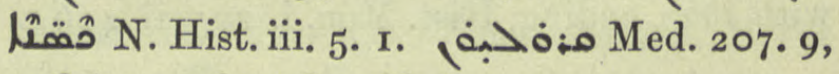
208 ter; $263.18,266.8$, I3.

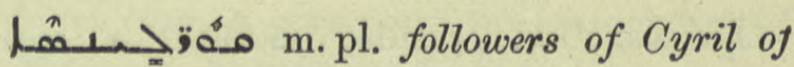
Alexandria, G. Busâmé 42. I9, fôn Coupes ii. 147 .

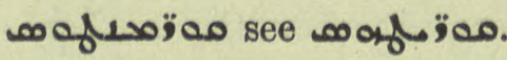

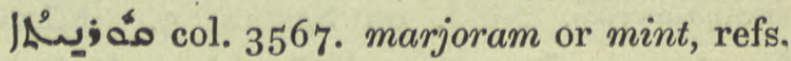
Natur 44. I, Med. 50. 10, 59. I 4, 80. 21 ,

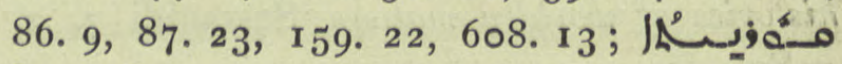
jow! ib. 308. 20, j;of? 5 ib.

إن col. 3567. a hammer. See denom. to hammer, col. $375^{2}$.

مه; معه pr.n.m. Cursus, Can. J. Tell. 22. See under

مـ Kـ Kápvkos, Corycus in Cilicia, Nöld. F. S. 470.54.

مه; Lor Lat. cursor, a courier, Stud. Syr. Rahm. ii. 3 and often.

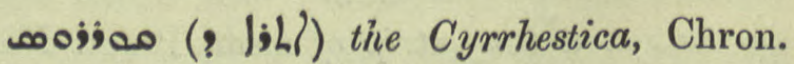
Min. 254. 24.

hosoingabitant of Cyrrhus, Chron.

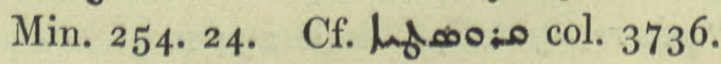




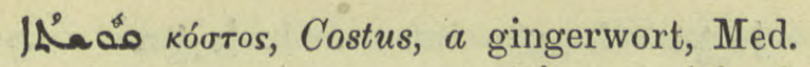
51. $2,13,52$. ib. 328. 7, 362. 12, قسط ib. 608. 17. Two refs. to this spelling are given under of of col. 3549 .

مُ col. 3569. a small bird, fly-catcher, Natur 25.9.

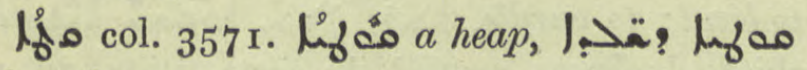
Dion. 89. 19.

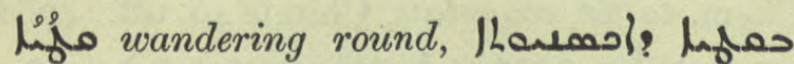
loa y gad o he had wandered about in exile, Dion. 69. 14.

aـ/4_ col. 3572. Kótauva, Cotenna in Pamphylia, Nöld. F. S. 472.86.

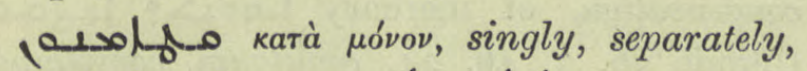
A. M. B. iv. 282 ; see oforo/flo col. 3454 .

مै a salve for stiff or painful muscles, Med. I46. го.

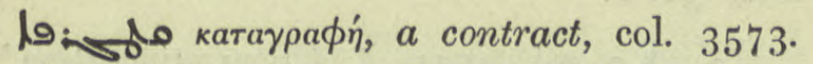
2 of par. Corr. ref. to Syr. Rom. Rechtsb. to

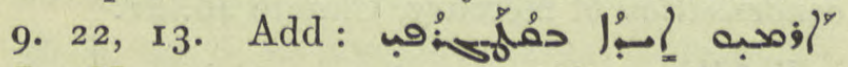
Jos. Narses 104. 4 ; a receipt, acknowledgement, Dion. 170.15 .

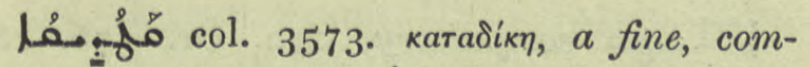

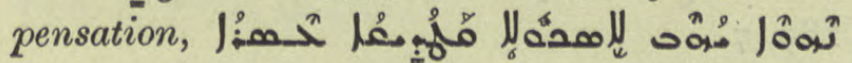

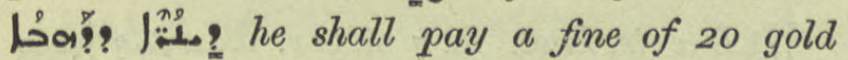
dinars to the School, Stat. Schol. Nis. 187 pen., 189. 14 .

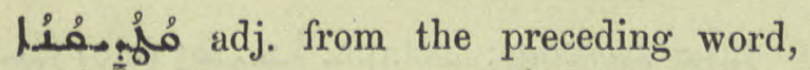

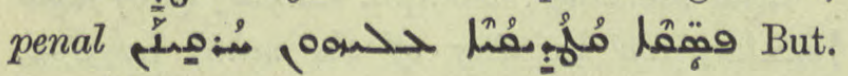
Sap. Philos. 2. $\mathrm{I}$.

مِئه col. 3573. r) Ar. linen, N. Hist. ii. iii. 3 , Med. 577. I I.

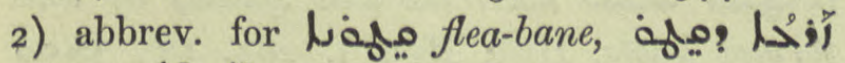
gloss to lin Med. 603 ult.

人ُ مُ cols. $357^{2}$, 3580. 2) Psyllium or Inula coryza, flea-bane, sool

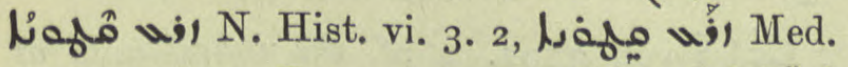

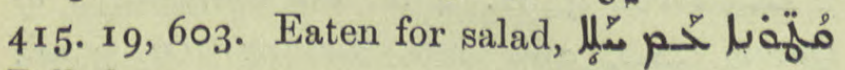
Pallad. 335. 17 .

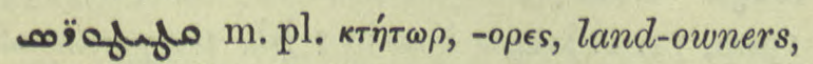
proprietors, Sev. Lett. I24. 20, 255.5 .

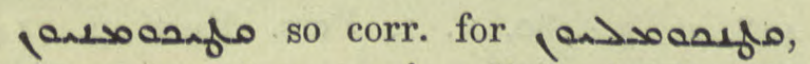

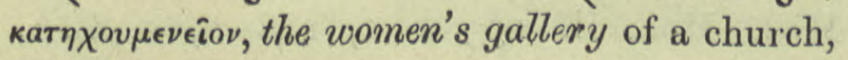
L.E.S. $33^{6 .} 8$.

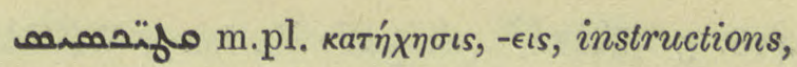
catechizings, Sev. Ant. Vit. 27 r. 6.

Mjo to cut down, fell trees. Cf. bop Levy NHeb. WB., Mand. לג, Arab. בשל (ij) the cedars have been felled, Coupes ii. $5^{2}$. 5, Nöld. in loc. WZKM. xii. $35^{8}$. n. I.

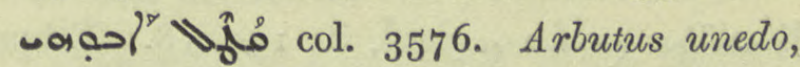
strawberry bush, Med. 264. I 7, Ar. PflnN. 334. ل لِ . concoction, Sev. Lett. 233. I I. 2) entangle-

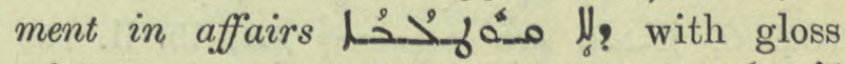

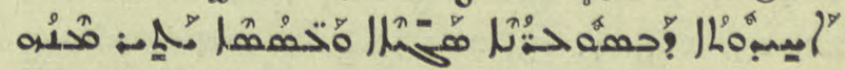
Hormizd 684 .

uم م col. 3579. ashy grey clouds, ef. IAw col. 3743 .

ميل ملّم

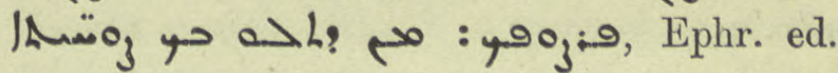
Lamy iv. 2 I9. 12.

من요 1.نo:? finely chopped pomegranates, Med. 213.19.

م f. pl. subst. insects, N. Hist, vii. 2. 2 bis.

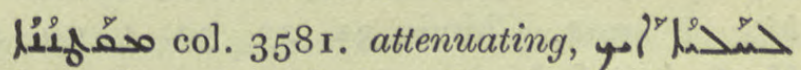

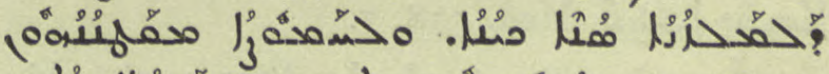

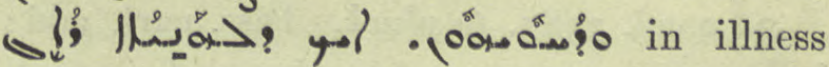
Nature hates sweet food as nauseating and desires as pleasant the sour which attenuates and expels vapours or humours, BH. Theol.

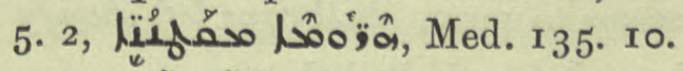

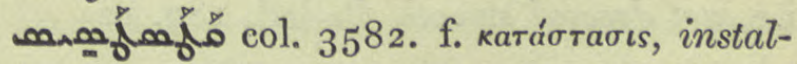
lation of an Emperor, Pet. Ib. 68. I I, of a Patriarch, ib. 7o. 3 ; state, condition,

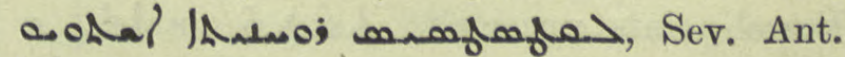
Vit. 48.13 .

u. o col. 2582. Arabisms. Astron. to

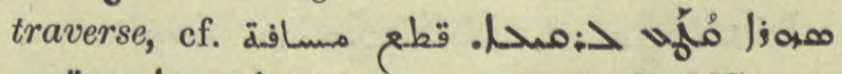
Rylands MS. 44, fol. 2 I a. With قط to impose taxes, Dion. 204. 23; often with lé to do damage by exaction, ib. 166. 3, 199. 5, 204. 19. 232 . I7; to cut purses, مُخْح

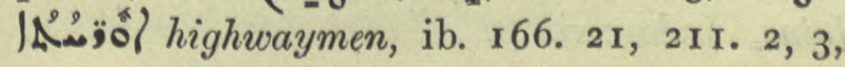
Q $\mathrm{q} 2$ 


\section{مח}

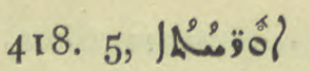

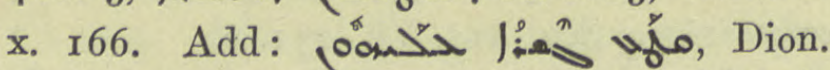

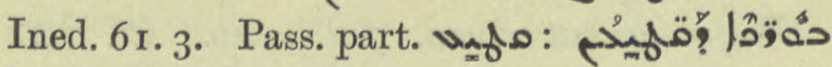
broken-kneed, Pallad. 726. 2. F. emph.

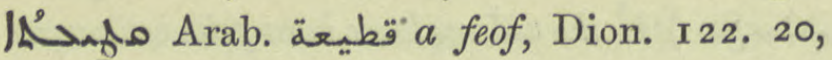
WZKM. x. 166, but an exaction, a tax,

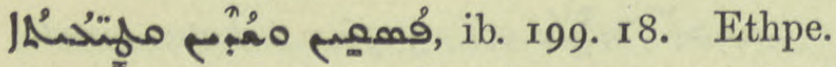

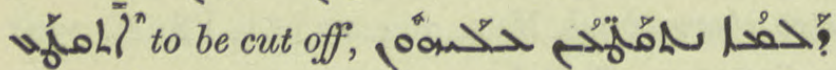

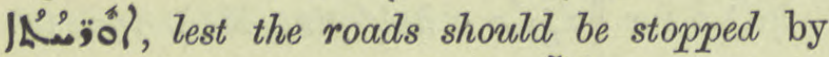
snow, Dion. 96. 7. Pael uํํ add: 1) to

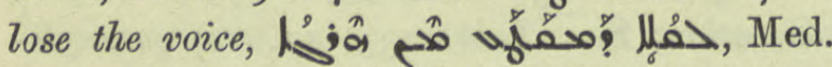

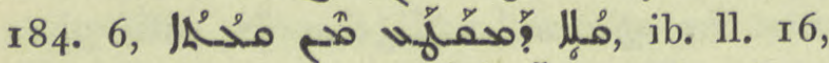
$22,23,185.4,13,22$; محمَح cut in pieces, Charms $\mathrm{I}_{3} \cdot 5$.

Lحُمُ add: rit. E.-Syr. an extra Hulala said at morning service during the Fast,

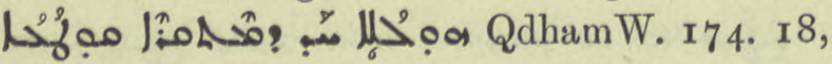
180. 3 .

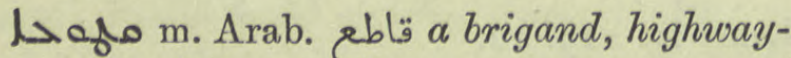

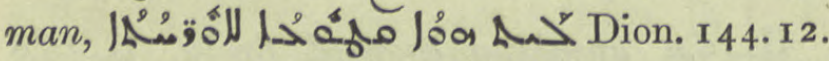

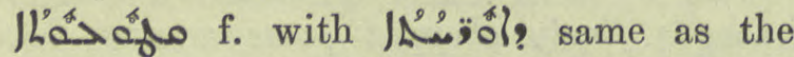
preceding word, viz. highway robbery, Dion. I9I. 22.

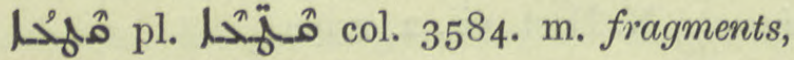
bits of brass, Chimie 31. 23, 32. 4, 99. 4, .

수 col. 3585 . to pluck. Pass. part. add: ي select and compact, Preface to Cod. Sachau I39, Tekkaf I 8 infra. Pael part. مبقص 5 of par. قدمَاْفُم seems to mean skull-caps left shaggy i.e. not dressed smooth, Pallad. I80. I2.

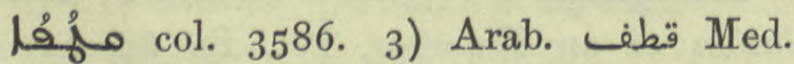
607. 6. Atriplex hortensis, garden orache.

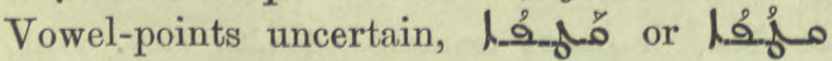

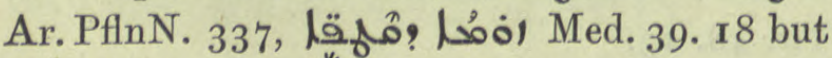

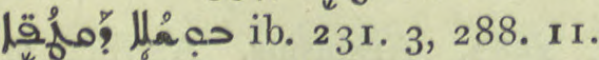

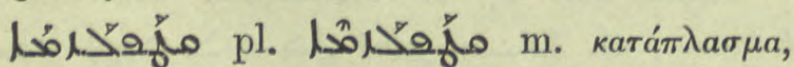
a cataplasm, plaster, poultice, Med. 58. 6, $65.9,80.23,23$ ०. $5,232.11$.

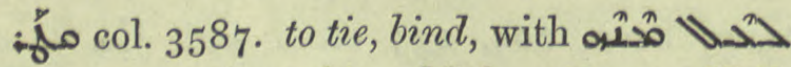
to hang up above him, Med. 568. 4. N.B.

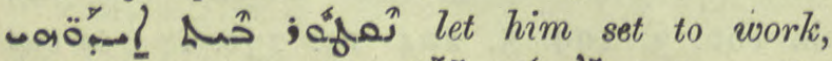

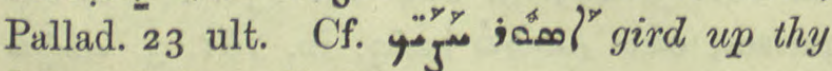
loins, Luke xvii. 8.

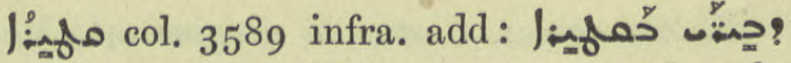
the oppressed, A.M.B. iv. 267. II. Metaph. forced citations, Sev.

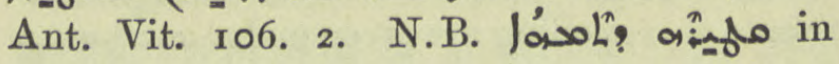
meditation the flesh is overcome by amazement,

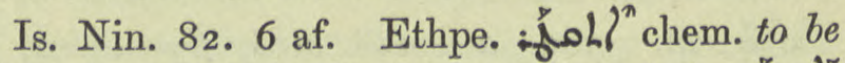
fixed, solidified, Chimie 47. 4, 6. Aph. مُ to bind; to curdle, 人?; thick darkness covered the earth, Dion. 57. I. Chem. to fix, coagulate, Chimie 46. ult., 47. I,

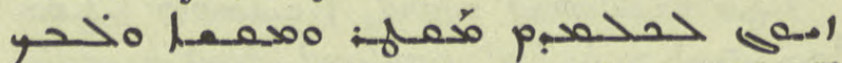
mercury fixes, solidifies and combines with all substances, ib. 1. 7 .

1:3ُ condensation, of mercury Lمo: Chimie 36. 7, 46. 24, amalgam, compound,

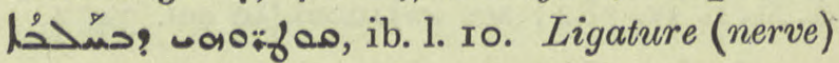

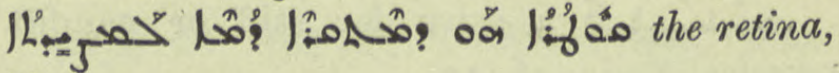
Med. 3r. I.

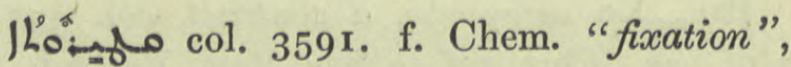
condensation of mercury, Chimie 46. 21 .

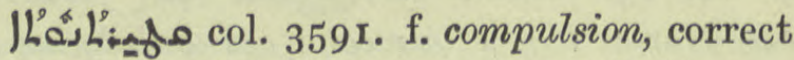
ref. It should be Apis م. 20, not Aphr.

ملئin compulsory, Syn. Or. 176. 18, I89. 7 .

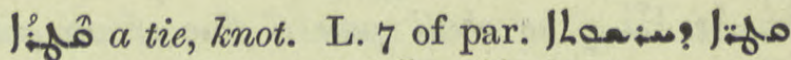
Charms 78. 18.4 قط a train of camels, Dion. 57. 8.

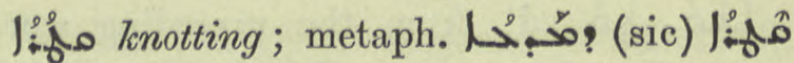

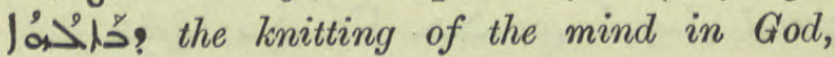
Pallad. 765 ult.

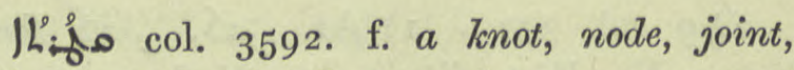
IL the seed sprang up and formed knots, Hist. Mon. i. 285. 17 ;

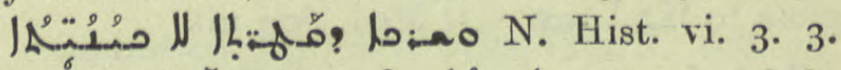

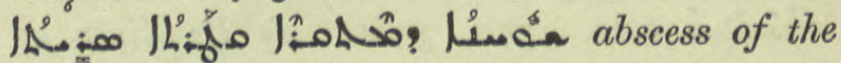
septum, Med. 62. 12, 63. 6, 9, 14 .

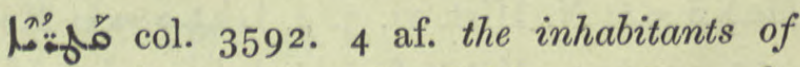
Qatar, ط.ق ald refs. Bahira 206. 3 af.,

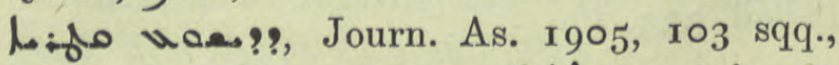

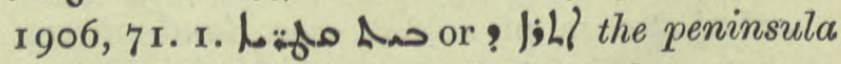
of Bahrein, A.M.B. i. 466 , Syn. Or. 215. 3, 448. n. 3 , 480. n. I, Pers. Mart. p. II 4 , Hist. Mon. ii. I53 n. I, I88 n, 2, Z.A. ix. $3^{6}$. 


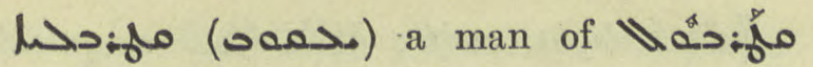
Qatrabul, south of Baghdad, see col. 3593, Journ. As. 1906, 73 .

إمidillage on the Zab, Chast. 60 pen.

ماهُ

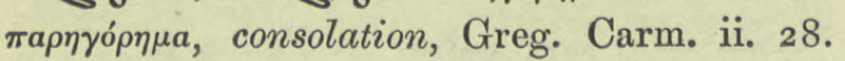
I6. Cf. col. 3595 infra.

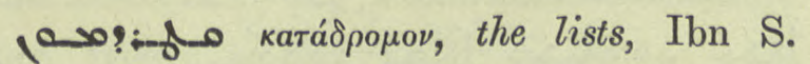
Thes. $5 v$.

م- مل col. 3596. kitpov, the citron, pl. ملمتم Chimie I2. 16, 271.9.

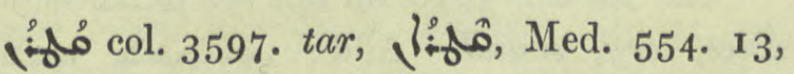
JAOS. xx. 189. 5 .

مُبنْ Kaido, nephew of Kublai Khan, Jab. 19. II.

مبـفة kouvóv, common, Nest. Hérac. 44. 4,6 .

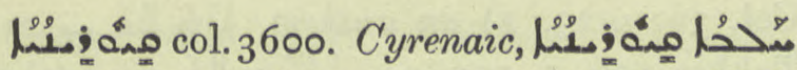
the milky sap of Asa foetida, Silphion, Med.

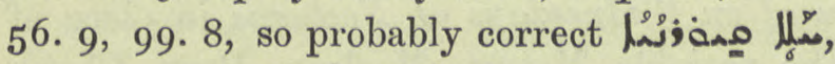
ib. $175 \cdot 15$. Cf.

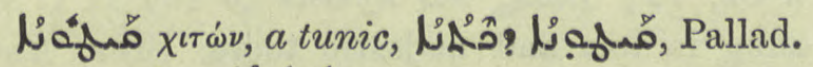

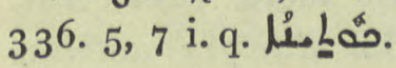

Lof of col. $3^{60}$ r. End of line 1, 1l. 2 and 3 . Dele yodh and trs. to لُمَّ a path, col. $35^{80}$.

مh Citium, a city of Cyprus, Nöld. F. S. i. $472.8 \mathrm{r}$.

Hor ol. $3^{601 . ~ 1) ~ k i т r a, ~ c r a v i n g ~ f o r ~ s o u r ~}$ food, Greg. Carm. i. 1 18. г 7, see /fto Suppl.

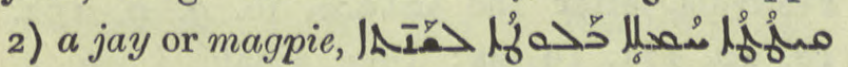
the magpie collects acorns for the year, N. Hist. vii. 4 . 4 .

مدحه Kai Khosru = Cyrus, ZDMG. lvii. 563.2.

مشحم col. 3602 med. Corr. (م) a a drain. م pr.n.m. K€ $\lambda_{\epsilon} \rho$, Celer, a servant of Theodora, Dion. Ined. 468 pen., مسخ:م صم var. محن;, Sev. Ant. Vit. 237. 2.

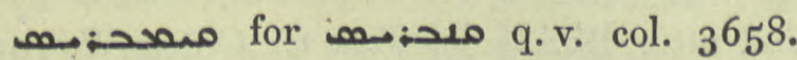

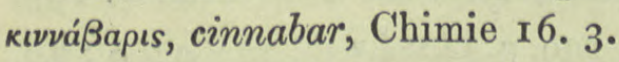

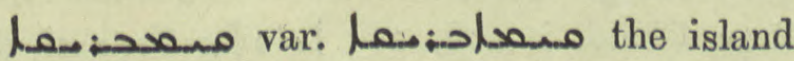
Cimbrica = Jutland, Jac. Edes. Hex. xxx. 8.
مئم: col. 3603. Cimolian earth, مبمهف

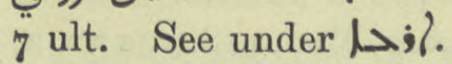

مصح: col.3604. cinnabar, Chimie 52.19.

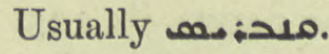

col. $3^{6} 59$ and Suppl.

an name of a stone used in the preparation of sulphur liquid for dyeing, Chimie 49. 7 .

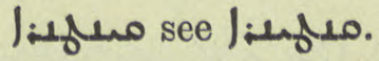

مُ a village in Adiabene, M. Z. I95. 5.

אivprots, motion or sensation, gloss Jhase is o Mart. Petri, R.O.C. iii. 47.

مده perh. a corruption of kaukias, the NE. wind. But a south wind, Med. 533. $\mathbf{r}$.

مُ

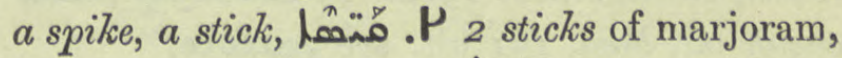
Med. 59. 7, 9; an oz. of مصمع/, in a list of measures, Chimie 69. I I, but prob. for مهمر a pint, ib. 23. I 5 , one oz. terebinth oil, Med. 60. I5, I 44. 6, 8 .

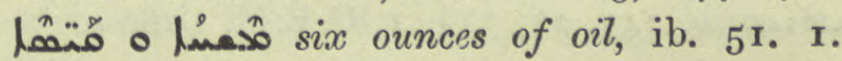

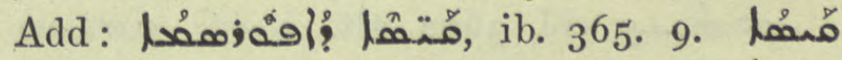

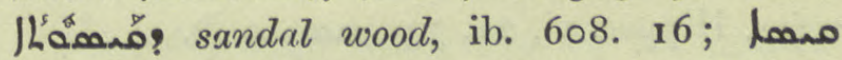
(נ: 1. 4 of par. epithet of gentian, Chimie 7. 12 ,

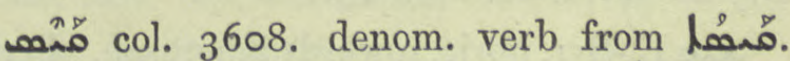
to stiffen, make rigid, paralysed, vovana o Med. I0. 4, II5. I3, II6.4, I0,

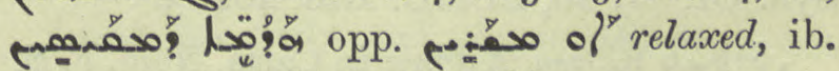

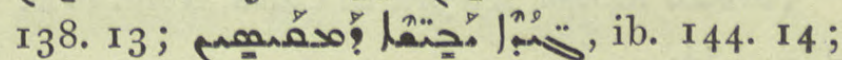

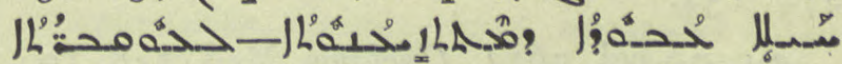

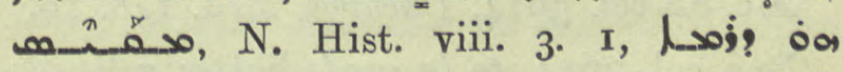
م BH.

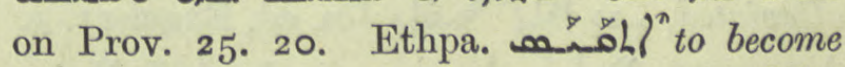
rigid, فำ

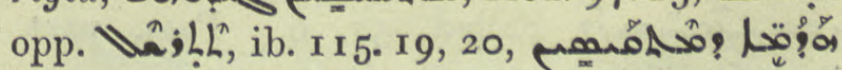
ib. 117.13 ; to have spasms, be in convulsions, Hippoc. v. 4. 62. To harden as the bark of

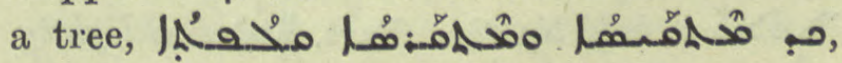
N. Hist. vi. 2. 3 .

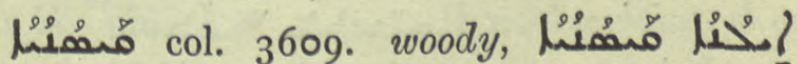
N. Hist. vi. 2. 2, /Rُ

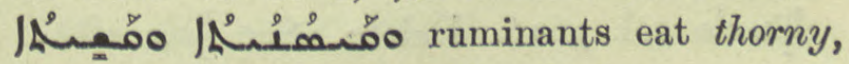
evoody and hard fodder, ib. vii. 6. 5. 


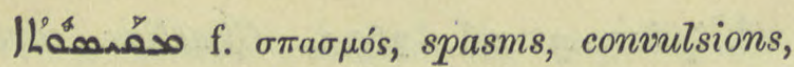
Hippoc. ii. 26 , iii. 24 , iv. $16,57,66-68$;

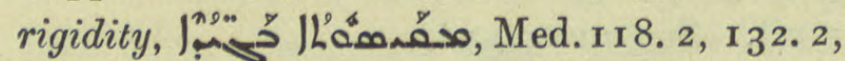

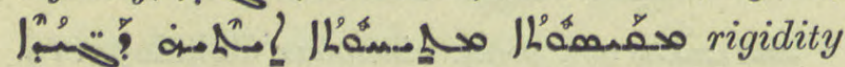
of the body is a tension of the nerves, ib. 135 . 16, I36. several times, I rigors, spasms, ib. 4. 9, 28. 5, 7, 1 1 7. I and often; col. 3697 under مaer.

مa Ká $\sigma \omega$, Cassa, a city of Pamphylia, Nöld. F. S. 472.87 .

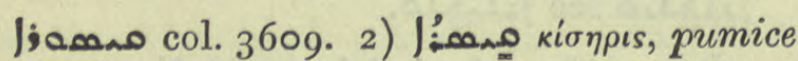

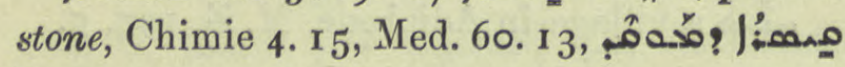
ib. 169.9 ; 174 ult.

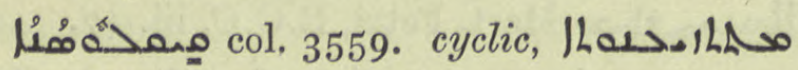

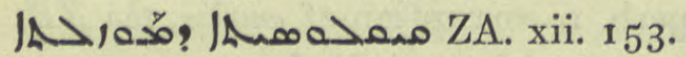

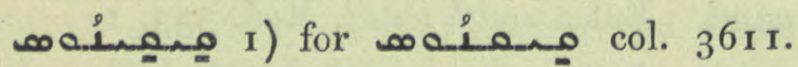

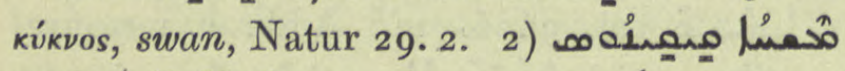
castor oil, Med. I5I ult. see ملمبه.

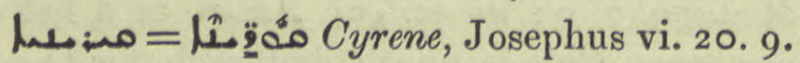

مـ col. 36r2. pr.n. Cyrus. After the ref. to C.B.V. add: Anecd. Syr. iii. 246, 9 ; من: ib. 245. 27, 314. I, C. B. M. $1060 a$.

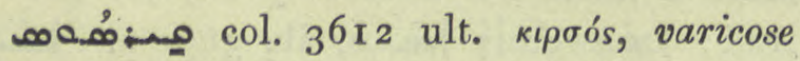
veins, Hippoc. vi. 2 I, 33, مد: معص ib.

مـي:مل Circaea, Enchanter's Nightshade, Med. 255. I3.

E.-Syr. bpric. towards Khorasan. Perh. the island Kas or Qis in the Persian Gulf, Syn. Or. 7 r, 28, ZDMG. xliii. 402. 3 .

مشA Kißvpa, Kibyra in Caria, Nöld. F. S. 474. 134 .

مص:00l. 36r4. Cachrys, Med. 148. 1 (о.

"

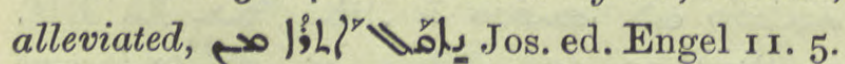

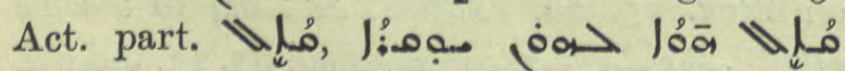
فㅁ. Pallad. 57. 6. Pass. part.

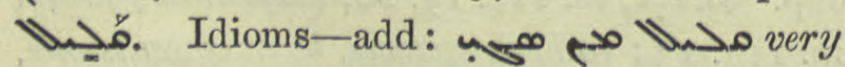
little, nothing whatever, L. E. S. 173.8 . Palp.

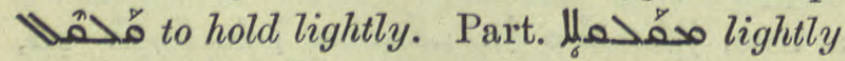
esteemed, Hist. Mon. ii. 105. 5 af. Ethpalpal

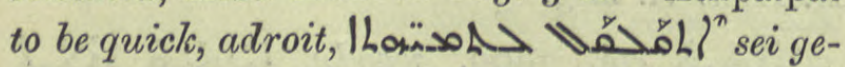
wandt in staunenswerthen Thaten, Tekkaf 155 .

لـ glue, col. $35^{19} 9$ and Suppl.

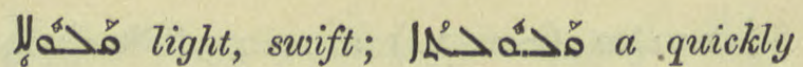
growing plant, N. Hist. vi. 2. 2.

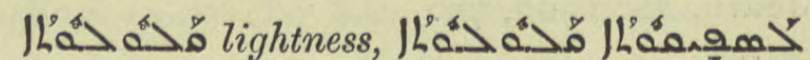
\قَ volatility accompanies emptiness, N. Hist.

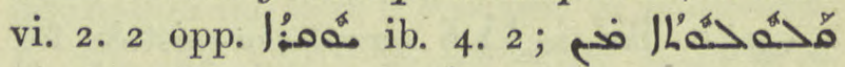

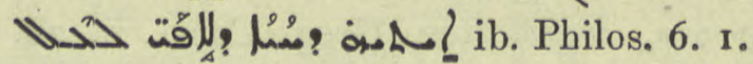

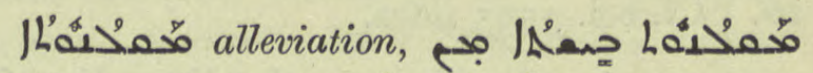

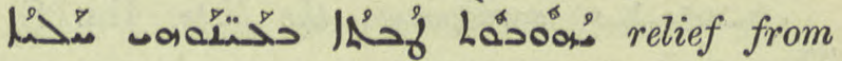
misfortune is sweeter in his eyes than a valuable present, B H. Pol. iii. i.

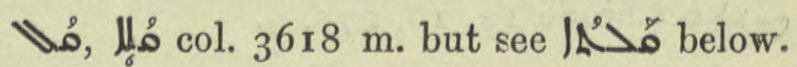
voice, cry, sound. Add: oه م>0م they went up at their cry, Jo. Eph. 207. 5 ;

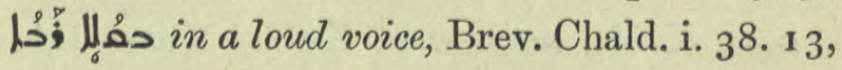
85. 4, 22, QdhamW. 131. 14, I35 ult., حمُ

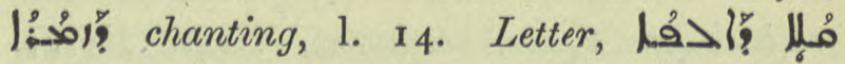

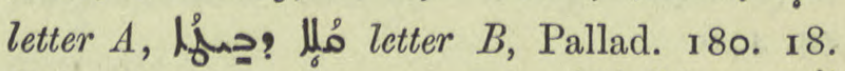

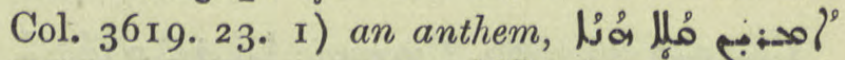

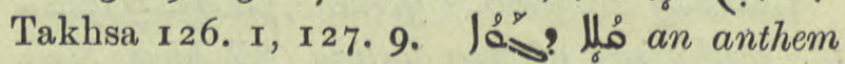
used on Wednesdays at Nocturns in place of the variable Motwa, Maclean, Brev. Chald. i. 82. 12; / قلا لـ hymns to the B. V.M. sung in low and majestic tones, Brev. Ant.

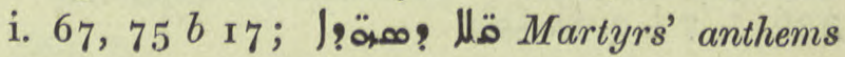
i.e. used on their days, QdhamW. 36. 16;

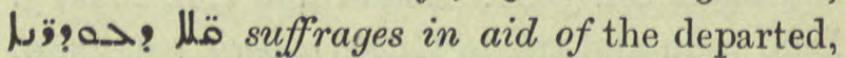

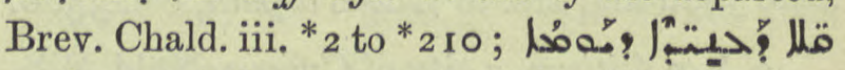
anthems of the Departed, QdhamW. 244. I 5 ;

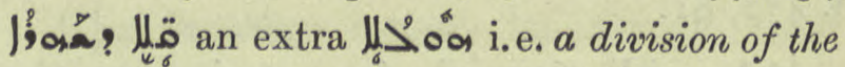
Psalter sung at Nocturns on Sundays and Festivals, after the fُLár, ib. 9. 9, 152. 18, 20, I7 1. 19 bis; Brev. Chald. i. 26. I, 89. I, 109. 4 af., I30. I, Takhsa 9r. 9.

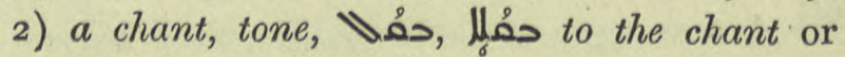
tone i. e. not monotoned, Brev. Chald. i. 29.

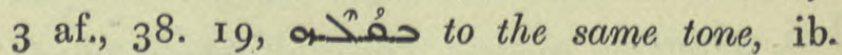

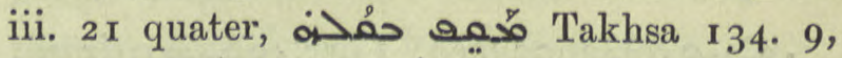
QdhamW. I53. 2, 5, 7, لِّم

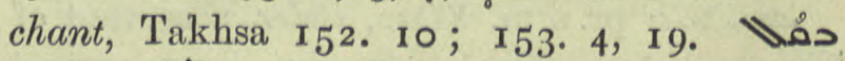

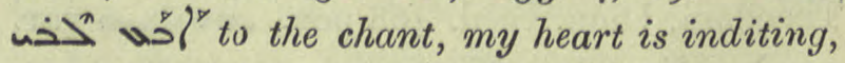
Brev. Chald.i. 63. 20; حمُلا ومستمدا ib. 5 o pen.

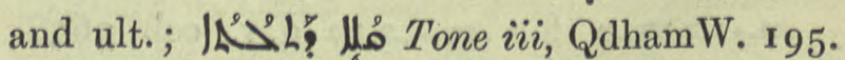

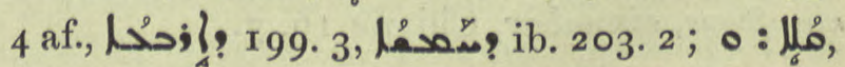
Brev. Chald. i. 108. 20 ; ا م : Tone $x i$, ib. 94. 20,95 pen., 97.17 . 
Emph. st. f. 1مْ مُ an anthem and proper psalms said at Nocturns after the بح

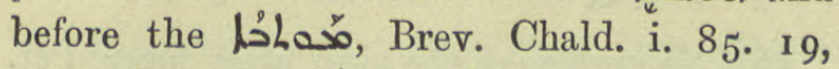

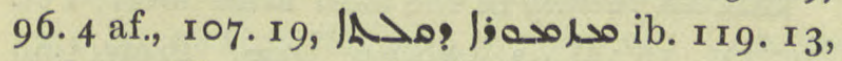
I28. 4 , iii. 23.4 af., QdhamW. 136. 2 ff., 

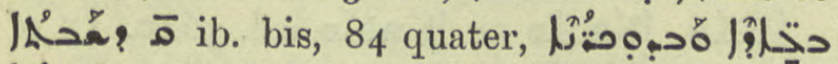

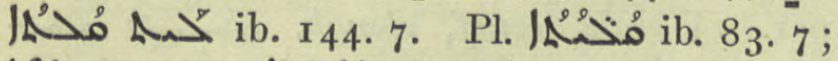

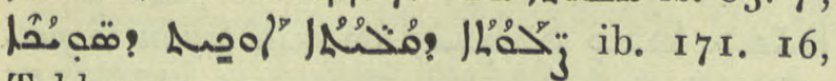
Takhsa 90. 3 .

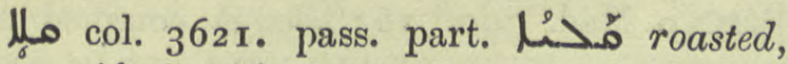

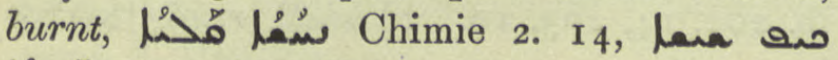

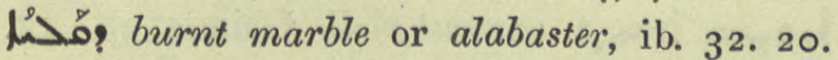

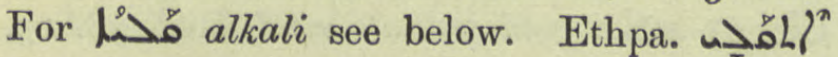
metaph. to be annoyed, vexed, arams und?

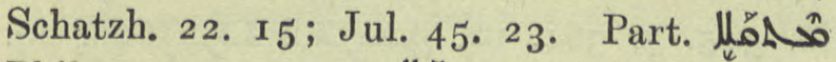

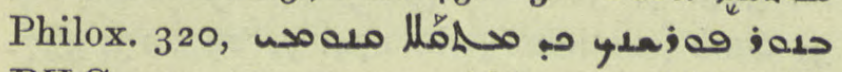
BH Carm. 73. I7 ; writhing, A. M. B. iii. 590 ult.

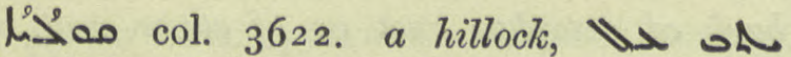

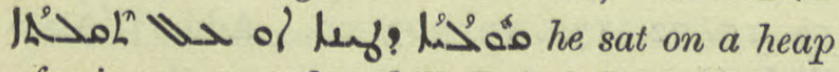
of mire or on a dunghill, P.A.O.S. xv. $14 \mathbf{I}$.

مـ calamint, Med. 608. I5 ;

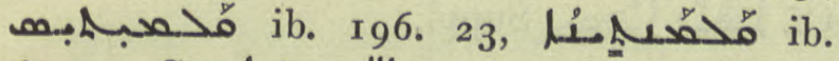

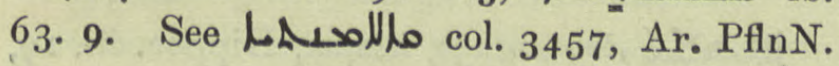
329 .

abracelet, Dion. I 79. I 5 , 17 .

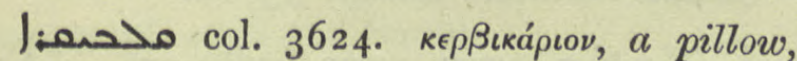

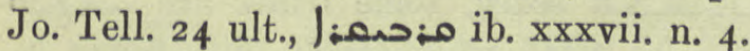

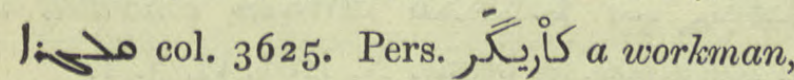
Nöld. on Dion. WZKM. x. 167; a bricklayer, Pallad. 182. 6, Brook's Chron. 573. 10; a bootmaker, A.M. B. vii. II6.

Ihoir the labour of a navvy, Dion. 95. 16.

مخب..n.m. ZDMG. xliii. 402 pen. perh. a mis-spelling of Calendion q.v. col. $3^{6} 3^{6}$.

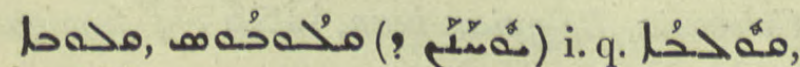

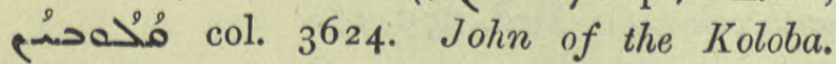
574. 18, 652. 9, محبهد ib. 650. r8. 19.

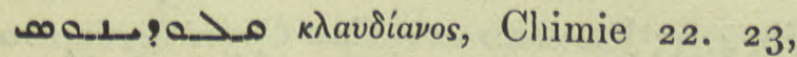

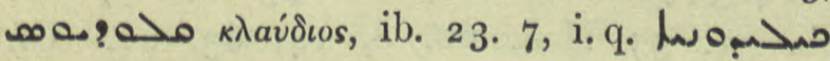
celandine, a yellow dye.

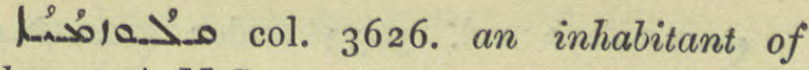
Clysma, A. M. B. v. 447 ult., 45 r. I.

L مخa Colonia, a bpric. in Cappadocia, De Goeje B. 64.5 .

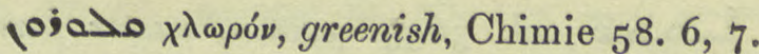

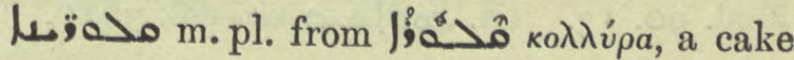
of bread. Collyrians, who offer each year a cake in the name of the B.V.M., Coupes ii. 139 .

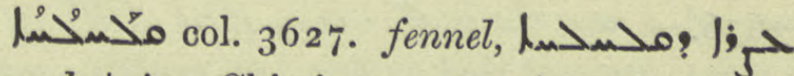
fennel juice, Chimie 5. 16. An equivalent for gum ammoniac and gum sandarach, $\mathrm{pl}$.

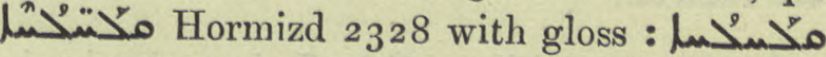

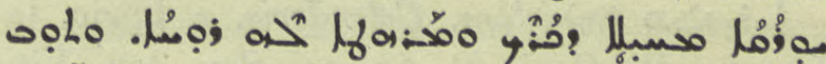

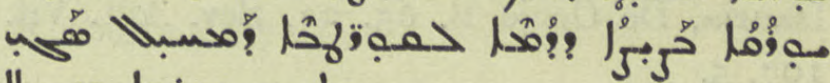
(

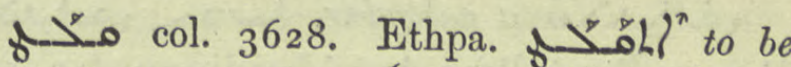

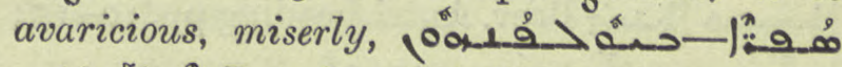
个

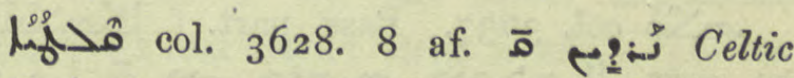

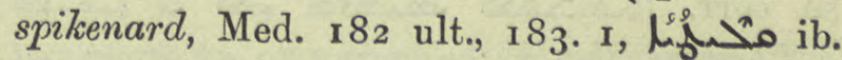
I 82 ult., 183. I, 290.10.

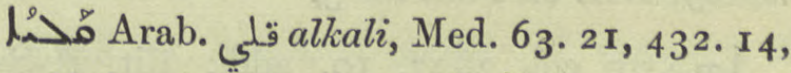

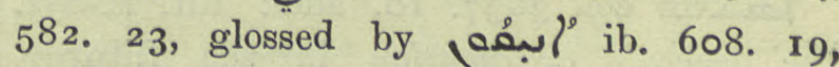

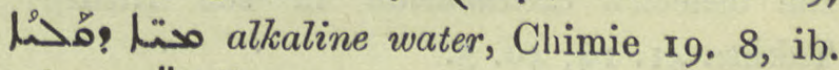
26. 20, 이 ib. 27.2.

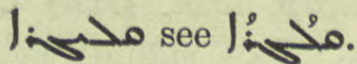

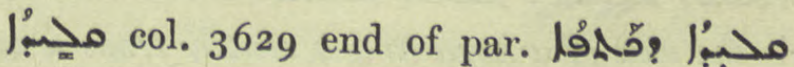
the clavicle, Med. 128. 8, 225. г3. A key =explanation, Chimie 56. 11 , محسم 1.24 ; مofle called might, a powerful preparation of copperas, saffron, and salt, ib. $57.18,19$.

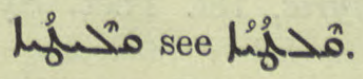

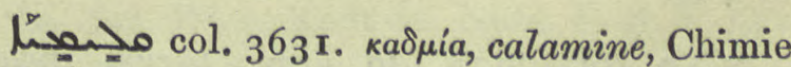
7. 8, مخدما ib. 2. 6.

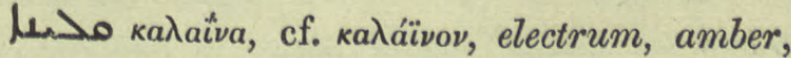

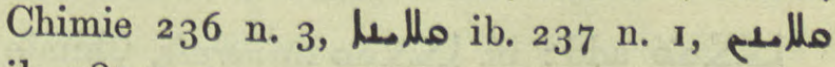
ib. 287 n. 3 .

قاليقلا Arab. Erzerum, Dion. 94. I I, 97. I trad. 82. n.

مخبم) مخمسمص. see. 


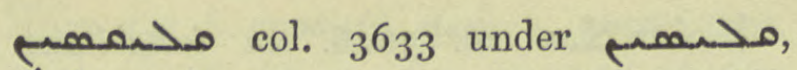

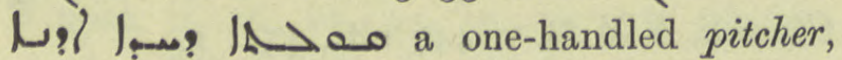
A.M.B. vii. 515, Pallad. 486. 4 .

hمبر col. 3635. croton, castor berry,

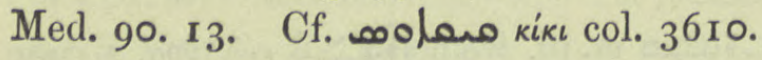

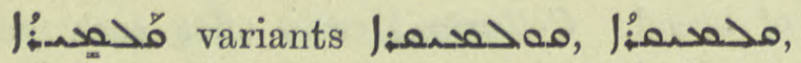

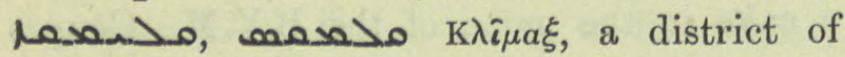
Scete, Pallad. r68. 4. Cf. مخعمدم: col. $3^{6} 3^{6}$.

calamint; see

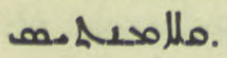

K $\eta \lambda \epsilon \in v \delta \rho \eta s$, Celenderis, a bpric. in Isauria, De Goeje B. 65. 22, Sev. Ant. Vit. 107.5 .

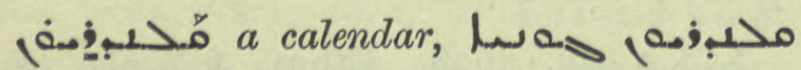
مrev. Ant.i.heading pp. 10-15;

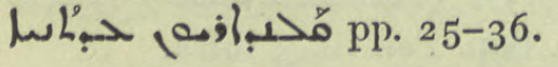

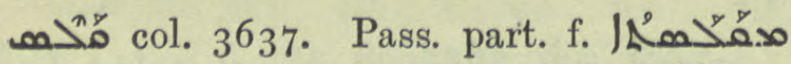

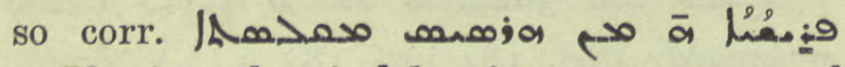
a Pharisee, that is belonging to an approved heresy, BH. on Phil. iii. 5 .

إم the deacon's exclamation in the Liturgy, Takhsa 4r. 14, 46. 5 .

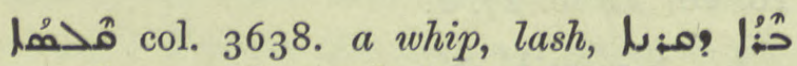

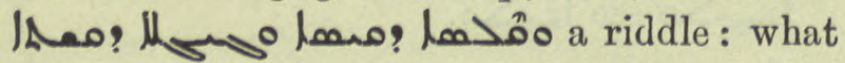
is the well of a horn and a switch from a tree and the circle of a bow? Answer: a curve, Brit. Mus. Or. 2084. 47 r. Perhaps

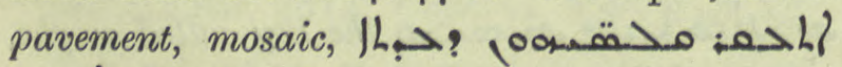
PI/o Chabot, Dion. 203. 20.

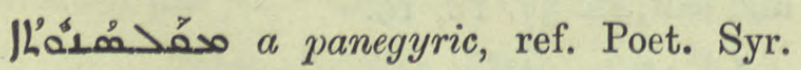
6. 4 af.

مذw col. 3638. to hurl, thrust away, فححم ححت: 97. 4 .

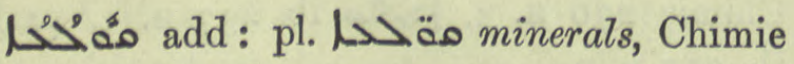
3. 6, shining ores, ib. 4. 4.

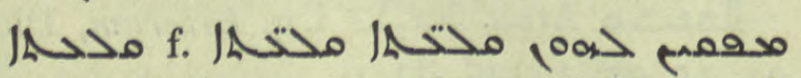
in time of plague they slung out the dead, "par groupes", Chabot, Dion. 39. 6.

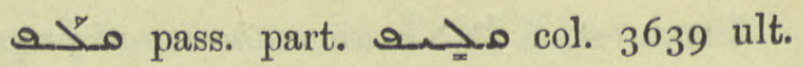
. 6 shelled beans, Med. 564. 6.

مُحَ peeting of the skin after fever, Pet.

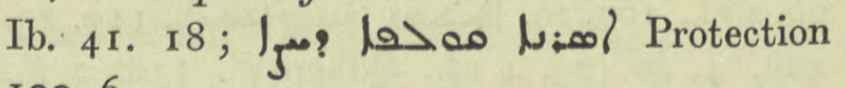
100. 6 .

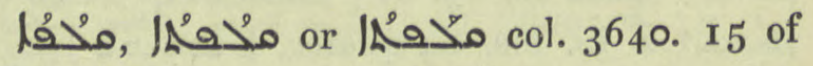
par. I) scale, scurf, slough, a  off in thick scales, Pet. Ib. 4I. I6; lỏá? لئ محَحْم Med. 590. I6; slough coughed up,

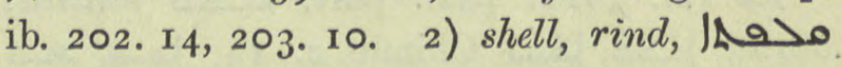
Loua llay? coconut-shell, Chimie 38. 13,

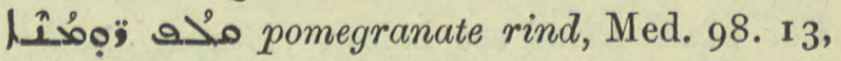

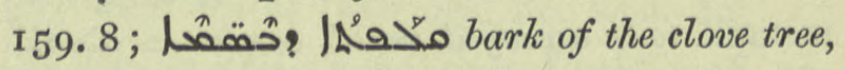
ib. 305. 7, 36r. 10, 608. 20. 3) bark (medicine), ib. 174. 7, 295. 13, 337 .

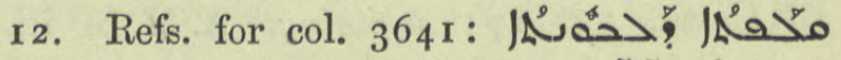

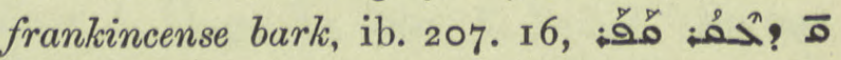
bark of camphor root or of caper root, ib. 59. I 5, 22, 172. 19, 303. 20. Add: مخحف2

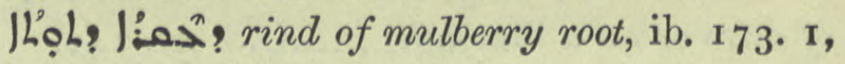

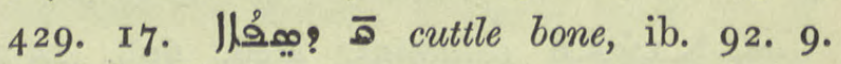

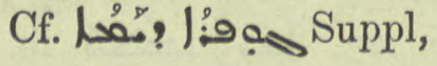

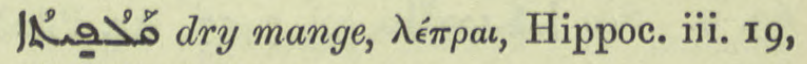
A.M.B. ii. 678 .

ال

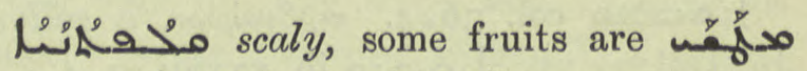
معَم concealed in a scaly pod, as beans, N. Hist. vi. 3. I.

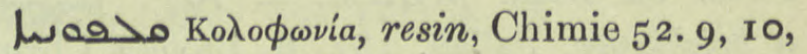
Med. I 46. 4, 375. 2 I, 609. 3, 4, 375. II. 2 I. See مصه col. 352 I.

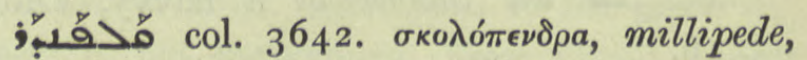
G. Warda ap. S. George ed. Folkm. 48 n. 2. White locusts, QdhamW. 186. 15.

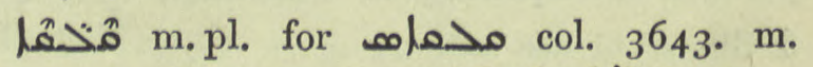

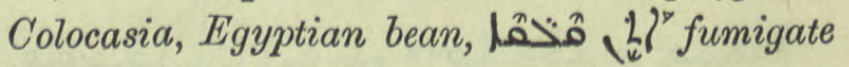
with colocasia, Med. 579. I5.

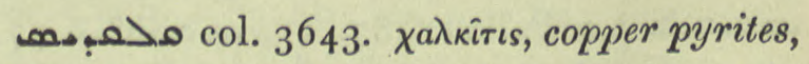
white vitriol, Chimie 2. 10, مذمبمس زاج القطر ib. 4.3 ; مخمب: Cyprian copperas, ib. 50. 5 ; Med. 60. 8, 14, 63. 2 I. Cf. مخمب:مص and مخمد. 


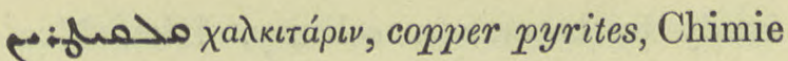
I4. 18 .

مخمد. copperas, green vitriol, Duval Notes, on زحمـب 5 زاج 5 but Chimie 5. 6 gives xá $\lambda_{\kappa a \nu} \theta o \nu$, as an equivalent for مخمبرم ib. 44. 24, I. q. the following.

col. 3644 . I. copperas. Add:

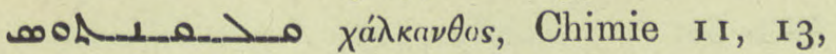
(ib. مخمعلم: ib. I7. 4.

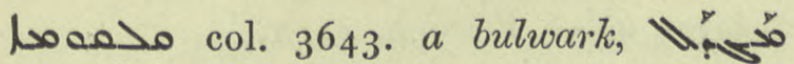
a a tower with a parapet or machicolations, S. Dan. $5^{2} b 4$.

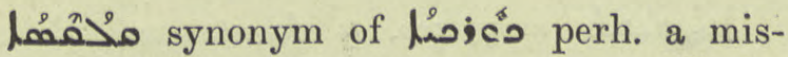

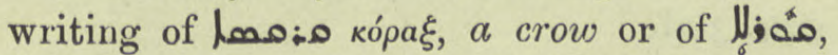
مَّمُ a crane, Hist. Mon. i. 2 I 9. 8, ii. 4 I 8 n., . 549. 7 .

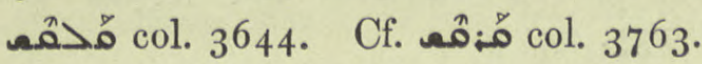

a cellarer, Pet. Ib, I8. 9.

مجخ: Delete par. and substitute $\kappa \epsilon \lambda \lambda a ́ p \iota o \nu$, provisions, Sanct. Vit. $284=$ A.M.B. iv. 323 ; مخi Anecd. Syr. iii. 340. I, Zach. Rhet. Brooks ii. 218.3 .

مُمحمبلا perh. Bengali Kamala, the red powder on the fruit of Rottlera Tinctoria, Med. 429. I 8.

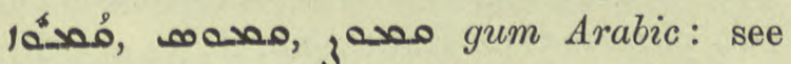
مo col. $353^{8}$ and Suppl.

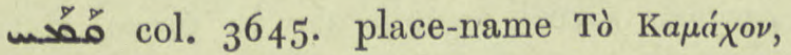
a fortress of Greater Armenia, on the W. bank of the Euphrates, now Gamash, Dion. 86. $2 \mathrm{I}, 88.3$.

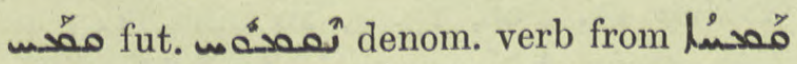
to grind flour, Med. 565. 9, 566. I I.

.

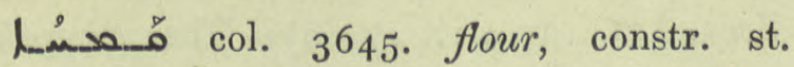

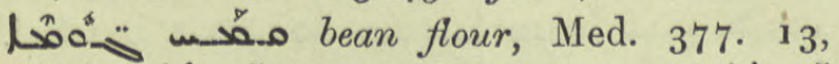

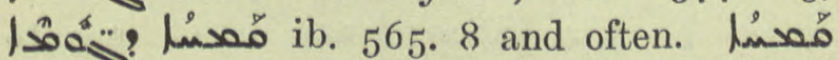

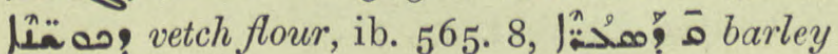

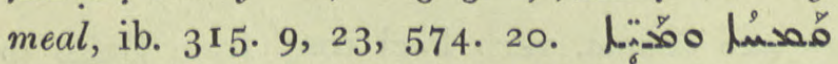
.

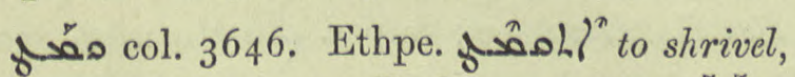
contract, Med. 76. 17. Pael pass. part.

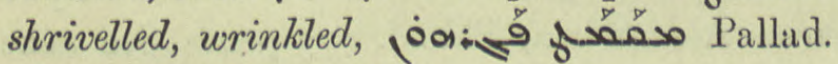
เo6. 4 = A. M. B. vii. 17 .

2716
مصله Arab. قمه a case, Pet. Ib. 39. II.

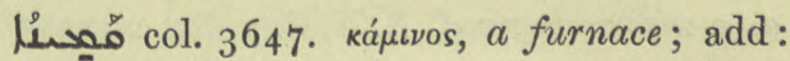
مa of the furnace of a still, Chimie 59. 4 ; h

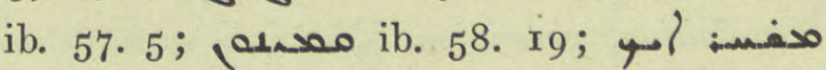

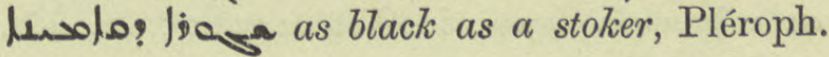
42. 8.

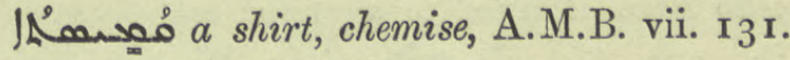

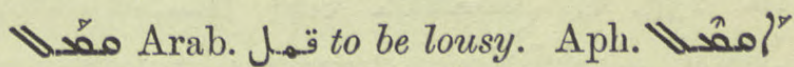

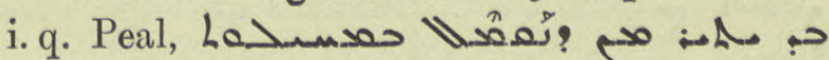
مuman hair becomes infected with lice by the failure of native heat, $\mathrm{N}$. Hist. vii. . 7 .

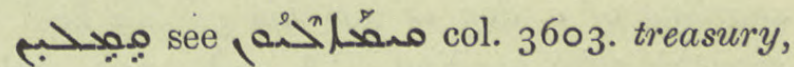
S. Dan. 14 a 7 af.

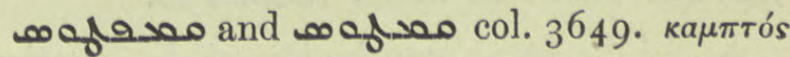
$=\kappa a \mu \pi \tau \dot{\eta} \rho$, a turning-post, goal. Metaph.

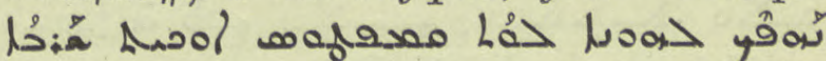
let us return to the point or to the subject, Bar Sal. in Lit. 2. 25 , مaم ib. 7 1. 2 I and often; Greg. Carm. ii. 38. 20.

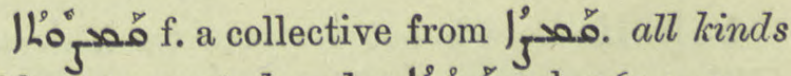

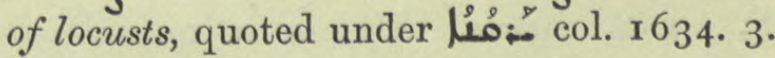

Jiso col. 3650. a girdle. A vestment of

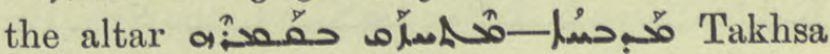
I 4 6. 18 .

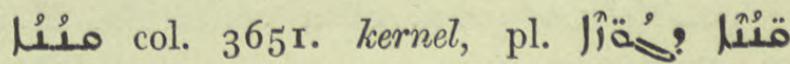
walnut kernels, Med. 555. 6, 577. 18, 583. 10.

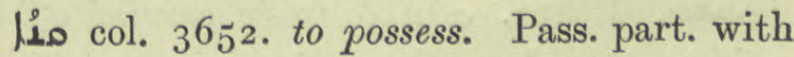

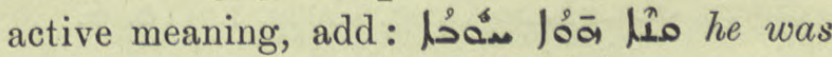

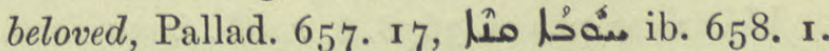
Ethpe. uيh to be gained, won over, w10h? (I) Mar Aba ii. 420 .

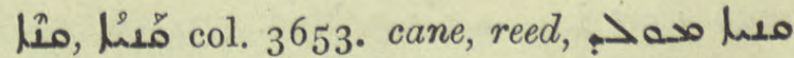
कla Jac. Edes. Hjelt in Nöld. F. S. i. 572.

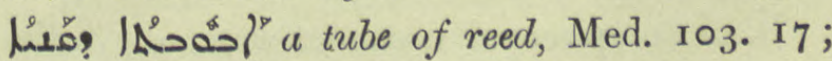

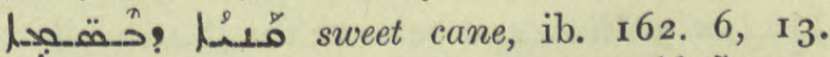
A splinter of cane, extract a splinter from the ear, ib. 559. I4.

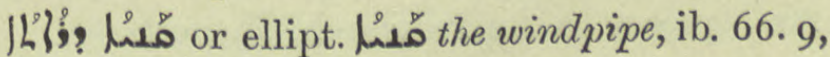

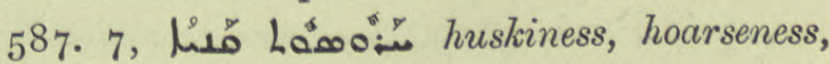

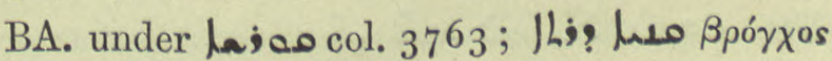
Hippoc. vi. 36, Jlo: $\overline{\text { s }}$ aiooiov, ib. v. 20. R $\mathrm{r}$ 


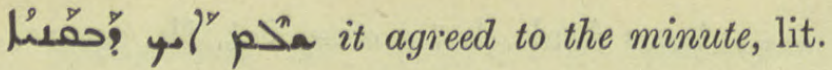
as a measuring rod, A.M.B. v. 81, 20.

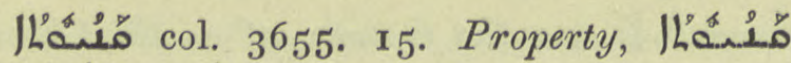
|Lُقُ

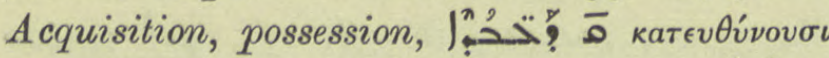

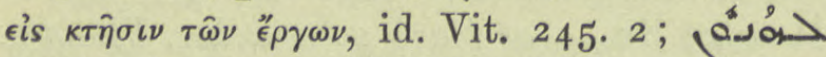

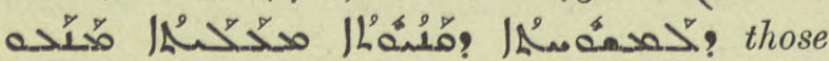
who have attained to supreme self-possession,

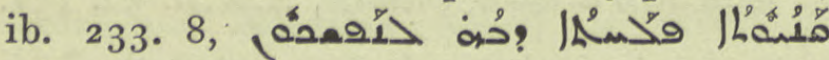

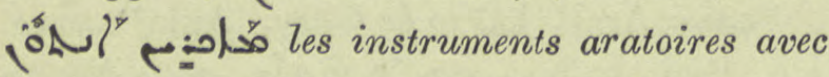
lesquels vous cultivez votre ame, ib. 1. ro.

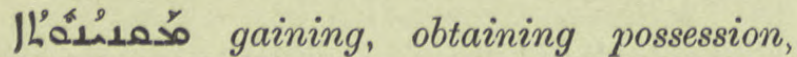

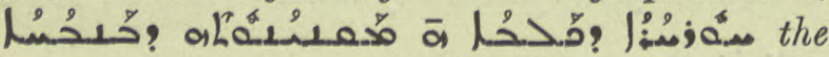
snarling of a dog is (at) his getting hold of something by barking, B A. col. I370 under

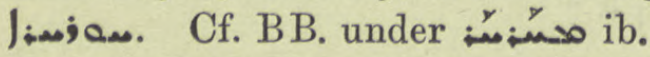

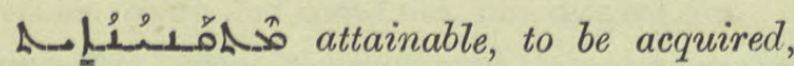

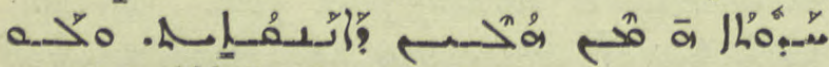

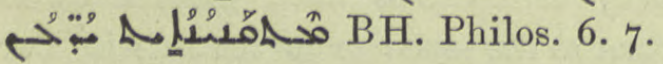

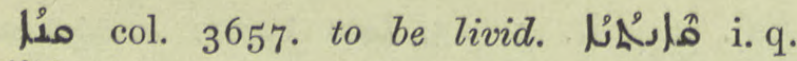

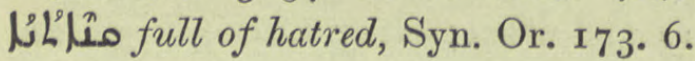

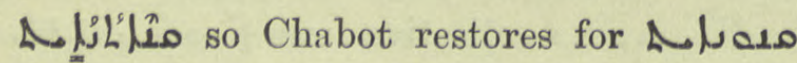
(MS.), with hatred. Unless it means like

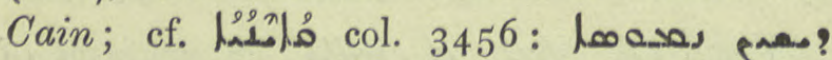

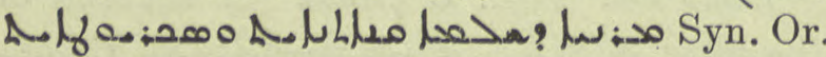
I72. 7 .

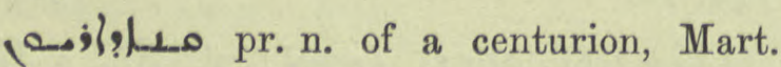
Pauli, R.O.C. iii. 54 .

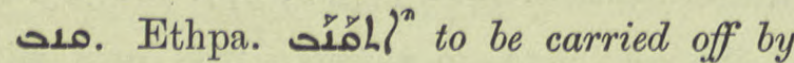
raiders, مه Greg. Carm. i. I 10. 6.

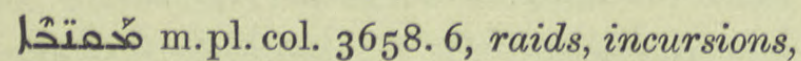
مهمتحا فischon. Min. 326. 16 $=$ Brooks Chron. 306 ult.

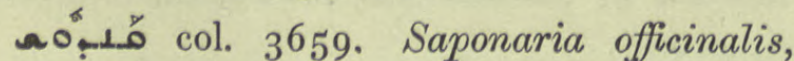
soap wort i.q. ميمبُْ0 Med. 53. 16, 55. 8, 56. I 7, 64. 1, Ar. PflnN. 306.

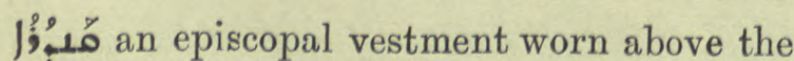

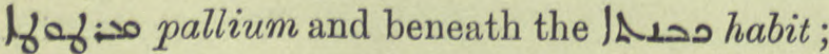
ref. Heavenly Intelligences as. Said in that book to be the sign of honour given by the Arab power to the Patriarch, Maclean.

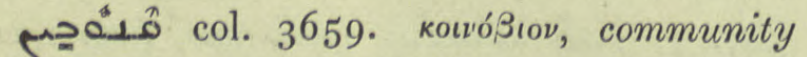
life, بـ when he had completed his training in the monastery and was ready to become a solitary, Hist. Mon. i. 218. I.

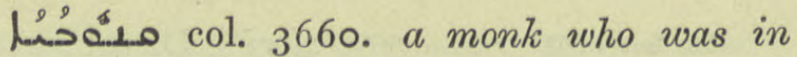
training in community life for the more advanced ascetic life, Hormizd 538 and $n$. p. 28

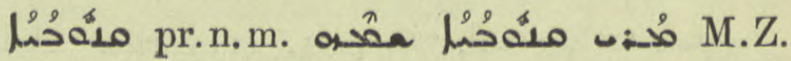
209. 5 .

kotvo-

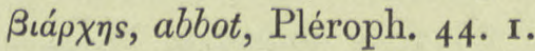

Canopus, Sev. Ant. Vit. I 7. II.

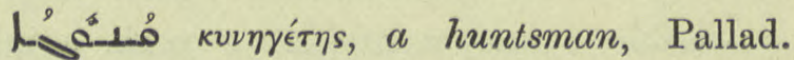
524.3 af.

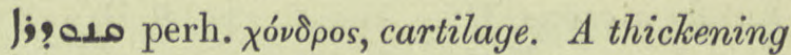

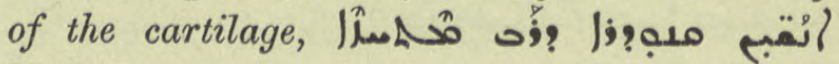
Med. I 55. 23.

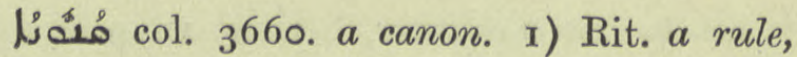
مدةقر Brev. Ant. i. Cal. ق ق Takhsa $16_{5}$ tit., twenty-seven of these rules follow to p. I70. 2) a response, مـة

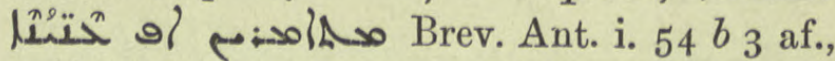
$59 b 5$ af. A psalm recited into portions after each of which follows a verse of a Canon, ib. $53 b$ infra, or an ejaculation e. g. two phrases then Hallelujah, ib. $54 a_{3}$, Brev. Chald. i. 316, 320 tit., 32 I. 13. 3) a hymn, canticle, Greek canons are in prose, Syriac are metrical, Or. Xt. iii. Iо3 n. I, cf. Brev. Ant. i. Cal. 55. The ending of a prayer, said with raised voice and marked by cymbals, Takhsa II. 4 af., I3. 4, 20 infra, I 43 med. and often. مُعبِ Aweful art Thou, the opening words of several canons for festivals, ib. 52. Ke.so.a? $\overline{5}$ the Creed, Brev. Ant.i. I $2 a$ I. 4)=6) in Thes. diptychs; مoa) Bar Sal.

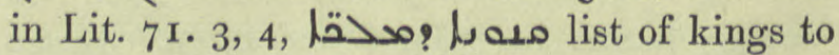
be prayed for, ib. 1. 27, 72. 4 .

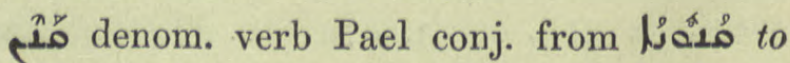

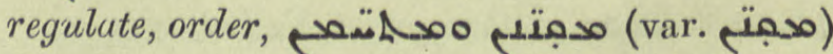
Sev. Lett. 22. 19; imper. مै Hormizd ${ }_{2} 5^{6} 3$.

مدفُْم col. 366r. delete "forte". Canopus near Alexandria, Pallad. 473 pen. See next article. 
مerde col. 366r. delete "Forte sit."

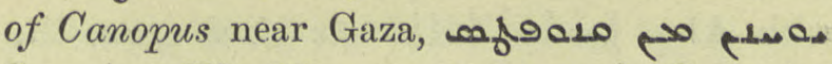
Sev. Ant. Vit. 86. 9, endos the Canopite, ib. 224. 4, I I, Nau on Sev. Vit. R. O.C. v. 8I, vii. $106 \mathrm{n.} 2$; it. Pet. Ib. 132.22.

مدهمe Canopus, a village 2 miles S. of Gaza, Pet. Ib. I 32.22.

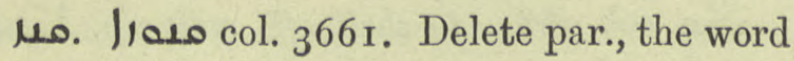
is lios a beak.

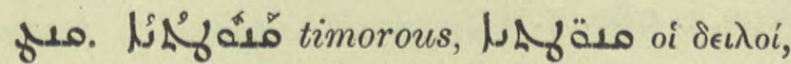

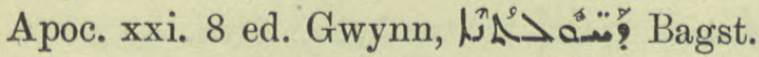

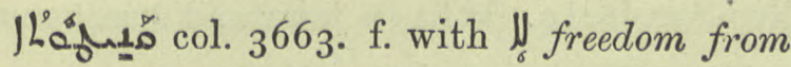
restraint, Sev. Lett. $264 \cdot 7,482.2$.

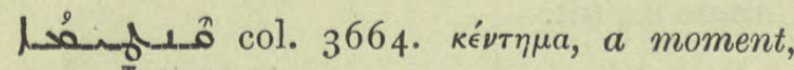

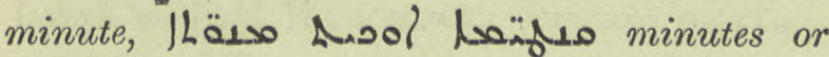
degrees, Georg. Arab. I I. I3, I 5, Med. $45^{2}$. A stop, point, Joar hool hand of Epiph. 15. 6, I6. I 4.

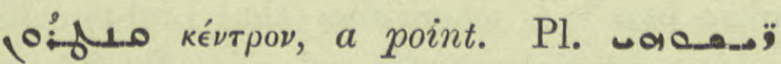

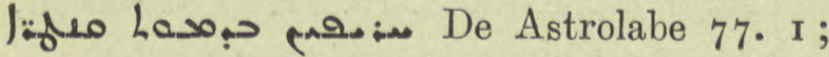
the quarters of the outer circle of the astrolabe are called Centres of life, of parents, of weddings of the midst of the earth and of the midst of

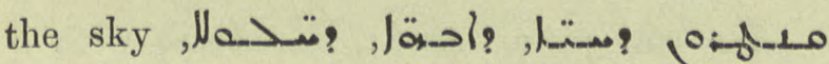
ib. $25^{1}, 25^{2}$, 254 , and $284 \mathrm{n}$.

م. مُ col. 3665. corrupt form of

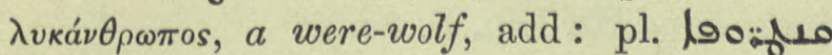
Hist. Mon. i. clxv. Syr. r, Hormizd 2018, sing. ib. 2058 , abs. st. مدلid ib. 2495. حـ مـ abusive name for a Jacobite shrine, ib. $270 \mathrm{r}$.

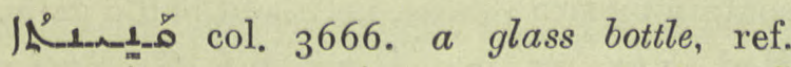

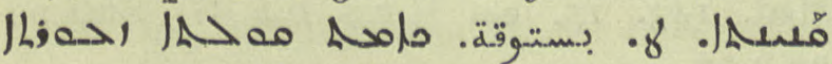

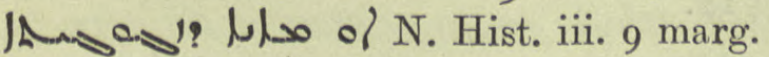

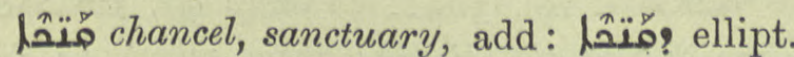
for Brev. Chald. i. 60. 5, 70. 12, $23^{8} .3$.

ـoc مـ col. 3667. I) substance, add:

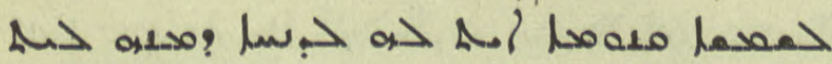
م Ephr. Ref. i. 50. 46, 5I. 5, 17, 25-27, 32, 43. A hard substance, a solid, مته مدا

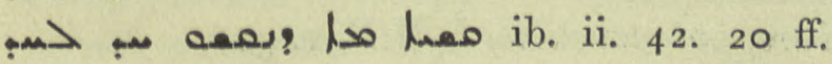

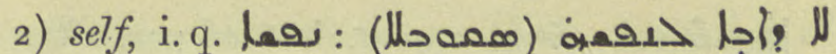

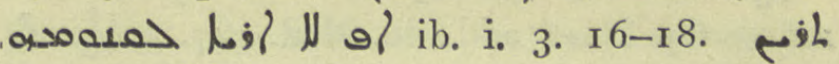

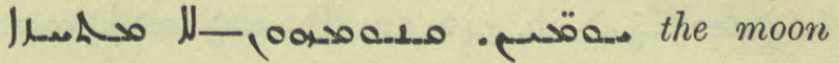
during two days is not visible, ib. 23. 7; H. of of the Ship (of Light) itself is not visible, ib. 26. 48, 27. 2.

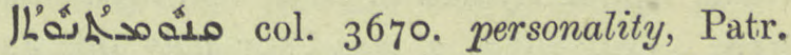

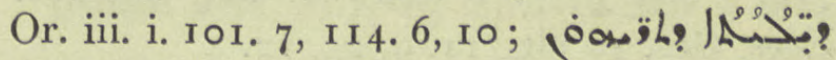

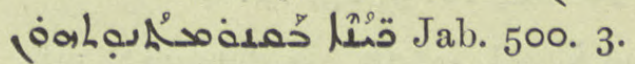

| Greg. Carm. i. $25 \cdot 9$.

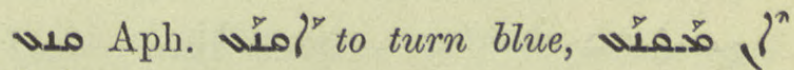
Chimie 48. ro.

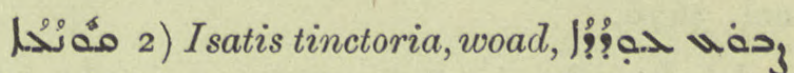
مبنح dip a wad of woad fibre, Med. 102. I I, I03. 2.

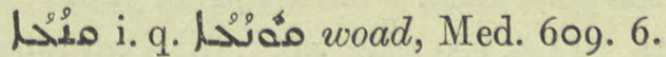

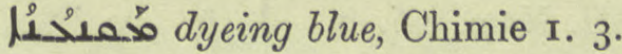

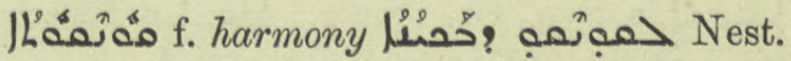
Hérac. 23. 13.

No10 Qanqal, village and monastery in Arzun on the river Sarbat, Chast. I3. IO, 2 I.

ملزم Zach. ed. 3672 ult. Pl.

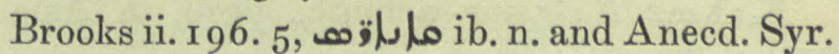
iii. $3^{2} 3.8$ add : $k a v a ̂ \lambda_{\epsilon s}$, Lat. canales, conduits, Guidi.

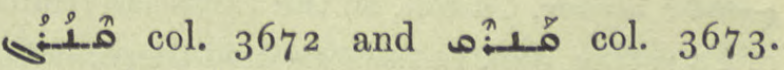

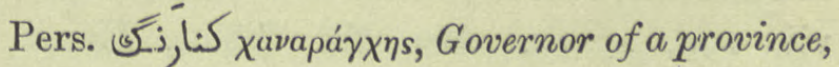
Lord of the Marches, Marquis, B HChr. 76. 9 and 4 af., Anecd. Syr. iii. 207. 7, مخ: مع ib. 206 ult., but مد:م Zach. ed. Brooks ii. 25.20 , 26. 3. Pr.n.m. مُ مُ B. O. iii. i. $485=$ Hist. Mon. i. I63. Cf. Tabari $44^{2}$ n. 5 .

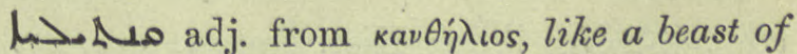
burden, Sev. Lett. 299. I 8.

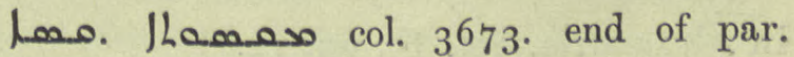
Add ref. Galen. ZDMG. xxxix. 243.

مُعب col. 3674 . Add: cap of a charioteer, Pléroph. 108. 3 .

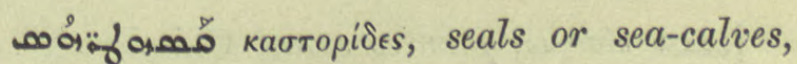
N. Hist. vii. $4 \cdot 3$. 


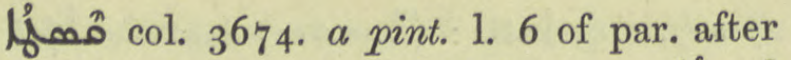
ref. to Op. Nest. add: id. Med. 447.5, مُمْهُ

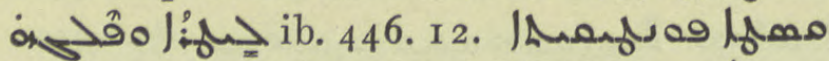

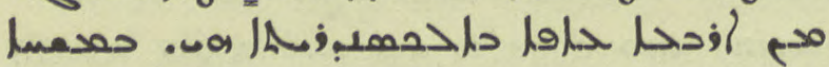
| lesole the Pontic pint is four times the Alexandrian i.e. eight Italian litres, Epiph. Symm. ii. 193. Cf. Metrologie 716. Col. $3^{675 .} 2$ vessel for holy oil, Jac. Edes. Can. ro ult., I 1. 9, 25, 12. 4 .

مُ Med. 49. 7, 19, 53. 16, 54. 6, 55. 6, 10, 263. 2, 9, 609. 8, 9 and often.

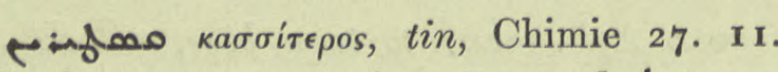

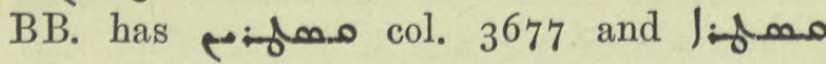
col. 3676 .

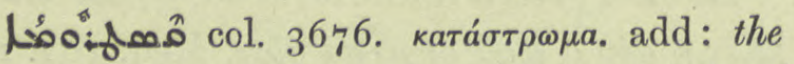
low partition wall to the right and left of the chancel gate, Hist. Mon. i. I 7 I. 6, 306. 19= ii. 342 n., 544 n. see plan vol. i. liii, Catholicos

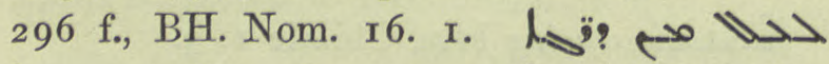
100.-6y Syn. Or. I70. 2. 1 م م Inscript. Sém. No. 52, Nöld. in loc. ZA. xxi. I6o.

معمدي col. 3497. 5 af. fumes given off by melting calamine, col. 3226.4 under / : 9 DBB. 1634. I3, App. I75.

لأهistr.nia, a bpric., prob. in Asia Minor, De Goeje B. 65 . 19.

? مصمب: col. 3678 . end of par. add:

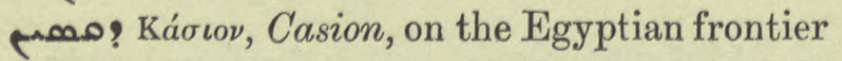
of Palestine, Jac. Baradaeus died there, R.O.C. vii. 199. 4, 12. See Mt. Casius, Herod. ii. 158 .

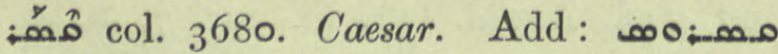
(i) the Emperor Nero, Mart. Luc. ed. Nau 158 bis.

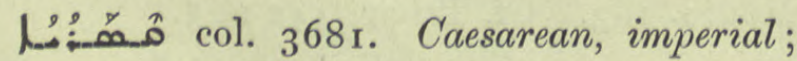

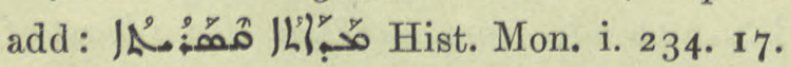

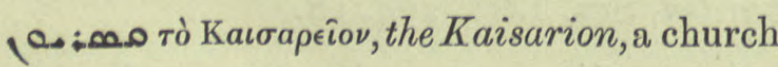
in Alexandria, Pet. Ib. 65. 12.

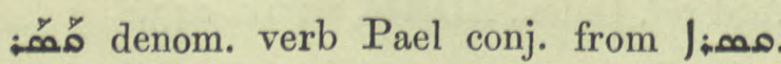
مחم: مa with pumice stone. Chimie 258 n. 1 .

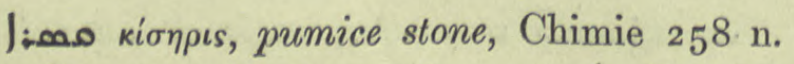

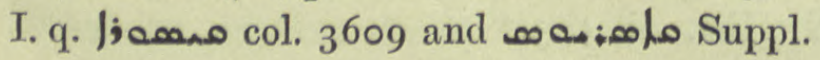

\section{randar}

L مُ col. 3682. to cry, scream. Act. part.

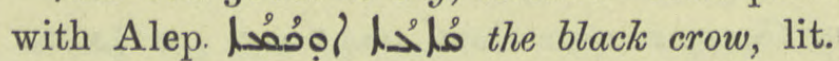
the screamer, Med. 592. I 7 .

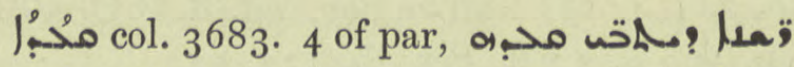
corr. translation to "the nobles in session with him”. Cf. Arab. قَعَدَ Duval Édesse 85 n. 3.

مُحنّ

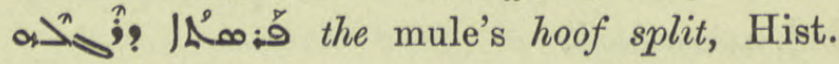
Mon. i. 280.7 .

| Neo-Syr. ملرo. It has a juicy and sweet root.

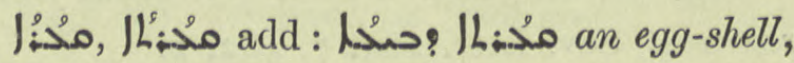
Chimie 84 ult.

محمح: , محن: concave as if Pael or Aphel part. same meaning; Klamroth on Arabic Euclid, ZDMG. xxxv. 300.

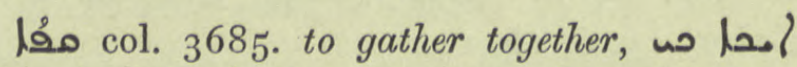

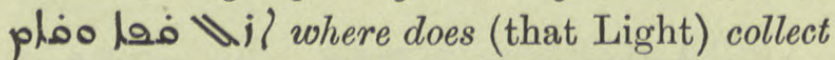
and stay? Ephr. Ref. i. I6. г1. To skim off, INOO مan Chimie 33. 12, 35. 10.

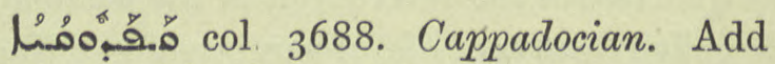

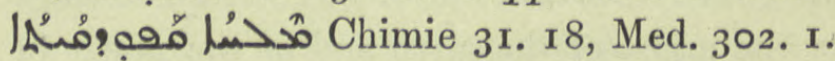

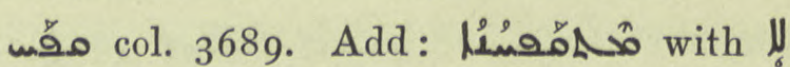

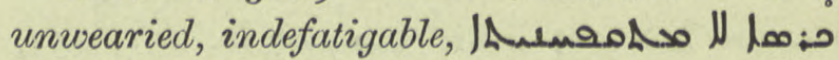

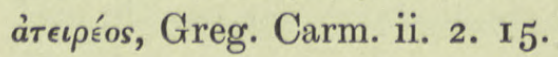

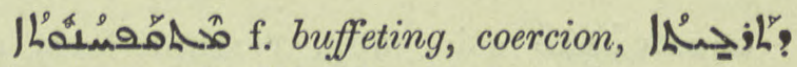
of the mind, Is. Nin. B. 8r. 6.

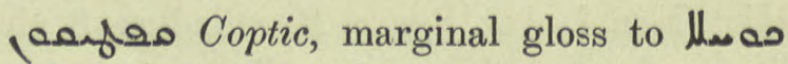
(נر) Chimie 47.5.

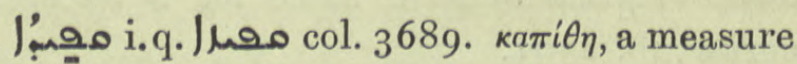

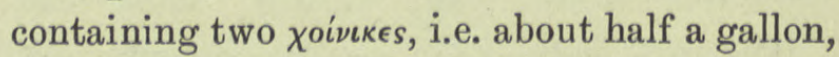

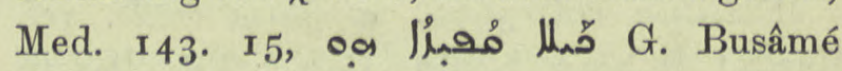
$27 b$ I 2 .

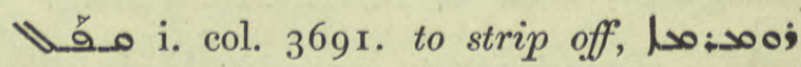

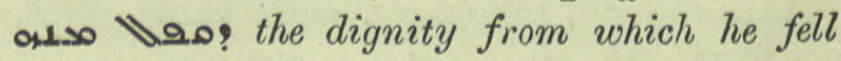
$=$ of which he was stripped, Ephr. ed. Lamy

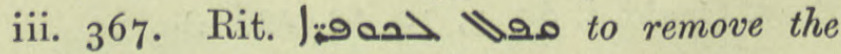
fair linen, Bar Sal. in Lit. 89. 4. Pass. part. موبn, ref. "مe saw that the roof was stripped off, Pallad. I8. I3.

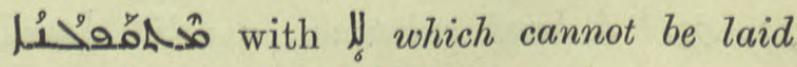
bare, indestructible, A.M. B. v. 506 . 


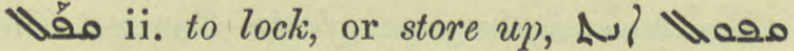
آحس

مقلا wa we ransack innermost recesses, Ephr. ed. Lamy iii. $40 \mathrm{I}, 56 \mathrm{I}$.

ไดُ

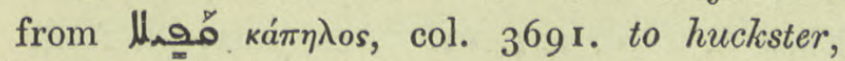
Sev. Lett. 501. 2.

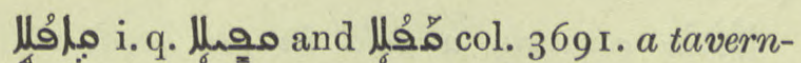
keeper; a huckster, Nars. ed. Ming. i. 360. Io.

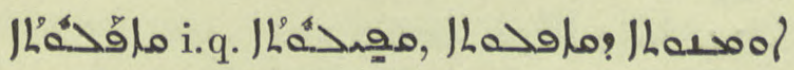
tavern-keeping, Nars. ed. Ming. 360. 10.

슬 like a petty trader, Sev. Lett. 388. 20.

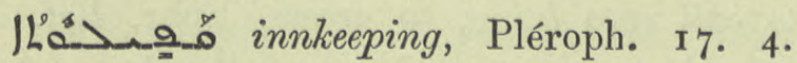
Metaph. adulteration, Sev. Lett. 8. 10.

وخحسه م

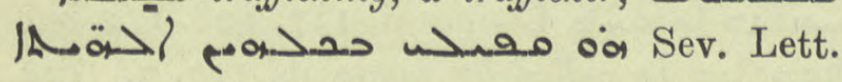
I45. 12 .

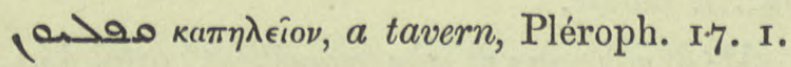

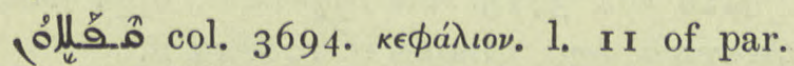
add: capital sum, principal, Sev. Lett. I I6. г. مقلll (مه ib. I 20. I6. (Ollawoo in sum, ib. 268. 2.

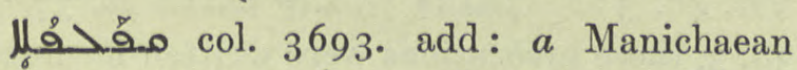

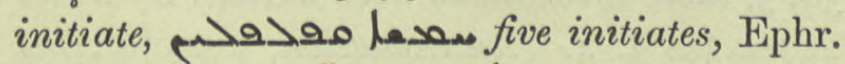
Ref. ii. 205. 7 ; lla

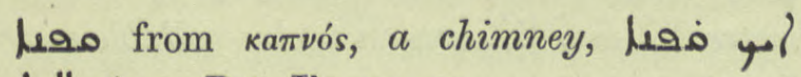

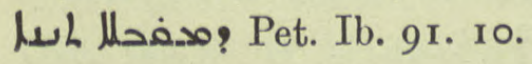

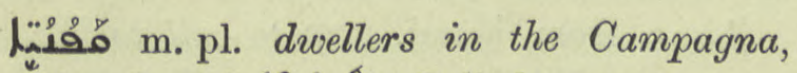

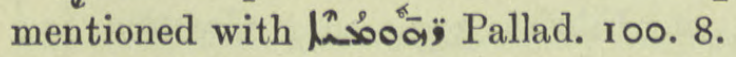

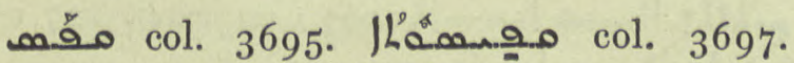

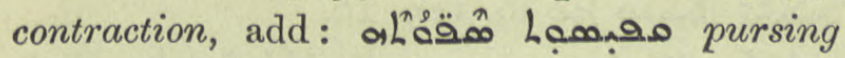
up the lips, But. Sap. Econ. iii. 2 : compression of wind in a narrow space, Ephr. Ref. ii.

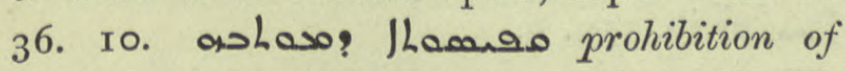
vagrancy to a monk, order to abide in his cell,

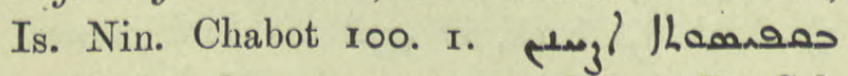
we have made a summary, an abstract, Bar Sal. in Lit. 94. 2.

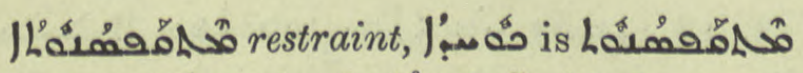
modesty is self-restraint from fear of committing culpable action, But. Sap. Eth. 2. 2.

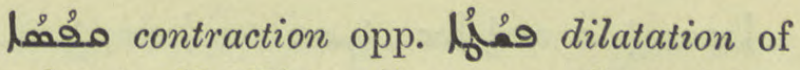
the heart, N. Hist. vii. I. 3. Convulsion, a spasm, Med. 146. I I.

col. 3698. a measure equalling 4 or 12 pints. Add : محصمبم oa

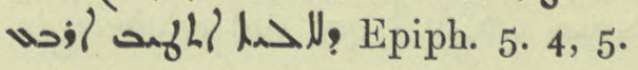

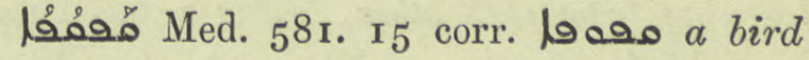
of night.

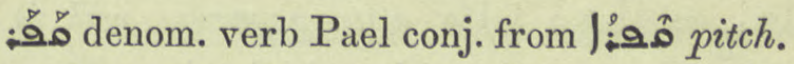

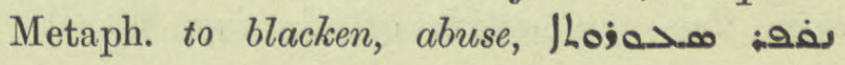
? مخل Greg. Carm. 105 pen.

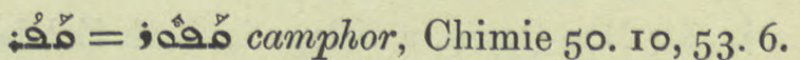

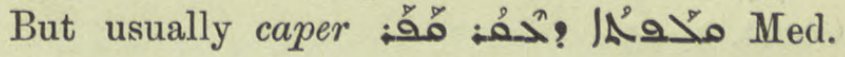
59. 16.

م مُه: Qaphrigawos, a people living to the south of the Pishon or Hedrinos? Chron. Min. 354. 3 .

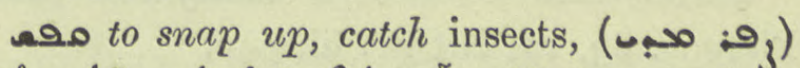

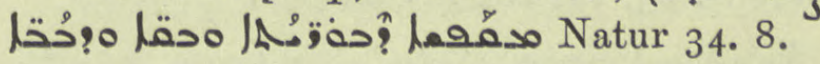

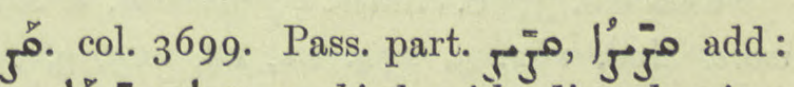
la G. Warda ed. A. Deutsch Io. 2 I.

ק a blind snake, lizard, Lev. xi. 30 Pseud. Jon. Nöld. in loc. ZDMG. xliv. 53 r.

爪 متر 35 f. pl. scrap-metal, clippings, filings, Chimie 45. I7. Perh. مكترمال

مُ مُ 3702. to break bread. Pass. part. friable, the fat of animals is N. Hist. vii. I. 5 .

مُ مُ fraction of the Eucharistic bread, refs. Takhsa 2 2. I I, 23. I 2, but the fracture, broken side of the host 1. $14,24.8,9$.

$1 L^{2}$ مُ Eucharistic bread, add: Takhsa 146. r 6 bis, I 66 ter, 167 quinquies. $1 L^{2}$ those who mix bread for the Eucharist, ib. I65. I5 ult.

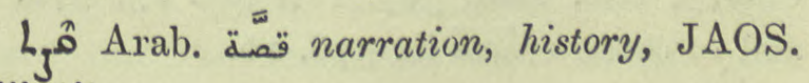
xiii. tit.

1 3704 . he who slays the sacrifice,

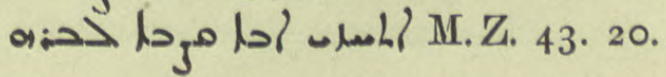




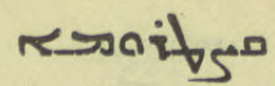

310

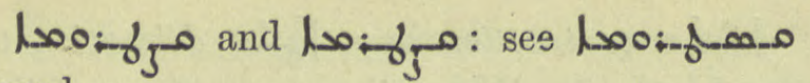
Suppl.

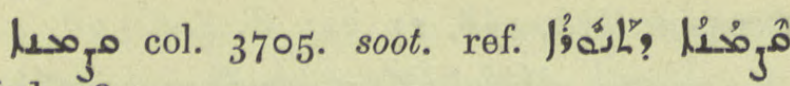
Med. 587,4 .

مَّ col. 3706. to be envious; animals are not envious in learning from each other, Natur 2. 6. Pass.

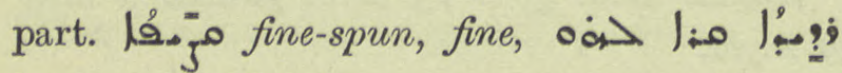

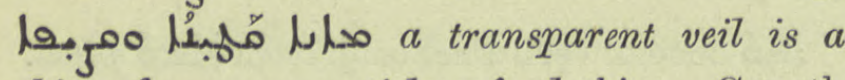
thin, fine-spun article of clothing, Ganath Busâmé $37 b 3$ af.

Qasra, a village in Beth Zabdai, Chast. 215.

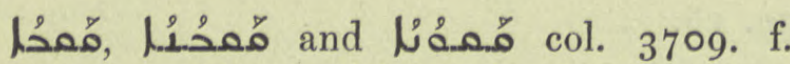

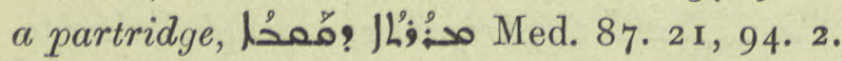
ผمْمُ ib. 553. 14, 558. 15, 16, 589 ult.

какó $\theta_{\epsilon \in}$, a malignant ulcer, Pléroph. 63. 12, R.O.C. iii. 342.

لِمَ 37 ro. Salsola fruticosa, a saltwort, Med. 162. 5, I 74. I I, 18, 609. 3, Ar. PflnN. 43. Cf. the following par.

> 37 iо. Cardamomum vulgare, cardamom, مُمفحُ (مed. I73 ult., I74. 4, 8, 263. I, 295. 14, 609. 3, Ar. PflnN. 296;

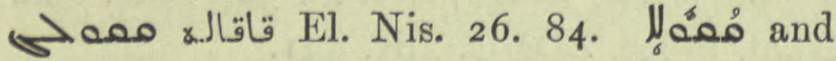

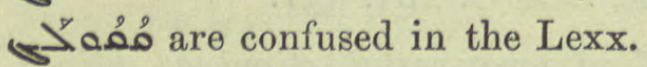

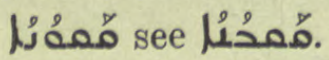

นـ как' exil. Name of an Egyptian sorceress, Kaki, Hist. Mon. i. clxv. 6 af.= Hormizd 2027.

حمA مع Beit Qouqa, a village of Adiabene near the Great Zab, Pers. Mart. n. I 7 15, Patr. Or. iii. i. 66. 6.

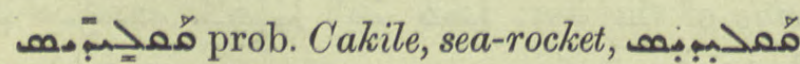

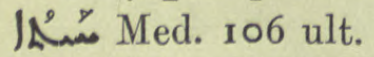

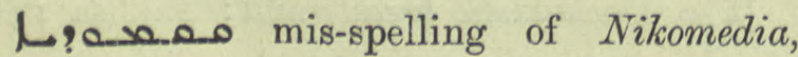
Nöld. F. S. i. 474 . I 35 .

مُمُ col. 37 1 Cherry, refs. Med. 367. I I, 369. 15, 370. 2 and so correct مُ مُ

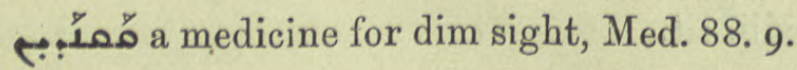
معمله = ممنُم a swan, Jac. Sar. Hom. iii. 92.6 .

\section{مit}

$1 L^{2}{ }^{\circ}$ Sap. Isag. ii. $\mathbf{~}$.

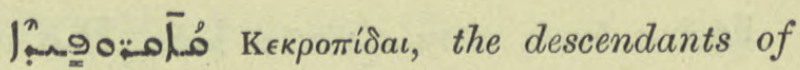
Cecrops, But. Sap. Isag. ii. I.

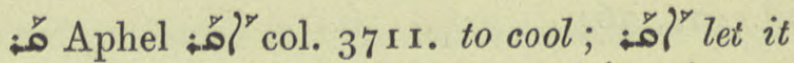

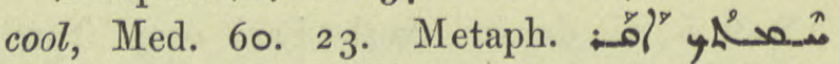
Ebed J. Card. ror. Ir. Act. part. pl. foods

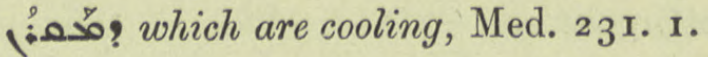

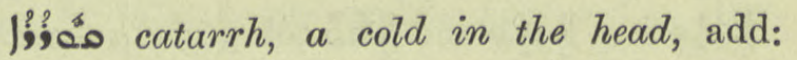
Hippoc. ii. 40, iii. 13, 19, 22 and gloss., Med.

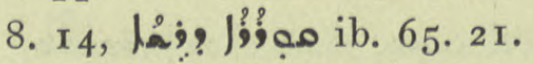

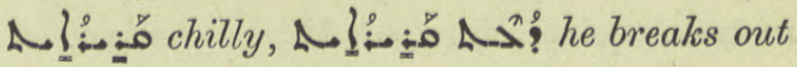
into a cold sweat, Med. 26. 3 .

م- col. 37r2. to call. Add: Mandaean to call into being, to create, Ephr. Ref. ii.

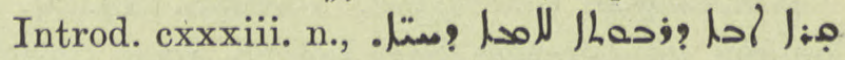

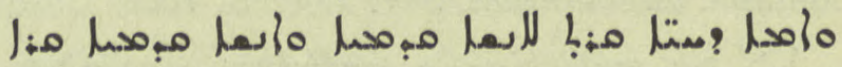
Coupes ii. I27, I 28. I, 2 but

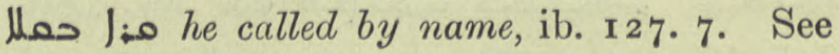
م: م: below.

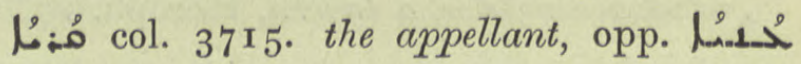
the respondent, Coupes ii. I 28 . $14 \mathrm{ff}$.

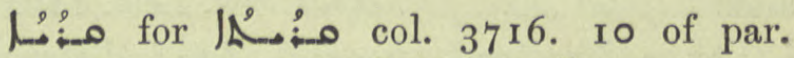
invocation. حمّ:ما (sic) Sev. Ant.

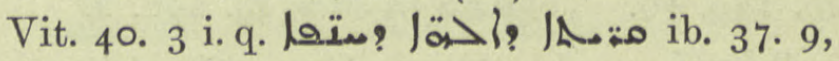
$3^{8 .} 3$, these invocations were written formulas

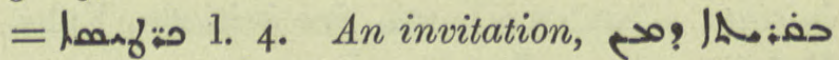
self-invited, Sev. Lett. 427. 2. Poetical

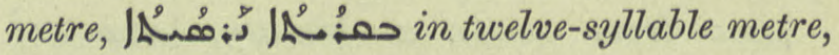
Jos. Narses I I I. 2.

م: from Mand. קרג to call to life, to create, cf. Fihrist 333. I9. Creation bl

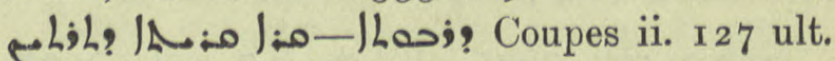

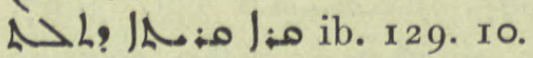

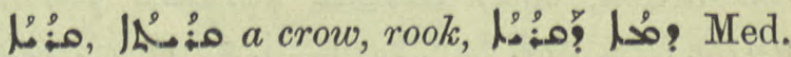

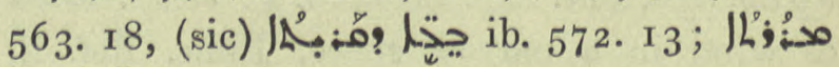

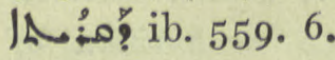

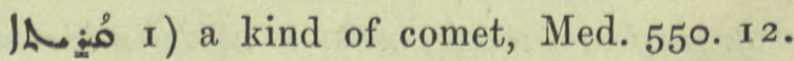
2) Arab. قر اقرى اقى back, his back pained.

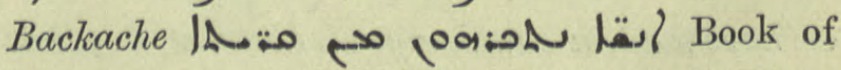
Shem I 10. I4. 3) col. 37 I7. a weevil, Dion,

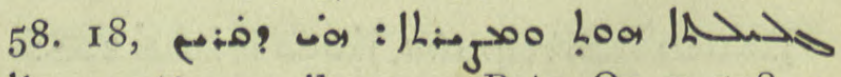

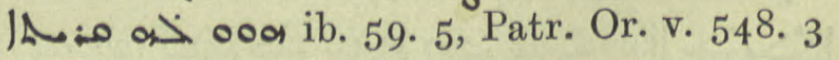

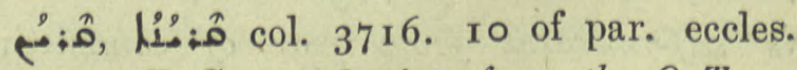
a lesson, E.-Syr. a lection from the O.T. or 
from the Acts, opp. Liñ a lection from the Epistles of S. Paul, Maclean.

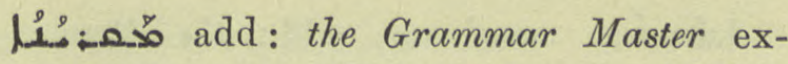
pounded the text of Holy Scripture, viz. how the words were to be vocalized, how to intone correctly; he also taught مزديل ?متحل choir reading i.e. musical notation, Barhad 383 . I2, 398 n., Journ. As. 1906, 275 n., Or. Xt. i.

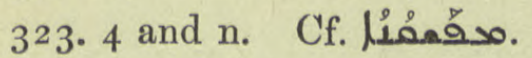

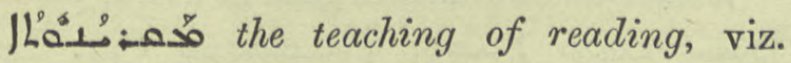
pronunciation and vocalization, Nars. ed. Ming. 33. I, Barḥad. Journ. As. I 905, I60 n.

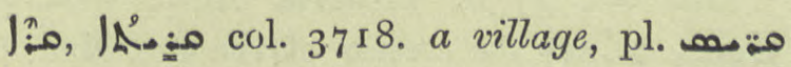
Dion. I17. I5.

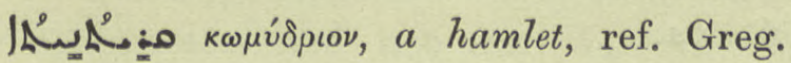
Carm. ii. 39. $\mathrm{r}$.

مـ م-أذفة مásos, sea crayfish or spiny lobster, N. Hist. vii. 4. I.

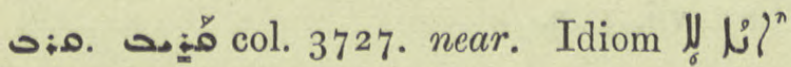
أمَّن I have nothing to do with it, it is no concern of mine, Pallad. 477. I2, لٕ لخ

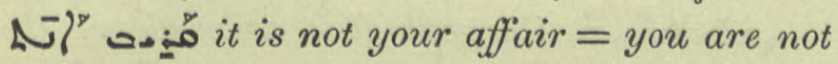
in fault, cf. Lat. affinis culpae, ib. 553. 7 ;

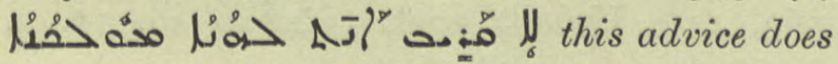
not concern you, A. M.B. v. $5^{27}$.

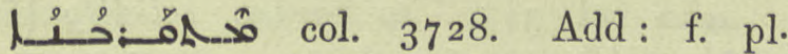

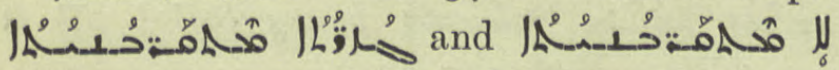
accessible and inaccessible islands, Jac. Edes. Hex. xix, 8, Io; Joghea> / from fellowship with heretics, Pléroph. I6. I 2. ג Sev. Ant. Hymns 228.

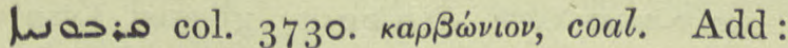

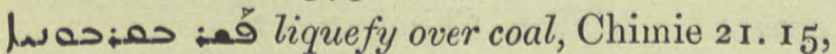
N. Hist. iv. I. I, Sev. Ant. Hom. 5 .

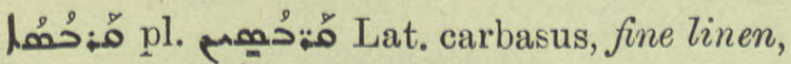

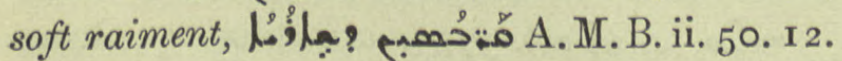

مث: Aph. مث: of thorn. To clear away thorns, to uproot,

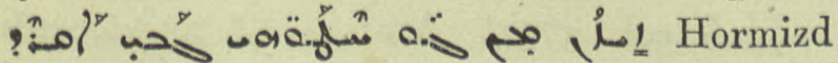
1307 .

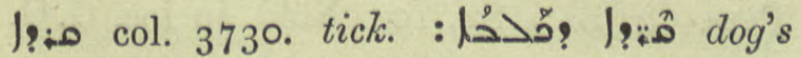
ticks, Med. 555. ult.

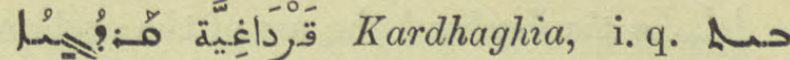
م: the district of Beit Kardagh in Marga, Hist. Mon. i. I83. 5 cf., ii. 330 n., 387 n.

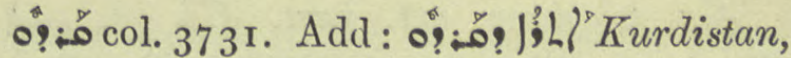
Hist. Mon. i. $3^{6} 5$. 19. A bpric. on the left bank of the Tigris opp. (ב⿳宀八工 bank, Syn. Or. 33. 22, 2 I 4. 4, 619. 4, ZDMG. xlii. 396.6 .

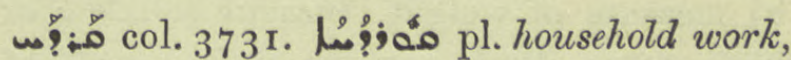

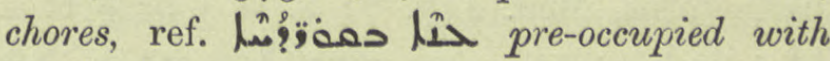
household matters, Poet. Syr. 36. 4 af.

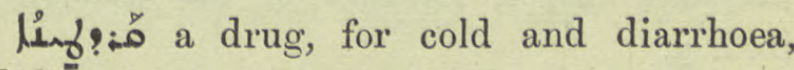
Med. 4 г 2. го.

م: مز: pr.n.m. for Rhadamanthus, Arist. Apol. «. I.

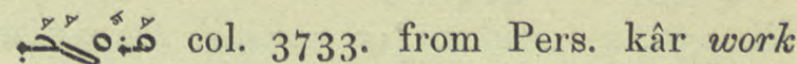
and bed master, Tabari $5 \circ 2$; a foreman, م: Syn. Or. 79. 20.

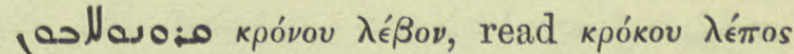
"écorce de safran" a symbolic name for sulphate or oxysulphate of arsenic or of antimony, Chimie $56.16,24,57.17$.

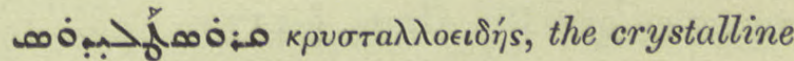
lens of the eye, Med. 68. I 5, 69. I5.

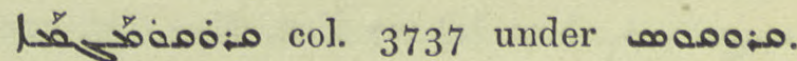
кроко́раүна, dregs of saffron oil, refs. Med. I62. 6; saffron oil, ib. 609. 4, 5 ;

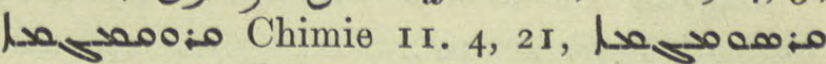
ib. 47 pen., مص مه ib. 247 med., مه: Med. I6r. 8.

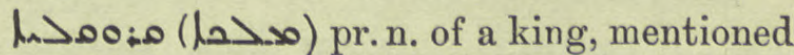
with Lُ S. George I $8 a$ II.

مث:Arab. قاروزه a bottle, Chimie 59. I6

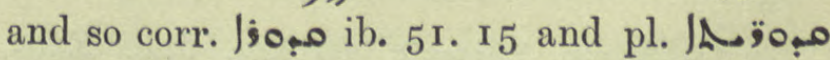

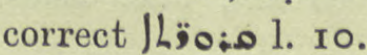

achoj col. 3738. кро́т⿻上丨 Duval, who says alchemists call it white tin or lead, Chimie 2 pen., cf. trad. 5.4 and n. 4 .

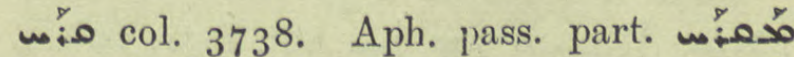
mentioned, referred to the Synod م.من: R. O. C. iv. $25^{6 .} 4$ af.

مُ مُ falling out of the eyelashes, Med. 83. 12. 


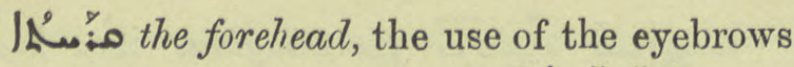
is to keep off from the eyes $1 \mathbf{L}^{2}$ N. Hist. ii. cap. iii, sect. 4. Cf.

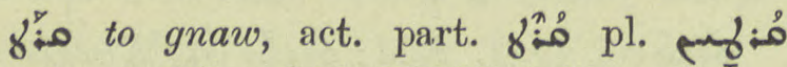
Warda ed. Hilgenfeld, ZDMG. lviii. 498.

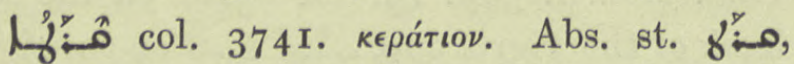
" حب0 "fenugrec", Nau, Pléroph. 36. 2, 37. 2. ชُ 2) measure of weight, I carat equals 4 or 6 barleycorns, Med. 447. 7 ; it is $\frac{1}{2}$ or $\frac{1}{4}$ of a فُ فُ î ?م- half a measure of the sanctuary, ib. 1.5 .

M: o col. 374r. the leek, ref. Med. 563. 2,

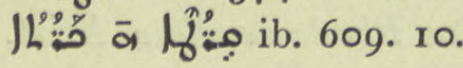

م: col. 3742. quoted from Anecd. Syr.

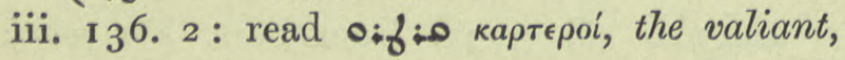
the fierce, Ahrens.

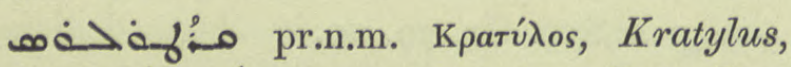
مu But. Sap. Periherm. I. 2.

ค. مै col. 3743. to cut off. A strengthened form of مf col. 3578 : see Duval on the insertion of ; Riš, Gram. § I I 7 .

سم

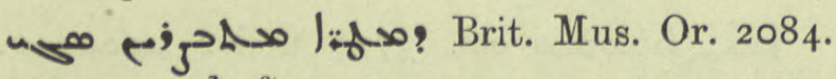
$2 v, 5 v$ and often.

مهز్مْ Carthamus tinctorius, Safflower, Bastard Saffron, ref. Med. 572. 17, Ar. PflnN. 2 I8.

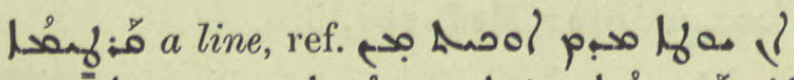

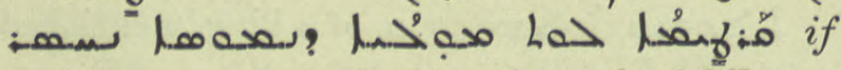
one jot or tittle be lacking from the fulfilment of the Law, G. Busâmé 82. 9 af.

مث: Lat. creta, chalk, Chimie 7. 20.

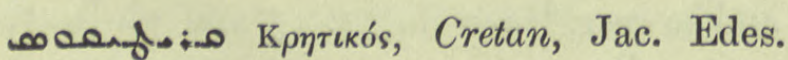
Hex. xi. I I.

مزيلما corruption of kptvîtos, crinitus, a comet, Sev. Lett. $3^{83}$. 10.

م: name of a province or district, Ant. Patr. 298. 9.

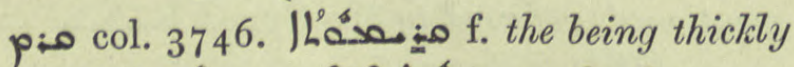

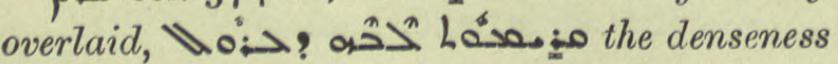
of an uncircumcised heart, But. Sap. Theol. 6. 4 infra.

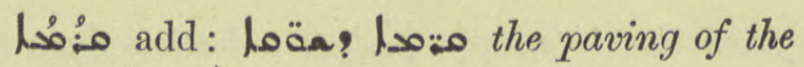
squares of a city, Jac. Edes. Hex. ii. 4.

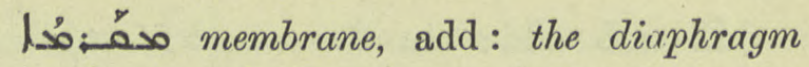
مدحما ف:0atr. Or. iii. i. III. 2.

إم fo a pitcher, Mar. Benj. 73. 3 = A.M.B. iii. 518 ; مَّمُ ib. 519.17 .

of a dittle pitcher, م- Sev. Lett. 423. 4 .

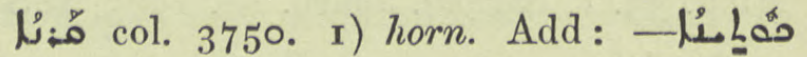
حُقْ the cornea of the eye, Med. 68, r8. 5) tip. Add : the claws of a crab, Natur 61. I4.

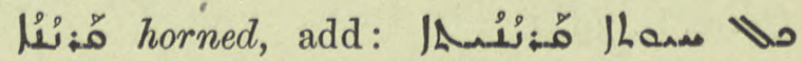

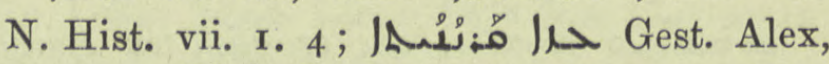

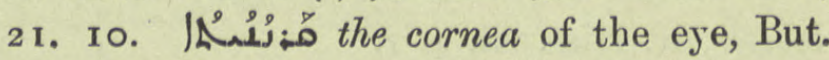
Sap. vii. I. 2.

IA مع col. 3752. a head veil, ref. A.M.B. vii. 118 .

فـ Caryophyllus aromaticus, the

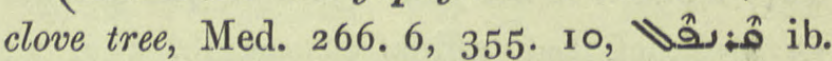
563. II. Usually spelt مُ مُ 89 bis, I7 74 . 12 and often.

مَّمُ

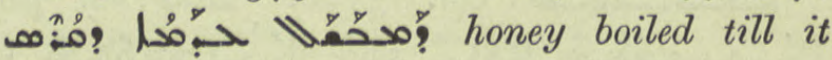
crystallizes, Med. 432. II.

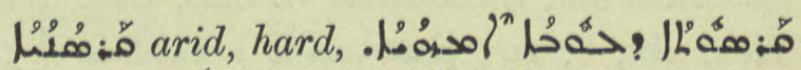

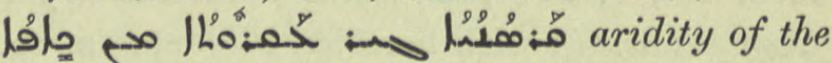
maternal bosom, for barrenness is harder than stone, G. Busâmé $7 \cdot 4$ af.

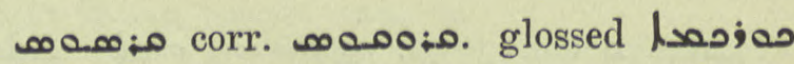
crocus i. e. saffron, Chimie 7. I2.

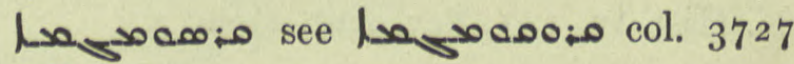
and Suppl.

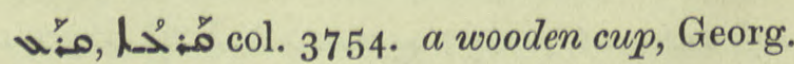
Ar. 35. 16. An alembic, Chimie 19. I7, 2 I. 23,22 . I, 4I. 6 .

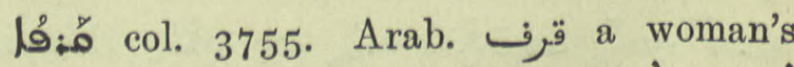

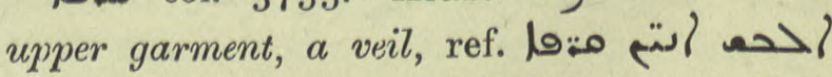
Patr. Or. iii. i. 84. 10; Nöld. in loc. G.G.A. $1907, x_{7} 8$. 


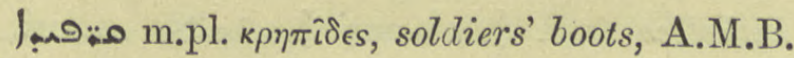
iii. 3.8 .

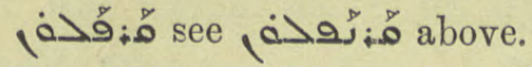

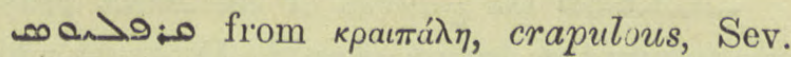
Ant. Vit. 23 I. 10.

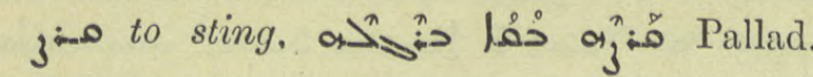
I 45 pen.

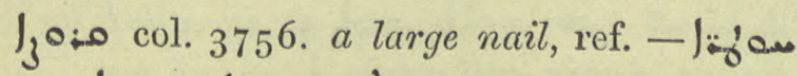

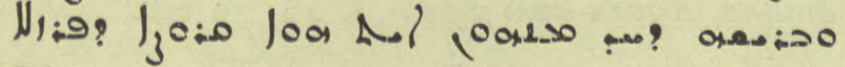
Dion. 48 . 18.

$1 L_{0}^{\circ}:$ : A. M. B. vii. 78 .

لإ. of hive stands. Med. 579. ro. L. 8 of par. scarcity of corn. The passage quoted from Aneed. Syr. is also found, Zach. Brooks ii. 174. 9 , 194. 2 .

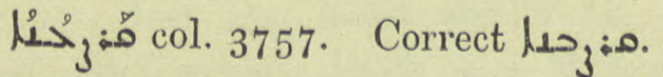

م many of those bae th 000 pro: Dion. R.O.C. ii. 64 . II.

متمعم col. 3758. a fugitive but pl. مخ:مب as from sing. م: Poet. Syr. 99. 8.

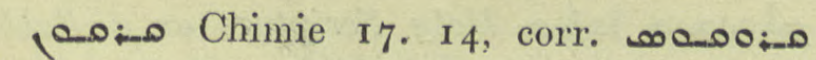
Crocus.

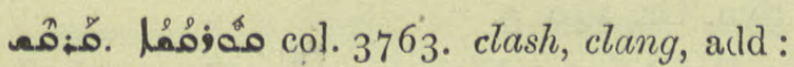

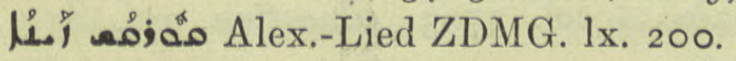

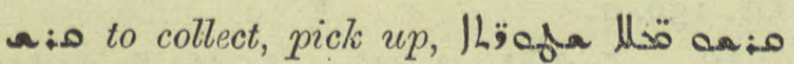

Dion. I 20. I 5 .

אaviotod cream, Med. 577. I.

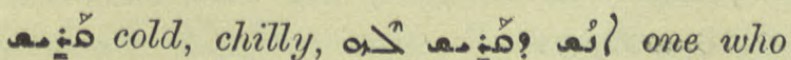
has a chill, Manichéisme ıоo. 18.

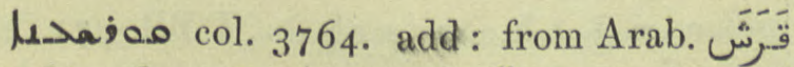

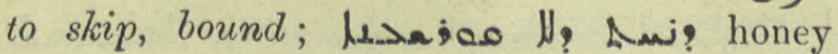
which flows down continuously, in a smooth stream, Geop. I18. 20.

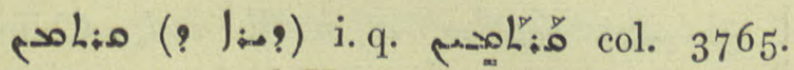
the monastery of Kartamin east of Mardin, Chron. Min. 207. 23, 216. 27 and often. unsolis the same, Dion. 69. I9.

h.1soh:-o m.pl. those of Kartamin, Dion. 69. 16.

2716

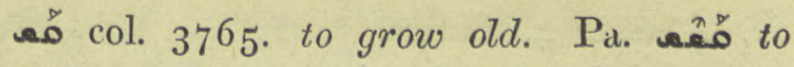
declare to be the elier; to give precedence, $\rightarrow$ مع of giving the elder brother's

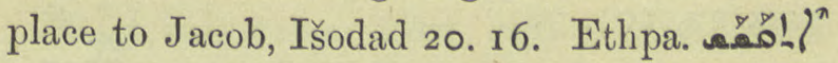

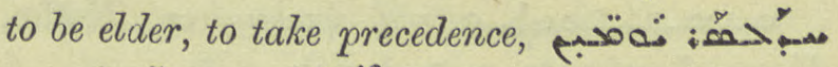

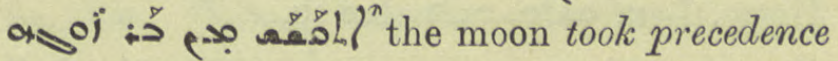
by twelve days of the sun, Jac. Sar. Hom. iii. 74. 6 ; Poet. Syr. 70. I.

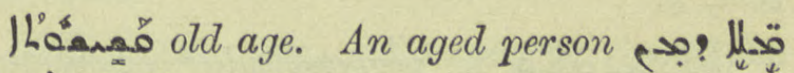
مُعبمer she remembered the words uttered by her aged relative, Pallad. I99. 7 .

Arab. خواس Qaś, bpric. in Seistan, on the river of the same name, SE. of Earah and W. of Bist, Syn. Or. 88 bis, 89 ter.

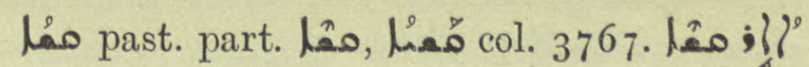

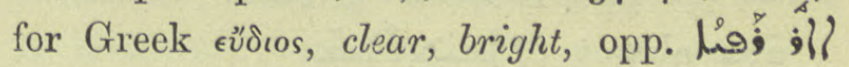

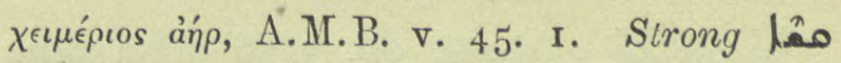

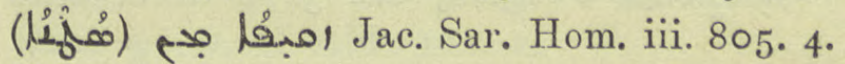

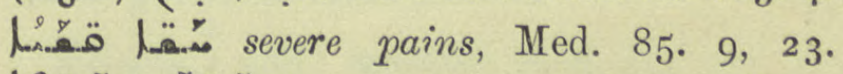

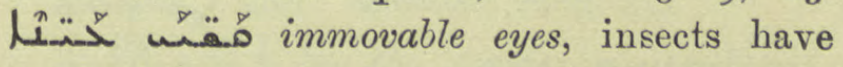
compound or simple eyes, the former are

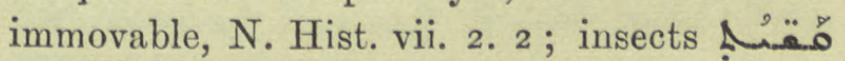

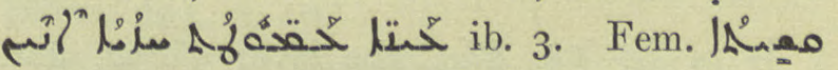
the sclerotic membrane of the eye, ib. vii. s. 2 ; م ventres constipés, Hippoc. iii. 5 .

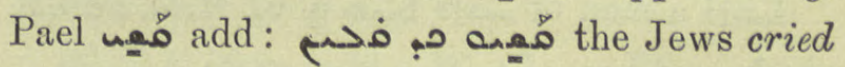
harder, Let Him be crucified, Stud. Syr. ii. $9 \cdot 9$.

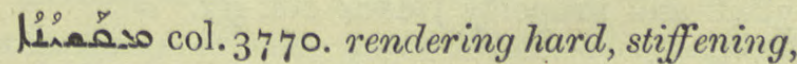

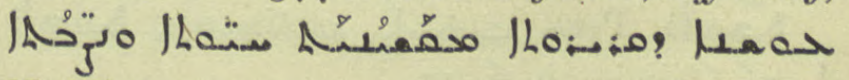
N. Hist. v. v. I.

|

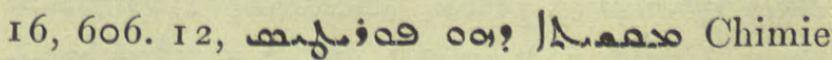
37. 12.

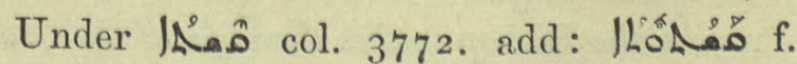
archery: a corps of archers, M.Z. 196. 20.

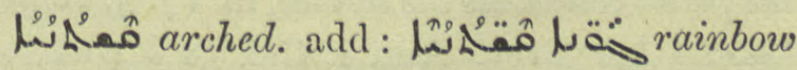
colours, N. Hist. v. 2. 3.

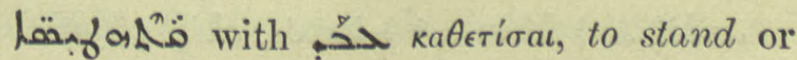
be placed perpendicularly, م. Hist. S S 


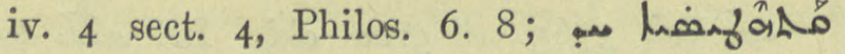
- o- 1 chambers of a furnace set perpendicularly one above the other, Chimie 100. 22 , 10I. 7 .

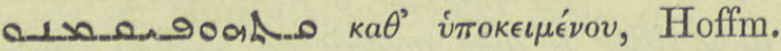
Herm. Lex. assanoagaho ib.

œ م مب م- مدم N. Hist. ii. v. sect. 4 .

LLa.

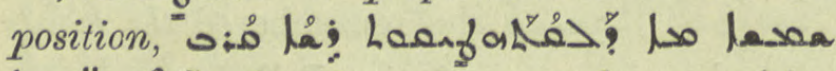

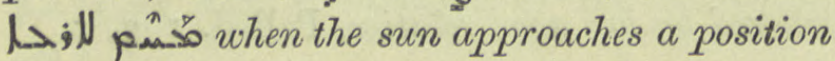
perpendicular to the earth it makes the earth

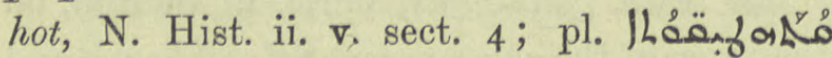
of the motions of the stars, ib. iv. iv. I.

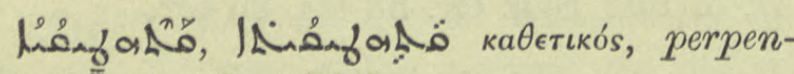
dicular, But. Sap. Philos. 5. 3.

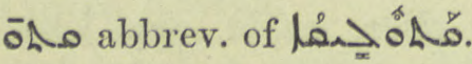

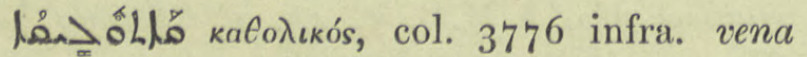

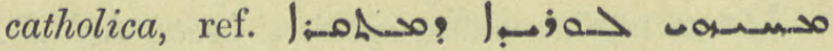
مه Sassanidi I4. I4, trad. 17 .

wan $>$ ollo the general or universal prayer recited by the deacon after the Diptychs while the priest prepares to consecrate the elements ملام

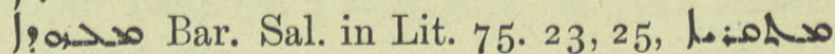
IANen oolo sol it is called by the simple, Again and again, 1. 26, 76 ter.

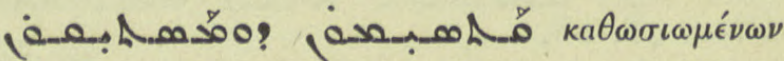
$\delta о \mu \epsilon \sigma \tau \iota \kappa \hat{\omega} \nu$, the Imperial bodyguard, Nest. Hérac. I70. 12.

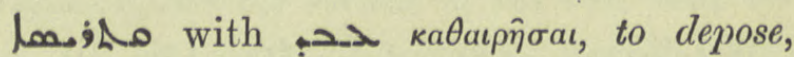
Sev. Lett. I72. 4, I 9 .

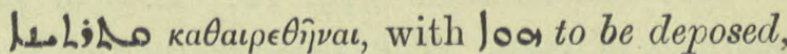

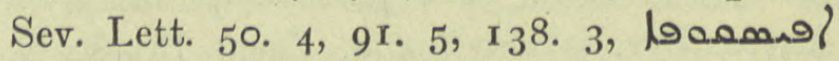

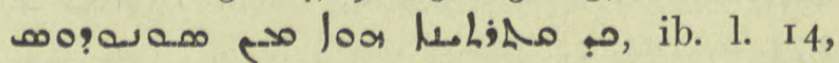
I 70. 9 .
J' or J' 'Pâ, Rha, the river Volga, Jac. Edes. Hex. xiii. I6, xxvi. Ir.

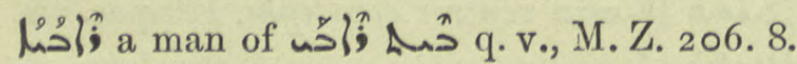
לे.

Ml; rice, Z.A. xxi. 207. Usually lio' col. $3^{84} 6$ and properly l10jol col. 93 .

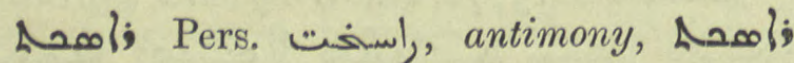
عُ Chimie 99. 6, 12.

ڤ': col. 3784. 27. Rit. the Bishop; the

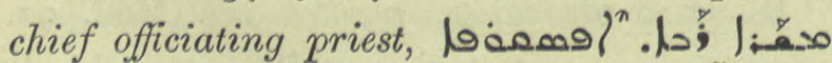

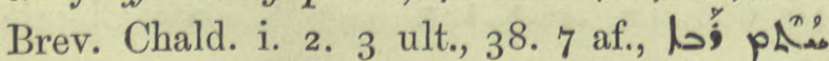

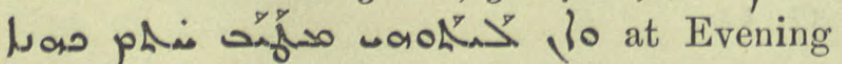
Prayers the Bishop makes the sign of the Cross i. e. concludes service and if no bishop is present the priest concludes the service, ib. $17.5, \mathrm{I} 8$ pen.

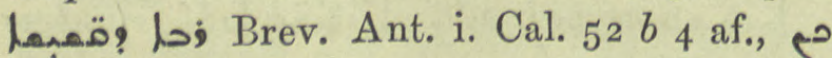

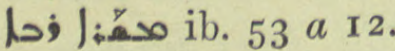

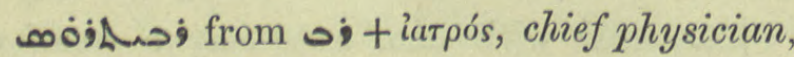

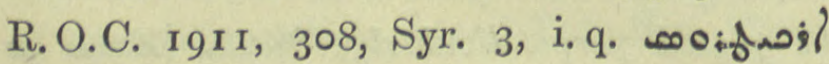
ib. 306.4 af.
L'ล์ oholy Scripture as a Rabbi teaches the duties of children and parents, Syn. Or. 140. 5 .

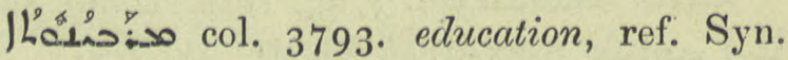
Or. I 56. 2.

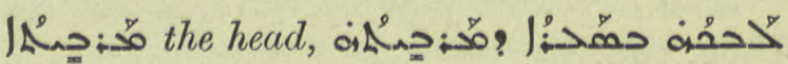
A.M.B. v. 604. I 4 .

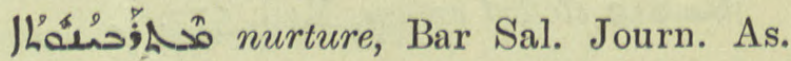
I 908 , I02. I I.

12 مis add: the waxing of the moon, under 20.

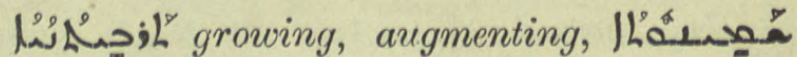

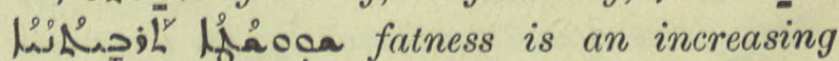
extension of the body, N. Hist, viii. 2. 3 .

; 294. n. I.

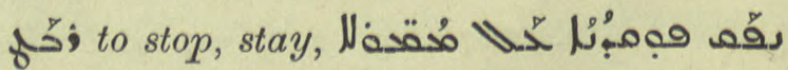

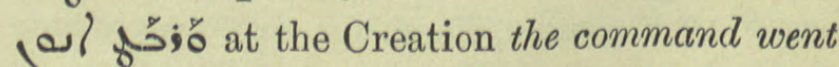
forth to the waters and He stayed them, Jac. Sar. Hom. iii. 44. I 5 . 
Waid Arab. Rabُ̂, name of two months of the Mohammedan lunar year, פדמ مب0مسا

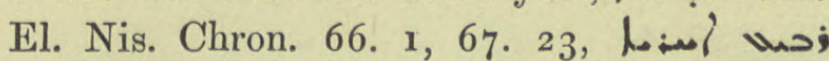
ib. 64 . 19.

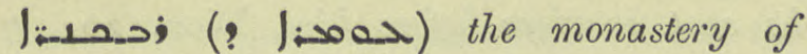
Rabkennaré, Journ. As. I 906, 109 n., I 10, I 4 .

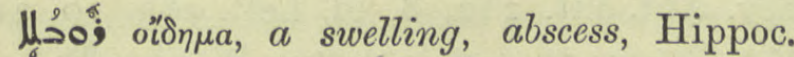
iv. 34 , v. $6 \mathrm{r}, 62$, Med. 66. 6 .

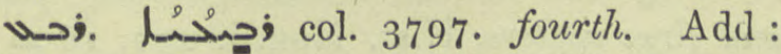

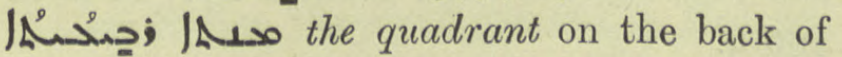
an astrolabe, De Astrolabe 258 bis. A vigil

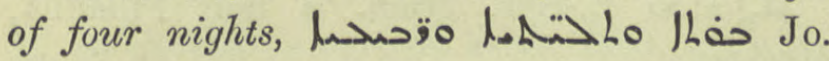
Tell. $23 \cdot 3$.

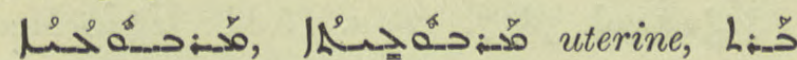
IA sister by the same mother, Jab. 456 .

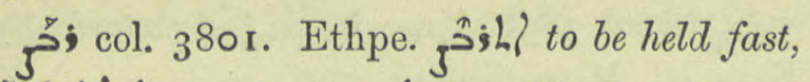

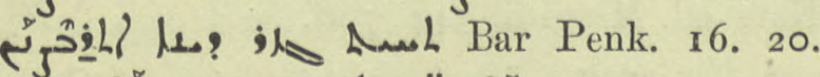

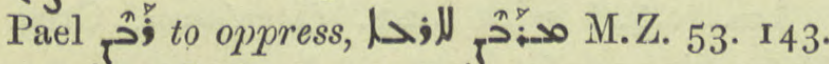

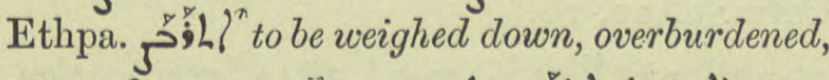

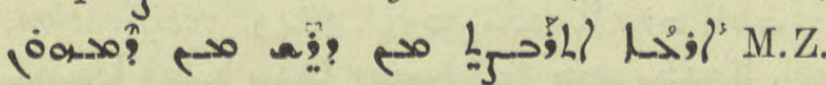
63.7 .

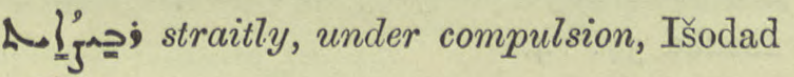
2 I. 17 .

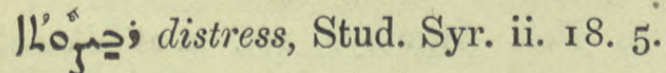

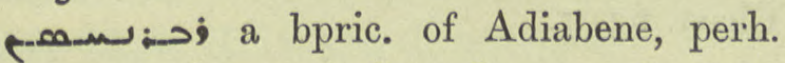

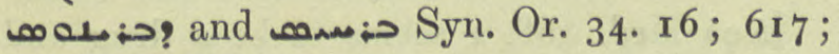
ZDMG. xliii. 394. 8.

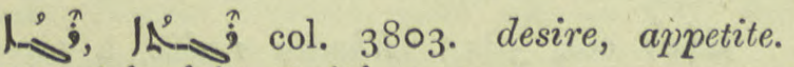

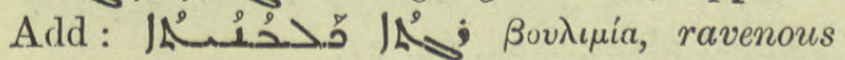
hunger, Med. 289. 9, 338. 19 and often;

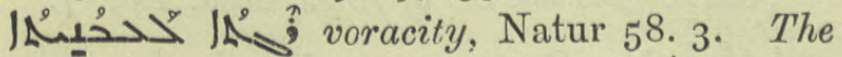

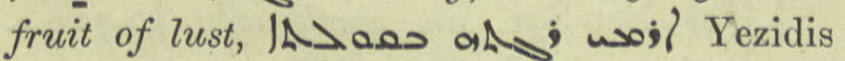
102. 8 .

13. Ethpa. us jL/ $^{n}$ col. 3806 . to flourish, to become fresh and green, of trees,

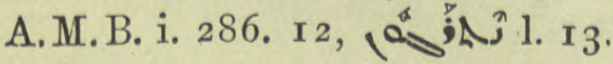

A

lool-s; col. 3807. 6. Correct: with حِحِ to distribute largess, Anecd. Syr. iii. 233.20 , 20 ib. 220 . 7 , same col. 1. $3^{\circ}$, corr. loọs' fóra, -as, largess, a donative. I. q. Aso; and aso' col. $3^{844}$.
مإِ col. $3^{807}$ lion lo $_{4}$ QdhamW. I87. I4.

lio فُ wrathful, QdhamW. I87. I5.

" ; col. 3809 . Corr. ref. to Act. Mart. i. 67. 19. Shaphel

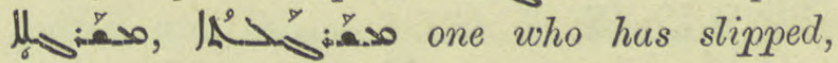
A

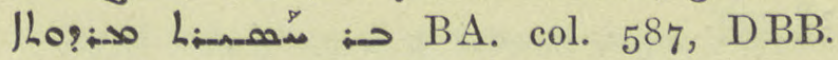
428 infra.

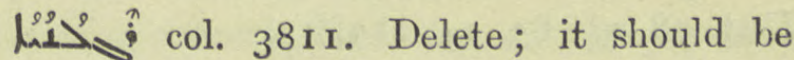
ר.

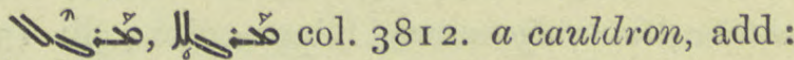

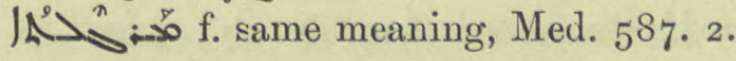

น earthquakes sometimes take place with concussion and by vertical slipping down, N. Hist. iv. I I. 2.

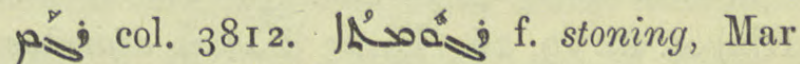
Qard. 92. I, ed. Abb. гоr. 2.

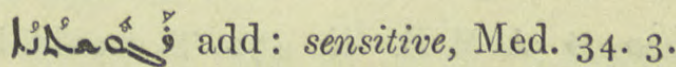

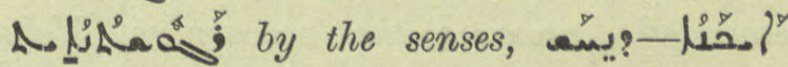

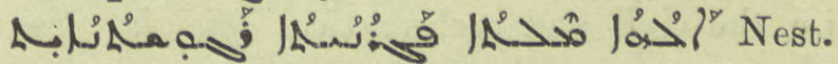
Hérac. 295. 9, го.

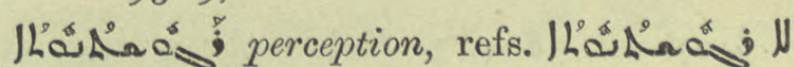

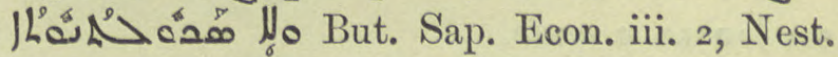
Hérac. 269. 2.

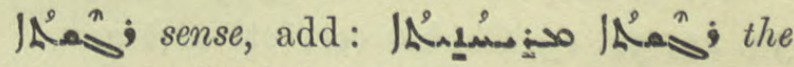
sense of smell, Med. 62. 7.

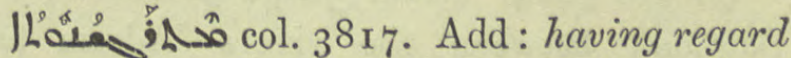

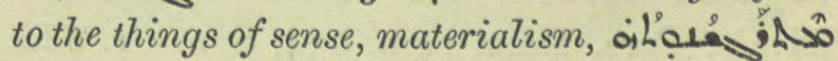
an? Pallad. 205. 5.

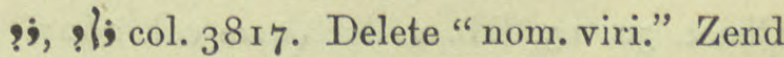
ratu, Lord. The governor of a province, a magistrate, official, Tabari 447 , Syn. Or. 77. $14,99.9$, trans. $3^{29}$ n. I. Mon. Syr. ii. 68. 16 so correct ;?; Jesus-Sabran 529 ult., 53 o bis, A.M.B. ii. 572 . I.

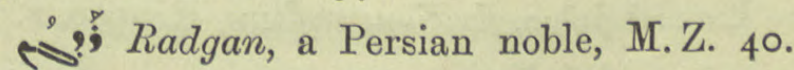
I 2.

9? from a Mandaean root, to rouse from

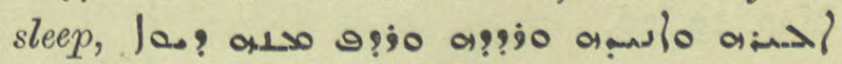
he wakened him and shook him and roused him and chased away the Devil from him, Theod. bar Choni in Coupes Pt. ii. I 30.4 af.

S S 2 


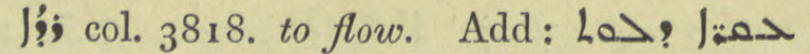

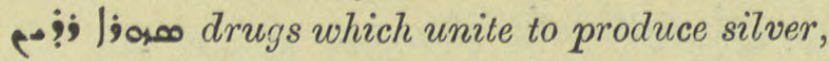

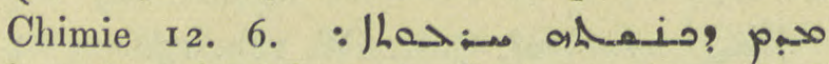

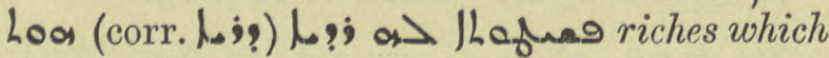
cunning has gathered, simplicity has dispersed, Philox. 79. I 9.

f:ơ? col. 3820. add: a traveller, Jab. 18 pen.

A. Lett. $78.3,185.14,2$ I I. I3.

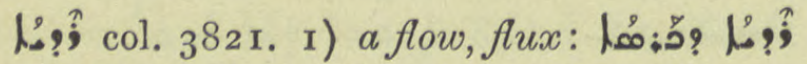

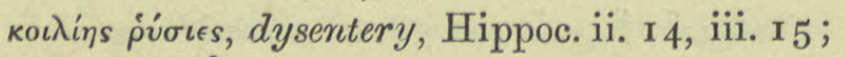
زُّ haemorrhage, ib. vii. 7. 2. 2) L L.

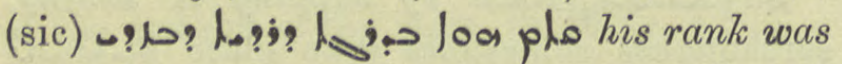
that of Governor of Beit Zabde, Mon. Syr. ii. 66. 26 .

1

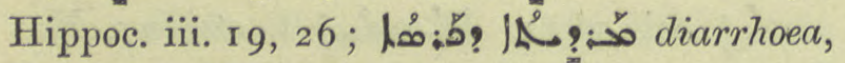
ib. vi. 5 o, Med. 364.4 .

فㅇ. A.M. iii. 470.8 should be

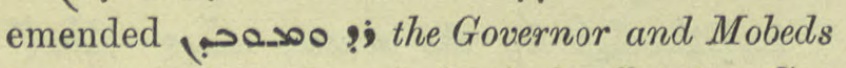
i.e. the civil and ecclesiastical officials. See :

Lo:' col. 3824.3 . Delete the article, and see

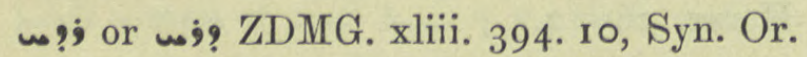
33. 29, 34. 19, Chabot corrects wị q. v.

9: col. 3824. Arab. اداد a province N. of Baghdad between the rivers 'Adaim and Diyala, Pers. Mart. 74 n. 634 , Syn. Or. 680. Or. Xt. i. 3ro. I, \}?; ib. 2 ; Jab. 2 I5. I4, Chast. 50. 15, BB. under 2000 col. 2607.

$\boldsymbol{\omega}$; Radani, bpric. of Beit Garmai, suffragan to Karka d' Beit Slok, Eranšahr 22, Syn. Or. 639. So Chabot corrects as ib. 43. I5. I. q. ?:ל col. 3782 .

Lxoo'; $\rho \in \hat{\varphi} \mu a$, fluxion, enflure, Hippoc. v. 9 .

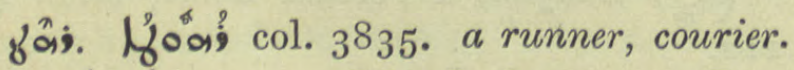
L Sol John the forerunner, Doc. Mon. 19.26.

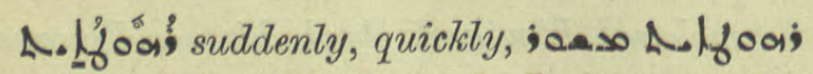
می مح: Our Lord made Lazarus spring quickly from the sepulchre, Doc. Mon. 20. 26.

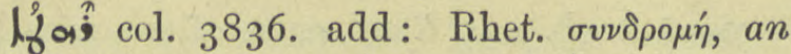
apparent concession, for Vit. 25 o. 4.

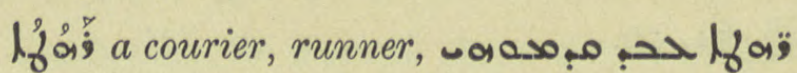
he sent couriers before him, Dion. 204. 7; a commissary, ib. I60. 23 , I61. I2. Hai; A مب: the Fore-runner, John the Baptist, Sev. Ant. Vit. 2 I 2. 8, id. Lett. 263 . ro, 2 I, Pet. Ib. 34. 19. 2) a rafter, $1 \mathrm{~L}^{2}$

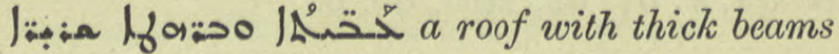
and firm rafters, Hist. Mon. i. 271, I6 = ii. $496 \mathrm{n}$.

lُfُa:- E. E.-Syr. gram. a half Pthakha, Maclean.

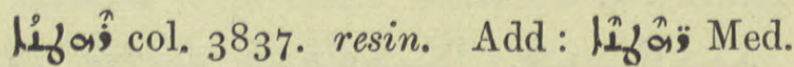

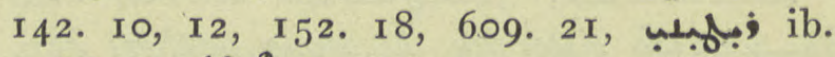
and l. I9, lif? ib. 40 r. I1.

J:Nars. ed. Ming. ii. 344. 7 af.

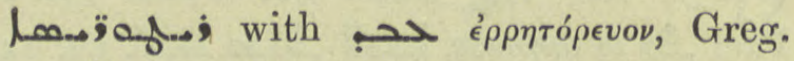
Carm. ii. 20. 16.

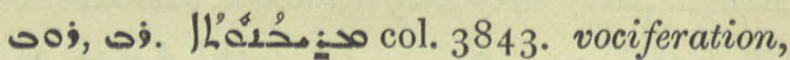

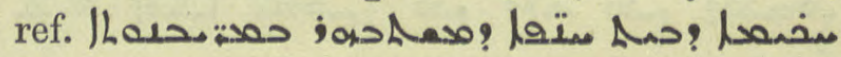
a heathen sage, glorying in vehement assertion, Išodad 37. I 4.

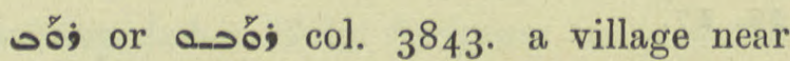
Anbar in Beit Aramaye, ref. so; Rewab? Chast. 63. 7 .

ذo: Med. 468. I seems to be a drug and not to be fُo thickened milk as col. $3^{843}$.

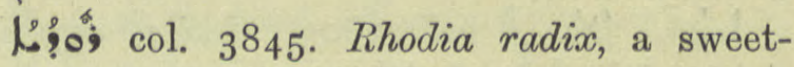
scented plant, Med. 64. 2 I.

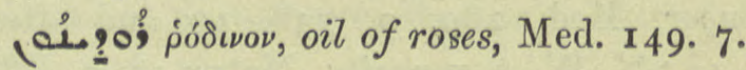

 Prognostications $3 r$ bis, $6 r, 7 v$.

مف: an animal with long sharp horns, Natur I 7 ult.

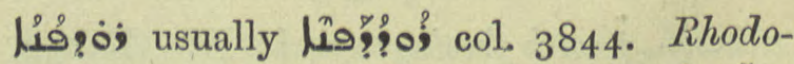

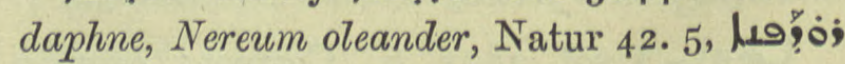
ib. 45.3 .

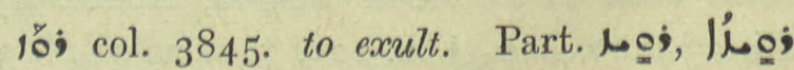

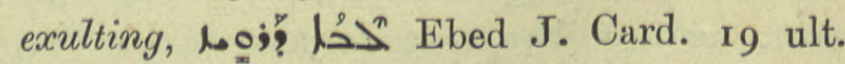
Pl. Jتم they who exult, Hormizd 168. 


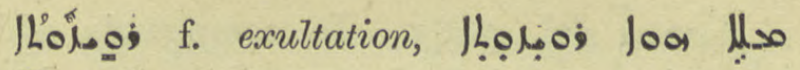
S. Dan. 7 I $a$ I 7 .

heo' col. 3848 . part, space. Add: conso' פ 2 the territory of the children of $\mathrm{Ham}$, Chron. Min. 356. ro, joakohim? L.os the inheritance of the Semites, ib. 1. 24. -90.:a a couple of uncanonical bishops consecrated an ignorant man and sent him out anywhither, nowhere in particular, cf. Arab. . ع. Išoyahb 68. 12.

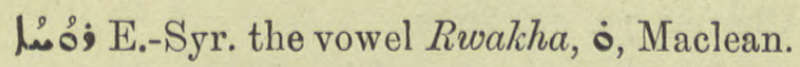

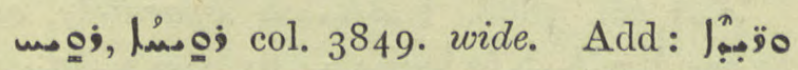
قo dilated or varicose veins, Med. I 7. I 8.

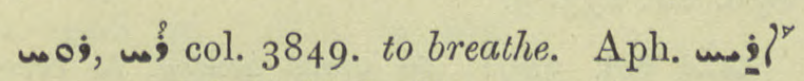
to cause to inhale, Med. 66. 22.

L spósirit, a spirit, considered as a cause

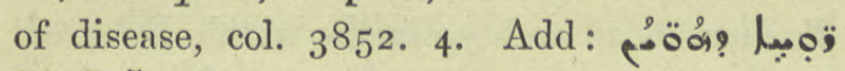

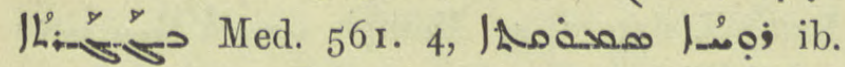
599. 2,1 ل

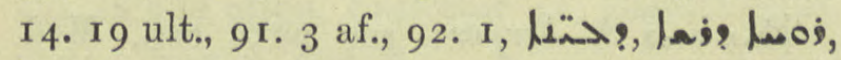
?. pains in the head, eyes, teeth, ib. 77 infra.

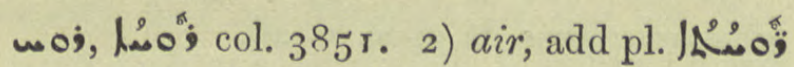
vapours, Chimie $3^{6 .} 4$.

|

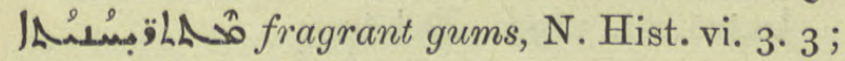
. 2.

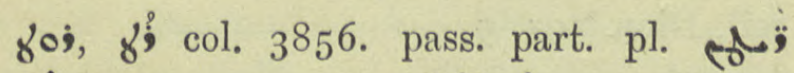

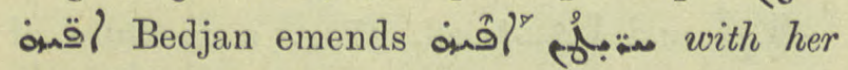
face scratched, A.M.B. iii. I 20. I.

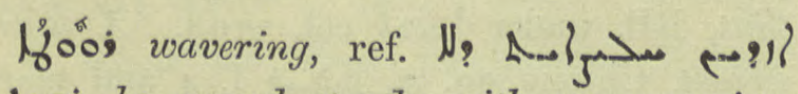
Hoo' be armed stoutly with no wavering, G. Warda ed. A. Deutsch 39. I I.

Hoํ a stake, a small plank; add: a thin, pliant branch of poplar or willow, Hist. Mon. i. $27 \mathrm{I}=$ ii. 496 n. 5, ef. bو jozy Suppl. i. $57 \mathrm{r}$.

No: m. pl. Lat. rotae, the rack or wheel, A. M.B. vi. 47 . 1 I.

ufö col. $3^{8} 57$ quoting BH. de Pl. $\mathbb{8}^{8}$. Emend مار gourds or cucumbers. The passage is from Arist. de Plantis i. 5 .
Loso; Rukut, bpric. in Seistan, perh. the

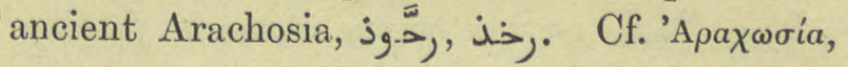

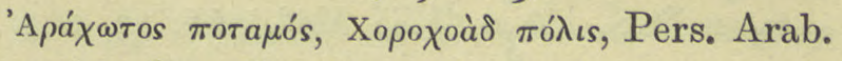
I 56 n., Syn. Or. 68 г ; 88. г 7, 89. г.

po;, p. p. liable to be

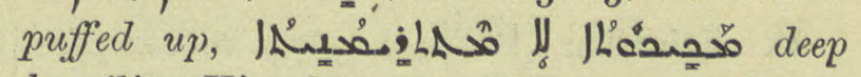
humility, Hist. Mon. i. 379. I.

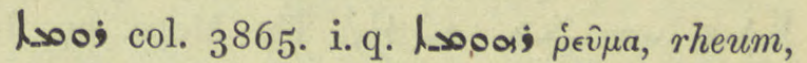

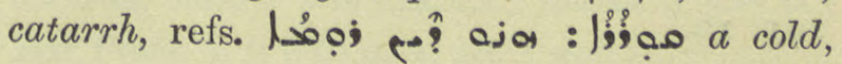

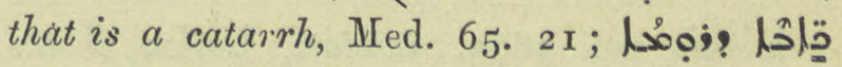

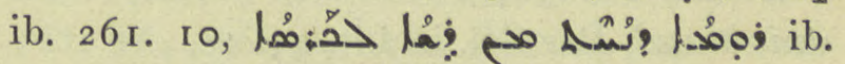

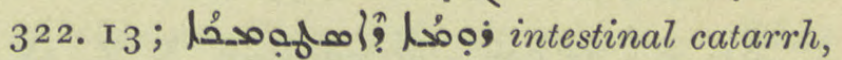

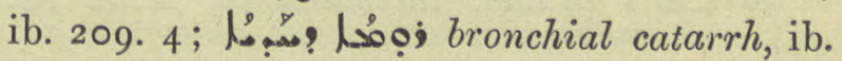
237. 10, 244. 2 I. Pl. Af pooj ib. 66. 10.

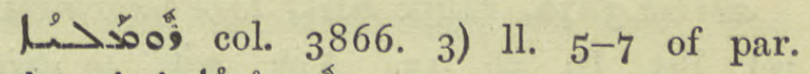
Delete, it is liحُ

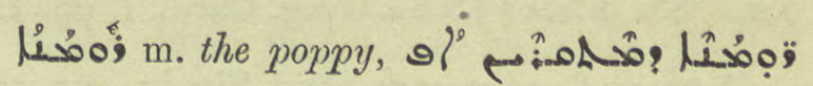

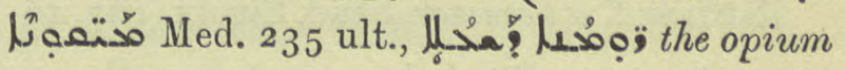

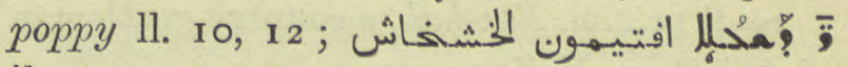
ib. 609. I6, I7.

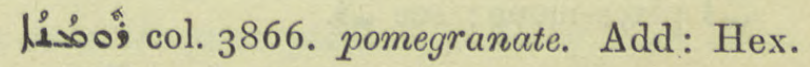
ค́оібкоs, Ex. xxviii. 33 .

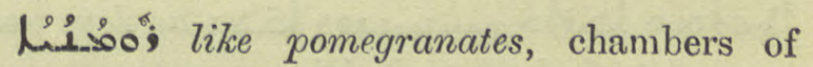

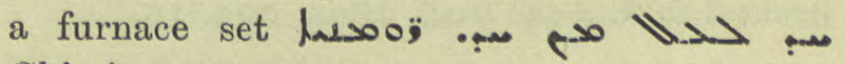
Chimie. 100. 22.

unso' Roumani, village in Adiabene, Chast. 8. 4 , ro. 7.

ـo' Arab. Rheum rliaponticum, garden Rhubarb, Med. 355. 13. See o\}; col. $378 \mathrm{I}$ where the spelling $\mathrm{Nol}$; is given.

م 0 os col. 3868 . Rhossus on the Syrian coast, Sev. Lett. 73 pen., Marg. 'Póoos.

œ vii. $\mathbf{r}$ I infra.

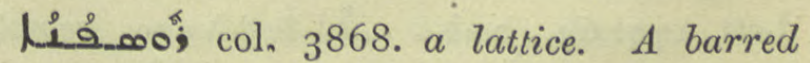
room, a prison,

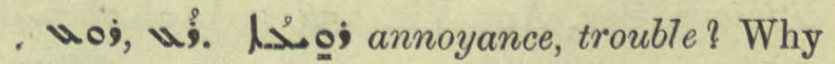
should I labour to collect many names لمدجدب-_oc. Jac. Edes. Hex. xxv. 5 .

19ீo' or lọ col. $3^{869}$. a moment. Ref. IA looj a short hour, a short moment, Ephr. Ref. ii. 197. 25 . 
மo;, م col. $3^{870 . ~}$

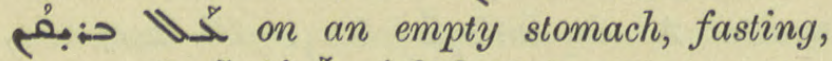

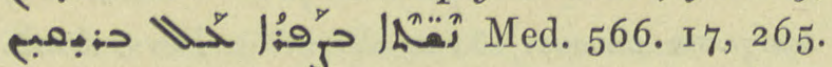

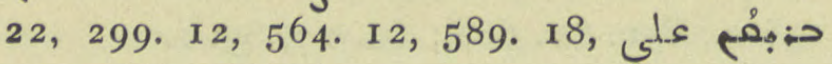
طوي الريق ib. 602. Ix.

j. Ethpalan to plot, refs. مصفئ Bar Penk. 34. 4, لlish The Mops. on Jo. 295. 10.

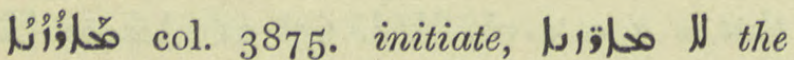
uninitiated, Bar Sal. in Lit. 47. 18.

чำ: I 18 .

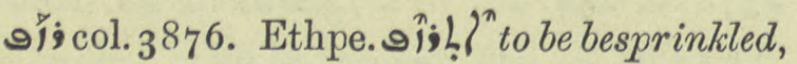
ref. Pet. Ib. 56. I5. Aph. فiं'? to drip, Greg. Carm. i. 99. I7.

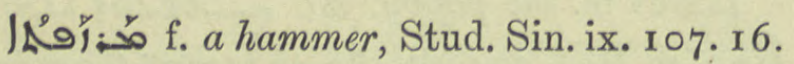

ara is pr.n.m. Razshah, a country gentleman of Adiabene, M.Z. I 4. 39.

פל place-name : see

w col. 3877 . to make circles. Ref. yol as dogs nose around to find the trail, Dion. 205. 15 .

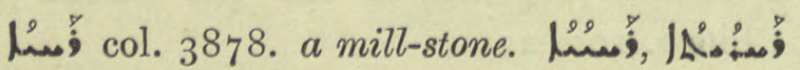
belonging to a mill,

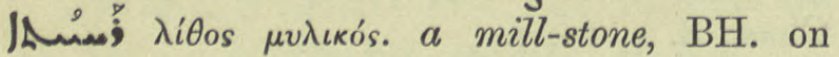
Luke xvii. 2.

أم Raḥta, a village in Adiabene, M.Z. 26. 150 .

pä; to be tender-hearted. Act. part. col. 3880, I3 compounds, add:

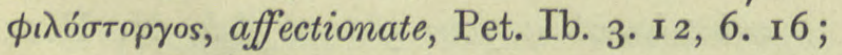

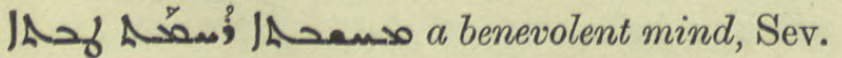

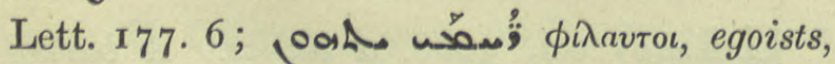

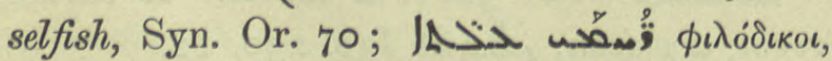
litigious, Sev. Ant. Vit. 253. 5, AnoLol pan фıло́нахоs, quarrelsome, ib. 22. I. Ethpa. prìl l $^{n}$ to receive mercy, be regarded with pity, (i) may the crops be blessed, Book of Shem i io pen.

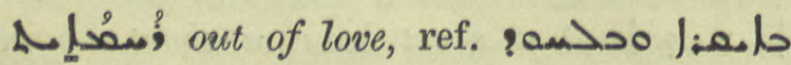
|مدام Loof's Nest. 388. 2.
12.

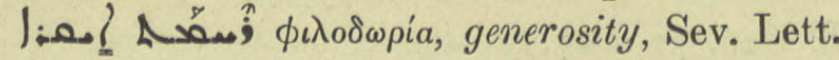

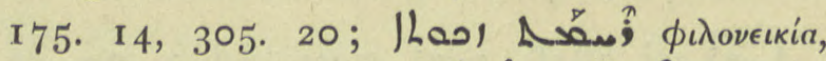
rivalry, ib. 229. 5 ; أمح

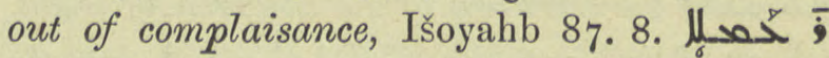

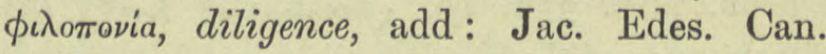
trad. I I. I I ; id. Hex. tit. and 9.

زمبمها Rhima, seventeenth bp. of Adiabene, M.Z. 64. I.

مâl priests and kings, Charms 73. 24; 80. I.

סمع separately, one

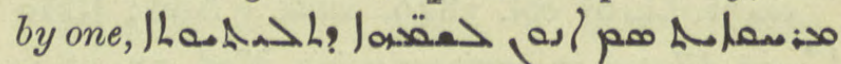
Syn. Or. $133 \cdot 9$.

;يُّ 夏 a young plant creeps and walks without feet, Cyrillona 594. 6. To move as an embryo in the womb, Ephr. ed. Lamy ii. 6r9. 3, 4. Metaph. e id. iii. 223.

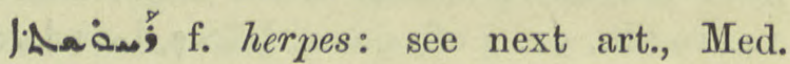
583. 17 .

Lـــ col. $389^{2}$. 3) herpès rongeant,

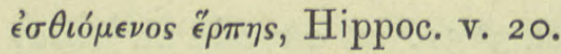

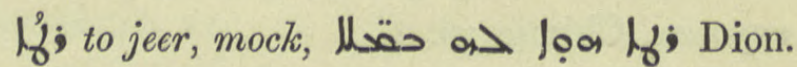
I 83 ult.

1 109 col. $3^{893}$. Delete.

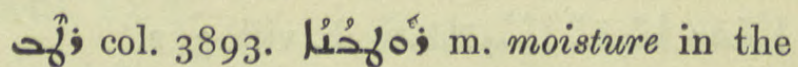
soil, Dion. 194. 9 .

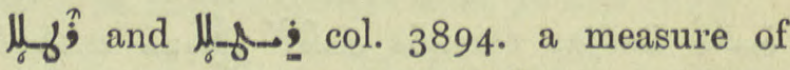
weight; $a$ rotl. Add: $2 \frac{1}{2} \mu / 3=$ I pint, BB. under مُحْ่ col. 3798 . A pound weight, Chimie 99. 3 , 川 贷; Med. 573. 16.

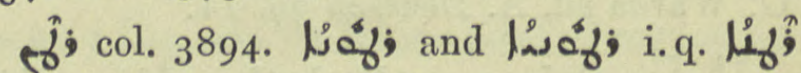
q.v. joras of; Bar Penk. 3. 3 .

偦; col. 3895 . Add : the murmured prayers

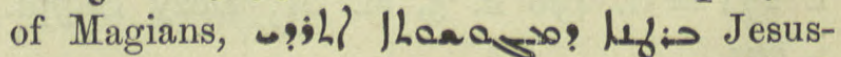

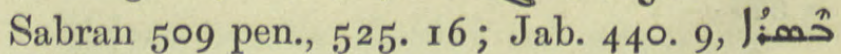
lifi? meat consecrated by Magian prayer, ib. 229. 3 .

1. m. m. pl. Rhatany or Ratany root, Krameria triandria, for hard spleen, Med. 40I. II. 


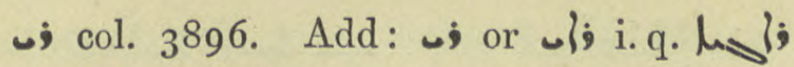
q.v. Rai or Rhages, a town of Upper Media, its ruins are about 25 miles SE. of Tehran, Syn. Or. $680 ; 43.20,60$. I 5 and so read for w ib. 33. 29, Hist. Mon. i. 270.5 , ii. 494 n., ZDMG. xliii. 396. 9, Or. Xt. i. 310. 2. The ethnic is ha.s.

Rî̀ 540. 4 .

ــ!̣\}a_; Rêw-Ardašir, later Rišahr, capital of Fars, on the frontier of Fars and Khuzistan, Tabari I 9. 4, Syn. Or. 680; 43. I I, 53. $18=$ trad. 300 . n. 4 and often, ZDMG. xliii. 396. 6, Hist. Mon. ii. 188 n. 4, Journ. As. 1906, 56. 5, Z. A. ix. $3^{6} 5$.

16 melting-pot, alembic, Chimie 38 . 8.

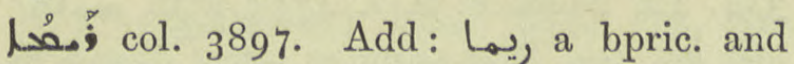
town of Mesene, under the Metropolitan of Pherat d'Maisan. Sapor I gave it the official name of Sad-i-Sapor, Syn. Or. $681 ; 33.24$, 34. I 2 and often, Eranšahr 4I, ZDMG. xliii. 394. $4,395.9,397.8$.

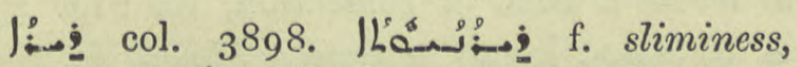

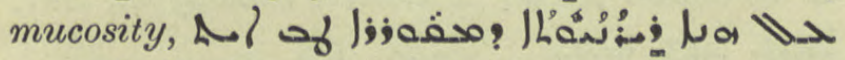
of seeds having a hard shell to protect a soft kernel, N. Hist. vi. 3. 2.

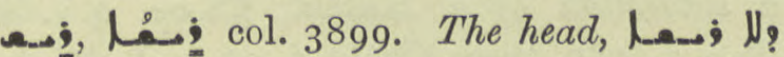
A cephala, Monophysite, Jo. Tell. 70. I. Under a) add: the point of a needle opp. مَمدَم

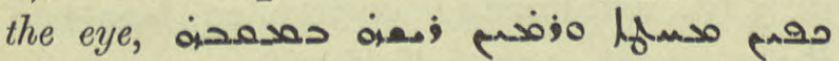
they bend a needle and put its point through the eye, Jac. Edes. Resol. I40. 3; the tip, ila> Natur 7. 12, Jo. Tell. 3. 20 ; a

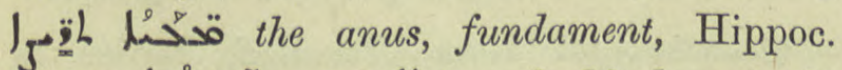

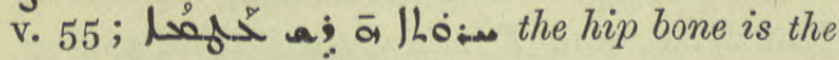
extremity of the thigh, Med. 604. I3; ia 织 the narrow apex of the heart, ib. ${ }^{2} 5^{2}$.

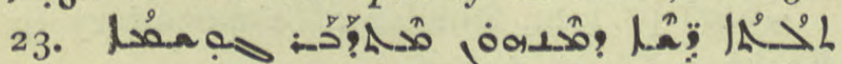
there are three heads or chief organs which

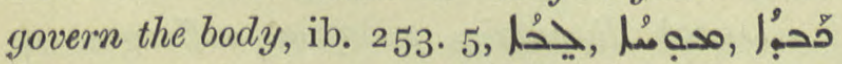
the heart, the brain, and the liver, 11. 6-9;

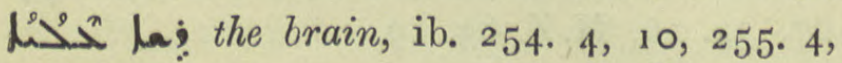


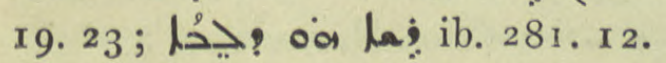

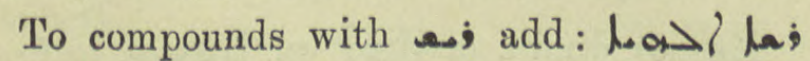

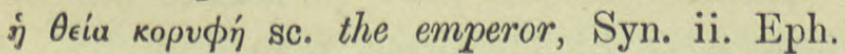

274. 19 ; | Stables, Patr. Or. v. 5, 755. 4, اA ib. 754. 8; مُحُ sore on the choroid membrane, Med. 77. 17;

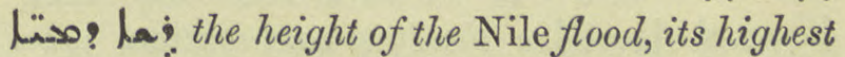

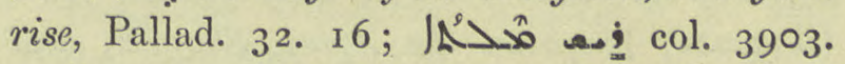
rudiments. Add: iAd D 0 at the top of her voice, Hist. Mon. i. 44. 10. An epitome,

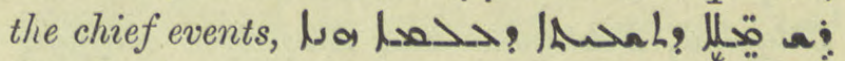
Bar Penk. I. 3 tit., n. 3, 28. 7 and infra, 30. 9, I I6. I 4, I 70. I4-I 6, I 97 n., Journ. As. I90;,

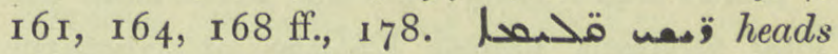
of districts, Dion. 128. 15, 199. 20. بل⿳⺈冂大 the first verse, Brev. Chald. i. $5^{8}$ pen., 60 ult.,

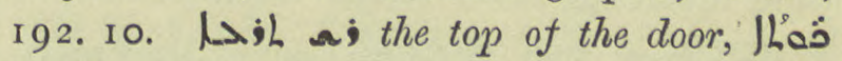
A 596. 4 .

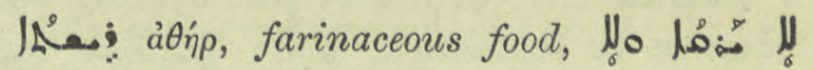
مَ A.M.B. v. I35. 7.

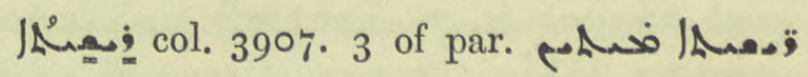

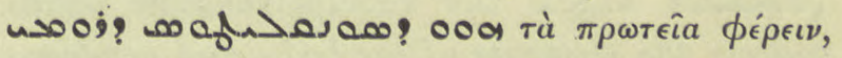
to take the first place $=$ to be of high rank,

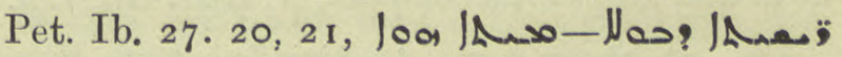
Sev. Ant. Vit. 17. 3. L. I5 An 15 Gen.

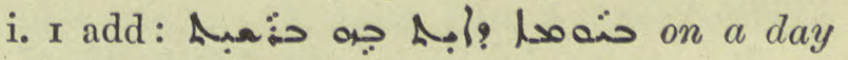
when In the beginning is said, Brev. Chald.

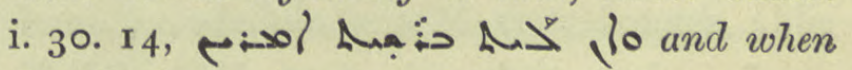
In the beginning is omitted, they say, ib. 29 pen.

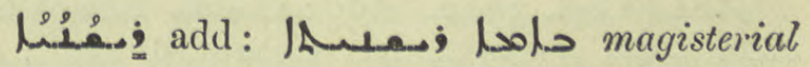
tribunal, Sev. Lett. I 28 ult.

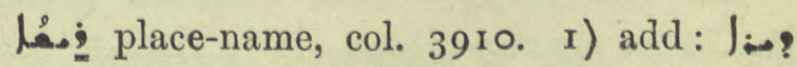
laj: M.Z. 211.4 ; village in Beit Garmai, Hist. Mon. i. 66. I6, Pers. Mart. 277.

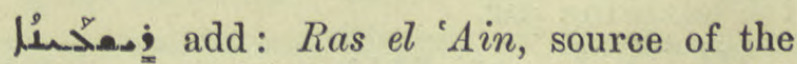
Khabor, cir. 80 kil. W. of Harran, Syn. Or. $68 \mathrm{r} ; 619.4$.

 39 10.

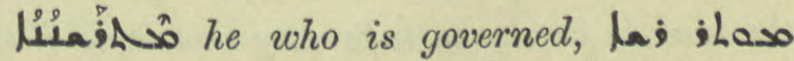

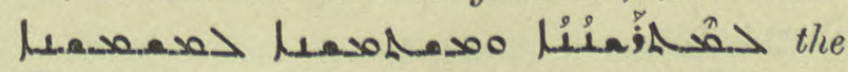
Governor is an advantage to the yoverned and so is the Master to the servants, But. Sap. Philos. i. 2. 
صô; col. 3913. to bestride, mount, ride. Imper. Mon. i. 165. 13. With físôl to take a course,

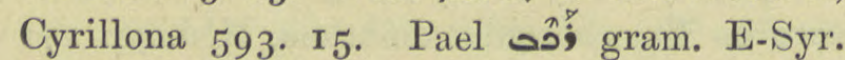

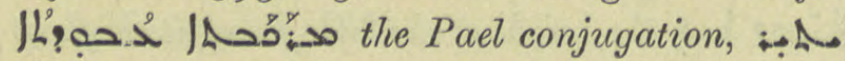

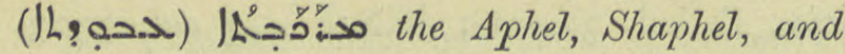

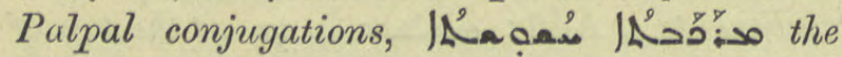

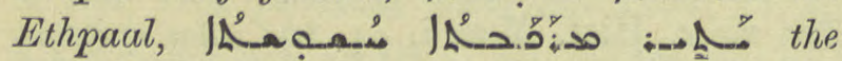
Ettaphal, Eshtaphal and Ethpalpal conjugations, Maclean.

12ْós's add: E-Syr. several portions of the long anthems named fölos at Nocturns are so called, hence of often means to the tune, Maclean.

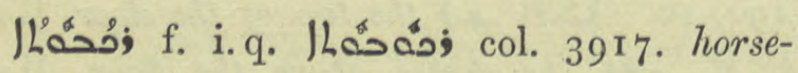
manship, Probus 86. I5.

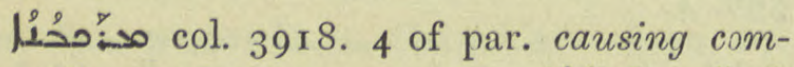
bination or coalescence, add:

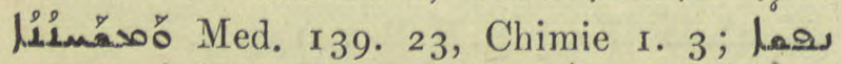

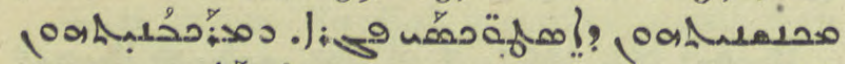
pas0o' Liso the vegetative soul which collects the elements of the body and combines them and keeps them compact, N. Hist. viii. 1. 4.

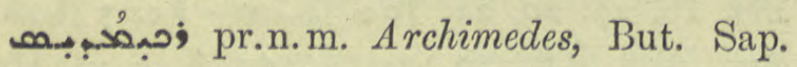
Philos. 6. 8.

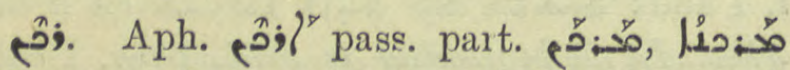
add: some letters of the alphabet os 100 $ح$ Save their sides bent, Hist. B.V.M. 68. 23 .

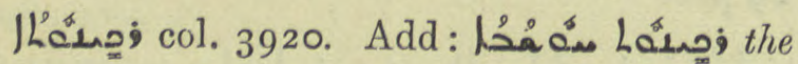
inctination of the thoughts, Is. Nin. B. 209. 4 af.

㶽

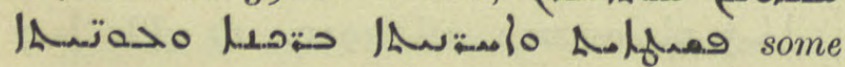
prayers are said simply and others to tones or chants, Bar Sal. in Lit. 76. 3, 4. See also the following par.

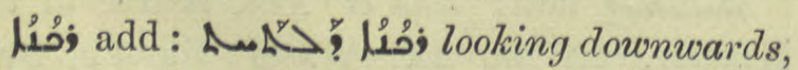

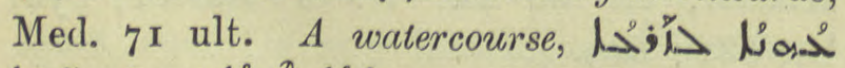

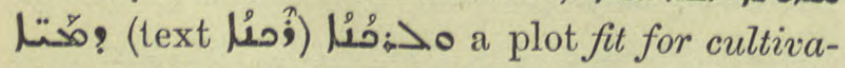
tion and irrigation, Pallad. 50. $17=$ A.M.B.

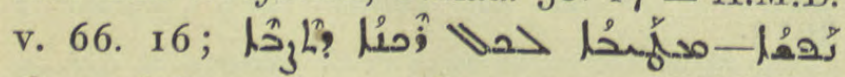
the soul is ready to take any course it likes, ib. 34 . I.

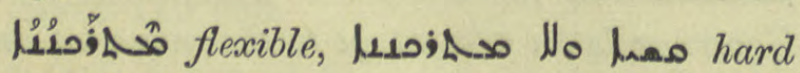
and inflexible, Cyr. 45. 29 ; with humble supplication, Jesus-Sabran 520.7.

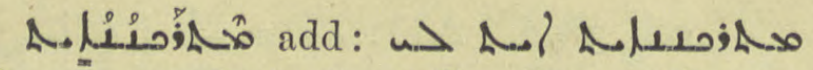
I am naturally inclined, Sev. Lett. I 28. 3.

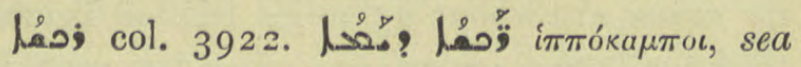
horses, Natur 48. 4, 50. 4 .

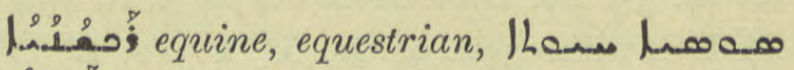

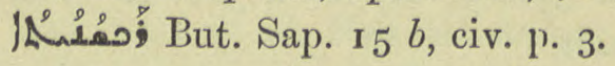

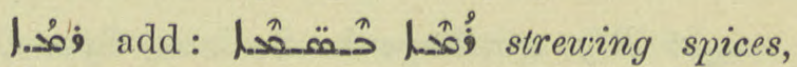

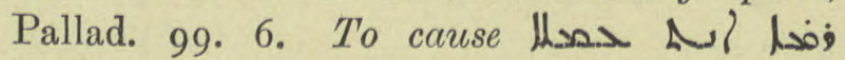
Lollo thou dost cause toil and weariness, Patr. Or. v. 5. 743. 4. Aph. w⿳亠口冋l" to lay down

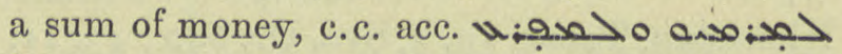
IA Sev. Ant. Hymns I38. To take,

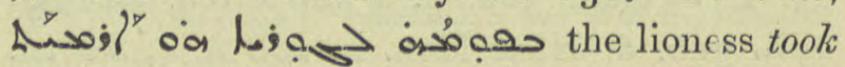
her cub in her mouth, Pallad. 289. 9. To

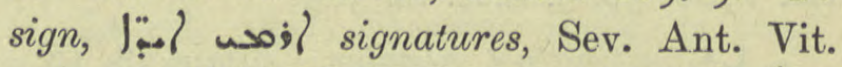
324. 12, Nöld. F. S. i. 475 ; with take his way, have recourse بs fioa.

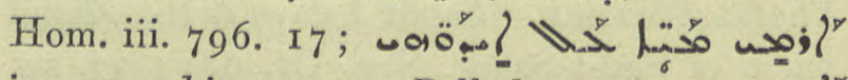
i.e. was his servant, Pallad. 5 ult. a.

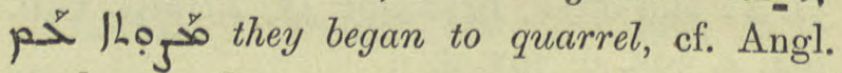
to throw a bone of contention, ib. 214. 23 ; - ahsar lo:s! to involve himself in, Sev. Lett. $213.8 / 9$.

1 1 col. 3928. a part of the Psalter, a subsection of a Hulala, بحمoa q. v. sub

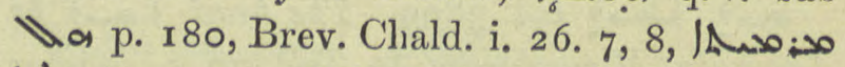

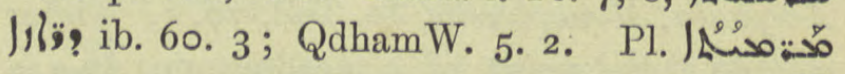

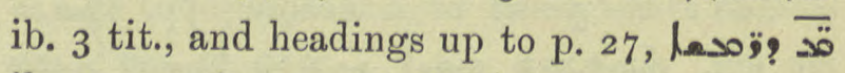
ib. I 1.20 tit., 132. 6, Takhsa 84 . 1 .

فำ the Mohammedan month, Ramadan, El. Nis. Chron. 65.9 i. q. $\sqrt{3}$ col. 3929.

?hiogoroj Ram Hormizd, bpric. in Khuzistan, now Ramiz, Tabari 46 n. 2, Syn. Or. $680 ; 73.8$, 110. 26.

- - ; pr.n.m. founder of a monastery in Kashkar, Chast. 69. 16.

Noso; Ramonin, a bpric. suffragan to Adiabene, Eranšahr 23 f., Syn. Or. 681 : 33 . 27 , 34. 16, M.Z. 63 . 19.

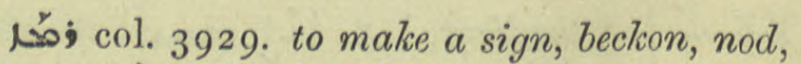

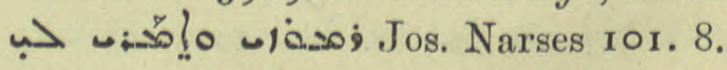

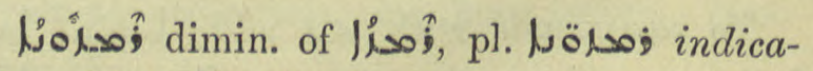
tions, hints, El. Tir. Gram. 3. I 4. 


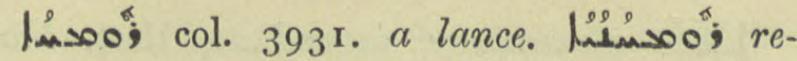

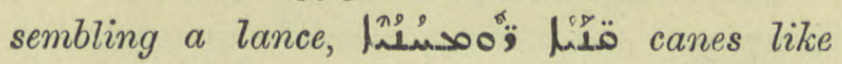
lances, N. Hist. vi. 2. 2.

织 Greg. Carm. ii. 4. 2 I.

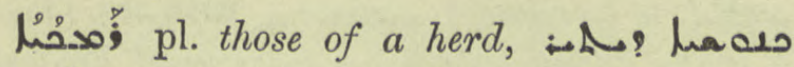

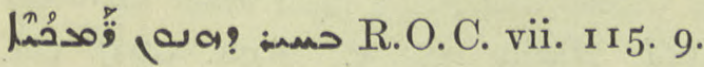

بـ بـ Jiفa), Mar Kardag 8. 10.

Uso; Ramla, village near of wa in Armenia, Patr. Or. v. 5. 707. 12.



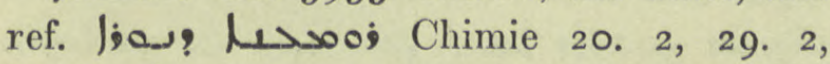
|

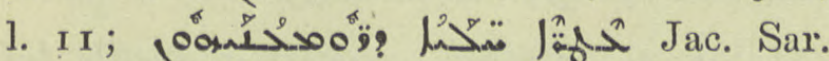
ZDMG. xii. I 22. 18 ; El. Nis. 3 o. 83 .

| Med. $5^{8} 58$.

L'j col. 3935. to think. Pass. part. L'j

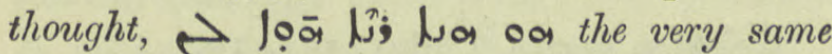
thought occurred to us, Gest. Alex.,257. I.

12.

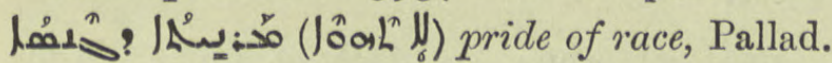
355.12.

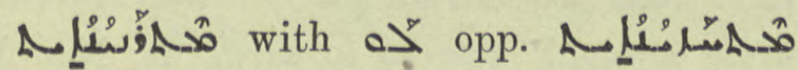
dreams are perceived not by thought but as it were by sight, N. Hist. viii. 3. 5 .

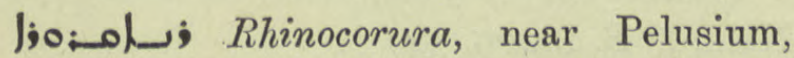
Pléroph. г3о. 7. Miswritten |jo:مis ib. I 28 ult.

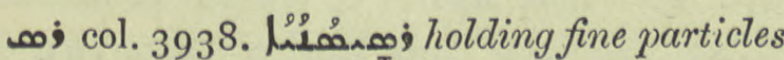
of moisture, damp, dewy,

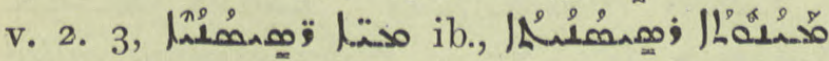

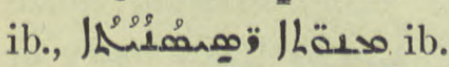

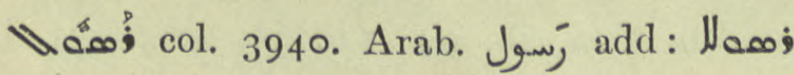
la $>$ i? the messenger of God = Mohammed, Dion. 6. 1,7 ; the messenger of an Emir, ib. 62 ult., 92 . I 7 .

گrassonin, perh. Ramonin, M. Z. 35. 69. Prob. NW. of Arbela.

M⿻rh. Arab. wa milk, Chimie 37. I. 2716

(ْْ col. 394 r. Inula helenium, elecampane. Ref. Med. I4I. 23, 237. 6, 305. I I.

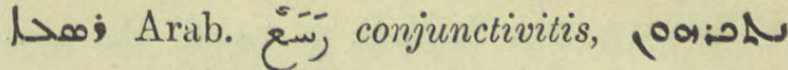
مه Bم Book of Shem, I IO. I5.

?-10 ; Rastegird, a village in Beit Nuhadra, Chast. 4. 4 af.

u' col. 394 r. Add: to pound, crush to

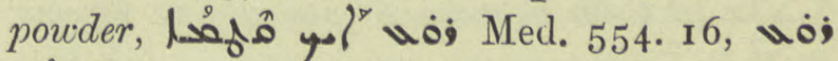

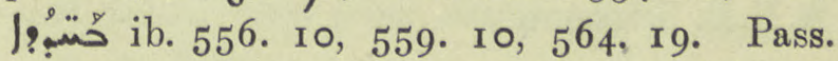

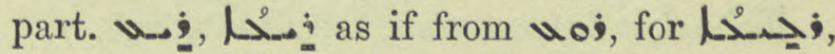

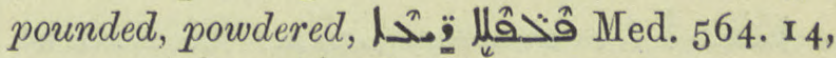

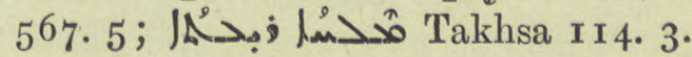

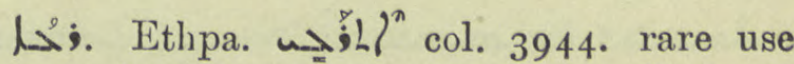

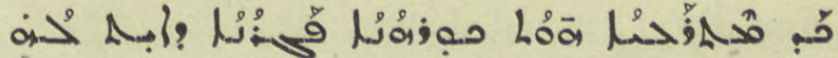
alleging a bodily disease, Pallad. 208. 2.

A.

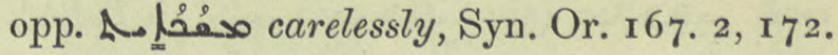
28, R. O.C. iv. 258 . 9.

A Nest. Hérac. I 35. I 2.

مُخحُم col. 3948. the mind, thought

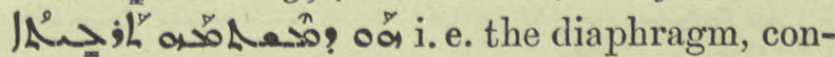
sidered to be the seat of the mind, Patr. Or. iii. i. I I 5. 4. Cf. Arist. Hist. Animal., i. xiv. 5,

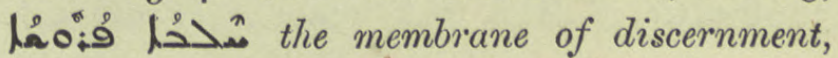

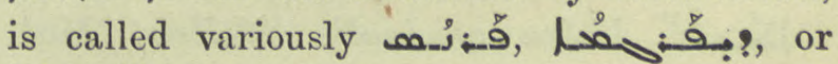

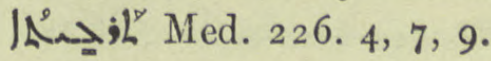

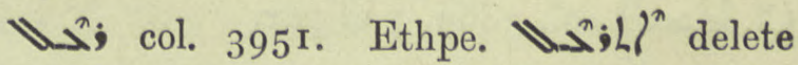
two lines and transpose both refs. to Ephr. to eight lines below, under Aphel. The third ref., to Jo. Eph., should be مند

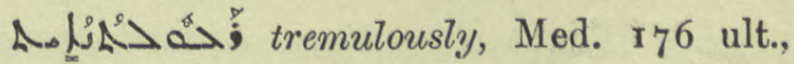
272 ult.

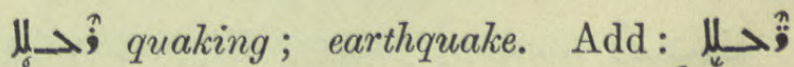

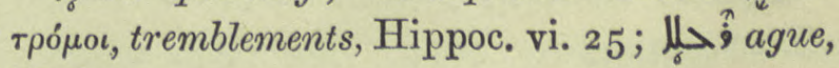
Med. 54. Iо.

$\Lambda \|_{6} \rightarrow \hat{i}$ vibratorily, with vibration or oscillation, of earthquakes, N. Hist. iv. I I. 2.

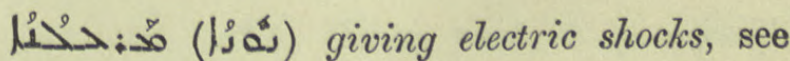
under كuppl. p. 204.

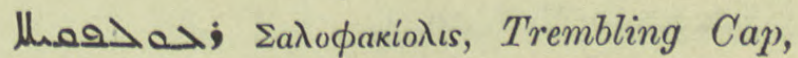
Shaky Cap, nickname of Timothy, Bp. of Alexandria, see under thog col. $3^{2}$ I9. Add: Anecd. Syr. iii. I33. 22, I64. Io. 


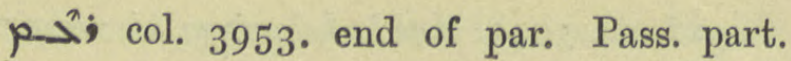
ليم: thunderstruck; lamenting, whining, Pallad. 13. $5=$ A.M.B. v. 14. 6, Gr. oikrpàs

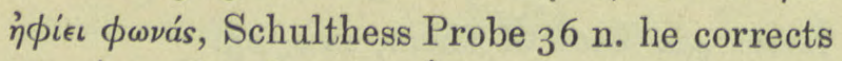

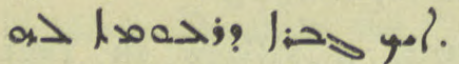

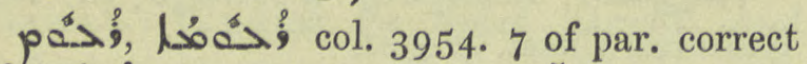

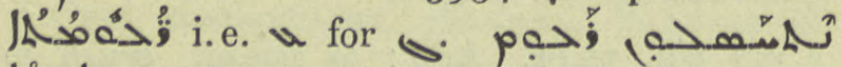
دlet them be weaned piteously in sorrow, A.M..B. ii. 109. 4.

L'ڤ்َ

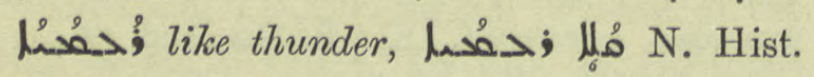
v. 4 . $\mathrm{I}$.

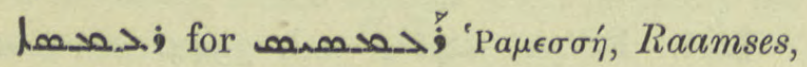
Hex. Ex. I, I I, ed. Lag.

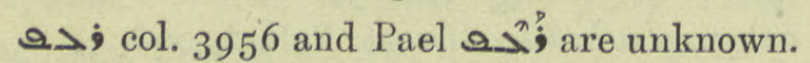
Perhaps for a... तy col. II 42 to upbraid, Dallad. 382. 16.

a $\rightarrow$ o; an Arabian monastery, C.B.M. 7 II $b$.

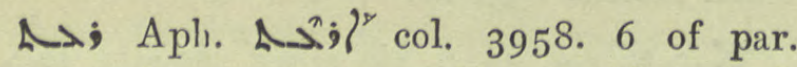
2) to make to palpitate, add ref. $\boldsymbol{\Delta}^{n} \boldsymbol{D}^{n} \boldsymbol{\sim}^{n}$ Med. 272 ult. 3) to be garrulous, BA. under L Lớ; col. 3868 ult.

|นㄱำ Malva officinalis, Mallow, Med. 563. 2 I marg. gloss x...b. $\overline{\text { ă }}$ ib. $5^{86}$ ult.

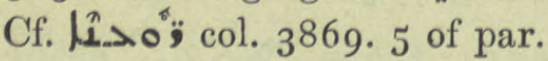

1

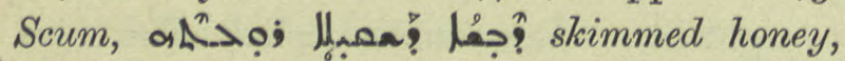
Med. 51. 7 .

1 refs. Med. 205. 13, 609. 18, 19.

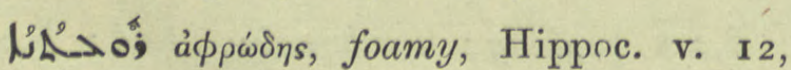
vii. 27 .

๑) Palp. ๑:๑ col. 3959. add: to move the wings, to flutter, a hawk will sail towards a south wind, Natur 33. 4 .

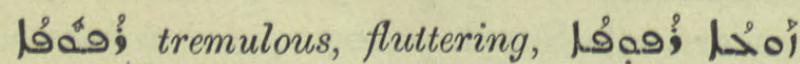

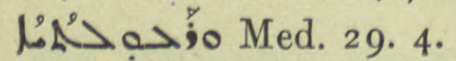

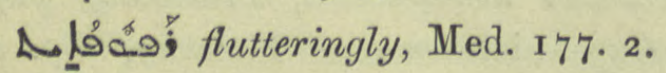

IAgag; f. a disease, perh. twitching, or blinking, Med. 584. 22. $\infty 9$ i

Lْفْó; momentary, immediate, Hist. Mon. i. 27.9 .

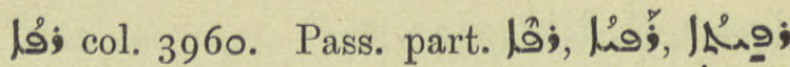

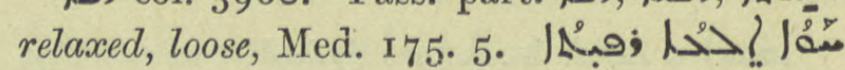
Eve, the loosened rib, S. Dan. 5 I $b$ 5. Ethpa.

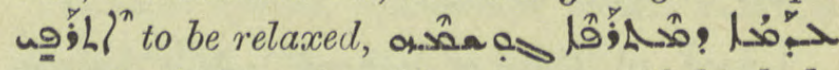
a snake before sloughing fasts until his body is relaxed, Natur 38. 5. Aph. u이" col.

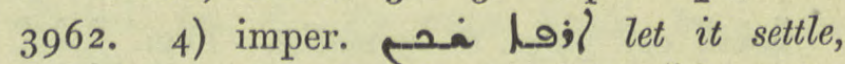
Chimie 35. I7. Rare uses: with مُ مُ to give tongue, utter loudly, Sev. Lett. I 19. I2, cf. 1. 17 of par. VHh. Mark xv. 37. | ?סע: wild asses which bound and snort, Pallad. 138. x6. To leave, digress Set us begin where we left off, Vit. Mon. 39. I r.

L’ڤ̊ó, m. softening, rendering ductile (of metals), Chimie 223 n. 3 .

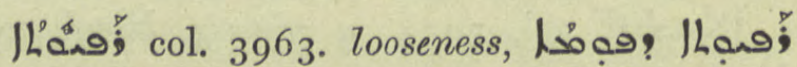
a relaxed mouth, Med. 168.9 ; liñ $;$; loosened teeth, ib. 169.18.

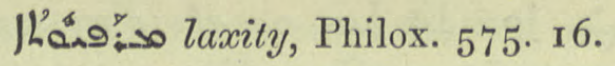

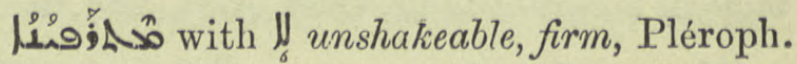
47. $\mathrm{I}$.

Lـُ col. 3964. a flock of birds. Add:

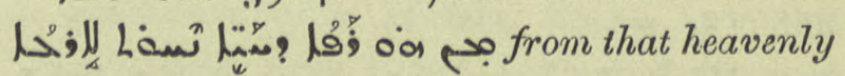
flight a monk returns to earth, Is. Nin. B. 277. 10.

(ه): 'Paфavéat, Raphana, in Coele-Syria, Nöld. F. S. i. 469. 34 .

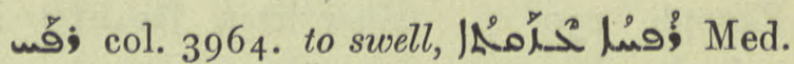
570. 20. Pael wă to be cracked or loosened,

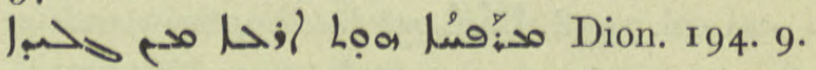

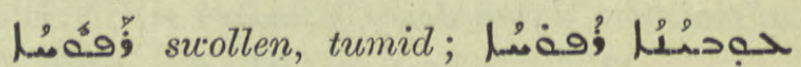

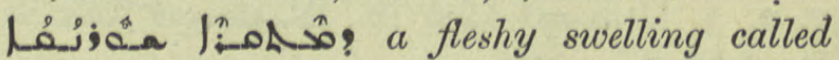

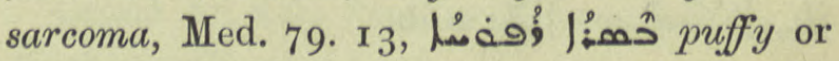
swollen flesh, ib. 94. I1 ; pl. swellings, ib. 97. 1 I, 157. I I.

Lo; col. $39^{6} 5$. Delete and emend in both

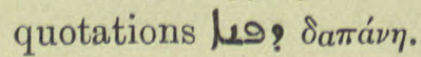

9. |

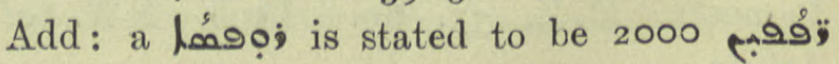
twinklings of the eye, Med. 53 r. I I but two

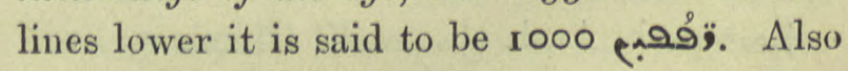




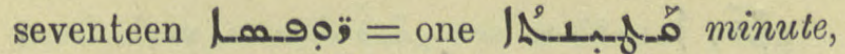

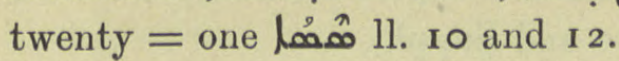

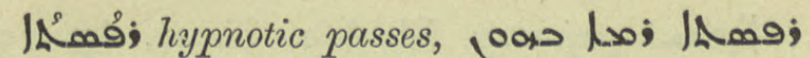
he made passes = cast a spell on them, Coupes ii. 154 ult.

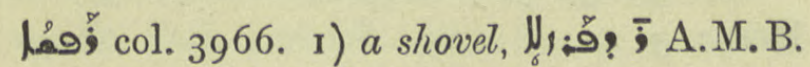
iii. $3^{84} \cdot 5 \cdot$ 2) 20

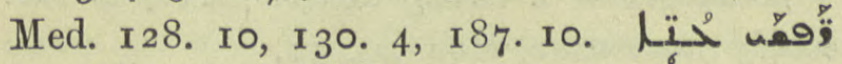
for divination, BHNom. ed. Bedj. I49. 5, Jac. Edes. Can. 27. 20.

3) col. 3968 . Pael 33 to crush. Pass. part. $33^{7}$ berries, Med. 302. $2 \mathrm{I}$.

$J_{33}^{\prime}$; contritio, Sym. Ps. lxxxix. 3 .

3\}; Arab. صin lead, Chimie 95. 21, 26 ,

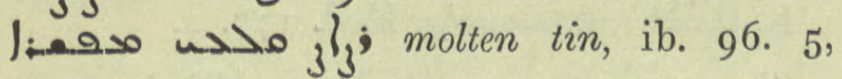

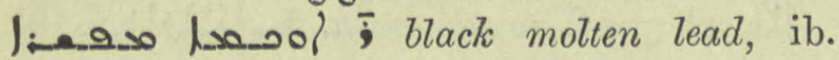

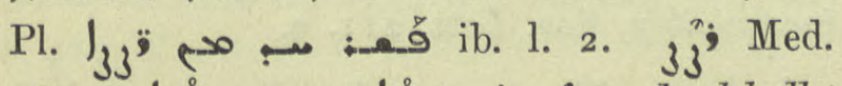

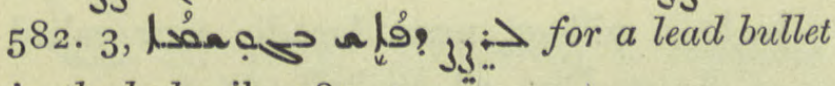
in the body, ib. $5^{87} \cdot 9$.

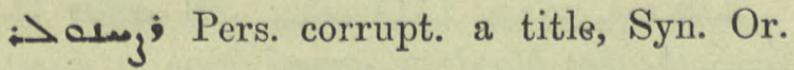
79. 22.

13. col. 3969. to trickle, drop, exude.

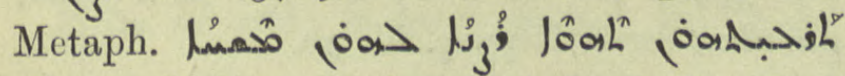
$1 L$ o. strenuous endeavour, A.M.B. v. I 7.5=Pallad. I5. 10.

9, col. 3970. Pass. part. $\boldsymbol{\theta}_{-3}$;. add: 茴 planks set close together, Poet.

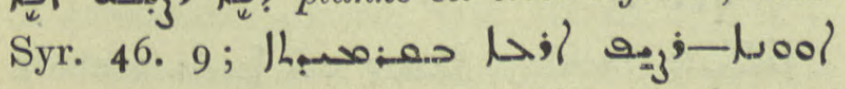
a compartment of a glass blower's furnace should be strengthened with a layer of potter's clay, "enduit d'une couche de terre à brique",

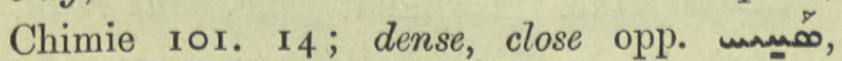
N. Hist. ii. v. sect. 4.

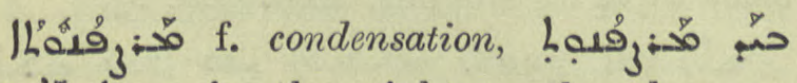
مهزן in the night earthquakes are violent by refrigeration of the earth's surface, N. Hist. iv. I I. 3 .

$\theta_{3}$; name of a village of Adiabene, Chast. 30. 17.
L. Mesopotamia, A.M.B. iii. $3^{2}$ I.

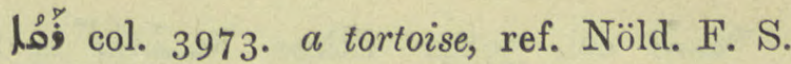
i. $55^{\mathrm{r}}$.

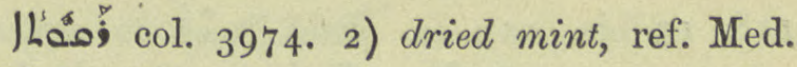
566. $17,609.20$.

น" adj. from Rakka, JAOS. xx. I89. 8,

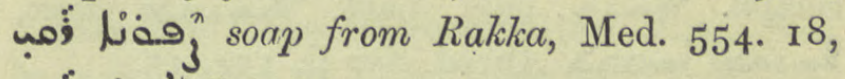
فํ.

مُّ col. 3975. the abdomen, epigastrium. Add: Med. 18. 22, 19. 9 and often, A.M.B.

i. 497 .

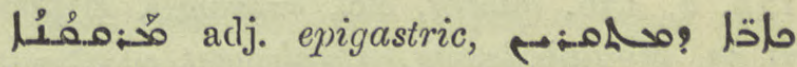

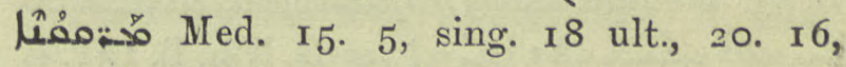

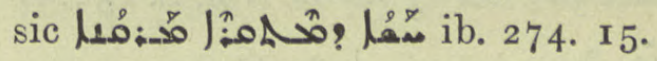

فُ Rakbakt, governor of Adiabene, M.Z. 6. 8, 7. 27 .

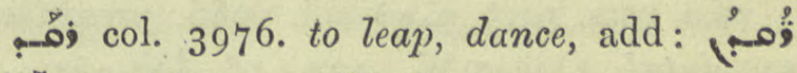

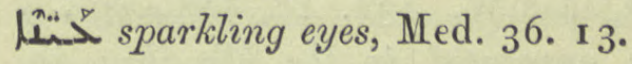

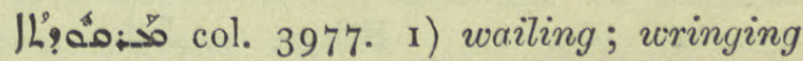

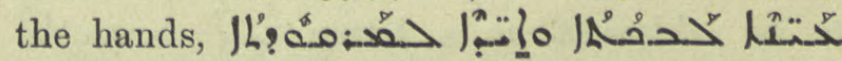
Ephr. Carm. Nis. I 4 I. 54. 2) dancing,

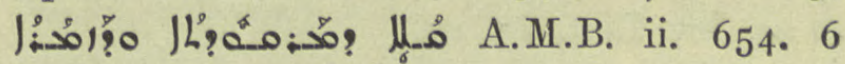
Bedjan suggests

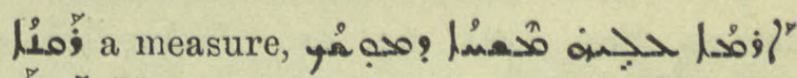

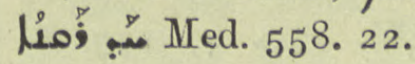

uم้ col. 3978. Add: to fix, wedge in, J

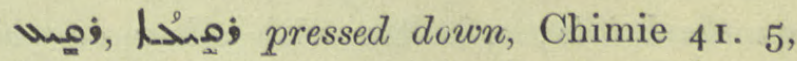
solidified (silver after melting) ومدحرا

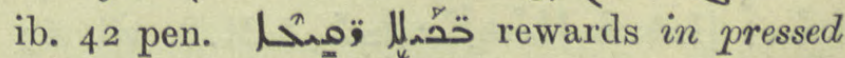
down measure, G. Busâmê 2. 1/2.

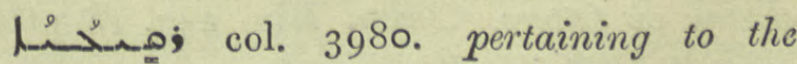
firmament, 每 reaching the boundaries of the firmament, Hist. B. V.M. I 20. 13 .

ค husked or pounded corn, Pallad. 3 I 2. I.

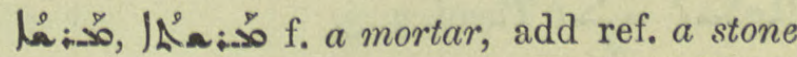

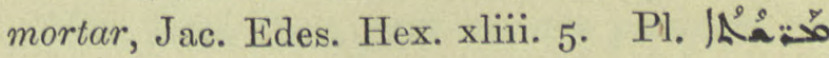
the broken portions of the Holy Bread, on

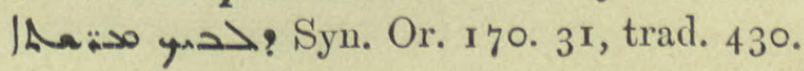


un: Reshi, village near Nineveb, M.Z. I6. 80 .

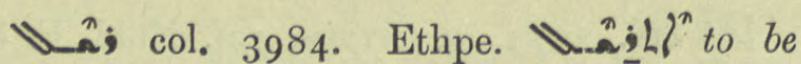

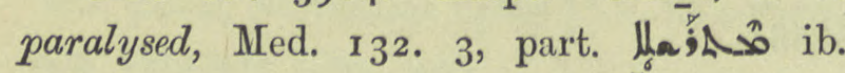
I18. I5 bis, I19. 4. Pael Vhä to soften, relax, act. part. فа Med. 277 II.

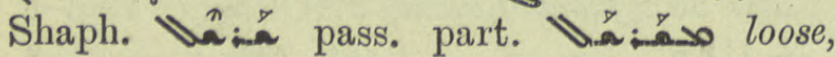
Anecd. Syr ii. I54. I5.

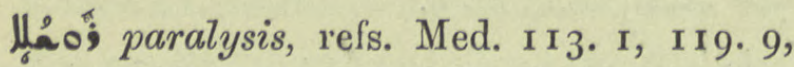
120. $14,126.3$, \&c.

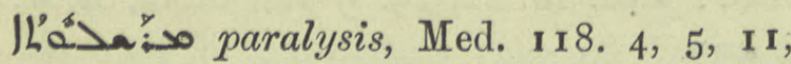
135. 2, 136. quater, \&c., àmóm $\lambda \eta \xi \iota s$, Hippoc.

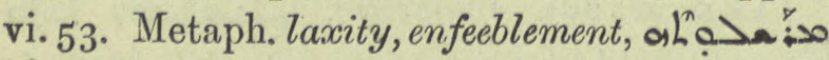
. بs. Nin. B. I3 I. I.

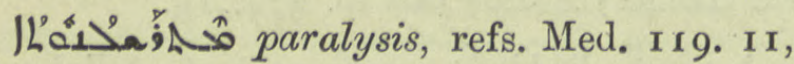
I78. 24 .

pá; pass. part. pån col. 3986. prescribed,

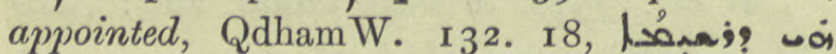
حس. as appointed in the Khudhra, ib.

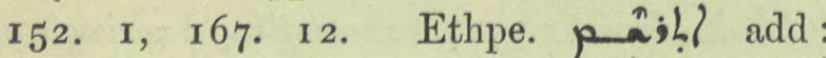
to be addressed as a letter, Lo>, the letter was addressed by mistake to us, Sev. Lett. 63.17 .

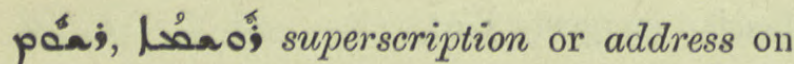
letters, Sev. Lett. 63. Ir. Index, I

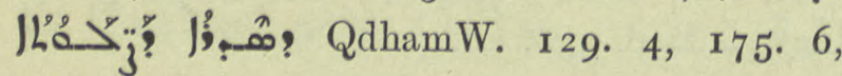
I 76 tit.

رُ on the bridle; a halter with silver or gold ornaments for the forehead of a horse, Dozy. A.M.B. vii. 63

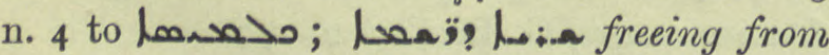
chains or fetters, Chron. Min. 247. 18 perh. corrupt. Cf. ZDMG. liv. 56o.

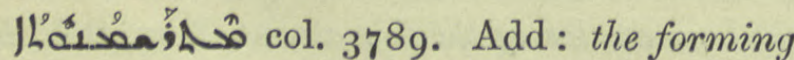

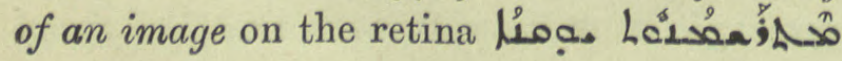

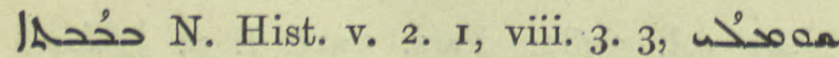

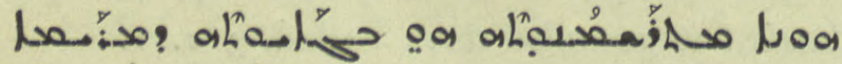
Ihodel the Theol. 5.3 .

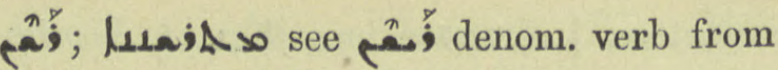
col. 3910 and Suppl.

Jhen' primacy, El. Nis. Chron. 65. 10, 66. 2 I and passim, see /Lُهُ 3909.

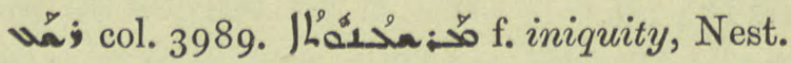
Hérac. 3. 2.

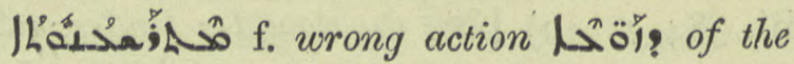
bodily movements, Med. 29. I I.

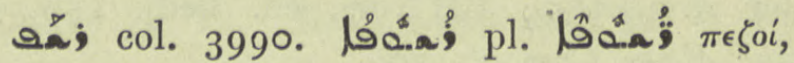
land animals, opp. Greg. Carm. ii. 3. 18.

فم. the first words, used as the name of a chant; see under s.

L' col. 399r. to tremble. L'L'\}' pulmonary,

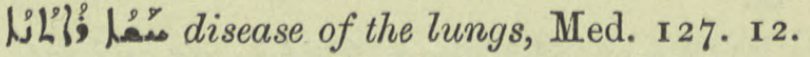

AlLớL' tremulously, Med. I77. 2.

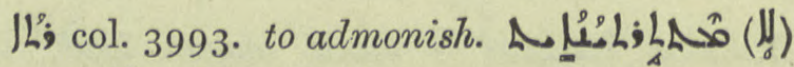
incorrigibly, Sev. Lett. 2 I r. 9.

JoL; a village near Amadia now called ديرى, Kal-w-Dim. ed. Bickell 1 27. 3.

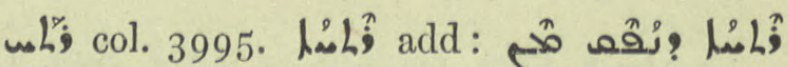
قحص the hot blood or gravy from a roasted liver, Med. 557. 2r, JAOS. xx. 193. 4; cf.

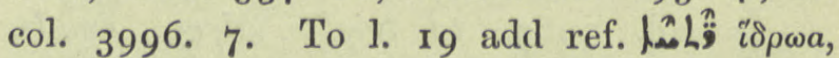
purulent sores, Hippoc. iii. 20.

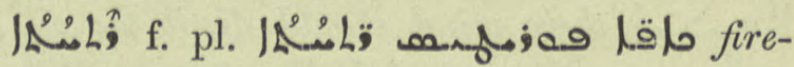
coloured stones, Chimie ror. 2 I.

Heno fervent. Correct ref. to Anecd. Syr.

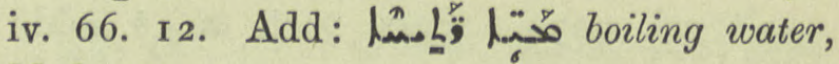
Med. 577. 7 .

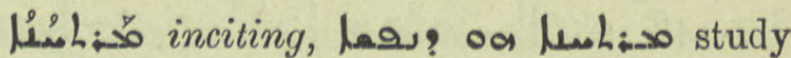
is an incentive to the soul, Is. Nin. Chab. 76. 3 af.

ษ Arab. see 1,j\}? /A>o? col. 933. An ingredient of black salve for wounds, Med. 586. I3.

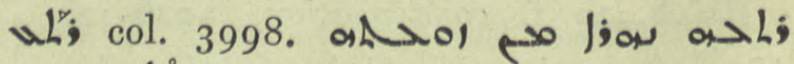
correct $\mathrm{w}_{\mathrm{A}} \boldsymbol{k}$, the river foams with agitation, Ephr. ed. Lamy i. ז 23.6. 
I a col. 4002 . almonds, Med. $57^{6}$ ult., $5^{82} .8$.

حــ frantically, as one possessed, ب0a went into a frantic rage, Sev. Lett. $3^{2}$. I.

شماهـ pr.n.m. Nöld. on Chwolson, ZDMG. xliv. 527. I4. Cf.

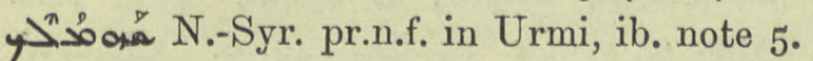

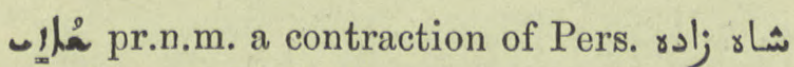
Shah Zada, A.M. B. iii. 473 .

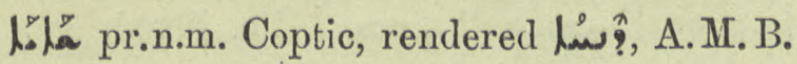
iii. 575 .

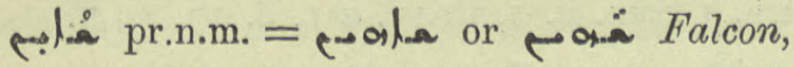
Fulk, A.M.B. iii. 473 ; but Sainus, B.O. iii. i. 4 I 3.6 .

Ma col. 4003. To borrow, to simulate,

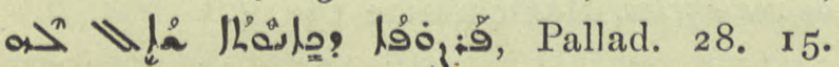

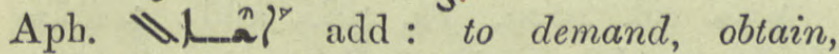

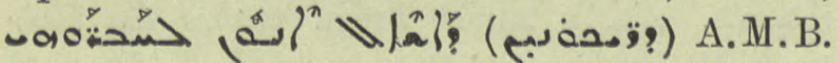
v. 556 .

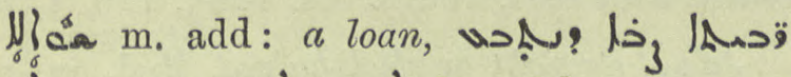

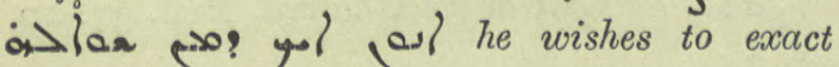
interest as arising from a loan of his, Sev. Lett. I 15 . I 7 .

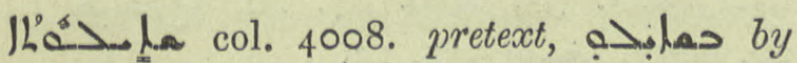

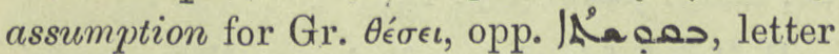
of Athanasius quoted, Nest. Hérac. 27 1. 2, 4,

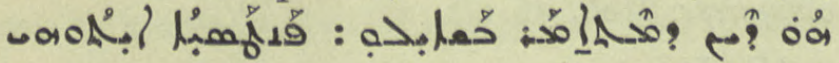

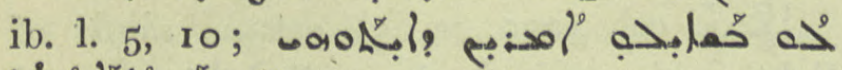

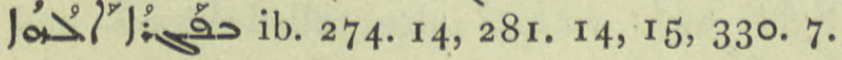

1요 263. 5 .

مـ: col. 4011. I. Add مغالحد read it as an interrogation, BH.

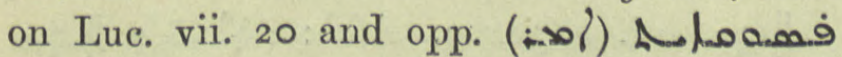
affirmatively, ib.

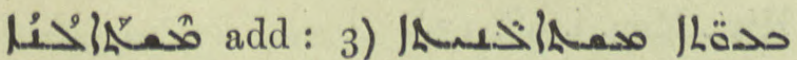
petitions of resignation of the office of a bishop, Sev. Lett. 53 ult. da. Ethpa. R.R aln col. 4013 add: to be planted out Gapeop. 72. 14.

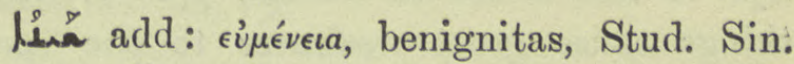

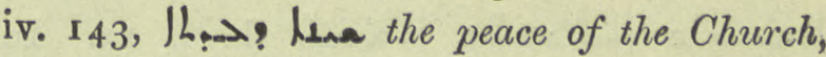
so the conversion of Constantine was called, Jul. $3 \cdot 4$.

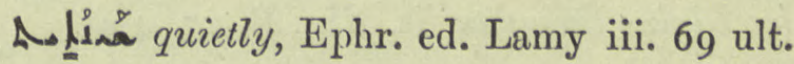

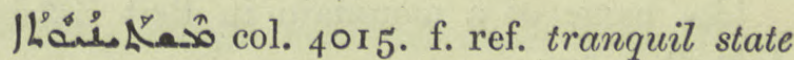

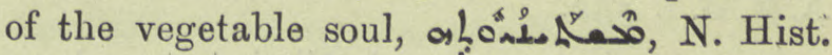
viii. 4.7 ult.

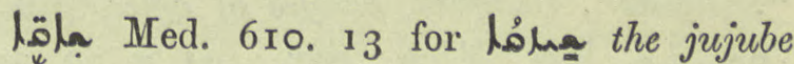
tree and fruit.

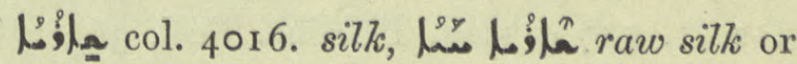
a live silkworm? remedy for shortness of breath and black bile, Med. 262. 24, ib. Lُ مُ ib. $265.9,266 . \mathrm{I}_{3}$.

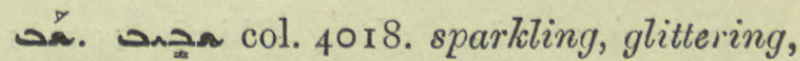

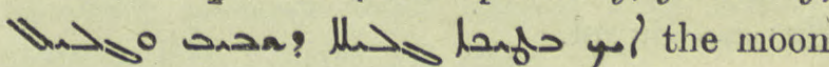
looks like a small round melon, round and glittering, Caus. Caus. 22 I. 3 .

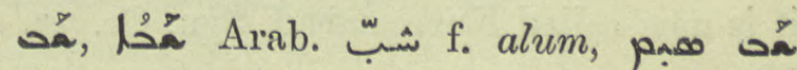
apply alum, Med. 562.8, 577. 18, 18 alum from Yemen, ib. 605 ult., Chimie 95.

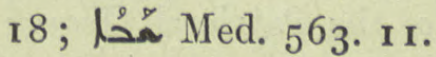

年 a week: see 4046 .

محُ col. 4018. Ethpe. ushaln to be led captive. Add : Afo' 川 rushing, Pallad. 70. I 7. Under 2) to be laid waste, devastated by a storm, ib. 197. 8. Under 3 ) end of par. bound to attend to their words, Hist. B.V.M. 204. II.

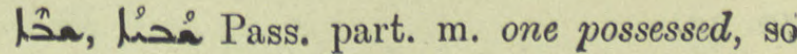

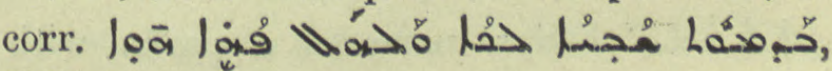
Pallad. 29I. Ir. 


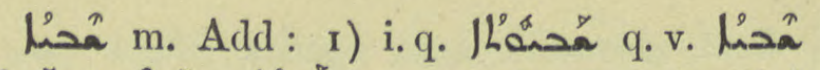

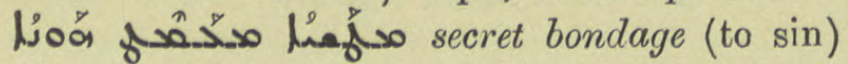
obscures the mind, Pallad. 508. 4. Dele "Vox suspecta".

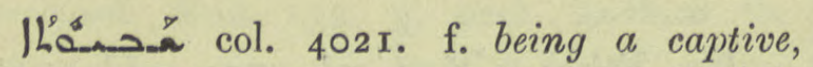

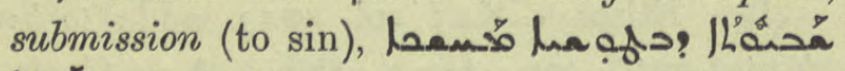
fróx, Pallad. 49 1.9. The quotation in Thes. Syr. is also found in Pallad. 758. $x$, three things cause great weakness to the soul,

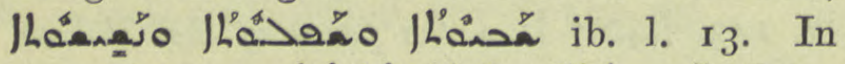

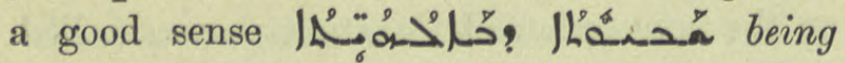
captivated by Divine considerations, possession by Divine thoughts, Is. Nin. B. 27. 4 af.

محُ name of a mountain in Beit Zabdai, A.M.B. i. $45^{2}$.

La col. 4021. 3) Chabot translates "insignes", insignia, badges of office, b으

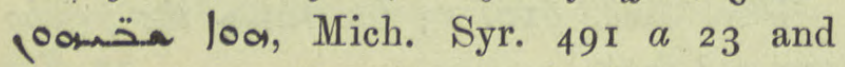
corrects to joorin two similar passages, 5 1 2. I, 5 77 a med. BH Chr. Eccl. $3^{6} 3$ ult., 365.1 .

محب لما cognomen of معسم, a hymn-writer, Journ. As. 1907, 390.

arab. شبة alum, Chimie 102. II. See an Suppl.

A. محمزסمهـ. The city was built by Sapor I, it is now called Kurremabad, Syn. Or. 37. 6 , $68 \mathrm{r}$.

; פabban Sapor, a monk contemp. of woal? of and of the Patriarch George 66I-68I, Journ. As. 1906, I I I ult., J J

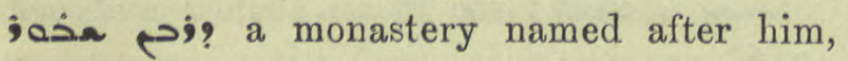
ib. 109 note.

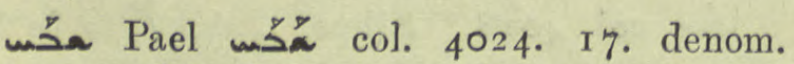
verb from linsos to repeat the doxology, say the gloria, refs. QdhamW. 44. 3 af., 46. I2, 47. 7. . to which the doxology is prefixed, to the tune, The right hand of Thy mercies, Maclean.

مُ 4026 med. 3) erroneously, for

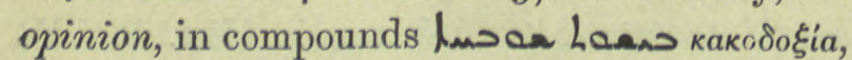
A.K.G.W. vii. (1904), 4, 8, 16, Pet. Ib. 70. 22.

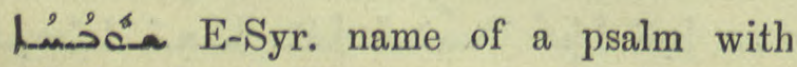
farcings sung at Nocturns on Sundays and

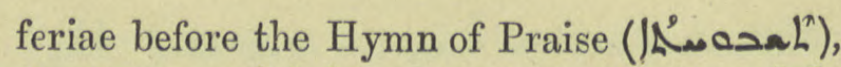

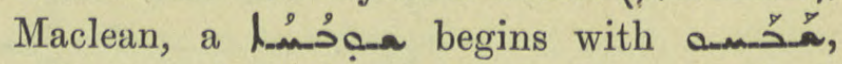
a /Arever Chald. i.

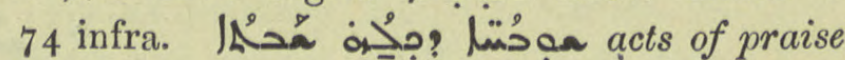

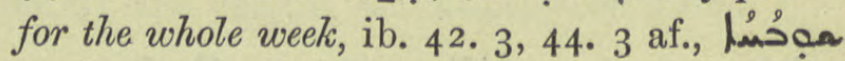
A pen., 243. 18, 260 antep. and pen., IL'a. !مسبهُ QdhamW. I7I. I ; ib. 86. 8, 87. 7 \&c.

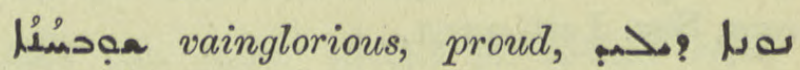
الم Ralands xliv $6 a$.

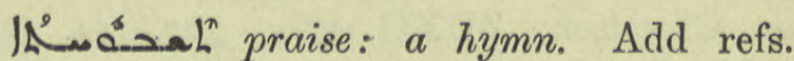
a hymn of praise, Takhsa I 49. 6, QdhamW.

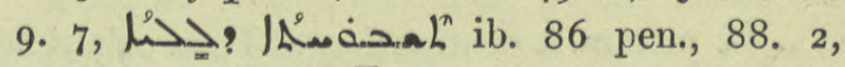
89. I, I9; 1:9y ? โ Brev. Chald. i. 50. I1 ;

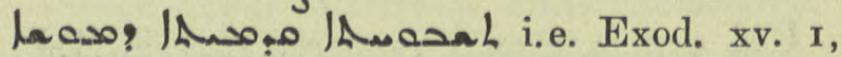

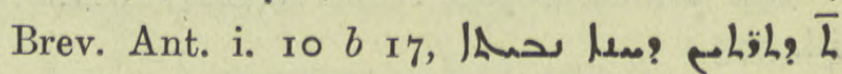
i. e. I Sam. ii. I, ib I I. $a$ I, 8. I3, I 9 ff.

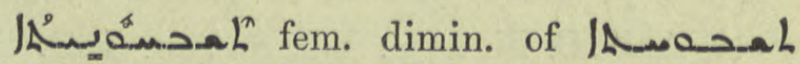
a refrain,

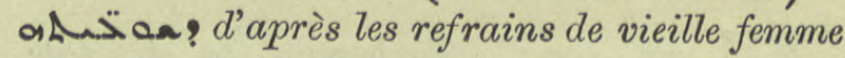
de ses cartes, Manichéisme I39. 4.

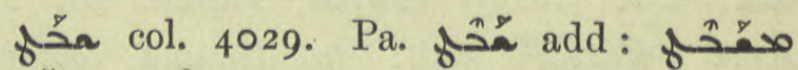
年

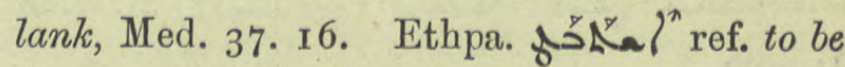
beaten with rods, Journ. As. $1895,119.152$.

ชั่์ straight or sleek-haired, Med. 37. 10, 38. 3 .

1 Ar.FischN. 35, Med. 87. 20, لرُ 138. 20, marg. أغ 555. 55 . 13 .

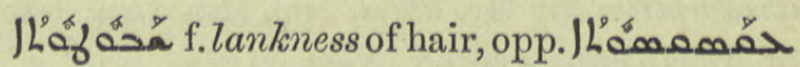
crispness, Med. 36 ult.

مُ col. 4030. m. 5) astron. shafts of light, add: محَّم are seen at dawn and eve to the right and left of the sun, N. Hist. v. 2. 4.

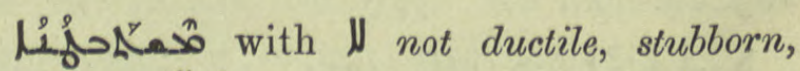
م: Greg. Carm. I I 8. 2.

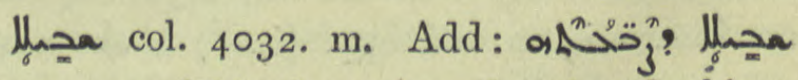
the traces of his fingers on the clay daubing, Hist. Mon. i. 98. 13.

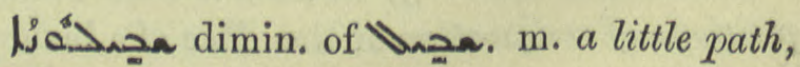
A.M.B. i. 483,484 . 


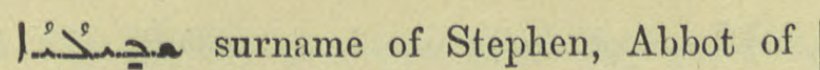
Mt. Casion, R.O.C. vii. I99. I I, A.D. 629.

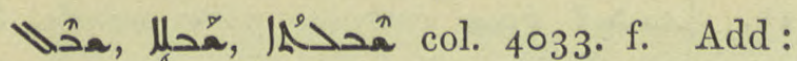

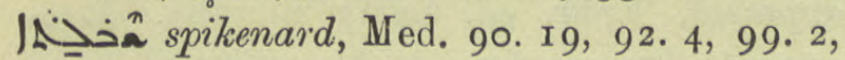
396. $23,397.3$, I $5,610.4$; Z.A. v. 394.

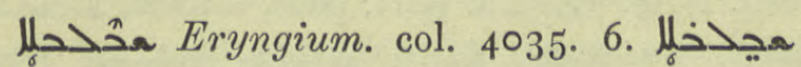
à Med. 6ro. 4.

I f. Eryngium, Med. 393. II,

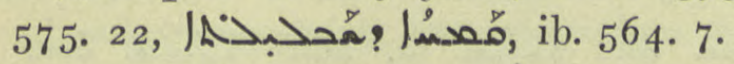

a a spot on Mt. Ararat, A.M.B. i. 450, Chast. $59,18$.

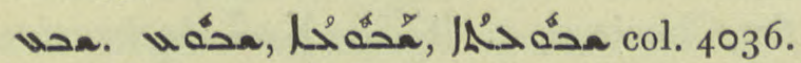
Add: E-Syr. a period of seven or fewer weeks,

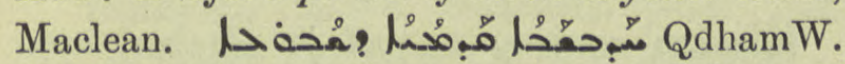

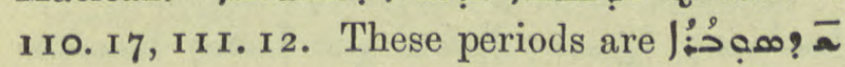

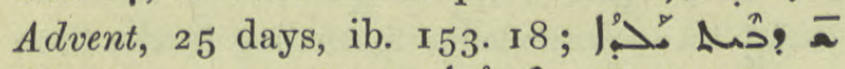
Christmas, 12 days; Lin? ? Epiphany,

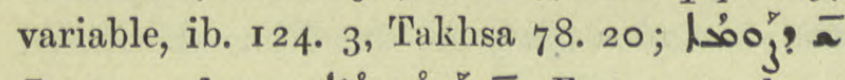

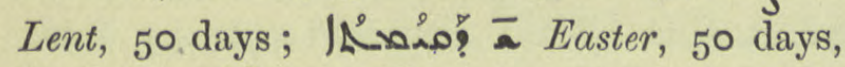
ib. 1. 21 ; بمخب̈n a weeks of the Apostles, 50 days beginning with Pentecost, ib. l. ult.,

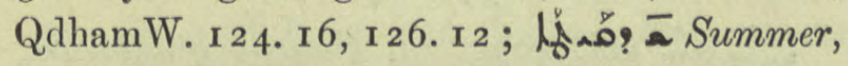
50 days, ib. I25. 2, I26. 9, Takhsa 78. 24 ;

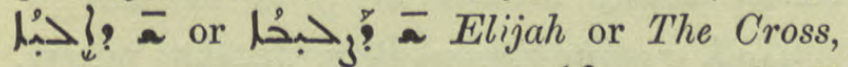

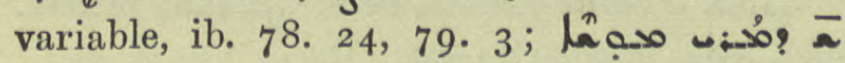
Mar Moses, not more than four weeks, omitted if Easter fall late; ILب Hallowing of the Church, 4 weeks before Advent, ib. 79. 6. See also Bar Penk. I7 I. 5, Brev. Chald. i. I 2. $13,18$.

นำ a add: the seven heavens of Bardesanes, Nars. ed. Ming. ii. 2 I8. 9 .

مح้م col. 4039. I5 . 3) to permit, give way, $\mathrm{Au}_{3}$ a a we did not permit him to have his way, Hist. B.V.M. 147 ult., $\ln _{3} \mathrm{D}_{3}$ ل alli ib. I48. 4 af.

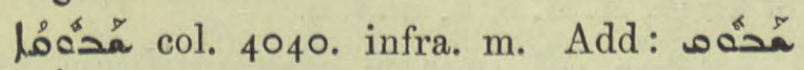
ल.j a small iron rod, Chimie $5^{2}$. I, 3.

1مُْْ one who pardons. Add: a deserter, one who abandons, Išoyahb. 138. 22.

ILoهo a f. deliverance, dismissal, Sev. Ant. Vit. 289.8 .

محُمُ m. 2) onrush, Josephus vi. 4 pen.; arting on fire or the taking hold of fire, ib. 1. 9 .

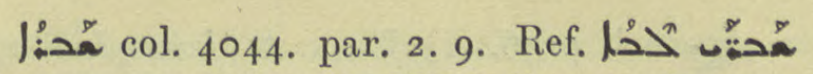
the innocent, Nars. ed. Ming. ii. 345 ult.

عُ pr.n.m. Boy, Inscript. Sém. No. 49, Z. A. xxi. 157 .

1 a col. 4045. Add: Eoßopíiss, a people of Ethiopia, Anecd. Syr. iii. 330. 2.

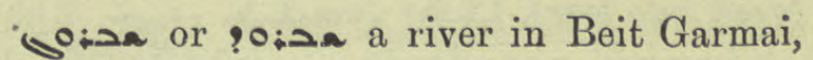
Chast. 46. I4, 19.

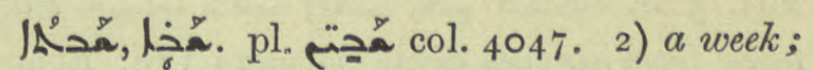
add: a company of monks having the same week of service, خحم:مب محتץ each consisting of fifty men, Anecd. Syr. ii. 209. 24;

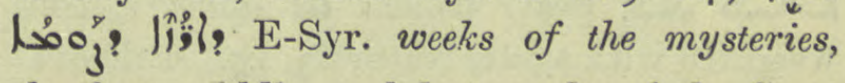
the first, middle, and last weeks of the Great Fast, QdhamW. 97. 13, 168 tit., 5.

Mecol. 405I. Correct to M Nest. 45.3 which has the same gloss as BB.

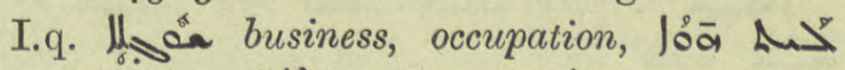

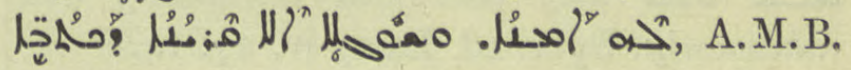
v. $439 . \mathrm{I}$.

pa col. 4051. to affect, injure, p. ע iो ? Aph. pinl to be occupied, y.as pal

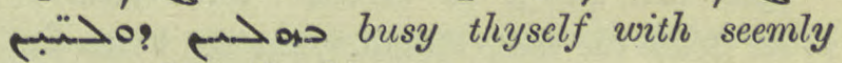
matters, BH. Stories 23. 104.

Lixar m. deception, add ref.

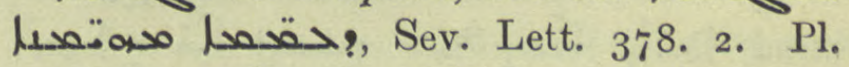
misfortunes, Sev. Ant. Vit. I 5 . I.

Jósang col. 4052. f. Ref. occupation, distraction of the human soul on account of its bond with the flesh diteranga, But. Sap. Theol. 5. 4 .

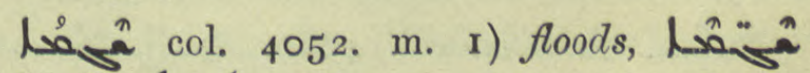

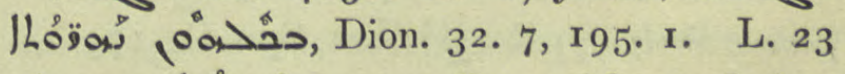
of par. lsos casually, without design,

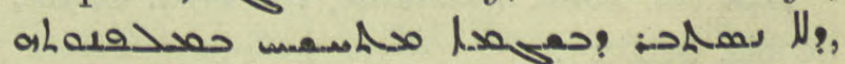
Theod. Mops. 293. 8. loges loa ll it was not an ordinary matter, M.Z. 2 I 6 . I 7 .

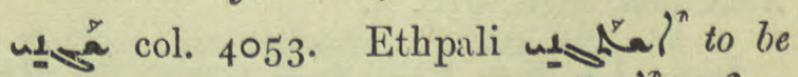
changed, different. Add: : $10 a>$ reptiles fled from Antony as if he smelt differently from other men, Pallad. 20. I5 = A.M.B. v. 24 . I.

شاهنشاء Pers. Shah-in-Shah, King of Kings, El. Nis. Chron. 96. 19. 


\section{is}

res col. 4056. I) Lexx. to descend, shoot

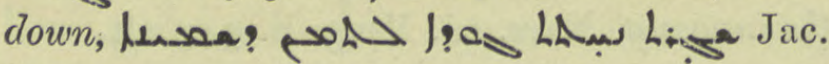
Sar. Hymn of the Palace which the Apostle Thomas built, ZDMG. xxviii. 618 , this must be imitated from the passage in Act. Apost. Apoc., see Thes. col. 4057 under Pa. 2) to

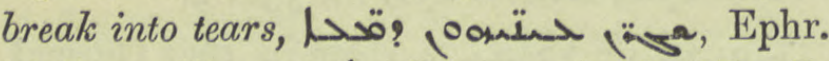

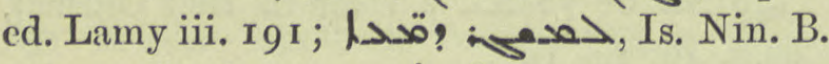
26I. 3 af. ad astears burst from his eyes, Sette Dorm. 2 1.1 38 . Ethpe.

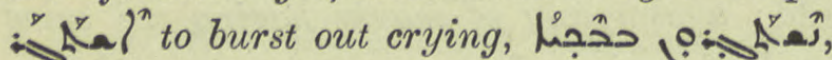
A.M.B. ii. 109.3. Pa.

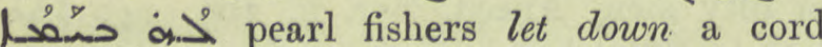
weighted with agate, Natur $6_{3}$. I 5. Ethpaual

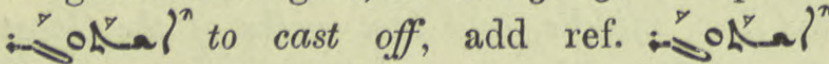

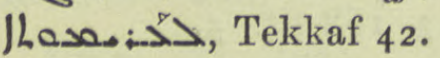

I? for for the gums, Med. 175. 5 .

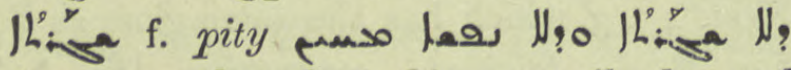
a- $\rightarrow$ ood they beat him mercilessly and inhumanly, Ephr. ed. Lamy iv. 27. 14.

ax. Liaǵ a col. 4061. tumult, disturbance. Add: loo:-D! henan troubles ab-

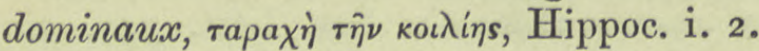

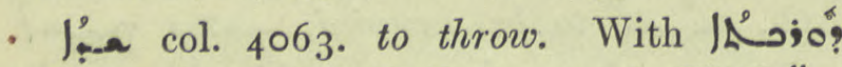
to set down the foot, Pallad. 744. 6. With 1 , he shouts aloud to the flock, Jul. 8. 17 . Pa. with lin to apply the

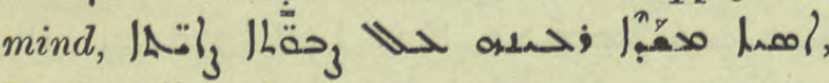
Is. Ant. ii. 108 pen.

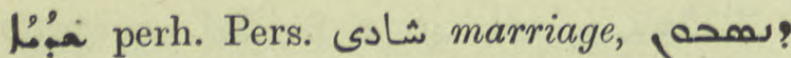
نقا ,للا a. BH. Journ. As. 1898, 94. 702, il permit aux prêtres et aux évéques de prendre des femmes impunément, Chabot.

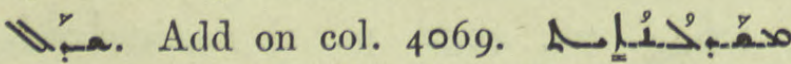

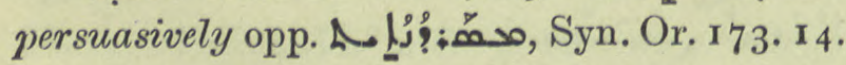

مُ col. 4069. haematite, ref. Med. 84 ter, 9 o bis, 610. 17 .

lone a plant, mentioned under l,j;oca Artemisia, Med. 6ro. 5 .

;. Pa. Pa with مُ0 she put her daughter before herself in good deeds and then followed her, Pallad. 204 pen.

شادُروان a fountain with jets of water falling into a basin, A.M.B. ii. 589. 9, Fraenk. in loc., Z.A. xvii. 190 .
Jane col. 407 I. to be quenched. To be

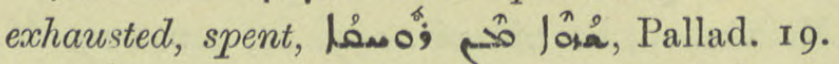

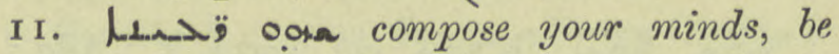
appeased, Ephr. ed. Lamy iii. 283. Loai

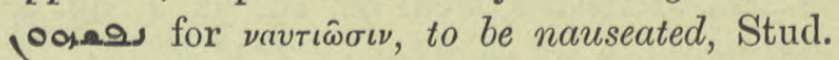

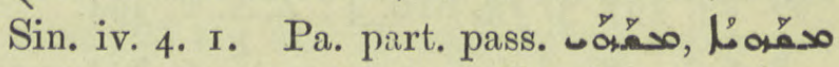
add: quiescent, inert, aight

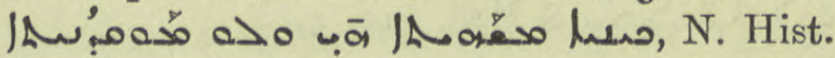
Bk. ii. cap. v. sect. 2.

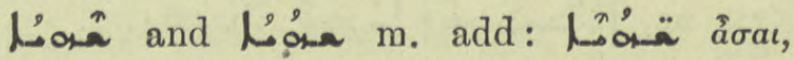
nausées, Hippoc. v. 58 ; بُ depression, Med. 284. 22. Jis than slow

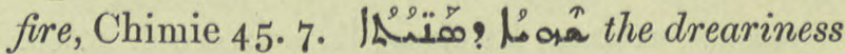
of hateful things, A.M.B. ii. roz.

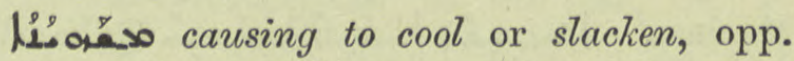

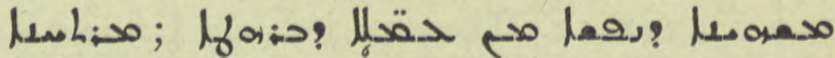

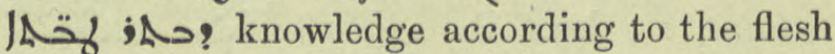
causes the soul to slacken from its labours in the race after the good, Is. Nin. ed. Chabot 76. 8,9 .

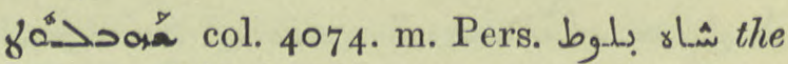
chestnut, ref. Med. 378. I 7 .

- for col. 4074 . m. Daucus gingidium, ref. Med. I 43. II I I62. 4.

بُ شاهـي a white falcon, pr.n.m. ef. (-a) a, Med. 589. 18.

شيمِ Amélineau 433 f. Exúatıs, Scete, A.M.B. iii. 574 , 4, v. 187.13 .

اشهل dark blue eyes, BB Merx Gram. 176 note 16 , red, reddened i.e. inflamed,

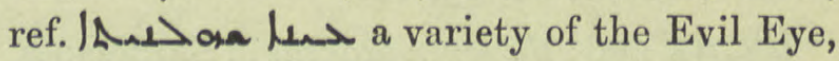
Charms 70. I, 87. 6 .

tuan col. 4122. i. q. atua a satrap, Charms 79. 16, 80. I, hij lo Jön lords and Aghas, ib. 83. 6.

شاهنشاهاء. Shahanshah =King of kings, A.M.B. ii. 668. 9.

(59ar col. 4075. m. conyza $=$ elecampane, fleabane, ref. مa فحي ز.بات يشبه القيسوم شابابج j, jóa a.coro, Med. 6ro. 8. Written  often. So correct for erean, ib. 265. 6.

ión col. 4075.

fó m. vigil. Add: àypvivia, insomnia, Hippoc. iii. 23, 30, Med. 3. 21, 4. 5, 37. I4, I36. 12. 
inxion

329

jǫ̛̣aำ The Secretary of State, Jab. 228. 4 af., 259.8 . I. q. - wogian col. 4076 .

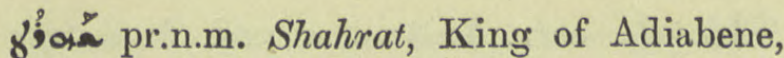
M.Z. 28. 9 .

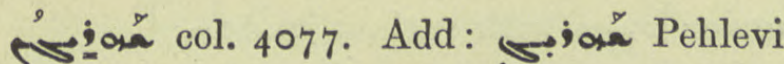
šahrîg, Pers. شه a district, Tabari 446, Pers. Mart. 239, Hist. Mon. I $5 \mathbf{I}=$ ii. 309 note. Sing. ib. 164. 7 ; usually pl. $1.17,198$ ter. Landed proprietors, of higher class than the Dihkâne lـoa! M.Z. 2 IO. II.

شه a a Persian month answering to August, Syn. Or. 68. 2 I.

شهرام E-Syr. bishopric, perh. في فيروز, Tabari I 23 , Syn. Or. rog. $6=366$.

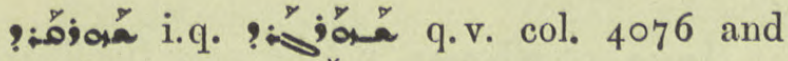

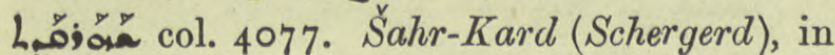
Beit Garmai, between Dakuk and Arbela, M.Z. 35. 70, a bishopric, ZDMG. xliii. 406. 8, Lia, ib. 394. 9, 399. 4, I6, 401. I, 402. I 2, Syn. Or. 33. 29 and often, El. Nis. Chron.

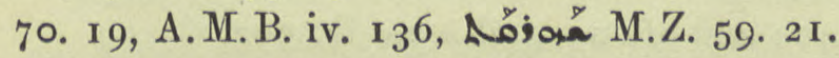

Ján col. 4077. to be at a moderate price; to be sufficient, Jân Ban Book of Shem

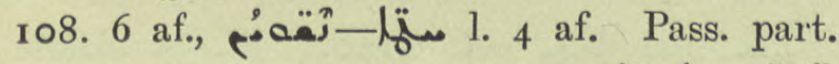
col. 4078. To compounds add: مارْ plants of the same species, N. Hist. iii. ii.

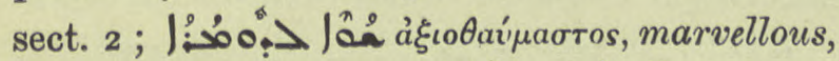

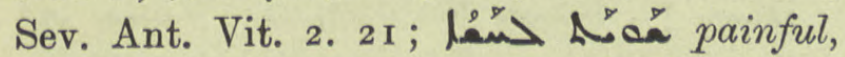

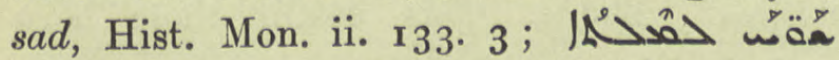
remarkable (parents), Sev. Ant. Vit. I I. 3 ; Ira: $\rightarrow$ Ao immediately, Sev. Lett. 196. 12. Ethpa. wo na $\left.\right|^{n}$ to be laid out for burial, waillo wohil L. E. S. i. ro8. 9.

$1 L^{2}{ }^{2}$ a col. 4082. f. add: moderate price,

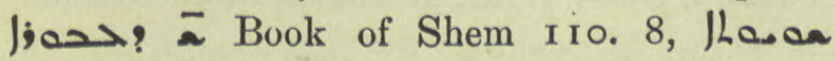

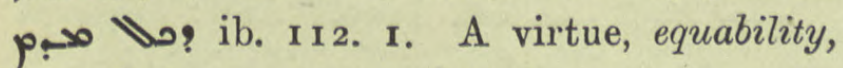
unruffled composure, Pallad. 7 7 2. I5. In

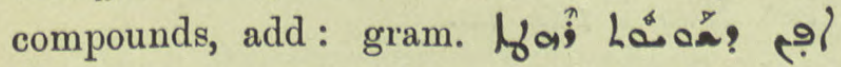
Âphen expresses equality of action $=\dot{\delta} \mu \nu \delta \rho o \mu i a$, BHGram. ed. Berth. 126, Hebraica iv. 169. $9 / 10$.

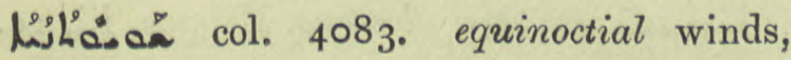

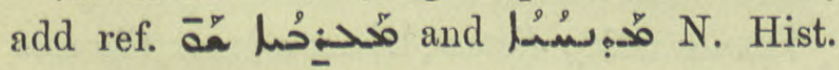
v. 3.2 .

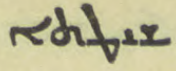

IR. abundance of food, add ref. Dion. 95. 5.

Lán col. 4084. equally. Add: equal parts, equal quantities, Chimie 43. 3, 47. 23 , 48. 3, \&c., Med. 59. 5, I 2, 570. I I, and often.

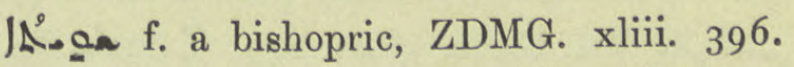

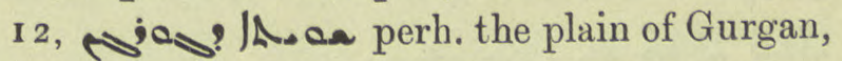
formerly Hyrcania, SE. of the Caspian, Syn. Or. 43. 20.

va col. 4085. Dosha ergot, a hard dry parasite of grass, Chimie 59. $2 \mathrm{r}$.

จan, to bray, rub down, rub fine, imper. Med. 184. 3, Chimie 23. 2,

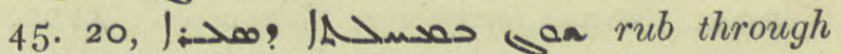
a hair sieve, ib. 53. Io. Pass. part. נلحدم)? \$n: a scoured alembic, ib. 35. 2.

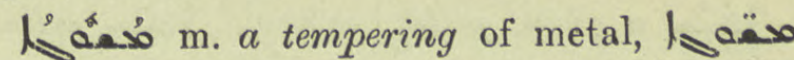

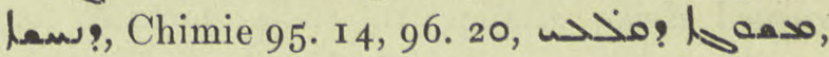
ib. 95.19 .

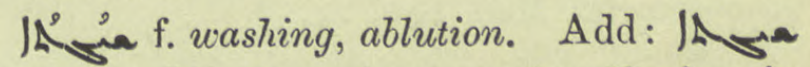
? yolks of eggs with alum, Chimie 95. 18.

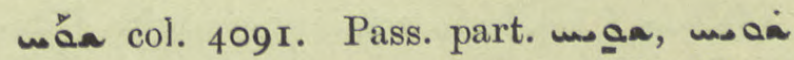

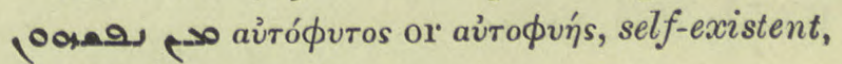
Manichéisme 97. 8.

JLán col. 4092. f. vitality, A.M.B. v.

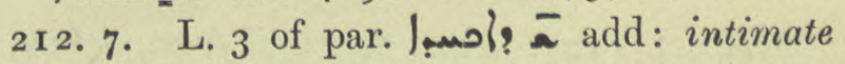
union of the body and soul, Sev. Ant. Hom. II. 5 .

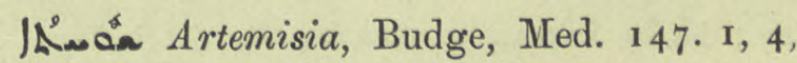
I7. Cf. Col. 4092.

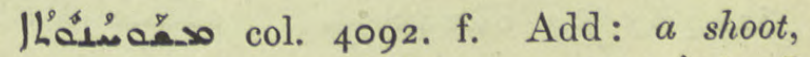
growth,

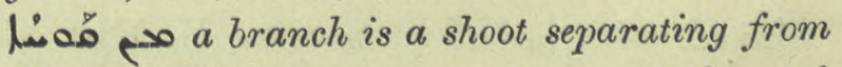
the stem, N. Hist. vi. 2, 3 ; م- 9 h

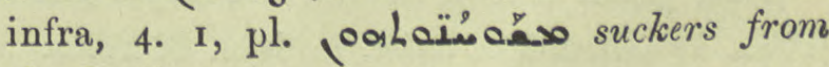
shrubs, ib.

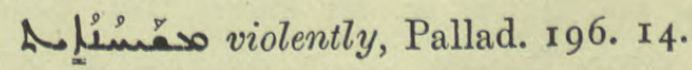

foa, fُ col. 4093 . Trs. from 1. 8 Rom. i. 22 and the following reference to col. 4 I 3 o. Col. 4094. Il. 3 and 5 correct final $L$ to $\gamma$.

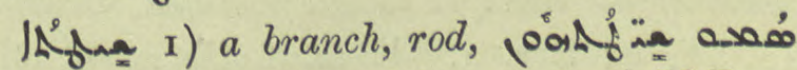
they laid down their fishing-rods, A.M. B. v. $\mathrm{U} \mathrm{u}$ 
532. 2) Arab. شتا شiآَتان winter; scarcity,

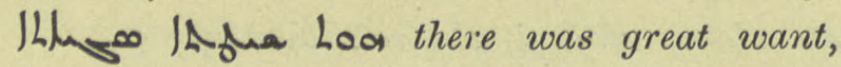
"il $y$ eut de nombreux accidents," Chabot, Dion. I 2 ult.

دهـب السه.m. sesame oil and balm, والبلسم BH. Carm. gloss. 249,

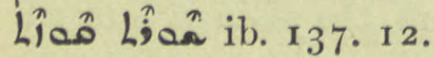

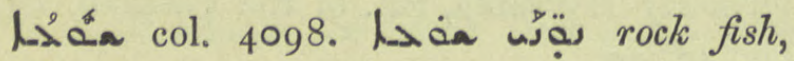
Med. 286.8.

 N. Hist. vii. 3 I ; 1 vi. 4 . $\mathrm{I}$.

פea. loa scrapings, filings, col. 4100.

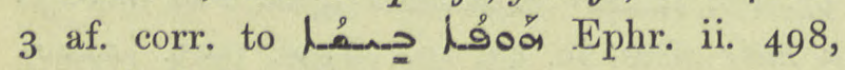
Fraenk. Z.A. xvii. 9. 50.

lمُa a plug, pellet, ofolll la pellets rained down similar to those formed for application to the eye, Pléroph. 22. I3, 23.9; suppositories, Med. I40. 14.

مَ' Anecd. Syr. iii. 24. 10, 14.

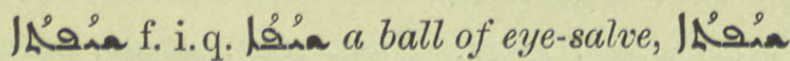
LLạ̈̂̉? Med. 82. 10.

人 BH. Stories 2 I. 95, 45 ult.

;ă col. 4 Io4. Add: to recoil from, refuse,

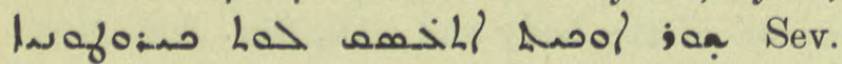

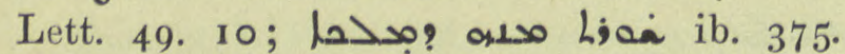
I7 ; ; Ethpali joLta ? $^{n}$ col. 4105.22 of par. to hide

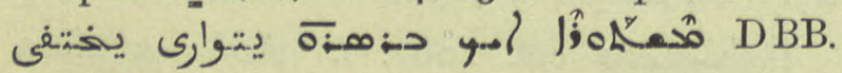
II 78. 8.

ןُّة with pl. meteors, N. Hist. v. 4. 3 tit.

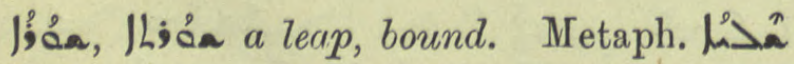

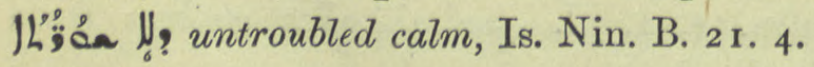

fica col. 4 roo. m. the navel, ref. Anot

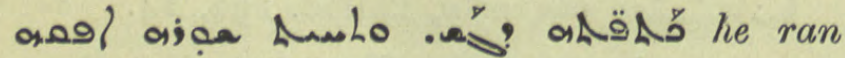
him through from the shoulder to the navel,

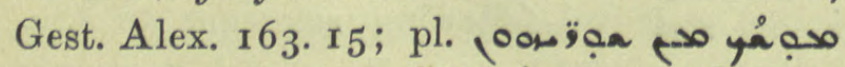

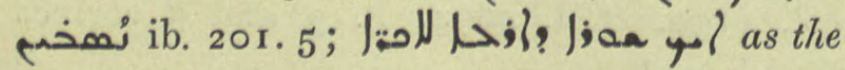
navel of the earth is to husbandmen, Charms 19. 16,94 Syr. 4. J'son f. the navel, add a second ref. Med. 569 , I3.

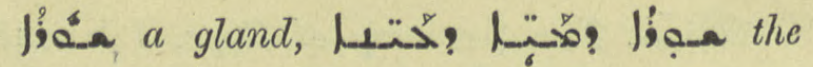
lachrymal gland, Med. 49. 23.

lágáa corr. losja a pod, husk, shell;

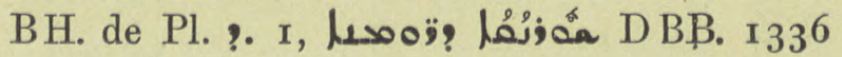

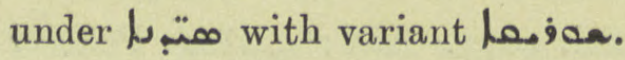

a col. 4108. 1) السوس (sovoa, Susa, bpric. near Karka d'Lédan, add Syn. Or. 683; 34. 4 and often., ZDMG. xliii. 393, Jab. 395 . 15. 2) Šus and Qal'at Šus, a fortress on a great height, near the Upper Zab, Pers. Mart. 200, 224 quoting Yakut, A.M.B. i. $4 \div 2$.

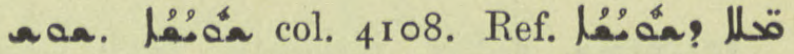
soothing words, blandishments, S. Dan. 6 I $b$, 7 af. of

Jaca or jldaca a lake near the Tigris, Jac. Edes. Hex. xxv. 2, cf. a a col. 4I08 and carea col. 4345 .

-fóar col. 4 108. Suster in Khuzistan. Jidaen Chast. 35. 4, JîAan ZDMG. xliii. 404 and often; مidaca ib. 393.

Hohaea Gentilic from ihnea, Mar Benj. 86. 5 .

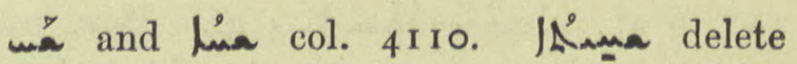
11. 3,4 , and 5 . The gloss. is under col. 1679 .

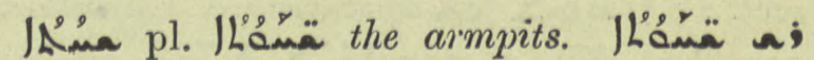
"lepra", Budge. This befalls those born early in Nisan, Med. 5 16. I7.

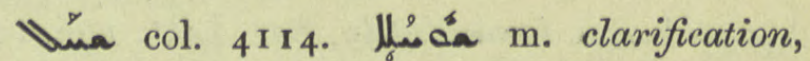
purification of light mingled with darkness, Manichéisme 144. 8.

بِ

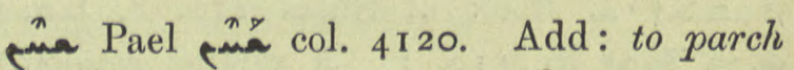
corn, معبه Hist. Mon. i. I64. Io.

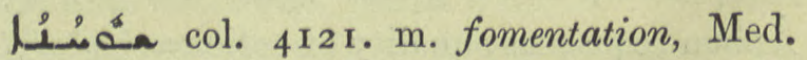

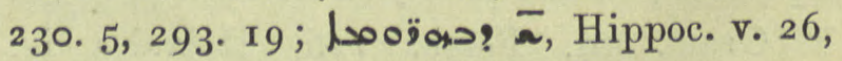
vi. 30 .



Lena wasting disease, lolasoo lina, Charms 3. 17 . 


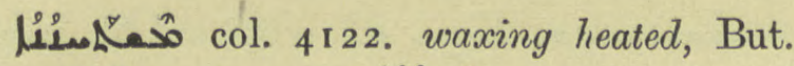

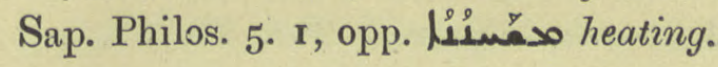

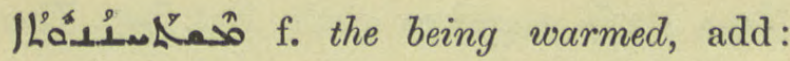
example of passive category, But. Sap. Isag.

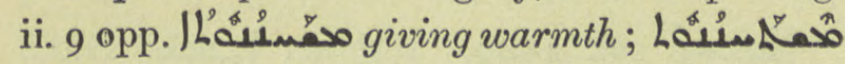

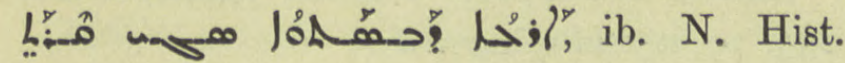
iv. $4 \cdot 3$.

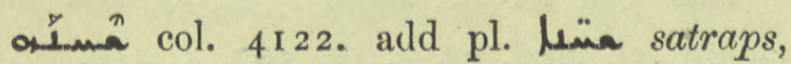
Charms 73. 24.

مشّم col. Pass. part. 4 I 23.

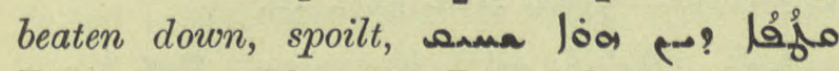
Rylands 44 fol. 5 b. Pounded, name of an eye salve, Med. 90. 7 .

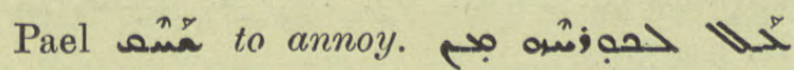

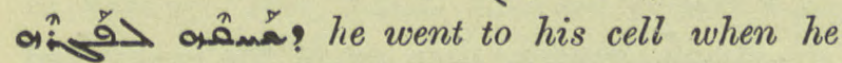
had tired out his body, A.M. B. vii. 78. I6. Aph. حمُحنُ why do you take so much trouble? Pallad. 7r. 8.

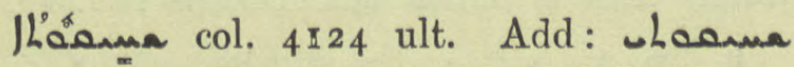
my affliction, Ephr. ed. Lamy iii. 491, مسبمa مص:, Ishoyahb 46. 9.

a col. 4I25. 5 of par. correct ref. to Chimie to 57. 2, I I. Add : to press together, crowd, مut Dion. 95. 3, 17 I. 3, 193. 6, 010: ib. 212.7.

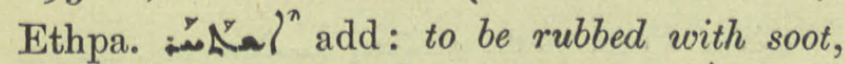
certain criminals had their heads shaven and then covered with soot, see Duval, Néoaraméen de Salamas 50. 6, trad. 37. I,

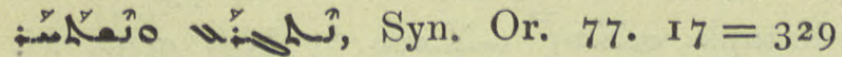
note 5 .

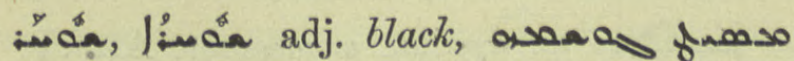
مa 0, Dion. I42. II, Brit. Mus. Or. 3337. I $7 v$; subst. charcoal, Barhad. 369.5 ; black

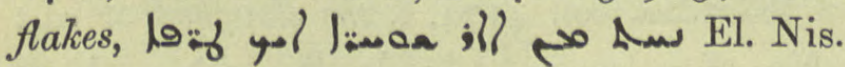
Chron. I 22.12.

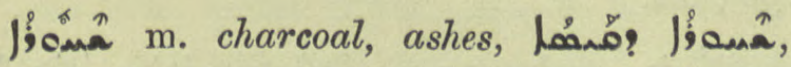
Med. 583. 22, 24, 584. 2, soot, بs jama

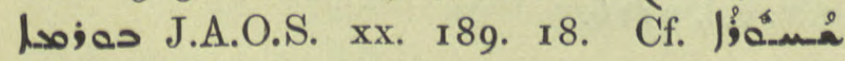
col. 4 I 27.20.

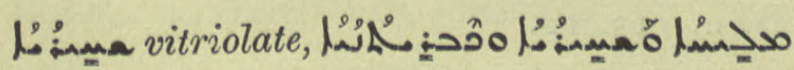
mineral springs are salt and vitriolate and sulphurous, N. Hist. ii. cap. v, sect. 4 .

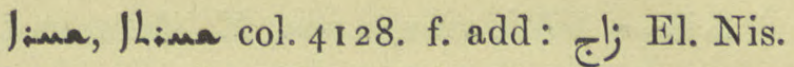
${ }^{2} 5.63$; i.q. Pers. شخار ashes for lye; vitriol, Lag. B.N. $3^{2}$ n.

An col. 4I 29. Add : to be marred, sullied,

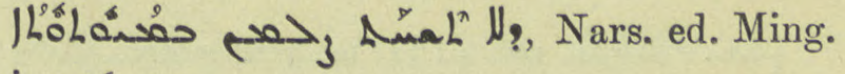
i. $35^{6 .} \mathrm{I} 3$.

jada prob. जrana parsnip, Med. 366. 21 .

مoda E-Syr. bpric., Conc. Jab. A. D. 420 , ZDMG. xliii. 395 infr.

0-far a village in gooas, Sassanidi 29.7.

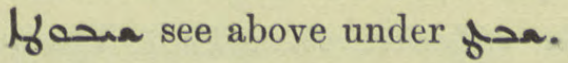

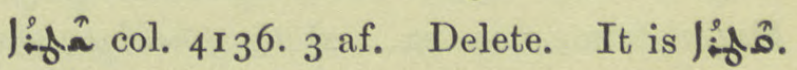

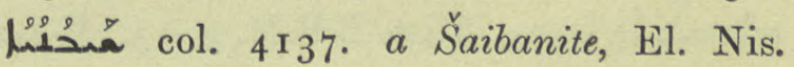
Chron. I74. I9.

(2) I) col. 4137. Syagrus, a kind of

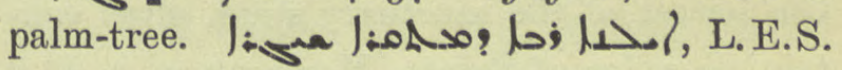
39. I I. 2) pl. the gums, ov̉入a, Hippoc.

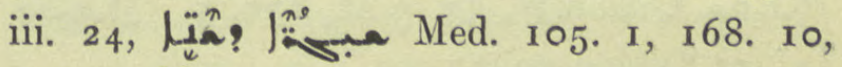
I75. 13 bis.

بa col. 4I39. a sheikh. Add: ò la dan y. Yezidis III. I I, Mt. Singar ${ }_{4} 6$ ult. Pl. JLمجة ib. 26. 6 af.

Sبchekhan, a district NE. of Mosul, Mt. Singar 24. n. 2.

J_Ln a monastery on the Lesser Zab, Chast. 6r. I.

يمتأمُ service berry, see col. $4^{\mathrm{I}} 3^{8}$.

in síp, terra Serica, China, Spic. Syr. I3. 22.

Lona oi $\Sigma \hat{\eta} \rho \epsilon s$, Seres, the Chinese, Jac. Edes. Hex. xiii. I5, Spic. Syr. I3. 24, 26, متح ib. 1. 2 I. Cf. Jؤ silk, col. 4016 .

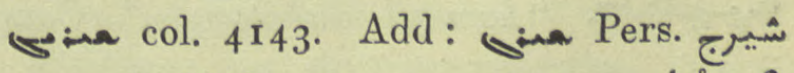

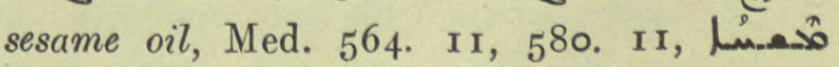

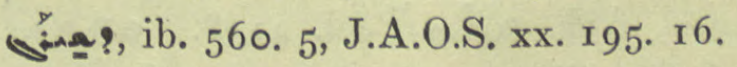

col. 4I44. Correct last line of par. to /A fan col. 4I 34 .

ش Arab. Aralectic pronunciation of جִ Gigouir now Shabshir in the province of Ménouf, Amél. $187 \mathrm{f}$., A.M.B. v. I 8 r. 10. 
Sišoh, village near Ma'altha, in the south of Beth Nuhadra, M.Z. 200. 9.

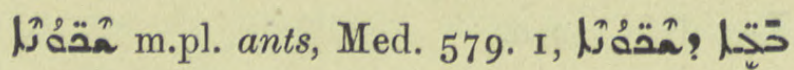
ib. $5^{80} .14$.

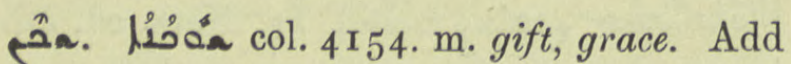

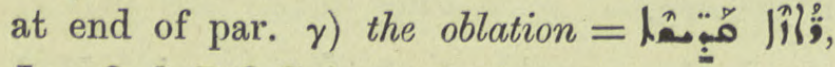
Jac. Ord. R.O.C. i. I3. 8 af.

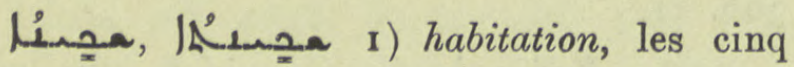

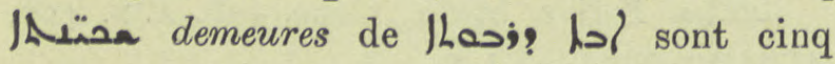

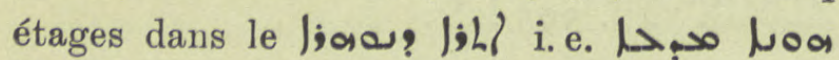

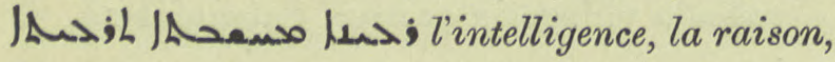
la pensée, la réflexion, la volonté, Cosmog. Manichéenne 1. 9, 20. 2) the Shekina, add: ahoa part of a church, the Mercy Seat=altar, Med. 596. 5. E.-Syr. a quasi-altar, set on the lowest of the sanctuary steps, often containing relics of Saints, Maclean : مُـإ مـ

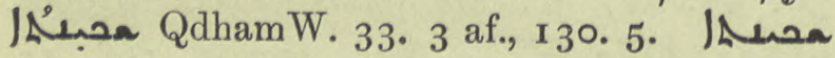
"פمبتمعل Takhsa 99. 17.

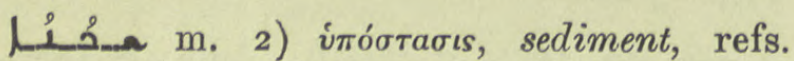

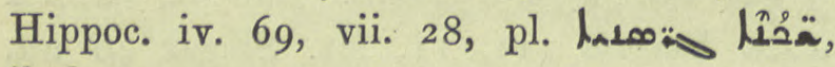
ib. 1. 29.

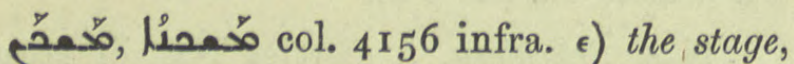

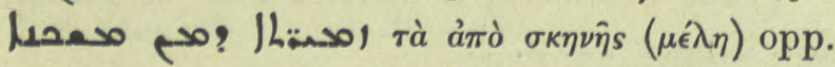
sacred verses, Sev. Ant. Vit. 244. 3, henfes Lصeso pomp and theatrical display, id. Lett. 297. 20 ; 0;-6il? ل 48. 12.

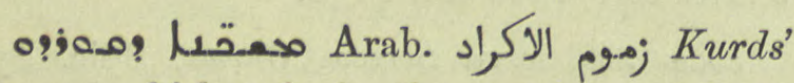
Camp, a bishopric in the province of Fars, Eranšahr 27, ZDMG. xliii. 396. 16, Syn. Or. $677 ; 43.24$.

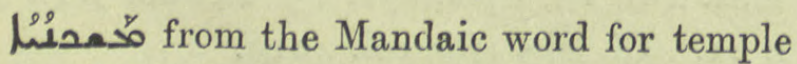
ovats "homme du temple," Coupes ii. 154,16 .

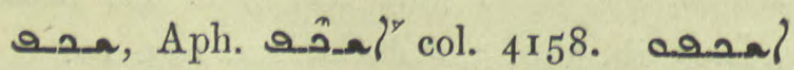
Anecd. Syr. iii. 257. 4. Correct esinal'they fixed stakes.

آمُ Arab. Arare, image. A metal standard with figure of a bird, Yezidi idols,

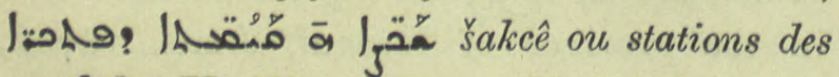
symboles, Yezidis r 12. го. Cf. مa below.

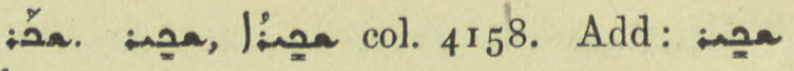

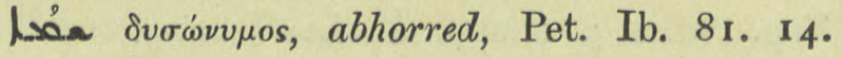
a vicious circle, But. Sap. Theol. I. 2 infr.

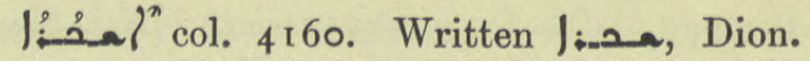
I 55. 19, a field of corn.

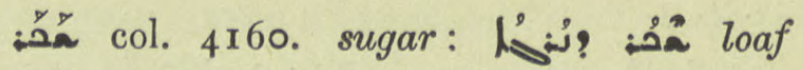
sugar, ref. Med. 92. 3. 10, 165. 4, 610. I7.

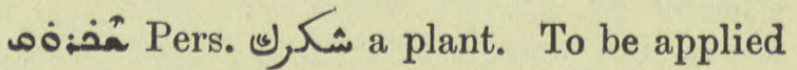
to sword-wounds, Med. $5^{8} 5$. Iо.

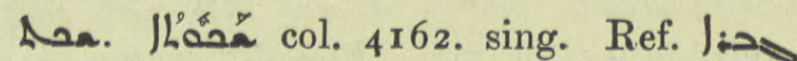
Jhena one sunk in tranquillity, in settled quiet,

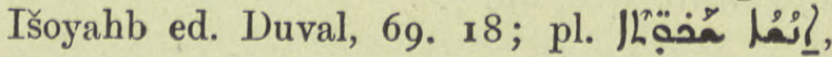
ib. 9 r. 3 .

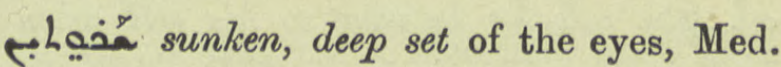
37. 13.

مُخح col. 4162. Add: a small stone, at a Yezidi wedding, the bridegroom

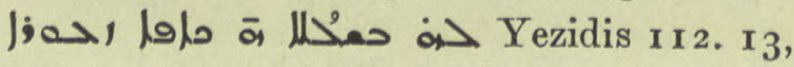

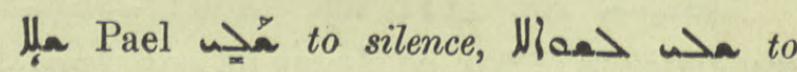
confute a question, Ephr. Ref. ii. 96. 23.

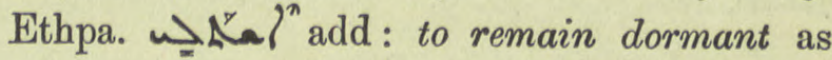

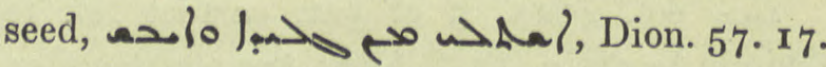

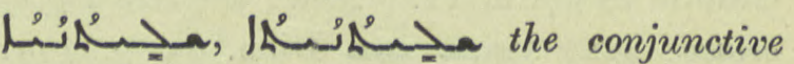
membrane of the eye, N. Hist. vii. I. 2.

مخدس Pass. part. a col. 4172. E.-Syr. the Apostle i.e. the Epistle for the day, always taken from St. Paul, Takhsa 4. 9, 18, 64. 2. Aph.

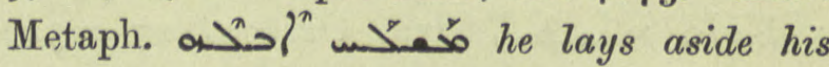
mourning, Ephr. ed. Lamy iii. 439. 4 .

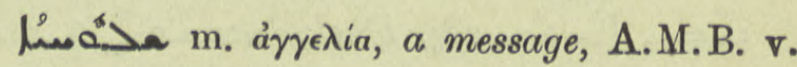
I70. 14.

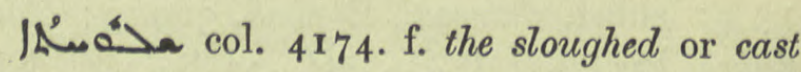

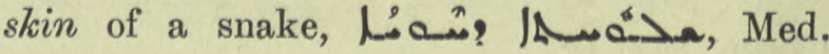
$5^{8} 3$ ult.

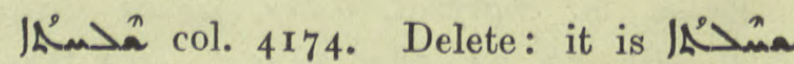
q. v. col. 4I16.

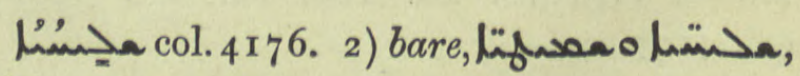
Dion. I1 3 . 12.

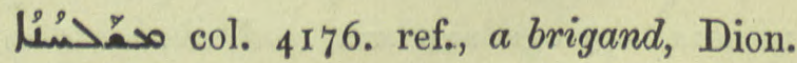
I27. 5 . 


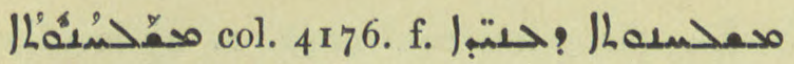
stripping the dead, Dion. 22 2. 6.

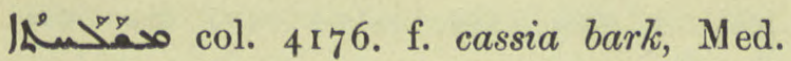
51. 3, I 3,52 . II, 297. 23,305 . I I, 13 and often.

Proper names: :

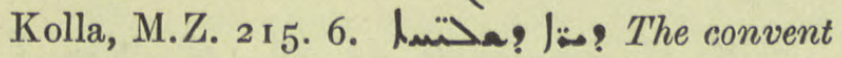
of the Apostles, in Khuzistan, Journ. As. x. vii. 111, rog.

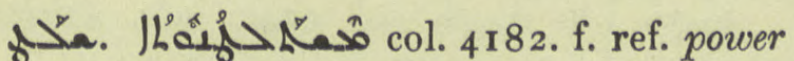
of the human soul over its forces, also of bad habits over the soul, N. Hist. viii. 4. I, Philos. 8. I.

مح้. Pael paz col. 4185. to salute, give

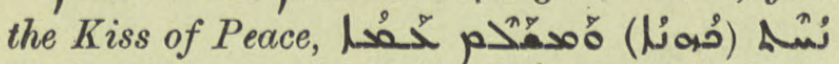

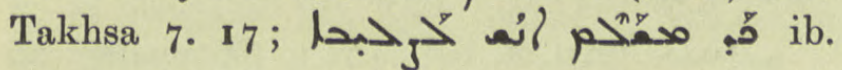
99. $13,15,17$.

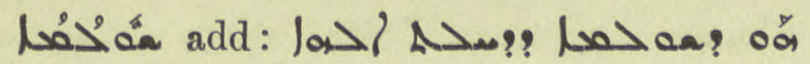
translating the Gr. formula $\delta$ îेs $\epsilon \dot{v} \sigma \epsilon \beta o v \hat{s}$ $\lambda \dot{\eta} \xi \epsilon \omega s$, Mélanges, Kugener R.O.C. v. 155 on Anecd. Syr. iii. 294. II.

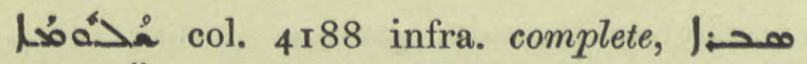
ال الl imperfect hope, Išoyahb 55. 27.

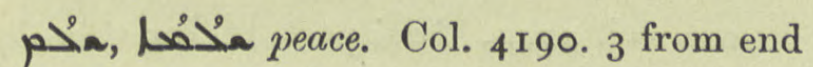

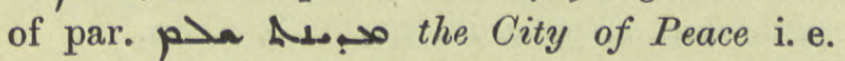
Baghdad, Chast. 34. 16.

Proper names: col. 4 I 95 infra. add Bishop of Karka de Ledan, Jab. 378. 2, ZDMG. xliii. 401 pen.

col. 4198. smallpox, ref. M.Z. 203.8.

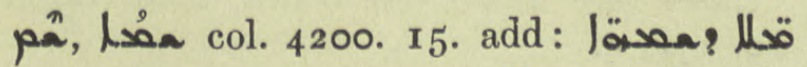

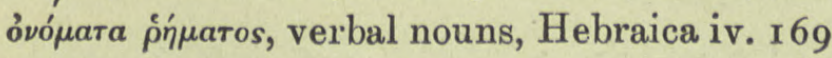
pen. and ult.

oh Mar Aba ii. Letter 3०5, 268.

คُ

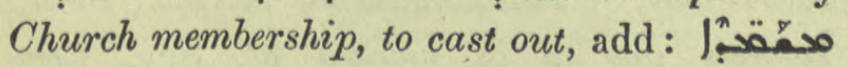

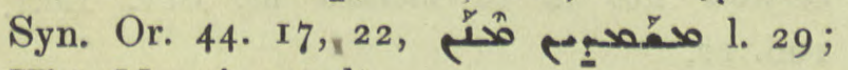
Hist. Mon. i. 39 ult.

A deprivation of priests orders, Syn. Or. $45 \cdot 2$.

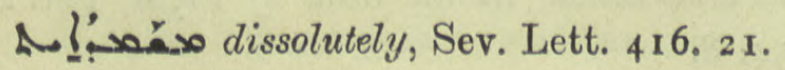

Sear. How col. $4207 . \mathrm{m}$. the strap? of a sandal, Sev. Ant. Hymns I32. note f.

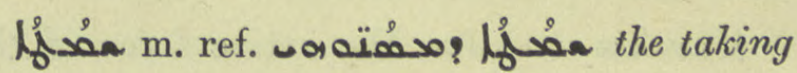
off of his shoes, BH. on Luc. iii. 16.

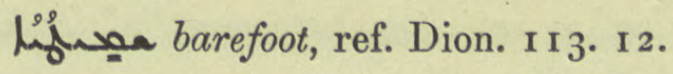

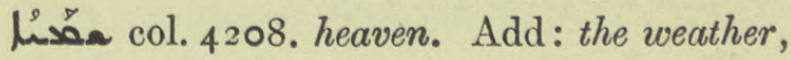
. 2 , Pallad. 33. 2 I

مấ

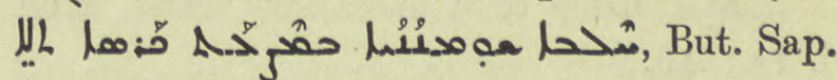
N. Hist. vii. 1. 3 .

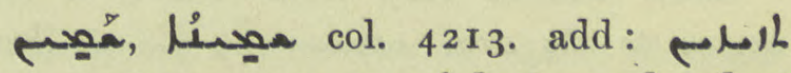
pangersty and robust habit, Sev. Ant. Hom. I I ult.

Wác col. 42 I 4. end of par. with o to be

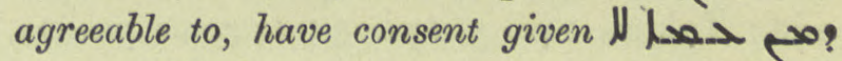
u., BH. Journ. As. 1898, 98. 869. Add:

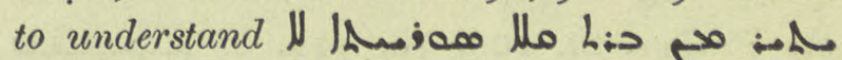
00a they understood no language beyond Syriac, Eus. Theoph. 189. 18.

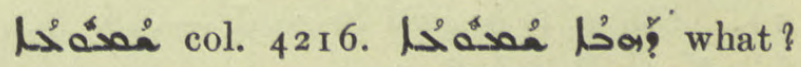
among the gifts of the Magi, G. Busâmé 64. 5 af.

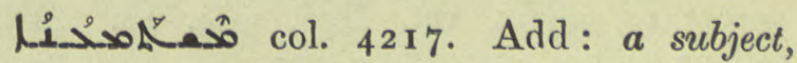
oJhax or David saw his subjects being destroyed by the death-dealing plague, Sev.

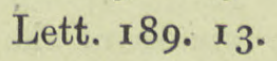

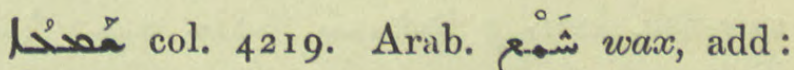
Med. $5^{81}$. 8, 586. 2, 5, 14, 15 .

مá Pael ar col. 4226. to send out.

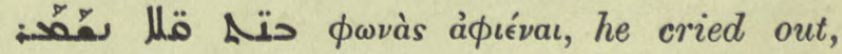

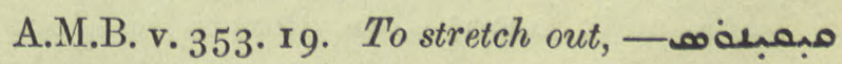

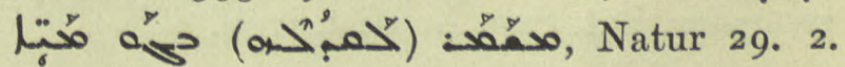
To discharge pus, deep-seated sores

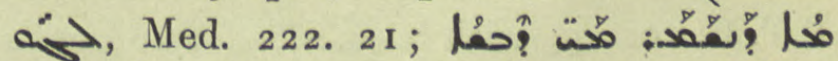

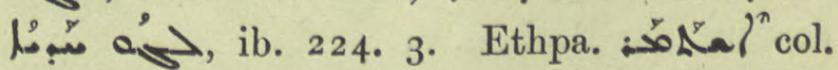
4221. l. I 8. T'o be discharged as matter or poison, Natur 4I. 3, Med. 223. II.

| . 


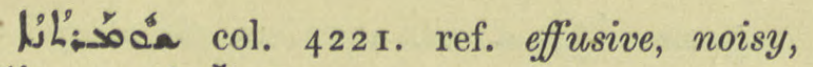

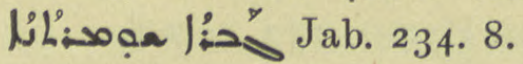

م omissive, the active force of the

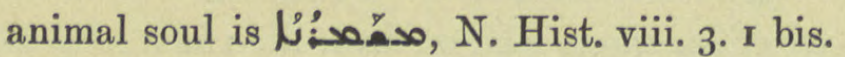

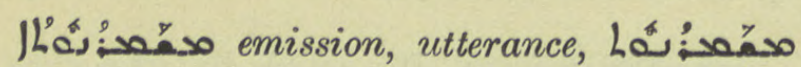

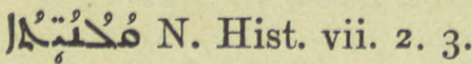

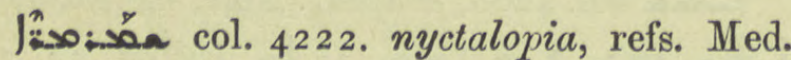
86. $20,88.23,89.5$.

مa name of a monastery, Hist. Mon. i. 143.16 .

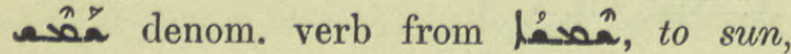

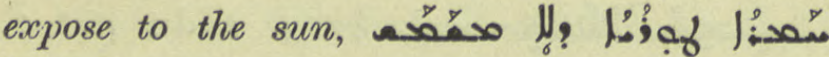
لم sountain wine which hath not fermented in the sun, Med. 302. 5 .

. add: Takhsa $\mathrm{I}_{4} 6.5, \mathbf{1} 70.5$; to recite psalms, 毛

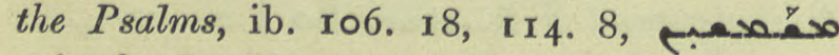
they recite Psalms phrase by phrase i.e. farcing after each, ib. 135 .

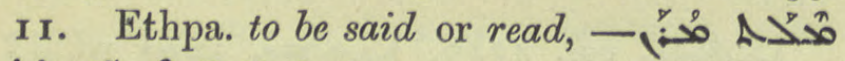

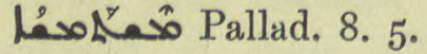

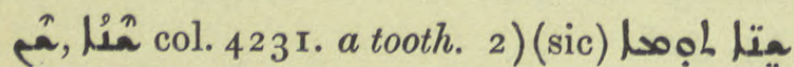
spikes of garlic, ref. Med. 564. 22. Add:

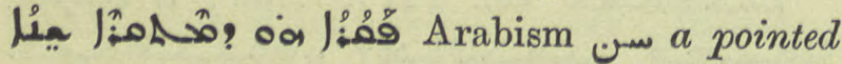
vertebra forming the apex of the cervical column, Med. I55. 22.

مُنُ pl. ple sharp spines of the

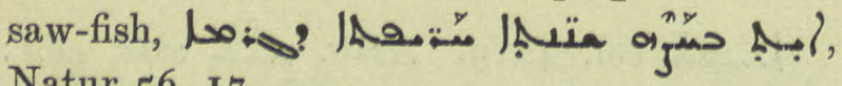
Natur 56. 17 .

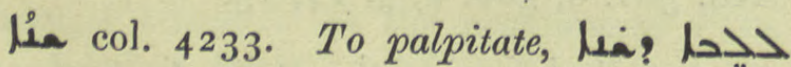

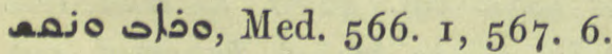

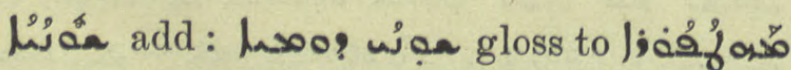
metaphor, But. Sap. N. Hist. iv. 5. 2.

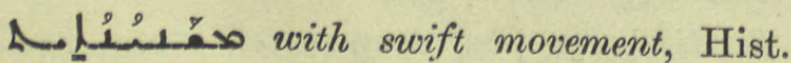

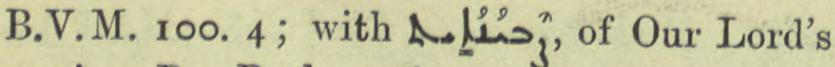
coming, Bar Penk. 9. 6.

I'ó col. 4239. change, Gaza MS. $217 b$; ref. هُ folly, But. Sap. Eth. iv. 4 .

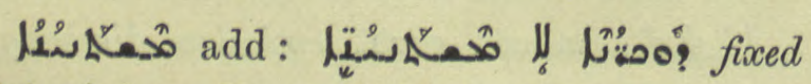
Saints' days, Brev. Chald. i. 486. 2.
Len the year. col. 4240. I. 4) locutions. مت some years after, Sev. Ant. Vit. 301. 4 and note, ib. ult.

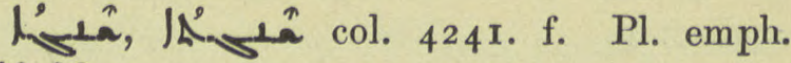

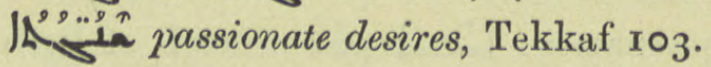

每 But. Sap. Eth. iv. 3 .

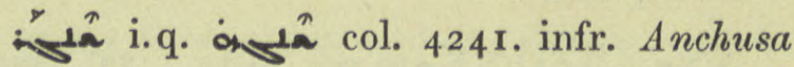

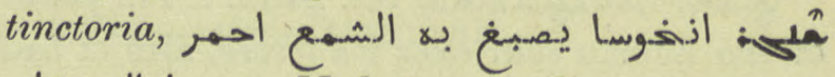

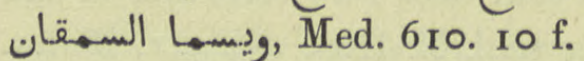

L. Lentilic from an inhabitant of Singar, Chast. 36. 9, pl. ib. 11. 10, I9.

r. a pr.n.m. col. 4243. Shenoudin, an Egyptian Monophysite monk, see Amélineau, Moines Égyptiens, Paris, r 889, Diosc. ed. Nau, 22. 2, 94. 3, R.O.C. xii. 32 , note 2.

بodıa Avesta shnûman, Pehlevi مaca, reconciliation, satisfaction. To say this was

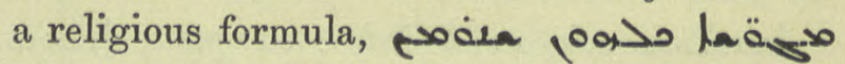
ححر ب. A.M. B. ii. 579. 2.

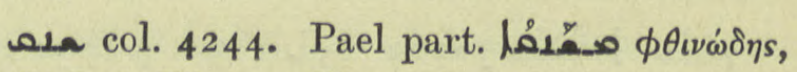
phthisical, Hippoc. iii, ro, iv. 8, v. 60.

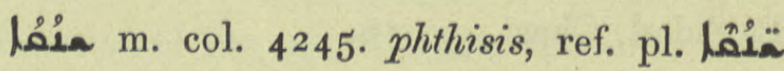
Med. 16. 19.

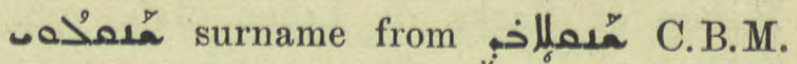
1067, No. 9, Chron. Sim. Sanqlawaja 5. 9. See infra.

12ْ col. 4240. tree lichen, Med. 89. I4, 20, 174.4 .

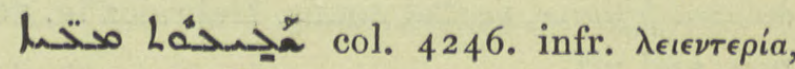
lientery, ref. Hippoc. iii. 21,29 , iv. 12, vi. I. 42 .

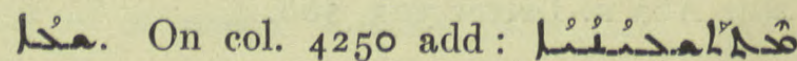

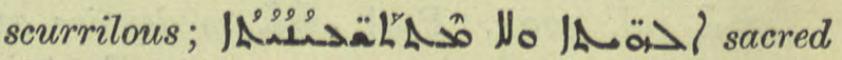
subjects not to be mocked at, Sev. Lett. 106. 12.

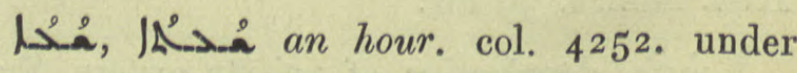

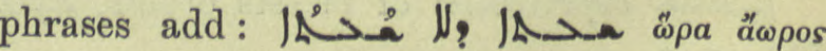
$=\mu \epsilon \sigma o v$ íkтเov, in the dead of night, Pet. Ib. 54.8 . 
احدا محتى "les temps d'une heure" are the degrees of the equator which answer to one hour, i.e. a twelfth part of the arc of the equator between sunrise and sunset, De Astrolabe $249.5,256$ bis, 282 note, 288 note.

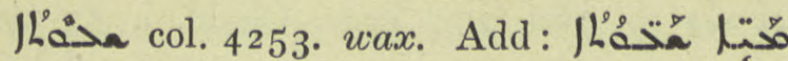
yellowish fluid from sores, Med. 554. I 2.

I'ớLó fa f. waxiness, thick waxen colour, Med. 557. 17.

فำ

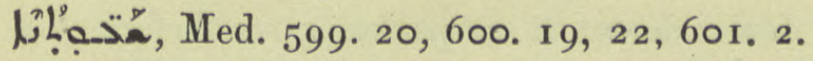

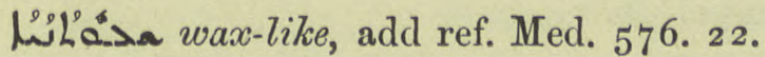

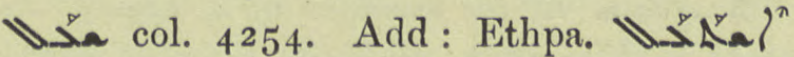
to be coughed up, Med. 201. 5 .

لـ up a fugitive, Dion. I I r. 5, 9, the Emir's portion of exactions, ib. I62. 2. 5 . For other instances of a for $\mathrm{e}^{\mathrm{cf} .}$ ib. 33. 6 and جعفر a alb. II5 f. Nöld. in loce.

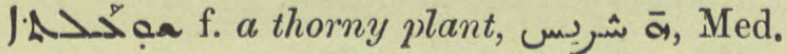
585.13 .

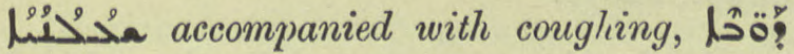

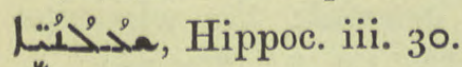

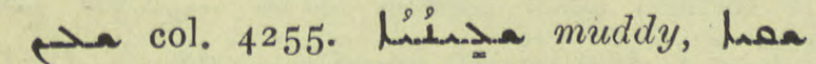
a محس 0 , Sev. Lett. 360. I.

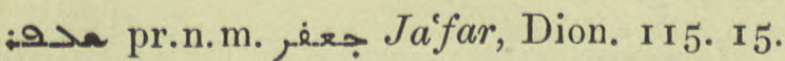

(- col. $4^{2} 5^{6}$. a mountain, Chast. 22 . ro, 50. $18,2 \mathrm{I}$.

Arab. Aُ شُ to look. To turn back,

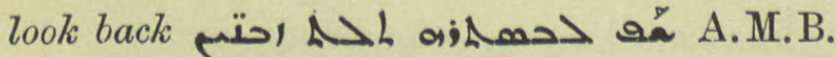

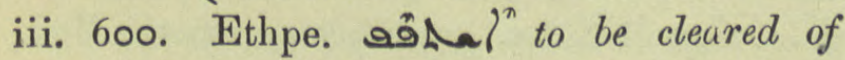
stones, נملهو ص: Geop. 14. 24. Aph. an ? with o to diverge from, Ephr. Ref. i.

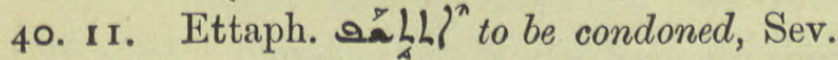
Lett. 140. 9 .

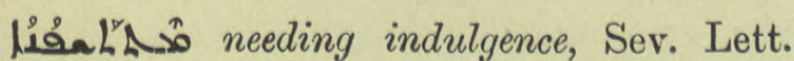
I63. II.

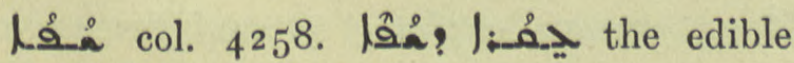
root of a small plant called in vulgar Arabic of Mosul 2 and in Modern Syriac

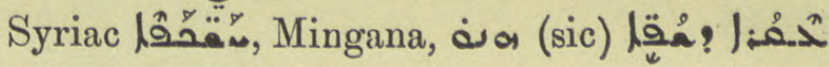
.

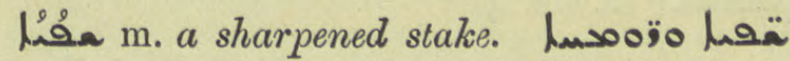
darts and spears, Josephus vi. I5 ult.

|ำกำ a relative adjective from Med. 600. 3 .

|م. of the slope or foot of a mountain,

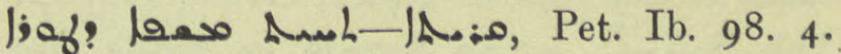
Cf. שִשִ to make steep or sloping, transl. 93 note.

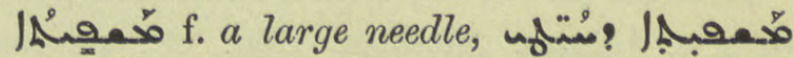

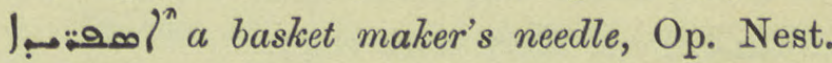
146. 5 .

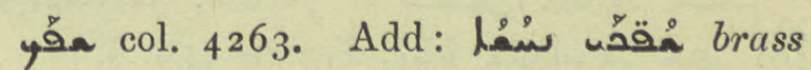
founders, Alex.-Lied. ZDMG. Ix. 8 I 9.

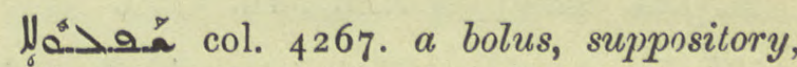
Med.

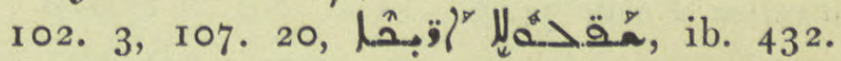

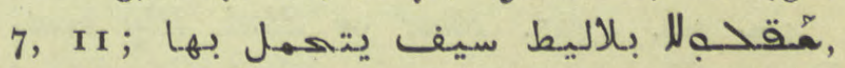
ib. 6 Iо. $\mathrm{I}_{4} \mathrm{f}$.

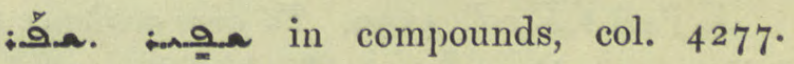

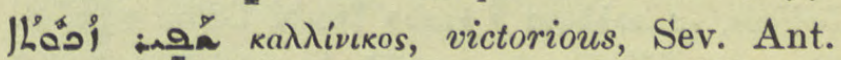

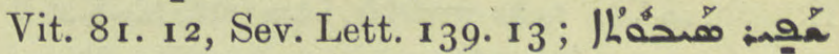

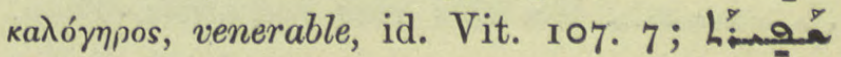

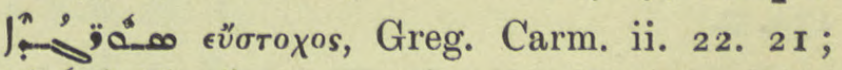

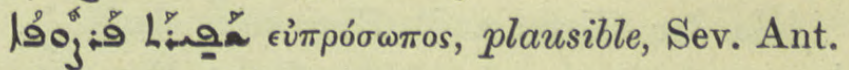
Hom. 58. 1, Letters 57. I r.

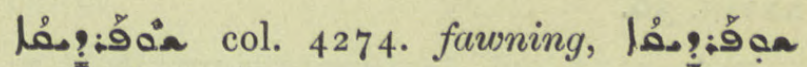
أم N. Hist. vii. I. I.

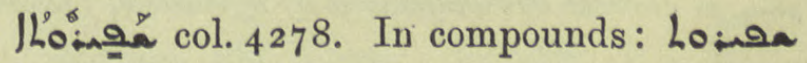

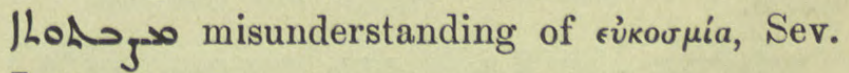
Lett. 79. I 9.

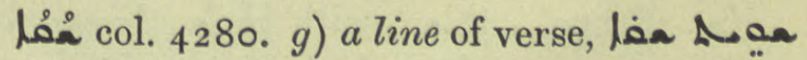
isocolon, a metre with four rests to a line,

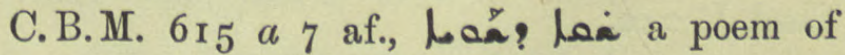
even lines opp. لl lon long lines mingled with shorter verses, Sev. Poet. $149 v$.

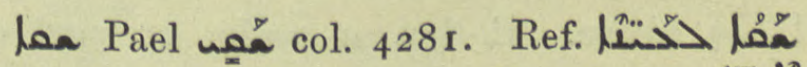
bathe the eyes, Med. 82. II. Ettaph. woal'L/"

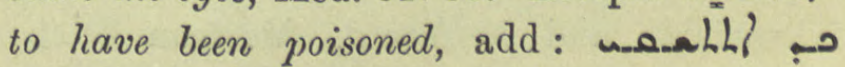

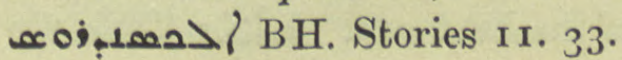




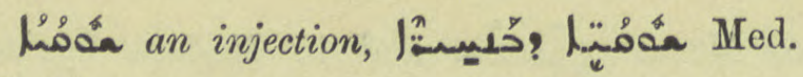
47. 15 .

هُ حمُ Ascalon, Diosc. ed. Nau 59. I 2 ,

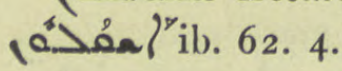

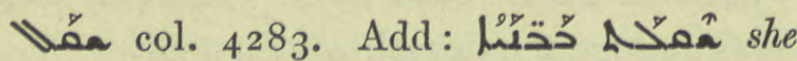
adopted, Pallad. I I 7. 3. Pass. part. col. 4286.

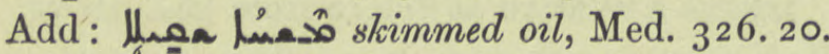

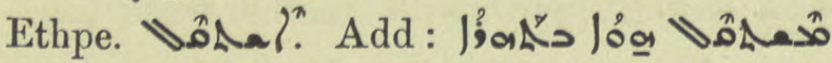
a) ${ }^{3}$ a he was rapt in ecstasy, Pallad. 108. I 2.

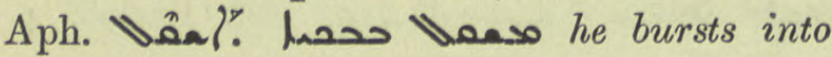
tears, Ephr. ed. Lamy iv. 8I. 4.

ممحب. pr.n.1. Šaqlaband, Journ. As. 1906, 76 i.q. Ja Saqlawa, Syn. Or. I I and مُعملاذب, Pérs. Mart. note 1847.

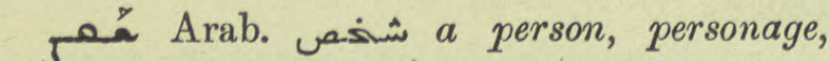

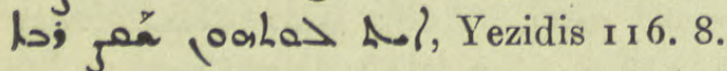

А.

|مُ col. 4297. pl.m. some bird, perh. a roller, Natur 34.3 .

a col. 4305. Shar or Shal, lily root, Budge, Med. 14 I. 23.

م Pael Pa col. 4299. To give credence to,

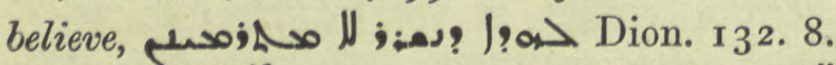
Ettaph. لل الم مد Nasas lya this was incredible, Dion. I32. 5 .

ק a for (Urim and) Thummim, J. xxviii. 30 .

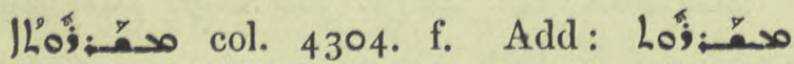
liñ 94. 8 .

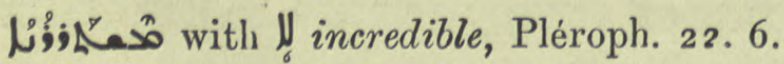

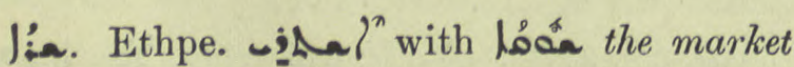

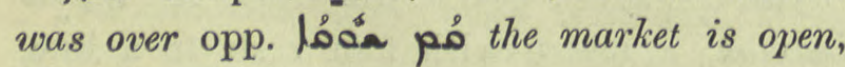
Ebed J. 240.

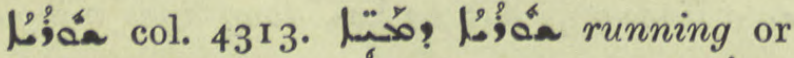
watering of the eyes, Med. 87. I4, cf. Jon ?ִ above. E.-Syr. a psalm or part of a psalm usually introducing an anthem, it is variable and is farced, Maclean Dict., Catholicos of the East 2 19. . proper shuraya, Takhsa 4. 6; 59. 8 af., I I I. 5; QdhamW. 7. I2, ult., 45. 4, 47. I2,

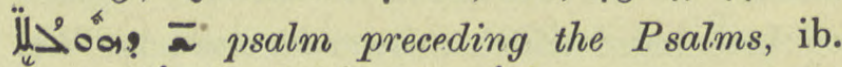
9. 5. 1he anthem and the collect "for help", l. 10; "مَّa

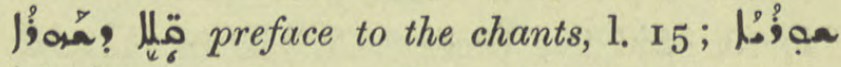

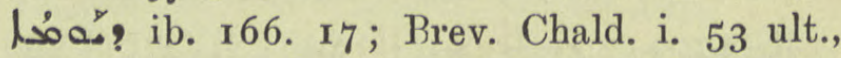
54. $7,63.18,65.15,19$ and often;

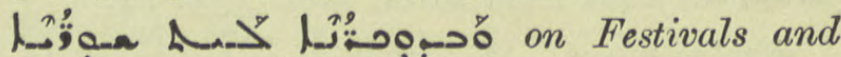
Commemorations (Feasts of Our Lord and Saints Days) anthems are said but no shuraye,

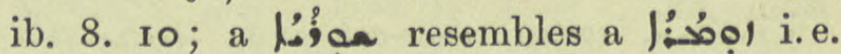
a few verses of a psalm sung before the Gospel, ib. 74. 3 .

A.

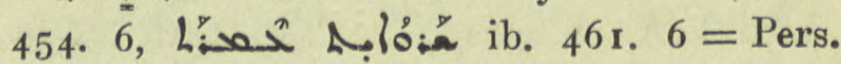
Mart. Ioo.

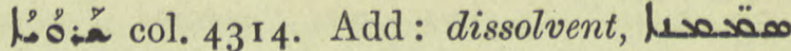

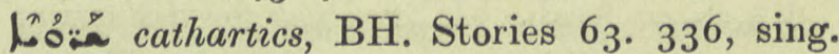
69.365 .

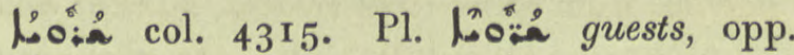
مُرَّهُ travellers, Jac. Sar. ZDMG. xii. I I8. 8.

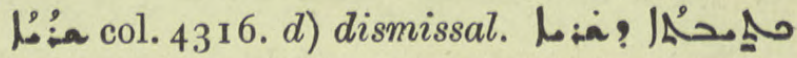
a dimissory letter, Sev. Lett. 49. 8.

often for silk: see col. 4016 and Suppl. above.

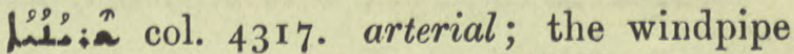

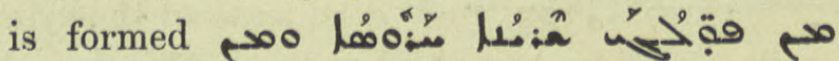
هist. N. Hist. vii. 1.3 .

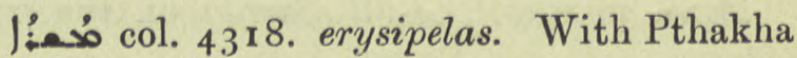

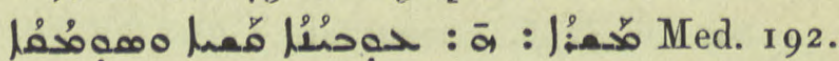

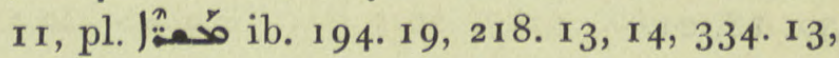
Hippoc. v. bis, vi. 24, vii. bis.

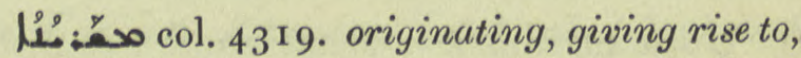

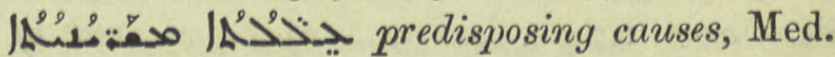
200. 1 .

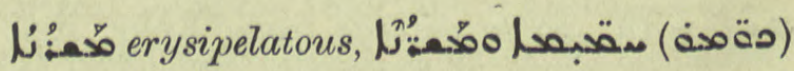

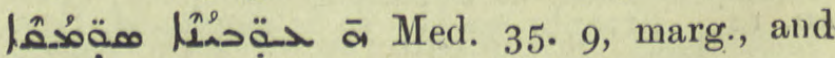

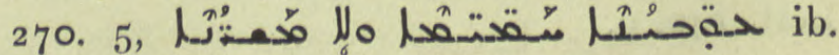
282. 17 .

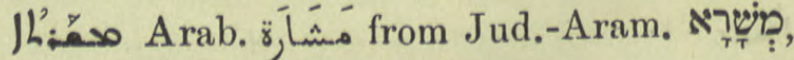
Mand. משארא, a plot of ground for sowing $\operatorname{cor} n$, Mand. Gram. I6o n. Cf. Ar. FremdW. 
rdix

129; DBB. 943. 7 gloss. to

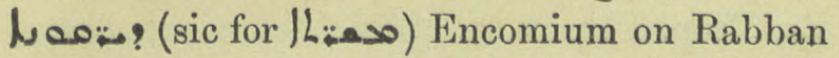
bar 'Idta, v. 670 ed. Budge, Lit. Centralblatt, 1903 .

Ihin f. prob. the navel, Jhan? |م: Charms 27 tit., and five times. Cf. |م

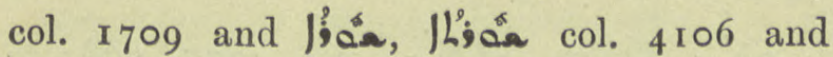
Suppl.

مَ pr.n.m. Mar Scheri, founder of the monastery on the hill of Dara, Chast. 2. 2 and 3 af.

L. a the Saraye, Chron. Min. 355. 20.

a col. 4332. to be dried up. Wow פ $a$ dried and salted fish, Alex.Lied Z.A. vi. 37I. I. Pass. part. aم:

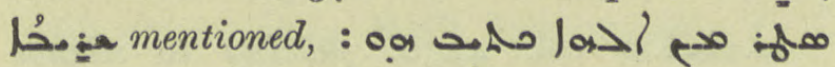

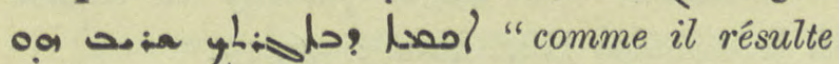
de ta lettre, le mot Dieu est omis", BH. Journ.

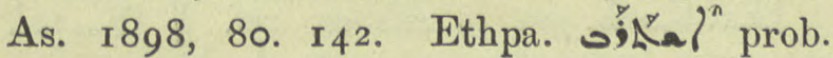

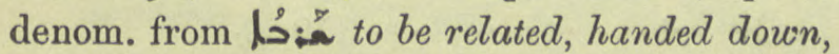
مهم ין Coupes ii. 157. 26 .

ฟ. a col. 4324. Add: name of the goddess worshipped at Arbela,

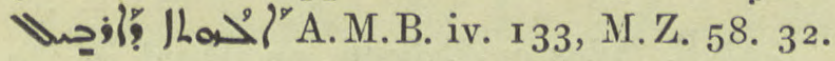

.

: refs. Hex. Jes. l. I I, Nars. ed. Ming. 326

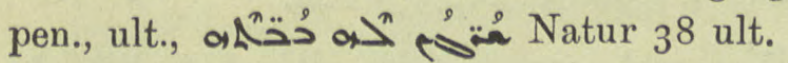

A col. 4326. 4 af. Sherda on the river Gozan, see onس Suppl. A.M.B. ii. 651. 10.

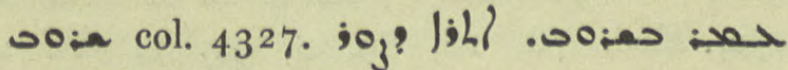
$1 \div y^{90}$ Nest. Chrest. 98. 24 I.

No:a m. dimin. a small plant, Protection

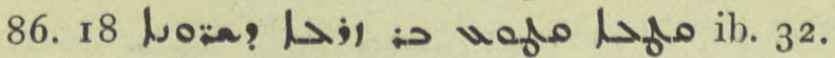

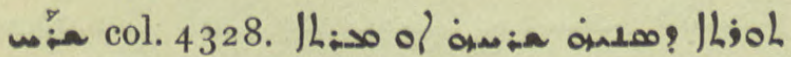
" the cow which hates or is excited towards her mistress", but the editor prefers the variant oitis of ais lacen "which hates its son or daughter", Protection 16. 8 af. Ethpa.

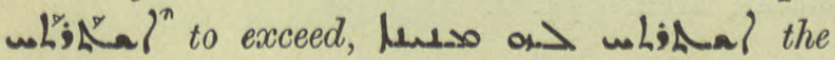
number of priests exceeded that of the laity, Is. Ant. ii. I 5 o. 17. s. ix

fơ col. 4330. m.pl. The Guards, refs. Dion. 135. 17, 20, 167.21; Polizei, Soldaten, Nöld. n.

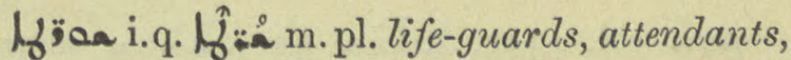
refs. Bar Penk. I 57 ult., I58. 3 , I 85 ; W Jjon over the service of the Guards, El. Nis. Chron. 192. 6, 220 . 14.

هُ: $\lambda$ od at length they come to -, Alex.Lied. 806 .

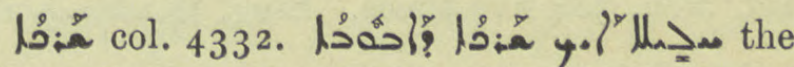
sting of a scorpion is hollow like the lower end of a flute, Natur $4 \mathrm{I}$. I.

1. eyelid, ref. Med. 94. 9 .

u. a col. 4334. to slip. Causative to make

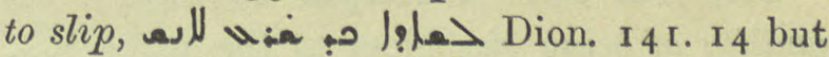

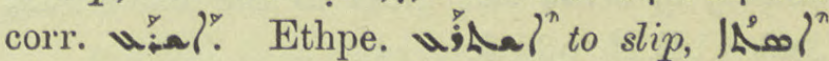

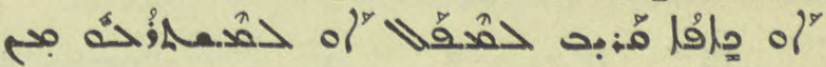

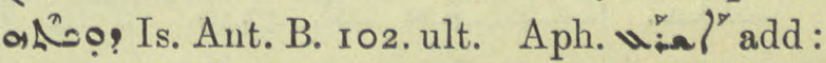

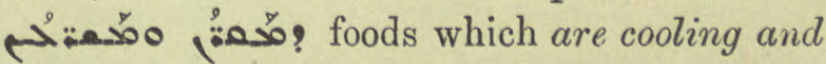
slip down easily, Med. 23 I. $\mathbf{~ . ~}$

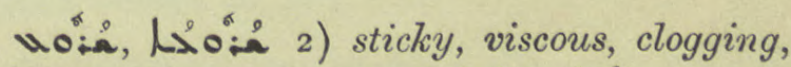

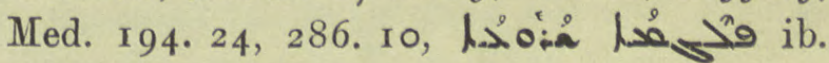
288. 6, 9 .

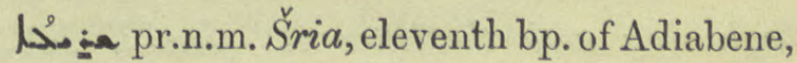
M.Z. 42. 2.

18ّ the plague. Disease in vineyards or crops, li:so facria o a Protection I 9. 8 .

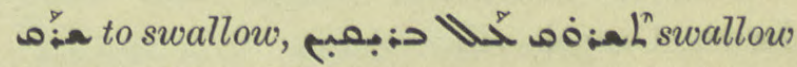
before breakfast, Med. 564. I 2.

1นُمـ col. 4339. 2) skin of a grape,

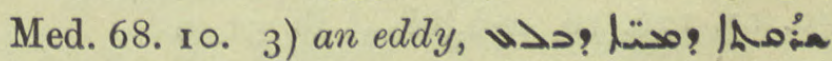
ا مe Loof's Nest. 374. 3 .

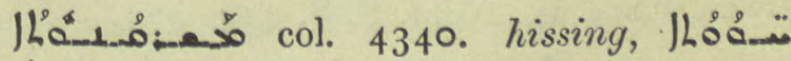

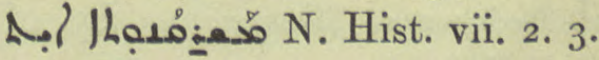

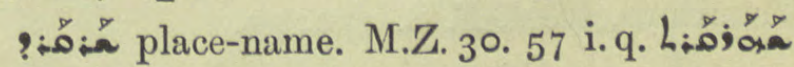
above and col. 4077 .

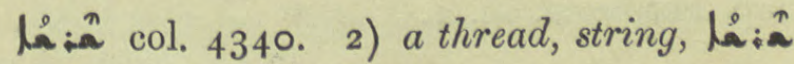
• 5 .

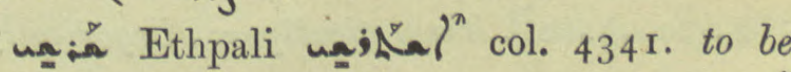

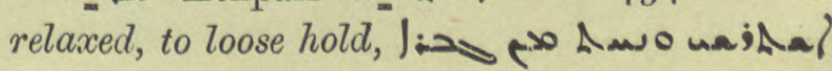
$\mathrm{x} x$ 
the snake uncoiled itself var. I:

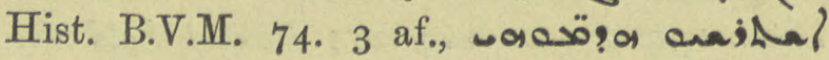
his limbs relaxed, became powerless, ib. I1 3.8.

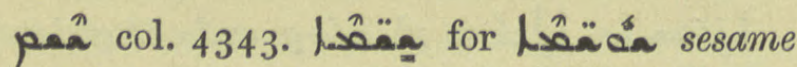
seed, Med. 553. 14, 576. 10, but مب مaمى ib. 39. 19 \&c.

Hesea cón col. 4343. Amomum: cardamom, refs. Med. I74. 4, 237. II, 318. 2, 361. II.

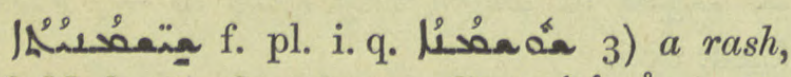

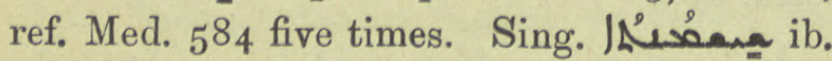

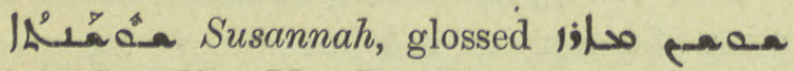
miraculous lily, Mar Bassus I 30.

Fisea i.q. Haca q.v. above and col. 4108. Shuster, Chast. 45. I 7.

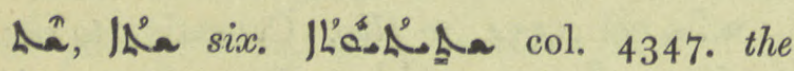
quality of being six, gloss to the name tha Seth, C.B.M. $985 b$.

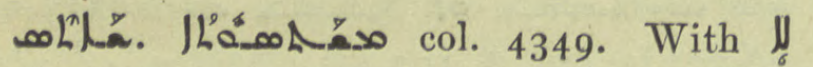

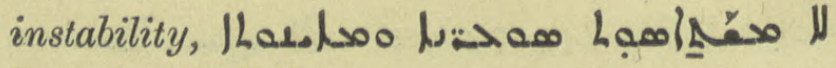
But. Sap. Eth. iv. 5 .

L2a col. 4349. to drink. Metaph. to swallow an affront, Anecd. Syr. ii. 198. Act. part.

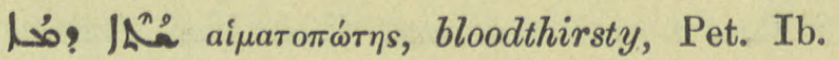
63.7 .

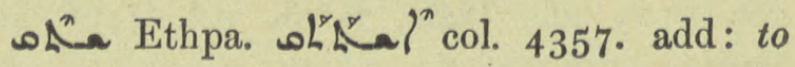
lose the power of speech when drunk, Hippoc. v. 4 , vii. 53 .

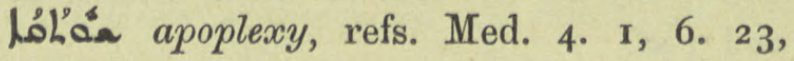
I18. $9,142.15$.

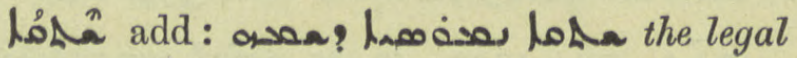
omission of the name of a heretic from the diptychs, Sev. Lett. 22. I 5 .

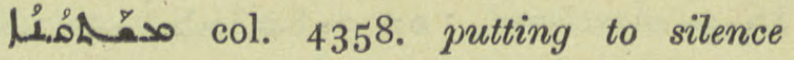

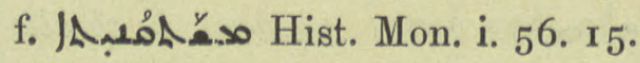

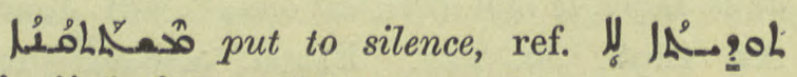

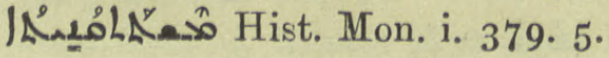

\section{$\therefore$ of $\because$}

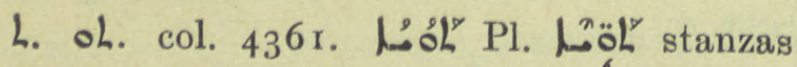

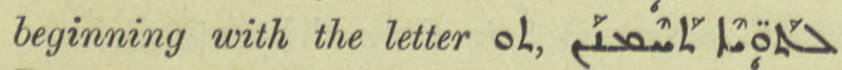
Proem. to Ebed. J. Card. 6. i I.

Yoo $3 L=1 \%$ ? $\left\{L^{n}\right.$ col. 4363 . Theodota mart., Lewis, Gospels viii.

1.96 Theodosia, virgin and martyr, Lewis, Gospels viii.

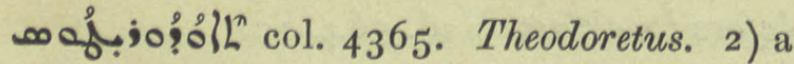
medicine named after its inventor, ref. Med.

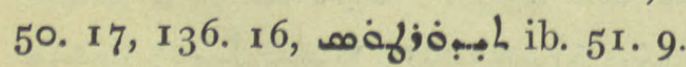

scefuoll Theantinus, bp. of Araxa in Lycia, Conc. ii., Nöld. F. S. 4r4. 474.

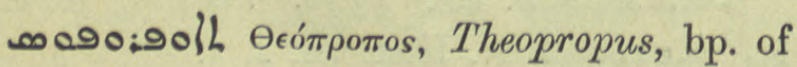
Trimithus, Nöld. F. S. 472.79.

๓ Sap. Periherm. iii. 2.

IAfo;o\} $L^{n}$ error, marg. JLand Warda $247 r$.

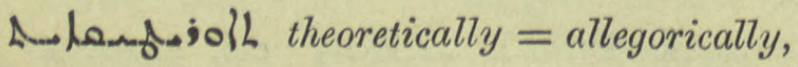
Bar. Sal. in Lit. 94. 3. a) iL Tylus or Talwan, an island in the Persian Gulf, Syn. Or. ${ }^{6} 6_{5}$ ult.

essoforsiL Themistius, bp. of Adrianople, Conc. ii. Nöld. F. S. 473 . 106.

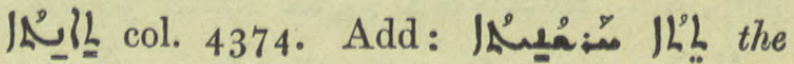
barren $f i g=$ the sycamore, Med. 576. I7, 582. 1 2. 2) a hard pustule on or near the eye, Med. 78. 18, 89. 8, 91. I, $/ L^{9} L^{n}$ idem 1. ult.

IL'gُ 3 L col. 4376. 3) the abdomen; ref. Patr.

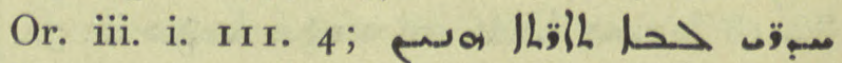

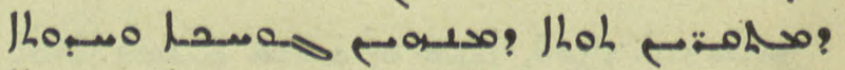
Jhastoo halsoo Hebraica (r888) iv. v. $218 \mathrm{ff}$; $228 \mathrm{f}$. Cf. Jhol below.

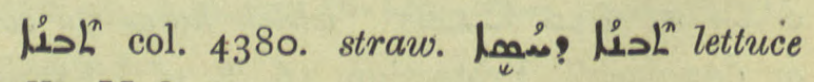
stalks, Med. 574. 2.

uح̌l col. 4380 . With lí.? to bring an action, Sev. Lett. 2 I 3.5 . 


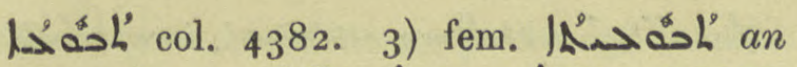

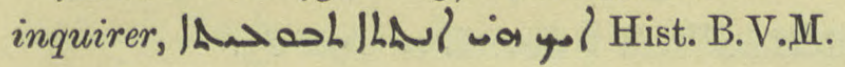
190. 2.

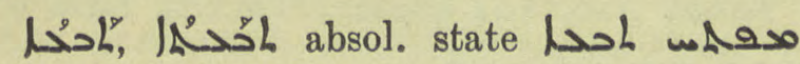
an occasion for argument, a plea, excuse, Ephr. Ref. ii. I42. I I Ming.; تهم vengeance for oneself, Pallad. 483. I3, I5;

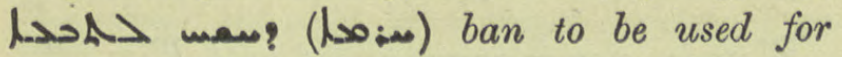
exorcizing, Charms in JAOS. xv. 286 ult.

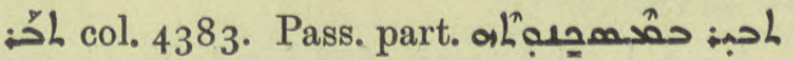
of shattered fortune, Pallad. 85 . 18.

$1 L^{\prime 2}: 2$ 2) hymni fracti, add: Or. Xt. v. 327 , No. 33, R.O.C. iv. 163. Sing. حهُ حُ

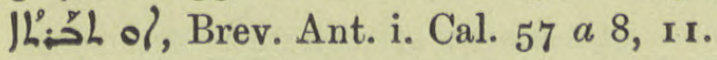

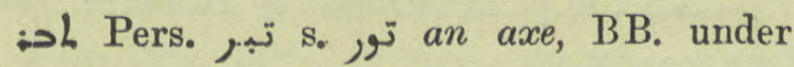
مُّم

J $: 2 L=$ = كَ arrears of taxes, Dion. $2 \propto_{4} \cdot 19$, Nöld. WZKM. x. 166.

صasol- L Tegapnos (Chabot) prob. for nowlsoll Theophanes, bp. of Bostra, Nöld.F.S. 469. 35 .

$p^{n} L^{\nu}$ denom. verb Pael conj. from lse $L^{n}$.

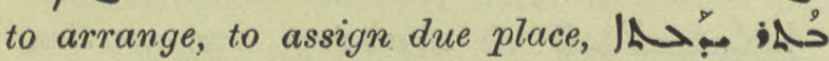

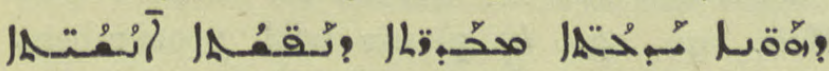
قَمْ after intellectual knowledge various scattered notices of human souls have their due place, But. Sap. Theol. 2. 4 .

$\therefore$ b col. $43^{88}$. denom. verb Paal conj. from

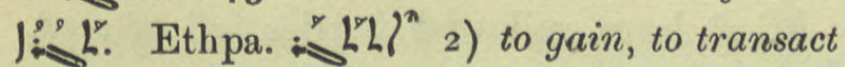

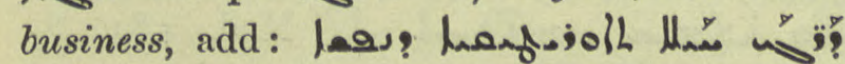
DDN of the degrees of contemplative power of the rational soul, the fourth is called

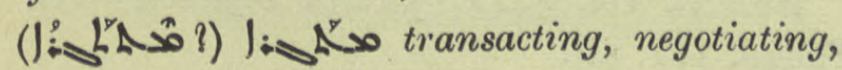

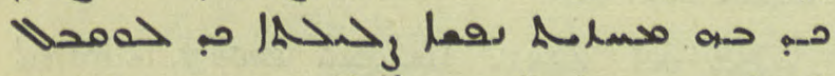

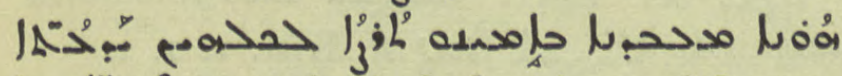

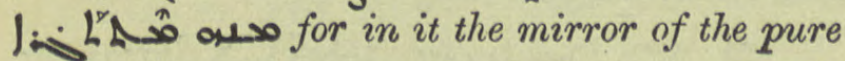
soul when it constantly looks towards the intelligent mind, gains all knowledge from it, N. Hist. viii. 4. 6.

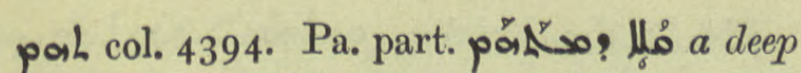
roar, Hist. B.V.M. 193. 7 .

ool particle col. 4400. L. 2 I of par. add: solo sol vulgar name for the nan $>0$ hlo or

2716 prayer for all, recited by the Deacon after Commemoration of special names in the Diptych, Bar Sal. in Lit. $75.22,26,76.26,77$ four times.

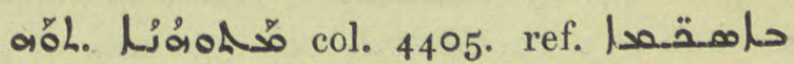
ر رoüh on startling pretexts, Išoyahb I 40.

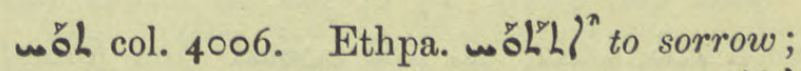

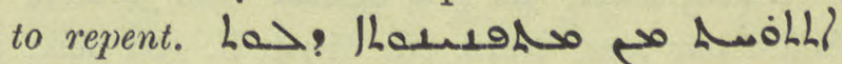

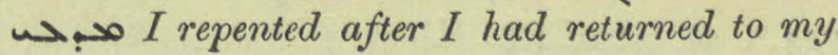
senses, Išoyahb $4^{2}$ pen.

yoh, y $L^{\prime}$ col. 4406. Pa. y.

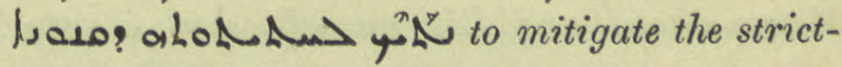
ness of the canon, Sev. Lett. I 50.4 af.

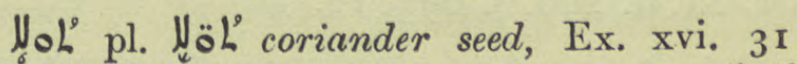
Urm., Med. $565.4,577 .{ }^{1} 5,587$ ult., لِّة $5^{2}$

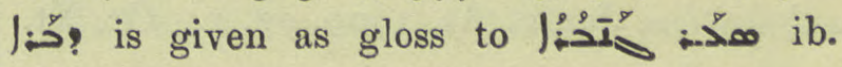
607. 10.

مة'ộ Givvos, thynnus, tunny, Menarat K. $49 a$, N. Hist. vii. 4.2 infr., Ar. FischN. ${ }_{5}^{6} 3$.

ool col. 44II. Turk. a gun. pounaol Charms 8. 5, I1. I5, I2. I0, I5; u löL bullets, ib. pen.

- $;$ ioh var. to olosof place-name, Anecd. Syr. iii. $320.1=$ Zach. Rhet. Brooks 205 ult.

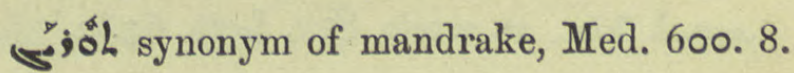

lổọ name of a potion, Med. 220. 10.

ăL col. 4414. Under Pael pass. part.

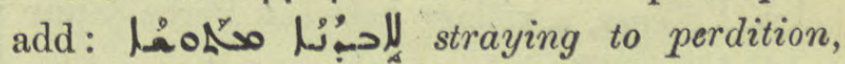
Is. Nin. B. 196.17 .

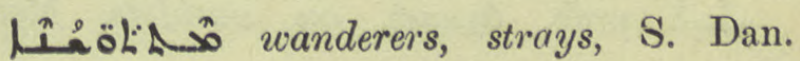
$5^{2} \quad b$ ult.

K'ớL col. 4415 ult. 3) piles, ref. Med. 583 .

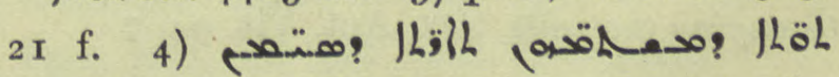
, حسدحا Ligpochondria, the soft part of the body immediately under the

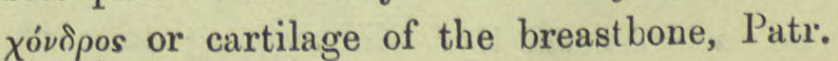
Or. iii. i. I I I. 4, BH. Hebraica, I880, 2 I 4 ,

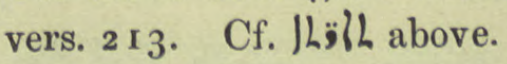

JLoL eaßaAi, Tabatha, a village near Gaza, R.O.C. v. $659 \cdot 3$.

KLĹL col. 44I6. deposit from a furnace, ref. Med. $59^{2} .8$.

$\mathbf{X} \quad \mathbf{2}$ 
a colLoL col. 4416. a kind of Euphorbia, ref. ف ف

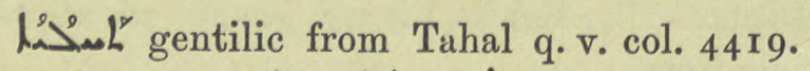

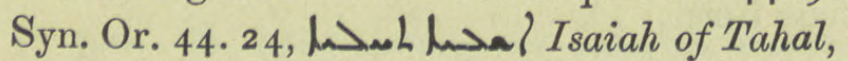
Išoyahb 133. 10, ZDMG. xliii. 396 ult.

phly col. 44I9. I7 of par. to forbid add:

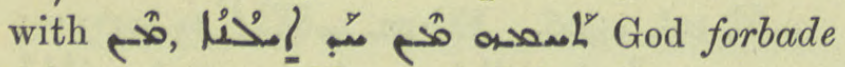
Adam one tree, Nars. ed. Ming. ii. 102 pen.,

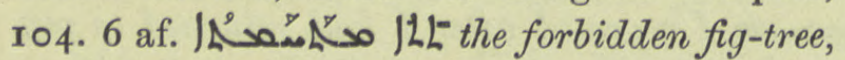
Hist. Mon. i. 135. 3. 2) to determine, appoint, مِسمَ Metropolitans and Bishops who had a prescribed right to vote for a new Catholicos, Or. Xt. ii. 290. 4, 292. I 2.

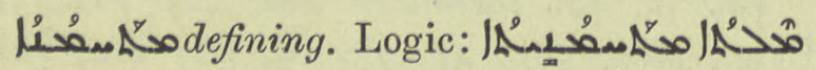
a definition, Journ. As. 1900. 90. 14, Or. Xt. ii. 66.17 .

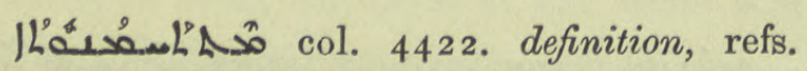

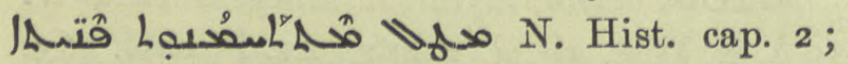
pt. 8 tit., ib. Philos. 6.5 .

15. col. 4422. that which is below, which follows, حص ح I I can repeat the following by heart, BH. Stories 51. 269.

boos Med. 577. 20 for lsoól garlic.

oosh teîs, an uncle, Sev. Lett. 67. 2.

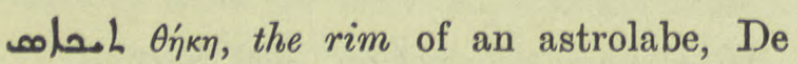
Astrolabe 81.

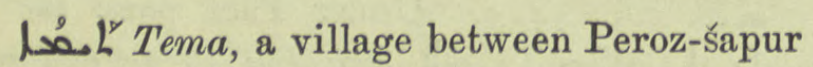
and Seleucia-Ctesiphon, Jab. 409. I.

yㅜㄴ prob. Onpıaḱ, an antidote, Med. 583. 19.

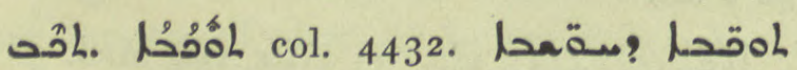
thronging thoughts, Sev. Ant. Vit. 94. 7

J'Lَم

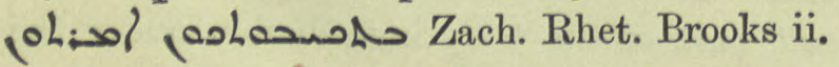
1 9.2 = Anecd. Syr. iii. 275. 23 so corr. poleswass.

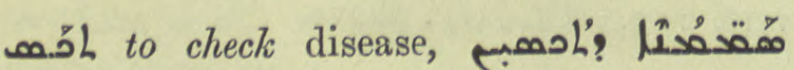

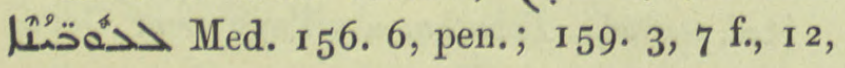
i61. 6.

J'a. 1.

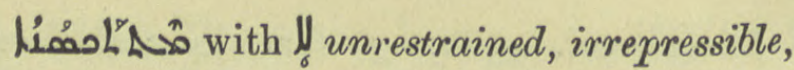

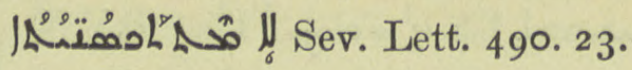

jaف jab. Io4 quater. Turkish form of jocóľ q. v. col. 4433.

للِ they removed the tray, BH. Stories 46. $24 \mathrm{I}$.

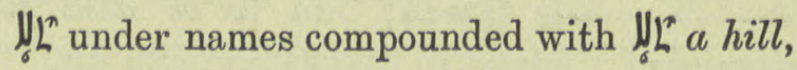
col. 4439. add :

L Thelabanda (Alabanda in Garcia), De Goeje B. 65 . 14.

L Tel-hadin, Sev. Lett. Ix7. 8, I 20. I.

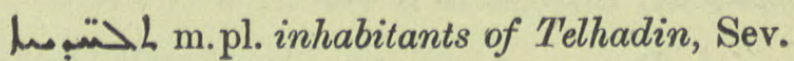
Lett. I 23.8.

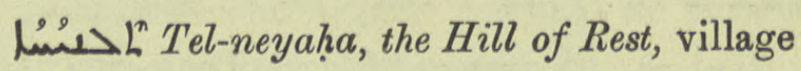
in Adiabene, M.Z. 32. 14, 56. 28.

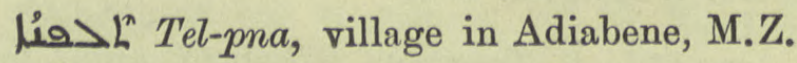
65 ult.

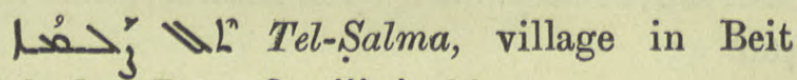
Nuhadra, Patr. Or. iii. i. 66. 7 .

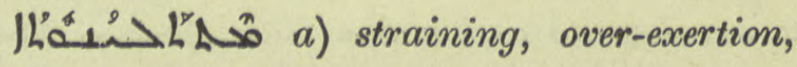
Med. 199. 23. b) plants with procumbent stems, as gourds and cucumbers, have

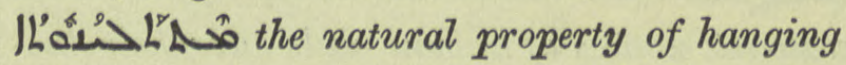
on to or supporting themselves by other bodies, N. Hist. vi. 2. 3 .

ل्रूّ ${ }^{n} \mathrm{pl}$. large yellowish berries full of seed, |⿹勹巳 Cf. ل्रol.

คُ Pallad. r8r. ult.

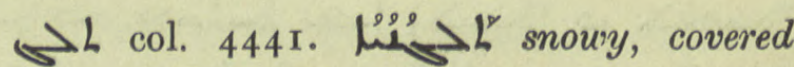
with snow, 每

Lاحس a blood vessel, Med. 202. I 4, 205. I and often,

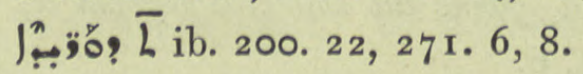

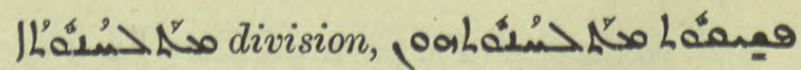
of water, N. Hist. ii. v. sect. 4 .

מo. $>$ h pr.n.m. Talidius, R.O.C. iii. 240 ult. 


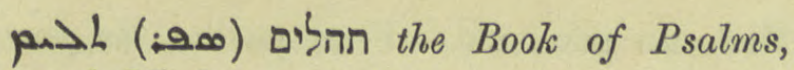
Eus. ed. Maclean 4I6. 8.

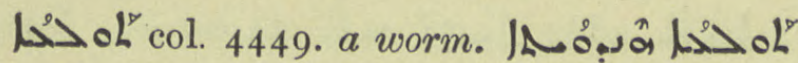
BA. the silkworm, ref. Natur 42. $16 \mathrm{ff}$.

مه threefold, Brev. Chald. IAwar we give Thee triple praise, Test. Dñi. 52. I I, 56. 7 af., 76.4 af.

LُRْ nights, Jo. Tell. 23. 3, quoted under حمحسر.

asol. an oly col. 4456. add: lans of of

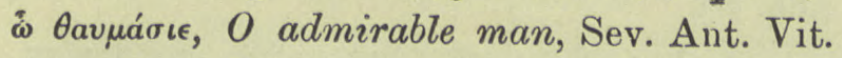
7. 5, Nest. Hérac. 24. I 5, 28 . I 5.

L'o\% from a blow on the head, Hippoc. vii. 4, I 2.

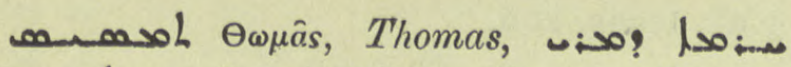
manch anathema of Mar Thomas, Charms 90. 6 af., JAOS. xv. 292 infra, 293. I.

15. Pael 325 col. 4463. Part. pl. smoky,

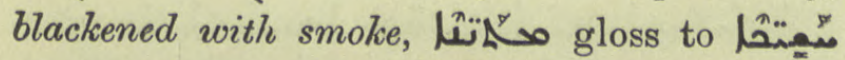

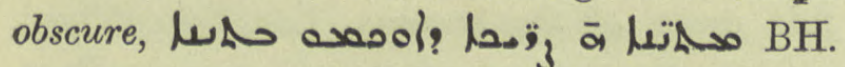
on Prov. xxii. 29, Ceriani LXX

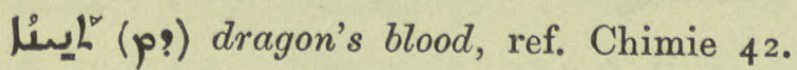

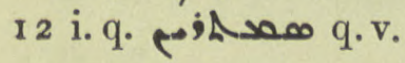

J'áúl smokiness, a feeling associated with heat in the stomach, Med. 29r. 10.

'ئ col. 447I. add: to become numb,

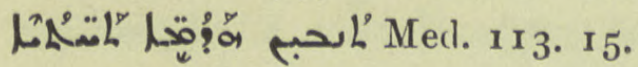

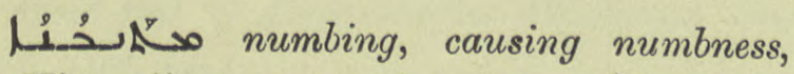
N. Hist. vii. 3. 3 quoted under نازمn.

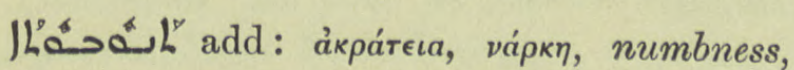
Hippoc. v. I5, 23, defined Med. 3. I5; I I I. I4, II2. 21 II3. I.

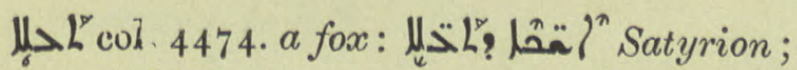
Ragwort, Med. 556. 16, 564. I I, JAOS. xx. x9I. 9.

فํํํ col. 4476. Ethpa. to begin to flow,

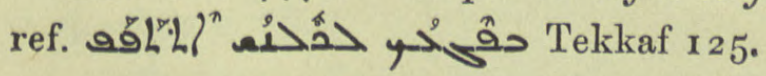

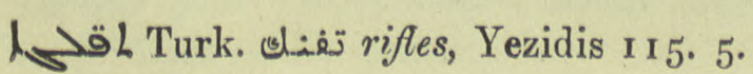

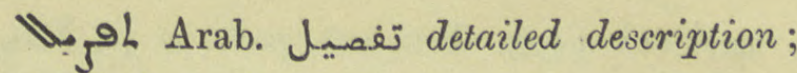
V.

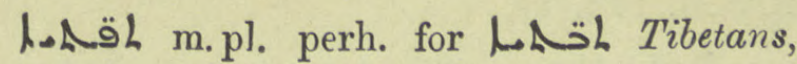
a race descended from Togarmah, Chron. Min. 355. I I.

Jooh marg. uooh Tekoa q.v. col. 4479. Pléroph. 58. 3. Mich. Syr. 208. $a$ 2, writes حash.

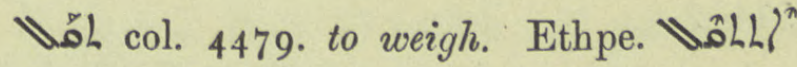
to be weighed down, oppressed $\mathrm{JL}$ erhe

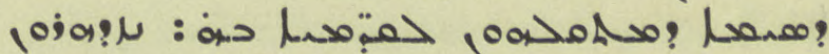

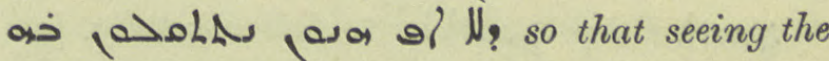
record of the burden on those of old they may beware of like oppression, Dion. I39. I, 2.

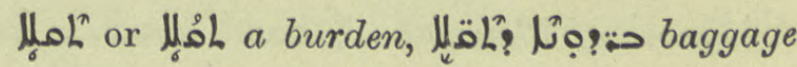
mules, Hist. Mon. i. 247. 3.

بم่ ing to the Book of Medicine the Mithqal of the Sanctuary equals twenty zuze or one dinar and the Mithqal used for weighing money or gems is one dirhem ơم

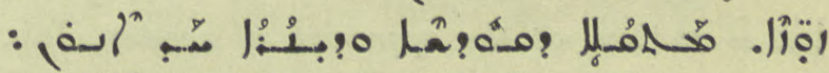

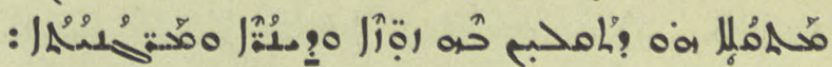
păg? Med. 447. 1-3.

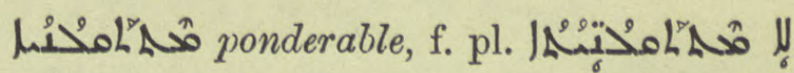
imponderable, Sev. Lett. $8_{5}$. 10.

مُ verbal adj.

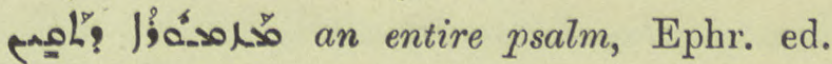
Lamy iv. 2 II. 20.

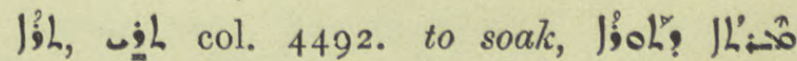

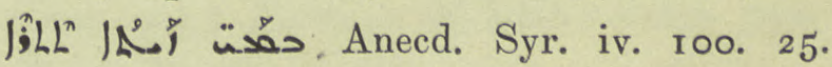

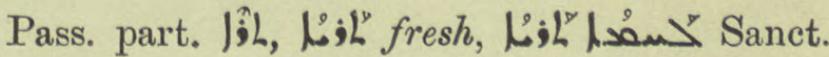

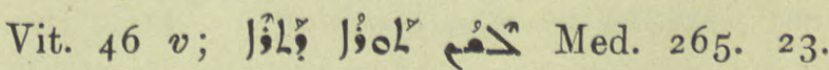

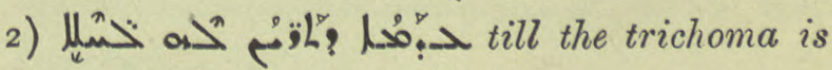
softened, Med, 92. I3.

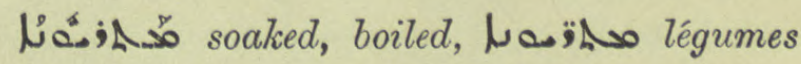
cuits à l'eau, S. Dan. in R.O.C. v. 393. 14, 398. 19.

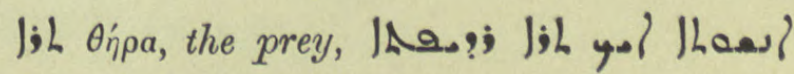
Ephr, ed. Lamy iv. 539. I3. 
هil col. 4495. Peal act. part. m. pl. لl

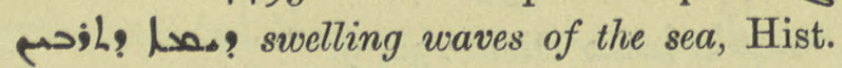
B.V.M. 125 . 12.

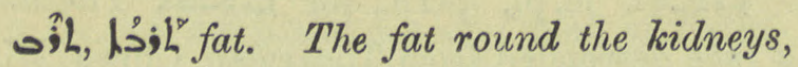

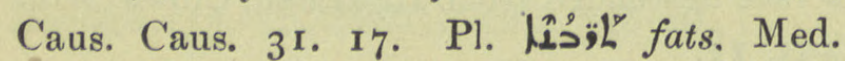

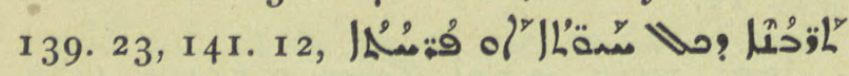
ib. I 53 . II.

|r. col. 4499. thick pottage, ref. Med. 574. 3 .

1soil Thermae, bpric. in Cilicia, De Goeje B. 64. 6 .

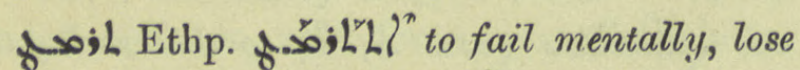

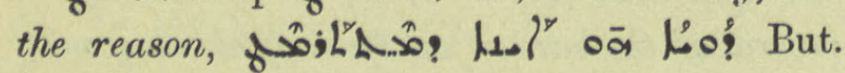
Sap. Eth. 1. 4.

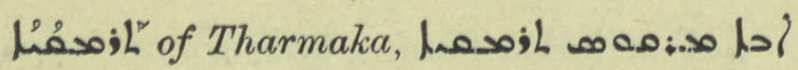
Charms 50. 18.

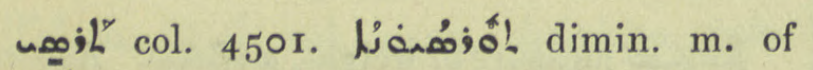
lịiớl victuals. A scanty meal, Hist. Mon. i. 126.19 .

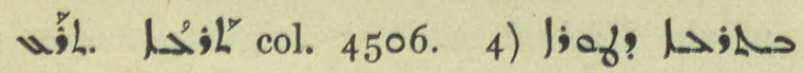
at the approach to the mountain, Chast. 26. I ;

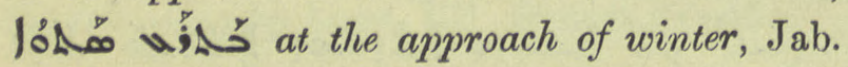

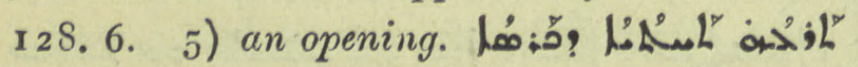

Med. 20 pen., ult. Vena portae, the portal vein, ib. 329. $5,33^{2} .16,33^{6} .23$.

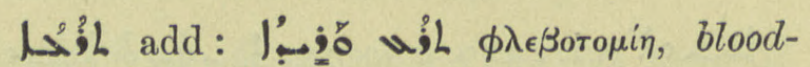
letting, Hippoc. vi. 45 .

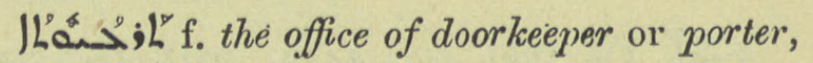
ILQُ

3h. $1 \mathrm{~L}^{\circ} \mathrm{g}$. $5 \mathrm{~L}$ col. $45^{\mathrm{II}}$. erectness of the

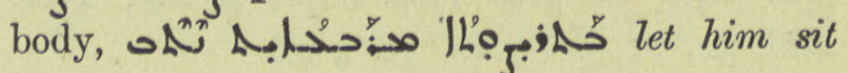
straight and foursquare, BH. Eth. 122. II.

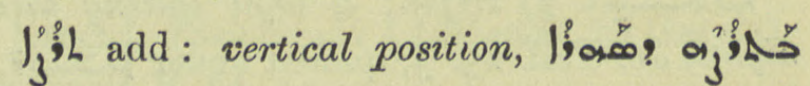
when the moon is straight overhead, Med. 588. 23 .

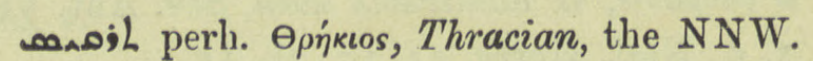
wind, Med. 533. 5 .

$\leftrightarrow L^{n}$ a village in the Holy Land but Gottheil transliterates TDS̈N Bahira 203 . 5 af., trans. 206.

VäL denom. verb Paal conj. from vǎh,

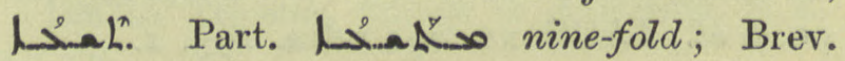
Chald. ish.

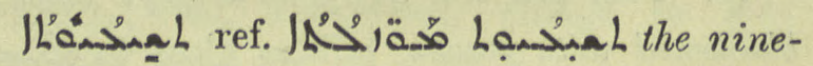
fold order of the heavens, But. Sap. Theol. 4. 7 ; the nine orders of angels, Brev. Chald. sh. 


\section{ADDENDA ET CORRIGENDA}

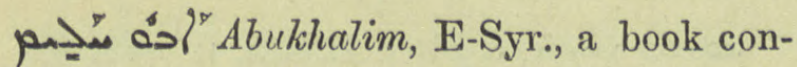
taining proper collects for festivals and some occasional offices, as that of the Prothesis before the Eucharist. The book is ascribed to Mar Elia, the third, Daily Offices, Glossary; Introd. xxviii; QdhamW. I62. I5.

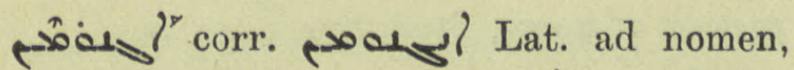

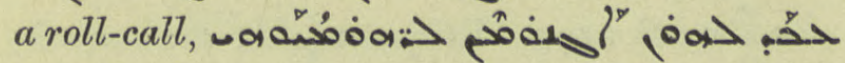
A.M.B.v. 536 ult., 537 . 7 , Fraenk. ZA.xvii. 85 .

P. $8 b$, oljandool oyster, Sev. Ant. Vit. 2I2. 2 .

P. I I a fujol Stud. Sin. ix. 16.

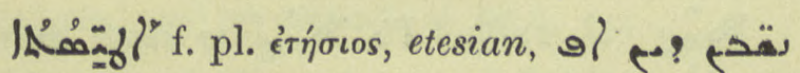
| Rylands MS. 44. 3 bis.

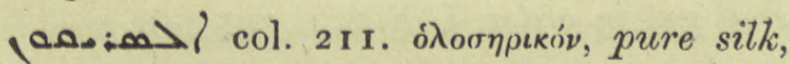
add ref. L.E. S. iii. 192. 10, (10, (1) 194. ro.

P. $21 \quad b \quad$ I3 af. /Ansog;as.

P. $27 b 7$ af. Complete ref. A.M. B. i. 476. 16.

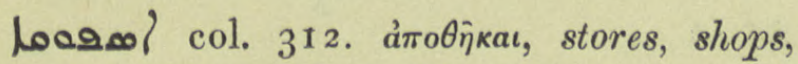
Descript. of Rome, Zach. Rhet. Brooks I 97 . I 6.

P. $3^{2} b_{7}$ af. $/ L_{0}$ j $^{*}$ " usually dough; a kneading-trough. A crate, IA a crate full of glass vessels, BHStories ror. $485,106.14$.

, the guards, Zach. Rhet. Brooks ii. 197. 9.

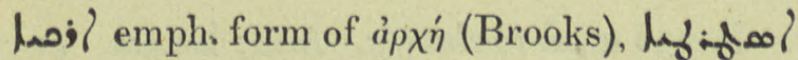
IDS hoil! charge of the great governorship i. e. a satrapy, L. E.S. i. $285 \cdot 4$.

P. $37 b$ г 3,14 . S. Fraenkel, ZDMG. liii., and so 1.5 af. should be S. not G.

P. 42 a 7 salis or Barbaiissus, i. q. د. p. 57 .

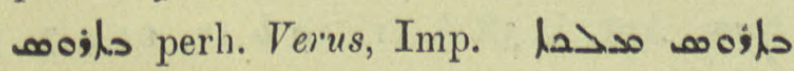
Theod. Mops. on Jo. 328 . 13.

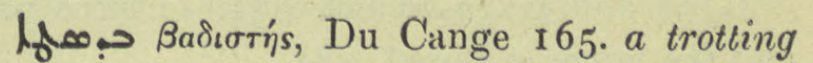
horse, S. Pelıg. I. $25=$ A.M.B. vi. 6. I 8 .

P. $47 b_{4}$. J 4 4) astron. add ref. Georg. Arab. 18. 5.

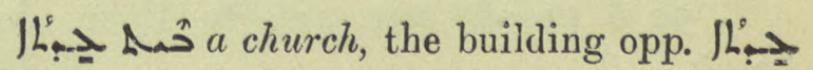
the congregation, Sev. Lett. $285.8,22$; pl.

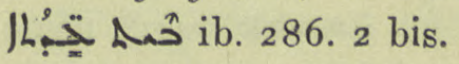

gase_sh pr.n. Buzurgmihr, Vizir of Khosroes Nushirwan, BH. Stories I 9. I, 20 bis, $2 \mathrm{I}$ ter.

רَّ Bardesanes, alphabet of, C.M.B.

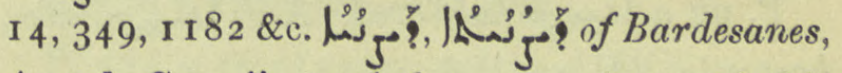
Anecd. Syr. ii. 13 infra, a specimen of this writing is given ib. Plate vii.

هـ. under lis l' 7 . 17 ; under loُ

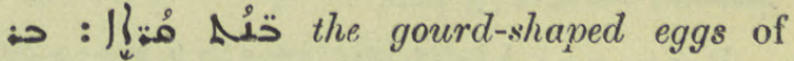
Trichocephalus, a tape-worm, Med. 424. 17 .

P. $59 b$ af. lhn for lla 6 o

In the beginning, the long farcing of l'salm $\mathrm{c}$ on great festivals. These farcings deal with the Creation and all begin with

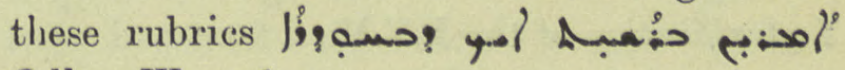
QdhamW. I 56. 5.

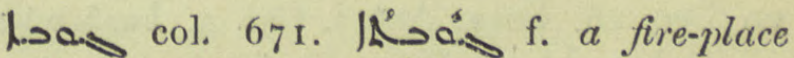
l إ. Eaph: Ref. ii. 35 ult.

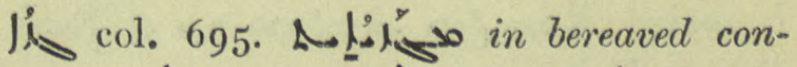

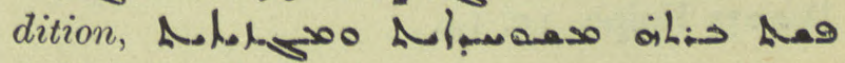
L.E.S. i. $185 \cdot 7$.

ym col. 703. to laugh; to sport, play,

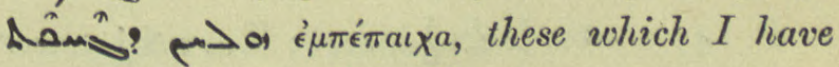
wrought in sport, Hex. Ex. x. 2.

P. 74 a med. $\mu_{6} \rightarrow$ a tortoise. Pl. A. M. B. vii, 25 .

P. 77 a, par. 9. add ref. L.E.S. i. 99. Io. 


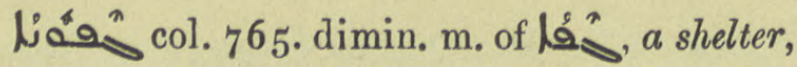
hut of branches, Hist. Mon. i. 98 .

P. 84 a I 4 لمحسو.

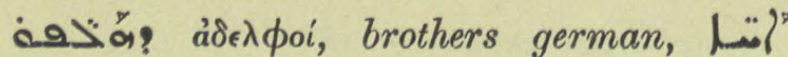

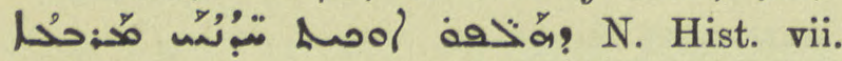
1. 3 infra.

Jifo! and Jifo: 0 col. 834. Delete par. and see under $\rho$ Suppl. 185 .

d ?li? Jab. 253. I, 264. ro and n. See

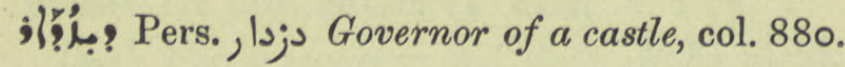

P. $92 b$ II af. corr. ref. to Ephr. Jos. 83 ult.

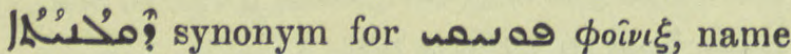
of a medicine, DBB. I576. 3 .

P. $94 b$ I6 af. $=$ col. 948 ult. misprint for 1) Delete; it is a mistake of Quatremère's for L $\mathbf{\Delta}$; ? which feeds.

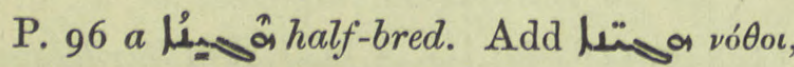
bastards, BH. on Heb. xii. 8.

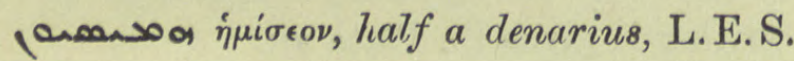
i. 212.4 .

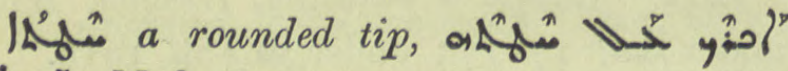

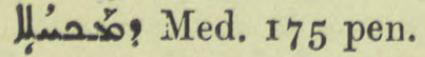

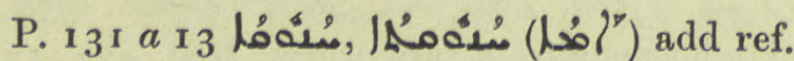
Protection 59. 2, 62, 78, 83 .

P. ${ }^{3} 35^{b}$ r 3 af. misprint, should be حيم

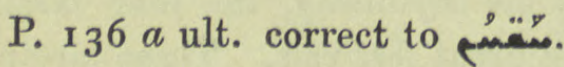

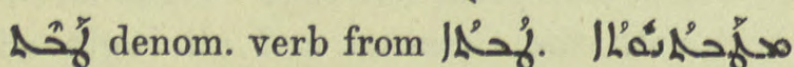
f. glorification of the B.V.M., a feast and word introduced by the Uniat Chaldaean Church, on Dec. 26, Brev. Ant. i. Cal. 5, Los Lafo" مخبL / Brev. Chald. i. مa tit., ff.

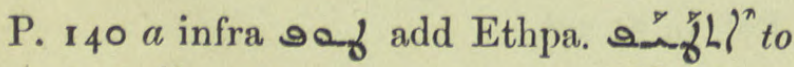

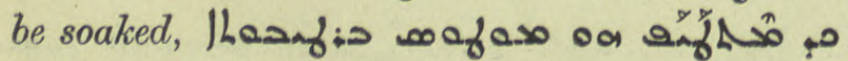
JAoo!? Epiph. x5. 14.

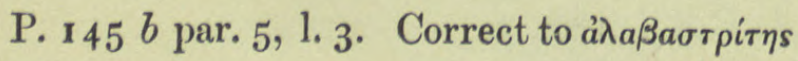
ib. 6,9 .

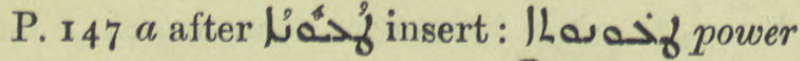
of bearing burdens, لlsos. $\bar{\jmath}$ Caus. Caus. 67. 22.

P. ${ }_{55} b$ par. 5 . Delete. It is a mistake for 1020 sُ red.
|⿳⺈:ّ add ref. Sev. Ant. Vit. 24. 3,

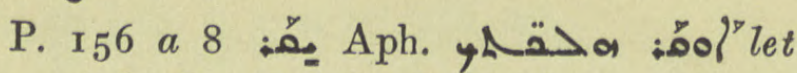
thy gait be dignified, Stud. Syr. i. 9. 4 .

P. $162 b$ par. 8. should be ) i\}r.

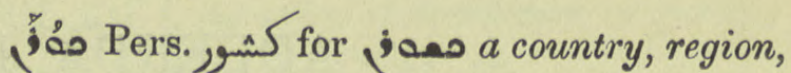
Nöld. on A.M.B. ii. 577. I.

ojo $\chi \hat{\omega}$ ôs, a place, L.E.S. ii. 328.8.

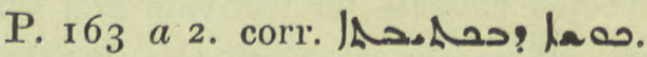

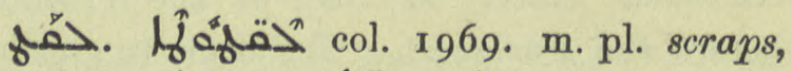
 in the desert used odd bits of sticks or stalks instead of a pen, Stud. Syr. i. 42. 9.

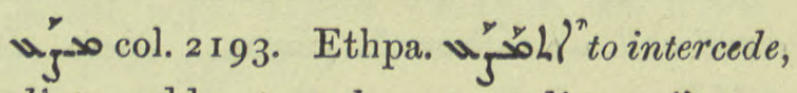
mediate, add: to take as mediator,

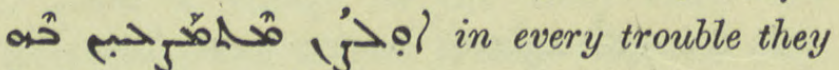
had recourse to him as a mediator, Hist. Mon. i. 98.9 .

M col. 2283 . Pa. W to clear away, arollo sidey swept and tidied and put in order, L.E.S. i. 237 pen.

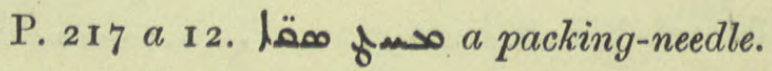

P. 2 I 7 × 6 . بمّ ref. to A.M.B. Supply v. $25 \cdot 7$.

كُقــبــفــا

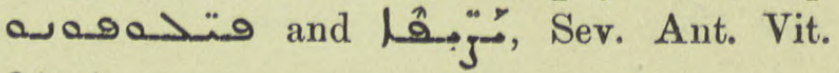
24. 4 .

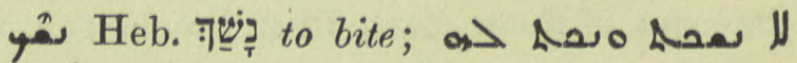
مخدم هم: let not a mad dog bite nor seize him, Protection I 8. 9.

P. $225 a$ delete last par. and $229 b$ add to par. 9 lasan? ha the 12 signs of the Zodiac, Rylands Bulletin, Vol. 4, 1917, I I6. I, I I, I 5 ff.

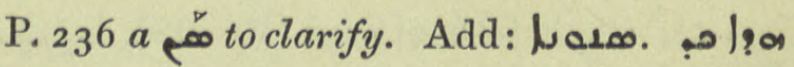
when black is laid on cunningly it enhances other colours, R.O.C. vii. II 4. 7. Nau suggests D. S. Margoliouth lesasan but is the word not related to oure?

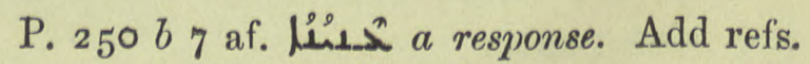
Brev. Ant. i. $58 b \times 1,59 b \times 7,6 \times a 7, \mathrm{pl}$. 1. $8, b 5$. 
P. $257 \propto$ 4. Delete Suppl.

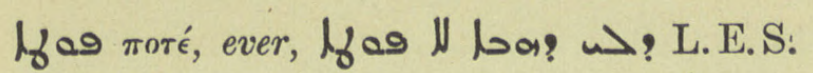
ii. 636.5 , o: ib. 637.9 .

هذمبر ? 2 L. E.S. ii. 539. 2.

-

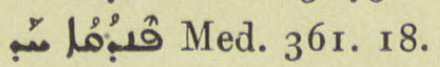

P. $270 b$ مُ و add : a mountain pass, gap,

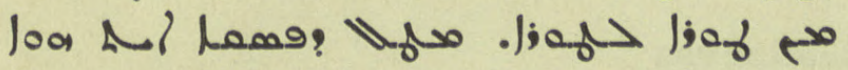
IA SOS.E. I. I32. II.

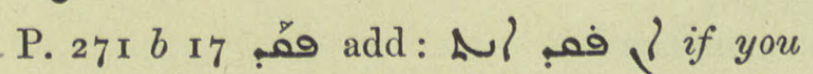
please, L.E.S. i. 79. 10, 80. 5, 1090 ib. 103. I I, u>000 188. 11, 900 (b) Father, be so good, =18. 6.

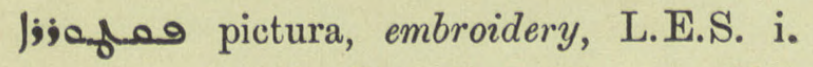
$23^{8}$ ult. $=$ Anecd. Syr. ii. 268. 13. (MS. (حملر) and so correct. p. $60 b$.

P. 273 a 6 af.

P. $276 b$ I 3 af. A.M.B. v.

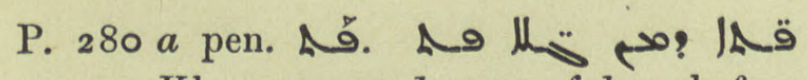
Khosroes set lumps of hay before Nu'man instead of bread, Chron. Min. 20. 3/4.

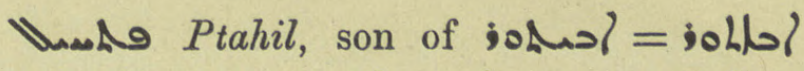
Coupes ii. I54. 8 and 3 af., commanded to create heaven, earth, and the human race. ib. 155.2.

P. 282 a 22 corr. مبپمِ

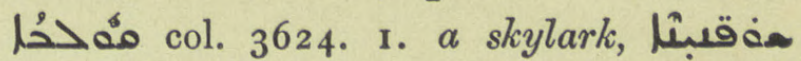
مac. Sar. Hom. iii. 92. 5 .

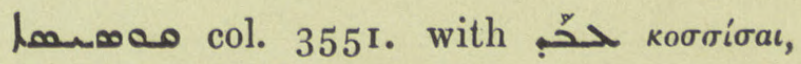
to box the ears, slap, L.E.S. iii. I 7 O. 10.

P. 299 a ult. jacsoando L.E.S. ii. 538. 8.

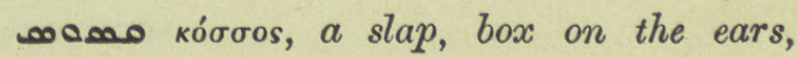

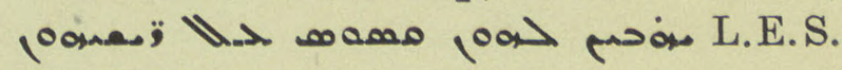
iii. 167.5 .

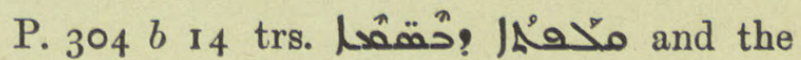
gloss. to 1. I5 before Med. 608. 20.

P. 320 b 18 corr. ثحص?

P. $323 \alpha$ I 6 af. corr. ref. to A.M.B. v.

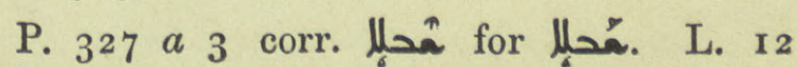

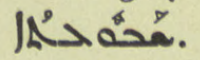

P. 328 a 1 2. Ethpe. - inal" add : with o to rage at, be enraged with, Dion. 236.2.

P. $332 a 2$ af. corr. 
PRINTED IN ENGLAND AT THE UNIVERSITY PRESS, OXFORD BY JOHN JOHNSON PRINTER TO THE UNIVERSITY 